\title{
A PHONOLOGICAL RECONSTRUCTION OF PROTO-HLAI
}

by

Peter K. Norquest

\author{
A Dissertation Submitted to the Faculty of the \\ DEPARTMENT OF ANTHROPOLOGY \\ In Partial Fulfillment of the Requirements \\ For the Degree of \\ DOCTOR OF PHILOSOPHY WITH A MAJOR IN ANTHROPOLOGY AND \\ LINGUISTICS \\ In the Graduate College \\ THE UNIVERSITY OF ARIZONA
}




\section{THE UNIVERSITY OF ARIZONA \\ GRADUATE COLLEGE}

As members of the Dissertation Committee, we certify that we have read the dissertation

prepared by Peter K. Norquest

entitled A Phonological Reconstruction of Proto-Hlai

and recommend that it be accepted as fulfilling the dissertation requirement for the

Degree of Doctor of Philosophy

Date: November 5, 2007

Jane Hill

Date: November 5, 2007

Diana Archangeli

Date: November 5, 2007

Graham Thurgood

Date: November 5, 2007

Michael Hammond

Date: November 5, 2007

Norma Mendoza-Denton

Final approval and acceptance of this dissertation is contingent upon the candidate's submission of the final copies of the dissertation to the Graduate College.

I hereby certify that I have read this dissertation prepared under my direction and recommend that it be accepted as fulfilling the dissertation requirement.

Date: November 5, 2007

Dissertation Director: Diana Archangeli

Date: November 5, 2007

Dissertation Director: Jane Hill 


\section{STATEMENT BY AUTHOR}

This dissertation has been submitted in partial fulfillment of requirements for an advanced degree at the University of Arizona and is deposited in the University Library to be made available to borrowers under rules of the Library.

Brief quotations from this dissertation are allowable without special permission, provided that accurate acknowledgment of source is made. Request for permission for extended quotation from or reproduction of this manuscript in whole or in part may be granted by the dead of the major departments or the Dean of the Graduate College when in his or her judgment the proposed use of the material is in the interest of scholarship. In all other instances, however, permission must be obtained from the author.

SIGNED: Peter K. Norquest 


\section{ACKNOWLEDGMENTS}

I would first like to acknowledge my committee members, who have provided me with support, encouragement, and a myriad of helpful comments and suggestions during the writing of this dissertation. In particular, I would like to thank my co-directors and advisors, Diana Archangeli and Jane Hill, for the amount of energy and time which they have offered me during the unique academic initiation of the dissertation process. I would also like to acknowledge Pittawawat 'Joe' Pittayaporn for his feedback on and careful critique of various parts of this dissertation, and for sharing with me his database of ProtoSouthwest Tai.

I would also like to acknowledge all of the Hlai language consultants on Hainan (listed in chapter one) who worked with me during my fieldwork in 2003-04, and in particular Lin Baosong (林保松), a native speaker of Ha Em who generously arranged (in cooperation with Wang Xueping (王學萍)) for my work with many of the other consultants with no request for compensation. I would also like to thank Wu Zhongyong (吳鐘勇), my primary Nadouhua consultant, for his patience during the long hours spent during our initial survey of his language. Thanks to Liu Jiansan (劉劍三) for his constructive dialogue and the gift of his self-authored Lingao dictionary. Finally, I want to acknowledge Wang Yuqiu (王裕秋), our liaison at Hainan Normal University, who regularly advocated for my family and without whom my fieldwork would not have been possible.

My fieldwork would also not have been possible with the support of NSF award no. 0236674 and a Fulbright fellowship to China, 2003-04.

Finally, I would like to acknowledge my family. My wife, Grace, and daughter, Vivian, have been extremely patient and supportive of me through my graduate career, often sacrificing their time with me so that I could work on my dissertation. My parents, Erik and Karen, have also been very supportive and encouraging, never expressing doubts about my chosen career path. And I can't forget our 'extended family', Chloe, Prince, Lila, Ares, Mo, and my wonderful garden for keeping me company on what would have otherwise been many lonely days of writing. 
This dissertation is dedicated to my wife, Grace, for her enduring support and companionship during our fieldwork in Hainan and the following years of writing this dissertation, and to my parents, Erik and Karen, for encouraging me to follow my own path of learning and discovery. 


\section{TABLE OF CONTENTS}

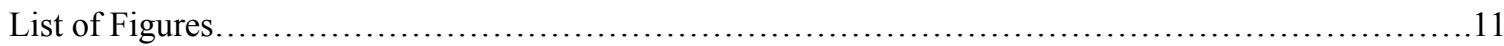

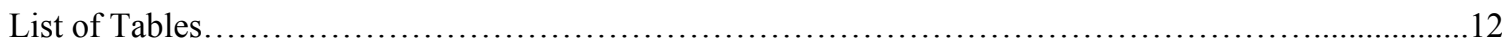

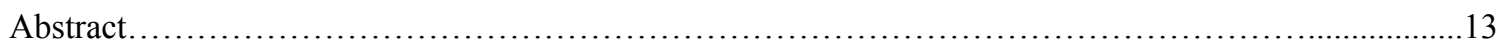

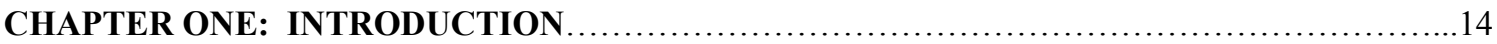

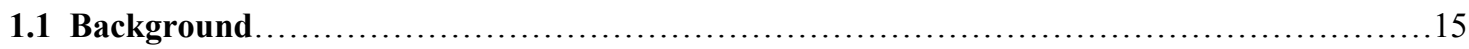

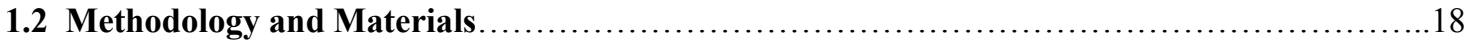

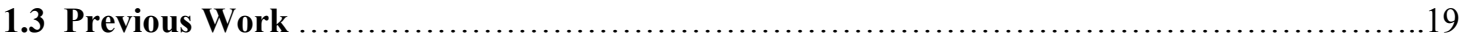

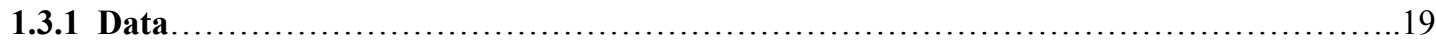

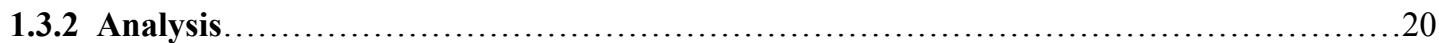

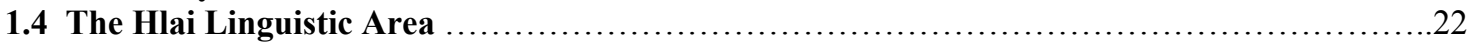

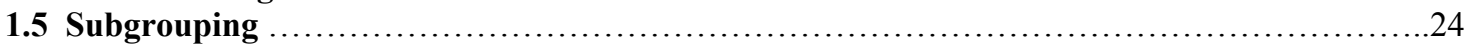

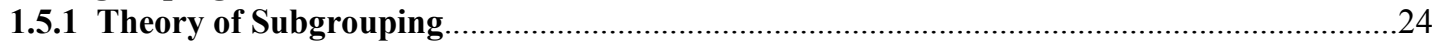

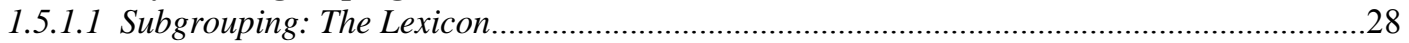

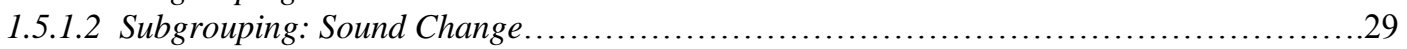

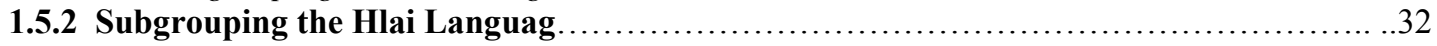

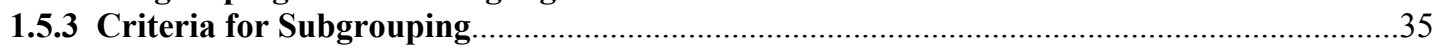

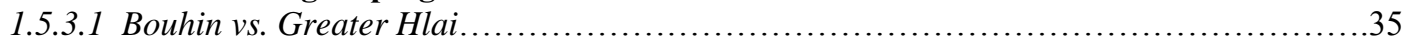

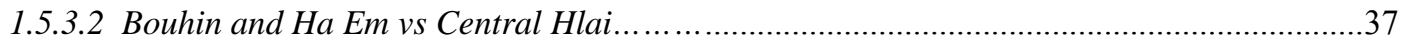

1.5.3.3 East Central Hlai vs North Central Hlai................................................. 38

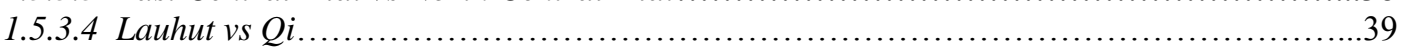

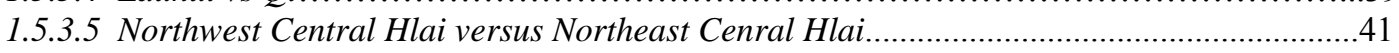

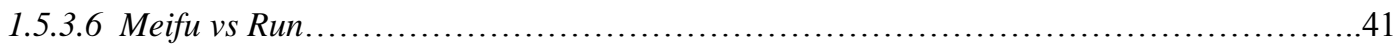

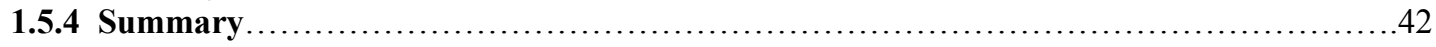

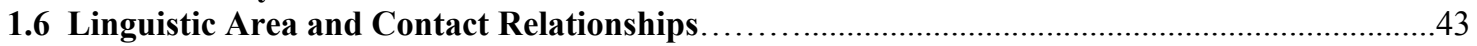

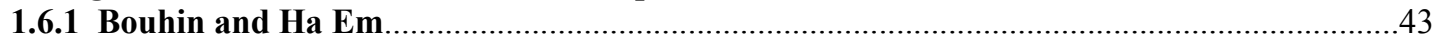

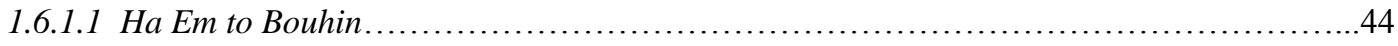

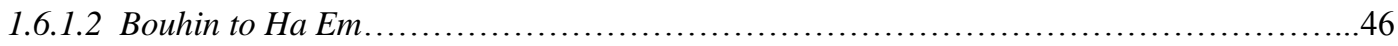

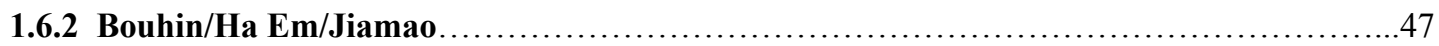

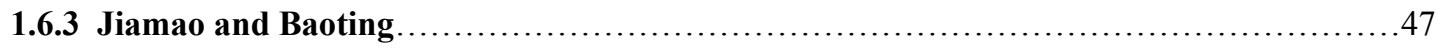

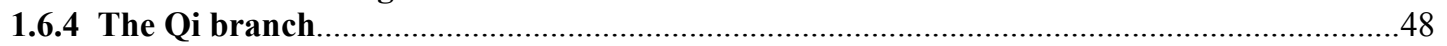

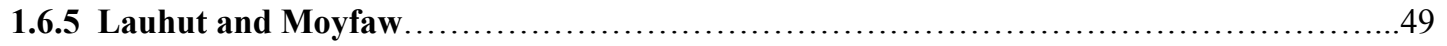

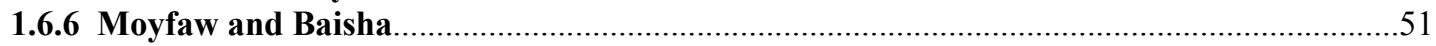

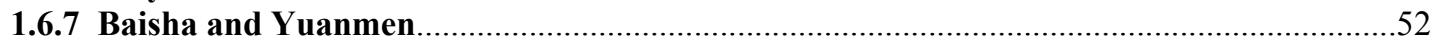

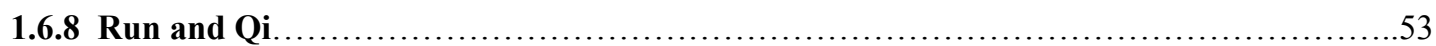

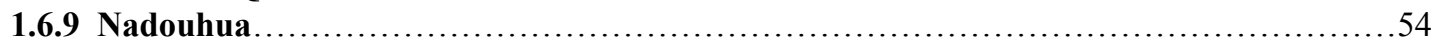

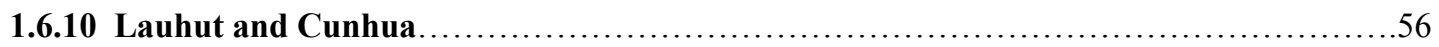

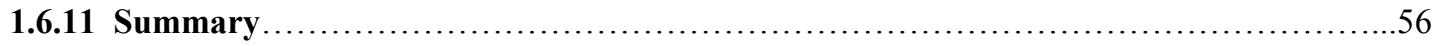

1.7 Theory of Language Change and Reconstruction..................................................

1.7.1 Principles of Language Change and Criteria for Reconstruction ........................59

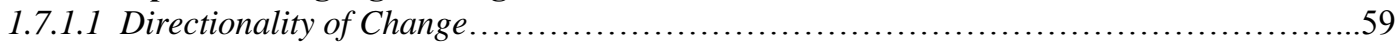

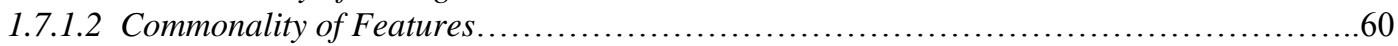

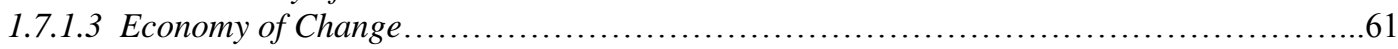

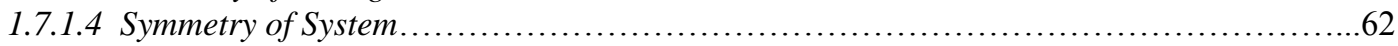

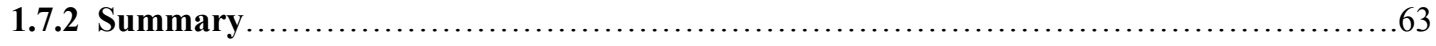

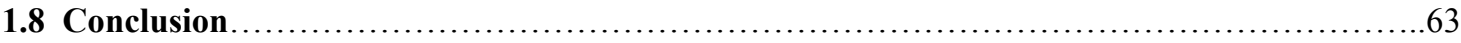

CHAPTER TWO: RECONSTRUCTION OF PROTO-HLAI INITIALS .........................65

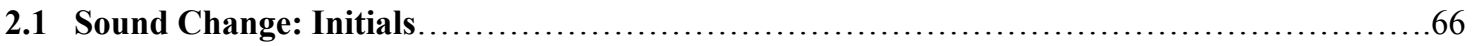


TABLE OF CONTENTS -- Continued

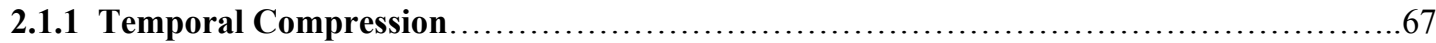

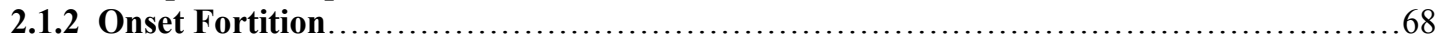

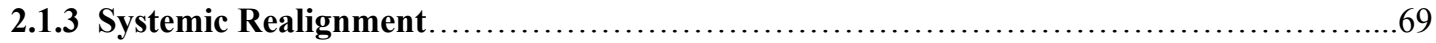

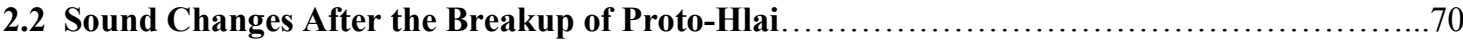

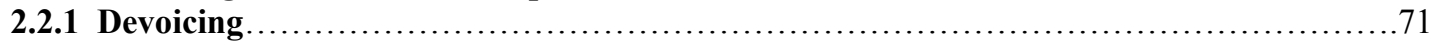

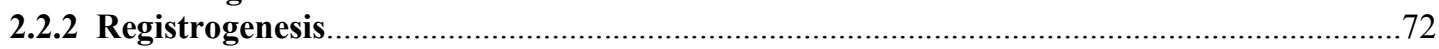

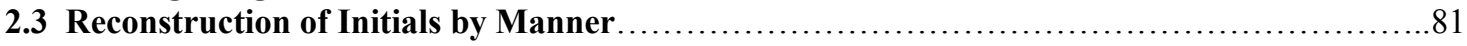

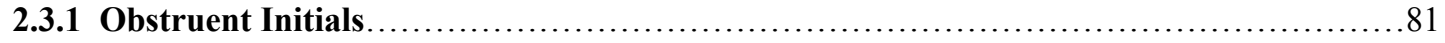

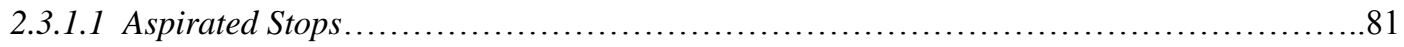

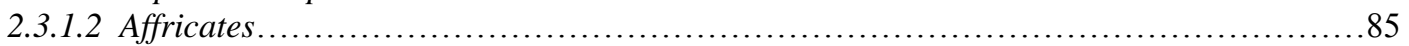

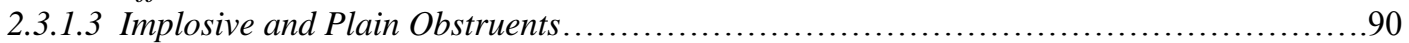

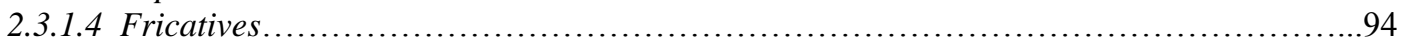

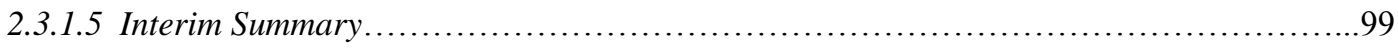

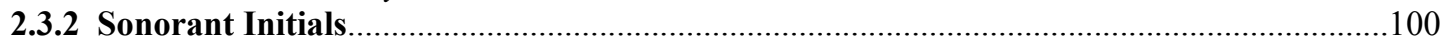

2.3.2.1 Preaspirated Nasals................................................................ 100

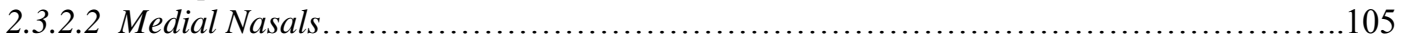

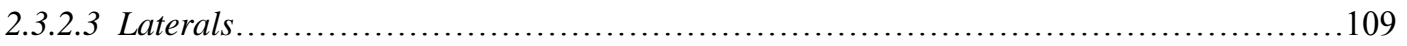

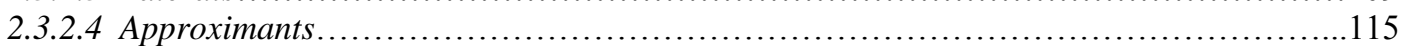

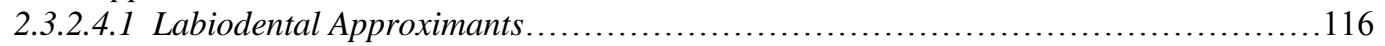

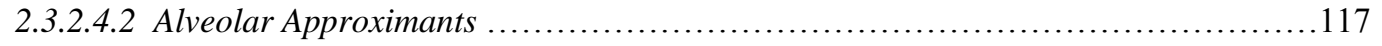

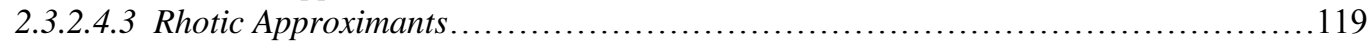

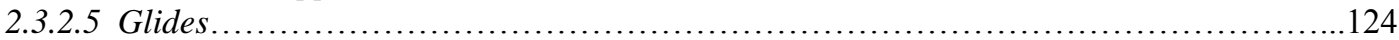

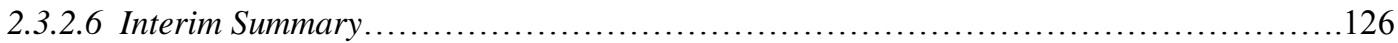

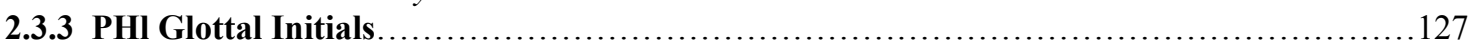

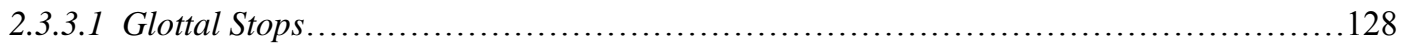

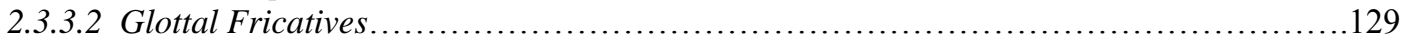

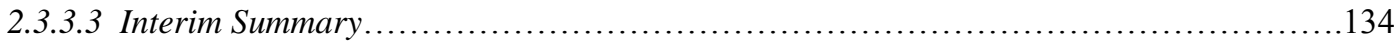

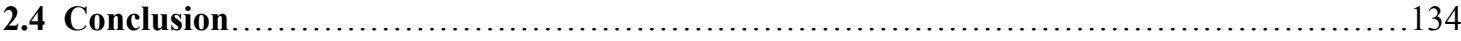

CHAPTER THREE: RECONSTRUCTION OF PROTO-HLAI RIMES ..........................144

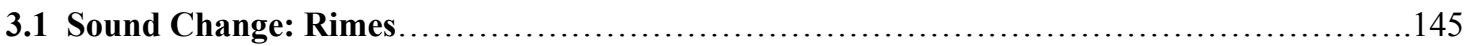

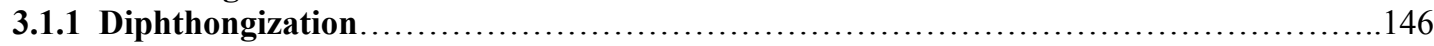

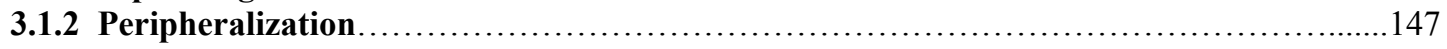

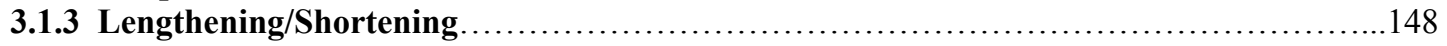

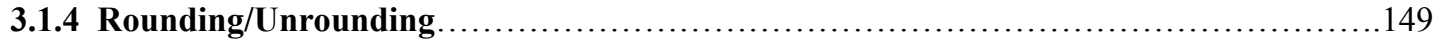

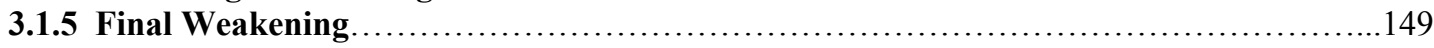

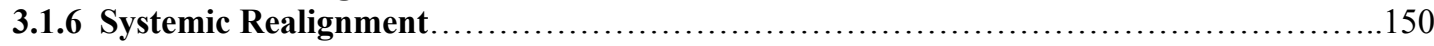

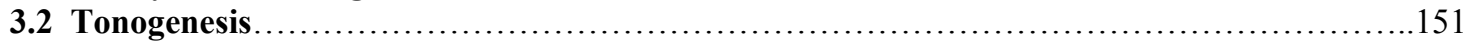

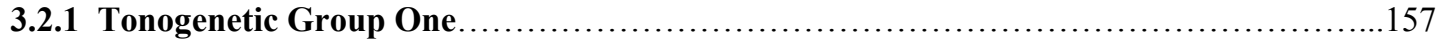

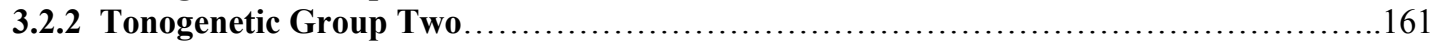

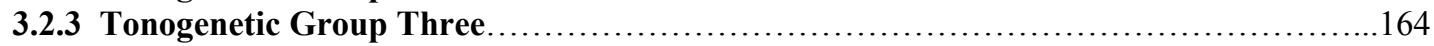

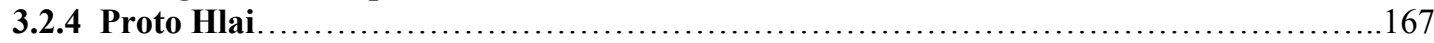

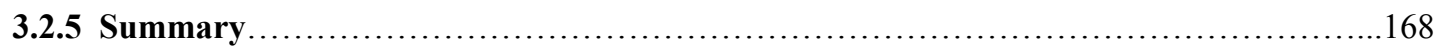

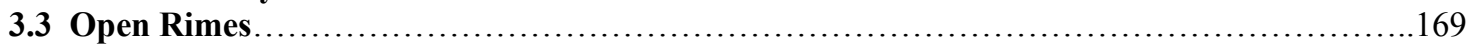

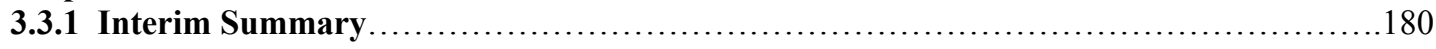

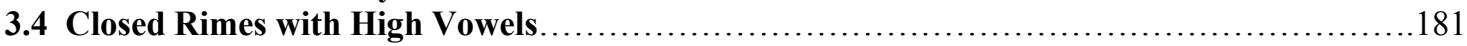

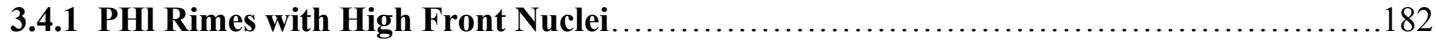

3.4.1.1 Long Rimes with High Front Vowels............................................... 183

3.4.1.2 Short Rimes with High Front Vowels.............................................. 185

3.4.2 Closed Rimes with High Back Unrounded Nuclei....................................... 191

3.4.2.1 Long Rimes with High Back Unrounded Vowels...................................... 192 
TABLE OF CONTENTS -- Continued

3.4.2.2 Short Rimes with High Back Unrounded Vowels...................................... 194

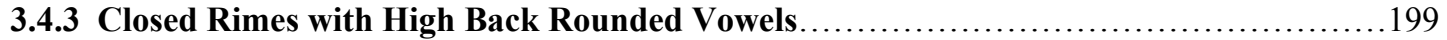

3.4.3.1 Long Closed Rimes with High Back Rounded Vowels...................................201

3.4.3.2 Short Closed Rimes with High Back Rounded Vowels..................................202

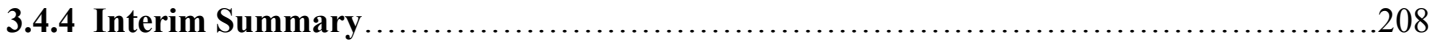

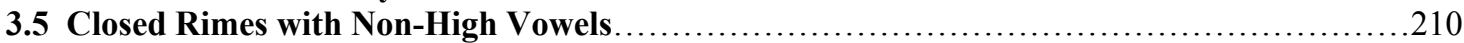

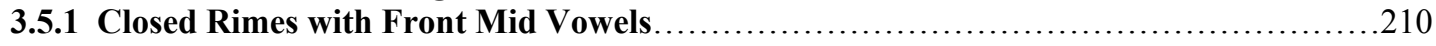

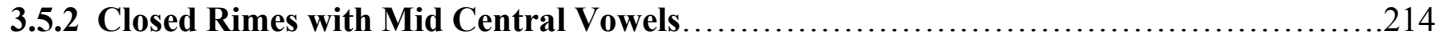

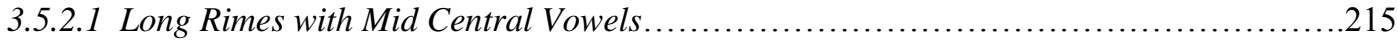

3.5.5.2 Short Rimes with Mid Central Vowels.......................................... 217

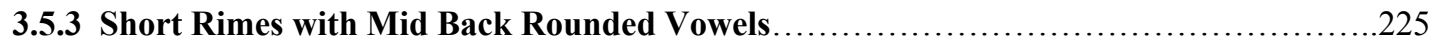

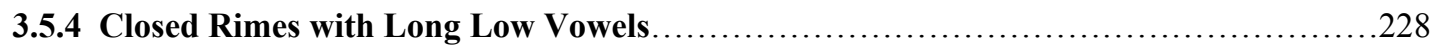

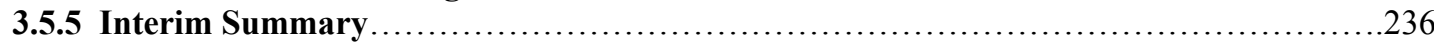

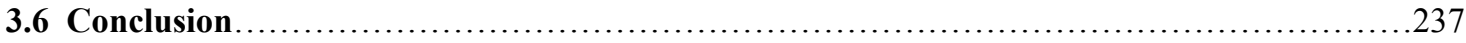

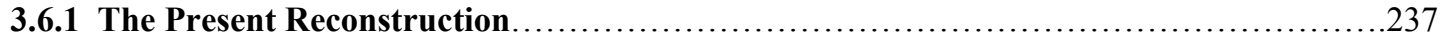

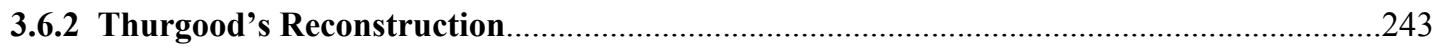

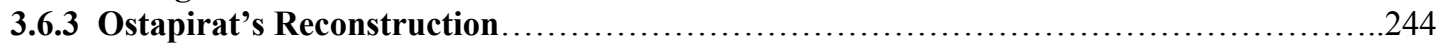

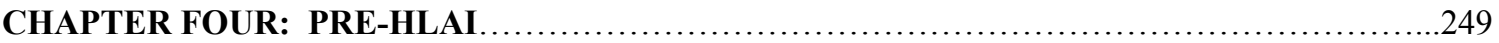

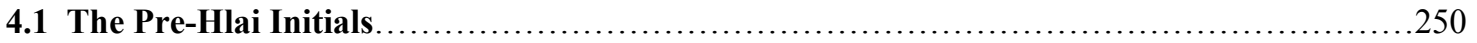

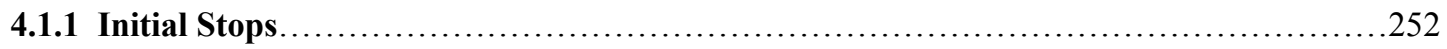

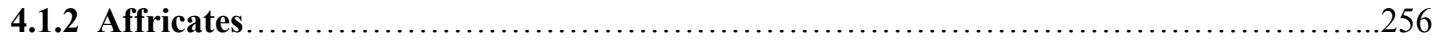

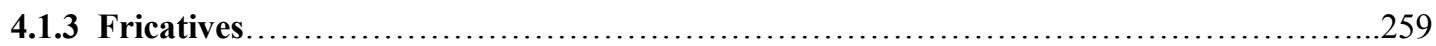

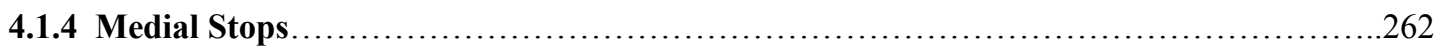

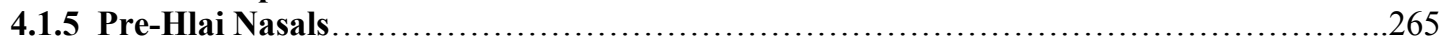

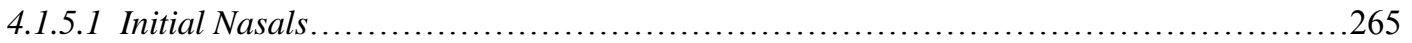

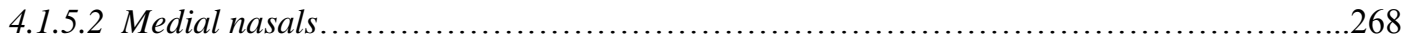

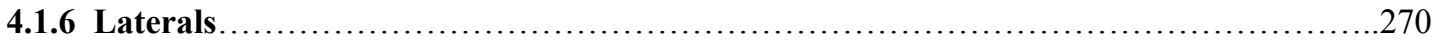

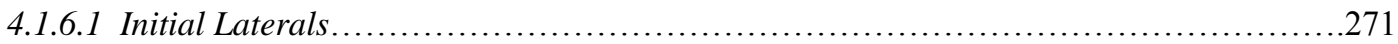

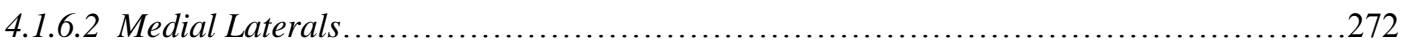

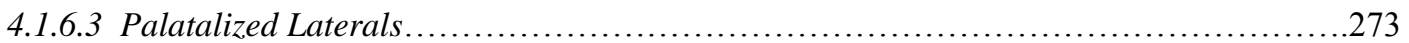

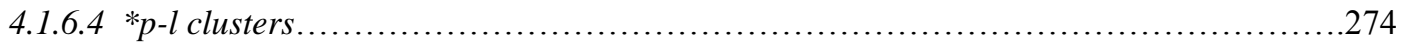

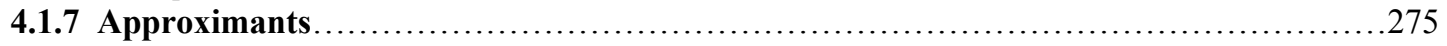

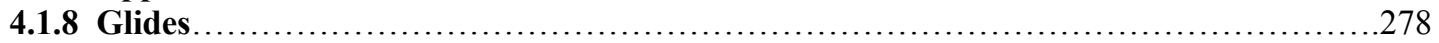

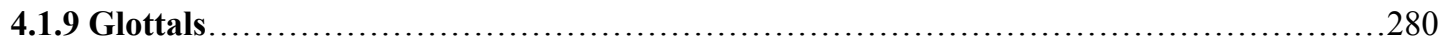

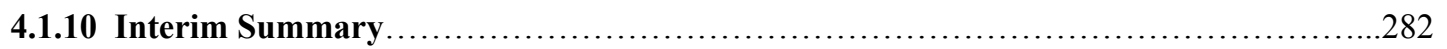

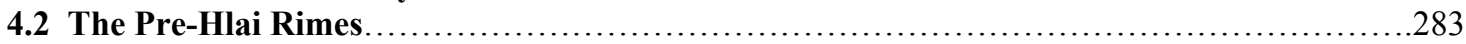

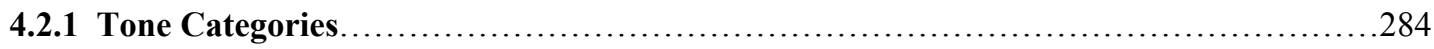

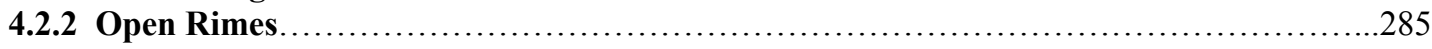

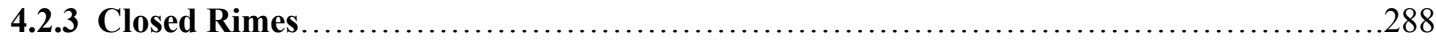

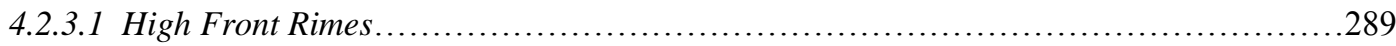

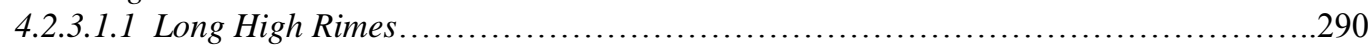

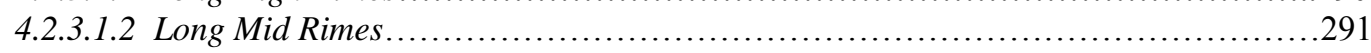

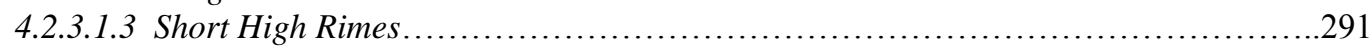

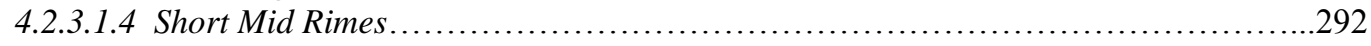

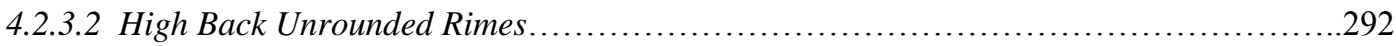

4.2.3.2.1 Long High Back Unrounded Rimes................................................2293

4.2.3.2.2 Long Mid Back Unrounded Rimes..................................................294

4.2.3.2.3 Short Back Unrounded Rimes............................................................2295

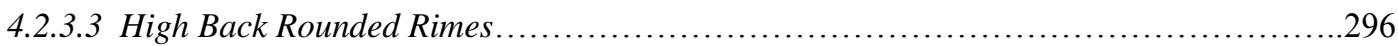


TABLE OF CONTENTS -- Continued

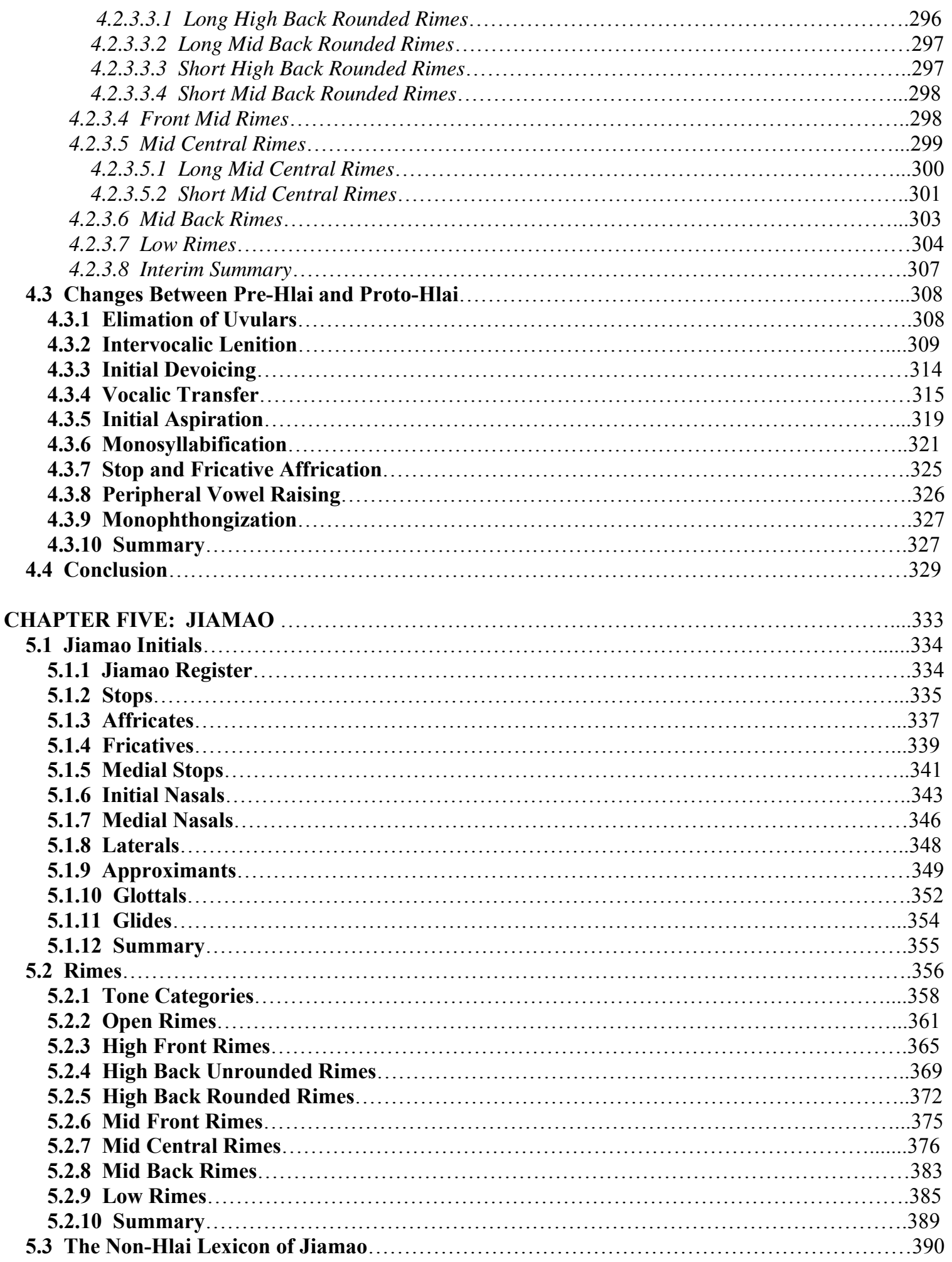


TABLE OF CONTENTS -- Continued

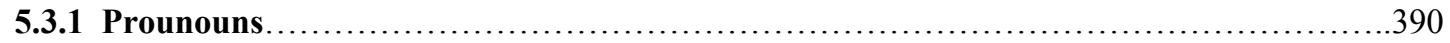

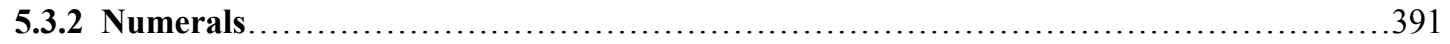

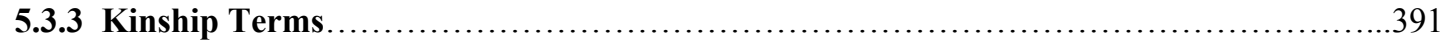

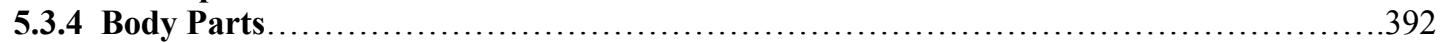

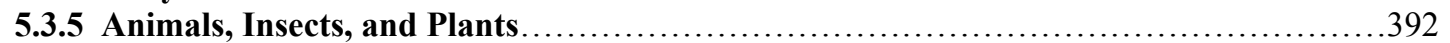

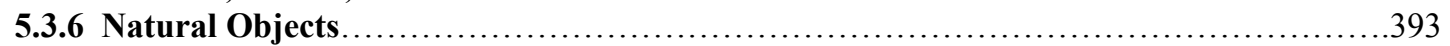

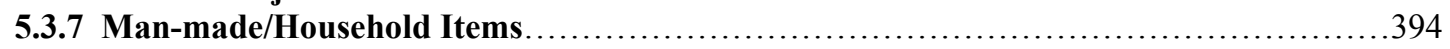

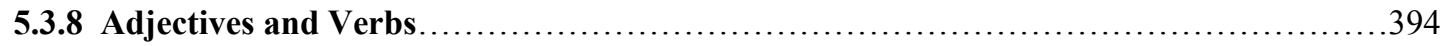

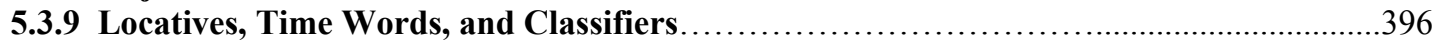

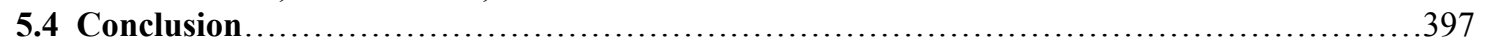

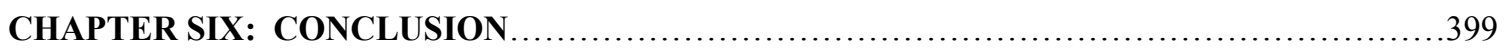

6.1 The Relationship between Hlai and Other Language Families of Southeast Asia...............399

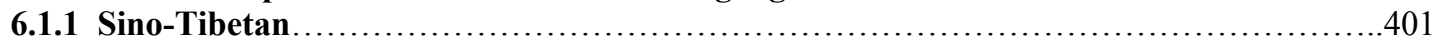

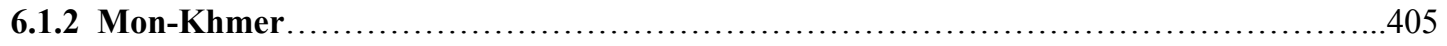

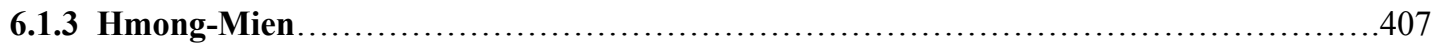

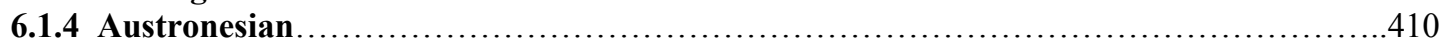

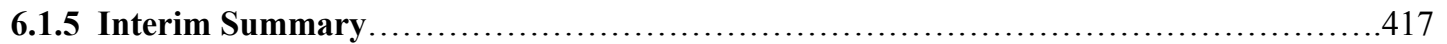

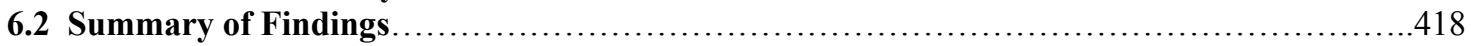

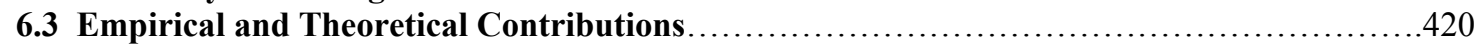

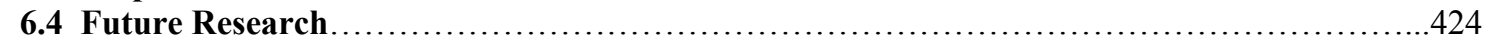

APPENDIX A: HLAI LANGUAGE DATA AND PROTO-HLAI RECONSTRUCTIONS...........426

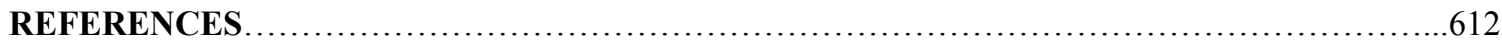




\section{LIST OF FIGURES}

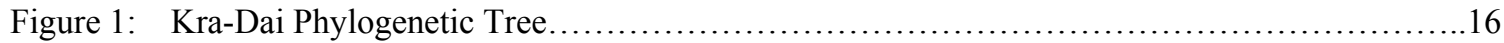

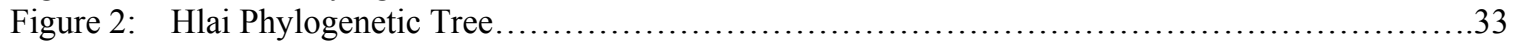

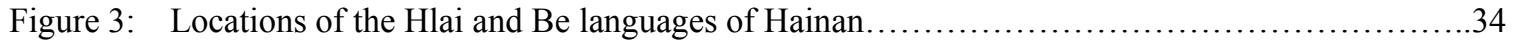

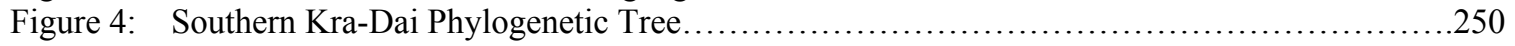




\section{LIST OF TABLES}

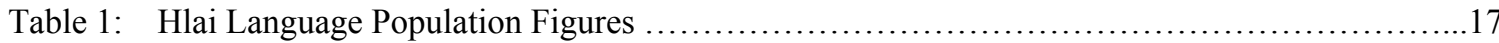

Table 2: Obstruent and Sonorant Initial Correlates with Register ................................. 73

Table 3: Comparison of Registrogenesis and Tonogenesis..........................................151

Table 4: Greater Hlai Distribution of Pure Vowels vs. Short Diphthongs...........................172 


\begin{abstract}
This dissertation presents a reconstruction of the phoneme inventory of Proto-Hlai, based on data from twelve Hlai languages spoken on Hainan, China. A classification of the Hlai languages is given with the innovations upon which it based, followed by a discussion of contact relationships and a discussion of reconstruction methodology. The inventory of Proto-Hlai initials is reconstructed, and original sesquisyllabic forms are shown to be necessary to account for the reflexes between the daughter languages; the initial inventory is also marked by the presence of aspiration on most consonants in word-initial position. This is followed by the reconstruction of the rime inventory, an outstanding features of which is two laryngeal components which are argued to have been the precursors to two of the synchronic tone categories in the daughter languages, and which conditioned segmental variation in most of the daughter languages. A comparison is made between Proto-Hlai, Proto-Be, and Proto-Southwest Tai, and a preliminary reconstruction of Proto-Southern Kra-Dai (the immediate ancestor of Proto-Hlai) is performed. When this reconstruction is compared with that of Proto-Hlai, it is shown that several important sound changes occurred in Pre-Hlai, including intervocalic obstruent lenition, vocalic transfer, aspiration of wordinitial consonants, and peripheral vowel raising. The language Jiamao is examined in detail, and it is argued that Jiamao is a non-Hlai language which has been in close contact with Hlai since the Pre-Hlai period. An examination of the correspondences between Jiamao and Hlai reveal at least two layers of Hlai loanwords in Jiamao, and evidence Jiamao was originally very different from Hlai structurally. Finally, the Proto-Hlai lexicon is compared with those of other Southeast Asian language phyla, and it is shown that Hlai retains evidence of shared lexicon (via either a genetic or contact relationship) with Sino-Tibetan, Mon-Khmer, Hmong-Mien, and Austronesian, the last of which is particularly striking. The dissertation concludes with a summary of findings, empirical and theoretical contributions, and suggestions for future research.
\end{abstract}




\section{CHAPTER ONE: INTRODUCTION}

The purpose of this dissertation is the reconstruction of the phonological system and lexical inventory of Proto-Hlai, one of the four main branches of the Kra-Dai phylum of Southeast Asia. This reconstruction is based primarily on the data found in Ouyang \& Zheng (1983), and has been supplemented by Ouyang (1998) as well as the author's own fieldwork in Hainan during the academic year of 2003-04. Data from a total of thirteen languages have been used in the reconstruction, all of which are spoken on the island of Hainan, China. The theoretical goal of this dissertation are to develop a theory of sound change with general principles that are applicable over the range of Hlai diachronic evolution, and the empirical goal is to implement these principles in explaining individual paths of phonological change which can be applied to the reconstruction of the Proto-Hlai phoneme inventory and lexicon.

This dissertation is a significant contribution to the field of Kra-Dai (and more generally Southeast Asian) historical linguistics, in that it provides a reconstruction of over one thousand Hlai vocabulary items which are thus available for comparison with forms in other languages and families both within and outside of the Kra-Dai phylum. It also contributes to historical reconstructive methodology by analyzing the types of change which have occurred between Proto-Hlai and its daughter languages, thereby creating an inventory and typology of sound change which is possible and especially applicable within Southeast Asia.

The reconstruction presented here has been divided between the system of initials (chapter 2) and the system of rimes (chapter 3), which form separate and largely exclusive systems. In addition to these, a discussion of Pre-Hlai, the stage prior to Proto-Hlai, is also included (chapter 4), accompanied by a comparison with Proto-Southwest Tai and Proto-Be. An analysis of the relationship between Jiamao, a non-Hlai language isolate, and the rest of Hlai with which it has been in longstanding contact is treated in

chapter 5. The conclusion of the dissertation (chapter 6) includes a discussion of prehistoric relationships with other Southeast Asian language families and phyla. 


\subsection{Background}

The Hlai family is a member of the Kra-Dai phylum (also commonly known as (Tai-)Kadai) of Southeast Asia. Although there is not uniform agreement yet among specialists regarding finer details of Kra-Dai subgrouping, there is unanimity that the following constituents exist:

$\begin{array}{ll}\text { Kra } & \text { Hlai } \\ \text { Kam-Sui } & \text { Tai } \\ \text { Lakkja } & \text { Be }\end{array}$

Of these six groups, Lakkja and Be are relatively small, consisting of only a few languages each. It is generally felt that Lakkja is closest to Kam-Sui, and that Be is closest to Tai, the only question being whether or not they are actually constituents of these respective larger families or coordinate with them. Ostapirat (2005) treats Lakkja as part of Kam-Sui, whereas Solnit (1988) and Hansell (1988) consider it coordinate with Kam-Sui; Thongkum (1992), on the other hand, considers Lakkja closer to Tai based on a count of 243 reconstructed Lakkja lexical items. When compared with the three traditional subgroups of Tai (Southwest, Central, and Northern), Be bears a resemblance specifically to Northern Tai, indicating that it may not only be part of the Tai group proper, but could form a subgroup with Northern Tai, having become divergent largely through its own displacement to Hainan; confirmation of this hypothesis, however, must await a full reconstruction of Proto-Tai (especially Northern Tai). Ostapirat (2005:108) also hypothesizes that at the highest levels of the Kra-Dai phylogenetic tree, Kra and Kam-Sui form a subgroup in opposition to another subgroup formed by Tai and Hlai, effectively constituting a Northern Kra-Dai versus a Southern Kra-Dai. Based on a count of the lexical items in Liang \& Zhang (1996) in which Hlai is cognate with one or more other Kra-Dai branches, Hlai was found to share lexical items exclusively with Tai in 42 instances, and exclusively with Kam-Sui in only 13 instances; this lends some credibility to Ostapirat's hypothesis. Bearing in mind that this classification is still tentative and awaiting final proof, the figure below represents a more articulated working model of the Kra-Dai family: 


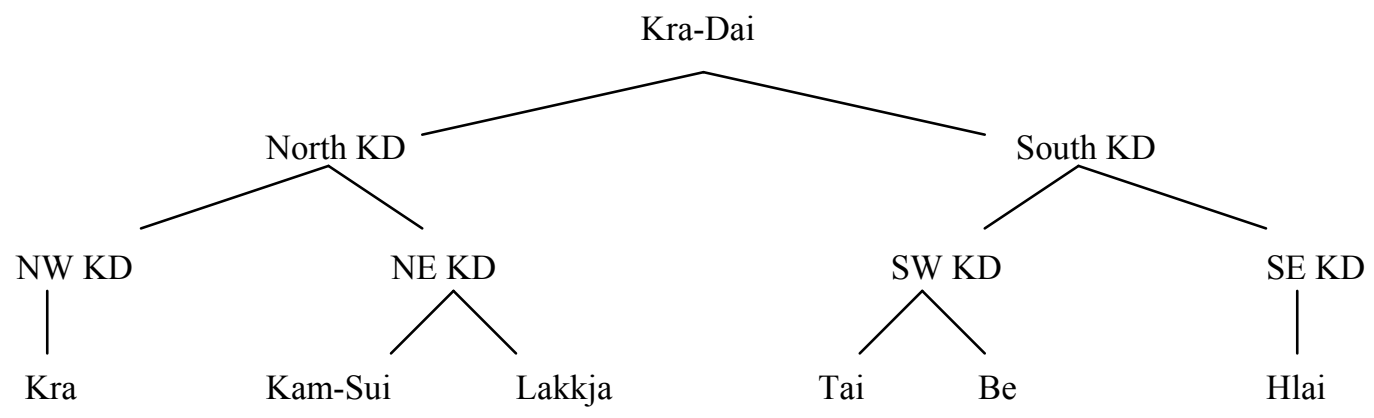

Although reconstructions of various degrees of detail exist for all of the families above, Proto-Hlai will be compared specifically with other Southern Kra-Dai proto-languages (Proto-Be and Proto-Southwest Tai) in chapter four, since they are tentatively assumed to be its closest relatives within Kra-Dai.

The Hlai languages are spoken exclusively on the island of Hainan, China. There is nothing to suggest that the speakers of the Hlai languages were not the first inhabitants of the island, the only other potential contenders being the speakers of the Jiamao language, which shows evidence of being in longstanding contact with Hlai, but is not actually a Hlai language itself. The Hlai are currently the largest 'minority group' on Hainan, which they share with various groups of Chinese (speaking varieties of Sinitic including Hakka, Yue (Danzhouhua), Southern Min (Hainanese), and Southwest Mandarin (Junhua)), as well as other ethnic groups including the Be (who also belong to the Kra-Dai phylum) in northern Hainan, the Utsat (whose language, Tsat, belongs to the Chamic subgroup of Austronesian) in southern Hainan, and pockets of Mien speakers (of the Hmong-Mien phylum), who were originally conscripted soldiers sent to Hainan by the Chinese to subdue the Hlai. In fact, the only major Southeast Asian language phylum which is not represented on Hainan is Austroasiatic. One other language, which must for now be considered an isolate, is Jiamao, mentioned above. Although it is shown in chapter five that there is very good reason to consider Jiamao to be ultimately of non-Hlai origin, speakers of Jiamao are considered to be part of the Hlai ethnic group by the Chinese government, and are counted as such in national censuses. Conversely, the speakers of Cunhua and Nadouhua, which I consider to be essentially Hlai languages based on their core vocabulary, are considered to be Chinese by the government and are counted as such. 
According to the 1990 census (Ostapirat 1993:1), the Hlai population was estimated to be $1,110,000$. However, a more conservative (and probably more accurate) number of 747,000 speakers is given in Shearer \& Hongkai (2002), which approaches 800,000 if the speakers of Cunhua and Nadouhua are included. The Hlai languages have traditionally been divided into five branches: Ha, Qi, Run, Meifu (which also includes the language referred to here as Changiiang), and Jiamao, with Cunhua and Nadouhua being outliers and falling outside of this classification. The primary groups classified here are shown below, with population figures adapted from Shearer \& Hongkai (2002: 88-90), and alternate names used in Ouyang \& Zheng (1983) and other sources given in parentheses:

Table 1: Hlai Language Population Figures

$\underline{\text { Family }}$ Group $\underline{\text { Language }}$

Hlai

\begin{tabular}{|c|c|c|}
\hline \multicolumn{2}{|c|}{ Bouhin (Heitu) } & 73,000 \\
\hline \multicolumn{2}{|c|}{ Ha Em (Zhongsha) } & 193,000 \\
\hline \multicolumn{2}{|c|}{ Lauhut (Baoding) } & 166,000 \\
\hline Qi & $\begin{array}{l}\text { Tongzha (Tongshi) } \\
\text { Zandui (Qiandui) } \\
\text { Baoting (Baocheng) }\end{array}$ & $\begin{array}{l}\frac{178,000}{125,000} \\
29,000 \\
24,000\end{array}$ \\
\hline Run & $\begin{array}{l}\text { Baisha } \\
\text { Yuanmen }\end{array}$ & $\begin{array}{l}\frac{44,000}{36,000} \\
8,000\end{array}$ \\
\hline Meifu & (Xifang) \& Changjiang & 30,000 \\
\hline $\mathrm{NWCH}$ & $\begin{array}{l}\text { Cunhua (Ngan Fon) } \\
\text { Nadouhua (Dongfang) }\end{array}$ & $\begin{array}{l}\frac{62,500}{60,000} \\
2,500\end{array}$ \\
\hline Jiamao & & 52,300 \\
\hline
\end{tabular}

As can be seen from the figure above, the Ha Em, Lauhut, and Qi groups are comparatively robust, while the Bouhin, Run, Meifu, NWCHl and Jiamao groups have fewer speakers. The Run and Meifu groups, along with NWCHl, are in closer contact with Chinese and their speakers are under greater pressure 
to shift to Chinese as a first language. Yuanmen, and Nadouhua especially, are critically endangered, and there is little evidence that the children of Nadouhua speakers are learning the language from their parents, many of whom are only semi-fluent themselves.

\subsection{Methodology and Materials}

Data for this dissertation is drawn largely from Ouyang \& Zheng (1983). Complimentary to this is Ouyang (1998), and $\mathrm{Fu}$ (1997). Fu (1990) was also consulted and used to establish an initial database on Nadouhua. These materials formed the core of the original database which was organized first by lexical items (alphabetical by the Chinese gloss), then by initials, rimes, and finally tone categories. Sound correspondences were listed, irregularities noted, and attempts were made to find causes for the irregularities. Some irregularities were the result of original mistakes in transcription; others were the result of borrowing (either from Chinese or between Hlai languages themselves); still others may be attributed to either idiosyncratic internal transmission errors or are otherwise still unexplained.

In addition to this, I also performed fieldwork in Hainan during the period September 2003-June 2004. During this time, fieldwork focused primarily on Nadouhua, although consultants were also located who spoke the ten varieties in Ouyang \& Zheng (1983), as well as Cunhua; additionally, the Changjiang language was 'discovered' and recorded for the first time. The data for this language are the most incomplete, since the Changjiang consultant only had eight hours during which to work, and attention was paid in that case to core Hlai vocabulary items. In all cases, data was elicited and recorded in a Word document, and occasional corrections made to already published material. A wordlist of 200 core items was established, and recordings were made for each language with at least one consultant. Recordings were done using a Shure BETA58a cardioid microphone and Speech Analyzer 2.5 software (SIL Speech Tools), recorded into a Dell Inspiron 600m laptop computer.

The language consultants who offered their valuable time and energy to this work are the following: 


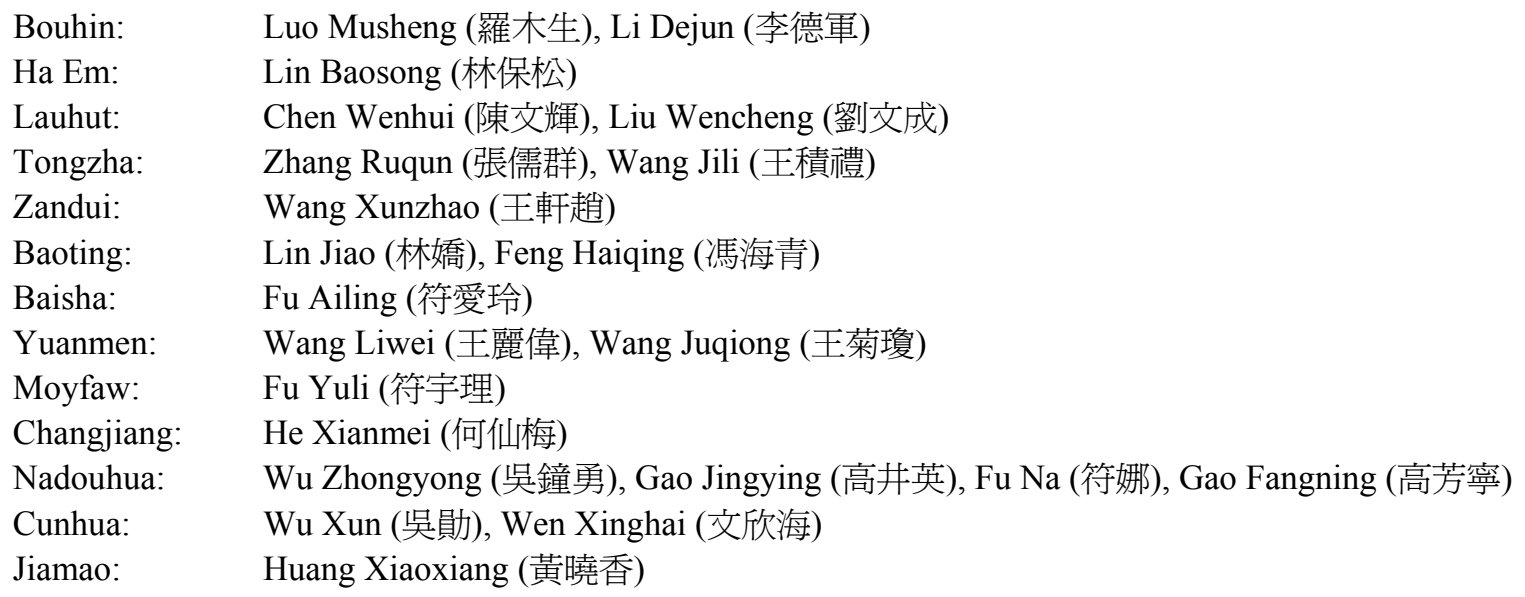

\subsection{Previous Work}

This section provides an overview of previous work on the Hlai languages, divided into two categories. The first category summarizes data which has been collected, described, and published. The second category includes publications which have performed some kind of analysis upon this data.

\subsubsection{Data}

Publications on what is now known as the Hlai languages began in the late nineteenth century, an overview of which is included in Ostapirat (1993: 11-18). The first modern large-scale collection of data was that of Savina (1931), in which he recorded two languages. The first, which he referred to as Southern Day, includes approximately 1,200 lexical items, and is essentially the same as Bouhin. The second, which he referred to as Central Hiai, includes a smaller number of words (150), and is obviously a Qi language, although it is more difficult to associate it with one of the three specific languages used in the present study. These languages were recorded in Vietnamese script, and must be read accordingly.

The next major publication of data was that of Wang \& Qian (1951), which records a dialect of Baisha. It is fairly similar to the dialect of Baisha recorded in Ouyang \& Zheng (1983), with the general 
exception that it records alveolopalatal affricates which have since become plain alveolar affricates, and the final $-l$ is transcribed in the place of $-u$ (this will be discussed more in chapter three).

Ouyang \& Zheng (1980) presented a limited amount of data for Lauhut (Baoding) and Tongzha (Tongshi). This was followed up by the much more comprehensive and massive study which they published as Ouyang \& Zheng (1983), which is a large and detailed reference on the Hlai languages that provides data on nine Hlai languages, plus Jiamao. It not only gives detailed phonetic descriptions of each of the languages, but also includes a total of 1,730 lexical items for all ten languages. A detailed language map of the Hlai languages on Hainan is also provided, along with the traditional classification mentioned at the beginning of this chapter.

$\mathrm{Fu}$ (1983) presented a short wordlist of Cunhua lexical items, the number of which was doubled in the follow-up publication of Ouyang \& Fu (1988). These short articles were finally followed by monographs on Cunhua, first $\mathrm{Fu}$ (1997) which is written by a native speaker of Cunhua, and then Ouyang (1998), a detailed treatment of Cunhua with comparative analysis and hypotheses about the history of Cunhua as a Chinese-Hlai mixed language. Finally, Fu (1990) presented a short wordlist for Nadouhua, also presumed to be a mixed language. It should be noted that the mixed status of both Cunhua and Nadouhua, while not pursued in detail here, has the potential for a sizable study in its own right.

\subsubsection{Analysis}

Benedict (1942) was the first publication to group Hlai (Li) with Tai, along with other lesserknown languages, in a new phylum which he called Tai-Kadai, where Tai was taken as one taxonomic unit, and the other languages (including Hlai, Gelao, Laqua, and Lachi) were placed under the umbrella term of Kadai. This term has been in common use since then, but is now in competition with another suggested name for the phylum, Kra-Dai (Ostapirat 2000), which is the term adopted here for the reasons argued therein. 
According to Ostapirat (1993:17), Shafer (1957) was the first to present comparative work on Hlai; this reference has unfortunately been unavailable to me.

Solnit (1982), using data from Ouyang \& Zheng (1980), treated registrogenesis and its connection with Hlai nasals and fricatives (Ostapirat 1993:17; this conference paper has also been unavailable to me).

Haudricourt (1984), reprinted as Haudricourt (1989), presented an article on Hlai tones, providing a further outline of registrogenesis for the Hlai languages in which he focuses primarily on initials, using data from Ouyang \& Zheng (1980).

Matisoff (1988) is the first large-scale reconstruction of the system of Proto-Hlai initials, based on Ouyang \& Zheng (1983). Matisoff excluded Jiamao data based on 'its extreme (and apparently unsystematic) aberrancy with respect to others (1988:289)'. He also did not have any access to data on Cunhua or Nadouhua.

Thurgood (1991a) was the next large comparative study using the data in Ouyang \& Zheng (1983). Using Matisoff (1988) as a place of departure, he proposed his own reconstruction of Hlai initials, and provided the first comprehensive reconstruction of the Hlai rimes. In this paper, Thurgood used additional data from both Cunhua and Nadouhua which had been unavailable to Matisoff. Thurgood (1991b) was the first paper to treat Jiamao and attempt to resolve its apparent inconsistencies with the other Hlai languages. He is also the first to suggest that Jiamao may have not originally been a Hlai language.

Peiros (1998) was the third scholar to present a reconstruction of the system of Hlai initials. His reconstruction diverges more from Matisoff (1988) than does Thurgood (1991a). He also suggests a vague outline of the Hlai rime system, but does not go into sufficient detail to be taken into account in this study. This represents the first serious effort to integrate the Hlai data into the greater Kra-Dai picture, with a reconstruction of Proto-Kra-Dai initials included.

Ostapirat (1993a) is a reconstruction of the Proto-Hlai rime system, along with the first classification of the Hlai languages which does not directly mirror that in Ouyang \& Zheng (1983). It is the best work to date on the background of Hlai studies. Ostapirat (1993b) provides an argument for dental and velar clusters in the Proto-Hlai initials, a hypothesis which he seems to have later abandoned. Ostapirat (1996) addresses the complicated issue of Kra-Dai - $u$, and examines the possibility (based largely on 
Wang \& Qian 1951, which describe a variety of Baisha) that final $-l$ is at least one source for this final glide. Ostapirat (2004) provides a revised reconstruction of both Proto-Hlai initials and rimes (the latter of which differing significantly in some respects with his proposed system in Ostapirat 1993a), and attempts to integrate the Jiamao data into the reconstruction. Finally, Ostapirat (2005) addresses the putative relationship between Kra-Dai and Austronesian, first advocated in Benedict (1942), arguing that the hypothesis should still be taken seriously, and representing the second major attempt at integrating the Hlai material into an overall Kra-Dai picture (although a comprehensive reconstruction is not included in this particular paper, the goal rather being to show that there are regular correspondences in specific areas between Kra-Dai phonemes on the one hand and Austronesian phonemes on the other).

\subsection{The Hlai Linguistic Area}

Hainan is an island with few natural barriers to prevent travel and/or interaction among its inhabitants, and as a result natives of Hainan are often at least bilingual, and often have a functional knowledge of three or even more languages. Although it is ultimately possible to subgroup the Hlai languages as shown in the preceding section, there is also evidence for contact relationships which are often both intense and longstanding. These relationships often betray themselves in identifiable loanwords, and are also attested to by consultants with which I have done fieldwork. The most important of these contact relationships are described in section 1.6. The sociolinguistic situation on Hainan has grown out of a complicated series of immigration and subsequent interaction (a process which intensified with Chinese immigration beginning in the Song dynasty (Kwok 2006: 202)), but Hainan is a terminal point for migration as a result of being an island off the Chinese mainland with no proximal geographic neighbors. The human mosaic resulting from this has created a more richly complicated system than many traditional continental models.

The unmarked situation in historical reconstruction has been that changes in one language or language branch which do not occur in another defines a point in time in which they have broken away from each other and are no longer in contact: 
(5)

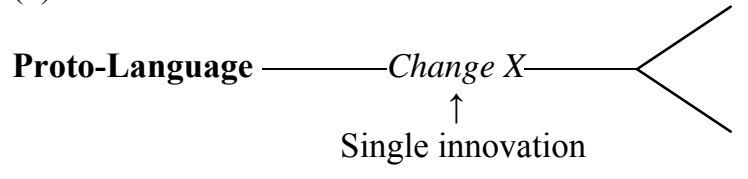

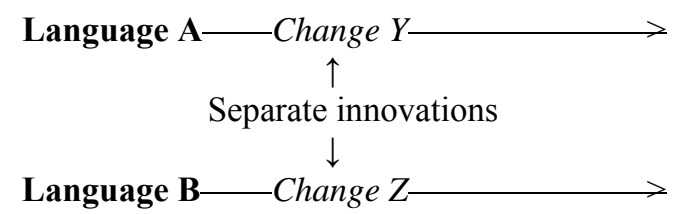

However, all evidence indicates that although there were some important changes which happened in PHl itself, when the proto-language branched into its daughter languages, there were new sound changes that seem to have originated in some area, gained momentum, and spread across the entire language area affecting it as essentially a single speech community. Further evidence for this comes from the fact that languages which arrived on Hainan in fairly recent times, such as the branch of Southern Min Chinese spoken on Hainan (Hainanese), have participated in more recent changes such as stop implosivization and desibilantization. This situation is more accurately portrayed in (6):

(6)

Proto-Hlai-

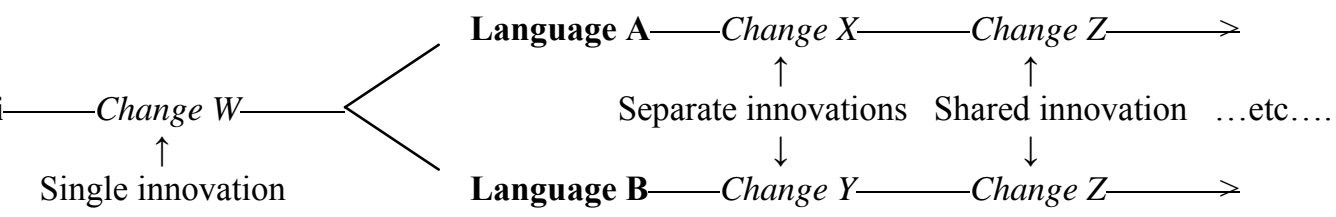

This fact is crucial to an understanding of $\mathrm{PHl}$ reconstruction, because it means that sound changes shared by daughter languages may not be a reliable indicator of their unity or disunity at the time the changes were effected. This is a very similar to the situation which Ross (1988: 9-11) models in Oceanic, in which he uses the term linkage to refer to '... a group of communalects which have arisen by dialect differentiation..'. He informally divides linkages into two kinds, the first being a chain where '...communalects are typically spread along a coastline, each related most closely to its neighbor on either side...', and the second being a network, where '...communalects are scattered over a land area or an archipelago, typically having neighbors on more than two sides, and often sharing different innovations with several of these.' The Hlai languages under discussion here fall very squarely under the second (network) model, and it is shown below that various degrees of interaction have continued between subgroups after their initial branching, leading primarily to lexical borrowing and to the diffusion of sound 
changes across already differentiated groups which are not necessarily close to each other in the Hlai family tree.

The fact that sound change within Hlai, and on Hainan in general, can be understood to entail a large component of diffusion and/or parallel innovation allows an important degree of theoretical freedom. Without this understanding, a complex history of apparently monolithic changes would need to be chronologically linearized, and artificially projected back further in time than was historically accurate. Moreover, if the assumption were made that every shared innovation was an inheritance, the phylogenetic tree of the Hlai languages would be mired in not just one or two, but a number of paradoxical subgroupings which could not all be true simultaneously.

\subsection{Subgrouping}

This section is divided into three parts. The first part (1.5.1) outlines and explains the theory of subgrouping which will be used to subgroup the Hlai languages, the second part (1.5.2) gives the Hlai family tree itself, and the third (1.5.3) implements this theory, offering the evidence for that tree at all nodes. When examples from the twelve Hlai language are given, they are given in this order:

(7) Order of the Hlai languages when given in examples

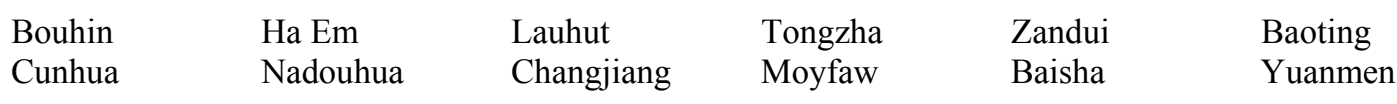

\subsubsection{Theory of Subgrouping}

The goal of this section is to explain the theory of subgrouping adopted in this study. Criteria are discussed which allow the discernment between (or the establishment of likelihood of) changes which have been inherited versus those which have been diffused through contact.

There are two objects which are available for analysis in phonological reconstruction: the sound changes which affect lexical items, and the lexical items themselves. In cases where there are either few 
lexical items recorded or where there is a range of items between languages, these cannot generally be used as a reliable criteria for subgrouping (Thurgood 1982: 251). In these cases, sound changes are the only viable object which can be used for subgrouping. It is fortunate that there is a large amount of lexical data available on the Hlai languages (the only exception presently being Changjiang, which there is nevertheless enough data for to be of use) to achieve results using the lexicon for subgrouping. This being the case, the lexicon will be examined first in view of its role in subgrouping, with sound change being treated thereafter.

Before proceeding, there is one general principle which can be outlined that applies to both the lexicon and to sound change. It may be asked whether retentions and innovations carry equal weight in subgrouping, and it has been convincingly argued (Thurgood 1982, Blust 1999, Campbell 2004) that innovations are a much better criteria than retentions, as all retentions may be potentially be inherited by all languages which have descended from some proto-language, but innovations are only inherited by those daughter languages which have descended from the parent at the particular place in the tree at which the innovation originated.

For example, assume that a proto-form for some lexical item is known. Compare the two trees below, where the bottom nodes are individual languages and where $\mathrm{X}$ is a retention and $\mathrm{Y}$ is an innovation. The fact that the first and second languages retain an inherited form in (8a) does not provide evidence that they should be subgrouped together; all that can be inferred is that the third language has innovated. However, in the case of (8b), the fact that both the second and third languages share an innovation (if it can be shown not to be a loan from one to the other) is evidence that they form a subgroup, and that the innovation can be reconstructed in an immediate ancestor:

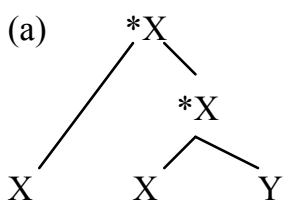

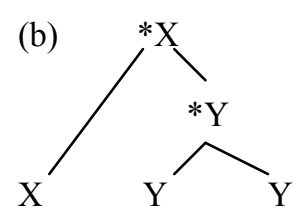

An example of this is given below, where the Proto-Hlai form for pound rice has been inherited in most languages, but where there was an innovation in Run which was inherited by the daughter languages, Baisha and Yuanmen: 
(9)

\begin{tabular}{|c|c|c|c|c|c|c|}
\hline Gloss & $\underline{\text { Proto-Hlai }}$ & $\underline{\text { Hlai language } \mathrm{d}}$ & & & & \\
\hline pound rice & $*^{t} c^{\mathrm{h}} \mathrm{a}: \mathrm{k}$ & ts $^{\mathrm{h}} \mathrm{a}: ?^{7} \quad$ ts $^{\mathrm{h}} \mathrm{e}: \mathrm{P}^{7}$ & $\mathrm{ts}^{\mathrm{h}} \mathrm{e}: \mathrm{k}^{7}$ & $\operatorname{ts}^{\mathrm{h}} \mathrm{e}: ?^{7}$ & $\operatorname{ts}^{\mathrm{h}} \mathrm{e}: ?^{7}$ & $\mathrm{ts}^{\mathrm{h}} \mathrm{e}: ?^{7}$ \\
\hline & & $\mathrm{ts}^{\mathrm{h}} \varepsilon \mathrm{k}^{2} \quad \mathrm{~s} \varepsilon \mathrm{P}^{4}$ & ts ${ }^{\mathrm{h}} \mathrm{e}: ?^{7}$ & ts $^{\mathrm{h}} \mathrm{u}:{ }^{2}$ & $\mathrm{p}^{\mathrm{h}} \mathrm{aw}^{3}$ & $\mathrm{p}^{\mathrm{h}_{\mathrm{a}} \mathrm{w}^{3}}$ \\
\hline
\end{tabular}

It must occasionally be asked if a reconstructed form is truly a descendant from Proto-Hlai or is a more recent innovation. Internally, the best criteria to use in identifying the most likely Proto-Hlai form is that which is reconstructible using the most diverse branches of the tree. Take the examples below, where the subgrouping has been established, but the proto-form is unknown and must be reconstructed based on the evidence of the three languages represented by the terminal nodes of the tree:
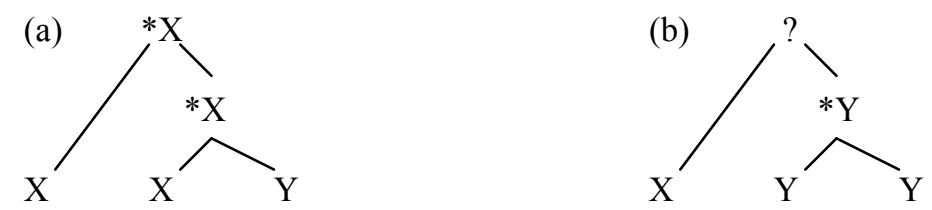

In (10a), the second and third languages disagree, and the proto-form for this group would not be reconstructible based merely on the evidence found between these two languages. However, thanks to the fact that the first language agrees with the second, the third language can be shown to have innovated, and the proto-form is reconstructible at all levels. In (10b), on the other hand, the immediate ancestor of the second and third languages can be reconstructed, but since the first language disagrees with them, it is unclear which daughter, if either, has inherited the original form from the proto-language, which is not reconstructible based on this evidence (this is most often a problem at the highest level of the tree).

The only way that innovation can be identified in a situation like that shown in (10b) above is if there is external evidence available. This is fortunately the case for several Hlai lexical items, where cognates can be found in other branches of Kra-Dai (see chapter four for several examples). An example of this is given below:

$\underline{\text { Gloss }}$

house $\underline{\text { Hlai language data }}$

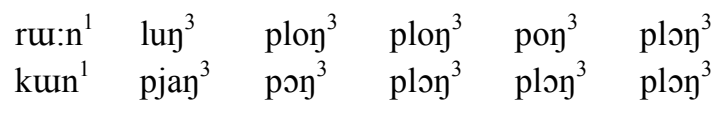


In this case, the evidence at face value seems to strongly indicate that the Proto-Hlai form should be reconstructed as *p-lon?, with innovations in Bouhin and Cunhua. However, there is evidence from both the Southwest Tai (*ruən) and Be (*ra:n) branches of Kra-Dai that the Bouhin form is the descendent of the original Hlai form. Since it is argued below that the initial split in Hlai is between Bouhin and the rest of the family (subsumed under a node called Greater Hlai), the Greater Hlai form can be shown to be an early innovation, which was then inherited regularly by all of the Greater Hlai daughter languages.

There is one other confounding factor in this otherwise straightforward approach to subgrouping described above, and that is borrowing, which can occur in two different scenarios: (1) borrowing between two related languages, and (2) mutual borrowing from a third language. Borrowing can introduce a noninherited lexical item into one or more languages, which can give the false appearance of shared inheritance if there are no criteria by which to identify the borrowed words. The second scenario described above is generally not problematic in the case of the Hlai languages, as the third language which two (or more) Hlai languages may borrow from is nearly always Chinese (of one variety or another, all spoken on Hainan). Recent Chinese loanwords in the Hlai languages are normally readily identifiable due to the fact that they are easily traced to the Chinese source of origin, and the reflexes in the Hlai languages are generally irregular and occasionally contain phonotactic patterns not normally allowed in Hlai. An example is given below:

\begin{tabular}{lllllll} 
Gloss & \multicolumn{3}{l}{ Hlai language data } \\
kick & $\mathrm{t}^{\mathrm{h}} \mathrm{i}: \mathrm{P}^{8}$ & $\mathrm{t}^{\mathrm{h}} \mathrm{am}^{2}$ & $\mathrm{t}^{\mathrm{h}} \mathrm{e}: \mathrm{k}^{8}$ & $\mathrm{t}^{\mathrm{h}} \mathrm{e}: \mathrm{P}^{7}$ & $\mathrm{t}^{\mathrm{h}} \mathrm{i}: \mathrm{P}^{9}$ & $\mathrm{t}^{\mathrm{h}} \mathrm{ek}{ }^{9}$ \\
& ---- & ---- & ---- & $\mathrm{t}^{\mathrm{h}} \mathrm{e}: \mathrm{k}^{8}$ & $\mathrm{t}^{\mathrm{h}} \mathrm{et}^{7}$ & $\mathrm{tia}^{7}$
\end{tabular}

Although all of the forms above except for the Ha Em form appear to be related at first glance, there are a number of irregularities which allow the identification of the rest of these words as loans. The first is that the tone indicated with 8 does not occur in Bouhin, Lauhut, or Moyfaw forms of native origin, nor does tone 9 normally occur in Zandui or Baoting forms. The Yuanmen initial fails to correspond to the initials of the other forms (it would also be aspirated), and while all of the rimes are permissible in the individual languages except the one in Baoting, they reflect a variety of earlier rimes including *i:k, *a:k and *it. 
Finally, these can be compared with Chinese 踢 (Mandarin $t^{h} i::^{1}$ ), to which the general shape of the Hlai forms conform.

A more problematic situation arises in the case of borrowing between one Hlai language and another. Ideally, the two languages in question will have undergone different paths of change, so that a loan into the borrowing language will be conspicuous due to one or more irregular reflexes which can be shown to be regular in the donor language. Several examples of this are discussed below in section 1.5.3. It is more difficult when words occur in languages with regular correspondences, which gives the impression of common inheritance, as in the following example (prefixes are omitted here for the sake of exposition):
$\underline{\text { Gloss }}$
Proto-Hlai
$\underline{\text { Hlai language data }}$

a fly

*hwa:y?

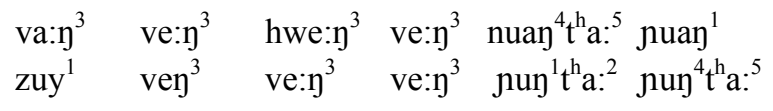

In this case, the Proto-Hlai form *hwa:y? has been inherited in most Hlai languages. However, a form reconstructible as *C-nu:n t $\mathrm{t}^{\mathrm{h}}$ a:h (literally 'rice mosquito') occurs in Zandui, Baisha, and Yuanmen, and is an innovation (the bare form mosquito has been adopted in Baoting). Although it will be argued below that Baisha and Yuanmen indeed form a subgroup (Run), Zandui is actually part of the Qi subgroup with Tongzha and Baoting. A case such as the one above can serve to confuse an attempt at subgrouping, and must be explained as either a very early loan from Run into Zandui or an independent calque which may have come about through participation in a language area. This kind of example is generally rare, and will not cause a critical misanalysis as long as the criteria outlined below are adhered to.

\subsubsection{Subgrouping: The Lexicon}

When using the lexicon for subgrouping, the following two rules are stipulated: 
Criteria for lexical subgrouping

(a) related languages share innovations not found in other languages

(b) the sound correspondences between these innovated cognates are regular

The rationale for (14a) is that an innovation which occurs at some intermediate level should be inherited by all daughter languages and only those languages. The rationale for (14b) is that irregular sound correspondences are more likely to indicate a lateral contact relationship (where one language has borrowed from another) rather than one of vertical inheritance.

If both of these criteria are met, then the only remaining consideration is to what extent (14a) holds true. Technically, two languages can be subgrouped together on the basis of a single lexical item which fulfills the requirements of (14) above. On a more practical level, however, confidence in the subgrouping increases in direct proportion to the amount of shared innovations which exist. One or two shared forms may be considered indicative of a subgroup, but twenty shared forms are more convincing. What is generally the case in the Hlai languages is that the greatest numbers of shared lexical innovations tend to be found at the lowest levels of the tree. Lexical innovations can still be identified at higher levels of the tree, but their frequency decreases and a greater reliance on sound change criteria is required.

\subsubsection{Subgrouping: Sound Change}

The general decision-making algorithm which has been used to decide between inherited sound changes and diffused sound changes has relied on a subjective assessment (backed up by typological data whenever possible) of which changes are likely to be rare, idiosyncratic, and less likely to diffuse, versus those which are more common and, once underway, will tend to apply wherever the relevant environment for such a change exists (in line with the argument in Blevins (2004) that the typological commonality of sound change correlates with its likelihood of independent occurrence). Data from the non-Hlai languages of Hainan have been informative in this regard, in that these languages have been receptive to certain sound changes (such as the shift from plain $p$ and $t$ to implosive 6 and $d$ in Hainanese, Be, and Mien, followed by 
the shift from $s$ to $t$ documented in Shintani (1991)), but resistant to others, and therefore offer evidence as to the likelihood that some change can occur through diffusion versus being restricted to inheritance only.

Examples of sound changes which are considered unlikely to diffuse across language boundaries include vocalic transfer (Benedict 1975) and rhinoglottophilia (Matisoff 1975). Vocalic transfer is a form of metathesis where the features of a high vowel preceding a stressed syllable are transferred onto the initial of that stressed syllable, in the form of a coarticulation. Rhinoglottophilia is the reinterpretation of the percepts of a glottal consonant as nasalization, being realized as a nasal segment where there was none in the direct ancestor of the language in question. Both of these changes, while not lacking precedent (especially within Southeast Asia), occur rarely enough that multiple separate occurrences seem unlikely, and therefore the languages which exhibit the change are likely to have inherited it from a common ancestor.

Examples of changes which appear to diffuse readily include the shift from palato-alveolar affricates and fricatives to alveolar affricates and fricatives, and registrogenesis. The first change involves the shift of the palato-alveolar phonemes $\mathrm{t} \int, \mathrm{t}^{\mathrm{h}}$ and $\int$ to the alveolar phonemes $\mathrm{ts}, \mathrm{ts}{ }^{\mathrm{h}}$ and $\mathrm{s}$. This change occurred in the majority of the Hlai languages irregardless of their relationship, and must therefore have been easily diffused. Registrogenesis is the development of high and low registers in correlation with the original voicing status (voiced or voiceless) of initial consonants. It only occurred in a subset of Hlai languages, but probably originated with Hainanese, and spread across contiguous languages, sometimes occurring in one language but failing to occur in another closely related language. It would therefore be imprudent to suggest these changes as criteria for subgrouping, but they fail to obscure the picture if they are identified as areal changes which are easily diffused.

It has sometimes been the case that a rarer kind of change has preceded a more general type of change, thereby removing the environment for the latter change. An example of this can be seen in the reflexes of the Proto-Hlai initial $* \mathrm{t}^{\mathrm{h}}$ : 
Proto-Hlai $\quad$ Hlai language data

$* \mathrm{t}^{\mathrm{h}}$

$\begin{array}{llllll}\text { ts }^{\mathrm{h}} & \text { ts }^{\mathrm{h}} & \text { ts }^{\mathrm{h}} & \text { ts }^{\mathrm{h}} & \text { ts }^{\mathrm{h}} & \text { ts }^{\mathrm{h}} \\ \mathrm{h} & \mathrm{h} & \text { ts }^{\mathrm{h}} & \text { ts }^{\mathrm{h}} & \text { ts }^{\mathrm{h}} & \text { ts }^{\mathrm{h}}\end{array}$

While the common change from $* t \int^{\mathrm{h}}$ to $t \mathrm{~s}^{h}$ has occurred in most languages, a different and lessexpected kind of change occurred in Cunhua and Nadouhua. The best explanation which can be offered for this is that a single change from $* \int^{\mathrm{h}}$ to $h$ occurred in the direct ancestor of Cunhua and Nadouhua (Northwest Central Hlai), after which the change from $* t \int^{h}$ to $t s^{h}$ diffused throughout the remaining subgroups and daughter languages at some later point.

Another example can be seen in the following reflexes of PHI * $\mathrm{lj}$ (the use of bold font indicates low register - see chapter two):

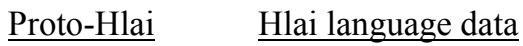

\begin{tabular}{llllll|}
$z$ & $z$ & $z$ & $t$ & $q$ & 1 \\
l & $z$ & $z$ & $z$ & $z$ & ts
\end{tabular}

Although there are two exceptions (Cunhua $\boldsymbol{I}$ and Yuanmen $\boldsymbol{t s}$ ), the majority of Hlai languages have undergone an identical change of * ${ }^{\mathrm{j}} \mathrm{j}$ to $z$. The Qi languages, on the other hand, all show the reflex 4 . It is therefore assumed that Proto-Qi $* 1 \mathrm{j}$ changed to $\mathrm{A}$ (via the intermediate stage of $\xi$ ) during the time that most if not all other Hlai languages still retained original $l j$. The change $* 1 j$ to $z$ then diffused throughout the remaining languages with the exception of Cunhua and Yuanmen.

As with lexical innovations, one unique phonological innovation between two languages is indicative of common descent from a mutual ancestor, but a collection of shared innovations is quite convincing. The set of innovations which characterize each subgroup are given in the next section in support of the Hlai family tree proposed below. 


\subsubsection{Subgrouping the Hlai Languages}

The subgrouping of Hlai languages adopted in this dissertation largely overlaps but is nonidentical with the traditional classification given in figure (3) above. One important change is the addition of a new language referred to here as Changjiang, following the tradition of associating a language with the area in which it is spoken. This language is closest to the one traditionally called Meifu (Xifang in Ouyang \& Zheng 1983), a term which I have retained for the subgroup, referring to the individual language itself as Moyfaw (the Hlai name from which Mandarin Meifu is derived). My Moyfaw-speaking consultants informed me that the Meifu group is generally more complex than has hitherto been implied in previous work, and that there may well be additional divisions within this group yet to be documented.

Another important difference is that I have included the northwestern languages Cunhua and Nadouhua in the Hlai family tree. The affiliation of these two languages has complex underpinnings, due to the fact that both languages are spoken by populations which are suspected to be of mixed Hlai-Chinese ancestry, and who currently consider themselves to be more ethnically aligned with the Chinese than the Hlai. Both Cunhua and Nadouhua have significant Chinese components of their vocabulary which are not found in the other Hlai languages, which supports the hypothesis of mixed ancestry and the intimate language contact which would have accompanied it ${ }^{1}$. However, in both cases, the core of the vocabulary is undeniably Hlai, a fact which indicates to me that if they are to be classified, it should be with the other Hlai languages.

Finally, I have broken up the traditional Ha subgroup into individual constituents, as there is sufficient phonological evidence to indicate that Bouhin, Ha Em, and Lauhut should be considered independent groups which do not share common innovations with each other. In fact, there is good reason to believe that the first primary split in the Hlai family is between Bouhin and all other languages. Ostapirat (1993) arrived at a similar conclusion, and separated Bouhin from the rest of the Ha group; he did not redo the classification as drastically as what is proposed here, and left it as a primary branch within what he called Southern Hlai, as opposed to a primary branch of Hlai proper. What actually seems to be

\footnotetext{
${ }^{1}$ Ouyang (1998) includes an excellent discussion of this issue for Cunhua (in Mandarin).
} 
the case is that the Hlai groups which were not in contact with other more recent (primarily Chinese) immigrants at the north-northeast contact zone on Hainan were grouped together under the cover-term $\mathrm{Ha}$; the other traditional groups (Qi, Run, and Meifu) were in contact with non-Hlai immigrants, and thereby recognized individually.

The Hlai phylogenetic tree argued for in this dissertation is given in (17), with alternative language names found in the literature given below the names used here (subgroup labels are explained below):

Figure 2: Hlai Phylogenetic Tree

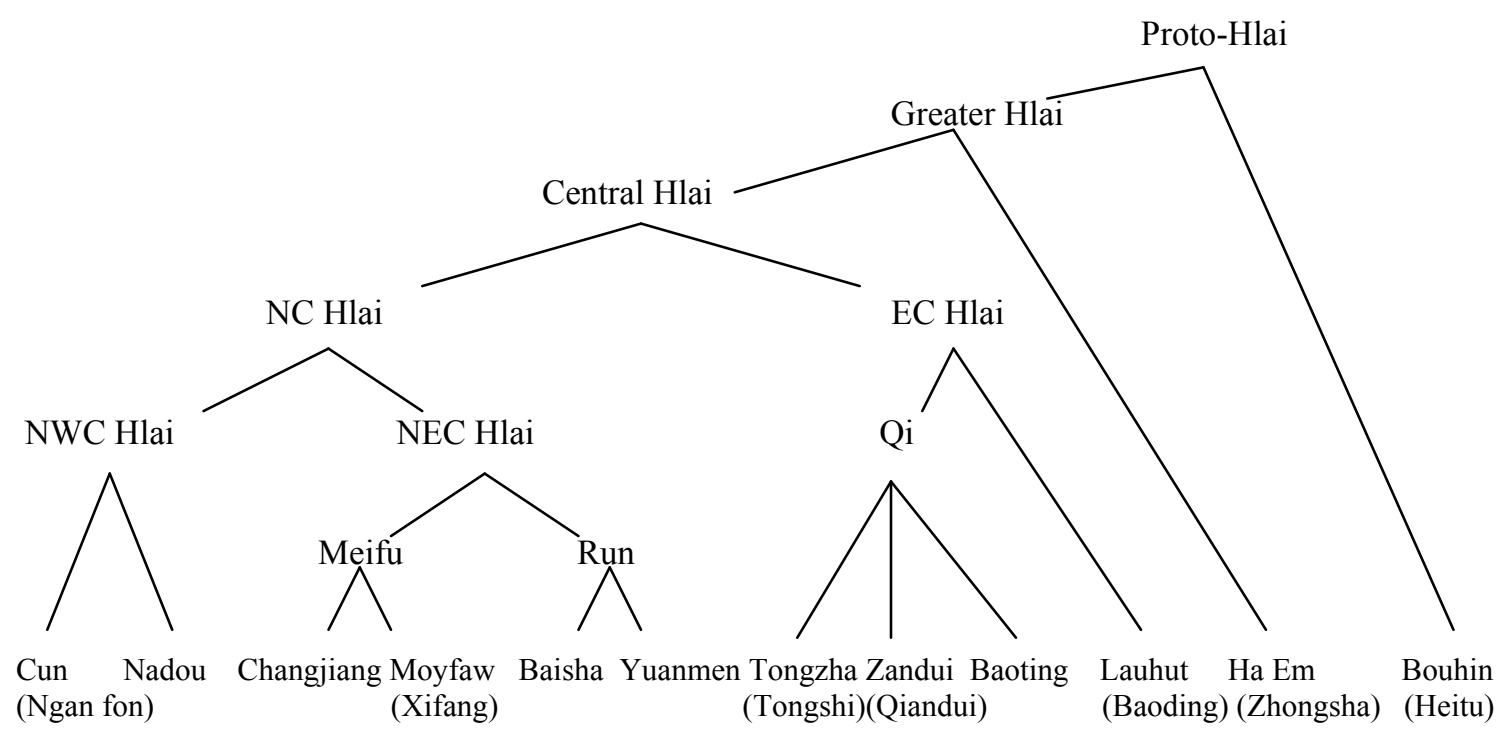

The specific language locations are given in the map below, adapted from Edmondson \& Solnit (1988). The only language not shown explicitly is Nadouhua, which is spoken close to the northwestern coast in Dongfang. This map is deceptive in one sense, in that it gives the illusion that languages are restricted to a particular area, which is somewhat true of the Central Hlai languages, but not of the rest. The languages Bouhin and Ha Em are spoken in great swaths throughout the western and southern parts of the island, penetrating north as far as many of the North Central Hlai languages, which form enclaves within them. Lauhut and Tongzha also cover fairly large areas, and Jiamao, while generally spoken in the southeastern end of the island, has pockets of speakers further north.

Figure 3: Locations of the Hlai and Be languages of Hainan 


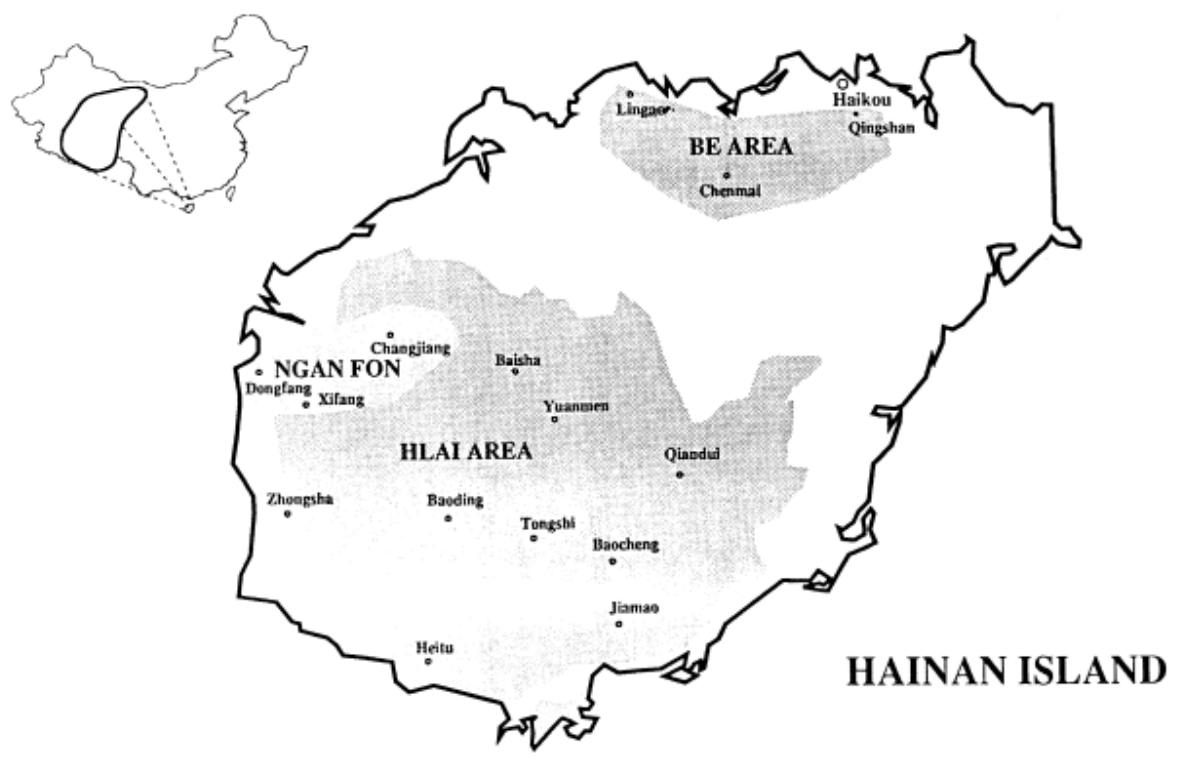

The hypothesis adopted here of the general pattern of population dispersal is the following. As the Proto-Hlai unity began to break up, the first division occurred between Bouhin and Greater Hlai. The Bouhin group was probably comparatively small, and restricted to the center of the island, from where it eventually expanded to the north and south (there are no Bouhin-speaking communities on either the western or eastern peripheries of the island).

The next schism occurred between Ha Em and Central Hlai, the latter apparently also originating in the center of the Hlai speaking area, to the east of Bouhin. Evidence for this is primarily derived from the fact that Ha Em is spoken in a large swath around the periphery of the Hlai language area, primarily along the west coast and through the southeast. Interaction between Ha Em and Jiamao must have begun at this time, as Ha Em is the most likely donor of more recent Hlai lexical items into Jiamao and probably bordered it almost exclusively before the intrusion of the Qi group into that part of the island.

The Central Hlai group then broke into (roughly) northern and eastern groups, with the North Central Hlai group overtaking area originally inhabited by Bouhin and Ha Em, and probably coming into 
contact relatively rapidly with non-Hlai Chinese immigrants to the north, leading to the genesis of Northwest Central Hlai (Cunhua and Nadouhua). Northeast Central Hlai eventually divided into the Meifu ${ }^{2}$ and Run groups.

The East Central Hlai group then bifurcated into Lauhut and Qi. Lauhut seems to have first expanded westward, and then to the north where it covered areas formerly occupied by West Central Hlai. The Qi languages eventually occupied a portion of the area which was once continuously occupied by Jiamao. It is difficult to subgroup the Qi group, as some evidence shows that Zandui groups with Tongzha, and other evidence shows that it groups with Baoting; for now, the Qi branch will be shown with a tripartite structure.

Finally, Meifu split into Changjiang (which became part of a linguistic area with Northwest Central Hlai), and Moyfaw (which aligned more closely with Run). The Run group itself divided into Baisha and Yuanmen, due at least partly to contact with Tongzha, with which Yuanmen shares several areal innovations (to be described below).

\subsubsection{Criteria for Subgrouping}

The key phonological distinctions used for subgrouping are given in this section, based on the criteria given above. Innovations used to distinguish between subgroups are shown here; differences in development between individual languages within the same subgroup will be dealt with later, as will sound changes that are the result of areal diffusion.

\subsubsection{Bouhin vs. Greater Hlai}

There are a number of important differences between Bouhin (BH) and the rest of the Hlai languages, in the categories of both initials and rimes. The most important distinction between Bouhin and

\footnotetext{
${ }^{2}$ The name Moyfaw is derived from PHl *C-mə:y t $\int^{\text {h }}$ wəw > PWCHl *?mə:y fəw 'below Chinese', or '(those) under the Chinese'.
} 
Greater Hlai (GHI) is found in the plain sonorants -- more specifically, the nasals and liquids. It will be argued in the following chapter that all plain sonorants, with the exception of the approximants, were automatically pre-aspirated at the stage of Proto Hlai. In Bouhin, the pre-aspirated sonorants either became deaspirated (in the case of the nasals) or hardened to an obstruent (in the case of the lateral). In Greater Hlai, on the other hand, the nasals all shifted to prenasalized stops (later becoming oral stops which finally devoiced) and the preaspirated lateral remained unchanged. In addition, PHl *r merged in Bouhin with PHl $*_{\varsigma}$, while it changed to a rhotic approximant in Greater Hlai:

\begin{tabular}{|c|c|}
\hline PHI & $\underline{\mathrm{BH}}$ \\
\hline *hm & $\mathrm{m}$ \\
\hline *hn & $\mathrm{n}$ \\
\hline *hn & $\mathrm{n}$ \\
\hline *hy & $\mathrm{y}$ \\
\hline${ }^{*} \mathrm{hl}$ & d \\
\hline *(h)r & r \\
\hline
\end{tabular}

In addition to this, there was a constraint which developed in Bouhin against fricatives; the defricativization of the labial fricatives are a specifically Bouhin innovation:

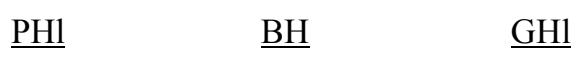

$\begin{array}{lll}*_{\mathrm{f}} & \mathrm{p}^{\mathrm{h}} & * \mathrm{f} \\ *_{\mathrm{C}-} \beta & \mathrm{p}(<\mathrm{b}<\beta) & * \mathrm{C}-\beta\end{array}$

Bouhin also shows marked differences in the rimes. In the cases of high vowels followed by a glottal element, those in Greater Hlai underwent diphthongization; the same vowels remained pure in Bouhin, only undergoing a later and more recent diphthongization:

\begin{tabular}{|c|c|c|}
\hline$\underline{\mathrm{PHl}}$ & $\underline{\mathrm{BH}}$ & $\underline{\mathrm{GHl}}$ \\
\hline$* \mathrm{i}: \mathrm{h} / \mathrm{P}$ & $\mathrm{ey}^{\mathrm{B} / \mathrm{C}} \quad\left(<\mathrm{i}:^{\mathrm{B} / \mathrm{C}}\right)$ & $* \partial y^{B / C}$ \\
\hline $\mathrm{u}: \mathrm{h} / \mathrm{?}$ & $\partial u^{\mathrm{B} / \mathrm{C}}\left(<\mathrm{w:}^{\mathrm{B} / \mathrm{C}}\right)$ & $* \partial u^{B / C}$ \\
\hline :h/2 & $\mathrm{ow}^{\mathrm{B} / \mathrm{C}}\left(<\mathrm{u}^{\mathrm{B} / \mathrm{C}}\right)$ & $*_{\partial w^{B / C}}$ \\
\hline
\end{tabular}


In rimes with a schwa nucleus closed by stops, Bouhin has undergone two innovative developments. In the short series of rimes, the nucleus was colored by the following stop (22a). This allowed the long series to shorten, and then undergo the vowel lowering which was the normal development for the short series in the other Hlai languages (22b):

$\begin{array}{lll}\underline{\mathrm{PHl}} & \underline{\mathrm{BH}} & \underline{\mathrm{GHl}} \\ \text { *əm } & \text { om } & \text { *əm } \\ \text { *əp } & \text { op } & \text { *əp } \\ \text { *ən } & \text { en } & \text { *ən } \\ \text { *ət } & \text { et } & \text { *ət } \\ \text { *əy } & \text { oy } & \text { *əy } \\ \text { *ək } & \text { ok } & \text { *ək }\end{array}$

(b)

\begin{tabular}{|c|c|c|}
\hline PHl & $\underline{\mathrm{BH}}$ & $\underline{\mathrm{GHl}}$ \\
\hline *ə:m & am & *ə:m \\
\hline *ə:p & ap & *ə:p \\
\hline *ə:n & an & *ə:n \\
\hline *a:t & at & $*_{\partial: t}$ \\
\hline $9: 1$ & an & *ə:y \\
\hline *ə:k & $\mathrm{ak}$ & $*_{\partial}: \mathrm{k}$ \\
\hline
\end{tabular}

The final crucial distinction between Bouhin and Greater Hlai, where Bouhin has remained conservative, is in the velar-closed rimes with long low nuclei. These remained unchanged in Bouhin, whereas they fronted in Greater Hlai to low front vowels.

\begin{tabular}{|c|c|c|c|}
\hline (23) & $\underline{\mathrm{PHI}}$ & $\underline{\mathrm{BH}}$ & $\underline{\mathrm{GH}}$ \\
\hline & *a:y & $\mathrm{a}: \mathrm{y}$ & ${ }^{*} \varepsilon: \eta$ \\
\hline & *a:k & a:P & $*^{*} \varepsilon: \mathrm{k}$ \\
\hline
\end{tabular}

\subsubsection{Bouhin and Ha Em vs Central Hlai}

There are certain initials in PHl which actually represent the initial of a main syllable preceded by a sesquisyllable. In general, there is a strong prohibition against liquid clusters in the Hlai languages. However, there is one exception, that being PHl *p-l. In Bouhin and Ha Em (HE), the initial consonant debuccalized and merged with PHI *C-1. In Central Hlai, on the other hand, the two consonants merged into a cluster (24a).

There are another set of initials which included coarticulated glides at the stage of Proto-Hlai. Of the three initials which had labiovelar glides, these were deleted in two of them (24b) (with the third, PHI *hyw, merging with *hw as it did in most other languages). 
In several other instances where a PHl presyllable contained a high vowel, it was merely dropped in Bouhin and Ha Em along with the presyllable initial; however, in Central Hlai, a form of metathesis called vocalic transfer occurred in which the preceding high vowel became realized as a secondary articulation on the main consonant, prior to the deletion of the presyllable itself (24c). This metathesis, as an innovation, is the most important diagnostic feature of the Central Hlai group:

\section{$\underline{\mathrm{PHl}}$}

(a)

(b)

$$
\begin{aligned}
& { }^{* \mathrm{p}-\mathrm{l}} \\
& { }^{*} \mathrm{t}^{\mathrm{h}} \mathrm{w} \\
& { }^{*} \mathrm{Cuyw}
\end{aligned}
$$

(c)

$\begin{array}{ll}* \text { Cur } & \text { 厂 } \\ * \text { Cur } & \text { 厂 }\end{array}$

*Cif

*Cuh

$*^{*} \mathrm{Ci}$ ?

$* \mathrm{Cu}$ ?
$\underline{\mathrm{BH}}$

1

$\mathrm{ts}^{\mathrm{h}}$

y

c

c

$\mathrm{h}$

$\mathrm{h}$

$?$

$?$
$\underline{\mathrm{HE}}$

$1 \quad * \mathrm{pl}$

ts $^{\mathrm{h}}$

y

c

g

$\mathrm{h}$

$\mathrm{h}$

$?$

$?$
$\underline{\mathrm{CHl}}$

*pl

$* \mathrm{t} \int^{\mathrm{h}} \mathrm{w}$

*Cunw

*Curw

*Cusw

${ }^{*}$ Cifj

*Cufiw

$* 2 \mathrm{j}$

$* ? \mathrm{w}$

\subsubsection{East Central Hlai vs North Central Hlai}

There are three main criteria which distinguish East Central Hlai (ECHl) from North Central Hlai (NCHl). One of the most important of these is the distinct developments of $\mathrm{PHl}{ }^{*} \mathrm{Cif}$ and ${ }^{*} \mathrm{Cuf}$, which were preserved in $\mathrm{ECHl}$ but were reinterpreted as preglottalized nasals in $\mathrm{NCHl}$ :

$\begin{array}{llll}\underline{\text { PHl }} & \underline{\mathrm{CHl}} & \underline{\mathrm{ECHl}} & \text { NCHl } \\ *_{\text {Cif }} & *_{\text {Cifj }} & *_{\text {Cifj }} & * \text { ? } \\ *_{\text {Cuf }} & *_{\text {Cufw }} & *_{\text {Cufw }} & * \text { ?pw }\end{array}$

A chain shift occurred in $\mathrm{NCHl}$ involving the rimes with high vowels, where rimes with long high vowels were shortened, and those with short high vowels were lowered to mid vowels; both categories were preserved in $\mathrm{ECHI}$ : 
(26)

\begin{tabular}{|c|c|c|c|}
\hline PHl & $\mathrm{CHl}$ & ECHI & $\underline{\mathrm{NCHl}}$ \\
\hline$*_{\mathrm{i}} \mathrm{C}$ & $*_{\mathrm{i}: \mathrm{C}}$ & $*_{\mathrm{i}: \mathrm{C}}$ & $*_{\mathrm{iC}}$ \\
\hline *u:C & *u:C & *u:C & ${ }^{*} \mathrm{uC}$ \\
\hline${ }^{*} \mathrm{u}: \mathrm{C}$ & $*_{\mathrm{u}: \mathrm{C}}$ & *u:C & $*_{\mathrm{uC}}$ \\
\hline$*_{\mathrm{iC}}$ & $*_{\mathrm{iC}}$ & $*_{\mathrm{iC}}$ & $*_{\mathrm{eC}}$ \\
\hline${ }^{*} \mathrm{wC}$ & $*^{\mathrm{uC}}$ & ${ }^{*} \mathrm{uC}$ & $* \gamma \mathrm{C}$ \\
\hline$*_{\mathrm{uC}}$ & $*_{\mathrm{uC}}$ & $* u C$ & $*_{\mathrm{oC}}$ \\
\hline
\end{tabular}

Finally, in ${ }^{\circ} \mathrm{oC}$ rimes where the coda was velar, the nucleus was lowered in $\mathrm{NCHl}$ but preserved in $\mathrm{ECHl}$ :

\begin{tabular}{|c|c|c|}
\hline PHl & $\mathrm{CHl}$ & ECHl \\
\hline *oy & *oy & *oy \\
\hline$*_{\mathrm{ok}}$ & $*_{\mathrm{ok}}$ & $*_{\mathrm{ok}}$ \\
\hline
\end{tabular}

\subsubsection{Lauhut vs Qi}

One key difference between the Lauhut and Qi subgroups is that the Qi languages all underwent registrogenesis (discussed more thoroughly in chapter two), which never occurred in Lauhut. Since registrogenesis most likely occurred fairly late in the history of the Hlai languages, however, it should not be relied on as a subgrouping criterion.

In the initials, there are four main differences between Lauhut and Qi, all involving coarticulated initials. First, the PHl affricate $* \mathrm{t}^{\mathrm{h}} \mathrm{w}$ became $f$ in Lauhut but merely reduced to $* \mathrm{t}^{\mathrm{h}}$ in the Qi languages. In the case of * $\mathrm{j}$, Lauhut simplified this to the glide *j (then becoming $\mathrm{z}$ ), while in Qi it became a voiced lateral fricative * ${ }^{*} . \mathrm{PHl}{ }^{*} \mathrm{Cirj}$ and ${ }^{*} \mathrm{Curw}$ became ${ }^{*} \mathrm{r}$ and ${ }^{*}$ rw respectively in ECHl, and both underwent fortition in Qi to *d and *v respectively, whereas the former remained $r$ and the latter simplified to $w$ in Lauhut. 
(28)

\begin{tabular}{|c|c|c|}
\hline PHI & ECHl & $\underline{\mathrm{LH}}$ \\
\hline$* t \int^{h} w$ & $* \mathrm{t}^{\mathrm{h}} \mathrm{W}$ & f \\
\hline$* 1 \mathrm{j}$ & $* \mathrm{lj}$ & $\mathrm{z}$ \\
\hline *Cirj & $*_{\mathrm{r}}$ & $\mathrm{r}$ \\
\hline *Curw & $*_{\mathrm{rw}}$ & $\mathrm{v}(<\mathrm{w})$ \\
\hline
\end{tabular}

The nucleus of the PHl rime *a:y backed and rounded in Lauhut, but lowered in Qi.

$\begin{array}{llll}\underline{\mathrm{PHl}} & \underline{\mathrm{ECHl}} & \underline{\mathrm{LH}} & \underline{\mathrm{Qi}} \\ \text { *ə:y } & \text { *ə:y } & \text { о:y } & \text { *a:y }\end{array}$

Final palatal stops in Qi depalatalized (as they did in Bouhin and Ha Em), but were preserved in Lauhut.

(30) Development of palatal finals in ECHl

\begin{tabular}{|c|c|c|c|}
\hline$\underline{\mathrm{PHl}}$ & $\underline{\mathrm{ECHl}}$ & $\underline{\mathrm{LH}}$ & $\underline{\text { Qi }}$ \\
\hline$*^{*} \mathrm{u}: \mathrm{n}$ & ${ }^{*} \mathrm{u}: \mathrm{n}$ & $\mathrm{u}: \mathrm{n}$ & $*^{u} \mathrm{u}: \mathrm{n}$ \\
\hline$*_{\mathrm{u}: \mathrm{c}}$ & ${ }^{*} \mathrm{u}: \mathrm{c}$ & $\mathrm{u}: \mathrm{c}$ & $*_{\mathrm{u}: \mathrm{t}}$ \\
\hline *un & *un & un & *un \\
\hline$* u c$ & $*_{\mathrm{uc}}$ & uc & $*$ ut \\
\hline *әn & *әр & an & $*_{\text {an }}$ \\
\hline$* \partial c$ & $* \partial c$ & $\mathrm{ac}$ & $*_{\text {at }}$ \\
\hline *a:p & *a:n & $\mathrm{a}: \mathrm{n}$ & $*_{\mathrm{a}: \mathrm{n}}$ \\
\hline *a:c & *a:c & $\mathrm{a}: \mathrm{c}$ & $*_{\mathrm{a}: \mathrm{t}}$ \\
\hline
\end{tabular}

Finally, rimes with long front high nuclei which were closed by velars diphthongized in Qi, but remained pure in Lauhut:

$\begin{array}{llll}\underline{\mathrm{PHl}} & \underline{\mathrm{ECHl}} & \underline{\mathrm{LH}} & \text { Qi } \\ *_{\mathrm{i}: \mathrm{y}} & *_{\mathrm{i}: \mathrm{y}} & \mathrm{i}: \mathrm{y} & *_{\mathrm{i} \partial \mathrm{y}} \\ *_{\mathrm{i}: \mathrm{k}} & *_{\mathrm{i}: \mathrm{k}} & \mathrm{i}: \mathrm{k} & *_{\mathrm{i} j \mathrm{k}}\end{array}$




\subsubsection{Northwest Central Hlai versus Northeast Cenral Hlai}

There are three criteria which allow the subgrouping of North Central Hlai into Northwest Central Hlai (NWCHl) and Northeast Central Hlai (NECHl). The first is the divergent development of PHl * $\mathrm{t}^{\mathrm{h}}$, which remained an affricate in NECHl, but which deaffricated to a fricative in NWCHl (a parallel development with Proto-Be) (32a). While $\mathrm{PHl} *$ s remained alveolar in NECHl, it interdentalized to * $\theta$ in $\mathrm{NWCHl}(32 \mathrm{~b})$. Finally, NWCHl is also the only subgroup to preserve evidence of $\mathrm{PHl}$ *hyw, where it became * $\mathrm{yw}$, as opposed to other languages in which it merged with PHl *hw (32c):

$\underline{\mathrm{PHl}} \quad \underline{\mathrm{NCHl}} \quad \underline{\mathrm{NWCHl}} \quad \underline{\mathrm{NECHl}}$

(a) $\quad * \int^{\text {h }}$

(b) $\quad * \mathrm{~s}$

(c) *hyw

$\begin{array}{lll}* \mathrm{t}^{\mathrm{h}} & *_{\mathrm{x}} & *_{\mathrm{t}}^{\mathrm{h}} \\ *_{\mathrm{s}} & *_{\theta} & *_{\mathrm{s}} \\ * \mathrm{hyw} & *_{\mathrm{yw}} & *_{\mathrm{w}}\end{array}$

The one distinction in rimes between the two branches is in $\mathrm{PHl} *$ in, the coda of which alveolarized in NECHI, but remained velar in NWCHI:

$\begin{array}{llll}\underline{\mathrm{PHl}} & \underline{\mathrm{NCHl}} & \underline{\mathrm{NWCHl}} & \underline{\mathrm{NECHl}} \\ *_{\text {in }} & *_{\text {en }} & *_{\text {en }} & *_{\text {en }}\end{array}$

\subsubsection{Meifu vs Run}

There are four different lines of evidence supporting the distinction between Meifu and Run in the initials. The first is $\mathrm{PH} * \mathrm{~s}$, which remained *s in Meifu but which affricated to ${ }^{*} \mathrm{ts}^{\mathrm{h}}$ in Run. The PHl palatalized coronals $* \mathrm{lj}$ and ${ }^{*} \mathrm{Cirj}$ developed differently; the first simplified to $*_{\mathrm{j}}$ in Meifu but remained unchanged in Run, whereas the latter depalatalized to the variants *d and *r in Meifu, but was in variation in Run between ${ }^{*} \mathrm{~d}$ and palatalized $*$ rj. Finally, NECHl ${ }^{*} \mathrm{C}-\beta$ and ${ }^{*} \mathrm{Curw}$ both simplified to $*$ ?w in Meifu, but became labial fricatives in Run: 


\begin{tabular}{|c|c|c|c|}
\hline PHI & NECHI & $\underline{\text { Meifu }}$ & $\underline{\text { Run }}$ \\
\hline$*_{\mathrm{s}}$ & $*_{\mathrm{s}}$ & $*_{\mathrm{s}}$ & $* \mathrm{ts}^{\mathrm{h}}$ \\
\hline$* \mathrm{lj}$ & $* 1 \mathrm{j}$ & $*_{j}$ & $* 1 \mathrm{j}$ \\
\hline${ }^{*}$ Cirj & $* \mathrm{rj}$ & $* \mathrm{~d} \sim \mathrm{r}$ & $* d \sim r j$ \\
\hline${ }^{*} \mathrm{C}-\beta$ & ${ }^{*} \mathrm{C}-\beta$ & $* ? \mathrm{w}$ & $* \beta$ \\
\hline *Cur & *Curw & $* ? \mathrm{w}$ & $*_{\mathrm{V}}$ \\
\hline
\end{tabular}

There are several rimes which underwent diphthongization in Run but which retained long pure nuclei in Meifu (35a). In addition, $\mathrm{PHl} *^{*}$ :k underwent the same changes as other ${ }^{*}$ ə:C rimes in Meifu, but shortened idiosyncratically in Run (35b):

$\underline{\text { PHI }} \underline{\text { NECHl }} \quad \underline{\text { Meifu }} \quad \underline{\text { Run }}$

(a)

\begin{tabular}{|c|c|c|c|}
\hline *ə:m & *o:m & $*_{\mathrm{o}}: \mathrm{m}$ & *uam \\
\hline *ə:p & $*^{\prime} \mathrm{p:p}$ & $*_{o: p}$ & *uap \\
\hline *ə:y & *o:y & $*_{0}: y$ & $*_{\text {uay }}$ \\
\hline *a:y & ${ }^{*} \varepsilon: \eta$ & $*_{e}: y$ & *ian \\
\hline *ə:k & *o:k & $*_{\mathrm{o}: \mathrm{k}}$ & *o? \\
\hline
\end{tabular}

\subsubsection{Summary}

In summary, there are a large number of sound changes (both initials and rimes) which separate Bouhin from the rest of the Hlai languages (Greater Hlai), and provide evidence that this is the first-order split in the Hlai family tree. The next split is between Ha Em and the rest of Hlai (Central Hlai), the principal criteria for which is the vocalic transfer which occurred in the latter. Central Hlai can be divided between North Central Hlai, in which rhinoglottophilia led to the merger of *Cifij and *Cufw with *C-n and ${ }^{*} \mathrm{C}-\mathrm{yw}$, and where the length difference in the high vowel rimes was replaced by a height difference, and East Central Hlai, in which neither of these changes occurred.

Within East Central Hlai, a number of sound changes distinguish Lauhut and Qi in both initials and rimes. The three Qi languages are not subgrouped here, due to the difficulty in separating inherited from diffused sound changes. There is a salient split in North Central Hlai between Northwest Central Hlai 
and Northeast Central Hlai, the former being distinguished primarily by the unique developments of PHl $* t \int^{\text {h }},{ }^{*}$, and *hyw, but also by a handful of other changes. Finally, Meifu and Run are distinguished by developments primarily in the approximants and the non-high rimes with grave codas.

No tonal information has been used in subgrouping. This is because, despite the existence of three distinct tonogenetic groups of languages (see chapter three), it appears to be the case that the tone system of a language developed largely in accordance with the linguistic area in which it participated, possibly long after the original breakup of Proto-Hlai. This assertion is in fact very plausible, in view of the degree to which it appears tonogenesis diffused throughout a large part of Southeast Asia, often cross-cutting language (and family) boundaries (see Enfield (2005) for an overview on diffusion in Southeast Asia).

\subsection{Linguistic Area and Contact Relationships}

This section is devoted to providing evidence for contact between various Hlai subgroups. This contact is most often betrayed through irregular reflexes which exist in lexical items in one language, which can be plausibly attributed to borrowing from a neighboring language in which the development was regular.

\subsubsection{Bouhin and Ha Em}

There is evidence for a period of contact, probably longstanding, between Bouhin and Ha Em beginning sometime after the subsequent changes which occurred in both after the initial breakup of PHI into Bouhin and Greater Hlai ${ }^{3}$. The direction of influence has largely been from Ha Em to Bouhin, which is evident through a number of items in Bouhin which show irregular correspondences in initials and/or rimes and/or tones, but which have exact matches in Ha Em.

\footnotetext{
${ }^{3}$ This was underscored by one of my two Bouhin consultants, whose father spoke Bouhin but whose mother spoke Ha Em, indicating that these two groups of speakers remain in close contact which includes intermarriage, at least in some areas.
} 


\subsubsection{Ha Em to Bouhin}

At some point in the individual development of Bouhin, there was a change from $\mathrm{PHl} * \mathrm{f}$ to aspirated $p^{h}$, and with one exception, all Bouhin words with this reflex appear to be otherwise regular. After this change, there were apparently a number of words borrowed from Ha Em beginning with $f$, which still obey the constraint against fricatives, but lack aspiration:

\begin{tabular}{|c|c|c|c|c|}
\hline Gloss & $\underline{\mathrm{PHl}}$ & $\underline{\mathrm{Ha}} \mathrm{Em}$ & Bouhin & Expected Bouhin \\
\hline hold in mouth & * fə:m & fo: $m^{1}$ & po: $m^{1}$ & $\mathrm{p}^{\mathrm{h}} \mathrm{am}^{1}$ \\
\hline side & $*_{\text {fa:y }}$ & fe: $y^{1}$ & pe: $\eta^{1}$ & $\mathrm{p}^{\mathrm{h}} \mathrm{a}: \mathrm{n}^{1}$ \\
\hline shoulder pole & $*_{\mathrm{fi}: \mathrm{k}}$ & fi: $: ?^{7}$ & pi: $?^{9}$ & $\mathrm{p}^{\mathrm{h}} \mathrm{i}: ?^{7}$ \\
\hline
\end{tabular}

In (37), there is an unexpected reflex $f$, which otherwise only appears in Bouhin in Chinese loanwords. The fact that these initials did not undergo the shift to $p$ in Bouhin could indicate that they have come into the language quite recently:

\begin{tabular}{|c|c|c|c|c|}
\hline Gloss & PHl & Ha Em & Bouhin & Expected Bouhin \\
\hline $\begin{array}{l}\text { k.o. reed } \\
\text { vicious }\end{array}$ & $\begin{array}{l}*_{\text {fa: }} \\
*_{\text {fan }}\end{array}$ & $\begin{array}{l}\text { fa: }{ }^{1} \\
\text { fan }^{1}\end{array}$ & $\begin{array}{l}\text { fa: } w^{1} \\
\text { fan }^{1}\end{array}$ & $\begin{array}{l}\mathrm{p}^{\mathrm{h}} \mathrm{a} \cdot \mathrm{w}^{1} \\
\mathrm{p}^{\mathrm{h}} \mathrm{en}^{1}\end{array}$ \\
\hline
\end{tabular}

The examples in (38) are of a variety of PHl preaspirated nasal initials, all of which are normally reflected in Bouhin by plain nasals, but which instead begin with plain stops, the normal reflex in Ha Em (and all other Hlai languages). These irregular initials are accompanied by irregular rimes in all but two cases:

\begin{tabular}{|c|c|c|c|c|}
\hline$\underline{\text { Gloss }}$ & $\underline{\mathrm{PHl}}$ & $\underline{\mathrm{Ha}} \mathrm{Em}$ & Bouhin & Expected Bouhin \\
\hline stupid & *hmə:y & po: $y^{1}$ & po: $y^{1}$ & ma:y ${ }^{1}$ \\
\hline widow(er) & *hmə:y? & po: $y^{3}$ & po: $y^{3}$ & ma: $y^{3}$ \\
\hline tadpole & *hnu:h & tow $^{2}$ & tow $^{2}$ & now $^{2}$ \\
\hline pocket & *hni:h & $\operatorname{tay}^{2}$ & $\operatorname{tay}^{2}$ & $n e y^{2}$ \\
\hline chop & *hnək & $\operatorname{tak}^{7}$ & $\operatorname{tak}^{7}$ & nok $^{7}$ \\
\hline carp & *hpa:t & tsa: $t^{7}$ & tsa: $t^{7}$ & na: $t^{7}$ \\
\hline
\end{tabular}


The following examples show cases where $\mathrm{PHl} * \mathrm{hl}$ is reflected by $t$ instead of the expected $d$; in these cases, I suggest that Bouhin, lacking the phoneme f, substituted an s for Ha Em f, which then underwent the regular Bouhin shift to $t$ :

\begin{tabular}{|c|c|c|c|}
\hline Gloss & $\underline{\mathrm{PHl}}$ & $\underline{\mathrm{Ha}} \mathrm{Em}$ & Bouhin \\
\hline hear it said & *hli:y & di: $y^{1}$ & ti: $\eta^{1}\left(<\operatorname{si}: \eta^{1}\right)$ \\
\hline plague & *hlu:n? & fu: $n^{3}$ & tu: $n^{3}\left(<\operatorname{su}: n^{3}\right)$ \\
\hline armspan & *hla:nf & fa: $n^{2}$ & ta: $\mathrm{n}^{2}\left(<\mathrm{sa}: \mathrm{n}^{2}\right)$ \\
\hline
\end{tabular}

Finally, the examples below are Bouhin forms which have $g$ as the reflex of PHl rhotics, instead of the expected $r$; there are several words in this category, and the examples below have been chosen because they have irregular rimes in Bouhin as well, reinforcing their identification as loans:

\begin{tabular}{|c|c|c|c|c|}
\hline Gloss & $\underline{\mathrm{PHl}}$ & $\underline{\mathrm{Ha}} \mathrm{Em}$ & Bouhin & Expected Bouhin \\
\hline pile up & *rə:p & go: $p^{7}$ & go: $p^{7}$ & $\operatorname{rap}^{7}$ \\
\hline cool (water) & *ron & $\operatorname{gan}^{1}$ & $\operatorname{gan}^{1}$ & $\operatorname{ren}^{1}$ \\
\hline not & *Curi:h & gay $^{2}$ & gay $^{2}$ & $\mathrm{rey}^{2}$ \\
\hline
\end{tabular}

There is one example where the Bouhin form indicates borrowing from $\mathrm{Ha} \mathrm{Em}$ in the rime $\mathrm{PHl}$ category *ok:

\begin{tabular}{|c|c|c|c|c|}
\hline$\underline{\text { Gloss }}$ & PHl & $\underline{\mathrm{Ha} E m}$ & $\underline{\text { Bouhin }}$ & Expected Bouhin \\
\hline drip (cntr) & ${ }^{*} \mathrm{k}^{\mathrm{h}} \mathrm{ok}$ & $\mathrm{k}^{\mathrm{h}} \mathrm{uk}^{7}$ & $\mathrm{k}^{\mathrm{h}} \mathrm{k}^{7}$ & $\mathrm{k}^{\mathrm{h}} \mathrm{ok}^{7}$ \\
\hline
\end{tabular}

There are a number of examples where $\mathrm{PHl}{ }^{*}$ : $\mathrm{C}$ has a reflex in Bouhin regular for $\mathrm{Ha} \mathrm{Em}$, but not for Bouhin itself:

\begin{tabular}{|c|c|c|c|}
\hline$\underline{\text { Gloss }}$ & $\underline{\mathrm{PH}}$ & $\underline{\mathrm{Ha} E m}$ & Bouhin \\
\hline bee & *kə:y & ko: $y^{1}$ & ko: $y^{1}$ \\
\hline hold in mouth & $*$ fə:m & fo: $m^{1}$ & po: $\mathrm{m}^{1}$ \\
\hline love (enjoy) & *?ə:p & 1o: $\mathrm{p}^{7}$ & 3o: $p^{7}$ \\
\hline sleep & *tढว:n & tso: $n^{1}$ & tso: $\mathrm{n}^{1}$ \\
\hline (tie) tight & $*_{\text {fə:t }}$ & fo: $t^{7}$ & $\mathrm{p}^{\mathrm{h}} \mathrm{o}: \mathrm{t}^{7}$ \\
\hline
\end{tabular}




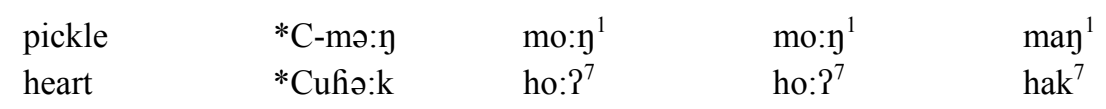

There are also several examples of $\mathrm{PHl} * \mathrm{a}: \mathrm{K}$, in which Bouhin would be expected to preserve the low vowel but shows a mid front vowel, of which two are given below:

\begin{tabular}{|c|c|c|c|c|}
\hline$\underline{\text { Gloss }}$ & $\underline{\mathrm{PHl}}$ & $\underline{\mathrm{Ha} E m}$ & Bouhin & Expected Bouhin \\
\hline shrimp & *Cura:y & re: $\eta^{1}$ & re: $\eta^{1}$ & ra: $y^{1}$ \\
\hline phlegm & *ha:k & he: $?^{7}$ & he: $?^{9}$ & ha: $:^{7}$ \\
\hline
\end{tabular}

Finally, there are a number of examples of $\mathrm{PHl} *_{\partial \mathrm{C}}$ appearing with unexpected reflexes in Bouhin; the vowels of these rimes were normally colored by the place of the Bouhin coda, but regularly lowered to *aC in $\mathrm{Ha} \mathrm{Em}$ :

\begin{tabular}{|c|c|c|c|c|}
\hline Gloss & $\underline{\mathrm{PHl}}$ & $\underline{\mathrm{Ha} E m}$ & Bouhin & Expected Bouhin \\
\hline empty & ${ }^{*}$ Curi:h & ray $^{2}$ & ray $^{2}$ & rey $^{2}$ \\
\hline crocodile & *ki:? & kay $^{3}$ & $\mathrm{kay}^{3}$ & key $^{3}$ \\
\hline village & *6u:? & $6 a w^{3}$ & $6 a w^{3}$ & $60 w^{3}$ \\
\hline care for & *đəр & dap $^{7}$ & dap $^{7}$ & dop $^{7}$ \\
\hline curse & $* t^{h} \partial n$ & $\mathrm{t}^{\mathrm{h}} \mathrm{an}{ }^{1}$ & $\mathrm{t}^{\mathrm{h}} \mathrm{an}{ }^{1}$ & $\mathrm{t}^{\mathrm{h}} \mathrm{e} \mathrm{n}^{1}$ \\
\hline gnat & ${ }^{*} \mathrm{C}$-mət & $\mathrm{mat}^{7}$ & $\mathrm{mat}^{7}$ & $\operatorname{met}^{7}$ \\
\hline groan & *kəy & $\operatorname{kay}^{1}$ & $\operatorname{kay}^{1}$ & $\operatorname{koy}^{1}$ \\
\hline stick to & ${ }^{*} \mathrm{p}^{\mathrm{h}} \partial \mathrm{k}$ & $\mathrm{p}^{\mathrm{h}} \mathrm{ak}^{7}$ & $\mathrm{p}^{\mathrm{h}} \mathrm{ak}^{7}$ & $\mathrm{p}^{\mathrm{h}} \mathrm{ok}^{7}$ \\
\hline
\end{tabular}

\subsubsection{Bouhin to Ha Em}

There is a limited set of examples which show Bouhin influence on Ha Em, all being of irregular intitials. It is probably significant that three of these six examples are female kinship terms, indicating a longstanding practice of intermarriage: 


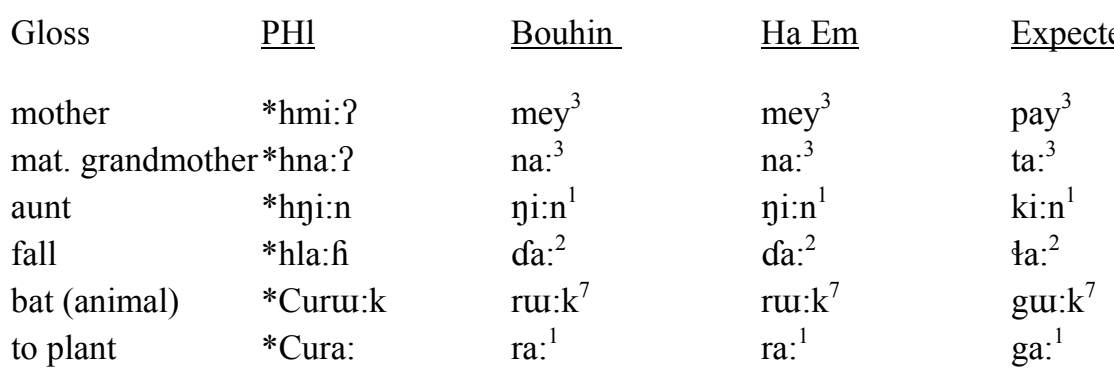

\subsubsection{Bouhin/Ha Em/Jiamao}

There are three examples shared only between Bouhin, Ha Em, and Jiamao; the Bouhin rimes in all three examples are irregular, which indicates that Jiamao was the likely donor first into Ha Em and then indirectly from Ha Em into Bouhin:

\begin{tabular}{|c|c|c|c|c|}
\hline Gloss & PHI & $\underline{\text { Jiamao }}$ & $\underline{\mathrm{Ha}} \mathrm{Em}$ & Bouhin \\
\hline exchange & *p-ləy & $60: \mathrm{k}^{7}$ & $6 o: ?^{7}$ & $60: ?^{7}$ \\
\hline well (n.) & $*^{t} \varphi^{\mathrm{h}} \mathrm{u}: \mathrm{y} ?$ & $t^{h} \circ y^{5}$ & $t^{h} u \eta^{2}$ & $t^{\mathrm{h}} u \eta^{2}$ \\
\hline be & ${ }^{*} \mathrm{C}$-mən & $\operatorname{tsay}^{1}$ & $\operatorname{tsan}^{3}$ & $\operatorname{tsay}^{3}$ \\
\hline
\end{tabular}

\subsubsection{Jiamao and Baoting}

There is more recent evidence of intimate contact between Jiamao and Baoting. This is evident through occasional forms in Baoting which show irregular correspondences, but which have a parallel in Jiamao ${ }^{4}$.

\footnotetext{
${ }^{4}$ This connection was again underscored by work with one of my two Baoting consultants, whose father spoke Baoting but whose mother spoke Jiamao.
} 


\begin{tabular}{|c|c|c|c|c|}
\hline Gloss & $\underline{\mathrm{PHl}}$ & $\underline{\text { Jiamao }}$ & Baoting & Expected Baoting \\
\hline roll around & *p-li:n & $\operatorname{lin}^{1}$ & li:n ${ }^{1}$ & pli:n ${ }^{1}$ \\
\hline non-rtns. rice & *tci:m? & tsiam $^{1}$ & tsiam $^{1}$ & tsi: $\mathrm{m}^{3}$ \\
\hline dirty & *si:k & $\mathrm{ts}^{\mathrm{h}} \mathrm{iak}^{7}$ & $\operatorname{tiak}^{9}$ & $\operatorname{tiak}^{7}$ \\
\hline solemn silence & $*$ ?ik & Pi:k ${ }^{9}$ & 2i: $\mathrm{k}^{7}$ & $\mathrm{Pik}^{7}$ \\
\hline blackhead & *Curup & $\operatorname{lup}^{8}$ & $\operatorname{lup}^{8}$ & $\operatorname{lup}^{8}$ \\
\hline grandfather & $*^{\mathrm{h}} \mathrm{u}: ?$ & pə:w ${ }^{4}$ & $\mathrm{p}^{\mathrm{h}} \partial: \mathrm{w}^{6}$ & $\mathrm{p}^{\mathrm{h}} \mathrm{aw} \mathrm{w}^{3}$ \\
\hline die out & $*$ tсәр & tsep ${ }^{7}$ & tsep $^{7}$ & $\operatorname{tsap}^{7}$ \\
\hline cloth & $*$ dəp & təp ${ }^{7}$ & dəp ${ }^{7}$ & $\mathrm{dap}^{7}$ \\
\hline alcohol & *C-na:w? & 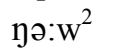 & yə:w ${ }^{5}$ & ya:w ${ }^{3}$ \\
\hline goose & *C-na:nh & ตə:n ${ }^{5}$ & yə: $n^{5}$ & ya: $n^{5}$ \\
\hline
\end{tabular}

\subsubsection{The Qi branch}

There has been a certain amount of interaction within the Qi branch in terms of contact. There seems to have been bidirectional influence between Tongzha and Zandui, and more unidirectional influence of Tongzha on Baoting. There do not seem to have been any obvious loans between Zandui and Baoting. The first set of examples in (48) are probable loanwords from Zandui into Tongzha:

\begin{tabular}{|c|c|c|c|c|}
\hline Gloss & $\underline{\mathrm{PHl}}$ & $\underline{\text { Zandui }}$ & $\underline{\text { Tongzha }}$ & Expected Tongzha \\
\hline flood & *vac & vat $^{8}$ & $\mathrm{vat}^{8}$ & $\mathrm{fat}^{8}$ \\
\hline knock & *6u:y? & buan $^{3}$ & buan $^{3}$ & $6 u: y^{3}$ \\
\hline distribute & *ku:h & ko: $:^{\circ}$ & kow $^{5}$ & $\mathrm{kaw}^{5}$ \\
\hline collapse & $* t^{h} \mathrm{u}: \mathrm{h}$ & $\mathrm{t}^{\mathrm{h}} \mathrm{O}:{ }^{5}$ & $\mathrm{t}^{\mathrm{h}} \mathrm{ow}^{5}$ & $\mathrm{t}^{\mathrm{h}} \mathrm{aw}{ }^{5}$ \\
\hline
\end{tabular}

The examples below, on the other hand, show loans from Tongzha into Zandui. The difference between the $k$ of Zandui scratch (in high register) is unexpected, as it does not show a specific correspondence to the Tongzha initial: 


\begin{tabular}{|c|c|c|c|c|}
\hline Gloss & PHl & Tongzha & Zandui & Expected Zandui \\
\hline kiss & * rju:c & tu: $t^{8}$ & tu: $t^{8}$ & $t^{h} u: t^{8}$ \\
\hline scratch & *runf & $\operatorname{gun}^{2}$ & $\operatorname{kun}^{5}$ & hun ${ }^{2}$ \\
\hline aim at & *rən & $\operatorname{gan}^{4}$ & $\operatorname{gan}^{4}$ & $\operatorname{han}^{4}$ \\
\hline cricket & *Curu:y? & fu: $\eta^{6}$ & fu: $\eta^{6}$ & fuan $^{6}$ \\
\hline spider & $* k^{h} \mathrm{u}: \mathrm{y}$ & $\mathrm{k}^{\mathrm{h}} \mathrm{u}: \mathrm{y}^{1}$ & $k^{h} u: \eta^{1}$ & $\mathrm{k}^{\mathrm{h}} \mathrm{uay}^{1}$ \\
\hline $\operatorname{mix}$ & *гәр & $\operatorname{rop}^{8}$ & $10 p^{9}$ & $1 a p^{8}$ \\
\hline care for (sprout) & $*$ dəp & dop $^{7}$ & dop $^{7}$ & $\mathrm{dap}^{7}$ \\
\hline
\end{tabular}

The following are examples of loans from Tongzha into Baoting:

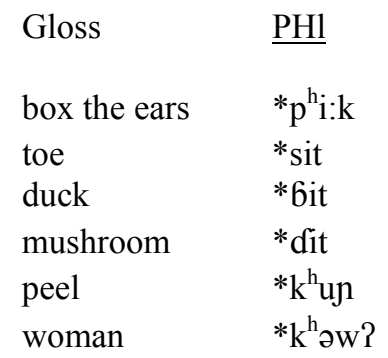

Tongzha

$\mathrm{p}^{\mathrm{h}} \mathrm{ia}^{7}$

tet $^{7}$

bet $^{7}$

$$
\operatorname{det}^{7}
$$

$\mathrm{k}^{\mathrm{h}} \mathrm{un}^{1}$

$\mathrm{k}^{\mathrm{h}} \mathrm{O}:^{5}$
Baoting

$\mathrm{p}^{\mathrm{h}}{ }_{\mathrm{ia}} \mathrm{P}^{7}$

tet $^{7}$

bet $^{7}$

$\operatorname{det}^{7}$

$\mathrm{k}^{\mathrm{h}} \mathrm{un}^{1}$

$\mathrm{k}^{\mathrm{h}} \mathrm{o}{ }^{5}$
Expected Baoting

$\mathrm{p}^{\mathrm{h}_{\text {iak }}}{ }^{7}$

tit $^{7}$

bit $^{7}$

$\mathrm{dit}^{7}$

$\mathrm{k}^{\mathrm{h}} \mathrm{un}^{1}$

$\mathrm{k}^{\mathrm{h}} \mathrm{0}:^{5}$

Finally, there is one example of a Jiamao loan into Zandui:

$\begin{array}{lllll}\text { Gloss } & \underline{\text { PHl }} & \underline{\text { Jiamao }} & \underline{\text { Zandui }} & \text { Expected Zandui } \\ \text { steel } & \text { Cufa:c } & \text { hua? }^{7} & \text { hua? }^{7} & \text { va:t }^{7}\end{array}$

\subsubsection{Lauhut and Moyfaw}

There are several lines of evidence which support a contact zone shared by Lauhut and the Meifu group. The first of these is the pattern of tone contour development, which in this case also included $\mathrm{Ha}$ Em: 
$(52)$

\begin{tabular}{|c|c|c|c|c|}
\hline Tone Category & $\underline{\mathrm{Ha} E m}$ & $\underline{\text { Lauhut }}$ & Moyfaw & Changjiang \\
\hline A & 53 & 53 & 53 & 53 \\
\hline B & 55 & 55 & 55 & 44 \\
\hline $\mathrm{C}$ & 11 & 11 & 24 & 22 \\
\hline D & 55 & 55 & 55 & $15 ?$ \\
\hline
\end{tabular}

The Ha Em and Lauhut tonal developments are identical, indicating a high degree of synchronization in the mechanisms which led to the genesis of contour tones. It can be seen that Moyfaw is nearly identical, the only exception being in category C. Changiiang is a bit less similar, with identical developments in category A and perhaps $\mathrm{B}$, but differences in $\mathrm{C}$ and $\mathrm{D}$. One explanation for this pattern is that the categories which show different contour tone patterns had already developed these before contact intensified; those which are the same, or nearly so, may have developed later and in tandem. This will be discussed more in chapter 3.

There are also several examples of unexpected reflexes in Moyfaw which can be explained as borrowings from Lauhut. The following are examples of initials which are unexpected in Moyfaw, but which have parallels in Lauhut:

\begin{tabular}{|c|c|c|c|c|}
\hline$\underline{\text { Gloss }}$ & $\underline{\mathrm{PH} 1}$ & $\underline{\text { Lauhut }}$ & Moyfaw & Expected Moyfaw \\
\hline arrow & $*$ si:p & ti:p $p^{7}$ & $\operatorname{tip}^{7}$ & $\operatorname{sip}^{7}$ \\
\hline calf & $*_{\text {r jin? }}$ & rej ${ }^{3}$ & ren ${ }^{3}$ & $\operatorname{ten}^{3}$ \\
\hline leopard & *Cufu:y? & hu: $y^{3}$ & huy $^{3}$ & yuy $^{3}$ \\
\hline clear & *ra:w & ga: $w^{1}$ & ya:w ${ }^{1}$ & $x a: w^{1}$ \\
\hline cast (sand) & *Curuy & vuy $^{1}$ & vuy $^{1}$ & yuy $^{1}$ \\
\hline
\end{tabular}

In addition to the examples above, the following examples can be added which show unexpected realizations of rimes: 


\begin{tabular}{|c|c|c|c|c|}
\hline Gloss & $\underline{\mathrm{PHl}}$ & $\underline{\text { Lauhut }}$ & Moyfaw & Expecte \\
\hline unhusked rice & ${ }^{*}$ C-mi:n? & $\mathrm{mi}: \mathrm{n}^{3}$ & $\min ^{3}$ & $\min ^{3}$ \\
\hline connect & $*_{\mathrm{t} c}^{\mathrm{h}} \mathrm{u}: \mathrm{n} ?$ & $\mathrm{ts}^{\mathrm{h}} \mathrm{u}: \mathrm{n}^{3}$ & $\mathrm{ts}^{\mathrm{h}} \mathrm{un}^{3}$ & $\mathrm{ts}^{\mathrm{h}} \mathrm{u \eta}^{3}$ \\
\hline tumor & $*$ fə:n & fo: $n^{1}$ & fon $^{1}$ & fo: $\eta^{1}$ \\
\hline just now & *Pən? & $\operatorname{Pan}^{3}$ & $\operatorname{Pan}^{3}$ & $2 a \eta^{3}$ \\
\hline coffin & *koyh & $\mathrm{kon}^{2}$ & $\operatorname{koy}^{2}$ & $\mathrm{k}^{2} \eta^{2}$ \\
\hline flea & *hmə:t & po: $t^{7}$ & po: $t^{9}$ & po: $\mathrm{k}^{7}$ \\
\hline greedy & *6วt & $6 \mathrm{at}^{7}$ & $6 a t^{7}$ & $6 \mathrm{ak}^{7}$ \\
\hline to dip & *dok & $\mathrm{dok}^{7}$ & $\mathrm{dok}^{7}$ & $\mathrm{dok}^{7}$ \\
\hline spade & $*_{t c} \mathrm{~h}_{\mathrm{a}} \mathrm{c}$ & $\mathrm{ts}^{\mathrm{h}} \mathrm{a}: \mathrm{c}^{7}$ & $\mathrm{ts}^{\mathrm{h}} \mathrm{a}: \mathrm{t}^{7}$ & $\operatorname{ts}^{\mathrm{h}} \mathrm{o}: \mathrm{t}^{7}$ \\
\hline vomit & $*_{\mathrm{fa}} \mathrm{k}$ & fe: $k^{7}$ & $\mathrm{fek}^{7}$ & fur: ${ }^{2}$ \\
\hline
\end{tabular}

\subsubsection{Moyfaw and Baisha}

Baisha has been described with a tone system with two correspondences in the A, B, and D categories, although it has never been clear what the basis of this distinction was (Matisoff 1988: 290), since it didn't seem to indicate a register split as is the case in most other languages with two tones per category. This 'tone split' in Baisha can, actually, be shown to be a result as evidence for loans from Moyfaw. Since Moyfaw and Baisha developed in very similar ways, evidence in itself for close contact between the two, it is difficult in most cases to detect loans based on unexpected segmental reflexes. However, the tone systems of each developed in a rather different manner, and it is this part of the phonology which betrays the loans. Compare the tone systems of the two languages ${ }^{5}$ :

\begin{tabular}{|c|c|c|c|}
\hline Tone Category & Baisha 1 & Baisha 2 & Moyfaw \\
\hline A & $11(1)$ & $51(4)$ & $53(1)$ \\
\hline B & $31(2)$ & $55(5)$ & $55(2)$ \\
\hline $\mathrm{C}$ & $33(3)$ & --- & $24(3)$ \\
\hline D & $11(8)$ & $55(7)$ & $55(7)$ \\
\hline
\end{tabular}

${ }^{5}$ The parenthesized numbers in the table below indicate the numbers given to each tone in Ouyang \& Zheng (1983), and which have been adopted in most subsequent citation of Hlai data. For example, the Baisha word for stone is listed as $t s^{h} i \eta^{4}$, which means that its actual tone value is a falling tone: $t s^{h} i \eta^{51}$. 
It can be seen that of the two Baisha tones per category, there is one in every instance except category $\mathrm{C}$ which corresponds in shape to its Moyfaw equivalent and one which does not. If the present hypothesis of borrowing is correct, then a number of words were loaned from Moyfaw into Baisha (note the high incidence of kinship terms below) in all categories, with the only caveat that in category $\mathrm{C}$, a tone (i.e. a pitch contour) had either not developed yet, or otherwise there was sufficient perceptual similarity for the Moyfaw tone to be assimilated as the native Baisha tone:

Gloss

(a)

$\begin{array}{ll}\text { stone } & *{ }^{\mathrm{h}}{ }^{\mathrm{h}} \mathrm{i} \text { : } \\ \text { o. bros wife } & * \text { tcu: } \\ \text { aunt } & * \text { hmi: } \\ \text { aunt } & * \text { hyi:n }\end{array}$

(b)

$\underline{\text { Gloss }}$

$\begin{array}{ll}\text { woman } & * \mathrm{k}^{\mathrm{h}} \text { əwh } \\ \text { finger } & * \text { lji:yh } \\ \text { shoulder } & * \text { va:h } \\ \text { branch (tree) } & * \text { C-ya:mh }\end{array}$

(c)

$\begin{array}{ll}\text { pat. grt grndpa } & * \mathrm{p}^{\mathrm{h}} \mathrm{ut} \\ \text { late } & * \mathrm{t}^{\mathrm{h}} \partial \mathrm{p} \\ \text { nose } & * \mathrm{k}^{\mathrm{h}} \partial \mathrm{t} \\ \text { ten } & * \mathrm{fu}: \mathrm{t}\end{array}$

Moyfaw

ts $^{\text {hin }}{ }^{1}$

tsow $^{1}$

pey $^{1}$

kin ${ }^{1}$

$\underline{\text { Moyfaw }}$

$\mathrm{k}^{\mathrm{h}} \mathrm{o}{ }^{2}$

$\mathrm{zin}^{2}$

va: ${ }^{2}$

ya: $m^{2}$

$\mathrm{p}^{\mathrm{h}} \mathrm{ok}^{7}$

ts $^{\mathrm{h}} \mathrm{ap}^{7}$

$\mathrm{k}^{\mathrm{h}} \mathrm{ak}^{7}$

fut $^{7}$
Baisha

ts $^{\text {h }}$ in $^{4}$

tsow $^{4}$

pey $^{4}$

kin $^{4}$

Baisha

$\mathrm{k}^{\mathrm{h}} \mathrm{o} \cdot{ }^{5}$

$\mathrm{zin}^{5}$

va: ${ }^{5}$

ya: $m^{5}$

$\mathrm{p}^{\mathrm{h}} \mathrm{ok}^{7}$

ts $^{\text {h }}$ ap $^{7}$

$\mathrm{k}^{\mathrm{h}} \mathrm{ak}^{7}$

fut $^{7}$
Expected Baisha

ts $^{\text {hin }}{ }^{1}$

tsow $^{1}$

pey $^{1}$

kin $^{1}$

Expected Baisha

$\mathrm{k}^{\mathrm{h}} \mathrm{o}:^{2}$

$\mathrm{zin}^{2}$

$\mathrm{va}^{2}$

ya: $m^{2}$

$\mathrm{p}^{\mathrm{h}} \mathrm{ok}^{8}$

ts $^{\text {h }}$ ap $^{8}$

$\mathrm{k}^{\mathrm{h}} \mathrm{ak}^{8}$

fut $^{8}$

One probable loan from Baisha to Moyfaw is the word snore:

$\underline{\text { Gloss }}$

snore
$\underline{\mathrm{PHl}}$

Cura:n
Baisha

fa: $y^{1}$
Moyfaw

fa: $y^{1}$
Expected Moyfaw

ya: $y^{1}$

\subsubsection{Baisha and Yuanmen}

There is also tonal evidence for loans from Baisha into Yuanmen. First, compare the tone values for the two languages: 


\begin{tabular}{llll} 
Tone Category & Baisha & & \multicolumn{2}{r}{ Yuanmen (High) } & Yuanmen (Low) \\
A & $11(1)$ & $42(1)$ & $11(4)$ \\
B & $31(2)$ & $51(5)$ & $131(2)$ \\
C & $33(3)$ & $44(3)$ & $13(6)$ \\
D & $11(8)$ & $55(7)$ & $13(8)$
\end{tabular}

It can be observed that Baisha tones A and B are closest in value to the Yuanmen tones of the same category in low register. Conversely, Baisha tone $\mathrm{C}$ is closest to Yuanmen tone $\mathrm{C}$ in high register, and Baisha tone D may, based on its low pitch level, be borrowed as the same tone in Yuanmen in low register. In fact, in the majority of cases in which the unexpected register occurs in Yuanmen, it has a parallel in Baisha with an identical or similar tone:

$\underline{\text { Gloss }}$

(a) gecko

(b)

pull tight
turtle
energy
rice

(c)

$\begin{array}{ll}\begin{array}{ll}\text { complete } \\ \text { armpit }\end{array} & * \text { C-mu: } \\ \text { brave water } & * \text { hnun? } \\ \text { stretch } & * \text { hja:? } \\ \text { Gloss } & \underline{\text { PHl }} \\ \text { have } & * \text { də:k } \\ \text { lash child } & * \text { fi:t } \\ \text { throw } & * \text { sə:p } \\ \text { bird } & * \text { səc }\end{array}$

*kunh

$* t^{\mathrm{h}} \mathrm{u}: \mathrm{h}$

$*^{\mathrm{h}} \mathrm{u}: \mathrm{h}$

$* \mathrm{t}^{\mathrm{h}} \mathrm{a}: \mathrm{h}$

hnun?

hja:?

PHl

\section{$\underline{\mathrm{PHl}}$ \\ Baisha}

*CuPa:w

*C-mu:y?

(d)

${ }^{*}$ sac

va: ${ }^{1}$

$\mathrm{kə \eta}^{2}$

$\mathrm{t}^{\mathrm{h}} \mathrm{aw}^{2}$

$\mathrm{k}^{\mathrm{h}} \mathrm{aw}^{2}$

$\mathrm{t}^{\mathrm{h}} \mathrm{a}:^{2}$

muy $^{3}$

yay $^{3}$

ton $^{3}$

$\mathrm{za}^{3}$

Baisha

๑ว ${ }^{8}$

$\mathrm{fit}^{8}$

ts $^{\text {h }}$ uap $^{8}$

ts $^{\text {hat }}{ }^{8}$ $\underline{\text { Yuanmen }}$

va: ${ }^{4}$

$k ә \eta^{2}$

$\mathrm{t}^{\mathrm{h}} \mathrm{ow}^{2}$

$\mathrm{k}^{\mathrm{h}} \mathrm{aw}^{2}$

$\mathrm{t}^{\mathrm{h}} \mathrm{a}:^{2}$

muy $^{3}$

yay $^{3}$

$\tan ^{3}$

$\mathrm{za}^{3}$

Yuanmen

do ${ }^{8}$

$\mathrm{fit}^{8}$

ts $^{\text {h }}$ uap $^{8}$

ts $^{\text {h }}$ at $^{8}$

\section{Expected Yuanmen}

va: ${ }^{1}$

$\mathrm{kə \eta}^{5}$

$\mathrm{t}^{\mathrm{h}} \mathrm{aw}^{5}$

$\mathrm{k}^{\mathrm{h}} \mathrm{aw}^{5}$

$\mathrm{t}^{\mathrm{h}} \mathrm{a}:^{5}$

mun $^{6}$

yay $^{6}$

tən $^{6}$

$\mathrm{za}:^{6}$

Expected Yuanmen

do $?^{7}$

$\mathrm{fit}^{7}$

ts $^{\mathrm{h}}$ uap $^{7}$

ts $^{\text {hat }}{ }^{7}$

\subsubsection{Run and Qi}

There seems to have been, from some point after the split in NWCHl between Meifu and Run, a certain degree of contact between the Run and Qi branches. This can be seen most clearly in the 
development of original complex initials (although there is some similarity with Meifu in the development of $\left.\mathrm{PHl}{ }^{*} \mathrm{rj}\right)$. NWCHl and Meifu have been included below for comparison to highlight the similarities between Run and Qi:

$\begin{array}{lllll}\text { PHl } & \text { NWCHl } & \text { Meifu } & \text { Run } & \text { Qi } \\ *_{\mathrm{fj}} & *_{\mathrm{fj}} & *_{\mathrm{c}} & *_{\mathrm{f}} & *_{\mathrm{f}} \\ *_{\mathrm{cj}} & *_{\mathrm{rj}} & *_{\mathrm{d} \sim \mathrm{r}} & *_{\mathrm{d} \sim \mathrm{rj}} & *_{\mathrm{d}} \\ *_{\text {Curw }} & *_{\mathrm{w}} & *_{\mathrm{r}} \mathrm{w} & *_{\mathrm{v}} & *_{\mathrm{v}}\end{array}$

There seems to be an especially intimate connection between Yuanmen and Tongzha, and it may be that interaction between Run and Qi (or even Tongzha specifically) is what led to the split between Baisha and Yuanmen within Run. Compare first the development of PHl *1j:

$\begin{array}{llll}\underline{\text { PHl }} & \text { Baisha } & \text { Yuanmen } & \text { Qi } \\ * 1 \mathrm{j} & * \mathrm{j} & * \mathrm{~d} z_{0} & * \mathrm{k}\end{array}$

Moreover, the development of tones as well as the occurrence of registrogenesis in Yuanmen seems to have been directly related to Tongzha:

\begin{tabular}{|c|c|c|c|c|}
\hline \multicolumn{2}{|c|}{$\underline{\text { Tone category }}$} & $\underline{\text { Baisha }}$ & $\underline{\text { Yuanmen }}$ & $\underline{\text { Tongzha }}$ \\
\hline \multirow[t]{2}{*}{ A } & $\mathrm{Hi}$ & 11 & 42 & 33 \\
\hline & Lo & & 11 & 11 \\
\hline \multirow[t]{2}{*}{ B } & $\mathrm{Hi}$ & 31 & 51 & 51 \\
\hline & Lo & & 131 & 121 \\
\hline \multirow[t]{2}{*}{ C } & $\mathrm{Hi}$ & 33 & 44 & 55 \\
\hline & Lo & & 13 & 14 \\
\hline \multirow[t]{2}{*}{$\mathrm{D}$} & $\mathrm{Hi}$ & 11 & 55 & 55 \\
\hline & Lo & & 13 & 13 \\
\hline
\end{tabular}

\subsubsection{Nadouhua}

In the case of Nadouhua, there have been loanwords borrowed from at least two sources. One has been Meifu - whether Changjiang or Moyfaw is more difficult to determine precisely, although other evidence indicates that Changjiang forms part of a speech area with Nadouhua, making it the more likely 
candidate. The following are examples, where Nadouhua exhibits unexpected initials that are similar to those in Meifu (reconstructed via the comparison of Changjiang and Moyfaw):

\begin{tabular}{|c|c|c|c|c|}
\hline$\underline{\text { Gloss }}$ & $\underline{\mathrm{PHl}}$ & $\underline{\text { Meifu }}$ & $\underline{\text { Nadouhua }}$ & Expected Nadouhua \\
\hline flower & $* \int^{\mathrm{h}} \varepsilon: \eta$ & $* \mathrm{ts}^{\mathrm{h}} \mathrm{e}: \mathrm{n}^{1}$ & $\mathrm{fen}^{1}\left(<\operatorname{sen}^{1}\right)$ & hen ${ }^{1}$ \\
\hline pus & * Curiw & *?wiw ${ }^{3}$ & Piw $^{3}$ & niw $^{3}$ \\
\hline know & *Curu: & *?wәu ${ }^{1}$ & ?we $?^{4}$ & $\eta \varepsilon P^{4}$ \\
\hline
\end{tabular}

Nadouhua has apparently also borrowed from Cunhua. The following examples show words with original $\mathrm{PHl} * \mathrm{r}$, which have apparently been borrowed from Cunhua when ${ }^{*} \mathrm{r}$ had already become $* \mathrm{~h}$, and Nadouhua (which did not have $G$ in its inventory) inserted an epenthetic glide which was linked to the features of the following vowel:

\begin{tabular}{|c|c|c|c|c|}
\hline$\underline{\text { Gloss }}$ & $\underline{\mathrm{PHl}}$ & $\underline{\text { Cunhua }}$ & Nadouhua & Expected Nadouhua \\
\hline sell & *ri:w? & hiw $^{4}$ & $\mathrm{zi}^{3}$ & $\mathrm{ni}^{3}$ \\
\hline spicy & $*_{\text {rit }}$ & het $^{4}$ & $\mathrm{ze}^{5}$ & $\eta e 2^{4}$ \\
\hline fat & *ru:y? & huy $^{4}$ & vuy $^{3}$ & puy $^{3}$ \\
\hline
\end{tabular}

To illustrate, the following scenario is hypothesized to have occurred:

$\begin{array}{llllll}\text { Gloss } & \text { Pre-Cunhua } & & \text { Pre-Nadouhua } & & \text { Nadouhua } \\ \text { sell } & \text { *hi:w? } & > & \text { *ji:w? } & > & \text { zi: }^{3} \\ \text { fat } & \text { *hu:y? } & > & \text { *wu:y? } & > & \text { vuy }^{3}\end{array}$

The following examples are of loans from Cunhua into Nadouhua where the latter shows unexpected rimes:

\begin{tabular}{|c|c|c|c|c|}
\hline$\underline{\text { Gloss }}$ & $\underline{\mathrm{PHl}}$ & $\underline{\text { Cunhua }}$ & $\underline{\text { Nadouhua }}$ & Expected Nadouhua \\
\hline flea & *hmə:t & $6 a t^{4}$ & $\mathrm{pa}^{4}$ & por ${ }^{4}$ \\
\hline son-in-law & *hlu: & $\mathrm{t} \theta \mathrm{ow}^{1}$ & low $^{2}$ & $l \varepsilon(\mathrm{w})^{2}$ \\
\hline do & $*_{v u: \mathrm{k}}$ & vok $^{4}$ & $\operatorname{vo}^{4}$ & $\operatorname{vu}^{4}$ \\
\hline vine basket & *6oy & $6 o y^{1}$ & $6 o y^{1}$ & $6 \circ y^{1}$ \\
\hline
\end{tabular}




\subsubsection{Lauhut and Cunhua}

Finally, it seems that Cunhua, like Moyfaw, borrowed from Lauhut in its recent history. The following are examples of unexpected correspondences in Cunhua initials, rimes, and/or tones which can plausibly be explained as borrowings from Lauhut:

\begin{tabular}{|c|c|c|c|c|}
\hline Gloss & $\underline{\mathrm{PHl}}$ & $\underline{\text { Lauhut }}$ & Cunhua & Expect \\
\hline weave (fabric) & *hwi: & hwey $^{1}$ & huy $^{5}$ & ${\mathrm{v} \varepsilon y^{1}}^{1}$ \\
\hline you $(\mathrm{pl})$ & *C-mu: & mәщ $^{1}$ & ma: ${ }^{1}\left(<\mathrm{mə}::^{1}\right)$ & mow $^{1}$ \\
\hline look & *kiw & kiw $^{1}$ & Piw $^{1}$ & koy $^{1}$ \\
\hline cotton & *6u:y ${ }^{\mathrm{C}}$ & 6u: $y^{3}$ & $6 u:(y)^{3}$ & $6 u y^{3}$ \\
\hline wear (lower) & ${ }^{*} \mathrm{p}^{\mathrm{h}} \mathrm{i}: \mathrm{n}$ & $\mathrm{p}^{\mathrm{h}} \mathrm{i}: \mathrm{n}^{1}$ & $\mathrm{p}^{\mathrm{h}} \mathrm{i}: \mathrm{n}^{1}$ & $\mathrm{p}^{\mathrm{h}} \mathrm{in}^{1}$ \\
\hline stick into & ${ }^{*} \mathrm{C}-\mathrm{pi}: \mathrm{p}$ & ni: $p^{7}$ & ni:p $p^{2}$ & yip $^{2}$ \\
\hline adze & $*^{p}{ }^{h} u: n$ & $\mathrm{p}^{\mathrm{h}} \mathrm{u}: \mathrm{n}^{1}$ & $p^{h} u: n^{3}$ & $\mathrm{p}^{\mathrm{h}} \mathrm{un}^{1}$ \\
\hline kiss & $*_{\text {rju:c }}$ & $\mathrm{ru}: \mathrm{c}^{7}$ & lu: $t^{2}$ & $\mathrm{zut}^{4}$ \\
\hline
\end{tabular}

\subsubsection{Summary}

Following the breakup of Proto-Hlai into various branches, and these branches into individual languages, it is apparent that there has been intimate contact between several non-sibling branches and languages around Hainan. This is evident not only through shared sound changes which have diffused from one language to another, but also through irregular reflexes in one language which can be explained through borrowing from another language in which those reflexes would be regular. The recognition of this contact is of the utmost importance in subgrouping, as it helps to differentiate sound changes which are more useful for subgrouping, and those which are more easily diffused.

Considerable contact has occurred between Bouhin and Ha Em, with most loanwords being borrowed into the former from the latter. A fair amount of more recent contact has occurred between Baoting and Jiamao, and there has been some contact as well within Qi between Tongzha and Zandui on the one hand and between Tongzha and Baoting on the other; there is also evidence of substantial contact between Run and Qi, and particularly between Yuanmen and Tongzha. In the West half of the island, there has been an interesting unidirectionality of influence, first of Lauhut on Moyfaw, from Moyfaw on Baisha, 
and finally from Baisha on Yuanmen. Lauhut has also contributed some loanwords to Cunhua, which in turn has loaned words to Nadou, which has also borrowed at least a few items from Meifu (or probably more specifically, Chanjiang). These contact relationships can be illustrated in the following simplified way (Changjiang and Moyfaw are collapsed below into Meifu (MF), due to the fact that there is not enough data available on Changjiang to make reliable inferences about contact):

Directionality of Contact Among the Hlai Languages

\begin{tabular}{|c|c|c|c|c|c|}
\hline & $\begin{array}{l}\text { Baisha } \\
36,000\end{array}$ & $\rightarrow$ & $\begin{array}{l}\text { Yuanmen } \\
8,000\end{array}$ & & \\
\hline & $\uparrow$ & & $\uparrow$ & & \\
\hline $\begin{array}{l}\text { Nadouhua } \leftarrow \\
2,500\end{array}$ & $\begin{array}{l}\text { Meifu } \\
30,000\end{array}$ & & $\begin{array}{l}\text { Tongzha } \\
125,000\end{array}$ & $\rightarrow$ & $\begin{array}{l}\text { Zandui } \\
29,000\end{array}$ \\
\hline$\uparrow$ & $\uparrow$ & & $\downarrow$ & & \\
\hline \multirow[t]{2}{*}{$\begin{array}{l}\text { Cunhua } \\
60,000\end{array}$} & $\begin{array}{l}\text { Lauhut } \\
166,000\end{array}$ & & $\begin{array}{l}\text { Baoting } \\
24,000\end{array}$ & $\leftarrow$ & $\begin{array}{l}\text { Jiamao } \\
52,300\end{array}$ \\
\hline & $\begin{array}{l}\text { Bouhin } \\
73,000\end{array}$ & $\leftarrow$ & $\begin{array}{l}\mathrm{Ha} \text { Em } \\
193,000\end{array}$ & & \\
\hline
\end{tabular}

It is interesting to note that when the chart above is compared with the population statistics in (3), there is an obvious and non-random correlation between population and what end of the arrow a language is on in a contact relationship. More specifically, the donor languages tend to have the highest populations, while the borrowing languages tend to have the smallest (note that the two most endangered languages, Yuanmen and Nadouhua, are exactly the two which are at the borrowing end of two contact relationships).

\subsection{Theory of Language Change and Reconstruction}

A central hypothesis about language change adopted here is that it is non-teleological, in line with Blevins (2004). It is often the case that languages are anthropomorphized, either purposefully or not, and it is said that some language 'does this' or 'does that', as if it were capable of conscious decision-making (for a critical view of this approach, see Enfield 2005). There is no 'hidden hand' assumed to be at work behind 
the direction of language change in the present work, and it is therefore possible for language change to potentially occur in favor of exotic changes as well as ordinary ones, towards asymmetry as well as towards symmetry. This does not negate the fact that there are typologically common changes and inventories which should be taken into consideration in reconstruction of both phoneme inventories as well as the various paths of change which phonemes can take individually or collectively. These typological commonalities are explained, however, through the inherent bias of the human articulatory and auditory systems, which are predisposed towards certain types of variation and error on both an individual level (where change is initiated) and at a community level (where change is conventionalized). It can therefore be anticipated that rare features of the inventory or particular asymmetries will be more unstable and prone to replacement due to these biases. It may also be supposed that change can be indirectly influenced in a psycholinguistic sense by the existence of categories with large numbers of exemplars, where change may be vectored toward such a category because of its high frequency (see the discussion in chapter two on systemic realignment in section 2.1.3).

I also subscribe in general to both the Evolutionary Phonology model and the arguments for it in Blevins (2004). The Evolutionary Phonology model states that the primary motivation for phonological change is imperfect transmission between the speaker and the listener/learner. The three categories of mistransmission are summarized below:

(a) Change: signal misheard by listener

(b) Chance: signal accurately heard, but intrinsically ambiguous

(c) Choice: multiple variants of signal available, and new exemplar is chosen

In theory, these explanations are entirely reasonable, and have the power to explain the bulk of phonological change. In practice, however, it is not always easy to decide which of these three possibilities is behind a particular change, although educated guesses can be made. I will therefore make the general assumption that the changes discussed in this dissertation are due to mistransmission, but will only categorize it according to Blevins' model when it is straightforward to do so. 


\subsubsection{Principles of Language Change and Criteria for Reconstruction}

Four basic principles about language change and reconstruction are presented below: directionality of change, commonality of features, economy, and symmetry. Although not identical, these assumptions are informed by the reconstruction methodology outlined in Campbell (2004: section 5.2). Examples are given for each to illustrate the principles under discussion.

\subsubsection{Directionality of Change}

Phonemes, or constructs which are comprised of phonemes such as the initial and the rime, can change in ways which are on a continuum of statistical probability, i.e. there are changes which are considered more likely, less likely, and impossible. This can be evaluated according to both phonetic (can it be explained via an understanding of the biases of the articulatory and auditory systems) as well as typological (is it commonly attested cross-linguistically) criteria, although an evaluation may be subjective in cases where there has not been much research into the type of change in question. For example, of the two changes listed below, the first (70a) is more likely, and the second (70b) less likely:
(a) $m>b$
(b) $b>m$

In evaluating sound change, those changes which are considered more likely are therefore explored first, and those which are considered less likely only entertained if and when there is strong evidence for them.

A corollary of this assumption is that changes will usually tend to occur one feature at a time. In other words, a change may involve place of articulation, or it may involve manner of articulation, but it is unlikely to involve both simultaneously. For example, the changes of a single feature in (71a) and (71b) below are more likely to occur than the simultaneous change of two features in (71c): 

(a) $t 6>t \int$
(b) $t c>t c^{\mathrm{h}}$
(c) $t_{6}>t^{\text {h }}$

This is not an absolute rule, however, as it is possible (especially in the case of perception-based change) for more than one feature to be affected at a time.

\subsubsection{Commonality of Features}

As stated above, it is expected that sound change will often involve a single feature at a time, although it may occasionally involve more; in no case is there any reason to believe that all features will change simultaneously. For this reason, the assumption is made that the reflexes of proto-phonemes in the daughter languages will preserve one or more features of the original phoneme in the proto-language from which they have evolved. In some cases, multiple identical features will have been retained, as in the following correspondence set of initials from the twelve Hlai languages used in this dissertation ${ }^{6}$ :

$\begin{array}{llllll}\mathbf{r} & \mathbf{r} & \mathrm{r} & \mathbf{r} & \mathbf{l} & \mathbf{l} \\ \mathbf{l} & \mathbf{l} & \mathbf{l} & \mathrm{r} & \mathrm{r} & \mathbf{r}\end{array}$

In this example, all of the reflexes are [coronal] and retain the feature [liquid], varying between being a lateral or a non-lateral (generally a tap or a trill). A reconstruction of a liquid phoneme is very secure in this instance, and the question is merely what kind of liquid it was, a decision which can be informed by inspecting its place in the overall inventory of reconstructed phonemes.

In other cases, common features are not preserved across the reflexes of the daughter languages, as in the following correspondence set:

$\begin{array}{llllll}\mathbf{f} & \boldsymbol{f} & \mathrm{v} & \mathbf{f} & \mathbf{f} & \mathbf{f} \\ \mathbf{v} & \mathbf{v} & \mathrm{kw} & \mathrm{g} & \mathrm{f} & \mathbf{f}\end{array}$

\footnotetext{
${ }^{6}$ The specific order and identity of these twelve languages is given in the next chapter.
} 
In this example, there are no features which can be considered common to all of the reflexes.

There are two taps, several labiodental fricatives, a velar fricative and a labialized velar stop. Since the place feature [labial] occurs in the majority of reflexes, it is likely that the proto-phoneme had some sort of a labial component. However, the two taps indicate that there was an alveolar component as well (and this still leaves the velar fricative and labialized velar stop needing explanation). The reconstruction ultimately adopted here is *Cur, a sesquisyllable with a high back rounded vowel preceding an alveolar tap which is the onset of the main syllable (see chapter two for the details of this reconstruction). This reconstruction is informed by both of the place features [labial] and [coronal], and the manner has been decided based on the principle of directionality described above.

This example also highlights another corollary principle of language change and reconstruction, which is the more heterogeneous the reflexes of the daughter languages, the more complex the original proto-phoneme, where complexity is defined by the total number of features represented in the original initial (i.e. plain $p$ is considered less complex than palatalized $p j$ ). One more example of this is the following:

$\begin{array}{llllll}\text { ts }^{\mathrm{h}} & \mathrm{ts}^{\mathrm{h}} & \mathrm{f} & \mathrm{ts}^{\mathrm{h}} & \mathrm{ts}^{\mathrm{h}} & \mathrm{ts}^{\mathrm{h}} \\ \mathrm{f} & \mathrm{f} & \mathrm{f} & \mathrm{f} & \mathrm{f} & \mathrm{pf}^{\mathrm{h}}\end{array}$

In this example, the one feature held in common across the reflexes of the daughter languages is that of frication. However, the place feature is split between [coronal] and [labial], with alveolar reflexes in five languages and labiodental reflexes in the others. Since there is no common place feature, and there is no typologically common change of either $* \mathrm{ts}^{\mathrm{h}}>f$ or $* \mathrm{f}>t \mathrm{~s}^{h}$, then the reconstruction of a complex protophoneme should be considered (in this case $* t \int^{h} \mathrm{w}$ - again, see chapter two for the details).

\subsubsection{Economy of Change}

In general, when more than one alternative is being considered, the one which involves the fewest steps of change should be favored. For example, it is known from comparative Tai work (see Li (1977)) 
that the original liquid of a stop-liquid cluster will become a glide, for example *kl $>k j$. Knowing this, and using again example (74) above, it would be logical to consider the following two potential reconstructions for this series of initials:
(a) $* \int^{\mathrm{h}} \mathrm{w}$
(b) $* t \int^{h} r$

The reason that one might consider the reconstruction in $(75 b)$ is that, since there is evidence for a labial component of the initial from amongst the daughter languages, this labial component could suggest that the proto-phoneme was a ${ }^{*} \mathrm{C}$-r cluster, where the ${ }^{*}$ lenited to $w$, influencing the outcome of change in the modern reflexes accordingly. Although this is not an unreasonable hypothesis from a typological standpoint, it suffers from the flaw that there is no direct evidence for a liquid, making the reconstruction of a ${ }^{*} \mathrm{C}-\mathrm{r}$ cluster the product of internal reconstruction, which is based on assumptions about the nature of the pre-proto-language. In fact, the ${ }^{*} \mathrm{C}$-r hypothesis is only one possible way in which this labial component may have arisen, but there are other possibilities such as the influence of a presyllable (as reconstructed for (73) above) or influence from a following vowel. Unless direct evidence comes to light for the reconstruction in (75b), the better reconstruction is therefore that in (75a), since that in $(75 b)$ requires an extra step to have taken place which is not necessary to account for the reflexes in the daughter languages.

\subsubsection{Symmetry of System}

As stated above, although there is not some conscious way in which a language organizes itself, the biases inherent in production and perception tend to lead to phonological inventories which are more balanced than not. For this reason, it is important to check the phoneme inventory for symmetry and make sure that all reconstructed phonemes have been aligned in both place and manner in as many instances as possible.

If this is done carefully, then there may be a residue of reconstructed phonemes which do not fit perfectly into the inventory. This is acceptable, since there are many instances of phonological inventories 
which aren't absolutely symmetrical, although typological data should be consulted since some asymmetries are more typologically common than others (for example, it is common to have an implosive series which includes only the bilabial and alveolar places of articulation (Maddieson 1984: 112).

\subsubsection{Summary}

The four principles of language change and criteria for reconstruction given above provide a consistent framework within which to objectively evaluate and determine the most appropriate reconstruction for a given set of data. The three kinds of possible mistransmission between speaker and listener/learner given in (69) above may be employed in the endeavor of reconstruction when constrained by these principles. More specifically, the principle of Directionality, which relies on a typological knowledge of common sound changes (mistransmissions), can narrow the set of candidate proto-phonemes from which the daughters have descended. The principle of Commonality serves to ensure that the total set of features of all daughter language reflexes is accounted for in the reconstructed phoneme. The principle of Economy serves as a counterbalance to Commonality, in that is requires that no more features are present in the reconstructed phoneme than are necessary to account for those features which are represented collectively in the daughter language reflexes. Finally, the principle of Symmetry serves as a systemic constraint on the entire reconstructed inventory, requiring the alignment of phonemes within the inventory (along such lines as the place and manner of articulation of consonants and the height and backness of vowels) and excluding gaps wherever possible, acknowledging asymmetry only when there is a compelling case to do so.

\subsection{Conclusion}

To summarize, the Hlai family is one of four major members of the Kra-Dai phylum of Southeast Asia. It is located on Hainan island, China, where speakers of Proto-Hlai probably comprised the founding population of the island. A total of twelve Hlai languages are presently in use, including the two mixed 
languages Cunhua and Nadouhua. In addition, the Jiamao language, an isolate, has been in contact with Hlai for what appears to have been quite a long time.

Due to the sociolinguistic situation on Hainan, the evolution of the Hlai languages must be understood to have involved both language branching and language contact. The issue of subgrouping was raised, and the subgrouping methodology discussed which will be used to separate inherited from diffused innovations when possible. More precisely, a phylogenetic tree of the Hlai languages is reconstructible when more improbable sound changes are used as criteria for assuming shared inheritance, while more common changes are considered to be likely to have diffused.

Languages in the southern end of the island which were isolated from contact with other non-Hlai languages have tended to remain homogeneous, while languages in the northern and eastern contact zones have tended to undergo fission. Using evidence from irregular correspondences, it is possible to demonstrate contact relationships between the Hlai languages, and also to identify which language has exerted asymmetrical influence on another.

Finally, a general theory of sound change was discussed, which states that sound change is nonteleological and is normally the result of mistransmission. The following criteria were identified for use in reconstruction: directionality of change, commonality of features, economy, and system symmetry.

The next two chapters will outline correspondence sets across the Hlai languages and show the reconstruction of the PHl phonological inventory. The system of initials is treated in chapter two, and the system of rimes in chapter three. 


\section{CHAPTER TWO: RECONSTRUCTION OF PROTO-HLAI INITIALS}

The primary goal of this chapter is to present the sets of initial correspondences which have been used to reconstruct the Proto-Hlai (PHl) inventory of initials, and explain reflexes of Proto-Hlai initials in the daughter languages if they have followed divergent paths. The reconstruction here will also be compared with those of Matisoff (1988), Thurgood (1994), Peiros (1998), and Ostapirat (2004), and these alternative reconstructions will be considered and discussed. It is the purpose of this chapter to motivate the reconstruction of Proto-Hlai proposed herein so that it may in turn be used for comparative work with other branches of Kra-Dai and/or used for more detailed study of specific types of change exemplified below.

The reconstruction in the chapter will be of Proto-Hlai, as opposed to Pre-Hlai which will be reconstructed in chapter four. Proto-Hlai is meant to be the best possible reconstruction of the single protolanguage which existed just prior to its first division into daughter languages. The reconstruction of the Proto-Hlai system of initials in this chapter will result in an inventory with distinctive asymmetries and gaps. The discussion of Pre-Hlai in chapter four will demonstrate how this system originated in a much more balanced and typologically common system.

Before a discussion and reconstruction of specific natural classes of initials is initiated, there are two preliminary issues which are addressed briefly below. The first is a reiteration of the theory of sound change adopted here. The second is a discussion of how two specific sound changes which occurred after the break-up of Proto-Hlai, initial obstruent devoicing and registrogenesis, interact with each other in ways that are meaningful in the context of Proto-Hlai reconstruction. With this background, we will be in a position to properly examine the various classes of initials themselves and the evolution of their individual members into their current forms in the daughter languages. 


\subsection{Sound Change: Initials}

In the reconstruction of Proto-Hlai initials undertaken in this chapter, the following criteria

described in chapter one are adhered to:

(i) Directionality of change: typologically natural changes are referred to and used as a model whenever possible; changes are assumed to occur one feature at a time unless evidence forces a different analysis.

(ii) Commonality of features: phonemes are reconstructed based on the features common between reflexes of daughter languages; greater heterogeneity of reflexes is taken to indicate greater complexity of the proto-phoneme.

(iii) Economy: a phoneme is reconstructed to the extent that it satisfactorily accounts for the posited change(s) between it and the reflexes of the daughter languages, and reconstructions assuming more changes than necessary are avoided.

(iv) Symmetry: the reconstructed inventory is checked to make sure that no symmetries have been overlooked in natural classes, either in place or in manner; it is accepted that parts of the inventory may be asymmetrical, and these are checked for typological naturalness.

Throughout the history of Hlai (including Pre-Hlai, Proto-Hlai, and the daughter languages), there are four main categories of sound change which are observable in the initials. These are (1) temporal compression, (2) gesture reduction, (3) onset fortition, and (4) systemic realignment. Of these four, temporal compression has been the most pervasive, and can be observed at all stages of the evolution of Hlai. Gesture reduction is most pronounced in Pre-Hlai, and will therefore be treated in chapter four.

Onset fortition can be observed in the transition from Pre-Hlai to PHl through a sound change referred to here as initial aspiration (see chapter four) and in the daughter languages. Systemic realignment is most prominent in the cases of the individual daughter languages, after the breakup of PHl. Temporal compression, onset fortition, and systemic realignment will each be exemplified below, using examples from this chapter. It is sometimes the case that a particular change can fall into more than one of these categories simultaneously. 


\subsubsection{Temporal Compression}

Bybee (2001) proposes that speech, like other motor activities, is an automated phenomenon, and as such is subject to the same kinds of overlap of originally linearized gestures. Bybee posits two main articulatory impetuses of sound change, one of which is temporal compression (the other being gesture reduction). Temporal compression occurs when the gestures connected to two segments that are linearized, and therefore discrete, begin to overlap as the time between the implementation of the first and second segments is decreased, leading to the deterioriation of their linearization. This can lead both to articulatorily-motivated sound change, as the two sets of gestures interfere with each other as their segments are compressed, as well as auditorily-motivated change as the percepts from each segment become confused in different ways over shorter durations. This often leads to various forms of coalescence, where features of each segment are preserved in the resulting single segment, although occasionally one segment is merely lost. Some of the most striking examples of temporal compression will be discussed in the chapter four; however, there are also sound changes treated in this chapter which can serve to illustrate this kind of sound change:

\begin{tabular}{|c|c|c|c|c|}
\hline \multicolumn{4}{|c|}{ Examples of temporal compression } & \multirow{2}{*}{$\begin{array}{l}\underline{\text { Source }} \\
\text { NWCHl, Meifu }\end{array}$} \\
\hline (a) & $\mathrm{fj}$ & $>$ & 6 & \\
\hline & $t \int^{h} w$ & $>$ & $\mathrm{f}$ & $\mathrm{NCHl}$ and Lauhut \\
\hline \multirow{2}{*}{ (b) } & nw & $>$ & $\mathrm{m}$ & Yuanmen \\
\hline & $\mathrm{lj}$ & $>$ & $\mathrm{d} z$ & Run \\
\hline \multirow[t]{3}{*}{ (c) } & hyw & $>$ & hw & Various \\
\hline & Cu.tw & $>$ & Pw & NECHl \\
\hline & Cufiw & $>$ & $3 \mathrm{w}$ & Western Qi \\
\hline \multirow[t]{3}{*}{ (d) } & ?1 & $>$ & 1 & All \\
\hline & $\mathrm{lj}$ & $>$ & 1 & Cunhua \\
\hline & $\mathrm{rj}$ & $>$ & $\mathrm{j}$ & Bouhin, Ha Em \\
\hline
\end{tabular}


(1a) shows examples of coalescence between a fricate (fricative or affricate) with a coarticulated glide, which result in another fricative that retains the manner of the original fricate but which has a place of articulation influenced by the following glide, shown in (2):

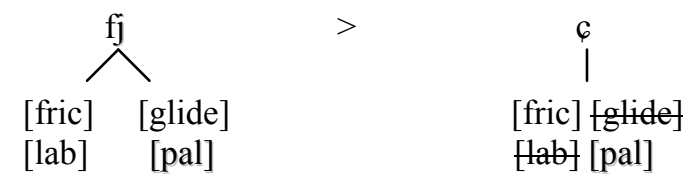

The examples in (1b) are of sonorants which coalesce with following glides, shown in (3):

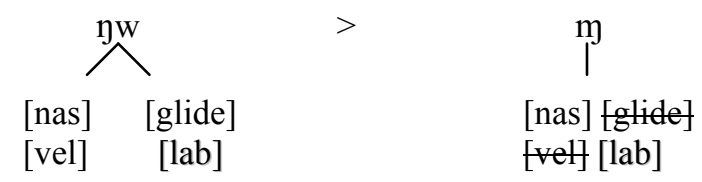

(1c) shows examples of complex segments in which the medial 'host' segment is lost at the expense of preserving the glottal element at the left edge, and the glide at the right, shown in (4):

(4)

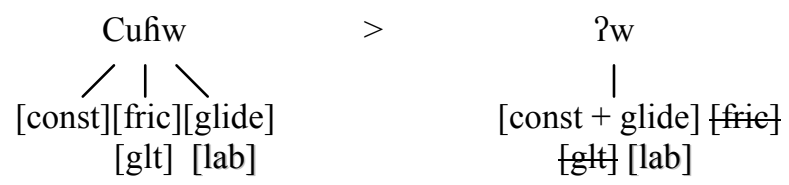

Finally, (1d) gives examples of complex segments in which one member is merely deleted, without involving modification of the other member, shown in (5):

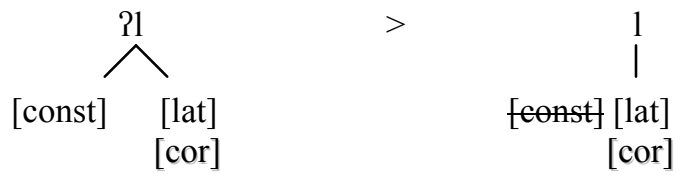

\subsubsection{Onset Fortition}

I also accept Blevins' (2004) proposal that fortition in onset position is a natural change. Some examples of fortition are provided here: 
Examples of fortition in onset position $\quad$ Source

$\begin{array}{lllll}\text { (a) } & \mathrm{hm} & > & \mathrm{b} & \\ & \mathrm{hn} & > & \mathrm{d} & \begin{array}{l}\text { Greater Hlai } \\ \text { Greater Hlai }\end{array} \\ \text { (b) } \quad \mathrm{f} & > & \mathrm{pf}^{\mathrm{h}} & \text { Yuanmen } \\ & \mathrm{s} & > & \mathrm{ts}^{\mathrm{h}} & \text { Run } \\ \text { (c) } \quad \mathrm{hl} & > & \mathrm{d} & \text { Bouhin } \\ & \mathrm{lj} & > & \mathrm{d} z & \text { Run } \\ & \mathrm{r} & > & \mathrm{d} & \text { Run, Meifu, Qi } \\ & & & & \\ \text { (d) } \quad \mathrm{hw} & > & \mathrm{v} & \text { All } \\ & \mathrm{hj} & > & \mathrm{z} & \text { All } \\ & \mathrm{v} & > & \mathrm{v} & \text { Various } \\ & \mathrm{I} & > & \mathrm{V} & \text { Various }\end{array}$

The first kind of fortition, illustrated in (6a), is an example in which preaspirated nasal stops change to prenasalized stops, with sonority decreasing in tandem with oral closure. Another kind, an increase in oral stricture in fricatives leading to their change to affricates, is shown in (6b). The development of oral closure in liquids is yet a third kind (6c). Finally, the narrowing of oral stricture in glides and approximants, giving rise to fricatives, is yet another example (6d). Several of the fortitions above are correlated with the the change called initial aspiration, discussed in chapter four.

\subsubsection{Systemic Realignment}

Finally, an important factor in sound change seems to be the psycholinguistic influence which preexisting categories (structural analogy: Blevins (2004: 153-55)) and/or categorical gaps exert over potential paths of change. The sound changes described below involve either whole merger of formerly distinct categories, or the influence of one category on the shift of another: 


\begin{tabular}{|c|c|c|c|c|c|}
\hline \multirow[t]{2}{*}{ (a) } & $v$ & $>$ & $\mathrm{v}$ & (v already in system) & Tongzha \\
\hline & $\mathrm{rj}$ & $>$ & $\mathrm{lj}$ & (lj already in system) & Yuanmen \\
\hline \multirow[t]{2}{*}{ (b) } & $\mathrm{v}$ & $>$ & $\mathrm{f}$ & (f already in system) & Tongzha, Run \\
\hline & 3 & $>$ & $\$$ & ( 1 already in system) & Qi \\
\hline \multirow[t]{2}{*}{ (c) } & Cifij & $>$ & $? \mathrm{n}$ & ( $\mathrm{p}$ already in system) & $\mathrm{NCHl}$ \\
\hline & Cufw & $>$ & ?yw & (?yw already in system) & $\mathrm{NCHl}$ \\
\hline \multirow[t]{2}{*}{ (d) } & Cu.nw & $>$ & ?w & ( $(w$ already in system) & NECHl \\
\hline & Curw & $>$ & $? \mathrm{w}$ & (?w already in system) & Meifu \\
\hline \multirow[t]{2}{*}{ (e) } & $\mathrm{s}$ & $>$ & $\mathrm{t}$ & (original $* \mathrm{t} \rightarrow \mathrm{t}^{\mathrm{h}}$ ) & Bouhin, Ha Em, ECHl \\
\hline & c & $>$ & 1 & (original *1 $\rightarrow \ddagger$ ) & Various \\
\hline
\end{tabular}

In (7a), approximants are merged with pre-existing categories in the initial inventory. (7b) gives examples of the devoicing of voiced obstruents, allowing a merger with existing voiceless obstruents. (7c) is an example of sesquisyllabic forms with fricatives hosting coarticulated glides preceded by merging with simpler, pre-existing segments. Examples of deletion of medial approximants allowing merger with a preexisting category are shown in (7d). Finally, (7e) provides three examples of gap-filling shifts, after these slots were vacated by their original occupants.

The three kinds of sound change detailed above provide an overview of the general mechanisms of change which have been at work within the Hlai languages throughout various points in their history. These sound changes seem to be general aspects of the dynamic sound system, which are always present as potential changes depending on the combination of the variables surrounding language transmission.

\subsection{Sound Changes After the Breakup of Proto-Hlai}

Although there are a number of different kinds of sound changes which have occurred in the history of the Hlai languages, not all of them have spread over equally large areas. This section describes two kinds of changes which have been widespread enough to affect a majority of the Hlai languages; more limited changes will be described individually in the discussion of initials itself. The two changes 
described below are also interactive, and understanding how this is so will clarify very much of the history of the Hlai initials.

\subsubsection{Devoicing}

There is evidence, both internal and external, that there has been a constraint against initial voiced obstruents in Hlai, for a very long time. In every instance that a sound change leads to a new initial voiced obstruent (or obstruent series), it is apparent that devoicing occurs either simultaneously or shortly thereafter. The instances in which this occurred in Pre-Hlai will be treated in chapter four.

Devoicing of obstruents after the break-up of Proto-Hlai has occurred in instances which have included all of the daughter languages, a subset, or individual cases. A good example is the class of medial glottal fricatives, which underwent devoicing as soon as the words which contained them lost their presyllables. This happened across the board with glottal fricatives preceded by non-high vowels, but only occurred outside of $\mathrm{NCHl}$ with those preceded by high vowels due to the rhinoglottophilic change which occurred in this branch:

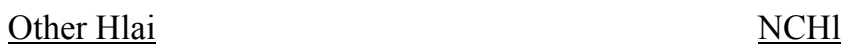

\begin{tabular}{|c|c|c|c|c|c|c|c|}
\hline$* \mathrm{~h}$ & $>$ & h & $>$ & $\mathrm{h}$ & $* h$ & $>$ & h \\
\hline *Cif & $>$ & $\mathrm{h}(\mathrm{j})$ & $>$ & $\mathrm{h}(\mathrm{j})$ & ${ }^{*} \mathrm{Cif}$ & $>$ & C-n \\
\hline *Cuf & $>$ & $\mathrm{f}(\mathrm{w})$ & $>$ & $\mathrm{h}(\mathrm{w})$ & *Cuf & $>$ & C-yw \\
\hline
\end{tabular}

Another example of widespread devoicing occurred in the case of $\mathrm{PHl}{ }^{*} \mathrm{C}-\beta$, which generally devoiced to ${ }^{*} \phi$ and then shifted to $f$ after the loss of the presyllable, except in the case of the Meifu branch, where it lenited to a glide:

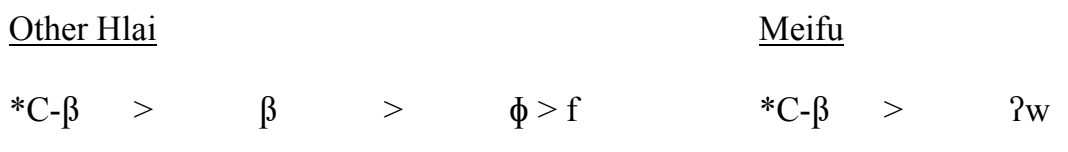


An example in which devoicing occurred on a smaller scale is in the Run branch, where $\mathrm{PHl}{ }^{*} \mathrm{rj}$ and ${ }^{*} \mathrm{r}$ underwent fortitions to voiced obstruents which subsequently underwent devoicing:

$\begin{array}{lllll}\text { Run } & & & & \\ *_{\mathrm{rj}} & > & \mathrm{d} & > & \mathrm{t} \\ *_{\mathrm{r}} & > & \mathrm{y} & > & \mathrm{x}\end{array}$

It is crucial to understand that registrogenesis (see below) occurred chronologically between earlier and later obstruent devoicings. Any obstruents which were devoiced prior to registrogenesis conditioned high register, irregardless of their earlier status as voiced obstruents. However, all obstruents which were voiced at the stage of registrogenesis conditioned low register, even if they subsequently devoiced. This can be illustrated in Run, using the reflex of PHl * rj above and that of PHl *hn (bold font indicates that a phoneme has conditioned low register):

$\begin{array}{lllllllll}\text { PHl } & & & \text { Devoicing } & & \text { Registrogenesis } & & \text { Devoicing } \\ * \mathrm{rj} & > & \mathrm{d} & > & \mathrm{t} & > & \mathrm{t} & > & \mathrm{t} \\ * \mathrm{hn} & > & \mathrm{hn}^{\mathrm{d}} & > & \mathrm{nd} & > & \mathbf{d} & > & \mathbf{t}\end{array}$

\subsubsection{Registrogenesis}

The Hlai languages, as is typical of the typological area in which they participate, are tone languages, and each word has an obligatory lexical tone. There are two components of the tone system: tone category, and register. The first of these is correlated with the rime, and in Kra-Dai studies, there are four original tone categories traditionally labeled A, B, C, and D. These categories are very likely inherited from the level of Proto-Kra-Dai itself. However, since they do not bear directly on the system of initials, they can be put aside until the discussion of PHl rimes in chapter three. Register (and the development thereof, registrogenesis), on the other hand, is correlated directly with the system of initials, and is therefore relevant to the present discussion of initials (for a more detailed comparison, see chapter three section 3.2). 
When there is a phonological register split in the Southeast Asian linguistic area, the two registers are usually referred to as 'high' register (with higher pitch across the rime) and 'low' register (with lower pitch across the rime). High register correlates with an original voiceless onset, and low register with an original voiced onset (Yip 2002: 33-38). In Southeast Asian historical linguistics, it is considered axiomatic that when there is a register split, high register indicates an originally voiceless initial, and low register indicates an originally voiced intitial. This is because in the process of registrogenesis, the natural lowering of F0 which accompanies voiced consonants becomes phonologized. For example:

(a) High register results from:

$\begin{array}{lllll}\mathrm{p} & \mathrm{t} & \mathrm{c} & \mathrm{k} & 2 \\ \mathrm{p}^{\mathrm{h}} & \mathrm{t}^{\mathrm{h}} & \mathrm{c}^{\mathrm{h}} & \mathrm{k}^{\mathrm{h}} & \\ \mathrm{f} & \mathrm{s} & \mathrm{c} & \mathrm{x} & \mathrm{h}\end{array}$

(b) Low register results from:

$\begin{array}{lllll}\mathrm{b} & \mathrm{d} & \mathrm{d} z & \mathrm{~g} & \\ \mathrm{v} & \mathrm{z} & \mathrm{z} & \mathrm{y} & \mathrm{h} \\ \mathrm{m} & \mathrm{n} & \mathrm{j} & \mathrm{y} & \\ & 1 & & & \\ \mathrm{w} & \mathrm{r} & \mathrm{j} & & \end{array}$

Although the classes of obstruents and sonorants function in rather different ways phonetically, there has nevertheless been a sort of compensatory adjustment in the register system so that the high and low pairs of the obstruent and sonorant categories are made equivalent, and a two-level register system results:

Table 2: Obstruent and Sonorant Initial Correlates with Register

\begin{tabular}{|l|l||l|l|}
\hline Obstruents & Register & Sonorants & Register \\
\hline \hline $\mathrm{p}$ & High & ?m & High \\
\hline $\mathrm{b}$ & Low & $\mathrm{m}$ & Low \\
\hline
\end{tabular}


This phenomenon is so regular, that when discrepancies occur between the syllable initial and expected tone register, a logical explanation has been formulated. This has proved to be extremely fruitful for diachronic exploration of Southeast Asian languages in the following two ways:

A. When voiceless obstruents are associated with low register, it is normally assumed that the obstruent was originally voiced, and underwent devoicing after registrogenesis:

$$
\begin{array}{ll}
\mathrm{p}^{\text {low }} & < \\
\mathrm{f}^{\text {low }} & <
\end{array}
$$

B. When voiced intitials are associated with high register, it is assumed that either there was originally a preceding voiceless consonant which conditioned the register and was subsequently lost, or that the glottis was held in a marked configuration (either spread or constricted) which preempted voicing. Depending on the nature of the initial inventory in question, these segments are usually represented as follows:

$$
\begin{aligned}
& \mathrm{b}^{\text {high }}<\quad * \mathrm{C}-\mathrm{b}, * \mathrm{~Pb} \\
& \mathrm{~m}^{\text {high }}<\quad * \mathrm{C}-\mathrm{m}, * \mathrm{Pm},{ }^{*} \mathrm{hm} \text {, } \\
& \mathrm{w}^{\text {high }}<\quad * \mathrm{C}-\mathrm{w}, * \text { ?w, *hw }
\end{aligned}
$$

Diachronically, the least sonorous segment controls the register in a complex initial with both voiceless and voiced elements. For example, a cluster such as $p l$ conditions high register, since the $p$ is voiceless which trumps the more sonorous liquid. A fully voiced initial cluster, such as $b l$, would condition low register, since neither segment is voiceless.

In the Hlai languages, registrogenesis only occurred after the breakup of $\mathrm{PHl}$, and even after the breakup of the Hlai subgroups into individual languages. It was very likely induced through contact with Hainanese, a variety of Southern Min (the first variety of Chinese to be spoken on Hainan, beginning in the Song dynasty). The strongest evidence for this is that those languages which underwent registrogenesis fall along the northern and north-eastern periphery of the Hlai-speaking area, where contact with Hainanese and other non-Hlai languages has been most intensive. It is also important to note that when languages underwent registrogenesis, they did not necessarily do it at the same time, so that some languages will show 
high register for the same lexical item, and some low, depending on which segmental and laryngeal changes had occurred by the time of the register split.

The Hlai languages can be divided between those which have undergone a register split, and those which have not. Moreover, not all languages that have undergone the split have done so in all four tone categories. Those which have undergone a split in all four categories are Yuanmen, Tongzha, Zandui, and Baoting (which, as shown in the previous chapter, are part of a linguistic area). Cunhua has a split in all categories except for B, and Nadouhua and Changjiang have a split in the A category, but not in B, C, or $\mathrm{D}^{7}$ :

Register split in the Hlai languages

(a) No register split

\begin{tabular}{|c|c|c|c|c|c|c|c|c|}
\hline Bouhin & $\mathrm{A}^{\text {high }}$ & $\mathrm{A}^{\mathrm{low}}$ & $\mathrm{B}^{\text {high }}$ & $\mathrm{B}^{\mathrm{low}}$ & $\mathrm{C}^{\text {high }}$ & $\mathrm{C}^{\text {low }}$ & $D^{\text {high }}$ & $\mathrm{D}^{\mathrm{low}}$ \\
\hline Ha Em & $\mathrm{A}^{\text {high }}$ & $\mathrm{A}^{\text {low }}$ & $\mathrm{B}^{\text {high }}$ & $\mathrm{B}^{\text {low }}$ & $\mathrm{C}^{\text {high }}$ & $\mathrm{C}^{\text {low }}$ & $\mathrm{D}^{\text {high }}$ & $\mathrm{D}^{\text {low }}$ \\
\hline Lauhut & $\mathrm{A}^{\text {high }}$ & $\mathrm{A}^{\text {low }}$ & $\mathrm{B}^{\text {high }}$ & $\mathrm{B}^{\text {low }}$ & $\mathrm{C}^{\text {high }}$ & $\mathrm{C}^{\text {low }}$ & $\mathrm{D}^{\text {high }}$ & $\mathrm{D}^{\text {low }}$ \\
\hline Moyfaw & $\mathrm{A}^{\text {high }}$ & $\mathrm{A}^{\mathrm{low}}$ & $\mathrm{B}^{\text {high }}$ & $\mathrm{B}^{\text {low }}$ & $\mathrm{C}^{\text {high }}$ & $\mathrm{C}^{\text {low }}$ & $\mathrm{D}^{\text {high }}$ & $\mathrm{D}^{\text {low }}$ \\
\hline Baisha & $\mathrm{A}^{\text {high }}$ & $\mathrm{A}^{\text {low }}$ & $\mathrm{B}^{\text {high }}$ & $\mathrm{B}^{\text {low }}$ & $\overline{C^{\text {high }}}$ & $\mathrm{C}^{\text {low }}$ & $\overline{D^{\text {high }}}$ & $\mathrm{D}^{\text {low }}$ \\
\hline
\end{tabular}

(b) Register split in category A only

Nadouhua

Changjiang

\begin{tabular}{|l|l|}
\hline $\mathrm{A}^{\text {high }}$ & $\mathrm{A}^{\text {low }}$ \\
\hline $\mathrm{A}^{\text {high }}$ & $\mathrm{A}^{\text {low }}$ \\
\hline
\end{tabular}

\begin{tabular}{|ll}
\hline $\mathrm{B}^{\text {high }}$ & $\mathrm{B}^{\text {low }}$ \\
\hline $\mathrm{B}^{\text {high }}$ & $\mathrm{B}^{\text {low }}$ \\
\hline
\end{tabular}

\begin{tabular}{|ll}
\hline $\mathrm{C}^{\text {high }}$ & $\mathrm{C}^{\text {low }}$ \\
\hline $\mathrm{C}^{\text {high }}$ & $\mathrm{C}^{\text {low }}$ \\
\hline
\end{tabular}

\begin{tabular}{|ll}
\hline $\mathrm{D}^{\text {high }}$ & $\mathrm{D}^{\text {low }}$ \\
\hline $\mathrm{D}^{\text {high }}$ & $\mathrm{D}^{\text {Iow }}$ \\
\hline
\end{tabular}

(c) Register split in categories A, C, D
Cunhua

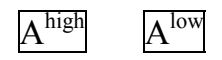
$\mathrm{B}^{\text {high }} \mathrm{B}^{\text {low }}$
$\mathrm{C}^{\text {high }} \quad \mathrm{C}^{\text {low }}$
$\mathrm{D}^{\text {high }} \quad \mathrm{D}^{\text {low }}$

(d) Register split in all categories

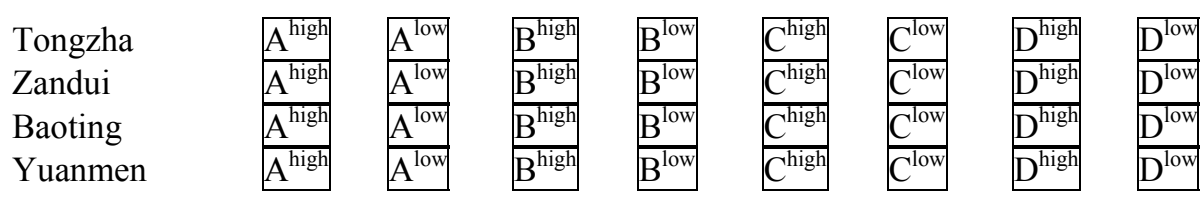

The figure below shows all of the languages which have undergone registrogenesis and the tone numbers which have been assigned to each tone in each register in the descriptive literature ${ }^{8}$ :

\footnotetext{
${ }^{7}$ This variation is explored more fully in the next chapter, where it is suggested that pre-existing phonation originating from final laryngeals may have blocked registrogenesis in categories B, C and D.

${ }^{8}$ The traditional numbering system which has been assigned to tones in the Hlai literature is less than optimal. The system is based on languages without a register split, where tone categories A, B, C, and D
} 
Tone numbers in registrogenetic languages

\begin{tabular}{|c|c|c|c|c|c|c|c|c|}
\hline$\underline{\text { Tone Category }}$ & $\underline{\text { Register }}$ & ${\underline{\text { Nadou }^{9}}}^{9}$ & Cjiang & Cunhua & $\underline{\text { Ymen }}$ & $\underline{\text { Tzha }}$ & $\underline{\text { Zdui }}$ & $\underline{\text { Bting }}$ \\
\hline \multirow[t]{2}{*}{ A } & High & 1 & 1 & 1 & 1 & 1 & 1 & 1 \\
\hline & Low & 4 & 4 & 4 & 4 & 4 & 4 & 4 \\
\hline \multirow[t]{2}{*}{ B } & High & 2 & 2 & 5 & 5 & 5 & 5 & 5 \\
\hline & Low & 2 & 2 & 5 & 2 & 2 & 2 & 2 \\
\hline \multirow[t]{2}{*}{$\mathrm{C}$} & High & 3 & 3 & 3 & 3 & 3 & 3 & 3 \\
\hline & Low & 3 & 3 & 4 & 6 & 6 & 6 & 6 \\
\hline \multirow[t]{2}{*}{ D } & High & 4 & 4 & 2 & 7 & 7 & 7 & 7 \\
\hline & Low & 4 & 4 & 4 & 8 & 8 & 8 & 8 \\
\hline
\end{tabular}

Although there is variation in tone contour across languages, a very salient property which can be seen is that tones in the high register, as the label suggests, are almost always higher in pitch level than those in the low register. The exception to this rule is in Nadouhua, where the overall contour of tone category A has settled at the bottom of the pitch range in the high register. This pattern fits with the model articulated in Thurgood (2002), which argues that low register has been conditioned by breathy voice (an assertion which is given additional strength by segmental reflexes of Zandui stops, to be described below). This is shown more clearly in the following figure, which shows the actual pitch values of the tones in the registrogenetic languages:

are assigned numbers $1,2,3$, and 4 respectively. In the registrogenetic languages in Ouyang \& Zheng (1983), this correlation was maintained, but an effort was made to correlate odd numbers with high register, and even numbers with low register. Therefore, category B appears 'flipped' when compared with categories $\mathrm{A}$ and $\mathrm{C}$. These categories were later applied to the less symmetrical tone/register categories in Cunhua and Nadouhua, making the system even more opaque. In assigning numbers to Changjiang, I have used the same system as Nadouhua, with which its history of registrogenesis is identical.

${ }^{9}$ Nadouhua and Changiiang tone 4 is always accompanied by glottal constriction within the rhyme. 
(18) Tone values in registrogenetic languages

\begin{tabular}{|c|c|c|c|c|c|c|c|}
\hline$\underline{\text { Language }}$ & $\underline{\text { Tone }}$ & $\underline{\text { High }}$ & $\underline{\text { Low }}^{10}$ & Language & $\underline{\text { Tone }}$ & $\underline{\text { High }}$ & $\underline{\text { Low }}$ \\
\hline Nadouhua & A & 11 & $21 ?$ & Tongzha & A & 33 & 11 \\
\hline Changjiang & A & 53 & $15 ?$ & & B & 51 & 121 \\
\hline \multirow[t]{3}{*}{ Cunhua } & A & 35 & 13 & & $\mathrm{C}$ & 55 & 14 \\
\hline & $\mathrm{C}$ & 42 & 13 & & $\mathrm{D}$ & 55 & 13 \\
\hline & $\mathrm{D}$ & 33 & 13 & Zandui & A & 33 & 11 \\
\hline \multirow[t]{7}{*}{ Yuanmen } & A & 42 & 11 & & B & 42 & 21 \\
\hline & B & 51 & 131 & & $\mathrm{C}$ & 35 & 213 \\
\hline & $\mathrm{C}$ & 44 & 13 & & $\mathrm{D}$ & 42 & 21 \\
\hline & $\mathrm{D}$ & 55 & 13 & Baoting & A & 44 & 22 \\
\hline & & & & & B & 53 & 31 \\
\hline & & & & & $\mathrm{C}$ & 35 & 213 \\
\hline & & & & & D & $53 / 44$ & 31 \\
\hline
\end{tabular}

The PHl initials themselves can be grouped into categories according to whether or not their reflexes triggered high or low register, and in which languages (low register is indicated with boxed phonemes).

The first category is comprised of those initials which unanimously indicate high register. The first subcategory within this group (19a) is comprised of original voiceless obstruents, including stops, affricates and fricatives. The second subcategory (19b) is comprised of glottal stop, both initial and medial. The third subcategory (19c) is comprised of the two implosive initials. The fourth subcategory (19d) has a single member, the preaspirated lateral. Finally, the fifth subcategory (19e) is comprised of originally voiced medials which became initial and devoiced in all languages prior to registrogenesis:

\footnotetext{
${ }^{10}$ There is one exceptional property which is shared by Nadouhua and Changjiang, indicating close areal contact at directly preceding and during the time of registrogenesis. This is the presence of glottal constriction in the low register of category A, where there was no historical laryngeal segment. What seems to have occurred here is that voiced initials became associated with laryngealization (Thurgood 1991: $4-8$ ), and resulted in the reinterpretation of this creaky voice as the presence of glottal constriction.
} 
(19)

$\underline{\text { PHl }} \underline{\underline{C u n}} \underline{\underline{N a d o u}} \underline{\mathrm{CJ}} \quad \underline{\mathrm{YM}} \quad \underline{\mathrm{TZ}} \quad \underline{\mathrm{ZD}} \quad \underline{\mathrm{BT}}$

(a)

$* \mathrm{p}^{\mathrm{h}}$
$* \mathrm{t}^{\mathrm{h}}$
$* \mathrm{t}^{\mathrm{h}}$
$* \mathrm{t}^{\mathrm{h}} \mathrm{w}$
$* \mathrm{t}^{\mathrm{h}}$
$* \mathrm{k}^{\mathrm{h}}$
$* \mathrm{t} \mathrm{c}$
$* \mathrm{k}$
$* \mathrm{f}$
$* \mathrm{fj}$
$* \mathrm{~s}$

$\begin{array}{lllllll}\mathrm{p}^{\mathrm{h}} & \mathrm{p}^{\mathrm{h}} & \mathrm{p}^{\mathrm{h}} & \mathrm{p}^{\mathrm{h}} & \mathrm{p}^{\mathrm{h}} & \mathrm{p}^{\mathrm{h}} & \mathrm{p}^{\mathrm{h}} \\ \mathrm{t}^{\mathrm{h}} \sim \mathrm{ts}^{\mathrm{h}} & \mathrm{t}^{\mathrm{h}} & \mathrm{t}^{\mathrm{h}} & \mathrm{t}^{\mathrm{h}} & \mathrm{t}^{\mathrm{h}} & \mathrm{t}^{\mathrm{h}} & \mathrm{t}^{\mathrm{h}} \\ \mathrm{h} & \mathrm{h} & \mathrm{ts}^{\mathrm{h}} & \mathrm{ts}^{\mathrm{h}} & \mathrm{ts}^{\mathrm{h}} & \mathrm{ts}^{\mathrm{h}} & \mathrm{ts}^{\mathrm{h}} \\ \mathrm{f} & \mathrm{f} & \mathrm{f} & \mathrm{pf}^{\mathrm{h}} & \mathrm{ts}^{\mathrm{h}} & \mathrm{ts}^{\mathrm{h}} & \mathrm{ts}^{\mathrm{h}} \\ \mathrm{ts}^{\mathrm{h}} & \mathrm{s} & \mathrm{ts}^{\mathrm{h}} & \mathrm{ts}^{\mathrm{h}} & \mathrm{ts}^{\mathrm{h}} & \mathrm{ts}^{\mathrm{h}} & \mathrm{ts}^{\mathrm{h}} \\ \mathrm{k}^{\mathrm{h}} & \mathrm{k}^{\mathrm{h}} & \mathrm{k}^{\mathrm{h}} & \mathrm{k}^{\mathrm{h}} & \mathrm{k}^{\mathrm{h}} & \mathrm{k}^{\mathrm{h}} & \mathrm{k}^{\mathrm{h}} \\ \mathrm{ts} & \mathrm{ts} & \mathrm{ts} & \mathrm{t} & \mathrm{ts} & \mathrm{ts} & \mathrm{ts}^{\mathrm{h}} \\ \mathrm{k} & \mathrm{k} & \mathrm{k} & \mathrm{k} & \mathrm{k} & \mathrm{k} & \mathrm{k} \\ \mathrm{f} & \mathrm{f} & \mathrm{f} & \mathrm{pf}^{\mathrm{h}} & \mathrm{f} & \mathrm{f} & \mathrm{f} \\ \mathrm{s} & \mathrm{f} & \mathrm{s} & \mathrm{pf}^{\mathrm{h}} & \mathrm{f} & \mathrm{f} & \mathrm{f} \\ \mathrm{t} \theta & \mathrm{f} & \mathrm{s} & \mathrm{ts}^{\mathrm{h}} & \mathrm{t} & \mathrm{t} & \mathrm{t}\end{array}$

(b) *?

${ }^{*} \mathrm{Ci}$ ?

$* \mathrm{Cu}$ ?

\begin{tabular}{|c|c|c|c|c|c|}
\hline$?$ & $?$ & $?$ & $?$ & $?$ & $?$ \\
\hline & $2 \mathrm{j}$ & $\mathrm{z}$ & $z$ & $\mathrm{z}$ & $\mathrm{z}$ \\
\hline & ?w & kw & $\mathrm{y}$ & ?w & $\mathrm{V}$ \\
\hline
\end{tabular}

(c) $\quad \begin{aligned} & * 6 \\ & *_{d}\end{aligned}$

$\begin{array}{lllllll}6 & 6 & 6 & 6 & 6 & 6 & 6 \\ d \sim \text { ts } & d & d & d & d & d & d\end{array}$

(d) $\quad *$ hl

$\begin{array}{llllllll}\mathrm{t} \theta & 1 & 1 & 1 & \& & 1 & 1\end{array}$

${ }^{*} \mathrm{C}-\beta$
$\quad * \mathrm{C}-\mathrm{h}$

$\begin{array}{lllllll}\text { f } & \text { f } & \text { kw } & \text { f } & \text { f } & \text { f } & \text { f } \\ \text { h } & \text { h } & \text { h } & \text { h } & \text { h } & \text { h } & \text { h }\end{array}$

The second category is comprised of those initials which only exhibit low register in Yuanmen; these are both medial glottal fricatives, which underwent a shift to medial nasals in NCHl:

$\begin{array}{llllllll}\underline{\text { PHl }} & \underline{\text { Cun }} & \underline{\text { Nadou }} & \underline{\text { CJ }} & \underline{\text { YM }} & \underline{\text { TZ }} & \underline{\text { ZD }} & \underline{\text { BT }} \\ { }^{*} \text { Cif } & \mathrm{n} & \mathrm{yj} & \mathrm{n} & \mathrm{n} & \mathrm{z} & \mathrm{z} & \mathrm{hj} \\ \text { *Cuh }^{\text {Cun }} & \mathrm{y} & \mathrm{g}(\mathrm{w}) & \mathrm{y} & \mathrm{m} & \mathrm{gw} & \mathrm{v} & \mathrm{hw}\end{array}$

The third category is comprised of initials which exhibit low register in only Yuanmen and Zandui; these are all medial sonorants: 
(21)

\begin{tabular}{|c|c|c|c|c|c|c|c|}
\hline$\underline{\mathrm{PHl}}$ & $\underline{\text { Cun }}$ & $\underline{\text { Nadou }}$ & $\underline{\mathrm{CJ}}$ & $\underline{Y M}$ & $\underline{\mathrm{TZ}}$ & $\underline{\mathrm{ZD}}$ & BT \\
\hline${ }^{*} \mathrm{C}-\mathrm{m}$ & $\mathrm{m}$ & $\mathrm{m}$ & $\mathrm{m}$ & $\mathrm{m}$ & $\mathrm{m}$ & $\mathrm{m}$ & $\mathrm{m}$ \\
\hline${ }^{*} \mathrm{C}-\mathrm{n}$ & $\mathrm{n}$ & $\mathrm{n}$ & $\mathrm{n}$ & n & $\mathrm{n}$ & $\mathrm{n}$ & $\mathrm{n}$ \\
\hline${ }^{*} \mathrm{C}-\mathrm{n}$ & $\mathrm{n}$ & $\mathrm{jj}$ & $\mathrm{n}$ & $\mathrm{n}$ & $\mathrm{n}$ & $\mathrm{n}$ & $\mathrm{n}$ \\
\hline${ }^{*} \mathrm{C}-\mathrm{y}$ & $\eta$ & y & $\eta$ & m & $\eta$ & $\mathrm{g}$ & $\mathrm{y}$ \\
\hline *Cuy & $\eta$ & yw & $\eta$ & $\mathrm{m}$ & yw & $y$ & yw \\
\hline${ }^{*} \mathrm{C}-1$ & 1 & 1 & 1 & 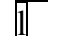 & 1 & & 1 \\
\hline
\end{tabular}

The fourth category is comprised of initials which exhibit low register in Cunhua, Nadouhua, and Changjiang; this includes the one true cluster in $\mathrm{PHl}$ :

$\begin{array}{llllllll}\underline{\text { PHl }} & \underline{\text { Cun }} & \underline{\text { Nadou }} & \underline{\text { CJ }} & \underline{\text { YM }} & \underline{\text { TZ }} & \underline{\text { ZD }} & \underline{\text { BT }} \\ { }^{* p-1} & 6 \sim 1 & \text { pj } & p & \text { pl } & \text { pl } & p & \text { pl }\end{array}$

The conditioning of low register is unexpected in this case, as an initial voiceless obstruent is expected to condition high register; an explanation for this is offered below in section 2.3.2.3.

The fifth category is comprised of initials which exhibit low register in Nadouhua and the Qi languages; it also includes only one member, medial *r:

\begin{tabular}{|c|c|c|c|c|c|c|c|}
\hline$\underline{\mathrm{PHl}}$ & $\underline{\text { Cun }}$ & $\underline{\text { Nadou }}$ & $\underline{\mathrm{CJ}}$ & $\underline{\mathrm{YM}}$ & $\underline{\mathrm{TZ}}$ & $\underline{\mathrm{ZD}}$ & $\underline{\text { BT }}$ \\
\hline${ }^{k} \mathrm{Cur}$ & $\mathrm{V}$ & m & kw & V & gw & $\mathrm{V}$ & hw \\
\hline
\end{tabular}

The sixth category consists of initials which exhibit high register only in Cunhua and Baoting; this includes the two PHl preaspirated glides:

\begin{tabular}{|c|c|c|c|c|c|c|c|}
\hline$\underline{\mathrm{PHl}}$ & Cun & $\underline{\mathrm{Nadou}}$ & $\underline{\mathrm{CJ}}$ & $\underline{\mathrm{YM}}$ & $\underline{\mathrm{TZ}}$ & $\underline{\mathrm{ZD}}$ & $\underline{\mathrm{BT}}$ \\
\hline${ }^{*} \mathrm{hj}$ & $\mathrm{Z}$ & $z$ & $\mathrm{Z}$ & $\mathrm{Z}$ & Z & $z$ & z \\
\hline *hw & $\mathrm{v}$ & $\mathrm{V}$ & $\mathrm{V}$ & $\mathrm{V}$ & $\mathrm{V}$ & $\mathrm{v}$ & $\mathrm{V}$ \\
\hline
\end{tabular}

The penultimate category consists of initials which exhibit low register in all but one language. This is a heterogeneous category which includes (a) the preaspirated labio-velar nasal, and (b) various approximants: 


$\underline{\text { PHl Cun }} \quad \underline{\text { Nadou }} \quad \underline{\mathrm{CJ}} \quad \underline{\mathrm{YM}} \quad \underline{\mathrm{TZ}} \quad \underline{\mathrm{ZD}} \quad \underline{\mathrm{BT}}$

(a) *hyw

\begin{tabular}{llllllll}
\hline$h$ & y & v & v & v & v & v
\end{tabular}

(b)

${ }^{* \mathrm{C}-\mathrm{v}}$
${ }^{* \mathrm{j}}$
${ }^{* \mathrm{Cur}}$
${ }_{\mathrm{r}}^{\mathrm{r}}$

\begin{tabular}{|lll}
\hline $\mathrm{v}$ & $\mathrm{v}$ & $\mathrm{v}$ \\
\hline $\mathrm{z}$ & $\mathrm{l}$ & $\mathrm{t}$ \\
\hline $\mathrm{v}$ & $\mathrm{v}$ & $\mathrm{kw}$ \\
\hline $\mathrm{h}$ & $\mathrm{y}$ & $\mathrm{g}$ \\
\hline
\end{tabular}

\begin{tabular}{llll}
$\mathrm{v}$ & $\mathrm{f}$ & $\mathrm{v}$ & $\mathrm{v}$ \\
$\mathrm{t}-\mathrm{ts}$ & $\mathrm{t}$ & $\mathrm{t}^{\mathrm{h}}$ & $\mathrm{t}$ \\
$\mathrm{f}$ & $\mathrm{f}$ & $\mathrm{f}$ & $\mathrm{f}$ \\
\hline $\mathrm{k}^{\mathrm{h}}$ & $\mathrm{g}$ & $\mathrm{h}$ & $\mathrm{h}$ \\
\hline
\end{tabular}

The final category consists of initials which conditioned low register in all languages; it includes (a) all of the initial preaspirated nasals, as well as (b) the palatalized lateral and (c) the tap:

$\underline{\mathrm{PHl}}$

$\underline{\text { Cun }} \quad \underline{\text { Nadou }} \underline{\text { CJ }} \quad \underline{Y M} \quad \underline{T Z} \quad \underline{\text { ZD }} \quad \underline{B T}$

(a)

$* \mathrm{hm}$
$* \mathrm{hn}$
$* \mathrm{hn}$
$* \mathrm{hy}$

\begin{tabular}{|lclllll|}
\hline 6 & $\mathrm{p}$ & $\mathrm{p}$ & $\mathrm{p}$ & $\mathrm{p}$ & $\mathrm{p}^{\mathrm{h}}$ & $\mathrm{p}$ \\
\hline $\mathrm{d} \sim \mathrm{ts}$ & $\mathrm{t}$ & $\mathrm{t}$ & $\mathrm{t}$ & $\mathrm{t}$ & $\mathrm{t}^{\mathrm{h}}$ & $\mathrm{t}$ \\
$\mathrm{ts}$ & $\mathrm{ts}$ & $\mathrm{ts}$ & $\mathrm{ts}$ & $\mathrm{ts}$ & $\mathrm{ts}^{\mathrm{h}}$ & $\mathrm{ts}$ \\
\hline $\mathrm{k}$ & $\mathrm{k}$ & $\mathrm{k}$ & $\mathrm{k}$ & $\mathrm{k}$ & $\mathrm{k}^{\mathrm{h}}$ & $\mathrm{k}$ \\
\hline
\end{tabular}

(b) $\quad * 1 j$

\begin{tabular}{|lllllll}
\hline 1 & z & z & ts & t & A & A \\
\hline
\end{tabular}

(c) $\quad *$ C-

\begin{tabular}{|llllllll}
\hline 1 & 1 & 1 & $\mathrm{r}$ & $\mathrm{r}$ & 1 & 1 \\
\hline
\end{tabular}

In summary, the languages above which have undergone registrogenesis are crucial to the reconstruction of $\mathrm{PHl}$, as they provide evidence for the voicing (or lack thereof) of initials at the point of registrogenesis. This evidence is still sometimes indirect however, given the fact that registrogenesis occurred after the second devoicing, which eliminated the context for low register in some originally voiced initials. This is the topic to which we now turn. 


\subsection{Reconstruction of Initials by Manner}

The various categories of $\mathrm{PHl}$ initials will be reconstructed in this section. The data from the twelve Hlai languages in Ouyang \& Zheng (1983) and from my own fieldwork are presented. In addition, the reflexes of the Bouhin ('Southern Hlai') and Qi ('Central Hlai') languages recorded in Savina (1931) and the Baisha dialect recorded in Wang \& Qian (1951) are presented. The order of presentation will be that of obstruents (divided into stops, affricates, and fricatives), followed by sonorants (divided into nasals, liquids, approximants, and glides). The primary difference between obstruents and sonorants is in the development of the aspirated initials; aspirated obstruents remained so in both primary branches of Hlai, whereas aspirated sonorants developed differently in Bouhin and in Greater Hlai. These reconstructions are discussed below. Where changes from the proto-form have occurred, they are discussed in detail within the typology of changes outlined in section 2.2 above, and the assumed paths of change are outlined. Three examples of each initial will be provided. Finally, the reconstruction proposed here will be compared with the earlier reconstructions of Matisoff (1988), Thurgood (1993), Peiros (1998), and Ostapirat (2004). In order to facilitate exposition, the initials will be subdivided into obstruents, sonorants, and glottals (which are set apart because of the way in which they pattern with the sonorants, despite being technically classified as obstruents).

\subsubsection{Obstruent Initials}

There are four classes of PHl obstruent initials: aspirated stops, aspirated affricates, implosive/plain voiceless stops, and fricatives. These will be treated in turn below.

\subsubsection{Aspirated Stops}

The reflexes of the PHl aspirated stops are shown below in (27). With the single exception of the Cunhua reflexes for the alveolar stop, the modern reflexes of the stops are homogeneous and 
straightforward in their development from PHl. Note that all reflexes in the daughter languages show aspiration; note also that all reflexes are in the high register in languages which distinguish between high and low, indicating original voiceless onsets at the stage of registrogenesis:

Reflexes of PHl voiceless stops

\begin{tabular}{|c|c|c|c|c|c|c|c|c|c|c|c|}
\hline$\underline{B h i n}$ & $\underline{\mathrm{Ha} E m}$ & $\underline{\text { Lhut }}$ & $\underline{\text { Tzha }}$ & $\underline{\text { Zdui }}$ & $\underline{\text { Bting }}$ & $\underline{\mathrm{Cun}^{11}}$ & $\underline{\text { Nadou }}$ & $\underline{\text { Cjiang }}$ & $\underline{\text { Mfaw }}$ & $\underline{\text { Baisha }}$ & $\underline{\text { Ymen }}$ \\
\hline$p^{h}$ & $p^{h}$ & $p^{h}$ & $p^{h}$ & $p^{\mathrm{h}}$ & $p^{h}$ & $p^{\mathrm{h}}$ & $p^{\mathrm{h}}$ & $p^{\mathrm{h}}$ & $p^{h}$ & $p^{\mathrm{h}}$ & $p^{h}$ \\
\hline$t^{h}$ & $\mathrm{t}^{\mathrm{h}}$ & $\mathrm{t}^{\mathrm{h}}$ & $\mathrm{t}^{\mathrm{h}}$ & $\mathrm{t}^{\mathrm{h}}$ & $\mathrm{t}^{\mathrm{h}}$ & $t^{h} \sim t s^{h}$ & $\mathrm{t}^{\mathrm{h}}$ & $\mathrm{t}^{\mathrm{h}}$ & $\mathrm{t}^{\mathrm{h}}$ & $\mathrm{t}^{\mathrm{h}}$ & $\mathrm{t}^{\mathrm{h}}$ \\
\hline $\mathrm{k}^{\mathrm{h}}$ & $\mathrm{k}^{\mathrm{h}}$ & $\mathrm{k}^{\mathrm{h}}$ & $\mathrm{k}^{\mathrm{h}}$ & $\mathrm{k}^{\mathrm{h}}$ & $\mathrm{k}^{\mathrm{h}}$ & $\mathrm{k}^{\mathrm{h}}$ & $\mathrm{k}^{\mathrm{h}}$ & $\mathrm{k}^{\mathrm{h}}$ & $\mathrm{k}^{\mathrm{h}}$ & $\mathrm{k}^{\mathrm{h}}$ & $\mathrm{k}^{\mathrm{h}}$ \\
\hline \multicolumn{4}{|c|}{ S. Hlai (Savina) } & \multicolumn{4}{|c|}{ C. Hlai (Savina) } & \multicolumn{4}{|c|}{ Baisha (Wang \& Qian) } \\
\hline & $\mathrm{f}$ & & & & $\mathrm{p}^{\mathrm{h}}$ & & & & $\mathrm{p}^{\mathrm{h}}$ & & \\
\hline & $t^{h}$ & & & & $\mathrm{t}^{\mathrm{h}}$ & & & & $\mathrm{t}^{\mathrm{h}}$ & & \\
\hline & $\mathrm{x}$ & & & & $\mathrm{x}$ & & & & $\mathrm{k}^{\mathrm{h}}$ & & \\
\hline
\end{tabular}

Based on the nearly uniform reflexes in (27) above, a series of aspirated stops is reconstructed.

The reconstructions which are adopted here are shown below in (28):

$$
\begin{aligned}
& { }^{*} \mathrm{p}^{\mathrm{h}} \\
& * \mathrm{t}^{\mathrm{h}} \\
& * \mathrm{k}^{\mathrm{h}}
\end{aligned}
$$

With one exception in Cunhua (see below), there has been no essential change between PHI and the reflexes of the daughter languages. Based on Savina's transcription, the velar aspirated stop lenited to a fricative in both his Southern and Central Hlai languages, and the bilabial aspirated stop in his Southern Hlai did so as well.

The only problematic development in the aspirated stops series is in Cunhua, where there are split reflexes between $t^{h}$ and $t s^{h}(29 \mathrm{a})$. This phenomenon is also seen in other alveolar series, with split reflexes in Cunhua for PHl *d ( $d$ and $t s)(29 \mathrm{~b}), \mathrm{PHl} * \mathrm{~s}\left(t \theta\right.$ and $\left.t s^{h}\right)(29 \mathrm{c})$, and only examples of $t s$ for *hn (where $d$ is expected) (29d):

\footnotetext{
${ }^{11}$ Where space requires, 'Cunhua' and 'Nadouhua' will be abbreviated as 'Cun' and 'Nadou', respectively.
} 
(29) Palatal variation in Cunhua alveolar initials

$\underline{\text { Gloss }} \quad \underline{\mathrm{PHl}} \quad \underline{\text { Cunhua }} \quad \underline{\text { Gloss }} \quad \underline{\mathrm{PHl}} \quad \underline{\text { Cunhua }}$

(a)

\begin{tabular}{|c|c|c|c|c|c|}
\hline $\begin{array}{l}\text { answer } \\
\text { dam } \\
\text { fall }\end{array}$ & $\begin{array}{l}* \mathrm{t}^{\mathrm{h}} \text { in } \\
* \mathrm{t}^{\mathrm{h}} \mathrm{\partial} \text { :m? } \\
* \mathrm{t}^{\mathrm{h}} \mathrm{ok}\end{array}$ & $\begin{array}{l}\mathrm{t}^{\mathrm{h}} \mathrm{en}^{1} \\
\mathrm{t}^{\mathrm{h}} \mathrm{am}^{3} \\
\mathrm{t}^{\mathrm{h}} \mathrm{ok}^{2}\end{array}$ & $\begin{array}{l}\text { half } \\
\text { rice } \\
\text { wrap }\end{array}$ & 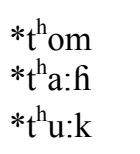 & $\begin{array}{l}\text { ts }^{\mathrm{h}} \mathrm{om}^{1} \\
\text { ts }^{\mathrm{h}} \mathrm{o}::^{5} \\
\text { ts }^{\mathrm{h}} \mathrm{ok}^{2}\end{array}$ \\
\hline castrate & *du:n & dun ${ }^{1}$ & want & *dur: & tsow $^{1}$ \\
\hline forest & *da:w? & $\mathrm{daw}^{3}$ & boil & *da:n & $\operatorname{tss}^{1}$ \\
\hline have & *du:k & dok $^{2}$ & rear & *du:p & tsuən $^{3}$ \\
\hline wild & *su:n & t $\theta$ uən ${ }^{1}$ & step & *sur:m & $\mathrm{ts}^{\mathrm{h}} \mathrm{am}^{1}$ \\
\hline water buffalo & *suy? & $\mathrm{t} \theta$ əy ${ }^{3}$ & wart & *su:c & ts $^{\mathrm{h}} \mathrm{u} \mathrm{t}^{2}$ \\
\hline \multirow[t]{3}{*}{ ripe } & *sur:k & $\mathrm{t} \theta$ wək ${ }^{2}$ & bird & $*_{\mathrm{s} ə \mathrm{c}}$ & $\mathrm{ts}^{\mathrm{h}} \mathrm{iat}^{2}$ \\
\hline & & & top & *hnw: & tsow $^{4}$ \\
\hline & & & six & *hnom & tsem $^{4}$ \\
\hline
\end{tabular}

There is, however, no split in the ${ }^{*} \mathrm{C}-\mathrm{n}$ category, where Cunhua reflexes are always $n$, so it appears that this phenomenon targets initial consonants, and not medials. Although there is not direct evidence for it, this variation seems to be between the original alveolar and palatal place of articulation, as Cunhua $t s^{h}$ and $t s$ are reflexes of earlier $* t_{6}{ }^{\mathrm{h}}$ and $*$ tc respectively. There does not seem to be any discernible conditioning factor in the rhymes following these initials which might have led to secondary palatalization, or any other kind of affrication.

In addition, there are two other non-alveolar initials in which Cunhua shows evidence of secondary palatalization. There are two instances where this variation occurs in two items with original initial labiovelar glides, where the normal reflex is $v$ (the vowel in left is irregular):

Palatal Variation in Cunhua Initial Labiovelar Glides

$\begin{array}{lll}\underline{\text { Gloss }} & \underline{\text { PHl }} & \text { Cunhua } \\ \text { left } & \text { *hwi: } & \text { zay }^{1} \\ \text { banana } & \text { *hwa:k } & \text { zek }^{2}\end{array}$


There are only two cases of which I am aware which involve palatalization of a medial, which in both instances is originally a velar nasal. The palatalization of the one in straw was probably due to the original following high front vowel:

Palatalization of Cunhua medial velar nasals

$\begin{array}{lll}\text { Gloss } & \text { PHl } & \text { Cunhua } \\ \text { envy } & * \text { C-na:yf } & \text { nay }^{5} \\ \text { straw } & * \text { Cunin } & \text { nen }^{3}\end{array}$

The reconstructions of Matisoff, Thurgood, Peiros, and Ostapirat are compared below with the one presently being presented:

\begin{tabular}{|c|c|c|c|}
\hline$\underline{\text { Matisoff }}$ & Thurgood & $\underline{\text { Peiros }}$ & $\underline{\text { Ostapirat }}$ \\
\hline${ }^{*} \mathrm{p}^{\mathrm{h}}$ & $* p^{h}$ & $* p^{h}$ & $*(?) \mathrm{p}$ \\
\hline$* \mathrm{t}^{\mathrm{h}}$ & $* \mathrm{t}^{\mathrm{h}}$ & $* \mathrm{t}^{\mathrm{h}}$ & $*(?) \mathrm{t}$ \\
\hline${ }^{*} \mathrm{k}^{\mathrm{h}}$ & $* \mathrm{k}^{\mathrm{h}}$ & ${ }^{*} \mathrm{k}^{\mathrm{h}}$ & $*_{\mathrm{k}}$ \\
\hline
\end{tabular}

Matisoff's, Thurgood's, and Peiros' reconstructions are all in agreement with the present one. That of Ostapirat is similar to the reconstruction of Pre-Hlai provided in chapter four; it violates Commonality by omitting the ubiquitous presence of aspiration from the reconstruction, and cannot be arrived at merely through the comparative method. The optional glottal stops in his reconstruction are dependent on two different Jiamao reflexes, which I will provide an alternative explanation for in chapter five.

Examples of the PHl stops are given below. The examples below and hereafter will be presented with languages in the following $\operatorname{order}^{12}$ :

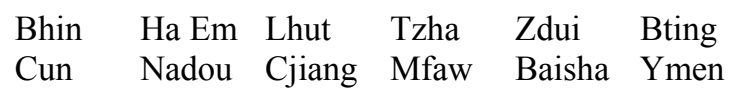

\footnotetext{
${ }^{12}$ Beginning here, forms which are suspected to be loans from another Hlai language due to the irregular correspondences discussed in chapter one are placed in parentheses.
} 
Examples of aspirated stops

\begin{tabular}{|c|c|c|c|c|c|c|c|c|c|c|c|}
\hline (a) & & & $* p^{h}$ & & & (b) & & & $* \mathrm{t}^{\mathrm{h}}$ & & \\
\hline 翅膀 & wing & & ${ }^{*} \mathrm{p}^{\mathrm{h}} \mathrm{i}: \mathrm{k}$ & & & 屁 & fart & & $* t^{h} \mathrm{u}: \mathrm{t}$ & & \\
\hline $\mathrm{p}^{\mathrm{h}} \mathrm{i}: \mathrm{k}^{7}$ & $\mathrm{p}^{\mathrm{h}} \mathrm{i}: \mathrm{P}^{7}$ & $\mathrm{p}^{\mathrm{h}} \mathrm{i}: \mathrm{P}^{7}$ & $\mathrm{p}^{\mathrm{h}} \mathrm{ia}{ }^{7}$ & $\mathrm{p}^{\mathrm{h}} \mathrm{ia} \mathrm{P}^{7}$ & $\mathrm{p}^{\mathrm{h}} \mathrm{iak}^{7}$ & $\mathrm{t}^{\mathrm{h}} \mathrm{u}: \mathrm{t}^{7}$ & $t^{h} u: t^{7}$ & $t^{h} u: t^{7}$ & $t^{h} u: t^{7}$ & $t^{h} u: t^{7}$ & $\mathrm{t}^{\mathrm{h}} \mathrm{u}: \mathrm{t}^{7}$ \\
\hline $\mathrm{p}^{\mathrm{h}}$ iək $^{2}$ & $\mathrm{p}^{\mathrm{h}_{\mathrm{i}} \mathrm{P}^{4}}$ & $\mathrm{p}^{\mathrm{h}_{\mathrm{i}} \mathrm{P}^{7}}$ & $\mathrm{p}^{\mathrm{h}} \mathrm{ik}^{7}$ & $\mathrm{p}^{\mathrm{h}} \mathrm{it}^{8}$ & $\mathrm{p}^{\mathrm{h}_{\mathrm{i}} \mathrm{P}^{7}}$ & $t^{h} u \partial t^{2}$ & $t^{h} u ?^{4}$ & $\mathrm{t}^{\mathrm{h}} \mathrm{ut}^{7}$ & $\mathrm{t}^{\mathrm{h}} \mathrm{ut}^{7}$ & $t^{h} u t^{8}$ & $\mathrm{t}^{\mathrm{h}} \mathrm{ut}^{7}$ \\
\hline 沙 & sand & & ${ }^{*} \mathrm{p}^{\mathrm{h}} \mathrm{u}: \mathrm{h}$ & & & 飯 & rice & & $*^{\mathrm{h}} \mathrm{h}: \mathrm{h}$ & & \\
\hline$\left(\mathrm{p}^{\mathrm{h}} \mathrm{aw}^{2}\right)$ & $\mathrm{p}^{\mathrm{h}} \mathrm{aw}^{2}$ & $\mathrm{p}^{\mathrm{h}} \mathrm{ow}^{2}$ & $\mathrm{p}^{\mathrm{h}} \mathrm{aw}{ }^{5}$ & $\mathrm{p}^{\mathrm{h}} \mathrm{o}:{ }^{5}$ & $\mathrm{p}^{\mathrm{h}} \mathrm{0}:^{5}$ & $\mathrm{t}^{\mathrm{h}} \mathrm{a}::^{2}$ & $\mathrm{t}^{\mathrm{h}} \mathrm{a}:{ }^{2}$ & $\mathrm{t}^{\mathrm{h}} \mathrm{a}:{ }^{2}$ & $\mathrm{t}^{\mathrm{h}} \mathrm{a}::^{5}$ & $\mathrm{t}^{\mathrm{h}} \mathrm{a}:{ }^{5}$ & $\mathrm{t}^{\mathrm{h}} \mathrm{a}:{ }^{5}$ \\
\hline $\mathrm{p}^{\mathrm{h}} \mathrm{a}:^{5}$ & $\mathrm{p}^{\mathrm{h}} \mathrm{aw}^{2}$ & $\mathrm{p}^{\mathrm{h}} \mathrm{aw}^{2}$ & $\mathrm{p}^{\mathrm{h}} \mathrm{aw}^{2}$ & $\mathrm{p}^{\mathrm{h}} \mathrm{aw}^{2}$ & $\mathrm{p}^{\mathrm{h}} \mathrm{aw}^{5}$ & $\operatorname{ts}^{\mathrm{h}} \mathrm{s}:{ }^{5}$ & $\mathrm{t}^{\mathrm{h}} \mathrm{a}:{ }^{2}$ & $\mathrm{t}^{\mathrm{h}} \mathrm{a}:{ }^{2}$ & $\mathrm{t}^{\mathrm{h}} \mathrm{a}:{ }^{2}$ & $\mathrm{t}^{\mathrm{h}} \mathrm{a}:{ }^{2}$ & $\mathrm{t}^{\mathrm{h}} \mathrm{a}:{ }^{2}$ \\
\hline 名字 & name & & $*^{\mathrm{h}} \mathrm{a}: \mathrm{y}$ & & & 鍋 & pot & & $* t^{\mathrm{h}} \partial \mathrm{w}$ & & \\
\hline $\mathrm{p}^{\mathrm{h}} \mathrm{a}: \mathrm{y}^{1}$ & $\mathrm{p}^{\mathrm{h}} \mathrm{e}: \mathrm{y}^{1}$ & $p^{h} e: y^{1}$ & $\mathrm{p}^{\mathrm{h}} \mathrm{e}: \mathrm{y}^{1}$ & $p^{h} e: y^{1}$ & $\mathrm{p}^{\mathrm{h}} \mathrm{e}: \mathrm{y}^{1}$ & $\mathrm{t}^{\mathrm{h}} \mathrm{aw}{ }^{1}$ & $\mathrm{t}^{\mathrm{h}} \mathrm{aw}{ }^{1}$ & $\mathrm{t}^{\mathrm{h}} \mathrm{aw}{ }^{1}$ & $\mathrm{t}^{\mathrm{h}} \mathrm{aw}{ }^{1}$ & $\mathrm{t}^{\mathrm{h}} \mathrm{aw}{ }^{1}$ & $\mathrm{t}^{\mathrm{h}} \mathrm{aw}{ }^{1}$ \\
\hline $\begin{array}{l}\mathrm{p}^{\mathrm{h}} \varepsilon \eta^{1} \\
\text { (c) }\end{array}$ & $\mathrm{p}^{\mathrm{h}} \mathrm{e} \eta^{1}$ & $\mathrm{p}^{\mathrm{h}} \mathrm{e}: \mathrm{y}^{1}$ & $\begin{array}{l}\mathrm{p}^{\mathrm{h}} \mathrm{e}: \mathrm{y}^{1} \\
{ }^{*} \mathbf{k}^{\mathbf{h}}\end{array}$ & $\mathrm{p}^{\mathrm{h}_{\text {ian }}}{ }^{1}$ & $\mathrm{p}^{\mathrm{h}_{\mathrm{iay}}}{ }^{1}$ & $\mathrm{ts}^{\mathrm{h}} \mathrm{aw}^{1}$ & $\mathrm{t}^{\mathrm{h}} \mathrm{aw}^{1}$ & $\mathrm{t}^{\mathrm{h}} \mathrm{aw}^{1}$ & $\mathrm{t}^{\mathrm{h}} \mathrm{aw}^{1}$ & $\mathrm{t}^{\mathrm{h}} \mathrm{aw}^{1}$ & $\mathrm{t}^{\mathrm{h}} \mathrm{aw}{ }^{1}$ \\
\hline 藍色 & blue & & ${ }^{*} \mathrm{k}^{\mathrm{h}} \mathrm{i}: \mathrm{w}$ & & & & & & & & \\
\hline $\mathrm{k}^{\mathrm{h}} \mathrm{i}: \mathrm{w}^{1}$ & $\mathrm{k}^{\mathrm{h}} \mathrm{i}: \mathrm{w}^{1}$ & $\mathrm{k}^{\mathrm{h}} \mathrm{i}: \mathrm{w}^{1}$ & $\mathrm{k}^{\mathrm{h}} \mathrm{i}: \mathrm{w}^{1}$ & $\mathrm{k}^{\mathrm{h}} \mathrm{iw}^{1}$ & $\mathrm{k}^{\mathrm{h}} \mathrm{i}: \mathrm{w}^{1}$ & & & & & & \\
\hline $\mathrm{k}^{\mathrm{h}} \mathrm{iw}^{1}$ & $\mathrm{k}^{\mathrm{h}} \mathrm{i}:{ }^{1}$ & $\mathrm{k}^{\mathrm{h}} \mathrm{iw}^{1}$ & $\mathrm{k}^{\mathrm{h}} \mathrm{iw}^{1}$ & $\mathrm{k}^{\mathrm{h}} \mathrm{iw}^{1}$ & $\mathrm{k}^{\mathrm{h}} \mathrm{iw}^{1}$ & & & & & & \\
\hline 腳 & leg & & ${ }^{*} \mathrm{k}^{\mathrm{h}} \mathrm{ok}$ & & & & & & & & \\
\hline $\mathrm{k}^{\mathrm{h}} \mathrm{ok}^{7}$ & $\mathrm{k}^{\mathrm{h}} \mathrm{uk}^{7}$ & $\mathrm{k}^{\mathrm{h}} \mathrm{ok}^{7}$ & $\mathrm{k}^{\mathrm{h}} \mathrm{ok}^{7}$ & $\mathrm{k}^{\mathrm{h}} \mathrm{O} ?^{7}$ & $\mathrm{k}^{\mathrm{h}} \mathrm{o \textrm {k } ^ { 7 }}$ & & & & & & \\
\hline$\left(\mathrm{k}^{\mathrm{h}} \mathrm{ok}^{2}\right)$ & $\left(\mathrm{k}^{\mathrm{h}} \mathrm{O} \mathrm{P}^{4}\right)$ & $\mathrm{k}^{\mathrm{h}} \mathrm{ok}^{7}$ & $\mathrm{k}^{\mathrm{h}} \mathrm{ok}^{7}$ & $\mathrm{k}^{\mathrm{h}} \mathrm{o \textrm {k } ^ { 8 }}$ & $\mathrm{k}^{\mathrm{h}} \mathrm{ok}^{7}$ & & & & & & \\
\hline 輕 & light & & ${ }^{*} \mathrm{k}^{\mathrm{h}} \mathrm{u}: ?$ & & & & & & & & \\
\hline $\mathrm{k}^{\mathrm{h}} \partial \mathrm{u}^{3}$ & $\mathrm{k}^{\mathrm{h}} \mathrm{au}{ }^{3}$ & $\mathrm{k}^{\mathrm{h}} \mathrm{au}{ }^{3}$ & $\mathrm{k}^{\mathrm{h}} \mathrm{au}{ }^{3}$ & $\mathrm{k}^{\mathrm{h}} \mathrm{au}{ }^{3}$ & $\mathrm{k}^{\mathrm{h}} \mathrm{au}{ }^{3}$ & & & & & & \\
\hline $\mathrm{k}^{\mathrm{h}} \mathrm{o} \mathrm{:}^{\mathrm{l}}$ & $\mathrm{k}^{\mathrm{h}} \mathrm{aw}^{3}$ & $\mathrm{k}^{\mathrm{h}} \mathrm{au}{ }^{3}$ & $\mathrm{k}^{\mathrm{h}} \partial \mathrm{u}^{3}$ & $\mathrm{k}^{\mathrm{h}} \mathrm{au}{ }^{3}$ & $\mathrm{k}^{\mathrm{h}} \mathrm{au}{ }^{3}$ & & & & & & \\
\hline
\end{tabular}

\subsubsection{Affricates}

The reflexes of the PHl affricates are somewhat more complicated than those of the stops, and perhaps the most difficult task is in assigning values for place of articulation to each series. The reflexes of the three PHl affricates are given below: 
Reflexes of the PHI affricates

$\begin{array}{llllllllllll}\text { Bhin } & \text { Ha Em } & \underline{\text { Lhut }} & \underline{\text { Tzha }} & \underline{\text { Zdui }} & \underline{\text { Bting }} & \underline{\text { Cun }} & \underline{\text { Nadou }} & \underline{\text { Cjiang }} & \underline{\text { Mfaw }} & \text { Baisha } & \underline{\text { Ymen }} \\ \text { ts }^{\mathrm{h}} & \mathrm{ts}^{\mathrm{h}} & \mathrm{ts}^{\mathrm{h}} & \mathrm{ts}^{\mathrm{h}} & \mathrm{ts}^{\mathrm{h}} & \mathrm{ts}^{\mathrm{h}} & \mathrm{h} & \mathrm{h} & \mathrm{ts}^{\mathrm{h}} & \mathrm{ts}^{\mathrm{h}} & \mathrm{ts}^{\mathrm{h}} & \mathrm{ts}^{\mathrm{h}} \\ \mathrm{ts}^{\mathrm{h}} & \mathrm{ts}^{\mathrm{h}} & \mathrm{f} & \mathrm{ts}^{\mathrm{h}} & \mathrm{ts}^{\mathrm{h}} & \mathrm{ts}^{\mathrm{h}} & \mathrm{f} & \mathrm{f} & \mathrm{f} & \mathrm{f} & \mathrm{f} & \mathrm{pf}^{\mathrm{h}} \\ \mathrm{ts}^{\mathrm{h}} & \mathrm{ts}^{\mathrm{h}} & \mathrm{ts}^{\mathrm{h}} & \mathrm{ts}^{\mathrm{h}} & \mathrm{ts}^{\mathrm{h}} & \mathrm{ts}^{\mathrm{h}} & \mathrm{ts}^{\mathrm{h}} & \mathrm{s} & \mathrm{ts}^{\mathrm{h}} & \mathrm{ts}^{\mathrm{h}} & \mathrm{ts}^{\mathrm{h}} & \mathrm{ts}^{\mathrm{h}}\end{array}$

\section{$\underline{\text { S. Hlai (Savina) }}$}

\section{$\int$}

C. Hlai (Savina)

\section{Baisha (Wang \& Qian)}

$\mathrm{t} \int^{\mathrm{h}}$
$\mathrm{f}$
$\mathrm{t} \int^{\mathrm{h}}$

I reconstruct these three series at two places of articulation: postalveolar for the first two and palatal for the third. The two postalveolar initials are distinguished between plain and labialized:

$$
\begin{aligned}
& * t \int^{h} \\
& * t \int^{h} w \\
& * t 6^{h}
\end{aligned}
$$

Post-alveolar and palatal affricates are reconstructed based on the witness of Savina and Wang \& Qian given above ${ }^{13}$, and it is apparent that the shift to the alveolar place of articulation is a recent change.

The inventory of possible coarticulations for ${ }^{*} \int^{\mathrm{h}}$ is defective, including $w$ but excluding $j$. This is the same pattern as occurs with $\mathrm{PHl} *_{\mathrm{r}}$ in Central Hlai:

$$
\begin{array}{lll}
* \mathrm{t}^{\mathrm{h}} & * \int^{\mathrm{h}} \mathrm{w} & \left(\text { no } * \mathrm{t} \int^{\mathrm{h}} \mathrm{j}\right) \\
* \mathrm{r} & * \text { Curw } & (\text { no } * \mathrm{rj})
\end{array}
$$

\footnotetext{
${ }^{13}$ There is one complication of this scenario involving Savina's 1931 transcription of Bouhin and a Qi language, which predates the collection of data in Ouyang \& Zheng (1983) by at least two decades. Savina records the reflexes of both Proto-Hlai $* t_{6}{ }^{\mathrm{h}}$ and ${ }^{*} \mathrm{t}^{\mathrm{h}}$ as $\int(<\mathrm{s}>$ in the Vietnamese orthography he was employing), in occasional variation with $s<\mathrm{x}>$. One interpretation of this fact is that Savina was recording real fricatives, and that his data are in conflict with that of Ouyang \& Zheng (1983). An alternative interpretation is that Savina used the Vietnamese letters which came closest to the Hlai affricates; since Vietnamese script only allows for one affricate (unaspirated tc, written $<\mathrm{c}>$ ), which Savina used consistently to represent Bouhin and Qi $t \epsilon$, he may have selected the closest (but suboptimal) Vietnamese characters possible for $t f^{h}$ and $t s^{h}$, namely $<$ s $>$ and $<\mathrm{x}>$, respectively.
} 
While these two phonemes do not synchronically form a natural class, it will be shown in chapter four that $\mathrm{PHl} * \mathrm{t}^{\mathrm{h}}$ is the reflex of an earlier retroflex affricate, which would form a natural class with $\mathrm{PHl}{ }_{\mathrm{r}}$ (see chapter four, section 4.1.2).

The developments of $* \int^{\mathrm{h}}$ and $* t \int^{\mathrm{h}} \mathrm{w}$ are divergent in the daughter languages, with essentially two alternate paths in each case. The most common development of $* t \int^{\text {h }}$ was to $t s^{h}$, merging with the reflexes of $* \mathrm{tc}^{\mathrm{h}}$, presumably through an intermediate stage of $* \mathrm{t}^{\mathrm{h}}$ :

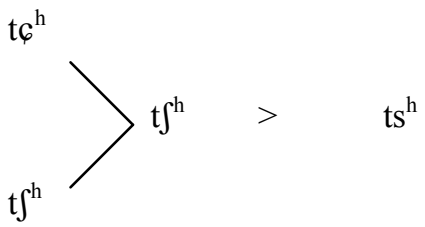

The other reflex of $* \int^{\mathrm{h}}$, in NWCHl, is $h$. Since $h$ is otherwise only a reflex of $* \mathrm{~h}$ in NWCHl, it provides a point of departure for how this change might have occurred. While a change from $* t^{\mathrm{h}}$ to $h$ seems rather awkward, there is actually a parallel available in Spanish which provides a model. Bradley and Delforge (2006) show that the Northern Spanish of the $16^{\text {th }}-17^{\text {th }}$ centuries underwent the following changes:

$\begin{array}{llll}\text { dental: } & \text { S } & > & \theta \\ \text { alveolar: } & \text { S } & > & \text { S } \\ \text { prepalatal: } & \text { S } & > & \mathrm{x}\end{array}$

If a parallel is made with developments in $\mathrm{NWCHl}$, then the one significant development that must have happened was the deaffrication of $* \mathrm{t}^{\mathrm{h}}$ to $* \int$ before the merger with $* \mathrm{tc}^{\mathrm{h}}$ could occur, which happened in all other languages. Comparing this with the development of $*_{s}$ and $*_{\varphi}$, a close parallel can be made with the Northern Spanish case:

\begin{tabular}{|c|c|c|c|c|c|c|c|}
\hline alveolar: & $\mathrm{s}$ & $>$ & S & $>$ & $\theta$ & $>$ & $\mathrm{t} \theta$ (Cun)/f (Nadou) \\
\hline retroflex: & $\mathrm{t} \int^{\mathrm{h}}$ & $>$ & $\int$ & $>$ & $\mathrm{x}$ & $>$ & $\mathrm{h}$ \\
\hline palatal: & 6 & $>$ & 6 & $>$ & $\int$ & $>$ & $\mathrm{s}$ \\
\hline
\end{tabular}


Alternatively, it can also be proposed that $\int$ merely debuccalized, changing directly from $\int$ to $h .^{14}$ There is presently not enough evidence to distinguish between these two alternatives.

The development of $* \int^{\mathrm{h}} \mathrm{w}$ also followed two distinct paths, these being more evenly distributed across languages. In Bouhin, Ha Em, and Qi, the labiovelar glide was simply lost, and $* t \int^{\text {hh }}$ merged with $* \mathrm{t} \int^{\mathrm{h}}$, developing in the same way. However, in Lauhut and $\mathrm{NCHl}$, this phoneme developed into a bilabial fricative, merging with $* f$. The hypothesis I present here is that in these languages, there was a devoicing (natural in the environment following a fricative) and narrowing in aperture of the glide. As temporal compression occurred, there was a coalescence of the fricative element of $* \int$ and the place features of *w, resulting in merger with the already existing category $* f$. This is compared with the development of the other two Hlai affricates below:

\begin{tabular}{|c|c|c|c|}
\hline$* \mathrm{t}^{\mathrm{h}}$ & $>$ & $\mathrm{t} \int^{\mathrm{h}}$ & $>$ \\
\hline$* t \int^{\mathrm{h}} \mathrm{w}$ & $>$ & $t \int^{h} w$ & $>$ \\
\hline$* t \varphi^{\mathrm{h}}$ & $>$ & $\mathrm{t} \int^{\mathrm{h}}$ & $>$ \\
\hline
\end{tabular}

A comparison with other reconstructions is given below:

$\begin{array}{llllll}\text { (a) } & --- & --- & -- & * \text {-t- } & * t^{\mathrm{h}} \\ \text { (b) } & *_{\mathrm{sr}} & *_{\mathrm{sr}} & *_{\mathrm{sw}} & * \text {-ut- } & * \mathrm{t}^{\mathrm{h}} \mathrm{w} \\ \text { (c) } & *_{\mathrm{ts}}^{\mathrm{h}} & *_{\mathrm{ts}}^{\mathrm{h}} & *^{\mathrm{h}} & \mathrm{c}^{\mathrm{h}} & * \mathrm{t}^{\mathrm{h}}\end{array}$

None of Matisoff, Thurgood, or Peiros reconstruct an independent phoneme for the series of correspondences I reconstruct as $* \mathrm{t}^{\mathrm{h}}$, owing to the fact that it can only be distinguished from $\mathrm{PHl} * \mathrm{tc}^{\mathrm{h}}$ by reflexes in NWCHl and Jiamao. Cunhua and Nadouhua were unavailable to Matisoff, and Jiamao was not used by either Matisoff or Thurgood (or presumably Peiros) due to its general complexity.

\footnotetext{
${ }^{14}$ It should be noted that this is the same reflex as that found in the Be languages, which also have $h$.
} 
Matisoff and Thurgood agree in reconstructing *sr for the series which I reconstruct as $* t^{\mathrm{h}} \mathrm{w} .{ }^{*} \mathrm{sr}$ fits into a category in Matisoff's system which also includes * fr and *vr, where fricatives may form licit clusters with a following *r, making the cluster *sr a bit less striking if still typologically rare. Although Matisoff does not explicitly explain how *sr gave rise to the dual reflexes of $t s^{h}$ and $f$, I infer that a shift of *r to $w$ must be assumed. The alternative account proposed here is therefore more economical on internal grounds, since it already assumes a labial component ${ }^{*} \mathrm{w}$ which influenced the shift to $f$. Peiros reconstructs a labialized alveolar fricative, which is closer to my reconstruction in that it assumes original labialization, as opposed to a ${ }^{*} \mathrm{C}-\mathrm{r}$ cluster.

Both Matisoff and Thurgood reconstruct alveolar $* \mathrm{ts}^{\mathrm{h}}$ for the third set of correspondences, and I differ merely in reconstructing a palatal affricate, as this fits better into the overall system of $\mathrm{PHl}$, as well as explaining such things as the $\mathrm{NWCHl}$ chain shift and the postalveolar values recorded for these consonants in Savina (1931) and Wang \& Qian (1951). Peiros reconstructs an aspirated palatal stop, which I reconstruct at the Pre-Hlai stage, not Proto-Hlai (see chapter four); reconstruction of a stop in PHl violates Commonality since there are no stops represented amongst the reflexes of the daughter languages.

Ostapirat reconstructs medial *-t- for the first series, and medial *-t- preceded by *u for the second. These are an integral part of Ostapirat's system, in which stops become affricated intervocalically before the loss of a presyllable in an originally sesquisyllabic word. For the third series, he reconstructs a plain palatal stop. As with the PHl stops above, aspects of Ostapirat's reconstruction correspond better with the reconstruction for Pre-Hlai which I present in chapter four, and his reconstructed series of stops also violates Commonality.

Examples of the PHl affricates are given below, in the following order:

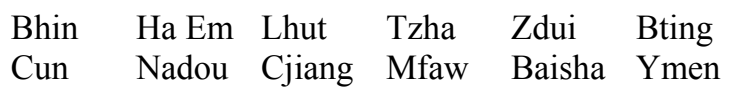


(42) Examples of aspirated affricates

\begin{tabular}{|c|c|c|c|c|c|c|c|c|c|c|c|}
\hline (a) & & & $* t \int^{h}$ & & & (b) & & & $* t \int^{h} w$ & & \\
\hline 花 & flower & & $* t \int^{h} a: \eta$ & & & 織 & weave & fabric) & $* t \int^{h} w u:$ & & \\
\hline $\mathrm{ts}^{\mathrm{h}} \mathrm{e}: \mathrm{y}^{1}$ & $\mathrm{ts}^{\mathrm{h}} \mathrm{e}: \mathrm{\eta}^{1}$ & $\mathrm{ts}^{\mathrm{h}} \mathrm{e}: \mathrm{y}^{1}$ & $\mathrm{ts}^{\mathrm{h}} \mathrm{e}: \mathrm{y}^{1}$ & $\mathrm{ts}^{\mathrm{h}} \mathrm{e}: \mathrm{y}^{1}$ & $\mathrm{ts}^{\mathrm{h}} \mathrm{e}: \mathrm{\eta}^{1}$ & ts $^{\mathrm{h}} \mathrm{u}: ?^{7}$ & ts $^{\mathrm{h}} \mathrm{u}: ?^{7}$ & fur:k ${ }^{7}$ & (fur: $?^{7}$ ) & ts $^{\mathrm{h}} \mathrm{uua}^{7}$ & ts $^{\mathrm{h}} \mathrm{u}: ?^{7}$ \\
\hline hey ${ }^{1}$ & $\left(\mathrm{fe \eta}^{1}\right)$ & $\mathrm{ts}^{\mathrm{h}} \mathrm{e}: \mathrm{y}^{1}$ & $\mathrm{ts}^{\mathrm{h}} \mathrm{e}: \mathrm{y}^{1}$ & ts $^{h_{\text {ian }}}{ }^{1}$ & 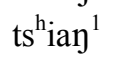 & fuək ${ }^{2}$ & fur ${ }^{4}$ & fur ${ }^{7}$ & furk $^{7}$ & funk $^{8}$ & $\mathrm{pf}^{\mathrm{h}} u \mathrm{P}^{7}$ \\
\hline 眼睛 & eye & & $* t \int^{\mathrm{h}} \mathrm{a}:$ & & & 三 & three & & $*_{\mathrm{t}} \int^{\mathrm{h}} \mathrm{wu} ?$ & & \\
\hline $\begin{array}{l}\text { ts }^{\mathrm{h}} \mathrm{a}:{ }^{1} \\
\text { ho: }\end{array}$ & $\begin{array}{l}\text { ts }^{\mathrm{h}} \mathrm{a}:{ }^{1} \\
\mathrm{ha}:{ }^{1}\end{array}$ & $\begin{array}{l}\text { ts }^{\mathrm{h}} \mathrm{a}:{ }^{1} \\
\text { ts }^{\mathrm{h}} \mathrm{a}:{ }^{1}\end{array}$ & $\begin{array}{l}\text { ts }^{\mathrm{h}} \mathrm{a}:{ }^{1} \\
\text { ts }^{\mathrm{h}} \mathrm{a}:{ }^{1}\end{array}$ & $\begin{array}{l}\text { ts }^{\mathrm{h}} \mathrm{a}:{ }^{1} \\
\text { ts }^{\mathrm{h}} \mathrm{a}:{ }^{1}\end{array}$ & $\begin{array}{l}\text { ts }^{\mathrm{h}} \mathrm{a}:{ }^{1} \\
\text { ts }^{\mathrm{h}} \mathrm{a}:{ }^{1}\end{array}$ & $\begin{array}{l}\text { ts }^{h} u:^{3} \\
\mathrm{fu}:{ }^{3}\end{array}$ & $\begin{array}{l}\text { ts }{ }^{h} u:^{3} \\
\text { fo: }{ }^{3}\end{array}$ & $\begin{array}{l}\mathrm{fu}:^{3} \\
\mathrm{fu}:^{3}\end{array}$ & $\begin{array}{l}\text { ts }^{h} u:^{3} \\
\text { fu: }:^{3}\end{array}$ & $\begin{array}{l}\text { ts }^{h} u:^{3} \\
\text { fu: }:^{3}\end{array}$ & $\begin{array}{l}\operatorname{ts}^{h} u::^{3} \\
\text { pf }^{h} u:^{3}\end{array}$ \\
\hline 抬 & lift (2) & & $*_{\mathrm{t}} \int^{\mathrm{h}} \mathrm{a}: \mathrm{m}$ & & & 下面 & bottom & & $*_{\mathrm{t}} \int^{\mathrm{h}} \mathrm{w} \partial \mathrm{w}$ & & \\
\hline $\begin{array}{l}\text { ts }{ }^{\mathrm{h}} \mathrm{a}: \mathrm{m}^{1} \\
\text { hom }^{1}\end{array}$ & $\begin{array}{l}\text { ts }^{\mathrm{h}} \mathrm{a}: \mathrm{m}^{1} \\
\text { han }^{1}\end{array}$ & $\begin{array}{l}\mathrm{ts}^{\mathrm{h}} \mathrm{a}: \mathrm{m}^{1} \\
\mathrm{ts}^{\mathrm{h}} \mathrm{a}: \mathrm{m}^{1}\end{array}$ & $\begin{array}{l}\text { ts }^{\mathrm{h}} \mathrm{a}: \mathrm{m}^{1} \\
\mathrm{ts}^{\mathrm{h}} \mathrm{a}: \mathrm{m}^{1}\end{array}$ & $\begin{array}{l}\text { ts }^{\mathrm{h}} \mathrm{a}: \mathrm{m}^{1} \\
\mathrm{ts}^{\mathrm{h}} \mathrm{a}: \mathrm{m}^{1}\end{array}$ & $\begin{array}{l}\text { ts }^{\mathrm{h}} \mathrm{a}: \mathrm{m}^{1} \\
\mathrm{ts}^{\mathrm{h}} \mathrm{am}^{1}\end{array}$ & $\begin{array}{l}\mathrm{ts}^{\mathrm{h}} \mathrm{aw}^{1} \\
\text { faw }\end{array}$ & $\begin{array}{l}\mathrm{ts}^{\mathrm{h}} \mathrm{aw}{ }^{1} \\
\text { faw }{ }^{1}\end{array}$ & $\begin{array}{l}\text { fow }^{1} \\
\text { faw }^{1}\end{array}$ & $\begin{array}{l}\text { ts }^{\mathrm{h}} \mathrm{aw}{ }^{1} \\
\text { faw }\end{array}$ & $\begin{array}{l}\mathrm{ts}^{\mathrm{h}} \mathrm{aw}^{1} \\
\text { faw }\end{array}$ & $\begin{array}{l}\text { ts }^{\mathrm{h}} \mathrm{aw}^{1} \\
\text { pfaw }^{1}\end{array}$ \\
\hline (c) & & & $* t \varphi^{h}$ & & & & & & & & \\
\hline 洞 & hole & & $* t c^{h} u: \eta ?$ & & & & & & & & \\
\hline ts ${ }^{h} u: \eta^{3}$ & ts $^{h} \mathrm{u}: \mathrm{y}^{3}$ & $\mathrm{ts}^{\mathrm{h}} \mathrm{u}: \mathrm{y}^{3}$ & $t^{h} \mathrm{u}: \eta^{3}$ & ts $^{\text {h }}$ uan $^{3}$ & ts ${ }^{h} u: y^{3}$ & & & & & & \\
\hline $\mathrm{ts}^{\mathrm{h}} \mathrm{oy}{ }^{3}$ & $\operatorname{sun}^{3}$ & ts $^{h} u y^{3}$ & ts $^{\mathrm{h}} \mathrm{un}^{3}$ & ts $^{\mathrm{h}} u \mathrm{y}^{3}$ & ts $^{\mathrm{h}} \mathrm{un}^{3}$ & & & & & & \\
\hline 硬 & hard & & $* \mathrm{tc}^{\mathrm{h}}$ ə:n? & & & & & & & & \\
\hline $\mathrm{ts}^{\mathrm{h}} \mathrm{an}^{3}$ & $\mathrm{ts}^{\mathrm{h}} \mathrm{O}: \mathrm{n}^{3}$ & $\mathrm{ts}^{\mathrm{h}} \mathrm{o}: \mathrm{n}^{3}$ & ts $^{\mathrm{h}} \mathrm{o}: \mathrm{n}^{3}$ & ts ${ }^{h} \rho: n^{3}$ & ts ${ }^{\mathrm{h}} \mathrm{o}: \mathrm{n}^{3}$ & & & & & & \\
\hline $\mathrm{ts}^{\mathrm{h}} \mathrm{an}^{3}$ & son $^{3}$ & ts $^{\mathrm{h}} \mathrm{o}: \mathrm{y}^{3}$ & ts $^{\mathrm{h}} \mathrm{o}: \mathrm{y}^{3}$ & ts $^{\text {h }}$ uan $^{3}$ & $\mathrm{ts}^{\mathrm{h}} \mathrm{u}: \mathrm{n}^{3}$ & & & & & & \\
\hline 木杵 & pestle & & $*_{\mathrm{t}}{ }^{\mathrm{h}} \mathrm{a}: \mathrm{k}$ & & & & & & & & \\
\hline $\mathrm{ts}^{\mathrm{h}} \mathrm{a}: ?^{7}$ & ts $^{\mathrm{h}} \mathrm{e}: ?^{7}$ & $\mathrm{ts}^{\mathrm{h}} \mathrm{e}: \mathrm{k}^{7}$ & ts ${ }^{\mathrm{h}} \mathrm{e}: ?^{7}$ & $\mathrm{ts}^{\mathrm{h}} \mathrm{e}: ?^{7}$ & ts ${ }^{\mathrm{h}} \mathrm{e}: ?^{7}$ & & & & & & \\
\hline $\mathrm{ts}^{\mathrm{h}} \varepsilon \mathrm{k}^{2}$ & $s \varepsilon ?^{4}$ & ts $^{\mathrm{h}} \mathrm{e}: ?^{7}$ & ts $^{\mathrm{h}} \mathrm{ur}:{ }^{2}$ & $\operatorname{ts}^{\mathrm{h}} \mathrm{e} \mathrm{P}^{8}$ & ts $^{\mathrm{h}} \mathrm{ia}{ }^{7}$ & & & & & & \\
\hline
\end{tabular}

\subsubsection{Implosive and Plain Obstruents}

The PHI plain and implosive stops and affricate are treated in this section. The modern reflexes of these initials are also very homogeneous. There is a noticeable difference in development between the anterior and posterior places of articulation, with the former being represented by implosive stops in the daughter languages, but the latter by plain obstruents. 
(43) Reflexes of PHl implosive and plain obstruents

\begin{tabular}{|c|c|c|c|c|c|c|c|c|c|c|c|}
\hline BHin & $\underline{\mathrm{HaEm}}$ & $\underline{\text { LHut }}$ & $\underline{\text { Tzha }}$ & $\underline{\text { Zdui }}$ & Bting & $\underline{\text { Cun }}$ & $\underline{\text { Nadou }}$ & $\underline{\text { Cjiang }}$ & $\underline{\text { Mfaw }}$ & $\underline{\text { Baisha }}$ & $\underline{\text { Ymen }}$ \\
\hline 6 & 6 & 6 & 6 & 6 & 6 & 6 & 6 & 6 & 6 & 6 & 6 \\
\hline d & d & d & d & d & d & $d \sim t s$ & d & d & d & d & d \\
\hline ts & ts & ts & ts & ts & ts & ts & ts & ts & ts & ts & $\mathrm{t}$ \\
\hline $\mathrm{k}$ & $\mathrm{k}$ & $\mathrm{k}$ & $\mathrm{k}$ & $\mathrm{k}$ & $\mathrm{k}$ & $\mathrm{k}$ & $\mathrm{k}$ & $\mathrm{k}$ & $\mathrm{k}$ & $\mathrm{k}$ & $\mathrm{k}$ \\
\hline \multicolumn{4}{|c|}{ S. Hlai (Savina) } & \multicolumn{4}{|c|}{ C. Hlai (Savina) } & \multicolumn{4}{|c|}{ Baisha (Wang \& Qian) } \\
\hline & $b$ & & & & $b$ & & & & $\mathrm{p}$ & & \\
\hline & d & & & & d & & & & $\mathrm{t}$ & & \\
\hline & ts & & & & ts & & & & t $\int$ & & \\
\hline & $\mathrm{k}$ & & & & $\mathrm{k}$ & & & & $\mathrm{k}$ & & \\
\hline
\end{tabular}

I reconstruct implosive stops at the bilabial and coronal places of articulation, but a plain affricate and stop at the palatal and velar places of articulation:

(44) $\begin{aligned} & * 6 \\ & * \mathrm{~d} \\ & { }_{\mathrm{t} c} \\ & { }_{\mathrm{k}}\end{aligned}$

The areal dispersion of the implosive series across Hainan may have begun with the Hlai languages, and was the impetus for one of the greater areal changes which swept across unrelated languages of the entire island, bringing about the genesis of implosive initials in unrelated languages such as Hainanese, Hainan Mien (Yao), and Lingao (Shintani (1991)). Regarding the variation in Cunhua reflexes, see section 2.4.1 above.

For the third and fouth series of correspondences, I reconstruct *tc and *k. The development of *tc followed the uniform shift of all palatal affricates first to postalveolar affricates, then to alveolar affricates. The only variation in the reflexes of the daughter languages is that of Yuanmen in the palatal series, which is $t$ instead of the expected ts. This is an example of systemic realignment, where there was systemic gap of an alveolar stop in the plain stop series, which was filled by the change of *tc to $t$. The general path of change for the plain voiced series is shown below: 


$$
\text { PH1 Depalatalization Alveolarization (Desibilantization: Yuanmen) }
$$

$*_{t 6} \quad>\quad$ t $5>$ ts $\quad(>\quad$ t $)$

A typological parallel for this stop series can be found in Vietnamese (Ferlus 1992a), where in the time between Middle Vietnamese and modern Vietnamese, the plain bilabial and alveolar stops became imploded, while the postalveolar and velar stops did not (the postalveolar later de-affricated, filling the vacancy left by the original $t$ after it became $d$, similar to the case in Yuanmen). The series which resulted shows a cut-off at the same place of articulation, where all post-alveolars fail to undergo implosivation:

\begin{tabular}{cc} 
Middle Vietnamese & Modern Vietnamese \\
\cline { 2 - 2 } $\mathrm{p}$ & $\mathrm{b}<\mathrm{b}>$ \\
$\mathrm{t}$ & $\mathrm{d}<\mathrm{d}>$ \\
ts & $\mathrm{t}$ \\
$\mathrm{k}$ & $\mathrm{k}$
\end{tabular}

Likewise, at some point in the history of Khmer (Ferlus 1992b: 83), all prevocalic (that is, mainsyllable initial) plain labial and alveolar stops became imploded while the palatal and velar stops remained unchanged:

\begin{tabular}{cc} 
Pre-Khmer & Modern Khmer \\
\cline { 2 - 2 } $\mathrm{p}$ & 6 \\
$\mathrm{t}$ & $\mathrm{d}$ \\
$\mathrm{c}$ & $\mathrm{c}$ \\
$\mathrm{k}$ & $\mathrm{k}$
\end{tabular}

This asymmetry in the initial inventories of the above languages can be explained phonetically through the fact that palatal and velar implosives are more typologically marked, a result of the increase in difficulty articulating implosives as one moves further back in the vocal tract (Ladefoged \& Maddieson (1996: 82)).

A comparison of reconstructions is given below: 
(a) $\quad * 6$

(b) $\quad * d$

(c) $* \mathrm{ts}_{\mathrm{s}}$

(d) $\quad * \mathrm{k}$
*6 $\quad * 2 b$

$*_{d} \quad * ? d$

*ts

*k
$*(?) \mathrm{b}$

$*(?) \mathrm{d}$

$*_{\mathrm{f}}$

*g
*6

$*^{*}$

*t6

*k

Matisoff and Thurgood both reconstruct $* 6$ and $* d$ for the first two series of correspondences, and

$*_{\text {ts }}$ and $* \mathrm{k}$ for the second. All are in general agreement with the present reconstruction, although I also consider the reconstruction of a palatal affricate for the third series more appropriate than an alveolar one, for the same reasons given in section (2.4.2) above.

I consider Peiros' reconstruction of the first two series to be technically correct, but at a point in time previous to $\mathrm{PHI}$; the reconstruction of $* \mathrm{~Pb}$ and $* ? \mathrm{~d}$ also violates Commonality, since the reflexes of the daughter languages uniformly indicate implosives. He reconstructs voiceless palatal and velar stops for the second series; I reject the reconstruction of a palatal stop for the reasons given in the previous section.

Ostapirat reconstructs the plain voiced stops $* \mathrm{~b},{ }^{*} \mathrm{~d},{ }^{*} \mathrm{f}$ and $*_{\mathrm{g}}$. I consider this to be roughly approximate to a stage of Pre-Hlai, as there is no evidence for voiced initials amongst the reflexes of the daughter languages. This is another violation of Commonality since there is no synchronic evidence for plain voiced stops. The variation between preglottalized and plain *b and *d in Ostapirat's reconstruction is due to two distinct correspondences each in Jiamao.

Examples of the PHl voiced stops are given below, in the following order:

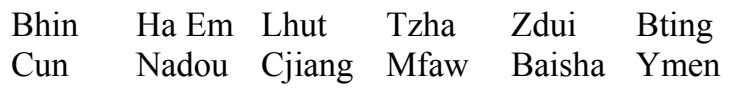


(49) Examples of PHl implosives, plain stop and affricate

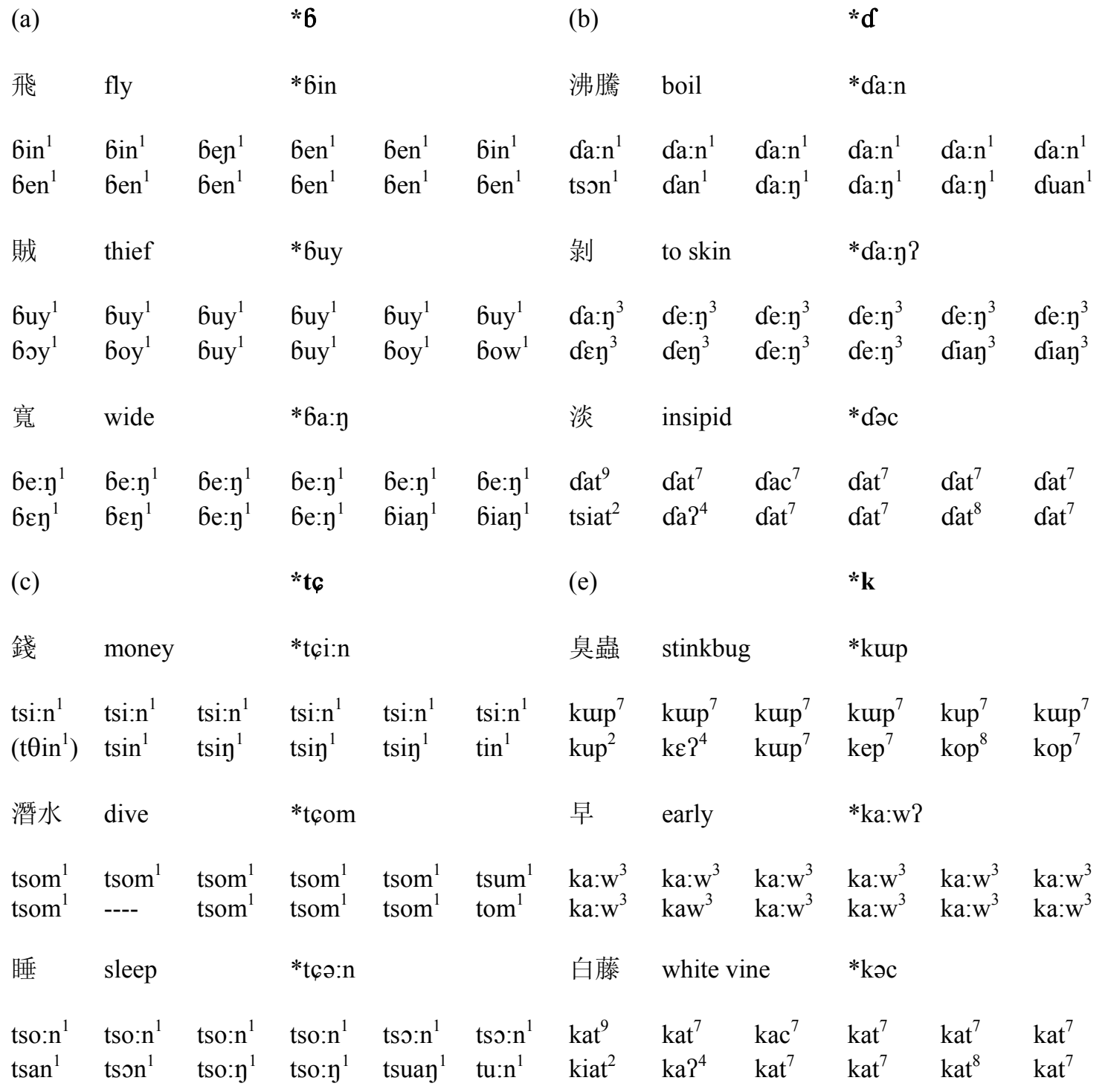

\subsubsection{Fricatives}

The reflexes of the PHl fricatives are the following: 
(50) Reflexes of PHl fricatives

$\begin{array}{llllllllllll}\text { BHin } & \text { HaEm } & \underline{\text { LHut }} & \underline{\text { Tzha }} & \underline{\text { Zdui }} & \text { Bting } & \underline{\text { Cun }} & \text { Nadou } & \underline{\text { Cjiang }} & \underline{\text { Mfaw }} & \text { Baisha } & \underline{\text { Ymen }} \\ \mathrm{p}^{\mathrm{h}} & \mathrm{f} & \mathrm{f} & \mathrm{f} & \mathrm{f} & \mathrm{f} & \mathrm{f} & \mathrm{f} & \mathrm{f} & \mathrm{f} & \mathrm{f} & \mathrm{pf}^{\mathrm{h}} \\ \mathrm{p}^{\mathrm{h}} & \mathrm{f} & \mathrm{f} & \mathrm{f} & \mathrm{f} & \mathrm{f} & \mathrm{s} & \mathrm{f} & \mathrm{s} & \mathrm{s} & \mathrm{f} & \mathrm{pf}^{\mathrm{h}} \\ \mathrm{p} & \mathrm{f} & \mathrm{f} & \mathrm{f} & \mathrm{f} & \mathrm{f} & \mathrm{f} & \mathrm{f} & \mathrm{kw} & \mathrm{r} & \mathrm{f} & \mathrm{f} \\ \mathrm{t} & \mathrm{t} & \mathrm{t} & \mathrm{t} & \mathrm{t} & \mathrm{t} & \mathrm{t} \theta & \mathrm{f} & \mathrm{s} & \mathrm{s} & \mathrm{ts}^{\mathrm{h}} & \mathrm{ts}^{\mathrm{h}}\end{array}$

\section{$\underline{\text { S. Hlai (Savina) }}$}

$$
\begin{aligned}
& p^{h} \\
& p^{h} \\
& p \\
& t
\end{aligned}
$$

C. Hlai (Savina)

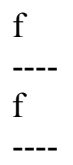

Baisha (Wang \& Qian)

f
f
t
th

I propose the following reconstructions, where ${ }^{*} \mathrm{C}$ - indicates the initial in the first syllable of a sesquisyllabic form:

$$
\begin{aligned}
& *_{\mathrm{f}} \\
& *_{\mathrm{fj}} \\
& { }^{*} \mathrm{C}-\beta \\
& { }_{\mathrm{s}} \mathrm{s}
\end{aligned}
$$

$\mathrm{PHl} * \mathrm{f}$ is reflected as such in all of the daughter languages except Yuanmen ${ }^{15}$ and Bouhin.

Regarding affrication at this place of articulation, there is a typological parallel in Hainanese (Norman 1969:

40), in which former Proto-Min aspirated stops have lenited to affricates:

$\begin{array}{cc}\text { Proto-Min } & \text { Hainanese } \\ * \mathrm{p}^{\mathrm{h}} & \mathrm{f} \sim \mathrm{pf} \sim \mathrm{pf}^{\mathrm{h}} \sim \phi \sim \mathrm{p} \Phi \\ * \mathrm{t}^{\mathrm{h}} & \mathrm{h} \\ * \mathrm{ts}^{\mathrm{h}} & \mathrm{s} \\ * \mathrm{k}^{\mathrm{h}} & \mathrm{x}\end{array}$

The evolution of this fricative has therefore probably taken a course such as the following:

$\begin{array}{llllll}\text { Bouhin } & * \mathrm{f} & > & \mathrm{pf}^{\mathrm{h}} & > & \mathrm{p}^{\mathrm{h}} \\ \text { Yuanmen } & * \mathrm{f} & > & \mathrm{pf}^{\mathrm{h}} & > & \mathrm{pf}^{\mathrm{h}} \\ \text { Other Hlai } & * \mathrm{f} & > & \mathrm{f} & > & \mathrm{f}\end{array}$
${ }^{15}$ The Yuanmen reflex, which is listed in Ouyang \& Zheng (1983) as $\mathrm{f}^{(\mathrm{h})}$, is actually described as an
affricate $\mathrm{pf}^{(\mathrm{h})}$ 
Palatalized $* \mathrm{fj}$ must be reconstructed in order to account for the alveolar fricatives in NCHl, and is reflected according to the correspondences given above in only one item, tooth. There is evidence for two more items with this initial based solely on Jiamao evidence (see chapter five). The development of $* \mathrm{fj}$ was the following:

$\begin{array}{lllllllll}\text { NWCHl, Meifu } & * \mathrm{fj} & > & \mathrm{fj} & > & 6 & > & \int & \mathrm{s} \\ \text { Other Hlai } & * \mathrm{fj} & > & \mathrm{f} & & & & \end{array}$

I reconstruct the third series as $* \mathrm{C}-\beta$, as there is evidence for a glottal stop derived from the initial consonant of ${ }^{*} \mathrm{C}-\beta$ in the Meifu branch. In most languages, the presyllable was eventually lost and $\beta$ devoiced to $\phi$ in accordance with the constraint against initial voiced obstruents and merged with $f$. The distinction between original $* \mathrm{f}$ and $* \beta$ is preserved in both Bouhin and Yuanmen, where reflexes of PHl $* \mathrm{f}$ are aspirated, but those of $\mathrm{PHI} * \mathrm{C}-\beta$ are not:

\begin{tabular}{llllll} 
Bouhin & $* f$ & $>$ & $\mathrm{pf}^{\mathrm{h}}$ & $>$ & $\mathrm{p}^{\mathrm{h}}$ \\
& $* \beta$ & $>$ & $\phi$ & $>$ & $\mathrm{p}$ \\
\multirow{4}{*}{ Yuanmen } & $*_{\mathrm{f}}$ & $>$ & $\mathrm{pf}^{\mathrm{h}}$ & $>$ & $\mathrm{pf}^{\mathrm{h}}$ \\
& $* \beta$ & $>$ & $\phi$ & $>$ & $\mathrm{pf}$
\end{tabular}

The single exception to the development of $* \mathrm{C}-\beta$ into a labiodental fricative is in Meifu, where there was a change instead to $* \mathrm{C}$-w through a stage of lenition; this contrasts with the development in the other Hlai languages, where the presyllable was lost, leaving $\beta$ vulnerable to devoicing in initial position (see section 2.2.1):

$\begin{array}{lllllll}\text { Meifu } & * \mathrm{C}-\beta & > & * \mathrm{C}-\mathrm{w} & > & * \mathrm{pw} & >\mathrm{kw}(\mathrm{CJ}) / \mathrm{\gamma}(\mathrm{MF}) \\ \text { Other Hlai } & * \mathrm{C}-\beta & > & * \beta & > & * \phi & >\end{array}$

Although there is no direct evidence (i.e. low register) for the voiced status of medial * $\beta$, it is in line with the principle of Economy since the change to Proto-Meifu $*$ ?w requires a single lenition of 
fricative to glide, and the change to $\phi$ in other languages requires the loss of the presyllable (with concomitant devoicing).

There was a shift of PHl *s to $t$ throughout all of Hlai except NCHl. It most likely did so through an intermediate stage of $*$ ts, as in the Vietnamese example above. *s underwent affrication to $t \int^{h}$ in the Run branch (where it has merged with the reflex of $* \varphi^{\mathrm{h}}$ ). It remains $s$ in the Meifu branch, but shifted to $\theta$ in NWCHl with affrication to $t \theta$ in Cunhua and a subsequent shift to $f$ in Nadouhua. These developments are illustrated below:

$\begin{array}{llllll}\text { NWCHl } & *_{\mathrm{s}} & > & \theta & > & \mathrm{t} \theta \text { (Cun)/f (Nadou) } \\ \text { Run } & *_{\mathrm{s}} & > & \mathrm{t} \mathrm{h}^{\mathrm{h}} & > & \mathrm{ts}^{\mathrm{h}} \\ \text { Meifu } & *_{\mathrm{s}} & > & \mathrm{s} & > & \mathrm{s} \\ \text { Other Hlai } & *_{\mathrm{s}} & > & \mathrm{ts} & > & \mathrm{t}\end{array}$

A comparison of reconstructions is given below:

\begin{tabular}{|c|c|c|c|}
\hline Matisoff & Thurgood & Peiros & Ostapirat \\
\hline$*_{f}$ & $* p$ & $*_{\mathrm{f}}$ & *-p- \\
\hline --- & --- & --- & *-ip- \\
\hline$* \mathrm{fr}$ & $*$ pr & *hw & *-up- \\
\hline$*_{\mathrm{S}}$ & $*_{\mathrm{s}}$ & $*_{\mathrm{S}}$ & $*_{\mathrm{s}}$ \\
\hline
\end{tabular}

Matisoff and Peiros reconstruct the first series as *f, and Thurgood as *p. I assume that the evidence Thurgood relies on to reconstruct a stop instead of a fricative is the Yuanmen reflex $\mathrm{pf}^{\mathrm{h}}$ and the Bouhin reflex $\mathrm{p}^{\mathrm{h}}$ under the assumption that they were retentions; as explained above, I understand these instead to be innovations (secondary fortitions), and the change of $* p>f$ violates Directionality. Neither of them reconstruct anything for what is reconstructed here as $*_{\mathrm{fj}}$ (which is very rare). Ostapirat reconstructs both as a medial *-p-, the latter with a preceding front high vowel, which fits into his system as a stop which undergoes frication intervocalically; without additional evidence for this reconstruction, this violates Commonality, since the majority of reflexes indicate $* f$. 
The third series is reconstructed as *fr by Matisoff, *pr by Thurgood (parallel to their *f and *p, respectively), and *hw by Peiros. I presume that Matisoff and Thurgood both reconstruct an *r cluster to account for the initial in Moyfaw $(\gamma)$, which is the regular reflex of either *r or *r-clusters in many parts of Kra-Dai, including Hlai (see below). However, the Changjiang reflex $k w$ (which neither Matisoff nor Thurgood were aware of), when presented next to Moyfaw $y$, indicates that the Meifu reflex was an original $* ? w$. The principle of Economy can therefore be invoked, since less change is involved between ${ }^{*} \mathrm{C}-\beta$ and the reflexes of the daughter languages than between *fr or *pr and the same reflexes. Ostapirat's reconstruction of a medial consonant for the second series is legitimate given the Meifu data, but we differ in our reconstruction of the manner of the consonant itself. Ostapirat does also not explain the important distinction between aspirated and unaspirated reflexes in Bouhin and Yuanmen.

There is complete agreement about the reconstruction of the second series as *s.

Examples of the $\mathrm{PHl}$ fricatives are given below, in the following order:

$\begin{array}{llllll}\text { Bhin } & \text { Ha Em } & \text { Lhut } & \text { Tzha } & \text { Zdui } & \text { Bting } \\ \text { Cun } & \text { Nadou } & \text { Cjiang } & \text { Mfaw } & \text { Baisha } & \text { Ymen }\end{array}$

(59) Examples of $\mathrm{PHl}$ fricatives

$$
\text { 天 }
$$

(a)

雨 (p

$$
\text { fən }
$$$$
\left(\mathrm{pa}::^{3}\right) \quad \mathrm{fa}:^{3}
$$$$
\text { fo: }{ }^{3} \text { fa: }{ }^{3}
$$

$* \mathbf{f}$

皮 $\quad$ skin (of fruit) $\quad *$ fa:k

$\mathrm{p}^{\mathrm{h}} \mathrm{a}: \mathrm{P}^{7} \quad$ fe: $?^{7} \quad$ fe:k $\mathrm{k}^{7}$

$f \varepsilon k^{2} \quad f \varepsilon ?^{4} \quad$ fe: $?^{7}$

*fun

fun

*fa:?

fa: ${ }^{3}$

fe: $?^{7}$ (b)

成熟 ripe

tur: $?^{7}$ tur: $?^{7}$

fun $^{1}$ fun

fon ${ }^{1}$

$\mathrm{pf}^{\mathrm{h}}$ ən $^{1}$

t $\theta$

水牛

water buffalo

$\mathrm{fa}:{ }^{3}$

tuy $^{3}$

fa: ${ }^{3}$

fa: ${ }^{3}$

fe: $?^{7}$

fur: ${ }^{2} \quad$ fe ${ }^{8}$ pf $\mathrm{a}^{\mathrm{h}}$

$t \theta o y^{3}$

洗 wash

fe: $?^{7} \quad \operatorname{tak}^{7}$

$\left(\right.$ pfia? $\left.^{7}\right) \mathrm{t} \mathrm{ak}^{2}$ tuy $^{3}$

foy ${ }^{3}$

tuy $^{3}$

suy $^{3}$

(1)

to: $:^{7}$

fว $?^{4}$ to: $\mathrm{k}^{7}$

so: $\mathrm{k}^{7}$
${ }^{*} \mathbf{S}$

*sur:k

tur:k ${ }^{7}$

tur: $?^{7} \quad$ tua $^{7}$ tur: $?^{7}$

suk $^{7} \quad$ ts $^{\mathrm{h}} \mathrm{uk}^{8} \quad$ ts $^{\mathrm{h}} \mathrm{u}$ ? $^{7}$

*suy?

tuy $^{3} \quad$ tuy $^{3} \quad$ tuy $^{3}$ suy $^{3} \quad$ ts $^{\mathrm{h}} \mathrm{oy}^{3} \quad \mathrm{ts}^{\mathrm{h}} \mathrm{ow}^{3}$

$*_{\mathrm{s}}$ :k

to: $2^{7} \quad$ to: $:^{7} \quad$ to: $:^{7}$

so: $k^{7} \quad$ ts $^{\mathrm{h}} \curvearrowright ?^{8} \quad$ ts $^{\mathrm{h}} \mathrm{\rho}^{7}$ 
(b)

牙齒 tooth $\quad *$ fjən

$p^{\mathrm{h}} \mathrm{en}^{1} \quad \operatorname{fan}^{1} \quad \operatorname{fan}^{1} \quad f a$

$\operatorname{sen}^{1} \quad$ fen $^{1} \quad \operatorname{sen}^{1}$
$* \mathbf{f j}$

$\operatorname{sen}^{1}$ (c)

熟 ripe, mature *C-ßuy

$\operatorname{fan}^{1} \quad \operatorname{fan}^{1} \quad$ puy

$\mathrm{fay}^{1} \quad \mathrm{pf}^{\mathrm{h}} \mathrm{an}^{1}$ foy ${ }^{1}$

fuy ${ }^{1}$

foy ${ }^{1}$

fuy $^{1}$

kuy

fuy

juy ${ }^{1}$ fuy $^{1}$

foy ${ }^{1}$

fuy $^{1}$

九 nine ${ }^{*} \mathrm{C}-\beta \mathrm{ur}:$ ?

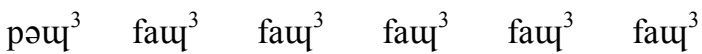

--- faw $^{3}$ kwau $^{3}$ уәщ ${ }^{3}$ faщ $^{3}$ fau $^{3}$

村庄 village $\quad *$ C- $\beta \mathrm{a}: \mathrm{n}$

---- $\quad$ fa: $n^{1} \quad$ fa: $n^{1} \quad$ fa: $n^{1}$

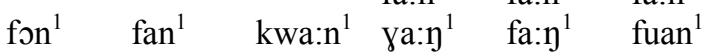

\subsubsection{Interim Summary}

A total of four classes of initials have been reconstructed in this subsection: aspirated stops, aspirated affricates, implosives and the plain stop and affricate, and fricatives. This half of the initial inventory is shown below:

$\begin{array}{lllll}* \mathrm{p}^{\mathrm{h}} & * \mathrm{t}^{\mathrm{h}} & * \mathrm{t} \mathrm{f}^{\mathrm{h}}(\mathrm{w}) & * \mathrm{tc}^{\mathrm{h}} & * \mathrm{k}^{\mathrm{h}} \\ * \mathrm{6} & * \mathrm{c} & & * \mathrm{t} \mathrm{c} & * \mathrm{k} \\ * \mathrm{f}(\mathrm{j}) & * \mathrm{~s} & & & \\ * \mathrm{C}-\beta & & & \end{array}$

There are several interesting asymmetries which are observable. The first is that implosive and plain initials are in complimentary distribution, according to anteriority of place of articulation. The distribution of the implosives is not typologically abnormal per se, as it is quite common for languages to have implosives at only the bilabial and alveolar places of articulation (Maddieson 1984: 112); it is less usual for these phonemes to lack plain counterparts with which they contrast (although less so in Southeast Asia, as shown above in examples (46-47). The contrast between a plain series and an aspirated series of stops, on the other hand, is very common (ibid: 39 ). 
The second is that the fricative series is defective according to the same criteria: fricatives exist at anterior places of articulation, but not posterior ones. This is also typologically regular, with the three most common fricatives being $s, \int$, and $f$ (ibid: 50 ).

The third is that there is only one medial oral fricative which can be reconstructed, at the most anterior (bilabial) place of articulation. This is a very particular property of the inventory, and it is difficult to assess how regular it may be in a typological sense. It is argued in chapter four that this phoneme is the sporadic result of intervocalic lenition of Pre-Hlai *C-b, with no such lenition having occurred at other places of articulation.

Finally, coarticulations are very restricted, with palatal coarticulation being limited to ${ }^{*}$, and labiovelar coarticulation limited to $* \int^{\mathrm{h}}$. Palatalization of labials is not uncommon, but it is less common for postalveolars to have secondary articulations (ibid: 38 ). These asymmetries are discussed and explained in chapter four, section 4.3.10.

\subsubsection{Sonorant Initials}

The sonorants are divided here into five categories: preaspirated nasals, medial nasals, laterals, approximants and preaspirated glides.

\subsubsection{Preaspirated Nasals}

The most common reflexes in this series are of plain stops in low register. Only one language (Bouhin) has retained nasal reflexes. The reflexes in the other Hlai languages are relatively uniform, with only slight variations. The reflexes of the fifth series are quite different than those of the first four (bold font again indicates that the initial is correlated with low register): 
(61) Reflexes of PHl preaspirated nasals

$\underline{\text { BHin }} \underline{\text { Ha Em }} \underline{\text { LHut }} \underline{\text { Tzha }} \underline{\text { Zdui }} \underline{\text { Bting }} \underline{\text { Cun }} \underline{\text { Nadou }}$ Cjiang Meifu $\underline{\text { Baisha }} \underline{\text { Ymen }}$

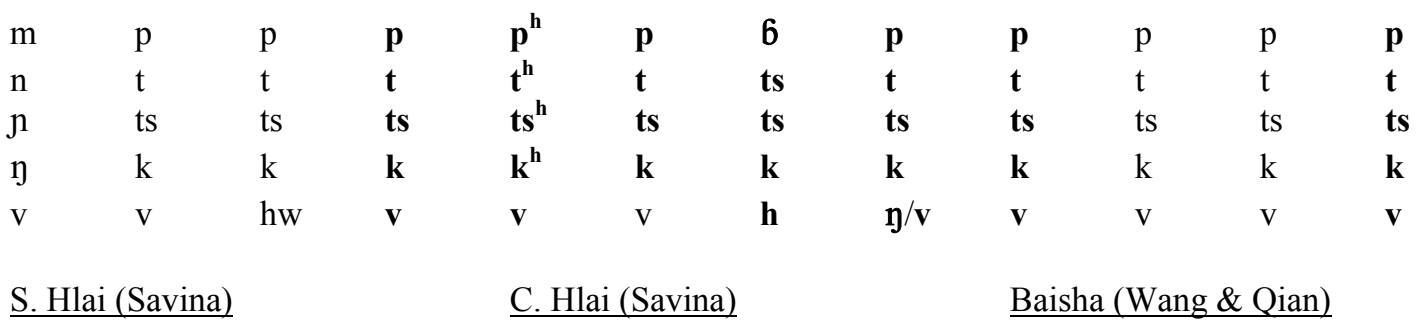

S. Hhai (Savina)

$\begin{array}{ll}\mathbf{p} & \mathrm{p} \\ \mathbf{t} & \mathrm{t} \\ --- & - \\ \mathbf{k} & \mathrm{k} \\ \mathbf{v} & \mathrm{v}\end{array}$

I propose the reconstruction of a series of preaspirated nasals for these series:

*hm
*hn
*hn
*hy
*hyw

The Nadouhua reflexes of *hyw may be conditioned by the following rime, as the following examples illustrate:

$$
\text { Split reflexes of PHl *hyw in Nadouhua }
$$

\begin{tabular}{|c|c|c|c|c|c|}
\hline$\underline{\text { Gloss }}$ & $\underline{\mathrm{PHl}}$ & $\underline{\text { Nadouhua }}$ & $\underline{\text { Gloss }}$ & $\underline{\mathrm{PHl}}$ & Nadouhua \\
\hline yam & *hywə:y & рәу⿰ ${ }^{4}$ & day & *hywən & $\operatorname{van} 2^{4}$ \\
\hline carry (water) & *hywə:y? & рəу⿰㇒⿻土一⿱乛龰 & sparse & *hywa:n? & $\operatorname{van}^{3}$ \\
\hline ghost & *hywə:t & yo $P^{4}$ & & & \\
\hline
\end{tabular}

My hypothesis is that preaspiration (which was lost in Bouhin) conditioned the reanalysis of original nasals as prenasalized stops in the Greater Hlai languages. I propose that this change occurred because Hlai nasals were truly preaspirated, and not merely voiceless. Bhaskararao \& Ladefoged (1991) compare these two types of nasals, and find that preaspirated nasals in Angami (a Tibeto-Burman language 
of North Eastern India) are aspirated throughout the duration of the nasal, and that ' ... before the voicing for the vowel begins, the oral occlusion is released while air is still flowing out through the nose. The auditory impression is that there is an epenthetic voiceless plosive after the voiceless nasal and before the vowel.' This auditory impression could then lead to reanalysis and to the leftward migration of the velic opening, and the accompanying leftward migration of velic closure, resulting in the 'poststopping' of the nasals (Ladefoged \& Maddieson 1996: 127-8):

$$
\mathrm{hm}>\mathrm{hm}^{\mathrm{b}}>\mathrm{mb}>\mathrm{b}
$$

During their duration as prenasalized voiced stops, these initials conditioned low register, only becoming voiceless stops when the nasal component of the initial was lost.

In general, the development of this class of initials was ultimately to plain voiceless stops, in low register if the language had undergone registrogenesis. Besides Bouhin, there are two other exceptions to this. The first is that in Cunhua: instead of plain stops in low register, there are implosives in low register at the bilabial and alveolar places of articulation. This seems to be due to the fact that the Cunhua labial and alveolar reflexes participated in a late wave of implosivation, perhaps at the time that implosives were entering the inventories of other non-Hlai languages around the island. The second exception is Zandui, the reflexes of which are aspirated instead of plain stops. I believe that the explanation for the aspirated Zandui initials is that they developed a particularly strong breathy-voiced release during their time as voiced stops. This breathy voice translated into aspiration after devoicing.

Below I show the various changes described above, using the labial nasals to stand for nasals at all places of articulation:

$\begin{array}{llllllll}\text { Cunhua } & * \mathrm{hm} & > & \mathrm{mb} & > & \mathbf{b} & > & \mathbf{6} \\ \text { Zandui } & * \mathrm{hm} & > & \mathrm{mb} & > & \mathbf{b}^{\mathrm{h}} & > & \mathbf{p}^{\mathbf{h}} \\ \text { Other Hlai } & * \mathrm{hm} & > & \mathrm{mb} & > & \mathbf{b} & > & \mathbf{p}\end{array}$

The regular development of *hyw was to merge with $\mathrm{PHl} *$ hw. In NWCHl, however, it seems to have been reanalyzed as $\mathrm{h}$ in Cunhua; in Nadouhua, $\not w$ merely deaspirated: 


$\begin{array}{lllllll}\text { NWCHl } & * \text { hyw } & > & h(\text { Cun }) / \text { yw (Nadou) } & > & \text { h }(\text { Cun }) / \mathbf{y} / \mathbf{v}(\text { Nadou }) \\ \text { Other Hlai } & * \text { hyw } & > & \text { hw }>\quad h w / v & & \end{array}$

A comparison of reconstructions is given below:

\section{Comparison of reconstructions}

\begin{tabular}{|c|c|c|c|}
\hline$\underline{\text { Matisoff }}$ & Thurgood & $\underline{\text { Peiros }}$ & Ostapirat \\
\hline$* m b$ & ${ }^{*} \mathrm{~m} ?$ & $*_{\mathrm{m}}$ & ${ }^{*}$-m- \\
\hline *nd & $*_{n}$ ? & $*_{n}$ & $*$-n- \\
\hline *ndz & --- & $* \mathrm{n}$ & $*_{-\mathrm{n}}$ \\
\hline *ng & $* \eta ?$ & $* \eta$ & $*-1-$ \\
\hline --- & --- & --- & --- \\
\hline
\end{tabular}

Matisoff (1988) reconstructs the prenasalized stops *mb, *nd, *ndz, *ng, for the first four series; this is the same as the present reconstruction of Greater Hlai, but cannot account for Bouhin (the change *mb > m violates Directionality), and therefore can’t represent PHl. Thurgood (1994) reconstructs nasals with laryngeal constriction, ${ }^{*} \mathrm{~m} ?,{ }^{*} \mathrm{n}$, and ${ }^{*} \mathrm{y}$, with nothing reconstructed at the palatal place of articulation (he reserves this slot in his system for another series of correspondences). Peiros reconstructs plain nasals, which I consider appropriate for Pre-Hlai, not Proto-Hlai (see chapter four); the reconstruction of plain nasals also lacks the explanatory power of inherent in the reconstruction of preaspirated nasals in providing a mechanism for change to oral stops. Ostapirat reconstructs medial *-m-, *-n-, *-n-, *-n-, and *-w-. He does this to distinguish this nasal series from what I reconstruct as the preglottalized nasal series (see below), arguing that initial nasals developed an allophonic glottal stop, another violation of Directionality. None of Matisoff, Thurgood, Peiros, or Ostapirat distinguish *hyw from *hw, probably because the only reflexes which distinguish the two are found in NWCHl.

Examples of the PHl preaspirated nasals are given below, in the following order:

$\begin{array}{llllll}\text { Bhin } & \text { Ha Em } & \text { Lhut } & \text { Tzha } & \text { Zdui } & \text { Bting } \\ \text { Cun } & \text { Nadou } & \text { Cjiang } & \text { Mfaw } & \text { Baisha } & \text { Ymen }\end{array}$


(68) Examples of PHl preaspirated nasals

(a)

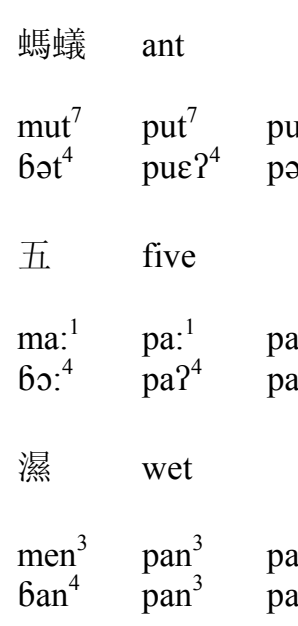

(c)

粽子 dumpling

--- $\quad$---- $\quad$ tsi:t ${ }^{7}$

tsit $^{4} \quad$ tsi? $^{4} \quad$----

身

ләщ ${ }^{1}$ tsəu $^{1}$ tsәщ ${ }^{1}$

--- ---- tsəư ${ }^{4}$

自己 self

--- tsau $^{3}$ tsau ${ }^{3}$ *hm

*hmuc

$\begin{array}{lll}\text { put }^{8} & \text { p ut }^{\mathrm{h}} & \text { put }^{8} \\ \text { pot }^{7} & \text { pot }^{8} & \text { pot }^{8}\end{array}$

*hma:

pa: ${ }^{4} \quad$ pa $:^{4} \quad$ pa:

pa: ${ }^{1}$ pa: ${ }^{1}$ pa: ${ }^{4}$

*hmən?

$\operatorname{pan}^{6}$

pay $^{3}$

*hn

*hji:t

$\begin{array}{ll}p^{h} a n^{6} & \operatorname{pan}^{6} \\ \operatorname{pay}^{3} & \operatorname{pan}^{6}\end{array}$

(d)

針 needle

tsi:t ${ }^{8} \quad$ ts $^{\text {h }} \mathrm{i}: \mathrm{t}^{8} \quad$ tsi: $\mathrm{t}^{8}$

tsit $^{7} \quad$ tsit $^{8} \quad$ tsit $^{8}$

*hјu:

tsəư ${ }^{4}$

tsəu' ${ }^{1}$ tsəuा

*hрu:?

草

grass

$\operatorname{kan}^{3}$

$\operatorname{kan}^{3} \quad \operatorname{kan}^{3}$ *hn

*hniw

tiw $^{4} \quad \mathrm{t}^{\mathrm{h}} \mathrm{iw}^{4} \quad$ tiw $^{4}$

tiw ${ }^{1}$ tew ${ }^{1}$ ti: ${ }^{4}$

*hnom

tom $^{4} \quad \mathrm{t}^{\mathrm{h}} \mathrm{om}^{4} \quad$ tum $^{4}$ tom $^{1}$ tom ${ }^{1}$ tom $^{4}$

*hna:w?

ta: $w^{6} \quad \mathrm{t}^{\mathrm{h}} \mathrm{a}: \mathrm{w}^{6} \quad$ ta: $\mathrm{w}^{6}$

ta: $w^{3} \quad$ ta: $w^{3} \quad$ ta: $w^{6}$

*hy

*hyuc

$\mathrm{kut}^{8} \quad \mathrm{k}^{\mathrm{h}} \mathrm{ut}^{8} \quad \mathrm{kut}^{8}$

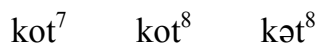

*hyu:h

$\mathrm{kaw}^{2} \quad \mathrm{k}^{\mathrm{h}} \mathrm{o}:^{2} \quad \mathrm{k}:^{2}$

$\mathrm{kaw}^{2} \quad \mathrm{kaw}^{2} \quad \mathrm{kaw}^{2}$

*hyən?

$\operatorname{kan}^{6} \quad \mathrm{k}^{\mathrm{h}} \mathrm{an}^{6} \quad \mathrm{kan}^{6}$

$\mathrm{ka \eta}^{3} \quad \mathrm{ka \eta}^{3} \quad \mathrm{kan}^{6}$ 


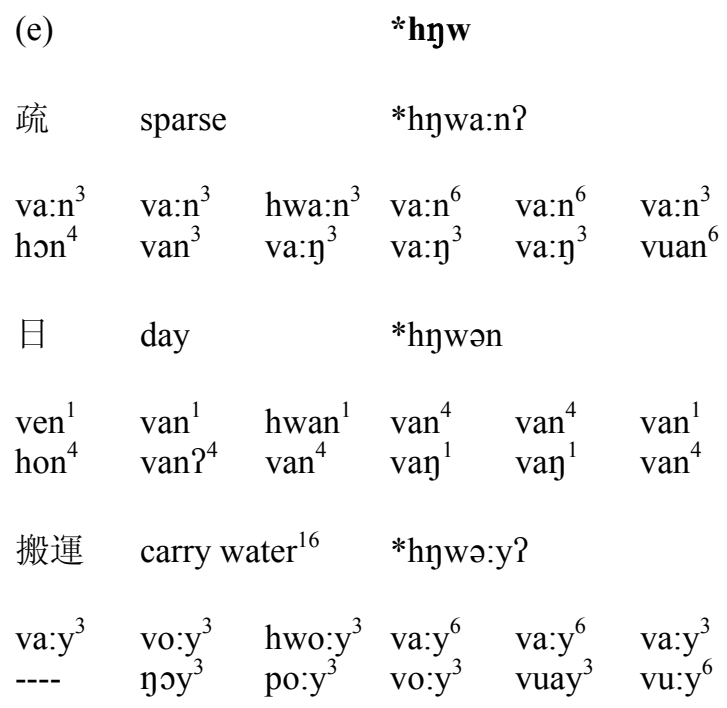

\subsubsection{Medial Nasals}

This set of correspondences is very uniform acoss the daughter languages, which exclusively show nasal reflexes. Given the nature of nasals as a class of sonorants, however, there are a surprisingly low number of languages in which these nasals conditioned low register (indicated by bold font):

(69) Reflexes of PHl medial nasals

\begin{tabular}{|c|c|c|c|c|c|c|c|c|c|c|}
\hline$\underline{\mathrm{BHin}}$ & $\underline{\mathrm{Ha}} \mathrm{Em}$ & $\underline{\text { LHut }}$ & Tzha & $\underline{\text { Zdui }}$ & Bting & Cun & $\underline{\text { Nadou }}$ & Cjiang & Meifu & Baisha \\
\hline $\mathrm{m}$ & $\mathrm{m}$ & $\mathrm{m}$ & $\mathrm{m}$ & m & $\mathrm{m}$ & $\mathrm{m}$ & $\mathrm{m}$ & $\mathrm{m}$ & $\mathrm{m}$ & $\mathrm{m}$ \\
\hline $\mathrm{n}$ & $\mathrm{n}$ & $\mathrm{n}$ & $\mathrm{n}$ & $\mathbf{n}$ & $\mathrm{n}$ & $\mathrm{n}$ & $\mathrm{n}$ & $\mathrm{n}$ & $\mathrm{n}$ & $\mathrm{n}$ \\
\hline $\mathrm{n}$ & $\mathrm{n}$ & $\mathrm{n}$ & $\mathrm{n}$ & $\mathbf{n}$ & $\mathrm{n}$ & $\mathrm{n}$ & $\mathrm{jj}$ & $\mathrm{n}$ & $\mathrm{n}$ & $\mathrm{n}$ \\
\hline$\eta$ & $\eta$ & $y$ & $\eta$ & y & $\eta$ & $\eta$ & $\eta$ & $\mathrm{y}$ & $\mathrm{y}$ & $\eta$ \\
\hline$\eta$ & y & jw & yw & y & $\eta(w)$ & $\eta$ & yw & $\eta$ & $\mathrm{y}$ & $\eta$ \\
\hline
\end{tabular}

\section{$\underline{\text { S. Hlai (Savina) }}$}

$\mathrm{m}$
$\mathrm{n}$
$\mathrm{n}$
$\mathrm{y}$
$\mathrm{y}$

C. Hlai (Savina)

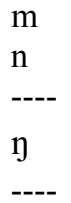

\section{Baisha (Wang \& Qian)}

$\mathrm{m}$
$\mathrm{n}$
$\mathrm{n}$
$\mathrm{y}$
----

\footnotetext{
${ }^{16}$ The initial in the Changjiang form for this word is irregular. It reflects an earlier *m, which was the result of a mistransmission of $* \mathrm{yw}$, providing unexpected confirmation of this reconstruction.
} 
My solution to this problem is to reconstruct a set of medial nasals (which eventually became preglottalized via temporal compression):

$$
\begin{aligned}
& { }^{*} \mathrm{C}-\mathrm{m} \\
& { }^{*} \mathrm{C}-\mathrm{n} \\
& { }^{*} \mathrm{C}-\mathrm{n} \\
& { }^{*} \mathrm{C}-\mathrm{n} \\
& { }^{*} \mathrm{Cun}
\end{aligned}
$$

In order to explain their tonal behavior (and adhere to Commonality by taking this into account), I suggest that these nasals were either still medial in some or all cases, or had become preglottalized by the time of registrogenesis, in which case high register would be expected. Moreover, the two languages which do have reflexes in low register, Yuanmen and Zandui, could be explained as having experienced early deglottalization of these sonorants prior to registrogenesis. This explanation gains further weight when it is noticed that these two languages are located at the geographic edges of Hainanese-speaking areas (in which a simple inventory of three plain nasals --- $m, n, \eta$--- is the norm) where an 'exotic' feature such as preglottalization might be expected to erode in the face of language contact. This is illustrated below in (71): $\underline{\text { PHl }} \underline{\text { Registrogenesis }}$

$\begin{array}{llllllll}\text { Ymen, Zdui } & * \mathrm{C}-\mathrm{m} & > & \mathrm{Pm} & > & \mathbf{m} & > & \mathbf{m} \\ \text { Other Hlai } & * \mathrm{C}-\mathrm{m} & > & \mathrm{Pm} & > & \mathrm{m} & > & \mathrm{m}\end{array}$

The medial nasals have shown a remarkable degree of stability within the daughter languages. The only change which occurred (late, but across-the-board) is the loss of the initial glottal stop, which became possible without merger with the preaspirated nasals in all but Bouhin due to the shift of the latter to prenasalized stops in Greater Hlai. Preglottalization also acted as a buffer against change, preserving these sonorants intact segmentally when compared to their preaspirated counterparts: 


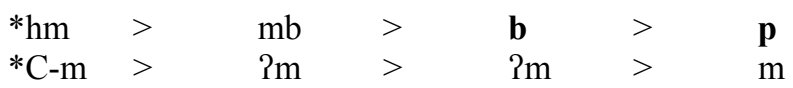

During the development of $* \mathrm{Cuy}$, vocalic transfer occurred at the stage of Central Hlai:

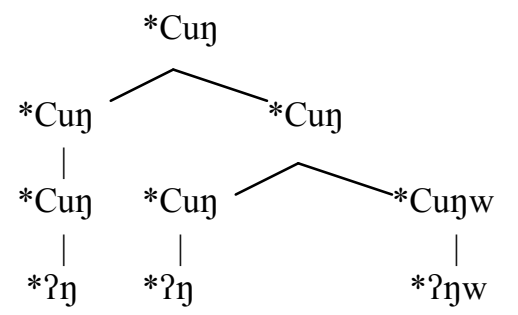

BH HE Central Hl

In terms of language-specific reflexes, $*$ ?m, $*$ ?n, and $* ? \eta$, have all developed in a straightforward way. The palatal nasal *2p has 'broken' to $\eta j$ in Nadou, becoming parallel with $\eta g w$, and depalatalized to $n$ in Zandui, most likely as a result of language contact. The CHl labiovelar nasal *2yw has merged (or is in the process of merging) with the reflexes of $* ? \eta$ several languages; it became labiodentalized in Yuanmen ${ }^{17}$.

The various reconstructions of this class of nasals are given below:

\section{Comparison of reconstructions}

$\underline{\text { Matisoff }} \underline{\text { Thurgood }} \underline{\text { Peiros }} \quad \underline{\text { Ostapirat }} \quad \underline{\text { PHl }}$

\begin{tabular}{|c|c|c|c|c|}
\hline$*_{\mathrm{m}}$ & $* \mathrm{~m}$ & ${ }^{*} \mathrm{C}-\mathrm{m}$ & $* \mathrm{~m}$ & ${ }^{*} \mathrm{C}-\mathrm{m}$ \\
\hline (b) & $*_{\mathrm{n}}$ & $*^{*} \mathrm{C}-\mathrm{n}$ & *n & ${ }^{*} \mathrm{C}-\mathrm{n}$ \\
\hline (c) & $* \mathrm{n}$ & ${ }^{*} \mathrm{C}-\mathrm{n}$ & $* \mathrm{n}$ & ${ }^{*} \mathrm{C}-\mathrm{n}$ \\
\hline (d) & $* \eta$ & ${ }^{*} \mathrm{C}-\mathrm{y}$ & $* \eta$ & $*^{*} \mathrm{C}-\mathrm{y}$ \\
\hline *yw & $* \eta \mathrm{w}$ & ${ }^{*} \mathrm{C}-\mathrm{nw}$ & $*_{\eta \mathrm{w}}$ & ${ }^{*}$ Cun \\
\hline
\end{tabular}

Matisoff (1988), Thurgood (1994), and Ostapirat (2004) all reconstruct these as the plain sonorants *m, *n, *n, *n, and *nw, respectively. However, it is very problematic to reconstruct these as plain sonorants in view of their registral behavior, as only two out of seven of the registrogenetic languages

\footnotetext{
${ }^{17}$ Yuanmen $m$ recorded by Ouyang and Zheng has merged completely with $m$ in the speech of my Yuanmen consultants.
} 
show evidence of original voiced initials, which is what one would otherwise expect for plain sonorants.

Ostapirat (2004:131) explains this as initial sonorants developing an allophonic glottal stop in initial position; however, this sort of change seems unnatural and is unprecedented in Southeast Asia, as far as I am aware.

Peiros' reconstruction of medial sonorants preceded by an initial stop is identical with the present reconstruction, save for the difference in *Cuy, where it is assumed here that the labiovelar reflex only developed through vocalic transfer in the Central Hlai languages.

Examples of the PHl medial nasals are given below, in the following order:

$\begin{array}{llllll}\text { Bhin } & \text { Ha Em } & \text { Lhut } & \text { Tzha } & \text { Zdui } & \text { Bting } \\ \text { Cun } & \text { Nadou } & \text { Cjiang } & \text { Mfaw } & \text { Baisha } & \text { Ymen }\end{array}$

(75) Examples of PHl medial nasals

(a)

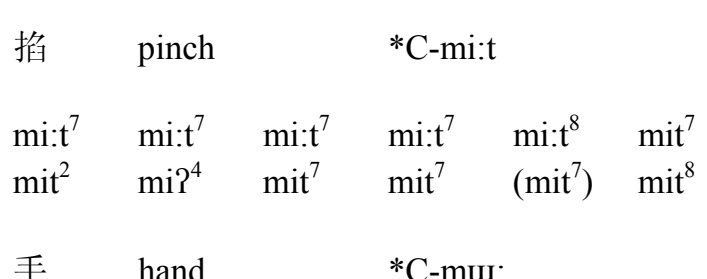

mәч' ${ }^{1}$ mәщ $^{1}$ məщ $^{1}$

mow $^{1} \quad \mathrm{mew}^{1} \quad---\mathrm{m}^{1}$

官 official

$\operatorname{mun}^{1} \operatorname{mun}^{1} \operatorname{mun}^{1}$

$\operatorname{mon}^{1} \quad$ muen $^{1}$ mon $^{1}$
$* \mathrm{C}-\mathrm{m}$

"C-mu:

mәщ ${ }^{1}$ mәщ $^{4}$ mәщ $^{1}$ nay

mәш ${ }^{1}$ тәщ $^{1}$ mәщ $^{4}$ nay $^{1}$

${ }^{*} \mathrm{C}$-mun

mun $^{1}$ mun

moy $^{1}$

(b)
竹箏 bamboo shoot *C-nur:y

nu: ${ }^{1} \quad$ nu: $:^{1} \quad$ nu: $y^{1}$

nuəy ${ }^{1}$ ney ${ }^{1}$ nuy $^{1}$

皮膚 skin

no: $\eta^{1}$

noy ${ }^{1}$

水 water

nam $^{3}$

$\operatorname{nan}^{3}$ no:n

no:n

nu: $\eta^{1}$ nua ${ }^{4}$ nu: $\eta^{1}$

nuy ${ }^{1}$ nuy ${ }^{1}$ nuy ${ }^{4}$

${ }^{*}$ C-nə:y

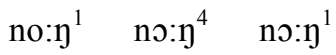

no:y ${ }^{1}$ nuay ${ }^{1}$ nuay $^{4}$

${ }^{*}$ C-nəm?

nom $^{3} \quad$ nam $^{3} \quad$ nam $^{6} \quad$ nam $^{3}$

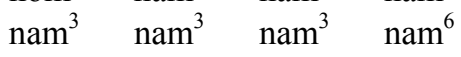


(c)

\begin{tabular}{|c|c|c|c|c|c|c|c|c|c|c|c|}
\hline 蚊子 & \multicolumn{2}{|c|}{ mosquito } & \multicolumn{3}{|c|}{ *C-лnu:y } & 哭 & \multicolumn{2}{|l|}{ cry } & \multicolumn{3}{|c|}{ *C-ni:? } \\
\hline nu: ${ }^{1}$ & лu: $y^{1}$ & лu: $\eta^{1}$ & лu: $y^{1}$ & nuan $^{4}$ & лu: $\eta^{1}$ & ney $^{3}$ & pay $^{3}$ & pay $^{3}$ & nay $^{3}$ & pay $^{6}$ & nay $^{3}$ \\
\hline $\operatorname{noy}^{1}$ & niw $^{1}$ & nuy $^{1}$ & nuy $^{1}$ & nup $^{1}$ & nup $^{4}$ & nay $^{3}$ & nay $^{3}$ & nay $^{3}$ & nay $^{3}$ & nay $^{3}$ & jay $^{6}$ \\
\hline 鹽 & \multicolumn{2}{|l|}{ salt } & \multicolumn{3}{|c|}{${ }^{*} \mathrm{C}$-лna:w? } & 肝 & \multicolumn{2}{|l|}{ liver } & \multicolumn{2}{|c|}{ *C-ya:n } & \\
\hline na:w ${ }^{3}$ & na:w ${ }^{3}$ & na: $w^{3}$ & na: $w^{3}$ & na:w ${ }^{6}$ & na: $w^{3}$ & ya:n ${ }^{1}$ & ya:n ${ }^{1}$ & па:n ${ }^{1}$ & ya:n ${ }^{1}$ & ya:n ${ }^{4}$ & ya:n ${ }^{1}$ \\
\hline na:w ${ }^{3}$ & рja: ${ }^{3}$ & na: $w^{3}$ & na: ${ }^{3}$ & na:w ${ }^{3}$ & na:w ${ }^{6}$ & yon ${ }^{1}$ & $\operatorname{yan}^{1}$ & ya: ${ }^{1}$ & ya: ${ }^{1}$ & ya: ${ }^{1}$ & juan $^{4}$ \\
\hline 月(亮) & \multicolumn{2}{|c|}{ month/moon } & \multicolumn{2}{|c|}{ *C-ла:n } & & 鰓 & gill & & \multicolumn{2}{|c|}{ *C-na:k } & \\
\hline na:n ${ }^{1}$ & na:n ${ }^{1}$ & na:n ${ }^{1}$ & na: $n^{1}$ & na: $n^{4}$ & na: ${ }^{1}$ & па: $2^{7}$ & ye: $?^{7}$ & ne: $\mathrm{k}^{7}$ & ye: $?^{7}$ & ye $: 2^{8}$ & ye: $?^{7}$ \\
\hline non ${ }^{1}$ & njan ${ }^{1}$ & ne: $n^{1}$ & na:1 ${ }^{1}$ & ла: $y^{1}$ & juan $^{4}$ & jek ${ }^{2}$ & ye $e^{4}$ & ye: $?^{7}$ & yu: $:^{2}$ & ye? ${ }^{8}$ & jia ${ }^{8}$ \\
\hline (e) & & & *Cun & & & & & & & & \\
\hline 稻草 & \multicolumn{2}{|c|}{ rice straw } & \multicolumn{3}{|c|}{ *Cunin? } & & & & & & \\
\hline $\operatorname{nin}^{3}$ & $\operatorname{gin}^{3}$ & nwin $^{3}$ & ---- & ---- & ---- & & & & & & \\
\hline yen ${ }^{3}$ & jwen ${ }^{3}$ & yen $^{3}$ & yen $^{3}$ & yen $^{3}$ & $\operatorname{men}^{6}$ & & & & & & \\
\hline \multicolumn{3}{|c|}{ 戴 (帽子)wear } & \multicolumn{2}{|c|}{ *Cunəw? } & & & & & & & \\
\hline naw $^{3}$ & naw $^{3}$ & jwow $^{3}$ & ---- & ро: ${ }^{6}$ & пว: ${ }^{3}$ & & & & & & \\
\hline yaw $^{3}$ & yo: ${ }^{3}$ & yo: $:^{3}$ & pwo: ${ }^{3}$ & yo: ${ }^{3}$ & mo: ${ }^{6}$ & & & & & & \\
\hline 枕 & \multicolumn{2}{|c|}{ rest head } & \multicolumn{2}{|c|}{ *Cuna:n } & & & & & & & \\
\hline ya:n ${ }^{1}$ & ya:n ${ }^{1}$ & ya:n ${ }^{1}$ & jwa:n ${ }^{1}$ & puan $^{4}$ & ya:n ${ }^{1}$ & & & & & & \\
\hline ---- & ---- & ya:n ${ }^{1}$ & ya:n ${ }^{1}$ & ya:n ${ }^{1}$ & muan $^{4}$ & & & & & & \\
\hline
\end{tabular}

\subsubsection{Laterals}

There are altogether four kinds of laterals which can be reconstructed for $\mathrm{PHl}$ : 
(76) Reflexes of PHl laterals

\begin{tabular}{|c|c|c|c|c|c|c|c|c|c|c|c|}
\hline$\underline{\text { BHin }}$ & $\underline{\mathrm{HaEm}}$ & $\underline{\text { LHut }}$ & $\underline{\text { Tzha }}$ & $\underline{\text { Zdui }}$ & Bting & $\underline{\text { Cun }}$ & $\underline{\text { Nadou }}$ & $\underline{\text { Cjiang }}$ & $\underline{\text { Mfaw }}$ & Baisha & $\underline{\text { Ymen }}$ \\
\hline d & t & 1 & 1 & 4 & 1 & $\mathrm{t} \theta$ & 1 & 1 & 1 & 1 & t \\
\hline 1 & 1 & 1 & 1 & I & 1 & 1 & 1 & 1 & 1 & 1 & 1 \\
\hline Z & $\mathrm{z}$ & $\mathrm{z}$ & 4 & 1 & 4 & I & $\mathbf{z}$ & $\mathbf{Z}$ & $\mathrm{z}$ & $\mathrm{z}$ & ts \\
\hline 1 & 1 & $\mathrm{pl}$ & $\mathrm{pl}$ & $\mathrm{p}$ & $\mathrm{pl}$ & $6 \sim 1$ & pj & $\mathbf{p}$ & $\mathrm{pl}$ & $\mathrm{pl}$ & $\mathrm{pl}$ \\
\hline
\end{tabular}

\section{$\underline{\text { S. Hlai (Savina) }}$}

$$
\begin{aligned}
& \mathrm{d} \\
& \mathrm{l} \\
& \mathrm{j} \\
& 1
\end{aligned}
$$

\section{Hlai (Savina)}

$\mathrm{tl}$
1
$\mathbf{t}^{\mathrm{h}}$
$\mathrm{pl}$

\section{Baisha (Wang \& Qian)}

4
1
3
pl

I treat the first two series of laterals as analogous to the preaspirated and preglottalized nasals, respectively, reconstructing preaspirated and medial laterals. I reconstruct a palatalized lateral for the third series, and a medial lateral with preceding bilabial stop for the final series:

$$
\begin{aligned}
& { }^{*} \mathrm{hl} \\
& { }^{*} \mathrm{C}-1 \\
& { }^{*} \mathrm{j} \\
& { }^{*} \mathrm{p}-1
\end{aligned}
$$

The lateral liquid seems to have accommodated preaspiration more easily than the nasals, and shows uniform reflexes as a lateral fricative in all languages except NWCHl and Bouhin. In Cunhua, $f$ shifted to $t \theta$, merging with the reflex of original *s. In Nadouhua, 4 appears to have merely lost its aspiration, but must have done so fairly recently after it conditioned high register reflexes. There was a different kind of development in Bouhin:

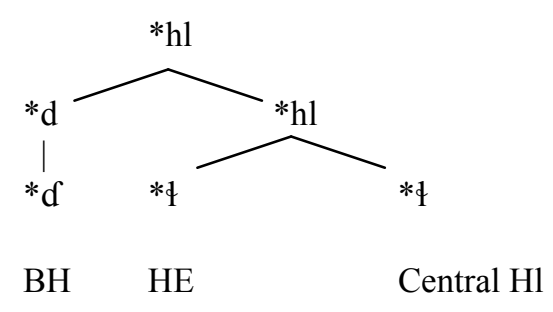


That is, Bouhin $h l$ hardened to $d$, after which it merged with $d$ (whether it first devoiced to $t$ as an intermediate stage is difficult to tell).

The development of the medial laterals in the Greater Hlai languages is similar to the development of the nasals, and a contrast with the preaspirated laterals was maintained in the following way:

$$
\begin{array}{lllll}
* \mathrm{hl} & > & 4 & > & 4 \\
* \mathrm{C}-\mathrm{l} & > & 21 & > & 1
\end{array}
$$

In the case of $* \mathrm{lj}$, the majority of languages have lost all traces of the original lateral, and simplified the cluster to a palatal glide (which then evolved into the weak fricative $z$ ). The three exceptions to this are Cunhua, Yuanmen, and the Qi branch. Cunhua merely simplified in the opposite direction, maintaining the lateral but losing the palatal glide. In Yuanmen, the reflex of *lj merged with one of the two reflexes of $*_{r j}$ which were apparently in free variation in Run. The fact that this did not occur in Baisha indicates that after the split of Run into Baisha and Yuanmen, *lj quickly developed into *j in Baisha, so that the $* 1 \mathrm{j}$ which developed in turn from * rj filled a gap. In Yuanmen on the other hand, * $1 \mathrm{j}$ was maintained, and merged with one of the reflexes of * rj before ultimately hardening to $d z$. This is shown below:

\begin{tabular}{|c|c|c|c|c|c|c|c|c|c|}
\hline \multirow[t]{2}{*}{ Baisha } & $* 1 \mathrm{j}$ & $>$ & $\mathrm{lj}$ & $>$ & $\mathrm{j}$ & $>$ & Z & $>$ & z \\
\hline & $*_{r j}$ & $>$ & $r \sim r j$ & $>$ & $\mathrm{d} \sim \mathrm{lj}$ & $>$ & $\mathrm{t} \sim \mathrm{dz}$ & $>$ & $\mathrm{t} \sim \mathrm{ts}$ \\
\hline \multirow[t]{2}{*}{ Yuanmen } & $* 1 j$ & $>$ & $\mathrm{lj}$ & $>$ & $\mathrm{lj}$ & $>$ & dz & $>$ & ts \\
\hline & $*_{r j}$ & $>$ & $r \sim r j$ & $>$ & $\mathrm{d} \sim \mathrm{lj}$ & $>$ & $\mathrm{t} \sim \mathbf{d} \mathbf{z}$ & $>$ & $t \sim t s$ \\
\hline
\end{tabular}

The reflexes in the languages of the Qi branch show a unique occurrence of the preaspirated lateral $\Varangle$ in low register. I hypothesize that $* 1 \mathrm{j}$ developed narrower stricture in its palatal glide, becoming * lz. This was in turn reinterpreted as a voiced lateral fricative ${ }^{*} \mathfrak{3}$, which conditioned low register before participating in the third devoicing, merging with $\$$ in high register: 


$$
* \mathrm{lj} \quad>\quad 1 / 2 \quad>\quad 3 \quad>\quad+1
$$

The cluster *p-l occupies a very unique position in the PHl phoneme inventory, as it continues to do today in the majority of the daughter languages. It is the single example of a stop-liquid cluster, more common in various Tai languages (and in Proto-Tai), but non-existant in PHl except in this one case.

The only problem with the reconstruction of this cluster as *p-l is the fact that it is in low register in three of the four registrogenetic $\mathrm{NCHl}$ languages, where *p is expected to condition high register. I propose that this represents an areal innovation amongst these three languages, and that when original *p-1 became a 'tight' cluster due to temporal compression, that the bilabial stop became voiced under the influence of the abutting lateral. This proposed evolution of *p-l is shown below:

$\begin{array}{llllllll}\text { Cunhua } & * \mathrm{p}-1 & > & \mathrm{bl} & > & \mathbf{b l} & > & \mathbf{6} \sim \mathbf{l} \\ \text { Nadouhua } & * \mathrm{p}-1 & > & \mathrm{bl} & > & \mathbf{b j} & > & \mathbf{p j} \\ \text { Changjiang } & * \mathrm{p}-1 & > & \mathrm{bl} & > & \mathbf{b} & > & \mathbf{p} \\ \text { Other CHl } & * \mathrm{p}-1 & > & \mathrm{pl} & > & \mathrm{pl} & > & \mathrm{pl} \sim \sim \mathrm{p}\end{array}$

As with other sesquisyllabic forms, it was normal for the sesquisyllable to merely be dropped in Bouhin and Ha Em, whereas the two consonants merged into a cluster in Central Hlai due to temporal compression:

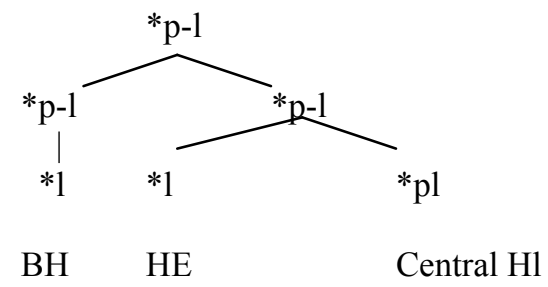

A comparison of reconstructions is given below: 


$$
\text { Comparison of reconstructions }
$$

\begin{tabular}{|c|c|c|c|}
\hline$\underline{\text { Matisoff }}$ & Thurgood & $\underline{\text { Peiros }}$ & Ostapirat \\
\hline *A & *\$? & $* \mathrm{hl}$ & $*_{-1}$ \\
\hline *1 & $* 1$ & ${ }^{*} \mathrm{C}-1$ & $*_{1}$ \\
\hline$*_{\mathrm{z}}$ & $* 1 \mathrm{j} ?$ & $*_{\mathrm{z}}$ & $*_{- \text {il- }}$ \\
\hline$*_{\mathrm{p} l}$ & $*_{\mathrm{pl}}$ & $*_{\mathrm{p}}$ & $*_{\mathrm{p}-1}$ \\
\hline
\end{tabular}

Matisoff reconstructs the first series as *4, and Peiros *hl, in line with the present reconstruction. It is unclear to me why Thurgood reconstructs * $\{$, indicating glottal constriction, although he may be relying on the evidence of the Bouhin reflex $d$. Ostapirat reconstructs medial *-1-, which underwent intervocalic fortition as a result of its medial position on analogy with his intervocalic nasal series.

Matisoff, Thurgood, and Ostapirat reconstruct *1 for the second series, whereas Peiros reconstructs ${ }^{*} \mathrm{C}-1$, in line with the present reconstruction. The reason for not reconstructing this as an initial lateral is the same as the case of the plain nasals - the occurrence of all forms in high register except for Yuanmen and Zandui, indicating that the $\mathrm{PHl}$ form was originally sesquisyllabic and began with a voiceless obstruent. The occurrence of this initial in the low register or Yuanmen and Zandui finds its explanation in the same circumstances as the preglottalized nasals - early deglottalization occurred before registrogenesis, most likely as a result of contact with non-Hlai languages.

Matisoff and Peiros reconstruct * $\mathrm{z}$ for the third series, which I consider untenable given the lateral reflexes which exist in Cunhua and the Qi branch (thereby violating Commonality, as well as Directionality in the case of the postulated change $*_{\mathrm{z}}>\emptyset$ ). Thurgood reconstructs $*^{*} \mathrm{j}$ ? and Ostapirat medial $*_{\text {-il-, }}$ both of which are closer to the present reconstruction. I differ with Ostapirat on account of the Bouhin and Ha Em evidence, which shows that the PHl initial must have already been palatalized before the breakup of the proto-language.

Matisoff, Thurgood, and Peiros all reconstruct *pl for the final series, and Ostapirat reconstructs *p-l, indicating an original sesquisyllable. These are all in basic agreement with the reconstruction 
proposed here, although I agree with Ostapirat that the PHl form specifically represents a sesquisyllable, not a tight cluster ${ }^{18}$.

Examples of the PHI laterals are given below, in the following order:

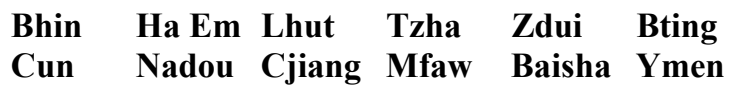

(85) Examples of PHl laterals

(a)

深

dak

$\mathrm{t} \mathrm{ak} \mathrm{k}^{2} \quad \mathrm{loP^{4 }} \quad$ to: $\mathrm{k}^{7}$

二 $\quad$ two $\quad$ *hlu:?

dow $^{3} \quad$ faw $^{3}$

$\mathrm{t} \theta \mathrm{a}:(\mathrm{y})^{3}$ law

血. blood

da: $t^{7} \quad$ ta: $t^{7}$

$\mathrm{t} \theta \mathrm{ot}^{2} \quad l a \mathrm{P}^{4}$ taw $^{3}$

taw $^{3}$

$* h l$

*hlə:k

to: $?^{7}$

to: $\mathrm{k}^{7} \quad$ to $^{8} \quad$ to ${ }^{7}$

law

taw $^{3} \quad$ taw $^{3}$

*hla:c

fa:c $\mathrm{c}^{7}$

to: $\mathrm{t}^{7}$ ta: $t^{7}$

to: $\mathrm{t}^{7}$ (b)

(長)大 big

$\operatorname{lun}^{1}$

$\log ^{1}$

鱗 fish scale

lap ${ }^{7}$

$\operatorname{lap}^{2}$

lo: $p^{7}$

$101^{4}$

lo: $\mathrm{p}^{7}$

lo: $\mathrm{p}^{7}$

遠 far

lay $^{1}$

lay $^{1}$

$\operatorname{luy}^{1}$

$\log ^{1}$

luy ${ }^{1}$

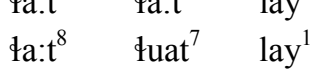

*C-I

*C-lun

$\log ^{1} \quad \operatorname{lug}^{4} \quad \operatorname{lug}^{1}$

$\log ^{1} \quad \operatorname{lug}^{1} \quad \operatorname{lug}^{4}$

*C-lə:p

lo: $p^{7} \quad$ lo: $p^{8} \quad$ lo: $p^{7}$

lo: $p^{7} \quad$ luap $^{8} \quad$ luap $^{8}$

*C-ləy

$\begin{array}{llll}\text { lay }^{1} & \text { lay }^{1} & \text { lay }^{4} & \text { lay } \\ & \\ \text { lay }^{1} & \text { lay }^{1} & \text { lay }^{1} & \text { lay }^{4}\end{array}$

${ }^{18}$ Pittayawat Pittayaporn suggests the alternative reconstruction * $\mathrm{Pbl}$ (pc, 12/14/06). While I do not adopt this reconstruction here, it is one explanation for the voicing in Cunhua, Nadouhua, and Changjiang; extraHlai comparison will eventually be necessary in order to choose between these two options. 
(c)

\begin{tabular}{|c|c|c|c|c|c|c|c|c|c|c|c|}
\hline 螞蟥 & \multicolumn{2}{|c|}{ water leech } & \multicolumn{3}{|l|}{ *ljiy } & 嫩 & \multicolumn{2}{|l|}{ tender } & \multicolumn{3}{|l|}{ *p-lu:k } \\
\hline $\operatorname{zin}^{1}$ & $\operatorname{zin}^{1}$ & $\operatorname{zin}^{1}$ & $\sin ^{4}$ & $\operatorname{tin}^{4}$ & $\sin ^{4}$ & lu: $?^{7}$ & lut: $?^{7}$ & plu: $\mathrm{k}^{7}$ & plu: $?^{7}$ & ---- & plu: $?^{7}$ \\
\hline $\operatorname{lin}^{4}$ & zen $2^{4}$ & ---- & $\operatorname{zen}^{1}$ & zen $^{1}$ & $\operatorname{tsen}^{4}$ & luək $^{4}$ & pju $^{4}$ & $\operatorname{pur}^{7}$ & pluk $^{7}$ & ---- & plur $^{8}$ \\
\hline 脖子 & neck & & $*$ ljoy? & & & 房子 & house & & ${ }^{*}$ p-loy? & & \\
\hline zoy $^{3}$ & zuy $^{3}$ & zoy $^{3}$ & $\operatorname{tog}^{6}$ & $\operatorname{tog}^{6}$ & ło $y^{6}$ & $\operatorname{lug}^{3}$ & $\operatorname{luy}^{3}$ & ploy $^{3}$ & ploy $^{3}$ & poy $^{3}$ & ploy $^{3}$ \\
\hline $1 \circ \eta^{4}$ & $z_{a y}^{3}$ & juan $^{3}$ & $\mathrm{zoy}^{3}$ & $\mathrm{zoy}^{3}$ & $\operatorname{tson}^{6}$ & ---- & pjay $^{3}$ & pэy ${ }^{3}$ & $\mathrm{plo \eta}^{3}$ & ploy $^{3}$ & ploy $^{3}$ \\
\hline 耳朵 & ear $^{19}$ & & *ljəy & & & 瞎 & blind & & *p-la:w & & \\
\hline 1 & zay $^{1}$ & zay $^{1}$ & tay $^{4}$ & tay $^{4}$ & tay ${ }^{4}$ & la:w ${ }^{1}$ & la: $w^{1}$ & pla:w ${ }^{1}$ & pla:w ${ }^{1}$ & pa:w ${ }^{1}$ & pla:w ${ }^{1}$ \\
\hline & nay ${ }^{4}$ & zay $^{4}$ & zay $^{1}$ & zay $^{1}$ & tsay $^{4}$ & $6 a: w^{4}$ & pjaw $^{4}$ & pa: $w^{4}$ & pla:w ${ }^{1}$ & pla:w ${ }^{1}$ & pla:w ${ }^{1}$ \\
\hline
\end{tabular}

\subsubsection{Approximants}

The set of combined approximants (including the rhotic) is large, owing to the fact that some may occur plain as well as accompanied by one or both of two coarticulated glides. The plain approximants developed in a rather straightforward manner; those with secondary articulations, on the other hand, seem to have been very unstable and often follow very divergent paths. Only the coronal approximants were able to support coarticulations, there being no evidence for coarticulated labiovelar approximants.

There is a certain asymmetry in this class of initials, in that there is evidence in Meifu for an initial glottal stop at the onset of some, but not others. The hypothesis presented here is that all of these initials were once medial, an issue that is explored in chapter four. For now, an initial consonant is automatically reconstructed in all instances.

The reflexes of the PHl approximants are given below:

\footnotetext{
${ }^{19}$ The Nadouhua initial is irregular, having assimilated under the influence of the initial syllable of this word, the full form being nu ${ }^{2}$ nay ${ }^{4}$
} 
(86) Reflexes of the PHl approximants

\begin{tabular}{|c|c|c|c|c|c|c|c|c|c|c|c|}
\hline BHin & $\underline{\mathrm{HaEm}}$ & $\underline{\text { LHut }}$ & $\underline{\text { Tzha }}$ & $\underline{\text { Zdui }}$ & Bting & Cun & $\underline{\text { Nadou }}$ & $\underline{\text { Cjiang }}$ & $\underline{\text { Mfaw }}$ & $\underline{\text { Baisha }}$ & $\underline{Y m e n}$ \\
\hline $\mathrm{v}$ & $\mathrm{V}$ & $\mathrm{v}$ & f & $\mathbf{v}$ & $\mathrm{v}$ & $\mathbf{v}$ & $\mathbf{v}$ & $\mathbf{v}$ & $\mathrm{V}$ & $\mathrm{V}$ & $\mathbf{v}$ \\
\hline $\mathrm{r}$ & $r$ & $\mathrm{r}$ & $\mathbf{r}$ & 1 & I & I & I & I & $\mathrm{r}$ & $\mathrm{r}$ & $\mathbf{r}$ \\
\hline z & $\mathrm{z}$ & $r$ & $\mathbf{t}$ & $\mathbf{t}^{\mathbf{h}}$ & $\mathbf{t}$ & $\mathbf{z}$ & I & $\mathbf{t}$ & $t$ & $\mathrm{t} \sim \mathrm{ts}$ & $t \sim t s$ \\
\hline $\mathrm{r}$ & $\mathrm{r}$ & $\mathrm{v}$ & f & f & f & $\mathbf{v}$ & $\mathbf{v}$ & $\mathrm{kw}$ & $y$ & f & f \\
\hline $\mathrm{r}$ & $\mathrm{g}$ & $\mathrm{g}$ & $\mathbf{g}$ & h & $\mathbf{h}$ & h & $y$ & g & $\mathrm{x}$ & $\mathrm{x}$ & $\mathrm{k}^{\mathrm{h}}$ \\
\hline $\mathrm{r}$ & $\mathrm{g}$ & $\mathrm{gw}$ & gw & $\mathbf{v}$ & hw & $\mathbf{v}$ & $\mathrm{y} / \mathrm{v}$ & $\mathrm{kw}$ & $\gamma$ & $\mathrm{v}$ & $\mathrm{v}$ \\
\hline
\end{tabular}

$\underline{\text { S. Hlai (Savina) }}$

$\mathrm{v}$
$\mathrm{d}(\mathrm{r})$
$\mathrm{j}$
$\mathrm{d}(\mathrm{r})$
$\mathrm{d}(\mathrm{r})$
$\mathrm{d}(\mathrm{r})$

C. Hlai (Savina)

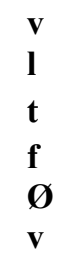

Baisha (Wang \& Qian)

$v$
$b$
$t \sim t s$
$f$
$x$
$v$

I reconstruct the following approximants for the sets of correspondences above:

$$
\begin{aligned}
& *^{*} \mathrm{r} \\
& { }_{r} \mathrm{r} \\
& { }^{*} \mathrm{rj} \\
& { }^{*} \mathrm{Cur} \\
& { }^{*} \mathrm{r} \\
& { }^{*} \mathrm{Cur}
\end{aligned}
$$

The palatalized approximant $*$ rj and the sesquisyllabic * Cur and *Cur must be reconstructed in order to account completely for the wide range of reflexes in the daughter languages, thus adhering to Commonality.

\subsection{Labiodental Approximants}

Excepting Cunhua, Lauhut and Tongzha, the reflexes of * $v$ have merged almost completely with those for *hw. The Cunhua reflex is $v$ in low register (compared to $v$ in high register from *hw), the Lauhut reflex is $v$ (as opposed to $h w$ from $*$ hw), and the Tongzha reflex is $f$ in low register (instead of $v$ 
from $* \mathrm{hw})$. The parallel changes in these three languages which show a distinction between $* v$ and $* \mathrm{hw}$ are shown below:

$\begin{array}{llllll}\text { Cunhua } & * v & > & v & > & \text { v } \\ & * h w & > & \text { hw } & > & \text { v } \\ \text { Lauhut } & * v & > & v & > & \text { v } \\ & * h w & > & \text { hw } & > & \text { hw } \\ \text { Tongzha } & * v & > & \text { v } & > & \text { f } \\ & * h w & > & \text { w } & > & \text { v }\end{array}$

Hlai $v$ is generally a very weak fricative in all languages, so that the change from $* v$ to $v$ would have required only a slight increase in stricture.

\subsection{Alveolar Approximants}

In the case of $\mathrm{PHl} * \mathrm{r}$, the flap is still retained in a several languages. The most common changes are from $*_{r}$ to $l$ and from $*_{r}$ to a trilled $r$. For the second and third series, I reconstruct $*_{r j}$ and $*^{C} \mathrm{Cur}$ respectively. Based on the Bouhin and Ha Em reflexes (which preserve evidence of only a palatal glide), * rj was a tap with a palatal coarticulation in PHl itself. The same was not true of *Cur, which was the initial of sesquisyllabic words, and did not undergo vocalic transfer until the divergence of Central Hlai:

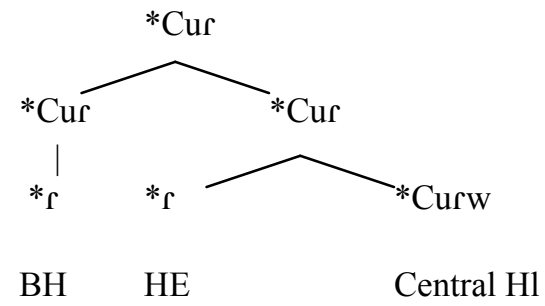

The development of $*_{r j}$ and $*^{C}$ ur has been roughly parallel in Central Hlai. There seems to be a general division between languages in which these initials remained approximants or were reduced to 
glides, and those in which they underwent fortition to voiced obstruents. The first category includes Lauhut and NWCHI; fortition did not occur in these languages:

\section{Evolution of * rj and *Cur: no fortition of approximant}

\begin{tabular}{|c|c|c|c|c|c|}
\hline Lauhut & $*_{r j}$ & $>$ & f & $>$ & $\mathrm{r}$ \\
\hline & *Curw & $>$ & $\mathrm{w}$ & $>$ & $\mathrm{v}$ \\
\hline Nadouhua & $*_{r j}$ & $>$ & f & $>$ & I \\
\hline & $* \mathrm{Curw}$ & $>$ & w & $>$ & $\mathbf{v}$ \\
\hline Cunhua & $*_{r j}$ & $>$ & $\mathrm{j}$ & $>$ & $\mathbf{z}$ \\
\hline & *Curw & $>$ & $\mathrm{w}$ & $>$ & $\mathbf{v}$ \\
\hline
\end{tabular}

In all instances, the presyllable of *Curw was lost. In Nadouhua and Lauhut, *rj lost its palatal coarticulation (it was retained and the tap was lost in Cunhua), but in all cases the tap of Central Hlai *Curw was lost, leaving only the labiovelar glide.

The second category includes the NECHl and Qi subgroups, where the approximant portion of $*_{\mathrm{rj}}$ and (with the exception of Meifu) *Curw did undergo fortition. A chain shift is observable, as it was in these three branches (as well as Lauhut) that original $*_{r}$ became trilled $*_{r}$, allowing original $*_{r j}$ to depalatalize to $*_{r}$ and fill the vacancy left by this change. It was this tap (and its labialized counterpart) which underwent fortition, leading to final stops and fricatives in the daughter languages:

\begin{tabular}{|c|c|c|c|c|c|c|c|c|}
\hline \multirow[b]{2}{*}{$*_{r}$} & \multirow[b]{2}{*}{$>$} & \multirow[b]{2}{*}{$\mathrm{r}$} & \multirow[b]{2}{*}{$>$} & \multirow[b]{2}{*}{$\mathrm{r}$} & \multicolumn{3}{|c|}{$1^{\text {st }}$ Devoicing } & $2^{\text {nd }}$ Devoicing \\
\hline & & & & & $>$ & $\mathrm{r}$ & $>$ & $\mathrm{r}$ \\
\hline$*_{r j}$ & $>$ & c & $>$ & c & $>$ & d & $>$ & $\mathbf{t}$ \\
\hline *Curw & $>$ & $\mathrm{C}-\mathrm{w}$ & $>$ & $2 \mathrm{w}$ & $>$ & $2 \mathrm{w}$ & $>$ & $\mathrm{kw} / \mathrm{y}$ \\
\hline$*_{r}$ & $>$ & $r$ & $>$ & $\mathrm{r}$ & $>$ & $\mathrm{r}$ & $>$ & $\mathrm{r}$ \\
\hline$*_{r j}$ & $>$ & $\mathrm{\rho} \sim \mathrm{lj}$ & $>$ & $\mathrm{d} \sim \mathrm{lj}$ & $>$ & $\mathrm{t} \sim \mathrm{d} \bar{F}$ & $>$ & $t \sim t s$ \\
\hline *Curw & $>$ & $\mathrm{rw}$ & $>$ & rw & $>$ & $(d) \mathrm{v}$ & $>$ & f \\
\hline$*_{r}$ & $>$ & $\mathrm{r}$ & $>$ & $\mathrm{r}$ & $>$ & $\mathrm{r}$ & $>$ & $\mathrm{r}$ \\
\hline$*_{r j}$ & $>$ & c & $>$ & f & $>$ & $\mathrm{d}$ & $>$ & $\mathbf{t}^{(\mathrm{h})}$ \\
\hline *Curw & $>$ & rw & $>$ & rW & $>$ & $(\mathrm{d}) \mathrm{v}$ & $>$ & f \\
\hline
\end{tabular}


The general tendency was, more specifically, for ${ }^{*} s$ to harden to $d$. If $*_{s}$ hardened prior to registrogenesis (as in Run), it underwent devoicing at this point and shows high register reflexes; if it hardened after registrogenesis (as in Meifu and Qi), then it conditioned low register reflexes before finally devoicing. There is variation in Run between the reflexes $t$ (high register) and ts (low register) for which there seems to be no discernible conditioning environment. What is evident is that based on the Yuanmen reflex of $* 1 \mathrm{j}$, the earlier form of variation was between ${ }^{*}$ (in which case the palatal glide was lost) and ${ }^{1} \mathrm{j}$ (in which case it was maintained).

The identical development of * Curw in both Run and Qi, where there was a change to an intermediate stage of $* v$ before finally devoicing to $f$ in low register, perhaps occurred through an initial change to $* d v$ if there was any parallel with the development of $*^{*}$ to $* d$. In Meifu, however, there was a development directly to $* ? \mathrm{w}$, paralleling the case of $*$ Curw below, where the glottal stop was preserved at the expense of the approximant.

\subsection{Rhotic Approximants}

The first division within Hlai occurred early (as detailed in chapter 1), with the Bouhin reflex of *r being an alveolar tap, but I hypothesize that *r shifted to a retroflex * $*_{I}$ in Greater Hlai, which later underwent a change in the daughter languages to a voiced velar fricative $\mathrm{y}$ :

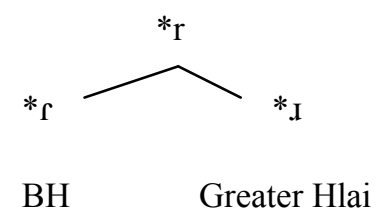

Vocalic transfer in *Cur did not occur outside of Central Hlai, however, so that the timing of the changes in *r itself and vocalic transfer occurred in the following way, with the presyllable leaving no trace in Bouhin and Ha Em, allowing the merger of original *r and *Cur in these two languages: 
(93)

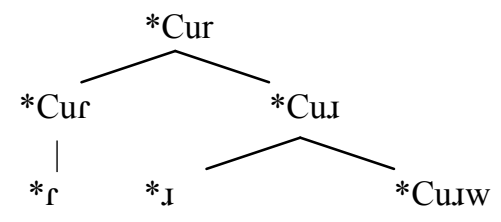

BH HE Central Hl

The Nadouhua reflexes seem, as in the case of PHl *hyw, to be conditioned by the following rime, with $v$ occurring before $a$, and $\eta$ occurring elsewhere:

(94)

\begin{tabular}{|c|c|c|c|c|c|}
\hline Gloss & $\underline{\mathrm{PHl}}$ & $\underline{\text { Nadouhua }}$ & $\underline{\text { Gloss }}$ & $\underline{\mathrm{PHl}}$ & Nadouhua \\
\hline with & *Curu: & yew ${ }^{4}$ & to plant & *Cura: & $\operatorname{va}^{4}$ \\
\hline run & *Curu:h & $\eta \varepsilon w^{2}$ & & & \\
\hline head & *Curəw? & пю: ${ }^{3}$ & & & \\
\hline
\end{tabular}

The timing of the change from $*_{I}$ to $*_{y}$ seems to have varied in its relationship to registrogenesis.

The change from *Cu.w to *?w occurred in all NCHl languages except for Nadouhua. The various paths of change are given below:

\section{Devoicing $\quad \underline{\text { Registrogenesis }}$}

\begin{tabular}{|c|c|c|c|c|c|c|c|c|c|}
\hline \multirow[t]{2}{*}{ Run } & $*_{\mathrm{I}}$ & $>$ & 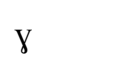 & $>$ & $\mathrm{x}$ & $>$ & $\mathrm{x}$ & $>$ & $\mathrm{x} / \mathrm{k}^{\mathrm{h}}$ \\
\hline & ${ }^{*}$ Cu.tw & $>$ & $? \mathrm{w}$ & $>$ & ?w & $>$ & $? \mathrm{w}$ & $>$ & $\mathrm{v}$ \\
\hline \multirow[t]{2}{*}{ Moyfaw } & $*_{\mathrm{I}}$ & $>$ & Y & $>$ & $\mathrm{x}$ & $>$ & $\mathrm{x}$ & $>$ & $\mathrm{x}$ \\
\hline & *Cu.tw & $>$ & Pw & $>$ & $2 \mathrm{w}$ & $>$ & $3 \mathrm{w}$ & $>$ & $y$ \\
\hline \multirow[t]{2}{*}{ Changjiang } & $*_{\mathrm{I}}$ & $>$ & $\mathrm{I}$ & $>$ & $\mathrm{I}$ & $>$ & Y & $>$ & g \\
\hline & ${ }^{*}$ Cu.rw & $>$ & Pw & $>$ & $\mathrm{kw}$ & $>$ & $\mathrm{kw}$ & $>$ & $\mathrm{kw}$ \\
\hline \multirow[t]{2}{*}{ Other $\mathrm{CHl}$} & $*_{\mathrm{I}}$ & $>$ & $\mathrm{I}$ & $>$ & $\mathrm{I}$ & $>$ & $\mathrm{y}$ & $>$ & $\mathbf{g} / \mathbf{h} / \mathfrak{n}$ \\
\hline & $*$ Cusw & $>$ & $\mathrm{I}(\mathrm{w}) / \mathrm{w}$ & $>$ & $\mathrm{y}(\mathrm{w}) / \mathrm{w}$ & $>$ & $\mathrm{y}(\mathbf{w}) / \mathbf{w}$ & $>$ & $\mathbf{g}(\mathbf{w}) / \mathbf{h}(\mathbf{w}) / \mathbf{y} / \mathbf{v}$ \\
\hline
\end{tabular}

A comparison of reconstructions is given below: 
(96) Comparison of reconstructions

\begin{tabular}{|c|c|c|c|c|c|}
\hline & $\underline{\text { Matisoff }}$ & Thurgood & $\underline{\text { Peiros }}$ & $\underline{\text { Ostapirat }}$ & $\underline{\mathrm{PHI}}$ \\
\hline (a) & $*_{\mathrm{v}}$ & ${ }^{*} \mathrm{w} ?$ & $*_{\mathrm{v}}$ & $*_{-v-}$ & $* v$ \\
\hline (b) & $*_{\mathrm{r}}$ & $* \mathrm{r} ?$ & $* \mathrm{r}$ & $*_{-r}-$ & $*_{r}$ \\
\hline (c) & --- & --- & --- & *-ir- & $*_{r j}$ \\
\hline (d) & $*_{\mathrm{vr}}$ & *pr? & $*_{\mathrm{zW}}$ & $*$-ur- & ${ }^{*} \mathrm{Cur}$ \\
\hline (e) & $* 3^{\prime} / * y$ & $* 3^{2 / *} \mathrm{y}$ & ${ }^{*} \mathrm{rw} /{ }^{*} \mathrm{\gamma}$ & $*_{-\mathrm{R}-} / *_{-} \mathrm{g}-$ & $*_{\mathrm{r}}$ \\
\hline (f) & $* \mathrm{gw}$ & $*_{\mathrm{yw}}$ & $*_{\mathrm{yw}}$ & $*$-uR-/*-ug- & ${ }^{*} \mathrm{Cur}$ \\
\hline
\end{tabular}

Matisoff and Peiros reconstruct the first series as *v (which in the present system would have devoiced to $f$ in the daughter languages), and Thurgood as *w? (which fails to explain the Tongzha reflex). Ostapirat's reconstruction of intervocalic *-v- is in agreement with mine, except that it is positionally more properly attributed to a stage of Pre-Hlai (see chapter four).

Matisoff and Peiros reconstruct *r, Thurgood *r?, and Ostapirat medial *-r- for the second series.

The problem with the reconstruction of * $\mathrm{r}$ for this series in the present reconstruction is that there is a more suitable candidate for ${ }^{*} \mathrm{r}$ based on the series of correspondences in (86). A tap, however, is consistent with the evidence if it can be assumed that it can change to a trill; given the propensity within the Hlai languages toward airstream activity in the initials (i.e. the aspirated obstruents and preaspirated sonorants), this seems entirely reasonable.

None of Matisoff, Thurgood, or Peiros offer a reconstruction for the third series, although Matisoff notes a couple of examples and states that a cluster must be reconstructed; Ostapirat proposes medial *-irfor this. I prefer to reconstruct a palatalized tap for this series, which allows a more direct explanation for how it can fill the gap left by former * $\mathrm{s}$ in the languages where this occurs, it is a better candidate for fortition to $* \mathrm{~d}$ (satisfying Directionality), and it is more compatible with palatalization than *r.

For the fourth series, Matisoff reconstructs *vr, Thurgood *pr? (in parallel with their *fr and *pr, respectively), Peiros * zw (parallel to his *sw), and Ostapirat medial *-ur-. Matisoff and Thurgood probably set up ${ }^{*}$-clusters here as well, based on a combination of labiodental reflexes and Moyfaw $\delta$, reconstruction of an *r-cluster here is again a violation of Economy, since the reconstruction of a labial 
element is all which is necessary to explain the modern reflexes. I prefer to reconstruct $*$ Cur, which has the benefit of explaining the lack of labialization in Bouhin and Ha Em, its parallel development (in terms of fortition) with $* r j$, and the Meifu reflex $* ? w$, which is understandable under the assumption of a presyllable initial pre-empting the medial tap.

Matisoff reconstructs the fifth series as either $* 3$ or ${ }^{*} \mathrm{\gamma}$, depending on the Bouhin reflex, and Thurgood does the same with near-identical $*^{*} 2$ and ${ }^{*} \mathrm{y}$; Peiros also adopts an alternation between *rw and ${ }^{*} \mathrm{\gamma}$, and Ostapirat reconstructs medial $*_{-\mathrm{R}-}$ or $*_{-} \mathrm{g}-$. These split reflexes are all based on the variation in Bouhin between $r$ and $g$, the latter of which I have identified in chapter one as the result of loans from Ha Em, which means that the second variant in each reconstruction is based on data which ultimately shouldn't be considered in the reconstruction. The reconstruction of a lateral $* \mathfrak{b}$ cannot account as elegantly for the split in reflexes between Bouhin (the change * 3 to $r$ violates Directionality) and Greater Hlai (the reconstruction ${ }^{*} \mathrm{\gamma}$ fits into Matisoff's and Peiros' system of voiced fricatives (along with $*_{\mathrm{v}}$ and $*_{\mathrm{z}}$ )). Ostapirat's reconstruction of a uvular trill seems awkward, given that there are no other uvulars in his system (a violation of Commonality). Peiros' *rw is closer to my reconstruction, although the reconstruction of a labiovelar coarticulation for this series (as opposed to the one below) seems unmotivated; Peiros' reconstruction of *rw contrasts with his *r, but is strange in that the reflexes between the two series have little in common except in Bouhin

The reconstruction of ${ }^{*} \mathrm{yw}$ (and $*$-ug-) for the final series is unmotivated, as it is again based on what I consider to be loans from Ha Em. I agree with Ostapirat, however, in positing a preceding $u$ for this initial.

Examples of the PHl approximants are given below, in the following order:

$\begin{array}{llllll}\text { Bhin } & \text { Ha Em } & \text { Lhut } & \text { Tzha } & \text { Zdui } & \text { Bting } \\ \text { Cun } & \text { Nadou } & \text { Cjiang } & \text { Mfaw } & \text { Baisha } & \text { Ymen }\end{array}$


(97) Examples of PHl approximants

(a)

上衣 clothes

ve: $\eta^{3} \quad$ ve: $\eta^{3} \quad$ ve: $\eta^{3}$

veๆ ${ }^{4} \quad$ veๆ ${ }^{3} \quad$ ve: $\eta^{3}$

肩膀 shoulder

$\mathrm{va}$ vo: 弓 vat $^{9} \quad$ vat $^{7} \quad \operatorname{vac}^{7} \quad$ fat $^{8}$ viat $^{4} \quad$ va? $^{4} \quad$ vat $^{7}$

窩

$\mathrm{ru}: 2^{7} \quad \mathrm{ru}: 2^{7} \quad \mathrm{ru} \cdot \mathrm{k}^{7}$ $\operatorname{lok}^{4} \quad \operatorname{lu} 2^{4} \quad \operatorname{lu}^{7}$

腸 intestine

ra: $y^{3} \quad$ ra: $y^{3} \quad$ ra: $y^{3}$ la: $(y)^{4} \quad$ lay $^{3}$ la: $y^{3}$

星星 star

ra: ${ }^{1} \quad$ ra: ${ }^{1}$ la:w ${ }^{4} \quad$ la? $^{4}$
${ }^{*} v$

*va:1?

fe:1 ve:1

*va:h

fa: ${ }^{2}$

$\mathrm{va}^{2}$

va:

va:

*vəc

fat $^{8}$

vat $^{7}$

$*_{r}$

*ru:k

ru:?

$\operatorname{ruk}^{7} \quad \operatorname{ruk}^{8} \quad \operatorname{ruP}^{8}$

*ra:y?

vat $^{8}$

vat $^{8}$

vat

vat $^{8}$

$\mathrm{va}^{.5}$

$\mathrm{va}^{2}$

zur:?

(b)

${ }^{*} \mathbf{r j}$

笑 laugh

* rja:w

e: $\eta^{3} \quad z a: w^{1} \quad z a: w^{1} \quad r a: w^{1}$

za: $w^{4} \quad$ la? $^{4} \quad$ ta: ${ }^{1}$

玩 play

*jju:k

ta: $w^{4} \quad \mathrm{t}^{\mathrm{h}} \mathrm{a}: \mathrm{w}^{4} \quad$ ta: $\mathrm{w}^{4}$

ta: ${ }^{1} \quad$ tsa: $^{1} \quad$ tsa: ${ }^{4}$

u: $?^{8} \quad \mathrm{t}^{\mathrm{h}} \mathrm{ua}^{8} \quad$ tu: $?^{8}$

$\operatorname{wk}^{7} \quad\left(\mathrm{tuk}^{7}\right) \quad----$

壞 bad

$*_{\text {rja:k }}$

ru: $\mathrm{k}^{7}$

tuk $^{7}$

$\mathrm{za}: 2^{7} \quad \mathrm{ze}: ?^{7} \quad$ re: $\mathrm{k}^{7}$

$l \varepsilon \mathrm{k}^{4} \quad l e ?^{4}$

te: $?^{7}$

te: $?^{8} \quad \mathrm{t}^{\mathrm{h}} \mathrm{e}: \mathrm{P}^{8} \quad$ te $: ?^{8}$

tur: ${ }^{2} \quad$ te $^{8} \quad\left(\right.$ tia? $\left.^{7}\right)$

(e)

*Cur

*Curi:p

生 raw

vi:p $p^{7}$

ri: $p^{7} \quad$ ri: $p^{7}$

vip $^{4}$

bone

kip

fi: $p^{8} \quad$ fi: $p^{8} \quad$ fi: $p^{8}$

yip $^{7} \quad$ fip $^{8} \quad$ fip $^{8}$

ra:y ${ }^{6} \quad$ la:y ${ }^{6} \quad$ la:y ${ }^{6} \quad$ ru: $?^{7} \quad$ ru: $?^{7}$

ra: $y^{3}$

ra: $y^{3}$

ruay $^{6}$

vurot

*ra:w

地 earth

ku?

*Curur:k

fur:? fua $^{8}$ fu: $?^{8}$ yuk $^{7} \quad$ fuk $^{8} \quad$ fur $?^{8}$

*Curən

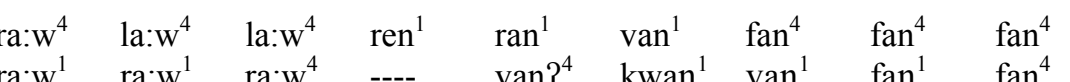




\begin{tabular}{|c|c|c|c|c|c|c|c|c|c|c|c|}
\hline (d) & & & $* \mathbf{r}$ & & & (f) & & & ${ }^{*}$ Cur & & \\
\hline 辣 & spicy & & $*_{\text {rit }}$ & & & 跑 & run & & *Curu: & & \\
\hline $\begin{array}{l}\text { rit }^{7} \\
\text { het }^{4}\end{array}$ & $\begin{array}{l}\text { git }^{7} \\
\left(\mathrm{ze}^{5}\right)\end{array}$ & $\begin{array}{l}\operatorname{gec}^{7} \\
\left(\text { git }^{7}\right)\end{array}$ & $\begin{array}{l}\text { get }^{8} \\
\text { xet }^{7}\end{array}$ & $\begin{array}{l}\text { het }^{8} \\
\text { xet }^{8}\end{array}$ & $\begin{array}{l}\mathrm{hit}^{8} \\
\mathrm{k}^{\mathrm{h}} \mathrm{et}^{7}\end{array}$ & $\begin{array}{l}\text { row }^{2} \\
\text { vaw }^{5}\end{array}$ & $\begin{array}{l}\text { gow }^{2} \\
\text { yew }\end{array}$ & $\begin{array}{l}\text { gow }^{2} \\
\text { kow }^{2}\end{array}$ & $\begin{array}{l}\text { gow }^{2} \\
\text { gow }^{2}\end{array}$ & ---- & $\begin{array}{l}\text { how }^{2} \\
\text { vow }^{5}\end{array}$ \\
\hline & net & & *rə:y? & & & 頭 & head & & *Curəv & & \\
\hline$a: y^{3}$ & go: $y^{3}$ & go: $y^{3}$ & ga: ${ }^{6}$ & ha: $y^{6}$ & ha: $y^{6}$ & raw $^{3}$ & gaw $^{3}$ & gwow $^{3}$ & go: ${ }^{6}$ & ho: ${ }^{6}$ & ho: ${ }^{6}$ \\
\hline 1०:(y) & yəy ${ }^{3}$ & go: $y^{3}$ & $x o: y^{3}$ & xuay $^{3}$ & $\mathrm{k}^{\mathrm{h}} \mathrm{u}: \mathrm{y}^{3}$ & vaw $^{3}$ & yo: ${ }^{3}$ & ko: $:^{3}$ & yo: ${ }^{3}$ & vo: ${ }^{3}$ & vo: $:^{3}$ \\
\hline 自們 & \multicolumn{2}{|c|}{ we (incl) } & *row & & & 種 & \multicolumn{2}{|c|}{ to plant $^{20}$} & \multicolumn{2}{|l|}{ *Cura: } & \\
\hline $\mathrm{ru}:{ }_{5}^{1}$ & $\begin{array}{l}\text { gaw }^{1} \\
\text { yaw }{ }^{4}\end{array}$ & $\begin{array}{l}\text { ga: }^{1} \\
\text { gaw }^{4}\end{array}$ & $\begin{array}{l}\text { gaw }^{4} \\
\text { xaw }^{1}\end{array}$ & $\begin{array}{l}\text { haw }{ }^{4} \\
\text { хә: }\end{array}$ & $\begin{array}{l}\text { how }^{4} \\
\mathrm{k}^{\mathrm{h}} \mathrm{o}^{7}\end{array}$ & $\begin{array}{l}\text { ra: }{ }^{1} \\
\text { vo: }{ }^{4}\end{array}$ & $\begin{array}{l}\left(\mathrm{ra}^{.1}\right) \\
\mathrm{va}^{4}{ }^{4}\end{array}$ & $\begin{array}{l}\text { gwa: }{ }^{1} \\
\text { ko: }\end{array}$ & $\begin{array}{l}\text { go: }{ }^{6} \\
\text { ya: }{ }^{1}\end{array}$ & $\begin{array}{l}\text { va: }{ }^{4} \\
\text { va: }{ }^{1}\end{array}$ & $\begin{array}{l}\text { hwa: }{ }^{4} \\
\text { va: }{ }^{4}\end{array}$ \\
\hline
\end{tabular}

\subsubsection{Glides}

The PHl glides were originally preaspirated, and their reflexes are for the most part entirely straightforward.

Reflexes of PHl glides

$\underline{\text { BHin }} \underline{\text { Ha Em }} \underline{\text { LHut }} \underline{\text { Tzha }} \underline{\text { Zdui }} \underline{\text { Bting }} \underline{\text { Cun }} \underline{\text { Nadou }} \underline{\text { Cjiang }} \underline{\text { Meifu }} \underline{\text { Baisha }} \underline{\text { Ymen }}$

$\begin{array}{llllllllllll}\mathbf{Z} & \mathrm{Z} & \mathrm{Z} & \mathbf{Z} & \mathbf{Z} & \mathrm{Z} & \mathrm{Z} & \mathbf{Z} & \mathbf{Z} & \mathrm{Z} & \mathrm{Z} & \mathbf{Z} \\ \mathrm{V} & \mathrm{V} & \text { hW } & \mathbf{V} & \mathbf{V} & \mathrm{V} & \mathrm{V} & \mathbf{v} & \mathrm{v} & \mathrm{V} & \mathrm{V} & \mathbf{v}\end{array}$

\section{$\underline{\text { S. Hlai (Savina) }}$}

j

V
C. Hlai (Savina)

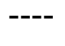

$\mathbf{v}$ $\underline{\text { Baisha (Wang \& Qian) }}$

3

$\mathrm{v}$

The following are reconstructed for the two series above:

$$
\begin{aligned}
& * \text { hj } \\
& * \text { hw }
\end{aligned}
$$

\footnotetext{
${ }^{20}$ The initials in the Nadouhua, Ha Em, and Zandui forms are all irregular, the reason probably being that they are the results of loans from other Hlai languages (in which they would be regular).
} 
As a general rule, the primary change which has occurred in the glides is an increase in stricture to the weak fricatives $z$ and $v$. It is possible that this was motivated by their preaspiration, which may have provided a percept of frication which became confused with the following glide, becoming susceptible to reanalysis as a fricative via temporal compression. In almost all cases, this change occurred before registrogenesis, creating an environment (voiced obstruents) which would condition low register.

The three exceptions to this scenario are Cunhua, Lauhut (for *hw only), and Baoting. In Cunhua and Baoting, the glides developed as described above except for the fact that they conditioned high register, not the expected low register which occurs in the other registrogenetic languages. I believe the best explanation for the registral behavior in these two languages is merely that they experienced the change from $h j / h w$ to $z / v$ after registrogenesis, since as preaspirated fricatives they would have conditioned high register. In the case of Lauhut $h w$, the best explanation is that it is conservative, and preserves the original reflex of $\mathrm{PHl} *$ hw. These developments are illustrated below:

\section{$\underline{\text { Registrogenesis }}$}

\begin{tabular}{|c|c|c|c|c|}
\hline Cunhua \& & $* h j$ & $>$ & hj & $>$ \\
\hline Baoting & *hw & $>$ & hw & $>$ \\
\hline \multirow[t]{2}{*}{ Lauhut } & *hj & $>$ & hj & $>$ \\
\hline & *hw & $>$ & hw & $>$ \\
\hline \multirow[t]{2}{*}{ Other Hlai } & $*$ hj & $>$ & Z & $>$ \\
\hline & ${ }^{*}$ hw & $>$ & $\mathbf{v}$ & $>$ \\
\hline
\end{tabular}

A Comparison of reconstructions is given below:

$\begin{array}{llllll}\text { (101) } & \underline{\text { Matisoff }} & \underline{\text { Thurgood }} & \underline{\text { Peiros }} & \underline{\text { Ostapirat }} & \underline{\text { PHl }} \\ \text { (a) } & *_{\mathrm{j}} & *_{\mathrm{j}} & *_{\mathrm{j}} & *_{-\mathrm{j}-} & * \mathrm{hj} \\ \text { (b) } & *_{\mathrm{w}} & *_{\mathrm{w}} & *_{\mathrm{w}} & *_{-\mathrm{w}-} & * \mathrm{hw}\end{array}$

Matisoff, Thurgood, and Peiros all reconstruct these two series as $*_{\mathrm{j}}$ and ${ }^{*} \mathrm{w}$, respectively. The weakness of this reconstruction is that it doesn't explain the registral behavior in Cunhua and Baoting, or the unique Lauhut reflex of $h w$ in the second series. 
Ostapirat reconstructs medial $*_{-j}$ - and $*_{-w}$-, positing intervocalic fortition of sonorants as he does in the case of the nasals and lateral. As in those cases, the fortition of a sonorant intervocalically is rather counter-intuitive.

Examples of the PHl glides are given below, in the following order:

\begin{tabular}{|c|c|c|c|}
\hline Shin & Ha Em & Lhut & Tzha \\
\hline Cun & Nadou & Cjiang & Mfav \\
\hline
\end{tabular}

(102) Examples of PHl glides

(a)

\begin{tabular}{|c|c|c|c|c|c|c|c|c|c|c|c|}
\hline 蛋/卵 & egg & & *hju:n & & & 彎 & crooked & & *hwə:y & & \\
\hline $\begin{array}{l}\text { zur:m } \\
\text { zum }^{1}\end{array}$ & 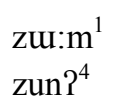 & $\begin{array}{l}\text { zu:m }{ }^{1} \\
\text { zum }^{1}\end{array}$ & $\begin{array}{l}\text { zu: } \mathrm{m}^{4} \\
\text { zum }^{1}\end{array}$ & $\begin{array}{l}\mathrm{zu}: \mathrm{m}^{4} \\
\mathrm{zum}^{1}\end{array}$ & $\begin{array}{l}\text { zur:m }{ }^{1} \\
\text { zum }^{4}\end{array}$ & voy ${ }^{3}$ & vay ${ }^{2}$ & $\begin{array}{l}\text { hwo: } \eta^{2} \\
\text { voy }{ }^{2}\end{array}$ & $\begin{array}{l}\text { vo: } \eta^{2} \\
\text { voy }\end{array}$ & $\begin{array}{l}\text { vo: } \eta^{2} \\
\text { voy }\end{array}$ & $\begin{array}{l}\text { vo: } y^{5} \\
\text { voy }\end{array}$ \\
\hline 老 & old & & *hja: & & & 贊揚 & praise & & *hwəy & & \\
\hline $\begin{array}{l}\mathrm{za}:{ }^{1} \\
\mathrm{zo}:{ }^{1}\end{array}$ & $\begin{array}{l}\mathrm{za}^{1}{ }^{4} \\
\mathrm{za}^{4}{ }^{4}\end{array}$ & $\begin{array}{l}\mathrm{za}^{1}{ }^{4} \\
\mathrm{za}^{4}\end{array}$ & $\begin{array}{l}\mathrm{za}^{4}{ }^{4} \\
\mathrm{za}:{ }^{1}\end{array}$ & $\begin{array}{l}\mathrm{za}^{4}{ }^{4} \\
\mathrm{za}:{ }^{1}\end{array}$ & $\begin{array}{l}\mathrm{za}^{1}{ }^{4} \\
\mathrm{za}:{ }^{4}\end{array}$ & $\begin{array}{l}\text { vay }^{1} \\
\text { vay }^{1}\end{array}$ & $\begin{array}{l}\text { vay }^{1} \\
\text { vay }^{4}\end{array}$ & $\begin{array}{l}\text { hway } \\
\text { vay }^{4}\end{array}$ & $\begin{array}{l}\text { vay }^{4} \\
\text { vay }^{1}\end{array}$ & $\begin{array}{l}\text { vay }^{4} \\
\text { vay }^{1}\end{array}$ & $\begin{array}{l}\text { vay }^{1} \\
\text { vay }^{4}\end{array}$ \\
\hline 羊 & sheep & & *hja:y & & & 芭蕉 & plantain $^{21}$ & & *hwa:k & & \\
\hline$: n^{1}$ & ze: $\eta^{1}$ & ze: $y^{1}$ & $z e: y^{4}$ & ze: $: \eta^{4}$ & $z e: y^{1}$ & va: $?^{7}$ & ve: $?^{7}$ & hwe:k ${ }^{7}$ & ve: $?^{8}$ & ve: $1^{8}$ & ve: $?^{7}$ \\
\hline $\mathrm{y}^{1}$ & $\mathrm{z \varepsilon \eta} \mathrm{P}^{4}$ & ---- & $z e: y^{1}$ & ziay $^{1}$ & zian $^{4}$ & $z \varepsilon k^{2}$ & ---- & ve: $?^{7}$ & vur: ${ }^{2}$ & $v e ?^{8}$ & $\operatorname{via}^{8}$ \\
\hline
\end{tabular}

\subsubsection{Interim Summary}

A total of five sets of sonorants have been reconstructed in this section: preaspirated and medial nasals, four kinds of laterals, approximants, and preaspirated glides. This half of the initial inventory is shown below:

${ }^{21}$ The Cunhua initial is irregular, the original ${ }^{*} \mathrm{w}$ apparently having been replaced by $j$, under the influence of the rime. 


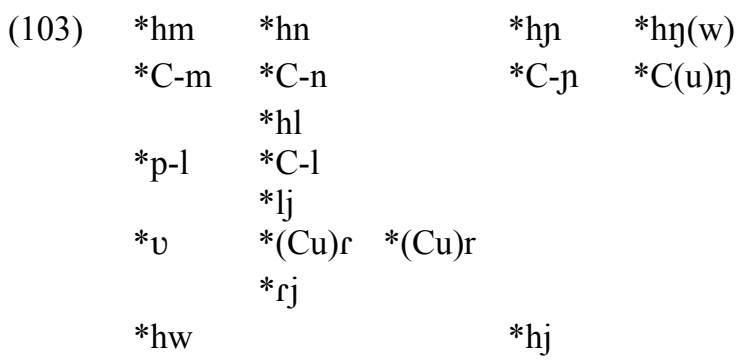

As in the case of the obstruents, there are a number of asymmetries which are of interest. The most glaring is the fact that there is no plain series of nasals and lateral (in initial position), or glides which contrast with the preaspirated series; this is typologically very uncommon (Maddieson 1984: 69).

The second is that the alveolar liquids exist in two varieties: plain and palatalized. In conjunction with this, presyllables with high back rounded vowels are reconstructible in the case of the velar nasal and coronal approximants; there are no corresponding presyllables reconstructible with high front vowels. Altogether, this indicates a preference for palatalization over labialization (the latter which occurs, nevertheless, with the velar nasal, a very common environment (ibid: 69)

Finally, the only presyllable initial to retain its place of articulation (in *p-l) is the most anterior, at the bilabial place of articulation. It may be hypothesized that this occurred because the articulation of [p] is independent of the tongue, and that other obstruents, the articulation of which required lingual gestures, debuccalized before [1] which requires one or more lingual constrictions. These asymmetries are discussed further in chapter four.

\subsubsection{PHI Glottal Initials}

There are two kinds of PHl glottals: glottal stops and glottal fricatives. Both the glottal stop and the glottal fricative occur in triplets: plain, preceded by ${ }^{*} \mathrm{Ci}-$, and preceded by ${ }^{*} \mathrm{Cu}-$. The reflexes of these six series are given below: 
(104) Reflexes of PHl glottal initials

\begin{tabular}{|c|c|c|c|c|c|c|c|c|c|c|c|}
\hline BHin & $\underline{\mathrm{HaEm}}$ & $\underline{\text { LHut }}$ & $\underline{\text { Tzha }}$ & $\underline{\text { Zdui }}$ & Bting & $\underline{\text { Cun }}$ & $\underline{\mathrm{Nadou}}$ & $\underline{\text { Cjiang }}$ & $\underline{\text { Mfaw }}$ & $\underline{\text { Baisha }}$ & $\underline{\text { Ymen }}$ \\
\hline$?$ & $?$ & $?$ & $?$ & $?$ & $?$ & $?$ & $?$ & $?$ & $?$ & $?$ & $?$ \\
\hline$?$ & $?$ & $\mathrm{Pj}$ & z & z & $\mathrm{Pj}$ & z & ij & z & z & z & z \\
\hline$?$ & $?$ & $3 \mathrm{w}$ & $\mathrm{gw}$ & $\mathrm{v}$ & ?w & $\mathrm{v}$ & Pw & $\mathrm{kw}$ & y & $\mathrm{v}$ & $\mathrm{v}$ \\
\hline $\mathrm{h}$ & $\mathrm{h}$ & $\mathrm{h}$ & $\mathrm{h}$ & $\mathrm{h}$ & $\mathrm{h}$ & $\mathrm{h}$ & $\mathrm{h}$ & $\mathrm{h}$ & $\mathrm{h}$ & $\mathrm{h}$ & $\mathrm{h}$ \\
\hline h & $\mathrm{h}$ & hj & $\mathrm{z}$ & z & hj & $\mathrm{n}$ & $\mathrm{jj}$ & $\mathrm{n}$ & $\mathrm{n}$ & $\mathrm{n}$ & $\mathrm{n}$ \\
\hline $\mathrm{h}$ & $\mathrm{h}$ & hw & gw & $\mathrm{v}$ & hw & $\mathrm{y}$ & $\mathrm{y}(\mathrm{w})$ & $y$ & $\eta$ & $\mathrm{y}$ & $\mathrm{m}$ \\
\hline
\end{tabular}

\section{$\underline{\text { S. Hlai (Savina) }}$}

\section{Hlai (Savina)}

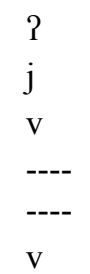

\section{Baisha (Wang \& Qian)}
$?$
3
$\mathrm{V}$
$\mathrm{h}$
$\mathrm{n}$
y

The reconstructions I propose for these series of correspondences are glottal stops and voiced fricatives, each plain as well as with with presyllables, the first with a preceding $i$, and the second with a preceding $u$ :

$$
\begin{aligned}
& * ? \\
& { }^{*} \mathrm{Ci} ? \\
& { }^{*} \mathrm{Cu} ? \\
& * \mathrm{~h} \\
& { }^{*} \mathrm{Cih} \\
& { }^{*} \mathrm{Cuh}
\end{aligned}
$$

\subsubsection{Glottal Stops}

The first series of reflexes is absolutely uniform throughout the daughter languages, and I reconstruct it as *2. I reconstruct the second and third series as ${ }^{*} \mathrm{Ci} i$ and ${ }^{*} \mathrm{Cu}$. The development of these two initials has paralleled that of the coronal approximants and glottal fricatives quite closely, in that the presyllables have been completely lost in Bouhin and Ha Em, but have left traces because of vocalic transfer in Central Hlai: 


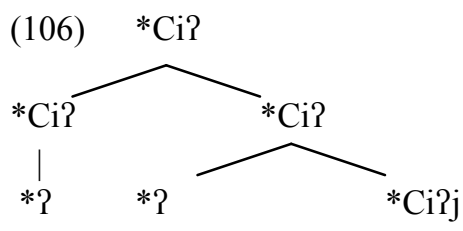

$\mathrm{BH} \quad \mathrm{HE} \quad \mathrm{CHl}$

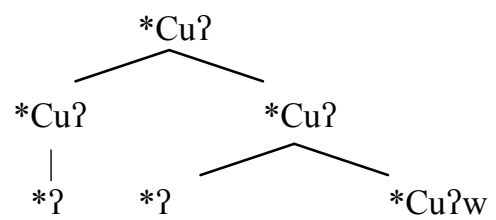

$\mathrm{BH} \quad \mathrm{HE} \quad \mathrm{CHl}$

Deglottalization has occurred in reflexes of both in six $\mathrm{CHl}$ languages, and for the palatal glide in Tongzha $^{22}$. In the majority of languages, when deglottalization has occurred, the glides have undergone an additional fortition to voiced fricatives, merging with the reflexes of the preaspirated glides (but being distinguished by register in most $\mathrm{CHl}$ languages).

In Meifu, $P w$ followed two different paths: in Changjiang, it was reanalyzed as $g w$ and devoiced to $k w$; in Moyfaw, it lost its glottal constriction while giving rise to the velar fricative $\gamma$, through deletion of the labial part of the original labiovelar coarticulation:

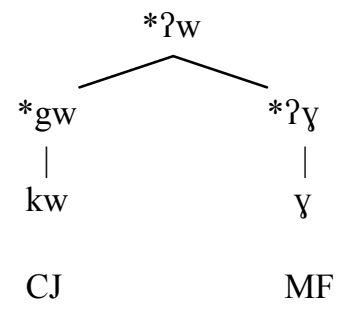

The reflexes of $* 2 \mathrm{w}$ in the Meifu branch are especially important, as they provide important information in the reconstruction of presyllables in PHl $* \mathrm{C}-\beta,{ }^{*} \mathrm{Cur}$, and (with Run and Cunhua) ${ }^{*}$ Cur above.

\subsubsection{Glottal Fricatives}

I reconstruct * $\mathrm{f}$ for the first series of correspondences in this group. Although this initial patterns in many ways with the class of approximants, the true approximants have in general developed into initials

\footnotetext{
${ }^{22}$ Note that deglottalization must have happened after registrogenesis in all languages in which it occurred, since none of the tonal reflexes indicate voiced initials.
} 
which conditioned low register at the time of registrogenesis. $\mathrm{PHl} * \mathrm{~h}$, on the other hand, did not condition low register in any languages. The reason that there is such an asymmetry in registral behavior when *h is compared with ${ }^{*} v$ and ${ }^{*} r$ is apparently that while ${ }^{*} v$ and ${ }^{*} r$ were true approximants, this fricative (as an obstruent) fit the environment for devoicing, changing * $h$ to $h$ across the board. The reason that this series of correspondences is not reconstructed merely as *h is that it interlocks into a system with *Cif and *Cuf, both of which are shown below as being necessarily voiced.

I believe that there are two things which suggest a reconstruction of * $\mathrm{Cif}$ and $* \mathrm{Cuh}$ for the second and third series. The first is external evidence, to be treated in chapter four. The other, which Matisoff (1988) notes, is that the place of articulation for these two initials (palatal and labiovelar) is very marked for obstruents or nasals, but typical of glides; he therefore suggests that the nasal reflexes in $\mathrm{NCHl}$ are the result of rhinoglottophilia, a phenomenon in which the percept of nasalization is usually triggered in the environment of laryngeals, leading to the phonologization of an actual nasal (Matisoff 1975). This is in essence the solution adopted here, where $\mathrm{PHl}{ }^{*} \mathrm{Cif}$ and ${ }^{*} \mathrm{Cuf}$ (after the application of $\mathrm{PCHl}$ vocalic transfer, rendering $* \mathrm{Cifj}$ and $* \mathrm{Cufw}$ ) were confused with $*$ ? $\mathrm{n}$ and $*$ ? $\mathrm{yw}$, the percept of nasalization originating in the temporal compression across the stop from the presyllable and the glottal fricative, but the place features being cued by the following glides. Bouhin and Ha Em did not participate in vocalic transfer, and the reflexes of * Cif and *Cuf merged with those of *f. These paths of change are outlined below:

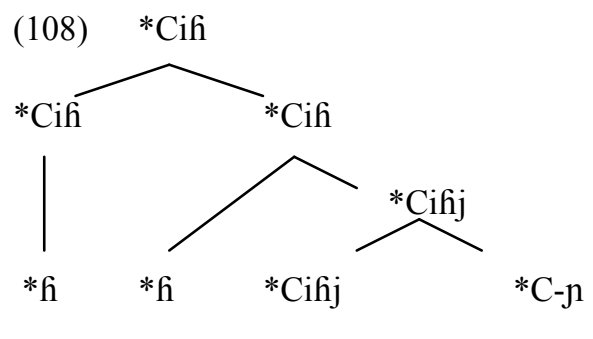

$\mathrm{BH} \quad \mathrm{HE} \quad \mathrm{ECHl} \quad \mathrm{NCHl}$

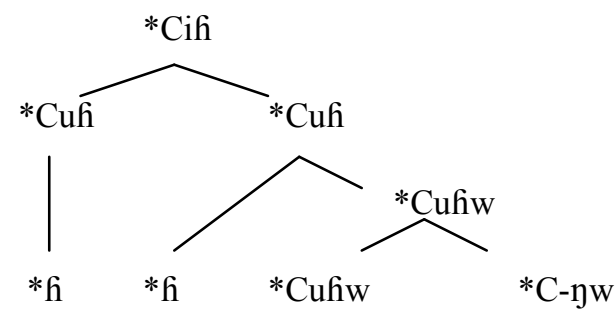

$\mathrm{BH} \quad \mathrm{HE} \quad \mathrm{ECHl} \quad \mathrm{NCHl}$ 
In ECHI, *Cifj and ${ }^{*} \mathrm{Cufw}$ generally followed roughly the same trajectory as * $\mathrm{h}$, losing the presyllable stop and devoicing to $h j$ and $h w$. There is variation in the reflexes for ${ }^{*}$ Cifij and ${ }^{*} \mathrm{Cufw}$ in Qi; in Tongzha and Zandui, *Cifj and ${ }^{*} \mathrm{Cufw}$ merged with ${ }^{*} \mathrm{Ci} i \mathrm{j}$ and ${ }^{*} \mathrm{Cu} P \mathrm{w}$, yielding $P j$ and $2 w$ respectively; in Baoting, on the other hand, *Cifj and *Cufw merely lost the presyllable and devoiced to $h j$ and $h w^{23}$ :
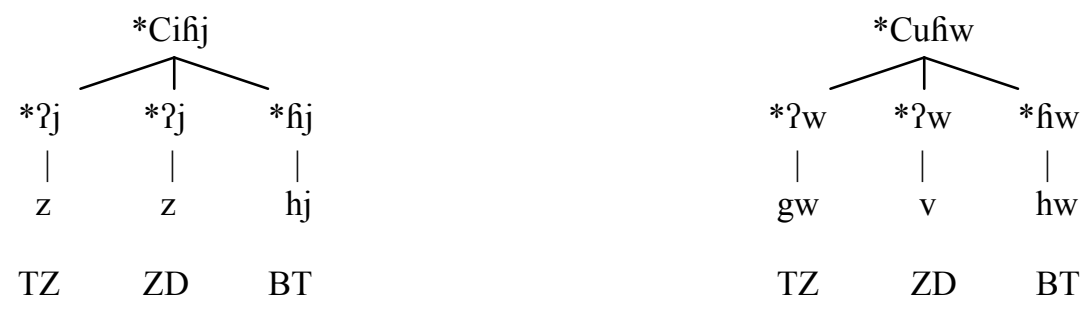

A comparison of reconstructions is given below:

\section{Matisoff}

Thurgood

$\underline{\text { Peiros }}$

$\underline{\text { Ostapirat }}$

$\underline{\mathrm{PHl}}$

(a) $\quad *$ ?

(b) $\quad * x j$

(c) $\quad * x w$

(a) $\quad * x$

(b) *hj

(c) $\quad * \mathrm{hw}$

\section{*?}

$--$

*xw

*x

*hn?

*hyw?
$* ?$

*?j

*?w

$* \mathrm{~h}$

*hp

*hyw

\section{*?}

*-i?-

*-u?-

*-k-

*-ik-

*-uk-
*?

*Ci?

${ }^{*} \mathrm{Cu}$ ?

$* \mathrm{~h}$

${ }^{*}$ Cif

*Cuf

There is complete unanimity in reconstructing *? for the first series.

Matisoff reconstructs the second and third series as *xj and *xw, while Thurgood does not reconstruct anything for the second but reconstructs the third as *xw. It is unclear to me what the motivation is for Matisoff and Thurgood to reconstruct initial velar fricatives for these correspondence sets, as a change from $x$ to $?$ is not a normal one typologically and a clear violation of Directionality (as well as Commonality). Peiros' reconstruction of preglottalized glides is similar to the present reconstruction, the difference being that it is equivalent with post-vocalic transfer Central Hlai. Ostapirat reconstructs medial *-iP- and *-u?-, which is only different from the present reconstruction notationally.

\footnotetext{
${ }^{23}$ In my own fieldwork, the initials produced by my Baoting consultant were still voiced $f$.
} 
Matisoff and Thurgood reconstruct * $\mathrm{x}$ and Peiros $* \mathrm{~h}$ for the first series; the uniform high register reflexes in the daughter languages are likely the main reason for Matisoff, Thurgood and Peiros all reconstruct this series with an original voiceless reflex. If * $\mathrm{x}$ is reconstructed, however, it presupposes complete debuccalization throughout all of the daughter languages, severely violating Commonality. Ostapirat reconstructs medial *-k-, which has a place in his system where intervocalic stops undergo affrication.

For the last two series, Matisoff reconstructs *hj and *hw, Thurgood *hn? and *hyw?, and Peiros *hn and *hyw. Matisoff's reconstruction is typologically reasonable when one assumes rhinoglotophilia, a phenomenon which he relies upon to explain the $\mathrm{NCHl}$ reflexes (as is done here). Thurgood and Peiros' reconstruction of a series of preaspirated nasals is very suspect, on the other hand, as they are the only preaspirated nasals in either of their reconstructions, and they occur at marked places of articulation. Ostapirat reconstructs medial $*-\mathrm{k}$ - preceded by $* \mathrm{i}$ and $* \mathrm{u}$ respectively $\left(*-\mathrm{ik}-,{ }^{*}\right.$-uk-). Although I disagree with his choice of consonants, our reconstructions agree in positing preceding high vowels.

Examples of the PHl glides are given below, in the following order:

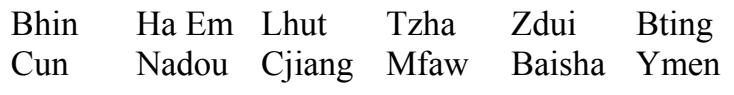


(111) Examples of PHl glottals

\begin{tabular}{|c|c|c|c|c|c|c|c|c|c|c|c|}
\hline (a) & & & $* ?$ & & & (b) & & & ${ }^{*} \mathrm{~h}$ & & \\
\hline 烤火 & light up & & *?um & & & 苦 & bitter & & *hə:m & & \\
\hline ?um ${ }^{2}$ & Pum ${ }^{2}$ & Pum ${ }^{2}$ & Pum 5 & Pum $^{5}$ & Pum $^{5}$ & ham $^{1}$ & ho: $\mathrm{m}^{1}$ & ho:m ${ }^{1}$ & ho:m $\mathrm{m}^{1}$ & hə:m $\mathrm{m}^{1}$ & ho: $\mathrm{m}^{1}$ \\
\hline Pom $^{5}$ & $\operatorname{Pun}^{2}$ & $\mathrm{Pom}^{2}$ & Pom $^{2}$ & $\mathrm{Pom}^{2}$ & $\mathrm{Pom}^{5}$ & $\operatorname{ham}^{1}$ & hon ${ }^{1}$ & ho:m ${ }^{1}$ & ho: $\mathrm{m}^{1}$ & huam $^{1}$ & huam $^{1}$ \\
\hline 吹 & blow & & *?u:h & & & 屎 & excres & & *ha:y? & & \\
\hline Pow $^{2}$ & Pow $^{2}$ & Pow $^{2}$ & Pow $^{5}$ & Pow $^{5}$ & Pow $^{5}$ & ha: $y^{3}$ & ha: $y^{3}$ & ha: $y^{3}$ & ha: $y^{3}$ & ha: $y^{3}$ & ha: $y^{3}$ \\
\hline low $^{5}$ & $1 \varepsilon w^{2}$ & Pow $^{2}$ & Pow $^{2}$ & Pow $^{2}$ & Pow $^{5}$ & ha: $(y)^{3}$ & hay $^{3}$ & ha: $y^{3}$ & ha: $y^{3}$ & ha: $y^{3}$ & huay $^{3}$ \\
\hline 下飯 & go with & (food) & * pu:n & & & 下巴 & chin & & *ha:y & & \\
\hline ?u:n ${ }^{1}$ & Pu:n ${ }^{1}$ & Pwen ${ }^{1}$ & Pu:n ${ }^{1}$ & Pu:n ${ }^{1}$ & Pu:n ${ }^{1}$ & ha: ${ }^{1}$ & he: $\eta^{1}$ & he: $\eta^{1}$ & he: $\eta^{1}$ & he: $\eta^{1}$ & he: $\eta^{1}$ \\
\hline Puən ${ }^{1}$ & Pun $^{1}$ & $\operatorname{Pon}^{1}$ & Pun $^{1}$ & $\operatorname{Pun}^{1}$ & Pun $^{1}$ & heๆ ${ }^{1}$ & hen ${ }^{1}$ & he: $\eta^{1}$ & he: $\eta^{1}$ & $\operatorname{hiay}^{1}$ & $\operatorname{hian}^{1}$ \\
\hline (c) & & & *Ci? & & & (d) & & & *Cif & & \\
\hline 木瓜/相 & 子 papay & $\mathrm{a} /$ cocon & It *Ci?u & & & 田埂 & field $r$ & & *Cihə:n & & \\
\hline Pun $^{2}$ & Pun $^{2}$ & Pjun $^{2}$ & $\operatorname{zun}^{5}$ & zun $^{5}$ & Pjumn $^{5}$ & han $^{1}$ & ho: $n^{1}$ & hjo: $n^{1}$ & $z o: n^{1}$ & $\mathrm{zo:n^{1 }}$ & hјว:n ${ }^{1}$ \\
\hline ---- & Pjen ${ }^{2}$ & ---- & ---- & $\left(\mathrm{zoy}^{5}\right)$ & $\mathrm{z}^{5} \mathrm{n}^{5}$ & $\operatorname{man}^{1}$ & non $^{1}$ & no: $\eta^{1}$ & no: $\mathrm{y}^{1}$ & nuay $^{1}$ & ju:n ${ }^{4}$ \\
\hline 吞 & swallow & & *Ci?ə & & & 茅草 & thatch & rass & *Cifia: & & \\
\hline 1o: $\mathrm{m}^{2}$ & 1o: $\mathrm{m}^{2}$ & Pjo:m² & $\mathrm{zo}: \mathrm{m}^{5}$ & $\mathrm{zo: \textrm {m } ^ { 5 }}$ & १јэ: $\mathrm{m}^{5}$ & ha: ${ }^{1}$ & ha: ${ }^{1}$ & hja: ${ }^{1}$ & $\mathrm{za}:^{1}$ & $\mathrm{za}:^{1}$ & hja: ${ }^{1}$ \\
\hline $\mathrm{zam}^{5}$ & ?jon ${ }^{2}$ & $\mathrm{zo}: \mathrm{m}^{2}$ & $\mathrm{zo}: \mathrm{m}^{2}$ & zuam $^{2}$ & zuam $^{5}$ & no: ${ }^{1}$ & пja: ${ }^{1}$ & je: ${ }^{1}$ & na: ${ }^{1}$ & na: ${ }^{1}$ & na: ${ }^{4}$ \\
\hline 打滾 & roll & & *Ci?o & & & 虫 & bug & & *Cifən & & \\
\hline $\operatorname{Pu\eta }^{3}$ & $\operatorname{Puy}^{3}$ & Pjon $^{3}$ & $\mathrm{zoy}^{3}$ & zon $^{3}$ & $\mathrm{joy}^{3}$ & hen $^{2}$ & $\operatorname{han}^{2}$ & $\operatorname{hjan}^{2}$ & $\operatorname{zan}^{5}$ & $\operatorname{zan}^{5}$ & $\operatorname{hjan}^{5}$ \\
\hline$z o \eta^{3}$ & ---- & $z_{0} \eta^{3}$ & $z O y^{3}$ & $z^{2} y^{3}$ & $z o \eta^{3}$ & $\operatorname{man}^{5}$ & yjen $^{2}$ & $\operatorname{nan}^{2}$ & $\operatorname{san}^{2}$ & $\operatorname{san}^{2}$ & $\operatorname{san}^{2}$ \\
\hline
\end{tabular}


(e)

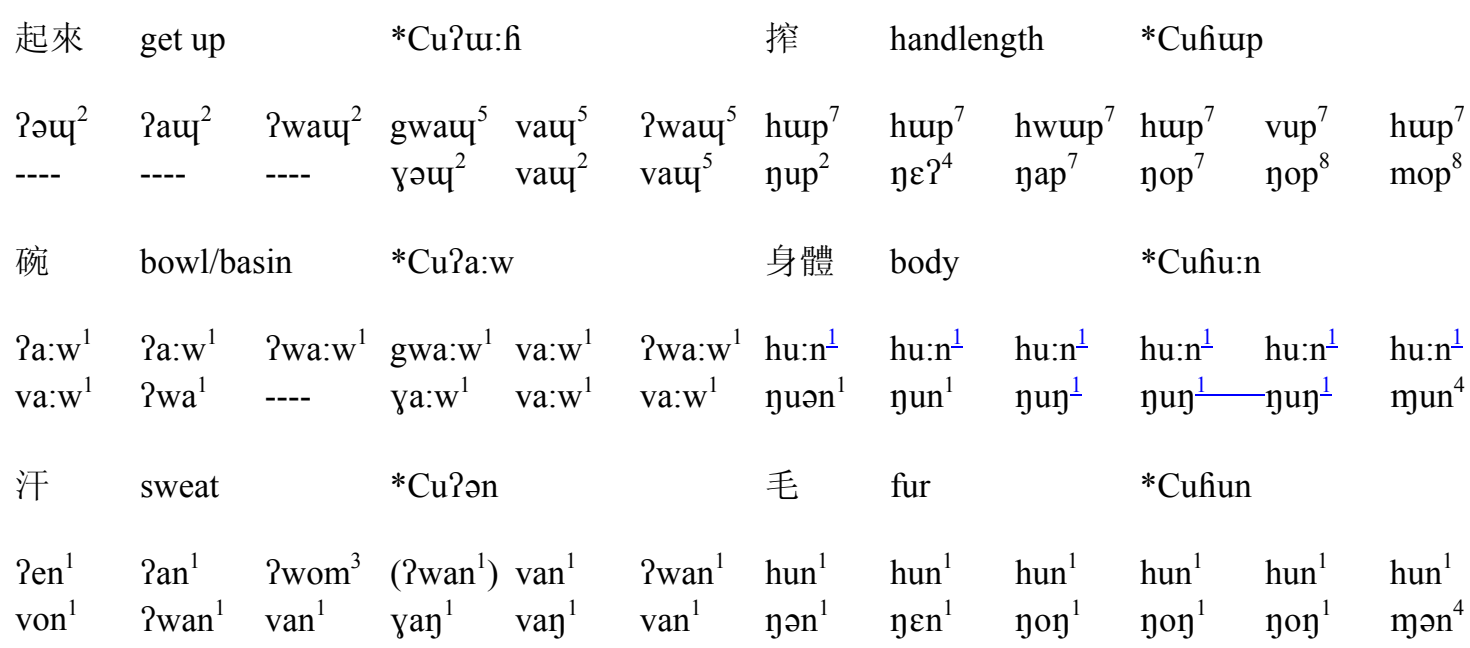

\subsubsection{Interim Summary}

There are two kinds of glottal initials which have been reconstructed in this section: stops and fricatives. These are both reconstructible with two kinds of presyllables, shown below:

(112) $\begin{array}{ll}? & \mathrm{f} \\ \mathrm{Ci} ? & \mathrm{Cif} \\ \mathrm{Cu} ? & \mathrm{Cuf}\end{array}$

Unlike the other classes of initials discusses above, the glottals are perfectly symmetrical, each existing in one of three configurations: plain, preceded by ${ }^{*} \mathrm{Ci}$, and preceded by ${ }^{*} \mathrm{Cu}$.

\subsection{Conclusion}

This chapter has presented arguments for the reconstruction of the various manner classes of $\mathrm{PHl}$ initials. It was proposed in the beginning of this chapter that the three primary classes into which most sound changes could be grouped were temporal compression, onset fortition, and systemic realignment. Temporal compression is a process which seems to have been occurring steadily in Hlai for a very long 
time, beginning at the Pre-Hlai stage (see chapter four). The outcome of temporal compression is normally the reduction of complex onsets to a single segment. This may occur through either the deletion of one part of a complex initial (by which is meant a cluster, a preglottalized or preaspirated consonant, or a consonant with a secondary articulation), or otherwise the coalescence of the features of two parts of a complex initial into a single segment. Onset fortition seems to have operated as a general mechanism to reduce sonority and increase stricture at the left edge of words, thereby increasing their general acoustic saliency. Finally, systemic realignment was a category preserving change, and either led to the merger of one category with another pre-existing category or to the repopulation of a previously vacated category.

Two interrelated kinds of sound change were also highlighted, which had an especially large effect on the initial inventories of the Hlai languages. These were the ongoing process of initial obstruent devoicing, and registrogenesis, which was probably a sound change which diffused into the Hlai-speaking area through contact with Hainanese, but which did not affect all Hlai languages. Registrogenesis did, however, record the voicing status of initials before the most recent initial obstruent devoicing and other relevant changes (such as the loss of glottal stop and preaspiration before sonorants) in the languages in which it did occur.

The PHl inventory of initials is given below:

(113) PHI Initial Inventory

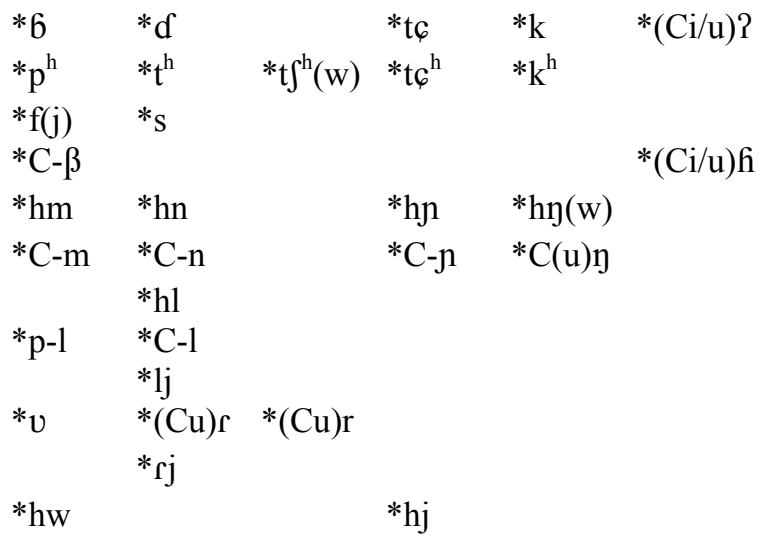


In terms of place, this inventory is rather normal typologically, with initial series represented at the bilabial, alveolar, postalveolar, palatal, velar, and glottal places of articulation. There are several gaps in the postalveolar series, and the fricative inventory is also skewed, being represented at the anterior places of articulation but not the posterior ones (with the exception of the glottal fricative) as discussed in section 2.3.1.4. The fact that the voiced glottal fricative *f does not co-occur with a voiceless counterpart *h is very normal (Maddieson 1984: 57).

There are five coarticulated initials reconstructed for PHl. The two coarticulated obstruents include $* t \mathrm{f}^{\mathrm{h}}$, which has a counterpart $* \int^{\mathrm{h}} \mathrm{w}$, and $* \mathrm{f}$, which had a counterpart $* \mathrm{fj}$. Neither $* \int^{\mathrm{h}} \mathrm{w}$ or $* \mathrm{fj}$ were very common, with only six examples of the former and one (and possibly two more, based on the Jiamao evidence given in chapter five) of the latter. Amongst the sonorants, *hy had a counterpart *hyw, and the tap $*^{\mathrm{r}}$ had a palatalized counterpart, ${ }^{*} \mathrm{rj}$. There was also a palatalized lateral $* 1 \mathrm{j}$, although it was not strictly the counterpart of $* \mathrm{hl}$ since there is no evidence that it was preaspirated. The only one of these initials which is clearly rare in a typological sense is $* t \int^{h} \mathrm{w}$.

There is also evidence for a set of sesquisyllabic forms with presyllables in various parts of the initial inventory. Besides the most conspicuous case of * $\mathrm{p}-1$, the evidence for these is often found in the Central Hlai languages, where vocalic transfer from high vowels became coarticulations on sonorants and glottal segments. Additional evidence is found in the nasal and lateral series, where the high register of most registrogenetic languages indicates the presence of a former syllable which eventually conditioned glottal constriction on the sonorant itself.

In terms of manner, strictly speaking, the PHl inventory is unremarkable. There is an opposition between aspirated and non-aspirated obstruents, a contrast in voice onset time which Maddieson (1984: 39) describes as the most common distinction if there are two series of obstruents (with a third series normally being 'glottalic', i.e. either ejective or implosive - in PHl, the implosive and plain series are in complimentary distribution as noted in section 2.3.1.4). However, one very striking aspect of the inventory is that aspiration extends to the sonorant series. That is, the glottis is only rarely in a neutral state, being spread in most cases. It is not the abundance in glottal specification that makes the $\mathrm{PHl}$ initial inventory 
seem strange per se; note the initial inventory of modern Sui, another Kra-Dai language of the Kam-Sui branch, which exhibits a system which seems quite similar to the Hlai inventory:

(114) Sui initial inventory (Edmondson, et al. (2001))

\begin{tabular}{|c|c|c|c|c|c|}
\hline $\mathrm{p}$ & $\mathrm{t}$ & ts & t6 & $\mathrm{k}$ & $\mathrm{q}$ \\
\hline $\mathrm{p}^{\mathrm{h}}$ & $\mathrm{t}^{\mathrm{h}}$ & $\mathrm{ts}^{\mathrm{h}}$ & $\mathrm{t} \varphi^{\mathrm{h}}$ & $\mathrm{k}^{\mathrm{h}}$ & $q^{h}$ \\
\hline $\mathrm{mb}$ & nd & & & $\mathrm{g}$ & $\mathrm{G}$ \\
\hline Pb & ?d & & & ?g & \\
\hline $\mathrm{m}$ & $\mathrm{n}$ & & $\mathrm{n}$ & $y$ & \\
\hline $\mathrm{hm}$ & $\mathrm{hn}$ & & hn & $\mathrm{h} \eta$ & \\
\hline ?m & ?n & & pn & $? \eta$ & \\
\hline $\mathrm{f}$ & & $\mathrm{S}$ & 6 & $\mathrm{x}$ & \\
\hline $\mathrm{V}$ & 1 & $\mathrm{z}$ & $\mathrm{j}$ & $\gamma$ & Б \\
\hline ?w & & & $\mathrm{Pj}$ & ?y & \\
\hline
\end{tabular}

The one crucial difference is that in Sui, there are neutral segments which contrast with the ones specified for either spread or constricted glottis; for example, Sui has both preaspirated and preglottalized nasals, but it also has a plain series. Proto-Hlai, on the other hand, lacks plain initials in the case of the nasals, lateral, and glides. The historical events in Pre-Hlai which led to this situation in PHl are examined in some detail in chapter four, section 4.3.

The reconstruction presented here has been compared with the reconstructions of Matisoff (1988), Thurgood (1994), Peiros (1998), and Ostapirat (2004); the differences between the present reconstruction and these alternative reconstructions have been discussed, and an argument presented for the former when it differs from the latter. The three reconstructions are provided in (116) below for reference, so that the similarities and differences between them may be easily compared (category labels are taken from the present reconstruction).

(115) Summary of Reconstructed Systems

(a) Aspirated Stops

$\begin{array}{lllll}\text { Matisoff } & \text { Thurgood } & \text { Peiros } & \text { Ostapirat } & \text { PHl } \\ * \mathrm{p}^{\mathrm{h}} & * \mathrm{p}^{\mathrm{h}} & * \mathrm{p}^{\mathrm{h}} & *(9) \mathrm{p} & * \mathrm{p}^{\mathrm{h}} \\ * \mathrm{t}^{\mathrm{h}} & * \mathrm{t}^{\mathrm{h}} & * \mathrm{t}^{\mathrm{h}} & *(9) \mathrm{t} & * \mathrm{t}^{\mathrm{h}} \\ * \mathrm{k}^{\mathrm{h}} & * \mathrm{k}^{\mathrm{h}} & * \mathrm{k}^{\mathrm{h}} & * \mathrm{k} & * \mathrm{k}^{\mathrm{h}}\end{array}$


(b) Affricates

\begin{tabular}{|c|c|c|c|}
\hline Matisoff & Thurgood & $\underline{\text { Peiros }}$ & $\underline{\text { Ostapirat }}$ \\
\hline -- & --- & --- & $*_{-} \mathrm{t}-$ \\
\hline$*_{\mathrm{sr}}$ & $*_{\mathrm{sr}}$ & $*_{\mathrm{sw}}$ & *-ut- \\
\hline$* \mathrm{ts}^{\mathrm{h}}$ & $* \mathrm{ts}^{\mathrm{h}}$ & $* \mathrm{c}^{\mathrm{h}}$ & $*_{\mathrm{c}}$ \\
\hline
\end{tabular}

(c) Fricatives

\begin{tabular}{|c|c|c|c|c|}
\hline Matisoff & Thurgood & $\underline{\text { Peiros }}$ & Ostapirat & PHl \\
\hline$*_{\mathrm{f}}$ & $* p$ & $*_{\mathrm{f}}$ & $*_{-}$- - & *f \\
\hline --- & --- & --- & *-ip- & $*_{\mathrm{fj}}$ \\
\hline$*_{\mathrm{fr}}$ & $* \mathrm{pr}$ & *hw & *-up- & ${ }^{*} \mathrm{C}-\beta$ \\
\hline$*_{\mathrm{s}}$ & $*_{\mathrm{S}}$ & $*_{\mathrm{s}}$ & $*_{\mathrm{s}}$ & $*_{\mathrm{S}}$ \\
\hline
\end{tabular}

(d) Implosives and Plain Stop and Affricate

\begin{tabular}{|c|c|c|c|}
\hline Matisoff & Thurgood & $\underline{\text { Peiros }}$ & Ostapirat \\
\hline$* 6$ & $* 6$ & $* ? b$ & $*(?) \mathrm{b}$ \\
\hline$*_{d}$ & $*_{d}$ & $* ? d$ & *(P)d \\
\hline$*_{\text {ts }}$ & $*$ ts & $*_{\mathrm{c}}$ & $*_{f}$ \\
\hline$*_{\mathrm{k}}$ & $*_{\mathrm{k}}$ & ${ }^{*} \mathrm{k}$ & $* g$ \\
\hline
\end{tabular}

(e) Preaspirated Sonorants

\begin{tabular}{|c|c|c|c|}
\hline Matisoff & Thurgood & $\underline{\text { Peiros }}$ & Ostapirat \\
\hline$*^{*} \mathrm{~b}$ & *m? & ${ }^{*} \mathrm{~m}$ & $*_{-\mathrm{m}}$ \\
\hline *nd & $*_{\mathrm{n}}$ ? & $* \mathrm{n}$ & $*$-n- \\
\hline$*_{\mathrm{ndz}}$ & --- & $* \mathrm{n}$ & $*-\mathrm{n}-$ \\
\hline *ng & $* \eta ?$ & $* \eta$ & $*-\eta$ \\
\hline --- & --- & --- & --- \\
\hline
\end{tabular}

(f) Medial Nasals

\begin{tabular}{|c|c|c|c|c|}
\hline Matisoff & Thurgood & $\underline{\text { Peiros }}$ & Ostapirat & $\underline{\mathrm{PHl}}$ \\
\hline$*_{m}$ & $*_{\mathrm{m}}$ & ${ }^{*} \mathrm{C}-\mathrm{m}$ & $*^{m}$ & ${ }^{*} \mathrm{C}-\mathrm{m}$ \\
\hline$* \mathrm{n}$ & $*_{\mathrm{n}}$ & ${ }^{*} \mathrm{C}-\mathrm{n}$ & $* \mathrm{n}$ & ${ }^{*} \mathrm{C}-\mathrm{n}$ \\
\hline$* \mathrm{n}$ & $* \mathrm{n}$ & ${ }^{*} \mathrm{C}-\mathrm{n}$ & $*_{\mathrm{n}}$ & ${ }^{*} \mathrm{C}-\mathrm{n}$ \\
\hline$* y$ & $* \eta$ & $* \mathrm{C}-\mathrm{n}$ & $* \eta$ & $* \mathrm{C}-\mathrm{n}$ \\
\hline *yw & *yw & $* \mathrm{C}-\mathrm{nw}$ & *yw & ${ }^{*} \mathrm{Cun}$ \\
\hline
\end{tabular}


(g) Laterals

\begin{tabular}{|c|c|c|c|}
\hline Matisoff & Thurgood & $\underline{\text { Peiros }}$ & $\underline{\text { Ostapirat }}$ \\
\hline$* 4$ & $* \notin ?$ & $* \mathrm{hl}$ & $*_{-1-}$ \\
\hline$* 1$ & $* 1$ & $*_{\mathrm{C}-1}$ & $* 1$ \\
\hline$* \mathrm{pl}$ & $* \mathrm{pl}$ & $*_{\mathrm{pl}}$ & $*_{\mathrm{p}-1}$ \\
\hline$*_{\mathrm{z}}$ & $* 1 \mathrm{j} ?$ & $*_{\mathrm{Z}}$ & $*_{-11-}$ \\
\hline
\end{tabular}

(h) Approximants

\begin{tabular}{|c|c|c|c|c|}
\hline$\underline{\text { Matisoff }}$ & Thurgood & $\underline{\text { Peiros }}$ & $\underline{\text { Ostapirat }}$ & $\underline{\mathrm{PHl}}$ \\
\hline$*_{\mathrm{V}}$ & $*_{\mathrm{w}} \mathrm{n}$ & $*_{\mathrm{V}}$ & $*-v-$ & $*_{v}$ \\
\hline$* \mathrm{r}$ & $*$ r? & $*_{r}$ & $*_{-r}-$ & $*_{r}$ \\
\hline --- & --- & --- & $*_{\text {-ir- }}$ & $*_{r j}$ \\
\hline$*_{\mathrm{vr}}$ & *pr? & $*_{\mathrm{ZW}}$ & $*$-ur- & *Cur \\
\hline$*^{*} / * y$ & ${ }^{*} \xi^{2} / * y$ & $*_{\mathrm{rw}} /{ }^{*} \mathrm{\gamma}$ & $*_{-\mathrm{R}-} / *_{-\mathrm{g}-}$ & $* \mathrm{r}$ \\
\hline${ }^{*} \mathrm{\gamma w}$ & ${ }^{*} \mathrm{\gamma w}$ & ${ }^{*} \mathrm{\gamma w}$ & *-uR-/*-ug- & *Cur \\
\hline
\end{tabular}

(i) Glides

\begin{tabular}{|c|c|c|c|c|}
\hline Matisoff & Thurgood & Peiros & Ostapirat & $\underline{\mathrm{PHl}}$ \\
\hline$*_{\mathrm{j}}$ & $*_{\mathrm{j}}$ & $*_{\mathrm{j}}$ & $*_{-\mathrm{j}-}$ & *hj \\
\hline$*_{\mathrm{W}}$ & $*_{\mathrm{W}}$ & $*_{\mathrm{W}}$ & $*_{-\mathrm{W}}$ & *hw \\
\hline
\end{tabular}

(j) Glottals

\begin{tabular}{|c|c|c|c|c|}
\hline Matisoff & Thurgood & $\underline{\text { Peiros }}$ & Ostapirat & $\underline{\mathrm{PHl}}$ \\
\hline$* ?$ & $* ?$ & $* ?$ & $* ?$ & $* ?$ \\
\hline$* x j$ & --- & $* ? \mathrm{j}$ & *-i?- & *Ci? \\
\hline$*_{\mathrm{XW}}$ & ${ }^{*} \mathrm{xW}$ & $* \mathrm{PW}_{\mathrm{w}}$ & *-u?- & ${ }^{*} \mathrm{Cu}$ ? \\
\hline$*_{\mathrm{x}}$ & $*_{\mathrm{x}}$ & $* \mathrm{~h}$ & $*_{-\mathrm{k}-}$ & $* h$ \\
\hline$*_{h j}$ & *hр? & $* \mathrm{hj}$ & $*_{-i k-}$ & *Cih \\
\hline *hw & *hyw? & *hyw & *-uk- & * Cuf \\
\hline
\end{tabular}

The first advantage which the present reconstruction has over those of Matisoff, Thurgood, Peiros, and in one instance Ostapirat, is the addition of several phonemes to the PHl inventory. Some of these were absent from their reconstructions because of lack of data - Changjiang in the case of Thurgood and Peiros, and Changjiang as well as Cunhua and Nadouhua in the case of Matisoff. Others were apparently overlooked. 
Other specific systemic problems can be discussed more easily when the initial inventories are organized and inspected. In doing so, I do my best to arrange the PHl phonemes in such a way as best represents the respective author's understanding of the overall system, and take full responsibility for any misanalysis. Matisoff's PHl initial inventory is shown below:

(116) Matisoff's PHl inventory

\begin{tabular}{|c|c|c|c|}
\hline$* 6$ & $*_{d}$ & $*_{\text {ts }}$ & ${ }^{*} \mathrm{k}$ \\
\hline${ }^{*} \mathrm{p}^{\mathrm{h}}$ & $* t^{h}$ & $*_{\mathrm{ts}}^{\mathrm{h}}$ & $* \mathrm{k}^{\mathrm{h}}$ \\
\hline${ }^{*} \mathrm{f}(\mathrm{r})$ & $* 1$ & $*_{s}(\mathrm{r})$ & ${ }^{*} \mathrm{x}(\mathrm{j} / \mathrm{w})$ \\
\hline$*_{v}(\mathrm{r})$ & ${ }^{*} 3$ & $*_{\mathrm{z}}$ & ${ }^{*} \mathrm{y}(\mathrm{w})$ \\
\hline *mb & *nd & *ndz & *yg \\
\hline *m & $* \mathrm{n}$ & $* \mathrm{n}$ & $* \eta(w)$ \\
\hline *hw & & *hj & \\
\hline${ }^{*} \mathrm{w}$ & $* 1$ & $*_{\mathrm{j}}$ & \\
\hline & $\begin{array}{l}{ }^{*} \mathrm{pl} \\
{ }^{*} \mathrm{r}\end{array}$ & & \\
\hline
\end{tabular}

Matisoff reconstructs an inventory with four main places of articulation (presuming an alignment between the sibilant and palatal initials), plus a glottal stop. It is generally symmetrical, with the following exceptions. The first is that there is a group of ${ }^{*} \mathrm{C}-\mathrm{r}$ clusters which are all fricative-initial $\left({ }^{*} \mathrm{fr},{ }^{*} \mathrm{sr}\right.$, and $\left.*_{\mathrm{vr}}\right)$; it is strange that there are no ${ }^{*} \mathrm{C}-\mathrm{r}$ clusers which begin with stops. As in my own system, labiovelar coarticulations are optional throughout much of the velar series; however, there is only a single palatal coarticulation $\left({ }^{*} \mathrm{xj}\right)$, which seems strange. Finally, it is noticeable that the plain stop $p$ occurs in the cluster *pl, but there is no plain counterpart *p. 
(117) Thurgood's PHl inventory

\begin{tabular}{|c|c|c|c|c|}
\hline${ }^{*} \mathrm{p}(1 / \mathrm{r})$ & & $*$ ts & *k & \\
\hline *pr? & & & & \\
\hline$* p^{h}$ & $* \mathrm{t}^{\mathrm{h}}$ & $* \mathrm{ts}^{\mathrm{h}}$ & $* \mathrm{k}^{\mathrm{h}}$ & \\
\hline & & $*_{s}(\mathrm{r})$ & $*_{x}(\mathrm{w})$ & \\
\hline & & & ${ }^{*} \mathrm{y}(\mathrm{w})$ & \\
\hline *m & $*_{\mathrm{n}}$ & $* \mathrm{n}$ & $*^{n}(\mathrm{w})$ & \\
\hline$*_{m}$ ? & $*_{n}$ ? & *hn? & $*_{y}$ ? & *hyw? \\
\hline & *ł? & & & \\
\hline & ${ }^{*} 3$ ? & & & \\
\hline & *lj? & & & \\
\hline & $*_{\mathrm{r}} \mathrm{P}$ & & & \\
\hline$*_{\mathrm{W}}{ }^{*}$. & $* 1$ & $*_{\mathrm{j}}$ & & \\
\hline
\end{tabular}

Thurgood also reconstructs an inventory with four main places of articulation plus glottal stop.

The first noticeable gap in this system is in the plain stops, where there is a conspicuous lack of a plain alveolar stop. There is a very pronounced asymmetry in the set of liquid clusters - with the sole exception of the typologically rare *sr, all liquid clusters begin with *p. Finally, there are a set of post-laryngealized initials which Thurgood represents with a final glottal stop, meant to indicate that the initial caused laryngealization across the entire syllable. Although I do not reconstruct post-laryngealization, these initials (with the exception of * 1 ) would all form a natural class of voiced sonorants in the present system; in Thurgood's system, the post-laryngealized initials do not seem to form a natural class, and the feature must therefore be stipulated. Complicating the situation, in the series of post-laryngealized nasals, there is a curious asymmetry between those with preaspiration (palatal and labiovelar) and those without it, which also seems to have no explanation. 
(118) Peiros's PHl inventory

\begin{tabular}{|c|c|c|c|c|c|}
\hline *6 & ${ }^{*} d$ & $*_{\mathrm{c}}$ & $*_{\mathrm{k}}$ & & $* \mathrm{P}(\mathrm{j} / \mathrm{w})$ \\
\hline${ }^{*} p^{h}$ & $* \mathrm{t}^{\mathrm{h}}$ & $* c^{\mathrm{h}}$ & $* \mathrm{k}^{\mathrm{h}}$ & & \\
\hline \multirow[t]{2}{*}{$*_{m}$} & $*_{n}$ & $* \mathrm{n}$ & $* \eta$ & & \\
\hline & & *hn & & *hnw & \\
\hline \multirow[t]{2}{*}{${ }^{*} \mathrm{C}-\mathrm{m}$} & ${ }^{*} \mathrm{C}-\mathrm{n}$ & $* \mathrm{C}-\mathrm{n}$ & ${ }^{*} \mathrm{C}-\mathrm{y}$ & ${ }^{*} \mathrm{C}-\mathrm{\eta w}$ & \\
\hline & ${ }^{*} \mathrm{C}-1$ & & & & \\
\hline * & $* 4$ & $*_{\mathrm{s}}(\mathrm{w})$ & & & ${ }^{*} \mathrm{~h}(\mathrm{w})$ \\
\hline & & ${ }^{*} \mathrm{z}(\mathrm{w})$ & ${ }^{*} \mathrm{y}(\mathrm{w})$ & & \\
\hline & $*_{\mathrm{r}}(\mathrm{w})$ & $* j$ & & & \\
\hline
\end{tabular}

Peiros reconstructs a system with five places of articulation including the glottal segments. It is generally symmetrical, and the primary strength of Peiros' system is the recognition of the distinction between an initial and medial sonorant series. The main weakness is the strange reconstruction of two preaspirated nasals at marked places of articulation. There is also quite widespread optional labiovelar coarticulation, but only one instance of palatal coarticulation (on the glottal stop).

(119) Ostapirat's PHl inventory

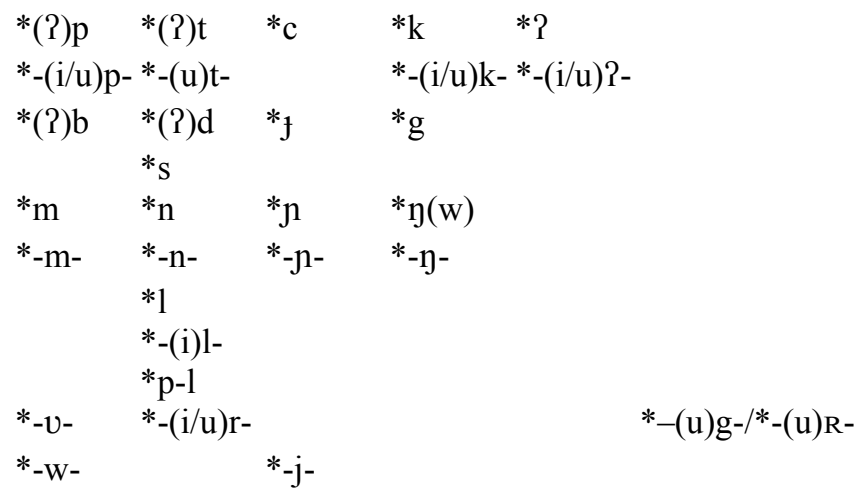

Ostapirat's system has several strengths. The primary one is the reconstruction of a distinction between initial and medial consonants. The latter are often reconstructed with preceding high vowels, which undergo vocalic transfer at stages subsequent to PHl. He also has a developed hypothesis of intervocalic affrication and fortition, for both obstruents and sonorants. The problem with this hypothesis is that the intervocalic changes which Ostapirat posits are often typologically odd, particularly in the case of fortition, where lenition is the norm intervocalically. The other significant weakness in my estimation is 
in the obstruent series, where Ostapirat posits optional preglottalized *?p, *?t, *?b, and *?d based on the Jiamao evidence. My primary reservation about this is due to the fact that I think the Jiamao variation does not reflect actual variation in the proto-language, but instead is due to layers of loanwords at different periods (see chapter five). Aside from this, while preglottalized voiced stops have been recorded in other Southeast Asian languages (see the Sui initial inventory above), preglottalized voiceless stops, to the best of my knowledge, are an anomaly.

In summary, the main advantages of the reconstruction proposed here are the addition of the postalveolar series, the absence of awkward *C-r clusters, the existence of presyllables before sonorants, some of which contained high vowels which conditioned vocalic transfer in Central Hlai, and an overall symmetry in both place and manner with few unexpected gaps.

The reconstructed inventory shown above in (113) was reconstructed, and some of the inconsistencies of the other systems of reconstruction avoided, through the use of the four principles outlined in chapter one: Directionality, Commonality, Economy, and Symmetry. Directionality is particularly important in constraining potential manner changes, as in the case of the fricatives. Commonality is important in maintaining the line between the reconstruction of a proto-language, and internal reconstruction of an earlier stage of that language. Economy is important in a similar way, in that it constrains reconstruction of initials so that they explain all, and only, the reflexes of the daughter languages, avoiding speculation about stages earlier than the proto-language. Finally, symmetry is an important constraint on the reconstructed inventory as a whole, suggesting gaps which are available to be filled, and otherwise highlighting asymmetries of the system which should be verified with typological data.

The purpose of the next chapter will be the reconstruction of the PHl system of rimes. The tone inventory will be treated first, after which the segmental portions of the rimes will be reconstructed and their subsequent paths of change outlined. 


\section{CHAPTER THREE: RECONSTRUCTION OF PROTO-HLAI RIMES}

The primary goal of this chapter is to present the sets of rime correspondences which have been used to reconstruct the $\mathrm{PHl}$ inventory of rimes, and explain reflexes of $\mathrm{PHl}$ rimes in the daughter languages if they have followed divergent paths. This will be done first for the PHl tone categories, and then for the segmental rimes. The reconstruction of the latter will also be compared with those of Thurgood (1994), Ostapirat (1993), and Ostapirat's revised (2004) reconstruction, and these alternative reconstructions will be considered and discussed. Although Peiros (1998) technically gives a reconstruction of the PHl rimes, he does not provide an overall system of rimes or give correspondence sets, so I am unfortunately unable to include his reconstruction in my comparison. The main objective of this chapter is to motivate the reconstruction of Proto-Hlai rimes proposed herein in a way that will allow further comparative work to be based on these results.

As with chapter two, the reconstruction in this chapter will be of Proto-Hlai, as opposed to PreHlai which will be reconstructed in the next chapter. The reconstruction of the Proto-Hlai system of rimes in this chapter will result in an inventory which is not as exotic as the PHI inventory of initials, but which nevertheless has its own idiosyncracies. The discussion of Pre-Hlai in the next chapter will demonstrate how this system originated in an earlier system which was ultimately simpler.

Before a discussion and reconstruction of specific natural classes of rimes is initiated, a further discussion of the currently adopted theory of sound change is necessary, accompanied by a discussion of how specific sound changes have interacted with each other. With this background, we will be in a position to properly examine the various classes of rimes themselves and the evolution of their individual members into their current forms in the daughter languages. 


\subsection{Sound Change: Rimes}

In the reconstruction of Proto-Hlai rimes undertaken in this chapter, the following criteria

described in chapter one are again adhered to:

(i) Directionality of change: typologically natural changes are referred to and used as a model whenever possible; changes are assumed to occur one feature at a time unless evidence forces a different analysis.

(ii) Commonality of features: phonemes are reconstructed based on the features common between reflexes of daughter languages; greater heterogeneity of reflexes is taken to indicate greater complexity of the proto-phoneme.

(iii) Economy: a phoneme is reconstructed to the extent that it satisfactorily accounts for the posited change(s) between it and the reflexes of the daughter languages, and reconstructions assuming more changes than necessary are avoided.

(iv) Symmetry: the reconstructed inventory is checked to make sure that no symmetries have been overlooked in natural classes, either in place or in manner; it is accepted that parts of the inventory may be asymmetrical, and these are checked for typological naturalness.

The rime is qualitatively different from the initial within the syllable, and the method of analysis must be modified accordingly; there are several ways in which vowels in particular undergo change which lack direct parallels with the initials. There is one parallel to be found with the initials, however, in the changes that involve final glottal segments. In the same way that glottal settings in the initial can affect pitch values (thereby controlling register), glottal settings in the coda can affect pitch values and trajectories. The way this occurs, as in the case of the initials, is through the medium of phonation. Thurgood (2002) provides a discussion of final laryngeals, and gives examples of laryngeals which either raise pitch or lower pitch at the end of a syllable. In the former case, abrupt glottal stop and nonbreathy $h$ are correlated with pitch raising or high pitch; in the latter case, creaky glottal stop and breathy $h$ are correlated with pitch lowering or low pitch. Kingston (2005) augments this with Athabaskan data, in which he argues that final glottal stop can condition either high or low pitch, depending on whether it is abrupt (conditioning tense voice on the preceding nucleus, leading to level high tone) or creaky (conditioning creaky voice, and depressing the tone). The changes just described which are applicable to the upcoming 
discussion of Hlai tonogenesis are shown below in (1a). Segmental changes posited here involving final glottal segments include the devoicing of final breathy $h$, the development of a creaky glottal stop from an abrupt glottal stop, and the loss of a glottal stop coarticulated with a final oral stop, all shown in (1b):

\begin{tabular}{|c|c|c|c|}
\hline \multirow[t]{4}{*}{ (a) } & *v? & $>$ & $\overline{\mathrm{v}} \mathrm{P}$ (high level) \\
\hline & $*_{\mathrm{vh}}$ & $>$ & $\overline{\mathrm{v}} \mathrm{h}$ (high level) \\
\hline & *vḥ & $>$ & vịn (falling) \\
\hline & *v? & $>$ & v? (falling) \\
\hline \multirow[t]{3}{*}{ (b) } & *vh & $>$ & vh \\
\hline & $*_{\mathrm{v}}$ ? & $>$ & $\mathrm{v} \stackrel{\sim}{?}$ \\
\hline & $*_{\mathrm{V}} \overparen{\mathrm{PC}}$ & $>$ & $\mathrm{vC}$ \\
\hline
\end{tabular}

The critical changes which have affected the rimes are (1) diphthongization, (2) peripheralization, (3) lengthening/shortening, (4) rounding/unrounding, (5) final weakening, and (6) systemic realignment (the last of which is actually the same as the change so-called in chapter two). These will each be explained and illustrated below.

\subsubsection{Diphthongization}

Diphthongization refers to any change which creates a salient sonority contour between a rime nucleus and coda, particularly if one does not exist at all prior to the change (2a). The most important constraint on this change is that the sonority of the nucleus must always be higher than that of the coda, and never vice versa, a favored syllable type which disfavors high nuclei. Diphthongization is one way to maximize this distinction, so that a syllable like key with a mid nucleus and high coda is preferable to ki:, with a high nucleus and no coda.

The most likely class of rimes to undergo this change is that of the pure open rimes, particularly the high ones which have a lower sonority profile in their nucleus, and the features of the coda which result from this change are always predicated upon the original features of the vowel. This is true also in the case 
of closed-syllable diphthongization, where high rimes lead initially to high-mid diphthongs (2b), and low rimes to high-low diphthongs (2c):

Examples of diphthongization

(a)

$\begin{array}{lll}\text { u: } & > \\ \text { o: } & >\end{array}$

(b)

i:n
u:k

(c)

$\begin{array}{lll}\varepsilon: y & > & \text { ian } \\ \text { ::y } & > & \text { uay }\end{array}$

$\underline{\text { Source }}$

Several

Cunhua

Qi

Zandui

Run

Yuanmen

The case in (2b) appears to be a case of feature delinking, where the distinctive features of the long vowel are maintained on the first half of the long vowel, but lost on the second half (3a). The case in (2c) appears to involve the splitting of the feature bundle, where the features [front], [back], and [round] are preserved on the first half of the long vowel, but the feature [low] is preserved on the second half (3b):

(a)

$\begin{array}{ccc}\mathrm{i}: \mathrm{y} & > & \text { iəy } \\ V & & \mid \neq \\ {[\mathrm{hi}]} & & {[\mathrm{hi}]} \\ \text { [front] } & & \text { [front] }\end{array}$

(b)

$\begin{array}{ccc}\varepsilon: n & > & \text { ian } \\ V & & / / \\ \text { [front] } & & \text { [front][low] } \\ \text { [low] } & & \end{array}$

\subsubsection{Peripheralization}

Peripheralization is the change in a vowel so that it moves from a more central position to a more peripheral position in the vowel space (peripheral here is used in the sense of Crothers (1978: 100), indicating vowels which are at the extremes of the acoustic vowel space). This can happen horizontally, as shown in the examples in (4a), or vertically, as in (4b): 
Examples of peripheralization

(a)

$\begin{array}{lll}\text { a:y } & > & \text { e:y } \\ \text { a:t } & > & \text { o:t } \\ & & \\ \text { e:w } & > & \text { i:w } \\ \text { ə:y } & > & \text { a:y }\end{array}$

Source

Several

Yuanmen

Yuanmen

Bouhin, Qi

It is not always clear why this change happens. It could be argued to maximize contrast, but it must then be recognized that this is not maximally functional, since vowel peripheralization results in the merger of formerly distinct categories at least as many times as it fills gaps in the rime inventory.

\subsubsection{Lengthening/Shortening}

The length of a rime can either be lengthened, as in (5a), or shortened, as in (5b):

$$
\text { Examples of lengthening and shortening } \quad \underline{\text { Source }}
$$

(a)

$\begin{array}{ll}\text { ew } & > \\ \text { ik } & >\end{array}$

(b)

$\begin{array}{lll}\mathrm{e}: \mathrm{w} & > & \text { ew } \\ \mathrm{a}: \mathrm{m} & > & \text { am }\end{array}$

Yuanmen

Tongzha, Zandui

Cunhua

Yuanmen

Lengthening may fill a gap in some instances, such as the example above involving Tongzha and Zandui where $* \mathrm{ik}$ lengthened to $i: k$ after original $* \mathrm{i}: \mathrm{k}$ had diphthongized to $i ə k$ and subsequently changed to ia? (also conforming to a general dispreference against short rimes with final velar stops). The same is true for shortening, as in the Yuanmen example above where long *a:m shortened to am. Since there was no originally short *am in the inventory, this filled a gap (although *əm eventually lowered, via vowel peripheralization, and merged with it). 


\subsubsection{Rounding/Unrounding}

An unrounded nucleus can be rounded, as in (6a), or a rounded nucleus unrounded (6b):

(6) Examples of rounding and unrounding $\quad \underline{\text { Source }}$

(a) $\quad$ əm $>$ om $\quad$ Lauhut

ə:y $\quad>\quad$ o:y $\quad$ Several

(b) un $>$ un Baoting

ot $>$ at $\quad$ Cunhua, Yuanmen

In the majority of cases, such as the Lauhut example above, rounding occurred in the environment of a labial (or occasionally velar) coda; conversely, unrounding primarily occurred in the environment of an alveolar or palatal coda. These can both be considered cases of assimilation. The one important exception is in the series of *ə:C rimes, where there was a backing and rounding of the nucleus in all Greater Hlai languages except Cunhua. This can be simultaneously considered a case of vowel peripheralization, where the backing is accompanied by automatic rounding.

\subsubsection{Final Weakening}

The complete deletion of codas occurs occasionally, although it is not common. There are two examples of glide deletion (7a) and one of stop deletion (7b). The dubuccalization of final stops is more common, with that of final $k(7 \mathrm{c})$ being particularly common, but debuccalization of all final stops occurring in one language $(7 d)$ : 


\begin{tabular}{|c|c|c|c|c|}
\hline \multicolumn{4}{|c|}{ Examples of coda deletion } & $\underline{\text { Source }}$ \\
\hline \multirow[t]{2}{*}{ (a) } & iw & $>$ & i: & Nadouhua \\
\hline & u:y & $>$ & u: & Yuanmen \\
\hline (b) & $\mathrm{a}: \mathrm{k}$ & $>$ & $\mathrm{w}:^{\mathrm{B}}$ & Moyfaw \\
\hline (c) & $\varepsilon: \mathrm{k}$ & $>$ & $\varepsilon: ?$ & Several \\
\hline (d) & $-\mathrm{p},-$ & & $-?$ & Nadouhua \\
\hline
\end{tabular}

\subsubsection{Systemic Realignment}

As in chapter two, systemic alignment occurs when there is categorical opportunity for change, either because an original member of the inventory has been vacated (8a), or otherwise because some member already exists, creating a stable category which may be a target for potential merger (8b):

$$
\text { Examples of systemic realignment } \quad \underline{\text { Source }}
$$

\begin{tabular}{|c|c|c|c|c|}
\hline \multirow[t]{2}{*}{ (a) } & $>$ & in & Baisha, Meifu & (original in $>$ en) \\
\hline & $>$ & $\mathrm{a}: y$ & Baisha, Meifu & (original a:y > $>$ :y) \\
\hline u:n & $>$ & u:n & Several & (u:n already in inventory) \\
\hline $\mathrm{a}: \mathrm{c}$ & $>$ & $\mathrm{a}: \mathrm{t}$ & Several & (a:t already in inventory) \\
\hline
\end{tabular}

It is important to reiterate that I do not consider these changes to be motivated by some 'hidden hand'; that is, a language will not anthropomorphically 'try' to fill a gap in an inventory in some directed way. Rather, it is the case that a certain amount of variation is always inherent within the speech community, and certain changes may be innovated and adopted as conventions depending on the potential amount of impedence generated by such functional considerations as maintaining category discreteness and so on. 


\subsection{Tonogenesis}

In traditional Kra-Dai historical linguistics (e.g. Gedney (1989), Edmondson \& Solnit (1988, 1997), it has been customary to represent the four tone categories by assigning the letters A-D, and to designate these categories as such in reconstructions, allowing the researcher to remain agnostic about the original values of the tone categories themselves. This is because the modern reflexes of these original values are always pitch contours carried on the nucleus of the syllable, occasionally accompanied by some sort of secondary phonation or constriction. Based on evidence in other Southeast Asian languages and language families, there is a speculative consensus which has developed that A and D were unmarked categories $^{24}$ (differentiated by the presence or absence of oral stop codas), and that the two marked tone categories B and C have arisen from marked glottal configurations in the rime of the syllable, via an intermediate stage of contrasting phonations. Although direct proof of this is often hard to come by, there has been some indirect evidence which suggests that words in category B were somehow associated with spread glottis and that words in category $\mathrm{C}$ were associated with constricted glottis.

This presents an interesting symmetry with registrogenesis, where the register split is also correlated with laryngeal distinctions. The similarities and differences are compared in the following chart (these are idealized simplifications for exposition -- for a full discussion see Thurgood (2002)):

Table 3: Comparison of Registrogenesis and Tonogenesis

\begin{tabular}{|c|c|c|c|c|}
\hline & \multicolumn{2}{|c|}{ Registrogenesis } & \multicolumn{2}{|c|}{ Tonogenesis } \\
\hline Syllable edge of origin & \multicolumn{2}{|c|}{ Left (initial) } & \multicolumn{2}{|c|}{ Right (coda) } \\
\hline General domain & \multicolumn{2}{|c|}{ Across rime } & \multicolumn{2}{|c|}{ Right edge of rime } \\
\hline Correlated with & \multicolumn{2}{|c|}{ Voicing } & \multicolumn{2}{|c|}{ Glottal stricture } \\
\hline Laryngeal opposition & Voiceless & Voiced & Constricted & Spread \\
\hline Effect on pitch & High & Low & High & Low \\
\hline Resulting in & \multicolumn{2}{|c|}{ High vs low pitch range } & \multicolumn{2}{|c|}{ Rising vs falling contour } \\
\hline
\end{tabular}

The modern Hlai languages do not preserve any segmental reflexes or phonation contrasts which could directly serve in the reconstruction of original laryngeal segments such as the ones described above,

\footnotetext{
${ }^{24}$ It will be shown below that the situation in category D is more complex.
} 
although their pitch values are suggestive. Based on this evidence alone, there is no compelling reason to reconstruct anything more than the traditional tone categories $\mathrm{B}$ and $\mathrm{C}$. However, there are split reflexes in the rimes of Greater Hlai, the Qi and NCHl branches, and Cunhua, which are correlated with tone category, and which provide rather direct evidence that final glottal segments need to be reconstructed for PHl. This is because there is little evidence that mere pitch differences themselves could condition segmental changes of the kind discussed shortly (apparent exceptions to this, such as Shuijingping Hmong (Mortensen 2006) and Fuzhouhua (Myers \& Tsay 2003), have alternative explanations, as shown by Mortensen (ibid)); however, these changes can be explained in a straightforward way if final glottal constituents of codas are assumed.

It might be suggested that these changes occurred during a stage of Hlai during which only phonation contrasts remained as evidence of former final laryngeals (as opposed to their simultaneous occurrence with them). The problem with this suggestion is that words in categories $\mathrm{B}$ and $\mathrm{C}$ pattern together segmentally in the evolution of rimes (at different times and in different subgroups and languages), and pattern against category A. If a phonation contrast was responsible, it is likely that the different phonations of categories $\mathrm{B}$ and $\mathrm{C}$ would affect rime nuclei differently, which is definitely not the case. An alternative hypothesis, which would group B and C together in contrast to A, is that original laryngeal segments existed in Proto-Hlai, and that these segments only conditioned pitch contrasts (and segmental changes) after the breakup into daughter languages.

One problem which this hypothesis might encounter is the lack of a typological example, where in some language these laryngeal segments exist as part of the rime and do not have to be inferred. It does seem to be the case that if the general tonogenetic mechanism under discussion here is valid, there must have been many such systems which existed historically in East and Southeast Asia, but which have since evolved into other types of systems (principally tone systems, although this is simplifying) and are no longer extant. One example which does exist, however, is Chepang, a Tibeto-Burman language of southcentral Nepal discussed in Weidert (1987: 8-9), which has the following system of syllable rimes: 


\begin{tabular}{|c|c|c|c|c|c|c|c|c|}
\hline (a) & $\mathrm{V}$ & $\mathrm{Vm}$ & $\mathrm{Vn}$ & Vin & $\mathrm{Vy}$ & Vl & $\mathrm{Vr}$ & Vy \\
\hline (b) & $V^{\mathrm{f}}$ & $\mathrm{Vm}^{\mathrm{f}}$ & $\mathrm{Vn}^{\mathrm{h}}$ & --- & $V y^{\mathrm{f}}$ & $\mathrm{Vl}^{\mathrm{fi}}$ & $\mathrm{Vr}^{\mathrm{fi}}$ & $V y^{h}$ \\
\hline (c) & $\mathrm{V}^{?}$ & $\mathrm{Vm}^{?}$ & $\mathrm{Vn}^{?}$ & $\operatorname{Vin}^{?}$ & $\mathrm{Vy}^{?}$ & $\mathrm{Vl}^{?}$ & $\mathrm{Vr}^{?}$ & $\mathrm{Vy}^{2}$ \\
\hline (d) & Vs & $\mathrm{Vp}$ & $\mathrm{Vt}$ & Vik & $\mathrm{Vk}$ & & & \\
\hline
\end{tabular}

This system mirrors the structure of the Hlai (and ultimately Kra-Dai) tone categories in that the first series (10a) and the fourth series (10d) could be considered laryngeally unmarked, whereas the second and third series (10b-c) are laryngeally marked categories, corresponding to spread and constricted glottis, respectively. Also, Weidert says of the second series that ' $[\mathrm{T}]$ he rhymes with breathy phonation $\left[{ }^{-\mathrm{f}}\right]$ quickly fade into voicelessness', indicating that voicing is not sustained as long as spread glottis in this environment. Recognizing the potential variation in the phonetic realization of syllable-final laryngeal gestures, Chepang can thus be taken as a general model of rime structure for present purposes.

The first set of evidence for final laryngeal segments within Hlai is found in the Greater Hlai branch. High vowels which were otherwise open underwent two distinct paths of development, depending on whether or not they were closed by laryngeal segments. Those which were closed by laryngeals diphthongized, in contrast to those which weren't which remained pure (although they diphthongized at a later point in time):

$\underline{\mathrm{PHl}} \quad \underline{\mathrm{GH}}$

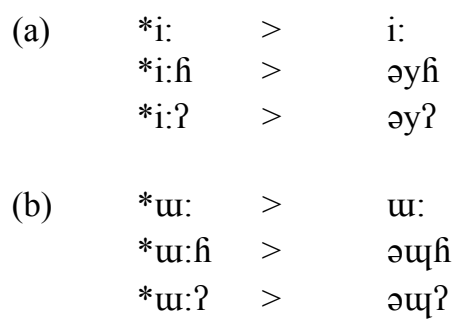

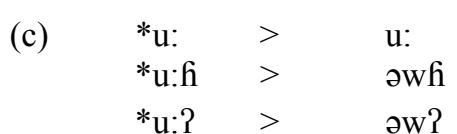


The original *u: rimes in categories B and C became further differentiated in Cunhua (12a), Zandui (12b), and Baoting (12c), presumably conditioned by these laryngeal segments or their successors. More specifically, it is entirely possible that during the process of tonogenesis, the final laryngeal $-h$ (category B) conditioned breathy phonation back into the rime, (possibly mirrored by $-?$ (category C) conditioning creaky voice in Cunhua in the same environment), which in turn affected the perception of the rimes and subsequent reanalysis (a phenomenon very common in e.g. Mon-Khmer). The following paths of change are inferred, and are intended to be the most parsimonious explanation for the derivation of these disparate reflexes:

\begin{tabular}{|c|c|c|c|c|c|c|c|}
\hline \multirow[t]{3}{*}{ (a) } & *u: & $>$ & u: & $>$ & u: & $>$ & ow \\
\hline & *u:h & $>$ & วwh & $>$ & əֶwh & $>$ & $a:^{\mathrm{B}}>\mathrm{a}:^{\mathrm{B}}$ \\
\hline & $* u: ?$ & $>$ & วw? & $>$ & әщ? & $>$ & ay $^{\mathrm{C}}$ \\
\hline \multirow[t]{3}{*}{ (b) } & *u: & $>$ & u: & $>$ & u: & $>$ & ow \\
\hline & *u:h & $>$ & owh & $>$ & อ़ & $>$ & o: ${ }^{\mathrm{B}}$ \\
\hline & *u:? & $>$ & วw? & $>$ & әw? & $>$ & $a w^{\mathrm{C}}$ \\
\hline \multirow[t]{3}{*}{ (c) } & $* \mathrm{u}:$ & $>$ & $\mathrm{u}:$ & $>$ & $\mathrm{u}:$ & $>$ & ow \\
\hline & *u:h & $>$ & owf & $>$ & כִwh & $>$ & $0:^{\mathrm{B}}>0:^{\mathrm{B}}$ \\
\hline & $*_{u}: ?$ & $>$ & วw? & $>$ & วw? & $>$ & $\mathrm{aw}^{\mathrm{C}} / \mathrm{ow}$ \\
\hline
\end{tabular}

The PHl diphthong * 2 w underwent monophthongization in the Qi and $\mathrm{NCHl}$ branches (possibly excluding Cunhua) if it was closed by a final laryngeal, but remained a diphthong if it was open:

$\begin{array}{lll}\text { *əw } & > & \text { *ow } \\ \text { *əwh } & > & \text { *o:h } \\ \text { *əw? } & > & \text { *o:? }^{2}\end{array}$

Cunhua also shows variation its reflexes of PHl *ən, between on in category A (14a) and an in categories B/C (14b): 
Examples of split Cunhua reflexes of $\mathrm{PH} *$ on by tone category

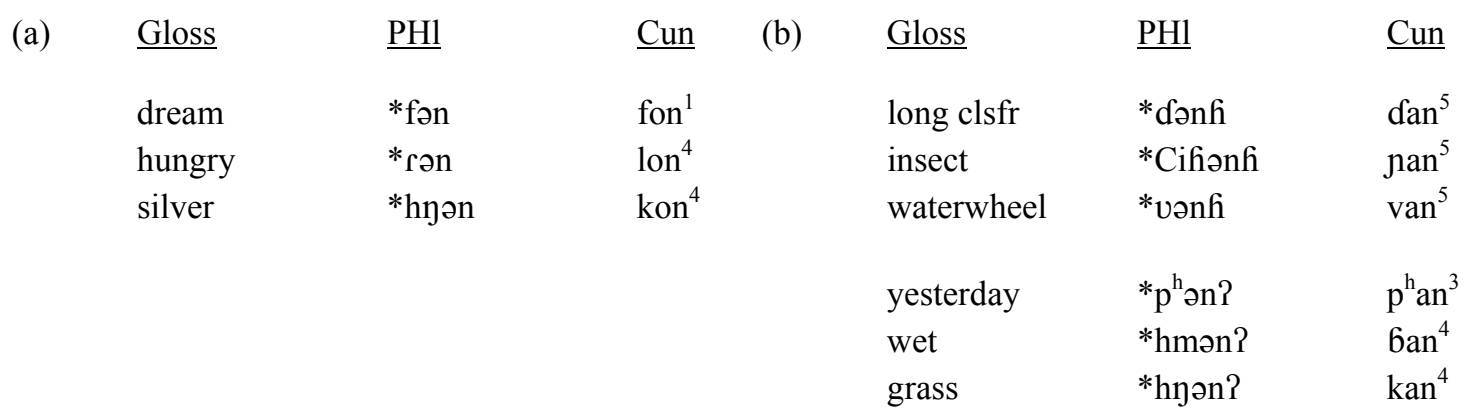

In other words, lowering of the vowel occurred when the coda included a laryngeal segment; otherwise, rounding occurred instead.

In the GHl, Qi/Run, and Cunhua examples shown above, it is preferable to hypothesize that conditioning environments were created via the presence/absence of a final glottal segment, not merely by pitch contour. This is the primary reason that I reconstruct final glottal segments, which only later develop into pitch contours.

The second reason for doing so is the lack of uniformity in the reflexes of the four tonal categories amongst the modern daughter languages. If Hlai tone categories consisted merely of pitch contour alone at the PHl stage, then a certain degree of similarity might be expected amongst daughter languages. This is, however, not the case. The reflexes of the modern languages are given below, with the standard East Asian pitch system used where the lowest pitch is 1 and the highest is $5^{25}$ :

\footnotetext{
${ }^{25}$ Using the pitch values 1, 3, and 5, examples of pitch contours in this system are as follows: $11 \mathrm{~J}$ (low level), $33 \dashv$ (mid level), 557 (high level), $13 \lambda$ (low rising), $35 \neg$ (high rising), $31 \mathrm{~V}$ (low falling) $53 \mathrm{y}$ (high falling), and so on.
} 


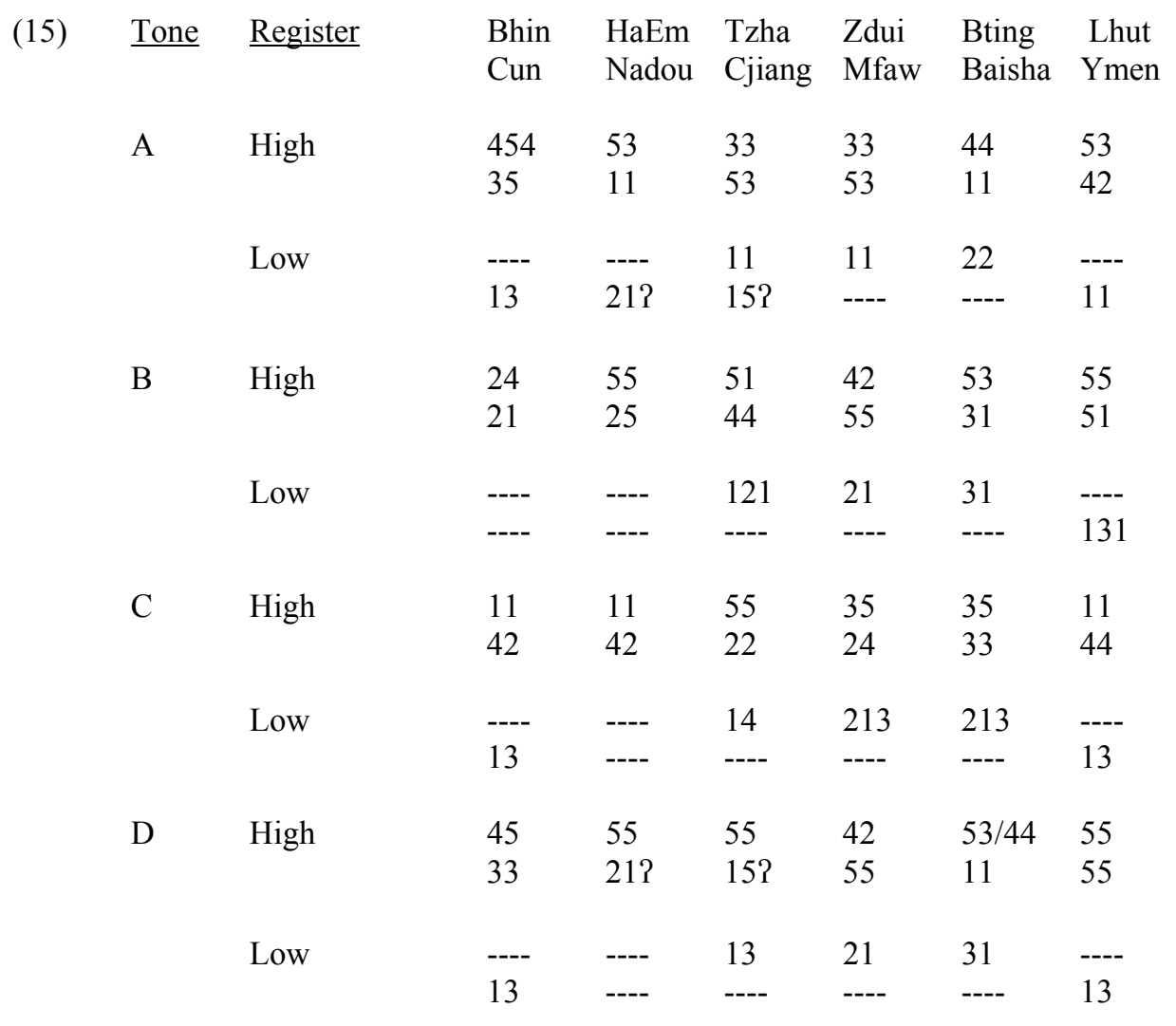

In reconstructing the pre-tonal system of PHl and its subgroups, I make the following crucial

assumptions:

(1) In sibling languages, tones of the same contour (even if they occur at different relative heights) may be assumed to have descended from a parent language with the same contour tone. If there are different tone contours within the same category, then the tones of the daughter language must have developed independently from a segment and/or phonation type in the parent language.

(2) Following Thurgood (2002), I make the assumption that high or rising tones (i.e. tones with a high pitch target) are derived from an abrupt glottal stop ? or a voiceless glottal fricative $h$. Low or falling tones (i.e. tones with a low pitch target) are derived from former creaky voice or breathy voice/voiced glottal fricative 6 . Dovetailing this model is Kingston (2005), which argues that an original final glottal stop can condition either high or low pitch, depending on whether or not it conditions tense voice or creaky voice in the preceding vowel.

(3) Category D tones can either have high targets or otherwise have mid to low targets. I follow Thurgood (2002) in positing that a tone D word with a high pitch target indicates that the final stop of that word was articulated with simultaneous glottal closure; conversely, an item with a mid to low pitch target did not have this accompanying glottal closure. 
(4) I take as a model Honda (2005) which shows that in the Vietic branch of Mon-Khmer, tonogenesis occurred earlier in category $\mathrm{C}$ than it did in category B. I therefore do not make the assumption that tonogenesis occurred simultaneously in all categories in all languages.

Based on the pitch contours given above, it seems as though tone categories have developed at least partly on an areal basis, in three general groups. These are: Group 1, including Bouhin, Ha Em, Lauhut, and Meifu; Group 2, including Qi and Run, and Group 3, comprising NWCHl.

\subsubsection{Tonogenetic Group One}

The tones of the first group are repeated below:

\begin{tabular}{|c|c|c|c|c|c|}
\hline (16) & $\underline{\text { Bhin }}$ & $\underline{\mathrm{HaEm}}$ & $\underline{\text { Lhut }}$ & $\underline{\text { Mfaw }}$ & Cjiang \\
\hline A & 454 & 53 & 53 & 53 & 53 (high register)/ $15 ?$ (low register) \\
\hline B & 24 & 55 & 55 & 55 & 44 \\
\hline $\mathrm{C}$ & 11 & 11 & 11 & 24 & 22 \\
\hline $\mathrm{D}$ & 45 & 55 & 55 & 55 & $15 ?$ \\
\hline
\end{tabular}

Before discussing the specific nature of the $\mathrm{B}$ and $\mathrm{C}$ categories, it will be useful to have an overview of the entire system. There is a discernible pattern in the tone categories of this group: category $\mathrm{A}$ is high falling, categories $\mathrm{B}$ and $\mathrm{D}$ are high level, and category $\mathrm{C}$ is low level (there are exceptions to each of these generalizations, which are assumed to be the result of later change and are treated below).

The first observation which can be made is that there is an opposition between the categories with high pitch (A, B, and D), and that with low pitch (C). This suggests an initial height-based pitch opposition, indicating that $\mathrm{C}$ is marked in some way, probably as a result of a phonation difference: 
Reconstructed Group One pitch trajectories

High (unmarked) Low (marked)
A: *53
B: *55
D: *55
C: *11

The second observation is that categories B and D pattern together in a nearly identical way (Changjiang being the one exception). This suggests that the two categories shared some similarity, and the most likely candidate for this similarity is closure by a voiceless coda:

\section{Reconstructed Group One tone categories}

High Low
$\mathrm{A}: * \mathrm{~V}^{53}$
$\mathrm{C}: * \mathrm{~V}^{11}$
B: * $\mathrm{VC}^{55}$
$\mathrm{D}: * \mathrm{VC}^{55}$

In general, the pitch at the left edge of the rime can be hypothesized to have been raised to the high end of the pitch range, in order to maximize contrast with the low pitch onset in category $\mathrm{C}$. The differences between the pitch trajectories in the high category can be explained in this way by suggesting that level high pitch was maintained when closed by a voiceless coda, but that lack of such a coda led to natural pitch declination and ultimately to a falling tone.

Based on the criteria stipulated at the beginning of this section, there are two interpretations for both categories B and C. The first possibility in category B is that it ended in a voiceless laryngeal fricative $h$, and the second is that it ended in a glottal stop. The first possibility in category $\mathrm{C}$ is that the phonation was creaky, and the second that it was breathy.

Based on the Moyfaw evidence discussed below, it will be tentatively assumed that pitch was lowered in category $\mathrm{C}$ due to creaky voice. The decision between final $h$ and $P$ in category B is therefore made tentatively in favor of the former, under the assumption that the creaky voice of category $\mathrm{C}$ originated in a former glottal stop. The initial state for tonogenetic group one is therefore the following: 
Reconstructed Group One tone category precursors

High Low
A: $* \mathrm{~V}^{53}$
$\mathrm{C}: * \mathrm{~V}^{11}$
$\mathrm{B}: * \mathrm{Vh}^{55}$
D: *V $\widehat{P C}^{55}$

The changes in the individual languages can now be treated. The Ha Em and Lauhut systems preserved the pitch values in (16) above, and therefore do not need to be discussed further save to point out that the postulated final glottal stop in category B and creaky voice in category $\mathrm{C}$ were both eventually lost, leaving only the pitch distinction behind.

The Bouhin differences can be explained in two steps. The first was that, after the eventual deletion of the glottal coda in category B, the height of the pitch was no longer constrained on the right edge, and the pitch drifted downward, causing a distinction between categories B and D. The second step was the addition of an initial raise in the high tones to the top of the pitch range, something which served to shorten the duration of the falling tone in category A, leading to a circumflex tone. This is an interesting example of what Pittayaporn (2007) calls peak-sliding, in which the peak of a tonal contour slides rightward. The only difference here being that high level tones (categories B and D) are affected as well as the contour tone (category A). These two changes are shown below:

$\underline{\text { Bouhin }}$

$\begin{array}{llllll}\mathrm{A} & * \mathrm{~V}^{53} & > & \mathrm{V}^{53} & > & \mathrm{V}^{4-54} \\ \mathrm{~B} & * \mathrm{Vh}^{55} & > & \mathrm{V}^{44} & > & \mathrm{V}^{2-4} \\ \mathrm{C} & * \mathrm{~V}^{11} & > & \mathrm{V}^{11} & > & \mathrm{V}^{11} \\ \mathrm{D} & * \widetilde{V}^{2} \mathrm{TC}^{55}> & \mathrm{VC}^{55} & > & \mathrm{VC}^{4-5}\end{array}$

The only additional change which occurred in Moyfaw was in category $\mathrm{C}$, in which a rising tone developed. I propose that the motivation for this was the development of creaky voice into a final glottal stop, which then proceeded to raise the pitch at the end of the rime. The entire tone then underwent raising after the loss of creaky voice: 
(21) Moyfaw

$\mathrm{C} \quad \mathrm{V}^{11} \quad>\quad \mathrm{V}^{13}>\mathrm{V}^{24}$

The situation in Changjiang is the most complex. There was no change in category A, and what appears to have happened in categories B and C is that the pitch 'centralized', lowering in B and raising in $\mathrm{C}$, after the loss of final $* \mathrm{~h}$ and creaky voice respectively. The most dramatic reflex of category D exists in Changjiang, where the glottal constriction can be heard in the modern language and is very pronounced, having raised the pitch to an extraordinarily high level.

The development of a register distinction in unique in this group, and Changjiang register patterns closely with Nadouhua, indicating that speakers of Changjiang became disassociated with Group One after tonogenesis and closer to Group Three by the time of registrogenesis. The fact that a register split is only extant in category A indicates that registrogenesis was probably blocked in the other three categories.

Thurgood (1991:4-8) proposes that in Nadouhua, originally voiced initials conditioned laryngealization which bled into the nucleus and was ultimately reanalyzed as glottal constriction. This is also an acceptable hyptothesis for Changjiang, which shows the same basic pattern. It can then be suggested that this laryngealization (which was probably originally breathy voice) successfully spread through the rime in category A, but was blocked by pre-existing glottal codas in categories B and D and by creaky voice in category C. The reanalysis of a final glottal stop in category A led to a merger with words in category D (the $G$ in the examples in low register represents the voicing of the original onset):

\section{Changjiang}

\section{High Register}

\begin{tabular}{|c|c|c|c|c|c|c|c|c|c|c|}
\hline $\mathrm{V}^{53}$ & $>$ & $\mathrm{V}^{53}$ & $>$ & $\mathrm{V}^{53}$ & A2 & $\mathrm{V}^{53}$ & $>$ & h. $\underline{.}^{53}$ & $>$ & $V P^{15}$ \\
\hline $\mathrm{Vh}^{55}$ & $>$ & $\mathrm{V}^{44}$ & $>$ & $\mathrm{V}^{44}$ & B2 & $\mathrm{Vh}^{55}$ & $>$ & hVh & $>$ & $\mathrm{V}^{44}$ \\
\hline $\mathrm{V}^{11}$ & $>$ & $\mathrm{V}^{22}$ & $>$ & $\mathrm{V}^{22}$ & $\mathrm{C} 2$ & $\mathrm{~V}^{11}$ & $>$ & $6 \underline{V}^{11}$ & $>$ & $\mathrm{V}^{22}$ \\
\hline$\tilde{\mathrm{V}} \widetilde{\mathrm{PC}}^{55}$ & $>$ & $V \widetilde{P C}^{15}$ & $>$ & $\mathrm{V} \widetilde{P C}^{15}$ & D2 & $\tilde{\mathrm{V}} \widetilde{P C}^{55}$ & $>$ & $\tilde{\mathrm{fV}} \overline{\mathrm{PC}^{55}}$ & $>$ & $\mathrm{V} \overline{\mathrm{PC}}^{15}$ \\
\hline
\end{tabular}




\subsubsection{Tonogenetic Group Two}

The tones of the group two languages are repeated below:

\begin{tabular}{|c|c|c|c|c|c|}
\hline$\underline{\text { Register }}$ & $\underline{\text { Baisha }}$ & $\underline{\text { Ymen }}$ & $\underline{\text { Tzha }}$ & $\underline{\text { Zdui }}$ & $\underline{\text { Bting }}$ \\
\hline High & 11 & 42 & 33 & 33 & 44 \\
\hline Low & & 11 & 11 & 11 & 22 \\
\hline High & 31 & 51 & 51 & 42 & 53 \\
\hline Low & & 131 & 121 & 21 & 31 \\
\hline High & 33 & 44 & 55 & 35 & 35 \\
\hline Low & & 13 & 14 & 213 & 213 \\
\hline High Long & 11 & 55 & 55 & 42 & 53 (long)/44 (short) \\
\hline Low & & 13 & 13 & 21 & 31 \\
\hline
\end{tabular}

Although there are exceptions, an overview of this group indicates that the following general pattern can be posited as the initial state in the four tone categories:

\section{Reconstructed Group Two pitch trajectories}
A: *33
B: *51
$\mathrm{C}: * 55$
D: *55

It can be safely assumed that mid level tone existed in category A simply because it was unmarked.

The falling tone in category B indicates a tone depressor at the right edge of the rime, which in the parameters established above can be either breathy voice $(*-h)$ or a creaky glottal stop $(*-?)$. Categories $\mathrm{C}$ and D pattern together (although this has been obscured somewhat by later changes), and the high level tone reconstructed in these categories is taken here to reflect tense voice, correlated with a final voiceless glottal stop. If this is so, then the choice for the final element in category B can be tentatively suggested to be *-h: 
Reconstructed Group Two tone category precursors
A: $* \mathrm{~V}^{33}$
B: $* \mathrm{Vh}^{51}$
$\mathrm{C}: * \underline{\mathrm{V}} \mathrm{P}^{55}$
D: * $\underline{\mathrm{V}}^{55}$

With this original configuration as a hypothesis, it is possible to move on and treat changes in the individual languages. Beginning with Baisha, there are two differences which stand out when compared to the rimes in (25) above. The first is that category D is the same as category A, not category C, and the second is that all of the pitch levels are quite low compared to their counterparts in other languages. I propose that the reason for the first difference is the loss of simultaneous glottal closure associated with final oral stops (and as a result, the tense voice which accompanied it). The reason for the second is that, for some reason, the whole pitch system was 'down-stepped', so that the relationships between the tones remained identical, but they became low when compared with other Hlai languages:

(26) Evolution of Baisha tone categories

$\begin{array}{lllll}\mathrm{A}: * \mathrm{~V}^{33} & > & \mathrm{V}^{33} & > & \mathrm{V}^{11} \\ \mathrm{~B}: * \mathrm{~V} \mathrm{f}^{51} & > & \mathrm{V}^{51} & > & \mathrm{V}^{31} \\ \mathrm{C}: * \underline{\mathrm{V}} ?^{55} & > & \underline{\mathrm{V}} 1^{55} & > & \mathrm{V}^{33} \\ \mathrm{D}: * \underline{\mathrm{V}}^{55} & > & \mathrm{VC}^{33} & > & \mathrm{VC}^{11}\end{array}$

This downstep can actually be verified through Wang \& Qian's (1951) data, where they give the following values for the Baisha tone categories:

(27) Baisha tone values from Wang \& Qian (1951)
A: $\dashv(33)$
B: Y $(53) \sim \sqrt{ }(31)$
C: 1 (55)
D: $\dashv(33)$

In Yuanmen, the trajectory of pitch in category A became falling, although the reasons for this are unclear. After the loss of the glottal stop in category $\mathrm{C}$, there was a slight declination in the overall pitch in category C. Registrogenesis seems to have occurred before these changes applied, as it acted upon a 
system quite similar to the one reconstructed in (25). The convex tone in the low register of category B is the result of the depression of pitch by the voiced initial, which then needed to rise to a mid target before it could fall again:

\section{Evolution of Yuanmen tone categories}

\section{High register}

\begin{tabular}{|c|c|c|c|c|c|}
\hline Al & $* \mathrm{~V}^{33}$ & $>$ & $\mathrm{V}^{33}$ & $>$ & $\mathrm{V}^{42}$ \\
\hline B1 & $* \mathrm{Vh}^{51}$ & $>$ & $\mathrm{Vh}^{51}$ & $>$ & $\mathrm{V}^{51}$ \\
\hline $\mathrm{C} 1$ & $* \underline{\mathrm{V}} \mathrm{P}^{55}$ & $>$ & $\mathrm{V}^{55}$ & $>$ & $\mathrm{V}^{44}$ \\
\hline$D 1$ & $* \underline{\mathrm{V}} \overline{\mathrm{PC}}^{55}$ & $>$ & $\mathrm{V} \widetilde{R C}^{55}$ & $>$ & $\mathrm{VC}^{5}$ \\
\hline
\end{tabular}

\section{$\underline{\text { Low register }}$}

$\begin{array}{llll}\mathrm{A} 2 & * \mathrm{~V}^{33}> & \mathrm{hV}{ }^{11}> & \mathrm{V}^{11} \\ \mathrm{~B} 2 & * \mathrm{~V} h^{51}> & \mathrm{hV} h^{1-31}> & \mathrm{V}^{131} \\ \mathrm{C} 2 & * \underline{\mathrm{V}}^{55}> & \mathrm{hV} 1^{13}> & \mathrm{V}^{13} \\ \mathrm{D} 2 & * \underline{\mathrm{V}}^{13} \mathrm{C}^{55}> & \mathrm{h} \underline{\mathrm{V}}^{13}> & \mathrm{VC}^{13}\end{array}$

The Tongzha situation is very similar to the Yuanmen one, indicating that they were in close interaction during the phase of tongogenesis. The only significant changes were the slight raise in pitch targets in the low register of category $\mathrm{C}$, and the lowering of the peak of the circumflex tone in the low register of category B (what Pittayaporn (2007) refers to as contour reduction):

\section{Evolution of Tongzha tone categories}

High register

\section{$\underline{\text { Low register }}$}

\begin{tabular}{|c|c|c|c|c|c|c|c|c|c|c|c|}
\hline A1 & $* V^{33}$ & $>$ & $\mathrm{V}^{33}$ & $>$ & $\mathrm{V}^{33}$ & A2 & $* \mathrm{~V}^{33}$ & $>$ & $6 \mathrm{~V}^{11}$ & $>$ & $\mathrm{V}^{11}$ \\
\hline B1 & $* \mathrm{Vh}^{51}$ & $>$ & $\mathrm{Vh}^{51}$ & $>$ & $\mathrm{V}^{51}$ & B2 & $* \mathrm{Vh}^{51}$ & $>$ & $\mathrm{fVh}^{1-31}$ & $>$ & $\mathrm{V}^{121}$ \\
\hline $\mathrm{C} 1$ & $* \underline{\mathrm{V}} \mathrm{P}^{55}$ & $>$ & $\underline{\mathrm{V}} \mathrm{P}^{55}$ & $>$ & $\mathrm{V}^{55}$ & $\mathrm{C} 2$ & $* \underline{\mathrm{V}} 1^{55}$ & $>$ & $\hat{h} \underline{V} 2^{13}$ & $>$ & $\mathrm{V}^{14}$ \\
\hline D1 & $* \widehat{V}^{5 C^{55}}$ & $>$ & $\underline{\mathrm{V}} \widehat{P C}^{55}$ & $>$ & $\mathrm{VC}^{55}$ & D2 & $* \underline{\mathrm{V}}^{55}$ & $>$ & $\mathrm{f} \underline{\underline{\mathrm{PC}}} \overline{\mathrm{C}}^{13}$ & $>$ & $\mathrm{VC}^{13}$ \\
\hline
\end{tabular}

The Zandui and Baoting developments were very similar, which indicates that they were also in close contact with each other during tonogenesis. The hypothesized changes which were common to both of them were (1) the loss of the final glottal fricative in category B, which shortened the trajectory of the fall, (2) the loss of tense voice in category $\mathrm{C}$ which led to a new rising tone, (3) the development of the final glottal stop in category D to a creaky stop ${ }^{26}$, leading to a falling tone and merging with category B, and (4) the genesis of a convex tone in the low register of category $\mathrm{C}$, as a result of the movement of the

\footnotetext{
${ }^{26}$ This is also what happened to final glottal stops in neighboring Jiamao (see chapter 5).
} 
low pitch target away from the left edge of the rime. The one change specific to Zandui was the downstep in pitch of the falling tones in categories B and D in both registers:

(30) Evolution of Zandui tone categories

$\underline{\text { High register }}$

\section{$\underline{\text { Low register }}$}

\begin{tabular}{|c|c|c|c|c|c|c|c|c|c|c|c|}
\hline A1 & $* \mathrm{~V}^{33}$ & $>$ & $\mathrm{V}^{33}$ & $>$ & $\mathrm{V}^{33}$ & A2 & $* \mathrm{~V}^{33}$ & $>$ & $6 \mathrm{~V}^{11}$ & $>$ & $\mathrm{V}^{11}$ \\
\hline B1 & $* V h^{51}$ & $>$ & $\mathrm{V}^{53}$ & $>$ & $\mathrm{V}^{42}$ & B2 & $* \mathrm{Vh}^{51}$ & $>$ & $6 V^{31}$ & $>$ & $\mathrm{V}^{21}$ \\
\hline $\mathrm{C} 1$ & $* \underline{\mathrm{V}} \mathrm{P}^{55}$ & $>$ & $V 2^{35}$ & $>$ & $\mathrm{V}^{35}$ & $\mathrm{C} 2$ & $* \underline{\mathrm{V}} 2^{55}$ & $>$ & hV $P^{13}$ & $>$ & $\mathrm{V}^{2-13}$ \\
\hline D1 & $* \underline{\mathrm{V}} \widetilde{\mathrm{PC}}^{55}$ & $>$ & $\mathrm{V} \widetilde{\mathfrak{R}}^{53}$ & $>$ & $\mathrm{VC}^{42}$ & $\mathrm{D} 2$ & $* \underline{\mathrm{V}}^{55}$ & $>$ & $\mathrm{h} \underline{\underline{\underline{2}}} \mathrm{C}^{31}$ & $>$ & $\mathrm{VC}^{21}$ \\
\hline
\end{tabular}

There were two changes unique to Baoting. The first was the split of category D into two subgroups based on rime length, with the short rimes developing a level tone as a result of their short duration and merging with category A. These two tones (A and short rimes in D) then raised in pitch:

(31) Evolution of Baoting tone categories

$\underline{\text { High register }}$

\begin{tabular}{|c|c|c|c|c|c|c|c|c|c|c|}
\hline A1 & $* \mathrm{~V}^{33}$ & $\mathrm{~V}^{33}$ & $>$ & $\mathrm{V}^{44}$ & A2 & $* \mathrm{~V}^{33}$ & $>$ & $\mathrm{hV}^{11}$ & $>$ & $\mathrm{V}^{22}$ \\
\hline B1 & $* V h^{51}$ & $\mathrm{~V}^{53}$ & $>$ & $\mathrm{V}^{53}$ & B2 & $* \mathrm{Vh}^{51}$ & $>$ & $6 V^{31}$ & $>$ & $\mathrm{V}^{31}$ \\
\hline $\mathrm{C} 1$ & $* \underline{\mathrm{V}} 2^{55}$ & $V 1^{35}$ & $>$ & $\mathrm{V}^{35}$ & $\mathrm{C} 2$ & $* \underline{\mathrm{V}} \mathrm{P}^{55}$ & $>$ & hV $1^{13}$ & $>$ & $\mathrm{V}^{2-13}$ \\
\hline D1L & $*{ }^{*} \widehat{P C}^{55}$ & $\underline{V}: \widehat{\sim}^{53}$ & $>$ & $V: C^{53}$ & $\mathrm{D} 2$ & *V $\overline{P C}^{55}$ & $>$ & $6 \underline{\underline{Z} \mathrm{C}^{31}}$ & $>$ & $\mathrm{VC}^{31}$ \\
\hline D1S & & $\underline{\underline{2}} \mathrm{~T}^{33}$ & $>$ & $\mathrm{VC}^{44}$ & & & & & & \\
\hline
\end{tabular}

\subsubsection{Tonogenetic Group Three}

Low register

The Cunhua and Nadouhua tone systems are repeated below. Both languages have register splits, but Cunhua shows them in all categories except B, whereas Nadouhua only shows a split in A: 
Tone values in the $\mathrm{NWCHl}$ languages

\begin{tabular}{|c|c|c|c|}
\hline$\underline{\text { Tone }}$ & $\underline{\text { Register }}$ & $\underline{\text { Cun }}$ & $\underline{\text { Nadou }}$ \\
\hline \multirow[t]{2}{*}{ A } & High & 35 & 11 \\
\hline & Low & 13 & $21 ?$ \\
\hline B & & 21 & 25 \\
\hline \multirow[t]{2}{*}{$\mathrm{C}$} & High & 42 & 42 \\
\hline & Low & 13 & (42) \\
\hline \multirow[t]{2}{*}{ D } & High & 33 & 212 \\
\hline & Low & 13 & (211) \\
\hline
\end{tabular}

There is significant divergence between these two languages, the only common point being the development of category $\mathrm{C}$ in high register. Reconstruction in this small group is admittedly speculative, and based to a large extent on the results of reconstruction in the first two tonogenetic groups. The tentative reconstructed system for this group is as follows:

Reconstructed Group Three tone category precursors

$\begin{array}{ll}\text { A1 } & * \mathrm{~V}^{33} \\ \mathrm{~B} 1 & * \mathrm{~V}^{21} \\ \mathrm{C} 1 & * \mathrm{~V} ?^{42} \\ \mathrm{D} 1 & * \mathrm{~V} \mathrm{PC}^{55}\end{array}$

In other words, category B is reconstructed with a final breathy laryngeal which has conditioned breathy voice back into the rime nucleus, category $\mathrm{C}$ with a creaky glottal stop, and category $\mathrm{D}$ with simultaneous glottal closure.

The Cunhua changes are shown below. In high register, category A developed a rising contour, possible in analogy with the new rising tone which emerged in low register during registrogenesis. Pitch was low in category B due to its breathy phonation, and slightly falling. The creaky glottal stop of category C was lost early, leaving only the pitch contour, and the coarticulated glottal stop in category D was lost. During registrogenesis, the lowering induced by voicing was concentrated at the left edge of the rime, and neutralized the original pitch disctinctions in all categories except B, which was not affected (probably because it was still breathy): 


$$
\text { Evolution of Cunhua tone categories }
$$

$\underline{\text { High register }}$

\begin{tabular}{|c|c|c|c|c|c|c|c|c|c|c|}
\hline$* \mathrm{~V}^{33}$ & $>$ & $\mathrm{V}^{35}$ & $>$ & $\mathrm{V}^{35}$ & A2 & $* \mathrm{~V}^{33}$ & $>$ & $6 V^{13}$ & $>$ & $\mathrm{V}^{13}$ \\
\hline *Ṿh $h^{21}$ & $>$ & $V h^{21}$ & $>$ & $\mathrm{V}^{21}$ & B2 & $*{ }^{*} h^{21}$ & $>$ & hழ̣̣ $h^{21}$ & $>$ & $\mathrm{V}^{21}$ \\
\hline$* \mathrm{~V} ?^{42}$ & $>$ & $\mathrm{V}^{42}$ & $>$ & $\mathrm{V}^{42}$ & $\mathrm{C} 2$ & $* \mathrm{~V} \mathfrak{2}^{42}$ & $>$ & $6 V^{13}$ & $>$ & $\mathrm{V}^{13}$ \\
\hline *V $\widetilde{\mathrm{PC}}^{55}$ & $>$ & $\mathrm{VC}^{33}$ & $>$ & $\mathrm{VC}^{33}$ & D2 & *V $\widetilde{\mathrm{PC}}^{55}$ & $>$ & $\mathrm{hVC}^{13}$ & $>$ & $\mathrm{VC}^{13}$ \\
\hline
\end{tabular}

There were significant changes in Nadouhua. In high register, there seems to have been a drop in pitch in category A until it reached the bottom of the pitch range. In category B, I propose that the breathy segment at the right edge devoiced, reversing the pitch trajectory as it created a new high target. Category C developed in the same way as Cunhua, merely losing the creaky glottal stop. Finally, the final stops in category $\mathrm{D}$ became creaky, lowering the pitch.

In low register, initial voicing induced breathy phonation, which was apparently blocked from spreading in categories B-D by final segments (indicating that registrogenesis and tonogenesis were occurring simultaneously and could therefore interact). It spread successfully in category A, however, and the phonation became creaky, lowering pitch and eventually being reinterpreted as a creaky glottal stop. As mentioned above, this register pattern is nearly identical to that of Changiiang, with the exception that Nadouhua developed a creaky glottal stop which lowered pitch, whereas Changjiang developed an abrupt glottal stop which raised it; low register category A rimes merged with category D in both languages.

Evolution of Nadouhua tone categories

\section{$\underline{\text { High register }}$}

\begin{tabular}{|c|c|c|c|c|c|}
\hline A1 & $* \mathrm{~V}^{33}$ & $>$ & $\mathrm{V}^{33}$ & $>$ & $\mathrm{V}^{11}$ \\
\hline B1 & *Vִ $h^{21}$ & $>$ & $\mathrm{Vh}^{25}$ & $>$ & $\mathrm{V}^{25}$ \\
\hline $\mathrm{C} 1$ & $* \mathrm{~V} ?_{\sim}^{42}$ & $>$ & $\mathrm{V} \mathfrak{\sim}^{42}$ & $>$ & $V^{42}$ \\
\hline D1 & *V $\widetilde{P C}^{55}$ & $>$ & $\mathrm{V} \widehat{? C}^{21}$ & $>$ & $V ?^{21}$ \\
\hline
\end{tabular}

\section{$\underline{\text { Low register }}$}

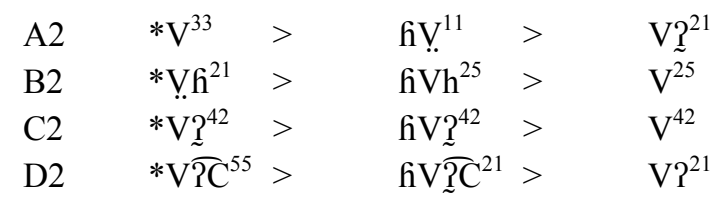




\subsubsection{Proto Hlai}

Moving finally to a reconstruction of the Proto-Hlai system, the following reconstruction is proposed based on the evidence from these three groups:

Reconstruction of Proto-Hlai tone category precursors

\begin{tabular}{|c|c|c|c|c|}
\hline & $\underline{\mathrm{PHl}}$ & Group 1 & Group 2 & Group 3 \\
\hline A & ${ }^{*} \mathrm{~V}$ & $* \mathrm{~V}^{53}$ & $* \mathrm{~V}^{33}$ & $* \mathrm{~V}^{33}$ \\
\hline B & *Vh & $* \mathrm{Vh}^{55}$ & $* V h^{51}$ & $* \bigvee h^{21}$ \\
\hline $\mathrm{C}$ & *V? & $* \stackrel{V}{ }^{11}$ & $* \underline{\mathrm{V}} \mathrm{P}^{55}$ & $* \mathrm{~V} \mathfrak{2}^{42}$ \\
\hline D & *V $\overline{P C}$ & $* \widehat{V}^{55}$ & $* \underline{\mathrm{V}}^{55}$ & *V $\widetilde{\mathrm{PC}}^{55}$ \\
\hline
\end{tabular}

This system is very close to that which has been inferred in much of the Kra-Dai literature (i.e. Gedney (1989), Edmondson \& Solnit $(1988,1997))$, with the exception that the laryngeal segment in category B is breathy * $\mathrm{h}$ as opposed to voiceless *h. This system is essentially identical to that of Chepang noted at the beginning of this section.

The changes between the PHl system and each group will now be briefly discussed. The first two changes which occurred in group one were (1) the breathy fricative $h$ devoiced to $h$ in category B and (2) the glottal stop in category $\mathrm{C}$ became creaky and eventually spread throughout the nucleus, lowering the overall pitch to the bottom of the pitch range. The pitch in categories A, B, and D was raised to the top of the pitch range, presumably to maximize contrast with category $\mathrm{C}$. This high pitch remained level where it was closed by a final glottal (categories B and D), but falling pitch arose in category A as a result of natural declination:

Evolution of tone categories in Group One

$\begin{array}{llllll}\mathrm{A} & * \mathrm{~V} & > & * \mathrm{~V} & > & * \mathrm{~V}^{53} \\ \mathrm{~B} & * \mathrm{Vh} & > & * \mathrm{Vh} & > & * \mathrm{Vh}^{55} \\ \mathrm{C} & * \mathrm{~V} ? & > & * \mathrm{~V} ? & > & * \mathrm{~V}^{11} \\ \mathrm{D} & * \mathrm{~V} \widehat{\mathrm{PC}} & > & * \mathrm{~V} \overline{\mathrm{PC}} & > & * \mathrm{~V}^{5} \overline{\mathrm{C}}^{55}\end{array}$


In the second group, the only development was that of tense voice (correlated with the presence of glottal stops in categories $\mathrm{C}$ and $\mathrm{D}$ ) which raised the pitch across the nucleus:

$$
\text { Evolution of tone categories in Group Two }
$$

$\begin{array}{llll}\mathrm{A} & * \mathrm{~V} & > & * \mathrm{~V}^{33} \\ \mathrm{~B} & * \mathrm{~V} h & > & * \mathrm{~V} h^{51} \\ \mathrm{C} & * \mathrm{~V} \mathrm{P} & > & * \underline{\mathrm{V}}{ }^{55} \\ \mathrm{D} & * \mathrm{~V} \widehat{\mathrm{PC}} & > & * \underline{\mathrm{V}} \mathrm{C}^{55}\end{array}$

Finally, the developments which occurred in group three were the spread of breathy voice back into the rime nucleus in category B (lowering the pitch of the overall rime), and the development of creakiness in the glottal stop which led to a falling contour in category C. This falling contour was raised in pitch to increase contrast with the lower pitch of category B:

Evolution of tone categories in Group Three

$\begin{array}{llllll}\mathrm{A} & * \mathrm{~V} & > & * \mathrm{~V} & > & * \mathrm{~V}^{33} \\ \mathrm{~B} & * \mathrm{Vh} & > & * \mathrm{~V} h & > & * \mathrm{~V} \mathrm{~h}^{21} \\ \mathrm{C} & * \mathrm{~V} ? & > & * \mathrm{~V} ? & > & * \mathrm{~V} ?^{42} \\ \mathrm{D} & * \mathrm{~V} \widehat{\mathrm{TC}} & > & * \mathrm{~V} \widetilde{\mathrm{TC}} & > & * \widetilde{V}^{55}\end{array}$

\subsubsection{Summary}

Based on the segmental variations within the rime in Greater Hlai and Cunhua, as well as the variation in pitch realization between the three major areal tone groups, the reconstruction of PHl final glottal segments is deemed to be necessary. Once this has been recognized, the tone values in the individual languages are seen to be rich in information which can be used to reconstruct the PHl pre-tone system, as well as its intermediate stages, following the guidelines provided at the beginning of this section. The PHl pre-tone system has been reconstructed with four categories: unmarked (category A), final breathy glottal fricative (category B), final glottal stop (category C), and final glottalized oral stop (category D). Given the typology of tone change outlined in (1), it was seen above that the principles used for segmental 
reconstruction (Directionality, Commonality, Economy and Symmetry) are of use in the reconstruction of the precursors of the tone categories as well, providing useful constraints on that process.

\subsection{Open Rimes}

There are a total of five open rimes which can be reconstructed for the PHl inventory. These include three high vowels, the front mid vowel, and one low vowel. Of these five vowels, the vowel *e: is quite rare, and likely occurred in $\mathrm{PHl}$ as the result of loans into the proto-language from one or more outside sources (although this is speculation). The reflexes of the $\mathrm{PHI}$ open rimes are given below; note that the first three sets of reflexes (reconstructed below as high vowels) only occur in tone category A:

(40) Reflexes of the $\mathrm{PHl}$ open rimes

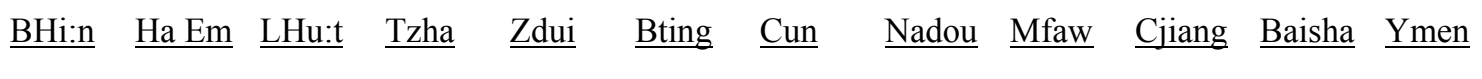

ey ey ey ey ey ey $\quad$ ey $\quad$ ey ey ey ey ey

әщ әщ әщ әщ әщ әщ

ow ow ow ow ow ow ow $\varepsilon w$ ow

e: $\quad$ e: e: e: e: e: --- --- e: $\quad$ e: e:

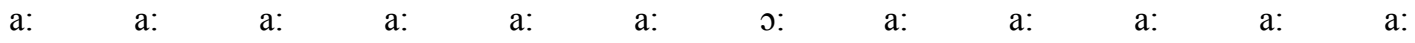
S. Hlai (Savina)
C. Hlai (Savina)
Baisha (Wang \& Qian)

ey

үщ

ew

$\varepsilon$ :

a: ey

rщ

aw

a: عy

วу

OW

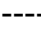

a:

The reconstructions for these vowels proposed here are given below:

$$
\begin{aligned}
& *_{\mathrm{i}}: \\
& \text { *u: }^{\mathrm{u}}: \\
& \text { *u: }^{\mathrm{u}} \\
& \text { *e: }^{\mathrm{e}} \\
& \text { *a: }^{\mathrm{a}}
\end{aligned}
$$


Although the reconstruction of the high vowels may appear to violate the principle of

Commonality (pure high vowels are not generally reflected in the reflexes of the daughter languages), it will be demonstrated below that these vowels must have been monophthongs at the stage of Proto-Hlai, and that high vowel diphthongization was a feature which either occurred independently or diffused between the subgroups.

There is also evidence for PHl high monophthongs in several instances where diphthongization failed to occur. The one relevant here is of four Bouhin examples in which high vowels failed to diphthongize:

\begin{tabular}{|c|c|c|}
\hline Gloss & $\underline{\mathrm{PHl}}$ & $\underline{\text { Bouhir }}$ \\
\hline aunt & *hmi: & mi: ${ }^{1}$ \\
\hline you & *C-mu: & mu: $:^{1}$ \\
\hline seven & $* t^{h} \mathrm{u}:$ & $\mathrm{t}^{\mathrm{h}} \mathrm{u}::^{1}$ \\
\hline eight & *ru: & $\mathrm{ru}:{ }^{1}$ \\
\hline
\end{tabular}

The category of high vowels is complicated in another way, as they each have two series of reflexes apiece: one in tone category A (shown above in (40)), and one in tone categories B and C; moreover, the vowel *u: has two different sets of reflexes between categories B and C in Zandui, Baoting and Cunhua:

Reflexes of the $\mathrm{PHI}$ open rimes

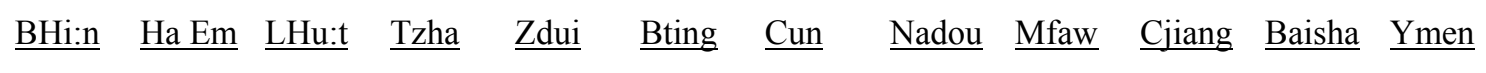

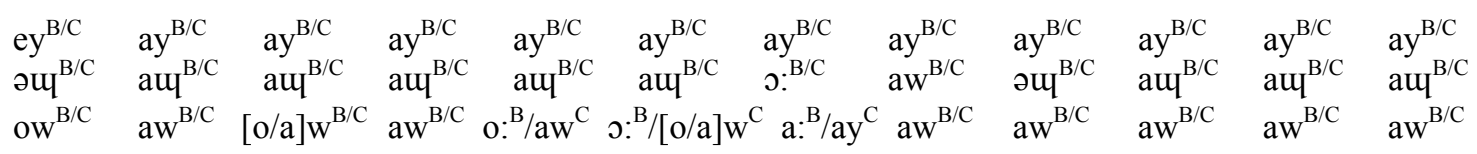

\section{$\underline{\text { S. Hlai (Savina) }}$}

$$
\begin{aligned}
& \mathrm{ey}^{\mathrm{B} / \mathrm{C}} \\
& \mathrm{rum}^{\mathrm{B} / \mathrm{C}} \\
& \mathrm{ew}^{\mathrm{B} / \mathrm{C}}
\end{aligned}
$$

\section{Hlai (Savina)}

$$
\begin{aligned}
& \text { ay }^{\mathrm{B} / \mathrm{C}} \\
& \gamma \mathrm{w}^{\mathrm{B} / \mathrm{C}} \\
& 0:^{\mathrm{B}} / \mathrm{aw}^{\mathrm{C}}
\end{aligned}
$$

Baisha (Wang \& Qian)

$$
\begin{aligned}
& a: y^{B / C} \\
& a: 1^{B / C} \\
& b w^{B / C}
\end{aligned}
$$

The reconstructions for these vowels in the marked tone categories proposed here are given below: 


$$
\begin{aligned}
& *_{\mathrm{i}: \mathrm{h} / \mathrm{?}} \\
& \text { *uth/? }^{\mathrm{u}} \mathrm{u}: \mathrm{h} / \mathrm{?}
\end{aligned}
$$

With the exception of Bouhin, the development of the high vowels was dependent on their tone category. With few exceptions (given shortly), the presence of a final glottal element conditioned diphthongization in Greater Hlai. The differences in development between Bouhin (44) and Greater Hlai (45) are shown below:

$$
\underline{\text { Bouhin }}^{27}
$$

\section{(a)}

$\begin{array}{lll}* \mathrm{i}: & > & \mathrm{i}: \\ * \mathrm{u}: & > & \mathrm{u}: \\ * \mathrm{u}: & > & \mathrm{u}:\end{array}$

\section{Greater Hlai}

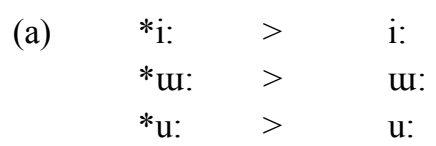

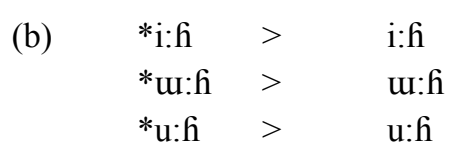

(c)

$\begin{array}{lll}* \mathrm{i}: \mathrm{P} & > & \mathrm{i}: \mathrm{l} \\ *_{\mathrm{u}: \mathrm{P}} & > & \mathrm{u}: ? \\ *_{\mathrm{u}: \mathrm{l}} & > & \mathrm{u}: \mathrm{l}\end{array}$

(b)

$\begin{array}{lll}* \mathrm{i}: \mathrm{h} & > & \text { әуh } \\ * \mathrm{u}: \mathrm{h} & > & \text { əuһ } \\ * \mathrm{u}: \mathrm{h} & > & \text { əwh }\end{array}$

(c)

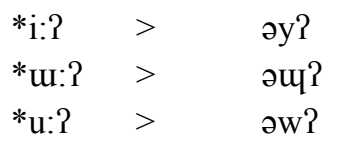

This is the central reason for the reconstruction of pure high vowels at the Proto-Hlai stage. If the dipthongs *ey, *əu, and *ow were reconstructed, the change to Greater Hlai *əy, *əu, and *əw would not only be more awkward, but the * əu in category A and the * əu in categories B and C would have undergone merger. Since this is clearly not the case, the best solution is the reconstruction of originally pure vowels which underwent two different kinds of diphthongization at two different times.

The consequence of this change was that originally pure high vowels diphthongized and merged with original short diphthongs in Greater Hlai category A, producing the following distribution:

${ }^{27}$ There is an additional reflex of *u:h in Bouhin, that of aw; I presume this to be (as with much other variation in Bouhin to be discussed below) due to contact with Ha Em. The reason for this assumption is that in all cases of apparent unconditioned variation in Bouhin, one of the variants is always identical to a Ha Em source, the speech community of which is also adjacent to that of Bouhin; this is very reminiscent of the situation with PHI *r and *Cur in chapter 2, where Bouhin shows unconditioned variation between the reflexes $\rho$ and $g$. I assume that the same explanation applies here, namely that the words with the reflex of $a w$ are loans from Ha Em, with which Bouhin seems to have been in rather direct contact throughout much of its history. 
Table 4: Greater Hlai Distribution of Pure Vowels vs. Short Diphthongs

\begin{tabular}{|c|c|c|c|}
\hline Rime & Tone $\mathrm{A}$ & Tone $\mathrm{B}$ & Tone $\mathrm{C}$ \\
\hline$*_{\mathrm{i}}$ & $\mathrm{X}$ & & \\
\hline *u: & $\mathrm{X}$ & & \\
\hline *u: & X & & \\
\hline *əy & $\mathrm{X}$ & $\mathrm{X}$ & $\mathrm{X}$ \\
\hline *əu & & $\mathrm{X}$ & $\mathrm{X}$ \\
\hline *ow & X & $\mathrm{X}$ & $\mathrm{X}$ \\
\hline Final & $-\varnothing$ & $-h$ & $-?$ \\
\hline
\end{tabular}

If this hypothesis is correct, then the first implication is that short mid central diphthongs in tone category A are original; the same diphthongs in category B and C, however, have two potential sources: original diphthongs and original pure vowels which underwent diphthongization (and ultimately losing their final laryngeal segments at the end of the process of tonogenesis described above, completely merging with their counterparts in category A). Since * əu does not occur in category A, the second implication is that there was no original Pre-Hlai *əu् category; all members in this category are derived from an original *u:h/2. The original inventory of pure high vowels and short diphthongs in PHl was therefore the following:

$$
\begin{array}{lll}
* \mathrm{i}: & * \mathrm{u}: & * \text { u: } \\
* \text { * } & & * \text { ə }
\end{array}
$$

The pure high vowels which remained in the Hlai languages after the diphthongization in Greater Hlai then underwent subsequent diphthongization themselves. In most cases of pure vowels in tone category A, the nucleic schwa was colored by the following glide and shifted to the corresponding midvowel which shared the same feature in frontness/backness. The majority evolution of the high vowels is shown below (48a) and compared with the simultaneous evolution of the short diphthongs in Greater Hlai (48b):

\begin{tabular}{|c|c|c|c|c|c|c|}
\hline (a) & $*_{\mathrm{i}}:$ & $>$ & ey & (b) & *əy & $>$ \\
\hline & *u: & $>$ & ә૫ & & *əઢ & $>$ \\
\hline & *u: & $>$ & ow & & *əw & $>$ \\
\hline
\end{tabular}


The only exceptions to the development shown in (48) occurred in Cunhua and Nadouhua, subsequent to diphthongization. In the case of Cunhua, ${ }^{*} u$ : rounded and merged with ${ }^{*} \mathrm{u}:$ :

$$
\text { Cunhua: } \quad * u \text { u: } \quad>\quad \text { u: }>\text { ow }>\text { ow }
$$

In the case of Nadouhua, a partial merger occurred, with *u: falling into variation between $u$ : and $u:$; all cases of short $ə$ were fronted to $\varepsilon$, preempting the otherwise ubiquitous assimilation of schwa to the following glide. The final velar glide of $\varepsilon \Psi$ was then lost, leaving long $\varepsilon:$ :

\begin{tabular}{|c|c|c|c|c|c|c|c|c|c|}
\hline \multirow[t]{3}{*}{ Nadouhua: } & $*_{\mathrm{i}}$ & $>$ & i: & $>$ & әу & $>$ & عy & $>$ & عy \\
\hline & *u: & $>$ & u: u: & $>$ & әщ $\sim \partial W$ & $>$ & $\varepsilon \Psi \sim \varepsilon W$ & $>$ & $\varepsilon: \sim \varepsilon W$ \\
\hline & $*^{*} \mathrm{u}:$ & $>$ & u: & $>$ & əw & $>$ & $\varepsilon \mathrm{W}$ & $>$ & $\varepsilon \mathrm{W}$ \\
\hline
\end{tabular}

A final exception occurred in Moyfaw, where the nucleus of *əu remained a mid vowel, failing to lower to ащ.

This kind of stress-correlated diphthongization can be found elsewhere in Southeast Asia. A good example of this is Chamic, where the shift from largely penultimate stress in Proto-Malayo-Polynesian (PMP) to final stress brought about the same situation by the time of Proto-Chamic (Thurgood (1999)): High vowel diphthongization in Proto Chamic

\begin{tabular}{|c|c|c|c|c|}
\hline$\underline{\text { Gloss }}$ & PMP & & $\underline{\text { Pre-Chamic }}$ & $\underline{\text { Proto-Chamic }}$ \\
\hline thorn & *duRi & $>$ & *durî: & *durəy \\
\hline dig & *kali & $>$ & *kali: & *kaləy \\
\hline $\operatorname{man}$ & *laki & $>$ & *lakí: & *lakəy \\
\hline Gloss & $\underline{\mathrm{PMP}}$ & & Pre-Chamic & Proto-Chamic \\
\hline $\operatorname{dog}$ & *asu & $>$ & *asú: & *?asəw \\
\hline widowed & *balu & $>$ & *balú: & *baləw \\
\hline know; able & $*$ tahu & $>$ & *tahú: & *thow \\
\hline
\end{tabular}

Further development of *u:h occurred in Zandui and Baoting: 


$$
* \text { u:h } \quad>\quad \text { owh } \quad>\quad \text { o:h }
$$

The three different developments of *u: in Cunhua were also very striking:

\begin{tabular}{|c|c|c|c|c|c|}
\hline *u: & $>$ & u: & $>$ & әW & $>$ \\
\hline *u:h & $>$ & əwh & $>$ & awh & \\
\hline *u:? & $>$ & วw? & $>$ & au? & \\
\hline
\end{tabular}

After *u:h/2 merged with *əw, the latter developed in Lauhut in two ways depending on the initial it followed, with the nucleus either lowering (54a) or backing under the influence of a preceding labial (54b):

(54) Examples of PHl * əwh/? in Lauhut

$\begin{array}{ll}\text { (a) Non-labial(ized) initials } & \text { (b) Labial(ized) initials }\end{array}$

\begin{tabular}{|c|c|c|c|c|c|}
\hline$\underline{\text { Gloss }}$ & $\underline{\mathrm{PHl}}$ & $\underline{\text { Lauhut }}$ & $\underline{\text { Gloss }}$ & $\underline{\mathrm{PH} 1}$ & $\underline{\text { Lauhut }}$ \\
\hline few & * rjəwh & $\operatorname{raw}^{2}$ & pile & $* p^{\mathrm{h}}$ əw? & $\mathrm{p}^{\mathrm{h}} \mathrm{ow}^{3}$ \\
\hline banyan & $* \mathrm{t}^{\mathrm{h}}$ วw? & $t^{h} a^{3}$ & hot & $* t \int^{h}$ wəw? & fow $^{3}$ \\
\hline male inlaw & *hjəw? & $\mathrm{zaw}^{3}$ & handle & *Cufiəwh & hwow $^{2}$ \\
\hline
\end{tabular}

This was also true of Baoting in *u:? rimes in category C (55a-b):

(55) Examples of PHl *u:? in Baoting

$\begin{array}{ll}\text { (a) Non-labial(ized) initials } & \text { (b) Labial(ized) initials }\end{array}$

\begin{tabular}{|c|c|c|c|c|c|}
\hline$\underline{\text { Gloss }}$ & $\underline{\mathrm{PHl}}$ & Baoting & $\underline{\text { Gloss }}$ & $\underline{\mathrm{PHl}}$ & Baoting \\
\hline ash & *su:? & $\operatorname{taw}^{3}$ & stab & ${ }^{*} \mathrm{p}^{\mathrm{h}} \mathrm{u}: ?$ & $\mathrm{p}^{\mathrm{h}} \mathrm{ow}^{3}$ \\
\hline two & *hlu:? & law ${ }^{3}$ & taboo & ${ }^{*} \mathrm{C}-\mathrm{mu}: ?$ & mow $^{3}$ \\
\hline kill & *C-hu:? & haw $^{3}$ & boil & *6u:? & $60 w^{3}$ \\
\hline
\end{tabular}

The only major exceptions to Greater Hlai diphthongization in categories B and C are the following: 
(56)

\begin{tabular}{|c|c|c|c|}
\hline Gloss & $\underline{\mathrm{PHl}}$ & $\underline{\text { Gloss }}$ & PHl \\
\hline this & ${ }^{*}$ C-ni:h & tadpole & *hnu:h \\
\hline thin & ${ }^{*} \mathrm{C}-\mathrm{li}: ?$ & run & *Curu:h \\
\hline point & *sur:? & blow & *?u:h \\
\hline
\end{tabular}

In addition, the following items may be counterexamples as well; all of them except one are found only in Bouhin and Ha Em, one being found only in Lauhut and Baisha:

$\begin{array}{ll}\text { Gloss } & \underline{\text { PHl }} \\ \text { older sister } & * \text { ?i:h } \\ \text { trip clsfr } & * 6 \text { ur:h } \\ \text { shadow } & *[\text { hw/v]u:h }\end{array}$

$\begin{array}{ll}\underline{\text { Gloss }} & \underline{\mathrm{PHl}} \\ \text { non-gltns. rice } & * \mathrm{C}-\mathrm{mu}: ? \\ \text { one } & * \mathrm{t} \varphi^{\mathrm{h}} \mathrm{ur}: ?\end{array}$

There is also one unique example of a high vowel which failed to diphthongize at all (58a), another example in which the high vowel failed to diphthongize in the majority of languages (58b), and one in which it failed to diphthongize in Qi (58c):

(a) $\quad$ E $\quad$ three $\int^{\mathrm{h}} \mathrm{wu}: ?$

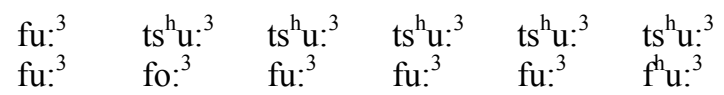

(b)

\begin{tabular}{|c|c|c|c|c|c|}
\hline 乳房 & breast & & *tci:h & & \\
\hline $\begin{array}{l}\text { tsi: }^{2} \\
\text { tscy }^{1}\end{array}$ & $\begin{array}{l}\text { tsi: }^{2} \\
\text { tscy }^{1}\end{array}$ & $\begin{array}{l}\left(\text { tsey }^{1}\right) \\
\text { tsey }^{1}\end{array}$ & $\begin{array}{l}\text { tsi: }^{5} \\
\text { tsey }^{1}\end{array}$ & $\begin{array}{l}\text { tsi: }^{5} \\
\text { tsi: }^{3}\end{array}$ & $\begin{array}{l}\text { tsi: }^{5} \\
\text { ti }^{3}\end{array}$ \\
\hline 母親 & mother & & *hmi:? & & \\
\hline $\begin{array}{l}\text { mey }^{3} \\
6 a y^{4}\end{array}$ & $\begin{array}{l}\left(\mathrm{mey}^{3}\right) \\
\text { may }^{1}\end{array}$ & pay $^{3}$ & $\begin{array}{l}\text { pi: }^{6} \\
\text { pay }^{3}\end{array}$ & $\begin{array}{l}\mathrm{p}^{\mathrm{h}} \mathrm{i}:{ }^{6} \\
\left(\mathrm{me}:^{1}\right)\end{array}$ & $\begin{array}{l}\text { pi: } \\
\left(\text { mey }^{6}\right)\end{array}$ \\
\hline
\end{tabular}

There is very little to note in the development of the non-high vowels. There do not seem to have been any changes correlated with tone category as in the case of the high vowels. The single change which occurred in the *a: rime category was the backing in Cunhua to 0 : 
The three reconstructions under comparison are presented below:

$$
\text { Thurgood Ostapirat (1993) Ostapirat (2004) }
$$

\begin{tabular}{|c|c|c|c|c|}
\hline (a) & $*_{\mathrm{ei}}$ & *әy & $* \mathrm{i}:$ & *i: \\
\hline (b) & *วu & *əu & $*_{\mathrm{i}}$ : & *u: \\
\hline (c) & *ou & *əw & $* \mathrm{u}:$ & $* \mathrm{u}:$ \\
\hline (d) & *ay & $*_{\text {ey }}$ & *ay & $* \mathrm{i}: \mathrm{h} / \mathrm{i}$ \\
\hline (e) & *au & $*_{\text {вu् }}$ & $* a l$ & *u:h/? \\
\hline (f) & $---/ *$ aw & $*_{-j-a w} / *_{0 w}$ & $*_{\mathrm{iw}} / *_{\mathrm{aw}}$ & *u:h/ ? \\
\hline g) & --- & --- & --- & *e: \\
\hline (h) & $*_{\mathrm{a}}$ & $*_{\mathrm{a}}$ & *a: & $*_{\mathrm{a}}:$ \\
\hline
\end{tabular}

In the case of the high vowels in category A, Thurgood reconstructs what I consider to be the final stage in the development of these vowels, although his reconstruction does obey the principle of Commonality. The recontruction in Ostapirat (1993) is what I consider to be an intermediate stage, where the vowels have already diphthongized but before the nucleus has been colored under the influence of the following glide. This seems a reasonable reconstruction at first glance given the reflexes in the daughter languages; however, it fails to account for the Bouhin data, which strongly indicate that pure vowels must be reconstructed at the PHl stage. Ostapirat (2004) reconstructs a system similar to the one presented here, the only difference $\left({ }^{*} \mathrm{i}:\right.$ vs. $\left.{ }^{*} \mathrm{u}:\right)$ being essentially notational.

Neither Thurgood nor Ostapirat distinguish the reflexes of the high vowels in categories B/C from those of the short mid diphthongs; this is almost certainly because the only language which can be used to distinguish between these two series is Bouhin, the witness of which is occasionally compromised because of its contact relationship with Ha Em. Ostapirat's (2004) reconstruction of *al is based on the data in Qian \& Wang (1951), in which they transcribe what I assume to have been Baisha *au as a:l; my assumption is that this transcription did not reflect a genuine $l$, but is in error due to confusion over the perception of final $u$. The reason for this is that Proto Kra-Dai final *1 is preserved in two other Kra-Dai languages: Saek, a Northern Tai language, and Laha, a Kra language. The following forms show cases in these languages where final $* 1$ is preserved, and their Hlai cognates: 


\begin{tabular}{|c|c|c|c|}
\hline (60) & $\underline{\text { Saek }}$ & Laha & PHl \\
\hline body louse & $\mathrm{ml} \varepsilon 1^{4}$ & $\mathrm{mdal}^{\mathrm{A} 2}$ & $* \mathrm{t}^{\mathrm{h}} \partial \mathrm{n}$ \\
\hline to fly & burl $^{1}$ & ---- & $*_{6 \text { in }}$ \\
\hline field dike & $\mathrm{yal}^{4}$ & ---- & *Cifłə:n \\
\hline be slippery & $\mathrm{mlul}^{5}$ & --- & *C-nu:nf \\
\hline stone & ri: $1^{2}$ & --- & $* \mathrm{tc}^{\mathrm{h}} \mathrm{i}: \mathrm{n}$ \\
\hline to teach & so: $1^{2}$ & --- & $*$ sun \\
\hline wasp & $\mathrm{t}^{\mathrm{h}} \mathrm{i}: 1^{4}$ & ---- & $* \mathrm{t}^{\mathrm{h}}$ in \\
\hline cast, sow & va: $1^{6}$ & ---- & *fə:nh \\
\hline (hard, solid, & ke: $1^{6}$ & ---- & $*_{\text {tc }}{ }^{\mathrm{h}} \partial: \mathrm{n}$ ) \\
\hline
\end{tabular}

In these examples, it is apparent that the regular reflex of Kra-Dai final $*_{1}$ is PHl final $*_{n}$. It is therefore untenable to suggest that the series reconstructed here as *u:h/? be reconstructed with a final lateral in Hlai.

Thurgood doesn't reconstruct anything for the rime I reconstruct as *u:f (which is also relatively rare, with only nine clear examples). Ostapirat (1993) reconstructs a diphthong preceded by a palatal glide for this series, with the understanding that the glide has 'colored' the diphthongs in Zandui and Baoting. Ostapirat (2004) reconstructs the diphthong *iw, based partially on Jiamao evidence, but doesn't offer the path by which it would have evolved into the reflexes of the daughter languages; this generally odd change violates Directionality.

Neither Thurgood nor Ostapirat reconstruct a PHI phoneme for the seventh series of correspondences, probably owing to the fact that it is very rare (only three examples, all given below). There is a consensus in reconstructing the final series as *a:.

Examples of the PHl glides are given below, in the following order:

\begin{tabular}{|c|c|c|c|}
\hline un & Ha Em & $\begin{array}{l}\text { Lhut } \\
\text { Ciiana }\end{array}$ & \\
\hline
\end{tabular}




\section{Examples of $\mathrm{PHI}$ open rimes}

(a)

\begin{tabular}{|c|c|c|c|c|c|c|c|c|c|c|c|}
\hline 榕樹 & banya & & $*_{\mathrm{ri}}$ & & & 被子 & comfor & & fi:? & & \\
\hline $\begin{array}{l}\text { rey }^{1} \\
\text { (huy }^{4} \text { ) }\end{array}$ & $\begin{array}{l}\text { gey }^{1} \\
\text { yey? }\end{array}$ & $\begin{array}{l}\text { gey }^{1} \\
\text { gey }^{4}\end{array}$ & $\begin{array}{l}\text { gey }^{4} \\
\text { xey }^{1}\end{array}$ & $\begin{array}{l}\text { hey }{ }^{4} \\
\text { xey }\end{array}$ & $\begin{array}{l}\text { hey }^{4} \\
\left(\text { tsey }^{4}\right)\end{array}$ & $\begin{array}{l}\text { pey }^{3} \\
\text { t } \theta a y^{3}\end{array}$ & $\begin{array}{l}\text { fay }^{3} \\
\text { fay }^{3}\end{array}$ & $\begin{array}{l}\text { fay }^{3} \\
\text { fay }^{3}\end{array}$ & $\begin{array}{l}\text { fay }^{3} \\
\text { fay }^{3}\end{array}$ & $\begin{array}{l}\text { fay }^{3} \\
\text { fay }^{3}\end{array}$ & $\begin{array}{l}\text { fay }^{3} \\
\left(\text { fuy }^{3}\right)\end{array}$ \\
\hline 火 & fire & & $*_{\mathrm{fi}}$ & & & 疥瘡 & small s & & C-ni:? & & \\
\hline$\left(\right.$ pey $\left.^{1}\right)$ & $\begin{array}{l}\text { fey }^{1} \\
\text { fey }^{1}\end{array}$ & $\begin{array}{l}\text { fey } \\
\text { fey }^{1}\end{array}$ & $\begin{array}{l}\text { fey } \\
\text { fey }^{1}\end{array}$ & $\begin{array}{l}\text { fey } \\
\text { fey }^{1}\end{array}$ & $\begin{array}{l}\text { fey } \\
\mathrm{f}^{\mathrm{h}} \mathrm{ey}^{1}\end{array}$ & ney ${ }^{3}$ & nay $^{3}$ & $\begin{array}{l}\text { nay }^{3} \\
\text { nay }^{3}\end{array}$ & $\begin{array}{l}\text { nay }^{3} \\
\text { nay }^{3}\end{array}$ & $\begin{array}{l}\text { nay }^{6} \\
\text { nay }^{3}\end{array}$ & $\begin{array}{l}\text { nay }^{3} \\
\text { nay }^{6}\end{array}$ \\
\hline 瘦 & thin & & ${ }^{*} \mathrm{C}-1 \mathrm{i}: ?$ & & & 哭 & cry & & C-ni:? & & \\
\hline$l e y^{3}$ & $l e y^{3}$ & $l e y^{3}$ & $1 e y^{3}$ & ley ${ }^{6}$ & $l e y^{3}$ & yey $^{3}$ & yay $^{3}$ & yay $^{3}$ & yay $^{3}$ & yay $^{6}$ & yay $^{3}$ \\
\hline $1 \varepsilon y^{3}$ & $1 \varepsilon y^{3}$ & $1 e y^{3}$ & $1 e y^{3}$ & $1 e y^{3}$ & $l e y^{6}$ & yay $^{3}$ & yay $^{3}$ & yay $^{3}$ & yay $^{3}$ & yay $^{3}$ & yay $^{6}$ \\
\hline (c) & & & *u: & & & (d) & & & *u:h/? & & \\
\hline 葉子 & leaf & & *6ur: & & & 乾涸 & dry & & $*^{\mathrm{h}} \mathrm{u}: \hat{h}$ & & \\
\hline бәщ ${ }^{1}$ & бәщ ${ }^{1}$ & бәщ ${ }^{1}$ & бәщ ${ }^{1}$ & бәч ${ }^{1}$ & бәщ ${ }^{1}$ & $\mathrm{k}^{\mathrm{h}} \partial \mathrm{u}^{2}$ & $\mathrm{k}^{\mathrm{h}} \mathrm{au}{ }^{2}$ & $\mathrm{k}^{\mathrm{h}} \mathrm{au}{ }^{2}$ & $\mathrm{k}^{\mathrm{h}} \mathrm{au}{ }^{5}$ & $\mathrm{k}^{\mathrm{h}} \mathrm{au}{ }^{5}$ & $\mathrm{k}^{\mathrm{h}} \mathrm{au} \mathrm{u}^{5}$ \\
\hline bow $^{1}$ & $6 \varepsilon w^{1}$ & 6әш ${ }^{1}$ & бәщ ${ }^{1}$ & бәш ${ }^{1}$ & бәш ${ }^{1}$ & $\mathrm{k}^{\mathrm{h}} \mathrm{0}:^{5}$ & $\mathrm{k}^{\mathrm{h}} \mathrm{aw}^{2}$ & $\mathrm{k}^{\mathrm{h}} \mathrm{au}{ }^{2}$ & $\mathrm{k}^{\mathrm{h}} \partial \mathrm{u}^{2}$ & $\mathrm{k}^{\mathrm{h}} \mathrm{au}{ }^{2}$ & $\mathrm{k}^{\mathrm{h}} \mathrm{au}{ }^{5}$ \\
\hline 要 & want & & *dur: & & & 矮 & short & & $* t^{h} w: ?$ & & \\
\hline Фәщ ${ }^{1}$ & dəщ $^{1}$ & dəu $^{1}$ & Һә૫ ${ }^{1}$ & бəщ ${ }^{1}$ & ---- & $\mathrm{t}^{\mathrm{h}} \partial \mathrm{u}^{3}$ & $\mathrm{t}^{\mathrm{h}} \mathrm{au}{ }^{3}$ & $\mathrm{t}^{\mathrm{h}} \mathrm{au}{ }^{3}$ & $\mathrm{t}^{\mathrm{h}} \mathrm{au}{ }^{3}$ & $\mathrm{t}^{\mathrm{h}} \mathrm{au}{ }^{3}$ & $\mathrm{t}^{\mathrm{h}} \mathrm{au} \mathrm{c}^{3}$ \\
\hline tsow $^{1}$ & $\mathrm{~d} \varepsilon:^{1}$ & dəщ ${ }^{1}$ & Һәщ ${ }^{1}$ & Һәщ ${ }^{1}$ & Һәщ ${ }^{1}$ & $\mathrm{t}^{\mathrm{h}} \mathrm{s} \mathrm{s}^{\mathrm{s}}$ & $\mathrm{t}^{\mathrm{h}} \mathrm{aw}^{3}$ & $\mathrm{t}^{\mathrm{h}} \mathrm{au}{ }^{3}$ & $\mathrm{t}^{\mathrm{h}} \partial \mathrm{u}^{3}$ & $\mathrm{t}^{\mathrm{h}} \mathrm{au}{ }^{3}$ & $\mathrm{t}^{\mathrm{h}} \mathrm{au}^{3}$ \\
\hline 女婿 & son-in & & *hlu: & & & 輕 & light & & ${ }^{*} \mathrm{k}^{\mathrm{h}} \mathrm{u}: ?$ & & \\
\hline Фəщ ${ }^{1}$ & фәщ ${ }^{1}$ & фәщ ${ }^{1}$ & фәщ ${ }^{1}$ & фәщ ${ }^{1}$ & фәщ ${ }^{1}$ & $\mathrm{k}^{\mathrm{h}} \partial \mathrm{u}^{3}$ & $\mathrm{k}^{\mathrm{h}} \mathrm{au}{ }^{3}$ & $\mathrm{k}^{\mathrm{h}} \mathrm{au}{ }^{3}$ & $\mathrm{k}^{\mathrm{h}} \mathrm{au}{ }^{3}$ & $\mathrm{k}^{\mathrm{h}} \mathrm{au}{ }^{3}$ & $\mathrm{k}^{\mathrm{h}} \mathrm{au}{ }^{3}$ \\
\hline t $\theta$ ow $^{1}$ & --- & Һәщ ${ }^{1}$ & фәщ ${ }^{1}$ & фәщ ${ }^{1}$ & фәщ ${ }^{1}$ & $\mathrm{k}^{\mathrm{h}} \mathrm{o}:^{{ }^{\mathrm{t}}}$ & $\mathrm{k}^{\mathrm{h}} \mathrm{aw}^{3}$ & $\mathrm{k}^{\mathrm{h}} \mathrm{au}{ }^{3}$ & $\mathrm{k}^{\mathrm{h}} \partial \mathrm{u}^{3}$ & $\mathrm{k}^{\mathrm{h}} \mathrm{au}{ }^{3}$ & $\mathrm{k}^{\mathrm{h}} \mathrm{au}{ }^{3}$ \\
\hline
\end{tabular}




\begin{tabular}{|c|c|c|c|c|c|c|c|c|c|c|c|}
\hline (e) & & & *u: & & & (f) & & & *u:h & & \\
\hline 斑鳩 & dove, pi & geon & ${ }^{*} \mathrm{k}^{\mathrm{h}} \mathrm{u}$ : & & & 沙 & sand & & ${ }^{*} p^{h} u: h$ & & \\
\hline $\mathrm{k}^{\mathrm{h}} \mathrm{ow}{ }^{1}$ & $\mathrm{k}^{\mathrm{h}} \mathrm{ow}^{1}$ & $\mathrm{k}^{\mathrm{h}} \mathrm{ow}^{1}$ & $\mathrm{k}^{\mathrm{h}} \mathrm{ow}^{1}$ & $\mathrm{k}^{\mathrm{h}} \mathrm{ow}^{1}$ & $\mathrm{k}^{\mathrm{h}} \mathrm{ow}^{1}$ & $\left(\mathrm{p}^{\mathrm{h}} \mathrm{aw}{ }^{2}\right)$ & $\mathrm{p}^{\mathrm{h}} \mathrm{aw}^{2}$ & $\mathrm{p}^{\mathrm{h}} \mathrm{ow}^{2}$ & $\mathrm{p}^{\mathrm{h}} \mathrm{aw}{ }^{5}$ & $\mathrm{p}^{\mathrm{h}} \mathrm{o}::^{5}$ & $\mathrm{p}^{\mathrm{h}} \mathrm{s}^{5}$ \\
\hline$\left(\mathrm{k}^{\mathrm{h}} \mathrm{ow}^{5}\right)$ & $\mathrm{k}^{\mathrm{h}} \varepsilon \mathrm{w}^{1}$ & $\mathrm{k}^{\mathrm{h}} \mathrm{ow}^{1}$ & $\mathrm{k}^{\mathrm{h}} \mathrm{ow}^{1}$ & $\mathrm{k}^{\mathrm{h}} \mathrm{ow}^{1}$ & $\mathrm{k}^{\mathrm{h}} \mathrm{ow}^{1}$ & $\mathrm{p}^{\mathrm{h}} \mathrm{a}:{ }^{5}$ & $\mathrm{p}^{\mathrm{h}} \mathrm{aw}^{2}$ & $\mathrm{p}^{\mathrm{h}} \mathrm{aw}^{2}$ & $\mathrm{p}^{\mathrm{h}} \mathrm{aw}^{2}$ & $\mathrm{p}^{\mathrm{h}} \mathrm{aw}^{2}$ & $\mathrm{p}^{\mathrm{h}} \mathrm{aw}^{5}$ \\
\hline 跟 & with & & *Curu: & & & 年 & year & & *hmu:h & & \\
\hline row $^{1}$ & row $^{1}$ & vow $^{1}$ & fow $^{4}$ & ---- & $\left(\right.$ vow $\left.^{1}\right)$ & mow $^{2}$ & paw $^{2}$ & pow $^{2}$ & paw $^{2}$ & $\mathrm{p}^{\mathrm{h}} \mathrm{o}:^{2}$ & po: $:^{2}$ \\
\hline vow $^{4}$ & $\left(\eta \varepsilon w 2^{4}\right)$ & gow $^{4}$ & yow $^{1}$ & $\left(\right.$ fow $\left.^{4}\right)$ & fow $^{1}$ & $6 a:^{5}$ & paw $^{2}$ & paw $^{2}$ & paw $^{2}$ & paw $^{2}$ & paw $^{2}$ \\
\hline 黃蜂 & wasp & & *p-lu: & & & 睡 & sleep (lie & & *hyu:h & & \\
\hline low $^{1}$ & low $^{1}$ & plow $^{1}$ & plow $^{1}$ & pow $^{1}$ & plow $^{1}$ & now $^{2}$ & $\mathrm{kaw}^{2}$ & $\mathrm{kaw}^{2}$ & $\mathrm{kaw}^{2}$ & $\mathrm{k}^{\mathrm{h}} \mathrm{o}:{ }^{2}$ & $\mathrm{ko}::^{2}$ \\
\hline $\operatorname{low}^{4}$ & --- & pow $^{1}$ & plow $^{1}$ & plow $^{1}$ & plow $^{1}$ & $\mathrm{ka}:^{5}$ & $\mathrm{kaw}^{2}$ & $\mathrm{kaw}^{2}$ & $\mathrm{kaw}^{2}$ & $\mathrm{kaw}^{2}$ & $\mathrm{kaw}^{2}$ \\
\hline (g) & & & ${ }^{*} \mathbf{u}: ?$ & & & (h) & & & *e: & & \\
\hline 刺 & prick (fi & nger) & ${ }^{*} p^{h} u: ?$ & & & 鵝 & goose & & ${ }^{*}$ C-ye: & & \\
\hline$\left(\mathrm{p}^{\mathrm{h}} \mathrm{aw}{ }^{3}\right)$ & $\mathrm{p}^{\mathrm{h}} \mathrm{aw}^{3}$ & $\mathrm{p}^{\mathrm{h}} \mathrm{ow}^{3}$ & $\mathrm{p}^{\mathrm{h}} \mathrm{aw}^{3}$ & $\mathrm{p}^{\mathrm{h}} \mathrm{aw}^{3}$ & $\mathrm{p}^{\mathrm{h}} \mathrm{ow}^{3}$ & ye: ${ }^{1}$ & ye: ${ }^{1}$ & ye: ${ }^{1}$ & ---- & ---- & ---- \\
\hline $\mathrm{p}^{\mathrm{h}} \mathrm{ay}^{3}$ & $\mathrm{p}^{\mathrm{h}} \mathrm{aw}^{3}$ & $\mathrm{p}^{\mathrm{h}} \mathrm{aw}^{3}$ & $\mathrm{p}^{\mathrm{h}} \mathrm{aw}{ }^{3}$ & $\mathrm{p}^{\mathrm{h}} \mathrm{aw}^{3}$ & $\mathrm{p}^{\mathrm{h}} \mathrm{aw}^{3}$ & ---- & ---- & ye: ${ }^{1}$ & ye: ${ }^{1}$ & ---- & ---- \\
\hline 殺 & kill & & *hu:? & & & 背着手 & hands in & back & *p-le: & & \\
\hline how $^{3}$ & haw $^{3}$ & haw $^{3}$ & haw $^{3}$ & haw $^{3}$ & haw $^{3}$ & ---- & ---- & ple: ${ }^{1}$ & ple: ${ }^{1}$ & pe: ${ }^{1}$ & ple: ${ }^{1}$ \\
\hline & haw $^{3}$ & haw $^{3}$ & haw $^{3}$ & haw $^{3}$ & haw $^{3}$ & ---- & ---- & ---- & ple: ${ }^{1}$ & ple: ${ }^{1}$ & ple: $^{1}$ \\
\hline 二 & two & & *hlu:? & & & 糍粑 & rice cake & & *C-ne:h & & \\
\hline dow $^{3}$ & faw ${ }^{3}$ & taw ${ }^{3}$ & taw ${ }^{3}$ & taw $^{3}$ & $\mathrm{daw}^{3}$ & ---- & ne: ${ }^{2}$ & ---- & ne: ${ }^{5}$ & $\left(\right.$ ne: $\left.:^{5}\right)$ & ne: ${ }^{5}$ \\
\hline t $\theta a:(y)^{3}$ & law ${ }^{3}$ & $\mathrm{faw}^{3}$ & $\mathrm{daw}^{3}$ & $\mathrm{daw}^{3}$ & $\mathrm{faw}^{3}$ & ---- & ---- & ---- & ---- & ne: ${ }^{2}$ & ---- \\
\hline
\end{tabular}




\begin{tabular}{|c|c|c|c|c|c|}
\hline (i) & & & *a: & & \\
\hline 厚 & thick & & ${ }^{*} \mathrm{C}$-na: & & \\
\hline $\begin{array}{l}\text { na: }{ }^{1} \\
\text { nə: }\end{array}$ & $\begin{array}{l}\text { na: }{ }^{1} \\
\text { na: }{ }^{1}\end{array}$ & $\begin{array}{l}\text { na: }{ }^{1} \\
\text { na: }{ }^{1}\end{array}$ & $\begin{array}{l}\text { na: }{ }^{1} \\
\text { na: }{ }^{1}\end{array}$ & $\begin{array}{l}\text { na: }{ }^{4} \\
\text { na: }{ }^{1}\end{array}$ & $\begin{array}{l}\text { na: }{ }^{1} \\
\text { na: }\end{array}$ \\
\hline 眼睛 & eye & & $* \mathrm{t}^{\mathrm{h}} \mathrm{a}:$ & & \\
\hline $\begin{array}{l}\text { ts }^{\mathrm{h}} \mathrm{a}^{1}{ }^{2} \\
\mathrm{~h}:^{1}\end{array}$ & $\begin{array}{l}\text { ts }^{\mathrm{h}} \mathrm{a}:{ }^{1} \\
\text { ha: }\end{array}$ & $\begin{array}{l}\text { ts } \mathrm{ts}^{\mathrm{h}} \mathrm{a}:{ }^{1} \\
\text { ts } \mathrm{h} a:{ }^{1}\end{array}$ & $\begin{array}{l}\text { ts }{ }^{\mathrm{h}} \mathrm{a}:{ }^{1} \\
\text { ts } \mathrm{h} a:{ }^{1}\end{array}$ & $\begin{array}{l}\text { ts }{ }^{\mathrm{h}} \mathrm{a}:{ }^{1} \\
\text { ts } \mathrm{h} a:{ }^{1}\end{array}$ & $\begin{array}{l}\text { ts }^{\mathrm{h}} \mathrm{a}:{ }^{1} \\
\text { ts }{ }^{\mathrm{h}} \mathrm{a}:{ }^{1}\end{array}$ \\
\hline 種 & to plant & & *Cura: & & \\
\hline $\mathrm{ra}:{ }_{4}^{1}$ & $\begin{array}{l}\left(\mathrm{ra}:{ }^{1}\right) \\
\mathrm{va}^{4}{ }^{4}\end{array}$ & $\begin{array}{l}\text { gwa: }{ }^{1} \\
\text { ko: }\end{array}$ & $\begin{array}{l}\text { gwa: }{ }^{4} \\
\text { ya: }{ }^{1}\end{array}$ & $\begin{array}{l}\left(\mathrm{va}:{ }^{4}\right) \\
\text { va: }{ }^{1}\end{array}$ & $\begin{array}{l}\text { hwa: }{ }^{4} \\
\text { va: }{ }^{1}\end{array}$ \\
\hline
\end{tabular}

\subsubsection{Interim Summary}

The reconstruction of five open rimes is possible, with the front mid vowel *e: being marginal:

$\begin{array}{ll}\text { (62) } \quad *_{\mathrm{i}}: & * \mathrm{ur}: \quad * \mathrm{u}: \\ & \left({ }_{\mathrm{e}}:\right)\end{array}$

(*e:) $\quad *_{\mathrm{a}}$ :

The high vowels have undergone parallel developments in category A, with the original high tending towards diphthongization; those in categories B and C developed differently in Bouhin and Greater Hlai, however, and it is this asymmetry between the two highest branches of Hlai which allows the original symmetry of the vowel inventory to be recovered. Due to its low frequency, the front mid vowel is considered to be of probable secondary origin, implying an original four-vowel system which consisted of three high vowels and one low vowel. This type of four-vowel system, while not typologically common, is nevertheless attested in other synchronic language systems (Maddieson 1984: 126). 


\subsection{Closed Rimes with High Vowels}

To make the treatment of the closed rimes more manageable, they will be divided into those with high nuclei in the present section and those with non-high nuclei in section 4.5. In addition, those rimes in this section will be divided between high front nuclei (4.4.1), high back unrounded nuclei (4.4.2), and high back rounded nuclei (4.4.3).

There are two series of closed rimes with high nuclei. The general pattern which exists is an opposition between short high versus short mid vowels in $\mathrm{NCHl}$, as opposed to short versus long high vowels in other Hlai:

(a) $\underline{\mathrm{NCHl}}$ :

$\begin{array}{lll}\mathrm{iC} & \mathrm{vs} & \mathrm{eC} \\ \mathrm{wC} & \mathrm{vs} & \mathrm{rC} \\ \mathrm{uC} & \mathrm{vs} & \mathrm{oC}\end{array}$

(b) Other Hlai:

$\begin{array}{lll}\text { i:C } & \text { vs } & \text { iC } \\ \text { u:C } & \text { vs } & \text { uC } \\ \text { u:C } & \text { vs } & \text { uC }\end{array}$

The two choices for reconstruction are between an original opposition between high and mid vowels (where $\mathrm{NCHl}$ would be conservative), and an original opposition in vowel length of high vowels (where other Hlai would be conservative). I have chosen the latter, for two reasons. The first is that of the various branches of Hlai, $\mathrm{NCHl}$ has undergone considerably more contact with the Non-Hlai languages of Hainan, none of which have an opposition in vowel length to the best of my knowledge. This would therefore be an exotic feature which may be expected to be modified under the pressure of language contact. The second reason is the pattern in (63a) is restricted to a specific subgroup, making it very likely that this was an innovation at the level of Proto-NCHl. In fact, the Baisha data in Qian \& Wang (1951) strongly suggest that the change in (63a) above was the result of a chain shift, as the patterns in high vowel rimes they record follow the following pattern:

$$
\begin{array}{lll}
\text { i:C } & \text { vs } & \text { eC } \\
\text { uC } & \text { vs } & \text { œC } \\
\text { u:C } & \text { vs } & \text { oC }
\end{array}
$$


In other words, it appears as though the short high rimes first lowered, and that the long high rimes only shortened afterward (apparently beginning with *u:C). It may therefore be suggested that only the short rime lowering occurred at the level of Proto- $\mathrm{NCHl}$, and that long rime shortening proceeded on an individual basis after the breakup of $\mathrm{NCHl}$ into daughter branches.

\subsubsection{PHI Rimes with High Front Nuclei}

There are altogether fourteen series of correspondences which I reconstruct with high front nuclei, seven long and seven short; their correspondences are given below:

Reflexes of rimes with high front nuclei

\begin{tabular}{|c|c|c|c|c|c|c|c|c|c|c|c|}
\hline BHi:n & HaE:m & $\underline{\mathrm{LHu}}: \mathrm{t}$ & Tzha & $\underline{\text { Zdui }}$ & Bting & Cun & $\underline{\text { Nadou }}$ & Cjiang & Mfaw & Baisha & $\underline{\text { Ymen }}$ \\
\hline i:w & i:w & i:w & i:w & iw & i:w & iw & i: & iw & iw & iw & iw \\
\hline i:m & i:m & i:m & i:m & $\mathrm{i}: \mathrm{m}$ & $\mathrm{i}: \mathrm{m}$ & $\mathrm{im}$ & in & $\mathrm{im}$ & $\mathrm{im}$ & $\mathrm{im}$ & im \\
\hline i:n & i:n & i:n & i:n & i:n & i:n & in & in & in & in & in & in \\
\hline i:y & i:n & $\mathrm{i}: \mathrm{y}$ & ian & iay & $\operatorname{ian}$ & iəy & in & in & in & in & in \\
\hline i:p & $i: p$ & $i: p$ & i:p & i:p & i:p & ip & i? & ip & ip & ip & ip \\
\hline $\mathrm{i}: \mathrm{t}$ & $\mathrm{i}: \mathrm{t}$ & $\mathrm{i}: \mathrm{t}$ & i:t & $\mathrm{i}: \mathrm{t}$ & $\mathrm{i}: \mathrm{t}$ & it & i? & it & it $\sim \mathrm{ik}$ & it & it \\
\hline $\mathrm{i}: ?$ & $\mathrm{i}: ?$ & $\mathrm{i}: \mathrm{k}$ & ia? & ia? & iak & iək & i? & i? & $\mathrm{ik}$ & it & i? \\
\hline iw & iw & iw & iw & iw & iw & әу & iw & iw & iw & ew & i:w \\
\hline $\mathrm{im}$ & $\mathrm{im}$ & $\mathrm{im}$ & $\mathrm{im}$ & $\mathrm{im}$ & im & em & $\varepsilon \mathrm{n}$ & em & em & em & em \\
\hline in & in & ej & en & en & in & en & en & en & en & en & en \\
\hline in & in & in & in & in & in & en & en & en & en & en & en \\
\hline ip & ip & ip & ip & ip & ip & ep & e? & ep & ep & ep & ep \\
\hline it & it & ec & et & et & it & et & e? & et & et & et & et \\
\hline ik & ik & ik & $\mathrm{i}: ?$ & $\mathrm{i}: ?$ & $\mathrm{ik}$ & --- & --- & --- & $\mathrm{ik}$ & --- & et \\
\hline
\end{tabular}


S. Hlai (Savina)
iw
iem
ien
ien
iep
iet
i:
iw
in
$----$
it
ek

C. Hlai (Savina)

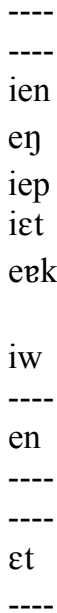

Baisha (Wang \& Qian)

iw

i:m

i:y

i:y

i:p

i:t

i:t

eW

en

(i:p)

et

The reconstructions I propose here are therefore the following:

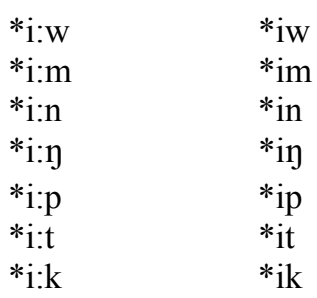

The reconstruction of these rimes is readily constrained by the principle of Symmetry, as is true for all of the other classes of closed rimes.

\subsubsection{Long Rimes with High Front Vowels}

As discussed above, long rimes in NCHl languages shortened:

$* \mathrm{i}: \mathrm{C}>\mathrm{iC}$

There is some evidence that the diphthongs in $\mathrm{NCHl}$ were exempted from this otherwise pervasive pattern. The Nadouhua reflex of PHl *i:w is $i$ : with the final glide lost after a long nucleus but not after a short one. In addition, the reversal of features in the Cunhua short diphthong may have occurred at the time 
when the nucleus was still high, allowing the originally long diphthong to shorten only after this change had occurred. In addition, the Meifu short diphthong seems to have not undergone vowel lowering, leading to a merger between the long and short series. These scenarios are shown below:

$\begin{array}{llllll}\text { Cunhua: } & \text { i:w } & > & \text { i:w } & > & \text { iw } \\ & \text { iw } & > & \text { uy } & > & \text { oy } \\ \text { Nadouhua: } & \text { i:w } & > & \text { i: } & & \\ & \text { iw } & > & \text { iw } & & \\ \text { Meifu: } & \text { i:w } & > & \text { iw } & & \\ & \text { iw } & > & \text { iw } & & \end{array}$

In Zandui, the long diphthong merged with its short counterpart as it did in the Meifu branch:

$\begin{array}{llll}\text { Zandui } & \text { i:w } & > & \text { iw } \\ & \text { iw } & > & \text { iw }\end{array}$

There were other changes in stop-closed rimes. In the Qi branch, there was a diphthongization in the rimes with velar finals, where original long $* \mathrm{i}$ : broke first to $i$, and then underwent schwa lowering to ia:

$\begin{array}{lllll}* \mathrm{i}: \mathrm{y} & > & \text { iə刀 } & > & \text { ia } \\ *_{\mathrm{i}: \mathrm{k}} & > & \text { iək } & > & \text { iak }\end{array}$

The nuclei of Cunhua rimes with velar codas also diphthongized to iə, following the general Hlai dispreference for short rimes in this environment:

$\begin{array}{lll}* \mathrm{i}: \mathrm{y} & > & \text { iə̀ } \\ * \mathrm{i}: \mathrm{k} & > & \text { iək }\end{array}$

In Nadouhua, there was an across-the-board merger of labial codas with alveolar codas; in addition, all final stops debuccalized to a glottal stop: 
(72)

$\begin{array}{lll}*_{\text {im }} & > & \text { in } \\ *_{\text {in }} & > & \text { in } \\ *_{\text {in }} & > & \text { in } \\ *_{\text {ip }} & > & \text { i? } \\ *_{\text {it }} & > & \text { i? } \\ *_{\text {ik }} & > & \text { i? }\end{array}$

There was a merger of the alveolar nasal with the velar in Moyfaw:

$*$ in $>$ in

Finally, Baisha underwent an interesting cross-merger, where (as in Meifu) the alveolar nasal merged with the velar, but where the velar stop merged with the alveolar, under the influence of the preceding vowel:

$\begin{array}{lll}* \text { in } & > & \text { in } \\ *_{\text {ik }} & > & \text { it }\end{array}$

\subsubsection{Short Rimes with High Front Vowels}

There were a number of individual developments of short rimes in the Hlai subgroups and daughter languages. The lowering to mid-vowels in $\mathrm{NCHl}$ mentioned earlier was universal:

$*_{\mathrm{iC}}>\mathrm{eC}$

A similar but more restricted change occurred in Lauhut, where alveolar-final rimes became palatalized as the vowel itself lowered to $e$ :

$\begin{array}{lll}* \text { in } & > & \text { en } \\ * \text { it } & > & \text { ec }\end{array}$

The Tongzha and Zandui alveolar-final rimes also lowered to $e$, while *ik lengthened to $i: k$, filling the gap left by original $* \mathrm{i}: \mathrm{k}$ (shown in (77) for comparison): 
(77)

$\begin{array}{lllll}*_{\text {in }} & > & \text { en } & & \\ *_{\text {it }} & > & \text { et } & & \\ & & & & \\ *_{\text {ik }} & > & \text { i:k } & > & \text { i:? } \\ *_{\text {i:k }} & > & \text { iək } & > & \text { ia? }\end{array}$

There appears to have been raising of the vowel in Moyfaw before the velar as well (there are no Changjiang cognates, so it cannot be determined if this occurred at the level of the Meifu branch or not). Since there are only two examples, however, it should be kept in mind that these may have been loans from a Lauhut: ek $>$ ik

A merger of velar final rimes with alveolar final rimes occurred in NECHI: ey $>$ en

This was mirrored by the same merger of the stops in Yuanmen:
ek $>$
et

Finally, the Yuanmen diphthong lengthened, creating an environment in which raising occurred (see section 4.5.1):

ew $>$ e:w $>$ i:w

A comparison of reconstructions is given below: 
Thurgood Ostapirat (1993) Ostapirat (2004) $\underline{\mathrm{PHl}}$

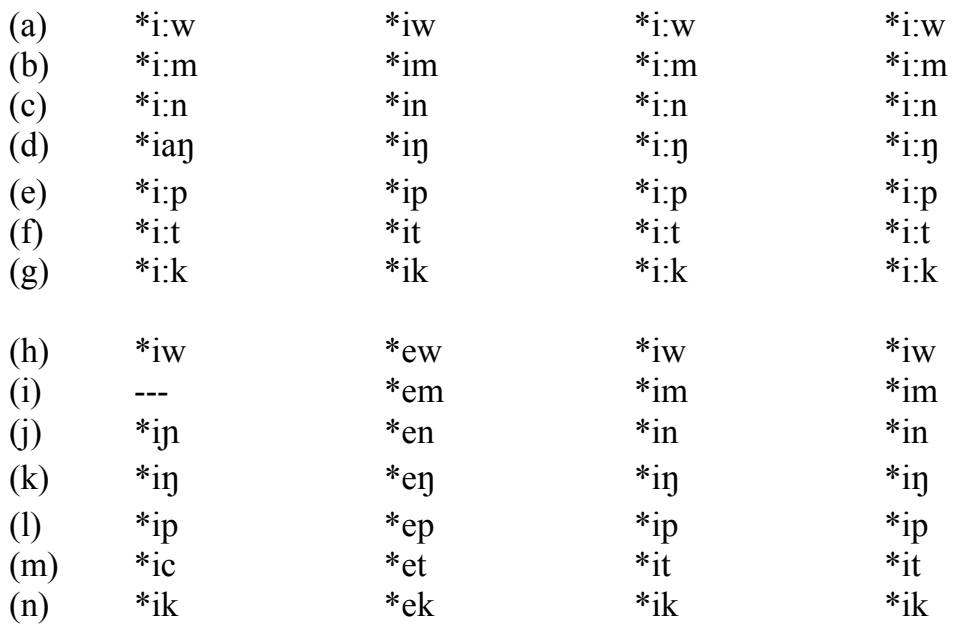

In general, Thurgood's reconstruction agrees with the present one in positing a length distinction.

There are three differences between Thurgood's reconstruction and the present one. The first is that he reconstructs *iay in (82d), based on the Qi evidence. I consider this a secondary development, and it is also at odds with the fact that he reconstructs *i:k instead of *iak for the sixth series, violating Symmetry. The second difference is that he does not reconstruct anything in (82i). Finally, he reconstructs $* \mathrm{in}$ and $*$ ic in $(82 \mathrm{j})$ and $(82 \mathrm{~m})$, whereas I consider the palatalization of the codas under the influence of the vowel to be a secondary development in Lauhut.

Ostapirat's (2004) reconstruction agrees with the present one in positing a length distinction, whereas Ostapirat (1993) reconstructed a distinction between high and mid nuclei. Given their individual assumptions, Ostapirat's two reconstructions are both straightforward.

Examples of the PHl closed rimes with high front nuclei are given below, in the following order:

$\begin{array}{llllll}\text { Bhin } & \text { Ha Em } & \text { Lhut } & \text { Tzha } & \text { Zdui } & \text { Bting } \\ \text { Cun } & \text { Nadou } & \text { Cjiang } & \text { Mfaw } & \text { Baisha } & \text { Ymen }\end{array}$


Examples of PHl closed rimes with high front nuclei

(a)

\begin{tabular}{|c|c|c|c|c|c|c|c|c|c|c|c|}
\hline 藍色 & blue & & ${ }^{*} \mathrm{k}^{\mathrm{h}} \mathrm{i}: \mathrm{w}$ & & & 兒媳/妻 & 子 $\mathrm{dtr}$ in & law/wif & $*$ C-liw & & \\
\hline $\mathrm{k}^{\mathrm{h}} \mathrm{i}: \mathrm{w}^{1}$ & $\mathrm{k}^{\mathrm{h}} \mathrm{i}: \mathrm{w}^{1}$ & $\mathrm{k}^{\mathrm{h}} \mathrm{i}: \mathrm{w}^{1}$ & $\mathrm{k}^{\mathrm{h}} \mathrm{i}: \mathrm{w}^{1}$ & $\mathrm{k}^{\mathrm{h}} \mathrm{iw}^{1}$ & $\mathrm{k}^{\mathrm{h}} \mathrm{i}: \mathrm{w}^{1}$ & $\operatorname{liw}^{1}$ & $\operatorname{liw}^{1}$ & $\operatorname{liw}^{1}$ & $\operatorname{liw}^{1}$ & $\operatorname{liw}^{4}$ & liw $^{1}$ \\
\hline $\mathrm{k}^{\mathrm{h}} \mathrm{iw}^{1}$ & $\mathrm{k}^{\mathrm{h}} \mathrm{i}:{ }^{1}$ & $\mathrm{k}^{\mathrm{h}} \mathrm{iw}^{1}$ & $\mathrm{k}^{\mathrm{h}} \mathrm{iw}^{1}$ & $\mathrm{k}^{\mathrm{h}} \mathrm{iw}^{1}$ & $\mathrm{k}^{\mathrm{h}} \mathrm{iw}^{1}$ & $10 y^{1}$ & $\operatorname{liw}^{1}$ & $\operatorname{liw}^{1}$ & $\operatorname{liw}^{1}$ & lew ${ }^{1}$ & li:w ${ }^{4}$ \\
\hline 貓 & cat & & ${ }^{*} \mathrm{C}-\mathrm{mi}: \mathrm{v}$ & & & 老鼠 & mouse & & *hniw & & \\
\hline mi:w ${ }^{2}$ & mi:w ${ }^{2}$ & mi:w ${ }^{2}$ & mi: $w^{5}$ & $\operatorname{miw}^{2}$ & mi:w & niw $^{1}$ & tiw $^{1}$ & tiw $^{1}$ & tiw $^{4}$ & $\mathrm{t}^{\mathrm{h}} \mathrm{iw}^{4}$ & tiw $^{4}$ \\
\hline $\mathrm{miw}^{5}$ & & $\operatorname{miw}^{2}$ & $\operatorname{miw}^{2}$ & $\left(\operatorname{miw}^{1}\right)$ & $\operatorname{miw}^{2}$ & tsoy ${ }^{4}$ & $\operatorname{tiw}^{4}$ & tiw $^{4}$ & tiw $^{1}$ & tew ${ }^{1}$ & $\mathrm{ti}: \mathrm{w}^{4}$ \\
\hline 賣 & sell & & *ri:w? & & & 尖刀 & & & *ljiw? & & \\
\hline ri: $w^{3}$ & gi: $w^{3}$ & ---- & gi: $w^{6}$ & hiw $^{6}$ & gi: ${ }^{6}$ & ziw $^{3}$ & ziw $^{3}$ & $\mathrm{ziw}^{3}$ & $\operatorname{tiw}^{6}$ & iww $^{6}$ & iww $^{6}$ \\
\hline hiw $^{4}$ & $\mathrm{zi}^{3}$ & $\operatorname{giw}^{4}$ & $\mathrm{xiw}^{3}$ & $\mathrm{xiw}^{3}$ & $\mathrm{k}^{\mathrm{h}} \mathrm{iw}^{3}$ & ---- & $\left(\mathrm{zcw}^{3}\right)$ & $\operatorname{liw}^{3}$ & ziw $^{3}$ & zew $^{3}$ & tsi: $w^{6}$ \\
\hline (c) & & & $* \mathrm{i}: \mathrm{m}$ & & & (d) & & & *im & & \\
\hline 塞 & plug up & & ${ }^{*} \mathrm{tc}^{\mathrm{h}} \mathrm{i}: \mathrm{m}$ & & & 嘗 & taste & & $*{ }^{\mathrm{t}} \varphi^{\mathrm{h}} \mathrm{im}$ & & \\
\hline ---- & ---- & $\mathrm{ts}^{\mathrm{h}} \mathrm{i}: \mathrm{m}^{1}$ & $\mathrm{ts}^{\mathrm{h}} \mathrm{i}: \mathrm{m}^{1}$ & $\mathrm{ts}^{\mathrm{h}} \mathrm{i}: \mathrm{m}^{1}$ & $\mathrm{ts}^{\mathrm{h}} \mathrm{i}: \mathrm{m}^{1}$ & $\operatorname{ts}^{\mathrm{h}} \mathrm{im}^{1}$ & $\operatorname{ts}^{\mathrm{h}} \mathrm{im}^{1}$ & $\operatorname{ts}^{\mathrm{h}} \mathrm{im}^{1}$ & $\left(\mathrm{ts}^{\mathrm{h}} \mathrm{em}^{1}\right)$ & $\mathrm{ts}^{\mathrm{h}} \mathrm{im}^{1}$ & ts $^{\mathrm{h}}{ }^{\mathrm{im}}{ }^{1}$ \\
\hline--- & $\sin ^{1}$ & $\left(\mathrm{ts}^{\mathrm{h}} \mathrm{im}^{2}\right)$ & ts $^{\mathrm{h}} \mathrm{i}: \mathrm{m}^{1}$ & $\mathrm{ts}^{\mathrm{h}} \mathrm{im}^{1}$ & $\mathrm{ts}^{\mathrm{h}} \mathrm{im}^{1}$ & ---- & ---- & ---- & $\mathrm{ts}^{\mathrm{h}} \mathrm{em}^{1}$ & $\mathrm{ts}^{\mathrm{h}} \mathrm{em}^{1}$ & $\mathrm{ts}^{\mathrm{h}} \mathrm{em}$ \\
\hline 舔 & lick & & *lji:mh & & & 項圈 & necklet & & ${ }^{*} \mathrm{k}^{\mathrm{h}} \mathrm{im}$ & & \\
\hline $\mathrm{zi}: \mathrm{m}^{2}$ & $\mathrm{zi}: \mathrm{m}^{2}$ & $\mathrm{zi}: \mathrm{m}^{2}$ & di: $\mathrm{m}^{2}$ & ---- & di: $\mathrm{m}^{2}$ & $\mathrm{k}^{\mathrm{h}} \mathrm{im}^{1}$ & $\mathrm{k}^{\mathrm{h}_{\mathrm{im}}}{ }^{1}$ & $\mathrm{k}^{\mathrm{h}_{\mathrm{im}}}{ }^{1}$ & $\mathrm{k}^{\mathrm{h}} \mathrm{im}^{1}$ & ---- & ---- \\
\hline$\left(1 \varepsilon \mathrm{m}^{5}\right)$ & $\left(\operatorname{lin}^{2}\right)$ & $\lim ^{2}$ & $\operatorname{nim}^{2}$ & $\operatorname{nim}^{2}$ & ---- & ---- & ---- & ---- & ---- & $\mathrm{k}^{\mathrm{h}} \mathrm{em}^{1}$ & ---- \\
\hline 分贈 & give as $\mathrm{g}$ & & *ri:m? & & & 禁母/方 & 疊 witch & /poison & *kim? & & \\
\hline$($ gi:m³ $)$ & gi:m³ & gi:m³ & $\begin{array}{l}\text { gi:m } \\
x^{6} i^{3}\end{array}$ & $\begin{array}{l}\text { hi:m6 } \\
\text { xim }^{6}\end{array}$ & $\begin{array}{l}\text { gi:m } \\
\mathrm{k}^{\mathrm{h}} \mathrm{im}^{3}\end{array}$ & $\begin{array}{l}\mathrm{kim}^{3} \\
\mathrm{kem}^{3}\end{array}$ & $\mathrm{kim}^{3}$ & $\begin{array}{l}\mathrm{kim}^{3} \\
\mathrm{kem}^{3}\end{array}$ & $\begin{array}{l}\mathrm{kim}^{3} \\
\mathrm{kem}^{3}\end{array}$ & $\begin{array}{l}\mathrm{kim}^{3} \\
\mathrm{kem}^{3}\end{array}$ & $\begin{array}{l}\mathrm{kim}^{3} \\
\mathrm{kem}^{3}\end{array}$ \\
\hline
\end{tabular}




\begin{tabular}{|c|c|c|c|c|c|c|c|c|c|c|c|}
\hline (e) & & & $* \mathbf{i}: \mathbf{n}$ & & & (f) & & & $*$ in & & \\
\hline 錢 & money & & $*_{\text {tci:n }}$ & & & 飛 & fly & & $* 6$ in & & \\
\hline tsi: ${ }^{1}$ & tsi: ${ }^{1}$ & tsi: ${ }^{1}$ & tsi:n ${ }^{1}$ & tsi: ${ }^{1}$ & tsi: ${ }^{1}$ & $\operatorname{bin}^{1}$ & $\operatorname{6in}^{1}$ & $6 e n^{1}$ & $6 \mathrm{en}^{1}$ & $6 e n^{1}$ & $\operatorname{6in}^{1}$ \\
\hline $\mathrm{t} \theta \mathrm{in}^{1}$ & $\operatorname{tsin}^{1}$ & $\operatorname{tsin}^{1}$ & $\operatorname{tsin}^{1}$ & $\operatorname{tsin}^{1}$ & $\operatorname{tin}^{1}$ & $6 \mathrm{en}^{1}$ & $6 \mathrm{en}^{1}$ & $6 e n^{1}$ & $\operatorname{ben}^{1}$ & $6 e n^{1}$ & $6 \mathrm{en}^{1}$ \\
\hline 石頭 & stone & & ${ }^{*} \mathrm{tc}_{\varphi}^{\mathrm{h}} \mathrm{i}: \mathrm{n}$ & & & 答應 & agree & & $* \mathrm{t}^{\mathrm{h}}$ in & & \\
\hline ts $^{\mathrm{h}} \mathrm{i}: \mathrm{n}^{1}$ & $\mathrm{ts}^{\mathrm{h}} \mathrm{i}: \mathrm{n}^{1}$ & $\mathrm{ts}^{\mathrm{h}} \mathrm{i}: \mathrm{n}^{1}$ & $\mathrm{ts}^{\mathrm{h}} \mathrm{i}: \mathrm{n}^{1}$ & $\mathrm{ts}^{\mathrm{h}} \mathrm{i}: \mathrm{n}^{1}$ & $\operatorname{ts}^{\mathrm{h}} \mathrm{i}: \mathrm{n}^{1}$ & $\mathrm{t}^{\mathrm{h}} \mathrm{in}^{1}$ & $\mathrm{t}^{\mathrm{h}} \mathrm{in}^{1}$ & $\mathrm{t}^{\mathrm{h}} \mathrm{e}{ }^{1}$ & $\mathrm{t}^{\mathrm{h}} \mathrm{en}^{1}$ & $\mathrm{t}^{\mathrm{h}} \mathrm{en}^{1}$ & $\mathrm{t}^{\mathrm{h}} \mathrm{in}^{1}$ \\
\hline $\sin ^{1}$ & $\sin ^{1}$ & ts $^{\mathrm{h}} \mathrm{in}^{1}$ & $\mathrm{ts}^{\mathrm{h}} \mathrm{in}^{1}$ & $\left(\mathrm{ts}^{\mathrm{h}} \mathrm{in}^{4}\right)$ & $\operatorname{ts}^{\mathrm{h}} \mathrm{in}^{1}$ & $\mathrm{t}^{\mathrm{h}} \mathrm{en}^{1}$ & $\mathrm{t}^{\mathrm{h}} \mathrm{en}^{1}$ & $\mathrm{t}^{\mathrm{h}} \mathrm{en}^{1}$ & $\mathrm{t}^{\mathrm{h}} \mathrm{en}^{1}$ & $\mathrm{t}^{\mathrm{h}} \mathrm{en}^{1}$ & $t^{h} e^{1}$ \\
\hline 舌頭 & tongue & & *hli:n? & & & 好 & good & & *hlin & & \\
\hline di: $n^{3}$ & ti: $n^{3}$ & ti: $n^{3}$ & ti: $n^{3}$ & ti: $n^{3}$ & ti: $n^{3}$ & $\operatorname{din}^{1}$ & $\operatorname{tin}^{1}$ & te ${ }^{1}$ & $\operatorname{ten}^{1}$ & $\operatorname{ten}^{1}$ & $\operatorname{tin}^{1}$ \\
\hline $\mathrm{t} \theta \mathrm{in}^{3}$ & $\operatorname{lin}^{3}$ & $\operatorname{tin}^{3}$ & $\operatorname{tin}^{3}$ & $\operatorname{tin}^{3}$ & $\operatorname{tin}^{3}$ & $\mathrm{t} \theta \mathrm{en}^{1}$ & len ${ }^{1}$ & $\operatorname{ten}^{1}$ & $\operatorname{ten}^{1}$ & ten ${ }^{1}$ & ten ${ }^{1}$ \\
\hline (g) & & & $* \mathrm{i}: \mathrm{n}$ & & & (h) & & & $*$ in & & \\
\hline 手指 & finger & & *lji:yh & & & 童山 & bare hill & & *kin & & \\
\hline $\mathrm{zi}: \mathrm{n}^{2}$ & zi: $\eta^{2}$ & zi: $\eta^{2}$ & $\operatorname{tian}^{2}$ & $\operatorname{tiay}^{2}$ & $4 i a \eta^{2}$ & $\mathrm{kin}^{1}$ & $\mathrm{kin}^{1}$ & $\operatorname{kin}^{1}$ & $\operatorname{kin}^{1}$ & $\operatorname{kin}^{1}$ & $\mathrm{kin}^{1}$ \\
\hline ---- & $\operatorname{zin}^{2}$ & $\operatorname{zin}^{2}$ & $\operatorname{zin}^{2}$ & $\left(\mathrm{zin}^{5}\right)$ & $\operatorname{tsin}^{2}$ & ---- & ---- & $\operatorname{ken}^{1}$ & $\operatorname{ken}^{1}$ & $\operatorname{ken}^{1}$ & $\operatorname{ken}^{1}$ \\
\hline 吊 & hang & & *ri:y? & & & 螞蟥 & leech & & *ljin & & \\
\hline ri: $y^{3}$ & ri: $\eta^{3}$ & ri: $y^{3}$ & $\operatorname{ria\eta }^{6}$ & $\operatorname{lia\eta }^{6}$ & $\operatorname{lia\eta }^{6}$ & $\mathrm{zin}^{1}$ & $\mathrm{zin}^{1}$ & $\operatorname{zin}^{1}$ & $\sin ^{4}$ & $\operatorname{tin}^{4}$ & $\operatorname{tin}^{4}$ \\
\hline $\operatorname{liə\eta }^{4}$ & $\operatorname{lin}^{3}$ & ---- & $\operatorname{rin}^{3}$ & $\operatorname{rin}^{3}$ & $\operatorname{rin}^{6}$ & $\left(\operatorname{lin}{ }^{4}\right)$ & $\operatorname{zeg} P^{4}$ & ---- & zen $^{1}$ & zen $^{1}$ & $\operatorname{tsen}^{4}$ \\
\hline \multicolumn{2}{|c|}{ 裂開/縫 } & & $* \mathrm{t}^{\mathrm{h}} \mathrm{i}: \mathrm{n}$ ? & & & 稻草 & straw & & \multicolumn{2}{|c|}{ *Cunin? } & \\
\hline $\mathrm{t}^{\mathrm{h}} \mathrm{i}: \mathrm{y}^{3}$ & $\mathrm{t}^{\mathrm{h}} \mathrm{i}: \mathrm{y}^{3}$ & $t^{\mathrm{h}} \mathrm{i}: \mathrm{y}^{3}$ & $\mathrm{t}^{\mathrm{h}} \mathrm{ia \eta ^{3 }}$ & $\mathrm{t}^{\mathrm{h}} \mathrm{iay^{3 }}$ & $\mathrm{t}^{\mathrm{h}} \mathrm{iay}^{3}$ & $\operatorname{yin}^{3}$ & $\operatorname{yin}^{3}$ & ywin $^{3}$ & $\operatorname{yin}^{3}$ & $\operatorname{yin}^{6}$ & $\operatorname{yin}^{3}$ \\
\hline ts $^{\text {hi }} i \partial \eta^{3}$ & --- & $\mathrm{t}^{\mathrm{h}} \mathrm{i} \mathrm{y}^{3}$ & $\mathrm{t}^{\mathrm{h}} \mathrm{i \eta ^{3 }}$ & $\mathrm{t}^{\mathrm{h}} \mathrm{i \eta ^{3 }}$ & $\mathrm{t}^{\mathrm{h}} \mathrm{i \eta ^{3 }}$ & nen $^{3}$ & ywen ${ }^{3}$ & yen $^{3}$ & yen ${ }^{3}$ & yen ${ }^{3}$ & men $^{6}$ \\
\hline
\end{tabular}


(i)

指甲 fingernail

li: $p^{7} \quad$ li: $p^{7} \quad$ li: $p^{7}$

$\operatorname{lip}^{2} \quad \operatorname{li}^{4} \quad \operatorname{lip}^{7}$

吳蚣 centipede

ri:p $p^{7} \quad$ ri: $p^{7} \quad$ ri: $p^{7}$

$\operatorname{lip}$

秕子 husk w/out rice *hli:p

ri:p $p^{8}$

rip $^{7}$

$\operatorname{rip}^{7} \quad \operatorname{rip}^{8}$

$\operatorname{lip}^{8}$ (j)

狗豆子

li:p $\quad$ li:p $p^{8} \quad$ li:p $p^{7} \quad$ ts $^{\mathrm{h}} \mathrm{ip}^{7} \quad \mathrm{ts}^{\mathrm{h}} \mathrm{ip}^{7} \quad \mathrm{ts}^{\mathrm{h}} \mathrm{ip}^{7}$

$1 /$ rip $^{8}$

- ---- ----

閃電 lightning

$* \operatorname{ljip}$

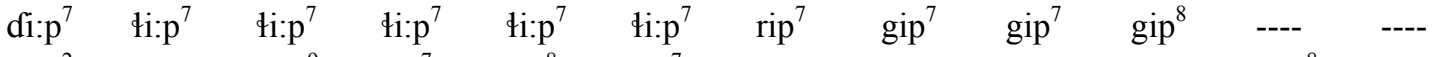
${\mathrm{t} \theta \mathrm{ip}^{2} \quad---\quad\left(\mathrm{ip}^{9}\right) \quad \text { tip }}^{9}$

(k)

*i:t

(1)

㱀紋 wrinkle

*C-mi:t

li:p $\mathrm{p}^{8} \quad$ zip $^{7}$

lep ${ }^{4}$

$\operatorname{zip}^{7}$

$\left(\right.$ fii $\left.^{5}\right) \quad$ zep $^{7}$

打獵 go hunting

$\operatorname{tip}^{7} \quad \operatorname{tip}^{7} \quad \operatorname{tip}^{7}$

zep $^{7} \quad$---- ----

掐 pinch

mi:t $\mathrm{t}^{7} \quad \mathrm{mi}: \mathrm{t}^{7} \quad \mathrm{mi}: \mathrm{t}^{7} \quad \mathrm{mi}: \mathrm{t}^{7} \quad \mathrm{mi}: \mathrm{t}^{8}$

mit $^{2} \quad \operatorname{mir}^{4} \quad$ mit $^{7}$

粽子 dumpling

*hpi:t

mit $^{7}$

$\left(\mathrm{mit}^{7}\right)$

mit $^{8}$

$\left(\right.$ nit $\left.^{9}\right) \quad$ nit

net $^{2} \quad$ ne? $?^{4} \quad$ net $^{7}$

端

$\begin{array}{lll}--- & --- & \text { tsi:t } \\ \text { tsit }^{4} & \text { tsi }^{4} & ----\end{array}$

tsi: $t^{8}$

tsit $^{7}$

ts $^{h} i: t^{8}$
tsit $^{8}$

tsi:t
tsit $^{8}$

$\mathrm{k}^{\mathrm{h}} \mathrm{it}^{7}$

$\mathrm{it}^{7}$

$\mathrm{k}^{\mathrm{h}} \mathrm{it}^{7}$

$\mathrm{k}^{\mathrm{h}} \mathrm{ec}$

店水

bail water

*hwi:t

辣 spicy

----

$\mathrm{k}^{\mathrm{h}} \mathrm{et}^{7}$
$\mathrm{k}^{\mathrm{h}} \mathrm{et}^{7}$

$\mathrm{k}^{\mathrm{h}} \mathrm{et}^{7}$

kit

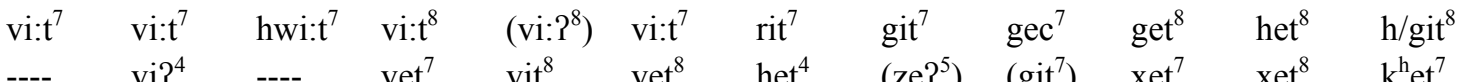




\begin{tabular}{|c|c|c|c|c|c|c|c|c|c|c|c|}
\hline \multicolumn{3}{|l|}{ (m) } & \multicolumn{3}{|l|}{$* \mathrm{i}: \mathrm{k}$} & \multicolumn{3}{|l|}{ (n) } & \multicolumn{3}{|l|}{$*_{\mathbf{i k}}$} \\
\hline 魚籠 & fish bo & & ${ }^{*} \mathrm{C}-\mathrm{li}:$ & & & 耙/掃 & rake/s & eep & $*_{\text {rjik }}$ & & \\
\hline li: $:^{7}$ & li $: ?^{7}$ & li: $\mathrm{k}^{7}$ & $\operatorname{lia}^{7}$ & $\operatorname{lia?^{8}}$ & $\operatorname{liak}^{7}$ & $\mathrm{zik}^{7}$ & $\mathrm{zik}^{7}$ & $\mathrm{rik}^{7}$ & $\mathrm{ti}: ?^{8}$ & $\mathrm{t}^{\mathrm{h}} \mathrm{i}: ?^{8}$ & $\mathrm{tik}^{8}$ \\
\hline $\operatorname{li\partial k}^{2}$ & $\operatorname{li}^{4}$ & $\operatorname{li}^{7}$ & $\operatorname{lik}^{7}$ & $\operatorname{lit}^{8}$ & $1 i i^{8}$ & ---- & ---- & ---- & ---- & ---- & tset $^{8}$ \\
\hline 翅膀 & wing & & ${ }^{*} \mathrm{p}^{\mathrm{h}} \mathrm{i}$ ik & & & 肅靜 & solen & silence & *?ik & & \\
\hline $\mathrm{p}^{\mathrm{h}} \mathrm{i}: \mathrm{P}^{7}$ & $\mathrm{p}^{\mathrm{h}} \mathrm{i}: \mathrm{P}^{7}$ & $\mathrm{p}^{\mathrm{h}} \mathrm{i}: \mathrm{k}^{7}$ & $\mathrm{p}^{\mathrm{h}} \mathrm{ia} ?^{7}$ & $\mathrm{p}^{\mathrm{h}} \mathrm{ia}^{7}$ & $\mathrm{p}^{\mathrm{h}} \mathrm{iak}^{7}$ & ---- & $2 \mathrm{ik}^{7}$ & $\mathrm{Pik}^{7}$ & Pi: $P^{7}$ & Pi: $P^{7}$ & $\left(2 \mathrm{i}: \mathrm{k}^{7}\right)$ \\
\hline $\mathrm{p}^{\mathrm{h}} \mathrm{i} \mathrm{k}^{2}$ & $\mathrm{p}^{\mathrm{h}} \mathrm{i} \mathrm{P}^{4}$ & $\mathrm{p}^{\mathrm{h}} \mathrm{i} \mathrm{P}^{7}$ & $\mathrm{p}^{\mathrm{h}} \mathrm{ik}^{7}$ & $\mathrm{p}^{\mathrm{h}} \mathrm{it}^{8}$ & $\mathrm{p}^{\mathrm{h}_{\mathrm{i}} \mathrm{P}^{7}}$ & ---- & ---- & ---- & $\left(\mathrm{Pik}^{7}\right)$ & ---- & --- \\
\hline 滿 & full & & $* \mathrm{t}^{\mathrm{h}} \mathrm{i}: \mathrm{k}$ & & & 小孩 & child & & ${ }^{*} \mathrm{C}$-lik & & \\
\hline $\mathrm{t}^{\mathrm{h}} \mathrm{i}: \mathrm{P}^{7}$ & $\mathrm{t}^{\mathrm{h}} \mathrm{i}: \mathrm{P}^{7}$ & $\mathrm{t}^{\mathrm{h}} \mathrm{i}: \mathrm{k}^{7}$ & $\mathrm{t}^{\mathrm{h}} \mathrm{ia} \mathrm{P}^{7}$ & $\mathrm{t}^{\mathrm{h}} \mathrm{ia}{ }^{7}$ & $\mathrm{t}^{\mathrm{h}} \mathrm{iak}^{7}$ & $\mathrm{lik}^{7}$ & $\mathrm{lik}^{7}$ & $\operatorname{lik}^{7}$ & li $: ?^{7}$ & li: $: 1^{8}$ & $\mathrm{lik}^{7}$ \\
\hline $\mathrm{t}^{\mathrm{h}} \mathrm{i}^{2}{ }^{2}$ & $\mathrm{t}^{\mathrm{h}_{i}} \mathrm{P}^{4}$ & $\mathrm{t}^{\mathrm{h}} \mathrm{i} ?^{7}$ & $\mathrm{t}^{\mathrm{h}} \mathrm{ik}^{7}$ & $\left(\mathrm{t}^{\mathrm{h}} \mathrm{i} \mathrm{P}^{8}\right)$ & $\mathrm{t}^{\mathrm{h}} \mathrm{i} \mathrm{P}^{7}$ & ---- & ---- & ---- & $\left(\operatorname{lik}^{7}\right)$ & ---- & ---- \\
\hline
\end{tabular}

\subsubsection{Closed Rimes with High Back Unrounded Nuclei}

The correspondences for the closed rimes with high back unrounded vowels are given below:

(84) Correspondences of closed rimes with high back unrounded nuclei

\begin{tabular}{|c|c|c|c|c|c|c|c|c|c|c|c|}
\hline HaE:m & $\underline{\text { BHi:n }}$ & $\underline{\text { LHu:t }}$ & $\underline{\text { Tzha }}$ & $\underline{\text { Zdui }}$ & $\underline{\text { Bting }}$ & $\underline{\text { Cun }}$ & $\underline{\mathrm{Nadou}}$ & $\underline{\text { Cjiang }}$ & $\underline{\text { Mfaw }}$ & $\underline{\text { Baisha }}$ & $\underline{\text { Ymen }}$ \\
\hline u:y & ---- & w:y & w:y & u:y & u:y & ---- & ---- & ---- & (uy) & ---- & ---- \\
\hline w:m & ш:m & ш:m & w:m & $\mathrm{u}: \mathrm{m}$ & u:m & um & un $\sim \varepsilon n$ & um $\sim$ um & a um um & a um um & $\mathrm{a} u \mathrm{um} \sim \mathrm{om}$ \\
\hline u:n & u:n & u:n & w:n & u:n & u:n & un & $\varepsilon n$ & wy & un & un & ən \\
\hline u:y & u:y & u:y & ш:y & uay & u:y & шәу & $\varepsilon \eta$ & un & un & uy & uy \\
\hline u:p & ш:p & u:p & u:p & $\mathrm{u}: \mathrm{p}$ & u:p & ---- & ---- & up & up & up & up \\
\hline w:t & u:t & u:t & u:t & u:t & u:t & ---- & ---- & --- & --- & wk & --- \\
\hline u:? & ш:? & w:k & ш:? & ша? & ш:? & uək & uP $\sim \varepsilon ?$ & ш? & uk & uk & u? \\
\hline um & wm & wm & um & um & um & $\mathrm{am} \sim \mathrm{om}$ & un & om $\sim$ em & om $\sim$ em & om & om \\
\hline un & un & un & un & un & un & un $\sim \partial n$ & $\varepsilon n$ & әท & әท & әๆ & ən \\
\hline un & wy & wy & wy & un & un & ---- & ---- & ---- & әฤ & วท & วท \\
\hline up & шр & wp & up & up & up & $\mathrm{up} \sim \varepsilon p$ & $\varepsilon ?$ & ap ep & op ep & op & op \\
\hline ut & ut & wt & ut & ut & ut & ut $\sim \partial t$ & $\varepsilon ?$ & ot & ək & ok & วt \\
\hline
\end{tabular}




\section{S. Hlai (Savina)}

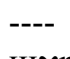

urm

urn

ury

urp

wrt

u:

um

un

wy

wt
C. Hlai (Savina)

urm

urn

ury
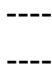

urk
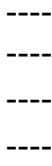

$---$
Baisha (Wang \& Qian)

u:m um
uy
uy
u:p
---
wk

----
œy
œy
op
œk

In general, the same patterns which applied to the class of rimes with high front vowels reoccur here. There is considerable variation in $\mathrm{NCHl}$ reflexes, presumably due to the marked nature of the high and mid back unrounded vowels. The reconstructions proposed here for these correspondences are given below:

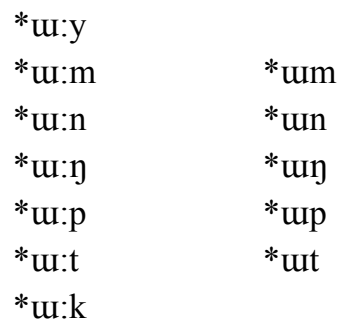

\subsubsection{Long Rimes with High Back Unrounded Vowels}

There have been very few changes in the long rimes of the non-NCHl languages, with the exception of Zandui. The three changes which have occurred in Zandui are that the vowel in the diphthong rounded, probably through dissimilation; the vowels in the rimes closed with labial stops became rounded under their influence; and the vowels in the rimes closed by velar stops have diphthongized, in the same way which occurred for those in section 4.4.1.1 above for the entire Qi branch: 
(86)

$$
\begin{aligned}
& \text { *u:y > u:y } \\
& \text { *u:m } \quad>\quad \text { u:m } \\
& \text { *u:p } \quad>\quad \text { u:p } \\
& \text { *ux:y > wəy }>\text { way } \\
& \text { *u:k }>\text { wək }>\text { ua? }
\end{aligned}
$$

In $\mathrm{NCHl}$, the regular shortening of long rimes occurred:

$$
\text { *u:C } \quad>\quad * u C
$$

The nucleus of the dipthong apparently rounded in Moyfaw; note, however, that since this is the only reflex of this rime in $\mathrm{NCHl}$, that it may be a loan:

$$
\text { wy }>\text { uy }
$$

The most common place of variation in $\mathrm{NCHl}$ is in the labial-final rimes, in which there was sporadic rounding of the vowel. This happened in all six NCHl languages, but could not have occurred at

\begin{tabular}{|c|c|c|c|c|c|}
\hline Gloss & $\underline{\text { Proto Hlai }}$ & & $\underline{\mathrm{NCHl}}$ & & Nadouhua \\
\hline weave & $* t \int^{h} w u r: k$ & $>$ & 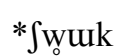 & $>$ & $\mathrm{fu}^{4}$ \\
\hline bone & *Curur:k & $>$ & *rwuk & $>$ & $v_{u} ?^{4}$ \\
\hline tender & *p-lur:k & $>$ & *pluk & $>$ & pju? $^{4}$ \\
\hline
\end{tabular}
the level of Proto Northern Hlai, since the variation does not hold across identical lexical items:

$\begin{array}{lll}\operatorname{mim}_{\operatorname{mon}} & > & \text { up }\end{array}$

Nadouhua also experienced rounding of vowel in $\mathrm{NCHl} u k$ rimes, under the influence of a preceding labial or labiovelar:

In addition, there were two cases of vowel lowering which occurred, in Nadouhua and in Yuanmen. In Nadouhua, anything which did not undergo secondary rounding shortened (merging with the 
PHl short * ${ }^{*} \mathrm{C}$ series) and lowered to a mid vowel, centralizing to schwa. Rimes with schwa, no matter their origin, then fronted to $\varepsilon \mathrm{C}$ (there are no examples of original up or ut in Nadouhua):

\begin{tabular}{|c|c|c|c|c|c|}
\hline um & $>$ & $\gamma n$ & $>$ & ən & $>$ \\
\hline un & $>$ & $\gamma n$ & $>$ & ən & $>$ \\
\hline un & $>$ & $\gamma \eta$ & $>$ & әך & $>$ \\
\hline uk & $>$ & $\gamma \mathrm{k}$ & $>$ & ək & $>$ \\
\hline
\end{tabular}

In Yuanmen, the labial-closed rimes which didn't round and alveolar-closed rimes shortened and lowered to merge with their counterparts from PHl *um/up and *un (there are no Yuanmen examples of original *ut):

$\begin{array}{lllll}\operatorname{um} & > & \gamma \mathrm{m} & > & \text { om } \\ \operatorname{up} & > & \text { rp } & > & \text { op } \\ \operatorname{un} & > & \text { rn } & > & \text { on }\end{array}$

\subsubsection{Short Rimes with High Back Unrounded Vowels}

The only change in this category which occurred in a non-NCHl language was again in Zandui, where the vowel became rounded under the influence of a following labial coda:

$\begin{array}{lll}{ }^{*} \text { um } & > & \text { um } \\ { }_{\text {uup }} & > & \text { up }\end{array}$

In $\mathrm{NCHl}$, the typical short-vowel lowering applied, with an additional centralization of the vowel in many cases; note that this was only possible after the lowering of original schwa nuclei (see section 4.5.2):

$\begin{array}{llll}{ }_{u \mathrm{uC}}> & \gamma \mathrm{C} & > & \partial \mathrm{C} \\ *_{\partial \mathrm{C}} & > & \mathrm{aC}\end{array}$


No NCHl language maintained an inventory of short $x C$ rimes. These rimes often fronted to $\partial C$ rimes as shown above; they were sometimes influenced further by preceding initials or rounded by following labials, and in some cases underwent idiosyncratic changes which seem to have been unmotivated. Complicating matters is the fact that some categories have few examples, so that the establishment of potential conditioning environments is problematic.

In Cunhua, the following variation occurs in $* r m$ :

$$
\mathrm{rm}>\mathrm{om} / \mathrm{\textrm {m }}>\mathrm{om} / \mathrm{am}
$$

There is only one example of original Northern Hlai *rm in Nadouhua, the reflex of which is $u m$.

In all other cases, the following shift occurred:

$\begin{array}{lllll}\gamma \mathrm{p} & > & \text { op } & > & \varepsilon ? \\ \gamma \mathrm{n} & > & \text { on } & > & \varepsilon \mathrm{n} \\ \gamma \mathrm{t} & > & \text { ot } & > & \varepsilon ?\end{array}$

The following changes occurred in the Meifu branch:

\begin{tabular}{|c|c|c|c|c|}
\hline $\mathrm{rm}$ & $>$ & әm & $>$ & $\mathrm{om} / \mathrm{em}$ \\
\hline$r p$ & $>$ & әр & $>$ & ap/ep (Changjiang), op/ep (Moyfaw) \\
\hline$\gamma n$ & $>$ & วn & $>$ & əฤ \\
\hline$\gamma t$ & $>$ & at & $>$ & (in Moyfaw) \\
\hline
\end{tabular}

Finally, the following occurred in the Run branch:

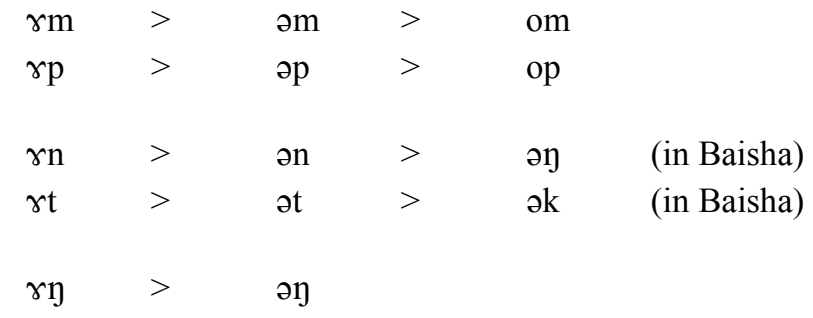

A comparison of reconstructions is given below: 


\begin{tabular}{|c|c|c|c|c|}
\hline (a) & --- & --- & $(* \mathrm{i}: \mathrm{y})$ & *u:y \\
\hline (b) & *uam (b) & *um & $* \mathrm{i}: \mathrm{m}$ & *u:m \\
\hline (c) & *u:n (b) & *un & $*_{\mathrm{i}: \mathrm{n}}$ & *u:n \\
\hline (d) & *u:y (b) & *un & $*_{i: y}$ & *u:y \\
\hline (e) & *uap (b) & *up & $* \mathrm{i}: \mathrm{p}$ & *u:p \\
\hline (f) & --- & --- & --- & *w:t \\
\hline (g) & *uak (b) & *uk & $* \mathrm{i}: \mathrm{k}$ & *u:k \\
\hline (h) & --- & $*^{\prime} r \mathrm{~m}$ & $*_{\mathrm{im}}$ & *um \\
\hline (i) & --- & $* \gamma n$ & $*$ in & *un \\
\hline (j) & --- & --- & --- & *un \\
\hline (k) & --- & $* \gamma p$ & $*$ ip & *up \\
\hline (1) & --- & $* \gamma t$ & $*_{\mathrm{it}}$ & *ut \\
\hline
\end{tabular}

Thurgood doesn't reconstruct anything in the (99h-1). For the first six series (excluding first and the sixth, for which there is only one clear example each), he reconstructs a series of either pure long $\mathrm{u}: \mathrm{C}$ rimes or diphthongized uaC rimes. These are all labeled with a (b) in his system, because they contrast with other rimes which are reconstructed identically based on other series of correspondences. These are assumed to be loans (Thurgood 1991: 19). It is unclear to me why there is an asymmetry between the rimes with velar codas, with a pure vowel being reconstructed for the third series (*u:y), but a diphthong for the sixth series (*uak), another violation of Symmetry.

As in the last section, Ostapirat's (1993) reconstruction is straightforward, positing a high vowel and mid vowel series based on the NCHl data. His (2004) reconstruction is identical with the one proposed here, save for the fact that he reconstructs high central vowels instead of high back vowels. He does not reconstruct anything in (99f) or (99j) as each is supported by only a single example, and his reconstruction of the first series is tentative (also based on only one example).

Examples of the closed rimes with high back unrounded vowels are given below, in the following order:

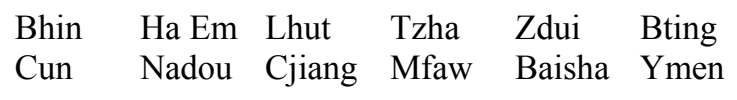


(a)

\begin{tabular}{llllll} 
竹子 & bamboo & \multicolumn{3}{c}{ *p-lu:yh } \\
& & & & \\
plu:y: & pu:y & plu:y & plu:y & ----- & lu: ${ }^{2}$ \\
---- & ---- & ---- & puy $\left.^{2}\right)$ & ---- & ----
\end{tabular}

(b)

*u:m

飽 $\mathrm{k}^{\mathrm{h}} \mathrm{um}^{1}$

full

$*^{\mathrm{h}} \mathrm{u}: \mathrm{m}$

(c)

烤火 warm by fire *?umf

$\mathrm{k}^{\mathrm{k}}$

$$
\text { 蛋/ }
$$

\begin{tabular}{|c|c|c|c|c|c|c|c|c|c|c|c|}
\hline $\begin{array}{l}\text { zur:m }{ }^{1} \\
\text { zum }^{1}\end{array}$ & $\begin{array}{l}\text { zur:m }{ }^{1} \\
\text { zun }^{4}\end{array}$ & $\begin{array}{l}\text { zu::m }{ }^{1} \\
\text { zum }^{1}\end{array}$ & $\begin{array}{l}\text { zur:m }{ }^{4} \\
\text { zum }^{1}\end{array}$ & $\begin{array}{l}\mathrm{zu}: \mathrm{m}^{4} \\
\mathrm{zum}^{1}\end{array}$ & $\begin{array}{l}\text { zur:m }{ }^{1} \\
\text { zum }^{4}\end{array}$ & $\begin{array}{l}\text { duum }^{3} \\
\text { t } \theta \mathrm{am}^{3}\end{array}$ & tumm ${ }^{3}$ & $\begin{array}{l}\text { tumm } \\
\text { tem }\end{array}$ & $\begin{array}{l}\text { tum } \\
\\
\mathrm{tem}^{3}\end{array}$ & $\begin{array}{l}4 u m^{3} \\
\text { tom }^{3}\end{array}$ & $\begin{array}{l}\text { tum } \\
\text { tom } \\
\text { to }\end{array}$ \\
\hline 爬 & crawl & & \multicolumn{3}{|c|}{ *Cuhu:m } & 烘 & \multicolumn{2}{|c|}{ warm by fire } & \multicolumn{2}{|c|}{ *Curum? } & \\
\hline $\begin{array}{l}\text { hu::m } \\
\text { num }^{1}\end{array}$ & hu::m¹ & $\begin{array}{l}\text { hwu:m }{ }^{1} \\
\text { yum }^{1}\end{array}$ & $\begin{array}{l}{ }^{1} \text { hu: }{ }^{1} \\
\text { yum }^{1}\end{array}$ & $\begin{array}{l}\text { vu:m }{ }^{1} \\
\text { yum }^{1}\end{array}$ & $\begin{array}{l}\text { hu:m }{ }^{1} \\
\text { mom }\end{array}$ & $\mathrm{rum}^{3}$ & gum $^{3}$ & $\begin{array}{l}\text { gum }^{3} \\
\text { kum }^{1}\end{array}$ & $\begin{array}{l}\text { gum }^{6} \\
\text { jum }^{3}\end{array}$ & ---- & hum ${ }^{6}$ \\
\hline (d) & & & *u:p & & & (e) & & & *up & & \\
\hline 鱉 & pt nosed & turtle & $* t^{h} u: p$ & & & 臭蟲 & stinkbug & & *kup & & \\
\hline$t^{h} u: p^{7}$ & $\mathrm{t}^{\mathrm{h}} \mathrm{u}: \mathrm{p}^{7}$ & $\mathrm{t}^{\mathrm{h}} \mathrm{u}: \mathrm{p}^{7}$ & $\mathrm{t}^{\mathrm{h}} \mathrm{u}: \mathrm{p}^{7}$ & $\mathrm{t}^{\mathrm{h}} \mathrm{u}: \mathrm{p}^{7}$ & $t^{h} u: p^{7}$ & kup $^{7}$ & $\operatorname{kup}^{7}$ & kup $^{7}$ & $\operatorname{kup}^{7}$ & $\operatorname{kup}^{7}$ & kup $^{7}$ \\
\hline ---- & ---- & ---- & $\mathrm{t}^{\mathrm{h}} \mathrm{up}^{7}$ & $\mathrm{t}^{\mathrm{h}} \mathrm{up}^{8}$ & $\mathrm{t}^{\mathrm{h}} \mathrm{up}^{7}$ & kup $^{2}$ & $k \varepsilon ?^{4}$ & $\left(\right.$ kup $\left.^{7}\right)$ & $\mathrm{kep}^{7}$ & kop $^{8}$ & $\mathrm{kop}^{7}$ \\
\hline 搓洗 & scrub & & *fju:p & & & 呷 & $\operatorname{sip}$ & & $*{ }^{t} c^{\mathrm{h}}$ up & & \\
\hline (pu: $p^{7}$ ) & fur:p $p^{7}$ & fur:p $p^{7}$ & fur:p $p^{7}$ & fu: $p^{7}$ & fur:p $p^{7}$ & ts $^{\mathrm{h}} u p^{7}$ & $\operatorname{ts}^{\mathrm{h}} u p^{7}$ & ts $^{h} u p^{7}$ & ts $^{\mathrm{h}} u \mathrm{p}^{7}$ & ts $^{h} u^{7}$ & ts $^{\mathrm{h}} \mathrm{up}^{7}$ \\
\hline ---- & ---- & fup $^{7}$ & fup $^{7}$ & fup $^{8}$ & fup $^{7}$ & $\mathrm{ts}^{\mathrm{h}} \mathrm{ip} \mathrm{p}^{2}$ & $\operatorname{se} ?^{4}$ & ---- & $\mathrm{ts}^{\mathrm{h}} \mathrm{op}{ }^{7}$ & ---- & ---- \\
\hline 稻剪 & rice knif & & $*^{\mathrm{h}} \mathrm{w}: \mathrm{p}$ & & & 搾 & handleng & gth & ${ }^{*}$ Cufiul & & \\
\hline & $k^{h} u: p^{7}$ & $k^{h} u: p^{7}$ & ---- & --- & --- & hup $^{7}$ & hupp $^{7}$ & hwup $^{7}$ & $\operatorname{hup}^{7}$ & $\operatorname{vup}^{7}$ & hupp $^{7}$ \\
\hline & ---- & $k^{h} u^{7}$ & $\mathrm{k}^{\mathrm{h}} \mathrm{up}^{7}$ & $\mathrm{k}^{\mathrm{h}} \mathrm{up}^{8}$ & $\mathrm{k}^{\mathrm{h}} \mathrm{up}^{7}$ & yup $^{2}$ & $\eta \varepsilon ?^{4}$ & $\operatorname{yap}^{7}$ & yop ${ }^{7}$ & yop $^{8}$ & mop $^{8}$ \\
\hline
\end{tabular}


(f)

\begin{tabular}{|c|c|c|c|c|c|c|c|c|c|c|c|}
\hline 䦔 & castrate & & *dur:n & & & 重 & heavy & & $* \mathrm{k}^{\mathrm{h}} \mathrm{un}$ & & \\
\hline $\begin{array}{l}\text { dur:n }{ }^{1} \\
\text { duun }^{1}\end{array}$ & du::n & $\begin{array}{l}\text { dur:n }{ }^{1} \\
\text { dury }^{1}\end{array}$ & $\begin{array}{l}\text { dur:n }{ }^{1} \\
\text { dury }^{1}\end{array}$ & $\begin{array}{l}\text { dur:n }{ }^{1} \\
\text { dury }^{1}\end{array}$ & $\begin{array}{l}\text { dur:n }{ }^{1} \\
\text { dən }{ }^{1}\end{array}$ & $\begin{array}{l}\mathrm{k}^{\mathrm{h}} \mathrm{un}^{1} \\
\mathrm{k}^{\mathrm{h}} \mathrm{on}^{1}\end{array}$ & $\begin{array}{l}\mathrm{k}^{\mathrm{h}} \mathrm{un}^{1} \\
\mathrm{k}^{\mathrm{h}} \varepsilon \mathrm{n}^{1}\end{array}$ & $\begin{array}{l}\mathrm{k}^{\mathrm{h}} \mathrm{uun}^{1} \\
\mathrm{k}^{\mathrm{h}} \partial \mathrm{y}^{1}\end{array}$ & $\begin{array}{l}\mathrm{k}^{\mathrm{h}} \mathrm{u} \mathrm{n}^{1} \\
\mathrm{k}^{\mathrm{h}} \partial \mathrm{y}^{1}\end{array}$ & $\begin{array}{l}\mathrm{k}^{\mathrm{h}} \mathrm{uun}^{1} \\
\mathrm{k}^{\mathrm{h}} \partial \mathrm{y}^{1}\end{array}$ & $\begin{array}{l}\mathrm{k}^{\mathrm{h}} \mathrm{un}^{1} \\
\mathrm{k}^{\mathrm{h}} \partial \mathrm{n}^{1}\end{array}$ \\
\hline 骟子 & \multicolumn{2}{|c|}{ pheasant } & \multicolumn{3}{|l|}{ *dur:n } & 淺 & \multicolumn{2}{|l|}{ shallow } & \multicolumn{2}{|c|}{$* \mathrm{t}^{\mathrm{h}} \mathrm{w}[:] \mathrm{n} ?$} & \\
\hline $\begin{array}{l}\text { dur:n } \\
\text { duun }^{1}\end{array}$ & dur:n ${ }^{1}$ & $\begin{array}{l}\text { dur:n }{ }^{1} \\
\text { dumm }^{3}\end{array}$ & $\begin{array}{l}\text { dur: }{ }^{1} \\
\text { duuy }^{1}\end{array}$ & $\begin{array}{l}\text { dur:n }{ }^{1} \\
\text { duuy }^{1}\end{array}$ & $\begin{array}{l}\text { du::n }{ }^{1} \\
\text { dən }{ }^{1}\end{array}$ & $\begin{array}{l}\mathrm{t}^{\mathrm{h}} \mathrm{u}: \mathrm{n}^{3} \\
\text { fumn }\end{array}$ & $\begin{array}{l}\mathrm{t}^{\mathrm{h}} \mathrm{un}^{3} \\
\mathrm{t}^{\mathrm{h}} \varepsilon \mathrm{n}^{3}\end{array}$ & $\begin{array}{l}\mathrm{t}^{\mathrm{h}} \mathrm{un}^{3} \\
\mathrm{t}^{\mathrm{h}} \partial \mathrm{y}^{3}\end{array}$ & $\begin{array}{l}t^{\mathrm{h}} \mathrm{un}^{3} \\
\mathrm{t}^{\mathrm{h}} \partial \mathrm{y}^{3}\end{array}$ & $\begin{array}{l}\mathrm{t}^{\mathrm{h}} \mathrm{un}^{3} \\
\mathrm{t}^{\mathrm{h}} \partial \mathrm{y}^{3}\end{array}$ & $\begin{array}{l}t^{h} u_{n}^{3} \\
t^{h} \partial n^{3}\end{array}$ \\
\hline 出/開 & \multicolumn{2}{|c|}{ exit/ open up } & \multicolumn{2}{|l|}{$* \mathrm{t}^{\mathrm{h}} \mathrm{u}: \mathrm{n}$} & & 刺 & \multicolumn{2}{|l|}{ thorn } & \multicolumn{2}{|c|}{ *Cufuun? } & \\
\hline $\begin{array}{l}\mathrm{t}^{\mathrm{h}} \mathrm{w}: \mathrm{n}^{1} \\
\text { ts }^{\mathrm{h}} \mathrm{un}^{1}\end{array}$ & $\begin{array}{l}\mathrm{t}^{\mathrm{h}} \mathrm{u}: \mathrm{n}^{1} \\
\mathrm{t}^{\mathrm{h}} \varepsilon \mathrm{n}^{1}\end{array}$ & $\begin{array}{l}t^{h} w: n^{1} \\
t^{h} w n^{1}\end{array}$ & $\begin{array}{l}t^{\mathrm{h}} \mathrm{w}: \mathrm{n}^{1} \\
\mathrm{t}^{\mathrm{h}} \mathrm{w} \mathrm{y}^{1}\end{array}$ & $\begin{array}{l}t^{\mathrm{h}} \mathrm{w}: \mathrm{n}^{1} \\
\mathrm{t}^{\mathrm{h}} \mathrm{w} \mathrm{y}^{1}\end{array}$ & $\begin{array}{l}\mathrm{t}^{\mathrm{h}} \mathrm{w}: \mathrm{n}^{1} \\
\mathrm{t}^{\mathrm{h}} \partial \mathrm{n}^{1}\end{array}$ & $\begin{array}{l}\text { hun } \\
\text { yun }\end{array}$ & $\begin{array}{l}\text { hun } \\
\text { yen }\end{array}$ & $\begin{array}{l}\text { hwum } \\
\text { nəy }\end{array}$ & $\begin{array}{l}\text { hurn }^{3} \\
\text { yəy }\end{array}$ & $\begin{array}{l}\text { hun }^{3} \\
\text { nəy }\end{array}$ & $\begin{array}{l}\text { hum }^{3} \\
\text { mən }\end{array}$ \\
\hline (h) & & & *u:t & & & (i) & & & ${ }^{*}$ ut & & \\
\hline \multicolumn{3}{|c|}{ 扒拉/撓 scratch } & \multicolumn{2}{|l|}{ *fiu:t } & & 曾祖母 & \multicolumn{2}{|c|}{ pat. grt grndma } & \multicolumn{2}{|l|}{ *tcuut } & \\
\hline hur: $t^{7}$ & hu: $\mathrm{t}^{7}$ & hu: $t^{7}$ & hu: $:^{7}$ & hur:t $t^{7}$ & hur:t ${ }^{7}$ & tsuut $^{7}$ & tsurt $^{7}$ & tsurt $^{7}$ & tsurt $^{7}$ & tsuut $^{7}$ & ---- \\
\hline \multirow[t]{7}{*}{---- } & ---- & ---- & ---- & hulk $^{8}$ & ---- & ---- & ---- & ---- & tsək $^{7}$ & $\left(\right.$ tsurk $\left.^{7}\right)$ & $\operatorname{tot}^{7}$ \\
\hline & & & & & & 嵝 & \multicolumn{2}{|c|}{ break (pull) } & $* t^{\mathrm{h}} u \mathrm{t}$ & & \\
\hline & & & & & & ---- & ---- & $t^{h} u t^{7}$ & $t^{h} u t^{7}$ & $t^{\text {h }} u t^{7}$ & $t^{h} u t^{7}$ \\
\hline & & & & & & ---- & ---- & ---- & $\mathrm{t}^{\mathrm{h}} \partial \mathrm{k}^{7}$ & $\mathrm{t}^{\mathrm{h}} \partial \mathrm{t}^{7}$ & $\mathrm{t}^{\mathrm{h}} \partial \mathrm{t}^{7}$ \\
\hline & & & & & & 層 & layer & & *hluit & & \\
\hline & & & & & & dutt $^{7}$ & turt ${ }^{7}$ & turt ${ }^{7}$ & turt ${ }^{7}$ & dutt ${ }^{7}$ & turt ${ }^{7}$ \\
\hline & & & & & & ---- & ---- & $4 \partial t^{7}$ & $\mathrm{l} \mathrm{k}^{7}$ & $4 \partial \mathrm{k}^{8}$ & $\mathrm{d \partial t}^{7}$ \\
\hline
\end{tabular}




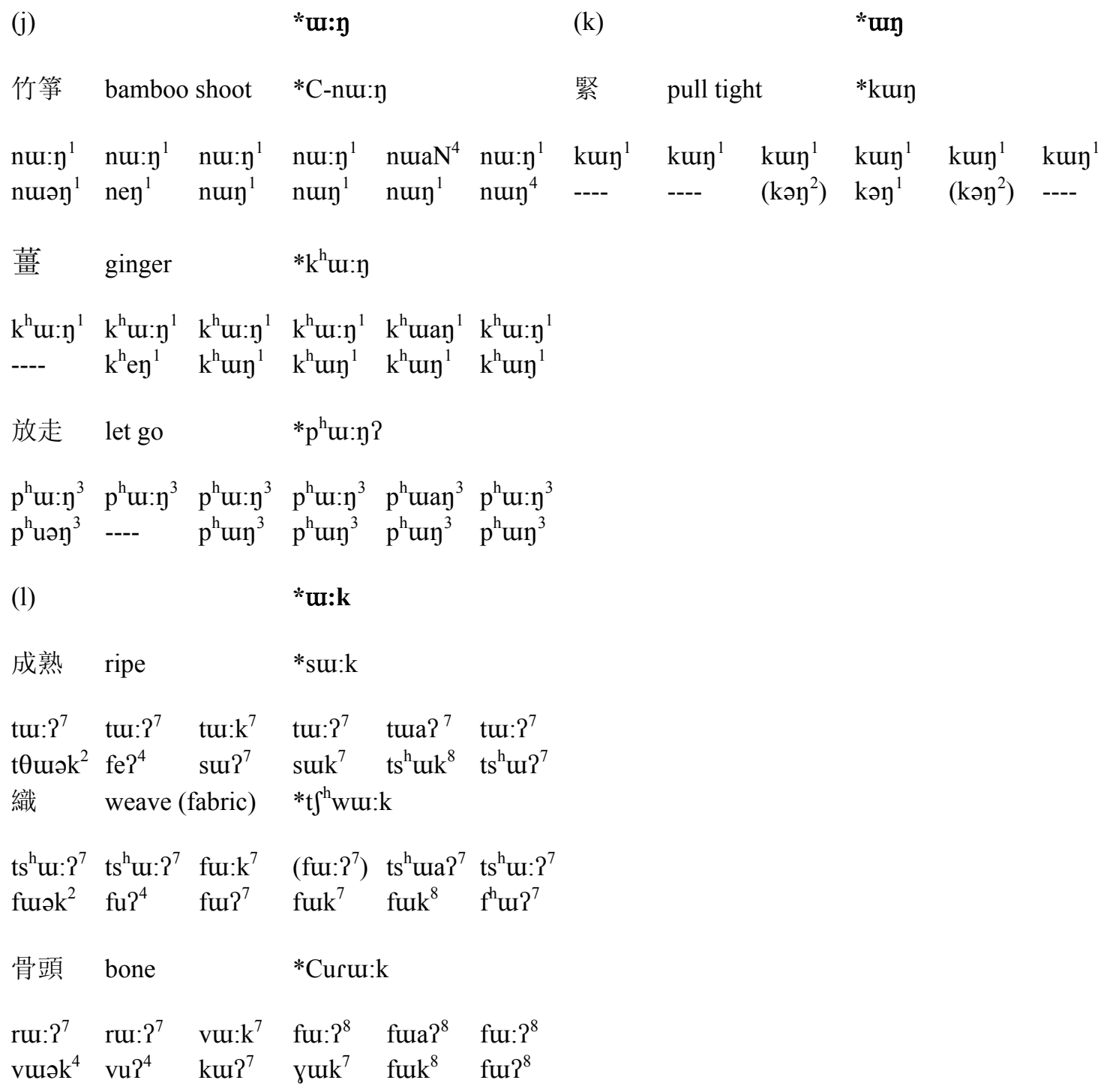

\subsubsection{Closed Rimes with High Back Rounded Vowels}

The correspondences for the closed rimes with high back rounded vowels are given below: 
(101) Reflexes of closed rimes with high back vowels in the Hlai languages

\begin{tabular}{|c|c|c|c|c|c|c|c|c|c|c|c|}
\hline HaE:m & $\underline{\text { BHi:n }}$ & $\underline{\mathrm{LHu}: \mathrm{t}}$ & $\underline{\text { Tzha }}$ & $\underline{\text { Zdui }}$ & Bting & $\underline{\text { Cun }}$ & $\underline{\text { Nadou }}$ & Cjiang & Mfaw & Baisha & $\underline{\text { Ymen }}$ \\
\hline u:y & u:y & u:y & u:y & u:y & u:y & uy & uy & uy & uy & uy & uy \\
\hline u:n & u:n & u:n & u:n & u:n & u:n & uən & un & un & un & un & un \\
\hline u:n & $\mathrm{u}: \mathrm{n}$ & $\mathrm{u}: \mathrm{n}$ & u:n & u:n & $\mathrm{u}: \mathrm{n}$ & uən & un & un & un & un & un \\
\hline u:y & $\mathrm{u}: \mathrm{y}$ & $\mathrm{u}: \mathrm{y}$ & $\mathrm{u}: \mathrm{y}$ & uay & $\mathrm{u}: \mathrm{y}$ & oy & un & un & un & un & un \\
\hline $\mathrm{u}: \mathrm{t}$ & u:t & u:t & $\mathrm{u}: \mathrm{t}$ & $\mathrm{u}: \mathrm{t}$ & u:t & uət & u? & u? & uk & uk & ut \\
\hline $\mathrm{u}: \mathrm{t}$ & u:t & u:c & u:t & u:t & u:t & uət & u? & ut & ut & ut & ut \\
\hline u:? & $\mathrm{u}: ?$ & $\mathrm{u}: \mathrm{k}$ & $\mathrm{u}: ?$ & ua? & u:? & ok & u? & u? & uk & uk & u? \\
\hline uy & uy & uy & uy & uy & uy & әу & oy & uy & uy & oy & ow \\
\hline un & un & un & un & un & un $\sim$ Un & ən & $\varepsilon n$ & oy & oy & oy & ən \\
\hline un & un & un & un & un & un & ---- & (u)En & on & on & on & ən \\
\hline un & un & oy & oy & un & un & oy & oy & oy & on & on & on \\
\hline ut & ut & ut & ut & ut & ut $\sim$ ut & วt & ---- & o? & ok & ok & tt \\
\hline ut & ut & uc & ut & ut & ut & at & (u) $\varepsilon ?$ & วt & ot & ot & tt \\
\hline
\end{tabular}

$\begin{array}{lll}\text { uoy } & \text { uy } & \text { uy } \\ \text { uon } & \text { uon } & \text { u:y } \\ ---- & --- & ---- \\ \text { uoy } & \text { uoy } & \text { u:y } \\ \text { uot } & \text { uot } & \text { u:k } \\ ---- & --- & -- \\ \text { u: } & \text { uok } & \text { u:k } \\ \text { uy } & \text { uy } & \text { oy } \\ \text { un } & \text { un } & \text { oy } \\ \text { un } & --- & --- \\ \text { un } & --- & \text { u:y } \\ \text { ut } & --- & \text { ok } \\ \text { ut } & ---- & \text { ot }\end{array}$

There are no examples of rimes with labial codas; in addition, there does not seem to be any evidence for a final series of correspondences supporting the reconstruction of PHl *uk. The reconstructions proposed here for the series above are the following: 
(102)

$\begin{array}{ll}\text { *u:y } & \text { *uy } \\ \text { *u:n } & \text { *un } \\ \text { *u: } & \text { *un } \\ \text { *u:n } & \text { *uy } \\ \text { *u:t } & \text { *ut } \\ \text { *u:c } & \text { *uc } \\ \text { *u:k } & \end{array}$

\subsubsection{Long Closed Rimes with High Back Rounded Vowels}

The most sweeping change in this series was the merger of the alveolar-final and palatal-final rimes, which occurred in Bouhin, Ha Em, and Qi:

$\begin{array}{lll}* \text { u:n } & > & \text { u:n } \\ * \text { u:c } & > & \text { u:t }\end{array}$

Besides this, the only other change which occurred outside of $\mathrm{NCHl}$ was the by now familiar diphthongization of the velar-final rimes in Zandui:

\section{Diphthongization before velars in Zandui}

$\begin{array}{lllll}* \mathrm{u}: \mathrm{y} & > & \text { uəg } & > & \text { uay } \\ * \mathrm{u}: \mathrm{k} & > & \text { uək } & > & \text { uak }\end{array}$

In $\mathrm{NCHl}$, the regular high vowel shortening occurred:

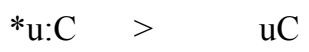

There was also a loss of palatal codas throughout NCHl. However, unlike other branches in which the final palatals merged with the final alveolars, the distinction between palatals and alveolars has been preserved by an initial merger of the final alveolars with the velars in Baisha and the Meifu branch:

\begin{tabular}{|c|c|c|}
\hline (106) & *un & $>$ \\
\hline & $*_{u t}$ & $>$ \\
\hline & *un & $>$ \\
\hline & $*_{\mathrm{uc}}$ & $>$ \\
\hline
\end{tabular}


Besides this, the only other changes were in NWCHI. In Cunhua, these rimes diphthongized before final alveolars, but lowered before final velars:

(107)

$\begin{array}{lll}* \text { un } & > & \text { uən } \\ * \text { ut } & > & \text { uət } \\ *_{\text {un }} & > & \text { uən } \\ *_{\text {uc }} & > & \text { uət } \\ *_{\text {un }} & > & \text { on } \\ * \text { uk } & > & \text { ok }\end{array}$

In Nadouhua, the palatals merged with the alveolars, and all oral stops lenited to glottal stop:

$\begin{array}{lll}* \text { un } & > & \text { un } \\ * \text { un } & > & \text { un } \\ * \text { un } & > & \text { up } \\ \text { *ut } & > & \text { u? } \\ *_{\text {uc }} & > & \text { u? } \\ *_{\text {uk }} & > & \text { u? }\end{array}$

3.4.3.2 Short Closed Rimes with High Back Rounded Vowels

In Bouhin, Ha Em and Qi, the same merger of alveolar and palatal codas occurred which was illustrated for the long rimes:

$\begin{array}{lll}* \text { un } & > & \text { un } \\ * \text { uc } & > & \text { ut }\end{array}$

However, the following reflexes are extant in Baoting; the causes underlying the variation are unclear:

$\begin{array}{lll}* \text { un } & > & \text { un } \sim \text { un } \\ * \text { un } & > & \text { un } \\ *_{\text {ut }} & > & \text { ut } \sim \text { ut } \\ *_{\text {uc }} & > & \text { ut }\end{array}$

In Lauhut and Tongzha, ${ }^{*} u$ lowered to $o$ before the velar coda: 
(111) *uy > oy

The regular $\mathrm{NCHl}$ vowel lowering occurred in this series:

(112) $* \mathrm{uC}>\mathrm{oC}$

The distinction between the $\mathrm{PHl} * \mathrm{uy} /{ }^{*} \mathrm{uk}$ series and the $\mathrm{PHl} *$ oy/ok series was maintained in

$\mathrm{NCHl}$ in the following way:

$\begin{array}{ll}* \text { uy } / \mathrm{uk}> & \text { oy } / \mathrm{ok} \\ *_{\text {oy }} / \mathrm{ok}> & \text { on } / \mathrm{ok}\end{array}$

The diphthong *uy never lowered in the Meifu branch, in parallel with *iw:

(114) *uy > uy

The same diphthong lengthened in Yuanmen (in parallel with ew), raising but in this case losing the final glide:
*uy $\quad>$
oy $>$
o:y $>$
u:y $>$
u: $\quad>$
ow

The same organizing principle which maintained the distinction between original alveolar and palatal codas in Baisha and the Meifu branch occurred here as well, as original alveolars merged with velars and original palatals became alveolars:

$\begin{array}{lll}* \text { un } & > & \text { oy } \\ * \text { ut } & > & \text { ok } \\ * \text { un } & > & \text { on } \\ * \text { uc } & > & \text { ot }\end{array}$

In NWCHl and Yuanmen, the final alveolars and palatals underwent the following mergers: 
(a) Cunhua and Yuanmen

$\begin{array}{lll}\text { on } & > & \text { on } \\ \text { ot } & > & \text { ot } \\ \text { of } & > & \text { on } \\ \text { oc } & > & \text { ot }\end{array}$

(b) Nadouhua:

$\begin{array}{lllll}\text { on } & > & \text { uən }> & (\mathrm{u}) \varepsilon \text { n } \\ \text { ot } & > & & (\text { no examples }) & \\ \text { on } & > & \text { uən }> & (\mathrm{u}) \varepsilon n \\ \text { oc } & > & \text { uət }>> & (\mathrm{u}) \varepsilon ?\end{array}$

A comparison of reconstructions is given below:

(118) Thurgood Ostapirat (1993) Ostapirat (2004) $\mathrm{PHl}$

\begin{tabular}{|c|c|c|c|c|}
\hline (a) & *u:y & *uy & $* u: y$ & *u:y \\
\hline (b) & *u:n (a) & *un & *u:n & *u:n \\
\hline (c) & --- & --- & --- & *u:p \\
\hline (d) & *u:y (a) & *uy & $* \mathrm{u}: \mathrm{y}$ & *u:y \\
\hline (e) & $*_{\mathrm{u}} \mathrm{t}$ & *ut & $*_{\mathrm{u}: \mathrm{t}}$ & *u:t \\
\hline (f) & $* \mathrm{u}: \mathrm{c}$ & --- & *u:c & ${ }^{*} \mathrm{u}: \mathrm{c}$ \\
\hline (g) & *uak (a) & *uk & ${ }^{*} \mathrm{u}: \mathrm{k}$ & $*^{\mathrm{u}}: \mathrm{k}$ \\
\hline (h) & *uy & *oy & *uy & *uy \\
\hline (i) & *un & *on & *un & *un \\
\hline (j) & --- & --- & --- & *un \\
\hline (k) & *oy & --- & *uy & *up \\
\hline (1) & *ut & --- & $* u t$ & \\
\hline (m) & *uc & $*_{\mathrm{ot}}$ & $*_{\mathrm{uc}}$ & \\
\hline
\end{tabular}

For long rimes, Thurgood reconstructs a similar pattern to the one proposed here, with the exception of the lack of a reconstruction in (118c) and the reconstruction of a diphthong in (118g). Those rimes marked with an (a) are in contrast with the same rime reconstructed in other sections. The reconstruction of *uak violates both Symmetry and Directionality, since a change from *u:k $>$ uak is much more likely than one from *uak $>u: k$. For short rimes, Thurgood's reconstruction is again similar to the present one, with the exception that he presents no reconstruction in (118j) (mirroring the lack of one in 
$(118 \mathrm{c}))$, and the reconstruction of *oy where I reconstruct *uy. This is likely due to the fact that the series with final palatal nasals are very rare - only three examples of long rimes, and four examples of short.

Ostapirat's (1993) reconstruction remains balanced under his guiding assumptions, but he misses several correspondences sets, failing to reconstruct anything for three out of four series where I reconstruct final palatals, as well as the tenth through twelfth series of correspondences. He amends this to a large degree in his (2004) reconstruction, which is identical with the one presented here except for the fact that the rimes ending in palatal nasals are still not reconstructed.

Examples of closed rimes with high back rounded vowels are given below, in the following order:

$\begin{array}{llllll}\text { Bhin } & \text { Ha Em } & \text { Lhut } & \text { Tzha } & \text { Zdui } & \text { Bting } \\ \text { Cun } & \text { Nadou } & \text { Cjiang } & \text { Mfaw } & \text { Baisha } & \text { Ymen }\end{array}$

(119) Examples of closed rimes with high back rounded vowels

(a)

鞭子

*u:y

whip

tsu: $y^{3} \quad$ tsu: $y^{3}$ tsuy

棉花 cotton

bu: $y^{3} \quad 6 u: y^{3}$ (6u:(y) $\left.)^{3}\right) 6 u y^{3}$

肥胖 fat

ru: ${ }^{3}$ gu:y

huy $^{4} \quad$ vuy $^{3}$

tsu:y ${ }^{3}$

tsuy

$$
6 u: y^{3}
$$

buy ${ }^{3}$

bu: $y^{3}$

buy ${ }^{3}$

gwey $^{3}$

gu: ${ }^{6}$
xuy $^{3}$ tçu:y?

tsu: ${ }^{3}$

*6u:y?

bu:y

*ru:y?

buy $^{3}$

6u: $y^{3}$

buy ${ }^{3}$

tsu: $y^{3}$

tuy $^{3}$

(b)

賊 thief

buy

boy ${ }^{1}$

buy

boy $^{1}$

腐爛

rotten

buy

buy $^{1}$

buy

buy ${ }^{1}$

boy $^{1} \quad$ bow $^{1}$

$\mathrm{t}^{\mathrm{h}} \mathrm{uy}^{1}$

$\mathrm{t}^{\mathrm{h}} \mathrm{uy}^{1}$

thuy $^{\text {h }}$

$\mathrm{t}^{\mathrm{h}}$ oy

$t^{\text {h }}$ uy $^{1}$

醉

drunk

$* t^{\mathrm{h}}$ uy

$\mathrm{t}^{\mathrm{h}} \mathrm{uy}$

$t^{\text {h }}{ }^{1}{ }^{1}$

thuy

$t^{\text {h }}{ }^{1}$

क

hu:y
xuy $^{3}$

hu: ${ }^{6}$

muy

puy

poy? $^{4}$ puy $^{1}$ *hmuy

$$
\text { puy }^{4} \quad \text { p }^{\text {h }}{ }^{4}{ }^{4} \quad \text { puy }^{4}
$$

puy $^{1}$ poy $^{1}$ pow $^{4}$ 
(c)

野

tu: tu: ${ }^{1}$ tu: $n^{1}$ tOuən ${ }^{1}$ fun $^{1} \quad \sup ^{1}$

身體 body

*Cufu:n

(d)

官ｏfficial

tu: $n^{1}$

tu:n ${ }^{1}$

----

mun

mu

mun $^{1}$ mun $^{1}$ muen $^{1}$ mon $^{1}$

語言ｌanguage

$* \mathrm{t}^{\mathrm{h}} \mathrm{un}$

$$
\text { hu: }
$$
juon $^{1}$ jun $^{1}$ yun

先/從前 first/in front

$* \mathrm{k}^{\mathrm{h}} \mathrm{u}: \mathrm{nh}$

毛 fur

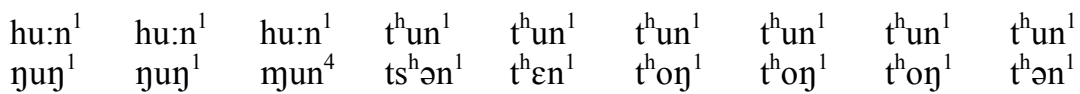

$\mathrm{k}^{\mathrm{h}} \mathrm{u} \cdot \mathrm{n}^{2}$ $\mathrm{k}^{\mathrm{h}} \mathrm{u}: \mathrm{n}^{5}$ $\mathrm{k}^{\mathrm{h}} \mathrm{u}: \mathrm{n}^{5}$ $\mathrm{k}^{\mathrm{h}} \mathrm{uan}^{5}$ k u:n $n^{2} \quad k u: n^{2}$ $\mathrm{k}^{\mathrm{h}} \mathrm{un}^{2}$ $\mathrm{k}^{\mathrm{h}} \mathrm{un}^{2}$

$\mathrm{k}^{\mathrm{h}} \mathrm{u}: \mathrm{n}^{\mathrm{s}}$

hun ${ }^{1}$

hun $^{1}$ $\eta \varepsilon n^{1}$

(f)

*u:t

Pu:t

消除

eliminate

hun
yoy $^{1}$

*Cuhun

發节

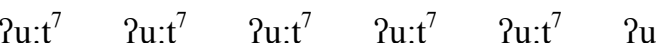

Puət $^{2} \quad$---- $\quad \mathrm{Pu}^{7} \quad \mathrm{Puk}^{7} \quad$---- $\quad$----

織 weave (net) $\quad * k^{\mathrm{h}} \mathrm{u} \cdot \mathrm{t}$

$k^{\mathrm{h}} \mathrm{u}: \mathrm{t}^{7} \quad \mathrm{k}^{\mathrm{h}} \mathrm{u}: \mathrm{t}^{7} \quad \mathrm{k}^{\mathrm{h}} \mathrm{u} \cdot \mathrm{t}^{7}$

$\mathrm{k}^{\mathrm{h}} \mathrm{uat}^{2} \quad---\quad \mathrm{k}^{\mathrm{h}} \mathrm{u}^{7}$

$\mathrm{k}^{\mathrm{h}} \mathrm{u} \cdot \mathrm{t}^{7}$

$\mathrm{k}^{\mathrm{h}} \mathrm{uk}^{7}$

$k^{h} u: t^{7}$

$\mathrm{k}^{\mathrm{h}} \mathrm{uk}^{8}$

$k^{h} u: t^{7} \quad p^{h} u t^{7}$

曾祖父 pat. grt grndpa

$\mathrm{p}^{\mathrm{h}} \mathrm{ut}^{7} \quad \mathrm{p}^{\mathrm{h}} \mathrm{ut}$

\section{$\mathrm{hut}^{7}$}

$\mathrm{put}^{\mathrm{h}}$

hun ${ }^{1}$

non ${ }^{1}$

hun $^{1}$ hun $^{1}$ yoy $^{1}$ mən $^{4}$

細 thin $\quad$ u

Pu:t

釘 to nail

$* \mathrm{t}^{\mathrm{h}} \mathrm{ut}$

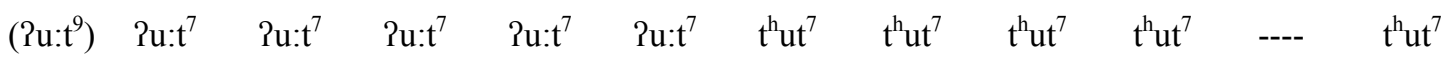
--- $\quad \mathrm{Pu}^{4} \quad \mathrm{Pu}^{7} \quad \mathrm{Puk}^{7} \quad \mathrm{Puk}^{8} \quad \mathrm{Put}^{7} \quad$---- $\quad$--- $\quad$---- $\quad \mathrm{t}^{\mathrm{h}} \mathrm{ok}^{7} \quad\left(\mathrm{t}^{\mathrm{h}} \mathrm{ok}^{7}\right) \quad \mathrm{t}^{\mathrm{h}} \partial \mathrm{t}^{7}$ 


\begin{tabular}{|c|c|c|c|c|c|c|c|c|c|c|c|}
\hline (g) & & & ${ }^{*} \mathrm{u}: \mathrm{n}$ & & & (h) & & & *un & & \\
\hline 脫 & take off & & *ku:n & & & 逃脫 & get awa & & ${ }^{*}$ C-lun? & & \\
\hline ku:n ${ }^{1}$ & ku:n ${ }^{1}$ & ku: ${ }^{1}$ & ku:n ${ }^{1}$ & ku: ${ }^{1}$ & ku: ${ }^{1}$ & $\operatorname{lun}^{3}$ & $\operatorname{lun}^{3}$ & $\operatorname{lun}^{3}$ & $\operatorname{lun}^{3}$ & $\left(\operatorname{lun}^{3}\right)$ & $\operatorname{lum}^{3}$ \\
\hline ---- & ---- & ---- & kun $^{1}$ & kun $^{1}$ & kun $^{1}$ & ---- & ---- & $\operatorname{lun}^{3}$ & $\operatorname{lon}^{3}$ & $\operatorname{lon}^{3}$ & $\operatorname{lon}^{6}$ \\
\hline 穿插 & & & $*_{\text {su:n }}$ & & & 背脊 & spine & & $* t^{\mathrm{h}}{ }^{\mathrm{h}} \mathrm{un} ?$ & & \\
\hline tu: $n^{1}$ & tu: ${ }^{1}$ & tu: ${ }^{1}$ & tu: ${ }^{1}$ & tu: $\mathrm{n}^{1}$ & tu: $\mathrm{n}^{1}$ & $\mathrm{ts}^{\mathrm{h}} \mathrm{un}^{3}$ & $\mathrm{ts}^{\mathrm{h}} \mathrm{un}^{3}$ & $\operatorname{ts}^{\mathrm{h}} \mathrm{un}^{3}$ & $\mathrm{ts}^{\mathrm{h}} \mathrm{un}^{3}$ & $\mathrm{ts}^{\mathrm{h}} \mathrm{un}^{3}$ & $\operatorname{ts}^{\mathrm{h}} \mathrm{un}^{3}$ \\
\hline ---- & ---- & $\operatorname{sun}^{1}$ & $\operatorname{sun}^{1}$ & ts $^{\mathrm{h}} \mathrm{n}^{1}$ & ts $^{\mathrm{h}} \mathrm{n}^{1}$ & ---- & $s \varepsilon n^{3}$ & ---- & $\operatorname{ts}^{\mathrm{h}} \mathrm{on}^{3}$ & $\operatorname{ts}^{\mathrm{h}} \mathrm{un}^{3}$ & $\operatorname{ts}^{\mathrm{h}} \mathrm{un}^{3}$ \\
\hline 下飯 & go with & & $*$ ?u:p & & & 噗 & & & * rjun? & & \\
\hline Pu:n ${ }^{1}$ & Pu: ${ }^{1}$ & Pwen $^{1}$ & Pu: $n^{1}$ & Pu: ${ }^{1}$ & Pu: ${ }^{1}$ & zun $^{3}$ & zun $^{3}$ & --- & $\operatorname{tun}^{6}$ & $t^{h} u n^{6}$ & $\operatorname{tun}^{6}$ \\
\hline ?uən ${ }^{1}$ & Pun $^{1}$ & Pon $^{1}$ & Pun $^{1}$ & Pun $^{1}$ & Pun $^{1}$ & ---- & ---- & $\left(\right.$ dun $\left.^{3}\right)$ & $\left(\tan ^{1}\right)$ & $\operatorname{ton}^{3}$ & $\operatorname{tən}^{3}$ \\
\hline (i) & & & $* u: c$ & & & (j) & & & $* u c$ & & \\
\hline 螢火蟲 & firefly & & *ku:c & & & 尾巴 & tail & & $*_{t} c^{h} u c$ & & \\
\hline ---- & ---- & $\mathrm{kuc}^{7}$ & $\mathrm{ku}: \mathrm{t}^{7}$ & ---- & ---- & $\mathrm{ts}^{\mathrm{h}} \mathrm{ut}^{\mathrm{7}}$ & ts $^{\mathrm{h}} \mathrm{ut}^{7}$ & $\mathrm{ts}^{\mathrm{h}} \mathrm{uc}^{7}$ & ts $^{\mathrm{h}} \mathrm{ut}^{7}$ & ts $^{\mathrm{h}} \mathrm{ut}^{7}$ & $\mathrm{ts}^{\mathrm{h}} \mathrm{utt}^{7}$ \\
\hline kuat $^{5}$ & ku2 $2^{5}$ & ---- & kut $^{7}$ & ---- & $\mathrm{kut}^{7}$ & $\mathrm{ts}^{\mathrm{h}} \partial \mathrm{t}^{2}$ & $\mathrm{~s} \varepsilon 2^{4}$ & $\mathrm{ts}^{\mathrm{h}} \partial \mathrm{t}^{7}$ & $\mathrm{ts}^{\mathrm{h}} \mathrm{ot}^{7}$ & $\mathrm{ts}^{\mathrm{h}} \mathrm{ot}^{8}$ & $\mathrm{ts}^{\mathrm{h}} \partial \mathrm{t}^{7}$ \\
\hline 瘊子 & wart & & $*_{\text {su:c }}$ & & & 螞蟻 & ant & & ${ }^{*}$ hmuc & & \\
\hline $\begin{array}{l}\text { tu: } t^{7} \\
\text { ts }^{h} u \partial t^{2}\end{array}$ & tu:t ${ }^{7}$ & tu: $c^{7}$ & $\begin{array}{l}\mathrm{tu}: \mathrm{t}^{7} \\
\mathrm{sut}^{7}\end{array}$ & ts $^{\mathrm{h}} \mathrm{ut}^{8}$ & $\begin{array}{l}\text { tu: } t^{7} \\
\text { ts }^{h} u^{7}{ }^{7}\end{array}$ & $\begin{array}{l}\mathrm{mut}^{7} \\
6 \partial t^{4}\end{array}$ & $\begin{array}{l}\text { put }^{7} \\
\text { pue } ?^{4}\end{array}$ & $\begin{array}{l}\text { puc }^{7} \\
\text { pət }^{7}\end{array}$ & $\begin{array}{l}\text { put }^{8} \\
\text { pot }^{7}\end{array}$ & $\begin{array}{l}p^{h} u t^{8} \\
\text { pot }^{8}\end{array}$ & $\begin{array}{l}\text { put }^{8} \\
\text { pot }^{8}\end{array}$ \\
\hline 吻 & kiss & & $*_{\text {rju:c }}$ & & & 針 & needle & & *hyuc & & \\
\hline$z u: t^{7}$ & $\mathrm{zu}: \mathrm{t}^{7}$ & $\mathrm{ru}: \mathrm{c}^{7}$ & $\mathrm{tu}: \mathrm{t}^{8}$ & $\left(\mathrm{tu}: \mathrm{t}^{8}\right)$ & tu: $t^{8}$ & put $^{7}$ & $\mathrm{kut}^{7}$ & $\mathrm{kuc}^{7}$ & $\mathrm{kut}^{8}$ & $\mathrm{k}^{\mathrm{h}} \mathrm{ut}^{8}$ & $\mathrm{kut}^{8}$ \\
\hline lu: $t^{2}$ & ---- & tut $^{7}$ & ---- & ---- & $\left(\right.$ tut $\left.^{7}\right)$ & ${\mathrm{k} \partial \mathrm{t}^{4}}^{4}$ & $\mathrm{k} \varepsilon \mathrm{P}^{4}$ & $\mathrm{k \partial t}^{7}$ & $\operatorname{kot}^{7}$ & $\operatorname{kot}^{8}$ & $\mathrm{k \partial t}^{8}$ \\
\hline
\end{tabular}




\begin{tabular}{|c|c|c|c|c|c|c|c|c|c|c|c|}
\hline (k) & & & *u:y & & & (1) & & & $*$ uy & & \\
\hline 䢃 & split & & *6u:y & & & (長)大 & big & & ${ }^{*}$ C-lur & & \\
\hline bu: $y^{1}$ & 6u: $y^{1}$ & bu: $y^{1}$ & bu: $y^{1}$ & buay $^{1}$ & bu: $y^{1}$ & $\operatorname{lug}^{1}$ & $\operatorname{luy}^{1}$ & $\log ^{1}$ & $\log { }^{1}$ & $\operatorname{lu\eta }^{4}$ & $\operatorname{luy}^{1}$ \\
\hline $6 o \eta^{1}$ & buy $^{1}$ & bun $^{1}$ & bun $^{1}$ & buy $^{1}$ & buy $^{1}$ & $\log ^{1}$ & $\log ^{1}$ & $\left(\operatorname{luy}^{1}\right)$ & $\operatorname{lon}^{1}$ & $\operatorname{luy}^{1}$ & $\operatorname{lun}^{4}$ \\
\hline 蚊子 & mosquit & & ${ }^{*}$ C-лu:y & & & 搖 & shake & & ${ }^{*}$ C-лn & & \\
\hline nu:y ${ }^{1}$ & nu: ${ }^{1}$ & nu:y ${ }^{1}$ & nu: $\eta^{1}$ & nuay $^{4}$ & nu:y ${ }^{1}$ & nog $^{2}$ & nuy $^{2}$ & nog $^{2}$ & nog $^{5}$ & nuy $^{2}$ & nuy $^{5}$ \\
\hline noy $^{1}$ & $\operatorname{niw}^{1}$ & nuy $^{1}$ & nuy $^{1}$ & nuy $^{1}$ & nun $^{4}$ & ---- & ---- & non $^{2}$ & noy $^{2}$ & nюn ${ }^{2}$ & nюท ${ }^{2}$ \\
\hline 洞 & hole & & $*_{\mathrm{t}} \boldsymbol{h}^{\mathrm{h}} \mathrm{u}: \mathrm{\eta} ?$ & & & 圈 & anima & pen & ${ }^{*} \mathrm{C}-\mathrm{lu}$ & & \\
\hline $\operatorname{ts}^{\mathrm{h}} \mathrm{u}: \mathrm{y}^{3}$ & $\mathrm{ts}^{\mathrm{h}} \mathrm{u}: \mathrm{y}^{3}$ & $\operatorname{ts}^{\mathrm{h}} \mathrm{u}: \mathrm{y}^{3}$ & ts $^{h} \mathrm{u}: y^{3}$ & ts $^{\text {h }}$ uay $^{3}$ & $\operatorname{ts}^{h} \mathrm{u}: \eta^{3}$ & $\operatorname{lun}^{2}$ & $\operatorname{lun}^{2}$ & $\operatorname{lo\eta }^{2}$ & $\log ^{5}$ & $\operatorname{lun}^{2}$ & $\operatorname{lun}^{5}$ \\
\hline $\mathrm{ts}^{\mathrm{h}} \mathrm{oy} \mathrm{y}^{3}$ & $\operatorname{sun}^{3}$ & $\mathrm{ts}^{\mathrm{h}} \mathrm{uy}^{3}$ & $\mathrm{ts}^{\mathrm{h}} \mathrm{u} \mathrm{y}^{3}$ & $\mathrm{ts}^{\mathrm{h}} \mathrm{u} \mathrm{y}^{3}$ & $\mathrm{ts}^{\mathrm{h}} \mathrm{uy}^{3}$ & ---- & $\log ^{2}$ & $\log ^{2}$ & $\log ^{2}$ & $\log ^{2}$ & $10 \eta^{2}$ \\
\hline (m) & & & $* \mathbf{u}: \mathbf{k}$ & & & & & & & & \\
\hline 窩 & nest & & *ru:k & & & & & & & & \\
\hline $\mathrm{ru}: \mathrm{P}^{7}$ & $\mathrm{ru}: \mathrm{P}^{7}$ & $\mathrm{ru}: \mathrm{k}^{7}$ & $\mathrm{ru}: \mathrm{P}^{8}$ & lua? ${ }^{8}$ & lu: $:^{8}$ & & & & & & \\
\hline $\operatorname{lok}^{4}$ & $\operatorname{lu} 1^{4}$ & $\operatorname{lu} 1^{7}$ & $\operatorname{ruk}^{7}$ & $\mathrm{ruk}^{8}$ & $\operatorname{ru}^{8}$ & & & & & & \\
\hline 包 & wrap & & $* \mathrm{t}^{\mathrm{h}} \mathrm{u}: \mathrm{k}$ & & & & & & & & \\
\hline$t^{h} u: ?^{7}$ & $t^{\mathrm{h}} \mathrm{u}: \mathrm{P}^{7}$ & $\mathrm{t}^{\mathrm{h}} \mathrm{u}: \mathrm{k}^{7}$ & $\mathrm{t}^{\mathrm{h}} \mathrm{u}: ?^{7}$ & $\mathrm{t}^{\mathrm{h}} \mathrm{ua} ?^{7}$ & $\mathrm{t}^{\mathrm{h}} \mathrm{u}: \mathrm{P}^{7}$ & & & & & & \\
\hline $\mathrm{ts}^{\mathrm{h}} \mathrm{ok}^{2}$ & $\mathrm{t}^{\mathrm{h}} \mathrm{u} \mathrm{P}^{4}$ & $\mathrm{t}^{\mathrm{h}} \mathrm{u} \mathrm{P}^{7}$ & $\mathrm{t}^{\mathrm{h}} \mathrm{uk}^{7}$ & $\mathrm{t}^{\mathrm{h}} \mathrm{uk}^{8}$ & $\mathrm{t}^{\mathrm{h}} \mathrm{u} \mathrm{P}^{7}$ & & & & & & \\
\hline 腦 & brain $^{28}$ & & *hlu:k & & & & & & & & \\
\hline du: $?^{7}$ & tu: $?^{7}$ & $\mathrm{tu}: \mathrm{k}^{7}$ & tu: $?^{7}$ & tua? ${ }^{7}$ & tu: $: ?^{7}$ & & & & & & \\
\hline $\mathrm{t} \theta \mathrm{ok}^{2}$ & nu? ${ }^{4}$ & $\mathrm{tu}^{7}$ & $\mathrm{uuk}^{7}$ & $\operatorname{tuk}^{8}$ & $\operatorname{tu}^{7}$ & & & & & & \\
\hline
\end{tabular}

\subsubsection{Interim Summary}

A total of three groups of rimes with high vowels have been reconstructed here: those with high front nuclei, those with high back unrounded nuclei, and those with high back rounded nuclei. Each of

\footnotetext{
${ }^{28}$ The Nadouhua form in this lexical set is irregular due to apparent spread of nasalization from the preceding member of the compound it is a part of, $y:^{3}{ }^{3}$ nu ${ }^{4}$ (literally 'head-brain').
} 
these series in turn has two subsets of rimes: those with long nuclei and those with short nuclei. The rimes which have been reconstructed in this section are repeated below:

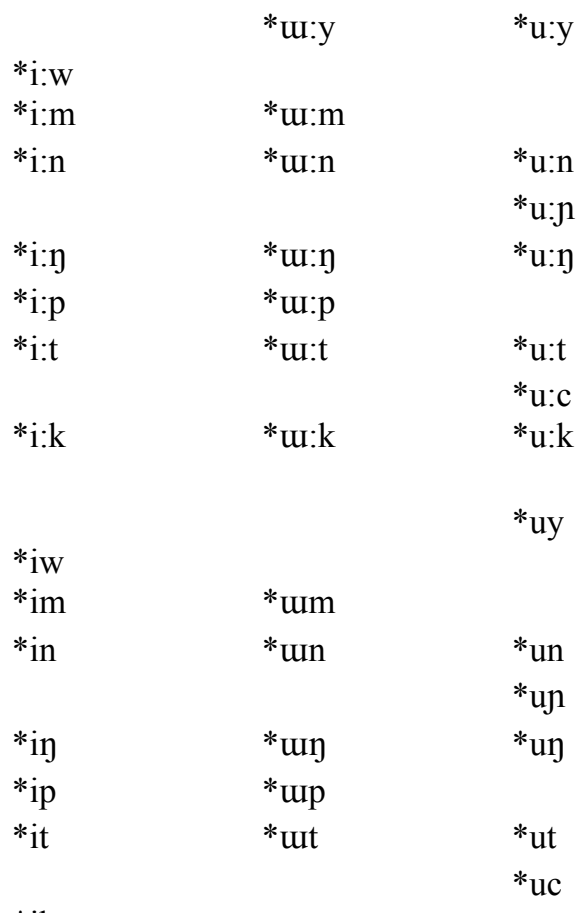

$* \mathrm{ik}$

There are two notable asymmetries in the rimes shown above, where the rimes with unrounded nuclei pattern in opposition to the rimes with rounded nuclei. The first is that unrounded nuclei can be followed by bilabial codas, but rounded nuclei cannot; the second is that rounded nuclei can be followed by palatal codas, but unrounded nuclei cannot (with the exception of the very marginal rime *u:y, which has only one example). In other words, there are natural co-occurrence restrictions preventing round vowels before bilabial codas, and non-round vowels before palatal codas

In addition to this, short rimes with velar stop codas are strongly disfavored (although *ik is reconstructible, it is very marginal in the actual lexical inventory). It is possible that original short rimes with high vowels underwent lengthening before velar stops, but this must remain speculation unless new evidence surfaces in favor of this hypothesis. 


\subsection{Closed Rimes with Non-High Vowels}

This section is subdivided into the following four subsections: closed rimes with front mid vowels (4.5.1), closed rimes with central mid vowels (4.5.2), closed rimes with back mid vowels (4.5.3), and finally, closed rimes with low vowels (4.5.4).

\subsubsection{Closed Rimes with Front Mid Vowels}

Although the patterns are robust enough to merit reconstruction, this category has far fewer examples than most other categories (as is the case with open front mid rimes in section 3.3 above). It is difficult to say whether or not there are velar-final series represented in this category, due to the fact that the $* \mathrm{a}: \mathrm{K}$ series and the putative $* \mathrm{e}: \mathrm{K}$ series have merged in all languages except for Bouhin, and words in Bouhin with an $* \mathrm{e}: \mathrm{K}$ rime may be loans from Ha Em (see section 4.5.4). Given the low frequency of the other rimes, it is statistically likely that there are a few actual $*_{\mathrm{e}} \mathrm{K}$ rimes which can be reconstructed, but that the majority of rimes in which Bouhin has an *e:K reflex are probably *a:K rimes where Bouhin has borrowed from Ha Em. All cases of *e:K rimes below will therefore be placed in parentheses, and reconstructions will place the vowel in brackets to indicate its indeterminacy.

(121) Reflexes of closed rimes with mid front vowels

\begin{tabular}{|c|c|c|c|c|c|c|c|c|c|c|c|}
\hline BHi:n & HaE:m & $\underline{\text { LHu:t }}$ & $\underline{\text { Tzha }}$ & $\underline{\text { Zdui }}$ & Bting & $\underline{\text { Cun }}$ & $\underline{\mathrm{Nadou}}$ & Cjiang & $\underline{\text { Mfaw }}$ & $\underline{\text { Baisha }}$ & $\underline{\text { Ymen }}$ \\
\hline e:w & e:w & e:w & e:w & e:w & e:w & ew & $\varepsilon \mathrm{W}$ & e:w & e:w & iaw & i:w \\
\hline e:m & $\mathrm{e}: \mathrm{m}$ & e:m & e:m & e:m & e:m & ---- & ---- & ---- & e:m & em & em \\
\hline e:p & e:p & e:p & e:p & e:p & e:p & $\varepsilon p$ & ---- & e:p & e:p & iap & ---- \\
\hline $\mathrm{e}: \mathrm{n}$ & e:n & e:n & e:n & e:n & e:n & $\varepsilon n$ & en & e:n & e:n & ian & i:n \\
\hline ---- & ---- & ---- & ---- & ---- & ---- & et & e? & ---- & ---- & et & $\mathrm{i}: \mathrm{t}$ \\
\hline (e:n & $e: \eta$ & e:y & e:n & e:y & e:n & $\varepsilon \eta$ & $\varepsilon \eta$ & e:y & e:y & ian & iay) \\
\hline (e:? & $\mathrm{e}: ?$ & e:k & $\mathrm{e}: ?$ & $\mathrm{e}: ?$ & e:? & $\varepsilon \mathrm{k}$ & $\varepsilon ?$ & $\mathrm{e}: ?$ & ш: & e? & ia?) \\
\hline
\end{tabular}


S. Hlai (Savina)

----
$\varepsilon \mathrm{m}$
$\varepsilon p$
----
---
$(\varepsilon \eta$
$(\varepsilon:$

C. Hlai (Savina)

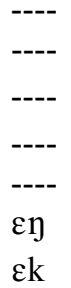

Baisha (Wang \& Qian)

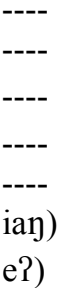

The reconstructions proposed for these correspondence series are given below:

$$
\begin{aligned}
& *_{\mathrm{e}} \mathrm{\textrm {w }} \\
& *_{\mathrm{e}} \mathrm{\textrm {m }} \\
& *_{\mathrm{e}} \mathrm{p} \\
& *_{\mathrm{e}} \mathrm{e} \mathrm{n} \\
& *_{\mathrm{e}} \mathrm{e} \mathrm{t} \\
& \left({ }^{*} \mathrm{e}: \mathrm{y}\right) \\
& \left({ }^{*} \mathrm{e}: \mathrm{k}\right)
\end{aligned}
$$

There have been few changes in these rimes, and those which have occurred all fall within NCHI. In NWCHl, there was a shortening which happened for all rimes:

$$
* \mathrm{e}: \mathrm{C} \quad \mathrm{eC} / \varepsilon \mathrm{C}
$$

In Baisha, the following asymmetrical development occurred:

$\begin{array}{rllll}* \text { (124): } & > & \varepsilon: \mathrm{w} & > & \text { iaw } \\ * \mathrm{e}: \mathrm{m} & > & \text { em } & > & \text { em } \\ * \mathrm{e}: \mathrm{p} & > & \varepsilon: \mathrm{p} & > & \text { iap } \\ * \mathrm{e}: \mathrm{n} & > & \varepsilon: \mathrm{n} & > & \text { ian } \\ * \mathrm{e}: \mathrm{t} & > & \text { et } & > & \text { et } \\ (* \mathrm{e}: \mathrm{n} & > & \varepsilon: \mathrm{n} & > & \text { iap }) \\ (* \mathrm{e}: \mathrm{k} & > & \text { ek } & > & \text { e? })\end{array}$

In Yuanmen, the bilabial-final rime was shortened, but the diphthong and alveolar-final rimes remained long and underwent raising; the velar final rimes (if legitimate) lowered and diphthongized: 
$(125)$

$\begin{array}{lll}* \mathrm{e}: \mathrm{w} & > & \text { i:w } \\ * \mathrm{e}: \mathrm{m} & > & \text { em } \\ * \mathrm{e}: \mathrm{p} & > & \text { (no examples) } \\ * \mathrm{e}: \mathrm{n} & > & \text { i:n } \\ * \mathrm{e}: \mathrm{t} & > & \text { i:t } \\ \left({ }^{*} \mathrm{e}: \mathrm{n}\right. & > & \text { ian }) \\ (* \mathrm{e}: \mathrm{k} & > & \text { ia? })\end{array}$

No comparison of reconstructions is given here, since neither Thurgood nor Ostapirat reconstruct anything for these series of rimes, the one exception being the putative *e:K series, which Ostapirat (1993) reconstructed as *-j-ay and *-j-ak, respectively, and the nasal final member for which Thurgood reconstructs *i:y.

Examples of these rimes are given below, in the following order:

$\begin{array}{llllll}\text { Bhin } & \text { Ha Em } & \text { Lhut } & \text { Tzha } & \text { Zdui } & \text { Bting } \\ \text { Cun } & \text { Nadou } & \text { Cjiang } & \text { Mfaw } & \text { Baisha } & \text { Ymen }\end{array}$

(126) Examples of closed rimes with mid front vowels

\begin{tabular}{|c|c|c|c|c|c|}
\hline (a) & & & ${ }^{*} \mathrm{e}: \mathrm{w}$ & & \\
\hline 鐵鏟 & iron sl & vel & $* \mathrm{t}^{\mathrm{h}} \mathrm{e}: \mathrm{w}$ & & \\
\hline & ---- & ---- & ---- & $\mathrm{t}^{\mathrm{h}} \mathrm{e}: \mathrm{w}^{1}$ & ---- \\
\hline & ---- & $\mathrm{t}^{\mathrm{h}} \mathrm{e}: \mathrm{w}^{1}$ & $\mathrm{t}^{\mathrm{h}} \mathrm{e}: \mathrm{w}^{1}$ & ---- & ---- \\
\hline 鈎 & knifeh & & *de:wh & & \\
\hline & de: $w^{2}$ & de: $w^{2}$ & ---- & ---- & ---- \\
\hline & ---- & ---- & de: $w^{2}$ & ---- & ---- \\
\hline & parrot & (reen) & *de:wh & & \\
\hline$: \mathrm{w}^{2}$ & de: $w^{2}$ & de: $w^{2}$ & de: $w^{5}$ & de: $w^{5}$ & de:w \\
\hline & ---- & de: $w^{2}$ & de: $w^{2}$ & diaw $^{2}$ & $d i: w^{5}$ \\
\hline
\end{tabular}


(b) $\quad *$ e:m $\quad$ (c) *e:p

癬 ringworm 旗 sunken -le:mhe:p

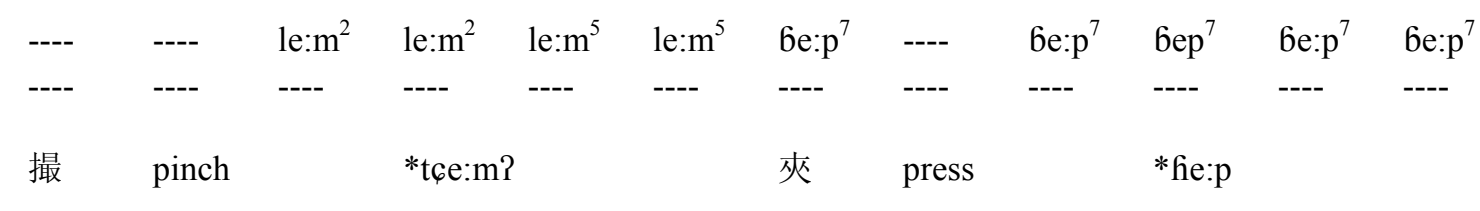

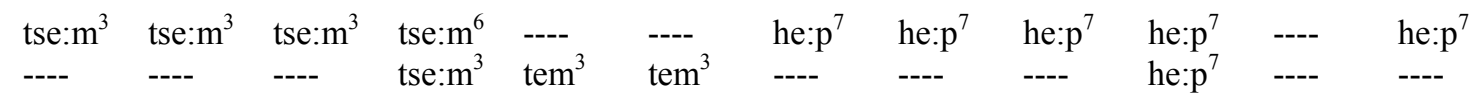

疊 pile (clfr) $\quad *$ C-le:p

$\begin{array}{llllll}\text { le: } p^{7} & \text { le: } p^{7} & \text { le: } p^{7} & \text { le: } p^{7} & --- & \text { le: } p^{7} \\ --- & --- & --- & --- & \text { liap }^{7} & ---\end{array}$

(d)

扁 flat

$6 e: n^{2} \quad 6 e \cdot n^{2} \quad 6 e \cdot n^{2}$

$6 \mathrm{En}^{2} \quad 6 \mathrm{en}^{2} \quad----$

木板 board *6e:nh

be: $n^{2} \quad$ be: $n^{2} \quad$ be: $n^{2} \quad$ be: $: n^{5} \quad$ be $: n^{5} \quad$ be: $n^{5}$

--- $\quad 6 n^{2} \quad 6 e: n^{2} \quad 6 e: n^{2} \quad----\quad---$

(f)

寬 $\quad$ wide $\quad * 6[\mathrm{e}]: \mathrm{y}$

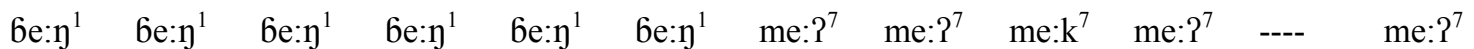

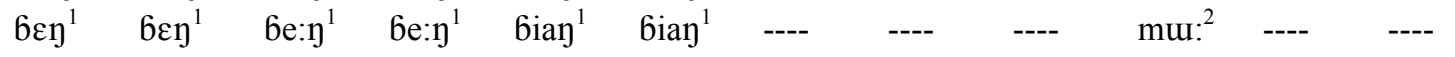

甜 sweet $*$ 干 $[\mathrm{e}]: \mathrm{n}$ 飯 dry rice ${ }^{*} \mathrm{C}-\mathrm{m}[\mathrm{e}]: \mathrm{k}$

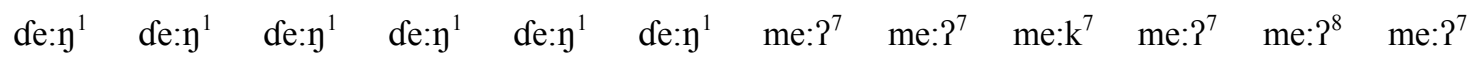

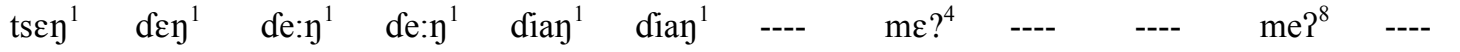

上衣 clothes $\quad * \mathrm{v}[\mathrm{e}]: \mathrm{n}$ 尋找 search $\quad * \mathrm{k}[\mathrm{e}]: \mathrm{k}$

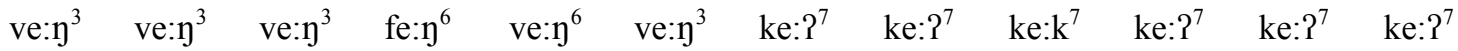

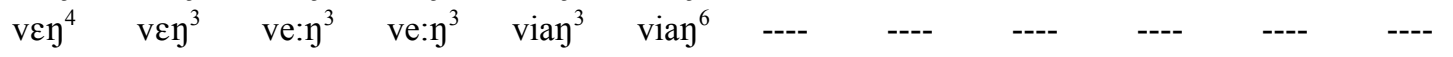




\subsubsection{Closed Rimes with Mid Central Vowels}

As in the case of rimes with high nuclei, there are both long and short rimes with mid central vowels, reflexes of which are given below:

(127) Reflexes of closed rimes with mid central vowels

\begin{tabular}{|c|c|c|c|c|c|c|c|c|c|c|c|}
\hline BHin & HaEm & $\underline{\text { LHut }}$ & Tzha & $\underline{\text { Zdui }}$ & Bting & $\mathrm{Cun}^{29}$ & $\underline{\text { Nadou }}$ & Cjiang & Mfaw & $\underline{\text { Baisha }}$ & $\underline{\text { Ymen }}$ \\
\hline a:y & o:y & o:y & a:y & a:y & a:y & o:(y) & әу & $0: y$ & o:y & uay & u:y \\
\hline am & $\mathrm{o}: \mathrm{m}$ & $\mathrm{o}: \mathrm{m}$ & $\mathrm{o}: \mathrm{m}$ & o:m & o:m & am & on & $\mathrm{o}: \mathrm{m}$ & $\mathrm{o}: \mathrm{m}$ & uam & uam \\
\hline an & o:n & o:n & o:n & o:n & o:n & an & on & $0: 1$ & o:y & uay & $\mathrm{u}: \mathrm{n}$ \\
\hline an & $0: 1$ & $0: \eta$ & $0: 1$ & $0: 1$ & $0: 1$ & ay & oy & $0: 1$ & $0: \eta$ & uan & uan \\
\hline ap & $o: p$ & $o: p$ & $o: p$ & o:p & o:p & ap & ว? & $o: p$ & $o: p$ & uap & uap \\
\hline at & o:t & o:t & o:t & o:t & o:t & at & o? & $\mathrm{o}: ?$ & $\mathrm{o}: \mathrm{k}$ & o? & $\mathrm{u}: \mathrm{t}(\sim \partial \mathrm{t}$ \\
\hline ak & $\mathrm{o}: ?$ & $\mathrm{o}: \mathrm{k}$ & o:? & ๑:? & ๑:? & ak & o? & o:? & $\mathrm{o}: \mathrm{k}$ & o? ว? & o? ว? \\
\hline ay & ay & ay & ay & ay & ay & ay & ay & ay & ay & ay & ay \\
\hline $\begin{array}{l}\text { aw } \\
\mathrm{aw}^{\mathrm{B} / \mathrm{C}}\end{array}$ & $\begin{array}{l}\mathrm{aw} \\
\mathrm{aw} \\
\mathrm{B} / \mathrm{C}\end{array}$ & $\begin{array}{c}\text { aw } \\
\text { ow/aw }\end{array}$ & $\begin{array}{l}\text { aw } \\
\mathrm{o}^{\mathrm{B} / \mathrm{C}}\end{array}$ & $\begin{array}{l}\text { aw } \\
\mathrm{o}:^{B / C}\end{array}$ & $\begin{array}{l}\text { aw } \\
o:^{B / C}\end{array}$ & $\begin{array}{l}\mathrm{aw} \\
\mathrm{aw} \\
\mathrm{B} / \mathrm{C}\end{array}$ & $\begin{array}{l}\text { aw } \\
o:^{B / C}\end{array}$ & $\begin{array}{l}\text { aw } \\
\mathrm{o}^{\mathrm{B} / \mathrm{C}}\end{array}$ & $\begin{array}{l}\text { aw } \\
\mathrm{o}^{\mathrm{B} / \mathrm{C}}\end{array}$ & $\begin{array}{l}\text { aw } \\
\mathrm{o}^{\mathrm{B} / \mathrm{C}}\end{array}$ & $\begin{array}{l}\text { aw } \\
\mathrm{o}^{\mathrm{B} / \mathrm{C}}\end{array}$ \\
\hline om & $\mathrm{am}$ & om & $\mathrm{am}$ & am & am & $\mathrm{am}$ & an & am & $\mathrm{am}$ & am & $\mathrm{am}$ \\
\hline en & an & an & an & an & an & on $/ \mathrm{an}^{\mathrm{B} / \mathrm{C}}$ & an & an & ay & an & an \\
\hline an & an & an & an & an & an & ian & an & an & an & an & an \\
\hline on & an & an & an & an & an & ey & an & ay & an & ay & oท \\
\hline op & ap & op & op & ap & ap & ap & $\mathrm{a} ?$ & ap & ap & ap & ap \\
\hline et & at & at & at & at & at & at & $\mathrm{a} ?$ & a? & ak & ak & at \\
\hline at & at & $\mathrm{ac}$ & at & at & at & iat & $\mathrm{a} ?$ & at & at & at & at \\
\hline ok & ak & ak & ak & ak & ak & ak & a? & ---- & $\mathrm{ok} \sim \mathrm{a}: \mathrm{k}$ & ak & ak \\
\hline
\end{tabular}

\footnotetext{
${ }^{29}$ The parentheses around the final glides in Cunhua indicates that these glides are pronounced in connected speech, but deleted in isolation. See Ouyang (1998: 19).
} 
S. Hlai (Savina)

ay

am

en

ay

ap

et

ek

ay

aw

aw

om

en

an

on

op

et

at
C. Hlai (Savina)

ay

om

วn

ว1

ว:

ay

aw

o:

am

an

$---$

an

ap

at

----
Baisha (Wang \& Qian)

uay

(om)

----

uan

uap

u:?

ay

ew

o:

am

aj

an

ap

ak

at

The reconstructions proposed here for these correspondences are the following:

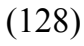

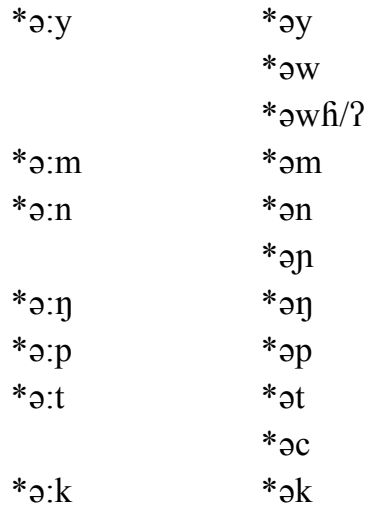

\subsubsection{Long Rimes with Mid Central Vowels}

In the case of the one long diphthong, the nucleus either lowered, backed and rounded, or in a few cases did both. In Cunhua, *ə:y would have been expected to lower to a:y; however, the failure of original 
PHI *a:y to back to ::y apparently prevented this, forcing a shift instead of *a:y to o:y. Both diphthongs lost their glide in isolation, retaining it only in connected speech as the first member of a compound word:

\begin{tabular}{|c|c|c|}
\hline * ə:y & $>$ & ๑:y \\
\hline *a:y & $>$ & $a: y$ \\
\hline
\end{tabular}

The most common change in the stop-closed rimes was backing/rounding to long $0:$; this failed to occur only in Cunhua and Bouhin:

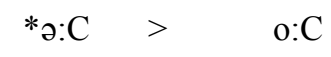

The nucleus in Zandui, Baoting, and Nadouhua lowered to o::

$\mathrm{o:C} \quad>\quad$ o:C

There were unique developments in the Run branch, which paralleled those of the front mid rimes in the last section. Although there were parallel developments in Baisha and Yuanmen in the labial-final and velar-final rimes, the development of the alveolar-final rimes was quite different. In Baisha (133a), the alveolar-nasal final rimes merged with the velar-nasal final rimes (only partial merger occurred in the case of original *ə:t, as variation between o? and $ว ?$ developed in the rime which backed from original $\left.*_{\curvearrowright}: k\right)$. In Yuanmen (133b), on the other hand, the alveolar-final rimes remained distinct from the velars after the backing of the vowel, and then underwent the raising of long mid-vowels which occurred throughout the Yuanmen rime inventory. There are two examples which have ət, in which case it can be assumed that Yuanmen o:t shortened to ot, which then underwent the regular change to $ə t$ : 
(132) Evolution of *ə:C in Run

(a) Baisha

\begin{tabular}{|c|c|c|c|c|}
\hline *ə:y & $>$ & ๑:y & $>$ & uay \\
\hline *ə:m & $>$ & ๑:m & $>$ & uam \\
\hline *ə:n & $>$ & ๑:ท & $>$ & uay \\
\hline *a:y & $>$ & ๑:y & $>$ & uan \\
\hline a.p & $>$ & ๑:p & $>$ & uap \\
\hline & $>$ & $\mathrm{o}: \mathrm{k}$ & $>$ & o? \\
\hline & $>$ & $\mathrm{o}: \mathrm{k} \sim 0: \mathrm{k}$ & $>$ & o? 0? \\
\hline
\end{tabular}

(b) Yuanmen

\begin{tabular}{|c|c|c|c|}
\hline *ə:y & $>$ & $0: y$ & u:y \\
\hline *a:m & $>$ & ๑:m & uam \\
\hline *ə:n & $>$ & o:n & u:n \\
\hline *ə:y & $>$ & o:y & uay \\
\hline ə:p & $>$ & ๑:p & uap \\
\hline *ə:t & $>$ & $o: t(\sim 0 t)>$ & u:t $(\sim \partial t)$ \\
\hline *ə:k & $>$ & $\mathrm{o}: \mathrm{k} \sim 0: \mathrm{k}>$ & о? ว? \\
\hline
\end{tabular}

Finally, there was a non-identical development in both Cunhua and Bouhin which had the same end result, leading to the merger in several categories between long *ə:C and short *əC. In Cunhua (133a), *ə:C lowered to a:C, and then shortened. In Bouhin (133b), * ${ }^{*}: \mathrm{C}$ first shortened to $ə \mathrm{C}$, and then lowered:

(133) Shortening and lowering of *ə:C in Cunhua and Bouhin

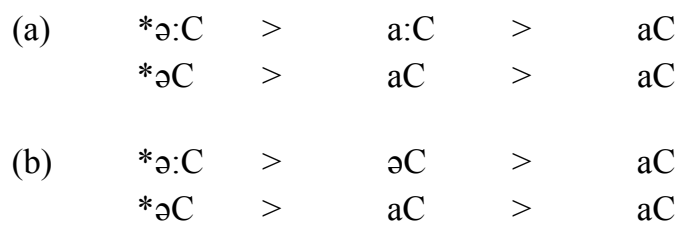

\subsubsection{Short Rimes with Mid Central Vowels}

The normal trajectory for the short diphthongs in tone category A was for the nucleus to lower to $a$ : 


$\begin{array}{lll}\text { *əy } & > & \text { ay } \\ \text { *əw } & > & \text { aw }\end{array}$

There are no reconstructible $\mathrm{PHl}$ rimes of the type * əyh or * əy? (see the next chapter for a possible explanation involving changes in Pre-Hlai). The development of *əwh/ 2 was rather different, in that monophthongization occurred in Qi as well as in $\mathrm{NCHl}$ :

$\begin{array}{lll}\text { *owh } & > & \text { o:h } \\ \text { *əw? } & > & \text { o:? }\end{array}$

The tone category-conditioned split in reflexes in Cunhua *əon was treated in section 4.2 above. Yet another unique development in Cunhua was that rimes with final velar nasals fronted and lengthened:

$$
\text { *əy } \quad>\quad \text { e:y }
$$

There was an interesting transfer in Cunhua of palatal features from the coda to the nucleus in rimes with palatal codas:

$\begin{array}{lllll}* \text { əл } & > & \text { an } & > & \text { ian } \\ * \partial \mathrm{c} & > & \text { ac } & > & \text { iat }\end{array}$

Yuanmen, Moyfaw, and Tongzha all have a single rime which underwent unpredicted backing and rounding, always before grave stops:

\section{Idiosyncratic backing and rounding}

$\begin{array}{llll}\text { Yuanmen: } & * \text { * } & > & * \text { o } \\ \text { Moyfaw: } & \text { *ək } & > & * \text { o } \\ \text { Tongzha: } & * ə p & > & \text { op }\end{array}$

Lauhut rimes backed and rounded when closed by a labial stop: 


$$
\begin{array}{lll}
* \text { *m } & > & \text { om } \\
* ə p & > & \text { op }
\end{array}
$$

Finally, the development of the nucleus in Bouhin was dependent upon the place of the final stop,

\begin{tabular}{|c|c|c|}
\hline (140) & *əm & $>$ \\
\hline & *əp & $>$ \\
\hline & *ən & $>$ \\
\hline & $*^{*} \partial t$ & $>$ \\
\hline & *əu & $>$ \\
\hline & $* \partial c$ & $>$ \\
\hline & *əy & $>$ \\
\hline & $*_{\partial k}$ & $>$ \\
\hline
\end{tabular}
as shown below:

\begin{tabular}{|c|c|c|c|c|}
\hline (141) & Thurgood & Ostapirat (1993) & Ostapirat (2004) & PHI \\
\hline (a) & ${ }^{*} \mathrm{o}: \mathrm{y}$ & $*_{-w}$-ay & *ə:y & *ə:y \\
\hline (b) & *uam (a) & $*_{\text {-w-em }}$ & *əm & *ə:m \\
\hline (c) & $*_{\mathrm{o}}: \mathrm{n}$ & $*_{\text {-w-en }}$ & *ən & $*_{\partial: n}$ \\
\hline (d) & *uan & 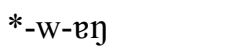 & *əy & *ə:y \\
\hline (e) & *uap (a) & *-w-ep & *əp & *ə:p \\
\hline (f) & $*_{0}: t$ & $*$-w-et & $* \partial t$ & *ə:t \\
\hline (g) & $*_{\mathrm{o}}: \mathrm{k}$ & $*_{-\mathrm{w}-\mathrm{ek}}$ & *ək & *ə:k \\
\hline (h) & *ay & $*_{\text {ey }}$ & *ay & *əy \\
\hline (i) & *aw & $*_{\mathrm{ew}}$ & $*$ aw & *ow \\
\hline (j) & $*_{\mathrm{o}}:$ & $*_{-w}-\mathrm{ew}$ & *əw & *əwh/? \\
\hline (k) & *am & $*_{\mathrm{pm}}$ & *am & *əm \\
\hline (l) & $*$ an & --- & $*$ an & *ən \\
\hline (m) & *an & $*_{\text {en }}$ & *an & *əл \\
\hline (n) & *an & $*_{p y}$ & *ay & *əy \\
\hline (o) & *ap & $*_{p p}$ & *ap & $* \partial p$ \\
\hline (p) & $*$ at & --- & *at & $* \partial t$ \\
\hline (q) & $* a c$ & $*_{\mathrm{et}}$ & $*_{\mathrm{ac}}$ & $* \partial c$ \\
\hline (r) & --- & --- & --- & $*_{\partial \mathrm{k}}$ \\
\hline
\end{tabular}

A comparison of reconstructions is given below: 
For the 'long' series of correspondences, Thurgood reconstructs a combination of $o$ : and $u a$ rimes (violating both Symmetry and Directionality). The rimes marked with an (a) contrast with the identical rimes which were reconstructed in section 4.4.2.3. For the 'short' series, Thurgood reconstructs short rimes with $a$, which I consider to be a later stage after the PHI nucleus lowered (and which fails to account for the Bouhin reflexes). He reconstructs the pure vowel $o$ : for the third member of this series, not recognizing the correlation with tone category.

Ostapirat (1993) reconstructs the 'long' series with a short $\mathfrak{e}$ preceded by a labiovelar glide, which presumably colored the reflexes of the daughter languages with back, rounded nuclei; this does not explain the length of the rimes, however, and is a violation of Directionality, as this kind of lengthening is without strong precedent. He reconstructs rimes with short $\boldsymbol{E}$, in order to distinguish this series from the one below which he reconstructs with $a$, in order to account for the difference in length in the modern languages. He adopts the same strategy in the tenth series of reconstructing a preceding labiovelar glide in order to explain the $o$ : reflexes in Qi and $\mathrm{NCHl}$.

Ostapirat (2004) reconstructs rimes with $ə$ in the first series, and rimes with $a$ in the second. This reconstruction is closer to the present one in vowel quality, but it still fails to explain the length difference between the two series, and especially the chain-shift in Bouhin. His reconstruction in (142j) (*əw) is identical with the present one.

Examples are given below, in the following order:

$\begin{array}{llllll}\text { Bhin } & \text { Ha Em } & \text { Lhut } & \text { Tzha } & \text { Zdui } & \text { Bting } \\ \text { Cun } & \text { Nadou } & \text { Cjiang } & \text { Mfaw } & \text { Baisha } & \text { Ymen }\end{array}$


(142) Examples of closed rimes with mid central nuclei

\begin{tabular}{|c|c|c|c|c|c|c|c|c|c|c|c|}
\hline (a) & & & *ə:y & & & (b) & & & *əy & & \\
\hline 繩子 & rope & & *đว:y & & & 雞 & chicke & & ${ }^{*} \mathrm{k}^{\mathrm{h}} \partial \mathrm{y}$ & & \\
\hline da:y ${ }^{1}$ & do: $y^{1}$ & do:y ${ }^{1}$ & da: ${ }^{1}$ & da: ${ }^{1}$ & da: ${ }^{1}$ & $\mathrm{k}^{\mathrm{h}} \mathrm{yy}^{1}$ & $\mathrm{k}^{\mathrm{h}} \mathrm{ay}{ }^{1}$ & $\mathrm{k}^{\mathrm{h}}$ ay ${ }^{1}$ & $\mathrm{k}^{\mathrm{h}}$ ay ${ }^{1}$ & $\mathrm{k}^{\mathrm{h}} \mathrm{ay}^{1}$ & $\mathrm{k}^{\mathrm{h}} \mathrm{ay}{ }^{1}$ \\
\hline dว:(y) ${ }^{1}$ & doy ${ }^{1}$ & do: $y^{1}$ & do: $y^{1}$ & duay $^{1}$ & du:y ${ }^{1}$ & $\mathrm{k}^{\mathrm{h}} \mathrm{ay}^{1}$ & $\mathrm{k}^{\mathrm{h}} \mathrm{ay}{ }^{1}$ & $\mathrm{k}^{\mathrm{h}} \mathrm{ay}{ }^{1}$ & $\mathrm{k}^{\mathrm{h}} \mathrm{ay}{ }^{1}$ & $\mathrm{k}^{\mathrm{h}} \mathrm{ay}^{1}$ & $\mathrm{k}^{\mathrm{h}} \mathrm{ay}{ }^{1}$ \\
\hline 多 & many & & *hlə:y & & & 黎族 & Hlai & & *hləy & & \\
\hline da: $y^{1}$ & ło: $y^{1}$ & to: $\mathrm{y}^{1}$ & $4 a: y^{1}$ & $4 a: y^{1}$ & 4a: ${ }^{1}$ & day $^{1}$ & tay $^{1}$ & tay $^{1}$ & tay $^{1}$ & tay ${ }^{1}$ & tay $^{1}$ \\
\hline $\mathrm{t} \theta \mathrm{o}:(\mathrm{y})^{1}$ & loy ${ }^{1}$ & ło: $y^{1}$ & ło: $y^{1}$ & łuay $^{1}$ & łu:y ${ }^{1}$ & $\mathrm{t} \theta a y^{1}$ & lay ${ }^{1}$ & tay ${ }^{1}$ & tay ${ }^{1}$ & tay ${ }^{1}$ & tay ${ }^{1}$ \\
\hline 網 & net & & *rə:y? & & & 耳朵 & ear & & *ljəy & & \\
\hline ra: $y^{3}$ & go: $y^{3}$ & go: $y^{3}$ & ga: ${ }^{6}$ & ha:y ${ }^{6}$ & ha:y ${ }^{6}$ & zay $^{1}$ & zay $^{1}$ & zay $^{1}$ & tay ${ }^{4}$ & $4 a y^{4}$ & tay $^{4}$ \\
\hline hว:(y) ${ }^{4}$ & 1วy ${ }^{3}$ & go: $y^{3}$ & $x o: y^{3}$ & xuay $^{3}$ & $\mathrm{k}^{\mathrm{h}} \mathrm{u}: \mathrm{y}^{3}$ & lay $^{4}$ & nay $?^{4}$ & zay $^{4}$ & zay $^{1}$ & zay $^{1}$ & tsay $^{4}$ \\
\hline (d) & & & *əw & & & (f) & & & *əwh & & \\
\hline 看 & mortar & & $*_{\text {rəw }}$ & & & 明年/ & next y & $\mathrm{r} / \mathrm{tmrw}$ & * həw & & \\
\hline raw $^{1}$ & $\operatorname{raw}^{1}$ & raw $^{1}$ & $\mathrm{raw}^{4}$ & law ${ }^{4}$ & law $^{4}$ & haw $^{2}$ & haw $^{2}$ & haw $^{2}$ & ho: ${ }^{5}$ & ho: ${ }^{5}$ & ho: ${ }^{5}$ \\
\hline $\operatorname{law}^{4}$ & law $2^{4}$ & $\mathrm{law}^{4}$ & raw $^{1}$ & raw $^{1}$ & $\mathrm{raw}^{4}$ & hiaw $^{5}$ & ho: $:^{2}$ & ho: ${ }^{2}$ & ho: ${ }^{2}$ & ho: ${ }^{2}$ & ho: ${ }^{5}$ \\
\hline 鍋 & pot & & $* \mathrm{t}^{\mathrm{h}} \partial \mathrm{w}$ & & & 山 & mount & & $* \mathrm{Cuh}$ & & \\
\hline $\mathrm{t}^{\mathrm{h}} \mathrm{aw}{ }^{1}$ & $\mathrm{t}^{\mathrm{h}} \mathrm{aw}{ }^{1}$ & $\mathrm{t}^{\mathrm{h}} \mathrm{aw}{ }^{1}$ & $\mathrm{t}^{\mathrm{h}} \mathrm{aw}{ }^{1}$ & $\mathrm{t}^{\mathrm{h}} \mathrm{aw}{ }^{1}$ & $\mathrm{t}^{\mathrm{h}} \mathrm{aw}{ }^{1}$ & haw $^{3}$ & haw $^{3}$ & hwow $^{3}$ & go: ${ }^{3}$ & vo: ${ }^{3}$ & ho: ${ }^{3}$ \\
\hline $\mathrm{ts}^{\mathrm{h}} \mathrm{aw}^{1}$ & $\mathrm{t}^{\mathrm{h}} \mathrm{aw}{ }^{1}$ & $\mathrm{t}^{\mathrm{h}} \mathrm{aw}{ }^{1}$ & $\mathrm{t}^{\mathrm{h}} \mathrm{aw}{ }^{1}$ & $\mathrm{t}^{\mathrm{h}} \mathrm{aw}{ }^{1}$ & $\mathrm{t}^{\mathrm{h}} \mathrm{aw}{ }^{1}$ & naw $^{3}$ & noy ${ }^{3}$ & yo: ${ }^{3}$ & yo: ${ }^{3}$ & yo: ${ }^{3}$ & mo: ${ }^{6}$ \\
\hline 角 & horn & & * how & & & 頭 & head & & *Curə & & \\
\hline haw $^{1}$ & haw $^{1}$ & haw $^{1}$ & haw $^{1}$ & haw $^{1}$ & haw $^{1}$ & $\mathrm{raw}^{3}$ & gaw $^{3}$ & gwow $^{3}$ & go: ${ }^{6}$ & ho: ${ }^{6}$ & ho: ${ }^{6}$ \\
\hline haw $^{1}$ & haw $^{1}$ & haw $^{1}$ & haw $^{1}$ & haw $^{1}$ & haw $^{1}$ & $\mathrm{vaw}^{3}$ & yo: $:^{3}$ & go: $:^{3}$ & уо: ${ }^{3}$ & vo: ${ }^{3}$ & vo: ${ }^{3}$ \\
\hline
\end{tabular}


(e)

$$
\text { 鋒利 }
$$

ts $^{\mathrm{h}} \mathrm{am}^{1} \quad$ ts $^{\mathrm{h}} \mathrm{o}: \mathrm{m}^{1} \quad$ ts $^{\mathrm{h}} \mathrm{o}: \mathrm{m}^{1}$ ts $^{\mathrm{h}} \mathrm{o}: \mathrm{m}^{1}$ ts $^{\mathrm{h}} \mathrm{o}: \mathrm{m}^{1}$ ts $^{\mathrm{h}} \mathrm{O}: \mathrm{m}^{1}$ dom ${ }^{3}$

ts $^{\mathrm{h}} \mathrm{am}^{1} \quad \operatorname{son}^{1} \quad$ ts $^{\mathrm{h}} \mathrm{o}: \mathrm{m}^{1} \quad \mathrm{ts}^{\mathrm{h}} \mathrm{o}: \mathrm{m}^{1}$ ts $^{\mathrm{h}} \mathrm{uam}^{1}$ ts $^{\mathrm{h}} \mathrm{uam}^{1}$-.

果子 fruit

$* \mathrm{t} \int^{\mathrm{h}} \partial: \mathrm{m}$

水 water

$\mathrm{ts}^{\mathrm{h}} \mathrm{am}^{1} \quad \mathrm{ts}^{\mathrm{h}} \mathrm{o}: \mathrm{m}^{1}$

ham

hon ${ }^{1}$

苦 bitter

ts $\mathrm{s}^{\mathrm{h}} \mathrm{O} \mathrm{m}^{1}$

ts ${ }^{\mathrm{h}} \mathrm{o}: \mathrm{m}^{1}$ ts ${ }^{\mathrm{h}} \mathrm{\rho}: \mathrm{m}^{1}$ ts ${ }^{\mathrm{h}} \mathrm{\jmath}: \mathrm{m}^{1}$ nom

(ham ${ }^{1}$ ) ts ${ }^{\mathrm{h}} \mathrm{o}: \mathrm{m}^{1}$ ts $^{\mathrm{h}} \mathrm{uam}^{1}$ ts $^{\mathrm{h}}$ uam $^{1}$ nam $^{3}$

*hə:m

嘴巴

$\operatorname{nam}^{3}$

$\operatorname{nan}^{3}$

mouth

dom

dam

$\operatorname{dam}^{3}$

dam

dam ${ }^{3}$

$\left(\right.$ dəm $\left.{ }^{3}\right)$

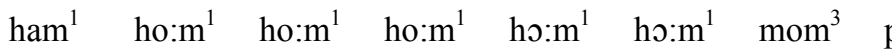

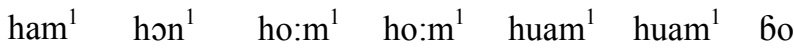

(g)

*ə:p

(h)

*sə:p

熄滅 die out

to:p

so:p

to: $\mathrm{p}^{7}$ to: $\mathrm{p}^{7}$

ts $^{\text {h uap }}{ }^{8}$ ts $^{\text {h }}$ uap $^{8}$

${ }^{*}$ C-lə:p

縫

鱗 fish scale

lo:p

10:p

10:p

$\operatorname{lap}^{7} \quad$ lo:

$\operatorname{lap}^{2} \quad 10 ?^{4}$

lo:p

lo: $\mathrm{p}^{7}$

luap

*rə:p

晚 late

tsap $^{7}$

$\operatorname{tsa}^{4}$

tsap $^{7}$

nom $^{3}$

$\operatorname{nam}^{3}$$$
\text { (nat }
$$

$\operatorname{nam}^{3} \quad$ nam $^{6} \quad$ nam $^{3}$

$\operatorname{nam}^{3} \quad \operatorname{nam}^{3} \quad \operatorname{nam}^{6}$

堆砌 pile up

$\left(\right.$ go: $\left.p^{7}\right)$ go: $p^{7}$ go: $p^{7}$

--- go: $p^{8} \quad$ ho: $p^{8} \quad$ ho: $p^{8} \quad$ ts $^{\text {h }}$ op $^{7}$ xо: $p^{7} \quad$ xuap $^{8} \quad \mathrm{k}^{\mathrm{h}} \mathrm{uap}^{7} \quad$---- tsop

*hm[ə/o]m?

$\operatorname{pam}^{6} \quad \mathrm{p}^{\mathrm{h}} \mathrm{am}^{6} \quad \mathrm{pam}^{6}$ pom $^{3} \quad$ pom $^{3} \quad$ pom $^{6}$

*əp

*tсәр

tsop $^{7}$ tsap $^{7} \quad$ tsep $^{7}$ tsap $^{7} \quad$ tsap $^{8} \quad \operatorname{tap}^{7}$

*C-люp

nop $^{7} \quad$ nap $^{8} \quad$ nap $^{7}$ nap $^{7} \quad$ nap $^{8} \quad$ nap $^{8}$

$*$ tc ${ }^{\mathrm{h}}$ әр

ts $^{\mathrm{h}}$ op $^{7} \quad \mathrm{ts}^{\mathrm{h} o p^{7}} \quad \mathrm{ts}^{\mathrm{h}} \mathrm{ap}^{7} \quad \mathrm{ts}^{\mathrm{h}} \mathrm{ap}^{7}$ ts $^{\mathrm{h}} \mathrm{ap}^{7} \quad \mathrm{ts}^{\mathrm{h}} \mathrm{ap}^{7} \quad \mathrm{ts}^{\mathrm{h}} \mathrm{ap}^{7} \quad \mathrm{ts}^{\mathrm{h}} \mathrm{ap}^{7}$ 
(i)

\begin{tabular}{|c|c|c|c|c|c|c|c|c|c|c|c|}
\hline 睡 & sleep & & *tढə:n & & & 秧/種 & seed & & $* \mathrm{f}[\mathrm{j}] ə n$ & & \\
\hline $\begin{array}{l}\left(\operatorname{tso}^{1}{ }^{1}\right) \\
\operatorname{tsan}^{1}\end{array}$ & $\begin{array}{l}\text { tso: }{ }^{1} \\
\text { tson }^{1}\end{array}$ & $\begin{array}{l}\text { tso: } n^{1} \\
\text { tso: } \eta^{1}\end{array}$ & $\begin{array}{l}\text { tso: } n^{1} \\
\text { tso: } \eta^{1}\end{array}$ & $\begin{array}{l}\text { tso:n }{ }^{1} \\
\text { tsuay }^{1}\end{array}$ & $\begin{array}{l}\text { tso: } n^{1} \\
\text { tu: } n^{1}\end{array}$ & $\begin{array}{l}p^{h} e^{1} \\
\text { fon }\end{array}$ & $\begin{array}{l}\operatorname{fan}^{1} \\
\operatorname{fan}^{1}\end{array}$ & $\begin{array}{l}\operatorname{fan}^{1} \\
\operatorname{fay}^{1}\end{array}$ & $\begin{array}{l}\text { fan }^{1} \\
\text { fay }^{1}\end{array}$ & $\begin{array}{l}\text { fan }^{1} \\
\text { fay }^{1}\end{array}$ & $\begin{array}{l}\operatorname{fan}^{1} \\
\operatorname{fan}^{1}\end{array}$ \\
\hline 田埂 & field rid & & *Cifə:n & & & 銀 & silver & & *hyən & & \\
\hline $\begin{array}{l}\operatorname{han}^{1} \\
\operatorname{nan}^{1}\end{array}$ & $\begin{array}{l}\text { ho:n }{ }^{1} \\
\text { non }^{1}\end{array}$ & $\begin{array}{l}\text { hjo: }{ }^{1} \\
\text { no: }{ }^{1}\end{array}$ & $\begin{array}{l}\text { zo:n }{ }^{1} \\
\text { no: }{ }^{1}\end{array}$ & $\begin{array}{l}\text { zo:n }{ }^{1} \\
\text { nuay }^{1}\end{array}$ & $\begin{array}{l}\text { hjo:n }{ }^{1} \\
\text { nu:n }\end{array}$ & $\begin{array}{l}\text { yen }^{1} \\
\text { kon }^{4}\end{array}$ & $\begin{array}{l}\operatorname{kan}^{1} \\
\operatorname{kan}^{4}{ }^{4}\end{array}$ & $\begin{array}{l}\operatorname{kan}^{1} \\
\operatorname{ka\eta }^{4}\end{array}$ & $\begin{array}{l}\operatorname{kan}^{4} \\
\operatorname{ka\eta }^{1}\end{array}$ & $\begin{array}{l}\mathrm{k}^{\mathrm{h}} \mathrm{an}^{4} \\
\operatorname{ka\eta }^{1}\end{array}$ & $\begin{array}{l}\operatorname{kan}^{4} \\
\operatorname{kan}^{4}\end{array}$ \\
\hline 竹子 & bamboo & (big) & *гə:nf & & & 日 & day & & *hywən & & \\
\hline $\begin{array}{l}\operatorname{ran}^{2} \\
\text { la: } n^{5}\end{array}$ & $\begin{array}{l}\text { ro: } n^{2} \\
l o n^{2}\end{array}$ & $\begin{array}{l}\text { ro: } n^{2} \\
\text { lo: } y^{2}\end{array}$ & $\begin{array}{l}\text { ro: } n^{1} \\
\text { ro: } y^{2}\end{array}$ & $\begin{array}{l}\text { lo: } n^{2} \\
\text { ruay }^{2}\end{array}$ & $\begin{array}{l}\text { lo: } n^{2} \\
\text { ru: } n^{2}\end{array}$ & $\begin{array}{l}\text { ven }^{1} \\
\text { hon }^{4}\end{array}$ & $\begin{array}{l}\operatorname{van}^{1} \\
\operatorname{van}^{4}\end{array}$ & $\begin{array}{l}\text { hwan }^{1} \\
\operatorname{van}^{4}\end{array}$ & $\begin{array}{l}\operatorname{van}^{4} \\
\operatorname{van}^{1}\end{array}$ & $\begin{array}{l}\operatorname{van}^{4} \\
\operatorname{van}^{1}\end{array}$ & $\begin{array}{l}\operatorname{van}^{1} \\
\operatorname{van}^{4}\end{array}$ \\
\hline (k) & & & $* \partial: t$ & & & (1) & & & $* \partial t$ & & \\
\hline 跳蚤 & flea & & hmə:t & & & 穿 & wear & & $* \mathrm{tc}^{\mathrm{h}} \partial \mathrm{t}$ & & \\
\hline $\begin{array}{l}\mathrm{mat}^{7} \\
6 a t^{4}\end{array}$ & $\begin{array}{l}\text { po:t }{ }^{7} \\
\left(\mathrm{pa}^{4}\right)\end{array}$ & $\begin{array}{l}\text { po:t } t^{7} \\
\left(\text { pət }^{9}\right)\end{array}$ & $\begin{array}{l}\text { po: }: t^{8} \\
\left(\text { po: }: t^{9}\right)\end{array}$ & $\begin{array}{l}\mathrm{p}^{\mathrm{h}} \mathrm{\rho}: \mathrm{t}^{8} \\
\text { po } ?^{8}\end{array}$ & $\begin{array}{l}\text { po:t }{ }^{8} \\
\left(\text { pət }^{8}\right)\end{array}$ & $\begin{array}{l}\text { ts }^{\mathrm{h}} \mathrm{et}^{7} \\
\text { ts }^{\mathrm{h}} \mathrm{at}^{2}\end{array}$ & $\begin{array}{l}\mathrm{ts}^{\mathrm{h}} \mathrm{at}^{7} \\
\mathrm{sa}^{4}\end{array}$ & $\begin{array}{l}\mathrm{ts}^{\mathrm{h}} \mathrm{at}^{7} \\
\mathrm{ts}^{\mathrm{h}} \mathrm{a} \mathrm{P}^{7}\end{array}$ & $\begin{array}{l}\mathrm{ts}^{\mathrm{h}} \mathrm{at}^{7} \\
\mathrm{ts}^{\mathrm{h}} \mathrm{ak}^{7}\end{array}$ & $\begin{array}{l}\mathrm{ts}^{\mathrm{h}} \mathrm{at}^{7} \\
\mathrm{ts}^{\mathrm{h}} \mathrm{ak}^{8}\end{array}$ & $\begin{array}{l}\text { ts }^{\mathrm{h}} \mathrm{at}^{7} \\
\text { ts }^{\mathrm{h}} \mathrm{at}^{7}\end{array}$ \\
\hline 啃 & gnaw & & ${ }^{*}$ Cufi:t & & & 鼻子 & nose & & ${ }^{*} \mathrm{k}^{\mathrm{h}} \partial \mathrm{t}$ & & \\
\hline hat ${ }^{7}$ & $\begin{array}{l}\text { ho:t }{ }^{7} \\
\left(\mathrm{yaP}^{5}\right)\end{array}$ & $\begin{array}{l}\text { hwo: } \mathrm{t}^{7} \\
\text { yo: } ?^{7}\end{array}$ & $\begin{array}{l}\text { go: } \mathrm{t}^{7} \\
\text { yo:k }\end{array}$ & $\begin{array}{l}\text { ho:t }{ }^{7} \\
\text { yo? }\end{array}$ & ho:t ${ }^{7}$ & $\begin{array}{l}\mathrm{k}^{\mathrm{h}} \mathrm{et}^{7} \\
\mathrm{k}^{\mathrm{h}} \mathrm{at}^{2}\end{array}$ & $\begin{array}{l}\mathrm{k}^{\mathrm{h}} \mathrm{at}^{7} \\
\mathrm{k}^{\mathrm{h}} \mathrm{a} \mathrm{P}^{4}\end{array}$ & $\begin{array}{l}\mathrm{k}^{\mathrm{h}} \mathrm{at}^{7} \\
\mathrm{k}^{\mathrm{h}} \mathrm{a} ?^{7}\end{array}$ & $\begin{array}{l}\mathrm{k}^{\mathrm{h}} \mathrm{at}^{7} \\
\mathrm{k}^{\mathrm{h}} \mathrm{ak}^{7}\end{array}$ & $\begin{array}{l}\mathrm{k}^{\mathrm{h}} \mathrm{at}^{7} \\
\mathrm{k}^{\mathrm{h}} \mathrm{ak}^{7}\end{array}$ & $\begin{array}{l}\mathrm{k}^{\mathrm{h}} \mathrm{at}^{7} \\
\mathrm{k}^{\mathrm{h}} \mathrm{at}^{7}\end{array}$ \\
\hline 蓋子 & lid & & hyə:t & & & 取/娶 & take/n & & ${ }^{*} \mathrm{hmot}$ & & \\
\hline ---- & ko: $\mathrm{t}^{7}$ & ko: $: t^{7}$ & ko:t $t^{8}$ & $\mathrm{k}^{\mathrm{h}} \mathrm{o}: \mathrm{t}^{\mathrm{g}}$ & $\mathrm{ko}: \mathrm{t}^{8}$ & ---- & $\mathrm{pat}^{7}$ & $\mathrm{pat}^{7}$ & pat $^{8}$ & $\mathrm{p}^{\mathrm{h}} \mathrm{at}{ }^{8}$ & pat $^{8}$ \\
\hline $\mathrm{kat}^{4}$ & $\mathrm{koP}^{4}$ & ko: $: 1^{7}$ & $\mathrm{ko}: \mathrm{k}^{7}$ & $\mathrm{kot}^{8}$ & $\mathrm{ku}: \mathrm{t}^{8}$ & $6 a t^{4}$ & $\mathrm{pa}^{4}$ & $\mathrm{pa}^{7}$ & $\mathrm{pak}^{7}$ & --- & ---- \\
\hline
\end{tabular}




\begin{tabular}{|c|c|c|c|c|c|c|c|c|c|c|c|}
\hline (m) & & & *əon & & & (n) & & & $* \partial c$ & & \\
\hline 打噴德 & sneeze & & *də๊ & & & 淡 & light & & *dəc & & \\
\hline ---- & $\operatorname{dan}^{1}$ & dap $^{1}$ & $\operatorname{dan}^{1}$ & $\operatorname{dan}^{1}$ & $\operatorname{dan}^{1}$ & $\left(d a t^{9}\right)$ & $\mathrm{dat}^{7}$ & $\mathrm{dac}^{7}$ & $\mathrm{dat}^{7}$ & $\mathrm{dat}^{7}$ & $\mathrm{dat}^{7}$ \\
\hline $\operatorname{dian}^{1}$ & ---- & ---- & ---- & $\operatorname{dan}^{1}$ & $\operatorname{dan}^{1}$ & tsiat $^{2}$ & $\mathrm{da}^{4}$ & $\mathrm{dat}^{7}$ & $\mathrm{dat}^{7}$ & $\mathrm{dat}^{8}$ & $\mathrm{dat}^{7}$ \\
\hline 選擇 & choose & & *hləл & & & 白藤 & white & ne (sm) & *kəc & & \\
\hline --.- & $\tan ^{1}$ & $\tan ^{1}$ & $\tan ^{1}$ & $\tan ^{1}$ & $\tan ^{1}$ & $\left(\mathrm{~kat}^{9}\right)$ & $\mathrm{kat}^{7}$ & $\mathrm{kac}^{7}$ & $\mathrm{~kat}^{7}$ & $\mathrm{~kat}^{7}$ & $\mathrm{~kat}^{7}$ \\
\hline --- & ---- & $\tan ^{1}$ & $\tan ^{1}$ & $\tan ^{1}$ & $\tan ^{1}$ & kiat $^{2}$ & $\mathrm{kaP}^{4}$ & $\mathrm{~kat}^{7}$ & $\mathrm{~kat}^{7}$ & $\mathrm{~kat}^{8}$ & $\mathrm{~kat}^{7}$ \\
\hline 脫 & remove & & ${ }^{*}$ C-ləлn? & & & 買 & buy & & $*_{t} \varphi^{\mathrm{h}} \partial \mathrm{c}$ & & \\
\hline $\operatorname{lan}^{3}$ & $\operatorname{lan}^{3}$ & $\operatorname{lan}^{3}$ & $\operatorname{lan}^{3}$ & ---- & $\operatorname{lan}^{3}$ & tshat $^{7}$ & tshat $^{7}$ & $\operatorname{tshac}^{7}$ & tshat $^{7}$ & tshat $^{7}$ & tshat $^{7}$ \\
\hline ---- & ---- & ---- & $\operatorname{lan}^{3}$ & $\operatorname{lan}^{3}$ & $\operatorname{lan}^{6}$ & $\operatorname{hiat}^{2}$ & ---- & tshiet $^{7}$ & tshat $^{7}$ & tshat $^{8}$ & tshat $^{7}$ \\
\hline (o) & & & *ə:y & & & (p) & & & *əy & & \\
\hline 樹漿 & sap & & $* \mathrm{t}^{\mathrm{h}} \partial: \mathrm{y}$ & & & 臉/前面 & face $/ \mathrm{fr}$ & & *dəท & & \\
\hline $\mathrm{t}^{\mathrm{h}} \mathrm{a} \mathrm{y}^{1}$ & $\mathrm{t}^{\mathrm{h}} \mathrm{o}: \mathrm{y}^{1}$ & $\mathrm{t}^{\mathrm{h}} \mathrm{o}: \mathrm{y}^{1}$ & $\mathrm{t}^{\mathrm{h}} \mathrm{o}: \mathrm{y}^{1}$ & $\mathrm{t}^{\mathrm{h}} \mathrm{O}: \mathrm{y}^{1}$ & $\mathrm{t}^{\mathrm{h}} \mathrm{o}: \mathrm{y}^{1}$ & don $^{1}$ & day $^{1}$ & day $^{1}$ & $\operatorname{dan}^{1}$ & $\operatorname{dan}^{1}$ & day $^{1}$ \\
\hline $\operatorname{ts}^{h} a a^{1}$ & $\mathrm{t}^{\mathrm{h}} \supset \mathrm{y}^{1}$ & $\mathrm{t}^{\mathrm{h}} \mathrm{o}: \mathrm{y}^{1}$ & $\mathrm{t}^{\mathrm{h}} \mathrm{o}: \mathrm{y}^{1}$ & $\mathrm{t}^{\mathrm{h}} \mathrm{uay}^{1}$ & th $^{\text {hua }}{ }^{1}$ & tse: $y^{1}$ & day $^{1}$ & day $^{1}$ & day $^{1}$ & $\operatorname{da\eta }^{1}$ & doy ${ }^{1}$ \\
\hline 皮膚 & skin & & ${ }^{*} \mathrm{C}-\mathrm{n}$ : $\mathrm{y}$ & & & 鼓 & drum & & ${ }^{*} \mathrm{C}-1 ə \eta$ & & \\
\hline $\operatorname{nay}^{1}$ & no: $y^{1}$ & no: $y^{1}$ & no: $y^{1}$ & n๑: $\eta^{4}$ & nว:1 ${ }^{1}$ & $\log ^{1}$ & $\operatorname{la\eta }^{1}$ & $\operatorname{la\eta }^{1}$ & $\operatorname{la\eta }^{1}$ & $\operatorname{lan}^{4}$ & $\operatorname{lay}^{1}$ \\
\hline $\operatorname{nan}^{1}$ & nэๆ ${ }^{1}$ & no: $y^{1}$ & no: $y^{1}$ & nuay $^{1}$ & nuay $^{4}$ & le ${ }^{1}$ & ---- & $\operatorname{lan}^{1}$ & $\operatorname{lan}^{1}$ & $\operatorname{lan}^{1}$ & $10 y^{4}$ \\
\hline 相同 & same & & 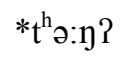 & & & po*田( & 期)field & & *?əท & & \\
\hline$t^{h} a y^{3}$ & $\mathrm{t}^{\mathrm{h}} \mathrm{o}: \mathrm{y}^{3}$ & $\mathrm{t}^{\mathrm{h}} \mathrm{o}: \mathrm{y}^{3}$ & $\mathrm{t}^{\mathrm{h}} \mathrm{o}: \mathrm{y}^{3}$ & $\mathrm{t}^{\mathrm{h}} \mathrm{s}: \mathrm{y}^{3}$ & $\mathrm{t}^{\mathrm{h}} \mathrm{s}: \mathrm{y}^{3}$ & $\operatorname{Poy}^{1}$ & $\operatorname{Pa\eta }^{1}$ & $\operatorname{Pa\eta }^{1}$ & $\operatorname{Pa\eta }^{1}$ & $\operatorname{Pa\eta }^{1}$ & $\operatorname{Pay}^{1}$ \\
\hline $\mathrm{ts}^{\mathrm{h}} \mathrm{an} \mathrm{y}^{3}$ & $\mathrm{t}^{\mathrm{h}} \mathrm{n}^{3}$ & ---- & $\mathrm{t}^{\mathrm{h}} \mathrm{o}: \mathrm{y}^{3}$ & $\mathrm{t}^{\mathrm{h}} \mathrm{uay}^{3}$ & $\mathrm{t}^{\mathrm{h}} \mathrm{uan}^{3}$ & $\operatorname{Peg}^{1}$ & --- & $\operatorname{Pa\eta }^{1}$ & $\operatorname{Pay}^{1}$ & Pay $^{1}$ & Poy ${ }^{1}$ \\
\hline
\end{tabular}




\begin{tabular}{|c|c|c|c|c|c|c|c|c|c|c|c|}
\hline (q) & & & *a:k & & & (r) & & & *ək & & \\
\hline 捉 & catch & & *hmə:k & & & 剁 & chop & & *hnək & & \\
\hline $\mathrm{nak}^{7}$ & po: $:^{7}$ & po:k ${ }^{7}$ & po: $: 1^{8}$ & $\mathrm{p}^{\mathrm{h}} \mathrm{\jmath}: ?^{8}$ & po: $1^{8}$ & $\left(\operatorname{tak}^{7}\right)$ & $\operatorname{tak}^{7}$ & $\operatorname{tak}^{7}$ & ---- & $\mathrm{t}^{\mathrm{h}} \mathrm{ak}^{8}$ & $\mathrm{tak}^{8}$ \\
\hline $6 a: k^{4}$ & po $?^{4}$ & po: $: 1^{7}$ & po: $\mathrm{k}^{7}$ & po? ${ }^{8}$ & po $?^{8}$ & $\mathrm{dak}^{2}$ & to $?^{5}$ & ---- & ---- & ---- & $\mathrm{tak}^{8}$ \\
\hline & wash & & *sə:k & & & 食指 & index & nger & $*_{\mathrm{t} \varphi}[\mathrm{\rho} / \mathrm{o}$ & & \\
\hline $\operatorname{tak}^{7}$ & to: $?^{7}$ & to: $\mathrm{k}^{7}$ & to: $: ?^{7}$ & to: $: ?^{7}$ & to: $: ?^{7}$ & $\left(\right.$ tsak $\left.^{9}\right)$ & $\operatorname{tsak}^{7}$ & $\mathrm{tsak}^{7}$ & $\mathrm{tsak}^{7}$ & tso? ${ }^{8}$ & $\operatorname{tsok}^{7}$ \\
\hline $\mathrm{ak}^{2}$ & fo $2^{4}$ & so: $:^{7}$ & so: $\mathrm{k}^{7}$ & $\operatorname{ts}^{\mathrm{h}} \curvearrowright ?^{8}$ & $\operatorname{ts}^{\mathrm{h}} \rho ?^{7}$ & tsak $^{4}$ & ---- & ---- & $\mathrm{ts} \mathrm{k}^{7}$ & tso ${ }^{8}$ & ---- \\
\hline & deep & & *hlə:k & & & 貼 & stick & & & ${ }^{*} \mathrm{p}^{\mathrm{h}} \partial \mathrm{k}$ & \\
\hline $\mathrm{dak}^{7}$ & to: $?^{7}$ & to: $\mathrm{k}^{7}$ & to: $?^{7}$ & ๖๖: $?^{7}$ & ๖๖: $:^{7}$ & $\mathrm{p}^{\mathrm{h}} \mathrm{ak}^{7}$ & $\mathrm{p}^{\mathrm{h}} \mathrm{ak}^{7}$ & $\mathrm{p}^{\mathrm{h}} \mathrm{a}: \mathrm{k}^{7}$ & $\mathrm{p}^{\mathrm{h}} \mathrm{ak}^{7}$ & $\mathrm{p}^{\mathrm{h}} \mathrm{ak}^{7}$ & $\mathrm{p}^{\mathrm{h}} \mathrm{ak}^{7}$ \\
\hline $\mathrm{ak}^{2}$ & $10 P^{4}$ & to: $?^{7}$ & to: $\mathrm{k}^{7}$ & to ${ }^{8}$ & to $2^{7}$ & ---- & --- & ---- & $\mathrm{p}^{\mathrm{h}} \mathrm{a}: \mathrm{k}^{7}$ & $\mathrm{p}^{\mathrm{h}} \mathrm{ak}^{7}$ & $\mathrm{p}^{\mathrm{h}} \mathrm{ak}^{7}$ \\
\hline
\end{tabular}

\subsubsection{Short Rimes with Mid Back Rounded Vowels}

The correspondences for short rimes with mid back round vowels are given below:

(143) Examples of short rimes with mid back round vowels

\begin{tabular}{|c|c|c|c|c|c|c|c|c|c|c|}
\hline BHi:n & $\underline{\mathrm{HaE}}: \mathrm{m}$ & $\underline{\mathrm{LHu}}: \mathrm{t}$ & $\underline{\text { Tzha }}$ & $\underline{\text { Zdui }}$ & Bting & Cun & $\underline{\text { Nadou }}$ & Cjiang & $\underline{\text { Mfaw }}$ & $\underline{\text { Baisha }}$ \\
\hline om & om & om & om & om & um & om & on & om & om & om \\
\hline oy & un & oy & oy & oy & oy & oy & ay & oy uay & on & on \\
\hline op & op & op & op & op & op & op & o? & op & op & op \\
\hline ok & uk & ok & ok & o? & $\mathrm{ok}$ & $\mathrm{ok}$ & a? & ०? & $\mathrm{ok}$ & ok \\
\hline
\end{tabular}

$\underline{\text { S. Hlai (Savina) }}$

\begin{tabular}{l} 
om \\
on \\
\hdashline ok
\end{tabular}
C. Hlai (Savina)

om

oy

ok

\section{Baisha (Wang \& Qian)}

om
oy
op
ok

The reconstructions proposed here for these series are the following:

\footnotetext{
${ }^{30}$ The Lauhut and Moyfaw rimes in this example are irregularly long.
} 
(144)

$$
\begin{aligned}
& \text { *om } \\
& \text { *op }^{\text {op }} \\
& \text { *oy }^{\text {oy }} \\
& \text { *ok }^{\text {o }}
\end{aligned}
$$

\begin{tabular}{|c|c|c|c|}
\hline \multirow[t]{5}{*}{ (145) } & \multicolumn{2}{|c|}{$\underline{\text { Pre-Hlai }}$} & Proto-Hlai \\
\hline & *on & $>$ & *ən \\
\hline & $*_{\text {ot }}$ & $>$ & * $\partial t$ \\
\hline & *oj & $>$ & *əј \\
\hline & $*_{\mathrm{oc}}$ & $>$ & $* \partial c$ \\
\hline
\end{tabular}

This class of rimes is defective in that it has no members with alveolar or palatal codas. I propose that if these additional rimes ever existed, they most likely merged with the class of short rimes with mid central vowels, in the following scenario:

As mentioned in section 4.4.3, there was a shift at the level of $\mathrm{NCHl}$ from mid back vowels to low back vowels before velar codas, which aided in keeping it distinct from the original $*_{\mathrm{uC}} \mathrm{class,}$ which lowered to ${ }^{\circ} \mathrm{C}$ in $\mathrm{NCHl}$ :

$\begin{array}{lll}* \text { oy } & > & \text { on } \\ *_{\text {ok }} & > & \text { ok }\end{array}$

There was sporadic dissimilation of the nucleus in Cunhua before labial codas:

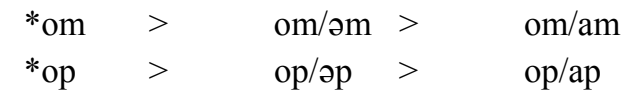

There are also three cases of fronting in Cunhua, two of them possibly conditioned by the initial:

$\begin{array}{lll}\text { Gloss } & \underline{\text { PHl }} & \text { Cunhua } \\ \text { bamboo } & \text { *dom } & \text { tsem }^{1} \\ \text { six } & \text { *hnom } & \text { tsem }^{4} \\ \text { thunder } & \text { *?om } & \text { Rem }^{1}\end{array}$

In Changjiang, there was sporadic lengthening of * 0 to $*$ $: y$, later diphthongizing to *uay: 


$$
* \text { oy } \quad>\quad \text { oy ว:y }>\quad \text { oy uay }
$$

Raising before velar codas occurred in Ha Em:

$\begin{array}{lll}* \text { oy } & > & \text { un } \\ *_{\text {ok }} & > & \text { uk }\end{array}$

Finally, the Baoting rimes ending in *-m raised to $u$, whereas the other three rimes lowered to $s$ :

$\begin{array}{lll}*_{\text {om }} & > & \text { um } \\ *_{\text {op }} & > & \text { op } \\ *_{\text {oy }} & > & \text { oy } \\ *_{\text {ok }} & > & \text { ok }\end{array}$

A comparison of reconstructions is given below:

(152) Thurgood $\underline{\text { Ostapirat (1993) Ostapirat (2004) }}$ PHl

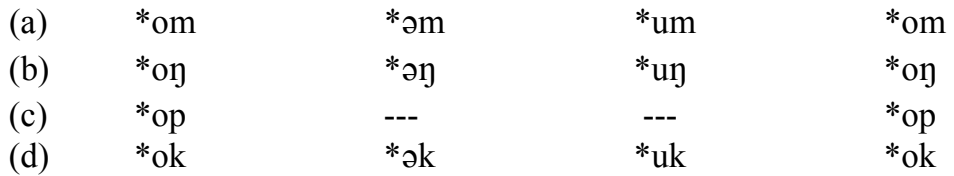

Thurgood's reconstructions are in agreement with the ones proposed here. Ostapirat (1993) reconstructed rimes with a mid central vowel, which became colored under the influence of the coda; these stand in contrast to the rimes he reconstructs in the last section with $\boldsymbol{E}$. He does not reconstruct anything for the third series. In his (2004) reconstruction, he instead reconstructs these rimes with a short $u$, which violates Economy since these rimes would have to lower twice in $\mathrm{NCHl}$ before velar codas; it also violates Commonality by assuming that the rimes in all daughter languages except Ha Em have all independently lowered.

Examples of the PHl glides are given below, in the following order:

\begin{tabular}{|c|c|c|c|}
\hline & & Lhut & Tzha \\
\hline un & Nadou & Cjiang & Mfav \\
\hline
\end{tabular}


(153) Examples of rimes with short mid rounded vowels

\begin{tabular}{|c|c|c|c|c|c|c|c|c|c|c|c|}
\hline (a) & & & *om & & & (b) & & & $*$ op & & \\
\hline 竹子 & bamboo & (big) & $*$ dom & & & 抱/背 & carry & & *?op & & \\
\hline dom $^{1}$ & dom $^{1}$ & dom $^{1}$ & dom $^{1}$ & dom $^{1}$ & dum $^{1}$ & ---- & Pop $^{7}$ & Pop $^{7}$ & Pop $^{7}$ & ---- & $2 o p^{7}$ \\
\hline tsem $^{1}$ & ---- & dom $^{1}$ & dom $^{1}$ & dom $^{1}$ & dom $^{1}$ & Pop ${ }^{2}$ & Po? ${ }^{4}$ & Pop ${ }^{7}$ & Pop ${ }^{7}$ & Pop ${ }^{8}$ & Pop $^{7}$ \\
\hline 瘍 & itch & & $*^{\mathrm{h}} \mathrm{om}$ & & & 拳頭 & fist & & ${ }^{*}$ Curop & & \\
\hline$\left(\mathrm{k}^{\mathrm{h}} \mathrm{um}^{1}\right)$ & $\mathrm{k}^{\mathrm{h}} \mathrm{um}^{1}$ & $\mathrm{k}^{\mathrm{h}} \mathrm{om}^{1}$ & $\mathrm{k}^{\mathrm{h}} \mathrm{om}^{1}$ & $\mathrm{k}^{\mathrm{h}} \mathrm{m}^{1}$ & $\mathrm{k}^{\mathrm{h}} \mathrm{m}^{1}$ & ---- & gop $^{7}$ & gop $^{7}$ & gop $^{8}$ & ---- & gop $^{8}$ \\
\hline $\mathrm{k}^{\mathrm{h}} \mathrm{om}^{1}$ & $\mathrm{k}^{\mathrm{h}}$ on $^{1}$ & $\mathrm{k}^{\mathrm{h}} \mathrm{om}^{1}$ & $\mathrm{k}^{\mathrm{h}} \mathrm{om}^{1}$ & $\mathrm{k}^{\mathrm{h}} \mathrm{om}^{1}$ & $\mathrm{k}^{\mathrm{h}} \mathrm{om}^{1}$ & ---- & --- & $\mathrm{kap}^{7}$ & yap $^{7}$ & vop $^{8}$ & vop $^{7}$ \\
\hline 六 & six & & *hnom & & & 捧 & handful & & ${ }^{*} \mathrm{k}^{\mathrm{h}} \mathrm{op}$ & & \\
\hline nom $^{1}$ & tom $^{1}$ & tom $^{1}$ & tom $^{4}$ & $\mathrm{t}^{\mathrm{h}} \mathrm{om}^{4}$ & tum $^{4}$ & ---- & $\mathrm{k}^{\mathrm{h}} \partial \mathrm{p}^{7}$ & $\mathrm{k}^{\mathrm{h}} \mathrm{op}^{7}$ & $\mathrm{k}^{\mathrm{h}} \mathrm{op}^{7}$ & $\mathrm{k}^{\mathrm{h}} \mathrm{ap}^{7}$ & $k^{h} \curvearrowright p^{7}$ \\
\hline tsem $^{4}$ & ton? $?^{4}$ & tom $^{4}$ & tom $^{1}$ & tom $^{1}$ & tom $^{4}$ & ---- & ---- & $\mathrm{k}^{\mathrm{h}} \mathrm{op}^{7}$ & $\mathrm{k}^{\mathrm{h}} \mathrm{op}^{7}$ & $\mathrm{k}^{\mathrm{h}} \mathrm{op}^{7}$ & $\mathrm{k}^{\mathrm{h}} \mathrm{op}^{7}$ \\
\hline (c) & & & *oy & & & (d) & & & $*_{0 k}$ & & \\
\hline 簛箕/笠 & 子 sieve & $(\lg )$ & $*$ doy? & & & 落 & fall & & $* \mathrm{t}^{\mathrm{h}} \mathrm{ok}$ & & \\
\hline$\left(\right.$ dun $\left.^{3}\right)$ & dun $^{3}$ & doy $^{3}$ & dog $^{3}$ & dog $^{3}$ & $d o y^{3}$ & $\mathrm{t}^{\mathrm{h}} \mathrm{ok}^{7}$ & $\mathrm{t}^{\mathrm{h}} \mathrm{uk}^{7}$ & $\mathrm{t}^{\mathrm{h}} \mathrm{ok}^{7}$ & $\mathrm{t}^{\mathrm{h}} \mathrm{k}^{7}$ & $\mathrm{t}^{\mathrm{h}} \mathrm{o} ?^{7}$ & $\mathrm{t}^{\mathrm{h}} \mathrm{ok}{ }^{7}$ \\
\hline do $y^{3}$ & $\mathrm{da \eta}^{3}$ & duan $^{3}$ & do $y^{3}$ & $d o y^{3}$ & do $y^{3}$ & $\mathrm{t}^{\mathrm{h}} \mathrm{ok}{ }^{2}$ & $\mathrm{t}^{\mathrm{h}} \mathrm{a} \mathrm{P}^{4}$ & $\mathrm{t}^{\mathrm{h}} \mathrm{o} ?^{7}$ & $\mathrm{t}^{\mathrm{h}} \mathrm{ok}$ & $\mathrm{t}^{\mathrm{h}} \mathrm{ok}^{8}$ & $\mathrm{t}^{\mathrm{h}} \mathrm{ok}^{7}$ \\
\hline 房子 & house & & ${ }^{*}$ p-loy? & & & 病 & sick & & $*_{\mathrm{t}} \varphi^{\mathrm{h}} \mathrm{ok}$ & & \\
\hline$\left.(\operatorname{lu})^{3}\right)$ & $\operatorname{luy}^{3}$ & ploy $^{3}$ & ploy $^{3}$ & pon $^{3}$ & $\mathrm{plo \eta}^{3}$ & $\mathrm{ts}^{\mathrm{h}} \mathrm{ok}^{7}$ & $\mathrm{ts}^{\mathrm{h}} \mathrm{uk}^{7}$ & $\mathrm{ts}^{\mathrm{h}} \mathrm{ok}^{7}$ & $\mathrm{ts}^{\mathrm{h}} \mathrm{ok}^{7}$ & $\operatorname{ts}^{\mathrm{h}} \mathrm{o} ?^{7}$ & $\mathrm{ts}^{\mathrm{h}} \mathrm{ok}{ }^{7}$ \\
\hline ---- & pjan $^{3}$ & poy $^{3}$ & $\mathrm{plop}^{3}$ & $\mathrm{plo \eta}^{3}$ & $\mathrm{plo \eta}^{3}$ & $\mathrm{sok}^{2}$ & $\mathrm{sa}^{4}$ & $\operatorname{ts}^{\mathrm{h}} \curvearrowright P^{7}$ & $\mathrm{ts}^{\mathrm{h}} \mathrm{ok}^{7}$ & $\mathrm{ts}^{\mathrm{h}} \mathrm{ok}$ & $\mathrm{ts}^{\mathrm{h}} \mathrm{ok}^{7}$ \\
\hline 脖子 & neck & & $* \operatorname{ljoy} ?$ & & & 腳 & leg & & ${ }^{*} \mathrm{k}^{\mathrm{h}} \mathrm{ok}$ & & \\
\hline $\mathrm{zoy}^{3}$ & zuy $^{3}$ & zoy $^{3}$ & ton $^{6}$ & $\operatorname{ton}^{6}$ & 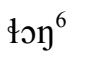 & $\mathrm{k}^{\mathrm{h}} \mathrm{ok}^{7}$ & $\mathrm{k}^{\mathrm{h}} \mathrm{uk}^{7}$ & $\mathrm{k}^{\mathrm{h}} \mathrm{ok}^{7}$ & $\mathrm{k}^{\mathrm{h}} \mathrm{ok}^{7}$ & $\mathrm{k}^{\mathrm{h}} \mathrm{o} ?^{7}$ & $\mathrm{k}^{\mathrm{h}} \mathrm{o \textrm {k } ^ { 7 }}$ \\
\hline $10 \eta^{4}$ & zay $^{3}$ & juan $^{3}$ & $\mathrm{zoy}^{3}$ & $z^{2} \eta^{3}$ & tsoy $^{6}$ & $\left(\mathrm{k}^{\mathrm{h}} \mathrm{ok}^{2}\right)$ & $\left(\mathrm{k}^{\mathrm{h}} \mathrm{o} 1^{4}\right)$ & $\mathrm{k}^{\mathrm{h}} \curvearrowright \mathrm{P}^{7}$ & $\mathrm{k}^{\mathrm{h}} \mathrm{ok}^{7}$ & $\mathrm{k}^{\mathrm{h}} \mathrm{ok}^{8}$ & $\mathrm{k}^{\mathrm{h}} \mathrm{o} \mathrm{k}^{7}$ \\
\hline
\end{tabular}

\subsubsection{Closed Rimes with Long Low Vowels}

The rimes with low nuclei occur in both long and short forms. The correspondence series for PH1 rimes with long low nuclei are given below: 
(154) Reflexes of PHl rimes with low vowels

\begin{tabular}{|c|c|c|c|c|c|c|c|c|c|c|c|}
\hline BHi:n & HaE:m & LHu:t & $\underline{\text { Tzha }}$ & $\underline{Z \text { dui }}$ & Bting & $\underline{\text { Cun }}$ & $\underline{\mathrm{Nadou}}$ & $\underline{\text { Cjiang }}$ & $\underline{\text { Mfaw }}$ & $\underline{\text { Baisha }}$ & $\underline{\text { Ymen }}$ \\
\hline$a: y^{B / C}$ & $a: y^{B / C}$ & $a: y^{B / C}$ & $\mathrm{a}: \mathrm{y}^{\mathrm{B} / \mathrm{C}}$ & $a: y^{B / C}$ & $a: y^{B / C}$ & $\mathrm{a}:(\mathrm{y})^{\mathrm{B} / \mathrm{C}}$ & $a y^{B / C}$ & $a: y^{B / C}$ & $a: y^{B / C}$ & $a: y^{B / C}$ & uay $^{\mathrm{B} / \mathrm{C}}$ \\
\hline a:w & $\mathrm{a}: \mathrm{w}$ & $\mathrm{a}: \mathrm{w}$ & $\mathrm{a}: \mathrm{w}$ & $\mathrm{a}: \mathrm{w}$ & $\mathrm{a}: \mathrm{w}$ & $\mathrm{a}: \mathrm{w}$ & $\mathrm{a}(\mathrm{w})$ & $\mathrm{a}: \mathrm{w}$ & $\mathrm{a}: \mathrm{w}$ & $\mathrm{a}: \mathrm{w}$ & $\mathrm{a}: \mathrm{w}$ \\
\hline $\mathrm{a}: \mathrm{m}$ & $\mathrm{a}: \mathrm{m}$ & $\mathrm{a}: \mathrm{m}$ & $\mathrm{a}: \mathrm{m}$ & $\mathrm{a}: \mathrm{m}$ & $\mathrm{a}: \mathrm{m}$ & $\mathrm{om}$ & an & $\mathrm{a}: \mathrm{m}$ & $\mathrm{a}: \mathrm{m}$ & $\mathrm{a}: \mathrm{m}$ & $\mathrm{am}$ \\
\hline $\mathrm{a}: \mathrm{n}$ & a:n & a:n & a:n & a:n & a:n & on & an & $a: y$ & $\mathrm{a}: \mathrm{y}$ & $\mathrm{a}: \mathrm{y}$ & uan \\
\hline a:n & a:n & $\mathrm{a}: \mathrm{n}$ & a:n & $\mathrm{a}: \mathrm{n}$ & a:n & on & on & a:n & a:n & $\mathrm{a}: \mathrm{n}$ & an \\
\hline a:y & e:y & e:y & e:y & e:y & e:y & $\varepsilon \eta$ & $\varepsilon \eta$ & e:y & e:y & ian & ian \\
\hline$a: p$ & a:p & a:p & $a: p$ & $a: p$ & $a: p$ & $\mathrm{op}$ & a? & $a: p$ & $a: p$ & $a: p$ & ap \\
\hline $\mathrm{a}: \mathrm{t}$ & a:t & a:t & $\mathrm{a}: \mathrm{t}$ & $\mathrm{a}: \mathrm{t}$ & a:t & ot & a? & $a: ?$ & $\mathrm{a}: \mathrm{k}$ & $\mathrm{a}: ?$ & uat \\
\hline a:t & $\mathrm{a}: \mathrm{t}$ & $\mathrm{a}: \mathrm{c}$ & $\mathrm{a}: \mathrm{t}$ & a:t & a:t & ot & a? & o:t & o:t & a:t & uat \\
\hline $\mathrm{a}: ?$ & e:? & e:k & e:? & e:? & e:? & $\varepsilon \mathrm{k}$ & $\varepsilon ?$ & e:? & u: & e? & ia? \\
\hline$\underline{\text { S. Hlai }}$ & avina) & & & C. $\mathrm{Hla}$ & (Savina & & & $\underline{\text { Baish }}$ & Wan & Qian) & \\
\hline
\end{tabular}

$\begin{array}{lll}\text { ay }^{\mathrm{B} / \mathrm{C}} & \mathrm{ay}^{\mathrm{B} / \mathrm{C}} & \mathrm{a}: \mathrm{y}^{\mathrm{B} / \mathrm{C}} \\ \text { aw } & \text { aw } & \text { aw } \\ \text { am } & \text { am } & \text { a:m } \\ \text { an } & \text { an } & \text { a:y } \\ \text { an } & \text { an } & ---- \\ \text { ay } \sim \text { y } & \varepsilon y & \text { iay } \\ \text { ap } & \text { ap } & \text { a:p } \\ \text { at } & --- & \text { a:? } \\ \text { at } & \text { at } & \text { uat } \\ \text { a: } \sim \varepsilon: & \text { ek } & \text { e? }\end{array}$

The reconstructions I propose for these are the following:

$$
\begin{aligned}
& \text { *a:y }{ }^{\mathrm{B} / \mathrm{C}} \\
& \text { *a:w } \\
& \text { *a:m } \\
& \text { *a:n } \\
& \text { *a:p } \\
& \text { *a:y } \\
& \text { *a:p } \\
& \text { *a:t } \\
& \text { *a:c } \\
& \text { *a:k }
\end{aligned}
$$

The earliest change which seems to have occurred is in the series closed by velar stops, between Bouhin and the rest of Hlai. Bouhin remained conservative, maintaining a low central nucleus, whereas the nucleus in the rest of post-PHl fronted to the front low vowel: 
Development of PHl *a:K in Bouhin and in other Hlai

\begin{tabular}{|c|c|c|c|c|}
\hline Bouhin: & *a:y & $>$ & a:y & \\
\hline & *a:k & $>$ & $\mathrm{a}: \mathrm{k}$ & (> \\
\hline Other Hlai: & *a:y & $>$ & $\varepsilon: \eta$ & \\
\hline & $* \mathrm{a}: \mathrm{k}$ & $>$ & $\varepsilon: \mathrm{k}$ & \\
\hline
\end{tabular}

These finals then proceeded to develop in various ways, as detailed below. As in several previous cases, Bouhin has two series of reflexes. The first, with the low central nucleus, have been inherited directly into Bouhin (157a); the second, with the mid front nucleus, always have a direct correspondent in Ha Em, and generally reflect loanwords (157b) (although a few may be legitimate cases of PHI *e:K rimes, as discussed in section 4.5.1):

(157) Examples of Bouhin inherited *a:K versus borrowed *a:K

\begin{tabular}{|c|c|c|c|c|c|c|}
\hline \multirow[t]{4}{*}{ (a) } & $\underline{\text { Gloss }}$ & $\underline{\mathrm{PHl}}$ & Bouhin & $\underline{\text { Gloss }}$ & $\underline{\mathrm{PHl}}$ & Bouhin \\
\hline & name & ${ }^{*} \mathrm{p}^{\mathrm{h}} \mathrm{a}: \mathrm{y}$ & $\mathrm{p}^{\mathrm{h}} \mathrm{a}: \mathrm{y}^{1}$ & flesh (of fruit) & ${ }^{*} \mathrm{C}$-ma:k & $\mathrm{ma}: ?^{7}$ \\
\hline & bamboo hat & *hla:y? & da: $: \eta^{3}$ & banana & *hwa:k & va: $1^{7}$ \\
\hline & chin & *ha:y & ha: $y^{1}$ & bad & $*_{r j a: k}$ & $\mathrm{za}: \mathrm{P}^{7}$ \\
\hline \multirow[t]{7}{*}{ (b) } & $\underline{\text { Gloss }}$ & $\underline{\mathrm{PHl}}$ & Bouhin & $\underline{\mathrm{Ha} E m}$ & & \\
\hline & wide & $* 6[\mathrm{e}]: \mathrm{n}$ & $6 e: y^{1}$ & $6 e: y^{1}$ & & \\
\hline & comb & $*_{\mathrm{s}}[\mathrm{e}]: \mathrm{y}$ & te: $\eta^{1}$ & te: $\eta^{1}$ & & \\
\hline & stir-fry & $* \mathrm{k}[\mathrm{e}]: \mathrm{y}$ & ke: $y^{1}$ & ke: $y^{1}$ & & \\
\hline & get & ${ }^{*} \mathrm{C}-\mathrm{m}[\mathrm{e}]: \mathrm{k}$ & me: $?^{7}$ & me: $?^{7}$ & & \\
\hline & phlegm & $* \mathrm{~h}[\mathrm{e}]: \mathrm{k}$ & he: $?^{9}$ & he: $?^{7}$ & & \\
\hline & pacify & $*_{\mathrm{r}}[\mathrm{e}]: \mathrm{k}$ & ge: $?^{7}$ & ge: $?^{7}$ & & \\
\hline
\end{tabular}

Note that there are other indications of loans in the examples above, including tone 9 in phlegm, as well as the initial $g$ in pacify, which does not occur in native Bouhin words.

Within $\mathrm{NCHl}$, the following changes occurred. In Cunhua, there was a backing of remaining low vowels in all positions, except in the diphthong where it remained unchanged. All rimes save the diphthong were subsequently shortened: 


$\begin{array}{lllll}* \mathrm{a}: \mathrm{y} & > & \mathrm{a}: \mathrm{y} & > & \mathrm{a}:(\mathrm{y}) \\ *_{\mathrm{a}: \mathrm{C}}> & \text { о:C } & > & \text { oC } \\ * \mathrm{a}: \mathrm{K} & > & \varepsilon: \mathrm{K} & > & \varepsilon \mathrm{K}\end{array}$

In Nadouhua, the low vowel was backed when it preceded the palatal nasal before the palatal merged with the alveolar series. I assume this failed to happen before the palatal stop because it participated in debuccalization before this change, bleeding the environment. All rimes were reduced in length:

$\begin{array}{lllll}* \mathrm{a}: \mathrm{C} & > & \mathrm{a}: \mathrm{C} & > & \mathrm{aC} \\ * \mathrm{a}: \mathrm{n} & > & \text { о: } \mathrm{n} & > & \text { on } \\ * \mathrm{a}: \mathrm{c} & > & \mathrm{a}: \mathrm{l} & > & \mathrm{a} ? \\ * \mathrm{a}: \mathrm{K} & > & \varepsilon: \mathrm{K} & > & \varepsilon \mathrm{K}\end{array}$

In the Meifu branch, a shift from alveolar to velar codas filled the gap left by the fronting of the nucleus in original PHl *a:y and *a:k. The timing of the loss of the palatal codas was different, so that the oral stop was lost in time to condition the raising and backing of the vowel, but the nasal coda was lost afterwards. The evolution of PHl *a:k in Moyfaw was highly irregular, ending in present-day $u:^{B}$. A transition through a final glottal stop, subsequently lost, presumably conditioned its merger with the Tone B category:

$$
\begin{aligned}
& \text { *a:n }>\text { a:y }>\text { a:y } \\
& * a: \text { n a:j }>\text { a:n } \\
& \text { *a:y }>\quad \varepsilon: \eta \quad>\quad \text { e:n } \\
& * a: t \quad>\quad \text { a:k }>\text { a:? (CJ)/a:k (MF) } \\
& * \text { a:c o:t }>>\text { o:t } \\
& * a: k \quad \text { s:k }>\quad \text { e:? (CJ)/ia? }>\mathrm{u}^{\mathrm{B}}{ }^{\mathrm{B}}(\mathrm{MF})
\end{aligned}
$$

Baisha alveolar codas also became velars before the loss of the palatal place of articulation in the codas, but after the fronting of the rimes in the original velar coda series. After this fronting occurred, the long vowel dipthongized before the nasal, whereas it shortened before the stop: 
$(161)$

$\begin{array}{lllll}* a: n & > & \text { a:n } & > & \text { a:n } \\ * a: n & > & \text { a:n } & > & \text { a:n } \\ * a: n & > & \varepsilon: n & > & \text { ian } \\ \text { *a:t } & > & \text { a:k } & > & \text { a:? } \\ \text { *a:c } & > & \text { a:t } & > & \text { a:t } \\ \text { *a:k } & > & \varepsilon: k & > & \text { e? }\end{array}$

Yuanmen *a:c merged with *a:t, and rimes before alveolar codas were backed and raised to $o: T$. *a:n merged with *a:n subsequent to this change, filling the gap left by original *a:n (similar to the Meifu branch above). All low non-central vowels then underwent diphthongization, while rimes with pure $a$ : were shortened:

\begin{tabular}{|c|c|c|c|}
\hline *a:y & $>$ & ०:y & $>$ \\
\hline$* a: w$ & $>$ & $\mathrm{a}: \mathrm{w}$ & $>$ \\
\hline *a:m & $>$ & $\mathrm{a}: \mathrm{m}$ & $>$ \\
\hline *a:n & $>$ & o:n & $>$ \\
\hline *a:p & $>$ & a:jn & $>$ \\
\hline *a:y & $>$ & $\varepsilon: \eta$ & $>$ \\
\hline *a:p & $>$ & $\mathrm{a}: \mathrm{p}$ & $>$ \\
\hline$* a: t$ & $>$ & $0: t$ & $>$ \\
\hline$* a: c$ & $>$ & $0: t$ & $>$ \\
\hline *a:k & $>$ & $\varepsilon: \mathrm{k}$ & $>$ \\
\hline
\end{tabular}

The evolution of rimes with long mid central vowels in Yuanmen is repeated here next to their counterparts with low central vowels, so that the movement of these nuclei can be compared. In particular, it can be seen that the shift *a:T $>\Im: T$ blocked the lowering of $*_{0}: C\left(\right.$ from $\left.{ }^{*} ə: T\right)>\jmath: C$ :

Development of *ə:C versus *a:C in Yuanmen

\begin{tabular}{|c|c|c|c|c|c|c|c|c|c|}
\hline *ə:y & $>$ & o:y & $>$ & u:y & *a:y & $>$ & 0:y & $>$ & uay \\
\hline *ə:m & $>$ & o:m & $>$ & uam & *a:m & $>$ & $\mathrm{a}: \mathrm{m}$ & $>$ & am \\
\hline *ə:n & $>$ & $\mathrm{o}: \mathrm{n}$ & $>$ & $\mathrm{u}: \mathrm{n}$ & *a:n & $>$ & o:n & $>$ & uan \\
\hline *ə:1 & $>$ & $0: 1$ & $>$ & uay & *a:y & $>$ & $\varepsilon: \eta$ & $>$ & ian \\
\hline *ə:p & $>$ & o:p & $>$ & uap & $* a: p$ & $>$ & $a: p$ & $>$ & ap \\
\hline *ə:t & $>$ & $\mathrm{o}: \mathrm{t}(\sim \mathrm{ot})$ & $>$ & $\mathrm{u}: \mathrm{t}(\sim \partial \mathrm{t})$ & $* a: t$ & $>$ & $0: t$ & $>$ & uat \\
\hline *ə:k & $>$ & $\mathrm{o}: \mathrm{k} \sim 0: \mathrm{k}$ & $>$ & ०P o? & $*_{\mathrm{a}: \mathrm{k}}$ & $>$ & $\varepsilon: \mathrm{k}$ & $>$ & ia? \\
\hline
\end{tabular}


As in the case of the other palatal-final rimes, there was a complete merger with the alveolar series in Bouhin, Ha Em, and Qi:

$\begin{array}{lll}* a: n & > & \text { a:n } \\ \text { *a:n } & > & \text { a:n } \\ & & \\ \text { *a:t } & > & \text { a:t } \\ \text { *a:c } & > & \text { a:t }\end{array}$

A comparison of reconstructions is given below:

(165) Thurgood

Ostapirat (1993) Ostapirat (2004) $\underline{\mathrm{PHl}}$

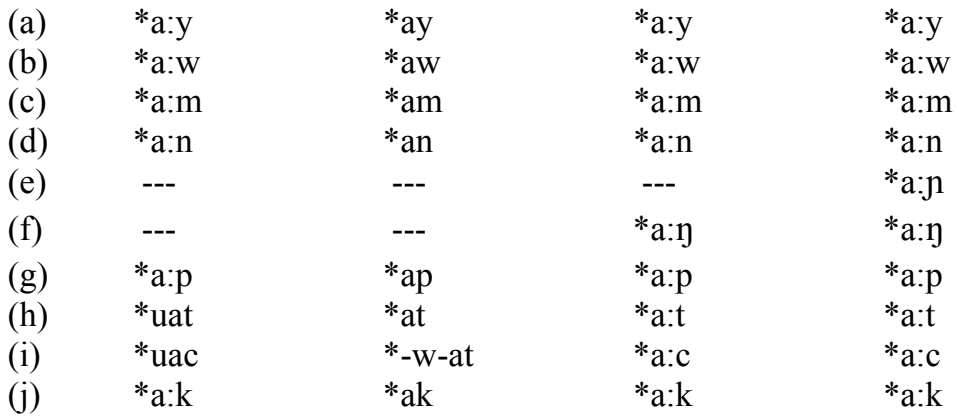

Thurgood generally reconstructs rimes with a long low vowel. He doesn't reconstruct anything in (165e-f) (having reconstructed *i:y where I reconstruct *a:y, which is listed in section 4.5.1 as more equivalent to *e:y), and he reconstructs diphthongs in (165h-i), violating Symmetry.

Ostapirat (1993) reconstructs plain low vowels, except in (165i) where he reconstructs a medial labiovelar glide to account for the reflexes where there are back vowels or diphthongs in $\mathrm{NCHl}$. He also fails to reconstruct anything in (165e-f). Ostapirat's (2004) reconstruction is identical to the present one, save for the omission of *a:n (of which there are only four examples altogether).

Examples of rimes with low vowels are given below, in the following order:

$\begin{array}{llllll}\text { Bhin } & \text { Ha Em } & \text { Lhut } & \text { Tzha } & \text { Zdui } & \text { Bting } \\ \text { Cun } & \text { Nadou } & \text { Cjiang } & \text { Mfaw } & \text { Baisha } & \text { Ymen }\end{array}$




\begin{tabular}{|c|c|c|c|c|c|c|c|c|c|c|c|}
\hline (a) & & & $* a: y$ & & & (b) & & & ${ }^{*} \mathbf{a}: \mathbf{w}$ & & \\
\hline 甘蔗 & & & *C-ma: & & & 流 & flow & & ${ }^{*} \mathrm{C}-\mathrm{ma}$ & & \\
\hline $\begin{array}{l}\left(\mathrm{may}^{3}\right) \\
\mathrm{ma}(\mathrm{y})^{3}\end{array}$ & $\begin{array}{l}\text { ma:y } y^{3} \\
\text { may }^{3}\end{array}$ & $\begin{array}{l}m a: y^{3} \\
m a: y^{3}\end{array}$ & $\begin{array}{l}\text { ma:y } y^{3} \\
m a: y^{3}\end{array}$ & $\begin{array}{l}\text { ma:y }{ }^{6} \\
m a: y^{3}\end{array}$ & $\begin{array}{l}\text { ma:y }{ }^{3} \\
\text { muay }^{6}\end{array}$ & $\begin{array}{l}\mathrm{ma}: \mathrm{w}^{1} \\
\mathrm{ma}: \mathrm{w}^{1}\end{array}$ & $\begin{array}{l}\text { ma:w }^{1} \\
\text { maw }^{1}\end{array}$ & $\begin{array}{l}\mathrm{ma}: \mathrm{w}^{1} \\
\mathrm{ma}: \mathrm{w}^{1}\end{array}$ & $\begin{array}{l}\mathrm{ma}: \mathrm{w}^{1} \\
\mathrm{ma}: \mathrm{w}^{1}\end{array}$ & $\begin{array}{l}\text { ma:w }{ }^{4} \\
m a: w^{1}\end{array}$ & $\begin{array}{l}m a: w^{1} \\
m a: w^{4}\end{array}$ \\
\hline 見 & see & & ${ }^{*}$ C-la:y & & & 星星 & star & & $*_{\text {ra:w }}$ & & \\
\hline $\begin{array}{l}\text { la: } y^{3} \\
\text { la:(y) }\end{array}$ & $\begin{array}{l}\text { la: } y^{3} \\
\text { lay }\end{array}$ & $\begin{array}{l}\text { la: } y^{3} \\
\text { la:y } y^{3}\end{array}$ & $\begin{array}{l}\text { la: } y^{3} \\
\text { la: } y^{3}\end{array}$ & $\begin{array}{l}\text { la: } y^{6} \\
\text { la:y }{ }^{3}\end{array}$ & $\begin{array}{l}\text { la: } y^{3} \\
\text { luay }\end{array}$ & $\begin{array}{l}\mathrm{ra}: \mathrm{w}^{1} \\
\mathrm{la}: \mathrm{w}^{4}\end{array}$ & $\begin{array}{l}\text { ra: }{ }^{1} \\
\operatorname{la}^{4}{ }^{4}\end{array}$ & $\begin{array}{l}r a: w^{1} \\
\text { la:w }{ }^{4}\end{array}$ & $\begin{array}{l}\text { ra: } w^{4} \\
\text { ra: } w^{1}\end{array}$ & $\begin{array}{l}\text { la: } w^{4} \\
\text { ra:w }{ }^{1}\end{array}$ & $\begin{array}{l}\text { la: } w^{4} \\
\text { ra: } w^{4}\end{array}$ \\
\hline 杘 & shit & & *ha:y? & & & 白 & white & & ${ }^{*} \mathrm{k}^{\mathrm{h}} \mathrm{a}: \mathrm{w}$ & & \\
\hline $\begin{array}{l}\text { ha:y } \\
\text { ha:(y) }\end{array}$ & $\begin{array}{l}\text { ha: } y^{3} \\
\text { hay }\end{array}$ & $\begin{array}{l}\text { ha:y }{ }^{3} \\
\text { ha:y } y^{3}\end{array}$ & $\begin{array}{l}\text { ha: } y^{3} \\
\text { ha: } y^{3}\end{array}$ & $\begin{array}{l}\text { ha:y }{ }^{3} \\
\text { ha: } y^{3}\end{array}$ & $\begin{array}{l}\text { ha:y }{ }^{3} \\
\text { huay }^{3}\end{array}$ & $\begin{array}{l}\mathrm{k}^{\mathrm{h}} \mathrm{a}: \mathrm{w}^{1} \\
\mathrm{k}^{\mathrm{h}} \mathrm{a}: \mathrm{w}^{1}\end{array}$ & $\begin{array}{l}\mathrm{k}^{\mathrm{h}} \mathrm{a}: \mathrm{w}^{1} \\
\mathrm{k}^{\mathrm{h}} \mathrm{aw}\end{array}$ & $\begin{array}{l}\mathrm{k}^{\mathrm{h}} \mathrm{a}: \mathrm{w}^{1} \\
\mathrm{k}^{\mathrm{h}} \mathrm{a}: \mathrm{w}^{1}\end{array}$ & $\begin{array}{l}\mathrm{k}^{\mathrm{h}} \mathrm{a}: \mathrm{w}^{1} \\
\mathrm{k}^{\mathrm{h}} \mathrm{a}: \mathrm{w}^{1}\end{array}$ & $\begin{array}{l}\mathrm{k}^{\mathrm{h}} \mathrm{a}: \mathrm{w}^{1} \\
\mathrm{k}^{\mathrm{h}} \mathrm{a}: \mathrm{w}^{1}\end{array}$ & $\begin{array}{l}\mathrm{k}^{\mathrm{h}} \mathrm{a}: \mathrm{w}^{1} \\
\mathrm{k}^{\mathrm{h}} \mathrm{a}: \mathrm{w}^{1}\end{array}$ \\
\hline (c) & & & $* a: m$ & & & (d) & & & *a:p & & \\
\hline 台 & lift (2) & & $*_{\mathrm{t}} \int^{\mathrm{h}} \mathrm{a}: \mathrm{m}$ & & & 甲虫 & cockroa & & $*_{\text {ra:p }}$ & & \\
\hline $\begin{array}{l}\text { ts }^{\mathrm{h}} \mathrm{a}: \mathrm{m}^{1} \\
\mathrm{~h}^{1} \mathrm{~m}^{1}\end{array}$ & $\begin{array}{l}\text { ts }^{\mathrm{h}} \mathrm{a}: \mathrm{m}^{1} \\
\mathrm{han}^{1}\end{array}$ & $\begin{array}{l}\text { ts }^{\mathrm{h}} \mathrm{a}: \mathrm{m}^{1} \\
\text { ts }^{\mathrm{h}} \mathrm{a}: \mathrm{m}^{1}\end{array}$ & $\begin{array}{l}\text { ts }^{\mathrm{h}} \mathrm{a}: \mathrm{m}^{1} \\
\text { ts }^{\mathrm{h}} \mathrm{a}: \mathrm{m}^{1}\end{array}$ & $\begin{array}{l}\text { ts }^{\mathrm{h}} \mathrm{a}: \mathrm{m}^{1} \\
\text { ts }^{\mathrm{h}} \mathrm{a}: \mathrm{m}^{1}\end{array}$ & $\begin{array}{l}\mathrm{ts}^{\mathrm{h}} \mathrm{a}: \mathrm{m}^{1} \\
\text { ts }^{\mathrm{h}} \mathrm{am}^{1}\end{array}$ & ra:p $p^{7}$ & $\begin{array}{l}\text { ra:p }{ }^{7} \\
\text { la } ?^{4}\end{array}$ & $\begin{array}{l}\text { ra: } p^{7} \\
\text { la: } p^{7}\end{array}$ & $\begin{array}{l}\text { ra: } p^{8} \\
\text { ra: } p^{7}\end{array}$ & $\begin{array}{l}\text { la:p }{ }^{8} \\
\text { ra:p }\end{array}$ & $\begin{array}{l}\text { la:p } p^{8} \\
\left.\text { (ra:p } p^{8}\right)\end{array}$ \\
\hline 問 & ask & & *ra:m & & & 挑 & carry on & shoulder & $* \mathrm{t}^{\mathrm{h}} \mathrm{a}: \mathrm{p}$ & & \\
\hline $\begin{array}{l}\left(\mathrm{ga:m}^{1}\right) \\
\text { høm }^{4}\end{array}$ & $\begin{array}{l}\text { ga:m }{ }^{1} \\
\operatorname{yan}^{4}{ }^{4}\end{array}$ & $\begin{array}{l}\text { ga: } m^{1} \\
\text { ga: }{ }^{4}\end{array}$ & $\begin{array}{l}\text { ga: } m^{4} \\
\text { xa:m }\end{array}$ & $\begin{array}{l}\text { ha: } m^{4} \\
\text { xa:m }\end{array}$ & $\begin{array}{l}\text { ha:m }{ }^{4} \\
\text { kham }^{1}\end{array}$ & $\mathrm{ts}^{\mathrm{h}} \mathrm{a}: \mathrm{p}^{7}$ & $\begin{array}{l}\text { ts }^{\mathrm{h}} \mathrm{a}: \mathrm{p}^{7} \\
\mathrm{ha}^{4} \mathrm{P}^{4}\end{array}$ & $\begin{array}{l}\text { ts }{ }^{\mathrm{h}} \mathrm{a}: \mathrm{p}^{7} \\
\text { ts }^{\mathrm{h}} \mathrm{a}: \mathrm{p}^{7}\end{array}$ & $\begin{array}{l}\text { ts }^{\mathrm{h}} \mathrm{a}: \mathrm{p}^{7} \\
\text { ts }^{\mathrm{h}} \mathrm{a}: \mathrm{p}^{7}\end{array}$ & $\begin{array}{l}\text { ts }^{\mathrm{h}} \mathrm{a}: \mathrm{p}^{7} \\
\text { ts }^{\mathrm{h}} \mathrm{a}: \mathrm{p}^{8}\end{array}$ & $\begin{array}{l}\text { ts }^{\mathrm{h}} \mathrm{a}: \mathrm{p}^{7} \\
\text { ts }^{\mathrm{h}} \mathrm{ap}^{7}\end{array}$ \\
\hline 跨 & step & & ${ }^{*}$ Cifa:n & & & 谷粒 & $\mathrm{pc}$ of ric & & * ha:p & & \\
\hline na:m² ${ }^{2}$ & $\begin{array}{l}\text { ha: } m^{2} \\
\text { yjen }\end{array}$ & $\begin{array}{l}\text { hja:m² } \\
\text { n } \varepsilon: m^{2}\end{array}$ & $\begin{array}{l}\text { za:m }{ }^{5} \\
\text { na:m }\end{array}$ & za:m & za:m & $\begin{array}{l}\text { ha:p }{ }^{7} \\
\text { hop }\end{array}$ & ha:p ${ }^{7}$ & $\begin{array}{l}\text { ha: } p^{7} \\
\text { ha: } p^{7}\end{array}$ & $\begin{array}{l}\text { ha: } p^{7} \\
\text { ha: } p^{7}\end{array}$ & $\begin{array}{l}\text { ha: }{ }^{7} \\
\text { ha: } p^{8}\end{array}$ & $\begin{array}{l}\text { ha:p }{ }^{7} \\
\text { hap }^{7}\end{array}$ \\
\hline
\end{tabular}




\begin{tabular}{|c|c|c|c|c|c|c|c|c|c|c|c|}
\hline (e) & & & *a:n & & & (f) & & & $*_{\mathbf{a}} \mathbf{t}$ & & \\
\hline 沸騰 & boil & & *da:n & & & 窮 & poor & & *va:t & & \\
\hline da: $n^{1}$ & da: $n^{1}$ & da: $n^{1}$ & da: $n^{1}$ & da: $n^{1}$ & da: $n^{1}$ & va:t ${ }^{7}$ & va:t ${ }^{7}$ & $v a: t^{7}$ & $f a: t^{8}$ & va: $t^{8}$ & va:t ${ }^{7}$ \\
\hline tson $^{1}$ & $\operatorname{dan}^{1}$ & da: $y^{1}$ & da: $y^{1}$ & da: $y^{1}$ & duan $^{1}$ & ---- & ---- & va: $?^{7}$ & $\mathrm{va}: \mathrm{k}^{7}$ & va: $?^{8}$ & vuat $^{8}$ \\
\hline 月(亮) & month/s & noon & ${ }^{*}$ C-ла: & & & 粘 & stick & & $* \mathrm{k}^{\mathrm{h}} \mathrm{a}: \mathrm{t}$ & & \\
\hline na: ${ }^{1}$ & na: ${ }^{1}$ & na: ${ }^{1}$ & na: ${ }^{1}$ & na: $n^{4}$ & na:n ${ }^{1}$ & ---- & $\mathrm{k}^{\mathrm{h}} \mathrm{a}: \mathrm{t}^{7}$ & $\mathrm{k}^{\mathrm{h}} \mathrm{a}: \mathrm{t}^{7}$ & $\mathrm{k}^{\mathrm{h}} \mathrm{a}: \mathrm{t}^{7}$ & $\mathrm{k}^{\mathrm{h}} \mathrm{a}: \mathrm{t}^{7}$ & $\mathrm{k}^{\mathrm{h}} \mathrm{a}: \mathrm{t}^{7}$ \\
\hline non $^{1}$ & njan $^{1}$ & ne: ${ }^{1}$ & na: $y^{1}$ & na: ${ }^{1}$ & nuan $^{4}$ & ---- & $\mathrm{k}^{\mathrm{h}} \mathrm{a} \mathrm{P}^{4}$ & $\mathrm{k}^{\mathrm{h}} \mathrm{a}: \mathrm{P}^{7}$ & $\mathrm{k}^{\mathrm{h}} \mathrm{a}: \mathrm{k}^{7}$ & $\mathrm{k}^{\mathrm{h}} \mathrm{a} \mathrm{r}^{8}$ & $\mathrm{k}^{\mathrm{h}} \mathrm{uat}^{7}$ \\
\hline 上 & above & & ${ }^{*} \mathrm{k}^{\mathrm{h}} \mathrm{a}: \mathrm{n}$ & & & 傳染 & infect & & ${ }^{*} \mathrm{k}^{\mathrm{h}} \mathrm{a}: \mathrm{t}$ & & \\
\hline $\begin{array}{l}\mathrm{k}^{\mathrm{h}} \mathrm{a}: \mathrm{n}^{1} \\
\mathrm{k}^{\mathrm{h}} \rightarrow \mathrm{n}^{1}\end{array}$ & $\begin{array}{l}\mathrm{k}^{\mathrm{h}} \mathrm{a}: \mathrm{n}^{1} \\
\mathrm{k}^{\mathrm{h}} \mathrm{an}^{1}\end{array}$ & $\begin{array}{l}\mathrm{k}^{\mathrm{h}} \mathrm{a}: \mathrm{n}^{1} \\
\mathrm{k}^{\mathrm{h}} \mathrm{a} \cdot \mathrm{n}^{1}\end{array}$ & $\begin{array}{l}\mathrm{k}^{\mathrm{h}} \mathrm{a} \cdot \mathrm{n}^{1} \\
\mathrm{k}^{\mathrm{h}} \mathrm{a} \cdot \mathrm{n}^{1}\end{array}$ & $\begin{array}{l}\mathrm{k}^{\mathrm{h}} \mathrm{a}: \mathrm{n}^{1} \\
\mathrm{k}^{\mathrm{h}} \mathrm{a} \cdot \mathrm{n}^{1}\end{array}$ & $\begin{array}{l}\mathrm{k}^{\mathrm{h}} \mathrm{a}: \mathrm{n}^{1} \\
\mathrm{k}^{\mathrm{h}} \mathrm{unn}^{1}\end{array}$ & $\mathrm{p}^{\mathrm{h}} \mathrm{a}: \mathrm{t}^{7}$ & $\mathrm{k}^{\mathrm{h}} \mathrm{a} \cdot \mathrm{t}^{7}$ & $\begin{array}{l}\mathrm{p}^{\mathrm{h}} \mathrm{a} \cdot \mathrm{t}^{7} \\
\mathrm{k}^{\mathrm{h}} \mathrm{a} \cdot ?^{7}\end{array}$ & $\begin{array}{l}\mathrm{k}^{\mathrm{h}} \mathrm{a} \cdot \mathrm{t}^{7} \\
\mathrm{k}^{\mathrm{h}} \cdot ?^{7}\end{array}$ & $\begin{array}{l}\mathrm{k}^{\mathrm{h}} \mathrm{a}: \mathrm{t}^{7} \\
\mathrm{k}^{\mathrm{h}} \mathrm{a} ?^{8}\end{array}$ & $\begin{array}{l}\mathrm{k}^{\mathrm{h}} \mathrm{a}: \mathrm{t}^{7} \\
\mathrm{k}^{\mathrm{h}} \mathrm{uat}^{7}\end{array}$ \\
\hline (g) & & & $* a: n$ & & & (h) & & & $* \mathbf{a}: \mathbf{c}$ & & \\
\hline *餽嘴 & greedy & & ${ }^{*}$ C-la: $\mathrm{n}$ & & & 停 & stop $(\mathrm{t}$ & n off) & ${ }^{*} \mathrm{C}-\mathrm{ya}: \mathrm{c}$ & & \\
\hline la: ${ }^{1}$ & la: ${ }^{1}$ & la: ${ }^{1}$ & la: $n^{1}$ & la: $n^{4}$ & la:n ${ }^{1}$ & ya:t ${ }^{7}$ & ya:t ${ }^{7}$ & ya: $\mathrm{c}^{7}$ & ya:t ${ }^{7}$ & ya:t ${ }^{8}$ & ya:t ${ }^{7}$ \\
\hline la: ${ }^{1}$ & $\operatorname{lon}^{1}$ & la: ${ }^{1}$ & la: ${ }^{1}$ & la: $n^{1}$ & $\operatorname{lan}^{4}$ & $\mathrm{yot}^{2}$ & ---- & yo: $: t^{7}$ & yo: $t^{7}$ & ya:t ${ }^{8}$ & yuat $^{8}$ \\
\hline 蟒蛇 & python & & ${ }^{*}$ C-na:j & & & 血 & blood & & *hla:c & & \\
\hline ---- & ---- & na: $\mathrm{n}^{3}$ & ---- & ---- & na: $n^{3}$ & da: $t^{7}$ & ta: $t^{7}$ & fa: $c^{7}$ & $4 a: t^{7}$ & fa: $t^{7}$ & fa: $t^{7}$ \\
\hline n๑n ${ }^{3}$ & non ${ }^{3}$ & ---- & ---- & ---- & ---- & $\mathrm{t} \theta \boldsymbol{\jmath t ^ { 2 }}$ & $l a 2^{4}$ & to: $t^{7}$ & to: $: t^{7}$ & fa: $t^{8}$ & tuat $^{7}$ \\
\hline 咬 & bite & & *hy[a:/ & $\ln ?$ & & & & & & & \\
\hline -- & $\mathrm{ka}: \mathrm{n}^{3}$ & $\mathrm{ka}: \mathrm{n}^{3}$ & $\mathrm{ka}: \mathrm{n}^{6}$ & $\mathrm{k}^{\mathrm{h}} \mathrm{a}: \mathrm{n}^{6}$ & $\mathrm{ka}: \mathrm{n}^{6}$ & & & & & & \\
\hline & ---- & $\operatorname{kan}^{3}$ & $\operatorname{kan}^{3}$ & $\operatorname{kan}^{3}$ & ---- & & & & & & \\
\hline
\end{tabular}


(i)

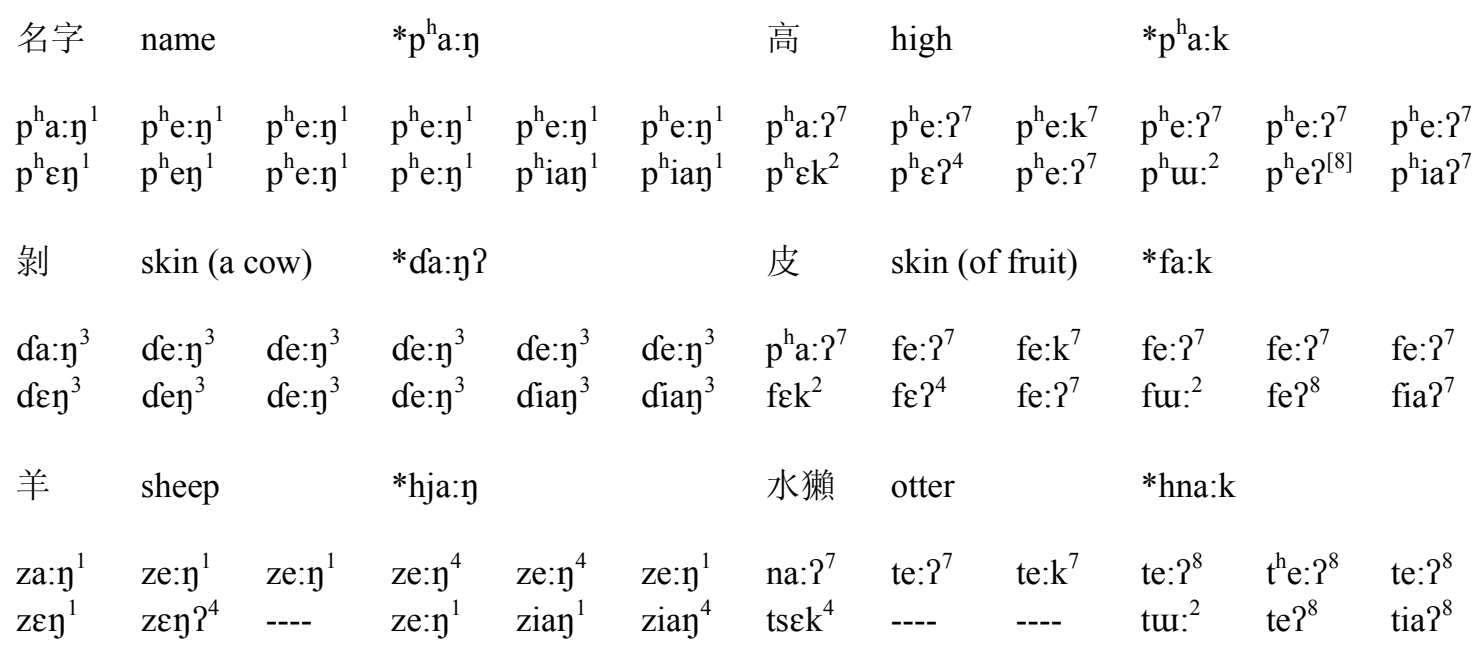

\subsubsection{Interim Summary}

Four series of rimes with non-high nuclei have been reconstructed in this section, listed below:

(167)

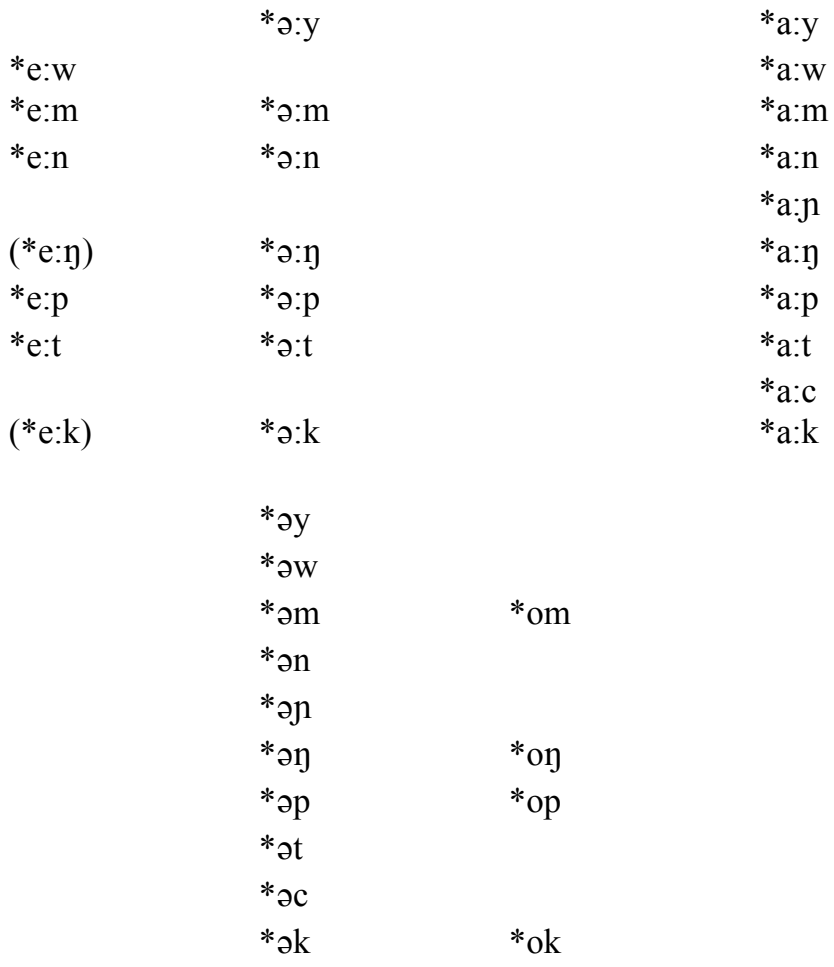


There are several asymmetries which exist, both regular and idiosyncratic. In the case of the former, it can be seen that no ${ }^{*} \mathrm{e}: \mathrm{C}$ rimes occur with palatal ending, a natural co-occurrence restriction; the lack of *ow can be explained the same way.

There are noticeable gaps of rimes with palatal endings in the ${ }^{*}$ : $\mathrm{C}$ and ${ }^{*} \mathrm{oC}$ series; there are also gaps for ${ }^{*}$ : w and alveolar-final rimes in the ${ }^{*} \mathrm{oC}$ series, none of which have readily apparent explanations (but note the hypothesis in (145) above).

Finally, there is an obvious distribution asymmetry in length for this set of rimes, where ${ }^{\mathrm{e}}: \mathrm{C}$ and $*_{\mathrm{a}}$ :C occur only long, and ${ }^{\mathrm{o}} \mathrm{C}$ occurs only short. There is no strong typological precedent for this, yet is seems to be consistent with other Southern Kra-Dai data (see next chapter).

\subsection{Conclusion}

The entire reconstructed system of $\mathrm{PHl}$ rimes is given and discussed in this section. A broader comparison of the three alternate systems of reconstruction is provided as well.

\subsubsection{The Present Reconstruction}

The system proposed here is given below. Any rimes which are reconstructed on the evidence of three or fewer examples are placed in brackets to indicate their marginality in the system. The rime categories are displayed according to coda (or lack thereof): 


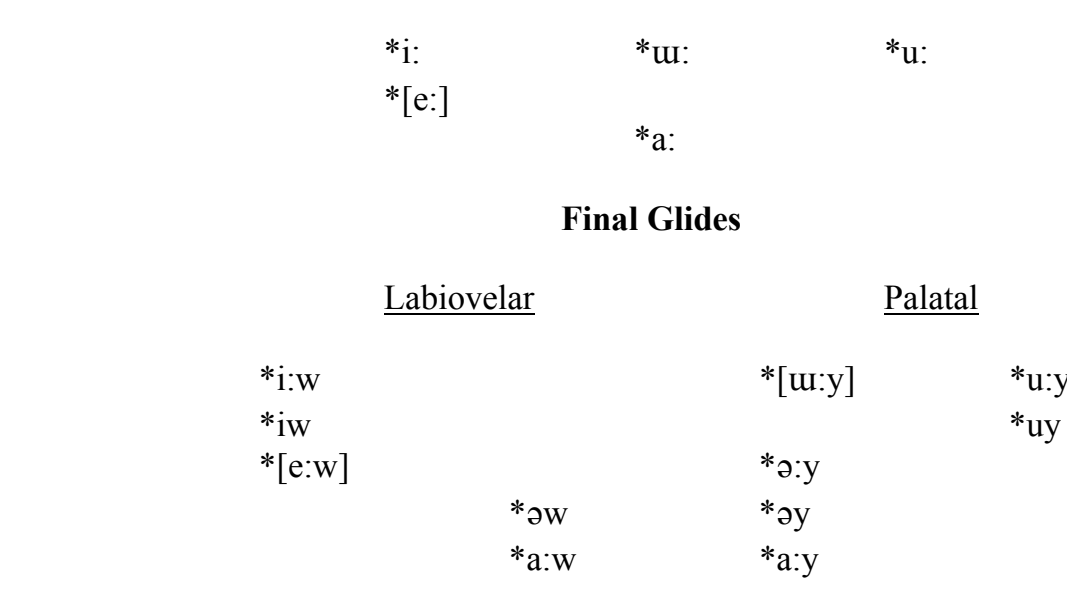

Labials

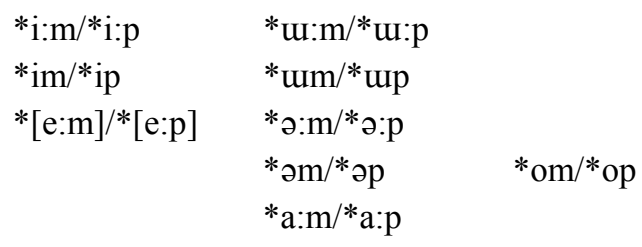

$\underline{\text { Palatals }}$

$* \partial \mathrm{j} /{ }^{*} \partial \mathrm{c}$

$* \mathrm{a}: \mathrm{j} / *_{\mathrm{a}}: \mathrm{c}$
Final Stops (Oral and Nasal)

\section{Open Rimes}

Alveolars

\begin{tabular}{|c|c|c|}
\hline \multirow{5}{*}{$\begin{array}{l}* \mathrm{i}: \mathrm{n} / *_{\mathrm{i}: \mathrm{t}} \\
*_{\mathrm{in} / * \mathrm{it}} \\
*[\mathrm{e}: \mathrm{n}] / *[\mathrm{e}: \mathrm{t}]\end{array}$} & *u:n/*[ur:t] & \multirow{2}{*}{$\begin{array}{l}* u: n / * u: t \\
* u n / * \text { ut }\end{array}$} \\
\hline & *un/*ut & \\
\hline & *ə:n/*ə:t & \\
\hline & *ən/*ət & \\
\hline & $* a: n / * a: t$ & \\
\hline
\end{tabular}

$\underline{\text { Velars }}$

\begin{tabular}{|c|c|c|c|}
\hline \multirow{5}{*}{$\begin{array}{l}*_{\mathrm{u}} \mathrm{p} / *^{*} \mathrm{u}: \mathrm{c} \\
*_{\mathrm{up}} / *_{\mathrm{uc}}\end{array}$} & $* \mathrm{i}: \mathrm{y} / * \mathrm{i}: \mathrm{k}$ & *u:y/*u:k & ${ }^{*} \mathrm{u}: \mathrm{y} /{ }^{*} \mathrm{u}: \mathrm{k}$ \\
\hline & $* \mathrm{in} / *[\mathrm{ik}]$ & $*[$ un $]$ & *up \\
\hline & ${ }^{*}[\mathrm{e}: \mathrm{n}] /\left[{ }^{*} \mathrm{e}: \mathrm{k}\right]$ & *ə:y/*ə:k & \\
\hline & & $* \partial \mathrm{y} / *[\partial \mathrm{k}]$ & *oy/*ok \\
\hline & & $* a: y / * a: k$ & \\
\hline
\end{tabular}

Final Laryngeals

$* \mathrm{~h}$

$* ?$

This system of rimes can be characterized generally as one with three levels of height and backness, a length distinction, glide codas at two places of articulation and stop (both nasal and oral) codas at four places of articulation. There are seven vowels, a number which is not highly marked (Maddieson 1984: 126). There are a number of gaps in the system, some of which are systematic, others of which are more idiosyncratic. These will be discussed in turn. 
The most obvious gap in the system is that of the low front and low back vowels. The most obvious gaps in the system are in the mid front and mid back vowels. The mid front vowels are exclusively long, few in number, and are generally very marginal within the system (see next chapter for more details). The mid back vowels on the other hand are robust and are etymologically of Kra-Dai origin; however, there are only short rimes in this category, creating a sharp asymmetry and a typologically rare situation since mid vowels tend to be long compared with their lower counterparts (ibid: 129). There are no high back rounded vowels preceding labial finals, due to a natural co-occurrence restriction (see section 3.4.4). Palatal finals occur only after high back vowels, short mid central vowels, and low vowels; it is unclear why there is an asymmetry in the mid central vowel category in this way. Short rimes with final velar stops exist, but are quite rare (particularly before oral velar stops). This reflects a strong preference for long nuclei before velar codas, and it is possible that some formerly short rimes lengthened in this environment. Finally, it is unclear why the rime *u:t is so rare, as there is nothing about the overall system which indicates that it should be so.

The reconstruction presented here has been compared with the reconstructions of Thurgood (1994), Ostpirat (1993), and Ostapirat (2004); the differences between the present reconstruction and these alternative reconstructions have been discussed in previous sections, and an argument presented for the former when it differs from the latter. The three reconstructions are provided below for reference, so that the similarities and differences between them may be easily compared (category labels are taken from the present reconstruction). As in the previous chapter, I do my best to arrange the system in question according to how I perceive the author's understanding of the system as a whole. Any mistakes in interpretation are my own. 
(169) Summary of Reconstructed Systems

(a) Open rimes

\begin{tabular}{|c|c|c|c|}
\hline Thurgood & Ostapirat (1993) & Ostapirat (2004) & $\underline{\mathrm{PHl}}$ \\
\hline$*_{\mathrm{ei}}$ & *əy & $*_{\mathrm{i}}:$ & $*_{\mathrm{i}:}$ \\
\hline *əu & *əu & $*_{\mathrm{i}}:$ & *u: \\
\hline$*_{\mathrm{ou}}$ & *əw & $*_{\mathrm{u}} \mathrm{u}$ & $* \mathrm{u}:$ \\
\hline *ay & $*_{\text {ey }}$ & *ay & $*_{\mathrm{i}: \mathrm{h} / \mathrm{R}}$ \\
\hline *au & $*_{\text {pu }}$ & $*$ al & *u:h/? \\
\hline --- & $*_{-j-a w}$ & $*_{\text {iw }}$ & *u:h \\
\hline *aw & ${ }^{*} \mathrm{ew}$ & *aw & $* \mathrm{u}: ?$ \\
\hline --- & --- & --- & $*_{\mathrm{e}}:$ \\
\hline$*_{\mathrm{a}}$ & $*_{\mathrm{a}}$ & $*_{\mathrm{a}}:$ & $*_{\mathrm{a}}:$ \\
\hline
\end{tabular}

(b) Closed rimes with high front nuclei

\begin{tabular}{|c|c|c|c|}
\hline Thurgood & Ostapirat (1993) & Ostapirat (2004) & PHl \\
\hline *i:w & $*_{\mathrm{iw}}$ & *i: $\mathrm{w}$ & $*_{\mathrm{i}: \mathrm{w}}$ \\
\hline$* \mathrm{i}: \mathrm{m}$ & $*_{\mathrm{im}}$ & $*_{\mathrm{i}: \mathrm{m}}$ & $*_{\mathrm{i}: \mathrm{m}}$ \\
\hline *i:n & $*_{\text {in }}$ & *i:n & $*_{\mathrm{i}: \mathrm{n}}$ \\
\hline *iay & $*_{\text {in }}$ & *i:y & $*_{i: y}$ \\
\hline$*_{\mathrm{i}: \mathrm{p}}$ & $*_{\text {ip }}$ & $*_{\mathrm{i}} \mathrm{p}$ & $*_{i: p}$ \\
\hline$*_{\mathrm{i}: \mathrm{t}}$ & $*_{\text {it }}$ & $*_{\mathrm{i}: \mathrm{t}}$ & $*_{\mathrm{i}: \mathrm{t}}$ \\
\hline$*_{\mathrm{i}: \mathrm{k}}$ & $*_{\mathrm{ik}}$ & $*_{\mathrm{i}: \mathrm{k}}$ & $* \mathrm{i}: \mathrm{k}$ \\
\hline *iw & *ew & *iw & *iw \\
\hline --- & $*_{\mathrm{em}}$ & $* \mathrm{im}$ & $*_{\mathrm{im}}$ \\
\hline$*_{\mathrm{in}}$ & *en & $*_{\text {in }}$ & $*$ in \\
\hline$*_{\text {in }}$ & *ey & *in & *in \\
\hline$*_{\mathrm{ip}}$ & $*_{\mathrm{ep}}$ & $*_{\text {ip }}$ & $*_{i p}$ \\
\hline$*_{\mathrm{ic}}$ & $*_{\text {et }}$ & $*_{\text {it }}$ & $*_{\mathrm{it}}$ \\
\hline *ik & *ek & $*_{\mathrm{ik}}$ & $* \mathrm{ik}$ \\
\hline
\end{tabular}


(c) Closed rimes with high back unrounded nuclei

Thurgood Ostapirat (1993) Ostapirat (2004) $\underline{\mathrm{PH1}}$

\begin{tabular}{|c|c|c|c|}
\hline--- & --- & $(* \mathrm{i}: \mathrm{y})$ & *uty \\
\hline *uam (b) & ${ }^{*}$ um & $* \dot{i}: \mathrm{m}$ & *u:m \\
\hline$*_{\mathrm{u}}: \mathrm{n}(\mathrm{b})$ & *uun & $*_{1}: n$ & *ui:n \\
\hline *u:p (b) & *un & *i: & *u:y \\
\hline *uap (b) & *up & $*_{\mathrm{i}: \mathrm{p}}$ & *u:p \\
\hline --- & --- & --- & *u:t \\
\hline *uak (b) & ${ }^{*}$ uk & $*_{\mathrm{i}: \mathrm{k}}$ & ${ }^{*} \mathrm{u}: \mathrm{k}$ \\
\hline--- & $* \gamma \mathrm{m}$ & $*_{\mathrm{im}}$ & \\
\hline --- & $* \gamma n$ & $*_{\text {in }}$ & *un \\
\hline--- & --- & --- & ${ }^{*}$ un \\
\hline --- & $* \gamma p$ & $*_{\text {ip }}$ & *up \\
\hline --- & $* \gamma t$ & $*_{\mathrm{it}}$ & *ut \\
\hline
\end{tabular}

(d) Closed rimes with high back rounded nuclei

Thurgood Ostapirat (1993) Ostapirat (2004) $\mathrm{PHl}$

\begin{tabular}{|c|c|c|}
\hline *u:y & *uy & *u:y \\
\hline *u:n (a) & *un & *u:n \\
\hline--- & --- & --- \\
\hline *u:y (a) & *up & *u:y \\
\hline${ }^{*} \mathrm{u}: \mathrm{t}$ & *ut & *u:t \\
\hline *u:c & --- & *u:c \\
\hline *uak (a) & *uk & *u:k \\
\hline *uy & *oy & *uy \\
\hline *un & *on & *un \\
\hline --- & --- & --- \\
\hline *oy & --- & *un \\
\hline *ut & --- & *ut \\
\hline *uc & $*_{\mathrm{ot}}$ & $* \mathrm{uc}$ \\
\hline
\end{tabular}

(e) Closed rimes with front mid nuclei

\begin{tabular}{|c|c|c|c|}
\hline --- & --- & --- & *e:w \\
\hline --- & --- & --- & *e:m \\
\hline --- & --- & --- & *e:p \\
\hline --- & --- & --- & $*_{\mathrm{e}: \mathrm{n}}$ \\
\hline --- & --- & --- & *e:t \\
\hline : & $*_{\text {-j-a }}$ & --- & (*e:y) \\
\hline (*a:k) & $*_{\text {-j-ak }}$ & --- & (*e:k) \\
\hline
\end{tabular}


(f) Closed rimes with central mid nuclei

\begin{tabular}{|c|c|c|c|}
\hline Thurgood & Ostapirat I & Ostapirat II & $\underline{\mathrm{PHl}}$ \\
\hline$*_{0}: y$ & *-w-ay & *ว:y & *ə:y \\
\hline *uam (a) & *-w-em & *əm & *ə:m \\
\hline *o:n & $*$-w-en & *ən & *ə:n \\
\hline *uay & *-w-ey & *əך & *ว:ท \\
\hline *uap (a) & $*$-w-ep & *əp & $*_{\partial: p}$ \\
\hline$*_{\mathrm{o}}: \mathrm{t}$ & $*-\mathrm{W}-\mathrm{et}$ & *ət & $*_{\partial: t}$ \\
\hline$*_{\mathrm{o}}: \mathrm{k}$ & *-w-ek & *ək & $*^{*} \partial: \mathrm{k}$ \\
\hline *ay & $*$ py & *ay & *əy \\
\hline *aw & ${ }^{*} \mathrm{ew}$ & $*$ aw & *ow \\
\hline$*_{\mathrm{o}}$ & *-W-ew & *ow & *əwh/? \\
\hline *am & $*_{\mathrm{pm}}$ & *am & *əm \\
\hline *an & --- & $*$ an & *ən \\
\hline *an & $*_{\mathrm{en}}$ & $*$ aj & *əј \\
\hline *ay & $*_{p y}$ & $*$ an & *əy \\
\hline *ap & $* p p$ & *ap & *əp \\
\hline$*_{\text {at }}$ & --- & $*$ at & *ot \\
\hline$*_{\mathrm{ac}}$ & $*_{\mathrm{pt}}$ & $*_{\mathrm{ac}}$ & $* \partial c$ \\
\hline --- & --- & --- & *ok \\
\hline
\end{tabular}

(g) Closed rimes with back mid nuclei

\begin{tabular}{|c|c|c|}
\hline Thurgood & Ostapirat I & Ostapirat II \\
\hline *om & *əm & $*_{\mathrm{um}}$ \\
\hline *on & *əy & *un \\
\hline *op & --- & --- \\
\hline$*_{\mathrm{ok}}$ & * $\mathrm{ok}$ & $*_{u k}$ \\
\hline
\end{tabular}

(h) Closed rimes with low nuclei

\begin{tabular}{|c|c|c|c|}
\hline Thurgood & Ostapirat I & Ostapirat II & $\underline{\mathrm{PHl}}$ \\
\hline$* a: y$ & *ay & $* a: y$ & $* a: y$ \\
\hline *a:w & *aw & $* a: w$ & *a:w \\
\hline$* \mathrm{a}: \mathrm{m}$ & *am & $* a: m$ & $* \mathrm{a}: \mathrm{m}$ \\
\hline *a:n & $*$ an & $* a: n$ & *a:n \\
\hline --- & --- & --- & *a:p \\
\hline$(* \mathrm{i}: \mathrm{y})$ & --- & *a:y & *a:y \\
\hline$* a: p$ & *ap & *a:p & $*_{\mathrm{a}} \mathrm{p}$ \\
\hline *uat & $*$ at & $*_{\mathrm{a}: \mathrm{t}}$ & $* a: t$ \\
\hline *uac & $*_{\text {-w-at }}$ & $* \mathrm{a}: \mathrm{c}$ & *a:c \\
\hline$* \mathrm{a}: \mathrm{k}$ & $*$ ak & $* \mathrm{a}: \mathrm{k}$ & $* \mathrm{a}: \mathrm{k}$ \\
\hline
\end{tabular}




\subsubsection{Thurgood's Reconstruction}

Thurgood's reconstructed rime inventory is shown below:

\section{Open Rimes}

$*_{\mathrm{a}} \quad *_{\mathrm{o}}$

\section{Final Glides}

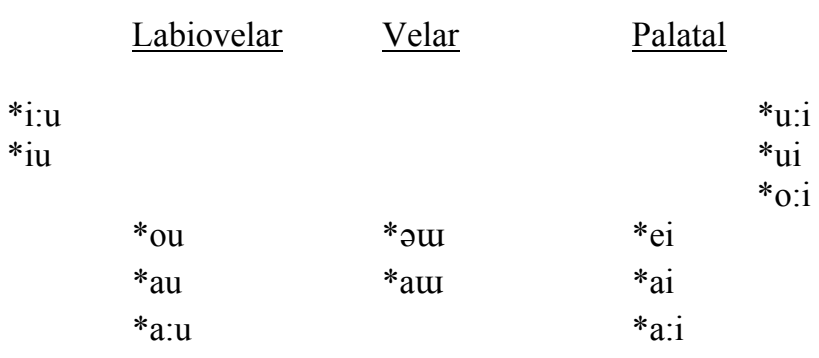

Final Stops (Oral and Nasal)

$\underline{\text { Labials }}$

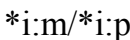

*ip

$$
\begin{aligned}
& \text { *uam (a/b)/*uap (a/b) } \\
& \text { *om } \\
& \begin{array}{l}
\text { *a:m/*a:p } \\
\text { *am/*ap }
\end{array}
\end{aligned}
$$

$\underline{\text { Palatals }}$

$* \mathrm{in} / *_{\mathrm{ic}}$

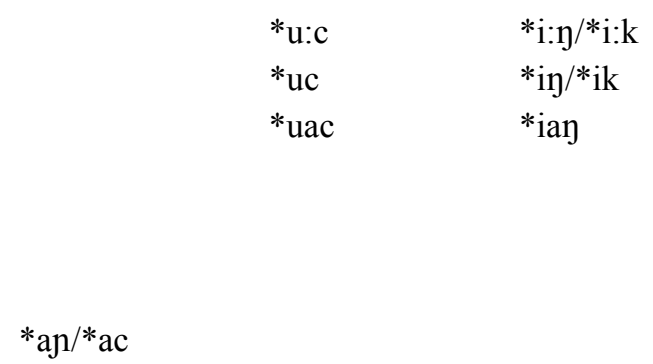

$\underline{\text { Alveolars }}$

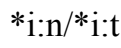

$\underline{\text { Velars }}$

$$
\begin{aligned}
& \text { *u:y (a/b) } \\
& \text { *uay } \\
& \text { *uak (a/b) } \\
& \text { *o:k }
\end{aligned}
$$$$
* \mathrm{a}: \mathrm{k}
$$$$
* \text { an }
$$

Thurgood's reconstruction of an original vowel length distinction is in alignment with the present reconstruction. His open rime category is typologically marked due to the lack of high vowels, the reflexes of which he reconstructs as diphthongs. I consider the greatest weakness of this system to be the 
duplication of rimes in the categories he labels (a) and (b), without adequate explanation about why the second series should be considered to reflect loanwords (as noted above, this violates the principles of Commonality and Symmetry). There is also an inconsistency in the reconstruction of pure long high vowels versus diphthongs in final nasal versus final oral stop categories, when the evidence seems to militate for symmetry between the two. He does not reconstruct precursors to the Hlai tone categories, which leaves the alternations in Greater Hlai, Qi, and Cunhua (and the symmetry in the rimes which they disguise) unaccounted for.

\subsubsection{Ostapirat's Reconstruction}

Ostapirat's (1993) system is shown next: 


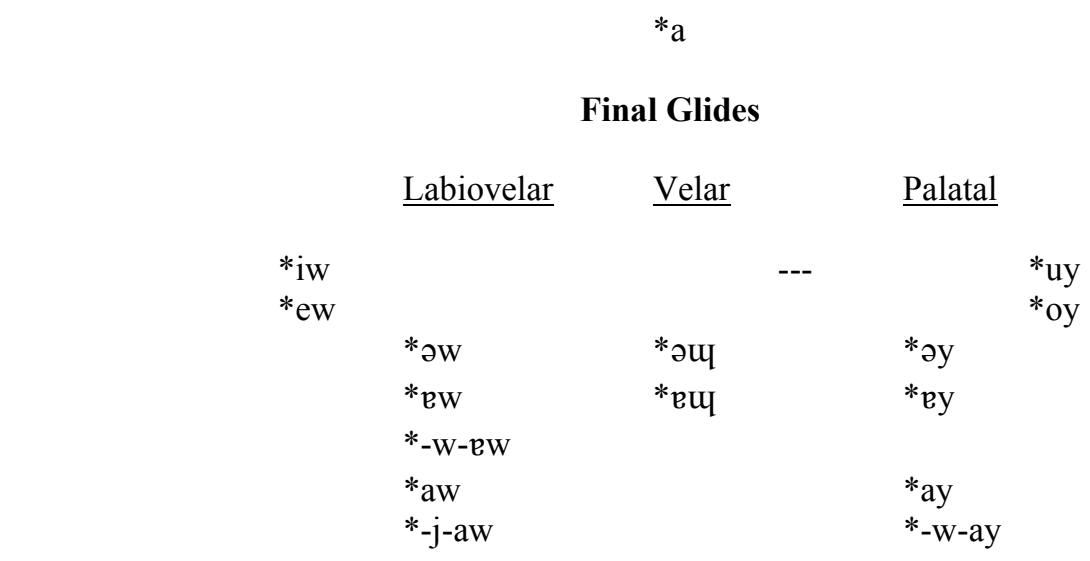

Final Stops (Oral and Nasal)

\section{$\underline{\text { Labials }}$}

$*_{\mathrm{im}} / *_{\mathrm{ip}}$
$*_{\mathrm{em}} / *_{\mathrm{ep}}$

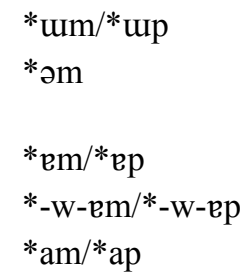

$\underline{\text { Alveolars }}$

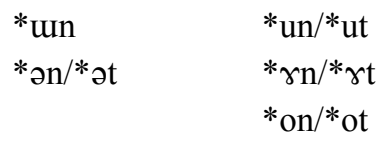

$*_{\mathrm{en}} /{ }^{*} \mathrm{et}$

*-w-en/*-w-et

$*$ an/*at

*-w-at

$\underline{\text { Velars }}$

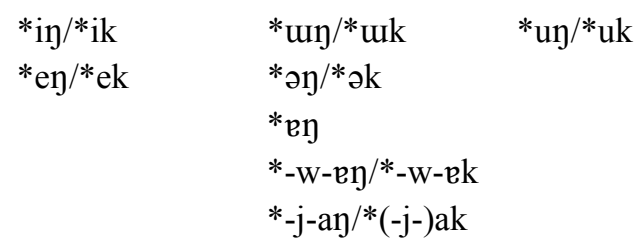

Ostapirat's (1993) system is generally symmetrical in several ways. One way in which his inventory is very strange is that it contains only one open rime $(* a)$. Although I disagree with the choice in reconstruction (and Ostapirat later revises this, see below), the decision to create a difference between high and mid vowels (as opposed to a vowel length distinction) based on the NCHl data is highly systematic, and it is interesting to see how this alternative works out. I consider the one significant shortcoming of the system to be the reliance on medial glides (-j- and -w-) to condition vowel quality and length distinctions, when more traditional explanations for vowel development are available (in my opinion this involves 
serious violations of Directionality). Ostapirat also fails to reconstruct final palatal stops, as well as any precursors to the Hlai tone categories, which leaves the alternations in Greater Hlai, Qi, and Cunhua unaccounted for.

In a major reworking of his (1993) hypothesis, Ostapirat's (2004) reconstruction is given below:

\begin{tabular}{|c|c|c|c|}
\hline \multicolumn{4}{|c|}{ Open rimes } \\
\hline & \multicolumn{2}{|c|}{$*_{\mathrm{i}}:$} & $* \mathrm{u}:$ \\
\hline & \multicolumn{2}{|c|}{$* \mathrm{a}:$} & \\
\hline \multicolumn{4}{|c|}{ Final Glides } \\
\hline \multicolumn{2}{|c|}{ Labiovelar } & & $\underline{\text { Palatal }}$ \\
\hline$*_{\mathrm{i}: \mathrm{w}}$ & & $(* \mathrm{i}: \mathrm{y})$ & \\
\hline \multirow[t]{5}{*}{$*_{\mathrm{iw}}$} & $*_{\mathrm{iw}}$ & & \\
\hline & & *ə:y & \\
\hline & *əw & & \\
\hline & *a:w & *a:y & \\
\hline & $*$ aw & *ay & \\
\hline
\end{tabular}

\section{Final Stops (Oral and Nasal)}

\section{$\underline{\text { Labials }}$}

$*$ i:m/*i:p

$*$ im/*ip

$---/$

$* \mathrm{i}: \mathrm{n} / *_{\mathrm{i}: \mathrm{t}}$
$* \mathrm{in} / *_{\mathrm{it}}$
$---/---$

$\underline{\text { Palatals }}$

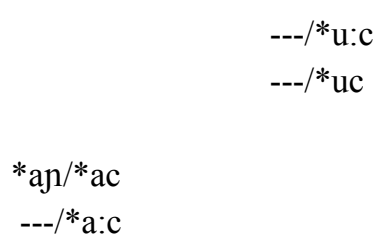

$\underline{\text { Alveolars }}$

*i:n/ --- $\quad$ *u:n/*u:t

$*_{\text {in }} / *_{\text {it }}$

$* \partial n / * \partial t$

$* a n / * a 1 / * a t$

$* \mathrm{a}: \mathrm{n} / *_{\mathrm{a}}: \mathrm{t}$

$\underline{\text { Velars }}$

\begin{tabular}{|c|c|c|}
\hline \multirow{2}{*}{$\begin{array}{l}* \mathrm{i}: \mathrm{y} / *_{\mathrm{i}: \mathrm{k}} \\
*_{\mathrm{i} y} / *_{\mathrm{ik}}\end{array}$} & $* \dot{\mathrm{i}}: \mathrm{y} / *_{\mathrm{i}}: \mathrm{k}$ & ${ }^{* u} \mathrm{u}: \mathrm{y} /{ }^{*} \mathrm{u}: \mathrm{k}$ \\
\hline & ---/ --- & *uy/*uk \\
\hline & *əy/*ək & \\
\hline & *ay/ --- & \\
\hline & $* \mathrm{a}: \mathrm{y} / *^{*} \mathrm{a}: \mathrm{k}$ & \\
\hline
\end{tabular}

This reconstruction is parallel in many ways with the one suggested in this dissertation. There is a full high vowel inventory (lacking the marginal *e:), a length distinction in the high vowels, and a general 
symmetry in the rime system as a whole, once co-occurrence constraints are taken into account. Weaknesses of this system include the lack of inclusion of some of the less well-represented rime categories which nevertheless show regular and expected patterns throughout the various Hlai languages; the reconstruction of a final lateral which is not justified by the overall evidence, despite the data in Wang \& Qian (1951), and the reconstruction of only short mid central vowels, which does not explain the long reflexes in the majority of the Hlai daughter languages (a violation of both Directionality and Commonality). Finally, there is no examination of the precursors to the Hlai tone categories, which forces the reconstruction of $*_{\mathrm{iw}}$ when there is actually no need for it, since this is in complimentary distribution with what is otherwise reconstructed as *aw.

In summary, this chapter has provided a comprehensive reconstruction of the PHl rime inventory. Besides cataloguing the major types of sound change which have occurred in the history of the Hlai languages, other major contributions include the evidence for and reconstruction of the segmental precursors of the $\mathrm{PHl}$ tone categories, outlining their subsequent development in the subgroups and daughter languages. A generally balanced system with typologically normal gaps has been reconstructed, which includes five vowels, two final glides, four places of articulation for final stops, and a vowel length distinction. Putting aside the set of changes which occurred quite early and distinguish Bouhin from Greater Hlai, it is almost invariably the $\mathrm{NCHl}$ languages which have undergone the most dramatic changes in the rime categories, while the other branches remain more conservative.

It has also been shown that the same four principles which apply to the reconstruction of initials apply equally well to the reconstruction of rimes. Directionality is important in constraining such processes as lengthening and diphthongization. Commonality is important in constraining the reconstruction to reflect the proto-language, and limiting the amount of internal reconstruction which is not appropriate at this level. Economy is important in checking the features of vowel nuclei, using the reflexes of the daughter languages to triangulate the appropriate point in the vowel space to reconstruct. Finally, Symmetry is particularly important in the case of the rimes, as the overall system is readily constrained by this principle, and its few asymmetrical aspects highlighted appropriately. 
The focus of the next chapter will be Pre-Hlai, the precursor to Proto-Hlai. This stage of Hlai is reconstructible using a combination of internal evidence as well as external evidence from other branches of Kra-Dai (in this case Be and Southwest Tai). Unlike chapters two and three, chapter four will treat both the initial system and the rime system, with the goal of reconstructing the earliest possible stage of Pre-Hlai, and then showing the changes which occurred between that stage and Proto-Hlai. 


\section{CHAPTER FOUR: PRE-HLAI}

The goal of this chapter is to develop a theory of the Pre-Hlai initial and rime inventories, and to trace the evolution of the Hlai initials and rimes from Pre-Hlai to Proto-Hlai.

To this end, cognates between Proto-Hlai, Proto-Be (PB), and Proto-Southwest Tai (PSWT) are compared in this section in order to develop a hypothesis of the original Pre-Hlai inventory of initials. Although reconstructions of other branches of Kra-Dai exist (most notably Proto-Kam-Sui (Thurgood 1988), Proto-Kra (Ostapirat 2000), and Proto-Lakkja (Theraphan 1992)), I do not refer to them here so as to keep interphyletic comparisons manageable, limiting the comparanda to other Southern Kra-Dai families.

It would be optimal to compare PHl with Proto-Tai itself. However, only one of the three primary branches of Tai has been reconstructed in any detail (Southwest Tai, Jonsson (1991); Pittayaporn (ms)); it is only when comparative reconstructions of Central Tai and Northern Tai are available that Proto-Tai itself

will be wholly reconstructible. For this reason, I have limited the Tai comparanda to Southwest Tai, using Pittayaporn's reconstruction as a primary source, since in my estimation it has surpassed earlier reconstructions of Southwest Tai in both scope and precision.

Proto-Be is a comparatively small language family also spoken on Hainan, and is reconstructed based on materials from five dialects from among a total of three daughter languages. A full discussion of the reconstruction of Proto-Be will be presented in another venue.

The tree below, focusing specifically on Southern Kra-Dai, shows which branches are being used in the present comparison: 
(1) Figure 4: Southern Kra-Dai Phylogenetic Tree

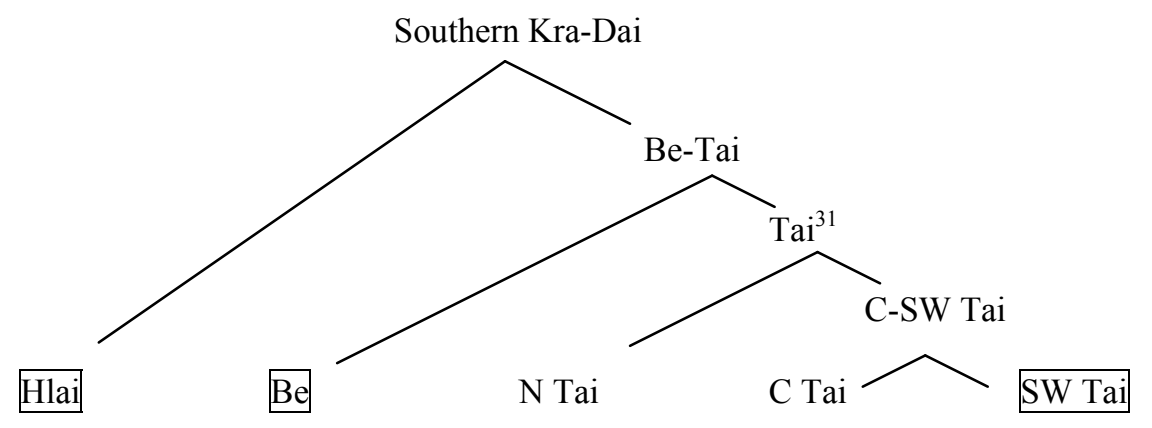

Section 4.1 gives a brief overview of the prosodic word shape inherited from Proto-Southern Kra-

Dai (PSKD), and presents a comparison between the PHl initials and the Proto-Be and Proto-Southwest Tai initials. Section 4.2 repeats this comparison for the rimes. Section 4.3 reviews and motivates the important changes which are hypothesized between Proto-Southern Kra-Dai and Proto-Hlai ${ }^{32}$.

\subsection{The Pre-Hlai Initials}

As alluded to in chapter two, the PHl phonological word consisted of two types: either (a) monosyllabic or (b) disyllabic. Disyllabic words were certainly iambic, as this stress type is usually a necessary precondition in Southeast Asia for an eventual transition to a strictly monosyllabic inventory:

(a)

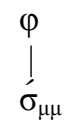

(b)

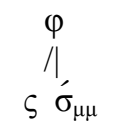

This first syllable in a disyllabic form ( $2 \mathrm{~b})$ is sometimes known as a semisyllable, presyllable, or minor syllable (in contrast with the second main syllable; the term presyllable will be adopted here), and the disyllabic foot was what is sometimes called sesquisyllabic ('syllable-and-a-half'), a term coined by James Matisoff in Matisoff (1973) (see also Svantesson (1983), Shaw (1993), Cho \& King (1996)).

${ }^{31}$ This is the original classification of Tai given in Li (1977). Pittayaporn (p.c. 9/9/07) suggests that SWT may actually be a subgroup of C Tai.

${ }^{32}$ As in chapters two and three, the four principles of language change and reconstruction given in chapter one are used in this chapter as well. 
The first hypothesis adopted here is that moraic weight became assigned exclusively to the main (rightmost) syllable, and that the first syllable in a disyllabic form lost the ability to host a mora. The presyllable could carry segmental features (the inventory of which would become gradually restricted over time), but was not associated with moraic content ${ }^{33}$. I hypothesize that this loss of and subsequent lack of a mora was correlated with the steady erosion of presyllables until their eventual extinction in the Hlai daughter languages. This kind of iambic system stands in contrast to other iambic systems which have remained more stable (such as those of the Semitic languages) and not been reduced to monosyllables. The progression from full presyllable to moraless presyllable to monosyllable is shown below ${ }^{34}$ :

(a)

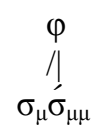

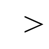

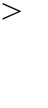

Example: Cirá:w $\quad>\quad$ C-rjá:w rjá:w

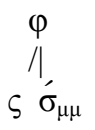

Example: Cirá:w $\quad>\quad$ C-rjá:w rjá:w

$>$

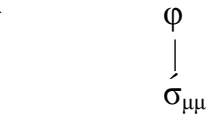

The existence of a length distinction in rimes suggests that codas were optionally moraic, bearing a mora in short rimes but not in long rimes ${ }^{35}$. PHl examples of each type are given below for monosyllabic words (3a) and sesquisyllabic words (3b), with the bimoraic domain of each word in brackets (remembering that the initial ${ }^{*} \mathrm{C}$ in the forms in $(3 \mathrm{~b})$ represents an initial consonant with unspecified features):

(a)

$\begin{array}{ll}\operatorname{dog} & * \mathrm{hm}[\mathrm{a}:] \\ \text { break } & * \mathrm{p}^{\mathrm{h}}[ə:] \mathrm{n} ? \\ \text { spine } & * \mathrm{tc}^{\mathrm{h}}[\mathrm{un}] ?\end{array}$

(b)

$$
\begin{aligned}
& \text { waist } \\
& \text { rough } \\
& \text { sore }
\end{aligned}
$$

${ }^{*}$ Cif[a:] $\mathrm{h}$

*Cur[a:]w

${ }^{*} \mathrm{Cu}$ ?[əw]

The second hypothesis adopted here is that the lowest unit of prosodic timing was the foot, a fact which would affect the organization of the segmental material associated to it. While this model predicts that the rime in both monosyllables and sesquisyllables should be identical, it also predicts three different

\footnotetext{
${ }^{33}$ I adopt Cho \& King's (2003) convention of showing a moraless sesquisyllable (or semisyllable) with an $\varsigma$. ${ }^{34}$ A form such as C-rjá:w in (3) may have been produced as [Cə̆rjá:w], with the intervening schwa existing solely as an artifact of phonetic implementation, but not represented in underlying representations.

${ }^{35}$ The laryngeal components of rimes in categories B and C do not appear to affect weight in any way, and are not considered to be potential mora-bearing units.
} 
types of initial consonants depending on their position within the foot. Under this model, the initial in a monosyllabic form lies at the edge of both the foot and the main syllable. On the other hand, there is an asymmetry in sesquisyllabic forms between the foot-initial consonant, which marks the edge of a prosodic timing category, and the initial consonant of the main syllable which does not (and now plays the ambiguous role of a syllable onset but a prosodicaly medial segment): The two types of feet in Pre-Hlai

$$
\begin{aligned}
& \text { Monosyllable } \\
& \text { Initial in main syllable domain } \\
& |--\downarrow---| \\
& {[(\mathrm{CV}:)]} \\
& |--\uparrow---|
\end{aligned}
$$

Initial in foot domain (b) Sesquisyllable

Initial in foot domain Medial in foot domain

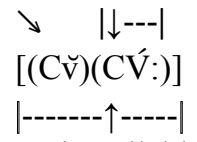

Initial in main syllable domain

It is shown below that each of these three kinds of initials has evolved along a different trajectory, each set eventually becoming disjunct with the other two.

The initials below are organized according to their Proto-Hlai categories. The PHl reconstructions are presented first, followed by PB reconstructions, PSWT reconstructions, and finally a tentative Proto Southern Kra-Dai (PSKD) reconstruction. Note that the PSKD reconstructions which follow are necessarily incomplete, due to their lack of input from Northern and Central Tai.

\subsubsection{Initial Stops}

When the aspirated initial stops reconstructed for PHl are compared with their PB and PSWT cognates, there are three patterns which emerge. The first is a correspondence between the PHl aspirated stops and PB/PSWT plain stops (6a), the second is with PB/PSWT voiced stops (6b), and the final only occurs at the velar place of articulation (6c), with PB/PSWT * $x$ on the one hand, and with $\mathrm{PB} * \mathrm{~g}$ and PSWT * $\mathrm{y}$ on the other: 
(6)

$\underline{\text { Proto-Hlai }} \underline{\text { Proto-Be }} \underline{\text { PSWT }} \underline{\text { PSKD }}$

(a)

$$
\begin{aligned}
& * \mathrm{p}^{\mathrm{h}} \\
& * \mathrm{t}^{\mathrm{h}} \\
& * \mathrm{k}^{\mathrm{h}} \\
& * \mathrm{k}^{\mathrm{h}}
\end{aligned}
$$

$*_{\mathrm{p}}$
$*_{\mathrm{t}}$
$*_{\mathrm{k}}$
$*_{\mathrm{k}}$

$* \mathrm{p}$
$* \mathrm{t}$
$* \mathrm{k}$
$* \mathrm{q}^{(\mathrm{h})}$

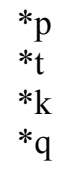

(b)

$$
\begin{aligned}
& *^{\mathrm{h}} \\
& * \mathrm{t}^{\mathrm{h}} \\
& * \mathrm{k}^{\mathrm{h}}
\end{aligned}
$$

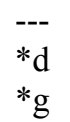

$* \mathrm{~b}$

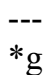

$* b$

$* d$

$* \mathrm{~g}$

(c)

$$
{ }^{*} \mathrm{k}^{\mathrm{h}}
$$

$*_{\mathrm{X}}$

$*^{\mathrm{x}}$

$* \mathrm{y}$

$*_{\mathrm{x}}$
$*_{\mathrm{y}}$

The first series (6a) is reconstructed here in PSKD as an original set of plain stops (which includes the uvular place of articulation), the second series as original voiced stops, and the third as a pair of velar fricatives. Under this hypothesis, PSWT is quite conservative, PB is nearly as conservative, but the PHI inventory is the end result of a series of mergers which occurred in Pre-Hlai. The merger of the uvular series with the velar series was the first (a merger shared with PB); the second is that the voiced obstruents merged with the voiceless obstruents, initiating a constraint on initial voiced obstruents in Hlai which has continued until the present day; the third was the merger of the velar fricatives with the velar stops. Finally, the entire series of PHl obstruents underwent what Ostapirat (2004) recognizes as redundant aspiration (what will be referred to below as initial aspiration), resulting from a general rule which affected all eligible initials in Pre-Hlai (see section 4.3). This series of changes is shown below:

$\underline{\text { PSKD }} \quad \underline{\text { Pre-Hlai }} \quad \underline{\text { Proto-Hlai }}$

(a)

$\begin{array}{lllll}* \mathrm{p} & > & * \mathrm{p} & > & * \mathrm{p}^{\mathrm{h}} \\ * \mathrm{t} & > & * \mathrm{t} & > & * \mathrm{t}^{\mathrm{h}} \\ * \mathrm{k} & > & * \mathrm{k} & > & * \mathrm{k}^{\mathrm{h}} \\ * \mathrm{q} & > & * \mathrm{k} & > & * \mathrm{k}^{\mathrm{h}}\end{array}$

(b)

$\begin{array}{lllll}* \mathrm{~b} & > & * \mathrm{p} & > & * \mathrm{p}^{\mathrm{h}} \\ * \mathrm{~d} & > & * \mathrm{t} & > & * \mathrm{t}^{\mathrm{h}} \\ * \mathrm{~g} & > & * \mathrm{k} & > & * \mathrm{k}^{\mathrm{h}}\end{array}$

(c)

$\begin{array}{lllll}* \mathrm{x} & > & * \mathrm{x} & > & * \mathrm{k}^{\mathrm{h}} \\ *_{\mathrm{y}} & > & * \mathrm{x} & > & * \mathrm{k}^{\mathrm{h}}\end{array}$


Examples of forms with original voiceless stops are given below. Most PSWT forms are those of Pittayaporn, although there are occasional forms taken from Jonsson (1991), and these will be indicated with a (J). In some cases where there is no PSWT form available, a Proto-Tai form exists based on Central and/or Northern Tai (Li (1977)), which is marked (Li):

\begin{tabular}{|c|c|c|c|c|c|}
\hline (8) & $\underline{\text { Gloss }}$ & $\underline{\text { Proto-Hlai }}$ & $\underline{\text { Proto-Be }}$ & $\underline{\text { PSWT }}$ & $\underline{\text { PSKD }}$ \\
\hline \multirow[t]{3}{*}{ (a) } & wing & ${ }^{*} p^{h} \mathrm{i}: \mathrm{k}$ & *pik & *pi:k & *pi:k \\
\hline & put down, let go & *p $p^{h} u: y ?$ & ${ }^{*} \operatorname{pian}^{\mathrm{X}}$ & --- & *puan ${ }^{\mathrm{C}}$ \\
\hline & male ancestor $\sim$ pat. gf & $* p^{h} u: ?$ & --- & ${ }^{*} \mathrm{pu}:^{\mathrm{B}}$ & $* p:^{B / C}$ \\
\hline \multirow[t]{3}{*}{ (b) } & shallow & $*^{\mathrm{h}} \mathrm{ur}: \mathrm{n}$ ? & *tur: $\mathrm{n}^{\mathrm{X}}$ & *tur:n ${ }^{\mathrm{C}}$ & $*_{\text {tur: }}{ }^{\mathrm{C}}$ \\
\hline & tree clsfr $\sim$ tree & $* t^{h} u: n ?$ & $*$ tuon $^{X}$ & $* \operatorname{ton}^{\mathrm{C}}$ & *to[: $] \mathrm{n}^{\mathrm{C}}$ \\
\hline & fall & $* \mathrm{t}^{\mathrm{h}} \mathrm{ok}$ & $*$ tok & $*$ tok & $*$ tok \\
\hline \multirow[t]{3}{*}{ (c) } & chicken & $* k^{\mathrm{h}} \partial \mathrm{y}$ & *kay & $*_{\text {kay }}{ }^{\mathrm{B}}$ & *kəy ${ }^{(\mathrm{B})}$ \\
\hline & eat & $* \mathrm{k}^{\mathrm{h}}$ ən & *kən & *kin & ${ }^{*} \mathrm{k}[\mathrm{j}] \partial n$ \\
\hline & old & *k $\mathrm{k}^{\mathrm{h}}$ owh & *ka: ${ }^{\mathrm{X}}$ & $*$ kaw $^{\mathrm{B}}$ & *kəw ${ }^{\mathrm{B}}$ \\
\hline \multirow[t]{2}{*}{ (d) } & ginger & *k $\mathrm{k}^{\mathrm{h}} \mathrm{w}: \eta$ & *kjay & *qi:y & *quay \\
\hline & arm & ${ }^{*} \mathrm{k}^{\mathrm{h}} \mathrm{i}: \mathrm{n}$ & *ke:n 'sleeve' & $* q^{h} \varepsilon: n$ & *qe:n \\
\hline
\end{tabular}

The voiced stops are less common than the voiceless stops:

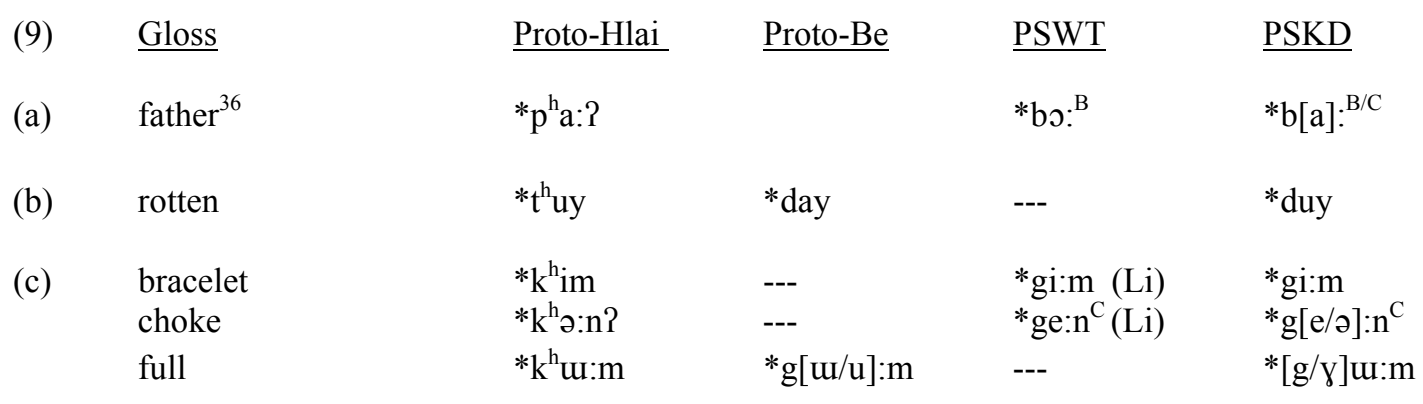

Examples of the reconstructed PSKD velar fricatives are given below:

${ }^{36}$ This comparison should be considered tentative, given the fact that this word is a 'nursery word', and the fact that $\mathrm{OC} *$ ba? could also potentially be the source of the word in either Hlai or Tai. 


\section{Gloss}

(a)

$$
\begin{aligned}
& \text { white } \\
& \text { dry } \\
& \text { light }
\end{aligned}
$$

(b)

dirty sweat
itch
fishy

Proto-Hlai

$\underline{\text { Proto-Be }}$

$\underline{\text { PSWT }}$

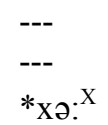

(*gə:y 'dust')

* guəm

--- *xa:w

${ }^{*}$ хач ${ }^{\mathrm{B}}$

---

*ylay
*yom
*ya:w

PSKD

*xa:w

*xu: ${ }^{\mathrm{B}}$

*xur: ${ }^{\mathrm{C}}$

*[d-] $\mathrm{\gamma i}^{37}$

*yom

* ya:w

The correspondences between initials in the following examples are more complex than those above; a brief attempt is made here to show how these correspondences may have arisen. The reader should remember that the analysis here is appropriate only for the amount of data presented, and the

\begin{tabular}{|c|c|c|c|c|}
\hline to spit & ${ }^{*} \mathrm{p}^{\mathrm{h}}[\mathrm{w}] \mathrm{i}: \mathrm{h}$ & --- & $* \mathrm{p}^{\mathrm{h}} \mathrm{i}^{\mathrm{B}}(\mathrm{Li})$ & ${ }^{*} \mathrm{p}^{\mathrm{h}}[\mathrm{w}] \mathrm{i} \mathrm{i}^{\mathrm{B}}$ \\
\hline incubate & ${ }^{*} \mathrm{p}^{\mathrm{h}} \mathrm{\partial :k}$ & *wak & $*_{\text {vak }}$ & $*[\mathrm{C}-] \mathrm{b} ə \mathrm{k}$ \\
\hline cheat & $* p^{h} \partial: \eta$ & --- & *bra:y & $* \operatorname{br}[\partial / a]: \eta$ \\
\hline land leech & $*^{\mathrm{h}} \mathrm{a}: \mathrm{k}$ & $*_{\text {ta: } \mathrm{k}}$ & $*$ da:k & *N-ta:k \\
\hline louse (body) & $* \mathrm{t}^{\mathrm{h}}$ ən & *də:n & $*_{\text {mlen }}$ & $*(\mathrm{~m}-) \mathrm{d}$ n \\
\hline rib & ${ }^{*} \mathrm{k}^{\mathrm{h}} \mathrm{a}: \mathrm{y}$ ? & --- & ${ }^{*} \mathrm{k}^{\mathrm{h}} \mathrm{ra}: \mathrm{y}^{\mathrm{C}}$ & ${ }^{*} \mathrm{k}^{(\mathrm{h})} \mathrm{ra}: \mathrm{y}^{\mathrm{C}}$ \\
\hline spider & $* \mathrm{k}^{\mathrm{h}} \partial \mathrm{w}$ & --- & *klwaw & *kuləw \\
\hline
\end{tabular}
eventual reconstruction of Proto-Tai will either confirm the present hypotheses or force their revision.

(11) Gloss $\underline{\text { Proto-Hlai }} \underline{\text { Proto-Be }} \underline{\text { PSWT }} \underline{\text { PSKD }}$

(11a) is a rare example of a correspondence set involving an aspirated PT initial. It is very possible that the source of the aspiration lies in the expressive, onomatopoeic nature of this word.

In (11b), the PHl form could descend from either an initial *p or *b, but the PB and PSWT forms reflect an intervocalic *-b-. The best explanation for this set, in my view, is that they represent an

\footnotetext{
${ }^{37}$ I tentatively reconstruct this as a sesquisyllabic word with initial $*$ d, which lenited and metathesized in
} Tai. 
ambiguously sesquisyllabic proto-form: either the Pre-Hlai form dropped an initial presyllable, or the PB and PSWT forms gained one after the breakup of PSKD.

The forms for cheat in (11c) may be cognate, and if so, provide evidence of an original *b in PreHlai; the difference in the rime vowels casts some doubt on this comparison, although it is possible that this difference was conditioned by the original rhotic preserved in the PSWT form

The evidence for the status of voicing in the initial in (11d) is conflicting, with a voiceless initial

in PB and a voiced initial in PSWT. I propose that one solution to this paradox is the original existence of an initial nasal, which was lost early in most branches, but lasted long enough in PSWT to lead to homorganic voicing of the initial.

The best account for body louse in (11e) may be that it was an original root beginning with $* \mathrm{~d}$ which underwent regular development in PHl and PB, but which was preceded in PSWT by an *m- prefix (which may have also colored the main vowel).

The word rib in (11f) indicates an original cluster in which the liquid was lost in Hlai; the aspiration of the initial is uncertain, since aspiration of plain initials in Hlai occurred automatically, and it will be shown below that *r-clusters in PSWT conditioned aspiration of the preceding initial.

\subsubsection{Affricates}

There are three sets of correspondences between PHl and PB/PSWT, falling into the same three categories as the stops: original voiceless obstruents, original voiced obstruents, and an original fricative:

$$
\underline{\text { Proto-Hlai }} \underline{\text { Proto-Be }} \underline{\text { PSWT }} \underline{\text { PSKD }}
$$

$$
\begin{aligned}
& * \mathrm{t} \int^{\mathrm{h}}(\mathrm{w}) \\
& * \mathrm{t}^{\mathrm{h}}
\end{aligned}
$$
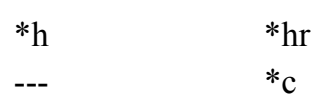

$* t$

(b)

$\begin{array}{ll}* \mathrm{t}^{\mathrm{h}}\left(/ *_{\mathrm{t} \varphi}^{\mathrm{h}}\right) & * \mathrm{~d} \\ * \mathrm{t} \varphi^{\mathrm{h}} & *_{\mathrm{z}}\end{array}$

(c) $* \mathrm{tc}^{\mathrm{h}}$

*s

$*_{\mathrm{c}}$
$*_{\mathrm{hr}}$
$*_{\mathrm{f}}$

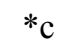


As in the case of the stops, it appears that PB and PSWT are generally conservative, whereas a series of mergers occurred in Pre-Hlai. Based on the PSWT evidence, a retroflex series is reconstructed here in PSKD, which affricated in all branches, eventually became alveo-palatal in PHI, and deaffricated to fricatives in PB and PSWT (it will be shown below that the regular PB reflexes of voiced fricatives are voiced stops). If this hypothesis is correct, the voiced retroflex fricative devoiced in Tai at some point previous to PSWT, merging with its voiceless counterpart. Additional evidence in Hlai for reconstruction of a retroflex series is found in the asymmetrical support of an optional labiovelar coarticulation $*_{t} \int^{\text {h }} \mathrm{w}$ but not a palatal coarticulation (first mentioned in chapter two, section 2.3.1.2). The same pattern is found in Central Hlai, where *rw is the only possible coarticulated rhotic (superscripted X indicates an impossible initial):

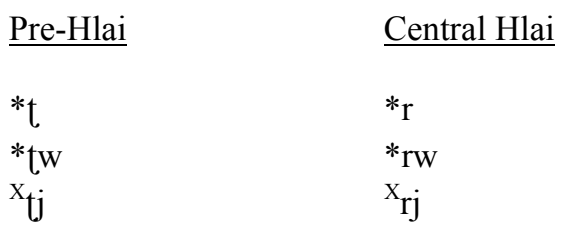

There are two cases in which PHl $* t \int^{h}$ w corresponds to PB *h and PSWT *hr (see (15b) below). Although it is tempting to see this as a case of vocalic transfer, the aspiration in the PHl reflex indicates that this initial was not originally word-medial. I tentatively suggest that the original rime in these cases had a rounded vowel, and that the labialization on the $\mathrm{PHl}$ initial is a result of the transfer of rounding from the rime. This provides an alternate explanation for what otherwise appears to be the only case of vocalic transfer of $* u$ in Pre-Hlai, in an unlikely environment (across an affricate).

The palatal reflexes in PSWT shown in (12a-b) offer proof of an original palatal stop series, which underwent affrication in Hlai. The PSKD palatal fricative ${ }^{*} 6$ followed the same trajectory in Hlai as the velar fricative $*_{\mathrm{x}}$, narrowing in stricture in Pre-Hlai and merging with the aspirated reflex of $*_{\mathrm{c}}$ by the time of PHl: 
(14)

\begin{tabular}{|c|c|c|c|c|}
\hline PSKD & & $\underline{\text { Pre-Hla }}$ & & $\underline{\text { Proto-Hlai }}$ \\
\hline$* t$ & $>$ & $* \operatorname{ts}(\mathrm{w})$ & $>$ & $* \mathrm{t} \int^{\mathrm{h}}(\mathrm{w})$ \\
\hline$*_{\mathrm{c}}$ & $>$ & $*_{\text {tc }}$ & $>$ & $* t^{\mathrm{h}}$ \\
\hline$* d$ & $>$ & *ts & $>$ & $* \mathrm{t} \int^{\mathrm{h}}\left(/ * \mathrm{t}^{\mathrm{h}}{ }^{\mathrm{h}}\right)$ \\
\hline$*_{j}$ & $>$ & $*$ t6 & $>$ & $*+c^{\mathrm{h}}$ \\
\hline$*_{6}$ & $>$ & $*_{6}$ & $>$ & $* t^{\mathrm{h}}$ \\
\hline
\end{tabular}

Examples of voiceless retroflex affricates and palatal stops are shown below:

(15) $\underline{\text { Gloss }}$

(a)

(b)

(c) fruit/testicles

carry on shoulder

weave (fabric) loom head louse

(to) wedge

\section{Proto-Hlai}

$*^{\mathrm{h}} \int^{\mathrm{h}} ə: \mathrm{m}$

$* t \int^{\mathrm{h}} \mathrm{a}: \mathrm{p}$

$* t \int^{\text {h }}$ wu:k

$* t \int^{\text {h }}$ wu:

$*_{t}{ }^{\mathrm{h}} \mathrm{i}: \mathrm{m}$

\section{Proto-Be}

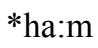

*ha:p

*huk

---

$--$

\section{$\underline{\text { PSWT }}$}

*hrəm

*hra:p

*hru:k

*hraw

$* \operatorname{cim}(\mathrm{J})$ $\underline{\text { PSKD }}$

*tə:m

*ta:p

*tu:k

*tu:

*ci:m

Examples of voiced $* d$ and $*^{*}$ are given below. There are a surprising number of examples of $* d$, but one reason for this may be that it has two potential origins: initial *d and intervocalic *t. Also note that the Hlai reflex of PSKD $* d$ becomes palatalized before the high vowel $*$ i:

\section{$\underline{\text { Gloss }}$}

(a)

$$
\begin{aligned}
& \text { stone } \\
& \text { take, carry }
\end{aligned}
$$

pain; (be) sick

(b)

$$
\begin{aligned}
& \text { taste } \\
& \text { hole }
\end{aligned}
$$

Proto-Hlai
${ }^{*} c^{\mathrm{h}} \mathrm{i}: \mathrm{n}$
*tc $^{\mathrm{h}} \mathrm{i}: \mathrm{w} ?$
*t $^{\mathrm{h}}{ }^{\mathrm{h}}$ ok

$*_{\text {t }}{ }^{\mathrm{h}}$ im

*tc $\mathrm{u}$ :y? $\underline{\text { Proto-Be }}$

*di:n

$*$ di:w

$* \mathrm{~d}$ k

${ }_{\text {*ə: }: y^{\mathrm{X}}}$
PSWT

*hrin

*hriw ${ }^{\mathrm{C}}$

---

$*_{\text {fim }}$

* ว $: \mathrm{y}^{\mathrm{B}}$ $\underline{\text { PSKD }}$

*di:n

$*$ di: ${ }^{\mathrm{C}}$

$* d[ə / \mathrm{o}] \mathrm{k}$

* fim

$*_{\mathrm{fo}}: \mathrm{y}^{\mathrm{B} / \mathrm{C}}$

Examples of the palatal fricative are given below. Whereas ${ }^{*} \varphi$ merged with $\mathrm{PHl} *^{*} \varphi^{\mathrm{h}}$, it depalatalized and merged with *s in PB/PSWT: 


\begin{tabular}{|c|c|c|c|c|}
\hline Gloss & Proto-Hlai & Proto-Be & PSWT & PSKD \\
\hline tail & $* c_{c}^{\mathrm{h}} \mathrm{uc}$ & $*$ suc & --- & $*_{6 u c}$ \\
\hline pestle; pound rice & $*_{\mathrm{t} c^{\mathrm{h}} \mathrm{a}: \mathrm{k}}$ & --- & $*_{\mathrm{sa}} \mathrm{k}$ & ${ }^{*} 6 \mathrm{a}: \mathrm{k}$ \\
\hline
\end{tabular}

The following correspondences are irregular:

\section{$\underline{\text { Gloss }}$}

(a) eye

(b) tongs
Proto-Hlai $\underline{\text { Proto-Be }}$

*ta:

$--$ $\underline{\text { PSWT }}$

*ta:

*gi:p $\underline{\text { PSKD }}$

*N-ta:

*gi:p

The word for eye (17a) developed from an original retroflex affricate based on the Hlai evidence, but irregularly in both PB and PSWT; I propose the solution to this was an original sesquisyllabic form with an initial nasal (for external reasons; see chapter 6); the same correspondence can be seen in the word for die in PB and PSWT, which are not cognate with Hlai forms for die.

The word tongs (17b) may be explainable through the irregular affrication of the initial velar in Pre-Hlai or PHl before the high vowel.

\subsubsection{Fricatives}

It has been shown above that the PSKD palatal and velar fricatives * 6 , ${ }^{\mathrm{x}}$ and ${ }^{*} \mathrm{y}$ merged with other Hlai categories, becoming $* \mathrm{tc}^{\mathrm{h}}$ and $* \mathrm{k}^{\mathrm{h}}$. The following are the correspondences for the PSKD fricatives which are still realized as such in PHI. PB initial voiced fricatives have closed in stricture to stops (19b), but there is PB evidence for medial labiodental fricatives (19c): 
(19)

$$
\text { Proto-Hlai }
$$

Proto-Be

PSWT

$\underline{\text { PSKD }}$

(a)

${ }^{*} \mathrm{f}$
${ }_{\mathrm{s}}$

$*_{\mathrm{f}}$
$*_{\mathrm{s}}$

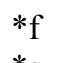

$*_{\mathrm{f}}$

$*_{\mathrm{s}}$

(b) $\begin{aligned} & *_{\mathrm{f}} \\ & *_{\mathrm{s}}\end{aligned}$

$* \mathrm{~b}$

$* \mathrm{~d}$

${ }^{*} \mathrm{~V}$
$*_{\mathrm{z}}$

$*_{\mathrm{v}}$
$*_{\mathrm{z}}$

$* \mathrm{f}$
$\quad * \mathrm{fj}$

$*_{\mathrm{W}}$

$*_{\mathrm{V}}$

${ }^{*} \mathrm{C}-\mathrm{v}$

${ }^{*} \mathrm{Civ}$

The only change which occurred in Hlai was the devoicing of the initial voiced fricatives, leading to their merger with the voiceless series.

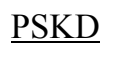

$\underline{\text { Pre-Hlai }}$

Proto-Hlai

(a)

$\begin{array}{ll}*_{\mathrm{f}} & > \\ *_{\mathrm{s}} & >\end{array}$

$*_{\mathrm{f}} \quad>$

$* f$

(b)

$\begin{array}{lllll}*_{\mathrm{V}} & > & *_{\mathrm{f}} & > & *_{\mathrm{f}} \\ *_{\mathrm{Z}} & > & *_{\mathrm{s}} & > & *_{\mathrm{s}}\end{array}$

(c)

${ }^{*} \mathrm{C}-\mathrm{v}>$
${ }^{*} \mathrm{Civ}>$

*f

$* f j$

Examples of the PSKD voiceless fricatives are given below:

$\underline{\text { Gloss }}$

(a) rain

millet

sky/cloud

(b) you (pl)

ripe water buffalo/cow
Proto-Hlai

$\underline{\text { Proto-Be }}$

*fwən

$*[\mathrm{f} / \mathrm{w}] \mathrm{a}: \mathrm{y}^{\mathrm{X}}$

*fa: ${ }^{\mathrm{X}}$

*su

*--

*say ${ }^{\mathrm{X}}$
PSWT

*frn

$*_{\text {fa: }}{ }^{\mathrm{C}}$

$*$ fa: ${ }^{\mathrm{C}}$

*su (Li)

*suk

--- $\underline{\text { PSKD }}$

$*$ fon

$*_{\text {fa: }}{ }^{\mathrm{C}}$

$* \mathrm{fa}:{ }^{\mathrm{C}}$

*su:

*sur:k

*suy ${ }^{\mathrm{C}}$

Examples of the PSKD voiced fricatives are given below: 
(22)

$\underline{\text { Gloss }}$

Proto-Hlai

$\underline{\text { Proto-Be }}$

$\underline{\text { PSWT }}$

PSKD

(a) sky/cloud

*fa:?

*ba: ${ }^{\mathrm{X}}$

$*_{\mathrm{va}}{ }^{\mathrm{C}}$

*va: ${ }^{\mathrm{C}}$

(b)

wash clothes

*sə:k

ash

*su:?

*dak

*zak

*zə:k

*də: ${ }^{X}$

$\left({ }^{*}\right.$ daw $\left.^{\mathrm{B}}\right)$

${ }^{*} \mathrm{zu}:{ }^{\mathrm{C}}$

Examples of medial voiced fricatives are given below:

Gloss

(a) fire

$$
\text { dream }^{38}
$$

(b)

tooth
seed

\section{Proto-Hlai}

*fi:

*fən

*fjən

$*_{\text {fjon }}$ $\underline{\text { Proto-Be }}$

*wey

*wə:n

*sjon

*wə:n $\underline{\text { PSWT }}$

*vay

*fan

*van

*van $\underline{\text { PSKD }}$

${ }^{*} \mathrm{C}$-vi:

${ }^{*} \mathrm{C}$-vən

*Civən

*Civən

The following items show irregular correspondences:

Gloss

(a) go word clsfr; mouth

(b) cast (seed)

(c) cockscomb

(d) vomit

(e) collect, pick up

\section{Proto-Hlai}

*fi:

*fa:k

*fə:nf

*fi:wh

*fa:k

*sip $\underline{\text { Proto-Be }}$

*pəy

*pa:k

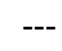

$*_{\text {si: }} \mathrm{x}$

*duak

$---$
PSWT

*pay

*pa:k

*hwa:n ${ }^{\mathrm{B}}$

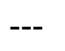

*ra:k

*kep
PSKD

*pwi:

*pwa:k

*[hw/v][ə/a $]: n^{\mathrm{B}}$

*[Civ]i: ${ }^{\mathrm{B}}$

*rwa:k

*[k/g]ip

The two items in (24a) may be best explained by positing an initial *pw, which delabialized in PL and PT, but which affricated to *pf and then simplified to *f in PHl.

The item in (24b) might be reconstructed with an original *hw, which hardened to ${ }^{*} \mathrm{v}$ in $\mathrm{PHl}$; the discrepancy in vowels casts doubt on its authenticity as a cognate set.

The initial in (24c) may be tentatively assumed to have been an original labiodental fricative, which underwent palatalization in PB (see tooth above).

\footnotetext{
${ }^{38}$ The PSWT voiceless reflex in this form is irregular.
} 
The word vomit (24d) is most easily explained by assuming an original ${ }^{*} \mathrm{r}$, and then positing an irregular change to *z in PB (which later became *d), as well as loss of *r and fortition of medial ${ }^{*} \mathrm{w}$ in $\mathrm{PHl}$, which led irregularly to a Pre-Hlai *va:k, then devoicing to *fa:k.

Finally, if the forms in (24e) are truly cognate, the PHl form may be the result of irregular palatalization before [i].

\subsubsection{Medial Stops}

The intervocalic stop correspondences which exist between PHI, PB and PSWT are the following:

\begin{tabular}{|c|c|}
\hline Proto-Hlai & Proto-Be \\
\hline
\end{tabular}

(a)

\begin{tabular}{|c|c|c|}
\hline$* 6$ & ${ }^{*} \mathrm{C}-\mathrm{b}$ & $* 2 b$ \\
\hline${ }^{*} \mathrm{C}-\beta$ & $\left(*^{*} \mathrm{v}\right)$ & $* ? b$ \\
\hline$*_{d}$ & ${ }^{*} \mathrm{C}-\mathrm{r}$ & $* ? d$ \\
\hline$*_{\text {tc }}$ & ${ }^{*} \mathrm{C}-\mathrm{j}$ & $* ? \mathrm{j}$ \\
\hline$*_{k}$ & --- & ${ }^{*} \mathrm{~g} \sim{ }^{*} \mathrm{k}^{\mathrm{h}}$ \\
\hline & ${ }^{*} 6$ & ${ }^{*} \mathrm{hr}$ \\
\hline
\end{tabular}

(b)

$$
* \mathrm{k}
$$

It is clear that intervocalic voicing has occurred in all three branches, and it is tempting to reconstruct it in PSKD itself; there are reasons why this is problematic, however, which are discussed below in section 4.1.7.

The development of the initials in (25a) in Hlai is given below; it is assumed that upon becoming initial, voiced initials devoiced in accordance with the global Hlai prohibition against voiced obstruent initials:

\begin{tabular}{|c|c|c|c|c|}
\hline PSKD & & Pre-Hlai & & Proto-Hlai \\
\hline${ }^{*} \mathrm{C}-\mathrm{p}$ & $>$ & ${ }^{*} \mathrm{C}-\mathrm{b}$ & $>$ & $* 6 \sim * C-\beta$ \\
\hline$*^{*} \mathrm{C}-\mathrm{t}$ & $>$ & ${ }^{*} \mathrm{C}-\mathrm{d}$ & $>$ & $*_{d}$ \\
\hline${ }^{*} \mathrm{C}-\mathrm{c}$ & $>$ & ${ }^{*} \mathrm{C}_{-\mathrm{f}}$ & $>$ & $* t c$ \\
\hline${ }^{*} \mathrm{C}-\mathrm{k}$ & $>$ & ${ }^{*} \mathrm{C}-\mathrm{g}$ & $>$ & $*_{\mathrm{k}}$ \\
\hline
\end{tabular}


At the bilabial place of articulation, all three branches show evidence for a medial *C-b. I propose that $\mathrm{PHl} * 6$ and $* \mathrm{C}-\beta$ both originate in Pre-Hlai $* \mathrm{C}-\mathrm{b}$, and that the majority change was to $\mathrm{PHl} * 6$; however, in a few instances, additional lenition occurred before the implosivization of ${ }^{*} \mathrm{C}-\mathrm{b}$, leading to $* \mathrm{C}-\beta$ (this is additional evidence that intervocalic voicing did occur in Pre-Hlai). At the alveolar place of articulation, both PHl and PSWT are reconstructible to medial $* \mathrm{C}$-d, but PB seems to have lenited even further to $* \mathrm{C}$-r. In the case of the palatal stops, both PB and PSWT have lenited first to voiced stops, then to sonorants, with $\mathrm{PHl}$ being the only branch retaining the original stricture of these phonemes. The reconstruction of ${ }^{*} \mathrm{C}-\mathrm{g}$ is more tenuous, with no PB examples, and one example of PSWT ${ }^{*} \mathrm{~g}$ and two of $* \mathrm{k}^{\mathrm{h}}$.

An outline of the differences in the developments in PB and PSWT is given below:

\begin{tabular}{|c|c|c|c|c|}
\hline$\underline{\text { PSKD }}$ & & $\underline{\text { Pre-Be }}$ & & Proto-Be \\
\hline$* \mathrm{C}-\mathrm{p}$ & $>$ & $* \mathrm{C}-\mathrm{b}$ & $>$ & $* \mathrm{C}-\mathrm{b}$ \\
\hline$* \mathrm{C}-\mathrm{t}$ & $>$ & $* \mathrm{C}-\mathrm{d}$ & $>$ & $* \mathrm{C}-\mathrm{r}$ \\
\hline${ }^{*} \mathrm{C}-\mathrm{c}$ & $>$ & $* \mathrm{C}-\mathrm{f}$ & $>$ & $* \mathrm{C}-\mathrm{j}$ \\
\hline$\underline{\text { PSKD }}$ & & \multicolumn{2}{|c|}{$\underline{\text { Pre-SWT }}$} & $\underline{\text { Proto-Southwest Tai }}$ \\
\hline${ }^{*} \mathrm{C}-\mathrm{p}$ & $>$ & $* \mathrm{C}-\mathrm{b}$ & $>$ & $* 2 \mathrm{~b}$ \\
\hline$* \mathrm{C}-\mathrm{t}$ & $>$ & $* \mathrm{C}-\mathrm{d}$ & $>$ & $* ? d$ \\
\hline$* \mathrm{C}-\mathrm{c}$ & $>$ & $* \mathrm{C}-\mathrm{f}$ & $>$ & $* ? \mathrm{j}$ \\
\hline${ }^{*} \mathrm{C}-\mathrm{k}$ & $>$ & $* \mathrm{C}-\mathrm{g} \sim \mathrm{g}^{\mathrm{f}}$ & & $*^{g} \sim \mathrm{k}^{\mathrm{h}}$ \\
\hline
\end{tabular}

The analysis of correspondences including $\mathrm{PHl} * \mathrm{k}$ is complicated by the other regular correspondence set $(24 b)$ in which PHl *k participates. The PB and PSWT evidence both indicate an *rcluster; the specifics of this cluster are more difficult to reconstruct, and for now it is merely reconstructed as *C-C.

Examples of the medial stops are given below: 


\section{Gloss}

(a)

fly
carry

(b)

$$
\text { village }
$$

times

(c)

$$
\begin{aligned}
& \text { face } \sim \text { nose } \\
& \text { sieve }
\end{aligned}
$$

gall bladder

(d)

$$
\begin{aligned}
& \text { stand } \\
& \text { step on }
\end{aligned}
$$

(e)

$$
\begin{aligned}
& \text { slant } \sim \text { tilt } \\
& \text { turbid } \\
& \text { crocodile }
\end{aligned}
$$

(f)

$$
\begin{aligned}
& \text { road } \\
& \text { jar, jug } \\
& \text { vine }
\end{aligned}
$$

Proto-Hlai

Proto-Be

*6in

*6i:k

*ba: $\mathrm{h}$

*C- $\beta a: n ?$

*C- $\beta$ ə: $\mathrm{y}$

*dəท

*doy?

*dəy

*tcu:n

*tcə:mf

$$
\text { *C-bjən }
$$

( $\left.{ }^{*} \mathrm{C}-[\mathrm{b}] \mathrm{i}: \mathrm{k}\right)$

$$
---
$$

$$
\text { (*C-wo: } \left.{ }^{\mathrm{X}}\right)
$$$$
*_{\text {və: }{ }^{[X]}}
$$

$\underline{\text { PSWT }}$

PSKD

*?bin

*?be:k

$* 2 \mathrm{ba}^{\mathrm{B}}$

${ }^{*}$ C-pin

${ }^{*} \mathrm{C}$-pe:k

*C-pa: ${ }^{\mathrm{B}}$

*2ba: ${ }^{\mathrm{C}}$

*C-pa: ${ }^{\mathrm{C}}$

${ }^{*} \mathrm{C}$-pə:y

---

*?day

*2doy ${ }^{\mathrm{C}}$

${ }^{*} \mathrm{C}$-təy

${ }^{*}$ C-rəy
${ }^{*}$ C-ro: $\eta^{\mathrm{X}}$

(*?bli:)

${ }^{*} \mathrm{C}$-ton ${ }^{\mathrm{C}}$

*C-rəy

* jui:n

${ }^{*} \mathrm{C}$-[n/j]u:n

---

*?ja:m ${ }^{\mathrm{B}}$ (Li)

${ }^{*} \mathrm{C}$-cun

*ki:y

*kunf

*k[i:]?

$--$

$--$

$--$

$*_{\mathrm{g} \varepsilon: \mathrm{y}}(\mathrm{J})$

$* \mathrm{k}^{\mathrm{h}} \mathrm{un}^{\mathrm{B}}$

$* \mathrm{k}^{\mathrm{h}} \varepsilon:^{\mathrm{C}}(\mathrm{J})$

${ }^{*} \mathrm{C}$-gun ${ }^{\mathrm{B}}$

${ }^{*} \mathrm{C}$-ge: ${ }^{\mathrm{C}}$

*ku:n
*kəy
*kəc

*6wən

*hron

*hray

${ }^{*} \mathrm{C}-\mathrm{Co}[:] \mathrm{n}$

${ }^{*} \mathrm{C}$-Cәy

*-

*C-Coc

The following example shows variation in place: palatal in PHI, alveolar in PSWT, and mixed reflexes in the Be daughter languages:
$\underline{\text { Gloss }}$
Proto-Hlai
$\underline{\text { Proto-Be }}$
$\underline{\text { PSWT }}$
$\underline{\text { PSKD }}$
extinguish
*tढəp
${ }^{*} \mathrm{C}-[\mathrm{r} / \mathrm{j}] \mathrm{ap}$
*?dap
${ }^{*} \mathrm{C}-[\mathrm{t} / \mathrm{c}] ə \mathrm{p}$

The following forms seem to show a difference between an original voiceless medial stop in PreHlai, and a voicless initial stop in PB/PSWT. One possible explanation is that the common ancestor of PB and PSWT lost original minor syllables before they could condition intervocalic voicing. However, there are at least three possible Chinese loans in the examples below (board, duck, and more than), indicating that a major (perhaps exclusive) source of this part of the lexical set may be loanwords. If this is true, then at least some of the PSKD forms below may not have actually existed: 
Gloss

(a) board

duck

crab

cotton

(b) castrate

soak

(c) chop

breath

(d) $\underline{\text { Proto-Hlai }}$ Proto-Be

*6e: $n^{\mathrm{B}}$

$*$ bit

*6u: ${ }^{\mathrm{B}}$

$* 6 u: y^{C}$

*du:n

*də:m ${ }^{\mathrm{c}}$

*tcək

*tcur:

${ }^{*} \mathrm{kua}^{\mathrm{C}}$ *pe:n ${ }^{\mathrm{X}}$

*pjət

---

*pu:y ${ }^{\mathrm{X}}$

*to:n

*cə: ${ }^{\mathrm{X}}$

$--$

$---$

*kua ${ }^{\mathrm{x}}$
PSWT

*pe: $\mathrm{n}^{\mathrm{C}}$

*pet

*pu:

---

*to:n

$--$

*cək (Li)

*аш

${ }^{*}$ kwa: ${ }^{B}$
PSKD

*(C-)p $\varepsilon: \mathrm{n}^{\mathrm{B} / \mathrm{C}}$

*(C-)pet

*(C-)pu: ${ }^{(\mathrm{B})}$

*(C-)pu:y ${ }^{\mathrm{C}}$

*(C-)to:n

$*(\mathrm{C}-)[\mathrm{t} / \mathrm{c}] \mathrm{\partial}: \mathrm{m}^{\mathrm{C}}$

*(C-)cək

*(C-)cu:

*(C-)kua ${ }^{\mathrm{C} / \mathrm{B}}$

\subsubsection{Pre-Hlai Nasals}

The correspondences between $\mathrm{PHl}$ and $\mathrm{PB} / \mathrm{PSWT}$ nasals is complicated by the fact that while there are correspondences between PHl initial nasals and PB/SWT initial nasals, as well as between PHI medial nasals and PB/SWT medial nasals, there are also mixed correspondences between initial and medial nasals from both branches. Examples are presented from a Hlai perspective, and two hypotheses are suggested in order to explain the mixed correspondences.

\subsubsection{Initial Nasals}

The following are the two sets of correspondences for the Hlai initial nasals ${ }^{39}$ :

\footnotetext{
${ }^{39}$ The H-initial forms in (32b) indicate original fricative-initial sesquisyllabic forms.
} 
(32)

$$
\text { Proto-Hlai }
$$

$\underline{\text { Proto-Be }}$

$\underline{\text { PSWT }}$

$\underline{\text { PSKD }}$

(a)

\begin{tabular}{|c|c|c|c|}
\hline *hm & $* \mathrm{~m}$ & $* m$ & $* \mathrm{~m}$ \\
\hline *hn & $* \mathrm{n}$ & *n & *n \\
\hline *hn & *n & $* \mathrm{n}$ & $* \mathrm{n}$ \\
\hline *hy & $* \eta$ & $* \eta$ & $* y$ \\
\hline *hyw & ${ }^{*} \mathrm{w}$ & *yw & *yw \\
\hline *hm & ${ }^{*} \mathrm{C}-\mathrm{m}$ & *hm & ${ }^{*} \mathrm{H}-\mathrm{m}$ \\
\hline *hn & ${ }^{*} \mathrm{C}-\mathrm{n}$ & *hn & *H-n \\
\hline *hy & --- & *hy & ${ }^{*} \mathrm{H}-\mathrm{n}$ \\
\hline *hyw & --- & *hw & ${ }^{*} \mathrm{H}-\mathrm{nw}$ \\
\hline
\end{tabular}

The only development between PSKD and PHI in the case of the first set of initials is the development of preaspiration by the time of $\mathrm{PHI}$ :

\begin{tabular}{|c|c|c|c|c|}
\hline PSKD & & Pre-1 & & Proto-Hlai \\
\hline$*_{\mathrm{m}}$ & $>$ & $*_{\mathrm{m}}$ & $>$ & $* \mathrm{hm}$ \\
\hline$*_{\mathrm{n}}$ & $>$ & $*_{\mathrm{n}}$ & $>$ & *hn \\
\hline$* \mathrm{n}$ & $>$ & $* \mathrm{n}$ & $>$ & *hn \\
\hline$* \eta$ & $>$ & $* \eta$ & $>$ & *hy \\
\hline *yw & $>$ & *yw & $>$ & *hnw \\
\hline
\end{tabular}

In the cases where $\mathrm{PHl}$ aspirated nasals correspond with PB/PSWT medial nasals ${ }^{40}$, the solution adopted here is that in PSKD, they were preceded by an initial fricative; this initial fricative conditioned high tone in PB and PSWT (and preaspiration in Li's Proto-Tai). This preaspiration led to a merger of these two categories of $\mathrm{PHl}$ nasals when plain initial nasals became preaspirated.

\begin{tabular}{|c|c|c|c|c|}
\hline PSKD & & Pre-Hlai & & Proto-Hlai \\
\hline *H-m & $>$ & *hm & $>$ & *hm \\
\hline *H-n & $>$ & *hn & $>$ & *hn \\
\hline$* \mathrm{H}-\mathrm{\eta}$ & $>$ & *hy & $>$ & *hy \\
\hline *H-nw & $>$ & *hךw & $>$ & *hyw \\
\hline
\end{tabular}

Examples of nasal-initial forms are given below:

${ }^{40}$ Many of these are animal names, and an animal prefix has been suggested to account for these (Ostapirat 2000, Thurgood 1988b). 
(35)

$\underline{\text { Gloss }}$

Proto-Hlai

Proto-Be

PSWT

PSKD

(a)

return/come

*hmu:

*mia

*hmu:m?

*mu: ${ }^{\mathrm{X}}$

*hmuc

*muac

\section{*mயә}

*mua

beard

(f)

(b) field

*hna:h

*nia

*hna:k

*na:k

*mum ${ }^{\mathrm{B}}$

*mot

*mu: $\mathrm{m}^{\mathrm{B} / \mathrm{C}}$

*moc

(c) shoot $\sim$ bow

*hpu:

*h[n]om?

*nว:

*na:

*na: ${ }^{(\mathrm{B})}$

*na:k

*na:k

dye

*hyən

*hyu:

*hyuc

$*[\mathrm{r} / \mathrm{j}] \mathrm{am}^{\mathrm{X}}$

*num

*nu:

(d) silver

sesame

needle

*hywən

*yว:n

---

*ya:c

*no:m ${ }^{\mathrm{C}}$

*no[:] $\mathrm{m}^{\mathrm{C}}$

(e) day

*wว:n

*yrn

*yən

* yua

*y[o:]c

*ywən

Examples of fricative initial forms with medial nasals are given below:

(36) Gloss

(a) $\operatorname{dog}$

$$
\text { pig }
$$

(b) mouse

(c) grey hair

(d) wind $\sim$ cold (sickness)

\section{Proto-Hlai}

*hma:

*hmo:t

*hməw

*hniw

*hnu:

*hyu:k

*hywə:t

\section{Proto-Be}

${ }^{*} \mathrm{C}$-ma:

${ }^{*} \mathrm{C}$-mat

${ }^{*} \mathrm{C}$-mo:

${ }^{*}$ C-nu:
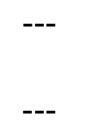

$---$ $\underline{\text { PSWT }}$

*hma:

*hmat

*hmu:

*hnu:

*hnuə

*hyo:k

*hwat $\underline{\text { PSKD }}$

*H-ma:

*H-mə:t

*H-mu:

*H-niw

*H-nua

*H-no:k

*H-ywa:t

The following word shows a more complex correspondence:

\section{Gloss}

smooth

The palatal nasal in the Hlai form above can be explained through the palatalization of the lateral and later coalescence of the two initial consonants: $\mathrm{ml}>\mathrm{mj}>\mathrm{n}>\mathrm{hn}$. 


\subsubsection{Medial nasals}

The medial nasal correspondence sets also split into two sets:

$\underline{\text { Proto-Hlai }} \quad \underline{\text { Proto-Be }} \quad \underline{\text { PSWT }} \quad \underline{\text { PSKD }^{41}}$

(a)

$\begin{array}{ll}{ }^{*} \mathrm{C}-\mathrm{m} & { }^{*} \mathrm{C}-\mathrm{m} \\ { }^{*} \mathrm{C}-\mathrm{n} & { }^{*} \mathrm{C}-\mathrm{n} \\ { }^{*} \mathrm{C}-\mathrm{n} & { }^{\mathrm{C}} \mathrm{C}-\mathrm{n} \\ { }^{*} \mathrm{C}-\mathrm{n} & { }^{*} \mathrm{C}-\mathrm{n}\end{array}$

(b)

$\begin{array}{ll}* \mathrm{C}-\mathrm{m} & * \mathrm{~m} \\ { }^{*} \mathrm{C}-\mathrm{n} & * \mathrm{n} \\ * \mathrm{C}-\mathrm{n} & *_{\mathrm{n}} \\ * \mathrm{C}-\mathrm{n} & * \mathrm{n}\end{array}$

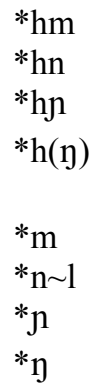

${ }^{*} \mathrm{C}-\mathrm{m}$

$* \mathrm{C}-\mathrm{n}$

${ }^{*} \mathrm{C}-\mathrm{n}$

$* \mathrm{C}-\mathrm{n}$

$* \mathrm{C}^{\mathrm{V}}-\mathrm{m}$

$* \mathrm{C}^{\mathrm{V}}-\mathrm{n}$

$* \mathrm{C}^{\mathrm{V}}-\mathrm{n}$

$* \mathrm{C}^{\mathrm{V}}-\mathrm{n}$

For the correspondence series in (38a), there are occasional instances of initial PB forms. I reconstruct sesquisyllabic forms with voiceless initials for this first set, with no changes between PSKD and PHI:

\begin{tabular}{|c|c|c|c|c|}
\hline PSKD & & Pre-Hlai & & Proto-Hlai \\
\hline${ }^{*} \mathrm{C}-\mathrm{m}$ & $>$ & $*^{\mathrm{C}}-\mathrm{m}$ & $>$ & ${ }^{*} \mathrm{C}-\mathrm{m}$ \\
\hline${ }^{*} \mathrm{C}-\mathrm{n}$ & $>$ & $* \mathrm{C}-\mathrm{n}$ & $>$ & $*^{*} \mathrm{C}-\mathrm{n}$ \\
\hline${ }^{*} \mathrm{C}-\mathrm{n}$ & $>$ & ${ }^{*} \mathrm{C}-\mathrm{n}$ & $>$ & ${ }^{*} \mathrm{C}-\mathrm{n}$ \\
\hline${ }^{*} \mathrm{C}-\mathrm{n}$ & $>$ & ${ }^{*} \mathrm{C}-\mathrm{n}$ & $>$ & ${ }^{*} \mathrm{C}-\mathrm{y}$ \\
\hline
\end{tabular}

There is a second set of correspondences (38b) where a PHl medial nasal corresponds with PB and PSWT initial nasals. It is notable that at the alveolar place of articulation, all of the PSWT initials belong to the class which $\mathrm{Li}(1977)$ reconstructs as PT *nR. I suggest that the PSKD form underlying this particular set of correspondences was a sesquisyllable with an originally voiced stop. These voiced stops devoiced in Pre-Hlai, becoming voiceless and conditioning high register; on the other hand, they conditioned low register in PB and PSWT, merging with monosyllabic items which had plain initial nasals.

${ }^{41} \mathrm{C}^{\mathrm{V}}$ here represents an originally voiced initial in a sesquisyllabic form. 
(40)

\begin{tabular}{|c|c|c|c|c|}
\hline PSKD & & Pre-Hla & & Proto \\
\hline$* \mathrm{C}^{\mathrm{V}}-\mathrm{m}$ & $>$ & ${ }^{*} \mathrm{C}-\mathrm{m}$ & $>$ & ${ }^{*} \mathrm{C}$ \\
\hline$* \mathrm{C}^{\mathrm{V}}-\mathrm{n}$ & $>$ & $* \mathrm{C}-\mathrm{n}$ & $>$ & ${ }^{*} \mathrm{C}$ \\
\hline${ }^{*} \mathrm{C}^{\mathrm{V}}-\mathrm{n}$ & $>$ & ${ }^{*} \mathrm{C}-\mathrm{n}$ & $>$ & ${ }^{*} \mathrm{C}-\mathrm{n}$ \\
\hline${ }^{*} \mathrm{C}^{\mathrm{V}}-\mathrm{n}$ & $>$ & ${ }^{*} \mathrm{C}-\eta$ & $>$ & $*^{\mathrm{C}}-\mathrm{n}$ \\
\hline
\end{tabular}

The following are examples of medial nasals preceded by originally voiceless initials:

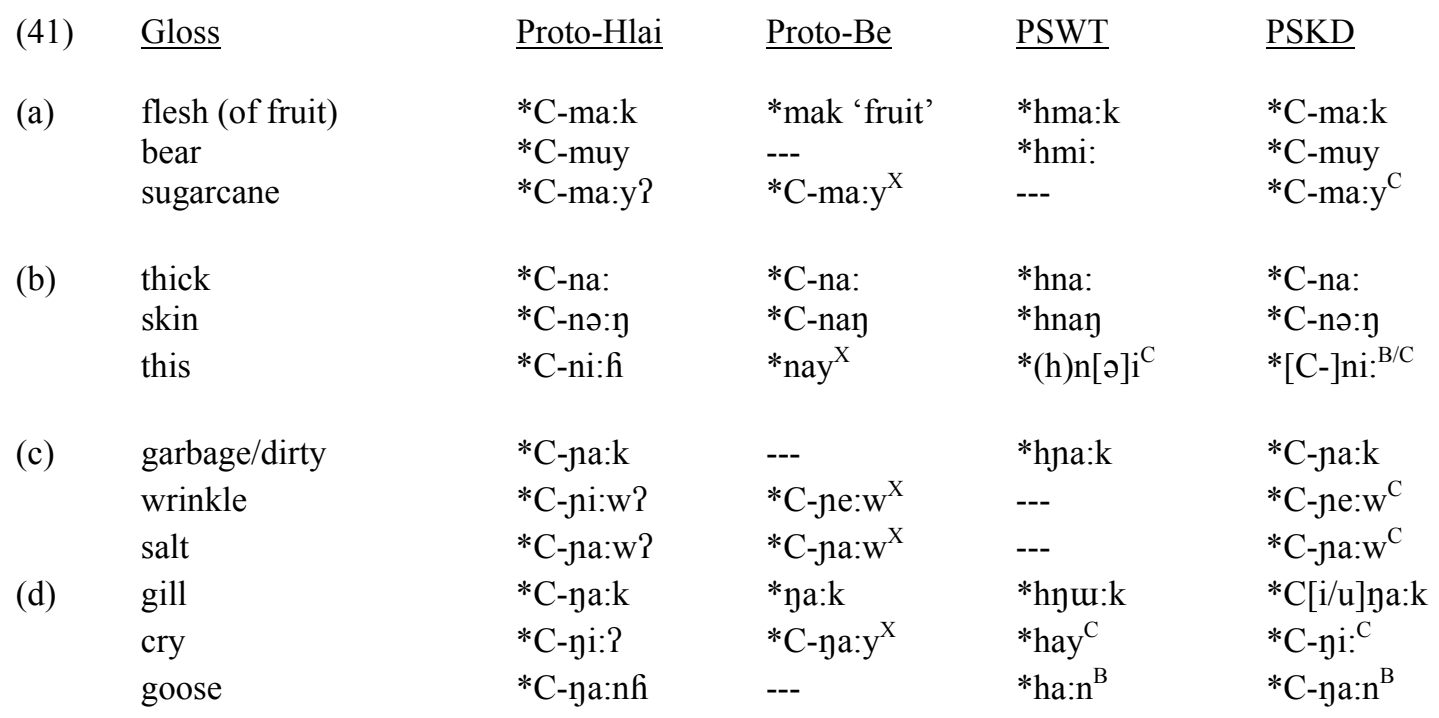

The following are examples of medial nasals preceded by originally voiced initials:

\begin{tabular}{|c|c|c|c|c|c|}
\hline (42) & $\underline{\text { Gloss }}$ & $\underline{\text { Proto-Hlai }}$ & $\underline{\text { Proto-Be }}$ & $\underline{\mathrm{PSWT}}$ & $\underline{\text { PSKD }}$ \\
\hline \multirow[t]{3}{*}{ (a) } & yam & *C-mən & *man & $*$ man & $* \mathrm{C}^{\mathrm{V}}$-mən \\
\hline & you & *C-mu: & *mə & *muin & $*^{\mathrm{V}}$-mu: \\
\hline & hand & *C-mu: & *mə: & *mu: & $* \mathrm{C}^{\mathrm{V}}$-mu: \\
\hline \multirow[t]{3}{*}{ (b) } & water & ${ }^{*} \mathrm{C}$-nəm? & $*_{\text {na: }}{ }^{\mathrm{X}}$ & $*_{\text {nam }}{ }^{\mathrm{C}}$ & $* \mathrm{C}^{\mathrm{V}}-\mathrm{n} \mathrm{m}^{\mathrm{C}}$ \\
\hline & bamboo shoot & *C-nu:y & *na:n & *nRa:y (Li) & $* \mathrm{C}^{\mathrm{V}}$-nuan ${ }^{\mathrm{C}}$ \\
\hline & stinger & ${ }^{*}$ C-nəy & & *lay & ${ }^{*} \mathrm{C}^{\mathrm{V}}$-nəy \\
\hline \multirow[t]{2}{*}{ (c) } & mosquito & *C-лu:n & *nuəy & *nuy & ${ }^{*} \mathrm{C}^{\mathrm{V}}$ - $\mathrm{nu}[:] \mathrm{y}$ \\
\hline & sew & ${ }^{* C}$-люp & *nap & *nip & $* \mathrm{C}^{\mathrm{V}}$-люp \\
\hline \multirow[t]{3}{*}{ (d) } & branch (tree) & *C-na:mh & --- & $*_{\text {ya:m}}^{\text {B }}(\mathrm{Li})$ & $* \mathrm{C}^{\mathrm{V}}$-na: $\mathrm{m}^{\mathrm{B}}$ \\
\hline & mute & ${ }^{*} \mathrm{C}$-nom & --- & *ywo[:] $\mathrm{m}^{\mathrm{C}}(\mathrm{Li})$ & $* \mathrm{C}^{\mathrm{V}}-\mathrm{n} \mathrm{m}^{(\mathrm{C})}$ \\
\hline & branch (road) & ${ }^{*} \mathrm{C}-$ na: $\mathrm{P}$ & --- & $*_{\text {ya }}{ }^{\mathrm{B}}(\mathrm{Li})$ & $* \mathrm{C}^{\mathrm{V}}-\mathrm{ya}:^{\mathrm{B} / \mathrm{C}}$ \\
\hline
\end{tabular}


A few exceptions which do not conform to the pattern above but which may still be cognate include the following:

\section{$\underline{\text { Gloss }}$}

(a) pickle

moon

(b)

climb crawl

(c) two days later

(d) straw

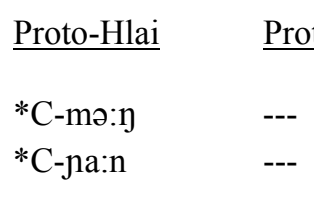

${ }^{*}$ C-na:n

*C-ju:

*Cuyin? $\underline{\text { Proto-Be }}$
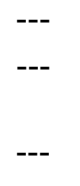

*rə:

*mu: $y^{\mathrm{x}}$
PSWT

*?blo:y

*?bluən

*gla:n

*ru:

$---$ $\underline{\text { PSKD }}$

${ }^{*} \mathrm{C}-\mathrm{bl}[\mathrm{\rho} / \mathrm{o}]: \mathrm{y}$

*C-b[i/u]la:n

*g-na:n

*C-mru:

${ }^{*} \mathrm{C}^{\mathrm{V}}$ unin $^{\mathrm{C}}$

The two words in (43a) are both potential examples of a correspondence between PHl ${ }^{*} \mathrm{C}-\mathrm{N}$ and PSWT * 2 bl. In both cases a cluster must be assumed, where either an original voiced stop nasalized in Hlai, or an original nasal stop denasalized in Tai. The palatal nasal in moon can be derived in the same manner as in smooth above, from the sequence C-ml > C-mj > C-n. If the two words in (43b) are cognate, it may be an example of lenition in PSWT of $*_{n}>* 1$. If the forms in $(43 \mathrm{c})$ are cognate, I propose an initial ${ }^{*} \mathrm{C}-\mathrm{mr}$ in PSKD. Finally, if $\mathrm{PB} * \mathrm{mu}: \mathrm{y}^{\mathrm{X}}$ is related to $\mathrm{PHl}{ }^{*} \mathrm{Cunin}^{\mathrm{C}}$ (43d), then the PB initial and rime may have both been influenced by the preceding presyllable vowel.

\subsubsection{Laterals}

There are several correspondence sets for the PHl lateral series, owing largely to the fact that PSKD medial *1 had the potential to combine into various clusters and/or was occasionally preempted by an initial consonant. The four PHl lateral series will therefore be subdivided and treated individually in this section. 


\subsubsection{Initial Laterals}

The four sets of correspondences for $\mathrm{PHl}$ *hl are listed below:

\begin{tabular}{|c|c|c|c|}
\hline$\underline{\text { Proto-Hlai }}$ & $\underline{\text { Proto-Be }}$ & $\underline{\text { PSWT }}$ & ${\underline{\text { PSKD }^{42}}}$ \\
\hline *hl & $* 1$ & $* 1$ & $*_{1}$ \\
\hline *hl & ${ }^{*} \mathrm{C}-1$ & $* \mathrm{hl}$ & ${ }^{*} \mathrm{H}-1$ \\
\hline *hl & $* p$ & $*(\mathrm{p}) 1$ & $*_{\mathrm{p}-1}$ \\
\hline *hl & $*_{\mathrm{m}}$ & $* 1$ & $*_{\mathrm{m}}-1$ \\
\hline
\end{tabular}

The first series provides straightforward evidence for initial PSKD *1, the second series for a fricative initial presyllable, the third for a *p-initial presyllable, and the final series for an *m-initial presyllable. Developments of these series into PHl are given below, in which the labial-initial series loses its presyllable between PSKD and Pre-Hlai, and the plain lateral becomes preaspirated by the time of ProtoHlai:

$\begin{array}{lllll}\underline{\text { PSKD }} & & \text { Pre-Hlai } & & \text { Proto-Hlai } \\ * 1 & > & * 1 & > & * \text { hl } \\ * \mathrm{H}-1 & > & * \mathrm{hl} & > & * \mathrm{hl} \\ *_{\mathrm{p}-1} & > & * 1 & > & * \mathrm{hl} \\ * \mathrm{~m}-\mathrm{l} & > & * 1 & > & * \mathrm{hl}\end{array}$

Examples of these series are given below:

$\underline{\text { Gloss }}$

(a) tongue

child

deep

(b) hat (bamboo)

many

son-in-law
Proto-Hlai

*hli:n?

*hlur:k

*hlə:k

*hla:y?

*hlə:y

*hlu: $\underline{\text { Proto-Be }}$

*li: $\mathrm{n}^{\mathrm{X}}$

*luk

*lak

*C-la: ${ }^{\mathrm{X}}$

$---$

*C-la:
PSWT

*li:n ${ }^{\mathrm{C}}$

*lu:k

*lrk

*hla:y

---
PSKD

*li:n ${ }^{\mathrm{C}}$

*lu:k

*lə:k

*H-la:y ${ }^{\mathrm{C}}$

*H-lə:y

*H-lu:

${ }^{42} \mathrm{C}^{\mathrm{V}}$ here represents an originally voiced initial in a sesquisyllabic form. 
(c) blood

(d) spittle

$$
\text { deaf }
$$

*hla:c

*hla:

*hlə:y

*hlə:k *pa:c

*pa:

*may

*mak *luat

*pla:

*la:y

(*hnuək) *p[i/u]la:c

*p-la:

*m-lə:y

*m-lə:k

The following two examples are exceptional:

$\underline{\text { Gloss }}$

(a) Hlai Tai

(b) chaff

\section{Proto-Hlai}

*hləy

*hli:p $\underline{\text { Proto-Be }}$

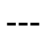

${ }^{*} \mathrm{C}$-lip $\underline{\text { PSWT }}$

*day

*kle:p $\underline{\text { PSKD }}$

*[1]əy

*[k-]le:p

The autonym in (47a) is in variation between Pre-Hlai $* 1$ and Tai $* d$; if these two forms are truly cognate, then an original lateral is implied which hardened irregularly to $* \mathrm{~d}$ in Tai. The expected PHl reflex of the word chaff in $(47 \mathrm{~b})$ is $* \mathrm{C}-1$, and this word apparently lost its presyllable in Pre-Hlai.

\subsubsection{Medial Laterals}

The correspondence sets for the PHl medial laterals are the following:

\begin{tabular}{|c|c|c|c|}
\hline Proto-Hlai & Proto-Be & PSWT & PSKD \\
\hline$*^{\mathrm{C}}-1$ & ${ }^{*} \mathrm{C}-1$ & *hl & ${ }^{*} \mathrm{C}-1$ \\
\hline${ }^{*} \mathrm{C}-1$ & $*^{*} \mathrm{C}-1$ & *kl & ${ }^{*} \mathrm{k}-1$ \\
\hline${ }^{*} \mathrm{C}-1$ & $*_{\mathrm{r}}$ & $* 1$ & $* \mathrm{C}^{\mathrm{V}}-1$ \\
\hline
\end{tabular}

The Hlai developments are analogous to the nasals in the last section, voiced and voiceless presyllable obstruents devoice and merge, with no other changes occurring between PSKD and PHl (save for loss of place information for the presyllable initial):

$\begin{array}{lllll}\underline{\text { PSKD }} & & \text { Pre-Hlai } & & \text { Proto-Hlai } \\ { }^{*} \mathrm{C}-1 & > & * \mathrm{C}-1 & > & * \mathrm{C}-1 \\ { }^{*} \mathrm{k}-1 & > & * \mathrm{k}-1 & > & * \mathrm{C}-1 \\ { }^{*} \mathrm{C}-1 & > & * \mathrm{C}-1 & > & * \mathrm{C}-1\end{array}$


Examples are given below:

$\underline{\text { Gloss }}$

(a) big

$$
\text { return }
$$

(b)

far
drum
forget
fingernail
small

\section{Proto-Hlai}

*C-lun

*C-lur:y

${ }^{*}$ C-ləy

*C-ləy

*C-lu:mh

*C-li:p

${ }^{*}$ C-lik

\section{Proto-Be}

--- *hluəy

*C-lun

${ }^{*}$ C-ləy

${ }^{*} \mathrm{C}$-lon

$--$

*klay

*klo:y

*lui:m

*lep

(*ri:p) $\underline{\text { PSKD }}$

${ }^{*} \mathrm{C}-1[\mathrm{u}] \mathrm{y}$

*C-lu[:]

*k-ləy

*k-1[o:] $\mathrm{y}$

${ }^{*} \mathrm{C}^{\mathrm{V}}$-lut:m${ }^{(\mathrm{B})}$

${ }^{*} \mathrm{C}^{\mathrm{V}}$-le[:]p

${ }^{*} \mathrm{C}^{\mathrm{V}}$-lek

The following form is exceptional, in that is shows variation between a lateral and rhotic medial liquid. Since the PB form indicates a lateral, I make the tentative assumption that there was confusion between liquids in Tai:

$\underline{\text { Gloss }}$

lazy
Proto-Hlai

*C-la:n?
Proto-Be

*la: ${ }^{\mathrm{X}}$ $\underline{\text { PSWT }}$

*gra: $\mathrm{n}^{\mathrm{C}}$ $\underline{\text { PSKD }}$

*g-la:n ${ }^{\mathrm{C}}$

\subsubsection{Palatalized Laterals}

The following series are nearly identical to those in the last subsection, the difference being the presence of a high front vowel in the presyllable which conditioned vocalic transfer in Hlai:

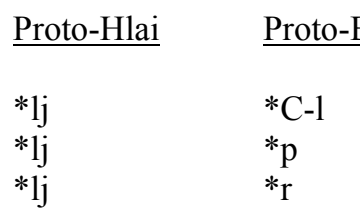

$\underline{\text { PSWT }}$

$*$ hl

$*$ pl $\underline{\text { PSKD }}$

$*^{*}$ Cil

*pil

${ }^{*} \mathrm{C}^{\mathrm{V}} \mathrm{il}$

The development of these forms in Hlai is as follows:

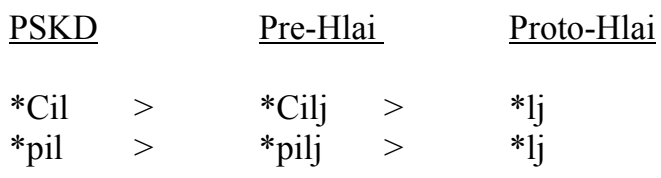




$$
* \mathrm{C}^{\mathrm{V}} \mathrm{il} \quad>\quad * \mathrm{Cilj} \quad>\quad * \mathrm{lj}
$$

Examples are given below:

$\underline{\text { Gloss }}$

(a) yellow remainder gadfly

(b) leech

(c) steal

\section{$\underline{\text { Proto-Hlai }}$}

*lja:y

*lja:

*lja:k

*ljin

*ljək
Proto-Be

$\underline{\text { PSWT }}$

*C-la:n

$---$

$---$

*pin

*rok
PSKD

*hluəy *Cila:y

*hluə *Cila:

*hluək *Cila:k

*pli:y $\quad$ *pili[:]

*lak $\quad * \mathrm{C}^{\mathrm{V}} \mathrm{il}$ ək

The following form shows a wide range of variation, even within the Hlai languages themselves:

$\underline{\text { Gloss }}$

lightning
Proto-Hlai

$*[\mathrm{C}-][\mathrm{j}] \mathrm{ip}$ $\underline{\text { Proto-Be }}$

${ }^{*}$ C-ljap
PSWT

*mle:p $\underline{\text { PSKD }}$

*m-le:p

\subsubsection{4*p-l clusters}

This series of PHl laterals participates in an extremely heterogeneous group of correspondences with PB/PSWT, and it appears that in at least some cases the PHl labial element may be secondary. The PSKD reconstructions below are extremely tentative:

$\begin{array}{llll}\underline{\text { Proto-Hlai }} & \underline{\text { Proto-Be }} & \underline{\text { PSWT }} & \underline{\text { PSKD }} \\ { }_{* \text { p-1 }} & --- & *(\mathrm{p}) 1 & * \mathrm{p}-1\end{array}$

There was no change between PSKD and PHI:

\begin{tabular}{|c|c|c|}
\hline PSKD & Pre-Hlai & Proto-Hlai \\
\hline *p-1 & $*_{\mathrm{p}-1}$ & $* \mathrm{p}-1$ \\
\hline
\end{tabular}

There is only one illustrative example of each of the above series: 
$\underline{\text { Gloss }}$

(a) swim $\sim$ float

(b) termite
Proto-Hlai

$\underline{\text { Proto-Be }}$

PSWT

$\underline{\text { PSKD }}$

*p-li:

*p-lu:k

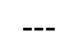

*puk *lo:y

*pluək (J) $*[p-] l o: y$

*p-lu:k

In addition to the forms above, the following sets exist as well:

$\underline{\text { Gloss }}$

(a) forehead

(b) near

(c) hornet

simmer
Proto-Hlai

*p-la:?

*p-lu:?

*p-lu:

*p-lom ${ }^{\mathrm{C}}$ $\underline{\text { Proto-Be }}$

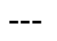

${ }^{*} \mathrm{C}-1$ ə: ${ }^{\mathrm{X}}$

$\left({ }^{*}\right.$ da: $\left.{ }^{\mathrm{X}}{ }\right)$

---
PSWT

${ }^{*} \mathrm{p}^{\mathrm{h}} \mathrm{a}: \mathrm{k}$

*klau ${ }^{\mathrm{C}}$

*to: ${ }^{\text {B }}$

$*$ tom $^{\mathrm{B}}$ $\underline{\text { PSKD }}$

*p-[1/r]a:k

${ }^{*} \mathrm{C}$-Іәч ${ }^{\mathrm{C}}$

*[p-]to: ${ }^{(\mathrm{C})}$

$*[p-] \operatorname{tom}^{B / C}$

The form in (59a) shows another confusion between the medial liquids *1 and *r, the latter having conditioned the aspiration in the PSWT initial. In the word near (59b), it appears as though there is an original root *ləư ${ }^{\mathrm{C}}$ which was prefixed independently in each of the two branches of PSKD. Finally, the two examples in (59c) show the same correspondence; if these are truly related, then one hypothesis is that PSWT retained the original roots, and that secondary prefixes were added in Pre-Hlai, resulting in the lenition of $*$ to $* 1$.

\subsubsection{Approximants}

The correspondences for PHl approximants are given below. The PB and PSWT reflexes indicate that the PHl labiovelar and alveolar approximants descend from original intervocalic voiced stops which underwent lenition in Hlai: 
(60)

$\underline{\text { Proto-Hlai }} \underline{\text { Proto-Be }} \underline{\text { PSWT }} \underline{\text { PSKD }}$

(a) $* v$

${ }^{*} \mathrm{C}$-b

$* ? b \sim * \mathrm{v}$

${ }^{*} \mathrm{C}-\mathrm{b}$

(b)

${ }^{*} r$
${ }^{*} \mathrm{rj}$
${ }^{*} \mathrm{Cur}$
${ }^{*} \mathrm{C}(\mathrm{u}) \mathrm{r}$

*(C-)r

*?d

$*[\mathrm{C}-] \mathrm{r}$

*C-r

*(C-)r

$*_{\mathrm{j}}$

*?d

${ }^{*} \mathrm{r}$

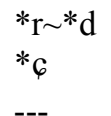

${ }^{*} \mathrm{r}$

${ }^{*} \mathrm{C}^{\mathrm{h}}$

${ }^{*} \mathrm{Cl}$
${ }^{*} \mathrm{C}$-d

${ }^{*} \mathrm{Cid}$

*Cud

${ }^{*} \mathrm{C}(\mathrm{u}) \mathrm{d}$

$* r$

*C-r

${ }^{*} \mathrm{C}-\mathrm{R}$

The reflexes of PSKD ${ }^{*} \mathrm{C}-\mathrm{p} /{ }^{*} \mathrm{C}-\mathrm{t}$ and $\mathrm{PSKD}{ }^{*} \mathrm{C}-\mathrm{b} /{ }^{*} \mathrm{C}-\mathrm{d}$ apparently merged in PSWT, although there is one PSWT reflex $* \mathrm{v}$ of PSKD $* \mathrm{C}-\mathrm{b}$ which indicates further lenition ${ }^{43}$. There is also evidence for an original intervocalic retroflex $* \mathrm{~d}$, reflected in PSWT *r.

The reflexes of the plain rhotic in PHl and PSWT are straightforward. PB has two reflexes with one example each; the reflex $* \mathrm{~d}$ probably reflects a secondary development to earlier $* \mathrm{z}$. The reflexes of the medial rhotic have merged with those of the initial rhotic in $\mathrm{PHl}$; the regular reflex in $\mathrm{PB}$ for a ${ }^{*} \mathrm{C}-\mathrm{r}$ cluster is $* 6$, whereas in PSWT it conditions aspiration of the initial. There is another set of correspondences between $\mathrm{PHI} * \mathrm{r}$ and $\mathrm{PSWT} * \mathrm{Cl}$, where the identity of the original liquid is unclear.

The posited developments between PSKD and PHl are as follows:

$\underline{\text { PSKD }} \quad \underline{\text { Pre-Hlai }} \quad \underline{\text { Proto-Hlai }}$

\begin{tabular}{|c|c|c|c|c|}
\hline${ }^{*} \mathrm{C}-\mathrm{b}$ & $>$ & ${ }^{*} \mathrm{C}-\mathrm{v}$ & $>$ & $* v$ \\
\hline${ }^{*} \mathrm{C}-\mathrm{d}$ & $>$ & ${ }^{*} \mathrm{C}-\mathrm{r}$ & $>$ & $*_{r}$ \\
\hline$* \mathrm{Cid}$ & $>$ & ${ }^{*}$ Cirj & $>$ & $*_{r j}$ \\
\hline${ }^{*} \mathrm{Cud}$ & $>$ & ${ }^{*} \mathrm{Cur}$ & $>$ & $* \mathrm{Cur}$ \\
\hline${ }^{*} \mathrm{Cud}$ & $>$ & ${ }^{*} \mathrm{Cur}$ & $>$ & ${ }^{*} \mathrm{Cur}$ \\
\hline$*_{\mathrm{r}}$ & $>$ & $*_{\mathrm{r}}$ & $>$ & $*_{\mathrm{r}}$ \\
\hline${ }^{*} \mathrm{C}-\mathrm{r}$ & $>$ & $*^{*} \mathrm{C}-\mathrm{r}$ & $>$ & $*_{\mathrm{r}}$ \\
\hline
\end{tabular}

${ }^{43}$ The standard reflex at the alveolar place of articulation is what Li (1977) reconstructs as *?dR, which indicates lenition in Northern Tai as well. 
Examples are given below:

(62)

(a)

(b)

(d) house we (incl)

know

(e) taro

ask

to plant

(f)

empty
head/hairknot
round

\section{$\underline{\text { Proto-Hlai }}$}

*va:h

*vว:n

*ri:n?

*a:w

*ra:

* rja:w

*rja:k

*Curu:

*Curur:k

*Curi:p

*rəy

* rac

*Cura:

*ru:n (Bouhin) * *ra:n

*row

*Curu:

*ra:k

*ra:m

*Cura:

*ru:?

*Curəw?

*Curom

\section{$\underline{\text { Proto-Be }}$}

${ }^{*}$ C-bia ${ }^{\mathrm{X}}$

$---$

${ }^{*}$ C-ri: ${ }^{\mathrm{X}}$

$--$

*ra:

*[C-]riaw

$--$

*[d/r] $]$ :

${ }^{*} \mathrm{C}$-rik

*C-rjəp

*C-rəy

(*[r]ət)

*rua

*d[ə/a $] \mathrm{w}$

(*hu: ${ }^{\mathrm{X}}$ )

* cak

---

* ca:

$\left({ }^{*} \text { ha: } w^{\mathrm{X}}\right)^{44}$

PSWT

*2ba: ${ }^{\mathrm{B}}$

*van

*--

*?dau

$---$

*ja:k

(*2blua:)

*?du:k

*?dip

*ray

* rət (Li)

*ruo

*ruən

*raw

${ }^{*}$ ru: ${ }^{\mathrm{C}}$

$*^{\mathrm{h}}{ }^{\mathrm{h}}$ uək

* $\mathrm{t}^{\mathrm{h}} \mathrm{a}: \mathrm{m}$

---

*plaw ${ }^{\mathrm{B}}$

*klaw ${ }^{\mathrm{C}}$

*klom $\underline{\text { PSKD }}$

${ }^{*} \mathrm{C}-\mathrm{ba} \mathrm{:}^{\mathrm{B}}$

*C-bə:n

${ }^{*} \mathrm{C}$-di: ${ }^{\mathrm{C}}$

${ }^{*} \mathrm{C}$-da:w

*C-d[əu]

*Cida:w

*Cida:k

*Cudu:

*Cudur:k

*Cudi[:]p

*C-dəy

*C-dac

*Cuda:

*ruan

*row

${ }^{*} \mathrm{Cur}[\mathrm{u} / \mathrm{u}]:^{(\mathrm{C})}$

*pira:k

*t-ra:m

*Cura

${ }^{*} \mathrm{p}-\mathrm{Ru}:^{\mathrm{B} / \mathrm{C}}$

*kuRəw ${ }^{\mathrm{C}}$

*kuRom

There is one group of irregular correspondences:

Gloss
hair
centipede
frog

\section{Proto-Hlai}

$\underline{\text { Proto-Be }}$

$\underline{\text { PSWT }}$

$\underline{\text { PSKD }}$

*[r/hn]om
*ri:p $^{\text {rit: }}$
*ri:t $^{2}$

*--

${ }^{*} \mathrm{p}^{\mathrm{h}}$ om

*p-dom

$*^{\mathrm{h}} \mathrm{ep}(\mathrm{Li})$

*k-de[:]p

$* \mathrm{k}^{\mathrm{h}}$ wiat

*k-de:t

\footnotetext{
${ }^{44}$ Although this PB form appears to be superficially similar to the PHI and PSWT forms, it is probably a
} borrowing from Hainanese haw $\mathrm{J}$. 
The Hlai reflexes of these three words indicate original PSKD $* \mathrm{C}-\mathrm{d} /{ }^{*} \mathrm{C}-\mathrm{d}$, but the PSWT reflexes indicate an original ${ }^{*} \mathrm{C}$-r. I tentatively suggest that the Hlai reflexes are regular, and that these are forms with medial $* \mathrm{~d}$ which underwent lenition to ${ }^{*} \mathrm{r}$ in Tai, conditioning aspirated initials.

\subsubsection{Glides}

There is an asymmetry in the glides, in that the labiovelar glide is generally well represented at the PSKD level, but the palatal glide is can be reconstructed in only one word which is almost certainly a loan (sheep), and may not be reconstructible back to PSKD itself (see chapter six, section 6.1). The existence of this phoneme in PSKD is therefore questionable without further reinforcement from other branches of KraDai. The other PSKD initial to which PHl *hj corresponds is medial ${ }^{*} \mathrm{C}-\mathrm{f}$.

The labiovelars, while not numerous, are nevertheless evident in the PSKD lexicon. There are two categories which can be reconstructed. The first is the plain labiovelar glides, which have been inherited in the daughter languages unchanged. The second is a set of medial glides which I reconstruct here as having been originally preceded by fricatives, employing the same logic as with the other sonorants.

The correspondences for the PHl glides are given below:

\begin{tabular}{|c|c|c|c|}
\hline$\underline{\text { Proto-Hlai }}$ & $\underline{\text { Proto-Be }}$ & $\underline{\text { PSWT }}$ & $\underline{\text { PSKD }}$ \\
\hline *hj & $*_{\mathrm{Z}}$ & --- & $*_{\mathrm{j}}$ \\
\hline *hj & ${ }^{*} \mathrm{C}-\mathrm{j}$ & $* ? j$ & ${ }^{*} \mathrm{C}-\mathrm{f}$ \\
\hline *hw & ${ }^{*} \mathrm{~W}$ & $*_{\mathrm{W}}$ & ${ }^{*} \mathrm{~W}$ \\
\hline *hw & ${ }^{*} \mathrm{C}-\mathrm{w}$ & $* \mathrm{hw}$ & $* \mathrm{H}-\mathrm{w}$ \\
\hline
\end{tabular}

The developments from PSKD were the following: 
(65)

\begin{tabular}{|c|c|c|c|c|}
\hline PSKD & & Pre-Hl & & Prot \\
\hline$*_{\mathrm{j}}$ & $>$ & $*_{\mathrm{j}}$ & $>$ & ${ }^{*} \mathrm{hj}$ \\
\hline${ }^{*} \mathrm{C}-\mathrm{f}$ & $>$ & ${ }^{*} \mathrm{C}-\mathrm{j}$ & $>$ & ${ }^{*} \mathrm{hj}$ \\
\hline${ }^{*} \mathrm{w}$ & $>$ & ${ }^{*} \mathrm{~W}$ & $>$ & \\
\hline$* \mathrm{H}-\mathrm{w}$ & $>$ & $* \mathrm{hw}$ & $>$ & $* \mathrm{~h}$ \\
\hline
\end{tabular}

At some point in Pre-Hlai, the medial palatal approximant ${ }^{*} \mathrm{C}-\mathrm{\jmath}$ lenited to a glide and lost its presyllable, merging with Pre-Hlai $* \mathrm{j}$. This is motivated by the fact that in the comparisons below with PB and PSWT, the reflex of PSKD intervocalic * $*_{\mathrm{f}}$ is PHI $*$ hj, not preglottalized $* 2 \mathrm{j}$, which would be expected if the lenition to a glide had occurred before the deletion of an original presyllable. This can be compared to the case of PHl *v and *hw, which followed similar but ultimately non-identical trajectories:

(66)

\begin{tabular}{|c|c|c|c|c|c|c|}
\hline \multicolumn{6}{|c|}{$\underline{\text { Pre-Hlai }}$} & $\underline{\mathrm{PHI}}$ \\
\hline${ }^{*} \mathrm{C}-\mathrm{j}$ & $>$ & $*_{j}$ & $>$ & $* \mathrm{j}$ & $>$ & *hj \\
\hline$*_{\mathrm{j}}$ & $>$ & $*_{\mathrm{j}}$ & $>$ & $* \mathrm{j}$ & $>$ & *hj \\
\hline${ }^{*} \mathrm{C}-\mathrm{v}$ & $>$ & $*_{v}$ & $>$ & $*_{v}$ & $>$ & $* v$ \\
\hline$*_{\mathrm{W}}$ & $>$ & $*_{\mathrm{W}}$ & $>$ & $*_{\mathrm{W}}$ & $>$ & *hw \\
\hline
\end{tabular}

Examples are given below:

Gloss

(a) sheep

(b) medicine

pull/stretch

egg

(c) bail water pool, whirlpool

fly (n.)

(d)

\begin{abstract}
typhoon/wind left side
\end{abstract}

\begin{tabular}{|c|c|c|c|}
\hline Proto-Hlai & Proto-Be & PSWT & $\underline{\mathrm{PSKD}}$ \\
\hline *hja:y & $*_{\text {zuay }}$ & --- & $*_{\mathrm{j} a: y}$ \\
\hline *hja: & ${ }^{*} \mathrm{C}$-jia: & *2ja: & *C-ұa: \\
\hline *hji:t & --- & *2jiat & ${ }^{*} \mathrm{C}-\mathrm{ji}: \mathrm{t}$ \\
\hline *hju:m & *C-лwәm & --- & ${ }^{*} \mathrm{C}-$-jo:m \\
\hline *hwi:t & --- & $*_{\text {wit }}(\mathrm{Li})$ & *wi:t \\
\hline *hwa:y & --- & *wəy (Li) & 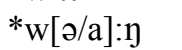 \\
\hline *hwa:y? & ${ }^{*}$ wa: $y^{\mathrm{X}}$ & --- & $*_{w a} y^{\mathrm{C}}$ \\
\hline *hwənf & ${ }^{*}$ C-wa: ${ }^{\mathrm{X}}$ & --- & $* \mathrm{H}-\mathrm{w}[\mathrm{\partial} / \mathrm{a}] \mathrm{n}^{\mathrm{B}}$ \\
\hline *hwi:y & *C-wi:n & --- & *H-wi:y \\
\hline
\end{tabular}




\subsubsection{Glottals}

The correspondence series in which the PHl glottals participate are given below:

$\underline{\text { Proto-Hlai }} \underline{\text { Proto-Be }} \underline{\text { PSWT }} \underline{\text { PSKD }}$

(a)

$\begin{array}{ll}*_{\mathrm{h}} & *_{\mathrm{g} \sim \mathrm{y}} \\ { }^{*} \mathrm{Cih} & *_{\mathrm{z} \sim \mathrm{\gamma}} \\ { }^{*} \mathrm{Cuh} & *_{\mathrm{g}}\end{array}$

(b) $* \mathrm{~h}$

$* h$

$*_{\mathrm{W}}$

*g

$\left({ }^{*} \mathrm{~g}\right)$

$*^{2}$

$$
* \gamma
$$

$$
\begin{aligned}
& { }^{*} \mathrm{C}-\mathrm{g} \\
& { }^{*} \mathrm{Cig} \\
& { }^{*} \mathrm{Cug}
\end{aligned}
$$

(c) $\quad * ?$

$* 2 / \varnothing$

$* \mathrm{q}^{\mathrm{h}}$

$* \mathrm{k}^{\mathrm{h}}$

*C-q

*C-G

${ }^{*} \mathrm{Cu}$ ?

---

$* ?$

*?

*?

${ }^{*} \mathrm{Cu}$ ?

There are several sets of correspondences for the PHl glottal fricative ${ }^{*} \mathrm{C}(\mathrm{i} / \mathrm{u}) \mathrm{h}$. The most

predominant reflexes in PB and PSWT are ${ }^{*} \mathrm{~g}$ and ${ }^{*} \mathrm{\gamma}$ respectively, allowing the reconstruction of PSKD medial $*$ g, filling a gap left in the precursors to the $\mathrm{PHl}$ approximant inventory. There are two additional series in which $\mathrm{PHl} * \mathrm{C}(\mathrm{i} / \mathrm{u}) \mathrm{h}$ participates, which I reconstruct as a pair of medial uvulars (voiceless and voiced) which merged in Pre-Hlai when they underwent intervocalic lenition. The glottal stop series is

\begin{tabular}{|c|c|c|c|c|}
\hline PSKD & & Pre-Hla & & $\underline{\text { Proto-Hlai }}$ \\
\hline${ }^{*} \mathrm{C}-\mathrm{g}$ & $>$ & ${ }^{*} \mathrm{C}-\mathrm{y}$ & $>$ & $* h$ \\
\hline${ }^{*} \mathrm{Cig}$ & $>$ & *Ciy & $>$ & ${ }^{*} \mathrm{Cih}$ \\
\hline${ }^{*} \mathrm{Cug}$ & $>$ & ${ }^{*}$ Cuy & $>$ & *Cuf \\
\hline${ }^{*} \mathrm{C}-\mathrm{q}$ & $>$ & *C-в & $>$ & $* 6$ \\
\hline${ }^{*} \mathrm{C}-\mathrm{G}$ & $>$ & *C-в & $>$ & $* h$ \\
\hline *? & $>$ & $* ?$ & $>$ & $* ?$ \\
\hline${ }^{*} \mathrm{Cu} ?$ & $>$ & ${ }^{*} \mathrm{Cu}$ ? & $>$ & *Cu? \\
\hline
\end{tabular}
unremarkable, $\mathrm{PH} * ?$ and $* \mathrm{Cu} ?$ corresponding with $\mathrm{PB} * ? / \varnothing$ and PSWT $* ?$. The changes from PSKD to $\mathrm{PHl}$ are given below:

Examples are given below: 
(70)

$\underline{\text { Gloss }}$

(a) chin

$$
\text { pinch }
$$

thatch grass

field ridge

handlength

smoke

body/person

(b) thigh

horn

fur

(c)

excrement
bitter
dig hole

(d)

bathe
bowl
crow

(e)

open
Proto-Hlai

*ha:y

*he:p

*Cifia:

*Cifə:n

*Cufurp

*Cufə:n

*Cufu:n

*ha:

*how

*Cufun

*ha:y?

* ศว:m

*Cifuut

*?a:p

*?a:yf

*?a:k

*CuPa:h $\underline{\text { Proto-Be }}$

*ya:y

*ge:p

*zia

* yian

$---$

*guan

(*hu:n)

*wa:

*wa:w

*wuən

*ga:y

*ga:m

*gu:c

$\begin{array}{ll}--- & * 2 a: p \\ --- & * 2 a: y^{\text {B }} \\ *[?] \mathrm{a}[:] \mathrm{k} & ---\end{array}$

*2a: ${ }^{\mathrm{C}}$

* $\mathrm{k}^{\mathrm{h}} \mathrm{om}$

${ }^{*} \mathrm{k}^{\mathrm{h}} \mathrm{ut}$

C-G[a:]y

${ }^{*} \mathrm{C}-\mathrm{G}[\mathrm{o} / \mathrm{o}]: \mathrm{m}$

*Cigut

*?a:p

*?a: $y^{\mathrm{B}}$

*?a:k

*CuPa: ${ }^{\mathrm{B} / \mathrm{C}}$

The following examples are irregular:

Gloss

(a) $\mathrm{I}$

(b) thorn

(c) frog
Proto-Hlai

*hu:

*Cufum

*?up
Proto-Be

(*ha:w)

*?un

*op
PSWT

$* \mathrm{k}[\mathrm{u}: / \mathrm{aw}]$

$\underline{\mathrm{PSKD}}$

${ }^{*} \mathrm{C}-\mathrm{ku}$ :

The initials of the $1^{\text {st }}$-person pronoun in (71a) are difficult to reconcile, but I suggest that the Hlai velar stop was originally intervocalic, and shifted irregularly to a uvular before undergoing intervocalic lenition: C-ku: > C-gu: > C-Gu: > C-fiu:. The PB initial indicates that it is borrowed from Hlai. It is possible that the forms in (71b) are unrelated; if they are cognate, the PB initial is irregular. Finally, it is 
possible but unsure that the forms for frog in (71c) are related. If so, the initial $* \mathrm{k}^{45}$ was irregularly debuccalized to *? in Pre-Hlai, and lost altogether in PB (which may be borrowed from Hlai).

\subsubsection{Interim Summary}

Through the comparison of PHl with PB and PSWT, it is possible to reconstruct a general first approximation of the PSKD initials. There is evidence that the PSKD prosodic word inventory was essentially the same as that posited for Pre-Hlai and Proto-Hlai, consisting of both monosyllabic words and disyllabic, sesquisyllabic words. The number of exceptional examples to otherwise general correspondence sets indicates that the evolution of initials in the various branches of PSKD has not always been similar, nor has it been without variation both between and within the daughter languages. The system of PSKD initials which has been reconstructed in this section, is shown below:

$\underline{\text { PSKD Initial Consonants }}$

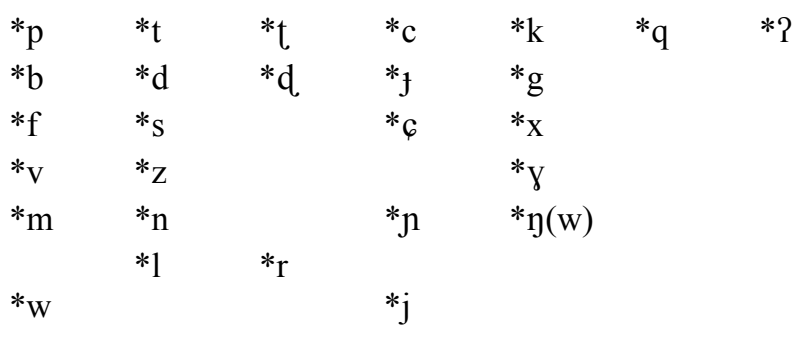

PSKD Medial Consonants

\begin{tabular}{|c|c|c|c|}
\hline${ }^{*} \mathrm{C}-\mathrm{p}$ & ${ }^{*} \mathrm{C}-\mathrm{t}$ & ${ }^{*} \mathrm{C}-\mathrm{c}$ & ${ }^{*} \mathrm{C}-\mathrm{q}$ \\
\hline${ }^{*} \mathrm{C}-\mathrm{b}$ & ${ }^{*} \mathrm{C}(\mathrm{i} / \mathrm{u}) \mathrm{d} * \mathrm{C}(\mathrm{u}) \mathrm{d}$ & $*{ }^{C}-\mathrm{f}$ & $*^{\mathrm{C}}(\mathrm{i} / \mathrm{u}) \mathrm{g} * \mathrm{C}-\mathrm{G}$ \\
\hline $\begin{array}{l}{ }^{*} \mathrm{C}(\mathrm{i}) \mathrm{V} \\
{ }^{*} \mathrm{C}^{(\mathrm{V})}-\mathrm{m} \\
\end{array}$ & $\begin{array}{l}* \mathrm{C}^{(\mathrm{V})}-\mathrm{n} \\
{ }^{*} \mathrm{C}^{(\mathrm{V})}(\mathrm{i}) 1 * \mathrm{C}(\mathrm{u}) \mathrm{r} \\
{ }^{*} \mathrm{p}(\mathrm{i}) 1 \\
* \mathrm{k}-1\end{array}$ & $* \mathrm{C}^{(\mathrm{V})}-\mathrm{n}$ & $* C^{(V)}-1$ \\
\hline *H-m & ${ }^{*}{ }^{*}{ }_{H}-n$ & & ${ }^{*} \mathrm{H}-\mathrm{n}(\mathrm{w})$ \\
\hline & & & \\
\hline
\end{tabular}

${ }^{45}$ External evidence from Kam-Sui and Kra support the hypothesis that the original initial in this word was *k. 
It is evident that the position of a consonant in a prosodic word has determined its evolution from PSKD to PHI. This is most glaring in the case of obstruents, but the same is also true of sonorants, the latter being particularly permeable to vocalic transfer within sesquisyllabic words. The specific Pre-Hlai changes which have been shown in the initials in this section will be thoroughly discussed in section 4.3.

The PSKD initial inventory itself is on balance more typologically normative than Hlai, primarily because it has plain obstruents and sonorants in word-initial position where PHl has aspirated obstruents and preaspirated sonorants. It has a wider inventory of place features (with retroflex in place of postalveolar and the addition of a uvular series), but nothing that isn't attested in attested language inventories.

The gaps which exist in word-initial position are not unusual. There is no evidence for a retroflex fricative series, a voiced palatal fricative, or a voiced uvular stop and fricative series; these are less common places of articulation where gaps are more likely to occur. The gaps in medial position include a plain retroflex stop and most fricatives (only the voiced labiodentals fricative is recsonstructible). I do not have an explanation for why these are absent, but further Kra-Dai comparative work may yet discover evidence for them.

\subsection{The Pre-Hlai Rimes}

In comparing PHl rimes with those of PB and PSWT, it becomes apparent (more so than in the case of the initials) that the PB correspondences are sometimes irregular, and this may be due to the fact that some of the forms have been borrowed from Hlai. Because of this, only irregularities in correspondence between Hlai and PSWT will be treated in any detail; irregularities in PB will be explored more fully in another venue.

This section is divided into three subsections. The first will compare and discuss the PSKD tone categories. The second section will be devoted to the open rimes, and the final section will focus on the closed rimes. 


\subsubsection{Tone Categories}

In the traditional parlance of Kra-Dai tone categories $(\mathrm{A}, \mathrm{B}, \mathrm{C}, \mathrm{D})$, the correspondences between PHl, PB, and PSWT are the following:

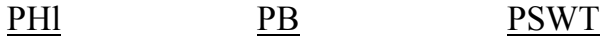

$\begin{array}{lll}\text { A } & \text { A } & \text { A } \\ \text { B } & \text { X } & \text { B } \\ \text { C } & \text { X } & \text { C } \\ \text { D } & \text { D } & \text { D }\end{array}$

The $\mathrm{PB}$ tone category $\mathrm{X}$ above is the designation used here for the marked tone category which corresponds to both $\mathrm{PHI}$ and PSWT categories B and C. It is apparent that PB category X represents a merger between the original categories $\mathrm{B}$ and $\mathrm{C}$.

In chapter three, the PHl tone system was examined, and tentatively reconstructed with category B correlated with spread glottis, and category $\mathrm{C}$ correlated with constricted glottis. Although the details of $\mathrm{PB}$ reconstruction will be treated elsewhere, it can be mentioned here that PB category $\mathrm{X}$ can be reconstructed as originally correlated with constricted glottis. Using the same glottal segments to represent this correlation as in chapter three, the relationship between PHl and PB can be restated as follows ${ }^{46}$ :

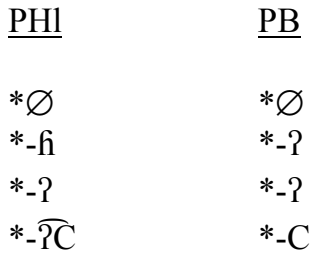

In other words, tone $\mathrm{B}$ spread glottis merged with tone $\mathrm{C}$ constricted glottis in $\mathrm{PB}$. Based on the PHl and PB evidence, the PSKD tone categories can tentatively be reconstructed as the following:

\footnotetext{
46 The tone categories of PSWT have not yet been reconstructed with any specific values, and PSWT will therefore not be compared with $\mathrm{PHl}$ and $\mathrm{PB}$.
} 
(75)

\begin{tabular}{|c|c|c|}
\hline PHI & $\underline{\mathrm{PB}}$ & $\underline{\text { PSKD }}$ \\
\hline${ }^{*} \varnothing$ & $* \varnothing$ & $* \varnothing$ \\
\hline *-h & $*_{-} ?$ & $*$-h \\
\hline$*_{-} ?$ & $*_{-} ?$ & $*_{-} ?$ \\
\hline *-TC & $*_{-} \mathrm{C}$ & $*-\widehat{P C}$ \\
\hline
\end{tabular}

The only change would have been in category B, where PSKD *-h voiced by the time of PHl, but merged with *-? in PB. This tentative reconstruction must await complete validation until the

reconstruction of the Proto-Tai tone system. Because of this, tone category letters will continue to be used with PB, PSWT, and PSKD forms.

\subsubsection{Open Rimes}

Having discussed the facts above regarding the PHI open rimes, a comparison with PB and PSWT may now be done. There are two sets of correspondences which appear upon comparison of the data; the first set in (76a) are the primary correspondences, while those in (76b) are more complex:

$\underline{\text { Proto-Hlai }} \underline{\text { Proto-Be }} \underline{\text { PSWT }} \underline{\text { PSKD }}$

(a)

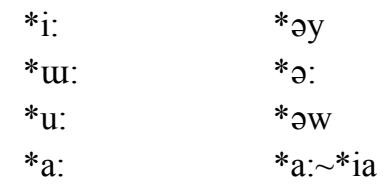

(b)

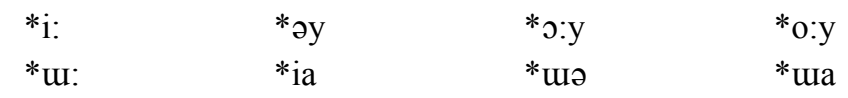

The examples in (76a) allow straightforward reconstruction of the three high vowels and the low vowel. There is variation in PSWT in the high vowel series between pure vowels and diphthongs which seems to be unconditioned; there is also occasional variation in vowel length and height in PB. There is variation in the PB reflex of PSKD *a:, between *a: and *ia. The latter will be shown below to descend from Pre-Be *ua; the reason for this variation in PB is unknown at this time. 
There have been no changes between PSKD and PHI:

\begin{tabular}{|c|c|c|c|c|}
\hline$\underline{\text { PSKD }}$ & & $\underline{\text { Pre-Hlai }}$ & & $\underline{\text { Proto-Hlai }}$ \\
\hline$* \mathrm{i}:$ & $>$ & $*_{\mathrm{i}}:$ & $>$ & $*_{\mathrm{i}:}$ \\
\hline *u: & $>$ & *u: & $>$ & *u: \\
\hline$* u:$ & $>$ & *u: & $>$ & $* \mathrm{u}:$ \\
\hline *a: & $>$ & $* \mathrm{a}:$ & $>$ & $* \mathrm{a}:$ \\
\hline
\end{tabular}

The examples in (76b) are more complex, and are the reflexes of a combination of diphthongs and high-low vowel sequences. There are two examples of the first correspondence set, which are very tentatively reconstructed as *o:y. For the second set, the hypothesis presented here is that at least one source of PHl *u: originates in an earlier *ua (for more evidence, see section 4.2.4 below):

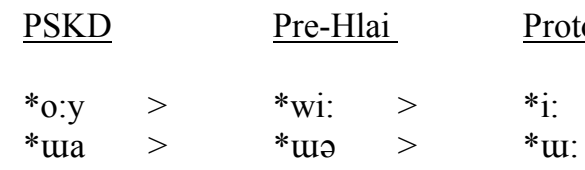

Examples are given below, first of the correspondence sets of (76a) in (79), then of (76b) in (80):

\section{$\underline{\text { Gloss }}$}

(a) go

fire

cry

(b) hand

leaf

near

(c) head louse

urine

turtle

(d) eye

thigh

$\operatorname{dog}$

(e) cogon grass

shoulder

field

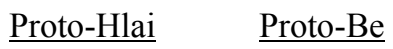

*fi:

*fi:

${ }^{*} \mathrm{C}-$ ni:?

${ }^{*} \mathrm{C}$-mu:

*C-bur:

*p-lu:?

$* t \int^{\mathrm{h}} \mathrm{wu}$ :

*C-du:

$* \mathrm{t}^{\mathrm{h}} \mathrm{u}: \mathrm{h}$

$* t \int^{h} a:$

*ha:

*hma:

*Cifia:

*va:h

*hna:h

*pəy
${ }^{* w e y ~}$
*C-ya:y

*mə:

*bə:

${ }^{*} \mathrm{C}-1$ - ${ }^{\mathrm{X}}$

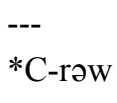

$--$

*ta:

*wa:

${ }^{*} \mathrm{C}$-ma:

*zia

${ }^{*} \mathrm{C}-\mathrm{bia}^{\mathrm{X}}$

*nia $\underline{\text { PSWT }}$

*pay

*vay

*hay ${ }^{\mathrm{C}}$

*mu:

*?bau

*klaw

*hraw

---

taw $^{\mathrm{B}}$

*ta:

*qa:

*hma:

*ya:

*?ba: ${ }^{\mathrm{B}}$

*na:
PSKD

*pwi:

*vi:

${ }^{*} \mathrm{C}-\mathrm{ni}:{ }^{\mathrm{C}}$

${ }^{*} \mathrm{C}^{\mathrm{V}}$-mu:

*C-pu:

${ }^{*} \mathrm{C}-1 \mathrm{u}:{ }^{\mathrm{C}}$

$* t(w) u:$

*C-tu:

$*$ tu: ${ }^{\mathrm{B}}$

*N-ta:

${ }^{*} \mathrm{C}$-qa:

*H-ma:

*Ciga:

${ }^{*} \mathrm{C}-\mathrm{ba}{ }^{\mathrm{B}}$

$*_{\text {na }}{ }^{(B)}$ 
(80)

$\underline{\text { Gloss }}$

Proto-Hlai

Proto-Be

PSWT

$\underline{\text { PSKD }}$

(a) shellfish

swim; float

$* c_{6}{ }^{\mathrm{h}} \mathrm{i}$ :

*həy

*hro:y

*to:y

*p-li:

$--$

*10:y

*[p-]lo:y

(b)

come/return
top

*hmu:

*mia

*muə

*mua

*hnu:

*hnuə

*H-nua

There are a number of exceptions to the above correspondences:

$\underline{\text { Gloss }}$

(a) shy

(b) mother

crocodile

wasp

(c) child

forehead

(d) shoot

you

(e) spirit

(f) sesame

(g) which

(h) remainder

boat

\section{Proto-Hlai}

*ri:

*hmi:?

*k[i:]?

*p-lu:

${ }^{*} \mathrm{C}$-di?

*p-la:?

*hpu:

*C-mu:

*Cufur:

*hyu:

*ra:

*lja:

*Cura: $\underline{\text { Proto-Be }}$

PSWT

*ra:y (Li)

*ma: ${ }^{\mathrm{X}}$

---

$\left(*\right.$ da: $\left.w^{\mathrm{X}}\right)$

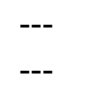

*nə:

*mə

'bow'

'bow'

*nuy

*mun

--- *qwan

*ya:

*?dau

*hluə

* rшə $\underline{\text { PSKD }}$

*r[ə]y

*me: ${ }^{\mathrm{B} / \mathrm{C}}$

${ }^{*} \mathrm{C}$-ge: ${ }^{\mathrm{C}}$

$*\left[\mathrm{p}-\right.$ to $^{(\mathrm{B})}$

*C-tek

${ }^{*} \mathrm{p}$-[1/r]a:k

*nu:

${ }^{*} \mathrm{C}^{\mathrm{V}}$-mu:

*Cugən

*[i/u]na:

${ }^{*} \mathrm{C}-\mathrm{d}[\mathrm{a}:]$

*Cila:

*Cuda:

The Tai rime in the word shy (81a) is irregularly low and long in comparison with the PHl rime *i:. The three words in $(81 \mathrm{~b})$ have very unexpected correspondences in PSWT, and may represent original PSKD *e: and $*_{0}$ :, where the mid vowels underwent regular raising in Pre-Hlai to high vowels. The words in $(81 \mathrm{c})$, if they are cognate, show an irregular lenition of final *-k in Hlai to an early glottal stop, which then conditioned tone C. The forms in (81d) both have well-known irregular nasal endings; there is a similar case in (81e) where, if cognate, it is possible that the final nasal was lost in Hlai. The mismatch of the vowels in sesame (81f) imply an earlier Pre-Hlai * yua, possibly a result of an earlier presyllable which did 
not effect vocalic transfer in Tai. The PSWT rime in $(81 \mathrm{~g})$ is irregular, and normally derives form earlier *əư; the fact that this word is a function word may explain the irregular development. Finally, the two words in (81h) are both cases of vocalic transfer in PB (boat) and PSWT. The outcome in PB is analogous to that which occurred in Central Hlai; it seems that in PSWT, the vocalic transfer of either of the high vowels $*_{\mathrm{i}}$ and ${ }^{*} \mathrm{u}$ has the potential to raise the final vowel *a:, resulting in the diphthong *uə. More evidence for this will be shown in section 4.2.4.

\subsubsection{Closed Rimes}

In the case of the closed rimes, the one overarching question which can be asked is: what is the origin of the length distinction? When the PHI data is compared with PB and PSWT, there is a general tendency (albeit with several exceptions) for vowel length to correlate, the one exception being the mid central vowels, where PSWT has only a short category. One possibility is that there may have been an inherited feature which existed in, and was inherited from, Proto-Southern Kra-Dai, that led to, but which was not itself, a length distinction. There does not yet seem to be specific clues as to what this feature was, however.

The most obvious candidate is stress, the presence of which is often typologically and phonetically associated with long rimes (see for example Hayes (1995)), and the absence of which is associated with short rimes. Since it has been argued in the first half of this chapter that Pre-Hlai (and by inference its predecessor) possessed an inventory which included at the very least bisyllabic words, a stress distinction would have been possible (but would have necessarily preceded the shift to iambic rhythm). However, there is one significant obstacle to this hypothesis, which is that it predicts long rimes for all monosyllabic forms, which should have borne a default stress. Since there are several examples of originally

monosyllabic words with short rimes, this is a serious problem and indicates that this hypothesis is probably incorrect, at least at the stage under discussion. 
The other possibility is that the length distinction was already present in Proto-Southern Kra-Dai, was inherited in the daughter languages, and discrepancies which exist in length are due to secondary changes which occurred after the break-up of Southern Kra-Dai into its daughter branches. In lieu of the problem with the stress-to-weight hypothesis, this is the hypothesis which will be adopted here, with the length distinction reconstructed in the Pre-Hlai rimes.

\subsubsection{High Front Rimes}

Comparisons in this section will not be subdivided according to the rime coda as was done in chapter three, as there are not enough examples to justify this approach. The rime categories are instead grouped together by nucleus only.

The correspondences for this category are given below:

\begin{tabular}{|c|c|c|c|}
\hline Proto-Hlai & Proto-Be ${ }^{47}$ & $\underline{\text { PSWT }}$ & $\underline{\text { PSKD }}$ \\
\hline$*_{\mathrm{i}} \mathrm{C}$ & $*_{\mathrm{i}: \mathrm{C}}$ & $* \mathrm{i}: \mathrm{C} \sim \mathrm{iC}$ & $*_{\mathrm{i}: \mathrm{C}}$ \\
\hline$*_{\mathrm{i}} \mathrm{C}$ & $*_{\mathrm{e}: \mathrm{C}}$ & $*_{\mathrm{e}: \mathrm{C}}$ & $*_{\mathrm{e}} \mathrm{C}$ \\
\hline$*_{\mathrm{iC}}$ & $*_{\mathrm{j} \partial \mathrm{C} \sim \mathrm{iC}}$ & $*_{\mathrm{iC}}$ & $*_{\mathrm{iC}}$ \\
\hline$*_{\mathrm{iC}}$ & $* \mathrm{j} \partial \mathrm{C}$ & $*_{\mathrm{eC}}$ & $*_{\mathrm{eC}}$ \\
\hline
\end{tabular}

It is apparent that the major change which has occurred in this rime category has been the merger in Hlai of the PSKD high and mid rimes, due to the raising of the latter category:

$\begin{array}{lllll}\underline{\text { PSKD }} & & \text { Pre-Hlai } & & \text { Proto-Hlai } \\ *_{\mathrm{i}: \mathrm{C}} & > & *_{\mathrm{i}: \mathrm{C}} & & *_{\mathrm{i}: \mathrm{C}} \\ *_{\mathrm{e}: \mathrm{C}}> & *_{\mathrm{i}: \mathrm{C}}> & *_{\mathrm{i}: \mathrm{C}} \\ *_{\mathrm{iC}}> & *_{\mathrm{iC}}> & *_{\mathrm{i} C} \\ *_{\mathrm{e} C}> & *_{\mathrm{iC}}> & *_{\mathrm{i} C}\end{array}$

${ }^{47}$ It should be noted that internal reconstruction within Proto-Be allows the interpretation of *jəC as an earlier Pre- $\mathrm{Be} * \mathrm{eC}$ in all environments except before velar codas. $\mathrm{PB} * \mathrm{j} \partial \mathrm{C}$ will be used here in order to stay consistent with the period of PB forms being compared. 
This raises the question of why there still exist ${ }^{*} \mathrm{e}: \mathrm{C}$ rimes in $\mathrm{PHl}$ (no short ${ }^{*} \mathrm{eC}$ rimes are reconstructed). This question will be treated in section 4.2.6.

\subsection{Long High Rimes}

In the correspondences for the $* \mathrm{i}: \mathrm{C}$ rime series, there is variation between long $(84 \mathrm{a})$ and short (84b) rimes in PSWT; PB rimes are long, with the exception of wing. In the case that the PB is long and the PSWT form is short, secondary shortening can be postulated in PSWT (or in Proto-Tai). In the absence of PB evidence, or where PB agrees with PSWT (as in live, raw), it is ambiguous whether there was secondary lengthening in Hlai or secondary shortening in the ancestor of PB and PSWT.

\begin{tabular}{|c|c|c|c|c|}
\hline$\underline{\text { Gloss }}$ & Proto-Hlai & $\underline{\text { Proto-Be }}$ & $\underline{\text { PSWT }}$ & $\underline{\text { PSKD }}$ \\
\hline reap & $*^{*} \mathrm{k}^{\mathrm{h}} \mathrm{i}$ wh & --- = & $*^{k} \mathrm{ki}: w^{\mathrm{B}}(\mathrm{Li})$ & ${ }^{*} \mathrm{ki}: \mathrm{w}^{\mathrm{B}}$ \\
\hline mat grass & *sji:w? & *ri:w ${ }^{\mathrm{X}}$ 'mat' & --- & ${ }^{*} \mathrm{C}[\mathrm{i}] \mathrm{di}: \mathrm{w}^{\mathrm{C}}$ \\
\hline lick & *lji:mf & $* \operatorname{li}: \mathrm{m}^{\mathrm{X}}$ & --- & ${ }^{*} \mathrm{C}^{\mathrm{V}} \mathrm{ili}: \mathrm{m}^{\mathrm{B}}$ \\
\hline tongue & *hli:n? & $* \operatorname{li}: \mathrm{n}^{\mathrm{X}}$ & *li: $\mathrm{n}^{\mathrm{C}}$ & $* \operatorname{li}: \mathrm{n}^{\mathrm{C}}$ \\
\hline hang & *ri:p? & ${ }^{*} \mathrm{C}-$ ri: $: \mathrm{y}^{\mathrm{X}}$ & --- & ${ }^{*} \mathrm{C}-\mathrm{di}: \mathrm{y}^{\mathrm{C}}$ \\
\hline left side & *hwi:y & *C-wi:y & --- & *H-wi:y \\
\hline tongs & $* c^{h} \mathrm{i}: \mathrm{p}$ & --- & *gi:p & $*_{\text {gi:p }}$ \\
\hline wing & ${ }^{*} \mathrm{p}^{\mathrm{h}} \mathrm{i} \mathrm{k}$ & $*_{\text {pik }}$ & * pi:k & * pi:k \\
\hline satisfied & $* \mathrm{t}^{\mathrm{h}} \mathrm{i}: \mathrm{k}$ & $* \mathrm{ti}: \mathrm{k}$ & -- & $*$ ti:k \\
\hline take $\sim$ carry & $* \mathrm{c}^{\mathrm{h}} \mathrm{i}: \mathrm{w} ?$ & *di:w 'lead' & *hriw ${ }^{\mathrm{C}}$ & $* \mathrm{di}: \mathrm{w}^{\mathrm{C}}$ \\
\hline plug up/(to) wedge & $*^{\mathrm{h}} \mathrm{i}: \mathrm{m}$ & --- & $* \operatorname{cim}(\mathrm{J})$ & $* \operatorname{ci}[:] \mathrm{m}$ \\
\hline $\begin{array}{l}\text { stone } \\
\text { sing }\end{array}$ & $*^{\mathrm{h}} \mathrm{i}$ i:n & *di:n & *hrin & $*$ di:n \\
\hline live, raw & *Curi:p & ${ }^{*} \mathrm{C}$-rjəp & *?dip & *Cudi[:]p \\
\hline peel cut off & *hli:t & --- & $* \operatorname{lit}(\mathrm{Li})$ & $* \operatorname{li}[:] \mathrm{t}$ \\
\hline bail water & *hwi:t & --- & *wit (Li) & ${ }^{*}$ wi[:] $]$ \\
\hline
\end{tabular}

(a) reap

mat grass

(b) 


\subsection{Long Mid Rimes}

In this category, there is regular agreement between PB and PSWT before sonorants, but the PB forms have high reflexes before stops; it is unclear whether or not this is always a result of borrowing from Hlai, or whether it is conditioned. The forms in (84b) indicate secondary shortening in PSWT. Finally there are three words $(84 \mathrm{c})$ in which there is an unexpected diphthong *iəC in PSWT:

$\underline{\text { Gloss }}$

(a)

wrinkle

cat

arm

slant $\sim$ tilt

chaff

carry

(b) centipede

fingernail

(c) blue

pull/stretch

frog

\section{$\underline{\text { Proto-Hlai }}$}

${ }^{*}$ C-ni:w?

*C-mi:wh

${ }^{*} \mathrm{k}^{\mathrm{h}} \mathrm{i}$ :

*ki:y

*hli:p

*C-bi:k

*ri:p

*C-li:p

${ }^{*} \mathrm{k}^{\mathrm{h}} \mathrm{i}$ :

*hji:t

*ri:t

\section{$\underline{\text { Proto-Be }}$}

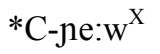

*[C-]me:w

*ke:n

---

(*C-lip)

$\left({ }^{*} \mathrm{C}-[\mathrm{b}] \mathrm{i}: \mathrm{k}\right)$

*rjəp

(*ri:p)

*he:w

---

$---$
PSWT

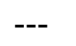

*me:w

${ }^{*} \mathrm{q}^{\mathrm{h}} \mathrm{\varepsilon}: \mathrm{n}$

$*_{\mathrm{g} \varepsilon: \mathrm{y}}(\mathrm{J})$

*kle:p

*?be:k

* $\mathrm{k}^{\mathrm{h}}$ ep (Li)

*lep

*xiow

*?jiat

* $\mathrm{k}^{\mathrm{h}}$ wiat $\underline{\text { PSKD }}$

${ }^{*} \mathrm{C}-$-ne: $\mathrm{w}^{\mathrm{C}}$

${ }^{*} \mathrm{C}^{\mathrm{V}}$-me: $\mathrm{w}^{(\mathrm{B})}$

*qe:n

*ge:y

*[k-]le:p

${ }^{*}$ C-pe:k

*q-de[:]p

$* \mathrm{~d}-\mathrm{le}[:] \mathrm{p}$

*xe:w

*C-je:t

$*$ q-de:t

\subsection{Short High Rimes}

PSWT vowel length agrees with PHl in (85a), but not in (85b). There are two exceptional forms in $(85 \mathrm{c})$ where the Hlai forms have a short $*_{\mathrm{iC}}$ rime, but the PB and PSWT forms have * $\mathrm{u}$. In the first case, it appears that the vowel was merely pre-empted by the coda in Be and SWT; in the second case, the Be form (if it is cognate) may have been influenced by the presyllable vowel: 
(86) $\quad$ Gloss

(a) taste
fly

(b) bracelet water leech

(c) mouse straw
Proto-Hlai

$*_{t}{ }^{\mathrm{h}}$ im

*C-bin

$*^{*}{ }^{h}$ im

*ljin

*hniw

*Cunin? $\underline{\text { Proto-Be }}$

PSWT

PSKD

*C-bjən

*--

*C-nu:

*mu: $\mathrm{y}^{\mathrm{X}}$ $*_{\text {fim }}$

*?bin

*gi:m (Li)

*pli:y

*hnu:

$--$ $*_{\text {fim }}$

${ }^{*}$ C-pin

*gi[:]m

*p[i]li[:]y

*H-niw

${ }^{*} \mathrm{C}^{\mathrm{V}}$ unin $^{\mathrm{C}}$

\subsection{Short Mid Rimes}

The forms with regular correspondences in this category are given below in (87a). The two forms in (87b) provide conflicting evidence for the length of the original PSKD rime:

$\underline{\text { Gloss }}$

(a) pick up

spicy

duck

mushroom

small

(b) lightning

wasp
Proto-Hlai $\quad \underline{\text { Proto-Be }}$

*sip

*rit

*C-bit

*C-dit

*C-lik

*[C-]1[j]ip

$* t^{\mathrm{h}}$ in

*C-ljap

$---$
PSWT

*kep

${ }^{*}$ p $\mathrm{h}$ et

*pet

*hret

*dlek (Li)

*mle:p

$* t \varepsilon: n$ $\underline{\text { PSKD }}$

*kep

*p-ret

*(C-)bet

$*[\mathrm{C}] \mathrm{et}$

*d-lek *m-le[:]p

*te[:]n

\subsubsection{High Back Unrounded Rimes}

The correspondences between $\mathrm{PHI}$ and PB/PSWT are presented below:

\begin{tabular}{|c|c|c|c|}
\hline Proto-Hlai & Proto-Be & PSWT & PSKD \\
\hline *u:C & *u:C & *u:C & *u:C \\
\hline *u:C & various & $*_{\mathrm{u}} \mathrm{C}$ & *u:C \\
\hline *u:C & $* \mathrm{iaC} \sim * \mathrm{a}: \mathrm{C}$ & *แəC & *uaC \\
\hline *uC & $*_{u} \mathrm{u}: \mathrm{C}$ & $*_{\omega} \cdot \mathrm{C}$ & $*_{\mathrm{u}}[\cdot] \mathrm{C}$ \\
\hline
\end{tabular}


There is a larger-than-average degree of variation and indeterminacy in this rime class, and the various instances of this will be discussed below. The evolution of Hlai from PSKD is given below, although the third row is unenlightening due to the uncertainty of the PSKD reconstruction:

\begin{tabular}{|c|c|c|c|}
\hline PSKD & Pre-Hlai & & Proto-Hlai \\
\hline *u:C & *u:C & $>$ & *u:C \\
\hline *uaC & *uəC & $>$ & *ur:C \\
\hline${ }^{*} \mathrm{ur}[:] \mathrm{C}$ & *uC & $>$ & $* u \mathrm{uC}$ \\
\hline
\end{tabular}

\subsection{Long High Back Unrounded Rimes}

In about half of the examples below (90a), PHl *u:C corresponds to PSWT *u:C (with some variation in PB), and these can be reconstructed as PSKD *u:C. There are also several cases where PHl *u:C rimes correspond with *u:C rimes in Be and/or SWT. In three of these cases (90b-c), the final is a velar stop; since PSWT has no examples of *u:k, it can be inferred that original *u:k rounded to *u:k in PSWT. However, in the example in (90c), it seems likely that the original rime was *u:k, and that the rounding transferred to the initial in Hlai, thereafter triggering dissimilation in the rime. Finally, the two examples in (90d) are indeterminate in the identity of the PSKD vowel:

\begin{tabular}{|c|c|c|c|c|}
\hline$\underline{\text { Gloss }}$ & $\underline{\text { Proto-Hlai }}$ & $\underline{\text { Proto-Be }}$ & $\underline{\text { PSWT }}$ & $\underline{\text { PSKD }}$ \\
\hline forget & *C-lu:mf & --- & *lur:m & ${ }^{*} \mathrm{C}^{\mathrm{V}}-\mathrm{lu}: \mathrm{m}^{(\mathrm{B})}$ \\
\hline full & $*^{\mathrm{h}} \mathrm{u}: \mathrm{m}$ & $* g[\mathrm{u} / \mathrm{u}]: \mathrm{m}$ & --- & *[g/y]u:m \\
\hline smooth & *hyuu:n & --- & ${ }^{*}$ mlur: ${ }^{\mathrm{B}}$ & $*^{*}$ mlur: $\mathrm{n}^{[\mathrm{B}]}$ \\
\hline shallow & $*^{\mathrm{h}} \mathrm{u}$ :n? & *tur: $\mathrm{n}^{\mathrm{X}}$ & *tur:n ${ }^{\mathrm{C}}$ & $*_{\text {tuu }: n^{C}}$ \\
\hline return & *C-lu:y & ${ }^{*}$ C-lum & --- & *C-lu[:]n \\
\hline child & *hlu:k & *luk & *lu:k & *lut:k \\
\hline bone & *Curw:k & ${ }^{*} \mathrm{C}$-rik & *?du:k & *Cudur:k \\
\hline weave (fabric) loom & $*_{\mathrm{t}} \int^{\mathrm{h}}$ wur:k & *huk & *hru:k & $*$ tu:k \\
\hline beard & *hmu:m? & ${ }^{*} \mathrm{mu}: \mathrm{m}^{\mathrm{X}}$ & --- & $* \mathrm{~m}[\mathrm{u} / \mathrm{u}]: \mathrm{m}^{\mathrm{C}}$ \\
\hline pull (scratch) $\sim$ scrape & $*^{\mathrm{h}} \mathrm{u}: \mathrm{t}$ & --- & *xu:t & $* x[\mathrm{u} / \mathrm{u}]: \mathrm{t}$ \\
\hline
\end{tabular}

(a)

(b)

(c)

\footnotetext{
pull (scratch) scrape
}

*hmu:m?
${ }^{*} \mathrm{k}^{\mathrm{h}} \mathrm{ur}: \mathrm{t}$

*xu:t

$* \mathrm{~m}[\mathrm{u} / \mathrm{u}]: \mathrm{m}^{\mathrm{C}}$ 


\subsection{Long Mid Back Unrounded Rimes}

The data implies, and there exists evidence, that the rimes in (91a) below are derived from a form with an original sequence of high-low vowels. Compare the PHl forms with *u:C rimes below with the Middle Chinese forms (Schuessler 2007) from which they are probably borrowed:

\begin{tabular}{|c|c|c|}
\hline Gloss & PHI & $\underline{\mathrm{MC}}$ \\
\hline two (a pair) & *C-lu:yf & $* \operatorname{liaj}^{\mathrm{B}}$ \\
\hline measure (rice) & *C-lu:y? & $* \operatorname{liay}^{\mathrm{C}}$ \\
\hline sword & *ku:mh & ${ }^{*}$ kiam $^{\mathrm{C}}$ \\
\hline ginger & $*^{\mathrm{h}} \mathrm{u}: \mathrm{y}$ & *kiay \\
\hline release & $*^{\text {*h }} u: \eta ?$ & *puay \\
\hline
\end{tabular}

What appears to have happened is that original *ia and *ua merged with *ia in both Hlai and Be, with the high vowel assimilating to the low central vowel. In Hlai, the assumption must then be made that the low half of the diphthong was eventually raised, perhaps through an intermediate stage (92) such as the one shown in the upcoming Tai examples. In Be, on the other hand, the high central vowel eventually fronted (93):

\begin{tabular}{|c|c|c|c|c|c|c|}
\hline$* \mathrm{iaC}$ & $>$ & *waC & $>$ & *шәC & $>$ & w:C \\
\hline *iaC & $>$ & *waC & $>$ & * шәC & $>$ & w:C \\
\hline *uaC & $>$ & *waC & $>$ & *шә & $>$ & w:C \\
\hline$* \mathrm{iaC}$ & $>$ & $*_{\mathrm{iaC}}$ & $>$ & $*_{\mathrm{iaC}}$ & & \\
\hline$*_{\mathrm{uaC}}$ & $>$ & $* \mathrm{iaC}$ & $>$ & $* \mathrm{iaC}$ & & \\
\hline
\end{tabular}

The Proto-Be form of house (94b) further suggests that in at least some cases, this complex nucleus may be the result of vocalic transfer of a high vowel from a former presyllable, and that this transfer is not always uniform in all three branches.

Finally, the words in (94c) appear to have originally been Pre-Hlai *u:C rimes which changed irregularly to *u:C rimes: 


\section{$\underline{\text { Gloss }}$}

(a)

(b)

(c)

egg

$$
\begin{aligned}
& \text { put down, let go } \\
& \text { ginger }^{48} \\
& \text { house } \\
& \text { bamboo shoot }
\end{aligned}
$$

castrate

\section{Proto-Hlai}

Proto-Be

PSWT

PSKD

${ }^{*} p^{h} u: y ?$

${ }^{*} k^{h} u: y$

${ }^{*} \operatorname{pia\eta }^{\mathrm{X}}$

*kjay

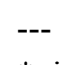

*qi:y

*puan $^{\mathrm{C}}$

*quan

*ru:n (Baohin) *ra:n

*C-nu:y

*na:y

*ruən

*nRa:y (Li)

*C[i/u]ra:n

${ }^{*} \mathrm{C}^{\mathrm{V}}[\mathrm{i} / \mathrm{u}]$ na:y

*hju:m

*C-du:n
$---$

*to:n
${ }^{*} \mathrm{C}$-jo[:]m

\begin{tabular}{|c|c|c|c|c|}
\hline$\underline{\text { Gloss }}$ & $\underline{\text { Proto-Hlai }}$ & $\underline{\text { Proto-Be }}$ & $\underline{\text { PSWT }}$ & $\underline{\text { PSKD }}$ \\
\hline handlength & *Cufiup & --- & *уu:p & *Cugu[:]p \\
\hline break (by pulling) & $*_{\mathrm{t}}^{\mathrm{h}} \mathrm{ut}$ & *tu:t 'split' & --- & $* \operatorname{tur}[:] \mathrm{t}$ \\
\hline heavy & $*^{\mathrm{h}}{ }^{\mathrm{u}}$ un & *xə:n & --- & *xu[:]n \\
\hline thorn & *Cufiun? & *?un & --- & ${ }^{*} \mathrm{Cu}[\mathrm{h}] \mathrm{un}^{(\mathrm{C})}$ \\
\hline frog & *?up & *op & *kop & $*[?] \mathrm{op}$ \\
\hline porcupine $\sim$ pangolin & *C-mun? & ${ }^{*} \mathrm{C}-\mathrm{mi}: \mathrm{n}^{\mathrm{X}}$ & $* \mathrm{hmen}^{\mathrm{C}}$ & $* \mathrm{C}-\mathrm{mVn}{ }^{\mathrm{C}}$ \\
\hline
\end{tabular}

*(C-)to:n

\subsection{Short Back Unrounded Rimes}

There doesn't seem to be a general PB/PSWT rime class which corresponds with PHI *uC:

The two forms in (95a) suggest an original *u vowel, but the length is uncertain. The PB form in (95b) is parallel with the PB reflex of PSKD *ur:, PB * $*$ :; what might condition the difference between this and *u:C is unclear. The word thorn in (95c), if the words are cognate, may have rounded in PB under the influence of an original presyllable vowel. The word frog in (95d) may be due to irregular unrounding of the nucleus: *?op $>*$ ?up $>*$ ?up. Finally, if porcupine and pangolin are legitimate comparanda (which may be doubtful), it is unclear how the three-way discrepancy in rimes originated.

\footnotetext{
${ }^{48}$ The vowel in the PSWT is unexpected, and may be the result of independent borrowing from Chinese.
} 


\subsubsection{High Back Rounded Rimes}

In general, the correspondences in this rime category mirror those of the high front rimes:

\begin{tabular}{|c|c|c|c|}
\hline Proto-Hlai & Proto-Be & $\underline{\text { PSWT }}$ & $\underline{\text { PSKD }}$ \\
\hline *u:C & $* \mathrm{u}: \mathrm{C} \sim{ }^{*} \mathrm{uC}$ & $* \mathrm{u}: \mathrm{C} \sim * \mathrm{u} \partial \mathrm{C}$ & ${ }^{*} \mathrm{u}: \mathrm{C}$ \\
\hline${ }^{*} \mathrm{u}: \mathrm{C}$ & $*_{\mathrm{u}} \mathrm{C}$ & $* \mathrm{uC}$ & $* \mathrm{u}[:] \mathrm{C}$ \\
\hline$*_{\mathrm{u}}: \mathrm{C}$ & *ə:C & $*_{0}: \mathrm{C}$ & $*_{0}: \mathrm{C}$ \\
\hline$*_{\mathrm{u}: \mathrm{C}}$ & *uəC & $*_{\mathrm{oC}}$ & $*_{\mathrm{o}[:] \mathrm{C}}$ \\
\hline *uy & *ay & $* \mathrm{i}:$ & *uy \\
\hline$* \mathrm{uC}$ & $* \mathrm{u}: \mathrm{C} \sim * \mathrm{uC}$ & $* \mathrm{uC}$ & $* \mathrm{uC}$ \\
\hline$*_{\mathrm{uC}}$ & *uəC & $*_{\mathrm{o}} \mathrm{C}$ & $*_{\mathrm{o}} \mathrm{C}$ \\
\hline
\end{tabular}

As with the high front rimes, there was a merger of the PSKD mid back rimes with the high back rimes in Hlai, due to the raising of the mid back vowels to high back vowels:

$\begin{array}{lllll}\underline{\text { PSKD }} & & \text { Pre-Hlai } & & \text { Proto-Hlai } \\ *_{\mathrm{u}: \mathrm{C}} & > & *_{\mathrm{u}: \mathrm{C}} & & *_{\mathrm{u}: \mathrm{C}} \\ *_{\mathrm{o}: \mathrm{C}} & > & *_{\mathrm{u}: \mathrm{C}}> & * \mathrm{u}: \mathrm{C} \\ *_{\mathrm{uC}}> & *_{\mathrm{uC}}> & * \mathrm{uC} \\ *_{\mathrm{oC}} & > & *_{\mathrm{uC}} & > & * \mathrm{uC}\end{array}$

\subsection{Long High Back Rounded Rimes}

There are two sets of rime correspondences (98a-b) which I reconstruct as PSKD *u:C, which may be in complementary distribution according to the place of the coda. The forms in (98c) are ambiguous between long and short rimes, and the latter between a high and mid vowel:

\begin{tabular}{|c|c|c|c|c|}
\hline (98) $\quad \underline{\text { Gloss }}$ & $\underline{\text { Proto-Hlai }}$ & $\underline{\text { Proto-Be }}$ & $\underline{\text { PSWT }}$ & $\underline{\mathrm{PSKD}}$ \\
\hline cotton & *C-bu:y? & *pu:y ${ }^{\mathrm{X}}$ & --- & $*(\mathrm{C}-)$ bu:y \\
\hline stand & *tcu:n & ${ }^{*} \mathrm{C}-[\mathrm{n} / \mathrm{j}] \mathrm{u}: \mathrm{n}$ & *2ju:n & $*$ C-cu:n \\
\hline wart & *su:c & --- & $*[\mathrm{~s} / \mathrm{h}] \mathrm{u}: \mathrm{t}$ & *su:c \\
\hline termite & *p-lu:k & *puk & *pluək & *p-lu:k \\
\hline wrap & $* \mathrm{t}^{\mathrm{h}} \mathrm{u}: \mathrm{k}$ & *tuk & --- & $* \mathrm{tu}: \mathrm{k}$ \\
\hline
\end{tabular}


(c)

mosquito

${ }^{*}$ C-nu:y

*nuəy

*nuy

${ }^{*} \mathrm{C}^{\mathrm{V}}$ - $\mathrm{nu}[:] \mathrm{y}$

nest

*ru:k

*ruək

${ }^{*} \mathrm{C}-\mathrm{d}[\mathrm{u} / \mathrm{o}][:] \mathrm{k}$

\subsection{Long Mid Back Rounded Rimes}

The first correspondence set in this group (99a) can be unambiguously reconstructed with *o:C rimes. The second (99b) is ambiguous between long ${ }^{\circ} \mathrm{o}: \mathrm{C}$ and short ${ }^{*} \mathrm{OC}$ :

$\underline{\text { Gloss }}$

before

hole

copper

inside brain, marrow

grey hair

(b)

tree clsfr/trunk
road
body/person
fart

\section{Proto-Hlai}

$*^{\mathrm{h}} \mathrm{u}$ :nf

$*^{\mathrm{h}} \mathrm{u}: \mathrm{y}$ ?

*C-du:y

*?u:k

*hyu:k

$*^{\mathrm{h}} \mathrm{u}$ :n?

*ku:n

*Cufu:n

$* t^{\mathrm{h}} \mathrm{u}: \mathrm{t}$

\section{Proto-Be}

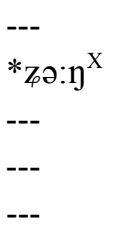

*tuən ${ }^{\mathrm{X}}$

*

(*hu:n)

*twat
PSWT

*ko: $n^{\mathrm{B}}$

$*^{2} \mathrm{y}: \mathrm{y}^{\mathrm{B}}$

*do:n

*?ว:k

*hyo:k

$* \operatorname{ton}^{\mathrm{C}}$

*hron

*yon

*tot $\underline{\text { PSKD }}$

*ko: ${ }^{\mathrm{B}}$

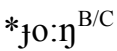

$*[\mathrm{C}-] \mathrm{do}: \mathrm{y}$

$*$ ?o:k

*H-no:k

*to[:] ${ }^{\mathrm{C}}$

*C-Co[:]n

*Cugo[:]n

*S-to[:]t

\subsection{Short High Back Rounded Rimes}

The rime in the first set of examples in this category (100a) can be reconstructed as *uy, having centralized and then lowered in PB. The second group can be reconstructed with *uC rimes. The final example is the lone example of $\mathrm{PHl} * \mathrm{uy}$, and is an exceptional correspondence.

$\underline{\text { Gloss }}$

(a) bear

drunk water buffalo $\sim$ cow

(b) warm

turbid

dig hole

take off

tail

\section{Proto-Hlai}

*C-muy

*hmuy

*suy?

*hlunf

*kunf

*Cifiut

*ljuc

$* \mathrm{c}^{\mathrm{h}} \mathrm{uc}$

\section{Proto-Be}

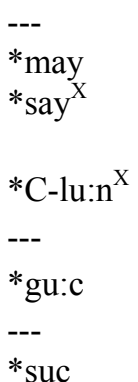

\section{PSWT}

*hmi:

---

$---$

*?un ${ }^{\mathrm{B}}$

${ }^{*} \mathrm{k}^{\mathrm{h}} \mathrm{un}^{\mathrm{B}}$

* $\mathrm{k}^{\mathrm{h}} \mathrm{ut}$

*hlwut (Li)

--- $\underline{\text { PSKD }}$

${ }^{*}$ C-muy

*muy

* suy ${ }^{\mathrm{C}}$

${ }^{*} \mathrm{H}-\operatorname{lun}^{\mathrm{B}}$

${ }^{*} \mathrm{C}$-gun ${ }^{\mathrm{B}}$

*Cigut

*Ciluc

* cuc 
(c) big $\quad *$ C-luy $\quad$--- $\quad *$ hluəy $\quad *$ C-luy

\subsection{Short Mid Back Rounded Rimes}

The majority of rimes in this category (101a) can be reconstructed as *oC. The length in (101b) is ambiguous, and the PB rime in (101c) seems to have undergone an irregular centralization and lowering:

$\underline{\text { Gloss }}$

(a) fur

rain

ant

(b) teach

(c) needle

Proto-Hlai
*Cufin
$*_{\text {fun }}$
*hmuc $^{\text {hmuc }}$
*sun $^{\text {*hyuc }}$

\subsubsection{Front Mid Rimes}

$\underline{\text { PSKD }}$

*wuən
$*$ fwən
$*$ muəc

q $^{\mathrm{h}}$ on

$*$ frn

*mot

*Cuqon

*fon

*moc

(*hu:n)

*so:n

$*_{\text {so[:]n }}$

*ya:c

$--$

There are only two examples of correspondences between PHl front mid vowels and PB/PSWT

forms. These correspondences are the following:

\begin{tabular}{|c|c|c|c|}
\hline Proto-Hlai & $\underline{\text { Proto-Be }}$ & $\underline{\text { PSWT }}$ & PSKD \\
\hline${ }^{*} \varepsilon: \mathrm{C}$ & $*_{\mathrm{e}: \mathrm{C}}$ & $*_{\varepsilon: C}$ & --- \\
\hline
\end{tabular}

The actual examples are the following:

$\underline{\text { Gloss }}$
board
press

\section{Proto-Hlai}

*6e:nh

*he:p

Proto-Be
${\text { *pe: }{ }^{\mathrm{X}}}^{\text {*ge:p }}$

$\underline{\text { PSWT }}$

${ }^{*} \mathrm{p} \varepsilon: \mathrm{n}^{\mathrm{C}}$

$---$ $\underline{\text { PSKD }}$

*pe:n?

${ }^{*}$ C-ge:p 
According to the discussion in section 4.2.3.1, these are exceptions to the rule that all Hlai front mid vowels raised to front high vowels in Pre-Hlai, which presents a conundrum. There are in fact only eight robust examples of PHl long front mid rimes, most of them without known Kra-Dai cognates (the two exceptions being the above board and press). One potential solution presents itself where most if not all of these are ultimately loans from Chinese, which entered Pre-Hlai after the raising of the front mid vowels:

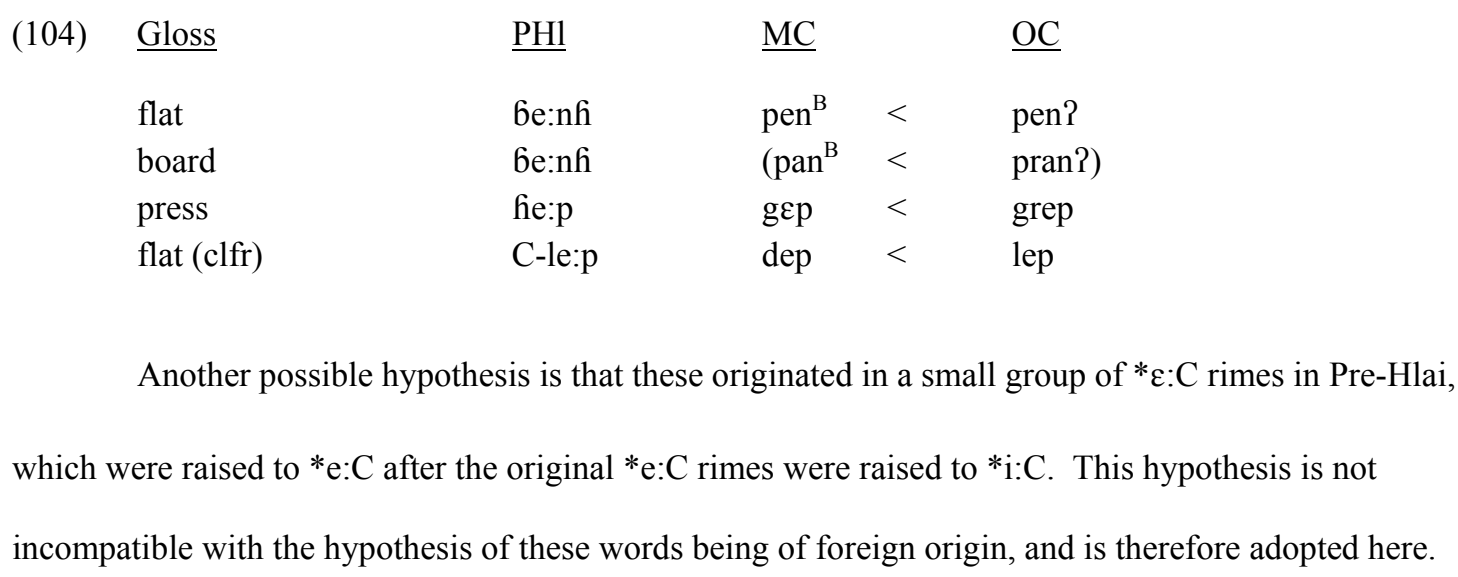

\subsubsection{Mid Central Rimes}

The reflexes of the mid central rimes, excepting some variation in PB for the PSKD short rimes, are for the most part straightforward. In general, the long and short rimes have merged completely in PSWT due to the shortening of the long rimes.

The case of PHl *ə:y is more complicated. It corresponds with PB and PSWT *a:y in all instances except saliva, in which it corresponds with PB *ay. It is tempting to suggest that PSKD *ə:y remained distinct from *a:y in Hlai, but lowered and merged with *a:y elsewhere. The only problem with this is the fact that there is a distributional gap in $\mathrm{PHl}$, where *a:y occurs only in categories B and C, with only one exceptional form (slow) in category A. This suggests that the original rime *a:y in category A raised in Hlai, merging with *ə:y. In light of the fact that there is no long PHI *ə:w reconstructed here, it is 
suggested that the Pre-Hlai development of the Hlai palatal-final diphthongs with mid central and low nuclei was the following:

\begin{tabular}{|c|c|c|c|}
\hline Pre-Hlai & $\underline{\text { Proto-Hlai }}$ & $\underline{\text { Pre-Hlai }}$ & $\underline{\text { Proto-Hlai }}$ \\
\hline *әу & *əy & *a:y > & *ə:y \\
\hline *əyh/? & *ə:yh/R & $* a: y h / 2>$ & *a:yh/2 \\
\hline
\end{tabular}

The following PSKD rimes are reconstructed under this hypothesis:

$\begin{array}{llll}\text { Proto-Hlai } & \text { Proto-Be } & \underline{\text { PSWT }} & \underline{\text { PSKD }} \\ *_{\partial: \mathrm{y}} & *_{\mathrm{a}[:] \mathrm{y}} & *_{\mathrm{a}: \mathrm{y}} & *_{\mathrm{a}: \mathrm{y}} \\ *_{\partial: \mathrm{C}} & *_{\mathrm{aC}} & *_{\mathrm{aC}} & *_{\partial: \mathrm{C}} \\ *_{\partial \mathrm{C}} & *_{\mathrm{a}[:] \mathrm{C} \sim *_{\partial}[:] \mathrm{C}} & *_{\mathrm{aC}} & *_{\partial \mathrm{C}}\end{array}$

Except for *a:y, the transition from PSKD to PHl was quite stable:

\begin{tabular}{|c|c|c|c|}
\hline$\underline{\text { PSKD }}$ & & Pre-Hlai & \\
\hline *a:y & $>$ & *a:y & $>$ \\
\hline *a:C & $>$ & *ə:C & $>$ \\
\hline əC & $>$ & & $>$ \\
\hline
\end{tabular}

\subsection{Long Mid Central Rimes}

Examples of the long mid central rimes are as follows:

(108) $\quad \underline{\text { Gloss }}$

(a) saliva

pass by

widow $^{49}$
Proto-Hlai

*hlə:y

*Curə:y

*hmə:y?

\section{Proto-Be}

*may

*ra:y

*C-ma:y ${ }^{\mathrm{X}}$
PSWT

*la:y

*kla:y

*hma:y $\underline{\text { PSKD }}$

*m-la:y

*kuRa:y

*H-ma:y ${ }^{\mathrm{C}}$

\footnotetext{
${ }^{49}$ This must be admitted as an exception to the hypothesis presented above.
} 
(b)

\begin{tabular}{|c|c|c|c|c|}
\hline fruit/testicles & $* t \int^{\mathrm{h}} \partial: \mathrm{m}$ & *ham & *hram & $*$ tə:m \\
\hline chop/slash & *və:n & --- & *van & ${ }^{*}$ C-bə:n \\
\hline field dike & *Cifə:n & --- & *yan & *Cigə: $\mathrm{n}$ \\
\hline smoke & *Cufə:n & $*_{\text {guan }}$ & *jwan & *Cugə:n \\
\hline skin & *C-nə:y & ${ }^{*} \mathrm{C}$-nay & *hnay & ${ }^{*}$ C-nə:n \\
\hline flea & *hmə:t & ${ }^{*} \mathrm{C}$-mat & *hmat & *H-mə:t \\
\hline wind; cold (sickness) & *hywə:t & --- & *hwat & *H-wə:t \\
\hline break off & $* c^{\mathrm{h}} \partial: \mathrm{k}$ & --- & *hrak & *to:k \\
\hline wash clothes & $*_{\mathrm{s}} \mathrm{s}: \mathrm{k}$ & $*$ dak & $*_{\text {zak }}$ & *zo:k \\
\hline deep & *hlə:k & *lak & 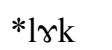 & *lo:k \\
\hline
\end{tabular}

There are several kinds of exceptions in this group. The first (109a) is a group of PB forms which have mid central rimes, some or all of which may be loans from Hlai. The second (109b) is a group of words which have irregularly low rimes in PSWT. The last three examples (109c) may all represent either irregular neutralization of the PHl nucleus, or otherwise irregular coloring of the nucleus in Tai:

Gloss
finish
times
soak
step on
sow, scatter
deceive
choke
bitter
pickle

\begin{tabular}{|c|c|c|c|}
\hline$\underline{\text { Proto-Hlai }}$ & $\underline{\text { Proto-Be }}$ & $\underline{\text { PSWT }}$ & $\underline{\text { PSKD }}$ \\
\hline *6ə:y? & ${ }^{*} \mathrm{C}-[\mathrm{b}] \partial: \mathrm{y}^{\mathrm{X}}$ & --- & ${ }^{*} \mathrm{C}-\mathrm{p} ә: \mathrm{y}^{\mathrm{C}}$ \\
\hline${ }^{*} \mathrm{C}-\beta \ni: \mathrm{y}$ & *va:y ${ }^{(\mathrm{X})}$ & --- & ${ }^{*}$ C-pə:y \\
\hline *da:m ${ }^{\mathrm{C}}$ & $* \cos : \mathrm{m}^{\mathrm{x}}$ & --- & $*(\mathrm{C}-)[\mathrm{t} / \mathrm{c}] \partial: \mathrm{m}^{\mathrm{C}}$ \\
\hline *tढə:mf & --- & *2ja:m ${ }^{\mathrm{B}}(\mathrm{Li})$ & ${ }^{*} \mathrm{C}-\mathrm{c}[\mathrm{\jmath} / \mathrm{a}]: \mathrm{m}^{\mathrm{B}}$ \\
\hline *fə:nh & --- & *hwa:n ${ }^{\mathrm{B}}$ & $*[\mathrm{f} / \mathrm{H}-\mathrm{w}][\mathrm{\rho} / \mathrm{a}]: \mathrm{n}^{\mathrm{B}}$ \\
\hline *p $\mathrm{p}^{\mathrm{h}} \partial: \mathrm{y}$ & --- & *bra:y & *br[ə/a]:n \\
\hline${ }^{*} \mathrm{k}^{\mathrm{h}} \mathrm{\partial}: \mathrm{n}$ ? & --- & $*_{\text {ge: }} \mathrm{n}^{\mathrm{C}}(\mathrm{Li})$ & $* \mathrm{~g}[\mathrm{e} / \partial]: \mathrm{n}^{\mathrm{C}}$ \\
\hline *hə:m & *ga:m & $*^{\mathrm{h}} \mathrm{om}$ & ${ }^{*} \mathrm{C}-\mathrm{G}[\curvearrowright: / \mathrm{o}] \mathrm{m}$ \\
\hline *C-mə:y & --- & *2blo:y & ${ }^{*} \mathrm{C}-\mathrm{bl}[\mathrm{\rho} / \mathrm{o}]: \mathrm{y}$ \\
\hline
\end{tabular}

\subsection{Short Mid Central Rimes}

Examples of the short mid central rimes are given below: 
(110)

$\underline{\text { Gloss }}$

far

gall bladder

chicken

we (incl)

horn

head hairknot

black

water

seed

tooth

yam

face/nose

burn/roast

forest $\sim$ mountain

cover

extinguish

rice

vine

strangle $\sim$ tight

chop/split
Proto-Hlai

*C-ləy

*dəy

*k $\mathrm{k}^{\mathrm{h}}$ y

*row

* frow

*Curəw?

*dəm?

${ }^{*} \mathrm{C}$-nəm?

*fjən

*fjon

*C-mən

*dəท

*rəy

*rəy

${ }^{*} \mathrm{k}^{\mathrm{h}}[\mathrm{\partial} / \mathrm{o}] \mathrm{p}$

*tcəp

*Curəp

*kəc

*rəc

*tcək
Proto-Be

*C-ləy

*C-rəy

*kay

$* \mathrm{~d}[\curvearrowright / \mathrm{a}] \mathrm{w}$

*wa:w

$\left(* \text { ha: }{ }^{\mathrm{X}}\right)^{50}$

${ }^{*}$ C-ram

*na:m ${ }^{\mathrm{X}}$

*wə:n

*sjon

*ma:n

*C-rəy

*C-rəy

* can

*xə[:]p

${ }^{*} \mathrm{C}$-[r/j]ap

*rap

* 6 ac

*[r]ət (D)

$---$
PSWT

PSKD

*klay

(*2bli:)

*kay ${ }^{\mathrm{B}}$

*k-ləy

*p-təy

*kəy ${ }^{(\mathrm{B})}$

*raw

* $\mathrm{q}^{\mathrm{h}}$ aw

*klaw ${ }^{\mathrm{C}}$

*row

*qow

*kurəw ${ }^{\mathrm{C}}$

*?dam

$* \mathrm{C}-[\mathrm{t} / \mathrm{d}] \partial \mathrm{m}^{[\mathrm{C}]}$

*nam ${ }^{\mathrm{C}}$

${ }^{*} \mathrm{C}^{\mathrm{V}}$-nəm ${ }^{\mathrm{C}}$

*van

*van

*man

*Cibən

*Cibən

${ }^{*} \mathrm{C}^{\mathrm{V}}$-mən

*?dan

*C-tən

${ }^{*} \mathrm{C}-\mathrm{d}$ y

*C-rəy

---

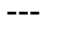

*?dap

*xəp

${ }^{*} \mathrm{C}$-[t/c]əp

*Cudəp

${ }^{*} \mathrm{C}-\mathrm{C} \partial \mathrm{c}$

---

*rət (Li)

${ }^{*} \mathrm{C}$-dəc

*cək (Li)

*(C-)cək

There are several sets of exceptions in this class as well. The first (111a) are probably originally original pure high vowels, which have diphthongized in Hlai. The second (111b) have high front vowels in PSWT, probably conditioned by a preceding palatal. The third (111c) are a pair of words where there are unexpected reflexes in both PB and PSWT. Body louse (111d) is a similar case, except that it has an irregular front vowel in PSWT. The final two examples (111e) contrast $\mathrm{PHl}{ }^{*} \partial \mathrm{C}$ with back vowels in PB/PSWT.

\footnotetext{
${ }^{50}$ Although the PB form looks very similar to the PHl and PSWT forms, it is ultimately more likely to be a borrowing from Hainanese haw $^{21}$.
} 
(111) Gloss

(a) you (pl)

stay, be at; alive

(b) eat

(c) silver day

(d) louse (body)

(e) drum mute
Proto-Hlai $\quad \underline{\text { Proto-Be }}$

*səw

*hməw

*hjow

${ }^{*} \mathrm{k}^{\mathrm{h}}$ ən

*C-nəp

*hyən

*hywən

$* \mathrm{t}^{\mathrm{h}}$ วn

*C-ləy

*C-nəm *su

*?mo:

${ }^{*} \mathrm{C}$-jəw ${ }^{\mathrm{X}}$

*kən

*nap

*yว:n

*wə:n

*də:n

*C-loy

$--$
PSWT

*su (Li)

*hmu:

*2ju: ${ }^{\mathrm{B}}$

*kin

*nip

*yrn

${ }^{*}$ wrn

*mlen

*klo:n

*ywo[:]m ${ }^{\mathrm{C}}(\mathrm{Li})$
PSKD

*su:

*H-mu:

${ }^{*} \mathrm{C}-\mathrm{ju}:^{(\mathrm{B})}$

${ }^{*} \mathrm{k}[\mathrm{j}] ə n$

${ }^{*} \mathrm{C}^{\mathrm{V}}$ - nәр

*yən

*ywən

*m-dən

$* \mathrm{k}-1[0] \mathrm{n}$

$* \mathrm{C}^{\mathrm{V}}-\mathrm{n}[0] \mathrm{m}$

\subsubsection{Mid Back Rimes}

The two main correspondence sets including $\mathrm{PHl}{ }^{*} \mathrm{OC}$ are the following:

\begin{tabular}{|c|c|c|c|}
\hline$\underline{\text { Proto-Hlai }}$ & $\underline{\text { Proto-Be }}$ & $\underline{\text { PSWT }}$ & $\underline{\text { PSKD }}$ \\
\hline${ }^{*} \mathrm{oC}$ & $*_{\mathrm{o}}[:] \mathrm{C}$ & $*_{\mathrm{oC}}$ & ${ }^{*} \mathrm{\jmath} \mathrm{C}$ \\
\hline${ }^{*} \mathrm{oC}$ & $* \partial[:] \mathrm{C}$ & $* a C$ & $*[\partial / \mathrm{\rho}] \mathrm{C}$ \\
\hline
\end{tabular}

The second of these indicates variation between PHI and PB/PSWT, not necessarily evidence for a separate set of rimes. Since the hypothesis in section 4.2.3.3 states that all Pre-Hlai ${ }^{\circ} \mathrm{oC}$ rimes raised to $*_{\mathrm{uC}}$, this set of correspondences cannot be reconstructed as ${ }^{\mathrm{o}} \mathrm{C}$ in PSKD. I therefore reconstruct a low back rime $*_{0} \mathrm{C}$, which raised to $*_{\mathrm{o}} \mathrm{C}$ in Hlai, filling the space vacated by original $*_{\mathrm{oC}}$ when it raised to $*_{\mathrm{uC}}$ :

$\underline{\text { PSKD }} \quad \underline{\text { Pre-Hlai }} \quad \underline{\text { Proto-Hlai }}$

$$
*_{\mathrm{o}} \mathrm{C} \quad *_{\mathrm{oC}}>*_{\mathrm{oC}}
$$

Examples of the correspondences in (112) are given below: 
Gloss

(a) hair

itch

simmer/boil

round

winnowing basket

fall

incubate

leg

(b) bran

neck
sieve
pain; sick
steal

Proto-Hlai

$\underline{\text { Proto-Be }}$

*s/hnom

* $\mathrm{k}^{\mathrm{h}} \mathrm{om}$

*p-lom?

*Curom

*doy?

$* t^{\mathrm{h}}$ ok

*C-mok

* $\mathrm{k}^{\mathrm{h}} \mathrm{ok}$

*rom

*ljoy?

$*[\mathrm{~d} / \mathrm{r}]$ on?

$* \mathrm{t}^{\mathrm{h}} \mathrm{ok}$

*ljok

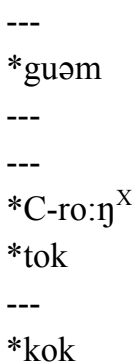

*-- $\operatorname{liən}^{\mathrm{X}}$

*ra:n

*dək

*rok
PSWT

*p ${ }^{\mathrm{h}}$ om

*yom

*tom ${ }^{\mathrm{C}}$

*klom

*?don ${ }^{\mathrm{C}}$

*tok

*hmok

---

*ram

---

$---$

$--$

*lak
PSKD

*p-dom

*yom

*[m-] tom ${ }^{\mathrm{C}}$

*kuRom

${ }^{*} \mathrm{C}$-ton ${ }^{\mathrm{C}}$

*tok

${ }^{*} \mathrm{C}-\mathrm{mok}$

*kok

*r[o/o $] \mathrm{m}$

${ }^{*} \mathrm{C}^{\mathrm{V}} \mathrm{il}[\mathrm{\rho} / \mathrm{\rho}] \mathrm{y}^{\mathrm{C}}$

*C-d[ə/o]y

$* \mathrm{~d}[\mathrm{\partial} / \mathrm{\rho}] \mathrm{k}$

$* \operatorname{dil}[\mathrm{\rho} / \mathrm{\rho}] \mathrm{k}$

There are only two sets of exceptions in this rime class. The first (115a) is two cases where

PSWT shows an unexpectedly high reflex *uC. The second $(115 b)$ is another two words in which ${ }^{*} \mathrm{C}$

may have sporadically lengthened in PSWT:

(115) Gloss

(a)

(b)

$$
\text { dye }
$$

hold w/ both hands

\section{Proto-Hlai}

*?om?

*6oy

*[hn]om?

* $\mathrm{k}^{\mathrm{h}} \mathrm{op}$
Proto-Be

---
$*[\mathrm{r} / \mathrm{j}] \mathrm{am}^{\mathrm{X}}$
*kup

$\underline{\text { PSWT }}$

*2um ${ }^{\mathrm{C}}$

*?bun (Li)

*no:m ${ }^{\mathrm{c}}$

*ko:p $\underline{\text { PSKD }}$

*?[u/o] $\mathrm{m}^{\mathrm{C}}$

${ }^{*} \mathrm{C}-\mathrm{p}[\mathrm{u} / \mathrm{s}] \mathrm{y}$

*nom ${ }^{\mathrm{C}}$

*k⿰p

\subsubsection{Low Rimes}

There are two primary kinds of correspondences including PHl low vowels: those with and

without the complications of vocalic transfer from preceding high vowels. These series are given below:

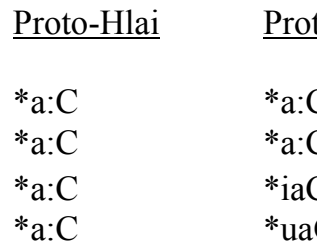

$\underline{\text { Proto-Be }}$

$* \mathrm{a}: \mathrm{C}$

$* \mathrm{a}: \mathrm{C}$

$* \mathrm{uaC}$ $\underline{\text { PSWT }}$

$* \mathrm{a}: \mathrm{C}$

*uəC

$--$

$* a: C$

\section{$\underline{\text { PSKD }}$}

$* \mathrm{a}: \mathrm{C}$

$*[\mathrm{i} / \mathrm{u}] \mathrm{Ca}: \mathrm{C}$

*iCa:C

$* \mathrm{uCa}$ :C 
The first series of the four above is by far the most numerous, and is uncomplicated. The second series is the next-best represented, and can be reconstructed as sesquisyllabic forms which had an initial high vowel that conditioned vocalic transfer and ultimate raising of the final vowel in PSWT with no effect in PB. The final two series are in the minority, and are examples of cases where vocalic transfer occurred in PB but not in PSWT, the converse of the second series. The evolution of these rimes in Hlai is straightforward:

$\begin{array}{llll}\underline{\text { PSKD }} & \underline{\text { Pre-Hlai }} & & \text { Proto-Hlai } \\ * \mathrm{a}: \mathrm{C}> & * \mathrm{a}: \mathrm{C}> & * a: \mathrm{C} \\ *[\mathrm{i} / \mathrm{u}] \mathrm{Ca}: \mathrm{C}> & * \mathrm{a}: \mathrm{C}> & * \mathrm{a}: \mathrm{C}\end{array}$

Examples are given below:

(118) $\underline{\text { Gloss }}$

(a)

sugarcane
star
fishy
salt
carry (2)
branch (tree)
ask
village
goose
lazy
chin
ribs
fly (n.)
carry on shoulder
bathe
yawn gasp
otter
sentence; mouth
land leech

\begin{tabular}{|c|c|c|c|}
\hline$\underline{\text { Proto-Hlai }}$ & $\underline{\text { Proto-Be }}$ & $\underline{\text { PSWT }}$ & $\underline{\text { PSKD }}$ \\
\hline${ }^{*}$ C-ma:y? & ${ }^{*} \mathrm{C}-\mathrm{ma}: \mathrm{y}^{\mathrm{X}}$ & --- & ${ }^{*} \mathrm{C}-\mathrm{ma}: \mathrm{y}^{\mathrm{C}}$ \\
\hline *ra:w & --- & *?da:w & ${ }^{*} \mathrm{C}$-da:w \\
\hline${ }^{*} \mathrm{k}^{\mathrm{h}} \mathrm{a}: \mathrm{w}$ & --- & *ya:w & *ya:w \\
\hline *C-na:w? & ${ }^{*}$ C-ла: $w^{X}$ & --- & ${ }^{*}$ C-ла:w ${ }^{C}$ \\
\hline${ }^{*} t \int^{h} a: m$ & *ha:m & *hra:m & $*$ ta:m \\
\hline *C-na:mf & --- & ${ }^{*}$ na:m ${ }^{\mathrm{B}}(\mathrm{Li})$ & $* \mathrm{C}^{\mathrm{V}}-$ na: $\mathrm{m}^{\mathrm{B}}$ \\
\hline *ra:m & --- & $*^{\mathrm{h}} \mathrm{a}: \mathrm{m}$ & $*_{\mathrm{t}-\mathrm{ra}: \mathrm{m}}$ \\
\hline${ }^{*} \mathrm{C}-\beta \mathrm{a}: \mathrm{n}$ & $\left(*\right.$ ?wว: $\left.{ }^{\mathrm{X}}\right)$ & *Pba: ${ }^{\mathrm{C}}$ & ${ }^{*}$ Cuba: ${ }^{\mathrm{C}}$ \\
\hline *C-na:nh & --- & $*$ ha: ${ }^{\text {B }}$ & $*^{*}$-ya: ${ }^{B}$ \\
\hline *C-la:n? & $* \operatorname{la}: n^{x}$ & $*_{\text {gra: }} \mathrm{n}^{\mathrm{C}}$ & ${ }^{*} \mathrm{~g}-\mathrm{Ra}: \mathrm{n}^{\mathrm{C}}$ \\
\hline *ha:y & *ya:N & *ga:y & ${ }^{*} \mathrm{C}$-ga: \\
\hline${ }^{*} \mathrm{k}^{\mathrm{h}} \mathrm{a}: \mathrm{y}$ ? & --- & ${ }^{*} \mathrm{k}^{\mathrm{h}} \mathrm{ra}: \mathrm{y}^{\mathrm{C}}$ & *kra: $\eta^{\mathrm{C}}$ \\
\hline *hwa:y? & $*_{w a}: y^{x}$ & --- & *wa: $\eta^{\mathrm{C}}$ \\
\hline$*_{t} \int^{h} a: p$ & *ha:p & *hra:p & $*$ ta:p \\
\hline *?a:p & --- & *?a:p & * $\mathrm{qa}: \mathrm{p}$ \\
\hline *hya:p & --- & *ya:p (Li) & *na:p \\
\hline *hna:k & *na:k & *na:k & *na:k \\
\hline *fa:k & *pa:k & *pa:k & *pwa:k \\
\hline$*^{\mathrm{h}} \mathrm{a} a \mathrm{k}$ & *ta:k & *da:k & *N-ta:k \\
\hline
\end{tabular}


(b)

$$
\begin{aligned}
& \begin{array}{l}
\text { moon } \\
\text { blood } \\
\text { yellow }
\end{array} \\
& \text { gadfly } \\
& \text { gill } \\
& \text { taro } \\
& \text { laugh } \\
& \text { fog } \\
& \text { boil }
\end{aligned}
$$

(c) laugh

(d) shrimp
vomit

*C-na:n
*hla:c
*lja:y
*lja:k
*C-ya:k
*ra:k

*Cira:w

*hya:w?

*C-da:n

*Cura:y

*fa:k

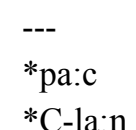

*C-la:y

*--
*aa:k
*a:k

*[C-]riaw

* niaw $^{\mathrm{X}}$

${ }^{*}$ C-rjan

*ruay

*duak

$\begin{array}{ll}\text { *2bluən } & \text { *C-b[i/u]la:n } \\ \text { *luət } & \text { *p[i/u]la:c } \\ \text { *hluəy } & \text { *Cila:y }\end{array}$

*hluək *Cila:k

*hyuak

$*^{\mathrm{p}}$ huək

${ }^{*} \mathrm{C}[\mathrm{i} / \mathrm{u}] \mathrm{na}: \mathrm{k}$

*p[i/u]ra:k

*Cida:w

*[i] ${ }^{*}{ }^{\mathrm{C}}{ }^{\mathrm{C}}$

${ }^{*}$ C[i]ta:n

*Cuda:y

*rwa:k

\begin{tabular}{|c|c|c|c|c|c|}
\hline (119) & $\underline{\text { Gloss }}$ & $\underline{\text { Proto-Hlai }}$ & $\underline{\text { Proto-Be }}$ & $\underline{\text { PSWT }}$ & $\underline{\text { PSKD }}$ \\
\hline (a) & more than & *kua? & $* \mathrm{kua}^{\mathrm{X}}$ & ${ }^{*} \mathrm{kwa}^{\mathrm{B}}$ & $*(\mathrm{C}-) \mathrm{kua}^{\mathrm{C} / \mathrm{B}}$ \\
\hline (b) & excrement & *ha:y? & $*_{\text {ga: }} \mathrm{y}^{\mathrm{X}}$ & ${ }^{*} \mathrm{k}^{\mathrm{h}} \mathrm{i}: \mathrm{C}$ & ${ }^{*} \mathrm{C}-\mathrm{Ga}: \mathrm{y}^{\mathrm{C}}$ \\
\hline (c) & orange/red & $* d[e]: n$ & ${ }^{*}$ C-rin & *?d $\varepsilon: y$ & $* \mathrm{C}-\mathrm{t}[\mathrm{e}]: \mathrm{y}$ \\
\hline (d) & pool, whirlpool & *hwa:y & --- & *wəy (Li) & ${ }^{*} w[ə / a:] \eta$ \\
\hline (e) & sheep & *hja:y & $*_{\text {zuay }}$ & --- & *ja:y \\
\hline (f) & tin iron & *hla:k 'tin' & --- & *hlek & --- \\
\hline
\end{tabular}

There are a small number of exceptions. The first (119a) is probably a loan from Chinese, and may not actually reconstruct back to PSKD. The second form (119b) has an irregular monophthong in PSWT. If the forms in $(119 \mathrm{c})$ are cognate, then this may be a rare instance of $\mathrm{PHl} * \mathrm{e}: \mathrm{y}$, but there is a discrepancy in vowel height in PB. The form in (119d) is irregular in both height and length. The word sheep in (119e) is very likely an old loan from Old Chinese *jay, and the PB medial *-u- is mysterious in this context. Finally, the word in (119f) has almost certainly been borrowed independently from Chinese, and no common ancestral form can be reconstructed. 


\subsubsection{Interim Summary}

The comparison of $\mathrm{PHl}$ rimes with those of $\mathrm{PB}$ and PSWT, as in the case of the initials, uncovers a core group of regular correspondences as well as a smaller but significant group of exceptions. This includes the tone categories, which generally match up regularly, but occasionally include mismatches between branches. These mismatches have not been explored here, as it is best to await the reconstruction of Proto-Tai and examine the place of PSWT therein before attempting to reconcile these mismatches at higher levels in the PSKD tree. It was demonstrated, however, that the PB tone system merged the PSKD $\mathrm{B}$ tone category with the $\mathrm{C}$ tone category, so that in effect tone category $\mathrm{B}$ was lost in $\mathrm{PB}$.

The tentative inventory of PSKD rimes, reconstructed here, which developed into the PHl rimes is the following:

\section{Open rimes}

$\begin{array}{lll}* \mathrm{i}: & *_{\mathrm{uu}} & *_{\mathrm{u}:} \\ \left(*_{\mathrm{e}}:\right) & *_{\mathrm{a}:} & \left(*_{\mathrm{o}}\right) \\ *_{\varepsilon}: & *^{\text {uи }} & \end{array}$

$\underline{\text { Closed rimes }}$

\begin{tabular}{|c|c|c|}
\hline$*_{\mathrm{i}} \mathrm{C}$ & *u:C & ${ }^{*} \mathrm{u}: \mathrm{C}$ \\
\hline$*_{\mathrm{iC}}$ & ${ }^{*} \mathrm{uUC}$ & $*_{\mathrm{uC}}$ \\
\hline$*_{\mathrm{e}} \mathrm{C}$ & $*_{\partial}: \mathrm{C}$ & $*_{0}: \mathrm{C}$ \\
\hline$*_{\mathrm{eC}}$ & $* \partial \mathrm{C}$ & $*_{\mathrm{oC}}$ \\
\hline${ }^{*} \varepsilon: C$ & $* \mathrm{a}: \mathrm{C}$ & \\
\hline & & $* \jmath \mathrm{C}$ \\
\hline
\end{tabular}

The most significant finding in this section is that the class of PSKD peripheral mid vowels underwent raising, merging with their high counterparts. It has also been hypothesized that PHI peripheral 
mid vowels must reflect original low vowels which raised to mid vowels, filling the gap left by the original mid vowels.

The reconstruction of the PSKD rime inventory ultimately reveals a nine-vowel system, with three levels of height and backness, the diphthong *ua, and a length distinction. The ${ }^{*} \varepsilon$ :C category is still marginal, and if ignored leaves an eight-vowel system which is essentially a seven-vowel system plus ${ }^{*} \mathrm{C}$. There is no present explanation for this asymmetry (however, comparison with Austronesian in chapter six indicates that $* \supset \mathrm{C}$ may be a variant of $* \partial \mathrm{C})$.

\subsection{Changes Between Pre-Hlai and Proto-Hlai}

The goal of this section is to move through the various changes which occurred after PSKD in PreHlai, leading ultimately to the inventory of initials and rimes reconstructible for Proto-Hlai. For every different change, the motivation for and mechanisms by which the change may have taken place are described if possible; consequences of the change are stated; and typologically relevant example from other languages are also provided.

\subsubsection{Elimation of Uvulars}

At some point in Pre-Hlai, uvular became defunct as a place of articulation. Evidence was presented above, based on the PSWT evidence, for the presence of the following categories in PSKD: *q, ${ }^{*} \mathrm{C}-\mathrm{q},{ }^{*} \mathrm{C}-\mathrm{G}$. The members of this category all initially merged with the velar series (systemic realignment), with the intervocalic velars later undergoing the normal change to glottal fricatives:

Pre-Hlai

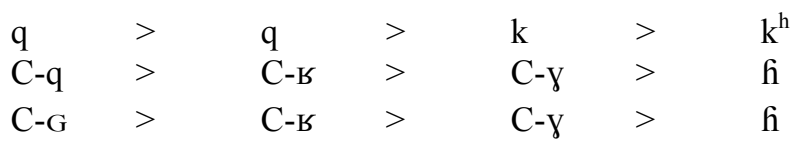

\section{Proto-Hlai}

$\mathrm{k}^{\mathrm{h}}$

h

h 
As presently reconstructed, there is no evidence for initial ${ }^{*} \mathrm{G}$ in the PSKD uvular inventory, which would have been expected to devoice and merge with * $\mathrm{q}$ in Pre-Hlai and be reflected as PHl * $\mathrm{k}^{\mathrm{h}}$. Further work in Tai or other Kra-Dai branches may uncover this evidence provided that the present reconstruction is accurate.

As a result of this change, the original Pre-Hlai oral stop inventory (122) was modified to that in (123):

$\begin{array}{lllll}\mathrm{p} & \mathrm{t} & \mathrm{c} & \mathrm{k} & \mathrm{q} \\ \mathrm{b} & \mathrm{d} & \mathrm{j} & \mathrm{g} & -- \\ \text { C-p } & \text { C-t } & \text { C-c } & \text { C-k } & \text { C-q } \\ \text { C-b } & \text { C-d } & \text { C-j } & \text { C-g } & \text { C-G }\end{array}$

$\begin{array}{llll}\mathrm{p} & \mathrm{t} & \mathrm{c} & \mathrm{k} \\ \mathrm{b} & \mathrm{d} & \mathrm{j} & \mathrm{g} \\ \mathrm{C}-\mathrm{p} & \mathrm{C}-\mathrm{t} & \mathrm{C}-\mathrm{c} & \mathrm{C}-\mathrm{k} \\ \mathrm{C}-\mathrm{b} & \mathrm{C}-\mathrm{d} & \mathrm{C}-\mathrm{j} & \mathrm{C}-\mathrm{g}\end{array}$

Examples are given below:

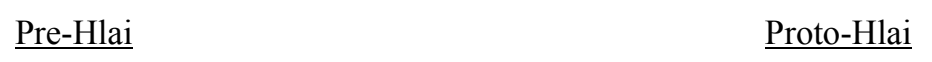

\begin{tabular}{|c|c|c|c|c|c|}
\hline (a) $\operatorname{arm}$ & *qe:n & $>$ & *ki:n & $>$ & ${ }^{*} \mathrm{k}^{\mathrm{h}} \mathrm{i}: \mathrm{n}$ \\
\hline thigh & ${ }^{*} \mathrm{C}-\mathrm{qa}:$ & $>$ & *C-ya: & $>$ & *ha: \\
\hline excrement & ${ }^{*} \mathrm{C}-\mathrm{Ga}: \mathrm{y} ?$ & $>$ & ${ }^{*}$-у-уа:y? & $>$ & *ha:y? \\
\hline
\end{tabular}

\subsubsection{Intervocalic Lenition}

It was shown in section 4.1 that Pre-Hlai medial obstruents underwent a process of intervocalic lenition. This intervocalic lenition can be seen as a reduction in the magnitude and/or increase in the sonority of intervocalic gestures, so that voiceless obstruents became voiced (125a), and voiced stops 
became approximants (125b). The evidence in section 4.1 shows that there was no lenition of intervocalic voiced fricatives (125c). The two kinds of intervocalic stop lenition ${ }^{51}$ are shown below:

(a) $\begin{aligned} \mathrm{CVpV} & >\mathrm{CVbV} \\ \mathrm{CVtV} & >\mathrm{CVdV} \\ \mathrm{CVcV} & >\mathrm{CV} \mathrm{V} \\ \mathrm{CVkV} & >\mathrm{CVgV} \\ \mathrm{CVfV} & >\mathrm{CVvV}\end{aligned}$

(b) $\begin{array}{ll}\mathrm{CVbV} & >\mathrm{CVuV} \\ \mathrm{CVdV} & >\mathrm{CV} \mathrm{V} \\ \mathrm{CV} \mathrm{V} V & >\mathrm{CVJV} \\ \mathrm{CVgV} & >\mathrm{CVhV} \\ \mathrm{CVvV} & >\mathrm{CVvV}\end{array}$

This is an archetypical example of temporal compression, resulting in a diminuition of duration of intervocalic consonants:

$$
\begin{gathered}
\varphi \\
/ \\
{\left[\varsigma \sigma_{\mu \mu}\right]} \\
/ \wedge \\
{[\mathrm{CvCV}]} \\
\uparrow
\end{gathered}
$$

Site of temporal compression

Lavoie (1999) argues that this kind of lenition can be seen primarily as a reduction in timing, where a shorter voiceless stop may be perceived as a voiced stop, and a shorter voiced stop may be perceived as an approximant. The consonants most vulnerable to lenition are those which occupy the onset position of the second syllable of a bisyllabic foot. It is important to note that her findings do not predict there to be a lenition from voiced stop to approximant through the intermediate stage of a fricative, but rather directly from one to the other.

The comparison with PB and PSWT (in which these categories merged) indicates that this occurred as a chain-shift in Hlai: original intervocalic voiced stops were first reanalyzed as approximants, leaving a gap in the system which was later filled when the original voiceless stops were reanalyzed as voiced:

${ }^{51}$ The lenition in (125b) was originally suggested in Ostapirat (2004); that in (125a) is suggested here for the first time. 

(i)
$\mathrm{CVbV}>$
$\mathrm{CVuV}$
(ii)
$\mathrm{CVpV}>$
$\mathrm{CVbV}$

The process of intervocalic lenition which Pre-Hlai medial consonants underwent is similar to changes which occurred in Spanish (Harris-Northall 1990:6). In Spanish, there was a three-way contrast between intervocalic geminates, voiceless consonants, and voiced consonants; geminates became singletons, voiceless stops became voiced, and voiced stops became voiced fricatives:

\begin{tabular}{|c|c|c|c|c|c|c|c|c|}
\hline \multicolumn{3}{|c|}{ Geminate stop to plain stop } & \multicolumn{3}{|c|}{ Plain stop to voiced stop } & \multicolumn{3}{|c|}{ Voiced stop to fricative } \\
\hline VppV & $>$ & $\mathrm{VpV}$ & $\mathrm{VpV}$ & $>$ & $\mathrm{VbV}$ & $\mathrm{VbV}$ & $>$ & $\mathrm{V} \beta \mathrm{V}$ \\
\hline $\mathrm{VttV}$ & $>$ & $\mathrm{VtV}$ & $\mathrm{VtV}$ & $>$ & VdV & $\mathrm{VdV}$ & $>$ & VðV \\
\hline VkkV & $>$ & $\mathrm{VkV}$ & $\mathrm{VkV}$ & $>$ & $\mathrm{VgV}$ & $\mathrm{VgV}$ & $>$ & $\mathrm{V} \otimes \mathrm{V}$ \\
\hline
\end{tabular}

Pre-Hlai did not have medial geminates, but otherwise the changes which it underwent seem to have been ultimately quite similar to the Spanish case.

Another typological parallel of this change is provided in Ferlus (1982), which describes intervocalic lenition in Vietnamese similar to that posited here for Pre-Hlai. In Vietnamese, intervocalic stops (both voiced and voiceless) were lenited to fricatives and approximants, some of which went on to merge with other phonemes. The schema which Ferlus outlines is the following (which I have modified by reversing the order he suggests for intervocalic voicing and lenition, avoiding spirantization of intervocalic voiceless stops):

\begin{tabular}{|c|c|c|c|c|c|c|}
\hline Proto-VN & & Voicing & & Lenition & & 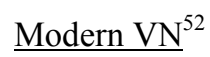 \\
\hline$-p / b-$ & $>$ & $-b-$ & $>$ & $-\beta-$ & $>$ & $\mathrm{v}$ \\
\hline$-t / d-$ & $>$ & $-d-$ & $>$ & -ð- & $>$ & $\mathrm{z}<\mathrm{d}>$ \\
\hline$-\mathrm{S} / \mathrm{C}-$ & $>$ & $-z-$ & $>$ & $-r-$ & $>$ & $\mathrm{z}<\mathrm{r}>$ \\
\hline$-\mathrm{t} \int / \mathrm{d} z-$ & $>$ & $-d 3-$ & $>$ & $-j-$ & $>$ & $\mathrm{z}<\mathrm{gi}>$ \\
\hline$-c / \mathfrak{y}^{-}$ & $>$ & $-\mathrm{y}^{-}$ & $>$ & $-j-$ & $>$ & $\mathrm{z}<\mathrm{gi}>$ \\
\hline$-\mathrm{k} / \mathrm{g}-$ & $>$ & $-g-$ & $>$ & $-8-$ & $>$ & $\mathrm{y}<\mathrm{g}(\mathrm{h})>$ \\
\hline
\end{tabular}

\footnotetext{
${ }^{52}$ The convention is followed here of placing orthography within angled brackets.
} 
Below are some examples of Vietnamese forms which have undergone lenition and subsequently lost their initial syllable. They are compared with Thavung, a related language (Hayes 1983) in which this intervocalic lenition has not occurred:

(130) Comparison of Vietnamese (lenited) with Thavung (non-lenited)

\begin{tabular}{|c|c|c|}
\hline English & Vietnamese & Thavun \\
\hline cotton & $<$ vảy $>$ [v-] & $\mathrm{kpa}: \mathrm{c}^{4}$ \\
\hline testicle & $<$ dáy $>$ [z-] & kta: $1^{1}$ \\
\hline paw & $<$ giò $>\quad[\mathrm{z}-1$ & kco \\
\hline bear & $<$ gâu $>$ [Y-] & $\mathrm{cku}^{4}$ \\
\hline
\end{tabular}

Intervocalic lenition may have also occurred in Proto-Min, in the case of what Norman (1986) calls the 'softened initials'. There is evidence in the Northern Min group for three series of initials which may be distinguished both segmentally and tonally: (a) aspirated, (b) plain, and (c) 'softened'. Each of these groups can be subdivided into groups (on the basis of register indicated by the tone) with original voiceless initials and with original voiced initials:

(131) Northern Min initial reflexes (adapted from Handel 2003)

$$
\text { JO JQ SB CC JY Proto-Min (present interpretation) }
$$

(a)

$\begin{array}{llllll}\mathrm{p}^{\mathrm{h}} & \mathrm{p}^{\mathrm{h}} & \mathrm{p}^{\mathrm{h}} & \mathrm{p}^{\mathrm{h}} & \mathrm{p}^{\mathrm{h}} & * \mathrm{p}^{\mathrm{h}} \\ \mathrm{t}^{\mathrm{h}} & \mathrm{t}^{\mathrm{h}} & \mathrm{t}^{\mathrm{h}} & \mathrm{t}^{\mathrm{h}} & \mathrm{t}^{\mathrm{h}} & * \mathrm{t}^{\mathrm{h}} \\ \mathrm{k}^{\mathrm{h}} & \mathrm{k}^{\mathrm{h}} & \mathrm{k}^{\mathrm{h}} & \mathrm{k}^{\mathrm{h}} & \mathrm{k}^{\mathrm{h}} & * \mathrm{k}^{\mathrm{h}}\end{array}$

(b)

$\begin{array}{llllll}\mathrm{p} & \mathrm{p} & \mathrm{p} & \mathrm{p} & \mathrm{p} & * \mathrm{p} \\ \mathrm{t} & \mathrm{t} & \mathrm{t} & \mathrm{t} & \mathrm{t} & * \mathrm{t} \\ \mathrm{k} & \mathrm{k} & \mathrm{k} & \mathrm{k} & \mathrm{k} & * \mathrm{k}\end{array}$

(c)

$\begin{array}{llllll}\mathrm{p} & \mathrm{p} & \mathrm{b}^{\mathrm{h}} & \mathrm{w} & \mathrm{w} & * \text {-p- } \\ \mathrm{t} & \mathrm{t} & \mathrm{d}^{\mathrm{h}} & 1 & 1 & * \text {-t- } \\ \mathrm{k} & \mathrm{k} & \mathrm{g}^{\mathrm{h}} & \varnothing & \varnothing & *-\mathrm{k}-\end{array}$

The five dialects above show segmental correspondences which fall into three groups for the 'softened' initials of (130c): (1) non-leniting (JO and JQ), (2) voicing (SB), and (3) leniting (CC and JY). Norman 
(1986) provides evidence for original presyllables (particularly nasals, based on Hmong-Mien loanwords) in this group of lexical items.

Handel (2003) argues convincingly that the tone reflexes of group (c) in Northern Min were conditioned by stops with an original breathy voice phonation; however, the segmental reflexes in the 'softening dialects', where what Handel reconstructs as originally breathy voiced stops are reflected as sonorants, remain unexplained. I believe that this is another example of the intervocalic lenition which we have been observing in other languages, and that they may have followed a similar progression as that postulated here for Hlai:

$\begin{array}{lllllll}-\mathrm{p}- & > & -\mathrm{b}- & > & -\mathrm{v}- & > & \mathrm{w} \\ -\mathrm{t}- & > & -\mathrm{d}- & > & -\mathrm{f}- & > & 1 \\ -\mathrm{k}- & > & \text {-g- } & > & -\mathrm{f}- & > & \varnothing\end{array}$

The differences in reflexes in these three groups were likely conditioned by the persistence of the presyllable. If presyllables were lost early, then little if any lenition occurred; if, on the other hand, presyllables persisted for a longer period of time, the environment was preserved which facilitated lenition, which proceeded to occur cyclically.

The Pre-Hlai lenition erased the class of intervocalic voiceless obstruents, and added a new series of approximants into the Hlai phoneme inventory which was allophonic (at this point) in word-medial position. In general, the word-initial (133a) and intervocalic (133b) obstruent inventories became disjoint, with the identity of initials becoming partially dependent on their prosodic position within the phonological word:

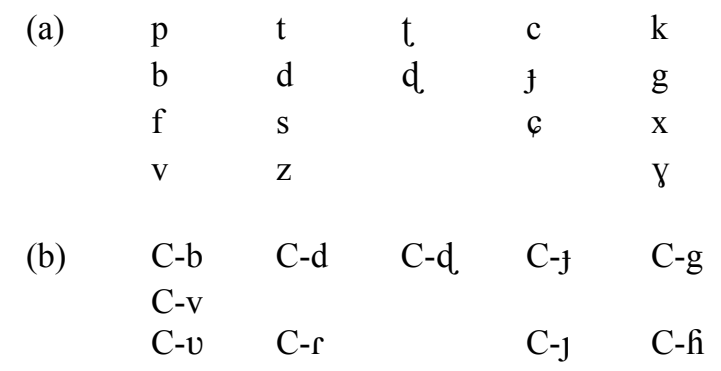


Examples are given below:

\section{Pre-Hlai}

$$
{ }^{*} \mathrm{C} \text {-pin }
$$$$
\text { *C-təy }
$$$$
\text { *C-cu:n }
$$

stand

slant

(b)

shoulder
hang
medicine
chin

${ }^{*} \mathrm{C}$-ke:y

*C-ba:h

${ }^{*}$ C-di:y?

${ }^{*} \mathrm{C}-\mathrm{ja}$ :

*C-ga:y
Proto-Hlai

*6in

*dəy

*tcu:n

*ki:y

*va:h

*ri:y?

*hja:

*ha:y

\subsubsection{Initial Devoicing}

The first instance of devoicing occurred in Pre-Hlai, affecting initial obstruents in both monosyllabic (135a-b) and sesquisyllabic words $(135 \mathrm{c}-\mathrm{d}$ - remember that $* \mathrm{H}$ - represents an initial fricative in a sesquisyllabic form). The cumulative list of devoiced initials from section 4.2 is given below. There is no direct evidence for (135d) as mergers in PHI, PB and PSWT would have obscured it as an independent category, but it is listed below as a logical possibility:

(a)

$\begin{array}{lll}* \mathrm{~b} & > & *_{\mathrm{p}} \\ *_{\mathrm{d}} & > & *_{\mathrm{t}} \\ *_{\mathrm{d}} & > & *_{\mathrm{t}} \\ *_{\mathrm{f}} & > & *_{\mathrm{c}} \\ *_{\mathrm{g}} & > & *_{\mathrm{k}}\end{array}$

(c)

$*^{\mathrm{V}}-\mathrm{C}>\quad * \mathrm{C}-\mathrm{C}$ (b)

$\begin{array}{lll}*_{\mathrm{v}} & > & *_{\mathrm{f}} \\ *_{\mathrm{z}} & > & *_{\mathrm{s}} \\ *_{\mathrm{y}} & > & *_{\mathrm{x}}\end{array}$

(d) $\quad * \mathrm{H}^{\mathrm{V}}-\mathrm{C}>\quad * \mathrm{H}-\mathrm{C}$

As a result of this change, the Pre-Hlai initial obstruent inventory (136) was modified to that in (137): 
(136)

$\begin{array}{ccccc}\mathrm{p} & \mathrm{t} & \mathrm{t} & \mathrm{c} & \mathrm{k} \\ \mathrm{b} & \mathrm{d} & \mathrm{d} & \mathrm{f} & \mathrm{g} \\ \mathrm{f} & \mathrm{s} & & \mathrm{c} & \mathrm{x} \\ \mathrm{v} & \mathrm{z} & & & \mathrm{d}\end{array}$

C-C

$\mathrm{C}^{\mathrm{V}}-\mathrm{C}$

$\mathrm{H}-\mathrm{C}$

$\mathrm{H}^{\mathrm{V}}-\mathrm{C}$

(137)

$\begin{array}{lllll}\mathrm{p} & \mathrm{t} & \mathrm{t} & \mathrm{c} & \mathrm{k} \\ \mathrm{f} & \mathrm{s} & & 6 & \mathrm{x}\end{array}$

C-C

$\mathrm{H}-\mathrm{C}$

Examples are given below:

\section{Pre-Hlai}

(a)

father
rotten
stone
hole
bracelet
sky
wash clothes
itch
yam
branch (tree)
fingernail

fingernail

*ba:?
*duy
*di:n
*fo:y?
*gim

*va:?

*zว:k

*yom

$* \mathrm{C}^{\mathrm{V}}$-mən

${ }^{*} \mathrm{C}^{\mathrm{V}}$-na:mh

${ }^{*} \mathrm{C}^{\mathrm{V}}$-le:p 
nasal (y), coronal approximants ( $(,, r)$, and glottals ( $(\mathrm{h}, ?)$. There were also a group of initials reconstructed in PHl which already hosted coarticulations:

$$
\begin{aligned}
& * \mathrm{fj} \\
& * 1 \mathrm{j} \\
& * \mathrm{cj}
\end{aligned}
$$

As discussed above, these are also cases of vocalic transfer. It appears as though vocalic transfer was first favored in the case of $*_{\mathrm{i}}$ preceding the coronal liquids $*^{1}$ and ${ }^{*} \mathrm{f}$, and therefore occurred early. There are only three known instances of $* \mathrm{fj}$, so it seems that these were rather exceptional cases, probably due to the fact that *f was an obstruent (it is apparent in general that more sonorous consonants are weaker barriers to vocalic transfer than less sonorous consonants):

$$
\begin{array}{lll}
\text { Civ } & > & \text { Civj } \\
\text { Cil } & > & \text { Cilj } \\
\text { Cir } & > & \text { Cirj }
\end{array}
$$

The asymmetry between the lengths of the two syllables in sesquisyllabic forms may have played a role in this change as well. In the examples below, the boundary between the two vowels of a word is shown in (141a) a full bisyllabic word where the medial consonant acts as a clearly defined boundary, and in (141b) the sesquisyllabic equivalent, where there is bleedthrough as the vowel space remains roughly proportionate in overall timing, despite the imbalance in syllable length:
(a)

$$
\begin{aligned}
& \text { Cilay } \\
& {[\mathrm{V} \mid \mathrm{V}]}
\end{aligned}
$$$$
>
$$
(b) Ciljá:n $[\mathrm{V} \mid \mathrm{V}]$

There are two typological parallels which can be cited as examples of intersyllabic vocalic transfer. The first is Proto-Paman, the roots of which were normally bisyllabic trochees, with contrastive vowel length in the first syllable (Smith (1997)). In several North Paman languages, including Awnțim, stress shift occurred and the final syllable became stressed. In words with a short initial syllable, the initial consonant was lost and features of the vowel were transferred into the remaining syllable (142a). In words 
with a long initial syllable, the same phenomenon occurred, but a vestigial schwa remained at the beginning of the word (142b).

(a)

English
mother's mother
skin
arm
3 sg obl.
sun
tooth

(b)

English
what
armpit
south
smoke
father's father
two

The second example is of the Oceanic languages Rotuman and Kwara'ae (see Blevins \& Garrett 1998, Heinz 2005, Norquest 2001, 2003), both of which have a register distinction where in the conservative (citation) register, syllables are of the shape $\mathrm{CV}$, but in the innovative (discourse) register, there is a metathesis of $C_{2}$ and $V_{2}$ within the foot. This is essentially the mirror-image of the vocalic transfer which is postulated for Hlai and shown in Awntim above, and is correlated with stress: trochaic in the case of Rotuman and Kwara'ae, as opposed to iambic in Hlai and Awntim. Note also that this case involves a shift from the syllable (separated by periods in the examples below) to the foot as the primary domain of timing and stress:

\footnotetext{
${ }^{53}$ Notice the lenition of Awntim intervocalic stops, similar to that posited for Hlai.
} 
$\underline{\text { Rotuman }}$

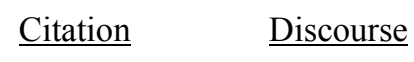

floor erroneous people

zealous

$$
\text { pu.pú.i }
$$$$
\text { se.sé.va }
$$

fa.mo.ri

fe.Péni

pu.púi
se.séav
fa.mǿr
fe.?én

to climb
to bail
seaweed
their name
$\underline{\text { Citation }}$

Kwara'ae

It is significant that in the data of both Blevins \& Garrett (1998) and Heinz (2005), words in the Kwara'ae discourse register show optional voiceless vowels following certain consonants in their corresponding position in the citation register:

(144) Kwara'ae (Blevins \& Garrett 1998: 530; Heinz 2004: 29)

\begin{tabular}{|c|c|c|c|c|c|}
\hline & $\underline{\text { Citation }}$ & $\underline{\text { Discourse }}$ & & $\underline{\text { Citation }}$ & Discourse \\
\hline cat & fúsi & húissi & fear & má?u & máựụ \\
\hline thin & kádo & káodo & wife & Páfe & Páenè \\
\hline name & sáta & sá:tạ & to burst & búsu & bú:su \\
\hline
\end{tabular}

This indicates the historical process by which this metathesis occurred involved an original articulation of $\mathrm{V}_{2}$ on both sides of $\mathrm{C}_{2}$, with later devoicing and loss of the latter half of $\mathrm{V}_{2}$ altogether. The corresponding situation in Hlai may have been similar, occurring on the opposite edge of the word:

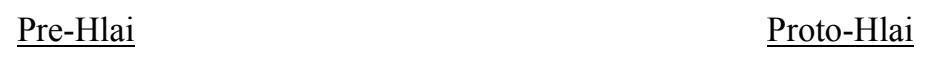

tooth

*Civán $\quad>\quad$ *Civján $\quad>\quad *$ fjón

The consequences of this change are that high vowel information from the presyllable became associated with the main syllable initial and was therefore preserved within the domain of the main syllable. This complicated the medial consonant inventory by creating a new class of palatalized medial consonants, shown in (146) below: 


$$
\begin{aligned}
& \begin{array}{lllll}
C-b & C-d & C-j & C-g & \\
C-v(j) & & & & C-h
\end{array} \\
& \text { C-m C-n } \quad \text { C- } n \quad \text { C- } n \\
& \text { C-v } \quad C-r(j) \quad \text { C- }- \\
& \text { C-1(j) C-r }
\end{aligned}
$$

Examples are given below:

Pre-Hlai

(a) tooth

(b) yellow

(c) laugh

\begin{tabular}{|c|c|c|c|}
\hline & & & $\underline{\text { Proto-Hlai }}$ \\
\hline$>$ & ${ }^{*} \mathrm{C}$-vjən & $>$ & $*_{\text {fjon }}$ \\
\hline$>$ & ${ }^{*} \mathrm{C}-\mathrm{lja}: \mathrm{y}$ & $>$ & *lja:y \\
\hline$>$ & ${ }^{*} \mathrm{C}-\mathrm{rja}: \mathrm{w}$ & $>$ & $*_{\text {rja:w }}$ \\
\hline
\end{tabular}

*Civən

*Cila:y

*Cida:w

\subsubsection{Initial Aspiration}

The hypothesis presented above in section 4.2 is that the more exotic aspects of the PHl consonant inventory were shaped by an increasing differentiation between the series of initial and medial consonants. More specifically, this differentiation occurred via a form of fortition of word-initial consonants, which involved increased airflow at the left edge of monosyllabic words leading to the aspiration of all initial consonants except the glottal stop. That this change did not affect presyllable consonants is demonstrated by the fact that $* \mathrm{p}-1$ did not change to ${ }^{*} \mathrm{p}^{\mathrm{h}}-1$. It can therefore be stated that aspiration only occurred at the left edge of a word which was aligned with the left edge of a main syllable (and was therefore correlated with stress) (148a); aspiration did not occur when if these categories were unaligned (148b):
(a) $\quad\left[\left(C V^{\prime}\right)\right]$
$\uparrow$
Target of aspiration
(b) $\quad[(\mathrm{C} \breve{v})(\mathrm{CV}:)]$ $\uparrow \uparrow$ Exempt from aspiration

The Pre-Hlai initial consonants which were affected are listed below: 
(a) Obstruents

$\begin{array}{lll}\mathrm{p} & > & \mathrm{p}^{\mathrm{h}} \\ \mathrm{t} & > & \mathrm{t}^{\mathrm{h}} \\ \mathrm{t} & > & \mathrm{t}^{\mathrm{h}} \\ \mathrm{c} & > & \mathrm{c}^{\mathrm{h}} \\ \mathrm{k} & > & \mathrm{k}^{\mathrm{h}}\end{array}$

(b) Sonorants

$\begin{array}{lll}\mathrm{m} & > & \mathrm{hm} \\ \mathrm{n} & > & \mathrm{hn} \\ \mathrm{j} & > & \mathrm{h} \\ \mathrm{y} & > & \mathrm{h} \eta \\ \mathrm{jw} & > & \mathrm{h} \eta \mathrm{w} \\ \mathrm{l} & > & \mathrm{hl} \\ \mathrm{w} & > & \mathrm{hw} \\ \mathrm{j} & > & \mathrm{hj}\end{array}$

Those which were not affected included the glottal stop, the rhotic liquid (which does not show any evidence of a distinction between initial and medial position in $\mathrm{PHl}$ ), and probably the fricatives (as they were already redundantly aspirated).

In light of the above discussion, the following description of Loloish (a branch of Tibeto-Burman) languages from Bradley (1978) is very interesting:

'One interesting phenomenon in Maru and several other Burmish languages is the existence of aspirated, glottalized, and voiced stops in all positions of articulation. The glottalized stops are the reflexes of certain *prefixed Proto-BL forms, while the aspirated stops are the reflexes of unprefixed *voiceless stops. This contrast is not found in Burmese dialects, which have merged the two manners of articulation to voiceless aspirated, as in 'standard' Burmese.' [emphasis mine]

Here, then, is an example in the Loloish family where there is a contrast between 'plain' initials which exhibit allophonic aspiration, and a glottalized set of initials, which is the result of original presyllables.

The most important change this effected was to modify the initial inventory of monosyllabic words from that in (150) to that in (151), leaving the only unaspirated initial the rhotic ${ }^{*} \mathrm{r}$ (which, if a trill, may have been redundantly aspirated despite specific evidence for this in the Hlai daughter languages):

$\begin{array}{lllllll}\mathrm{p} & \mathrm{t} & \mathrm{t} & \mathrm{c} & \mathrm{k} & & \text { ? } \\ \mathrm{f} & \mathrm{s} & & \mathrm{c} & \mathrm{x} & & \\ \mathrm{m} & \mathrm{n} & & \mathrm{n} & \mathrm{y} & \mathrm{yw} & \\ & \mathrm{l} & \mathrm{r} & \mathrm{j} & & & \end{array}$




$\begin{array}{llllllll}\text { (151) } & \mathrm{p}^{\mathrm{h}} & \mathrm{t}^{\mathrm{h}} & \mathrm{t}^{\mathrm{h}} & \mathrm{c}^{\mathrm{h}} & \mathrm{k}^{\mathrm{h}} & & \text { ? } \\ \mathrm{f} & \mathrm{s} & & 6 & \mathrm{x} & & \\ \mathrm{hm} & \mathrm{hn} & & \mathrm{hn} & \mathrm{hy} & \mathrm{hyw} & \\ & \mathrm{hl} & \text { (h)r } & & & & \\ & \mathrm{hw} & & & \mathrm{hj} & & & \end{array}$

Examples are given below:

\section{$\underline{\text { Pre-Hlai } \quad \text { Proto-Hlai }}$}

(a)

\begin{tabular}{|c|c|c|c|}
\hline wing & *pi:k & $>$ & ${ }^{*} \mathrm{p}^{\mathrm{h}} \mathrm{i}: \mathrm{k}$ \\
\hline fall & *tok & $>$ & $* \mathrm{t}^{\mathrm{h}} \mathrm{ok}$ \\
\hline carry on shoulder & $*$ ta:p & $>$ & $* t \int^{h} a: p$ \\
\hline (to) wedge & *ci:m & $>$ & $*_{\mathrm{t}} \varphi^{\mathrm{h}} \mathrm{i}: \mathrm{m}$ \\
\hline chicken & *kəy & $>$ & ${ }^{*} \mathrm{k}^{\mathrm{h}} \partial \mathrm{y}$ \\
\hline return/come & *mua & $>$ & *hmu: \\
\hline field & *na:h & $>$ & *hna:h \\
\hline shoot $\sim$ bow & *nur: & $>$ & *hnu: \\
\hline silver & *yən & $>$ & *hyən \\
\hline day & *ywən & $>$ & *hywən \\
\hline tongue & *li:n? & $>$ & *hli:n? \\
\hline sheep & $*_{j a: \eta}$ & $>$ & *hja:y \\
\hline bail water & $*_{\text {wi:t }}$ & $>$ & *hwi:t \\
\hline
\end{tabular}

\subsubsection{Monosyllabification}

Monosyllabification seems to have occurred in stages, with the loss of presyllables occurring early before the least sonorant medials, and later before more sonorant medials; with the exception of the initials which became implosives, all of these initials devoiced upon becoming initial. The sesquisyllabic words with medial obstruents which underwent this change in Pre-Hlai were the following:
(a) $\mathrm{C}-\mathrm{b}>6$
$\begin{array}{lll}\mathrm{C}-\mathrm{v} & >\quad \mathrm{v}>\mathrm{f}\end{array}$
C-d $>\quad$ d
Civj $>\quad v j>f j$
C-f $>\quad f>c$
$\mathrm{C}-\mathrm{g}>\mathrm{g}>\mathrm{k}$ 
The medial nasals and laterals still retained an unambiguous initial voiceless segment at the time of PHl, as indicated by their inclusion in the class of high register initials described in chapter two. ${ }^{54}$ The idiosyncratic lenition of *C-b to *C- $\beta$ must have occurred by this time, and it remained in sesquisyllabic form as well:

$$
\begin{array}{lll}
\mathrm{C}-\beta & > & \mathrm{C}-\beta \\
\mathrm{C}-\mathrm{m} & > & \mathrm{C}-\mathrm{m} \\
\mathrm{C}-\mathrm{n} & > & \mathrm{C}-\mathrm{n} \\
\mathrm{C}-\mathrm{n} & > & \mathrm{C}-\mathrm{n} \\
\mathrm{C}-\mathrm{n} & > & \mathrm{C}-\mathrm{\eta} \\
\mathrm{Cu \eta} & > & \mathrm{C} \text { } \\
\mathrm{C}-1 & > & \mathrm{C}-1
\end{array}
$$

Although there are only a subset of approximants and glottals which can be directly reconstructed with initial presyllables at the stage of Proto-Hlai (see chapter two), the systemic evidence and the development of Pre-Hlai posited here imply that all sonorants must have been potentially able to support presyllables at the stage of $\mathrm{PHl}$, even though direct evidence for them is not available in the reflexes of the modern languages. Those for which direct evidence for presyllables exists are the following:

$\begin{array}{lll}\text { Cur } & > & \text { Cur } \\ \text { Cur } & > & \text { Cur } \\ \text { Cif } & > & \text { Cif } \\ \text { Cuf } & > & \text { Cuf } \\ \text { Ci? } & > & \text { Ci? } \\ \text { Cu? } & > & \text { Cu? }\end{array}$

The initials for which evidence of a former presyllable is structural and ultimately indirect are the following:

\footnotetext{
${ }^{54}$ Where phonotactically possible, sonorants may have preserved an initial glottal stop as a vestige of the original presyllable initial, so that what is reconstructed in $\mathrm{PHl}$ as ${ }^{*} \mathrm{C}-\mathrm{m}$ may have actually been * $2 \mathrm{~m}$; I see no way to distinguish one possibility from another, so the former reconstruction is used.
} 


$\begin{array}{lll}\text { Cilj } & > & {[\mathrm{C}-] \mathrm{lj}} \\ \mathrm{C}-\mathrm{v} & > & {[\mathrm{C}-] \mathrm{v}} \\ \mathrm{C}-\mathrm{r} & > & {[\mathrm{C}-] \mathrm{r}} \\ \mathrm{C} i r j & > & {[\mathrm{C}-] \mathrm{rj}} \\ \mathrm{C}-\mathrm{j} & > & {[\mathrm{C}-] \mathrm{j}} \\ \mathrm{C}-\mathrm{r} & > & {[\mathrm{C}-] \mathrm{r}} \\ \mathrm{C}-\mathrm{h} & > & {[\mathrm{C}-] \mathrm{h}} \\ \mathrm{C}-\mathrm{P} & > & {[\mathrm{C}-] ?}\end{array}$

Since there is no direct evidence from the Hlai daughter languages supporting the reconstruction of the presyllable, these are reconstructed without presyllables in Proto-Hlai.

The motivation for this change involved the continuous phonetic weakening of the presyllable in sesquisyllabic forms, due to temporal compression acting on the foot and the ensuing overlap of gestures. A likely path of change is the devoicing of all minor syllable nuclei concomitant with extreme shortening. When the first and second consonants of the foot came into contact, place information from the first consonant could easily become unrecoverable through lack of a sufficient burst, leading to complete loss in pre-obstruent position (156a) and possibly to debuccalization in pre-sonorant position (157b):

(157) (a) Cəogá: > gá:

(b) Cọlá: > Plá:

Two typological examples, in which monosyllabification occurred in a way similar to that posited here for Hlai, are shown below. As mentioned previously, Proto-Chamic stress was iambic, and roots were typically bisyllabic (Thurgood 1999). When speakers of what became the language Tsat moved from mainland Southeast Asia to Hainan, there was pressure from language contact to reduce the bisyllabic lexicon to monosyllabic forms. When it was possible phonotactically for the first and second consonant of a word to form a cluster (a stop plus a liquid, the latter later leniting to a palatal glide), then the initial consonant was preserved (Figure 158a); this is contrary to the Hlai development, but similar to PSWT. If no cluster was phonotactically possible, then the initial consonant was lost entirely (Figure 158b). The first of the two vowels was lost in every instance. (Tsat also developed tone, in conformity with the language area into which it was integrated): 
(158) Reduction of bisyllabic forms to monosyllabic forms in Tsat

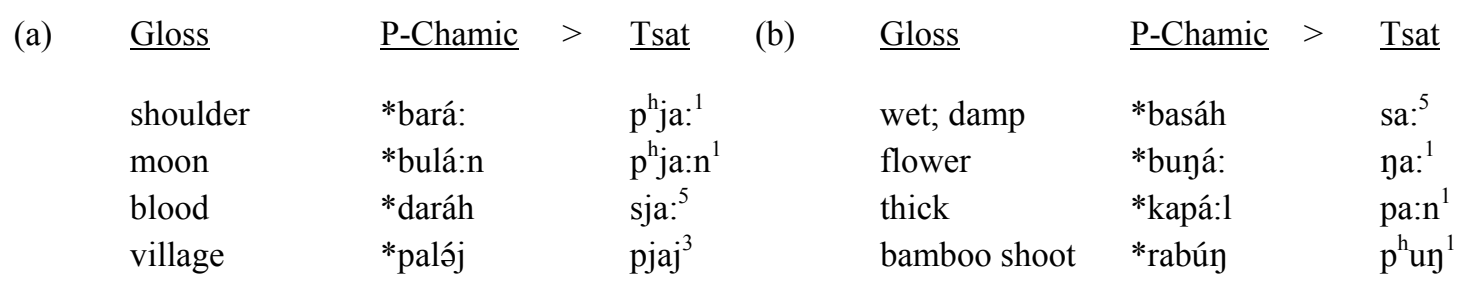

A Mon-Khmer example is the language Nhaheun, which descends from sesquisyllabic West

Bahnaric (Sidwell 2000), but has undergone monosyllabicization, with intervocalic lenition also occurring in some environments:

\begin{tabular}{|c|c|c|}
\hline Gloss & $\underline{\text { P-W. Bahnaric }}$ & Nhaheun \\
\hline termite & *kəntiár & tian \\
\hline skirt & *kədá:n & tá:y \\
\hline son-in-law & *pəsá:w & sá:w \\
\hline mortar & *tə2pál & dwáw \\
\hline crab & *kə?tá:m & grá:m \\
\hline onion & *kə?diám & griam \\
\hline stone & *təmó: & nwó: \\
\hline right side & *cəmá: & mmá: \\
\hline
\end{tabular}

The most important consequence of monosyllabification was the collapse of the originally separate initial and medial obstruent series (160); the plain stop inventory was repopulated, after the original initial stops had become aspirated. The following expanded inventory (161) was the result ${ }^{55}$ :

$\begin{array}{llllll}\mathrm{p}^{\mathrm{h}} & \mathrm{t}^{\mathrm{h}} & \mathrm{t}^{\mathrm{h}} & \mathrm{c}^{\mathrm{h}} & \mathrm{k}^{\mathrm{h}} & \text { ? } \\ \mathrm{f} & \mathrm{s} & & c & \mathrm{x} & \\ \mathrm{C}-\mathrm{b} & \mathrm{C}-\mathrm{d} & & \mathrm{C}-\mathrm{f} & \mathrm{C}-\mathrm{g} & \\ \mathrm{C}-\mathrm{v}(\mathrm{j}) & & & & & \end{array}$

(161) $\begin{array}{llllll}\mathrm{p}^{\mathrm{h}} & \mathrm{t}^{\mathrm{h}} & \mathrm{t}^{\mathrm{h}} & \mathrm{c}^{\mathrm{h}} & \mathrm{k}^{\mathrm{h}} & \text { ? } \\ \mathrm{b} & \mathrm{d} & & \mathrm{c} & \mathrm{k} & \\ \mathrm{f}(\mathrm{j}) & \mathrm{s} & & \mathrm{c} & \mathrm{x} & \end{array}$

\footnotetext{
${ }^{55}$ I make the assumption that the implosives $* 6$ and $* d$ originated at this point. It is possible that they passed through a medial stage of plain $* \mathrm{~b}$ and $* \mathrm{~d}$ after losing their presyllables, but the conditioning environement of an earlier presyllable consonant makes this seem less likely.
} 
Examples are given below:

$$
\text { Pre-Hlai }
$$

Proto-Hlai

overflow
gall bladder
step on
turbid
fire
tooth

${ }^{* C}$-ba:h
${ }^{* C}$-dəy
${ }^{* C}$-fə:mh
${ }^{* C}$-gunh
${ }^{* C}$-vi:
${ }^{* C}$-vjən

$>$
$>$
$>$
$>$
$>$
$>$

*?ba:h
*?dəy
*fə:mh
*gunh
*vi:
*vjən

$>\quad * 6 a: \mathrm{h}$

$>\quad *$ dəy

$>\quad *$ tढว:mh

$>\quad *$ kunh

$>\quad *$ fi:

$>\quad *$ fjon

\subsubsection{Stop and Fricative Affrication}

Toward the end of the Pre-Hlai period, but before Proto-Hlai, the retroflex and palatal obstruents underwent affrication. This was followed in the case of the retroflex by a shift to the palato-alveolar place of articulation, the first step in the loss of retroflex as a place of articulation (which would be completed with the loss of *r after the breakup of PHl):

$\begin{array}{lllll}\mathrm{t}^{\mathrm{h}} & > & \mathrm{ts}^{\mathrm{h}} & > & \mathrm{t}^{\mathrm{h}} \\ \mathrm{c}^{\mathrm{h}} & > & \mathrm{tc}^{\mathrm{h}} & & \\ \mathrm{c} & > & \mathrm{t} 6 & \end{array}$

At some point after initial obstruent devoicing, the dorsal fricatives underwent affrication and merged with their affricate or stop counterparts:

$\begin{array}{lll}6 & > & \mathrm{t} 6^{\mathrm{h}} \\ \mathrm{x} & > & \mathrm{k}^{\mathrm{h}}\end{array}$

These two forms of affrication modified the obstruent inventory from (164a) to (164b):

$\begin{array}{lllllll}\text { (a) } & \mathrm{p}^{\mathrm{h}} & \mathrm{t}^{\mathrm{h}} & \mathrm{t}^{\mathrm{h}} & \mathrm{c}^{\mathrm{h}} & \mathrm{k}^{\mathrm{h}} & \text { ? } \\ & \mathrm{b} & \mathrm{d} & & \mathrm{c} & \mathrm{k} & \\ & \mathrm{f}(\mathrm{j}) & \mathrm{s} & & 6 & \mathrm{x} & \\ \text { (b) } & \mathrm{p}^{\mathrm{h}} & \mathrm{t}^{\mathrm{h}} & \mathrm{t} \mathrm{f}^{\mathrm{h}} & \mathrm{tc}^{\mathrm{h}} & \mathrm{k}^{\mathrm{h}} & ? \\ & 6 & \mathrm{~d} & & \mathrm{tc} & \mathrm{k} & \\ & \mathrm{f}(\mathrm{j}) & \mathrm{s} & & & & \end{array}$


Examples are given below:

\section{$\underline{\text { Pre-Hlai }}$}

$\underline{\text { Proto-Hlai }}$

(a)

$\begin{array}{ll}\text { head louse } & *_{\text {tu: }} \\ \text { taste } & *_{\text {fim }} \\ \text { stand } & *_{\text {C-cu:n }} \\ & \\ \text { pestle } & *_{\text {ca: }} \\ \text { white } & *_{\text {xa: }}\end{array}$

$\begin{array}{ll}> & * \mathrm{t}^{\mathrm{h}} \mathrm{wu}: \\ > & * \mathrm{c}^{\mathrm{h}} \mathrm{im} \\ > & * \text { cu: } \\ & *^{\mathrm{c}} \mathrm{a}: \mathrm{k} \\ > & * x a: \mathrm{w}\end{array}$

$>\quad * t \int^{\text {h }} w u:$

$>\quad * t_{6}{ }^{\mathrm{h}}$ im

$>\quad$ *tcu:n

(b)

white

*xa:w

$\begin{array}{ll}> & * c^{\mathrm{h}} \mathrm{a}: \mathrm{k} \\ > & * \mathrm{k}^{\mathrm{h}} \mathrm{a}: \mathrm{w}\end{array}$

\subsubsection{Peripheral Vowel Raising}

The most sweeping change in the Pre-Hlai rime inventory was that of the raising of the peripheral (front and back) mid vowels, allowing their merger with the high vowels (167a). This stands in opposition to the central vowel series $\left({ }^{*}\right.$ ə), which did not merge with the high back unrounded vowel series $\left({ }^{*} u\right)$. If the hypothesis presented in section 4.2 is correct, this was part of a chain shift which allowed the low peripheral vowels to raise in turn and fill the gaps left by the original mid-vowels (167b):
(a) $\quad \mathrm{e}(: \mathrm{C})>\mathrm{i}(: \mathrm{C})$
$\mathrm{o}(: \mathrm{C})>\mathrm{u}(: \mathrm{C})$
(b) $\quad \varepsilon:(C)>e:(C)$
$\curvearrowright \mathrm{C}>\mathrm{oC}$

This change in the original Pre-Hlai rime inventory (168) resulted in the smaller inventory in (169):

$\begin{array}{llll}(168) & \mathrm{i}(: \mathrm{C}) & \mathrm{u}(: \mathrm{C}) & \mathrm{u}(: \mathrm{C}) \\ & \mathrm{e}(: \mathrm{C}) & \partial(: \mathrm{C}) & \mathrm{o}(: \mathrm{C}) \\ & \varepsilon:(\mathrm{C}) & \mathrm{a}(: \mathrm{C}) & \mathrm{o} \\ & & & \\ (169) & \mathrm{i}(: \mathrm{C}) & \mathrm{u}(: \mathrm{C}) & \mathrm{u}(: \mathrm{C}) \\ & \mathrm{e}:(\mathrm{C}) & \partial(: \mathrm{C}) & \mathrm{oC} \\ & & \mathrm{a}(: \mathrm{C}) & \end{array}$

Examples are given below: 


$$
\text { Pre-Hlai } \quad \text { Proto-Hlai }
$$

\begin{tabular}{|c|c|c|c|c|}
\hline \multirow[t]{4}{*}{ (a) } & wrinkle & ${ }^{*}$ C-ne:w? & $>$ & *C-ni:w? \\
\hline & small & $* \mathrm{C}^{\mathrm{V}}$-lek & $>$ & ${ }^{*} \mathrm{C}$-lik \\
\hline & before & *ko:nf & $>$ & ${ }^{*} \mathrm{k}^{\mathrm{h}} \mathrm{u}: \mathrm{nh}$ \\
\hline & needle & *yoc & $>$ & *hyuc \\
\hline \multirow[t]{2}{*}{ (b) } & board & ${ }^{*} \mathrm{C}-\mathrm{p} \varepsilon: \mathrm{nh}$ & $>$ & *6e:nf \\
\hline & brood & $*^{*} \mathrm{C}-\mathrm{mok}$ & $>$ & ${ }^{*} \mathrm{C}$-mok \\
\hline
\end{tabular}

\subsubsection{Monophthongization}

If the PSKD reconstruction in section 4.2 is correct, then the following two mergers took place in the rime inventory via monophthongization:

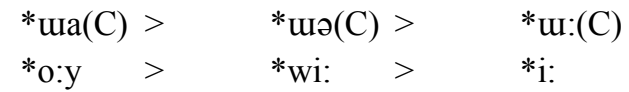

Examples are given below:

Pre-Hlai $\quad \underline{\text { Proto-Hlai }}$

\begin{tabular}{|c|c|c|c|c|c|c|}
\hline \multirow[t]{2}{*}{ (a) } & top & *H-nua & $>$ & *H-nшә & $>$ & *hnu: \\
\hline & put down, let go & *puan? & $>$ & *ршәу? & $>$ & *ph $u: \eta ? ?$ \\
\hline (b) & shellfish & *to:y & $>$ & *twi: & $>$ & $*_{\mathrm{t} \varphi^{\mathrm{h}} \mathrm{i}}$ \\
\hline
\end{tabular}

\subsubsection{Summary}

The goal of this section was to take the postulated PSKD initial and rime inventories as a starting point and illustrate the changes which have occurred throughout Pre-Hlai which led to the Proto-Hlai inventory. It has been shown that nine important changes occurred in Pre-Hlai. Those changes which affected the initials were the elimination of uvulars, intervocalic lenition, initial devoicing, vocalic transfer, word-initial aspiration, monosyllabification, and dorsal fricative affrication. Those changes which affected the rimes were peripheral vowel raising and monophthongization. The collective history of these changes 
is one in which category deletion and creation has led to alternating contractions and expansions in the initial inventory, and a reduction in the inventory of rimes.

The first contraction of the Pre-Hlai initial inventory occurred with the elimination of uvular as a valid place of articulation. The medial consonant inventory was then modified through intervocalic lenition, which eliminated the intervocalic voiceless stops, but added a new series of intervocalic approximants. The initial consonant inventory was reduced via the ongoing process of obstruent devoicing, where all voiced initial obstruents devoiced and merged with their voiceless counterparts. Vocalic transfer then expanded the medial consonant inventory by creating a set of consonants with secondary palatal articulations. Stresscorrelated word-initial aspiration increased the gap between the set of initial consonants and the medial consonants. The now very asymmetrical sets of initial and medial obstruents were merged as the latter lost their presyllables in the first wave of monosyllabification, while dorsal fricative affrication removed the posterior fricatives from the fricative series, merging them with the palatal affricate and the velar stop, respectively.

The set of Pre-Hlai rimes underwent a dramatic reduction as a result of peripheral vowel raising, where original peripheral mid nuclei merged with their high counterparts, and original peripheral low nuclei rose to fill their vacated positions in the vowel space. Individual monophthongizations led to the merger of *o:y and *ua with *i: and *u: respectively.

The set of changes described above explains most of the asymmetries in the $\mathrm{PHl}$ phoneme inventory. The skewing of the PHl fricative inventory is explained by dorsal fricative affrication, which led to the elimination of fricatives at anterior places of articulation. The presence of the palatalized labiodental fricative, lateral, and tap are the result of palatal vocalic transfer, which primarily targeted the latter two phonemes. Finally, the number of aspirated and preaspirated initials in proportion to plain initials is the result of initial aspiration, which aspirated all word-initial consonants but had no effect on medial consonants. The only aymmetry in the set of initials left unexplained is that of the PHl postalveolar series, which was argued to derive from an original retroflex series; it was shown above that there is no evidence for an original retroflex nasal, and the evidence for an original retroflex fricative is very sparse. 
The two asymmetries which exist in the $\mathrm{PHl}$ rime inventory, long *e:C rimes without short * $\mathrm{eC}$ rimes, and short $*_{\mathrm{oC}}$ rimes without long $*_{\mathrm{o}} \mathrm{C}$ rimes, has not been completely explained. In the case of the $*_{\mathrm{e}} \mathrm{C}$ rimes, it was seen that the evidence strongly suggests that these rimes are ultimately borrowed from Chinese. In the case of the ${ }^{\circ} \mathrm{oC}$ rimes, which derive from Pre-Hlai $*_{0} \mathrm{C}$, two comparisons with ProtoAustronesian suggest that they may have been derived from original *əC rimes before grave initials (the conditioning environment for the change from $* \partial \mathrm{C}$ to $* \jmath \mathrm{C}$ is unclear).

\subsection{Conclusion}

The main focus of this chapter has been to compare Proto-Hlai with Proto-Be and ProtoSouthwest Tai, in view of performing a preliminary reconstruction of Proto-Southern Kra-Dai. This reconstruction is not exhaustive, and cannot be until the reconstruction of Northern and Central Tai and, ultimately, of Proto-Tai itself. However, this preliminary reconstruction is sufficient for the primary purpose of this chapter, which was to uncover the changes which occurred in Pre-Hlai between ProtoSouthern Kra-Dai and Proto-Hlai.

Taking a bird's-eye view of the changes described in section 4.3, it can be generalized that there have been four main structural changes which have occurred between PSKD and PHI. The first of these, intervocalic lenition and subsequent vocalic transfer, was position-dependent and served to create important asymmetries between the inventory of initial and medial consonants. The second, initial aspiration, was also position dependent, and served to sharpen the asymmetry between the initial and medial consonants. The third, monosyllabification, was dependent on the sonority of the medial consonant in sesquisyllabic forms, and adjusted the ratio of monosyllabic words to sesquisyllabic words sharply in favor of the former. Finally, peripheral vowel raising led to a sharp reduction in the rime inventory, the final effect of which was to decrease the number of peripheral mid vowel rimes and eliminate the inventory of peripheral low vowel rimes. 
The reconstructed PSKD inventory of initials in (173) can be compared with that of PHl (174)

below. There are some noticeable gaps in the inventory which are not reconstructible with the amount of evidence examined in this chapter; some of these may be filled in the future with more work on Tai:

(173) PSKD Initial Consonants

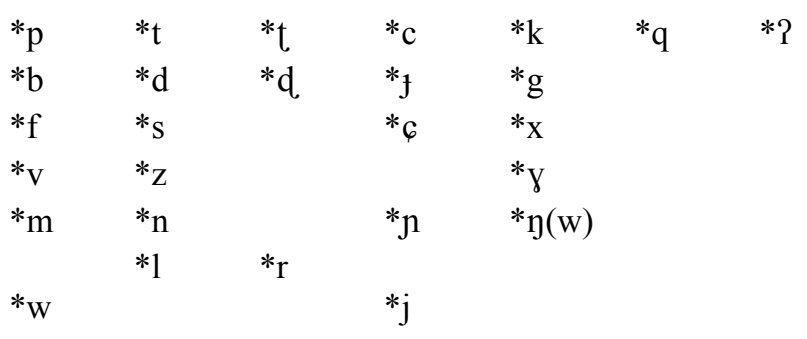

$\underline{\text { PSKD Medial Consonants }}$

\begin{tabular}{|c|c|c|c|}
\hline${ }^{*} \mathrm{C}-\mathrm{p}$ & ${ }^{*} \mathrm{C}-\mathrm{t}$ & ${ }^{*} \mathrm{C}-\mathrm{c}$ & $* \mathrm{C}-\mathrm{k} \quad * \mathrm{C}-\mathrm{q}$ \\
\hline$* \mathrm{C}-\mathrm{b}$ & ${ }^{*} \mathrm{C}(\mathrm{i} / \mathrm{u}) \mathrm{d} * \mathrm{C}(\mathrm{u}) \mathrm{d}$ & $* \mathrm{C}-\mathrm{f}$ & ${ }^{*} \mathrm{C}(\mathrm{i} / \mathrm{u}) \mathrm{g} * \mathrm{C}-\mathrm{G}$ \\
\hline $\begin{array}{l}{ }^{*} \mathrm{C}(\mathrm{i}) \mathrm{v} \\
{ }^{*} \mathrm{C}^{(\mathrm{V})}-\mathrm{m}\end{array}$ & $\begin{array}{l}{ }^{*} \mathrm{C}^{(\mathrm{V})}-\mathrm{n} \\
{ }^{*} \mathrm{C}^{(\mathrm{V})}(\mathrm{i}) 1 * \mathrm{C}(\mathrm{u}) \mathrm{r} \\
{ }^{*} \mathrm{p}(\mathrm{i}) 1 \\
* \mathrm{k}-\mathrm{l}\end{array}$ & $* \mathrm{C}^{(\mathrm{V})}-\mathrm{n}$ & $* C^{(\mathrm{V})}-\mathrm{n}$ \\
\hline *H-m & $\begin{array}{l}* \mathrm{H}-\mathrm{n} \\
*^{*} \mathrm{H}-\mathrm{l}\end{array}$ & & *H-n(w) \\
\hline
\end{tabular}

(174) PHl Initial Consonants

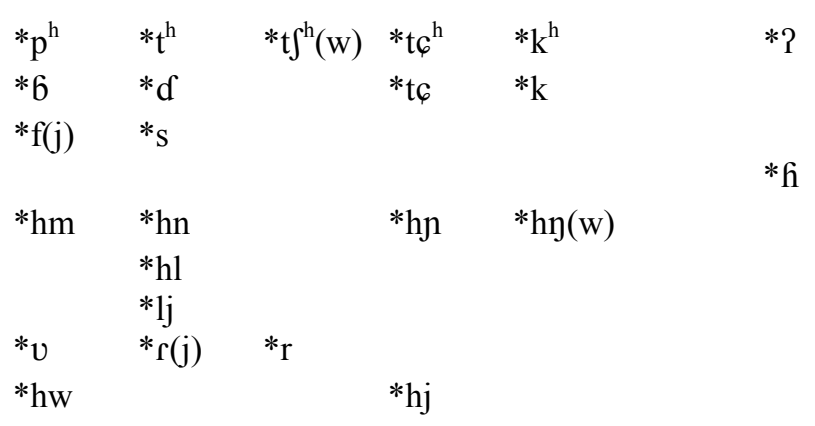

$\underline{\text { PHl Medial Consonants }}$

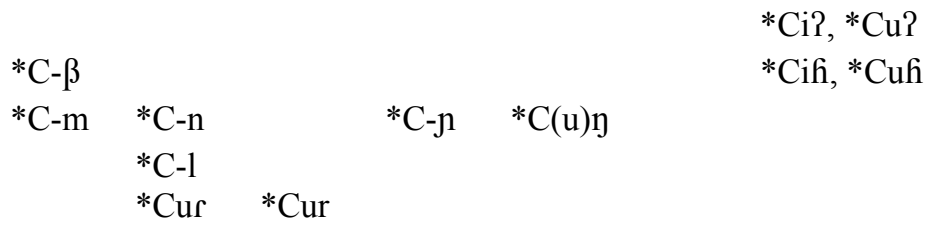


The differences between the PSKD rime inventory (175) and the PHl rime inventory (176) are also shown below. Since there is no evidence for changes in codas between PSKD and PHI, and the PSKD pretone system is too tentative to reconstruct without additional input from Tai, the exposition below will be simplified by writing -C for all codas and omitting tonal precursors:

(175) $\quad$ PSKD Open Rimes

\begin{tabular}{|c|c|}
\hline $\begin{array}{l}*_{\mathrm{i}}: \\
\left(*^{2} \mathrm{e}\right)\end{array}$ & *ur: \\
\hline${ }^{*} \varepsilon:$ & *a: \\
\hline
\end{tabular}

PSKD Closed Rimes

$\begin{array}{lll}*_{\mathrm{i}: \mathrm{C}} & *_{\mathrm{u}} \mathrm{C} & *_{\mathrm{u}: \mathrm{C}} \\ *_{\mathrm{iC}} & *_{\mathrm{u}} & *_{\mathrm{uC}} \\ *_{\mathrm{e}: \mathrm{C}} & *_{\partial} \mathrm{C} & *_{\mathrm{o}} \mathrm{C} \\ *_{\mathrm{e}} \mathrm{C} & *_{\partial \mathrm{C}} & *_{\mathrm{oC}} \\ *_{\varepsilon}: \mathrm{C} & *_{\mathrm{a}: \mathrm{C}} & \\ & & *_{\jmath \mathrm{C}}\end{array}$

*uaC

(176) PHl Open Rimes

$\begin{array}{ll}* \mathrm{i}: & * \mathrm{ul}: \\ * \mathrm{e}: & * \mathrm{u}:\end{array}$

$\underline{\text { PHl Closed Rimes }}$

$\begin{array}{lll}*_{\mathrm{i}: \mathrm{C}} & *_{\mathrm{u}: \mathrm{C}} & *_{\mathrm{u}: \mathrm{C}} \\ *_{\mathrm{iC}} & *_{\mathrm{u}} \mathrm{C} & *_{\mathrm{uC}} \\ *_{\mathrm{e}: \mathrm{C}} & *_{\partial} \mathrm{C} & \\ & *_{\partial \mathrm{C}} & *_{\mathrm{oC}} \\ & *_{\mathrm{a}: \mathrm{C}} & \end{array}$

The next section will be devoted to the treatment of Jiamao, its relationship with Hlai having been a continuing challenge in Hlai comparative work. It will be shown that Jiamao has borrowed from Hlai in 
both the Pre-Hlai and post-Proto-Hlai periods, and data from Jiamao is therefore important in supporting the reconstructions of both periods. 


\section{CHAPTER FIVE: JIAMAO}

The Jiamao language has been recognized since the publication of Ouyang \& Zheng (1983) as being somehow related to but very different from the other Hlai languages. There are at least three reasons for this. The first is that Jiamao shares less than half of its lexicon with the other Hlai languages; excluding more recent Chinese loans, this leaves a significant part of the lexicon (including some very basic vocabulary) with an unknown origin. The second is that Jiamao shows no robust pitch distinction between tone categories $\mathrm{A}, \mathrm{B}$, and $\mathrm{C}$, a distinction otherwise maintained robustly by all other Hlai languages (although this distinction is reflected in Jiamao vowel length). Finally, there is a one-to-many correspondence between Proto-Hlai initials and rimes on the one hand, and Jiamao initials and rimes on the other; some of these correspondences can in turn be shown to correlate with Pre-Hlai reflexes which predate PHl.

The hypothesis that Jiamao is not originally a Hlai language at all was first advanced in Thurgood (1992); it is suggested here that it is a (as of yet) language isolate which has been in long-term contact with Hlai; this hypothesis has the advantage of explaining the three facts listed above in the following way. First, the non-Hlai part of the Jiamao vocabulary which has not been borrowed from Chinese or other neighboring languages can be postulated as the oldest lexical stratum in Jiamao. Second, the distinction between the tone categories in vowel length as opposed to pitch can be understood to reflect a distinction that was salient to Hlai speakers in one way, but salient to speakers of Jiamao in another, and this is

reflected as such in the Jiamao reflexes; the inconsistency in tone category representation can be related to either misperception at the time of borrowing or to changes in representation of tone category at different points in time in the Hlai donor language. Finally, the one-to-many correspondences in both initials and rimes can be explained if the assumption is made that they represent different strata of loanwords into Jiamao from two or more distinct periods of contact.

This chapter is structured in the following way. The Jiamao initials will be treated in section 5.1, with an emphasis on multiple correspondences with Proto-Hlai; these multiple correspondences will be 
argued to reflect a distinction between borrowing from Pre-Hlai ${ }^{56}$ and borrowing from Proto-Hlai or its immediate daughter, Ha Em. The Jiamao rimes will be treated in section 5.2, beginning with a discussion of the tone categories, and then moving to the segmental component of the rimes, again showing that multiple correspondences can be explained according to the period during which the borrowing occurred. Finally, non-Hlai core lexical items will be given and discussed.

\subsection{Jiamao Initials}

This section begins with a discussion of register, which when understood, will help to clarify the discussion of segmental initials which follows. Jiamao probably underwent registrogenesis through participation in the same language area that it shares with the Qi languages. There is a register division in all tone categories, as well as a length distinction in category D in both registers, something which only exists otherwise in Baoting (and there only in high register).

\subsubsection{Jiamao Register}

The values of the tones in both high and low register as listed in Ouyang \& Zheng (1983) are given below (the tone numbers which are assigned therein to designate each tone category are given in parentheses):

\footnotetext{
${ }^{56}$ Citing Pre-Hlai forms is problematic from the perspective of the rime, because original Pre-Hlai peripheral mid-vowels can only be separated from their high counterparts using external Kra-Dai evidence; the same is true in differentiating original *ua and $*_{0}$ :y. Proto-Hlai peripheral mid vowel rimes therefore can't be used in the Pre-Hlai reconstructions.
} 
(1) Jiamao tone reflexes

$\begin{array}{lllll}\text { Tone Category } & \underline{\text { High }} & & \underline{\text { Low }} & \\ \text { A } & 55 & (1) & 11 & (4) \\ \mathrm{X} & 51 & (5) & 31 & (2) \\ \text { DLong } & 53 & (9) & 31 & (8) \\ \text { DShort } & 55 & (7) & 22 & (10)\end{array}$

The same convention which is used with Proto-Be is used here, namely the designation of the unmarked tone category as A, and the marked tone category as X. An important difference is that while PB tone $\mathrm{X}$ correlates directly with Hlai tone categories $\mathrm{B}$ and $\mathrm{C}$, Jiamao tone $\mathrm{X}$ does not show any such straightforward correlation (see section 5.2.1).

The same pitch depression associated with low register which is apparent in the registrogenetic Hlai languages is very marked in Jiamao, where all low register tones are clearly lowered versions of their high register counterparts. Jiamao register is valuable in reconstruction for the same reasons it is in the other Hlai languages, as it indicates the voicing status of initials at the time or registrogenesis, regardless of the status of their voicing in modern Jiamao.

With an understanding of Jiamao register in place, it is now possible to proceed to the Jiamao initials. These will be grouped by manner in the same way as the initials in chapters 2 and 4 . Since it is argued that the period of loans into Jiamao began at a stage before PHl, both Pre-Hlai and PHl reconstructions will be provided as appropriate.

\subsubsection{Stops}

The PHl stop correspondences with Jiamao are the following: 
(2)

\begin{tabular}{|c|c|c|}
\hline Pre-Hlai & $\underline{\mathrm{PHl}}$ & Jiamac \\
\hline$*_{p}$ & $* p^{h}$ & $6, \mathrm{p}^{\mathrm{h}}$ \\
\hline$*_{\mathrm{t}}$ & $* \mathrm{t}^{\mathrm{h}}$ & $d, t^{h}$ \\
\hline$*_{\mathrm{k}}$ & $* \mathrm{k}^{\mathrm{h}}$ & $\mathrm{h}, \mathrm{k}^{\mathrm{h}}$ \\
\hline
\end{tabular}

There is evidence above for two distinct layers of loans. The hypothesis presented here is that PreHlai plain stops were borrowed before initial aspiration occurred, and afterwards followed two distinct paths of development. The bilabial and alveolar stops underwent implosivization, in keeping with the general island-wide diffusion of this sound change. The velar stop, on the other hand, underwent lenition to a fricative, and eventually debuccalized. A second round of borrowing occurred after the aspiration of the Hlai initials, and occurred at the stage of PHl or after. These were all borrowed as aspirated initials, and remained unchanged. These two layers of Hlai loanwords into Jiamao and their subsequent developments are shown below:

\begin{tabular}{|c|c|c|c|c|c|c|}
\hline & $\underline{\text { Hlai }}$ & & $\underline{\text { Jiamao }}$ & $\underline{\text { Hlai }}$ & & $\underline{\text { Jiamao }}$ \\
\hline Pre-Hlai & $* p$ & $\rightarrow$ & $p>6$ & $*_{\mathrm{t}}$ & $\rightarrow$ & $t>d$ \\
\hline \multirow[t]{2}{*}{ PHl } & $*^{*} p^{h}$ & $\rightarrow$ & $\mathrm{p}^{\mathrm{h}}$ & $* \mathrm{t}^{\mathrm{h}}$ & $\rightarrow$ & $\mathrm{t}^{\mathrm{h}}$ \\
\hline & Hlai & & Jiamao & & & \\
\hline Pre-Hlai & $*_{\mathrm{k}}$ & $\rightarrow$ & $k>x$ & & & \\
\hline PHl & $* k^{h}$ & $\rightarrow$ & $\mathrm{k}^{\mathrm{h}}$ & & & \\
\hline
\end{tabular}

Examples are given below:

$\begin{array}{lll}\text { (a) } & \underline{\text { Pre-Hlai }} & \underline{\text { Jiamao }} \\ \text { slap } & \text { *pi:k } & 6 \text { ia }^{5} \\ \text { break } & \text { *pə:n? } & 6 \text { wan }^{1} \\ \text { branch (road) } & \text { *pa: } & \text { 6ow } \\ \text { (c) } & \text { Pre-Hlai } & \text { Jiamao } \\ \text { exit } & \text { *tur:n } & \text { dan }^{1} \\ \text { pot } & \text { *tow } & \text { daw } \\ \text { seven } & \text { *tu: } & \text { daw }\end{array}$

(b)

escape
hide
high

(d)

rotten

solid

to breed
$\underline{\mathrm{PH}}$

$*^{*} \mathrm{p}^{\mathrm{h}}$ iw

*ph

${ }^{*} p^{h} a: k$

$\underline{\mathrm{PH}}$

$* \mathrm{t}^{\mathrm{h}} \mathrm{uy}$

* $\mathrm{t}^{\mathrm{h}}$ ə:mf

$* t^{\mathrm{h}}$ a:y $\underline{\text { Jiamao }}$

$\mathrm{p}^{\mathrm{h}_{\text {iw }}}{ }^{1}$

$\mathrm{p}^{\mathrm{h}} \mathrm{ak}^{7}$

$\mathrm{p}^{\mathrm{h}} \mathrm{w}: \mathrm{:}^{5}$

$\underline{\text { Jiamao }}$

$\mathrm{t}^{\mathrm{h}} \mathrm{uyy}^{5}$

$t^{\mathrm{h}} \mathrm{o}: \mathrm{m}^{5}$

$t^{\mathrm{h}} \mathrm{e}: \mathrm{y}^{1}$ 


\begin{tabular}{|c|c|c|c|c|c|}
\hline (e) & $\underline{\text { Pre-Hlai }}$ & $\underline{\text { Jiamao }}$ & (f) & $\underline{\mathrm{PHl}}$ & $\underline{\text { Jiamao }}$ \\
\hline old & *kəwf & hi: ${ }^{1}$ & full & $*^{*}{ }^{h} \mathrm{u}: \mathrm{m}$ & $\mathrm{k}^{\mathrm{h}} \mathrm{om}^{1}$ \\
\hline nose & *kət & ho:t ${ }^{9}$ & chicken & $* \mathrm{k}^{\mathrm{h}} \partial \mathrm{y}$ & $\mathrm{k}^{\mathrm{h}} \mathrm{yy}^{1}$ \\
\hline leg & *kok & ho: $\mathrm{k}^{9}$ & ascend & $*^{\mathrm{h}} \mathrm{a}: \mathrm{n}$ & $\mathrm{k}^{\mathrm{h}} \mathrm{u} \mathrm{n}^{1}$ \\
\hline
\end{tabular}

There is one group of exceptions in the category of alveolar stops, in which palatalization occurred before an original high front vowel:

(5)

$\begin{array}{lll}\text { Gloss } & \underline{\mathrm{PHl}} & \underline{\text { Jiamao }} \\ \text { sound } & * \mathrm{t}^{\mathrm{h}} \mathrm{i}: \mathrm{w} & \mathrm{ts}^{\mathrm{h}} \mathrm{ew}^{1} \\ \text { chopsticks } & * \mathrm{t}^{\mathrm{h}} \mathrm{i}: \mathrm{p} & \mathrm{ts}^{\mathrm{h}} \mathrm{ep}^{7} \\ \text { full } & * \mathrm{t}^{\mathrm{h}} \mathrm{i}: \mathrm{k} & \mathrm{ts}^{\mathrm{h}} \mathrm{ia}^{5}\end{array}$

One other exceptional correspondence occurs in this category:

(6)

$\begin{array}{lll} & \underline{\text { Pre-Hlai }} & \underline{\text { Jiamao }} \\ \text { louse } & * \text { tən } & \text { ten }^{1}\end{array}$

The expected Jiamao initial for this word is $d$, and the actual initial and register for this word is indicative of ${ }^{*} \mathrm{C}$-d. As shown in the last chapter, the Pre-Hlai form is descended from an original $* \mathrm{~d}$; whether or not this somehow explains the unexpected Jiamao initial is unclear.

\subsubsection{Affricates}

The reflexes of the Hlai affricates are the following:

$\begin{array}{lll}\underline{\text { Pre-Hlai }} & \underline{\text { PHl }} & \underline{\text { Jiamao }} \\ * \mathrm{t} \int & * t \mathrm{t}^{\mathrm{h}}(\mathrm{w}) & \mathrm{t} \\ * \mathrm{t} \varphi & * \mathrm{t}^{\mathrm{h}} & \mathrm{ts}, \mathrm{ts}^{\mathrm{h}}\end{array}$


Jiamao, like Bouhin and $\mathrm{Ha} \mathrm{Em}$, has merged $\mathrm{PHl} * \mathrm{t}^{\mathrm{h}}$ and $* \mathrm{t}^{\mathrm{h}} \mathrm{w}$, preserving no evidence of the labiovelar coarticulation in the latter. There is only one extant reflex of these initials, indicating that they were either borrowed in the same way from both Pre-Hlai as well as PHl, or that what were originally two reflexes merged into a single reflex. I make the tentative assumption that this affricate aspirated after early instances of borrowing, shifted to a fricative $\int$, and participated in a chain shift with *s (see below), so that $\int$ shifted to $s$, which then participated in the regional shift of $s$ to $t$. The palatal affricate, if the one example below in (8) is indicative, was borrowed as a plain affricate from Pre-Hlai; after aspiration in PHl, the aspirated affricate was borrowed as such. Both remained unchanged until the shift of palatals to alveolars:

\begin{tabular}{|c|c|c|c|c|c|c|}
\hline & $\underline{\text { Hlai }}$ & & $\underline{\text { Jiamao }}$ & $\underline{\text { Hlai }}$ & & $\underline{\text { Jiamao }}$ \\
\hline Pre-Hlai & $* t \int$ & $\rightarrow$ & $\mathrm{t} \int^{\mathrm{h}}>\mathrm{s}>\mathrm{t}$ & $*_{\mathrm{c}}$ & $\rightarrow$ & $c>t 6>t s$ \\
\hline PHl & $* \operatorname{tr}^{\mathrm{h}}(\mathrm{w})$ & $\rightarrow$ & $t \int^{h}>s>t$ & $* t 6^{\mathrm{h}}$ & $\rightarrow$ & $\mathrm{t}^{\mathrm{h}}>\mathrm{ts} \mathrm{s}^{\mathrm{h}}$ \\
\hline
\end{tabular}

Examples are given below:

(9)
(a)

repay

sick

eye

(c)

burn
$\underline{\mathrm{PHl}}$

$* \mathrm{t} \int^{\mathrm{h}} \mathrm{\partial}: \mathrm{m}$ ?

$* \mathrm{t}^{\mathrm{h}} \mathrm{ok}$

$* t \int^{\mathrm{h}} \mathrm{a}$ :

$\underline{\text { Pre-Hlai }}$

*cuy? $\underline{\text { Jiamao }}$

tiam $^{1}$

ta: $\mathrm{k}^{9}$

tow ${ }^{1}$

$\underline{\text { Jiamao }}$

tsey $^{1}$ (b)

weave (fabric) head louse

below

(d)

shellfish

hole

wear
$\underline{\mathrm{PHl}}$

*t ${ }^{\mathrm{h}}$ wur:k *t $\int^{\mathrm{h}}$ wu: $*$ t $\int^{\text {h }}$ wow

PH1

$*{ }^{\mathrm{h}}{ }^{\mathrm{h}} \mathrm{i}$ :

$*_{t} c^{\mathrm{h}} \mathrm{u}: \mathrm{n}$ ?

$* t \varphi^{\mathrm{h}} \partial \mathrm{t}$ $\underline{\text { Jiamao }}$

ta: ${ }^{9}$

taw $^{1}$

ta: ${ }^{1}$

Jiamao

ts $^{\mathrm{h}} \mathrm{ay}^{1}$

ts ${ }^{\mathrm{h}} \mathrm{u}^{1}$

ts $^{\mathrm{h}} \mathrm{o}: \mathrm{t}^{9}$

There is one exceptional correspondence in this category:

$\underline{\text { Gloss }}$

tree
$\underline{\mathrm{PHl}}$

$* t \int^{h} \partial y$ $\underline{\text { Jiamao }}$

ts $^{\mathrm{h}}$ ay $^{1}$ 
This irregular initial probably indicates a relatively late date of borrowing, most likely from Baoting $t s^{h} a y^{1}$ (as Baoting is the language with which Jiamao has been in a more recent contact relationship).

\subsubsection{Fricatives}

The relexes of the Hlai fricatives in Jiamao are given below:

$\begin{array}{lll}\text { Pre-Hlai } & \text { Proto-Hlai } & \text { Jiamao } \\ *_{\mathrm{f}} & *_{\mathrm{f}} & \mathrm{p} \\ *_{\mathrm{C}-\mathrm{vj}} & *_{\mathrm{fj}} & \mathrm{ts}^{\mathrm{h}} \\ *_{\mathrm{s}} & *_{\mathrm{s}} & \mathrm{ts}^{\mathrm{h}} \\ { }^{\mathrm{C}} \mathrm{C}-\beta & { }^{\mathrm{C}} \mathrm{C}-\beta & \mathrm{f}\end{array}$

The most straightforward assumption in the case of the fricatives is that they were borrowed into Jiamao without modification, only later undergoing changes internal to Jiamao:

\begin{tabular}{|c|c|c|c|c|c|c|}
\hline & Hlai & & $\underline{\text { Jiamao }}$ & $\underline{\text { Hlai }}$ & & $\underline{\text { Jiamao }}$ \\
\hline Pre-Hlai & $*_{\mathrm{f}}$ & $\rightarrow$ & $f>p$ & ${ }^{*} \mathrm{C}-\mathrm{vj}$ & $\rightarrow$ & $\mathrm{vj}>\mathrm{f} \varphi>\mathrm{ts}^{\mathrm{h}}$ \\
\hline \multirow[t]{2}{*}{ PHl } & $\begin{array}{l}\downarrow \\
* f\end{array}$ & $\rightarrow$ & $f>p$ & $\stackrel{\downarrow}{\downarrow} \mathrm{fj}^{-}$ & $\rightarrow$ & $f j>f \varphi>t s^{h}$ \\
\hline & Hlai & & $\underline{\text { Jiamao }}$ & $\underline{\text { Hlai }}$ & & $\underline{\text { Jiamao }}$ \\
\hline Pre-Hlai & $*_{\mathrm{S}}$ & $\rightarrow$ & $s>t s^{h}$ & ${ }^{*} \mathrm{C}-\beta$ & $\rightarrow$ & $\beta>\phi>f$ \\
\hline PHl & $\begin{array}{r}\downarrow \\
*_{s}\end{array}$ & $\rightarrow$ & $\mathrm{s}>\mathrm{ts}^{\mathrm{h}}$ & $\begin{array}{c}\downarrow \\
* \mathrm{C}-\beta\end{array}$ & $\rightarrow$ & $\beta>\phi>f$ \\
\hline
\end{tabular}

The fricatives appear to have been stable throughout the first two waves of borrowing, with only one reflex apiece in Jiamao. PHl *f participated in a rather late change in Jiamao in which fricatives were hardened to stops, and the Jiamao reflex is therefore $p$. This gap was then available to be filled, so like Bouhin and Yuanmen, the only case in which Jiamao has a reflex $f$ is in the case of $* C-\beta$. There is more than one way that it can be conjectured that $* \mathrm{fj}$ led to $t s^{h}$ in Jiamao, but I hypothesize an intermediate stage of $f_{6}$ (with the aperture of the gliding assimilating to that of the preceding fricative). This is parallel to the 
development of $* \mathrm{t}^{\mathrm{h}} \mathrm{w}$ to $f$ in $\mathrm{NCHl}$ and Lauhut discussed in chapter two, and is similar to a change called High Vowel Frication in Bantu, an example of which is given in Shona (Mathangwane 1999: 88):

\begin{tabular}{|c|c|c|c|c|c|}
\hline Proto-Bantu & Shona & Example & Proto-Bantu & & $\underline{\text { Shona }}$ \\
\hline *pi & $\mathrm{s}^{\mathrm{w}}$ & stick & *-pímbo & $>$ & $\mathrm{s}^{\mathrm{w}} \mathrm{imbo}$ \\
\hline *tụ & $\mathrm{pf}$ & forge & *-tụ́d-a & $>$ & pfura \\
\hline
\end{tabular}

Unlike all other fricatives which hardened to plain stops, *s seems to have undergone an earlier change to an affricate (an identical shift with Run). This cleared the way for the sibilant chain shift mentioned above:

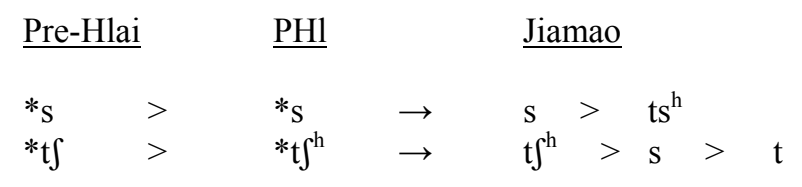

Examples are given below. Note that only three examples of * $\mathrm{fj}$ exist, and of these, there is only corroboration for this reflex in tooth in the NWCHl and Meifu branches:

(a)

go hairbun dream

(c)

poke a hole *sur:y? you (pl) thread needle $\underline{\text { PHl }} \underline{\text { Jiamao }}$

$*_{\text {fi: }} \quad$ pay $^{1}$

*fun?

*fən

poy ${ }^{1}$

po: ${ }^{1}$

$\underline{\text { Jiamao }}$

ts $^{\mathrm{h}} \mathrm{a}: \mathrm{y}^{5}$

ts $^{\text {h }}$ aw $^{1}$

ts $^{\mathrm{h}}$ o: $\mathrm{k}^{9}$ (b)

scrub

seed

tooth

(d)

done

village

nine $\underline{\text { Pre-Hlai }}$

*C-vjui:p

*C-vjən

*C-vjən

$\underline{\mathrm{PHl}}$

*C-ßuy

*C- $\beta$ a:n

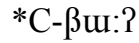

$\underline{\text { Jiamao }}$

ts $^{\text {h }}$ uəp ${ }^{9}$

ts $^{\mathrm{h}}$ an ${ }^{1}$

ts $^{\mathrm{h}} a \mathrm{y}^{1}$

$\underline{\text { Jiamao }}$

fuəy $^{1}$

fuən ${ }^{1}$

fə: ${ }^{1}$

There is one exceptional correspondence in this category:

$\underline{\text { Gloss }}$

return
$\underline{\mathrm{PH}}$

*sut $\underline{\text { Jiamao }}$

tsok $^{7}$ 
In the case of this word, the expected Jiamao initial is aspirated $t s^{h}$, and if related, it is unclear why it is unaspirated.

\subsubsection{Medial Stops}

The reflexes of the Hlai medial stops are the following. There are three reflexes each of $* 6$ and $* d$, and two each of *tc and *k (as in chapter two, a reflex in bold font indicates that it is correlated with low register):

\begin{tabular}{|c|c|c|}
\hline Pre-Hlai & $\underline{\mathrm{PH} 1}$ & $\underline{\text { Jiamac }}$ \\
\hline$* \mathrm{C}-\mathrm{b}$ & $* 6$ & $\mathrm{p}, 6, \mathbf{f}$ \\
\hline${ }^{*} \mathrm{C}-\mathrm{d}$ & ${ }^{*} d$ & $\mathrm{t}, \mathrm{d}, \mathrm{t}$ \\
\hline${ }^{*} \mathrm{C}-\mathrm{f}$ & $*_{t c}$ & ts, $\mathrm{ts}^{\mathrm{h}}$ \\
\hline${ }^{*} \mathrm{C}-\mathrm{g}$ & $*_{\mathrm{k}}$ & $\mathrm{k}, \mathrm{h}$ \\
\hline
\end{tabular}

Reflexes of words borrowed at the time of $\mathrm{PHl}$ or subsequently are straightforward. Those borrowed during the period of Pre-Hlai, however, show two sets of correspondences. In one case, the presyllable seems to have merely dropped off, leaving plain voiced stops which later devoiced. In the other, additional intervocalic lenition occurred, in which the stops shifted to voiced fricatives or, in the case of the alveolar, to a lateral. The palatal and velar fricatives devoiced before registrogenesis, whereas the bilabial fricative didn't devoice until afterward: 
(18)

\begin{tabular}{|c|c|c|c|c|c|c|}
\hline & $\underline{\text { Hlai }}$ & & $\underline{\text { Jiamao }}$ & $\underline{\text { Hlai }}$ & & $\underline{\text { Jiamao }}$ \\
\hline \multirow[t]{2}{*}{ Pre-Hlai } & ${ }^{*} \mathrm{C}-\mathrm{b}$ & $\rightarrow$ & $C-b>b>p$ & $* \mathrm{C}-\mathrm{d}$ & $\rightarrow$ & $C-d>d>t$ \\
\hline & $\downarrow$ & & $C-\beta>\beta>\mathbf{v}>\mathbf{f}$ & $\downarrow$ & & $\mathrm{C}-1>1>13>4$ \\
\hline \multirow[t]{2}{*}{ PHl } & *6 & $\rightarrow$ & 6 & $* d$ & $\rightarrow$ & d \\
\hline & $\underline{\text { Hlai }}$ & & $\underline{\text { Jiamao }}$ & Hlai & & $\underline{\text { Jiamao }}$ \\
\hline \multirow[t]{2}{*}{ Pre-Hlai } & $* \mathrm{C}-\mathrm{f}$ & $\rightarrow$ & $\mathrm{C}-\mathrm{y}_{\mathrm{f}}>\mathrm{d} \mathrm{f}_{\mathrm{p}}>\mathrm{ts}$ & ${ }^{*} \mathrm{C}-\mathrm{g}$ & $\rightarrow$ & $C-g>g>k$ \\
\hline & $\downarrow$ & & $\mathrm{C}-\mathrm{z}>\mathrm{z}>\mathrm{s}>\mathrm{ts}^{\mathrm{h}}$ & $\downarrow$ & & $\mathrm{C}-\mathrm{y}>\mathrm{y}>\mathrm{x}>\mathrm{h}$ \\
\hline PHl & $*_{\text {t6 }}$ & $\rightarrow$ & t $6>$ ts & $*_{\mathrm{k}}$ & $\rightarrow$ & $\mathrm{k}>\mathrm{k}$ \\
\hline
\end{tabular}

Examples are given below:

(a)

leaf

overflow

end

(c)

fly

thief

feed

(d)

porcupine

bamboo

dregs

(f)

castrate

fear

(g)

bear fruit

jump

extinguish
*C-bu:

${ }^{*} \mathrm{C}-\mathrm{ba}: \mathrm{h}$

*C-bə:y?

$\underline{\text { Pre-Hlai }}$

*C-bin

*C-buy

*C-bu:h

$\underline{\text { Pre-Hlai }}$

${ }^{*} \mathrm{C}$-dəy

${ }^{*} \mathrm{C}$-dom

${ }^{*}$ C-da:k

$\underline{\text { Pre-Hlai }}$

*C-du:n

${ }^{*} \mathrm{C}$-da:?

$\underline{\text { Pre-Hlai }}$

*C-dzi:y

*C-dzu:n?

${ }^{*} \mathrm{C}$-dzəp $\underline{\text { Jiamao }}$

pi: ${ }^{1}$

po: ${ }^{1}$

puy $^{5}$

$\underline{\text { Jiamao }}$

fin $^{4}$

fuy ${ }^{4}$

fo: ${ }^{4}$

$\underline{\text { Jiamao }}$

ti: ${ }^{1}$

təm ${ }^{1}$

tu: ${ }^{5}$

$\underline{\text { Jiamao }}$

$\operatorname{lum}^{4}$

to: ${ }^{4}$

$\underline{\text { Jiamao }}$

tsen $^{1}$

tsuən $^{1}$

tsep $^{7}$ (b)

decorate

duck

to hoe

(e)

bright

bamboo

ladle
PHl

*6i:nf

*6it

*6əwh $\underline{\text { Jiamao }}$

bion $^{5}$

bet $^{7}$

$60: w^{5}$ $\underline{\text { Jiamao }}$

$\operatorname{din}^{1}$

dum $^{5}$

do: $\mathrm{k}^{9}$ (h)

elder bros wife $\quad *$ C-dzu: stand

*C-dzu:n ts ${ }^{\mathrm{h}} \mathrm{u}:{ }^{1}$

ts $^{\mathrm{h}} \mathrm{u}: \mathrm{n}^{1}$ 
(i)

able

jar

grind (rice) $\underline{\text { Pre-Hlai } \quad \underline{\text { Jiamao }}}$

*C-gi:w

*C-gə:n?

*C-ga:nh

kอ: $n^{1}$

$\mathrm{ka}: \mathrm{n}^{5}$ (j)

yawn

Note that the timing of the devoicing of voiced stops which entered Jiamao in the first wave of borrowing must be timed against the changes which occurred with the plain voiceless stops which entered at the same time:

$$
\begin{aligned}
& \mathrm{p}>\mathrm{b} \\
& \mathrm{t}>\mathrm{d} \\
& \mathrm{k}>\mathrm{x} \\
& \mathrm{b}>\mathrm{p} \\
& \mathrm{d}>\mathrm{t} \\
& \mathrm{g}>\mathrm{k}
\end{aligned}
$$

\begin{tabular}{|c|c|c|c|}
\hline$(21)$ & $\underline{\text { Gloss }}$ & $\underline{\text { Pre-Hlai }}$ & $\underline{\text { Jiamao }}$ \\
\hline \multirow[t]{2}{*}{ (a) } & pheasant & ${ }^{*}$ C-du:n & $\mathrm{t}^{\mathrm{h}} \mathrm{un}^{4}$ \\
\hline & front & *C-dəワ & $\mathrm{t}^{\mathrm{h}}$ iə门 $^{4}$ \\
\hline \multirow[t]{2}{*}{ (b) } & soak & *C-də:m? & $\operatorname{tsiom}^{4}$ \\
\hline & light, insipid & $* \mathrm{C}$-dəc & $\mathrm{tsia}^{2}$ \\
\hline \multirow[t]{2}{*}{ (c) } & $\operatorname{sink}$ & *C-fə:n & $\operatorname{tsen}^{4}$ \\
\hline & grandma & *C-эu:? & tso: ${ }^{2}$ \\
\hline (d) & grasshopper & $*[\mathrm{C}-\mathrm{g} / \mathrm{y}] \mathrm{it}$ & $\mathrm{Pet}^{7}$ \\
\hline
\end{tabular}

There are several exceptional correspondences in this category, the reasons for which are unclear:

\subsubsection{Initial Nasals}

There are two correspondences for each of the Hlai initial nasals, with the exception of *hyw, which has followed the development of most other Hlai languages in merging with *hw. The reflexes of 
Pre-Hlai *n vary between $n$ and $n$, apparently conditioned by the following vowel $-n$ before low vowels and $n$ elsewhere.

\begin{tabular}{|c|c|c|}
\hline Pre-Hlai & PHI & $\underline{\text { Jiamao }}$ \\
\hline$*_{m}$ & $* \mathrm{hm}$ & $\mathbf{m}, \mathbf{p}$ \\
\hline$* \mathrm{n}$ & *hn & $\mathbf{n}, \mathbf{t}$ \\
\hline$* \mathrm{n}$ & *hn & $\mathrm{n} / \mathbf{n}, \mathbf{t s}$ \\
\hline$* \eta$ & *hy & $\mathbf{y}, \mathbf{k}$ \\
\hline *yw & *hyw & $\mathrm{V}$ \\
\hline
\end{tabular}

The development of the initial nasals is shown below. The reflexes in the second wave of borrowing indicate that this wave was not borrowed precisely at the time of PHl, but shortly thereafter from one of the daughter languages, almost certainly Ha Em (which was not only present in the same vicinity, but had evolved in a way that best explains the form of the Jiamao borrowings):

\begin{tabular}{|c|c|c|c|c|c|c|}
\hline & $\underline{\text { Hlai }}$ & & $\underline{\text { Jiamao }}$ & $\underline{\text { Hlai }}$ & & $\underline{\text { Jiamao }}$ \\
\hline Pre-Hlai & $*_{\downarrow}^{* m}$ & $\rightarrow$ & $\mathrm{m}>\mathbf{m}$ & ${ }^{* n}$ & $\rightarrow$ & $\mathrm{n}>\mathbf{n}$ \\
\hline \multirow[t]{2}{*}{ PHl } & $* \mathrm{hm}$ & $\rightarrow$ & $\mathrm{mb}>\mathrm{b}>\mathbf{p}$ & $*$ hn & $\rightarrow$ & $\mathrm{nd}>\mathrm{d}>\mathbf{t}$ \\
\hline & $\underline{\text { Hlai }}$ & & $\underline{\text { Jiamao }}$ & $\underline{\text { Hlai }}$ & & $\underline{\text { Jiamao }}$ \\
\hline Pre-Hlai & ${ }^{*} \mathrm{n}$ & $\rightarrow$ & $\mathrm{n}>\mathrm{n} / \mathbf{n}$ & $\begin{array}{r}* \eta \\
\downarrow\end{array}$ & $\rightarrow$ & $\mathfrak{y}>\boldsymbol{y}$ \\
\hline \multirow[t]{2}{*}{ PHl } & *hn & $\rightarrow$ & $\mathrm{n} \mid>\mathrm{d} \mathbf{z}_{\mathrm{p}}>\mathbf{t s}$ & *hy & $\rightarrow$ & ๆg $>$ g $>\mathbf{k}$ \\
\hline & $\underline{\text { Hlai }}$ & & $\underline{\text { Jiamao }}$ & & & \\
\hline Pre-Hlai & (*yw & $\rightarrow$ & $\mathrm{w}>\mathrm{hw}>\mathrm{v})$ & & & \\
\hline $\mathrm{PHl}$ & *hyw & $\rightarrow$ & $\mathrm{hw}>\mathrm{hw}>\mathrm{v}$ & & & \\
\hline
\end{tabular}

Examples are given below: 


\begin{tabular}{|c|c|c|c|c|c|}
\hline (a) & $\underline{\text { Pre-Hlai }}$ & $\underline{\text { Jiamao }}$ & (b) & $\underline{\mathrm{PHl}}$ & $\underline{\text { Jiamao }}$ \\
\hline beard & *mu:m? & mum $^{2}$ & ant & *hmuc & puət $^{8}$ \\
\hline come & *mu:n & muy $^{4}$ & wet & *hmən? & pə: $n^{4}$ \\
\hline mouth & *məm? & muəท ${ }^{4}$ & $\operatorname{dog}$ & *hma: & pow $^{4}$ \\
\hline (c) & $\underline{\text { Pre-Hlai }}$ & $\underline{\text { Jiamao }}$ & (d) & $\underline{\mathrm{PHl}}$ & $\underline{\text { Jiamao }}$ \\
\hline mos y. brother & *nut:? & ni: ${ }^{4}$ & long & *hna:w? & tow $^{4}$ \\
\hline $\operatorname{six}$ & $*$ nom & $n m^{4}$ & dragon & *hnəท & tə: $\eta^{4}$ \\
\hline otter & *na:k & nu: ${ }^{2}$ & wetfield & *hna:h & tow $^{4}$ \\
\hline (e) & $\underline{\text { Pre-Hlai }}$ & $\underline{\text { Jiamao }}$ & (f) & $\underline{\mathrm{PHl}}$ & $\underline{\text { Jiamao }}$ \\
\hline shoot & *nu: & $\mathrm{ni}^{4}$ & unhusked rice & *hn[e]:y & tsi: $y^{2}$ \\
\hline card (cotton) & *nu: & ni: $:^{4}$ & & & \\
\hline surround & *na:w? & nə:w ${ }^{2}$ & & & \\
\hline (g) & $\underline{\text { Pre-Hlai }}$ & $\underline{\text { Jiamao }}$ & (h) & $\underline{\mathrm{PHl}}$ & $\underline{\text { Jiamao }}$ \\
\hline aunt & *yi:n & niən ${ }^{2}$ & necklace & *hyi:w & kew $^{4}$ \\
\hline lay down & *yu:h & yo: ${ }^{4}$ & needle & *hyuc & kuət $^{8}$ \\
\hline \multirow[t]{4}{*}{ lid } & *yə:t & put $^{8}$ & fog & *hya:w? & $\mathrm{k} 0: \mathrm{w}^{4}$ \\
\hline & & & (i) & $\underline{\mathrm{PHl}}$ & $\underline{\text { Jiamao }}$ \\
\hline & & & wind & *hywə:t & vurat $^{7}$ \\
\hline & & & day & *hywən & vo: $n^{1}$ \\
\hline
\end{tabular}

There are several exceptional correspondences in this category as well:

\begin{tabular}{|c|c|c|c|}
\hline (25) & $\underline{\text { Gloss }}$ & $\underline{\mathrm{PHl}}$ & $\underline{\text { Jiamao }}$ \\
\hline \multirow[t]{5}{*}{ (a) } & hemp & hmi:n & $\operatorname{mian}^{1}$ \\
\hline & mother & hmi:? & ma: $y^{5}$ \\
\hline & slip(pery) & hpu:n & nuan $^{5}$ \\
\hline & glutinous rice & hya:? & now ${ }^{1}$ \\
\hline & frog (inedible) & hyu:h & yay $^{1}$ \\
\hline (b) & mouse & hniw & kew $^{4}$ \\
\hline (c) & yawn & hya:p & ho:p ${ }^{8}$ \\
\hline
\end{tabular}


The forms in (25a) are all in high register; the best explanation for this is that they were borrowed after initial aspiration, so that preaspirated nasals conditioned high register (and therefore before the shift to post-stopped nasals in Greater Hlai). The word yawn in (25c) indicates an irregular original glottal fricative, which may have formed under the presence of the preceding word in the opaque compound $h o:{ }^{5} h s: p^{8}$.

\subsubsection{Medial Nasals}

The correspondences of the Hlai medial nasals in Jiamao all occur in high register, indicating that they were still either medial or preglottalized at the time of registrogenesis. The same variation in the palatal series that occurred initially also occurs medially:

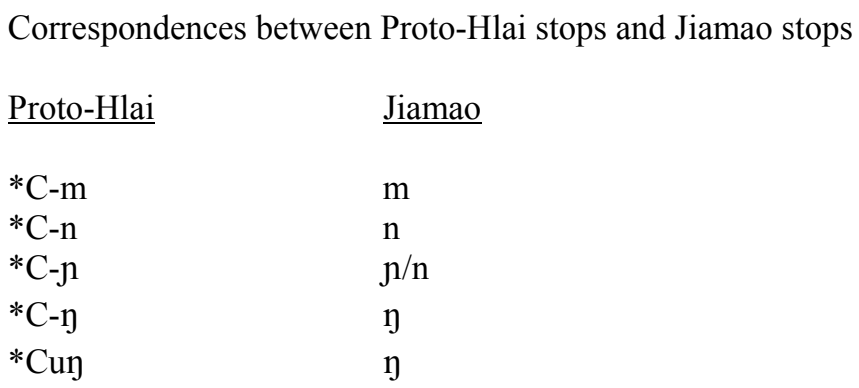

There was no change between the stages of Pre-Hlai and PHl, and there are therefore only one set of reflexes for each initial:

\begin{tabular}{|c|c|c|c|c|c|c|}
\hline & $\underline{\text { Hlai }}$ & & $\underline{\text { Jiamao }}$ & Hlai & & $\underline{\text { Jiamao }}$ \\
\hline Pre-Hlai & ${ }^{*} \mathrm{C}-\mathrm{m}$ & $\rightarrow$ & $\mathrm{C}-\mathrm{m}>\mathrm{m}$ & ${ }^{*} \mathrm{C}-\mathrm{n}$ & $\rightarrow$ & $C-n>n$ \\
\hline \multirow[t]{2}{*}{ PHl } & ${ }^{*} \mathrm{C}-\mathrm{m}$ & $\rightarrow$ & $\mathrm{C}-\mathrm{m}>\mathrm{m}$ & ${ }^{*} \mathrm{C}-\mathrm{n}$ & $\rightarrow$ & $C-n>n$ \\
\hline & $\underline{\text { Hlai }}$ & & $\underline{\text { Jiamao }}$ & $\underline{\text { Hlai }}$ & & $\underline{\text { Jiamao }}$ \\
\hline Pre-Hlai & ${ }^{*} \mathrm{C}-\mathrm{n}$ & $\rightarrow$ & $\mathrm{C}-\mathrm{n}>\mathrm{n} / \mathrm{n}$ & ${ }^{*} \mathrm{C}-\mathrm{n}$ & $\rightarrow$ & $C-\eta>y$ \\
\hline PHl & ${ }^{*} \mathrm{~d}-\mathrm{n}$ & $\rightarrow$ & $\mathrm{C}-\mathrm{n}>\mathrm{n} / \mathrm{n}$ & ${ }^{\downarrow} \mathrm{d}-\eta$ & $\rightarrow$ & $C-y>n$ \\
\hline
\end{tabular}




$\begin{array}{llll} & \underline{\text { Hlai }} & & \underline{\text { Jiamao }} \\ \text { Pre-Hlai } & * \text { Cuy } & \rightarrow & \text { C- } \mathrm{y}>\mathrm{y} \\ & \downarrow & & \\ \text { PHl } & * \text { Cuy } & \rightarrow & \text { C- }-\mathrm{y}>\mathrm{y}\end{array}$

Examples are given below:

\section{(a)}

poison

hand

foreigner

(c)

year before last

cow

moon

(e)

wild potato

rest head
$\underline{\mathrm{PHl}}$

${ }^{*}$ C-mi:n

*C-mu:

${ }^{*} \mathrm{C}$-mə:y

$\underline{\mathrm{PHl}}$

${ }^{*}$ C-nu:

${ }^{*} \mathrm{C}$-niw

*C-na:n

$\underline{\mathrm{PHl}}$

*Cuna:

*Cuna:n $\underline{\text { Jiamao }}$

mi:n ${ }^{5}$

ma: ${ }^{1}$

muəy $^{1}$

Jiamao

na: ${ }^{1}$

naw $^{1}$

nuən $^{1}$

$\underline{\text { Jiamao }}$

now $^{1}$

yuən $^{1}$ (b)

bamboo shoot

water

thick

(d)

tattoo face

cry

liver
$\underline{\mathrm{PHl}}$

*C-nu:y

*C-nəm?

*C-na:

PHI

*C-nu:

${ }^{*} \mathrm{C}-\mathrm{ni}:$ ?

*C-ya:n $\underline{\text { Jiamao }}$

na: ${ }^{1}$

na: ${ }^{1}$

now ${ }^{1}$

$\underline{\text { Jiamao }}$

yaw $^{5}$

ya:y ${ }^{1}$

yuən $^{1}$

There are two kinds of exceptional correspondences in this category:

$\underline{\text { Gloss }}$

(a)

bear
shake
salt
alcohol
branch (tree)

(b) wear (hat)

\section{$\underline{\text { Pre-Hlai }}$ \\ $\underline{\text { Jiamao }}$}

*C-muy

*C-nюnh

${ }^{*} \mathrm{C}-$-na:w?

*C-ya:wh

*C-na:mh

*Cunəw? muy $^{4}$

nuər ${ }^{4}$

no: ${ }^{4}$

yə: $w^{2}$

yəm ${ }^{2}$

niaw $^{1}$

In (29a), the register of these forms indicates that the nasals were initial, not medial; they may have been borrowed as such. The originally velar nasal in (29b) palatalized under the influence of the following rime. 


\subsubsection{Laterals}

The Jiamao correspondences for initial Hlai laterals are given below. In all cases, there is a oneto-one correspondence:

\begin{tabular}{|c|c|c|}
\hline Pre-Hlai & $\underline{\mathrm{PHI}}$ & $\underline{\text { Jiamao }}$ \\
\hline *1 & *hl & 4 \\
\hline *lj & $* \mathrm{lj}$ & ts \\
\hline${ }^{*} \mathrm{C}-1$ & $*^{*} \mathrm{C}-1$ & 1 \\
\hline$*_{p}-1$ & $*_{\mathrm{p}-1}$ & 1 \\
\hline
\end{tabular}

Amongst the four categories of laterals, the only one which underwent a significant change between Pre-Hlai and PHl is the plain lateral, which became aspirated before PHI. Although Jiamao has a modern aspirated reflex for this initial, the fact that it occurs in low register indicates that it must have been borrowed as a voiced segment, most probably a plain lateral. It could have later become aspirated in one of two ways: either (1) by first developing into the voiced lateral fricative $\mathcal{F}$ (the hypothesis advocated here), probably under the influence of Baoting and the other Qi languages which had inherited this initial as a reflex of * $1 \mathrm{j}$, or (2) by undergoing unconditioned spontaneous aspiration.

\begin{tabular}{|c|c|c|c|c|c|c|}
\hline & Hlai & & $\underline{\text { Jiamao }}$ & $\underline{\text { Hlai }}$ & & $\underline{\text { Jiamao }}$ \\
\hline Pre-Hlai & *1 & $\rightarrow$ & $1>b>4$ & $* 1 \mathrm{j}$ & $\rightarrow$ & $\mathrm{lj}>\mathrm{d} \mathrm{p}_{\mathrm{p}}>\mathrm{ts}$ \\
\hline \multirow[t]{2}{*}{ PHl } & $\begin{array}{c}\downarrow \\
* h l\end{array}$ & $\rightarrow$ & $1>b>4$ & $\begin{array}{r}\downarrow \\
* 1 \mathrm{j}\end{array}$ & $\rightarrow$ & $\mathrm{lj}>\mathrm{dz}>\mathrm{ts}$ \\
\hline & Hlai & & Jiamao & Hlai & & Jiamao \\
\hline Pre-Hlai & $\begin{array}{c}* \mathrm{C}-1 \\
\downarrow\end{array}$ & $\rightarrow$ & $\mathrm{C}-1>1$ & ${ }^{*} \mathrm{p}-1$ & $\rightarrow$ & $\mathrm{p}-1>1$ \\
\hline PHI & $*^{*} \mathrm{C}-1$ & $\rightarrow$ & $\mathrm{C}-1>1$ & $* \mathrm{p}-1$ & $\rightarrow$ & $\mathrm{p}-1>1$ \\
\hline
\end{tabular}

Examples are given below: 
(32)

(a)

$\underline{\mathrm{PHl}}$

tongue

brain

fish

*hli:n?

*hlu:k

*hla:

(c)

$\underline{\mathrm{PH}}$

\section{fingernail}

change clothes

uncover
*C-li:p

${ }^{*}$ C-low

${ }^{*} \mathrm{C}$-la:k $\underline{\text { Jiamao }}$

4i:n ${ }^{4}$

4u: $?^{8}$

fow ${ }^{4}$

$\underline{\text { Jiamao }}$

lep ${ }^{7}$

law ${ }^{1}$

lua $^{5}$ (b)

leech

neck

steal

(d)

bamboo

termite

near
$\underline{\mathrm{PHl}}$

*ljin

*ljoy?

*ljok

$\underline{\mathrm{PHl}}$

*p-lu:yf

*p-lu:k

*p-lur:?
Jiamao

tsi: $\eta^{4}$

tsay $^{2}$

tsa: $k^{8}$

$\underline{\text { Jiamao }}$

luәу ${ }^{5}$

lua ${ }^{5}$

la: ${ }^{1}$

There are several kinds of exceptional correspondences in this category, for reasons which are

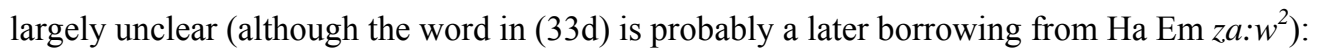

Gloss

(a)

$$
\text { warm }
$$

not know

(b) heart

(c) choose

(d) spade

(e) measure (rice)
$\underline{\mathrm{PHl}}$

*hlunf

*hlum?

*hla:w?

*hlən

*lja:wh

*C-lu:n?
Jiamao

$t^{\text {h }} u: n^{5}$

$t^{\text {h }}$ um $^{4}$

ts $^{\mathrm{h}_{\text {iaw }}}{ }^{1}$

$\operatorname{ts}^{4} n^{4}$

$z a: w^{4}$

๒๐: $\eta^{4}$

\subsubsection{Approximants}

The Jiamao reflexes of the Hlai approximants are given below. Single reflexes in Jiamao indicate stability of these intitials between Pre-Hlai and PHl. 


\begin{tabular}{|c|c|c|}
\hline Pre-Hlai & PHl & $\underline{\text { Jiamao }}$ \\
\hline${ }^{*} \mathrm{C}-\mathrm{v}$ & $*_{v}$ & $\mathbf{v}$ \\
\hline$*^{*} \mathrm{C}-\mathrm{r}$ & $*_{r}$ & 1 \\
\hline$*_{r j}$ & $*_{r j}$ & ts \\
\hline *Cur & * Cur & l \\
\hline$*_{\mathrm{r}}$ & $*_{\mathrm{r}}$ & $\mathrm{k}$ \\
\hline${ }^{*} \mathrm{Cur}$ & ${ }^{*}$ Cur & $\mathrm{k}$ \\
\hline
\end{tabular}

Developments of these initials are on balance similar to those in other Hlai languages. Jiamao patterns with Bouhin and $\mathrm{Ha} \mathrm{Em}$ in not undergoing vocalic transfer in the ${ }^{*} \mathrm{Cur}$ and ${ }^{*}$ Cur initials. The reflex of $* \mathrm{r}$ must have velarized very early to a velar fricative, devoicing before registrogenesis, and finally undergoing fortition to a stop, in the same manner as $f>p$ and $s>t$.

\begin{tabular}{|c|c|c|c|c|c|c|}
\hline & $\underline{\text { Hlai }}$ & & $\underline{\text { Jiamao }}$ & $\underline{\text { Hlai }}$ & & $\underline{\text { Jiamao }}$ \\
\hline Pre-Hlai & ${ }^{*} \mathrm{C}-\mathrm{v}$ & $\rightarrow$ & $v>\mathbf{v}$ & $*_{r j}$ & $\rightarrow$ & $\mathrm{rj}>\mathrm{d} \mathrm{F}_{\mathrm{p}}>\mathrm{ts}$ \\
\hline \multirow[t]{2}{*}{ PHl } & $\begin{array}{r}\downarrow \\
* v\end{array}$ & $\rightarrow$ & $v>\mathbf{v}$ & $\begin{array}{c}\downarrow \\
* r j\end{array}$ & $\rightarrow$ & $\mathrm{rj}>\mathrm{d} \mathrm{l}_{\mathrm{p}}>\mathrm{ts}$ \\
\hline & Hlai & & $\underline{\text { Jiamao }}$ & $\underline{\text { Hlai }}$ & & $\underline{\text { Jiamao }}$ \\
\hline Pre-Hlai & $\begin{array}{c}* \mathrm{C}-\varsigma \\
\downarrow\end{array}$ & $\rightarrow$ & $\mathrm{c}>\mathrm{l}$ & ${ }^{*} \mathrm{Cur}$ & $\rightarrow$ & $\mathrm{c}>\mathrm{I}$ \\
\hline \multirow[t]{2}{*}{ PHl } & $*_{r}$ & $\rightarrow$ & $\mathrm{c}>\mathrm{l}$ & $* \mathrm{Cur}$ & $\rightarrow$ & $f>1$ \\
\hline & $\underline{\text { Hlai }}$ & & $\underline{\text { Jiamao }}$ & Hlai & & $\underline{\text { Jiamao }}$ \\
\hline Pre-Hlai & $\begin{array}{c}*_{r} \\
\downarrow\end{array}$ & $\rightarrow$ & $\mathrm{y}>\mathrm{x}>\mathrm{k}$ & $\begin{array}{c}* \text { Cur } \\
\downarrow\end{array}$ & $\rightarrow$ & $\mathrm{y}>\mathrm{x}>\mathrm{k}$ \\
\hline PHl & $*_{r}$ & $\rightarrow$ & $y>x>k$ & $*$ Cur & $\rightarrow$ & $\mathrm{y}>\mathrm{x}>\mathrm{k}$ \\
\hline
\end{tabular}

Note that Jiamao $l$ in low register is always the reflex of PHl ${ }^{*}$, whereas Jiamao $l$ in high register is the reflex of $\mathrm{PHl} * \mathrm{C}-1$.

Examples are given below: 
(36)

\begin{tabular}{|c|c|c|c|c|c|}
\hline (a) & $\underline{\mathrm{PHl}}$ & $\underline{\text { Jiamao }}$ & (b) & $\underline{\mathrm{PHl}}$ & $\underline{\text { Jiamao }}$ \\
\hline length clsfr & *vən? & viən $^{4}$ & calf & $*_{\text {rjin? }}$ & $\operatorname{tsin}^{4}$ \\
\hline master & $*_{\text {va:y }}$ & vu: ${ }^{4}$ & sift & $*_{\text {rjow }}$ & tsi:w ${ }^{4}$ \\
\hline clothes & *va:y? & vo: ${ }^{4}$ & laugh & $*_{\text {rja:w }}$ & $\mathrm{tsu}^{4}$ \\
\hline (c) & $\underline{\mathrm{PHl}}$ & $\underline{\text { Jiamao }}$ & (d) & $\underline{\mathrm{PHl}}$ & $\underline{\text { Jiamao }}$ \\
\hline skirt & $*_{\text {ri:n? }}$ & $\operatorname{liən}^{2}$ & bone & *Curur:k & $\operatorname{liək}^{10}$ \\
\hline mortar & $*_{\text {row }}$ & lu: ${ }^{4}$ & earth & *Curən & $\operatorname{len}^{4}$ \\
\hline intestine & *ra:y? & luy ${ }^{4}$ & boat & *Cura: & $10:^{4}$ \\
\hline (e) & $\underline{\mathrm{PHl}}$ & $\underline{\text { Jiamao }}$ & (f) & $\underline{\mathrm{PHl}}$ & $\underline{\text { Jiamao }}$ \\
\hline sell & *ri:w? & ki:w ${ }^{1}$ & pus & *Curiw? & $\mathrm{ku}:{ }^{1}$ \\
\hline cicada & *rə:y & kuy $^{1}$ & head & *Curəw? & ki:w ${ }^{1}$ \\
\hline taro & $*_{\mathrm{ra}} \mathrm{k}$ & kw: ${ }^{5}$ & 100 & *Cura:n & kw:n ${ }^{1}$ \\
\hline
\end{tabular}

There are several kinds of exceptional correspondences in this category:

\section{Gloss}

(a)

(b)

$$
\text { poor }
$$

lizard magpie

(c) day bfr last

(d)

row
to lead
red

(e)

accumulate
cricket
not
curse

\section{$\underline{\mathrm{PHl}}$}

*va:t

*rju:

*ju:t

$*_{\text {ru:? }}$

*rə:yh

*ruy?

*ra:n?

*vu:m?

*Curu:y?

*su:y

*ra:? $\underline{\text { Jiamao }}$

fur: $t^{8}$

liə门 ${ }^{4}$

luot $^{8}$

tsa: ${ }^{4}$

tuy $^{5}$

luəy ${ }^{2}$

fu: $n^{4}$

vuram $^{1}$

fuəฺ ${ }^{4}$

vay $^{1}$

ha: ${ }^{4}$

Many of these exceptional reflexes remain mysterious. The two words in (37b) seem to have been borrowed as plain * $r$ as opposed to palatalized $*_{r j}$, and the last four words $(37 \mathrm{e})$ can all be considered recent loans from Baoting, as all of them are very close or exact matches of Qi reflexes. 


\subsubsection{Glottals}

The Jiamao reflexes of Hlai glottal fricatives and stops are given below:

\begin{tabular}{|c|c|c|}
\hline Pre-Hlai & $\underline{\mathrm{PHl}}$ & $\underline{\text { Jiamao }}$ \\
\hline$* \mathrm{f}$ & $* \mathrm{~h}$ & h \\
\hline${ }^{*} \mathrm{Cif}$ & ${ }^{*} \mathrm{Cif}$ & $\mathbf{h} / \mathbf{z}$ \\
\hline${ }^{*} \mathrm{Cuh}$ & ${ }^{*} \mathrm{Cuf}$ & h \\
\hline *? & $* ?$ & $?$ \\
\hline${ }^{*} \mathrm{Ci}$ ? & ${ }^{*} \mathrm{Ci} ?$ & $?$ \\
\hline${ }^{*} \mathrm{Cu} ?$ & ${ }^{*} \mathrm{Cu}$ ? & $?$ \\
\hline
\end{tabular}

Jiamao again patterns with Bouhin and Ha Em in not undergoing vocalic transfer in forms with medial glottal segments and preceding high vowels. There are two exceptions to this. One is ${ }^{*} \mathrm{Cif}$, where the Jiamao reflex is often $z$ in low register before low vowels; $z$ is also occasionally the reflex of * $\mathrm{h}$ if followed by a high front vowel in the rime. The other exception is *Ci?, for which there is only one Jiamao example. This example (steam) shows evidence for vocalic transfer, but it is also possible based on the rime that this is a relatively recent borrowing from Baoting. The Jiamao reflex of ${ }^{*} \mathrm{Ci}$ ? must therefore be considered to be very tentative.

\begin{tabular}{|c|c|c|c|c|c|c|}
\hline & Hlai & & $\underline{\text { Jiamao }}$ & Hlai & & $\underline{\text { Jiamao }}$ \\
\hline Pre-Hlai & $* h$ & $\rightarrow$ & $\mathrm{h}>\mathbf{h} / \mathbf{z}$ & $* ?$ & $\rightarrow$ & $?$ \\
\hline \multirow[t]{2}{*}{ PHl } & $\begin{array}{r}\downarrow \\
* h \\
h\end{array}$ & $\rightarrow$ & $\mathrm{h}>\mathbf{h} / \mathbf{z}$ & $\begin{array}{r}\downarrow \\
* ?\end{array}$ & $\rightarrow$ & $?$ \\
\hline & Hlai & & $\underline{\text { Jiamao }}$ & $\underline{\text { Hlai }}$ & & $\underline{\text { Jiamao }}$ \\
\hline Pre-Hlai & ${ }^{*} \mathrm{Cif}$ & $\rightarrow$ & $\mathrm{h}(\mathrm{j})>\mathbf{h} / \mathbf{z}$ & ${ }^{*}$ Ci? & $\rightarrow$ & --- \\
\hline \multirow[t]{2}{*}{ PHl } & *Cif & $\rightarrow$ & $\mathrm{h}(\mathrm{j})>\mathbf{h} / \mathbf{z}$ & ${ }^{*} \mathrm{Ci}$ ? & $\rightarrow$ & $(\mathrm{Pj}>\mathrm{ts})$ \\
\hline & $\underline{\text { Hlai }}$ & & $\underline{\text { Jiamao }}$ & $\underline{\text { Hlai }}$ & & $\underline{\text { Jiamao }}$ \\
\hline Pre-Hlai & ${ }^{*} \mathrm{Cuh}$ & $\rightarrow$ & $\mathrm{h}>\mathbf{h}$ & ${ }^{*} \mathrm{Cu} ?$ & $\rightarrow$ & $?$ \\
\hline PHl & ${ }^{*} \mathrm{Cuf}$ & $\rightarrow$ & $\mathrm{h}>\mathbf{h}$ & ${ }^{*} \mathrm{Cu} ?$ & $\rightarrow$ & $?$ \\
\hline
\end{tabular}


Examples are given below:

\begin{tabular}{|c|c|c|c|c|c|}
\hline (a) & $\underline{\mathrm{PHl}}$ & $\underline{\text { Jiamao }}$ & (b) & $\underline{\mathrm{PHl}}$ & $\underline{\text { Jiamao }}$ \\
\hline horn & *həw & haw $^{4}$ & field ridge & *Cifə:n & huan $^{4}$ \\
\hline shit & *ha:y? & huay $^{2}$ & leak & *Cifəр & ho:p ${ }^{9}$ \\
\hline step & *ha:mh & ho: $\mathrm{m}^{2}$ & crippled & *Cifa:yh & he: $y^{2}$ \\
\hline (c) & $\underline{\mathrm{PHl}}$ & $\underline{\text { Jiamao }}$ & (d) & $\underline{\mathrm{PHl}}$ & $\underline{\text { Jiamao }}$ \\
\hline spirit & *Cufu: & ha: ${ }^{4}$ & thatch grass & *Cifa: & zow $^{4}$ \\
\hline gourd & *Cufiu:p & huəp $^{8}$ & step & *Cifa:mh & $\mathrm{za}: \mathrm{m}^{5}$ \\
\hline bite & *Cufrə:t & huət $^{10}$ & bitter & *hə:m & $\operatorname{ziam}^{4}$ \\
\hline (e) & PHl & $\underline{\text { Jiamao }}$ & (f) & PHl & $\underline{\text { Jiamao }}$ \\
\hline wash & *?u:t & Puət $^{9}$ & steam & *CiPunf & $\operatorname{tsun}^{1}$ \\
\hline dense & *?ə:t & Pшәt ${ }^{7}$ & & & \\
\hline bathe & *Pa:p & Pup 9 & & & \\
\hline (g) & $\underline{\mathrm{PHl}}$ & $\underline{\text { Jiamao }}$ & & & \\
\hline get up & *Cu?ur:h & Pa: ${ }^{1}$ & & & \\
\hline open & *Cu?a:h & Row $^{1}$ & & & \\
\hline
\end{tabular}

There are several kinds of exceptional correspondences in this category:

\begin{tabular}{|c|c|c|c|}
\hline (41) & $\underline{\text { Gloss }}$ & $\underline{\text { Pre-Hlai }}$ & $\underline{\text { Jiamao }}$ \\
\hline \multirow[t]{3}{*}{ (a) } & I & *hu: & $\mathrm{kaw}^{1}$ \\
\hline & pluck/scratch & *hus:t & kurat $^{7}$ \\
\hline & hunchback $^{\mathrm{MR}}$ & *6วтก & $\mathrm{k} v: \mathrm{m}^{1}$ \\
\hline (b) & go & *hi: & hay $^{1}$ \\
\hline (c) & earthworm $(\lg )$ & *Cufən & $\operatorname{van}^{1}$ \\
\hline \multirow[t]{2}{*}{ (d) } & pond & *Cifu:yh & $2 j \ni y^{5}$ \\
\hline & tree heart & *Cuf⿵⺆ & $2 \mathrm{iat}^{7}$ \\
\hline (e) & swollen & *?un & fun $^{4}$ \\
\hline
\end{tabular}


Thurgood (p.c.) suggests that the first person pronoun in (41a) may be a loan from Utsat. The word in (41c) is a late loan, most likely from Baoting $h w a n^{1}$. Finally, the word in (41e) seems to have developed an excrescent fricative in initial position, which later devoiced.

\subsubsection{Glides}

The Jiamao reflexes of the Hlai glides are the following:

\begin{tabular}{|c|c|c|}
\hline Pre-Hlai & PHI & $\underline{\text { Jiamao }}$ \\
\hline$*_{\mathrm{j}}$ & *hj & ts \\
\hline${ }^{*} \mathrm{w}$ & *hw & $\mathrm{v}$ \\
\hline
\end{tabular}

The only difference between the Pre-Hlai and PHl glides is that the latter became preaspirated.

The Jiamao reflexes of the glides occur in high register, indicating that they were preaspirated at the time of registrogenesis. Any glides originally borrowed as unaspirated must have therefore later become preaspirated, merging with the preaspirated glides borrowed later.

\begin{tabular}{|c|c|c|c|c|c|c|}
\hline & Hlai & & $\underline{\text { Jiamao }}$ & Hlai & & Jiamao \\
\hline Pre-Hlai & $*_{j}$ & $\rightarrow$ & $\mathrm{j}>\mathrm{hj}>\mathrm{dz}>\mathrm{ts}$ & ${ }^{*} \mathrm{~W}$ & $\rightarrow$ & $\mathrm{w}>\mathrm{hw}>\mathrm{v}$ \\
\hline PHl & $\begin{array}{c}\downarrow \\
* \mathrm{hj}\end{array}$ & $\rightarrow$ & $\mathrm{hj}>\mathrm{d}_{\mathrm{p}}>\mathrm{ts}$ & *hw & $\rightarrow$ & $\mathrm{hw}>\mathrm{v}$ \\
\hline
\end{tabular}

Examples are given below:

\begin{tabular}{|c|c|c|c|c|c|}
\hline & $\underline{\mathrm{PHl}}$ & $\underline{\text { Jiamao }}$ & & $\underline{\mathrm{PHl}}$ & $\underline{\text { Jiamao }}$ \\
\hline egg & *hju:m & tsum $^{1}$ & a bundle of rice & *hwiw & vew $^{1}$ \\
\hline elder male inlaw & *hjəw? & tsi:w ${ }^{1}$ & ghost & *hwə:t & vurat $^{9}$ \\
\hline old & *hja: & tso: ${ }^{1}$ & plantain & *hwa:k & vua $^{5}$ \\
\hline
\end{tabular}

There are two exceptional correspondences in this category: 


$$
\underline{\text { Gloss }}
$$

$\underline{\mathrm{PH1}}$

$\underline{\text { Jiamao }}$

$\begin{array}{llll}\text { (a) } & \text { corner } & \text { *hju:y } & \text { tshuəy }^{1} \\ \text { (b) } & \text { stretch } & \text { *hja:? } & \text { tso: }{ }^{4}\end{array}$

The word in (45b) is irregular in register, and may represent a recently borrowed form.

\subsubsection{Summary}

In examining the initial consonants of Proto-Hlai and Jiamao, the largest complications in correspondences between the two can be explained in terms of a model in which there were two periods of borrowing from Hlai by Jiamao. The first period was in the latter part of the Pre-Hlai period, but before initial aspiration. The second period was around the time of reconstructed Proto-Hlai, and possibly extending into the early period of Proto-Hlai disintegration. When there is only a single Jiamao reflex for a particular Hlai initial, it is assumed that there was no change in Hlai between the two periods of Jiamao borrowing. When there are at least two Jiamao reflexes, the additional witness of Jiamao as external evidence for the various stages of Hlai is quite valuable. Despite the fact that regular correspondences between Jiamao and Hlai can be identified once the nature of the borrowing relationship has been explicated, there remains a residue of Jiamao forms which seem related to Hlai but have unexpected correspondences; these forms are explainable in at least some cases as due to errors in transmission during borrowing, and reinforce the hypothesis that Jiamao is a non-Hlai language which has been in intense contact with Hlai for quite some time. 


\subsection{Rimes}

In comparison with the Jiamao initials, the Jiamao rimes are much more complicated, and often seem to reflect more than two layers of loanwords. These layers can be sorted out to a certain degree using a combination of reference directly to various stages of Hlai, as well as relying on the Jiamao initials to provided constraints on linearization of borrowing, provided that there are two distinct layers in the class of initials in question.

There are still numerous exceptions to the generalizations which apply to different strata of vocabulary; it is my opinion that these can only be accounted for by both transmission errors at the time of the loans themselves as well as at least occasional idiosyncratic changes which occurred later, after the loans entered the language. Although in some rime categories it is possible to suggest a general progression of loanword strata, it is not presently possible to do this in all cases; where this stratification is evident, it is laid out in as much detail as possible.

Based upon comparison with the timeline of the Jiamao initials, the following general principles of Jiamao sound change chronology are relatively secure:

(i) There were two different changes involving schwa, one earlier, one later

(ii) Diphthongs in closed rimes originate in formerly pure vowels.

In the case of (i), the two changes are in keeping with similar changes which affected the Hlai languages, as well as Lingao to the north. These changes were the following, with (a) preceding (b):

$\begin{array}{ll}\text { (a) } & \partial>0 \\ \text { (b) } & \partial>\mathrm{a}\end{array}$

In the majority of cases, it is probably safe to assume that a Jiamao word with the first reflex is an earlier loan; the one exception to this is the Hlai rime category ${ }^{*} \mathrm{oC}$, which, given the phonetic similarity, may be suspected of being of more recent origin if it corresponds to a Jiamao rime $x$, either long or short. On the other hand, a Jiamao word with the latter reflex can not automatically be assumed to be a later loan, since it 
is only known that its individual path of development had led to a schwa nucleus by the time at which the change $\boldsymbol{\rho}>a$ occurred.

In the case of (ii), the generalization can be made that low-centered diphthongs of the type $\mathrm{VaC}$ (where $\mathrm{V}$ is some high vowel) originated in long low vowels, and that mid-centered diphthongs of the type $V \partial C$ (where $\mathrm{V}$ is some high vowel) originated in mid vowels (short in the case of unrounded nuclei, long in the case of rounded nuclei). Moreover, words with these diphthongs may be assumed not to originate in the most recent layer of loanwords, since more recent loanwords have often filled their original positions in the vowel space. The general schema is as follows:

$\begin{array}{lllll}\varepsilon: \mathrm{C} & >\mathrm{iaC} & \mathrm{eC} & > & \text { iəC } \\ \Lambda: \mathrm{C} & >\text { шаC } & \gamma \mathrm{C} & > & \text { шәC } \\ & & \text { оC } & > & \text { uəC }\end{array}$

The only regular exception to this rule is in the case of velar-final rimes which, as in the case of some of the Hlai languages, provided an environment for the diphthongization of long high vowels (where $K$ represents both oral and nasal velar stops):

$\begin{array}{lllll}\mathrm{i}: \mathrm{K} & > & \text { iəK } & > & \text { iaK } \\ \mathrm{u}: \mathrm{K} & > & \text { uəK } & > & \mathrm{a}: \mathrm{K} \\ \mathrm{u}: \mathrm{K} & > & \text { uəK } & > & \text { uaK }\end{array}$

There are two specific areas in which Jiamao is particularly sporadic in its correspondences, which suggest that the categories in question were not available in the early language. The first is the class of Hlai $u$ rimes, both open and closed, long and short. Reflexes of this rime class, while not completely random, are dispersed into an above-average number of categories, most often ending up in one of the Hlai $ə$ categories, but also into the $i$ and $u$ categories as well. This suggests that there was simply no original Jiamao $u$ category (although there certainly is in present-day Jiamao), and that words were assigned to other rime categories in accordance with how they were perceived at the time of borrowing. 
The second category which shows a large amount of unpredictable variation is vowel length. The correlation with Hlai vowel length improves in the more recent stratum of loans, but in the earlier stratum (or strata), it is noticeably variation in all categories except $a: C$, a category which contained only long members in PHI. Even in this category, Jiamao displays a sharp tendency to favor short rimes if the coda is a bilabial stop (nasal or oral).

Due to the difficulty of fixing rime strata to specific stages of Hlai, only Proto-Hlai forms will be compared below, even if their initials indicate a Pre-Hlai borrowing.

\subsubsection{Tone Categories}

The Jiamao table of tone values is repeated below:

$\begin{array}{lllll}\text { Tone Category } & \text { High } & & \underline{\text { Low }} & \\ \text { A } & 55 & (1) & 11 & (4) \\ \mathrm{X} & 51 & (5) & 31 & (2) \\ \text { DLong } & 53 & (9) & 31 & (8) \\ \text { DShort } & 55 & (7) & 22 & (10)\end{array}$

With the exception of the rimes which end in an oral stop (category D), the default category for borrowed Hlai vocabulary is Jiamao category A. There is a significant minority of forms in Jiamao category $\mathrm{X}$, which seem to be represented more or less equally across Hlai categories $\mathrm{A}, \mathrm{B}$, and $\mathrm{C}$. The only regular correlation with Jiamao category $\mathrm{X}$ is with long Hlai rimes with final velars, which lenited to glottal stops in Jiamao, conditioning tone $\mathrm{X}$ before finally disappearing:

(50) $\quad \mathrm{V}: \mathrm{k} \quad>\quad \mathrm{V}: \mathrm{P} \quad>\quad \mathrm{V}:^{\mathrm{X}}$

Examples of this are given below: 
(51)

$\begin{array}{lll}\underline{\text { Gloss }} & \underline{\mathrm{PHl}} & \underline{\text { Jiamao }} \\ \text { shoulder pole } & \text { fi:k } & \text { pia }^{5} \\ \text { termite } & \text { p-lu:k } & \text { lua }^{5} \\ \text { gill } & \text { C-ya:k } & \text { yua }^{5}\end{array}$

Examples of Jiamao category $\mathrm{X}$ in correlation with other Hlai tone categories are given below:

\begin{tabular}{|c|c|c|}
\hline Gloss & $\underline{\mathrm{PHl}}$ & $\underline{\text { Jiamao }}$ \\
\hline mountain valley & $\mathrm{k}^{\mathrm{h}} \partial: \mathrm{y}$ & $\mathrm{k}^{\mathrm{h}} \mathrm{\jmath}: \mathrm{y}^{5}$ \\
\hline burn & 6əy & $6 o y^{5}$ \\
\hline pickle & C-mə:n & mo: $n^{5}$ \\
\hline
\end{tabular}

(b)

$\begin{array}{lll}\text { cockscomb } & \text { fi:wh } & \text { pi:w } \\ \text { drink } & \text { ru:nf } & \text { ku:n } \\ \text { branch (tree) } & \text { C-ya:mf } & \text { yəm }^{2}\end{array}$

(c)

$\begin{array}{lll}\text { partridge } & \mathrm{t}^{\mathrm{h}} \mathrm{a}: \mathrm{n} \text { ? } & \text { da: } \mathrm{n}^{5} \\ \text { beard } & \text { hmu:m? } & \text { mum }^{2} \\ \text { neck } & \text { ljoy? } & \text { tsay }^{2}\end{array}$

It is possible that in a small number of cases, Jiamao category $\mathrm{X}$ represents faithful borrowings of Hlai category $\mathrm{C}$ words with glottal stop (the examples in 52c above all appear to be early loans). In general, however, this lack of correlation between tone categories throughout apparently all vocabulary strata is an important piece of evidence that the Hlai vocabulary in Jiamao is borrowed, not native, because it strongly indicates that the cues correlating with tone category were not perceived in the context in which these words were learned, and that the learners were biased towards other kinds of cues (such as vowel length, discussed below).

Although there is no discernible correlation between the Jiamao A and X categories on the one hand, and the Hlai A, B, and C categories on the other, this distinction seems to have been preserved in another way, where high vowels (53a-f) and short mid central rimes (53g-j) in Hlai tone categories B and C underwent lengthening, often followed by the lowering of the nucleus, but not always: 


\begin{tabular}{|c|c|c|c|c|c|c|}
\hline & $\underline{\mathrm{PHl}}$ & $\underline{\text { Jiamao }}$ & & & $\underline{\mathrm{PHl}}$ & $\underline{\text { Jiamao }}$ \\
\hline this & *C-ni:h & $\mathrm{n} \varepsilon:^{5}$ & (b) & $\begin{array}{l}\text { uncle } \\
\text { small sore } \\
\text { cry }\end{array}$ & $\begin{array}{l}{ }^{*} \mathrm{t}^{\mathrm{h}} \mathrm{i}: ? \\
{ }^{*} \mathrm{C}-\mathrm{ni}: ? \\
{ }^{*} \mathrm{C}-\mathrm{ni}: ?\end{array}$ & $\begin{array}{l}\text { ts }^{\mathrm{h}} \mathrm{a}: \mathrm{y}^{1} \\
\text { na:y } \\
\text { na: }{ }^{1}\end{array}$ \\
\hline get up & *Cu?u:h & Pa: ${ }^{1}$ & (d) & $\begin{array}{l}\text { short } \\
\text { near }\end{array}$ & $\begin{array}{l}*^{\text {h }} \text { w:? } \\
\text { *p-lu:? }^{*} \text { plu }\end{array}$ & $\begin{array}{l}\mathrm{t}^{\mathrm{h}} \mathrm{a}:{ }^{1} \\
\mathrm{la}:{ }^{1}\end{array}$ \\
\hline $\begin{array}{l}\text { blow } \\
\text { power } \\
\text { turtle }\end{array}$ & $\begin{array}{l}* ? u: h \\
* k^{h} u: h \\
* t^{h} u: h\end{array}$ & $\begin{array}{l}\text { Pa: } w^{1} \\
\mathrm{k}^{\mathrm{h}} \mathrm{a}: \mathrm{w}^{1} \\
\mathrm{da}: \mathrm{w}^{1}\end{array}$ & (f) & $\begin{array}{l}\text { ash } \\
\text { memorial } \\
\text { three }\end{array}$ & $\begin{array}{l}{ }^{*} \text { su:? } \\
{ }^{*} \mathrm{C}-\mathrm{mu}: ? \\
{ }^{*} \mathrm{t} \int^{\mathrm{h}} \mathrm{u} ?\end{array}$ & $\begin{array}{l}\operatorname{ts}^{\mathrm{h}} \mathrm{a}: \mathrm{w}^{1} \\
\mathrm{ma}: \mathrm{w}^{1} \\
\mathrm{ta}: \mathrm{w}^{1}\end{array}$ \\
\hline $\begin{array}{l}\text { fall down } \\
\text { tomorrow }\end{array}$ & $\begin{array}{l}\text { *dəwh } \\
\text { *həwh }\end{array}$ & $\begin{array}{l}\text { da:w }{ }^{1} \\
\text { ziaw }^{4}\end{array}$ & (h) & $\begin{array}{l}\text { Fas y. sis husb } \\
\text { head } \\
\text { fill w/ rice }\end{array}$ & $\begin{array}{l}\text { *hjəw? } \\
\text { *Curəw? } \\
\text { *k }^{\mathrm{h}} \text { əw? }\end{array}$ & $\begin{array}{l}\text { tsi:w } \\
\text { ki:w } \\
\text { hi:w }\end{array}$ \\
\hline $\begin{array}{l}\text { water } \\
\text { clothing clsfr } \\
\text { instruct }\end{array}$ & $\begin{array}{l}{ }^{*} \text { C-nəm? } \\
*_{\text {fən} ?} \\
*_{\text {C-nən? }}\end{array}$ & $\begin{array}{l}\text { na: }{ }^{1} \\
\text { pa: }{ }^{1} \\
\text { ya: }{ }^{1}\end{array}$ & (j) & $\begin{array}{l}\text { meat } \\
\text { wet } \\
\text { open (eyes) }\end{array}$ & $\begin{array}{l}\text { *rəm? } \\
\text { *hmən? } \\
\text { *C-ləø? }\end{array}$ & 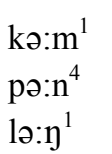 \\
\hline
\end{tabular}

The diphthongization and lengthening of the rimes in (53a-f) above is an exaggerated case of the diphthongization which occurred in Greater Hlai in the same environment.

It must be noted that from this point on, the terms Pre-Hlai and Proto-Hlai are replaced with Stratum 1 and Stratum 2 respectively, as the points of Jiamao borrowing from the point of view of the rimes is quite difficult to fix with any certainty. This is because for the initials, there is one important change (initial aspiration) which occurred toward the end of the Pre-Hlai period and therefore makes periodization rather easy; in the case of the rimes, these kinds of obvious changes are for the most part absent (it is likely that Jiamao borrowing began after peripheral vowel raising, which is the only real change which could have been used this way).

An example illustrating this point is the following:

\section{$\underline{\text { Gloss }}$}

(a)

(b) $\underline{\text { Pre-Hlai }}$

*tว:y

*tə:y?

$$
\text { jar }
$$

pickle
*C-gə:y?
*C-mə:y

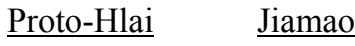

*t ${ }^{\mathrm{h}}$ ə:y

*th ${ }^{\mathrm{h}}$ ว:y?

da: ${ }^{1}$

$\mathrm{t}^{\mathrm{h}} \mathrm{a}:{ }^{1}$

$$
\text { *kə:n? }
$$

ko: ${ }^{1}$

*C-mə:y mว: $y^{5}$ 
It is argued below that the change * ${ }^{\prime}: \eta>a$ : preceded the change *ə:y $>0: \eta$ in Jiamao, so that the examples in (54a) above are assumed to have been borrowed at a period prior to those in (54b). However, the initial of sap indicates that it was borrowed in Pre-Hlai, before initial aspiration, whereas the initial of together indicates that it was borrowed after initial aspiration had already occurred. These would therefore be assigned to different strata of borrowing based on the initials, but it is clear that they both participated in the same change in the rime, which the items in (54b) did not, indicating that they were borrowed yet later. The only logical conclusion is that sap was borrowed first, followed by together, followed finally by jar and pickle. There are many instances of this phenomenon in the Jiamao lexicon, but although it complicates periodization, it is important to underscore that the periodization for the initials and that for the rimes never conflict, despite the difficulty in linearizing them absolutely. It is for this reason, therefore, that the labels Pre-Hlai and Proto-Hlai are replaced with Stratum 1 and Stratum 2 below.

\subsubsection{Open Rimes}

The open rimes, as in the case of the initials, generally have two discernible layers of loans with occasional exceptions. The Jiamao reflexes of the open rimes are shown below:

Correspondences between Proto-Hlai stops and Jiamao open vowels

\begin{tabular}{|c|c|}
\hline Proto-Hlai & $\underline{\text { Jiamao }}$ \\
\hline$*_{\mathrm{i}}:$ & $\mathrm{i}$ :, ay \\
\hline *u: & i:, a: \\
\hline *u: & u:, aw \\
\hline$* \mathrm{i}: \mathrm{h} / \mathrm{i}$ & a:y, ay \\
\hline *u: & a:, ə: \\
\hline *u:h & o:, a:w \\
\hline *u:? & $\mathrm{a}: \mathrm{w}$ \\
\hline *e: & $\varepsilon:$ \\
\hline *a: & ॰:, u:, \\
\hline
\end{tabular}

The loan chronology and subsequent changes of the Hlai open rimes are shown below: 


\begin{tabular}{|c|c|c|c|c|c|c|}
\hline & Hlai & & $\underline{\text { Jiamao }}$ & $\underline{\text { Hlai }}$ & & $\underline{\text { Jiamao }}$ \\
\hline Stratum 1 & $* \mathrm{i}:$ & $\rightarrow$ & i: > i: & $*_{\mathrm{i}: \mathrm{h} / \mathrm{P}}$ & & \\
\hline \multirow[t]{2}{*}{ Stratum 2} & $\begin{array}{c}\downarrow \\
\text { *əy }\end{array}$ & $\rightarrow$ & әy > ay & $\begin{array}{l}\downarrow \\
* \text { วyh/2 }\end{array}$ & $\rightarrow$ & ə:y > a:y \\
\hline & Hlai & & $\underline{\text { Jiamao }}$ & $\underline{\text { Hlai }}$ & & $\underline{\text { Jiamao }}$ \\
\hline Stratum 1 & $\begin{array}{c}* u: \\
\downarrow\end{array}$ & $\rightarrow$ & u: > i: & $\begin{array}{c}{ }^{*} u \mathrm{r}: \mathrm{h} / \mathrm{P} \\
\downarrow\end{array}$ & $\rightarrow$ & જщ > ә: \\
\hline \multirow[t]{2}{*}{ Stratum 2} & *әч & $\rightarrow$ & әщ > a: & *әцһ $/ 2$ & $\rightarrow$ & ә:પ્ > a: \\
\hline & $\underline{\text { Hlai }}$ & & $\underline{\text { Jiamao }}$ & $\underline{\text { Hlai }}$ & & $\underline{\text { Jiamao }}$ \\
\hline Stratum 1 & $\begin{array}{c}* \mathrm{u}: \\
\downarrow\end{array}$ & $\rightarrow$ & $\mathrm{u}:>\mathrm{u}:$ & $\begin{array}{c}* u: h(/ P) \\
\downarrow\end{array}$ & $\rightarrow$ & əw $>$ ow > \\
\hline \multirow[t]{2}{*}{ Stratum 2} & *əw & $\rightarrow$ & $\partial w>$ aw & *วwh/? & $\rightarrow$ & ə:w $>$ a:w \\
\hline & $\underline{\text { Hlai }}$ & & $\underline{\text { Jiamao }}$ & $\underline{\text { Hlai }}$ & & $\underline{\text { Jiamao }}$ \\
\hline Stratum 1 & & & & $* \mathrm{a}:$ & $\rightarrow$ & 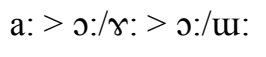 \\
\hline Stratum 2 & $* \mathrm{e}:$ & $\rightarrow$ & $\mathrm{e}:>\varepsilon:$ & $* \mathrm{a}:$ & $\rightarrow$ & $\mathrm{a}:>\mathrm{o}: / \mathrm{u}:>\mathrm{o}: / \mathrm{ow}$ \\
\hline
\end{tabular}

The two strata of loans pattern similarly throughout the high vowels. In the earliest layer, Hlai (or more specifically Greater Hlai) vowels were still pure. By the time of the second layer, these vowels had lengthened, and they underwent a process of diphthongization just as they did in the Hlai languages. The difference, however, is that in the Hlai languages they were kept distinct from the original series of short schwa-centered diphthongs (*əy and *əw) by lowering only to mid-vowels; in Jiamao, they lowered completely, allowing the two series to merge:

\begin{tabular}{|c|c|c|c|}
\hline$*_{\mathrm{i}}$ & $>$ & *əy & $>$ \\
\hline *u: & $>$ & * әш & $>$ \\
\hline *u: & $>$ & *əw & $>$ \\
\hline *əy & $>$ & *əy & $>$ \\
\hline * & $>$ & *əw & $>$ \\
\hline
\end{tabular}

The development of high vowels with final laryngeals is shown below. In the earlier stratum, the vowel was still pure, but diphthongized in Jiamao due to the final laryngeal. In the later stratum, it 
patterned with the other high vowels with final laryngeals, so it was borrowed as a diphthong and then lengthened, again due to the final laryngeal:

\begin{tabular}{|c|c|c|c|c|c|c|c|}
\hline \multirow[t]{3}{*}{ Stratum 1} & $* \mathrm{i}: \mathrm{h} / \mathrm{?}$ & $\rightarrow$ & --- & & & & \\
\hline & *u:h/? & $\rightarrow$ & ә૫ & $>$ & ru & $>$ & ə: \\
\hline & *u:h/1 & $\rightarrow$ & əw & $>$ & ow & $>$ & o: \\
\hline \multirow[t]{3}{*}{ Stratum 2} & *әућ/? & $\rightarrow$ & ə:y & $>$ & a:y & & \\
\hline & *əu $\mathrm{f} / \mathrm{P}$ & $\rightarrow$ & ə:પ & $>$ & $\mathrm{a}:$ & & \\
\hline & *əwh/R & & ə:W & $>$ & $\mathrm{a}: \mathrm{w}$ & & \\
\hline
\end{tabular}

Finally, the low vowel *a: has a total of four reflexes, two of them with specific conditioning factors. In the first layer, backing occurred; it was modified to a back unrounded vowel if preceded by a high back vowel, which then raised to a high vowel. In the second layer, *a: first raised to *o:, where it stayed if preceded by a palatal glide; otherwise it finally dipthongized to $2 w$ with subsequent coloring of the nucleus:

\begin{tabular}{|c|c|c|c|c|c|c|c|}
\hline \multirow[t]{2}{*}{ Stratum 1} & $\mathrm{a}:$ & $>$ & o: & $>$ & ๑: & $>$ & 0: \\
\hline & uCa: & $>$ & $\mathrm{uCr}$ : & $>$ & $\gamma:$ & $>$ & w: \\
\hline \multirow[t]{2}{*}{ Stratum 2} & a: & $>$ & o: & $>$ & әw & $>$ & ow \\
\hline & hja: & $>$ & hjo: & $>$ & hjo: & & \\
\hline
\end{tabular}

Examples are given below; exceptions are listed and discussed at the end:

(60)

(a)

mos y. bros wife *hmi:

(c)

$$
\text { leaf }
$$

shoot card (cotton)

$\underline{\mathrm{PHl}}$

*6u:

*hnu:

*hnu: $\underline{\text { PHl Jiamao }}$

\begin{abstract}
$$
\text { mi: }{ }^{4}
$$
mi: ${ }^{4}$
\end{abstract}

$\underline{\text { Jiamao }}$

pi: ${ }^{1}$

ni: ${ }^{4}$

ni: ${ }^{4}$ (b)

mollusk

go

fire

(d)

ladder

hand

spirit
$\underline{\mathrm{PH}}$

$* \mathrm{t}^{\mathrm{h}}{ }_{\mathrm{i}}$ :

*fi:

*fi:

$\underline{\mathrm{PH}}$

$*^{*} p^{h} u$ :

*C-mu:

*Cufur: $\underline{\text { Jiamao }}$

ts $^{\mathrm{h}}$ ay $^{1}$

pay ${ }^{1}$

pay $^{1}$

$\underline{\text { Jiamao }}$

6a: ${ }^{1}$

ma: ${ }^{1}$

ha: ${ }^{4}$ 
(e)

eight

knee

o. bros wife
$\underline{\mathrm{PHl}}$

*ru:

*C-ru:

*tcu: $\underline{\text { Jiamao }}$

$\mathrm{ku}:{ }^{1}$

lu: ${ }^{4}$

ts ${ }^{\mathrm{h}} \mathrm{u}{ }^{1}$ (f)

seven

head louse

I

(g)

uncle

small sore

cry

$\underline{\text { Jiamao }}$

(h)

nine

grandma

(j)

sleep (lie)

distribute

run
$\underline{\mathrm{PHl}}$

*C-ßu:?

*tcut?

$\underline{\mathrm{PH}}$

*hyu:h

*ku:h

*Curu:h (i)

short

near

get up

(k)

turtle

power

blow fว: ${ }^{1}$

tsə: ${ }^{2}$

$\underline{\text { Jiamao }}$

กว: ${ }^{4}$

ko: ${ }^{1}$

kə: ${ }^{1}$
(1)

ash

three

(m)

rice cake

$\underline{\text { Jiamao }}$

(n)

boat

overflow

grandpa

(p)

to plant
$\underline{\mathrm{PH}}$

*Cura:

*6a:h

*na:?

$\underline{\mathrm{PH}}$

*Cura:
$10:^{4}$

po: ${ }^{1}$

nว: ${ }^{4}$

$\underline{\text { Jiamao }}$

ku: ${ }^{1}$ (o)

branch (road)

dog

field

(q)

old

smoke medicine
$\underline{\mathrm{PHl}}$

$* \mathrm{t}^{\mathrm{h}} \mathrm{u}$ :

*t $\int^{\mathrm{h}} \mathrm{wu}$ :

*hu:

$\underline{\text { Pre-Hlai }}$

*tc $\mathrm{h}$ i:?

*C-ni:?

*C-ni:?

$\underline{\mathrm{PH}}$

*th $\mathrm{u}: ?$

*p-lut?

*Cu?u:h

$\underline{\mathrm{PH}}$

$*^{\mathrm{h}} \mathrm{u}: \mathrm{h}$

*k $\mathrm{k}: \mathrm{h}$

*?u:h

$\underline{\mathrm{PHI}}$

*su:?

*C-mu:?

*t $\int^{\mathrm{h}} \mathrm{wu}$ ?

$\underline{\mathrm{PHl}}$

*C-лe:h

$\underline{\mathrm{PH}}$

*pha:

*hma:

*hna:h

$\underline{\mathrm{PH}}$

*hja:

*hja:

*hja:
Jiamao

daw $^{1}$

taw ${ }^{1}$

kaw ${ }^{1}$

$\underline{\text { Jiamao }}$

ts ${ }^{\mathrm{h}} \mathrm{a}: \mathrm{y}^{1}$

na:y ${ }^{1}$

na:y ${ }^{1}$

Jiamao

$\mathrm{t}^{\mathrm{h}} \mathrm{a}:^{1}$

la: ${ }^{1}$

?a:

$\underline{\text { Jiamao }}$

da:w ${ }^{1}$

$\mathrm{k}^{\mathrm{h}} \mathrm{a}: \mathrm{w}^{1}$

?a:w ${ }^{1}$

Jiamao

ts ${ }^{\text {ha: }}{ }^{1}$

ma: ${ }^{1}$

ta: ${ }^{1}$

Jiamao

ne: ${ }^{2}$

$\underline{\text { Jiamao }}$

bow $^{1}$

pow ${ }^{4}$

tow ${ }^{4}$

$\underline{\text { Jiamao }}$

tso: ${ }^{1}$

tso: ${ }^{1}$

tso: ${ }^{1}$

There are a number of exceptions in this category, given below: 
(61)

Gloss

$\underline{\mathrm{PHl}}$

$\underline{\text { Jiamao }}$

(a) this

${ }^{*}$ C-ni:h

$\mathrm{n} \varepsilon:^{5}$

(b) navel

*Curu:

lo: ${ }^{2}$

return

*hmu:

pə: ${ }^{4}$

(c)

$\begin{array}{lll}\text { thin } & { }^{*} \text { ru: } & \text { kay }^{1} \\ \text { you } & { }^{*} \text { C-mu: } & \text { məy }^{1} \\ \text { look up at } & * \text { hyu:? } & \text { yəy }^{4} \\ \text { frog (inedible) } & * \text { hyu:h } & \text { yay }^{1}\end{array}$

(d) year

*hmu:h

ma: ${ }^{1}$

feed

*bu:h

fo: ${ }^{4}$

(e) two

*hlu:?

liaw $^{4}$

(f)

$\begin{array}{lll}\text { five } & * \text { hma: } & \mathrm{pu}^{4} \\ \text { mat. grandfather } & *{ }^{\mathrm{h}}{ }^{\mathrm{h}} \mathrm{a}: ? & \text { tə: }\end{array}$

The Jiamao reflex of the word in (61e) is what would normally be expected for PHl * ${ }^{*}$ wh/ $/ 2$ (see section

5.2.7 below), which indicates that this is how it was borrowed.

\subsubsection{High Front Rimes}

The correspondences between $\mathrm{PHl}$ and Jiamao rimes with high front nuclei are given below:

\begin{tabular}{|c|c|c|}
\hline (62) & Proto-Hlai & $\underline{\text { Jiamao }}$ \\
\hline \multirow[t]{7}{*}{ (a) } & $* \mathrm{i}: \mathrm{w}$ & ew, iw, i:w \\
\hline & $*_{\mathrm{i}: \mathrm{m}}$ & $\mathrm{em}$ \\
\hline & $* i: p$ & ep, ip \\
\hline & $*_{\mathrm{i}: \mathrm{n}}$ & iən, in, i:n \\
\hline & $*_{\mathrm{i}: \mathrm{t}}$ & et, it, i:t \\
\hline & $*_{\mathrm{i}: \mathrm{y}}$ & ey, ia, i:y \\
\hline & $* \mathrm{i}: \mathrm{k}$ & $\mathrm{ia}^{\mathrm{X}}, \mathrm{i}^{\mathrm{X}}$ \\
\hline
\end{tabular}

(b)

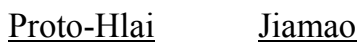

\begin{tabular}{|c|c|}
\hline$*_{\text {iw }}$ & ew, iw \\
\hline$*_{\mathrm{im}}$ & $\mathrm{em}, \mathrm{im}$ \\
\hline *ip & ep, ip \\
\hline$*$ in & in \\
\hline$*_{\text {it }}$ & et, it \\
\hline *in & i:y \\
\hline$* \mathrm{ik}$ & et, i:k, it \\
\hline
\end{tabular}


The Jiamao reflexes can be generally grouped into three sets in the case of the long rimes, and two in the case of the short rimes. The long rime reflexes could be interpreted in two ways - they could either reflect three strata of borrowing, or otherwise two of the reflexes could represent a single strata, with variation resulting from inconsistency at the point of borrowing. While recognizing both possibilities, I tentatively choose the latter hypothesis, under the assumption that there was a great deal of confusion in borrowing words with long rimes, some borrowed long and some borrowed short:

\begin{tabular}{|c|c|c|c|c|c|c|}
\hline & Hlai & & Jiamao & & & \\
\hline \multirow[t]{2}{*}{ Stratum 1} & $*_{\mathrm{i}: \mathrm{w}}$ & $\rightarrow$ & $\mathrm{i}: \mathrm{w}>\mathrm{iw}$ & & & \\
\hline & $\downarrow$ & & $\mathrm{iw}>\mathrm{ew}$ & & & \\
\hline \multirow[t]{2}{*}{ Stratum 2} & $*_{\mathrm{i}: \mathrm{w}}$ & $\rightarrow$ & i:w & & & \\
\hline & Hlai & & $\underline{\text { Jiamao }}$ & Hlai & & $\underline{\text { Jiamao }}$ \\
\hline \multirow[t]{3}{*}{ Stratum 1} & $*_{\mathrm{i}: \mathrm{m}}$ & $\rightarrow$ & --- & $*_{i: p}$ & $\rightarrow$ & $\mathrm{i}: \mathrm{p}>\mathrm{ip}$ \\
\hline & & $\searrow$ & & & $\searrow$ & \\
\hline & $\downarrow$ & & $\mathrm{im}>\mathrm{em}$ & $\downarrow$ & & ip $>$ ep \\
\hline \multirow[t]{2}{*}{ Stratum 2} & $*_{\mathrm{i}: \mathrm{m}}$ & $\rightarrow$ & --- & $*_{i: p}$ & $\rightarrow$ & --- \\
\hline & $\underline{\text { Hlai }}$ & & Jiamao & $\underline{\text { Hlai }}$ & & $\underline{\text { Jiamao }}$ \\
\hline \multirow[t]{3}{*}{ Stratum 1} & $*_{\mathrm{i}: \mathrm{n}}$ & $\rightarrow$ & $\mathrm{i}: \mathrm{n}>$ in & $*_{\mathrm{i}: \mathrm{t}}$ & $\rightarrow$ & $\mathrm{i}: \mathrm{t}>\mathrm{it}$ \\
\hline & & $\searrow$ & & & $\searrow$ & \\
\hline & $\downarrow$ & & in $>$ en $>$ iən & $\downarrow$ & & it $>$ et \\
\hline \multirow[t]{2}{*}{ Stratum 2} & $*_{i: n}$ & $\rightarrow$ & i:n & $*_{\mathrm{i}: \mathrm{t}}$ & $\rightarrow$ & i:t \\
\hline & $\underline{\text { Hlai }}$ & & $\underline{\text { Jiamao }}$ & $\underline{\text { Hlai }}$ & & $\underline{\text { Jiamao }}$ \\
\hline \multirow[t]{3}{*}{ Stratum 1} & $*_{\mathrm{i}: \mathrm{y}}$ & $\rightarrow$ & iəy $>$ ia & $*_{\mathrm{i}: \mathrm{k}}$ & $\rightarrow$ & iək $>$ ia $2>$ ia $^{x}$ \\
\hline & & $\searrow$ & & & $\searrow$ & \\
\hline & $\downarrow$ & & in $>$ en & $\downarrow$ & & --- \\
\hline Stratum 2 & $*_{\mathrm{i}: \mathrm{y}}$ & $\rightarrow$ & i:y & $*_{\mathrm{i}: \mathrm{k}}$ & $\rightarrow$ & $\mathrm{i}: \mathrm{k}>\mathrm{i}: 2>\mathrm{i}:{ }^{\mathrm{X}}$ \\
\hline
\end{tabular}

There was no similar confusion in the case of the short rimes: 
(64)

\begin{tabular}{|c|c|c|c|c|c|c|}
\hline & Hlai & & $\underline{\text { Jiamao }}$ & & & \\
\hline Stratum 1 & $*$ iw & $\rightarrow$ & iw $>$ ew & & & \\
\hline \multirow[t]{2}{*}{ Stratum 2} & $*_{\mathrm{iw}}$ & $\rightarrow$ & iw & & & \\
\hline & Hlai & & $\underline{\text { Jiamao }}$ & $\underline{\text { Hlai }}$ & & $\underline{\text { Jiamao }}$ \\
\hline Stratum 1 & $*$ im & $\rightarrow$ & $\mathrm{im}>\mathrm{em}$ & $*$ ip & $\rightarrow$ & ip $>$ ep \\
\hline \multirow[t]{2}{*}{ Stratum 2} & $* \mathrm{im}$ & $\rightarrow$ & $\mathrm{im}$ & $*$ ip & $\rightarrow$ & ip \\
\hline & $\underline{\text { Hlai }}$ & & $\underline{\text { Jiamao }}$ & $\underline{\text { Hlai }}$ & & $\underline{\text { Jiamao }}$ \\
\hline Stratum 1 & $\begin{array}{c}* \text { in } \\
\downarrow\end{array}$ & $\rightarrow$ & --- & $\begin{array}{c}* \text { it } \\
\downarrow\end{array}$ & $\rightarrow$ & it $>$ et \\
\hline \multirow[t]{2}{*}{ Stratum 2} & $*_{\text {in }}$ & $\rightarrow$ & in & $*_{\text {it }}$ & $\rightarrow$ & it \\
\hline & Hlai & & $\underline{\text { Jiamao }}$ & $\underline{\text { Hlai }}$ & & $\underline{\text { Jiamao }}$ \\
\hline Stratum 1 & *in & $\rightarrow$ & --- & $*_{\mathrm{ik}}$ & $\rightarrow$ & it $>$ et \\
\hline Stratum 2 & $\begin{array}{c}\downarrow \\
* \text { in }\end{array}$ & $\rightarrow$ & in > i:y & $\begin{array}{c}\downarrow \\
* \mathrm{ik}\end{array}$ & $\rightarrow$ & $\mathrm{ik}>\mathrm{i}: \mathrm{k}$ \\
\hline
\end{tabular}

After the first layer of borrowing, there was a lowering of the nucleus of short rimes and a shortening of the nucleus of long rimes, similar to what occurred in $\mathrm{NCHl}$ :

$$
\begin{array}{lll}
\mathrm{i}: \mathrm{C} & > & \mathrm{iC} \\
\mathrm{iC} & > & \mathrm{eC}
\end{array}
$$

This shift appears to have occurred early enough before the alveolar nasal to allow it to participate in the diphthongization of short mid vowels which affected the rimes ${ }^{*} \mathrm{eC}$ and ${ }^{*} \gamma \mathrm{C}$. Length distinctions seem to have remained intact in this final layer of borrowing.

Examples are given below. Stratum one borrowings corresponding to long rimes are given in (66a), and stratum two borrowings in (66b). Stratum one borrowings corresponding to short rimes are given in (66c), and stratum two in (66d): 
(66)

(a)

\begin{tabular}{|c|c|}
\hline $\begin{array}{l}\text { sound } \\
\text { blue } \\
\text { necklace }\end{array}$ & $\begin{array}{l}*{ }^{\mathrm{h}}{ }_{\mathrm{i}}: \mathrm{w} \\
{ }^{\mathrm{k}} \mathrm{k}_{\mathrm{h}}^{\mathrm{i}} \mathrm{w} \\
* \text { hyi:w }\end{array}$ \\
\hline plug up & *tch ${ }^{\mathrm{h}} \mathrm{i}: \mathrm{m}$ \\
\hline bear fruit & *tci:n \\
\hline stick into & ${ }^{*} \mathrm{C}-$-1:p \\
\hline chopsticks & $* \mathrm{t}^{\mathrm{h}} \mathrm{i}: \mathrm{p}$ \\
\hline fingernail & ${ }^{*} \mathrm{C}-\mathrm{li}: \mathrm{p}$ \\
\hline bail water & *hwi:t \\
\hline underwear & *fi:nh \\
\hline skirt & *si:n? \\
\hline decorate & *6i:nh \\
\hline finger & *lji:yh \\
\hline slap & ${ }^{*} \mathrm{p}^{\mathrm{h}} \mathrm{i}: \mathrm{k}$ \\
\hline full & $* \mathrm{t}^{\mathrm{h}} \mathrm{i}: \mathrm{k}$ \\
\hline shoulder pole & $*_{\mathrm{fi}: \mathrm{k}}$ \\
\hline
\end{tabular}

(b)

\begin{tabular}{|c|c|}
\hline cockscomb & *fi:wh \\
\hline wild & *lji:w \\
\hline sell & $*_{\text {ri:w? }}$ \\
\hline wear (lower) & $* p^{h} \mathrm{i}: \mathrm{n}$ \\
\hline tongue & *hli:n? \\
\hline ill omen & *hwi:n \\
\hline make way & ${ }^{*} \mathrm{p}^{\mathrm{h}} \mathrm{i}: \mathrm{y}$ ? \\
\hline rice wine & *6i:yh \\
\hline slant & *ki:n \\
\hline (c) & $\underline{\mathrm{PHl}}$ \\
\hline a bundle of rice & *hwiw \\
\hline mouse & *hniw \\
\hline & $* \mathrm{t} 6 \mathrm{im}$ \\
\hline
\end{tabular}

$\begin{array}{lll}\text { dogbean } & * \text { tc }{ }^{\mathrm{h}} \text { ip } & \text { ts }^{\mathrm{h}} \mathrm{ep}^{7} \\ \text { duck } & * \text { bit } & \text { bet }^{7} \\ \text { wrinkle } & * \text { C-jit } & \text { jet }^{7} \\ \text { grasshopper } & *[\mathrm{~g} / \mathrm{h} \eta] \text { it } & \text { Pet }^{7} \\ \text { sweep } & * \text { rjik } & \text { tset }^{10}\end{array}$

$\underline{\text { Jiamao }}$

ts $^{\mathrm{h}}$ ew ${ }^{1}$

$\mathrm{k}^{\mathrm{h}}$ ew ${ }^{1}$

kew ${ }^{4}$

ts ${ }^{\mathrm{h}} \mathrm{em}^{1}$

tsen $^{1}$

nep $^{7}$

ts $^{\mathrm{h}} \mathrm{ep}^{7}$

$\operatorname{lep}^{7}$

vet $^{7}$

pion $^{1}$

liən $^{2}$

6ion ${ }^{5}$

tsia $^{4}$

$6 \mathrm{ia}^{5}$

ts $^{\mathrm{h}} \mathrm{ia}^{5}$

pia $^{5}$

Jiamao

pi: ${ }^{5}$

tsi:w ${ }^{4}$

ki:w ${ }^{1}$

$p^{h}$ i:n ${ }^{1}$

fi: ${ }^{4}$

vi:n ${ }^{1}$

$\mathrm{p}^{\mathrm{h}} \mathrm{i}{ }^{1}$

bi: ${ }^{5}$

ki: ${ }^{1}$

$\underline{\text { Jiamao }}$

vew $^{1}$

kew ${ }^{4}$

tsem $^{4}$

$\operatorname{tset}^{10}$
$\underline{\mathrm{PHl}}$

Jiamao

able

*ki:w

kiw $^{1}$

hang

*ri:p?

$\operatorname{lin}^{4}$

centipede

*ri:p

$\operatorname{lip}^{8}$

to heat

$*_{\text {si:t }}$

ts $^{\text {h }}$ it $^{7}$

money

*tci:n

tsin ${ }^{1}$

roll (child)

*p-li:n

$\operatorname{lin}^{1}$

to tear

*C-ni:k

nit $^{7}$

$\underline{\mathrm{PHl}}$

Jiamao

be cheap $\quad * \mathrm{k}^{\mathrm{h}} \mathrm{i}: \mathrm{t} \quad \mathrm{k}^{\mathrm{h}} \mathrm{i}: \mathrm{t}^{9}$

wing

carry

${ }^{*} \mathrm{p}^{\mathrm{h}} \mathrm{i}: \mathrm{k}$

*6i:k

thick smoke

*di:k

$\mathrm{p}^{\mathrm{h}} \mathrm{i}:{ }^{5}$

fi: $:^{2}$

ti: ${ }^{2}$

(d)

$\underline{\mathrm{PHl}}$

Jiamao

escape

*p ${ }^{\text {hiw }}$

$\mathrm{p}^{\mathrm{h}} \mathrm{iw}^{1}$

poison

*kim?

$\operatorname{kim}^{1}$

a pinch

calf

fly

bright

lightning

throw away

*C-jim?

*rjin?

*6in

*din?

*ljip

* fit

$\operatorname{nim}^{1}$

$\operatorname{tsin}^{4}$

fin $^{4}$

$\operatorname{din}^{1}$

$\operatorname{lip}^{7}$

fit $^{7}$

leech *ljin

tsi: $\eta^{4}$

solemn silence * $\quad$ ik ?i:k ${ }^{9}$ 
There are a handful of exceptions in this category:

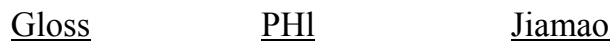

\begin{tabular}{|c|c|c|c|}
\hline (a) & $\begin{array}{l}\text { polished rice } \\
\text { hemp }\end{array}$ & $\begin{array}{l}\text { *tci:m? } \\
\text { *hmi:n }\end{array}$ & $\begin{array}{l}\operatorname{tsiam}^{1} \\
\operatorname{mian}^{1}\end{array}$ \\
\hline (b) & clean & ${ }^{*}{ }^{t}{ }^{\mathrm{h}}{ }_{\mathrm{i}: \mathrm{yh}}$ & ts $^{\mathrm{h}} \mathrm{iə \eta} \mathrm{y}^{5}$ \\
\hline (c) & & $\begin{array}{l}{ }^{*} \text { Curiw } \\
{ }^{*} \text { C-niw }\end{array}$ & $\begin{array}{l}\text { ku: } \\
\text { naw }^{1}\end{array}$ \\
\hline (d) & splash & ${ }^{*} c^{\mathrm{h}}{ }_{\text {inf }}$ & $\mathrm{ts}^{\mathrm{h}}{ }^{\mathrm{h}} \mathrm{t}^{\mathrm{T}}$ \\
\hline
\end{tabular}

The word in (67d) is one of two cases in Jiamao where the precursor to Tone B influenced transmission in such a way as to result in the replacement of the final nasal with a stop at the same place of articulation.

\subsubsection{High Back Unrounded Rimes}

The following are the correspondences long $u$ : $C$ rimes and short $u C$ rimes:

\begin{tabular}{|c|c|c|}
\hline (68) & $\underline{\text { Proto-Hlai }}$ & $\underline{\text { Jiamao }}$ \\
\hline \multirow[t]{6}{*}{ (a) } & *u:y & иәу \\
\hline & *u:m & um, om, ৩:m \\
\hline & *u:p & up, uəp \\
\hline & *u:n & un \\
\hline & *u:y & oy, a:y \\
\hline & *u:k & iək, a:k \\
\hline
\end{tabular}

(b)

It is more difficult to suggest a chronology for this series of rimes, although we can at least infer that rimes in $\supset$ and with diphthongs are not the most recent layer. It can also be assumed that mid vowels in some cases correspond to high vowels that were borrowed as short rimes and then underwent lowering, as in the case of the high front rimes above. I tentatively suggest the following chronology: 
(69)

\begin{tabular}{|c|c|c|c|c|c|c|}
\hline (a) & Hlai & & $\underline{\text { Jiamao }}$ & & & \\
\hline \multirow[t]{2}{*}{ Stratum 1} & *u:y & $\rightarrow$ & әу $>$ oy $>$ uәy & & & \\
\hline & $\underline{\text { Hlai }}$ & & $\underline{\text { Jiamao }}$ & Hlai & & $\underline{\text { Jiamao }}$ \\
\hline \multirow[t]{3}{*}{ Stratum 1} & *u:m & $\rightarrow$ & $\mathrm{u}: \mathrm{m}>\mathrm{um}$ & *u:p & $\rightarrow$ & $\mathrm{u}: \mathrm{p}>\mathrm{up}$ \\
\hline & & $\searrow$ & & & $\searrow$ & \\
\hline & $\downarrow$ & & $\partial \mathrm{m}>\mathrm{om}$ & $\downarrow$ & & әp $>$ op $>$ uəp \\
\hline \multirow[t]{2}{*}{ Stratum 2} & *u:m & $\rightarrow$ & ə:m > ๑:m & *u:p & $\rightarrow$ & --- \\
\hline & Hlai & & $\underline{\text { Jiamao }}$ & & & \\
\hline \multirow[t]{3}{*}{ Stratum 1} & *ur:n & $\rightarrow$ & u:y > wy & & & \\
\hline & & & әу $>$ a & & & \\
\hline & $\underline{\text { Hlai }}$ & & $\underline{\text { Jiamao }}$ & $\underline{\text { Hlai }}$ & & $\underline{\text { Jiamao }}$ \\
\hline Stratum 1 & *u:y & $\rightarrow$ & әу $>$ oท & *ur:k & $\rightarrow$ & $\partial \mathrm{k}>\mathrm{ek}>\mathrm{i} \partial \mathrm{k}$ \\
\hline Stratum 2 & $\begin{array}{l}\downarrow \\
* \text { wi:y }\end{array}$ & $\rightarrow$ & ə:y>a:y & 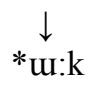 & $\rightarrow$ & ə:k $>\mathrm{a}: \mathrm{k}$ \\
\hline (b) & Hlai & & $\underline{\text { Jiamao }}$ & $\underline{\text { Hlai }}$ & & $\underline{\text { Jiamao }}$ \\
\hline Stratum 1 & *um & $\rightarrow$ & -- & *up & $\rightarrow$ & ə:p > $: p$ \\
\hline \multirow[t]{2}{*}{ Stratum 2} & $\stackrel{\downarrow}{*_{\mathrm{um}}}$ & $\rightarrow$ & um & $\begin{array}{c}\downarrow \\
* \text { up }\end{array}$ & $\rightarrow$ & -- \\
\hline & Hlai & & $\underline{\text { Jiamao }}$ & $\underline{\text { Hlai }}$ & & $\underline{\text { Jiamao }}$ \\
\hline Stratum 1 & *un & $\rightarrow$ & ə:n > o:n & *ut & $\rightarrow$ & at $>$ ot $>$ uət \\
\hline \multirow[t]{2}{*}{ Stratum 2} & $\begin{array}{c}\downarrow \\
*_{\text {un }}\end{array}$ & $\rightarrow$ & әy $>$ ay & $\begin{array}{c}\downarrow \\
*_{\text {ut }}\end{array}$ & $\rightarrow$ & -- \\
\hline & $\underline{\text { Hlai }}$ & & $\underline{\text { Jiamao }}$ & & & \\
\hline Stratum 1 & *un & $\rightarrow$ & -- & & & \\
\hline Stratum 2 & $\begin{array}{c}\downarrow \\
* \text { uy }\end{array}$ & $\rightarrow$ & un & & & \\
\hline
\end{tabular}

There is a tendency (but not an absolute rule) for back vowels to trigger velarization of an alveolar coda:

$$
\begin{aligned}
& \text { ш:n } \rightarrow \quad \text { ш:y }>\text { uท } \\
& \text { wn } \rightarrow \text { oy }>\text { ay }
\end{aligned}
$$


Examples are given below. Stratum one borrowings corresponding to long rimes are given in (71a), and stratum two borrowings in (71b). Statum one borrowings corresponding to short rimes are given in (71c), and stratum two in (71d):

(a)

bamboo

beard

egg

rice knife

rotten

full

wash

pt nosed turtle

pumpkin

come

castrate

pheasant

let go

child

bone

(c)

handlength

heavy

break (pull) $\quad * t^{\mathrm{h}} u t$

*fju:p

*th tu:p $^{\mathrm{h}}$

*dur:n

*du:n

$\underline{\mathrm{PHl}}$

$*^{\mathrm{h}}{ }^{\mathrm{u}}$ un $\underline{\text { Jiamao }}$

luəу ${ }^{5}$

*p-lu:yh

*hmu:m?

*hju:m

*k ${ }^{\mathrm{h}} \mathrm{u}: \mathrm{p}$

*t ${ }^{\text {h }}$ u:m

$*^{\mathrm{h}} \mathrm{w}: \mathrm{m}$

*Cufur:p

*hmu:n

*ph ${ }^{\mathrm{h}}$ :y?

*hlu:k

*Curw:k

tiək $^{8}$

$\operatorname{liək}^{10}$

*Cuhup

ho: $p^{8}$

$\mathrm{k}^{\mathrm{h}} \mathrm{p}: \mathrm{n}^{1}$

duət $^{9}$

$\operatorname{hup}^{7}$

dom ${ }^{1}$

$\mathrm{k}^{\mathrm{h}} \mathrm{om}^{1}$

ts $^{\text {h }}$ uəp ${ }^{9}$

duəp ${ }^{9}$

huəp ${ }^{8}$

mur ${ }^{4}$

twin ${ }^{4}$

$t^{\mathrm{h}} \mathrm{un}^{4}$

$60 \eta^{5}$

$\underline{\text { Jiamao }}$ (b)

$\underline{\mathrm{PHl}}$

$\underline{\text { Jiamao }}$

crawl

cheat

(d)

poke a hole
clear (sky)
bamboo shoot

ripe

weave (fabric)

not know

thorn

astringent

*Cufiun?

*hmun?

han ${ }^{4}$

pan $^{4}$

pull tight

*kun

kuin $^{5}$

There are various exceptions in this category: 
(72)

$\underline{\text { Gloss }} \quad \underline{\mathrm{PHl}} \quad \underline{\text { Jiamao }}$

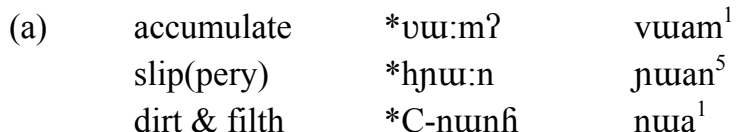

(b) two *C-lu:yh $\quad$ luan $^{5}$

ginger $\quad * \mathrm{k}^{\mathrm{h}} \mathrm{u}: \mathrm{y} \quad \mathrm{k}^{\mathrm{h}} \mathrm{u} \mathrm{y}^{5}$

measure (rice) $\quad{ }^{*}$ C-lu:y? $\quad$ ¡: $: \mathrm{y}^{4}$

These words in (72b) are borrowings of Chinese 兩, 薑 and 量 (Mandarin liang ${ }^{3}$, jiang $^{1}$ and liang $^{2}$ ), and may have been independently borrowed from Chinese instead of via Hlai.

\subsubsection{High Back Rounded Rimes}

The reflexes of the $u: C$ and $u C$ rimes are given below:

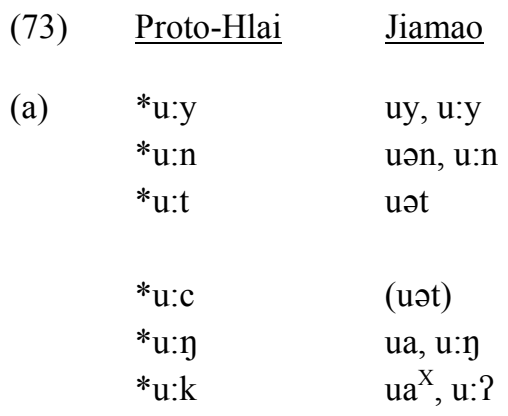

\begin{tabular}{|c|c|c|}
\hline \multirow[t]{5}{*}{ (b) } & *uy & ey, uəy, uy \\
\hline & *un & oy, uy, un \\
\hline & *ut & ok, ut \\
\hline & *un & on \\
\hline & *uc & uət, ut \\
\hline
\end{tabular}

These reflexes can also be organized into a rough framework of two layers, in which short reflexes which later lowered to mid-vowels are a distinguishing feature of the first layer: 
(74)

\begin{tabular}{|c|c|c|c|c|c|c|}
\hline (a) & $\underline{\text { Hlai }}$ & & $\underline{\text { Jiamao }}$ & & & \\
\hline Stratum 1 & $\begin{array}{c}* u: y \\
\downarrow\end{array}$ & $\rightarrow$ & uy & & & \\
\hline \multirow[t]{2}{*}{ Stratum 2} & *u:y & $\rightarrow$ & u:y & & & \\
\hline & $\underline{\text { Hlai }}$ & & $\underline{\text { Jiamao }}$ & $\underline{\text { Hlai }}$ & & $\underline{\text { Jiamao }}$ \\
\hline Stratum 1 & $\begin{array}{c}* u: n \\
\downarrow\end{array}$ & $\rightarrow$ & on $>$ uən & $\begin{array}{c}* u: t \\
\downarrow\end{array}$ & $\rightarrow$ & ot $>$ uət \\
\hline Stratum 2 & $* u: n$ & $\rightarrow$ & $\mathrm{u}: \mathrm{n}$ & $* u: t$ & $\rightarrow$ & --- \\
\hline
\end{tabular}

\begin{tabular}{|c|c|c|c|c|c|c|}
\hline & $\underline{\text { Hlai }}$ & & $\underline{\text { Jiamao }}$ & $\underline{\text { Hlai }}$ & & $\underline{\text { Jiamao }}$ \\
\hline Stratum 1 & *u:n & $\rightarrow$ & --- & ${ }^{*} \mathrm{u}: \mathrm{c}$ & $\rightarrow$ & (ot $>$ uət) \\
\hline \multirow[t]{2}{*}{ Stratum 2} & $*^{u}: \mathrm{n}$ & $\rightarrow$ & --- & $* \mathrm{u}: \mathrm{c}$ & $\rightarrow$ & \\
\hline & Hlai & & $\underline{\text { Jiamao }}$ & $\underline{\text { Hlai }}$ & & $\underline{\text { Jiamao }}$ \\
\hline Stratum 1 & *u:y & $\rightarrow$ & uəy $>$ ua & $* \mathrm{u}: \mathrm{k}$ & $\rightarrow$ & uə? $>$ ua $^{\mathrm{X}}$ \\
\hline Stratum 2 & ${ }^{*} \mathrm{u}: \mathrm{y}$ & $\rightarrow$ & $\mathrm{u}: \mathrm{y}$ & $* \mathrm{u}: \mathrm{k}$ & $\rightarrow$ & $\mathrm{u}: \mathrm{k}>\mathrm{u}: ?$ \\
\hline
\end{tabular}

(b)

Hlai $\quad \underline{\text { Jiamao }}$

\begin{tabular}{|c|c|c|c|c|c|c|}
\hline \multirow[t]{2}{*}{ Stratum 1} & *uy & $\rightarrow$ & u:y > uy & & & \\
\hline & $\downarrow$ & & әy $>$ ey & & & \\
\hline \multirow[t]{2}{*}{ Stratum 2} & *uy & $\rightarrow$ & оу > uəy & & & \\
\hline & $\underline{\text { Hlai }}$ & & $\underline{\text { Jiamao }}$ & \multicolumn{2}{|c|}{ Hlai } & $\underline{\text { Jiamao }}$ \\
\hline Stratum 1 & *un & $\rightarrow$ & әу $>$ ว & $*$ ut & $\rightarrow$ & $\partial \mathrm{k}>\mathrm{ok}$ \\
\hline \multirow[t]{2}{*}{ Stratum 2} & $\begin{array}{c}\downarrow \\
* \text { un }\end{array}$ & $\rightarrow$ & un $>$ un & $\begin{array}{c}\downarrow \\
* u t\end{array}$ & $\rightarrow$ & --- \\
\hline & Hlai & & $\underline{\text { Jiamao }}$ & Hlai & & $\underline{\text { Jiamao }}$ \\
\hline Stratum 1 & *un & $\rightarrow$ & ən $>$ on & $* u c$ & $\rightarrow$ & ot $>$ uət \\
\hline Stratum 2 & $\underset{*}{\downarrow}$ & $\rightarrow$ & --- & $\begin{array}{c}\downarrow \\
* \text { uc }\end{array}$ & $\rightarrow$ & ut \\
\hline
\end{tabular}

In the case of the short rimes, the back vowel conditioned velarization of alveolar codas: 


$$
\begin{array}{lll}
\text { un } & \rightarrow & \text { əy }>\text { on } \\
\text { un } & \rightarrow & \text { un }>\text { uy } \\
\text { ut } & \rightarrow & \text { ək }>\text { ok }
\end{array}
$$

Examples are given below. Stratum one borrowings corresponding to long rimes are given in (76a), and stratum two borrowings in (76b). Statum one borrowings corresponding to short rimes are given in (76c), and stratum two in (76d):

(a)

$$
\text { fat }
$$

wild

tree clsfr

body

fart

ten

wash

hole

white hair

termite

(c)

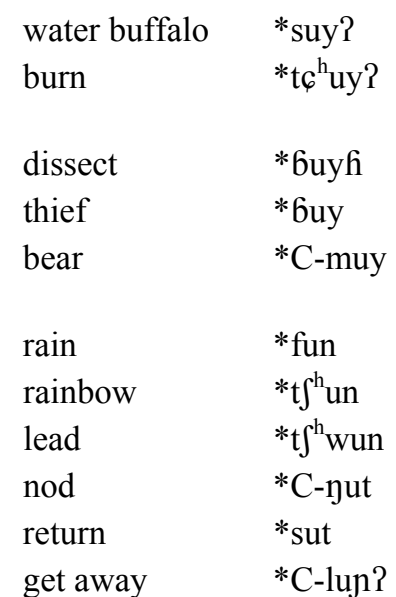

$\underline{\text { Jiamao }}$

$$
\text { kuy }^{1}
$$

ts $^{\mathrm{h}}$ uən ${ }^{1}$

duən ${ }^{1}$

huən $^{4}$

duət $^{9}$

puot $^{9}$

?uot ${ }^{9}$

$*$ fu:t

$*$ ?u:t

$*$ tc $\mathrm{h}: \mathrm{p}$ ?

*hyu:k

*p-lu:k

$\underline{\mathrm{PHl}}$

*C-lun?

ts $^{\mathrm{h}} \mathrm{ua}^{1}$

kua $^{2}$

lua $^{5}$

$\underline{\text { Jiamao }}$

ts $^{\mathrm{h}} \mathrm{ey}^{1}$

tsey ${ }^{1}$

puy ${ }^{1}$

fuy ${ }^{4}$

muy $^{4}$

poy ${ }^{1}$

ton ${ }^{1}$

ton ${ }^{1}$

$\mathrm{yok}^{7}$

tsok $^{7}$

$\operatorname{lon}^{1}$ (b)

$\underline{\mathrm{PHl}}$

${ }^{*} \mathrm{C}$-mu:y

*ru:nh

drink

sow (seed)

stand
Jiamao

\begin{tabular}{|c|c|c|}
\hline daughter & *?u:y & Pu: $:^{1}$ \\
\hline copper & *du:y & tu: $y^{1}$ \\
\hline wrap & $*^{\mathrm{h}} \mathrm{u}: \mathrm{k}$ & $t^{\text {h }} \mathrm{u}::^{9}$ \\
\hline brain & *hlu:k & tu: $:^{8}$ \\
\hline nest & $*_{\text {ru:k }}$ & $\operatorname{lu}::^{8}$ \\
\hline (d) & PHl & Jiamao \\
\hline
\end{tabular}

mu:y ${ }^{1}$

$\mathrm{ku}: \mathrm{n}^{5}$

mu: ${ }^{1}$

ts $^{\mathrm{h}} \mathrm{u}: \mathrm{n}^{1}$ 
Various kinds of exceptions are given below:

\begin{tabular}{|c|c|c|c|}
\hline (77) & $\underline{\text { Gloss }}$ & $\underline{\mathrm{PHl}}$ & $\underline{\text { Jiamao }}$ \\
\hline (a) & rotten & $* t^{\text {h }}$ uy & $t^{h} u y^{5}$ \\
\hline \multirow[t]{4}{*}{ (b) } & door & *C-mu:n & muan $^{1}$ \\
\hline & fold & *C-mu:n & ma: $n^{1}$ \\
\hline & parrot (black) & *tcu:nh & tsuay $^{5}$ \\
\hline & vine basket & *ku:p? & kuan $^{1}$ \\
\hline \multirow[t]{2}{*}{ (c) } & pond & *Cifu:yh & Pjoy ${ }^{5}$ \\
\hline & have & *du:k & tok $^{9}$ \\
\hline \multirow[t]{2}{*}{ (d) } & dust & *fu:p? & puəๆ $^{5}$ \\
\hline & cricket & *Curu:y? & fuəy ${ }^{4}$ \\
\hline \multirow[t]{4}{*}{ (e) } & swell & *Pun & fun $^{4}$ \\
\hline & steam & *CiPunh & $\operatorname{tsun}^{1}$ \\
\hline & blackhead & *Curut & $\operatorname{lup}^{8}$ \\
\hline & worship & $* p^{h} u c$ & $\mathrm{p}^{\mathrm{h}} \mathrm{ut}^{7}$ \\
\hline (f) & big & *C-lun & lo: ${ }^{1}$ \\
\hline (g) & fur & *Cufiun & ho:1 $\eta^{4}$ \\
\hline
\end{tabular}

\subsubsection{Mid Front Rimes}

The Jiamao reflexes of this small number of forms are all completely regular, and are presumably of recent origin given the lack of diphthongization of these rimes:

$\begin{array}{lll}\text { Proto-Hlai } & & \text { Jiamao } \\ \text { *e:m } & & \text { e:m } \\ *_{\text {*e:p }} & & \text { e:p } \\ \text { *e:n }^{\text {en }} & & \text { e:n }\end{array}$

(79)

$\underline{\text { Hlai }} \underline{\text { Jiamao }} \underline{\text { Hlai }} \underline{\text { Jiamao }}$

Stratum 2

$*_{\mathrm{e}: \mathrm{m}} \quad>$

e:m

e:p >

e:p

Hlai $\quad \underline{\text { Jiamao }}$

Stratum $2 \quad *_{\text {e:n }} \quad>\quad$ e:n 
Examples are given below:

$\begin{array}{lll} & \underline{\text { PHl }} & \underline{\text { Jiamao }} \\ \text { ringworm } & \text { *C-le:mh } & \text { le: } \mathrm{m}^{5} \\ \text { board } & * \text { be:nf } & \text { 6e: } \mathrm{n}^{5} \\ \text { sunken } & * \text { be:p } & \text { pe: } \mathrm{p}^{9}\end{array}$

The initial of the last form indicates an early level of borrowing; it is possible that this could merely be a mistransmission of the initial.

\subsubsection{Mid Central Rimes}

There is a very large amount of variation in this rime class, with the nucleus having been influenced by both the preceding initial as well as the tone category and the place of the coda. There is less variation in the long rimes; the variation in the short rimes, on the other hand, is unmatched (there are a total of seven 'regular' correspondences of *ən).

\begin{tabular}{|c|c|c|c|}
\hline Proto-Hlai & $\underline{\text { Jiamao }}$ & $\underline{\text { Proto-Hlai }}$ & $\underline{\text { Jiamao }}$ \\
\hline \multirow[t]{4}{*}{ *ə:y } & ey, uy, uy, uəy, a:y (b) & *әу & i:, ay, a:y \\
\hline & & *əu & ə:, a: \\
\hline & & *ow & u:, aw, a:w \\
\hline & & *əwh/? & iaw, i:w \\
\hline *a:m & o:m, iam, am & *əm & ə:m, a:m \\
\hline *ə:p & әp, ap & *əp & ๑:p, ep, әp, ap \\
\hline *ə:n & wan & *ən & ə:n, iən, uən, en, ə:n, an, a:n \\
\hline \multirow[t]{3}{*}{$*_{\partial}: t$} & wət, wat & $* \partial t$ & s:t, at \\
\hline & & *əл & wən, on \\
\hline & & $* \partial c$ & iət, et, a:t, at \\
\hline *a:y & oy, o:ท, a: & *əy & วท, ว:ท, ว:y, aฤ \\
\hline *ə:k & ok, o:k, a:, ak & *ək & ok, ak \\
\hline
\end{tabular}

The following tentative schema, as in the case of the $u$ rime class, should be considered only an approximation, with details to be worked out as work on Jiamao becomes more refined: 
(82)

\begin{tabular}{|c|c|c|c|c|c|c|}
\hline (a) & $\underline{\text { Hlai }}$ & & $\underline{\text { Jiamao }}$ & & & \\
\hline \multirow[t]{2}{*}{ Stratum 1} & *ə:y & $\rightarrow$ & uy, әу > ey & & & \\
\hline & $\downarrow$ & & uy, oy > uәy & & & \\
\hline \multirow[t]{2}{*}{ Stratum 2} & *ə:y & $\rightarrow$ & ə:y > a:y & & & \\
\hline & Hlai & & $\underline{\text { Jiamao }}$ & Hlai & & $\underline{\text { Jiamao }}$ \\
\hline \multirow[t]{3}{*}{ Stratum 1} & *ə:m & $\rightarrow$ & $\mathrm{om}>\partial \mathrm{m}>\mathrm{am}$ & *ə:p & $\rightarrow$ & $o p>\partial p>a p$ \\
\hline & & $\searrow$ & & & $\searrow$ & \\
\hline & $\downarrow$ & & ə:m > ๑:m & $\downarrow$ & & $o p>\partial p$ \\
\hline \multirow[t]{2}{*}{ Stratum 2} & *ə:m & $\rightarrow$ & $\varepsilon: \mathrm{m}>\mathrm{iam}$ & *ə:p & $\rightarrow$ & --- \\
\hline & $\underline{\text { Hlai }}$ & & $\underline{\text { Jiamao }}$ & $\underline{\text { Hlai }}$ & & $\underline{\text { Jiamao }}$ \\
\hline Stratum 1 & *ə:n & $\rightarrow$ & --- & *ə:t & $\rightarrow$ & $\gamma t>$ wat \\
\hline \multirow[t]{2}{*}{ Stratum 2} & $\begin{array}{c}\downarrow \\
* \partial: n\end{array}$ & $\rightarrow$ & $\Lambda: \mathrm{n}>$ uan & $\underset{* \partial: t}{\downarrow}$ & $\rightarrow$ & $\Lambda: t>$ uat \\
\hline & $\underline{\text { Hlai }}$ & & $\underline{\text { Jiamao }}$ & $\underline{\text { Hlai }}$ & & $\underline{\text { Jiamao }}$ \\
\hline \multirow[t]{2}{*}{ Stratum 1} & *ə:ท & $\rightarrow$ & 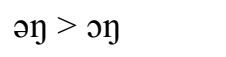 & $*_{\partial: \mathrm{k}}$ & $\rightarrow$ & $\partial \mathrm{k}>\partial \mathrm{k}$ \\
\hline & $\downarrow$ & & ว:ฤ> ว:ท & $\downarrow$ & & ə:k $>$ : $\mathrm{k}$ \\
\hline Stratum 2 & *ə:1 & $\rightarrow$ & ə:ๆ > a: & *ə:k & $\rightarrow$ & $\partial: \mathrm{k}>\mathrm{a}:$ \\
\hline (b) & $\underline{\text { Hlai }}$ & & $\underline{\text { Jiamao }}$ & $\underline{\text { Hlai }}$ & & $\underline{\text { Jiamao }}$ \\
\hline Stratum 1 & *əy & $\rightarrow$ & әy > i: & *әщ & $\rightarrow$ & әщ > ә: \\
\hline \multirow[t]{2}{*}{ Stratum 2} & *əy & $\rightarrow$ & әy > ay/a:y & *วน & $\rightarrow$ & ə: > a: \\
\hline & Hlai & & $\underline{\text { Jiamao }}$ & $\underline{\text { Hlai }}$ & & $\underline{\text { Jiamao }}$ \\
\hline Stratum 1 & $\begin{array}{c}* \partial \mathrm{w} \\
\downarrow\end{array}$ & $\rightarrow$ & əw > u: & $\begin{array}{c}* \text { əwh/? } \\
\downarrow\end{array}$ & $\rightarrow$ & $\varepsilon: \mathrm{w}>$ iaw \\
\hline \multirow[t]{2}{*}{ Stratum 2} & * วw & $\rightarrow$ & əw $>$ aw/a:w & *วwh/? & $\rightarrow$ & e:w > i:w \\
\hline & Hlai & & $\underline{\text { Jiamao }}$ & $\underline{\text { Hlai }}$ & & $\underline{\text { Jiamao }}$ \\
\hline \multirow[t]{2}{*}{ Stratum 1} & $* \partial \mathrm{m}$ & $\rightarrow$ & -- & $* ә p$ & $\rightarrow$ & op $>$ әp \\
\hline & $\downarrow$ & & -- & $\downarrow$ & & әp $>0: p$ \\
\hline
\end{tabular}




\begin{tabular}{|c|c|c|c|c|c|c|}
\hline Stratum 2 & $\begin{array}{l}* \text { *m } \\
\text { Hlai }\end{array}$ & $\rightarrow$ & $\begin{array}{l}\text { ə:m>a:m } \\
\underline{\text { Jiamao }}\end{array}$ & $\begin{array}{l}* \partial p \\
\text { Hlai }\end{array}$ & $\rightarrow$ & $\begin{array}{l}\text { әр }>\text { ap } \\
\underline{\text { Jiamao }}\end{array}$ \\
\hline \multirow[t]{2}{*}{ Stratum 1} & *ən & $\rightarrow$ & en/on $>$ iən/uən & *ət & $\rightarrow$ & --- \\
\hline & $\downarrow$ & & en, on $>$ o:n & $\downarrow$ & & $\partial t>0: t$ \\
\hline \multirow[t]{2}{*}{ Stratum 2} & *ən & $\rightarrow$ & $\partial[:] n>a[:] n$ & *ət & $\rightarrow$ & ot $>$ at \\
\hline & $\underline{\text { Hlai }}$ & & $\underline{\text { Jiamao }}$ & $\underline{\text { Hlai }}$ & & $\underline{\text { Jiamao }}$ \\
\hline Stratum 1 & $\begin{array}{c}* \partial \mathfrak{} \\
\downarrow\end{array}$ & $\rightarrow$ & rn $>$ wən & $\begin{array}{c}* \partial c \\
\downarrow\end{array}$ & $\rightarrow$ & et $>$ iət, $\partial t>$ at \\
\hline \multirow[t]{2}{*}{ Stratum 2} & *əј & $\rightarrow$ & -- & $* \partial c$ & $\rightarrow$ & et, ə:t $>$ a:t \\
\hline & $\underline{\text { Hlai }}$ & & $\underline{\text { Jiamao }}$ & Hlai & & $\underline{\text { Jiamao }}$ \\
\hline Stratum 1 & $\begin{array}{c}* \partial \eta \\
\downarrow\end{array}$ & $\rightarrow$ & $\partial[:] \eta>\curvearrowright[:] \eta$ & $\begin{array}{c}* \text { ək } \\
\downarrow\end{array}$ & $\rightarrow$ & ək $>$ ok \\
\hline Stratum 2 & *əท & $\rightarrow$ & oy $>$ an & *ək & $\rightarrow$ & ək $>$ ak \\
\hline
\end{tabular}

The various reflexes in the first stratum of *ə:y appear to be conditioned by the preceding initial, according to whether or not it is bilabial (uəy), alveolar (uxy), lateral (ey), or rhotic (uy) (see examples below in (87)).

There are a small number of rimes in modern Jiamao with a schwa nucleus. In order to explain their lack of lowering to $a$, I hypothesize that these forms had an $o$ nucleus until recently, when it dissimilated with the following coda, as in the following example:

$$
\text { ə:p }>\text { op }>\text { әp }
$$

The tendency for short rimes in Hlai tone category $\mathrm{C}$ to lengthen has already been mentioned above, and need not be repeated here. Another interesting development in the short diphthongs is the tendency in the earliest stratum to monophthongize when in category A:

$$
\begin{aligned}
& \text { әу }>\mathrm{i}: \\
& \text { əw }>\mathrm{u} \text { : }
\end{aligned}
$$


The nucleus of Hlai * əwh/? seems to have been prone to fronting in Jiamao. It fronted in Jiamao to $\varepsilon: w$, in the first stratum, and ultimately diphthongized. It fronted to $e: w$ in the the second stratum, undergoing raising:

\begin{tabular}{|c|c|c|c|}
\hline Stratum 1 & *əwh/R > & $\varepsilon: \mathrm{W}$ & $>$ \\
\hline Stratum 2 & *owh/R > & e:w & $>$ \\
\hline
\end{tabular}

Excluding cases where lengthening is induced via association with Hlai tone $\mathrm{C}$, there were two other situations in which lengthening occurred. The first was in the case of items participating in the change $\curvearrowright>\curvearrowright$ (86a), and the other was before oral palatal stops (86b):

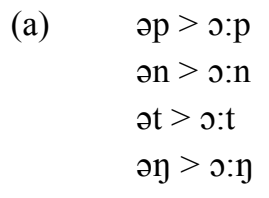

(b) $\quad$ oc $>a: t$

In some instances, rimes before an oral palatal stop were fronted:
(a) $\quad$ c $>$ et $>$ iət
(b) $\quad$ c $>$ et

Examples are given below. Stratum one borrowings corresponding to long rimes are given in (88a), and stratum two borrowings in (88b). Statum one borrowings corresponding to short rimes are given in (88c), and stratum two in $(88 \mathrm{~d})$ : 
(a)

rope
saliva
muntjac
many

cicada

iron

kidney

$$
\text { solid }
$$

close (eyes)

break

stay

to smoke

arrow

chew

dense

sap

together

skin

$$
\text { s. }
$$

wash

deaf

deep
$\underline{\mathrm{PHl}}$

* də:y

*hlə:y

*C-lə:y

*hlə:y

*rə:y

*ro:y

*C-nə:m

*th ə:mh

*hนə:p

*ph ว:n?

*t $\int^{h}$ ə:nh

*Cuhə:n

*C-nə:t

*Cuhə:t

*?ว:t

$* \mathrm{t}^{\mathrm{h}}$ ə:1

$* \mathrm{t}^{\mathrm{h}}$ ə:1?

*C-nə:1

*sə:k

*hlə:k

*hlə:k $\underline{\text { Jiamao }}$

tury ${ }^{1}$

tury ${ }^{4}$

ley ${ }^{1}$

tey ${ }^{4}$

kuy $^{1}$

kuy $^{1}$

$\operatorname{nam}^{1}$

$\mathrm{t}^{\mathrm{h}} \mathrm{o}: \mathrm{m}^{5}$

$\operatorname{nap}^{7}$

buan ${ }^{1}$

turan $^{1}$

huan ${ }^{4}$

yuət $^{7}$

huat ${ }^{10}$

?uət ${ }^{7}$

da: ${ }^{1}$

$\mathrm{t}^{\mathrm{h}} \mathrm{a}:{ }^{1}$

na:

ts ${ }^{\mathrm{h}} \mathrm{a}:^{5}$

$4 a:^{2}$

$4 a:^{2}$

(b)

hen

*rə:y

*hmə:y?

*C-ßə:y

*C-mə:y

foreigner

sharp

redeem

bitter

pile up

*rə:p

*tch ${ }^{\mathrm{h}}$ :m

*t $\int^{\mathrm{h}}$ ə:m?

*C-hə:m

Jiamao

ka: $y^{5}$

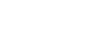


(c)

$\underline{\mathrm{PHl}}$

porcupine

gall bladder

*dəy

float

mortar

*6วw

*row

tomorrow

wear

banyan

* həwh

*Cunəw?

*th कw?

water

*C-nəm?

evening

$* t c^{\mathrm{h}}$ әp

sew

leak

*C-nәр

*Cifop

cloth

rice

* đəp

*Curəp

longyan

long/thin thing

long clsfr

louse

hungry

earth

tooth

feed (n.)

rub (rope)

dream

day

*6วn?

* dənh

*vən?

$* \mathrm{t}^{\mathrm{h}}$ ən

*C-rən

*Curan

*fjon

*k $\mathrm{k}^{\mathrm{h}}$ ən

*ph ${ }^{\mathrm{h}}$ วn

*fon

*hywan $\underline{\text { Jiamao }}$

ti: ${ }^{1}$

ti: ${ }^{1}$

fu: ${ }^{4}$

lu: ${ }^{4}$

ziaw $^{4}$

niaw $^{1}$

diaw $^{1}$

na:m ${ }^{1}$

ts ${ }^{\mathrm{h}} \mathrm{\rho}: \mathrm{p}^{9}$

лю: $p^{9}$

hว:p ${ }^{9}$

təp ${ }^{7}$

$1 ә p^{8}$

piən $^{1}$

tion $^{1}$

viən $^{4}$

$\operatorname{ten}^{1}$

$\operatorname{len}^{4}$

$\operatorname{len}^{4}$

ts $^{\mathrm{h}} \mathrm{an}^{1}$

han $^{1}$

6ว: $n^{1}$
pง:n ${ }^{1}$
vง:n

wear

gnat

nose

sneeze

pinch
$* \mathrm{tc}^{\mathrm{h}}$ ət

*C-mət

* $\mathrm{k}^{\mathrm{h}} \partial \mathrm{t}$

*də̄

* də๊? ts $^{\mathrm{h}} \mathrm{o:t^{9 }}$

ho: $t^{9}$

tuวn ${ }^{1}$

turon ${ }^{1}$ (d)

$\underline{\mathrm{PHl}}$

*kəy

*ləy

*C-ləy

*sow

*tow

* how

horn

*hjow?

*Curəw?

Fas y.
head

* $\mathrm{k}^{\mathrm{h}}$ əw?

fill $\mathrm{w} /$ rice

meat/wild pig *rəm?

care for (sprout) $*$ dəp

extinguish

*tсәр

hang up

*hyәp

livestock clsfr *hmən

*hmən

*hyən

silver

$*$ tc ${ }^{\mathrm{h}}$ วn

*fon

seed

move

*hənh

ts $^{\mathrm{h}} \mathrm{an}^{1}$

han $^{2}$ $\underline{\text { Jiamao }}$

$\mathrm{k}^{\mathrm{h}} \mathrm{yy}^{1}$

tay $^{4}$

lay $^{1}$

ts $^{\mathrm{h}} \mathrm{aw}^{1}$

daw ${ }^{1}$

haw $^{4}$

tsi:w ${ }^{1}$

ki:w ${ }^{1}$

hi:w ${ }^{1}$

$\mathrm{k} ә \mathrm{~m}^{1}$

dep $^{7}$

tsep $^{7}$

$\operatorname{kap}^{8}$

puən ${ }^{4}$

puən ${ }^{4}$

kuən $^{4}$

ts $^{\mathrm{h}} \mathrm{an}^{1}$

$\begin{array}{lll}\text { clothing clsfr } & *_{\text {fən}} \text { r } & \text { pa: }{ }^{1} \\ \text { instruct } & * \text { C-yən? } & \text { ya: }{ }^{1} \\ \text { stem } & * \text { C-yən } & \text { ya: }{ }^{1}\end{array}$

$\begin{array}{lll}\text { wet } & \text { *hmən? } & \text { pə: }{ }^{4} \\ \text { grass } & \text { *hyən? } & \text { kə: }{ }^{4}\end{array}$

close

*C-not

yat $^{7}$

choose

*hləj

tson ${ }^{4}$ 


\begin{tabular}{|c|c|c|c|c|c|}
\hline & $\underline{\mathrm{PHl}}$ & $\underline{\text { Jiamao }}$ & (d) & $\underline{\mathrm{PHI}}$ & $\underline{\text { Jiamao }}$ \\
\hline $\begin{array}{l}\text { sparrow } \\
\text { strangle }\end{array}$ & $\begin{array}{l}{ }^{*} \mathrm{p}^{\mathrm{h}} \partial \mathrm{c} \\
*_{\text {r }} \partial \mathrm{c}\end{array}$ & $\begin{array}{l}6 a t^{7} \\
\operatorname{lat}^{10}\end{array}$ & clear land & *hməc & pet $^{8}$ \\
\hline tree core & *Cufoc & $\operatorname{Pi\partial t}^{7}$ & buy & $* t \int^{h} \partial c$ & ta: $t^{9}$ \\
\hline red vine & *kəc & $\operatorname{kiat}^{7}$ & $\begin{array}{l}\text { forbidden food } \\
\text { drag out }\end{array}$ & $\begin{array}{l}{ }^{* C} \text {-nəc } \\
{ }^{*} \text { hwəc }\end{array}$ & $\begin{array}{l}\text { ya: } t^{9} \\
\text { va:t }\end{array}$ \\
\hline drum & *C-ləy & $10: y^{1}$ & burn & *6əy & $60 y^{5}$ \\
\hline pry & $*^{*} \mathrm{k}^{\mathrm{h}} \partial \eta \mathrm{h}$ & $\mathrm{k}^{\mathrm{h}} \mathrm{an}^{1}$ & dragon & *hnəy & tə: $\eta^{4}$ \\
\hline finger & $*_{\mathrm{t}}^{\mathrm{c} \partial \mathrm{k}}$ & tsok $^{7}$ & stick to & ${ }^{*} \mathrm{p}^{\mathrm{h}} \partial \mathrm{k}$ & $\mathrm{p}^{\mathrm{h}} \mathrm{ak}^{7}$ \\
\hline
\end{tabular}

There are several classes of exceptions in this group, as follows:

\begin{tabular}{|c|c|c|c|}
\hline (89) & Gloss & $\underline{\mathrm{PHl}}$ & $\underline{\text { Jiamao }}$ \\
\hline \multirow[t]{2}{*}{ (a) } & soak & *də:m? & tsiom $^{4}$ \\
\hline & $\operatorname{sink}$ & $*_{\text {tç:n }}$ & $\operatorname{tsen}^{4}$ \\
\hline \multirow[t]{2}{*}{ (b) } & mound & *Cifə:n & huən ${ }^{3}$ \\
\hline & betelnut & *C-lə:n? & luə习 ${ }^{1}$ \\
\hline (c) & lid & *hyə:t & yut $^{8}$ \\
\hline \multirow[t]{2}{*}{ (d) } & sift & *Cirəw & tsi:w ${ }^{4}$ \\
\hline & four & *t $\int^{\mathrm{h}} \partial \mathrm{w} ?$ & tiow $^{1}$ \\
\hline \multirow[t]{3}{*}{ (e) } & below & $* t \int^{\mathrm{h}}$ wəw & ta: ${ }^{1}$ \\
\hline & to hoe & *bowh & $60: w^{5}$ \\
\hline & fall down & *dəwh & da:w ${ }^{1}$ \\
\hline (f) & mouth & *hməm? & muəу ${ }^{4}$ \\
\hline (g) & bug & *Cifəənh & $\mathrm{zo}: \mathrm{t}^{8}$ \\
\hline (h) & groan & *kəy & kiəy $^{1}$ \\
\hline (i) & greedy & *6ət & $6 u t^{7}$ \\
\hline
\end{tabular}


The form in (89f) shows the same dissimilation between the initial and coda which occurred in Cunhua: mom $>$ moy $>$ muəy $^{4}$. The form in $(89 \mathrm{~g})$ is another form which is in tone category B in Hlai, and which seems to have conditioned the misperception of the final nasal as an oral stop in Jiamao.

\subsubsection{Mid Back Rimes}

The reflexes of the ${ }^{\circ} \mathrm{OC}$ rimes are given below:

\begin{tabular}{|c|c|}
\hline$\underline{\text { Proto-Hlai }}$ & $\underline{\text { Jiamao }}$ \\
\hline *om & um, əm, ৩:m, a:m, am \\
\hline$*_{o p}$ & up, op \\
\hline *on & 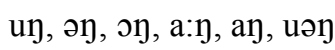 \\
\hline *ok & o:k, a:k \\
\hline
\end{tabular}

These reflexes overlap to a very large extent with the short $*_{\partial \mathrm{C}}$ rimes above, and it is likely that there was mistransmission in both directions, with both ${ }^{*} \mathrm{oC}$ rimes being borrowed as $\partial \mathrm{C}$, and ${ }^{*} \partial \mathrm{C}$ rimes as $o C$. This is also the one case where an $₫: C$ or $\supset$ reflex may not reflect descent from an earlier schwamedial form. A tentative schema of borrowing is given below:

\begin{tabular}{|c|c|c|c|c|c|c|}
\hline & $\underline{\text { Hlai }}$ & & $\underline{\text { Jiamao }}$ & $\underline{\text { Hlai }}$ & & $\underline{\text { Jiamao }}$ \\
\hline \multirow[t]{2}{*}{ Stratum 1} & *om & $\rightarrow$ & o:m, om > əm & $*$ *p & $\rightarrow$ & op \\
\hline & $\downarrow$ & & $\partial[:] \mathrm{m}>\mathrm{a}[:] \mathrm{m}$ & $\downarrow$ & & \\
\hline \multirow[t]{2}{*}{ Stratum 2} & *om & $\rightarrow$ & um & $*_{\mathrm{op}}$ & $\rightarrow$ & up \\
\hline & $\underline{\text { Hlai }}$ & & $\underline{\text { Jiamao }}$ & $\underline{\text { Hlai }}$ & & $\underline{\text { Jiamao }}$ \\
\hline \multirow[t]{2}{*}{ Stratum 1} & *oy & $\rightarrow$ & วy, oy $>$ əท & *ok & $\rightarrow$ & -- \\
\hline & $\downarrow$ & & $\partial[:] \mathrm{y}>\mathrm{a}[:] \mathrm{y}$ & $\downarrow$ & & ə:k > a:k \\
\hline Stratum 2 & *oy & $\rightarrow$ & un & $*^{o k}$ & $\rightarrow$ & o:k \\
\hline
\end{tabular}


There are two examples of rimes which I hypothesize to have been oC, only recently shifting to $ə \mathrm{C}$ :

$$
\begin{aligned}
& \text { om }>\text { əm } \\
& \text { oy }>\text { əท }
\end{aligned}
$$

There are also cases where an ${ }^{*} \mathrm{oC}$ rime either raised to $u C$ or was borrowed that way to begin with:

$$
\begin{aligned}
& \text { om }>\text { um } \\
& \text { op }>\text { up } \\
& \text { on }>\text { un }
\end{aligned}
$$

\begin{tabular}{|c|c|c|c|c|c|}
\hline (a) & $\underline{\mathrm{PHl}}$ & $\underline{\text { Jiamao }}$ & (b) & $\underline{\mathrm{PHl}}$ & $\underline{\text { Jiamao }}$ \\
\hline bamboo (big) & *dom & $\operatorname{təm}^{1}$ & bamboo (big) & $*$ dom & dum $^{5}$ \\
\hline husk & *rom & $\mathrm{kəm}^{1}$ & bury & *hlomf & tum ${ }^{4}$ \\
\hline $\operatorname{six}$ & *hnom & nəm ${ }^{4}$ & simmer & $*_{\mathrm{p}}$-lom? & $\mathrm{t}^{\mathrm{h}} u \mathrm{~m}^{4}$ \\
\hline thing clsfr & *hom & ko:m ${ }^{4}$ & ambush & $* \mathrm{t}^{\mathrm{h}} \mathrm{om} ?$ & $t^{\mathrm{h}} \mathrm{am}^{1}$ \\
\hline mute & $*$ C-nom & yว:m ${ }^{1}$ & itch & $*^{\mathrm{h}} \mathrm{om}$ & $\mathrm{k}^{\mathrm{h}} \mathrm{am}^{1}$ \\
\hline wine medicine & *Cufiom & hə: $\mathrm{m}^{4}$ & & & \\
\hline cover & ${ }^{*} \mathrm{k}^{\mathrm{h}} \mathrm{op}$ & $\mathrm{k}^{\mathrm{h}} \supset \mathrm{p}^{7}$ & carry & *?op & $\operatorname{Pup}^{7}$ \\
\hline winnow basket & *don? & tuəy ${ }^{1}$ & well & $* \mathrm{t}^{\mathrm{h}} \mathrm{oyh}$ & $\mathrm{t}^{\mathrm{h}} \supset \mathrm{y}^{5}$ \\
\hline to clean & *koy? & kuəๆ $^{1}$ & thing & *koy & $\mathrm{k}^{1}{ }^{1}$ \\
\hline shake & *C-noph & nuə๊ $^{4}$ & hunchback & *koy & $\mathrm{k}^{1} \mathrm{y}^{1}$ \\
\hline neck & *ljoy? & $\operatorname{tsan}^{2}$ & vine basket & *C-bon & $\operatorname{puy}^{1}$ \\
\hline resemble[same] & $*$ doy & day $^{1}$ & messy & *C-лор? & nuy $^{1}$ \\
\hline to fish & $*^{*} \operatorname{son} ?$ & $1 ə \eta^{2}$ & insert forcefully & $*_{t} c^{\mathrm{h}}$ oy? & $\operatorname{ts}^{\mathrm{h}} \mathrm{a}: \mathrm{y}^{5}$ \\
\hline fall & $* \mathrm{t}^{\mathrm{h}} \mathrm{ok}$ & dळ: $\mathrm{k}^{9}$ & sick & $* \mathrm{t}^{\mathrm{h}} \mathrm{ok}$ & ta: $\mathrm{k}^{9}$ \\
\hline monkey & ${ }^{*} \mathrm{C}$-nok & no:k ${ }^{9}$ & steal & $*$ ljok & tsa: $k^{8}$ \\
\hline leg & $* \mathrm{k}^{\mathrm{h}} \mathrm{ok}$ & ho: $\mathrm{k}^{9}$ & & & \\
\hline
\end{tabular}

Examples are given below. Stratum one borrowings are given in (94a), and stratum two borrowings in (94b):

There are a small number of exceptions in this category: 
$\underline{\text { Gloss }}$

$\underline{\mathrm{PHl}}$

$\underline{\text { Jiamao }}$

(a)

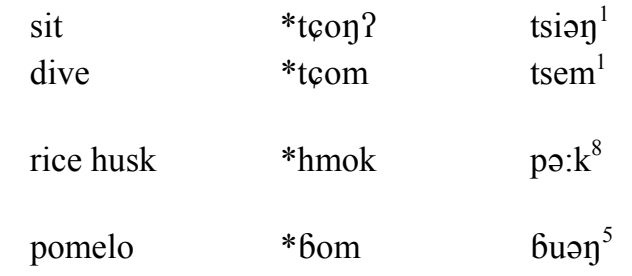

The nucleus of the two words in (95a) seems to have been fronted under the influence of the preceding initials. (95c) appears to be another case of dissimilation, as in the case of mouth above: 6om $>6$ on $>$ 6uəу ${ }^{5}$.

\subsubsection{Low Rimes}

The *a:C rimes, like the rimes at the other point of the cardinal vowel triangle, are more straightforward and predictable than rimes in other categories (although there is still an appreciable amount of variation). The reflexes of these rimes are given below:

\begin{tabular}{|c|c|c|}
\hline (96) & $\underline{\text { Proto-Hlai }}$ & $\underline{\text { Jiamao }}$ \\
\hline & $* a: y$ & uy, way, a:y \\
\hline & $* a: w$ & u:, u:w, ə:w, ॰:w, a:w \\
\hline & *a:m & əm, วm, ॰:m, a:m \\
\hline & *a:p & up, ว:p \\
\hline & *a:n & ய:n, uən, ว:n, a:n \\
\hline & $* \mathrm{a}: \mathrm{t}$ & (u:t) \\
\hline & $* \mathrm{a}: \mathrm{c}$ & uət, a:t \\
\hline & *a:y & ш:, ша, e:ๆ \\
\hline & $* \mathrm{a}: \mathrm{k}$ & u:, ша \\
\hline
\end{tabular}

One salient feature of the diphthongs is that there was an early stratum of *a:w rimes which were apparently borrowed as * $\partial \mathrm{w}$, later shortening to $u$ : 


$$
* \mathrm{a}: \mathrm{w} \rightarrow \text { əw }>\mathrm{u}:
$$

There seems to have been a strong tendency towards raising in the first stratum of *a:C loans, which followed this progression:

$$
* \mathrm{a}: \mathrm{C}>\gamma: \mathrm{C}>\mathrm{ur}: \mathrm{C}
$$

This was the first change which apparently led to the creation of a *u:C category in Jiamao, where none had existed before when Hlai * $\mathrm{w}[:] \mathrm{C}$ rimes were in the process of being borrowed (generally mixing with the Hlai *ə[:]C category).

The outcome of the second raising which occurred in the *a:C category at a later point in time depended on the codas:

$$
\begin{aligned}
& \text { a:y }>\Lambda: y>\text { may } \\
& \text { a:w }>0: w \\
& a: P>0: P \\
& a: T>0 T>\text { uəT } \\
& a: \eta>\varepsilon: \eta>e: \eta \\
& a: k>\Lambda: ?>\text { ma }^{B}
\end{aligned}
$$

The tendency for long rimes with bilabial codas to be shortened can be observed here as well:

$$
\begin{aligned}
& a: m>o m>2 m \\
& a: m>2 m>0 m \\
& a: p>\text { up }
\end{aligned}
$$

A schema of borrowing and subsequent changes is given below: 
$(101)$

\begin{tabular}{|c|c|c|c|c|c|c|}
\hline & $\underline{\text { Hlai }}$ & & $\underline{\text { Jiamao }}$ & Hlai & & $\underline{\text { Jiamao }}$ \\
\hline \multirow[t]{3}{*}{ Pre-Hlai } & $* a: y$ & $\rightarrow$ & r:y > uy & *a:w & $\rightarrow$ & әw > u:, $\gamma: w>u: w$ \\
\hline & & $\searrow$ & & & $\searrow$ & \\
\hline & $\downarrow$ & & $\Lambda: y>$ uay & $\downarrow$ & & o:W \\
\hline \multirow[t]{2}{*}{ PHl } & $* a: y$ & $\rightarrow$ & a:y & *a:w & $\rightarrow$ & ə:w, a:w \\
\hline & $\underline{\text { Hlai }}$ & & $\underline{\text { Jiamao }}$ & $\underline{\text { Hlai }}$ & & $\underline{\text { Jiamao }}$ \\
\hline \multirow[t]{3}{*}{ Pre-Hlai } & $* a: m$ & $\rightarrow$ & $\mathrm{om}>\partial \mathrm{m}$ & $* a: p$ & $\rightarrow$ & $\gamma: p>$ up \\
\hline & & $\searrow$ & & & $\searrow$ & \\
\hline & $\downarrow$ & & ๑:m, əm > & $\downarrow$ & & ๑:p \\
\hline \multirow[t]{2}{*}{ PHl } & $* \mathrm{a}: \mathrm{m}$ & $\rightarrow$ & $\mathrm{a}: \mathrm{m}$ & $* a: p$ & $\rightarrow$ & --- \\
\hline & $\underline{\text { Hlai }}$ & & $\underline{\text { Jiamao }}$ & $\underline{\text { Hlai }}$ & & $\underline{\text { Jiamao }}$ \\
\hline \multirow[t]{3}{*}{ Pre-Hlai } & $* a: n$ & $\rightarrow$ & $\gamma: w>u: n$ & *a:t/a:c & $\rightarrow$ & $\gamma: t>u: t$ \\
\hline & & $\searrow$ & & & $\searrow$ & \\
\hline & $\downarrow$ & & on $>$ uən & $\downarrow$ & & ot $>$ uət \\
\hline \multirow[t]{2}{*}{$\mathrm{PHl}$} & $* a: n$ & $\rightarrow$ & a:n & $* a: t / a: c$ & $\rightarrow$ & $\mathrm{a}: \mathrm{t}$ \\
\hline & $\underline{\text { Hlai }}$ & & $\underline{\text { Jiamao }}$ & $\underline{\text { Hlai }}$ & & $\underline{\text { Jiamao }}$ \\
\hline Pre-Hlai & $* a: y$ & $\rightarrow$ & $\tilde{\gamma}:>$ u: u? & $* \mathrm{a}: \mathrm{k}$ & $\rightarrow$ & $\gamma: 1>\omega:^{B}$ \\
\hline PHl & $* a: y$ & $\rightarrow$ & $\varepsilon: \eta>e: \eta$ & $* \mathrm{a}: \mathrm{k}$ & $\rightarrow$ & $\Lambda: P>$ шa $^{\mathrm{B}}$ \\
\hline
\end{tabular}

Examples of each stratum are given below. Stratum one borrowings are given in (102a), and stratum two borrowings in (102b): 
(102)

(a)

end

intestine

*6a:y?

*ra:y?

cold

sugarcane

shit

forehead

laugh

fishy

surround

alcohol

mountain fore

lift (2)

branch (tree)

carry on shoulder $* t^{\mathrm{h}} \mathrm{a}$ :p

yawn

partridge $\quad * \mathrm{t}^{\mathrm{h}} \mathrm{a}: \mathrm{n}$ ?

grind (rice)

red

100

snore

poor

blood

name

master

sweet

sheep

high

dregs

otter
${ }^{*} \mathrm{C}-$-ja:mf

${ }^{*} \mathrm{k}^{\mathrm{h}} \mathrm{a}: \mathrm{yh}$

${ }^{*} \mathrm{C}$-ma:y?

*ha:y?

*da:w

*Cira:w

${ }^{*} \mathrm{k}^{\mathrm{h}} \mathrm{a}$ :w

*hna:w?

*C-na:wh

*da:w?

*t $\int^{\text {ha }} a: m$

*hฤa:p

*ka:nf

*ra:n?

*Cura:n

*Cura:n

*va:t

*hla:c

*p ${ }^{\mathrm{h}}$ a:y

*va:y

*da:y

*hja:y

${ }^{*} \mathrm{p}$ a:k

*da:k

*hna:k $\underline{\text { Jiamao }}$

puy $^{5}$

luy ${ }^{4}$

$\mathrm{k}^{\mathrm{h}}$ way $^{1}$

muay ${ }^{1}$

hway $^{2}$

tu: ${ }^{1}$

tsu: ${ }^{4}$

hu: ${ }^{1}$

nə:พ ${ }^{2}$

yə: $w^{2}$

tw: ${ }^{1}$

tom ${ }^{1}$

nəm ${ }^{2}$

to: $p^{9}$

ho: $p^{8}$

da: $n^{5}$

ka: $n^{5}$

fu: $n^{4}$

ku: ${ }^{1}$

lu: ${ }^{4}$

fur:t ${ }^{8}$

4uət ${ }^{8}$

$\mathrm{p}^{\mathrm{h}} \mathrm{u}:{ }^{1}$

vu: ${ }^{4}$

tw: $?^{7}$

tsur: $?^{7}$

$p^{\text {h }} u:^{5}$

tw: ${ }^{5}$

nu: $:^{2}$ (b)

service

envy

*da:yf

*C-na:yf

da: $y^{5}$

ya: ${ }^{1}$
Jiamao

fog

salt

cotton

*hya:w?

$\mathrm{ko}: \mathrm{w}^{4}$

${ }^{*}$-nna:w?

*ha:w?

no: ${ }^{4}$

ho:w ${ }^{4}$

stew

spade

${ }^{*}$ C-na:wh

ya: ${ }^{5}$

*lja:wh

$\mathrm{za}: \mathrm{w}^{4}$

step

*C-ha:mf

ho: $\mathrm{m}^{2}$

step

*Cifia:mf

$\mathrm{za}: \mathrm{m}^{5}$

bathe

*?a:p

Pup 9

$\begin{array}{lll}\text { remainders } & *^{*} \text { da:n } & \text { duən }^{1} \\ \text { village } & { }^{*} \text { C- } \beta \text { a:n } & \text { fuən }^{1} \\ \text { moon } & { }^{*} \text { C-na:n } & \text { nuən }^{1}\end{array}$

stop (turn off)

${ }^{*}$ C-na:c

ya: $t^{9}$

ribs

* $\mathrm{k}^{\mathrm{h}} \mathrm{a}: \mathrm{y}$ ?

$\mathrm{k}^{\mathrm{h}} \mathrm{e}: \mathrm{y}^{1}$

crippled

*Cifa:yf

he: $\eta^{2}$

big brother

*?a:n?

ใe: ${ }^{1}$

$\begin{array}{lll}\text { land leech } & * t^{\text {h }} \text { a:k } & \text { dwa }^{5} \\ \text { gill } & * \text { C-na:k } & \text { yua }^{5} \\ \text { plantain } & * \text { hwa:k } & \text { vwa }^{5}\end{array}$


The following exceptions exist in this series of rimes:

\begin{tabular}{|c|c|c|c|}
\hline (103) & $\underline{\text { Gloss }}$ & $\underline{\mathrm{PHl}}$ & $\underline{\text { Jiamao }}$ \\
\hline (a) & smell & *ha:y & huәу ${ }^{4}$ \\
\hline (b) & yawn & *ka:w & ho: ${ }^{5}$ \\
\hline (c) & $\begin{array}{l}\text { white } \\
\text { long }\end{array}$ & $\begin{array}{l}*^{\mathrm{h}} \mathrm{a} \text { :w } \\
\text { *hna:w? }^{2}\end{array}$ & $\begin{array}{l}\mathrm{k}^{\mathrm{h}} \mathrm{ow}^{1} \\
\text { tow }^{4}\end{array}$ \\
\hline & steer & ${ }^{*} \mathrm{C}$-na:w & now $^{1}$ \\
\hline (d) & clothes & *va:y? & vo: ${ }^{4}$ \\
\hline (e) & testicle & *hma:n? & po: $n^{2}$ \\
\hline (f) & $\begin{array}{l}\text { millet } \\
\text { skin (of fruit) }\end{array}$ & $\begin{array}{l}\text { *fa:y? } \\
\text { *fa:k }\end{array}$ & $\begin{array}{l}\text { pua }^{1} \\
\text { pua }^{5}\end{array}$ \\
\hline (g) & $\begin{array}{l}\text { phlegm } \\
\text { toad }\end{array}$ & $\begin{array}{l}\text { *ha:k } \\
\text { *ra:k }\end{array}$ & $\begin{array}{l}\text { ha: } k^{8} \\
\text { la: } p^{8}\end{array}$ \\
\hline
\end{tabular}

The examples in (103c) all lost their labiovelar codas, either at the point of borrowing or sometime afterwards, and followed the regular development of *a: depending on the stratum in which they were borrowed.

\subsubsection{Summary}

The Jiamao to Hlai rime correspondences are significantly more complex than those of the initials, which may imply a number of possible facts. The first is that there may have been more than two discrete periods of borrowing, as implied by the initial correspondences, or that the two periods of borrowing may not have been discrete, and there may have been overlap. On the other hand, this high degree of variation in rime reflexes may indicate that the speakers of Jiamao were less adept at perceiving differences in the Hlai rime categories than those of the initials, and that the higher degree of confusion over rime identity resulted in the Hlai loans being dispersed cross-categorically in a less-discriminating fashion. 
It is apparent that part of the confusion in rime transmission involved the Hlai tone categories.

The fact that Hlai tone categories B and C correlate with Jiamao rime length in specific contexts mentioned above indicates that Thurgood (1991: 427) is correct when he suggests that Jiamao speakers 'focused on Hlai voice quality characteristics, ignoring pitch characteristics.'

\subsection{The Non-Hlai Lexicon of Jiamao}

A select group of Jiamao core vocabulary is discussed in this section, with the two-fold purpose of exemplifying its differences with Hlai on the one hand, and of highlighting the non-Hlai core vocabulary for other scholars who may see a connection with another language or language family.

\subsubsection{Prounouns}

The pronoun schema (including the deictics) for PHl and Jiamao is given below:

\begin{tabular}{|c|c|c|c|c|c|}
\hline (104) & PHl & $\underline{\text { Jiamao }}$ & & PHl & $\underline{\text { Jiamao }}$ \\
\hline $1 \mathrm{sg}$ & *hu: & kaw $^{1}$ & $2 \mathrm{sg}$ & *C-mu: & məy ${ }^{1}$ \\
\hline $1 \mathrm{pl} \mathrm{excl}$ & *fəw & Paw $^{1}$ & $2 \mathrm{pl}$ & *səw & tshaw $^{1}$ \\
\hline $1 \mathrm{pl} \mathrm{incl}$ & *row & tey ${ }^{1}$ & & & \\
\hline $3 \mathrm{sg}$ & *C-na: & $n e y^{5}$ & this (proximal) & *C-ni:h & $\mathrm{n} \varepsilon:^{5}$ \\
\hline \multirow[t]{2}{*}{$3 \mathrm{pl}$} & *ru:h & mow $^{5}$ & that (medial) & * Һәщи & $\mathrm{k} \varepsilon:^{4}$ \\
\hline & & & that (distal) & ${ }^{*} \mathrm{C}$-ma: $\mathrm{h}$ & mo: ${ }^{5}$ \\
\hline
\end{tabular}

Of the Jiamao personal pronouns given above, the only ones which seem to be directly related to the Hlai ones are in the second person (and even there, the rime of the 2sg pronoun is irregular). Although the 1sg pronoun appears related (more so because of its resemblance to Tai 1sg pronouns which are usually of the shape ku: or kaw), the resemblance may be deceptive, as the initial $k$ in Jiamao is normally a reflex of either $\mathrm{PHl} *_{\mathrm{g}}$ or ${ }^{\mathrm{r}} \mathrm{r}$ (although see the alternate explanation in section 5.1). Amongst the deictics, the 
proximal and distal pronouns appear to be related to Hlai (although in the case of the proximal pronoun, the vowel is irregular) but the medial appears to be unrelated.

\subsubsection{Numerals}

The PHl numerals and their Jiamao equivalents are given below:

\begin{tabular}{|c|c|c|c|c|c|}
\hline (105) & PHl & $\underline{\text { Jiamao }}$ & & PHl & $\underline{\text { Jiamao }}$ \\
\hline one & $*\left[\mathrm{tc}^{\mathrm{h}} \mathrm{ur}:\right]$ & ku: ${ }^{2}$ & $\operatorname{six}$ & *hnom & $n ə m^{4}$ \\
\hline two & *hlu:? & $\operatorname{tiaw}^{4}$ & seven & $* \mathrm{t}^{\mathrm{h}} \mathrm{u}:$ & daw $^{1}$ \\
\hline three & $* t \int^{h} w u ?$ & ta: $w^{1}$ & eight & *ru: & $\mathrm{ku}::^{1}$ \\
\hline four & *t $\int^{\mathrm{h}} \partial w ?$ & tiəw $^{1}$ & nine & *C- $\beta u$ :? & fə: ${ }^{1}$ \\
\hline five & *hma: & pu: ${ }^{4}$ & ten & $* f u: t$ & puot $^{9}$ \\
\hline 100 & ${ }^{*}$ Cura:n & kw: ${ }^{1}$ & & & \\
\hline
\end{tabular}

On the face of it, this semantic group seems to be more uniform, as all of the Jiamao numerals are related to their Hlai counterparts (the numeral one is only tentatively reconstructible in PHl, and shouldn't therefore be considered a counterexample). However, in cases where it is discernible, it becomes clear that these numerals were not all borrowed at the same time, in the same stratum. Focusing on the initials, it becomes clear that five (with an oral stop reflex), is part of a later stratum than six (with a nasal stop reflex); seven also appears to be part of an earlier stratum. Moving to the rimes (and remembering that as a category they are generally more complex), eight (with a pure vowel) appears to be earlier than that of seven (with a diphthong).

\subsubsection{Kinship Terms}

There are several kinship terms which stand out in Jiamao as being of non-Hlai origin: 
(106)

ancestor

wife

Father's y sister *fur? $\underline{\text { PHl Jiamao }}$ ${ }^{*} p^{h}$ ut $p^{h} u: ?$

pə: $w^{4}$ tuən ${ }^{4}$

na: $w^{5}$

fi: ${ }^{4}$
PHl

man

woman

child *pha:? C-ma:n *hmi:? $\mathrm{k}^{\mathrm{h}}$ əwh mu $^{2}$ ta: ${ }^{1}$

*lur:k C-lik

younger sibling *ru:y
Jiamao

$$
\mathrm{p}^{\mathrm{h}} \mathrm{u}^{1} \text { tsə: }{ }^{4}
$$

ment

$\operatorname{tiok}^{8}{ } \mathrm{lat}^{7}$ nuəy ${ }^{5}$

The words for man, woman, and child above are interesting for the reason that they all seem to have compounded the initial part of each Hlai form (also compounds themselves) to an older, native form; the rimes of the first two can be explained to be the result of neutralization, common in the case of the first member of Jiamao compound words.

\subsubsection{Body Parts}

There is a large amount of basic body terminology which is unique to Jiamao:

\begin{tabular}{|c|c|c|c|c|c|}
\hline (107) & $\underline{\mathrm{PHl}}$ & $\underline{\text { Jiamao }}$ & & $\underline{\mathrm{PHl}}$ & $\underline{\text { Jiamao }}$ \\
\hline face & *dəy & $\mathrm{p}^{\mathrm{h}} \mathrm{an}^{1}$ & chin & *ha:y & $\operatorname{ts}^{\mathrm{h}} \mathrm{\jmath}: \mathrm{y}^{1}$ \\
\hline ear & *ljəy & ko: ${ }^{1}$ & throat & $*^{*} \mathrm{k}^{\mathrm{h}} \mathrm{\partial}: \mathrm{k}$ & $10: k^{8}$ \\
\hline shoulder & *va:h & vet $^{10}$ & breast & $*_{\text {tci: }}$ & $\operatorname{nen}^{5}$ \\
\hline arm & ${ }^{*} \mathrm{k}^{\mathrm{h}} \mathrm{i}: \mathrm{n}$ & tur:n $n^{1}$ & lung & *kəwh & vuəך $^{4}$ \\
\hline sweat & *CuPən & Purat $^{7}$ & urine & *du: & tsem $^{5}$ \\
\hline
\end{tabular}

Of these, the words for face, shoulder, arm, and chin are particularly diagnostic of Southern KraDai languages, and the absence of the usual Kra-Dai forms here is conspicuous.

\subsubsection{Animals, Insects, and Plants}

Jiamao words for animals (a), insects (b), and plants (c), are compared below: 


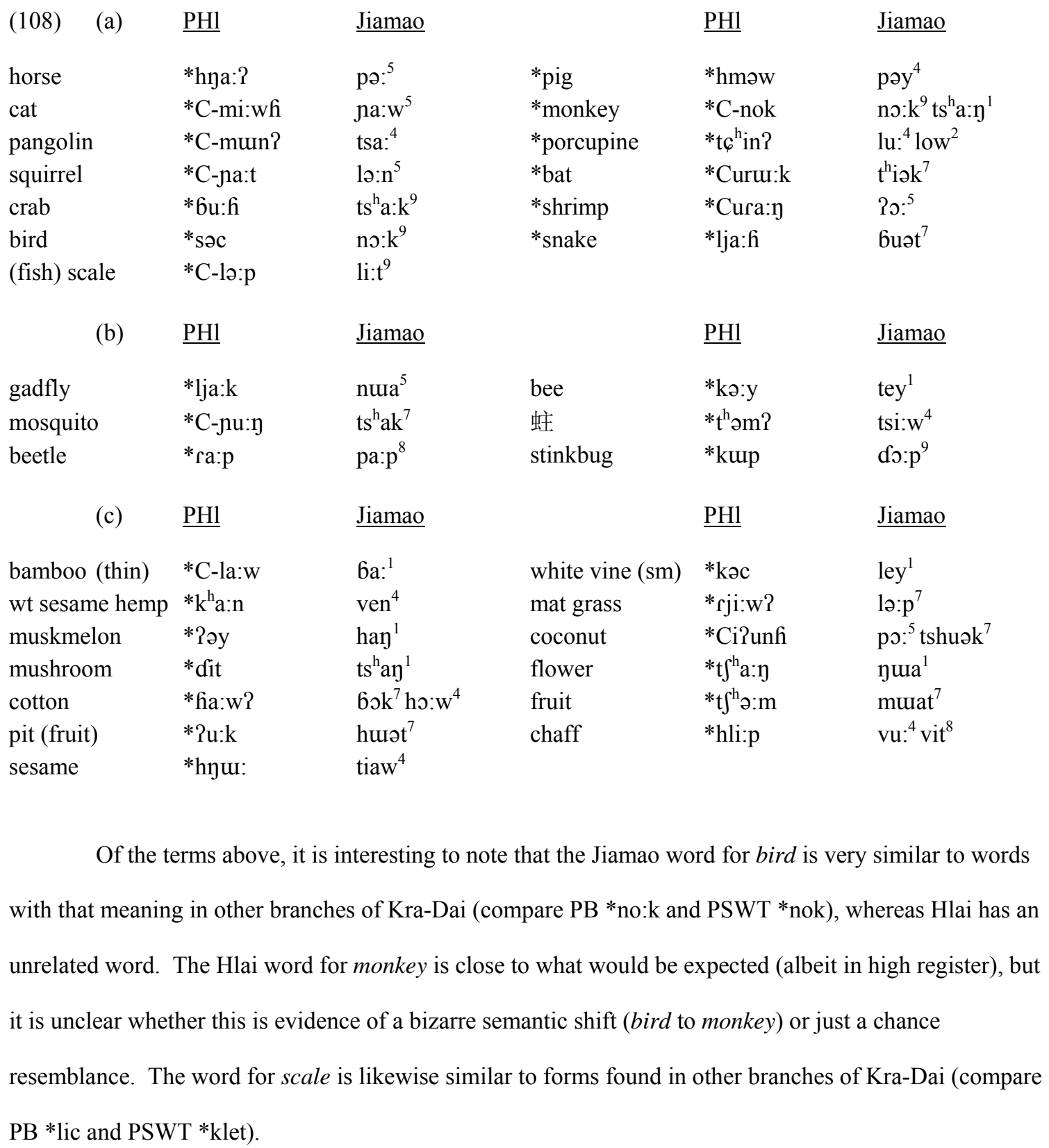

\subsubsection{Natural Objects}

The following words are common vocabulary falling under the general semantic umbrella of nature: 
(109)

$\begin{array}{ll}\text { sky } & * \text { fa:? } \\ \text { hail } & \text { *sinf } \\ \text { wild land } & \text { *hmu:n } \\ \text { mountain } & \text { *Cufow? } \\ \text { ash } & \text { *su:? }\end{array}$

\section{$\underline{\text { Jiamao }}$}

vuəy $^{1}$
ts $^{\text {a }}:^{5}$
fa:
tsow $^{1}$
fa: $^{1}$

$\underline{\mathrm{PHl}}$

star
frost
forest
typhoon
sand

Jiamao

tsap $^{7} \operatorname{tsin}^{5}$ no: $w^{4}$ vuəy ${ }^{1}$ yuən ${ }^{5}$ $\mathrm{p}^{\mathrm{h}} \mathrm{a}: \mathrm{y}^{1}$ dey $^{1}$

\subsubsection{Man-made/Household Items}

The following words relate to material culture, and are generally well-represented within the Hlai languages proper:

house

animal pen

single bench

cradle

bamboo hat

shoes

net (small)

fan

jar

sharp knife

\begin{tabular}{|c|c|}
\hline $\mathrm{PHl}$ & $\underline{\text { Jiamao }}$ \\
\hline$*_{\text {rut:n/*p-loy? }}$ & fur: ${ }^{2}$ \\
\hline *su:nh & yat $^{7}$ \\
\hline$* \mathrm{t}^{\mathrm{h}} \mathrm{imf}$ & yuən $^{1}$ \\
\hline *?u: & २ว: ${ }^{1}$ \\
\hline *hla:y? & kuən $^{3} 10: y^{5}$ \\
\hline *kə:m? & $1 \partial m^{5}$ \\
\hline *rə:y? & piw $^{1}$ \\
\hline *hwoy? & $\mathrm{p}^{\mathrm{h}} \mathrm{a \eta ^{5 }}$ \\
\hline *kəy & $l e y^{5}$ \\
\hline iw? & $\operatorname{tiak}^{8} \mathrm{k}^{\mathrm{h}} \mathrm{ua}$ \\
\hline
\end{tabular}

\begin{tabular}{|c|c|c|}
\hline & $\mathrm{PHl}$ & $\underline{\text { Jiamao }}$ \\
\hline child's house & *kuy? & do: ${ }^{1}$ \\
\hline trough & $*[\mathrm{t} S]^{\mathrm{h}} \mathrm{u}:$ & to: $\eta^{1}$ \\
\hline mat & $* t^{\mathrm{h}} \mathrm{w}: \mathrm{k}$ & tsijk $^{8}$ \\
\hline road & *ku:n & $\operatorname{tin}^{1}$ \\
\hline earring & *hwi:yh & ts $^{\text {h }}$ iaw ${ }^{1}$ \\
\hline arrow & $*_{\text {si:p }}$ & pe: ${ }^{1}$ \\
\hline fish basket & ${ }^{*} \mathrm{C}$-li:k & $6 a: w^{1}$ \\
\hline vine basket & $*_{\text {t } \varphi^{\mathrm{h}} \text { วwh }}$ & 6i: $n^{1} 10:{ }^{1}$ \\
\hline bowl/basin & *CuPa:w & la: $\mathrm{k}^{9}$ \\
\hline a handle (knife) & *fi:n? & buən ${ }^{1}$ \\
\hline
\end{tabular}

\subsubsection{Adjectives and Verbs}

The following long list of adjectives (111) and verbs (112) contain a large amount of basic vocabulary, some of which is regularly represented in all branches of Kra-Dai: 


\begin{tabular}{|c|c|c|c|c|c|}
\hline (111) & $\underline{\mathrm{PHl}}$ & $\underline{\text { Jiamao }}$ & & $\underline{\mathrm{PHl}}$ & $\underline{\text { Jiamao }}$ \\
\hline black & *dəm? & $\mathrm{ts}^{\mathrm{h}} \mathrm{ey}{ }^{1}$ & grey & *hma:wh & $\mathrm{p}^{\mathrm{h}} \mathrm{u}:{ }^{1}$ \\
\hline yellow & *lja:y & tsay $^{4}$ & indigo & $*_{\mathrm{t}} \mathrm{h}^{\mathrm{h}} \mathrm{i}: \mathrm{y}$ & ta: ${ }^{1}$ \\
\hline salty & *Cufa:n? & $\tan ^{2}$ & spicy & $*_{\text {rit }}$ & $\mathrm{kə \eta}^{5}$ \\
\hline good & $*$ hlin & $\operatorname{man}^{1}$ & bad & $*_{r j a: k}$ & Pay $^{1}$ \\
\hline old (thing) & *ka:w? & Pjo:t $t^{9}$ & new & *hma:n & taw $^{4}$ \\
\hline early & *ka:w? & puət $^{8}$ & late & $*$ fəy & liaw $^{1}$ \\
\hline fast & *hjin? & ts $^{\text {h }}{ }^{2}{ }^{5}$ & slow & $*_{\mathrm{si}}: ?$ & $t_{s} \eta^{2}$ \\
\hline wide & *6a:y & $\mathrm{vi}^{4}$ & narrow & *6i:p & vi: $n^{5}$ \\
\hline hard & $* \epsilon^{\mathrm{h}} \partial: \mathrm{n}$ ? & lə: $\mathrm{w}^{4}$ & soft & *hmu:t & puək $^{7}$ \\
\hline dense & $*^{\mathrm{h}} \mathrm{i}: \mathrm{t}$ & ni: $t^{9}$ & sparse & *hywa:n? & fuən $^{1}$ \\
\hline live, fresh & *Curi:p & tuət ${ }^{8}$ & rotten & *?u:n? & nu: $:^{8}$ \\
\hline horizontal & $*_{\sinh }$ & $\operatorname{lə\eta }{ }^{4}$ & vertical & *hən & tiam $^{1}$ \\
\hline blind & *p-la:w & $\mathrm{k}^{\mathrm{h}} \mathrm{iw}^{1}$ & lazy & ${ }^{*}$ C-la:n? & luam $^{2}$ \\
\hline beautiful & *hlin C-mu:n & $\mathrm{nok}^{7}$ & young & *hlur:k C-mu:n & mut $^{2}$ niaw ${ }^{1}$ \\
\hline hot & $* t \int^{h}$ wəw? & Pjuy $^{1}$ & cool (water) & $*$ rən & $6 e: k^{7}$ \\
\hline afraid & *da:? & to: ${ }^{4}$ & thin & *C-li:? & ya:w ${ }^{1}$ \\
\hline dry & *ra:nf & $\mathrm{k}^{\mathrm{h}} \mathrm{a}:^{1}$ & sterile (egg) & *Cura:w? & tsum $^{1} \operatorname{luə\eta }^{2}$ \\
\hline round & *Curom & la: $w^{1}$ & few & *rjəwh & tə: $\mathrm{k}^{8}$ \\
\hline empty & *Curayh & $\mathrm{k}^{\mathrm{h}} \mathrm{a} \mathrm{y}^{1}$ & straight & $*^{*}$-mu:c & kwi $^{5}$ \\
\hline fine & $*$ ?u:t & nә: $\mathrm{p}^{8}$ & & & \\
\hline (112) & $\underline{\mathrm{PHl}}$ & $\underline{\text { Jiamao }}$ & & $\underline{\mathrm{PHI}}$ & $\underline{\text { Jiamao }}$ \\
\hline do & *vu:k & len ${ }^{1}$ & understand & $* \mathrm{k}^{\mathrm{h}} \mathrm{u}: \mathrm{y}$ & $\min ^{4}$ tay $^{2}$ \\
\hline listen & *p-lu: & yey $^{2}$ & look & *kiw & may $^{1}$ \\
\hline eat & *C-lu:h & tey ${ }^{5}$ & swallow & *Cißə:mh & kuat $^{10}$ \\
\hline vomit & $*$ fa:k & $2 \circ y^{1}$ & lick & *lji:mh & te:p $p^{8}$ \\
\hline bite & *hya:n? & da: $n^{1}$ & hold in mouth & $*$ fə:m & tom ${ }^{5}$ \\
\hline hit & $*^{t^{h}} \mathrm{a}: y \mathrm{~h}$ & $\operatorname{təp}^{8}$ & cast (seed) & $*_{\text {fə:nh }}$ & $\operatorname{Pia}^{1}$ \\
\hline cut rice & $* t^{h}$ un & $\mathrm{p}^{\mathrm{h}} \mathrm{u}: \mathrm{w}^{1}$ & winnow & *fənh & $\mathrm{vat}^{7}$ \\
\hline light fire & *?umf & tu: ${ }^{5}$ & boil & *da:n & puot $^{8}$ \\
\hline flow & ${ }^{*} \mathrm{C}-\mathrm{ma}: \mathrm{w}$ & bo: $t^{8}$ & swim & *p-li: & $\operatorname{lon} n^{1}$ \\
\hline take & $*$ hmət & $\mathrm{mi}^{2}$ & pick up & $*_{\mathrm{t}} \mathrm{h}_{\mathrm{i}}^{\mathrm{i}} \mathrm{w}$ ? & $\mathrm{k}^{\mathrm{h}} \varepsilon:{ }^{5}$ lə: $\mathrm{w}^{4}$ \\
\hline kill & *hu:? & tse: ${ }^{2}$ & call & $*_{\text {tça:n }}$ & kow $^{4}$ \\
\hline play & *rju:k & liaw $^{1}$ & arrive & *da:n? & vow $^{1}$ \\
\hline get & ${ }^{*} \mathrm{C}$-ma:k & muən $^{1}$ & give & *du: & muan $^{1}$ \\
\hline answer & $* \mathrm{t}_{\mathrm{in}}$ & ho: $n^{4}$ & die & *la:wf; *lju:y & bo: $t^{9}$ \\
\hline fall & *hla:h & $3 a:^{5}$ & braid (lg) & $*_{\mathrm{p}-\mathrm{li}}$ & ta: $:^{1}$ \\
\hline weave (net) & $* k^{h} u: t$ & $\mathrm{ts}^{\mathrm{h}} \mathrm{a}:{ }^{1}$ & weave (fabric) & *hwi: & $\operatorname{ts}^{\mathrm{h}} \mathrm{up}^{7}$ \\
\hline
\end{tabular}




\subsubsection{Locatives, Time Words, and Classifiers}

Finally, the following are three groups of lexically closed groups, each with its own collection of basic vocabulary. The first group is locatives (113a), the second time words (113b), and the final one classifiers (113c):
(a)

$\underline{\mathrm{PH1}}$

$\underline{\text { Jiamao }}$

$\underline{\mathrm{PH}}$

Jiamao

top

front

side

*phi:? hnu:

law ${ }^{1}$ law $^{1}$

inside

$\mathrm{p}^{\mathrm{h}} \mathrm{a}: \mathrm{y}^{5} \mathrm{t}^{\mathrm{h}}$ iə门 ${ }^{4}$

outside

*phi:? Pu:k

ts $^{\mathrm{h}} \mathrm{u}:{ }^{1} \mathrm{ke}:{ }^{4}$

left

$$
\text { *fa:y }
$$

$\mathrm{p}^{\mathrm{h}} \mathrm{a}: \mathrm{y}^{5}$

opposite side

*lju:n

lo: $\mathrm{w}^{4}$

*phi:? hwi:y

hay $^{2}$ huəy $^{4}$

right

*hluunh

Pe:k ${ }^{9}$

everywhere

*hyom hya:y?

vow ${ }^{1}$ la: ${ }^{4}$

(b)

$\underline{\mathrm{PHl}}$

$\underline{\text { Jiamao }}$

$\underline{\mathrm{PHl}}$

$\underline{\text { Jiamao }}$

2 years ago

*hmu:h ru:

$\mathrm{mu}^{2} \operatorname{lut}^{10}$

3 days later

.

2 days ago

*hwan ru:

vว:n ${ }^{1}$ tsu: ${ }^{4}$

2 days later

*hwən Cufia:t

$\mathrm{ku}^{2} \mathrm{kut}^{10}$

from before

*tc ${ }^{\mathrm{h}} \mathrm{i} \mathrm{k}^{\mathrm{h}} \mathrm{u}: \mathrm{nh}$

ts ${ }^{\mathrm{h}}{ }^{1}$ vo: ${ }^{1}$ mo: ${ }^{5}$

last year

*hmu:h p ${ }^{\mathrm{h}}$ ən?

mu $^{2}$ lə:y ${ }^{4}$

*hmu:fi də̃y

ma: ${ }^{1} \mathrm{t}^{\mathrm{h}}$ iə门 ${ }^{4}$

morning

*hwən Cufiu:

$\mathrm{ku}^{2} \mathrm{ka}:^{2}$

next year

noon

*ka:w? dom

ziaw $^{4}$ hom $^{4}$

*t $\int^{\mathrm{h}} \mathrm{a}: \mathrm{h} \eta \mathrm{w}$

$\mathrm{kuu}^{2}$ duən ${ }^{5}$

(c)

$\underline{\text { Jiamao }}$

$\underline{\mathrm{PH}}$

$\underline{\text { Jiamao }}$

\begin{tabular}{|c|c|c|}
\hline trip clsfr & *ku:n & $\operatorname{lem}^{1}$ \\
\hline pile clfr & *C-le:p & $6 a: k^{9}$ \\
\hline land measure & $*_{\mathrm{t}} \varphi^{\mathrm{h}} \mathrm{a}: \mathrm{n}$ & huan $^{1}$ \\
\hline person clsfr & $*_{\mathrm{t} c \mathrm{u}: \mathrm{n}}$ & yuən ${ }^{1}$ \\
\hline bundle clsfr & *6i:k & puək $^{9}$ \\
\hline layer & *hlut & fur: $n^{4}$ \\
\hline piece (of) & $* \mathrm{t}^{\mathrm{h}} \mathrm{un}$ & $\mathrm{dat}^{7}$ \\
\hline
\end{tabular}

$\begin{array}{ll}\text { tree clsfr } & { }^{*} \mathrm{k}^{\mathrm{h}} \mathrm{ur}: \mathrm{yh} \\ \text { needle clsfr } & *{ }^{\mathrm{h}} \mathrm{u}: \mathrm{y} \\ \text { pile clsfr } & { }^{\mathrm{h}} \mathrm{p}^{\mathrm{h}} \text { əw? } \\ \text { speech clsfr } & * \text { fa:k } \\ \text { long thing clsfr } & * \text { Cifia:h } \\ \text { pair (of) } & * \text { hlu:m }\end{array}$

no: $w^{5}$

ts $^{\text {hin }}{ }^{5}$

tsu: $n^{5}$

tuəy ${ }^{5}$

tury ${ }^{1}$

Pum $^{1}$ 


\subsection{Conclusion}

Although there is no doubt about the significance of the Hlai component in the Jiamao vocabulary, the data in the previous sections indicate strongly that this vocabulary is ultimately borrowed, and that Jiamao was not originally a Hlai language (contra Ostapirat (2004)). The correspondence between Hlai and Jiamao initials indicate strongly that there have been at least two stages of borrowing, the first occurring during what was probably late Pre-Hlai, and the second during and/or shortly after the period of Proto-Hlai unity itself.

There is nothing to indicate that Jiamao began borrowing Hlai vocabulary until sometime after the first occurrence of devoicing, intervocalic lenition and the first instance of vocalic transfer occurred in PreHlai. It is possible that Jiamao came into contact with Hlai before the first monosyllabification which occurred before obstruents, and certainly before initial aspiration occurred in Hlai. It also seems unlikely that Jiamao began borrowing before peripheral mid-vowel raising, but given the large degree of variation in the Jiamao rime correspondences, it is difficult to be absolutely certain.

Concerning the origin of Jiamao, Thurgood (1991) mentions a comment made by Ni Dabai, where Ni says that:

'[T]he Jiamao were Hui, that is, Muslims, who originally came to Hainan in two waves, the first in 986 A.D. and 988 A.D. and the second in 1486.'

Thurgood then suggests, based on Jiamao register data, that this original Jiamao population was Austroasiatic. While there does not seem to be lexical evidence (as far as I am aware) to confirm this, Thurgood is the first person to draw attention to the fact that the Jiamao vocabulary represents (at least) two layers of borrowing. If the time frame suggested by $\mathrm{Ni}$ is correct (which only further research might be able to confirm), then the approximate 500 years between the two waves of immigration would serve as very convenient points with which to date Hlai itself to a certain extent, since the first dates (986-988 A.D.) would have placed the latter part of Pre-Hlai at about 1000 A.D., and the second date (1486 A.D.) would 
place the breakup of Proto-Hlai at a point not long before 1500 A.D. This approximately 500-year-long period encapsulates a number of sound changes which have been proposed in this latter period of Pre-Hlai and at the beginning of the Proto-Hlai disintegration, but not an unreasonable number. It may also be conjectured that sound change in Hlai accelerated in proportion to the amount of language contact into which its speakers entered into, which may have been minimal in the first millennia of the Hlai occupation of Hainan, but probably began to intensify during the time in question, and certainly becoming more intense by the time of Chinese involvement in the island.

At this point, there are only two words which may be traceable to another language phylum:

$\begin{array}{lll}\text { Gloss } & \underline{\text { Jiamao }} & \text { Pre-Jiamao } \\ \text { butterfly } & \text { 6ay }^{5} 6 u \mathrm{a}^{1} & * 6 ə \mathrm{y}^{\mathrm{x}} 6 \Lambda \text { :n } \\ \text { pig } & \text { pəy }^{4} & * \text { mb[o]y }\end{array}$

These are very reminiscent of Proto-Austronesian *qari-baybay ${ }^{57}$ and *babuy respectively, but without other comparisons they remain only chance resemblances at this point. It may be possible that they are borrowings from Utsat, a Chamic language spoken in southern Hainan. The Utsat word for pig is $p^{h} u y^{11}$, from an earlier *buy; the word for butterfly is presently unknown to me.

As Thurgood (1997: fn. 7) points out, Jiamao is of extreme value in the reconstruction of ProtoHlai, and the validation of the stage of Pre-Hlai which existed prior to initial aspiration is particularly important. It is vexing that the origin of the native Jiamao vocabulary is not more forthcoming, but future research (including non-linguistic data) may yet provide further clues into the elusive origin of this Southeast Asian language isolate.

${ }^{57}$ See the next chapter for the conventions used when citing Proto-Austronesian forms. 


\section{CHAPTER SIX: CONCLUSION}

This final chapter is divided into four sections. The first section places Hlai within the context of the Southeast Asian language area, and shared vocabulary (whether borrowed or potentially inherited from a common ancestor) will be examined and analyzed. The second section presents a summary of findings throughout the various parts of this dissertation. The third provides an overview of the empirical and theoretical contributions of this dissertation. The final section provides a brief discussion of future research.

\subsection{The Relationship between Hlai and Other Language Families of Southeast Asia}

There are five language phyla extant in Southeast Asia at present: Sino-Tibetan, Mon-Khmer, Hmong-Mien, Kra-Dai, and Austronesian. At present, there is no consensus view that any two or more of these phyla are genetically related, although relationships have been proposed in all directions, and some proposals have met with greater success (both in terms of acceptance as well as in quality of supporting data) than others. It is not the purpose of this section to argue for a genetic relationship between Kra-Dai and any other phylum. However, with a reconstruction of Proto-Hlai in hand, each potential relationship can be observed from the perspective of Hlai and the circumstantial evidence presented in such a way as to provide clues for future research. That is the purpose of this section, and each of the four non-Kra-Dai phyla will be treated individually with an eye to increasing the resolution of the prehistoric picture which involved Hlai, either individually or as part of Kra-Dai.

The place of Hlai in the Southeast Asian interphyletic picture is somewhat unique, given its presumed 3,500 year isolation on Hainan after moving from the mainland. Lexical connections with other phyla can be inferred in a number of ways, all of them interesting from a prehistoric perspective. First, shared words may have been inherited from the immediate ancestor of Hlai, Southern Kra-Dai, in which case they are of interest because of the antiquity of the contact they imply. If these words are unique to Hlai within Kra-Dai, on the other hand, the implication is that there was either contact with Hlai before speakers moved to Hainan, that there have been incursions from the mainland into Hainan, or (probably 
least likely) that Hlai has been carried back to the mainland at some time subsequent to the original migration of Hlai speakers to Hainan.

Before treating each phylum on an individual basis, it should be noted that Chinese plays a special role in this section due to its cultural prominence in Southeast Asia. While it would be much too hasty to presume that all loan relationships have been unidirectional when Chinese is involved in examples across multiple phyla, it is nevertheless probably safe to target Chinese as a donor in the majority of cases. The following examples ${ }^{58}$ include Tibeto-Burman because, although it is related to Chinese within Sino-Tibetan, it has been shown (see Sagart (1999)) that it (or its daughter branches) has also been the recipient of loans from Chinese:

$\underline{\mathrm{PHl}}$

old

duck

belt; rope

work

pass, beyond

bee

vegetable

goose

sheep

*k $^{\mathrm{h}}$ əwh
*6it $^{\text {*at }}$
*də:y
*koy $^{\text {*kua? }}$
*kə:y
*sa:y?
*C-ya:nh
*hja:y

\section{$\underline{\text { Old Chinese }}$}

*gwə?

${ }^{*} \mathrm{p}^{\mathrm{h}}$ it

*tas

*kon

*kuayh

---

$*_{\mathrm{ts}}^{\mathrm{h}}$ ə?

*yrans

$*[1 / \mathrm{j}] \mathrm{an}$

\begin{tabular}{l} 
Viet-Muon \\
\hline *gu $^{\mathrm{C}}$ \\
*wit \\
*tjəy \\
*kon \\
*kwa \\
*k way \\
--- \\
*hyan \\
---
\end{tabular}

$--$ $\underline{\text { Tibeto-Burman } \text { Hmong-Mien }}$

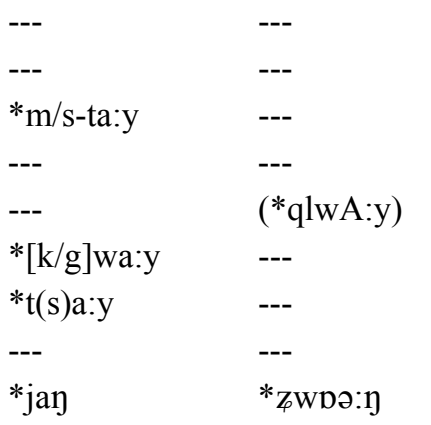

Finally, the last several examples do not include Chinese, but are notable items which are widespread in Southeast Asia:

$\begin{array}{ll}\text { fly (v. })^{59} & * \text { 6in } \\ \text { this } & * \text { C-ni:h } \\ \text { flash (lightning) } & *[\mathrm{C}-] \text { ljip } \\ \text { forest } & * \text { rəy }\end{array}$

$\underline{\text { TB }}$

*bjer

$---$

*ljap

$\underline{\mathrm{VM}}$
*pel
*əni
---
*rən

$\underline{\mathrm{WB}}$

$\underline{\mathrm{HM}}$

$---$

*?ne:y

*ne?

*yklay

${ }^{58}$ Sources for each of these reconstructions can be found in the sections below.

${ }^{59}$ As evidence for a final liquid for fly in Kra-Dai, see Saek burl ${ }^{1}$. 
Of all of the items listed above, there are equivalent forms found in Proto-Be and/or Proto-SWT in all except one case, representing either a PSKD etymology or otherwise indicating that early borrowing occurred separately after the break-up of PSKD, but probably before Hlai speakers entered Hainan. The one form which occurs only in Hlai is bee, a potential loan from Tibeto-Burman.

\subsubsection{Sino-Tibetan}

The following list of items are part of PSKD etymologies, most of which appear to be loans from Chinese, although it is possible that one or more may have gone in the other direction. Based on the progression from $\mathrm{OC}$ to $\mathrm{MC}$, it is likely that the $\mathrm{TB}$ word for ginger is also a borrowing:

\begin{tabular}{|c|c|c|c|c|c|}
\hline (3) & $\underline{\text { PSKD }}$ & $\underline{\mathrm{PHl}}$ & $\underline{\mathrm{OC}}$ & $\underline{\mathrm{MC}}$ & $\underline{\mathrm{TB}}$ \\
\hline put down, let go & ${ }^{*}$ puan ${ }^{\mathrm{C}}$ & *ph $u: \eta ?$ & *panh & ${ }^{*}$ puan $^{\mathrm{C}}$ & --- \\
\hline weave (fabric) & $*$ tu:k & $* t \int^{h} w u: k$ & $*$ tək & $*$ tcik & $*_{\mathrm{t}}(\mathrm{r}) \mathrm{ak}$ \\
\hline carry (on pole) & $*$ ta:m & $*_{\mathrm{t}} \int^{\mathrm{h}} \mathrm{a}: \mathrm{m}$ & *tlam & $*$ tam & --- \\
\hline chicken & *kəy ${ }^{(\mathrm{B})}$ & $*^{\mathrm{k}^{\mathrm{h}}} \partial \mathrm{y}$ & $* \mathrm{ke}$ & $* \mathrm{ke}$ & --- \\
\hline ginger & *quay & $*^{*} \mathrm{~h} u: y$ & *kan & *kiay & *kjan \\
\hline arm $\sim$ shoulder & *qe:n & ${ }^{*} \mathrm{k}^{\mathrm{h}} \mathrm{i}: \mathrm{n}$ & *ken & $*$ ken & --- \\
\hline dig hole & *Cigut & *Cifiut & *gut & $*_{\text {gut }}$ & --- \\
\hline buffalo & $*$ suy $^{\mathrm{C}}$ & *suy? & $*_{\text {s-jəy? }}$ & $*_{\mathrm{zi}} \mathrm{B}^{\mathrm{B}}$ & --- \\
\hline silver & *yən & *hyən & *yrən & *yin & *nul \\
\hline many, much & *H-la:y & *hlə:y & $*$ t-lay & *tay & --- \\
\hline remainder & *Cila: & *lja: & $*$ la & $* \mathrm{ja}$ & --- \\
\hline head & ${ }^{*}$ kurəw $^{\mathrm{C}}$ & *Curəw? & *hlu? & $*_{6 u^{B}}$ & --- \\
\hline
\end{tabular}

The following three words are similar to TB in rime; since there is a three way correspondence with a single TB initial cluster, it is likely that only one, if any, of the following words has a true relationship:

$\underline{\text { PSKD }} \quad \underline{\text { PHI }} \quad \underline{\text { TB }}$

$\begin{array}{llll}\text { shellfish } & * \text { to:y } & * \text { t }^{\mathrm{h}} \mathrm{i}: & * \text { k-roy } \\ \text { gall bile } & * \text { C-təy } & * \text { dəy } & * \text { k-rəy } \\ \text { horn } & * \text { C-qəw } & * \text { həw } & * \text { k-rəw }\end{array}$


The next Hlai examples have no PSKD ancestor, and it is likely that they represent loans from Chinese. Potential Pre-Hlai examples are given in place of PSKD:

\begin{tabular}{|c|c|c|c|c|c|}
\hline (5) & $\underline{\text { Pre-Hl }}$ & $\underline{\mathrm{PHl}}$ & $\underline{\mathrm{OC}}$ & $\underline{\mathrm{MC}}$ & $\underline{\mathrm{TB}}$ \\
\hline $\operatorname{man}$ & *pa:? & $* p^{h} a: ?$ & *pa & *pua & *pwa \\
\hline vehicle & $*$ cia & $* t^{\mathrm{h}}{ }^{\mathrm{h}} \mathrm{a}$ & *k-hla & $*_{t} \varphi_{\text {ja }}$ & -- \\
\hline lacquer & $*_{\mathrm{cit}}$ & $* \epsilon^{\mathrm{h}}[\mathrm{i} / \mathrm{\partial}] \mathrm{t}$ & $*_{\text {ts }}{ }^{\text {it }}$ & $*_{\mathrm{ts}}^{\mathrm{h}_{\text {it }}}$ & --- \\
\hline group & $*_{\text {gun }}$ & $*^{\mathrm{h}} \mathrm{un}$ & $*_{\text {gun }}$ & $*_{\text {gun }}$ & --- \\
\hline axe & *C-puah & *buah & *pa? & $*_{p u a}{ }^{\mathrm{B}}$ & *pwa \\
\hline float & *C-pəw & *6əw & $* b u$ & $* \mathrm{bu}$ & --- \\
\hline same together & ${ }^{*} \mathrm{C}$-toy & *doy & $*$ doy & $*$ doy & --- \\
\hline wade & ${ }^{*} \mathrm{C}$-cəm & *tcom & *dzam & *dziam & --- \\
\hline sword & ${ }^{*}$ C-kuamf & *ku:mf & *kams & ${ }^{*}$ kiam $^{\mathrm{C}}$ & --- \\
\hline work & ${ }^{*}$ C-kon & *koy & *kon & *kon & --- \\
\hline look up at & *yша? & *hyur:? & *yay? & $* \operatorname{yiay}^{\mathrm{B}}$ & --- \\
\hline horse $\sim 7^{\text {th }}$ branch & *ya:? & *hya:? & *ya? & $* \mathrm{ya}^{\mathrm{B}}$ & --- \\
\hline hear (said) & *(h)le:y & *hli:y & *hley & $* t^{h}$ en & --- \\
\hline measure (rice) & *C-luan? & *C-lu:y? & *rayh & $* \operatorname{lian}^{\mathrm{C}}$ & $*[\mathrm{~g} / \mathrm{k}]-\mathrm{ran}$ \\
\hline boar (wild) & $* \mathrm{~d}-\mathrm{l} \partial \mathrm{c}$ & $* \mathrm{C}-\mathrm{l}$ əoc & *d-lats & $*$ das & --- \\
\hline pair & *C-luanh & *C-lu:yh & *ran? & $* \operatorname{lian}^{B}$ & --- \\
\hline
\end{tabular}

The cases below are less more problematic, but still possibly related:

\begin{tabular}{|c|c|c|c|c|}
\hline (6) & $\underline{\text { Pre-Hl }}$ & $\underline{\mathrm{PHl}}$ & $\underline{\mathrm{OC}}$ & $\mathrm{MC}$ \\
\hline mat & *duak & $*^{\mathrm{h}} \mathrm{u}: \mathrm{k}$ & $*_{\text {s-lak }}$ & *ziak \\
\hline rest & $*_{\varphi \mathrm{u}: \mathrm{k}}$ & $*_{t} \varphi^{\mathrm{h}} \mathrm{u}: \mathrm{k}$ & $*_{\mathrm{s} ə \mathrm{k}}$ & $*_{\mathrm{sik}}$ \\
\hline lragon & *nəy & *hnəy & *roy & *lion \\
\hline nake & *Cila:h & *lja:h & *m-lay & $*_{\text {za }}$ \\
\hline wallow $\sim$ drink & *Cißə:mh & *Cißə:mf & *?əm? & * $\operatorname{Pim}^{\mathrm{B}}$ \\
\hline
\end{tabular}

Finally, the following are potential examples of possible loans between Hlai and TB:

\begin{tabular}{|c|c|c|c|}
\hline (7) & $\underline{\text { Pre-Hl }}$ & $\underline{\mathrm{PHl}}$ & $\underline{\mathrm{TB}}$ \\
\hline rice & *da:h & $*^{\mathrm{h}} \mathrm{a}: \mathrm{h}$ & $*$ dzja \\
\hline bee & ${ }^{*}$ C-ka:y & *kə:y & $*[\mathrm{k} / \mathrm{g}] \mathrm{wa}: \mathrm{y}$ \\
\hline brain & *lu:k & *hlu:k & $*_{\text {nuk }}$ \\
\hline fall & *la:h & *hla:h & $*[\mathrm{~g} / \mathrm{k}]-\mathrm{la}$ \\
\hline exchange & *p-ləy & *p-ləy & $* 1[\mathrm{a} / \mathrm{e}] \mathrm{y}$ \\
\hline iron $\sim$ copper & *rə:y & *rə:y & *g-rəy \\
\hline
\end{tabular}


It can be noted in the above example sets that there is a very patterned correspondence between the Chinese and Hlai tone categories. Category A in Hlai (the unmarked category) always corresponds to the same in Chinese, whereas the marked categories B and C correspond to marked categories in Chinese ${ }^{60}$. However, there are mixed correspondences between these two categories, which I interpret to indicate two layers of loanwords. The reason for this confusion may involve, at least partly, the beginning of a transition from final glottal segments to a phonation difference in one or both families. The older layer includes the following:

\begin{tabular}{|c|c|c|c|c|}
\hline$\underline{\text { Gloss }}$ & $\underline{\text { Pre-Hl }}$ & $\underline{\mathrm{PHI}}$ & Old Chinese & Late Han \\
\hline head & *kurəw? & *Curəw? & *hlu? & $* \varphi u^{B}$ \\
\hline buffalo & *suy? & $*_{\text {suy? }}$ & $*_{\text {s-jəy? }}$ & $*_{\mathrm{zi}^{\mathrm{B}}}$ \\
\hline horse $^{61}$ & *ya:? & *hya:? & * ya? & $*_{\eta a^{B}}$ \\
\hline look up at & *yua? & *hџu:? & *yay? & ${ }^{*} \operatorname{yiaj}^{\mathrm{B}}$ \\
\hline sword & *C-kuiamh & *ku:mh & *kams & ${ }^{*} \mathrm{kiam}^{\mathrm{C}}$ \\
\hline goose & *C-na:nh & *C-na:nh & *yrans & ${ }^{*}$ yan $^{\mathrm{C}}$ \\
\hline
\end{tabular}

What I interpret as a more recent layer, with 'reversed' tone category correspondences, includes the following:

\begin{tabular}{|c|c|c|c|c|}
\hline Gloss & $\underline{\text { Pre-Hl }}$ & $\underline{\mathrm{PHl}}$ & $\underline{\text { Old Chinese }}$ & Late Han \\
\hline axe & ${ }^{*} \mathrm{C}$-puah & *6uah & *pa? & ${ }^{*}$ pua $^{\mathrm{B}}$ \\
\hline pair & *C-luanh & *C-lut:yh & *ran? & $* \operatorname{lian}^{\mathrm{B}}$ \\
\hline swallow $\sim$ drink & *Cißə:mh & *Cißə:mh & *Pəm? & $* \operatorname{Pim}^{\mathrm{B}}$ \\
\hline put down, let go & *puay? & *phu:y? & *payh & ${ }^{*}$ puan $^{\mathrm{C}}$ \\
\hline pass, beyond & *C-kua? & *kua? & *kuayh & *kuay ${ }^{\mathrm{C}}$ \\
\hline
\end{tabular}

The following two examples are interesting, in that they both show evidence of OC medial *-1correlating with retroflexion, in Hlai in the first case, and in Chinese itself in the second:

\footnotetext{
${ }^{60}$ It should be noted that, due to what amounts to more or less a historical accident, Kra-Dai category B is usually taken to be associated with spread glottis as is Chinese category C, whereas Kra-Dai category C and Chinese category $\mathrm{B}$ are both associated with constricted glottis.

${ }^{61}$ It is interesting that the Hlai word horse does not correspond to the commonly used term OC *mra?, but rather to the $7^{\text {th }}$ earthly branch of the Chinese zodiac which is associated with the horse.
} 
$\underline{\text { Pre-Hl }} \quad \underline{\mathrm{PHl}} \quad \underline{\mathrm{OC}} \quad \underline{\mathrm{MC}}$

$\begin{array}{lllll}\text { carry (on pole) } & * \text { ta:m } & * \mathrm{t}^{\mathrm{h}} \mathrm{a}: \mathrm{m} & * \text { tlam } & * \text { tam } \\ \text { boar (wild) } & * \text { C-ləc } & * \text { C-lac } & * \text { d-lats } & * \text { das }\end{array}$

There are several cases where a Hlai form is reconstructed as originally sesquisyllabic, yet is comparable to monosyllabic Chinese forms. The following cases show exact identity in the initials of the main syllable:

\begin{tabular}{|c|c|c|c|c|}
\hline Pre-Hl & PHl & $\underline{\mathrm{OC}}$ & $\underline{\mathrm{MC}}$ & $\underline{\mathrm{TB}}$ \\
\hline *C-puah & *6uah & *pa? & ${ }^{*}$ pua $^{\mathrm{B}}$ & *pwa \\
\hline *C-ta:y & *đə:y & $*$ tas & $*$ tas & *m/s-ta:y \\
\hline${ }^{*}$ C-koy & *kon & *kon & *koy & --- \\
\hline *C-kuamh & *ku:mh & *kams & ${ }^{*} \mathrm{kiam}^{\mathrm{C}}$ & --- \\
\hline *C-kua? & *kua? & *kuayh & ${ }^{*}$ kuay ${ }^{C}$ & --- \\
\hline *CiPə:mh & *Cißə:mf & *ใəm? & $* \operatorname{Pim}^{\mathrm{B}}$ & --- \\
\hline *C-na:nf & *C-na:nh & *yrans & $* \operatorname{yan}^{C}$ & --- \\
\hline *C-luay? & ${ }^{*}$ C-lu:y? & *rayh & $* \operatorname{liay}^{C}$ & $*[\mathrm{~g} / \mathrm{k}]$-ran \\
\hline *C-luayh & *C-lu:yh & *ray? & $* \operatorname{liay}^{\mathrm{B}}$ & --- \\
\hline
\end{tabular}

The words below correspond to Chinese words with voiced initials; in addition to these, there is one TB comparison for bee which is indeterminate for voicing:

\begin{tabular}{|c|c|c|c|c|}
\hline (12) & $\underline{\text { Pre-Hl }}$ & $\underline{\mathrm{PHl}}$ & $\underline{\mathrm{OC}}$ & $\underline{\mathrm{MC}}$ \\
\hline float & ${ }^{*} \mathrm{C}$-pəw & *6əw & $* \mathrm{bu}$ & $*$ bu \\
\hline same together & ${ }^{*}$ C-toy & $*$ doy & $*$ don & $*$ don \\
\hline wade & ${ }^{*} \mathrm{C}$-cəm & $*$ tcom & $*$ dzam & $*$ dziam \\
\hline
\end{tabular}

Finally, the following sesquisyllabic form corresponds to a voiceless aspirated initial:

$\begin{array}{llll}\underline{\text { Pre-Hl }} & \underline{\text { PHl }} & \underline{\text { OC }} & \underline{\text { MC }} \\ *_{\text {C-pet }} & *_{\text {bit }} & *^{h^{h_{i t}}} & *^{h_{i t}}\end{array}$

duck

*p it

${ }^{*} \mathrm{p}^{\mathrm{h}}$ it 


\subsubsection{Mon-Khmer}

Much to the chagrin of Southeast Asian scholars, a full reconstruction of Mon-Khmer has yet to be achieved. However, even if it were available, it would likely represent a family of such antiquity that comparison with Hlai would be rather anachronistic in the context of a contact relationship. For the purposes of this chapter, reconstructions of three different Mon-Khmer subfamilies have been used: VietMuong (Thompson (1976)), West Bahnaric (Sidwell \& Jacq (2003)), and South Bahnaric (Sidwell (2000)).

The comparisons below involve only Viet-Muong. Of these, the following are particularly similar:

\begin{tabular}{|c|c|c|c|}
\hline (14) & $\underline{\text { Pre-Hl }}$ & $\underline{\mathrm{PHl}}$ & $\underline{\mathrm{VM}}$ \\
\hline deer & ${ }^{*} \mathrm{C}-\mathrm{d} ə: \mathrm{y} ?$ & *гə:y? & *?day \\
\hline hoe & ${ }^{*} \mathrm{C}-\mathrm{kwa}: \mathrm{k}$ & *kwa:k & *kuək \\
\hline mouth & $*_{\text {məm? }}$ & *hməm? & $*_{\text {mom }}$ \\
\hline good & *len & *hlin & *lej \\
\hline drum $^{62}$ & *C-ləy & $*$ C-ləy & $*_{\text {hədloy }}{ }^{\mathrm{B}}$ \\
\hline fast & $*_{\mathrm{j}}[\mathrm{e}] \mathrm{n} ?$ & *hj[i]n? & *hpen \\
\hline
\end{tabular}

The set of vocabulary below may also be related, although the resemblance is not as direct:

\begin{tabular}{|c|c|c|c|}
\hline (15) & $\underline{\text { Pre-Hl }}$ & $\underline{\mathrm{PHl}}$ & $\underline{\mathrm{VM}}$ \\
\hline insipid & ${ }^{*} \mathrm{C}$-təc & $*$ dəc & ${ }^{* m}$-lac \\
\hline dry & ${ }^{*}$ C-ta:w & *da:w & $*_{\text {t-raw }}{ }^{\mathrm{B}}$ \\
\hline fall & *la:h & *hla:h & $* \mathrm{hl}^{\mathrm{C}}$ \\
\hline & $*_{\mathrm{ja}}$ & *hja: & $*^{*} \mathrm{~g}^{\mathrm{h}} \mathrm{ja}$ \\
\hline
\end{tabular}

The following vocabulary items are found in West and/or South Bahnaric, and occasionally in Viet-Muong. The first set is made up of Hlai forms which are included in the PSKD reconstruction in chapter four:

${ }^{62}$ This word is hypothesized in chapter 4 to descend from PSKD *k-1[0] $\mathrm{y}$. 


\begin{tabular}{|c|c|c|c|c|c|}
\hline (16) & $\underline{\text { PSKD }}$ & $\underline{\mathrm{PHl}}$ & $\underline{\mathrm{WB}}$ & $\underline{\mathrm{SB}}$ & $\underline{\mathrm{VM}}$ \\
\hline cover & *xəр & $* k^{h} \partial p$ & *kəp & --- & --- \\
\hline scratch $\sim$ scrape & $*_{x}[\mathrm{u} / \mathrm{u}]: \mathrm{t}$ & ${ }^{*} \mathrm{k}^{\mathrm{h}} \mathrm{w}: \mathrm{t}$ & *krus:t & --- & --- \\
\hline crow & $*$ ?a:k & *?a:k & *k-1a:k & *kən?a:k & --- \\
\hline board & $*(\mathrm{C}-) \mathrm{p} \varepsilon: \mathrm{n}^{\mathrm{B} / \mathrm{C}}$ & *6e:nh & $*[t]$ pan & --- & --- \\
\hline winnowing fan & ${ }^{*} \mathrm{C}-\mathrm{toy}^{\mathrm{C}}$ & $*$ doy? & --- & *gədo:y & --- \\
\hline centipede & $* \mathrm{q}-\mathrm{de}[:] \mathrm{p}$ & $*_{\text {ri:p }}$ & *k? зер & *kər?ip & --- \\
\hline mother, female & $* \mathrm{me}^{\mathrm{B} / \mathrm{C}}$ & *hmi:? & *me:? & *me: & ${ }^{*}$ həmay $^{\mathrm{B}}$ \\
\hline ant $\sim$ termite & $*$ moc & *hmuc & *s-mo:c & *mo:c & *moc \\
\hline sesame & *yua & *hyu: & *r-na: & 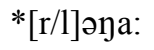 & --- \\
\hline widow(er) & $*^{H}$-ma:y ${ }^{\mathrm{C}}$ & *hmə:y? & *k-ma:y & --- & --- \\
\hline cat & ${ }^{*} \mathrm{C}^{\mathrm{V}}-\mathrm{me}: \mathrm{w}^{(\mathrm{B})}$ & *C-mi:wh & *me:w & --- & --- \\
\hline
\end{tabular}

The following items lack PSKD etymologies, and may therefore be loans into Hlai:

$\underline{\text { Pre-Hl }} \quad \underline{\text { PHl }} \quad \underline{\text { WB }} \quad \underline{\text { SB }} \quad \underline{\text { VM }}$

\begin{tabular}{|c|c|c|c|c|c|}
\hline throw & *pa:y? & $*^{\mathrm{h}} \mathrm{a}$ a:y? & *poy & --- & ${ }^{*} p^{h} \circ \eta^{B}$ \\
\hline close (mouth) & $*$ dəp & $* t^{\mathrm{h}} \partial \mathrm{p}$ & $*$ dap & --- & --- \\
\hline dry in sun & $* t i: y ?$ & $*_{t} \int^{\mathrm{h}} \mathrm{i}: \mathrm{y}$ ? & *tur:y & --- & --- \\
\hline blow & *?u:h & *?u:h & --- & *?uh & --- \\
\hline mushroom & ${ }^{*}$ C-tet & $*$ dit & $*$ p-se:t & *nose:t & --- \\
\hline hawk & ${ }^{* C}$-ci:w? & *tci: $\mathrm{w}^{63}$ & $*_{\text {cuw }}$ & --- & \\
\hline phlegm & ${ }^{*}$ C-ga:k & *ha:k & *kəha:k & --- & --- \\
\hline aunt & *mi: & *hmi: & $* \operatorname{mih}$ & --- & --- \\
\hline year & *mo:h & *hmu:h & *k-mっ: & --- & --- \\
\hline yawn $\sim$ gasp & *ya:p & *hya:p & *ha:p & *nəga:p & --- \\
\hline loose & *lo:n? & *hlu:y? & *r-10:y & --- & --- \\
\hline banyan & *ri: & *ri: & $*_{\text {y-rəy }}$ & $*_{\text {fəri: }}$ & --- \\
\hline pinch & ${ }^{*} \mathrm{C}$-me:t & ${ }^{*} \mathrm{C}$-mi:t & *c?be:t & --- & --- \\
\hline flash; open eyes & ${ }^{*}$ C-ləy? & ${ }^{*} \mathrm{C}$-ləy? & *b-la:y & --- & --- \\
\hline
\end{tabular}

The following forms are more problematic, but still represent possible cognates. The set (18) has PSKD etymologies, and the set in (19) does not and Pre-Hlai reconstructions are therefore given:

${ }^{63}$ This form is inferred from Lauhut (Ouyang (1992)), and it is unknown to what extent it is found in the other Hlai languages. 


\begin{tabular}{|c|c|c|c|c|c|}
\hline (18) & $\underline{\mathrm{PSKD}}$ & $\underline{\mathrm{PHl}}$ & $\underline{\mathrm{WB}}$ & $\underline{\mathrm{SB}}$ & \\
\hline soak & $*(\mathrm{C}-)[\mathrm{t} / \mathrm{c}] \partial: \mathrm{m}^{\mathrm{C}}$ & *də:m? & $*$ t-ram & *təram & --- \\
\hline black, dark & $*^{C} \mathrm{C}-[\mathrm{t} / \mathrm{d}] \mathrm{m}^{(\mathrm{C})}$ & *dəm? & *kəlam & --- & --- \\
\hline chin jaw & ${ }^{*} \mathrm{C}$-ga:y & *ha:y & *ka:y & *ka:y & --- \\
\hline thatch grass & *Ciga: & *Cifa: & --- & *gəja: & --- \\
\hline stinger & $* \mathrm{C}^{\mathrm{V}}$-nəy & *C-nəy & *kəlay & --- & --- \\
\hline (19) & $\underline{\text { Pre-Hl }}$ & $\underline{\mathrm{PHl}}$ & $\underline{\mathrm{WB}}$ & $\underline{\mathrm{SB}}$ & $\underline{\mathrm{VM}}$ \\
\hline descend & $*$ tu:? & $* t \int^{h} u: ?$ & $* \mathrm{c}$-ruh & --- & --- \\
\hline beetle termite & ${ }^{*} \mathrm{C}$-da:p & *ra:p & --- & *kəla:p & --- \\
\hline muntjac & *C-lə:y & ${ }^{*}$ C-lo:y & *k-ro:y & --- & --- \\
\hline $\operatorname{tin}$ & *la:k & *hla:k & $*_{\mathrm{t} \text {-ra:k }}$ & --- & --- \\
\hline
\end{tabular}

Based on the above comparisons, it appears that Hlai (as well as its ancestor PSKD) has been in contact with certain Mon-Khmer subgroups. The above examples suggest that one of these has been VietMuong, and there is a surprising amount of suggestive evidence that there has been at least as much with Bahnaric. This latter connection is especially important for the prehistory of Hlai, in that it suggests that Hlai must have once existed far enough south to engage in this contact, the alternative being that a group of Bahnaric speakers migrated at some point to Hainan.

\subsubsection{Hmong-Mien}

A relationship between Kra-Dai and Hmong-Mien was first suggested in Benedict (1942), where he included Hmong-Mien (Miao-Yao) in his Austro-Thai phylum. More recently, Kosaka (2002) published an article in which he identified a small group of vocabulary items which are suggestive of a relationship between Kra-Dai and Hmong-Mien, although whether this is due to a genetic relationship or to contact remains to be seen. The most comprehensive reconstruction of Hmong-Mien currently available is that of Wang and Mao (1995), and that is what is employed here for comparison. Wang and Mao (1995) is very useful in that it represents a large sample of core vocabulary across twenty-three Hmong-Mien languages. The major drawback of this work, in my opinion, is that the authors engage in a particular kind of 'protoform stuffing' whereby they do not appear to seek to account for intermediate processes between the proto- 
language and daughter languages that may explain what end up being sometimes very divergent realizations in phonetic form. Rather, in the case of the initials, they often merely linearize different reflexes, resulting in clusters that are ultimately unrealistic. This situation is not as bad with the rimes, but the system is still overburdened. The other major omission of this work is the reconstruction of tone categories, which are discussed in the introduction of the book but abandoned in the actual reconstruction. These shortcomings should be borne in mind during the presentation of data below.

At least one third of the potential cognates (using the term to include words which have a relationship either due to common descent or to borrowing) between Hmong-Mien and Hlai appear to be mutual borrowings from Chinese (although some of these are widespread area words, in which case Chinese is not the only candidate for the loan source $)^{64}$. Examples are given below:

\begin{tabular}{|c|c|c|c|c|}
\hline (20) & $\underline{\text { Gloss }}$ & $\underline{\mathrm{MC}}$ & $\underline{\mathrm{PHl}}$ & $\underline{\mathrm{PHM}}$ \\
\hline 放 & let go & ${ }^{*}$ puay $^{\mathrm{C}}$ & ${ }^{*} p^{\text {h}} u: \eta ?$ & *poəy \\
\hline 槽 & trough & --- & $* t \int^{h} \mathrm{u}:$ & *dðAw \\
\hline 乾淨 & clean & $*$ dziej $^{\mathrm{C}}$ & $*{ }^{h_{6}} \mathrm{i}: \mathrm{yh}$ & *ndðey \\
\hline 雞 & chicken & *ke & $*^{*}{ }^{\mathrm{h}} \partial \mathrm{y}$ & *qәу \\
\hline 姜 & ginger & *kiay & $* k^{h} u: y$ & $* q^{h}$ wjæy \\
\hline 斧子 & axe & ${ }^{*} \mathrm{pua}^{\mathrm{B}}$ & *6uah & *pəw \\
\hline 浮 & to float & $*$ bu & *6ow & $*_{\text {mbjow }}$ \\
\hline 錢 & coin, money & $*$ dzian & *tci:n & *dðIn \\
\hline 過 & to pass & * kuai $^{\mathrm{C}}$ & *kuə? & *qlwA:y \\
\hline 龍 & dragon & *lion & *hnəy & ${ }^{*}$ yglon, ${ }^{*}$ klju: \\
\hline 銀 & silver & *yin & *hyən & *nwe:n \\
\hline 染 & dye (cloth) & ${ }^{*} \mathrm{nam}^{\mathrm{B} / \mathrm{C}}$ & $*^{*}$-nom? & *nem \\
\hline 量 & (to) measure & $* \operatorname{liay}^{(\mathrm{C})}$ & *C-lu:y? & *luəy \\
\hline 兩 & pair & $* \operatorname{liaj}^{\mathrm{B}}$ & *C-lu:yh & *lwaəy \\
\hline 羊 & sheep & $*_{\text {jan }}$ & *hja:n & $*_{\text {ZWDə:y }}$ \\
\hline
\end{tabular}

The following forms may be related in some way. Due to small number of comparisons, along with the reservations expressed about the Hmong-Mien reconstruction, future work will be necessary to either confirm or deny these comparisons. The first group of words considered here are those which have a

\footnotetext{
${ }^{64}$ Note, however, Edmondson (to appear 2007), where the hypothesis is presented that Hmong-Mien is the closest relative to Sino-Tibetan within Southeast Asia. If this is correct, then some HM items which are taken to be Chinese loans might actually be cognates in the genetic sense of the word.
} 
PSKD pedigree, indicating that if borrowing occurred (in either direction), it may not have involved Hlai directly:

\begin{tabular}{|c|c|c|}
\hline$\underline{\text { PSKD }}$ & $\underline{\mathrm{PHl}}$ & $\underline{\text { PHM }}$ \\
\hline$*_{\mathrm{tu}}:^{\mathrm{B}}$ & $* \mathrm{t}^{\mathrm{h}} \mathrm{u}: \mathrm{h}$ & $* \mathrm{dAw}$ \\
\hline *xa:w & $* \mathrm{k}^{\mathrm{h}} \mathrm{a}: \mathrm{w}$ & *qləw \\
\hline *Civən & $*_{\text {fjon }}$ & *hmjnin \\
\hline *mua & *hmu: & *mu \\
\hline${ }^{*} \mathrm{C}^{\mathrm{V}}$-mu: & ${ }^{*} \mathrm{C}$-mu: & *mwu \\
\hline$*[\mathrm{C}-] \mathrm{ni}:{ }^{\mathrm{B} / \mathrm{C}}$ & *C-ni:h & *?ne:y \\
\hline${ }^{*}$ C-ла:w ${ }^{\mathrm{C}}$ & *C-jna:w? & *ntsaw \\
\hline${ }^{*} \mathrm{C}$-lu: ${ }^{\mathrm{C}}$ & *p-lu:? & *yklə \\
\hline *Cila:y & *lja:y & *GlwjAy \\
\hline *m-la: & *hla: & *mbdzaw \\
\hline *p-ret & $*_{\text {rit }}$ & *mbdza:t \\
\hline$*[\mathrm{C}-] \mathrm{ku}$ & *hu: & *kə:n \\
\hline${ }^{*} \mathrm{C}-\mathrm{G}[\mathrm{a}:] \mathrm{y}$ & *ha:y? & *qay \\
\hline${ }^{*} \mathrm{C}-\mathrm{G}[\mathrm{\jmath} / \mathrm{o}]: \mathrm{m}$ & *hə:m & *2i:m \\
\hline
\end{tabular}

The next items don't have PSKD etymologies, and Pre-Hlai forms are provided instead:

$\underline{\text { Pre-Hl }} \quad \underline{\text { PHl }} \quad \underline{\text { PHM }}$

\begin{tabular}{|c|c|c|c|}
\hline father & *pa:? & ${ }^{*} \mathrm{p}^{\mathrm{h}} \mathrm{a}: ?$ & *pwtsa \\
\hline cooking stove & $*_{\text {su:}}$ ? & $*_{\text {su:? }}$ & $*$ tsu \\
\hline in front & *C-təy & *dəy 'face' & *ndæ:y \\
\hline porcupine & $*$ C-təy & *dəy & *ndzoy \\
\hline female & *mi:? & *hmi:? & *mjnip \\
\hline pig & *məw & *hmow & $*$ mpp \\
\hline uncle (mos bro) & *nu:? & *hnu:? & $*_{\text {naw }}$ \\
\hline long & *na:w? & *hna:w? & *ntæ:w \\
\hline ear (of grain) & *na:n & *hna:y & *hnjey \\
\hline heart & *la:w? & *hla:w? & *plow \\
\hline $3 p$ pronoun & ${ }^{*}$ C-na: & *C-na: & *næn \\
\hline liver & *C-ya:n & *C-na:n & $* \eta k^{\mathrm{h}} 1 \mathrm{An}$ \\
\hline ear & *Ciləy & *ljəy & *mbdzAy \\
\hline neck & *Ciloy? & *ljoy? & *qlA:n \\
\hline scissors & *Ciliw? & *ljiw? & *nclji:w \\
\hline knee & *C-du: & *ru: & *dzwew \\
\hline
\end{tabular}


The forms below are superficially less similar, but may still represent a lexical relationship. The first group (23) has a PSKD etymology, and the second group, lacking this, includes Pre-Hlai reconstructions: $\underline{\text { PSKD }} \quad \underline{\text { PHI }} \quad \underline{\text { PHM }}$

\begin{tabular}{|c|c|c|c|}
\hline head louse & *twu: & $* t \int^{h}$ wu: & $* n t s^{\mathrm{h}}$ oy \\
\hline big & ${ }^{*} \mathrm{C}-1[\mathrm{u}] \mathrm{y}$ & ${ }^{*}$ C-lun & *hlu \\
\hline shoulder & ${ }^{*} \mathrm{C}-\mathrm{ba}:^{\mathrm{B}}$ & *va:h & $* \mathrm{bu}$ \\
\hline fly (insect) & *wa: $y^{\mathrm{C}}$ & *hwa:y? & *moəy \\
\hline (24) & $\underline{\text { Pre-Hl }}$ & $\underline{\mathrm{PHl}}$ & $\underline{\mathrm{PHM}}$ \\
\hline burn & $*$ cuy? & $*_{t} \varphi^{\mathrm{h}}$ uy? & ${ }^{*} \mathrm{p}^{\mathrm{h}} \mathrm{ss}^{\mathrm{h}} \mathrm{uy}$ \\
\hline to sink & ${ }^{*} \mathrm{C}$-cə:n & *tढə:n & $* \mathrm{dpn}$ \\
\hline sore (n.) & *C-na:y & *C-na:y & ${ }^{*} \mathrm{ts}^{\mathrm{h}} \mathrm{A}: \mathrm{y}$ \\
\hline fall (rain) & *C-lu:y & *C-lu:y & $*$ du:y \\
\hline to cut (scissors) & $*[\mathrm{C}-] \mathrm{r} ə \mathrm{p}$ & *rəp & *klep \\
\hline
\end{tabular}

Although some of the above examples are most likely chance resemblances, taken as a group, the comparisons above suggest some sort of relationship which transcends mutual contact with Chinese. The nature of this relationship will probably not be clarified further until a fine-tuning of the Hmong-Mien reconstruction and the finalization of a Kra-Dai reconstruction.

\subsubsection{Austronesian}

The comparison between Kra-Dai and Austronesian was brought to fore in Benedict (1942), where they served as the two main components of his Austro-Thai phylum, in which he also included HmongMien. The hypothesis has been extremely controversial, owing particularly to the fact that Benedict had generally very loose criteria for his comparisons, and eventually included an even more controversial branch, Japanese (Benedict 1991). Two notable recent contributions to the debate have been Thurgood (1994), in which it is argued that the similarities between Kra-Dai and Austronesian are due to an ancient 
contact relationship, and Ostapirat (2005), where it is argued that these reflect a genuine genetic relationship.

It is not the purpose of this section to take a stand on this issue, and it is my opinion that this matter should be examined thoroughly only when a Kra-Dai reconstruction exists that is on par with the present reconstruction of Proto-Austronesian (PAn), which is considerably more complete and wellunderstood. However, a comparison between PAn and Hlai is nevertheless interesting in its own right, not only because parallels exist in Hlai which are not found in other branches of PSKD, but also because the polysyllabic nature of PAn can serve as a platform to demonstrate the application of the sound changes discussed in chapter four. Most of the PAn lexical items below have been taken from Zorc (1995), although a few have been taken from the Austronesian basic vocabulary database (which can be found at the following URL: http://language.psy.auckland.ac.nz/austronesian/).

Before examining the data, a discussion of the nature of the PAn reconstruction employed here is necessary. In general, the system of Blust (1999) will be adopted, but with the following modifications based on a reinterpretation of the Formosan language data, to be explored in greater detail elsewhere:

$$
\begin{array}{ll}
\mathrm{C}=\mathrm{t} & \mathrm{N} / \mathrm{L}=\mathrm{l} \\
\mathrm{S}=\mathrm{S} & \mathrm{l}=\mathrm{r} \\
\mathrm{j}=\mathrm{d} & \mathrm{r}=--- \\
\mathrm{Z} / \mathrm{z}=\mathrm{f} &
\end{array}
$$

In other words, a retroflex series is introduced, a voiced palatal stop is added, and the system of liquids is reinterpreted so that original $* \mathrm{~N} /{ }^{*} \mathrm{~L}$ are reinterpreted as $* 1$, original $* 1$ is reinterpreted as ${ }^{*} \mathrm{r}$, and original PAn *r is considered secondary (there is little evidence for it in the traditional PAn reconstruction in any case).

It is significant that the majority of comparisons presented here are part of PSKD etymologies presented in chapter four. Note that many of the following have been proposed before by others: 


\begin{tabular}{|c|c|c|c|}
\hline (26) & $\underline{\text { PSKD }}$ & $\underline{\mathrm{PHl}}$ & $\underline{\text { PAn }}$ \\
\hline ancestor & $*_{p u}{ }^{\mathrm{B} / \mathrm{C}}$ & $* p^{h} u: ?$ & $* a(m) p u$ \\
\hline fart & $*$ S-to[:]t & $* \mathrm{t}^{\mathrm{h}} \mathrm{u}: \mathrm{t}$ & *qว̆(n)tut \\
\hline land leech & *N-ta:k & $* \mathrm{t}^{\mathrm{h}} \mathrm{a}: \mathrm{k}$ & *matək $\sim$ mətak \\
\hline head louse & $* t(w) u:$ & $* t \int^{h} w \partial w$ & $* \mathrm{kutu}$ \\
\hline eye & *N-ta: & $* \mathrm{t} \int^{\mathrm{h}} \mathrm{a}$ & *mata \\
\hline rib & $* \mathrm{k}^{(\mathrm{h})} \mathrm{ra}: \mathrm{\eta}^{\mathrm{C}}$ & $* \mathrm{k}^{\mathrm{h}} \mathrm{a}: \mathrm{\eta} ?$ & *tak(ə)Ran \\
\hline dirt on skin & $*[\mathrm{~d}-] \gamma \mathrm{\gamma i}:$ & $* \mathrm{k}^{\mathrm{h}} \partial \mathrm{y}$ & $*$ daki \\
\hline rain $\sim$ mist & $*_{\text {fon }}$ & $*$ fun & $*_{\mathrm{Ra}}+\mathrm{bun}$ \\
\hline fire & *C-vi: & $*$ fi: & *sapuy \\
\hline tooth & *Civən & $*_{\text {fjon }}$ & *nipən \\
\hline you (pl) & $*_{\mathrm{su}}$ & $*_{\text {səw }}$ & $*_{\mathrm{i}-(\mathrm{ka}) \mathrm{su}}$ \\
\hline black & $* \mathrm{C}-[\mathrm{t}] \partial \mathrm{m}^{(\mathrm{C})}$ & *đəm? & *qitəm \\
\hline gall bladder $\sim$ liver & *C-təy & * đəy & *qatəy \\
\hline otter & $*_{\text {na:k }}$ & *hna:k & *sanaq \\
\hline hand & $* \mathrm{C}^{\mathrm{V}}$-mu: & *C-mu: & *qa-rima \\
\hline you & ${ }^{*} \mathrm{C}^{\mathrm{V}}$-mu: & *C-mu: & *kamu \\
\hline bear & *C-muy & *C-muy & *tumay \\
\hline this & $*[\mathrm{C}-] \mathrm{ni}^{\mathrm{B} / \mathrm{C}}$ & *C-ni:h & *qa-ni \\
\hline water $\sim$ sea & $* \mathrm{C}^{\mathrm{V}}-$ nəm $\mathrm{m}^{\mathrm{C}}$ & ${ }^{*} \mathrm{C}$-nəm? & *tənəm \\
\hline weep & ${ }^{*} \mathrm{C}-\mathrm{ni}{ }^{\mathrm{C}}$ & *C-ni:? & $*$ tanis \\
\hline child & *lut:k & *hlut:k & *alak \\
\hline shoulder & ${ }^{*} \mathrm{C}-\mathrm{ba}:^{\mathrm{B}}$ & *va:h & *qabara \\
\hline raw, live & *Cudi[:]p & *Curi:p & *qudip \\
\hline shrimp & *Cuda:y & *Cura:y & *qudan \\
\hline star $\sim$ sun & ${ }^{*} \mathrm{C}$-da:w & $*_{\text {ra:w }}$ & *qa-(n)daw \\
\hline thigh & *C-qa: & *ha: & *paqas \\
\hline excrement & ${ }^{*} \mathrm{C}-\mathrm{G}[\mathrm{a}:] \mathrm{y}$ & *fha:y? & $*$ taqi \\
\hline I & $* \mathrm{C}-\mathrm{ku}:$ & *hu: & $* a k u$ \\
\hline head & ${ }^{*}$ kurəw $^{\mathrm{C}}$ & *Curəw? & $*$ quru \\
\hline to plant & *Cura & *Cura: & *mura \\
\hline
\end{tabular}

The following are a list of items which only appear in the Hlai branch of Southern Kra-Dai. Possible Pre-Hlai reconstructions are provided in place of PSKD forms: 


slap
rub rope $\sim$ weave
pinch
seven
three
sharp
five
six
mat. grandma
that
bury
fish scale
eight
sell

\begin{tabular}{|c|c|c|}
\hline Pre-Hl & $\underline{\mathrm{PHl}}$ & $\underline{\text { PAn }}$ \\
\hline *pi:k & ${ }^{*} \mathrm{p}^{\mathrm{h}} \mathrm{i}: \mathrm{k}$ & *pik \\
\hline *bən & $* p^{h}$ ən & *bəl+bəl \\
\hline *ti:p & $* \mathrm{t}^{\mathrm{h}} \mathrm{i}: \mathrm{p}$ & *a-tip $\quad$ (PMP) \\
\hline *tu: & $* \mathrm{t}^{\mathrm{h}} \mathrm{u}:$ & *pitu \\
\hline *tu:? & $* t \int^{h} \mathrm{u}: ?$ & *təru \\
\hline *fə:m & $* t \varphi^{\mathrm{h}} \partial: \mathrm{m}$ & *tajəm \\
\hline *ma: & *hma: & *rima \\
\hline *nom & *hnom & *2ənəm \\
\hline *na:? & *hna:? & *ina 'mother's sister' \\
\hline *C-na: & *C-na: & $*_{\text {i-na? }}$ \\
\hline *lomh & *hlomf & *taləm \\
\hline *C-lə:p & *C-lə:p & *quşəlap \\
\hline $\begin{array}{l}\text { *ru: } \\
\text { *ri:w? }\end{array}$ & $\begin{array}{l}\text { *ru: } \\
\text { *ri:w? }\end{array}$ & $\begin{array}{l}*_{\text {waru }} \\
*_{\text {sariw }}\end{array}$ \\
\hline
\end{tabular}

The following five forms are problematic for various reasons, and may be fortuitous resemblances in some or all cases; since all of the PSKD forms below were listed as problematic in chapter four, it is also possible that one branch of PSKD may have a true connection to the PAn form, while the other branch does not:

\begin{tabular}{|c|c|c|}
\hline$\underline{\mathrm{PSKD}}$ & $\underline{\mathrm{PHl}}$ & $\underline{\text { PAn }}$ \\
\hline$* k[j] ə n$ & ${ }^{*} \mathrm{k}^{\mathrm{h}} \partial \mathrm{n}$ & *ka?ən \\
\hline *pwa:k & *fa:k & *baq+baq \\
\hline *rwa:k & *fa:k & $*$ m-utaq $\sim$ ruac \\
\hline *p[i/u]ra:k & *ra:k & *biraq \\
\hline${ }^{*} \mathrm{C}-\mathrm{b}[\mathrm{i} / \mathrm{u}] \mathrm{la}: \mathrm{n}$ & *C-na:n & *bural \\
\hline
\end{tabular}

The following forms lacking PSKD etymologies are also problematic, but merit further investigation:

$\underline{\text { Pre-Hl }} \quad \underline{\text { PHl }} \quad \underline{\text { PAn }}$

one

person

ten

skin (of fruit) fruit

two

cicada

snake

go let's go

* cua?

*[?]a:w

*fu:t

*va:k

*lu:?

*ro:y

*Cila:h

*tc ${ }^{\mathrm{h}} \mathrm{u}:$ ?

PAn

*[?]a:w

$*_{\mathrm{i}+\mathrm{sa}}$

*fu:t

*tau

*fa:k

*hlu:?

*ro:y

*puruq

*buaq

${ }^{*} \mathrm{~d}_{3}$ usa

*lja:h

*lari

*C-Gi:

*hi:

* Surar

*aRi

(PHF) 
There are a few observations which can be made based on the examples above. The first is that the loss of initial syllables in polysyllabic forms seems to have been an ongoing process in Hlai and its predecessors for some time. The following Hlai-PAn comparisons are those in which the evidence indicates that the Hlai form has developed from an original monosyllabic form in Pre-Hlai:

$\underline{\text { Pre-Hl }} \quad \underline{\mathrm{PHl}} \quad \underline{\mathrm{PAn}}$

ancestor

seven

fart

land leech

eye

sharp

dirt on skin

rib

rain $\sim$ mist

bury *pu:?

*tu:

*tu:t

*ta:k

*ta:

*fว:m

*ki:

*ka:n?

*von

*lomf
${ }^{*} \mathrm{p}$ h $\mathrm{u}:$ ?

$* t^{\mathrm{h}} \mathrm{u}$ :

$* t^{\mathrm{h}} \mathrm{u}: \mathrm{t}$

$* \mathrm{t}^{\mathrm{h}} \mathrm{a}: \mathrm{k}$

$* t \int^{\mathrm{h}} \mathrm{a}$ :

*tch ${ }^{\mathrm{h}}$ : $\mathrm{m}$

* $\mathrm{k}^{\mathrm{h}} \mathrm{i}$ :

${ }^{*} \mathrm{k}^{\mathrm{h}} \mathrm{a}: \mathrm{n}$ ?

*fun

*hlomf $\underline{\text { PAn }}$

$* a(m) p u$

*pitu

*qă(n)tut

*matək mətak

*mata

*tajom

*daki

*tak(ə)Ray

$*_{\text {Ra }}$ +bun

*taləm

There are two examples which indicate that in forms with a sequence of two high back vowels, the second of the two underwent diphthongization:

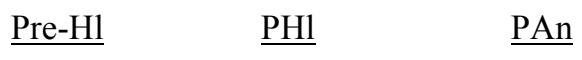

head louse

*twow

$* t \int^{\text {h }}$ wəw

*kutu

head

*Curəw?

*kurəw?

*quru

Bilabial stops, whether intervocalic voiceless or initial voiced, appear to have been prone to affrication:

$\begin{array}{lll}\text { Pre-Hl } & \underline{\text { PHl }} & \underline{\text { PAn }} \\ \text { *C-vi: } & \text { *fi: } & \text { *sapuy } \\ \text { *Civən } & \text { *fjən } & \text { *nipən } \\ \text { *[v]on } & \text { *fun } & \text { *Ra+bun } \\ \text { *[v]a:k } & \text { *fa:k } & \text { *baq-baq } \\ \text { *[v]a:k } & \text { *fa:k } & \text { *buaq }\end{array}$

The following two examples show that retroflexes merged with alveolars intervocalically: 


$\underline{\text { Pre-Hl }} \quad \underline{\text { PHl }} \quad \underline{\text { PAn }}$

$\begin{array}{llll}\text { gall bladder liver } & * \text { C-təy } & * \text { dəy } & * \text { qatəy } \\ \text { star } \sim \text { sun } & * \text { C-da:w } & * \text { ra:w } & * \text { qa-(n)daw }\end{array}$

The following two forms (keeping in mind that snake shows other irregularities) show one possible source of final $* \mathrm{~h}$ in Hlai tone category B:

$\underline{\text { Pre-Hl }} \quad \underline{\text { PHl }} \quad \underline{\text { PAn }}$

$\begin{array}{llll}\text { shoulder } & * \text { *-ba:h } & * \text { va:h } & * \text { *abara } \\ \text { snake } & * \text { Cila:h } & * \text { lja:h } & * \text { surar }\end{array}$

One form shows a possible source of final $* ?$ in tone category $\mathrm{C}$ :

$\begin{array}{lll}\underline{\text { Pre-Hl }} & \underline{\text { PHl }} & \underline{\text { PAn }} \\ *_{\text {C-ni:? }} & *_{\text {C-ni:? }} & \text { *tanis }\end{array}$

Finally, if the forms for bear and scale are valid comparanda, there appears to have been an unmotivated metathesis of the two vowels (it is assumed that scale first simplified in the following way: *qusəlap > *qəlap): $\underline{\text { Pre-Hl }} \underline{\text { PHl }} \underline{\text { PAn }}$

$\begin{array}{llll}\text { bear } & *[\text { ta }] \text { muy } & * \text { C-muy } & * \text { tumay } \\ \text { scale } & *[\text { qa]lo:p } & * \text { C-lə:p } & * \text { qusəlap }\end{array}$

The existence of the Hlai-PAn cognates above offers a unique opportunity to demonstrate the series of sound changes listed in chapter four. Keeping in mind that 'cognate' here is used to indicate two words that are related non-accidentally, either through common descent or through a language contact relationship, it can be inferred that these words existed at an early stage of Pre-Hlai (including potentially PSKD or higher) before the following changes took place. 


PAn
Intervocalic lenition
Elimation of uvulars
Peripheral vowel raising
Initial devoicing
Vocalic Transfer
Initial aspiration
Monosyllabification
Stop and fricative affrication
PHI

PAn

Intervocalic lenition

Elimation of uvulars

Peripheral vowel raising

Initial devoicing

Vocalic Transfer

Initial aspiration

Monosyllabification

Stop and fricative affrication

PHI

PAn

Intervocalic lenition

Elimation of uvulars

Peripheral vowel raising

Initial devoicing

Vocalic Transfer

Initial aspiration

Monosyllabification

Stop and fricative affrication

PHI

\begin{tabular}{|c|c|c|c|c|c|}
\hline weave & seven & eye & sharp & black & gall liver \\
\hline bəl & pitu & mata & tafom & qitəm & qatoy \\
\hline bən & tu: & ta: & fə:m & qidəm? & qadəy \\
\hline bən & tu: & ta: & fə:m & kidəm? & kadəy \\
\hline bən & tu: & ta: & fə:m & kidəm? & kadəy \\
\hline pən & tu: & ta: & cə:m & kidəm? & kadəy \\
\hline pən & tu: & ta: & cə:m & kidəm? & kadəy \\
\hline $\mathrm{p}^{\mathrm{h}}$ ən & $\mathrm{t}^{\mathrm{h}} \mathrm{u}:$ & $t^{\mathrm{h}} \mathrm{a}:$ & $\mathrm{c}^{\mathrm{h}}$ ə:m & kidəm? & kadəy \\
\hline $\mathrm{p}^{\mathrm{h}}$ ən & $\mathrm{t}^{\mathrm{h}} \mathrm{u}:$ & $t^{\mathrm{h}} \mathrm{a}:$ & $c^{\mathrm{h}} ə: \mathrm{m}$ & dəm? & dəy \\
\hline $\mathrm{p}^{\mathrm{h}}$ ən & $\mathrm{t}^{\mathrm{h}} \mathrm{u}$ : & $\mathrm{ts}^{\mathrm{h}} \mathrm{a}:$ & t $\varphi^{\mathrm{h}} \partial: \mathrm{m}$ & đəm? & dәу \\
\hline $\mathrm{p}^{\mathrm{h}}$ ən & $\mathrm{t}^{\mathrm{h}} \mathrm{u}:$ & $\mathrm{t}^{\mathrm{h}} \mathrm{a}:$ & $\mathrm{t} \varphi^{\mathrm{h}} \partial: \mathrm{m}$ & dəm? & dəy \\
\hline fire & tooth & shoulder & live & star $\sim$ sun & thigh \\
\hline sapuy & nipən & qabara & qudip & qa-daw & paqas \\
\hline Savi: & Civən & qava:R & quri:p & qara:w & рава: \\
\hline Savi: & Civən & kava:h & kuri:p & kara:w & pafia: \\
\hline Savi: & Civən & kava:h & kuri:p & kara:w & pafia: \\
\hline savi: & Civən & kava:h & kuri:p & kara:w & pafia: \\
\hline savi: & Civjən & kava:h & kuri:p & kara:w & pafia: \\
\hline savi: & Civjən & kava:h & kuri:p & kara:w & pafa: \\
\hline fi: & fjən & kava:h & kuri:p & kara:w & paha: \\
\hline fi: & fjən & kava:h & kuri:p & kara:w & pafia: \\
\hline fi: & fjən & C-va:h & Curi:p & C-ra:w & C-fha: \\
\hline five & six & otter & bury & sell & eight \\
\hline rima & Pənəm & şanaq & taləm & sariw & waru \\
\hline ma: & nom & na:q & lomf & sari:w? & waru: \\
\hline ma: & nom & na:k & lomf & sari:w? & waru: \\
\hline ma: & nom & na:k & lomf & sari:w? & waru: \\
\hline ma: & nom & na:k & lomf & sari:w? & waru: \\
\hline ma: & nom & na:k & lomf & sari:w? & waru: \\
\hline hma: & hnom & hna:k & hlomf & sari:w? & waru: \\
\hline hma: & hnom & hna:k & hlomf & sari:w? & waru: \\
\hline hma: & hnom & hna:k & hlomf & sari:w? & waru: \\
\hline hma: & hnom & hna:k & hlomf & ri:w? & ru: \\
\hline
\end{tabular}

weave seven eye sharp black gall liver

bəl pitu mata tafəm qitəm qatəy

bən tu: ta: fə:m qidəm? qadəy

bən tu: ta: fə:m kidəm? kadəy

bən tu: ta: fə:m kidəm? kadəy

pən tu: ta: cə:m kidəm? kadəy

pon tu: ta: cə:m kidəm? kadəy

$\mathrm{p}^{\mathrm{h}}$ ən $\quad \mathrm{t}^{\mathrm{h}} \mathrm{u}: \quad \mathrm{t}^{\mathrm{h}} \mathrm{a}: \quad \mathrm{c}^{\mathrm{h}}$ ə:m kidəm? kadəy

$p^{\text {h}}$ ən $t^{\text {h }} u: \quad t^{\text {ha: }} c^{\text {h }}$ ə:m dəm? dəy

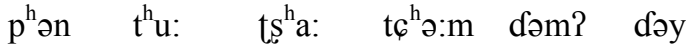

$p^{h}$ ən $\quad t^{\text {hu }}$ : $\quad \int^{\text {h }} a: \quad$ t6 $6^{\text {h }}: m$ dəm? dəy

fire tooth shoulder live star $\sim$ sun thig

sapuy nipən qabaRa qudip qa-daw paqas

Savi: Civən qava:R quri:p qara:w рава:

Savi:

Savi: Civən kava:h kuri:p kara:w pafa:

savi: Civən kava:h kuri:p kara:w paha:

savi: Civjən kava:h kuri:p kara:w paha:

şavi: Civjən kava:h kuri:p kara:w paha:

fi: fjon kava:h kuri:p kara:w pafa:

fi: fjən kava:h kuri:p kara:w pafa:

fi: fjən C-va:h Curi:p C-ra:w C-ha:

five six otter bury sell eight

rima ?ənəm sanaq taləm sariw waru

ma: nom na:q lomf sari:w? waru:

ma: nom na:k lomf sari:w? waru:

ma: nom na:k lomf sari:w? waru:

ma: nom na:k lomf sari:w? waru:

ma: nom na:k lomf sari:w? waru:

hma: hnom hna:k hlomf sari:w? waru:

hma: hnom hna:k hlomf sari:w? waru

hma: hnom hna:k hlomf ri:w? ru: 


PAn
Intervocalic lenition
Elimation of uvulars
Peripheral vowel raising
Initial devoicing
Vocalic Transfer
Initial aspiration
Monosyllabification
Stop and fricative affrication
PHI

\begin{tabular}{|c|c|c|c|c|c|}
\hline ear & this & water $\sim$ sea & weep & scale & head \\
\hline tumay & qa-ni & tənəm & tanis & quşəlap & quru \\
\hline muy & qani:h & tənəm? & tani:? & qalə:p & qurəw? \\
\hline tamuy & kani:h & tənəm? & $\tan \mathrm{i}: ?$ & k-lə:p & kurəw? \\
\hline tamuy & kani:h & tənəm? & tani:? & k-lə:p & kurəw? \\
\hline tamuy & kani:h & tənəm? & $\tan \mathrm{i}: ?$ & k-lə:p & kurəw? \\
\hline tamuy & kani:h & tənəm? & tani:? & k-lə:p & kurəw? \\
\hline tamuy & kani:h & tənəm? & $\tan \mathrm{i}: ?$ & k-lə:p & kurəw? \\
\hline tamuy & kani:h & tənəm? & tani:? & k-lə:p & kurəw? \\
\hline tamuy & kani:h & tənəm? & tani:? & k-lə:p & kurəw? \\
\hline muy & C-ni:h & C-nəm? & C-ni:? & C-lə:p & Curəw? \\
\hline
\end{tabular}

\subsubsection{Interim Summary}

Despite the fact that Hlai as a language group has been isolated on Hainan for a non-trivial amount of time, evidence has been provided in this section which indicates that there are vestiges of interaction with all four of the non-Kra-Dai language phyla of Southeast Asia which remain in the lexicon.

Given the cultural prominence of Sinitic in Southeast Asia, it is unsurprising to find that Hlai retains a number of words which indicate early interaction with Chinese, with tone correspondences indicating that this may have occurred at more than one point in history. It was also shown that there may have been at least limited interaction with Tibeto-Burman.

Evidence also exists which indicates interaction with Mon-Khmer, with the greatest amount appearing in Viet-Muong and West Bahnaric. As reconstructions of other Mon-Khmer families are finalized, it may become apparent that Hlai interaction was not limited to these two subgroups, or that interaction with Bahnaric may have occurred at a time preceding that during which West Bahnaric became an independent branch.

Although the present state of Hmong-Mien reconstruction makes comparison with Hlai difficult, it was nevertheless seen that a number of suggestive parallels exist that deserve further examination.

Finally, comparison with Austronesian offers a very satisfying number of lexical parallels, as well as additional confirmation for sound changes postulated in chapter four between the stages of PSKD and 
$\mathrm{PHl}$; in addition, comparison with Hlai can inform the reconstruction of the more problematic areas of the PAn consonant inventory.

\subsection{Summary of Findings}

There are a number of different issues which have been treated in this dissertation and, hopefully, clarified to some degree. The first of these is the phylogenetic structure of the Hlai language family. The evidence presented in chapter one provided evidence for a phylogenetic tree, based on innovations inferred from comparison of daughter branches and languages with Proto-Hlai. This tree retains all of the traditional groups from Ouyang \& Zheng (1983) except for the Ha branch, where it was shown that each 'Ha' language either represents (Bouhin and $\mathrm{Ha} \mathrm{Em}$ ) or is part of (Lauhut) a separate branch of Hlai, and that these are probably grouped together based on shared cultural context as opposed to actual common descent. It was also argued that vocalic transfer across approximants was more likely to be shared via common descent as opposed to cross-language diffusion, and that this criteria was therefore an excellent one for subgrouping the Central Hlai languages. It was shown that subsequent to the fission of Proto-Hlai into branches, and of these branches into daughters, that there were several points of intimated contact between various languages in different areas around the island. This contact shows up primarily through loan words, which are discernible through irregular reflexes of segments and tones, but also through various sound changes which have diffused within their respective areas.

An inventory of initials was reconstructed in chapter two for Proto-Hlai and evidence was provided for two types of words, monosyllabic and sesquisyllabic. There is occasionally evidence for the nature of the first vowel in a sesquisyllabic word, preserved in coarticulations on (primarily) approximants which occurred as a result of vocalic transfer at the Central Hlai level. There is a noticeable difference in the kinds of initials which are reconstructed as word-initial as opposed to syllable-initial within a sesquisyllabic word. Most in the former category are reconstructed as aspirated, whereas those in the second category (with the exception of the glottal stop) are more sonorous, and include only members which are voiced. Perhaps the most important force driving the evolution of Hlai initials was shown to be 
temporal compression, which led to further and further reduction of multiple segments in the onset until only a single segment remained.

The rime inventory is reconstructed in chapter three, including both the laryngeal as well as segmental components of the Proto-Hlai rimes. The four Kra-Dai tone categories were examined in the context of Proto-Hlai, and the three-way contrast in the first three categories (the fourth category ending in voiceless oral obstruents) was reconstructed as plain versus breathy voice versus constricted glottis. The segmental rime inventory was reconstructed, with three levels of height, three degrees of backness, and a length distinction in all closed rimes. Several places of interaction between rime nuclei and final laryngeal elements were highlighted, including the diphthongization of high vowels before laryngeals in Greater Hlai, the effects of laryngeals on specific rime categories in various subgroups and daughter languages.

Proto-Hlai was compared with Proto-Be and Proto-Southwest Tai, the only fully reconstructed branch of Tai, in chapter four. Comparison with these other two members of Southern Kra-Dai allowed a tentative reconstruction of Proto-Southern Kra-Dai, from which the general evolution to Proto-Hlai could be inferred. Several changes were illustrated involving the initials, the most far-reaching of which include the first initial devoicing, intervocalic lenition and initial aspiration. Two processes which began in PreHlai but were only completed after the breakup of Proto-Hlai were monosyllabification and vocalic transfer. The most fundamental change in the rimes was shown to be peripheral mid vowel raising, which led to the original mid-vowels $*_{e}$ and $*_{o}$ to rise and merge with original $*_{\mathrm{i}}$ and $*_{\mathrm{u}} \mathrm{u}$, allowing original $*_{\varepsilon}$ and $* \mathrm{~s}$ to rise in turn and fill their vacated positions.

The Jiamao language was the focus of chapter five. Jiamao has been a consistent enigma in the area of Hlai comparative work, and the idea originally advanced in Thurgood (1991) that Jiamao is originally a non-Hlai language was reinforced heavily in this chapter. Data was presented which shows that a subset of the Jiamao rimes have two reflexes of Proto-Hlai initials, indicating two layers of borrowing; the nature of these reflexes also indicate that the earliest contact with Jiamao occurred during a later period of Pre-Hlai. Comparison of the rimes supported this analysis and, if anything, showed that it may be necessary to invoke more than two strata of loanwords in order to explain the variation, although 
another source is undoubtedly transmission errors during borrowing. The fact that the Jiamao tone system is only loosely correlated with that of Proto-Hlai, along with the evidence given for a large group of core vocabulary of non-Hlai origin, served to underscore the ultimately non-genetic relationship between Jiamao and Hlai.

Finally, the comparison of Proto-Hlai with other reconstructed Southeast Asian proto-languages in this chapter has shown that Hlai and its ancestors have been anything but isolated in prehistory, and that contact has occurred at one time or another with some constituents of every one of the other Southeast Asian language phyla. Comparison between these families has in some cases been informative in both directions, and comparison with polysyllabic Proto-Austronesian in particular allowed a test of the Pre-Hlai sound changes postulated in chapter four.

\subsection{Empirical and Theoretical Contributions}

The empirical contributions of this data include the collection of the lexicon of Nadouhua, the documentation of Changjiang as a previously unrecorded Hlai language and the collection of much of its lexicon, and the double-checking of the data collected in Ouyang \& Zheng (1983) and Ouyang (1998) for

the other ten Hlai languages and Jiamao. Audio recordings of approximately two hundred basic vocabulary items were also made in all thirteen languages used in this dissertation with the help of the consultants listed in chapter one. The cumulative data for over 1,000 vocabulary items has been included in the appendix, with accompanying reconstructions based on the analysis given in this dissertation.

The collective study of the Hlai languages provides an excellent case study in the dynamics of language contact in a delimited linguistic area such as an island, and this dissertation has contributed to an better understanding of the complexities of language contact. While the products of contact with non-Hlai languages (especially Chinese) are easily recognized, the effects of intra-family language contact can be more difficult to distinguish and interpret. Because of the amount of contact which has taken place between the various Hlai languages, there are two principles which have been necessarily emphasized. The first is that when using lexical criteria for subgrouping, it is extremely important to check for regular 
correspondences. It was noted that in most cases, irregular correspondences are not random, but are the consequence of replacement by a cognate form from a related language. Irregular correspondences are therefore not only important to note for theoretical reasons, but also because they can provide crucial clues about a contact situation which has either gone unrecognized or has been underappreciated. The second principle is that when using phonological changes as criteria for subgrouping, care must be taken to separate less common changes which are likely one-time events from more common changes which are prone either to diffusion or to independent occurrence within languages. Less common changes which can be argued to have occurred before the diffusion of more general changes are particularly important evidence in subgrouping, as the likelihood is high that these kinds of changes are in evidence only in languages which have descended from a common ancestor. The implementation of the two principles above has allowed a subgrouping of the Hlai languages to be performed with high confidence, the most notable features of which are the recognition of the initial split between Bouhin and Greater Hlai, and the use of vocalic transfer as a crucial criterion for inclusion in the Central Hlai subgroup.

There are several theoretical contributions which have been made in this dissertation which pertain to an understanding of language change. One outstanding generalization is that speakers of languages in contact situations which are under pressure to conform to a particular template (the monosyllable in this case) may adopt different strategies on their way to convergence. For example, while consonant clusters were generally disfavored, the strategy adopted in Central Hlai to form monosyllables in the case of *p-1 sesquisyllables was to allow the formation of a cluster *pl, whereas the initial consonant was lost in Bouhin and Ha Em. A similar example occurred in the case of post-PHl vocalic transfer, where the information from an original high vowel in a presyllable was preserved as a coarticulation on the main syllable initial in Central Hlai; all information from these vowels was lost completely in Bouhin and Ha Em. This fact is of great value in the enterprise of phonological reconstruction, as the greater the degree of variation is between the daughter languages, the larger the total amount of information is which is retained form comparison.

Another important generalization which can be made based on the data in this dissertation is that the more heterogeneous the reflexes of the daughter language, the more complex the proto-phoneme is 
likely to be from which they descended. It was shown in chapter one that general similarity among the reflexes of the daughter languages is indicative that an identical, or nearly-identical, proto-phoneme can be reconstructed. When there is a large degree of variation, as in the case of the initials which either hosted coarticulations in Proto-Hlai or gained them later in Central Hlai, a more complex phoneme must necessarily be reconstructed in order to account for the variation. The reason for this is very likely that there are fewer opportunities for mistransmission in the case of simple phonemes, whereas the chances that mistransmission will occur in the case of complex phonemes is higher. This can be due to misperception on the part of the learner (Change in Blevins' (2004) model), to variation on the part of the speaker (Choice in the same model), or some combination of both.

A third generalization which can be made is that language change, being non-teleological, can sometimes drift away from the typological norm; the results of such a change may be inherently unstable, however, and further change back toward the typological norm may occur due to biases inherent in the articulatory and auditory systems. An example of this was the change called initial aspiration, which resulted in the aspiration of obstruents and the preaspiration of sonorants if they were initial both in the word and the main syllable. This change resulted in a typologically marked situation in Proto-Hlai, in which only preaspirated sonorants (with the possible exception of *r) occurred in this environment, to the exclusion of plain sonorants. However, this ended up being a temporary state of affairs, as the preaspirated nasals either became unaspirated (Bouhin) or became post-stopped nasals (Greater Hlai), and the glides were reanalyzed as weak fricatives, leaving the liquids (if $r$ is included) as the only sonorants which remain preaspirated in some of the daughter languages. In most cases the end results of these changes were either merger with existing categories or repopulation of former categories, a change referred to here as systemic realignment.

A fourth generalization is that when the evidence forces the conclusion that the same sound change has occurred multiple times in the history of a language family, it should be considered that a general constraint is involved. This was seen to be true in Hlai in the case of a constraint against voiced obstruents. It was shown in chapter four that PSKD voiced obstruents underwent devoicing in Pre-Hlai. It was also shown in chapter two that devoicing occurred again after the break-up of Proto-Hlai but before 
registrogenesis, and a third time after registrogenesis. Since there is no evidence which suggests that voiced obstruents (which arise as the result of sound change) remain voiced for any duration, the most economical analysis is therefore to recognize a general constraint against voiced obstruents which may apply whenever the sufficient environment is created; this is preferable to positing three separate obstruent devoicings which were ultimately unrelated.

There are three generalizations which can be made about vocalic transfer. The first is that in situations which involve prosodic change (i.e. the several examples give in chapter four, section 4.3.4), the linearization of consonant and vowel phonemes becomes more easily confused, often leading to a reanalysis of vowel position within the word. In Hlai, this led to a form of metathesis in which the features of original presyllable high vowels were reanalyzed as coarticulated glides hosted by the following consonant. The second generalization is that vocalic transfer interacts with sonority, and it was observed that the higher the sonority of a consonant, the greater the chances of vocalic transfer occurring across it (the one exception being the glides, which may have been exempt from this change due to their strong similarity with high vowels). The third generalization is that vocalic transfer did not occur simultaneously in the case of the two high vowels. In all but one case, vocalic transfer of the front vowel $i$ had already occurred in Pre-Hlai, but vocalic transfer of the back vowel $u$ did not occur until the break-up of Proto-Hlai, in Central Hlai, although it occurred in several of the same environments.

Finally, this dissertation has made theoretical contributions involving the reconstruction of two important constituents of the Proto-Hlai (and ultimately Kra-Dai) phonological word. The first is the presyllable and the sesquisyllabic word. Ostapirat $(2004,2005)$ presented the first formal reconstruction of Proto-Hlai in which the existence of sesquisyllabic forms were necessary to fully explain the reflexes of the daughter languages. This dissertation has elaborated on Ostapirat's important observation by refining the reconstruction of sesquisyllabic forms (chapter two) and using this knowledge to improve our understanding of Proto-Southern Kra-Dai word shape and related changes in Proto-Be and Proto-Southwest Tai (chapter four).

The second constituent which has been reconstructed is the category of word-final laryngeals which were the precursors of the tone categories B and C. The evidence supporting the reconstruction of final 
laryngeals was given in chapter three, and the methodology for the reconstruction of these laryngeals for Hlai was proposed here for the first time. Although the analysis provided here is exploratory, this is a significant achievement in Hlai comparative studies, and provides a model which can be used in other parts of the Kra-Dai family (and ultimately in other language families and phyla of Southeast Asia where paradigmatic tone categories exist).

\subsection{Future Research}

The purpose of this final section is to outline several possible ways for potential research to proceed, and reasons to do so. Focusing first on Hlai-internal research, the Lauhut dictionary compiled by Ouyang (1992) is solid proof that additional data collection is both possible and worthwhile. The dictionary is a valuable collection of the Lauhut lexicon which is probably nearly exhaustive. While it becomes obvious upon close inspection that a large portion of the modern lexicon contains borrowings from various Hainan Chinese sources, it also demonstrates that there are a number of native Hlai words which exist that were not available in Ouyang \& Zheng (1983). The dictionary therefore offers a readymade list of vocabulary which may be elicited in the other Hlai languages, offering the possibility of greatly expanding the inventory of Proto-Hlai forms included in this dissertation. It is also highly recommended that data collection is done in parts of Hainan which have not yet been sampled. The 'discovery' of Changjiang during the preparatory fieldwork for this dissertation, coupled with the anecdotal accounts of my consultants, indicates that the existence of as yet unrecorded Hlai languages or dialects is likely, and all of these have the potential to add evidence to the Proto-Hlai reconstruction (as well as other areas of inquiry), as Changjiang has done here. Finally, as the present reconstruction has focused solely on phonological reconstruction of the lexicon, there is obviously room for reconstruction in other parts of the grammar, most notably syntax. It of course goes without saying that sociolinguistic research will pay dividends, given the complex mosaic of ethnic and social groups which interact with each other across Hainan. As the Hlai languages in general, and some languages in particular (most notably Nadouhua and Yuanmen) are under pressure from Chinese, resulting in gradual but steady language-shift, any research yet 
to be done upon Hlai languages is better done sooner rather than later. This is especially true since, while Hlai (like many minority languages) is recognized by the Chinese government, there has never been a writing system developed for any of the Hlai languages and there is no apparent effort being made by the government toward language preservation.

Moving to the topic of Hlai-external research, the next important step in Kra-Dai comparative studies is to finalize reconstructions of the Central and Northern subgroups of Tai, so that a full Tai reconstruction can be performed which will finally be able to replace the tentative reconstruction done by Li (1977) which has filled this role since its publication. Northern Tai in particular will provide crucial evidence for this reconstruction, and will allow the question to be resolved of whether $\mathrm{Be}$ is ultimately a member of Tai, or a sister as it has been treated here. At this point, comparison with Hlai can be performed in full and a complete PSKD reconstruction performed. Within the area of Northern Kra-Dai, the reconstructions of Proto Kra (Ostapirat 2000), Proto Kam-Sui (Thurgood 1988) and Proto-Lakkja (Theraphan 1992) need to be revisited and brought current with advances in Southern Kra-Dai, including the theory developed here of sesquisyllabic proto-forms. Assuming the accuracy of Ostapirat's classification presented in chapter one, the comparison of Kra, Kam-Sui, and Lakkja will allow ultimately allow a reconstruction of Proto Northern Kra-Dai (PNKD), which can ultimately be compared with PSKD, allowing a reconstruction of Proto-Kra-Dai itself. It is only at this point that Kra-Dai can best be compared with other language phyla with an eye to uncovering the nature of their prehistorical relationships. The current state of Austronesian studies makes this a promising venture, with Sino-Tibetan studies advancing, and Mon-Khmer family reconstructions showing progress largely due to the work of Paul Sidwell. As noted, a new and more detailed reconstruction of Hmong-Mien is necessary before serious comparison with Kra-Dai will be possible; the materials exist, and once this undertaking is accomplished, the relevance to Kra-Dai could be pleasantly surprising. 


\section{APPENDIX A: HLAI LANGUAGE DATA AND PROTO-HLAI RECONSTRUCTIONS}

The data presented in this appendix is based on the wordlist published in Ouyang \& Zheng (1983), and has been supplemented by my fieldwork in Hainan during the academic year of 2003-2004. Lexical items are presented in both Chinese and English, and are in alphabetical order according to the standard Mandarin reading. The order in which the languages are given is the following:

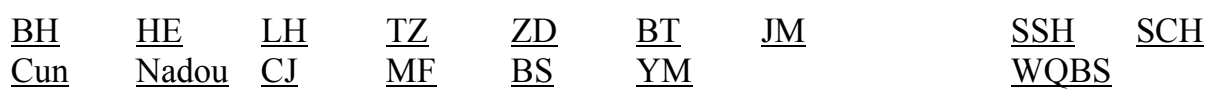

The three languages at the far right are from Savina's (1931) dictionary and Wang \& Qian's (1951) wordlist; the abbreviations are the following:

$$
\begin{aligned}
& \text { SSH }=\text { Savina's Southern Hlai (Bouhin) } \\
& \text { SCH }=\text { Savina's Central Hlai (Qi) } \\
& \text { WQBS }=\text { Wang \& Qian's Baisha }
\end{aligned}
$$

Savina's forms have been converted here to the International Phonetic Alphabet as closely as possible based on the Vietnamese script which he used for his transcriptions. The only instances in which this is non-transparent are of vowel length in diphthongs, where he uses the finals $<\mathrm{i}>$ and $<0>$ to indicate long nuclei, and the finals $<\mathrm{y}>$ and $<\mathrm{u}>$ to indicate short nuclei. For example, $<$ ai $>$ and $<$ ao $>$ indicate [a:y] and [a:w], and $<$ ay $>$ and $<$ au $>$ indicate [ay] and [aw] respectively. In the case of the tones, the following conventions have been adopted (where ' $v$ ' represent the rime nucleus):

$\begin{array}{llll}\text { Khong (mid level): } & \text { V } & \text { Huyen (low falling): } & \text { v } \\ \text { Sac (high rising): } & \text { V } & \text { Hoy (low rising): } & \text { V̌ } \\ \text { Nga (high broken): } & \text { V́ } & \text { Nang (low broken): } & \underline{\mathbf{v}}\end{array}$

Proto-Hlai forms are given to the immediate left of the lexical items. If there is agreement at the highest level of the Hlai phylogenetic tree (between Bouhin and one or more Greater Hlai languages), then a Pre-Hlai form is given to the left of the Proto-Hlai form. If not, then a proto-form is given (appropriate to 
the level to which it can be reconstructed), and it is indicated to the left which node of the tree the form is reconstructible to, using the following abbreviations:

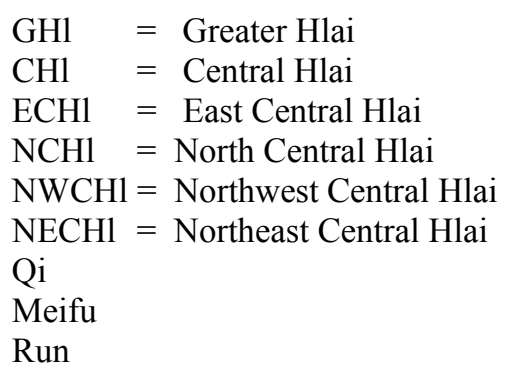

Forms which are not cognate with any other forms and which therefore do not contribute to reconstruction (both loanwords from Chinese and lexical isolates) have been removed for ease of exposition. Two important exceptions are Bouhin and $\mathrm{Ha} \mathrm{Em}$, which both form individual branches at a high level in the tree, the lexical isolates of which may therefore represent inherited Proto-Hlai forms even if they do not agree with Greater Hlai (in the case of Bouhin) or Central Hlai (in the case of Ha Em). The other exception is Jiamao, which appears without brackets when it appears to be a Hlai borrowing, but is placed in brackets otherwise. Forms which appear to be borrowed between Hlai languages (based on the criteria given in chapter one) are placed in parentheses. Irregular correspondences that are not obviously due to borrowing are placed in brackets.

Note that to simplify presentation, reconstructed Greater Hlai and Central Hlai forms are given as Proto-Hlai in the body of the dissertation. 


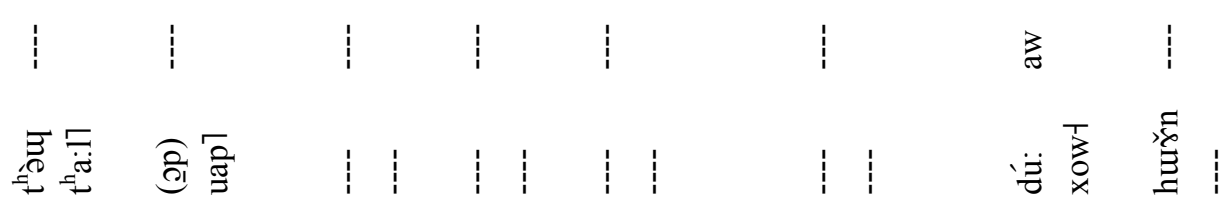

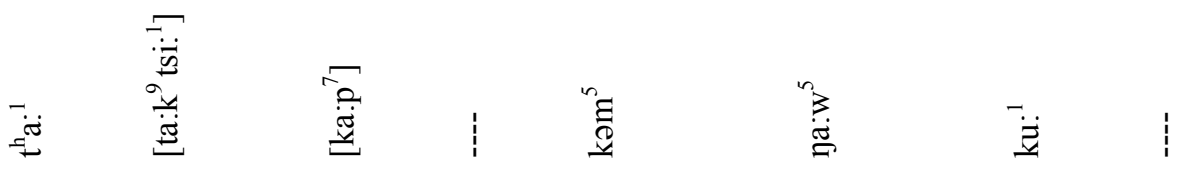

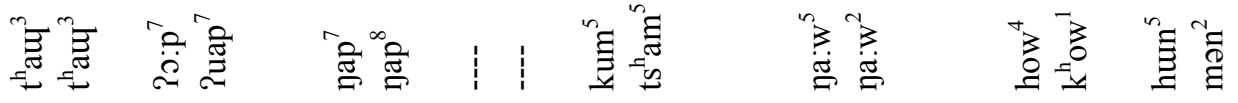

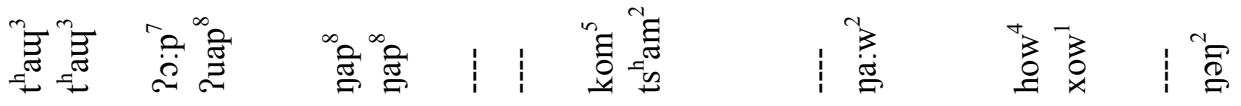

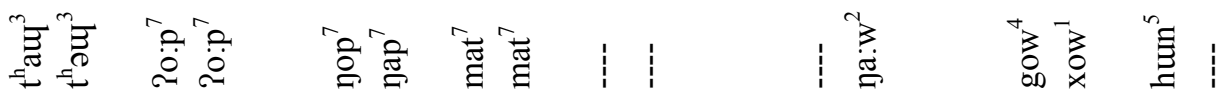

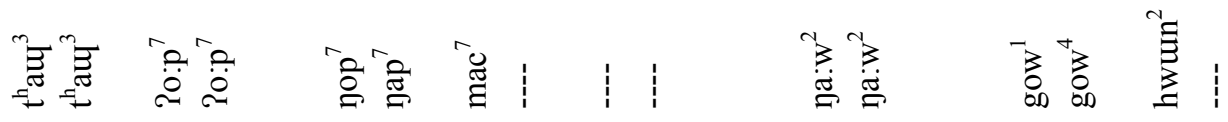

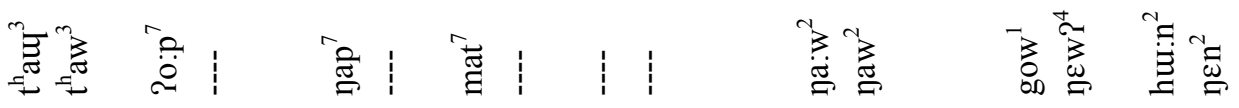

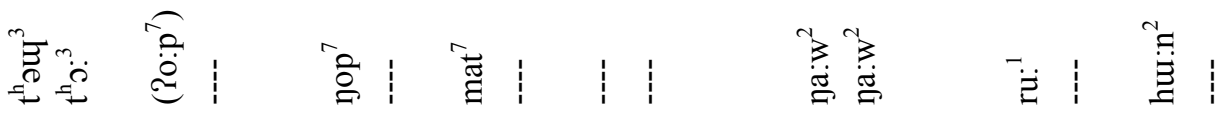

离

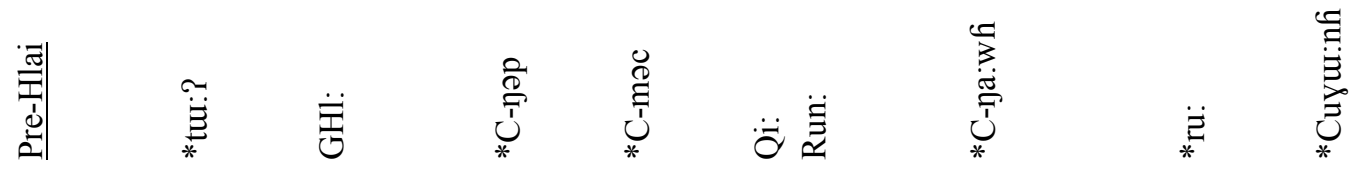

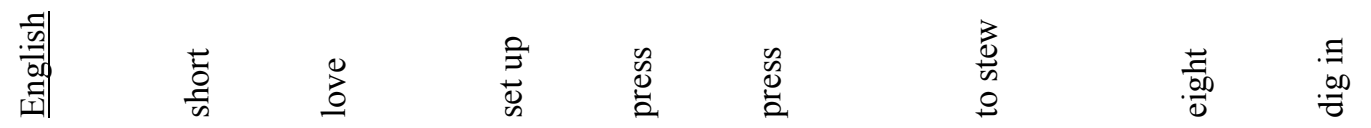

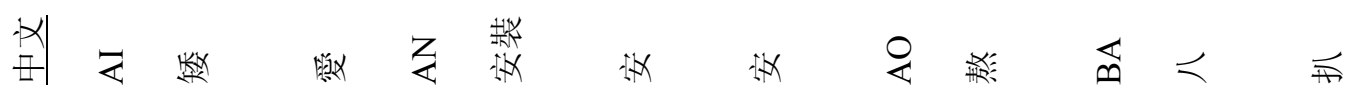




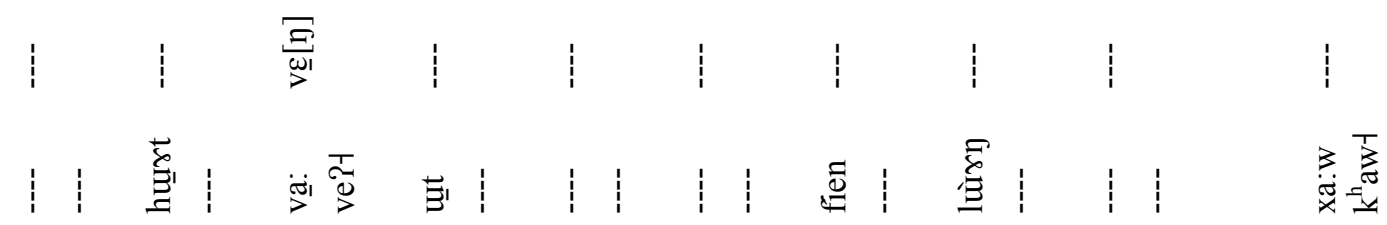

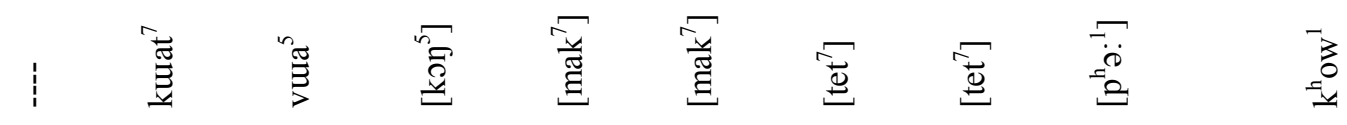

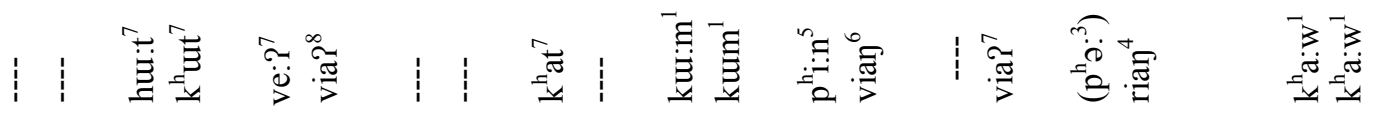

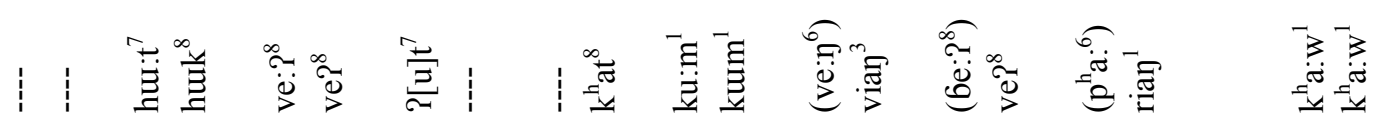

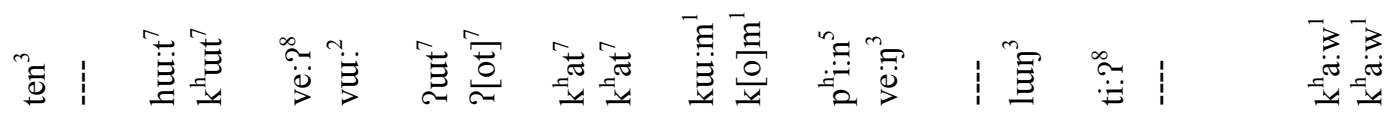

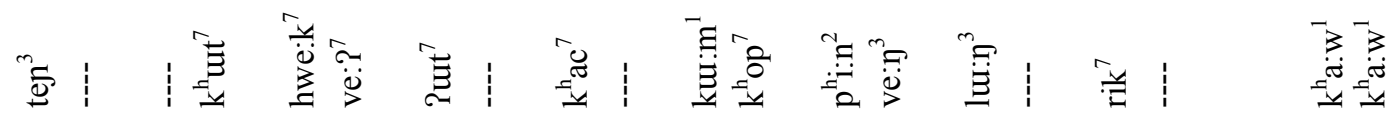

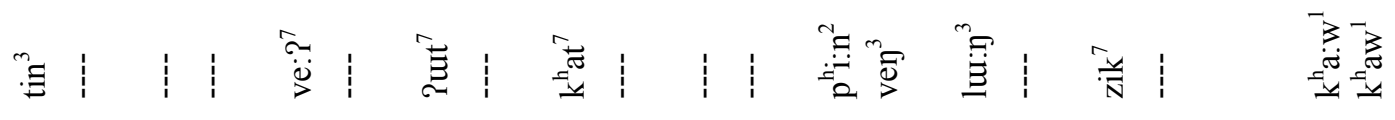

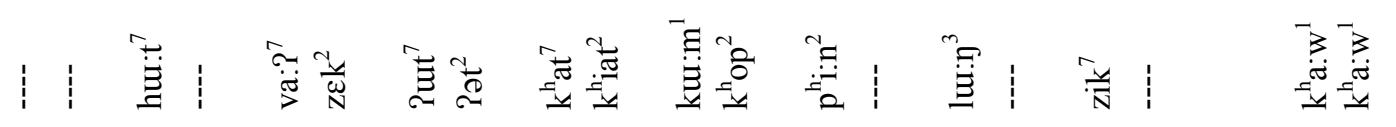

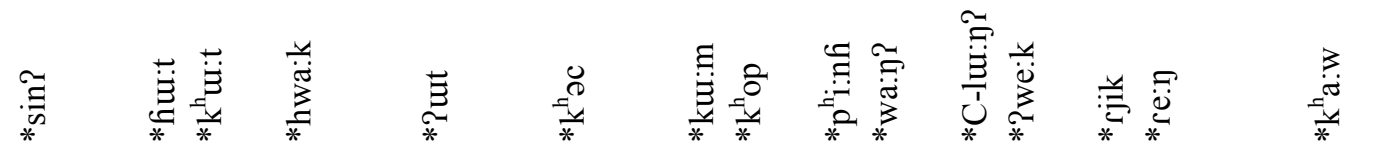

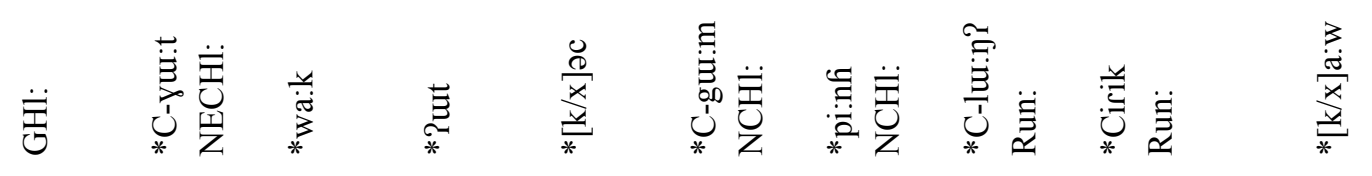

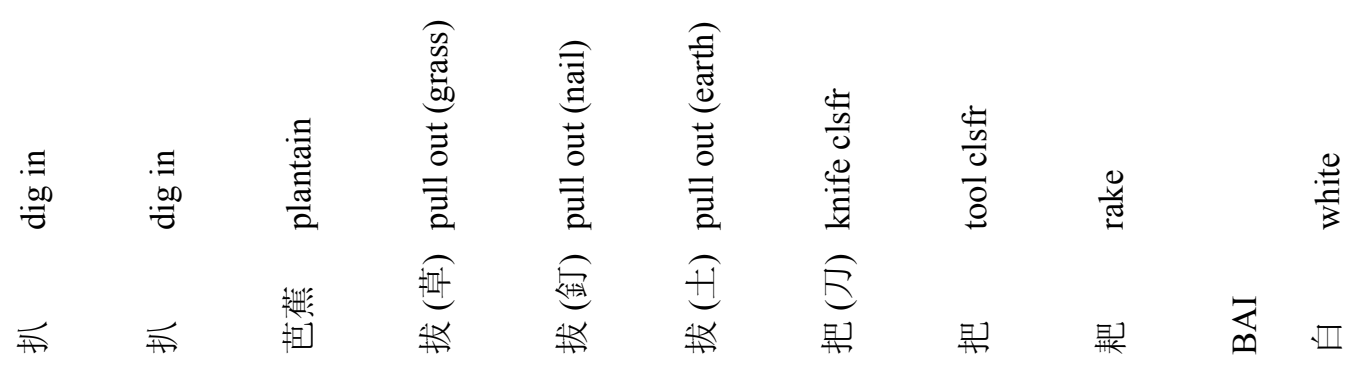




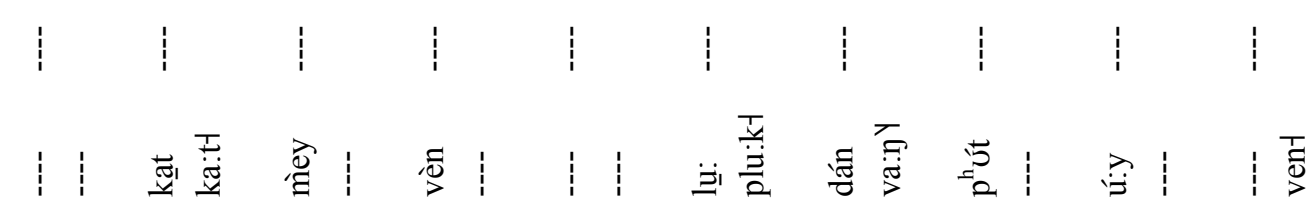

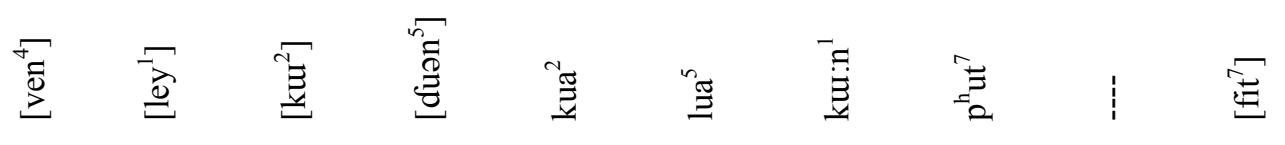

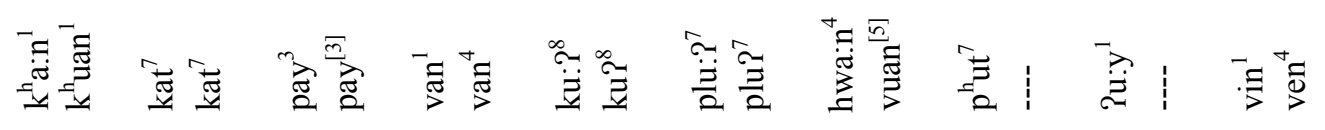

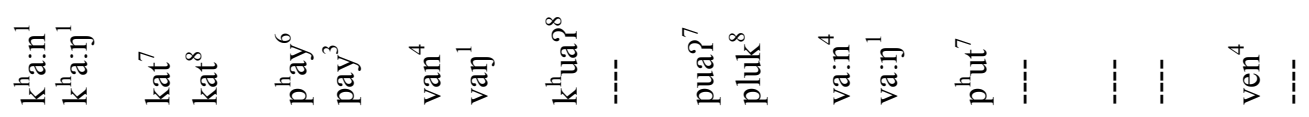

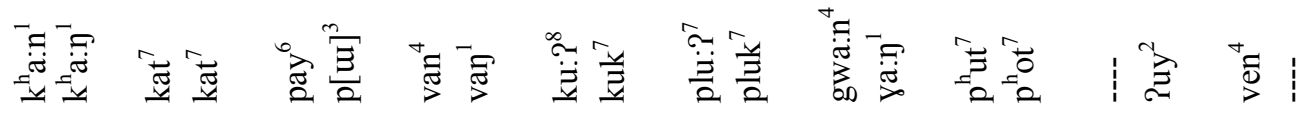

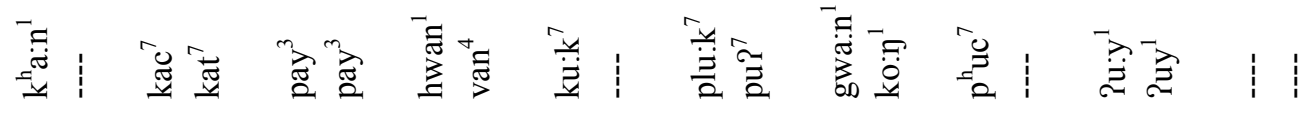

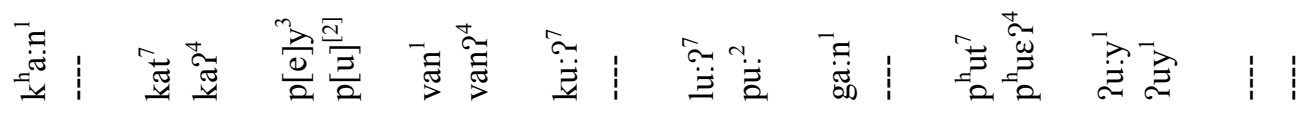

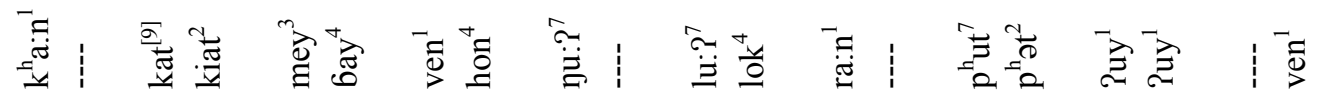

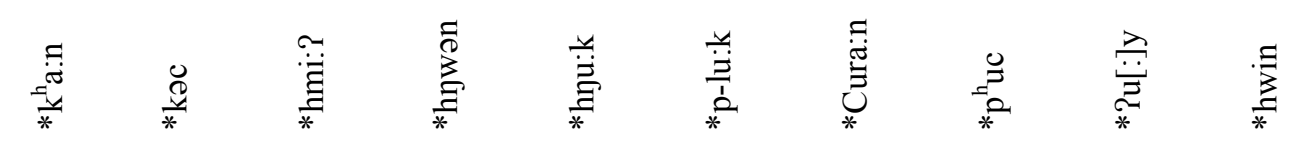

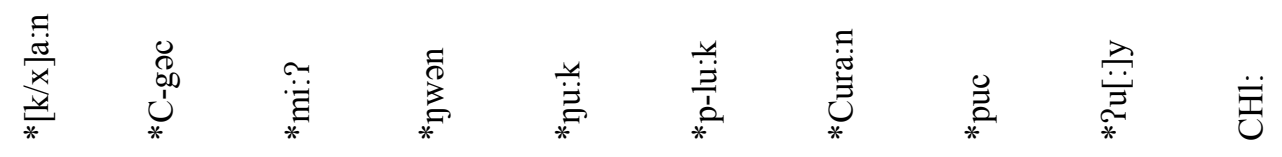

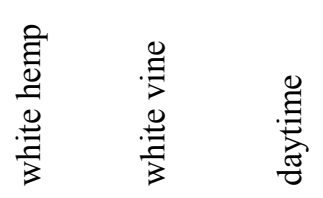
衰竞

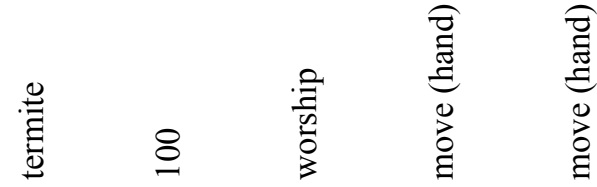
篮籍监

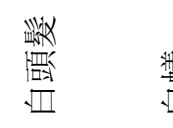
迹

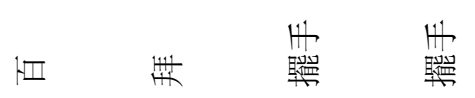




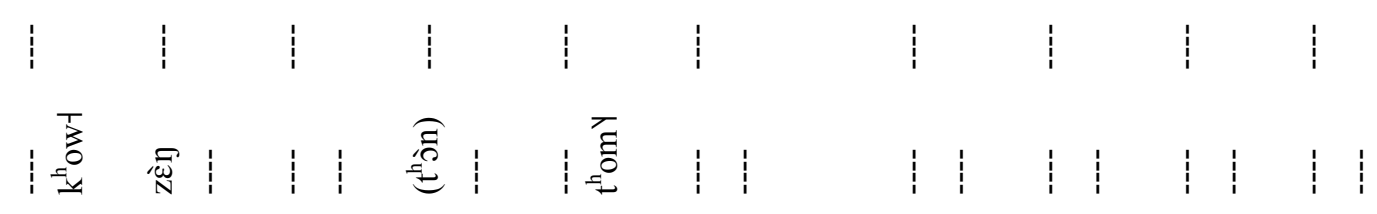

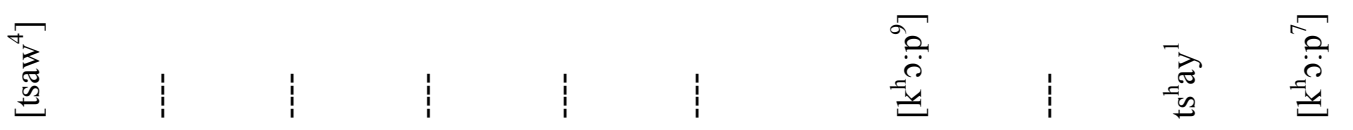

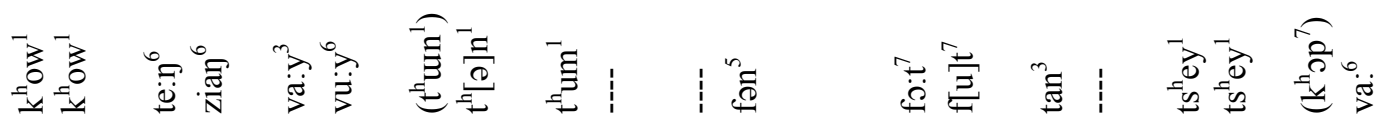

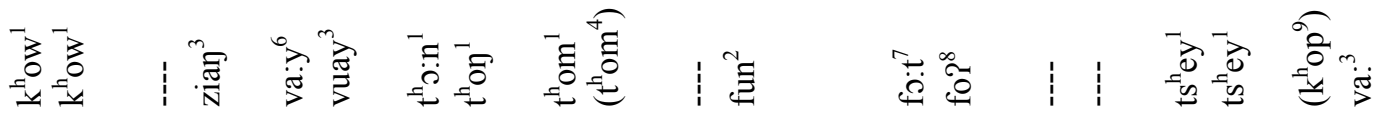

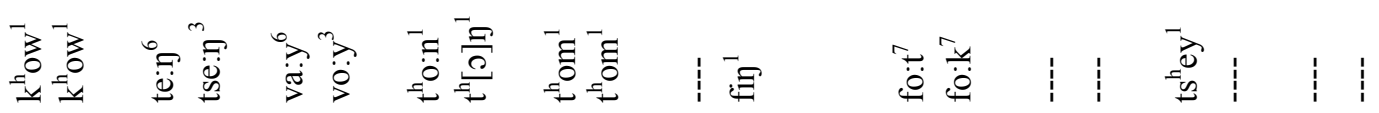

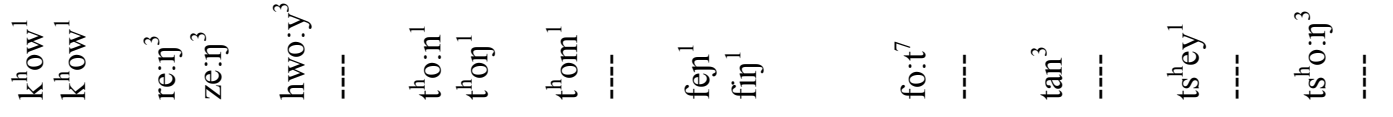

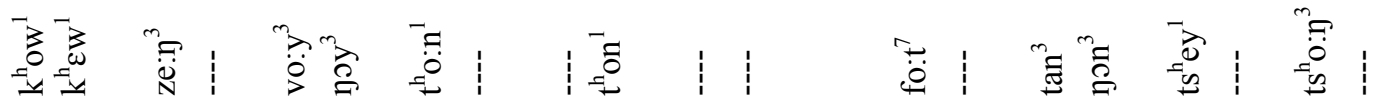

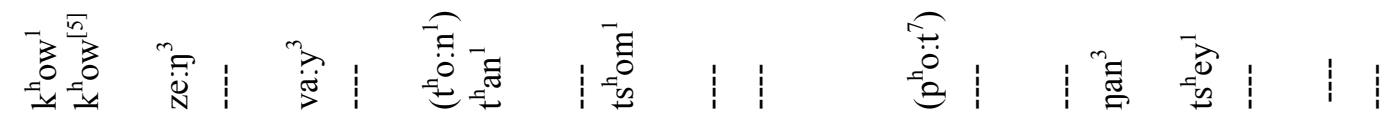

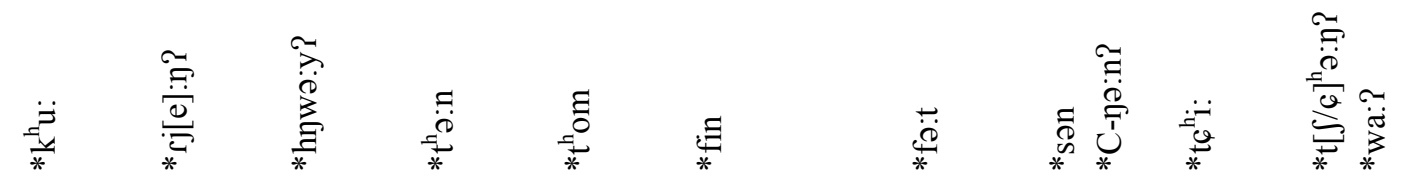

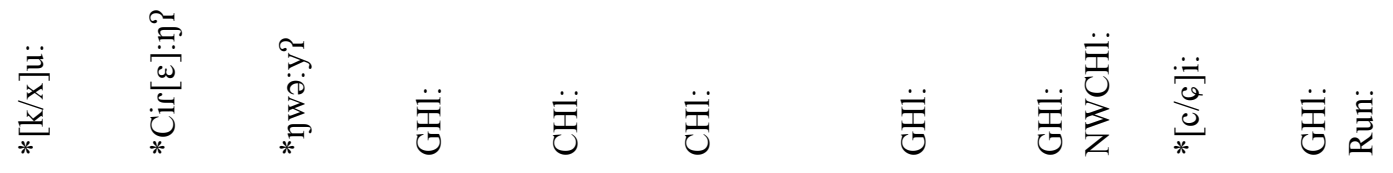

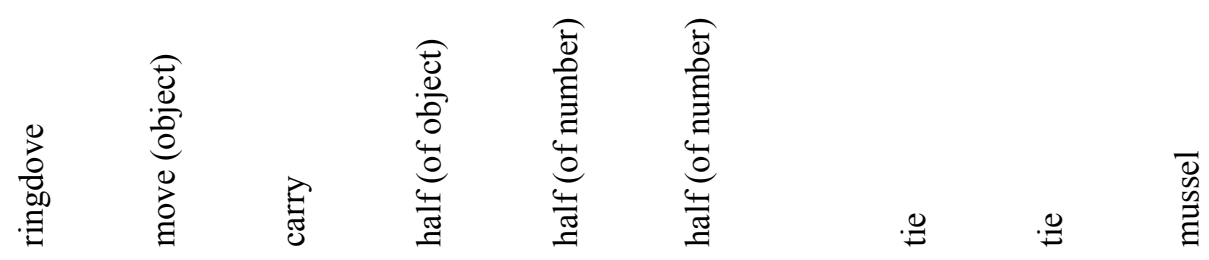

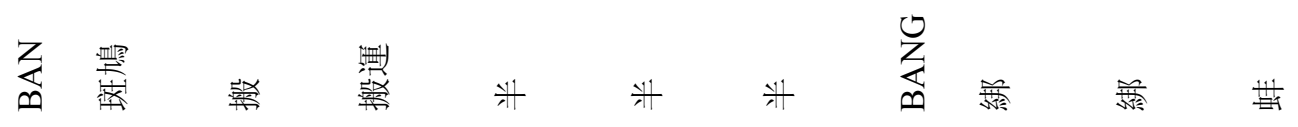




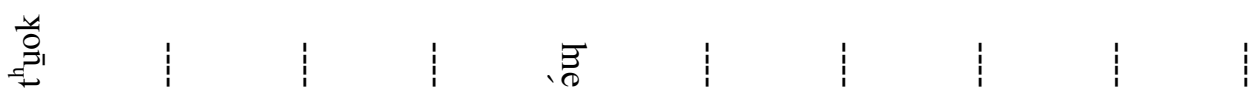

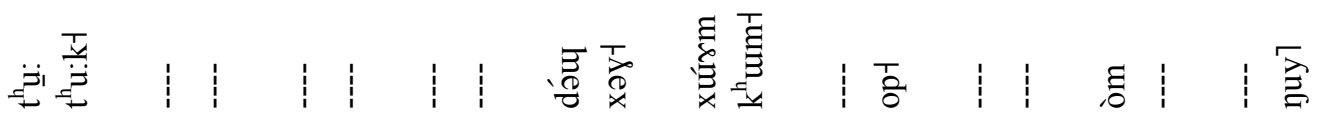

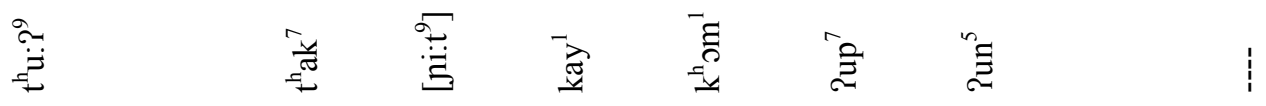

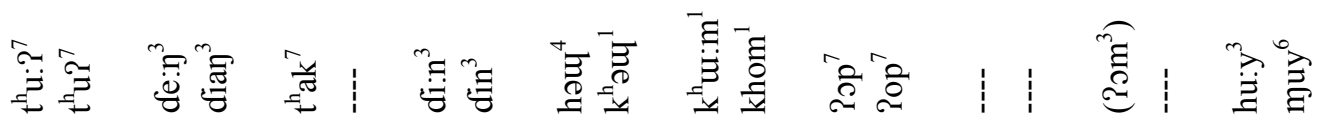

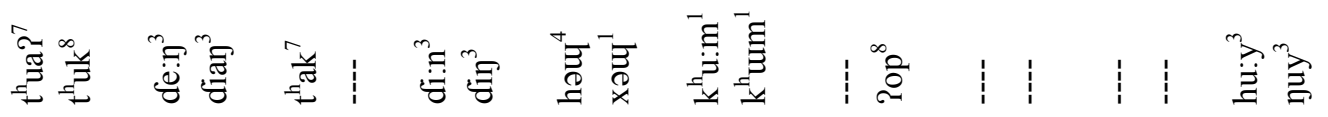

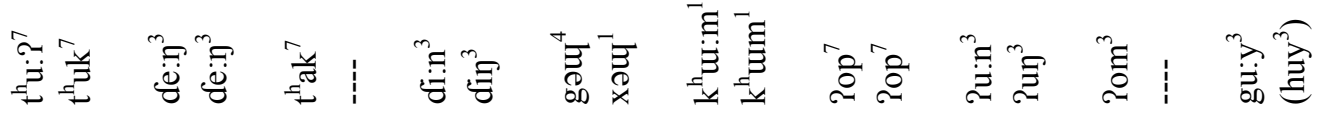

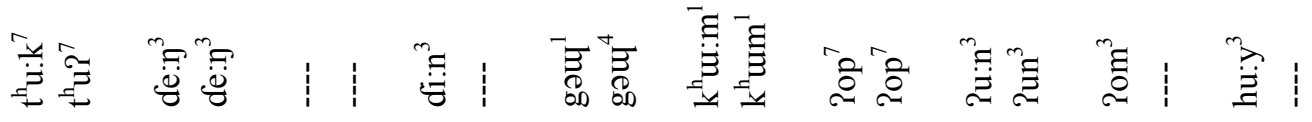

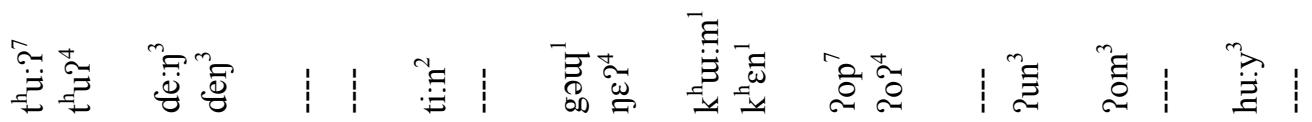

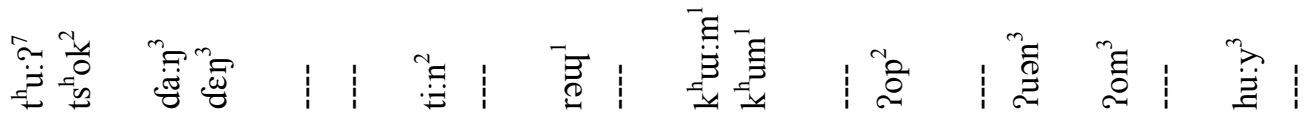

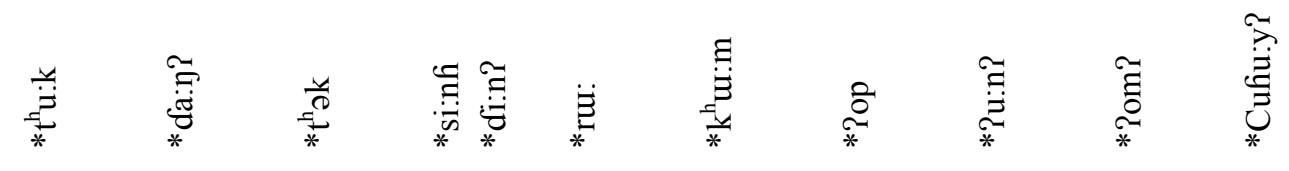

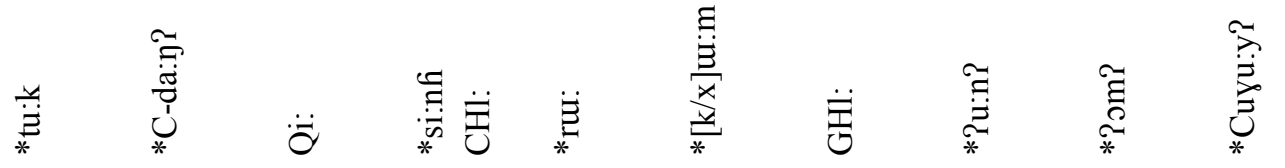

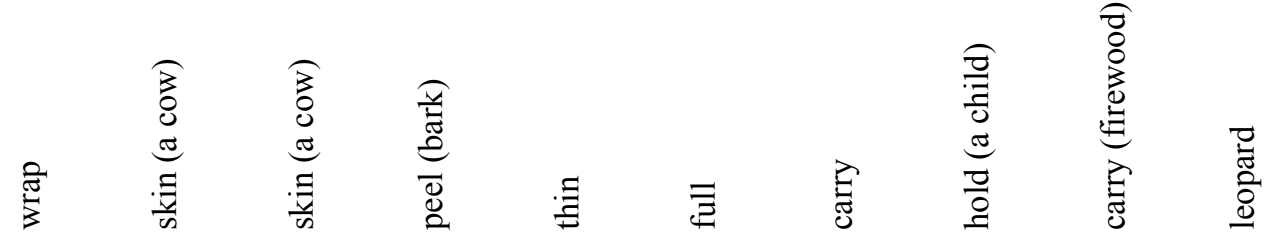

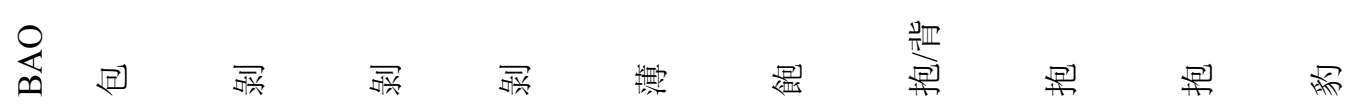




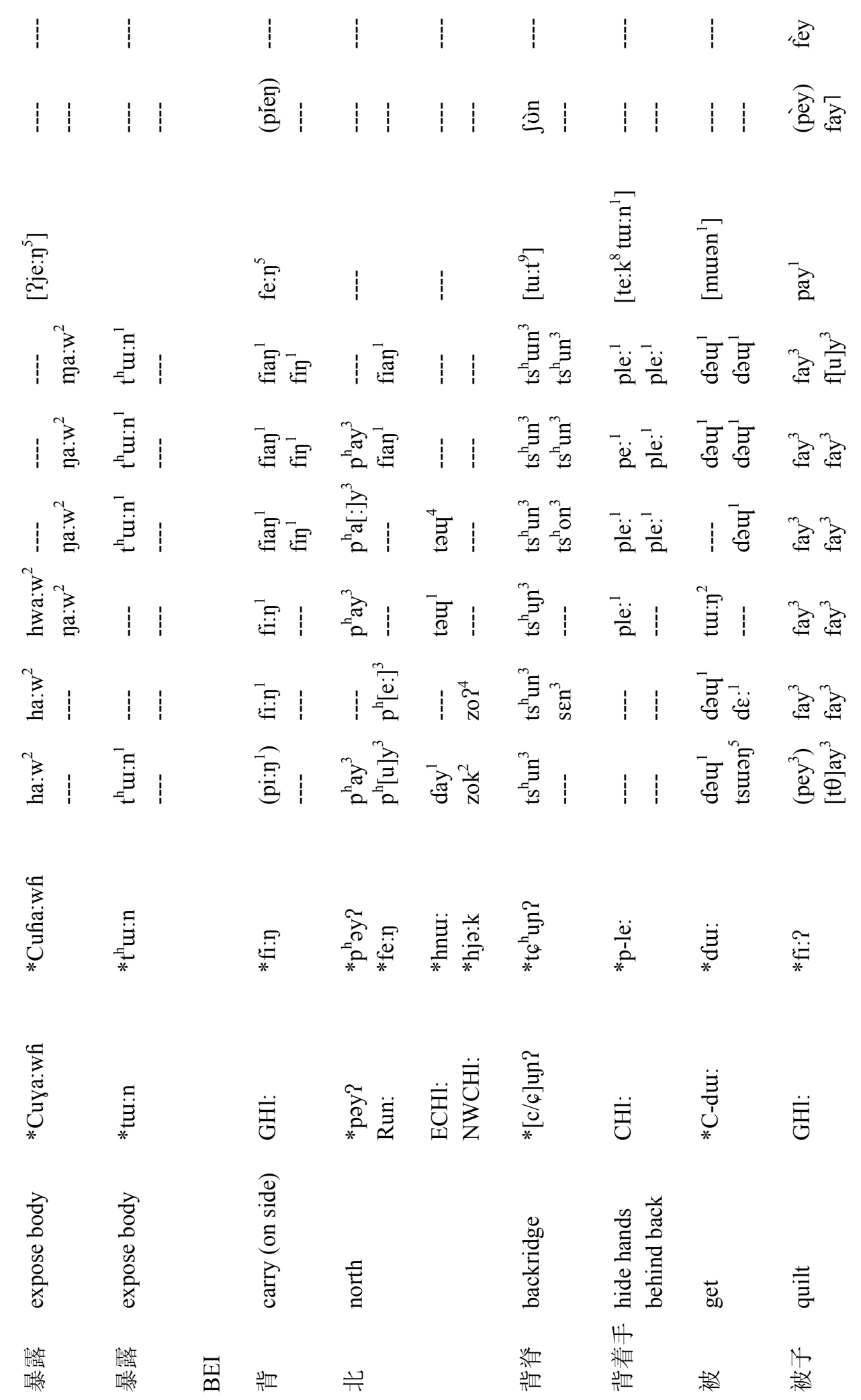




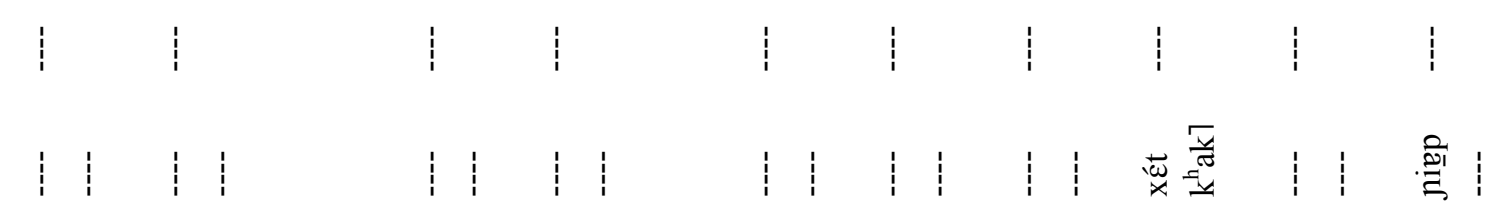

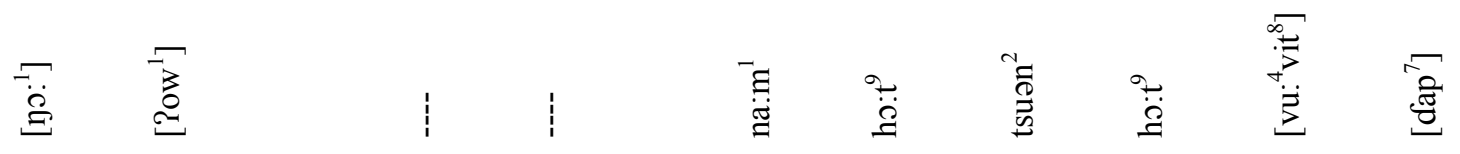

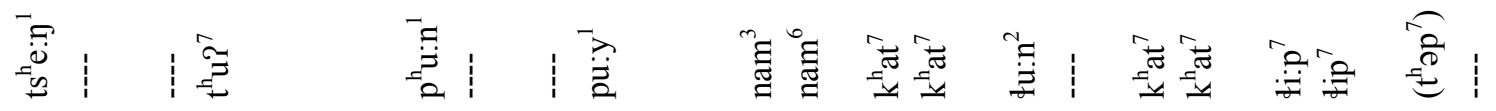

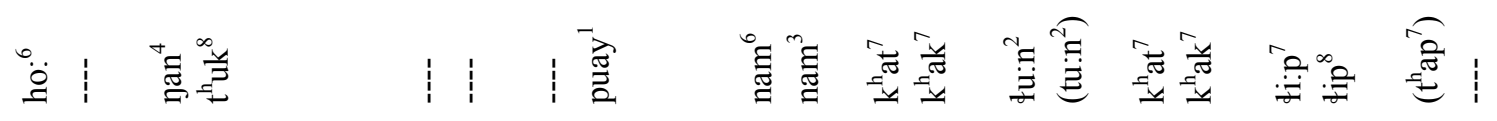

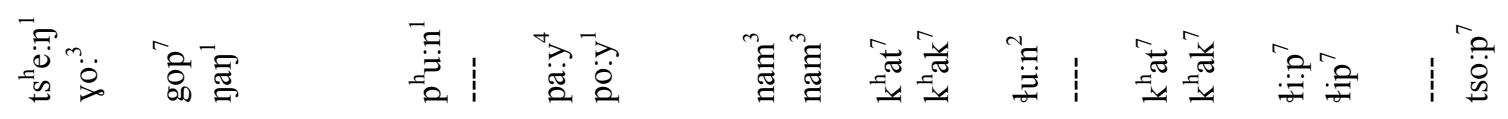

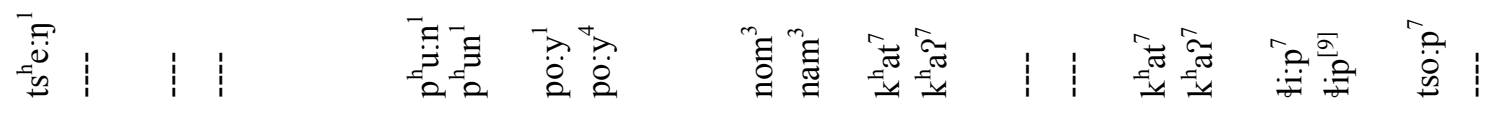

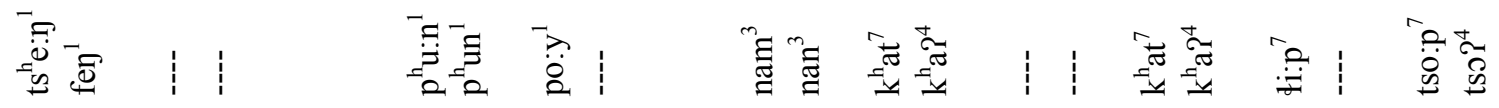

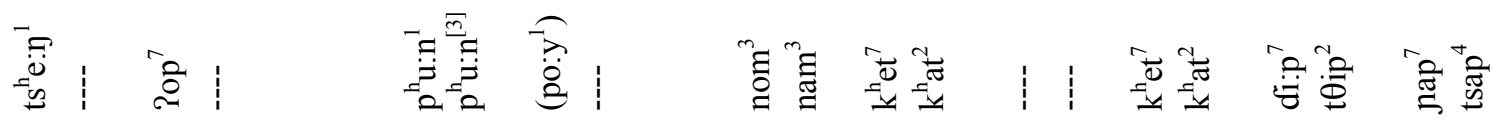

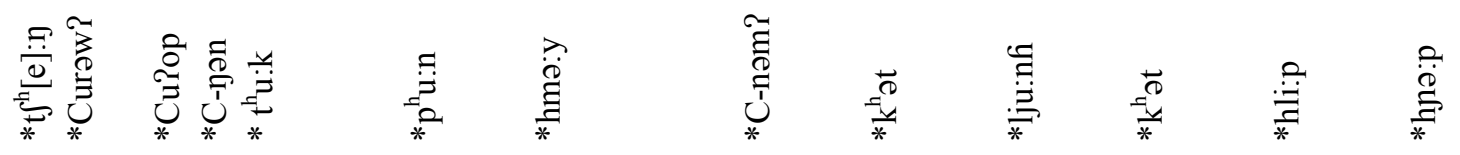

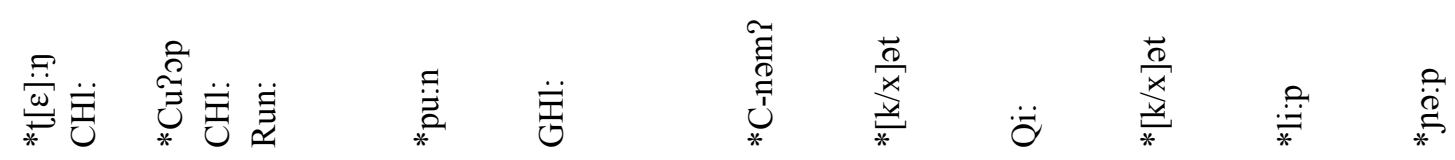

몰

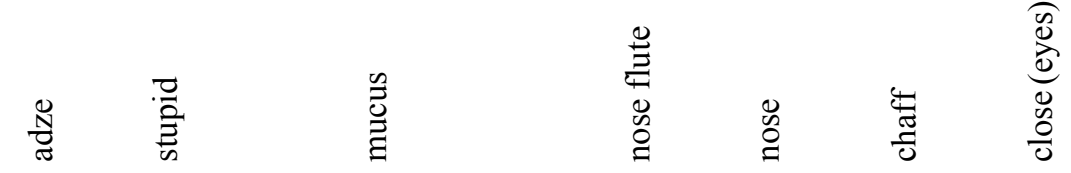

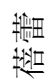

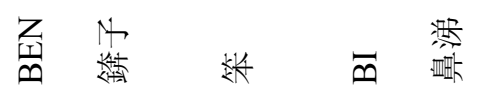

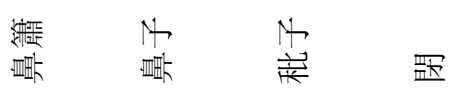




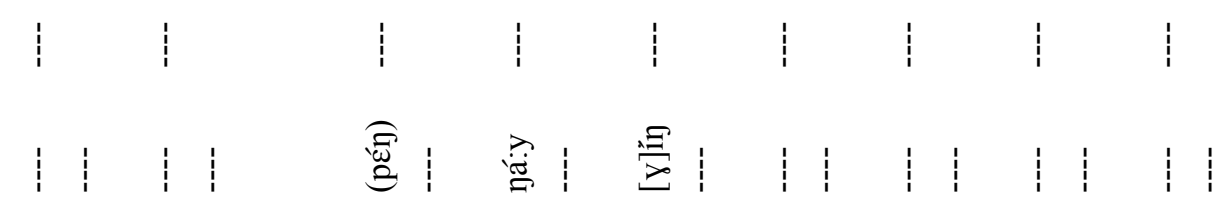

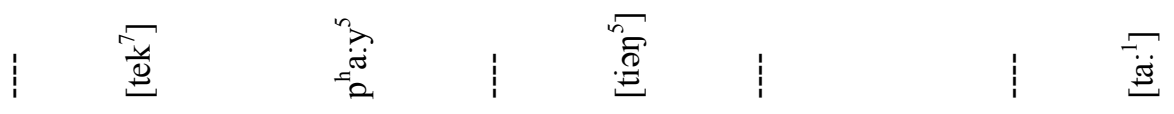

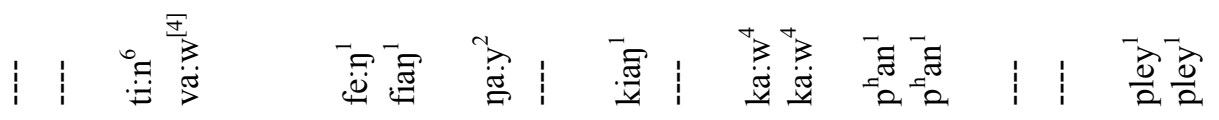

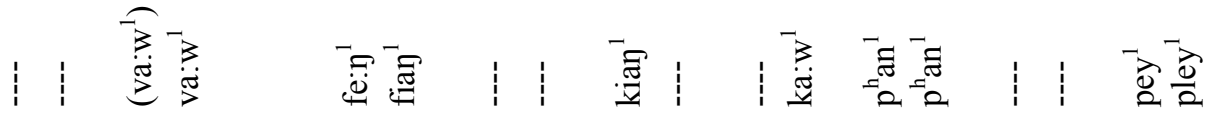

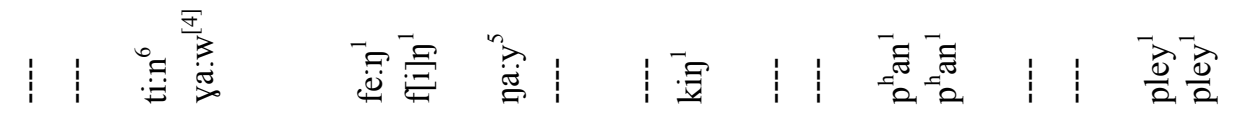

焉:

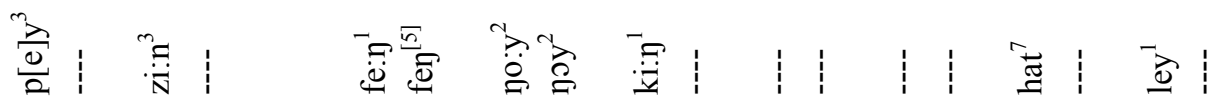

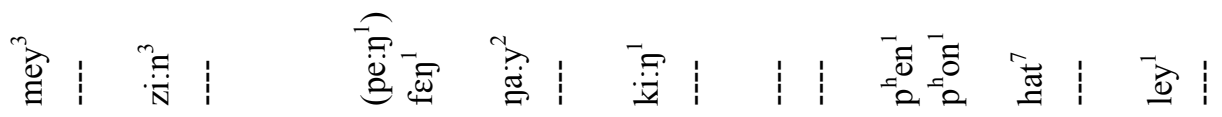

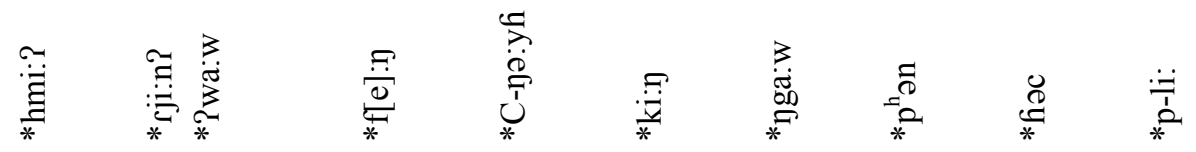

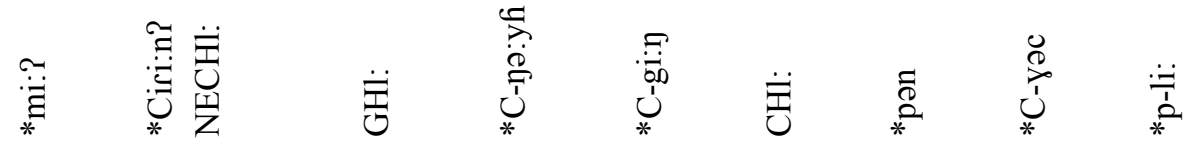

$\frac{8}{8}$

此

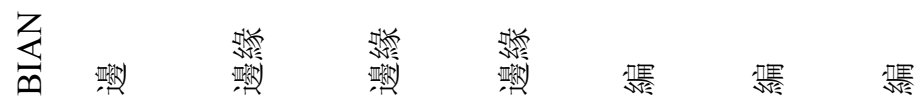




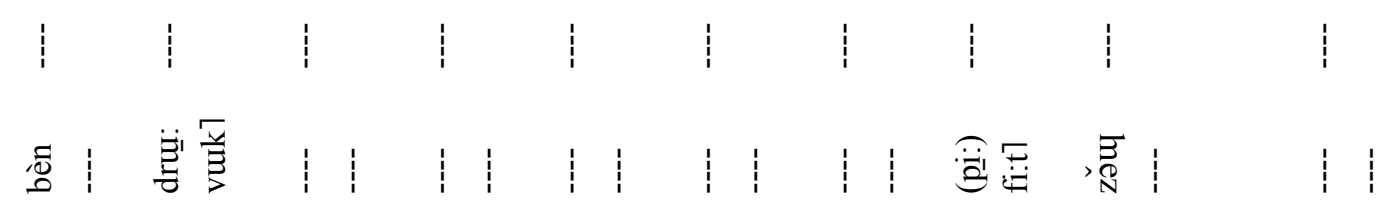

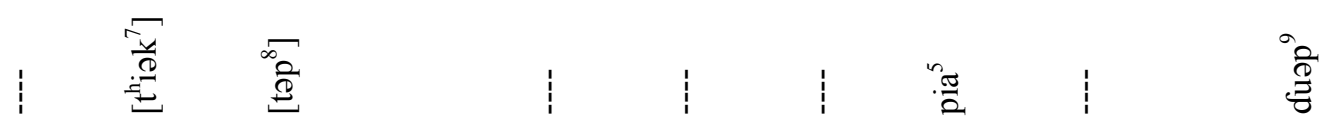

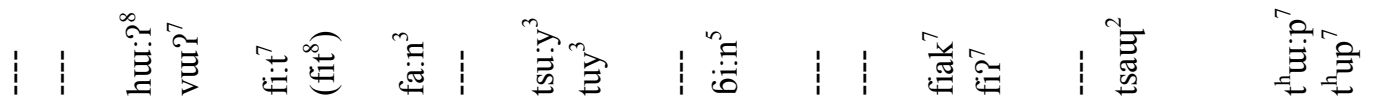

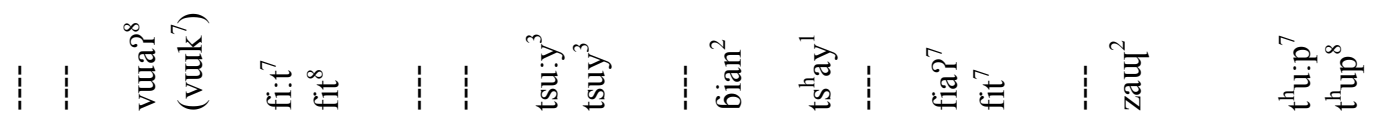

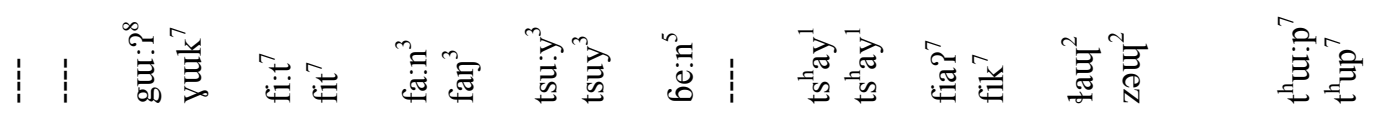

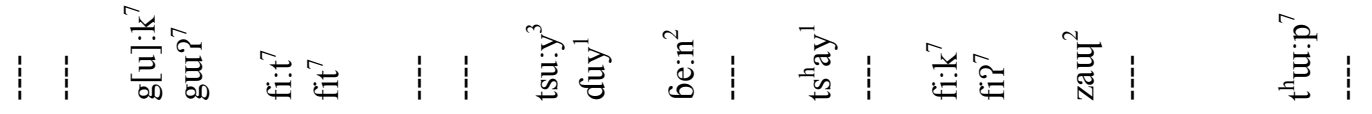

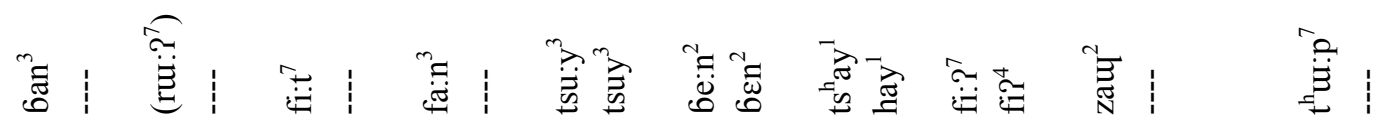

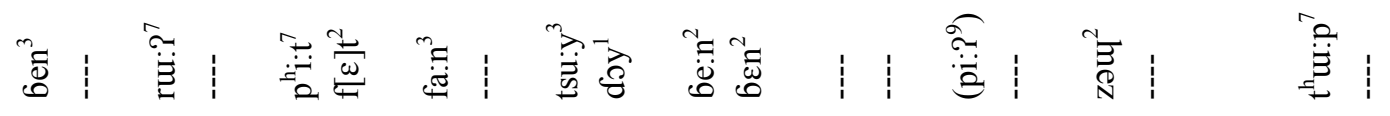

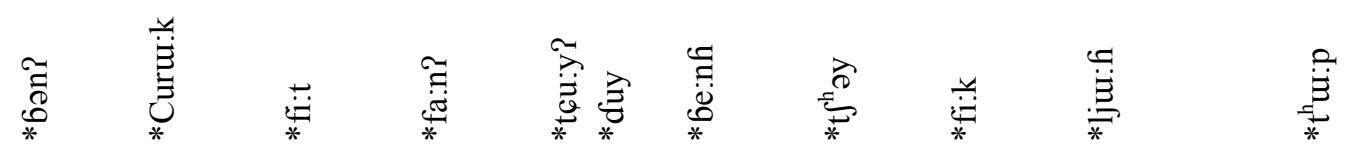

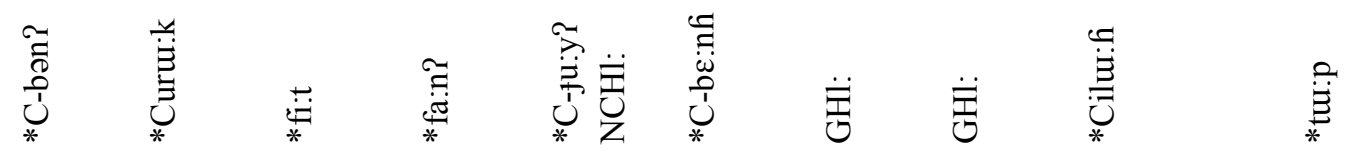

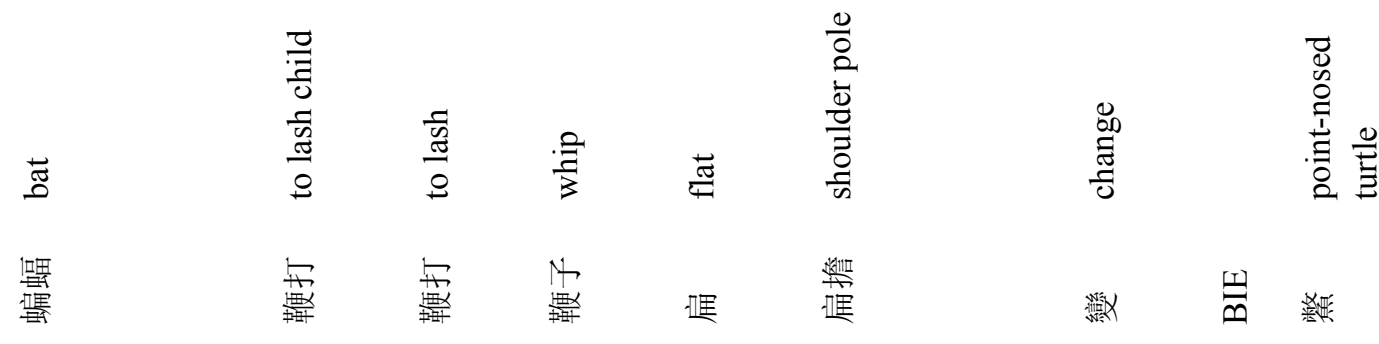




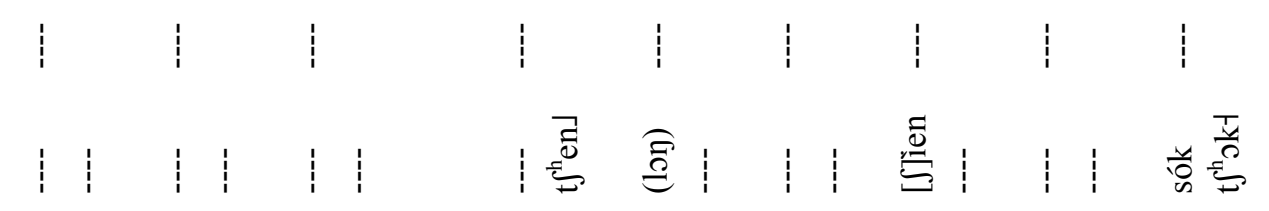

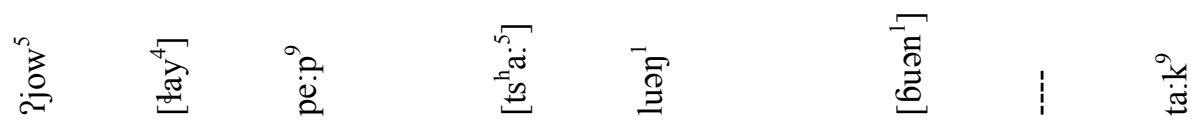

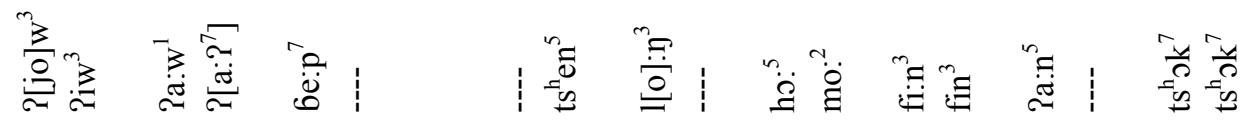

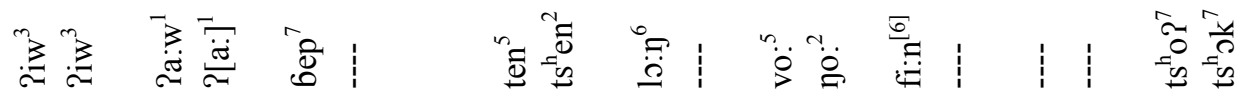

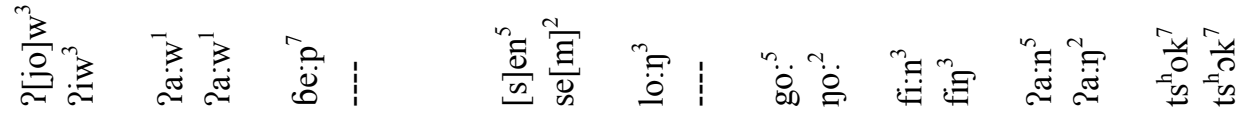

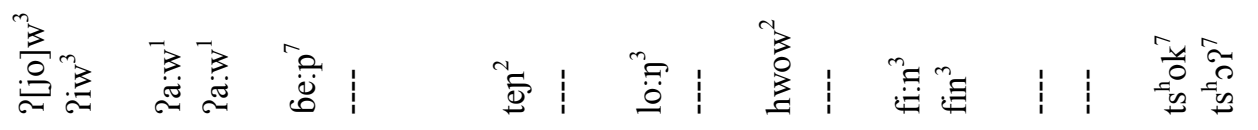

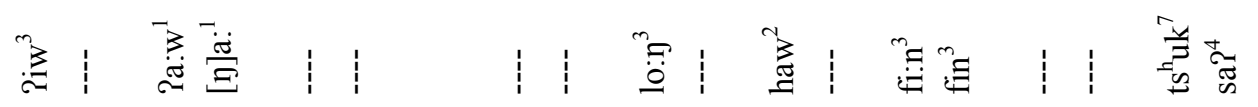

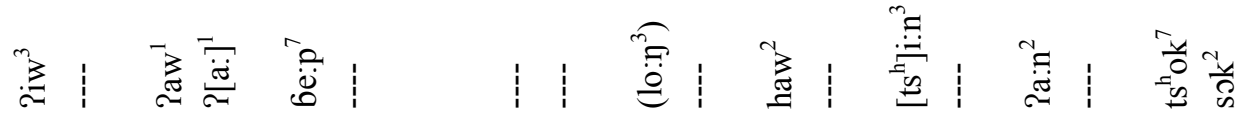

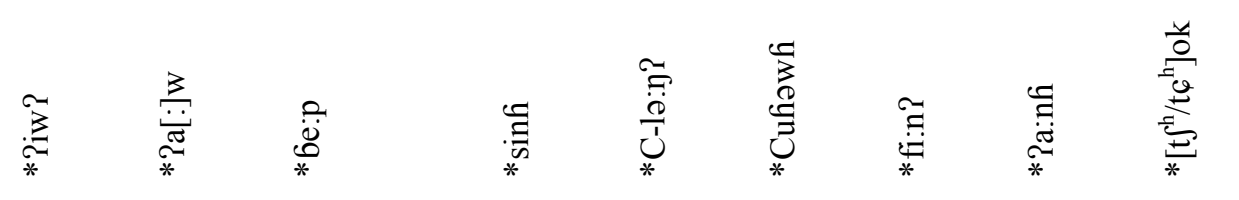

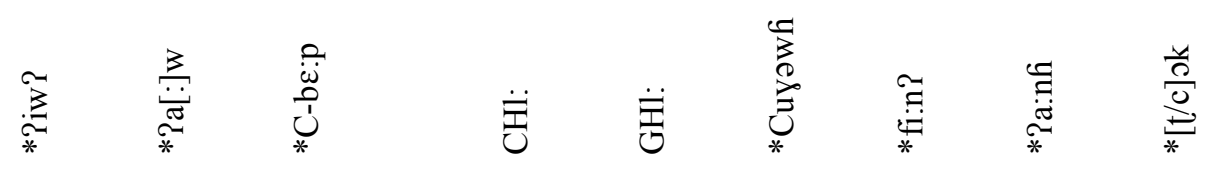

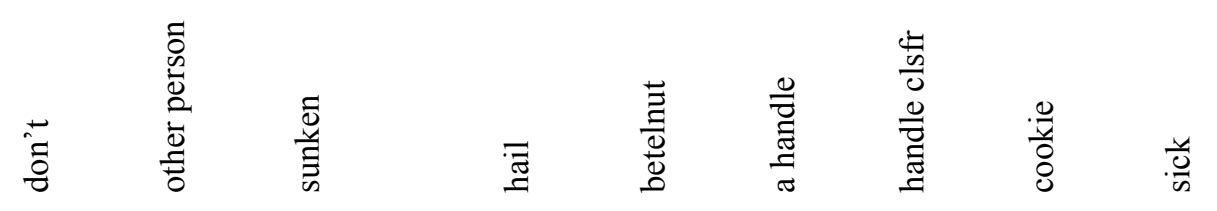

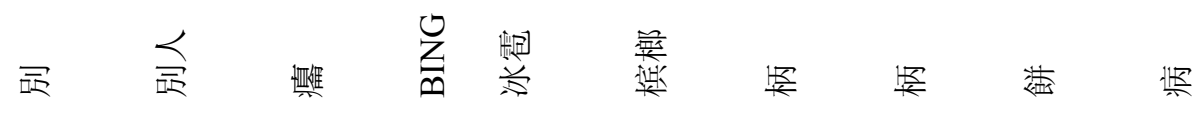




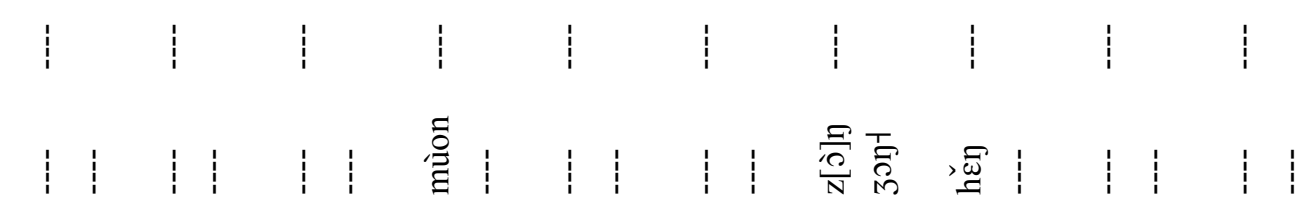

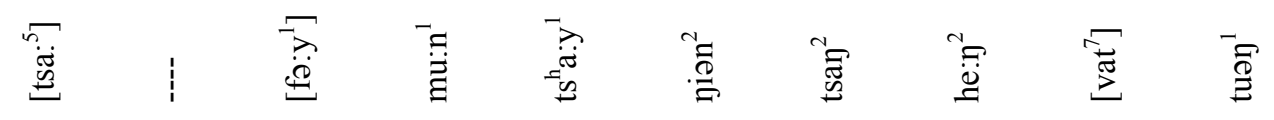

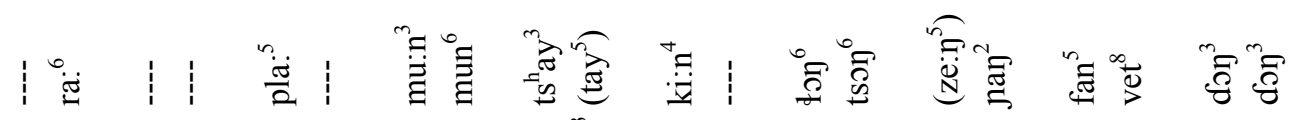

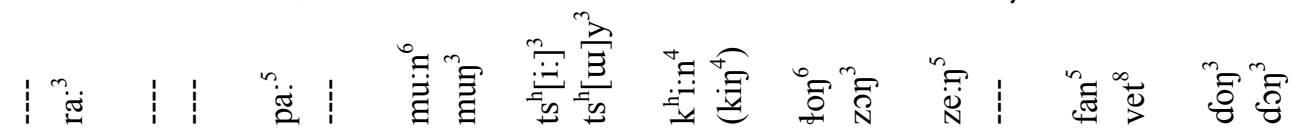

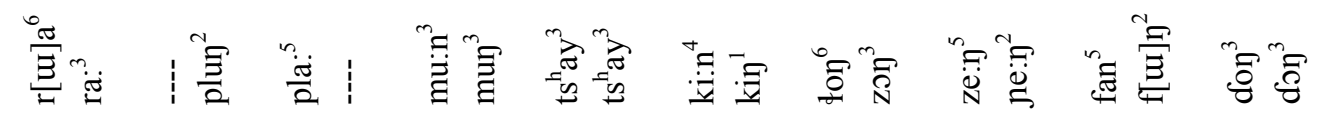

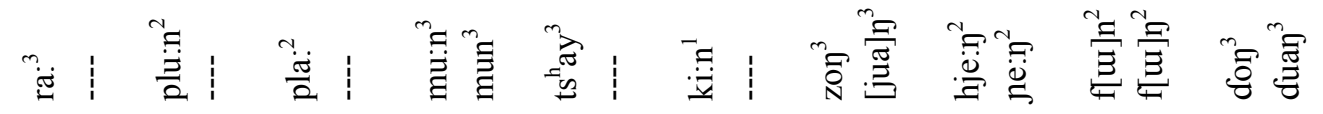

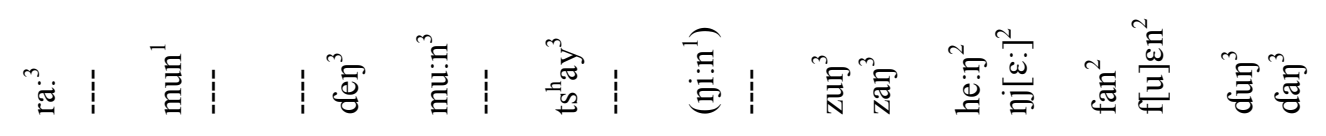

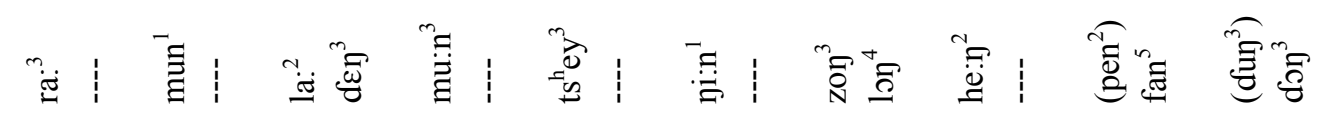

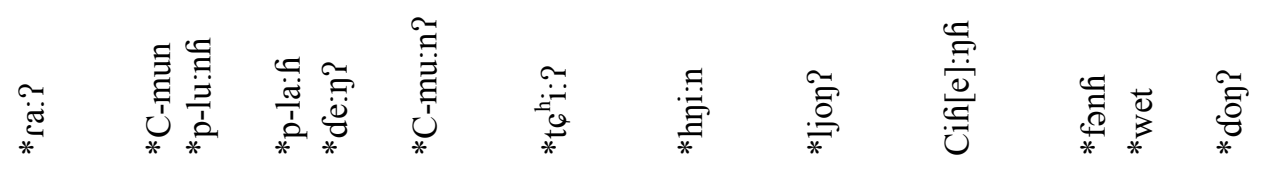

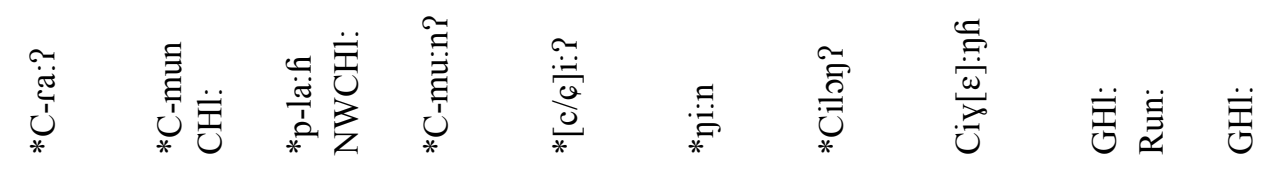

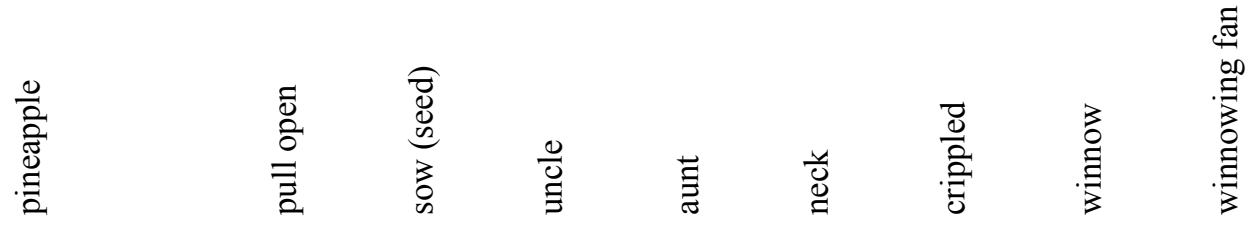

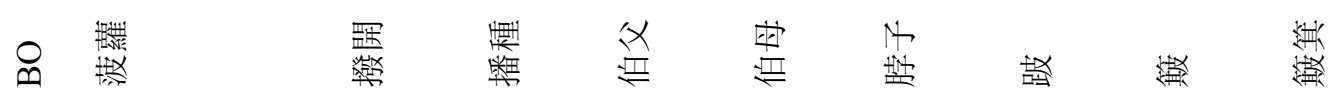


$1 \quad 1 \quad 101010$

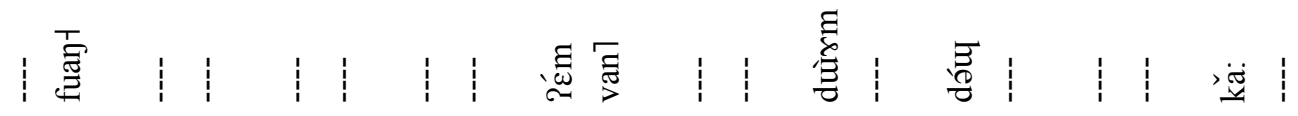

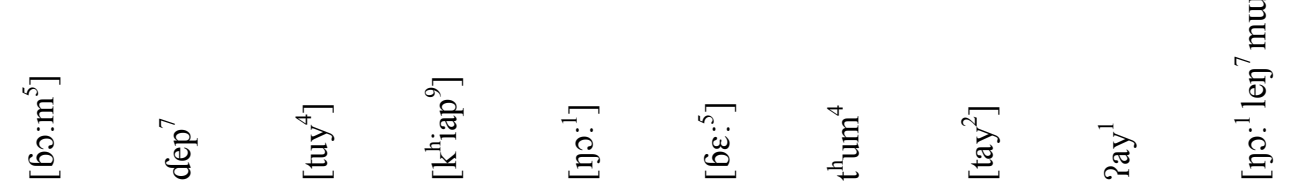

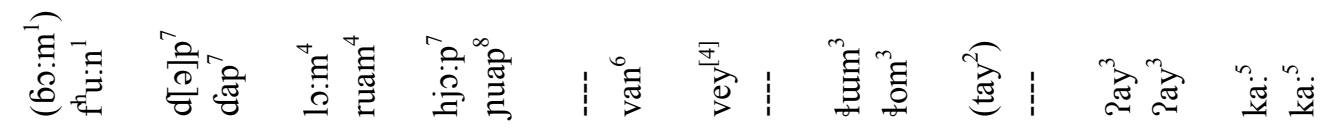

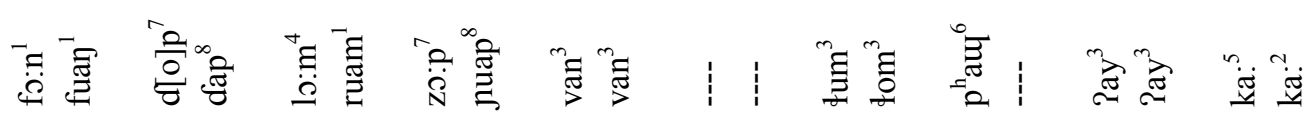

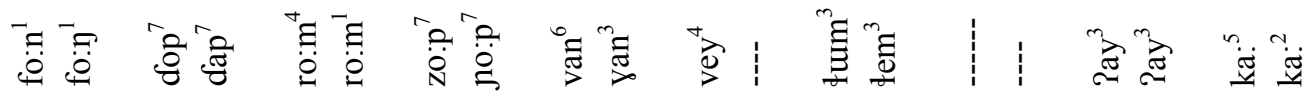

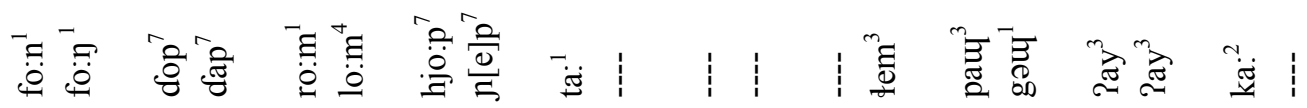

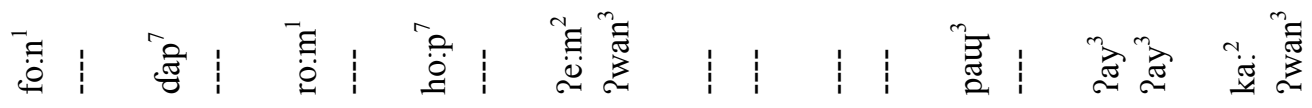

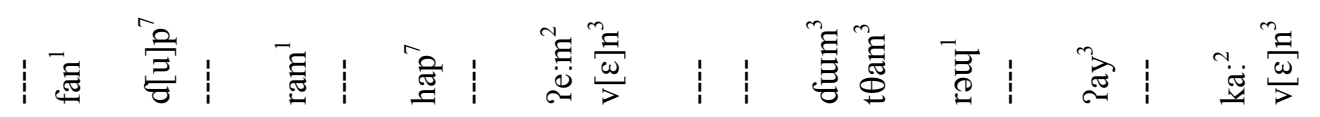

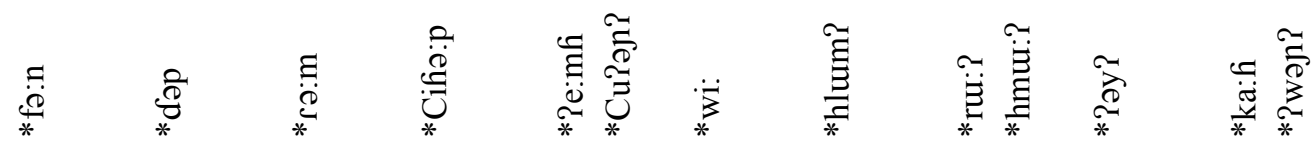

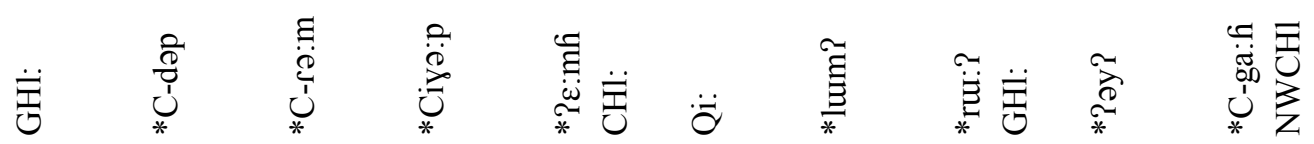

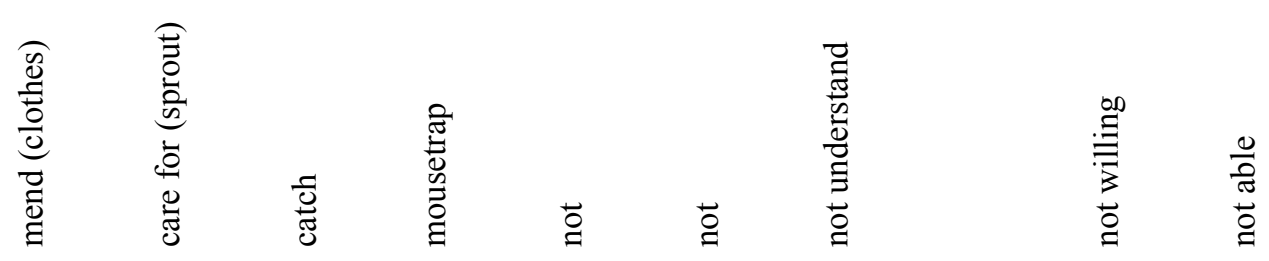

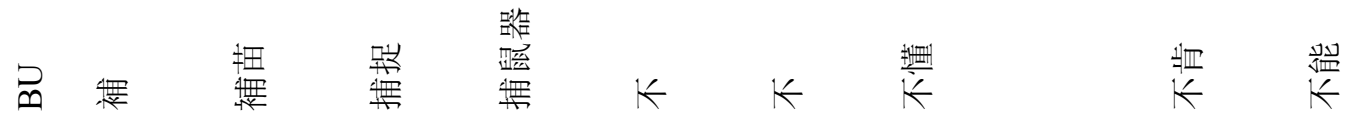




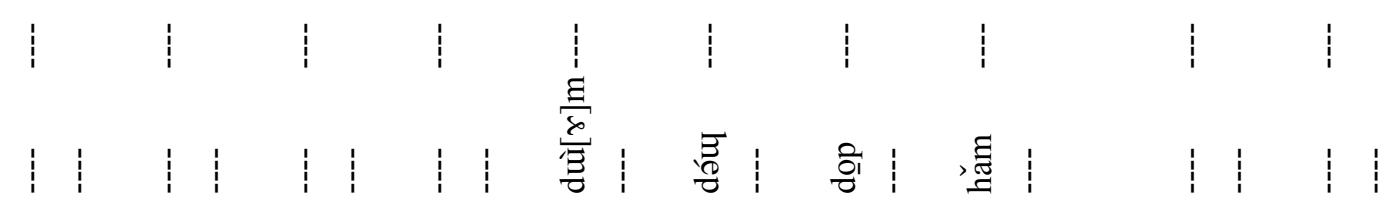

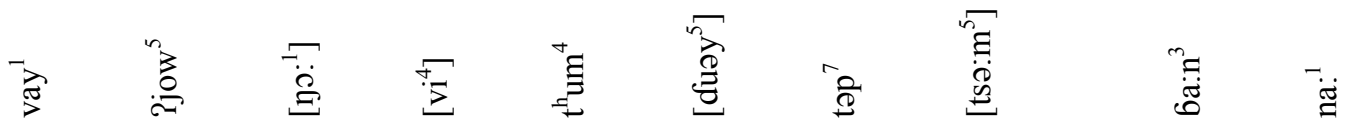

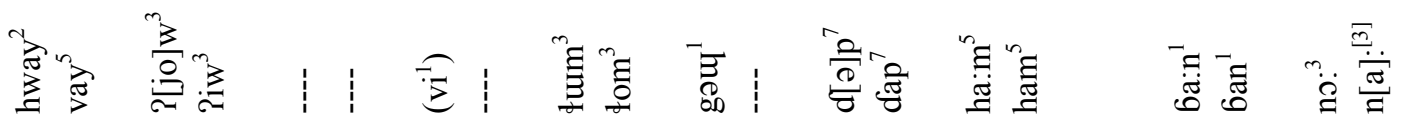

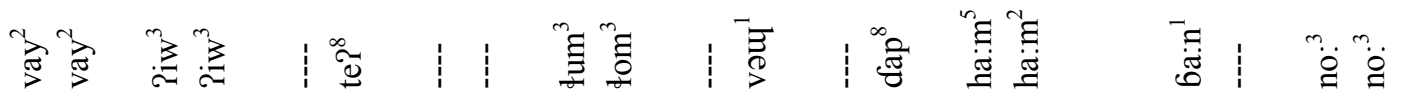

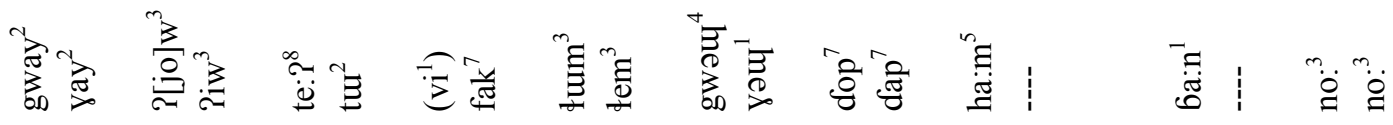

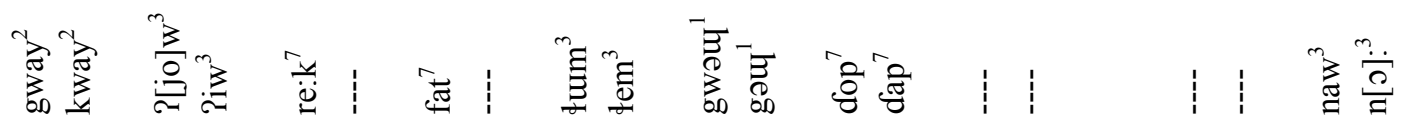

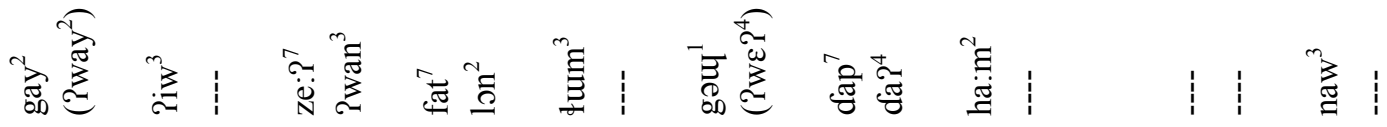

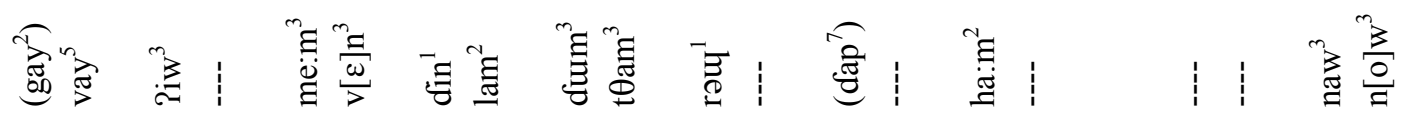

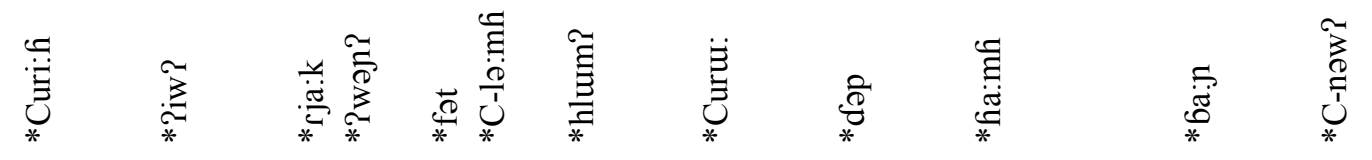

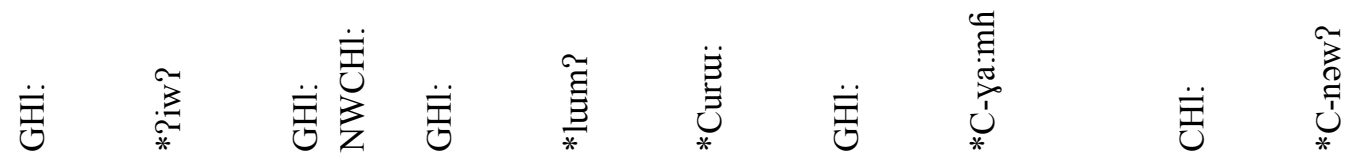

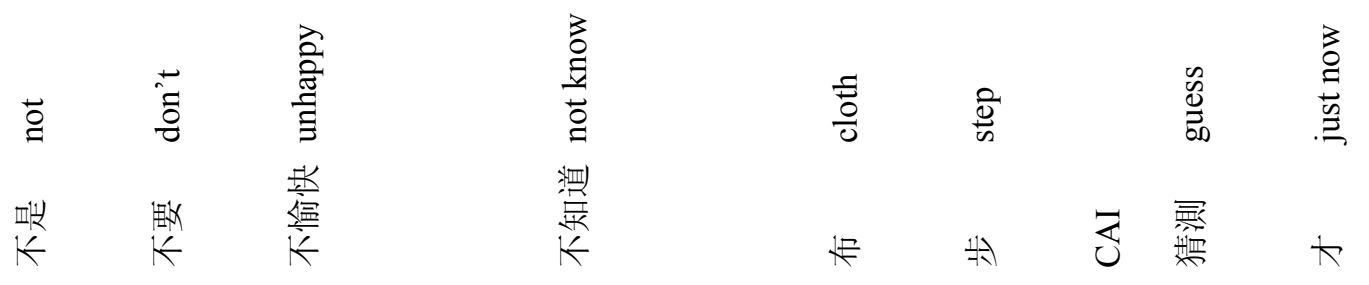



1111
$1 \quad 1$
需

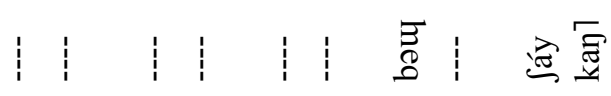

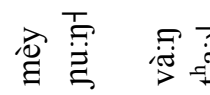
通|
\begin{tabular}{l|l|l} 
& $\bar{c}$ \\
\hline
\end{tabular}

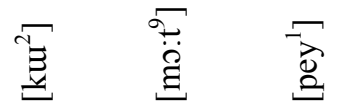
童

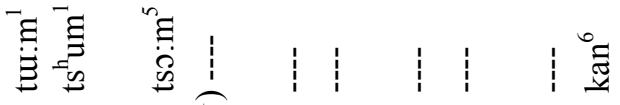

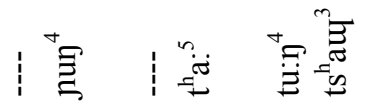

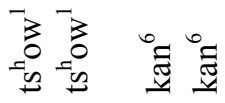

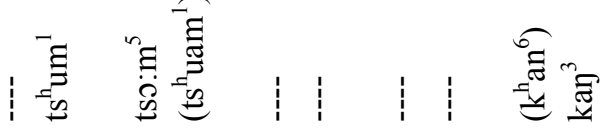

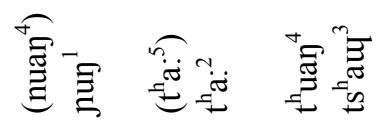

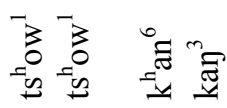
言言:

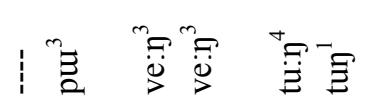

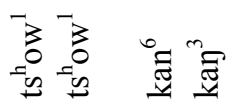

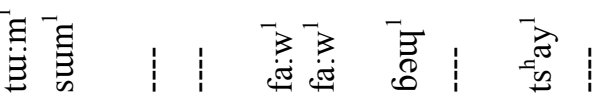

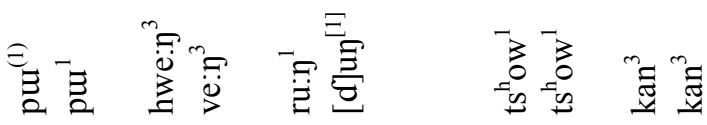

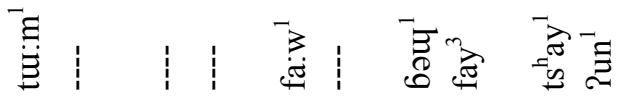

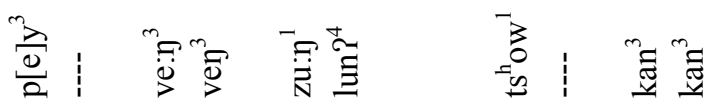

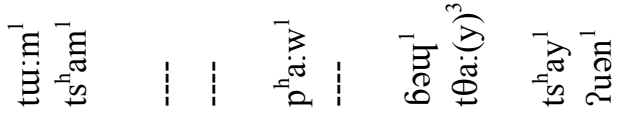

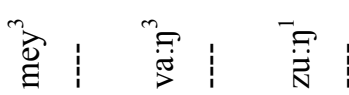
言|

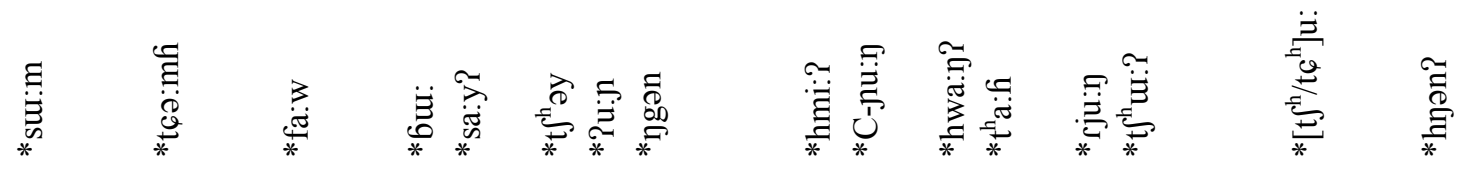

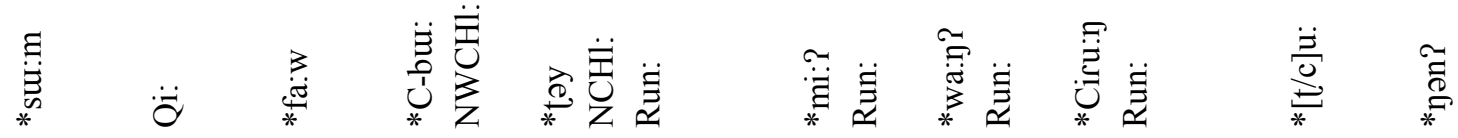

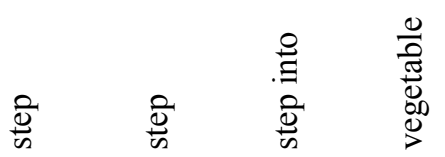
产

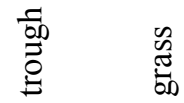
䁖䁖譄
否盍

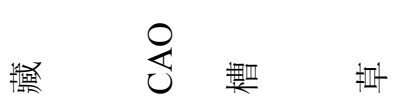




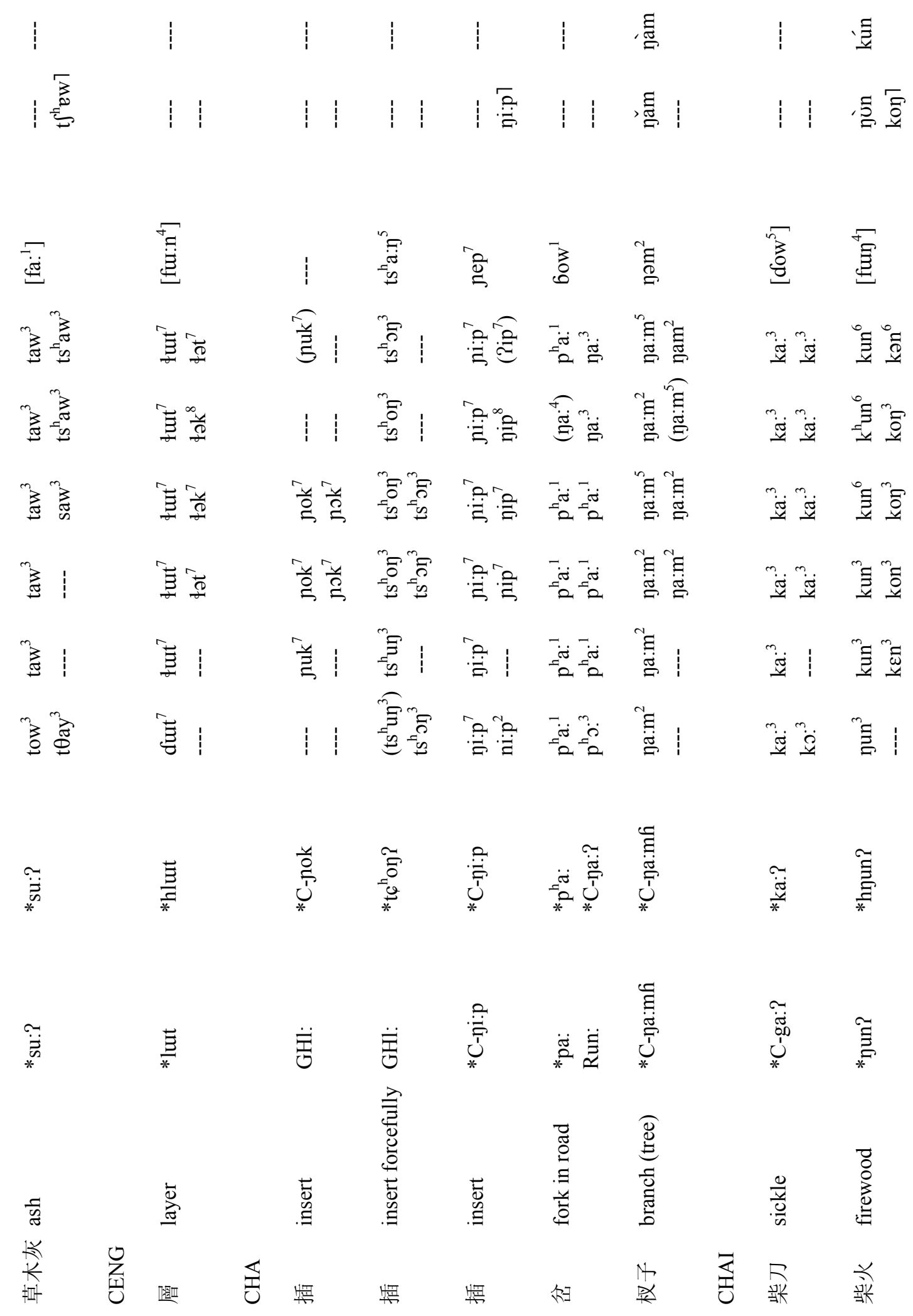




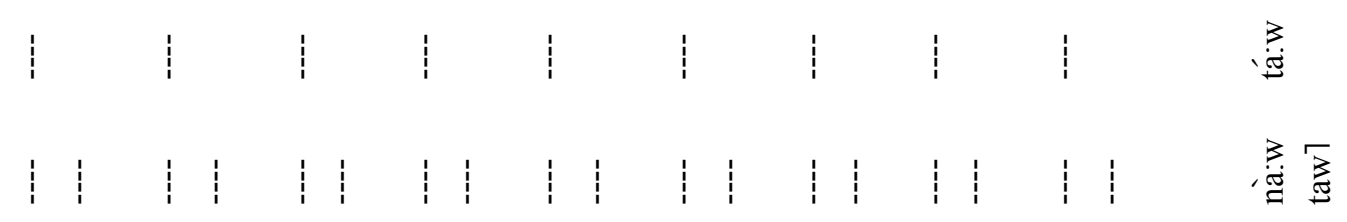

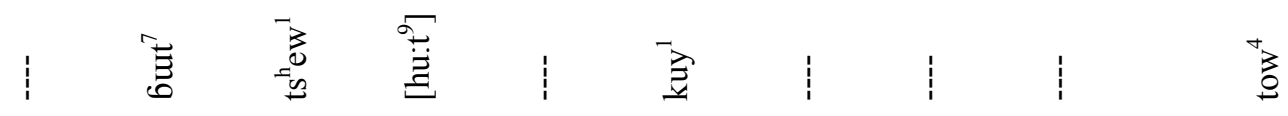

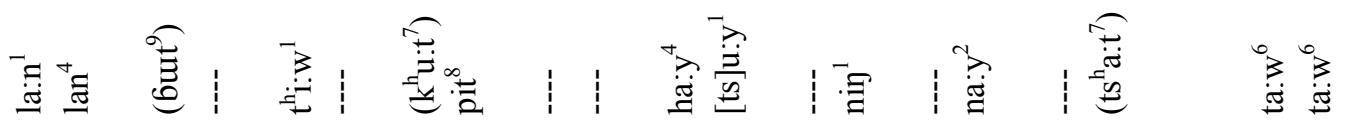

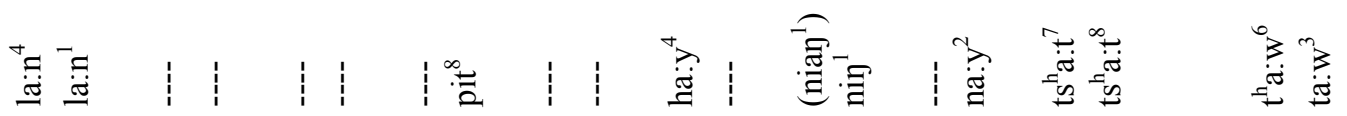

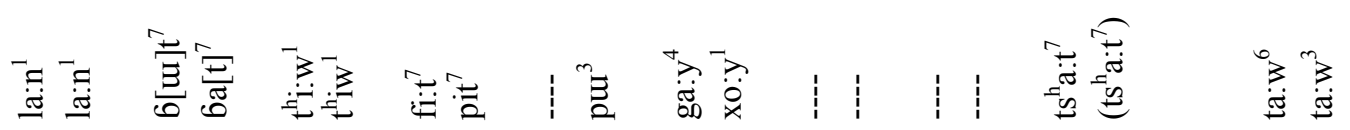

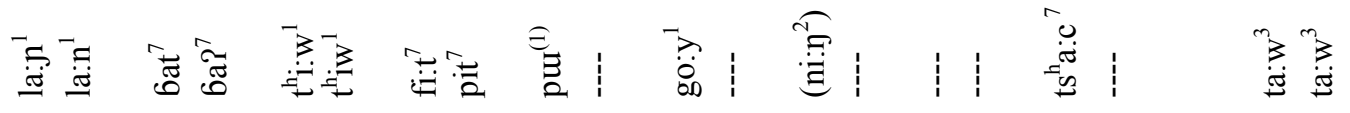

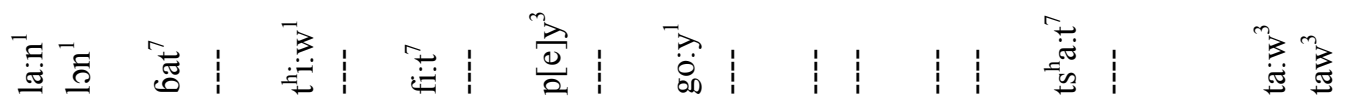

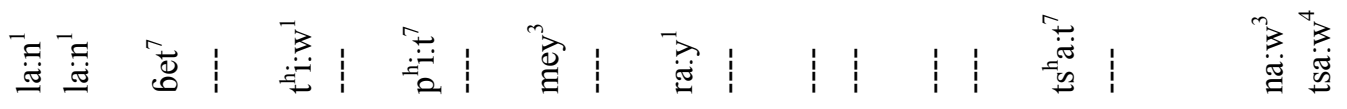

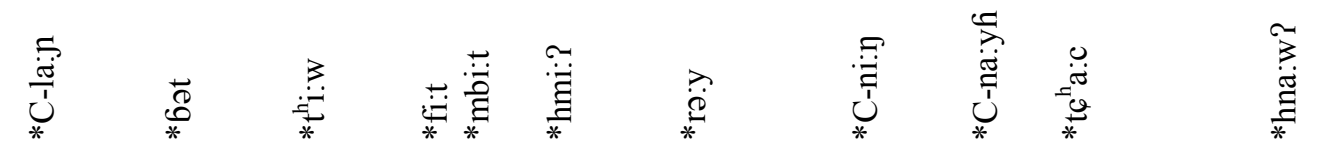

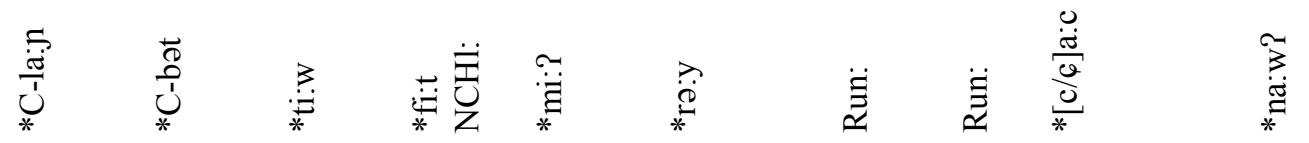

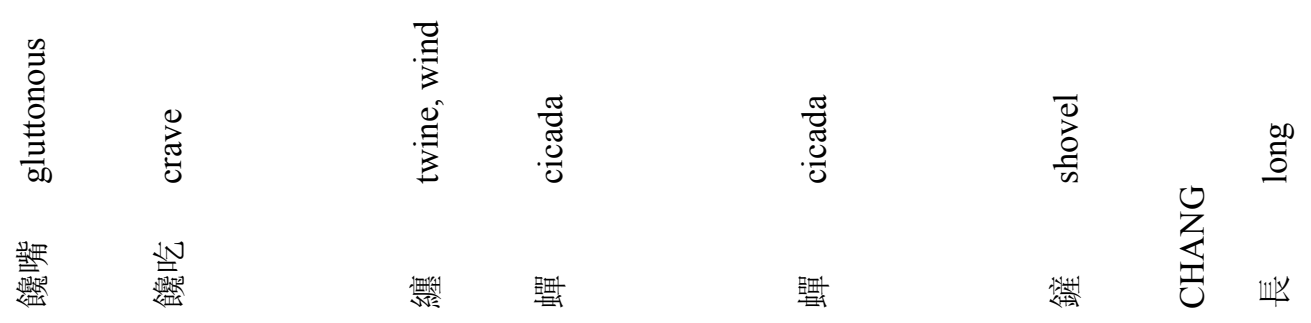




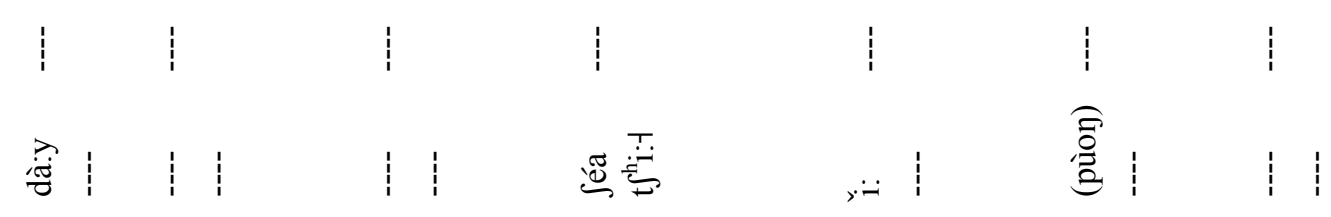

$\stackrel{+}{\Xi} \stackrel{+}{\Xi}$

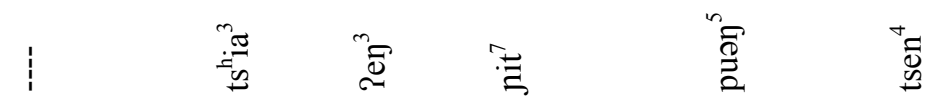

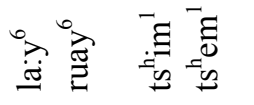

豆章

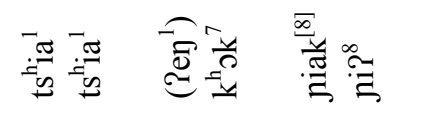

管震要

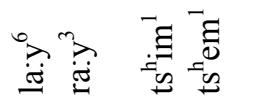

瞕

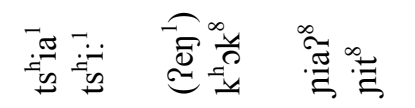

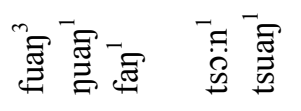

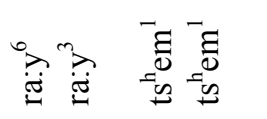

㰯

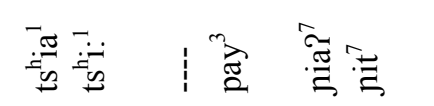

管率

整色言言

要豆

翌离

管|

管息

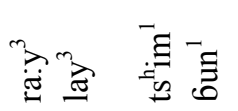

$\frac{7}{2-3}$

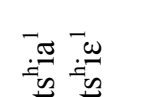

离

整重

管窒要

要

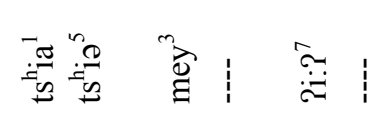

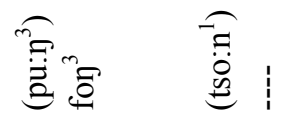

ํㅗㄹ

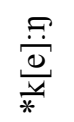

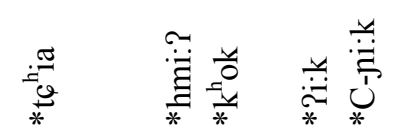

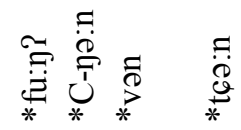

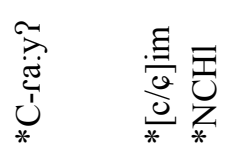

$\underset{*}{\stackrel{P}{\omega}}$

愛

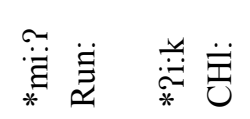

言善 善

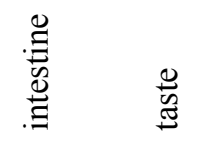

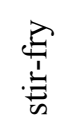

$\begin{array}{lll}\frac{0}{0} & \overline{0} & \stackrel{ \pm}{0} \\ \frac{0}{0} & \frac{0}{3} & \stackrel{0}{0}\end{array}$

$\stackrel{\vec{n}}{\frac{5}{0}}$

当

留测导

한

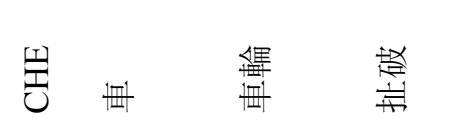

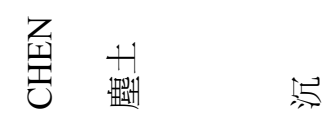




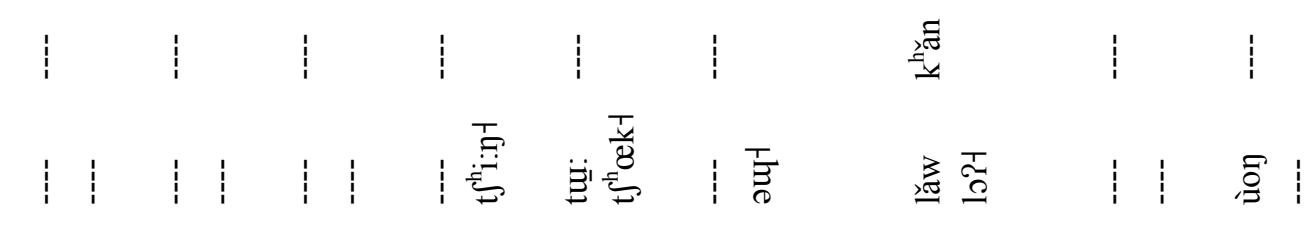

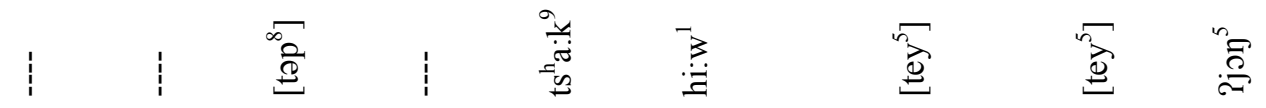

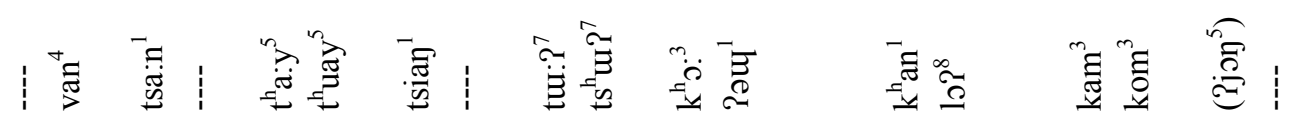

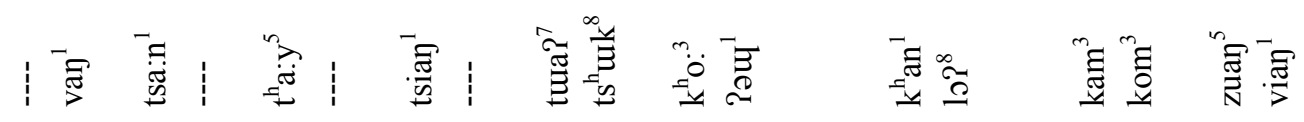

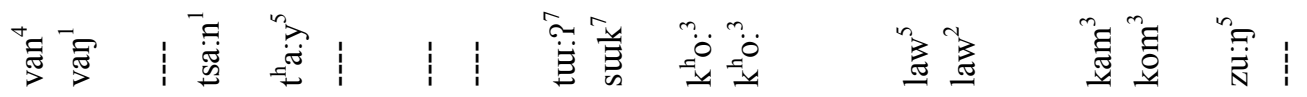

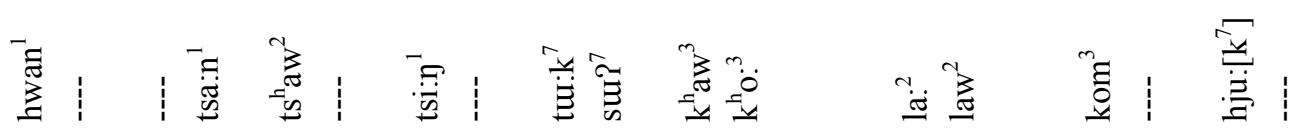

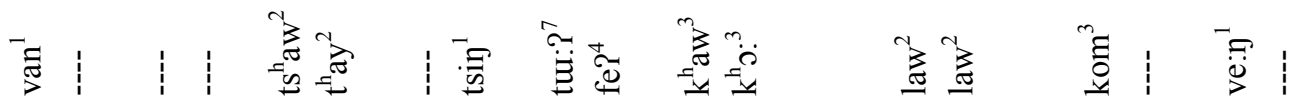

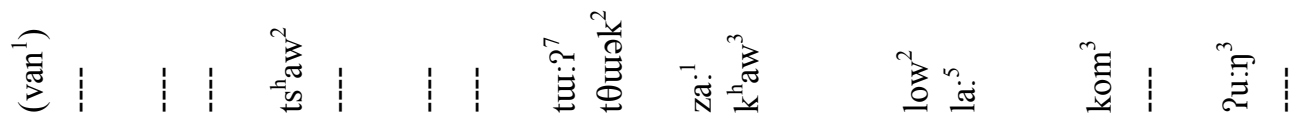

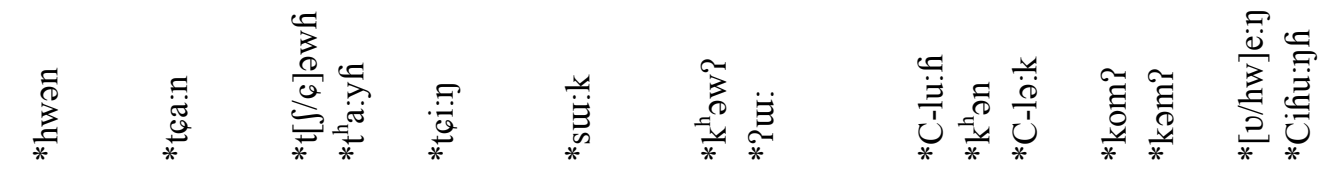

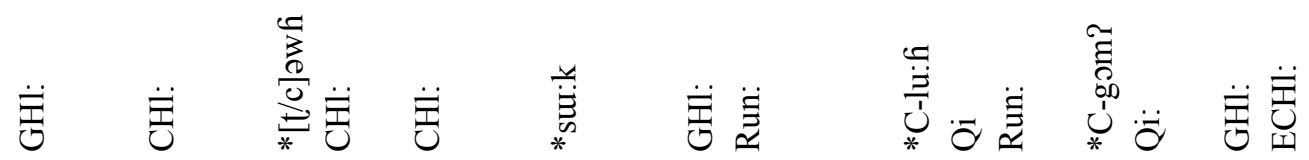

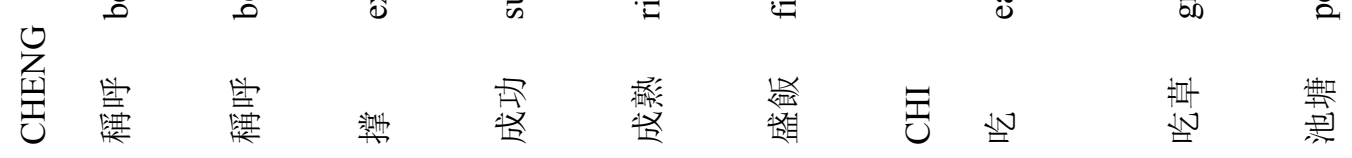




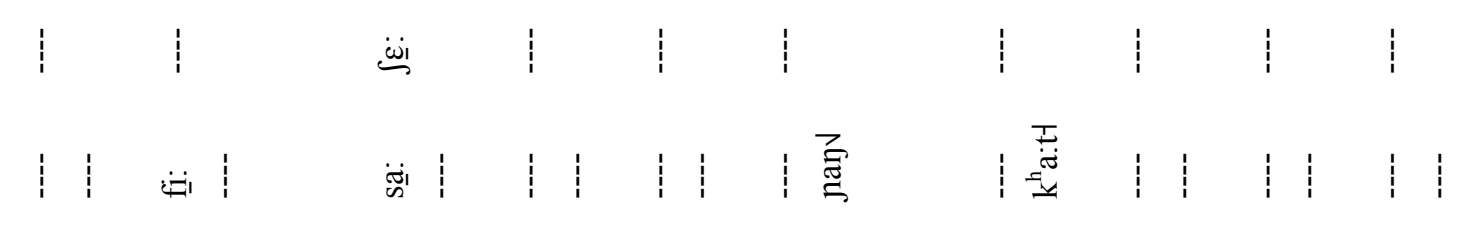

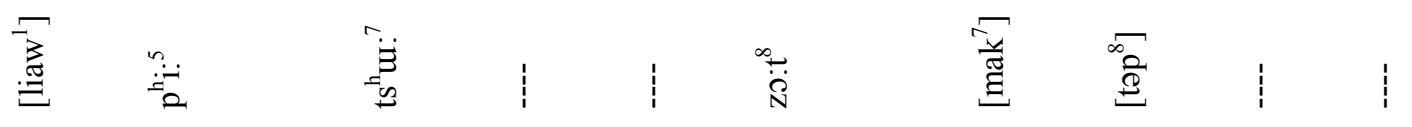

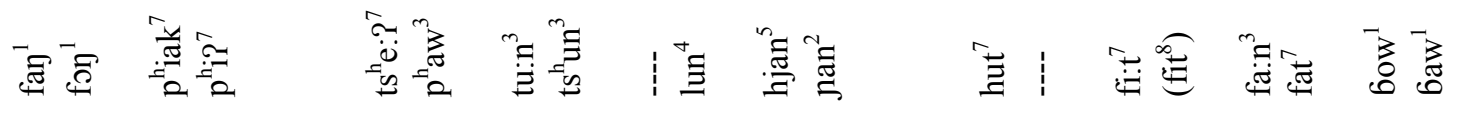

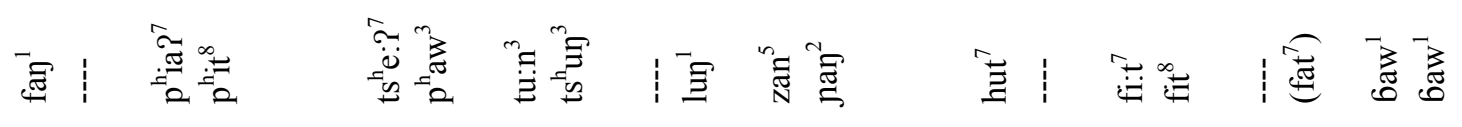

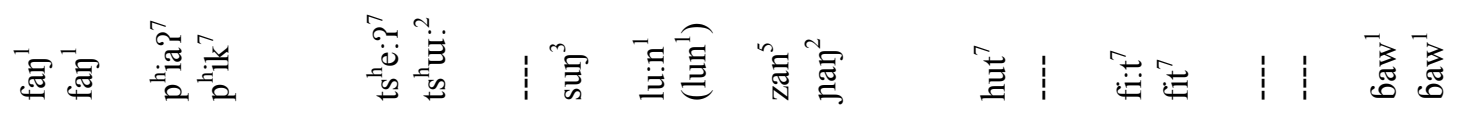

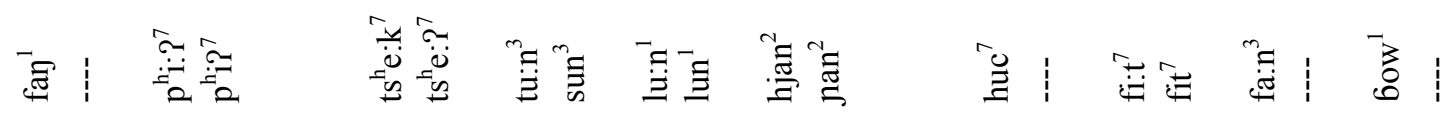

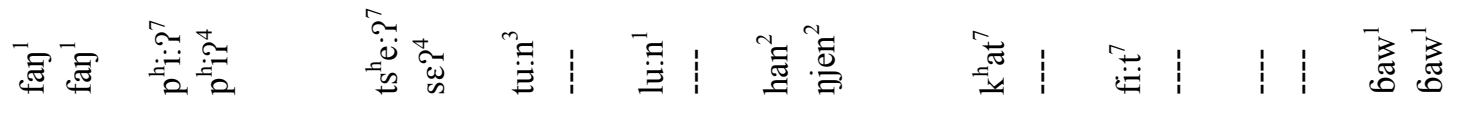

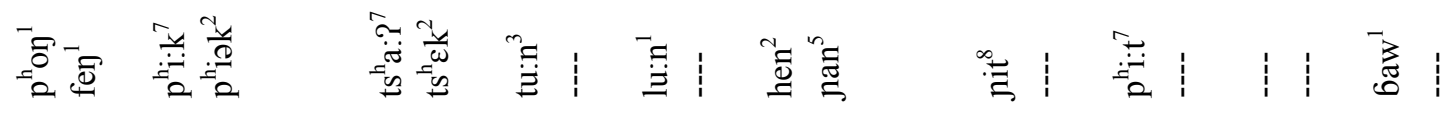

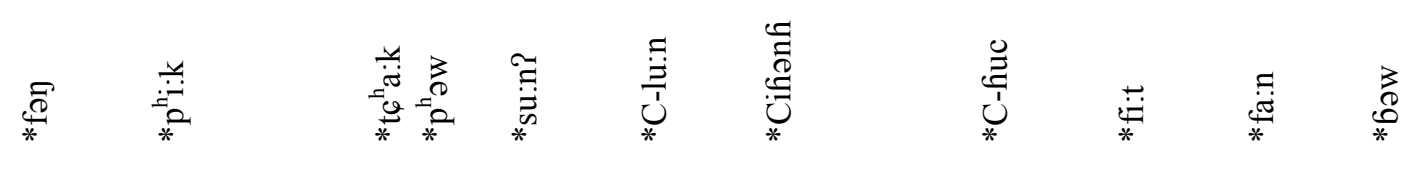

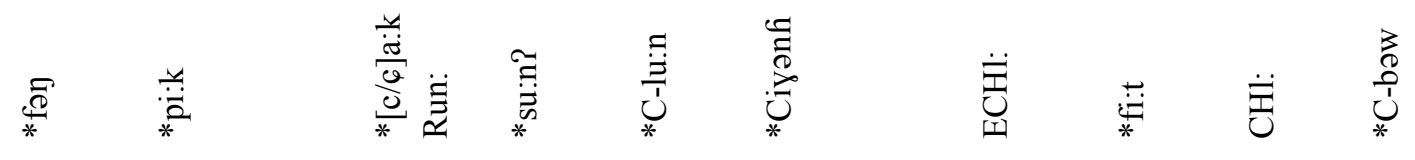

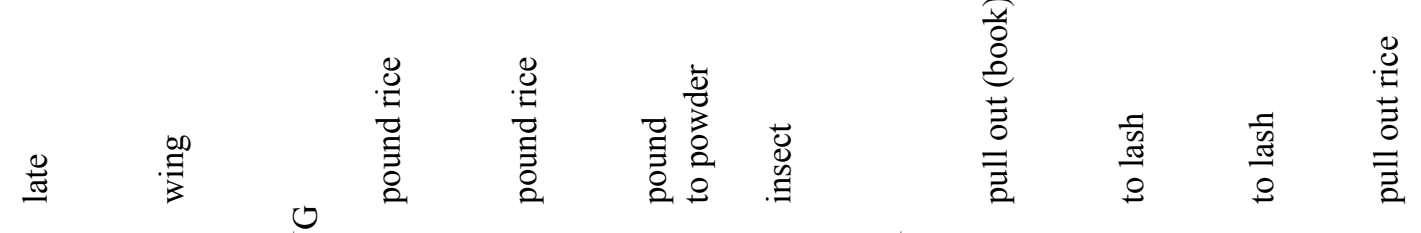

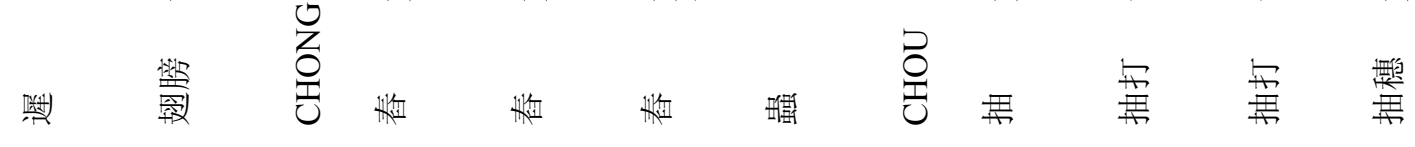




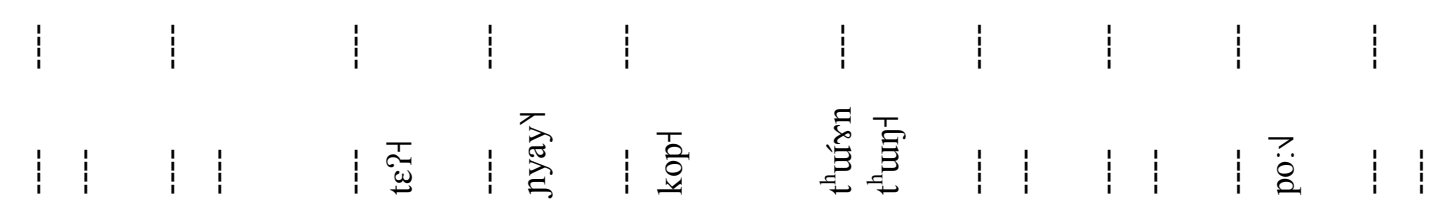

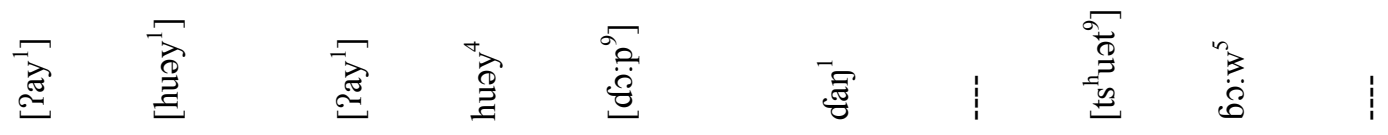

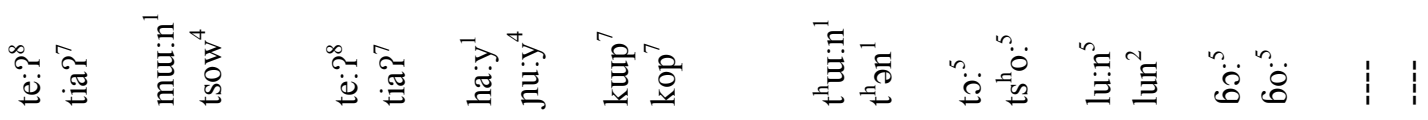

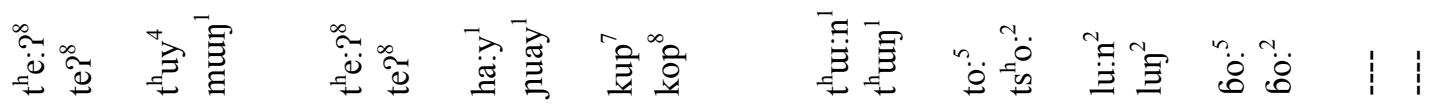

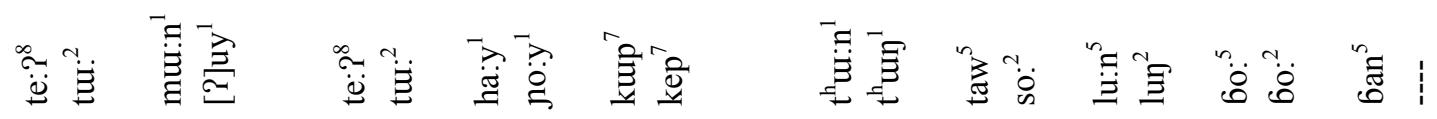

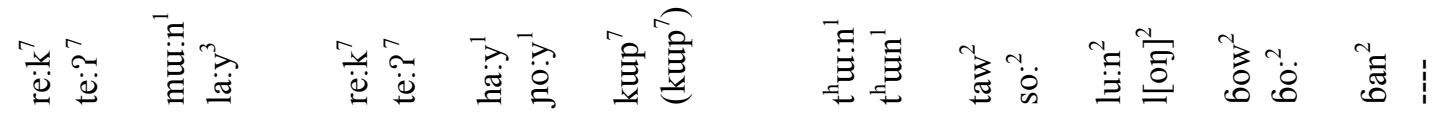

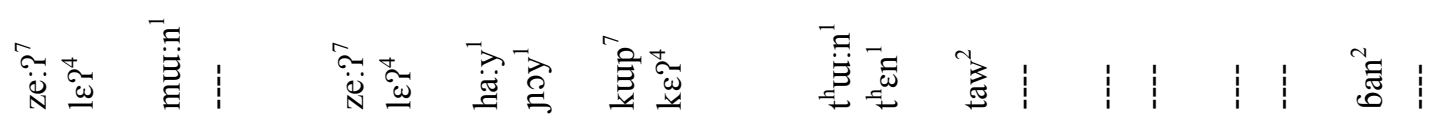

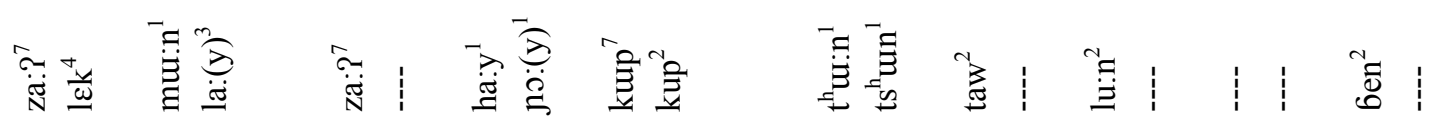

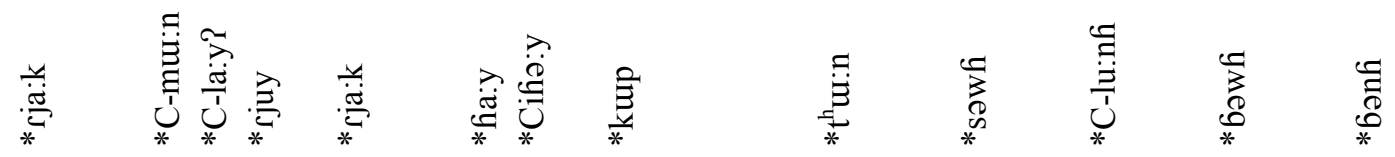

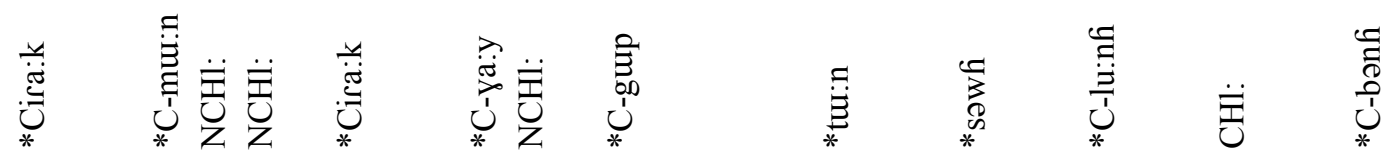

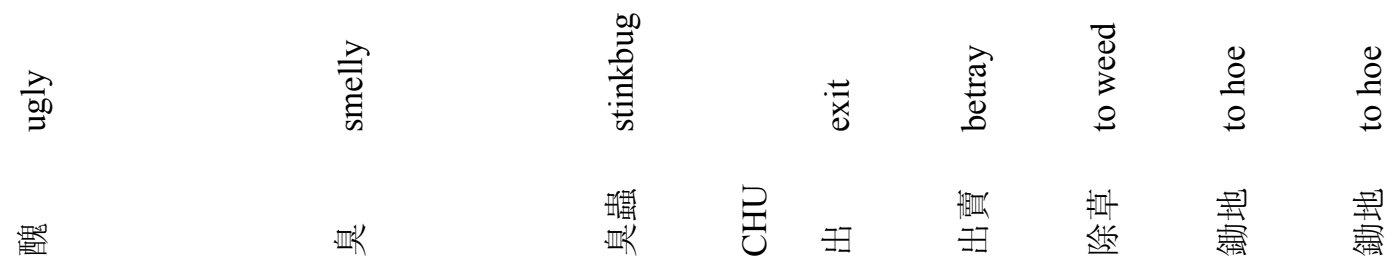




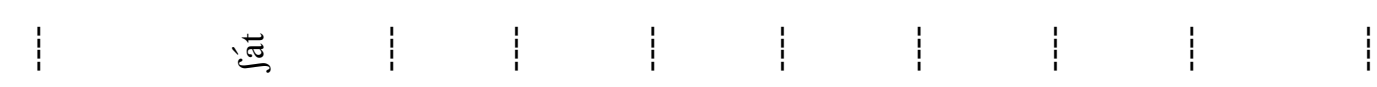

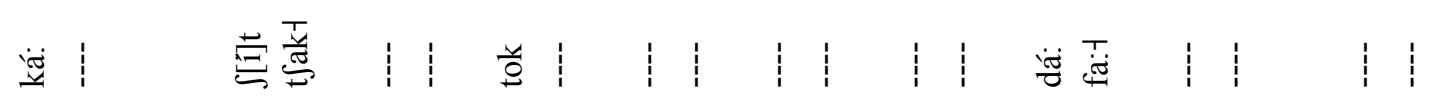

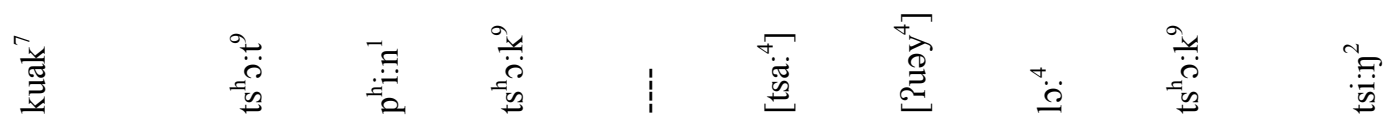

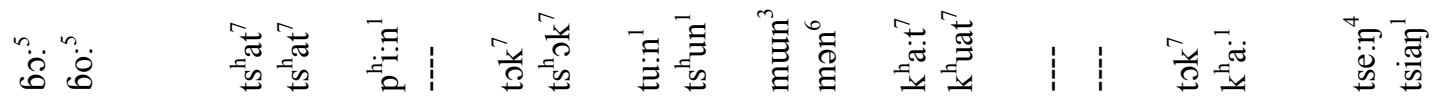

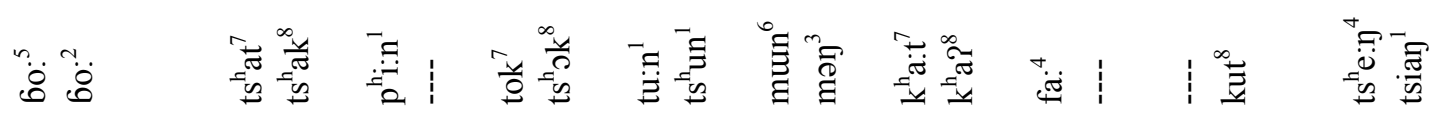

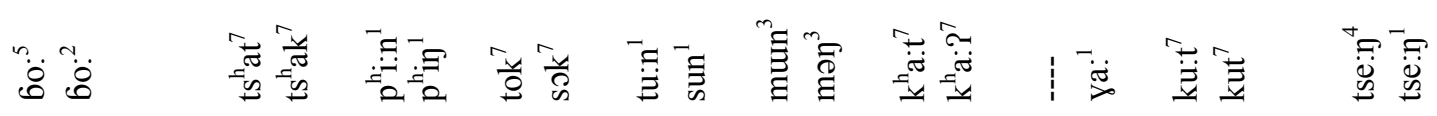

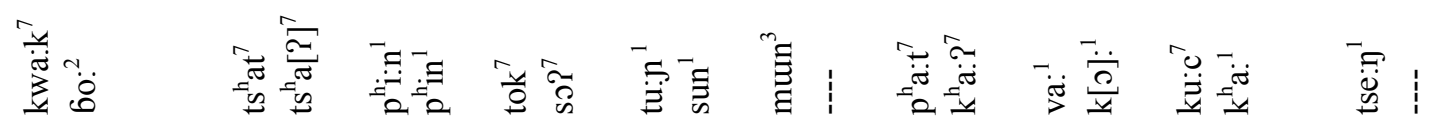

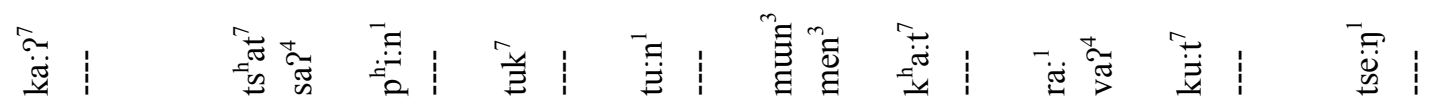

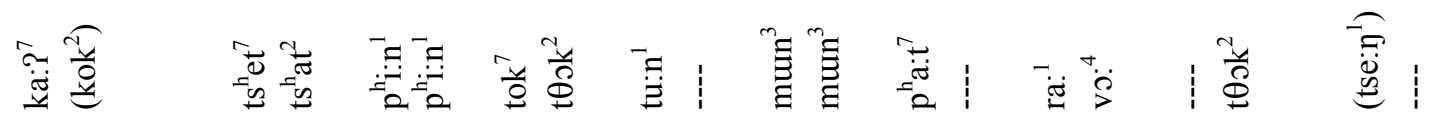

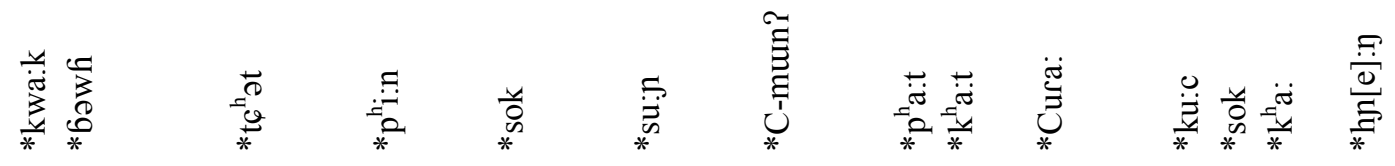

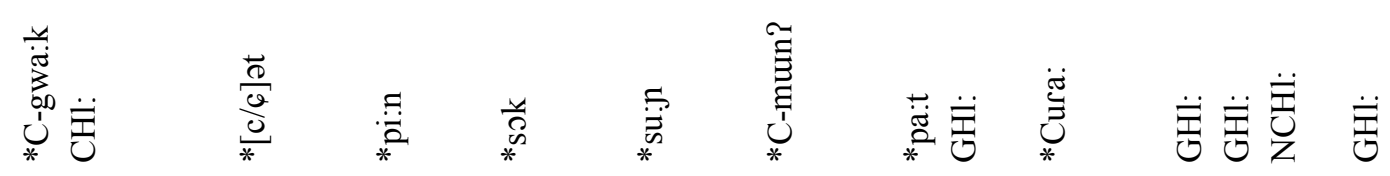

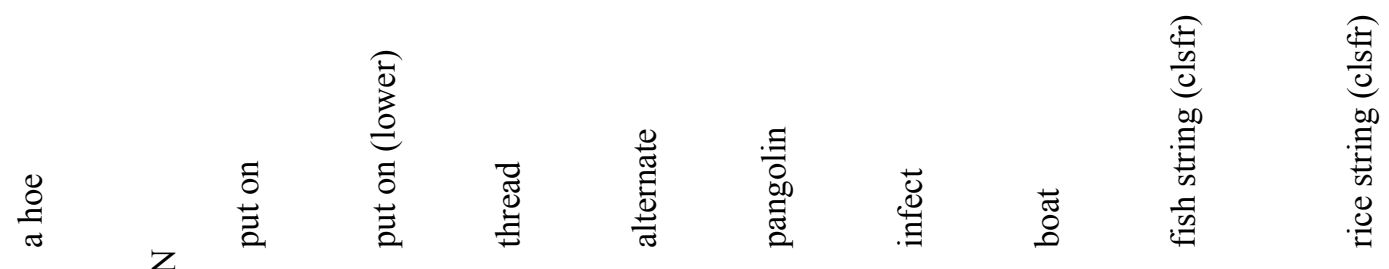

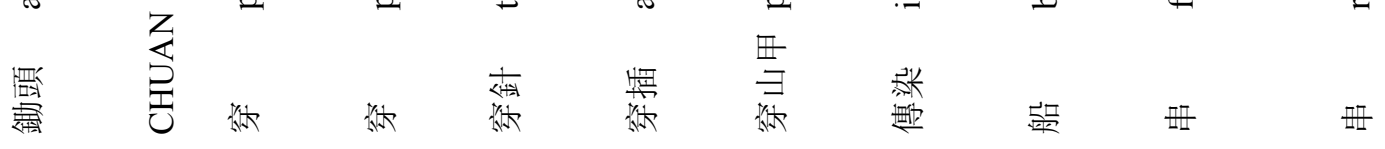




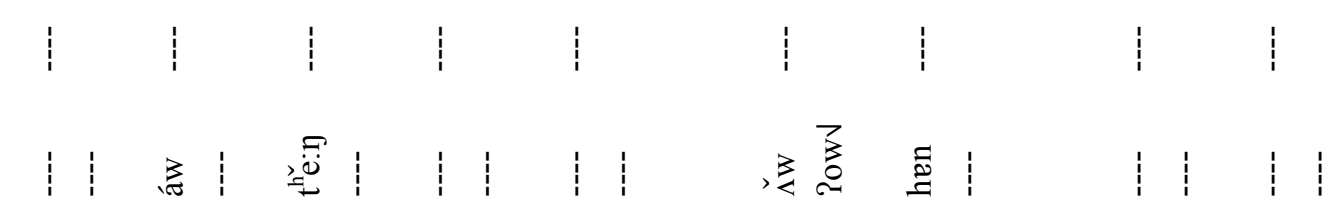

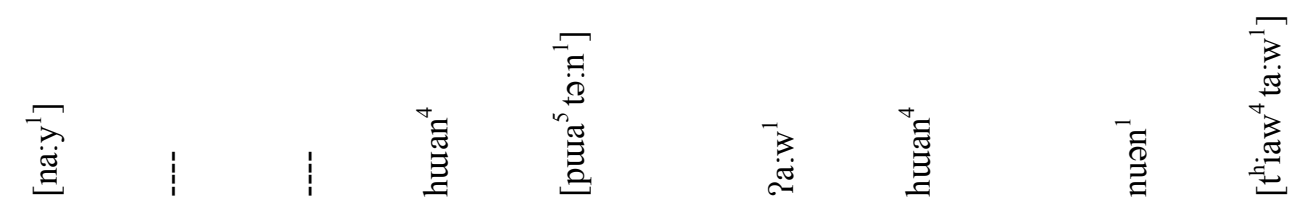

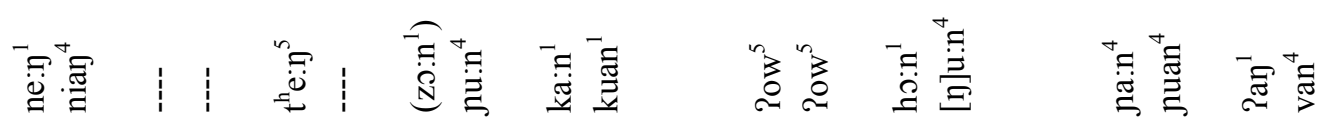

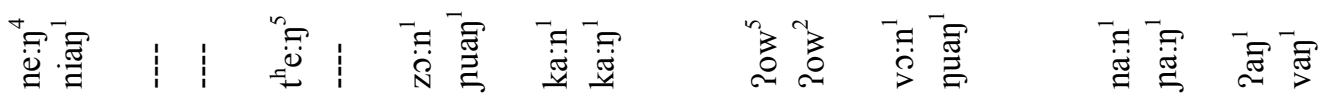

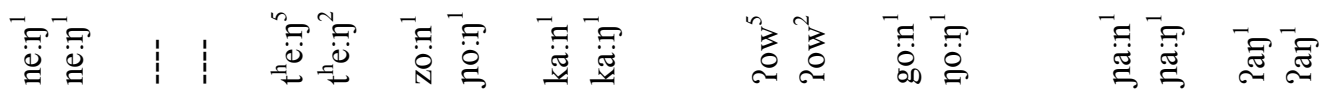

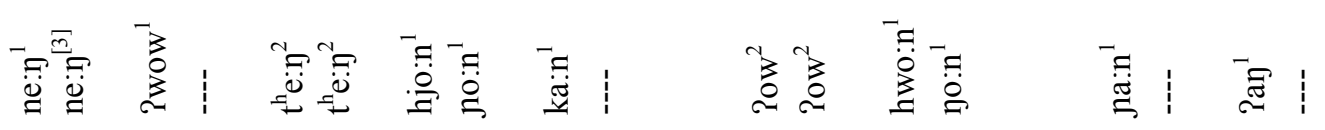

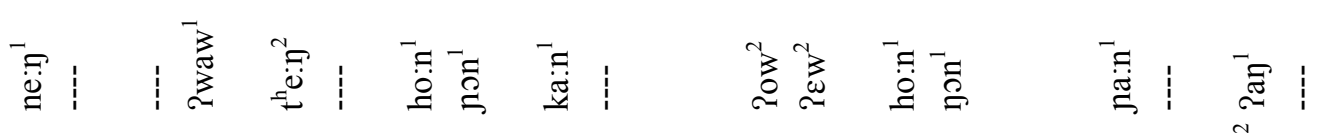

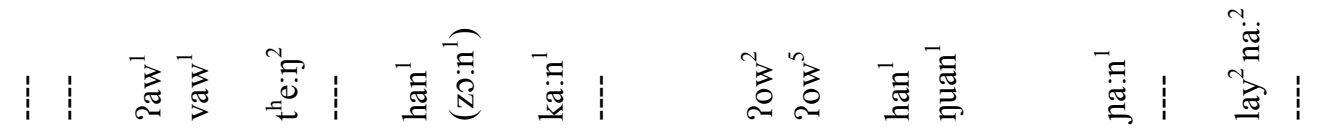

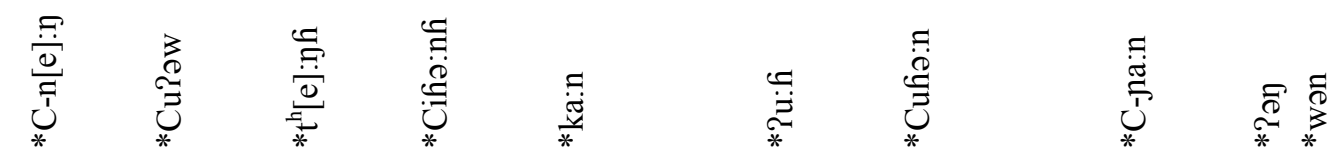

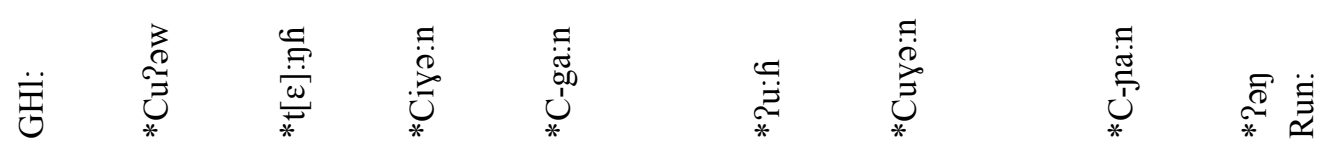

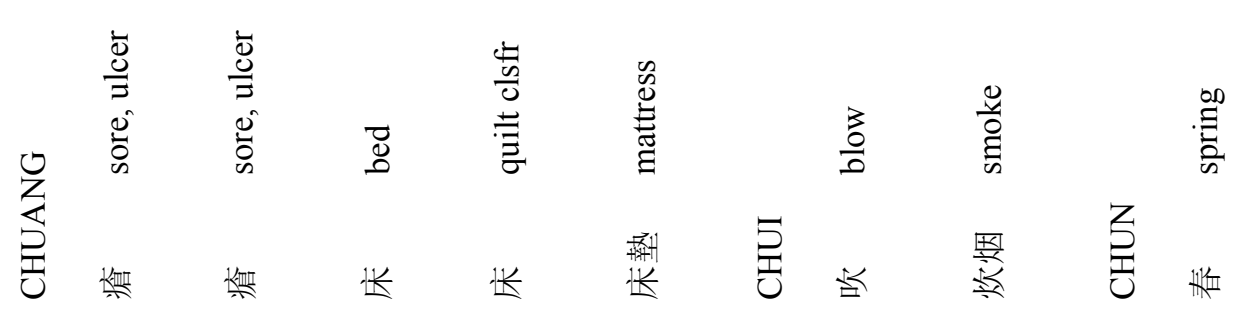




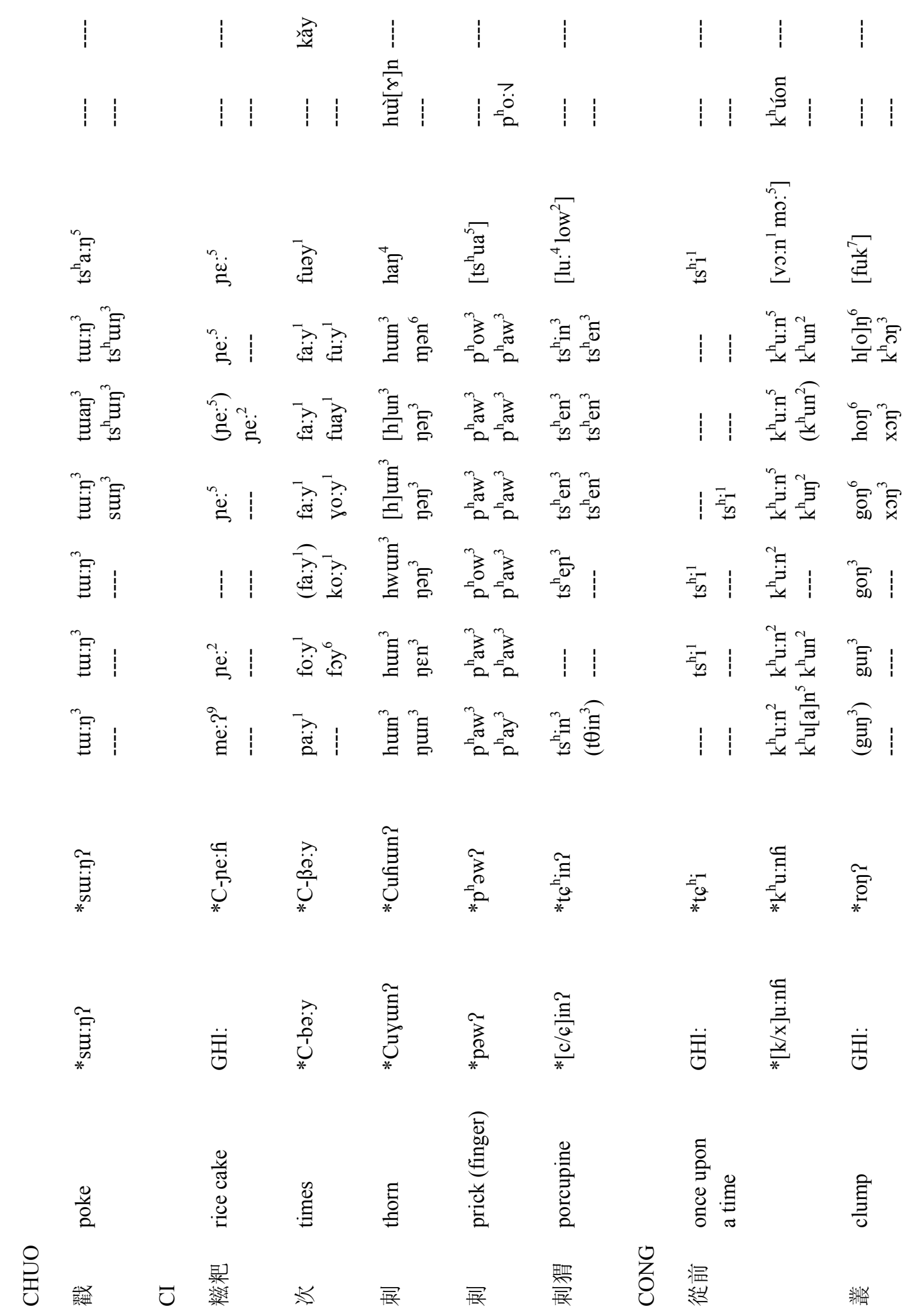




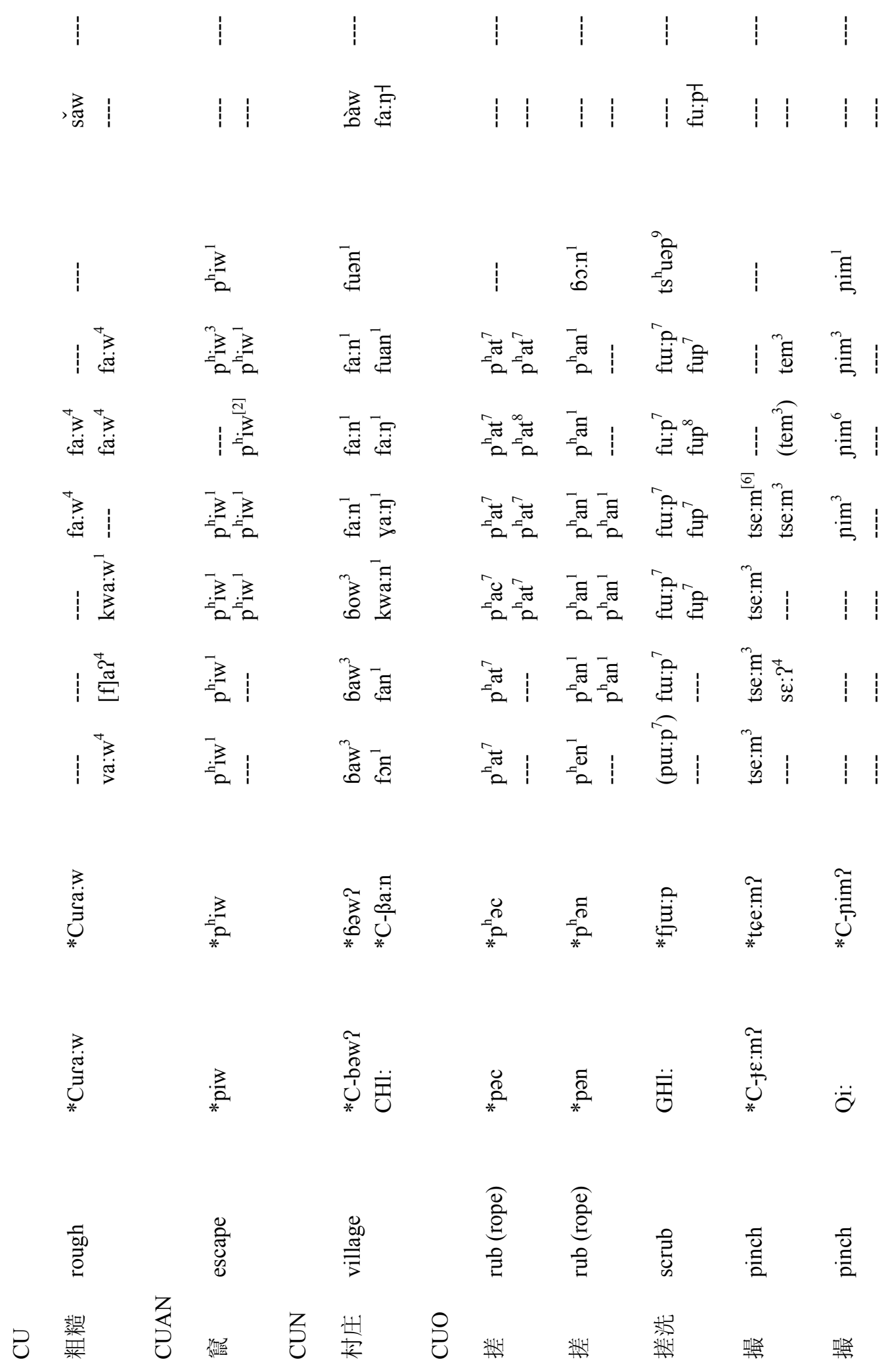




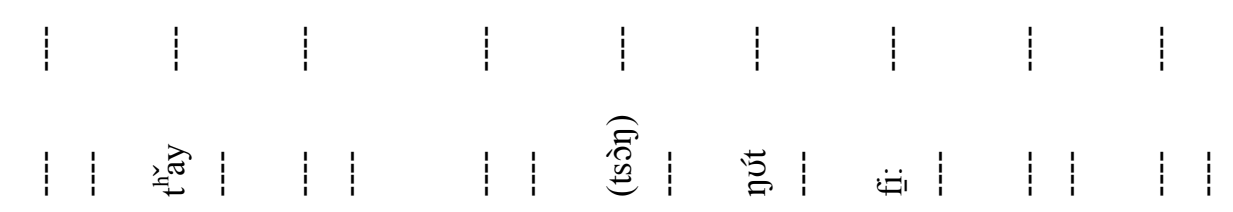

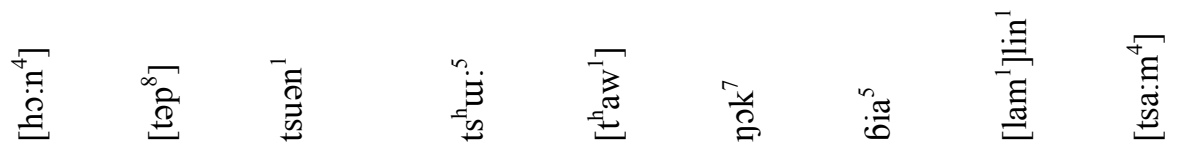

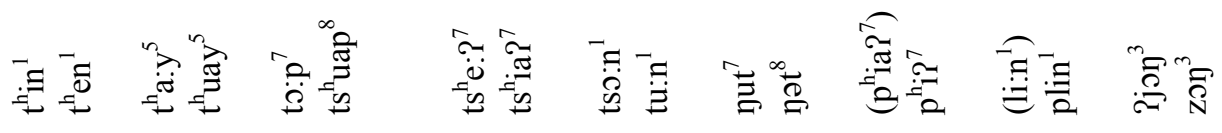

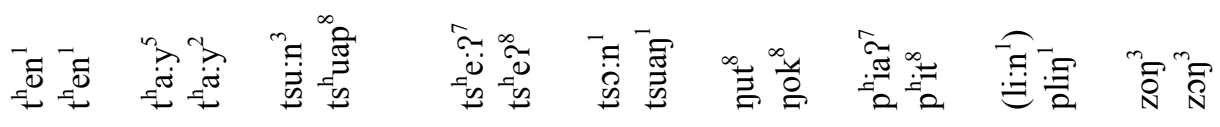

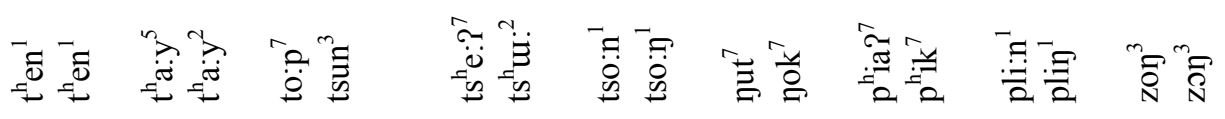

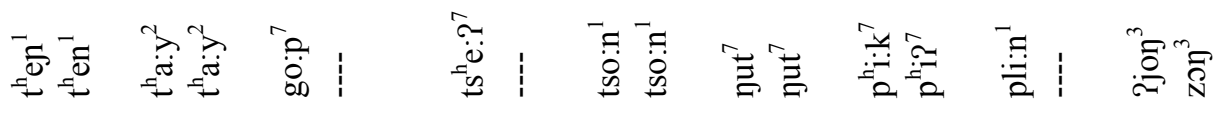

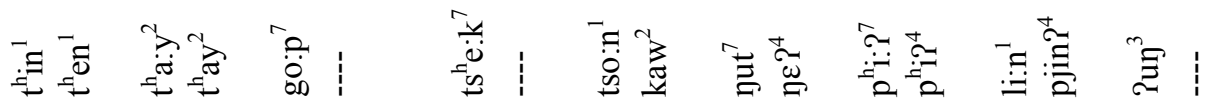

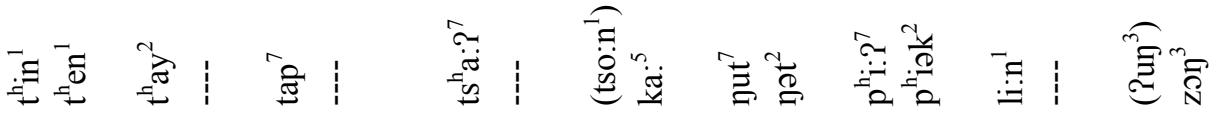

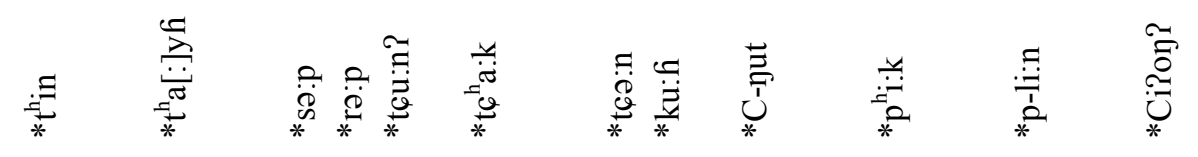

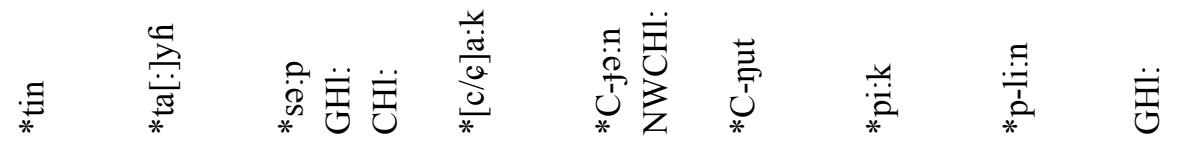
旁高旁竞

4
0
0
0
0

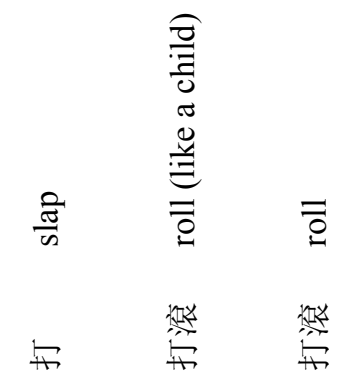

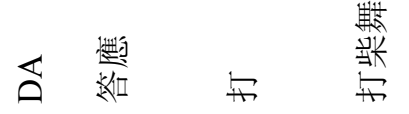
些 


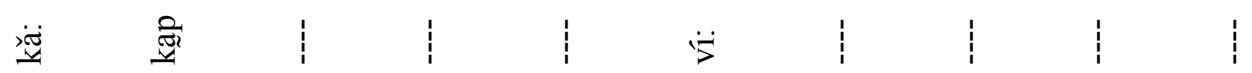

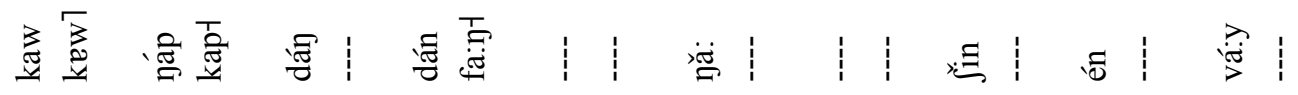

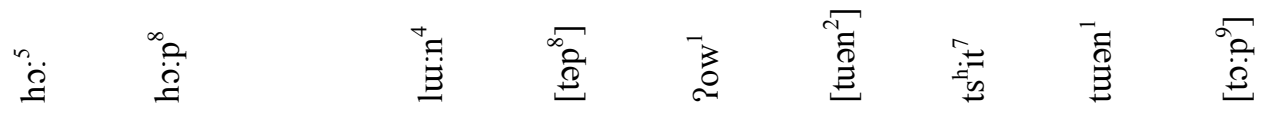

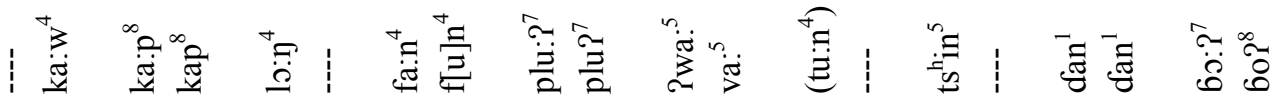

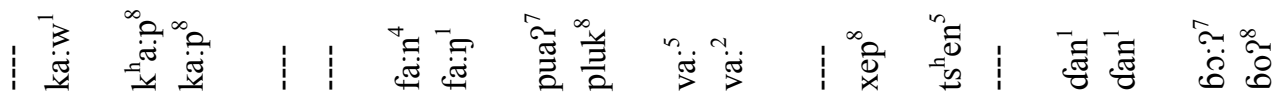

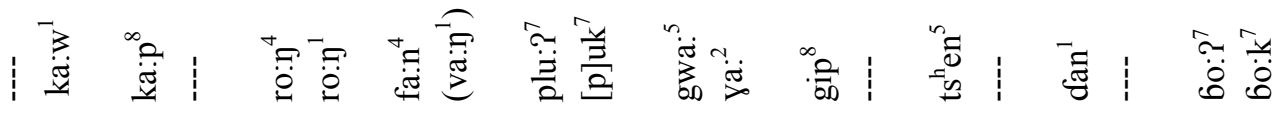

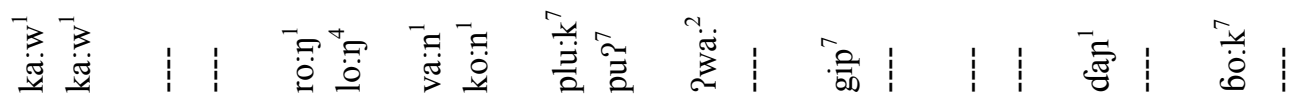

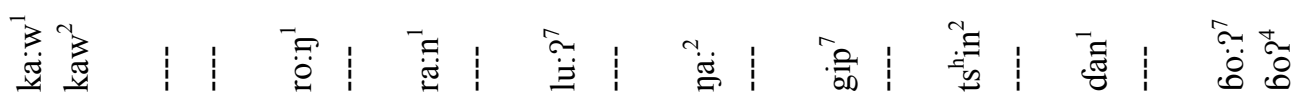

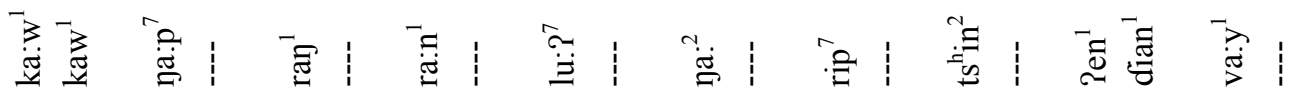

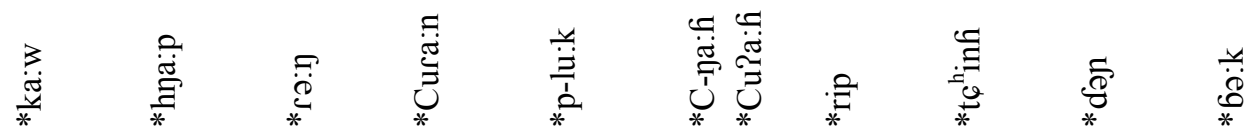

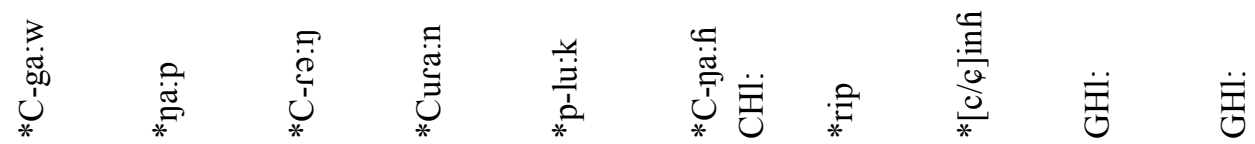

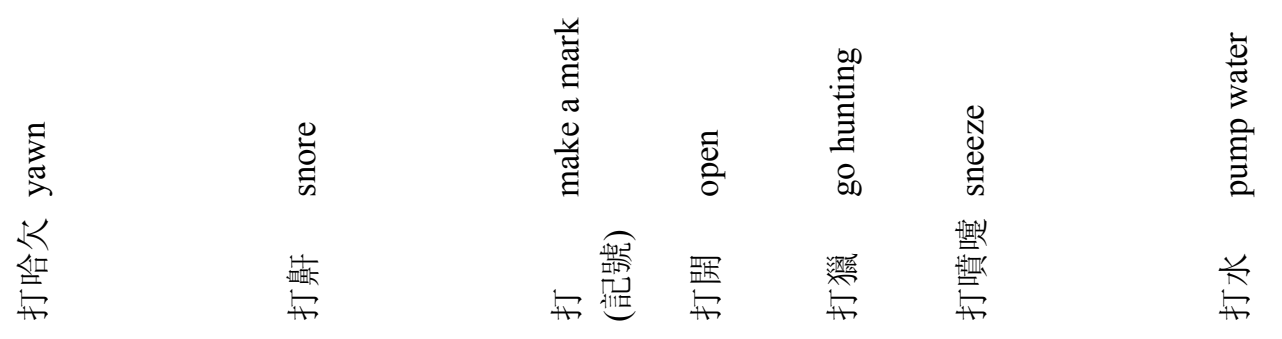




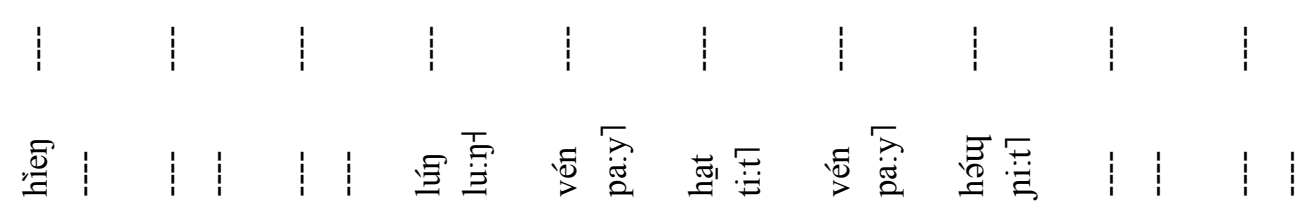

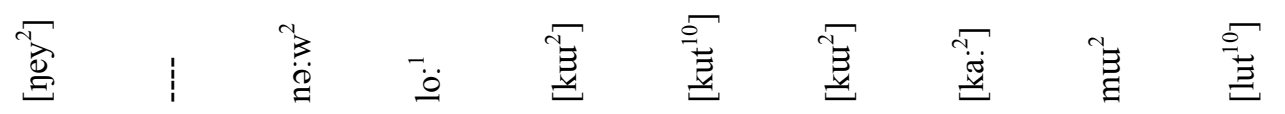

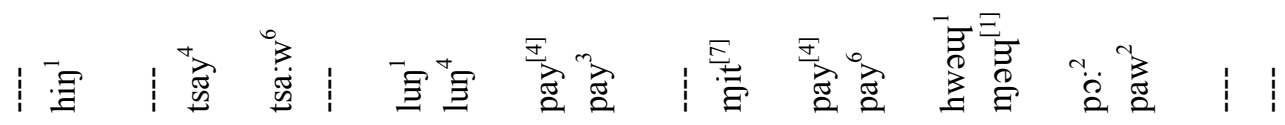

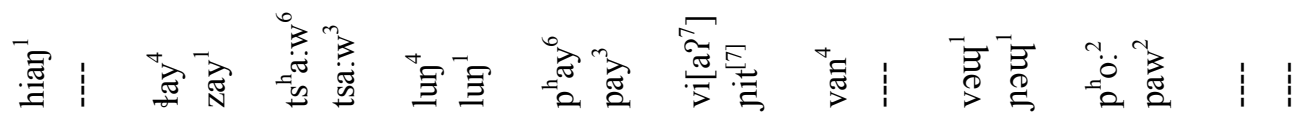

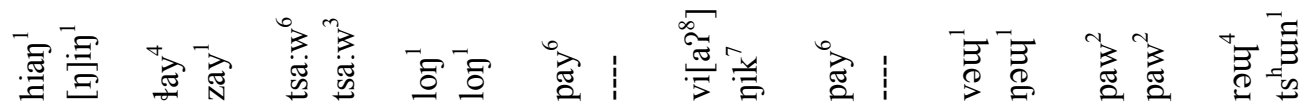

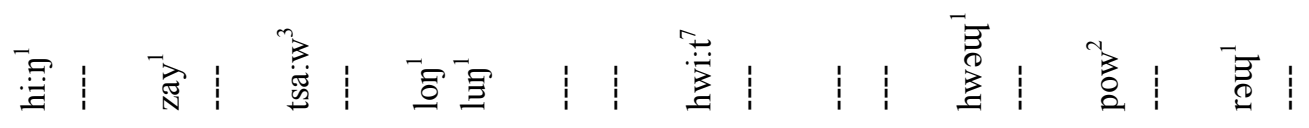

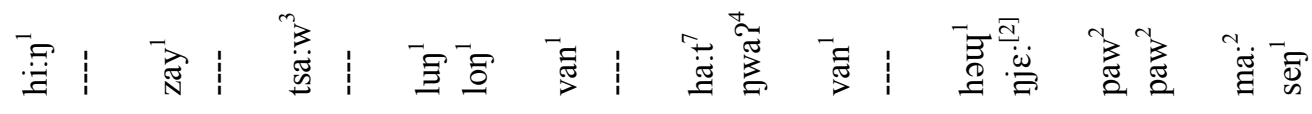

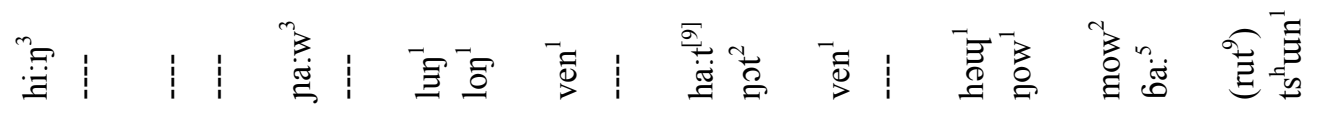

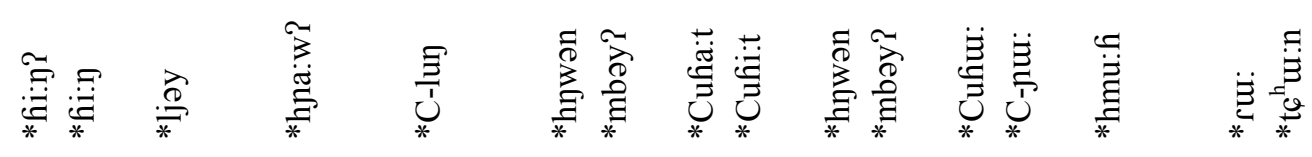

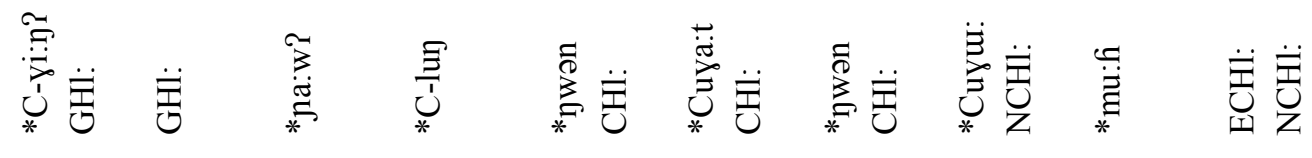

量

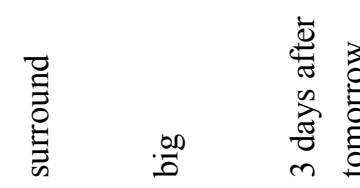

鼠

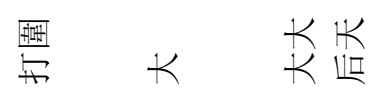

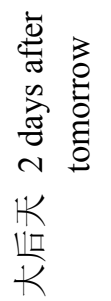

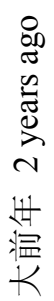




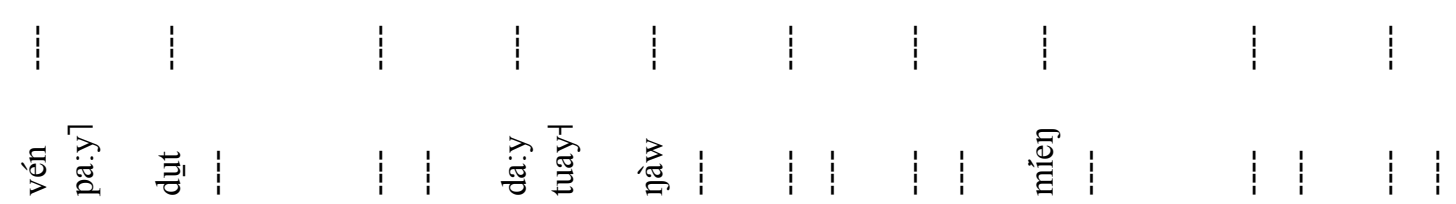

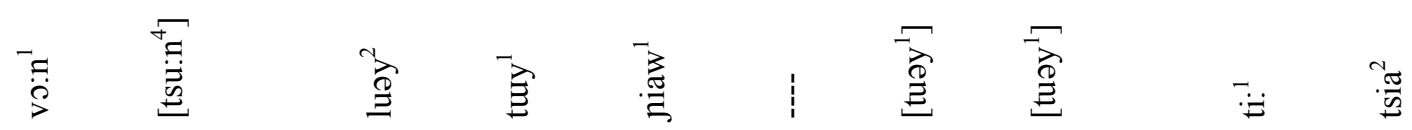

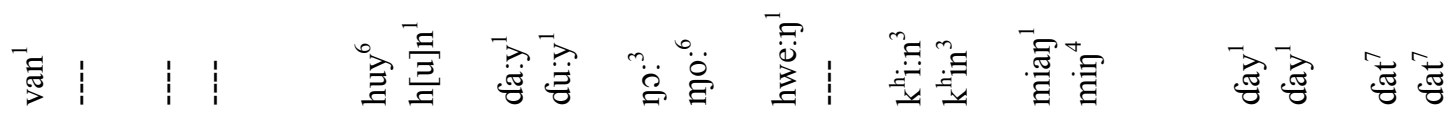

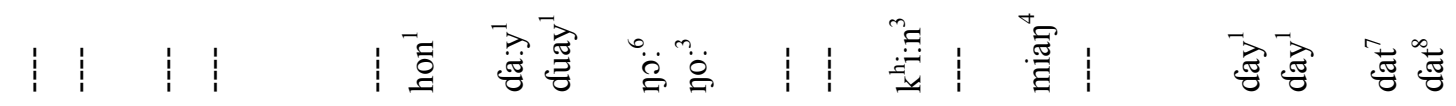

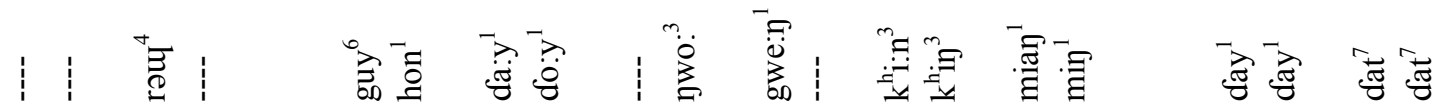

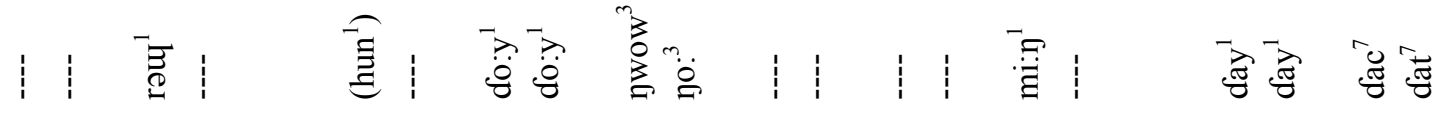

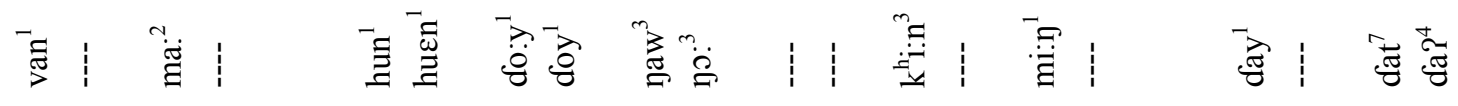

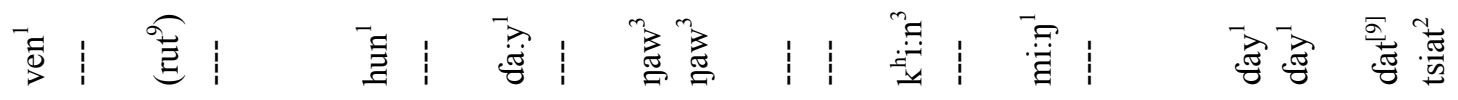

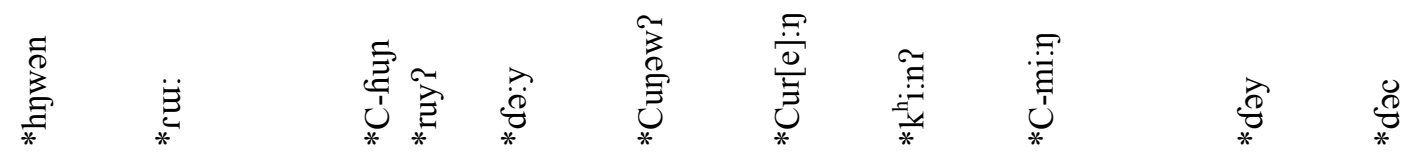

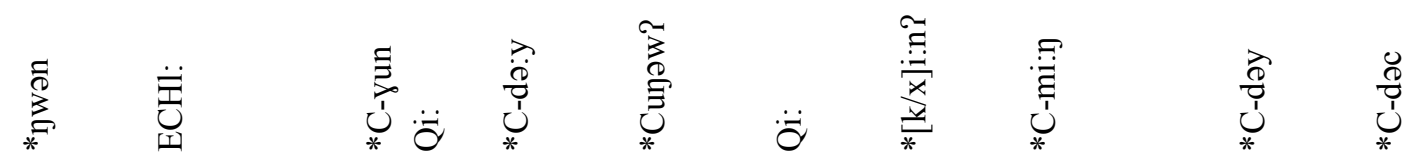

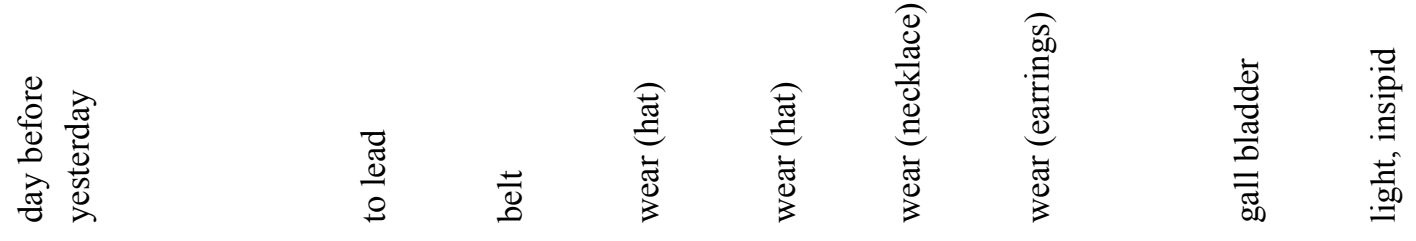

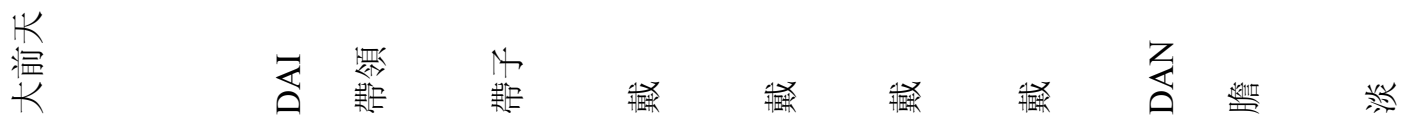




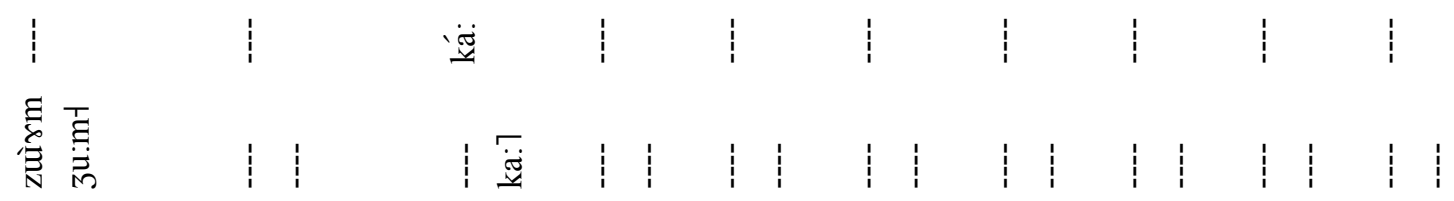

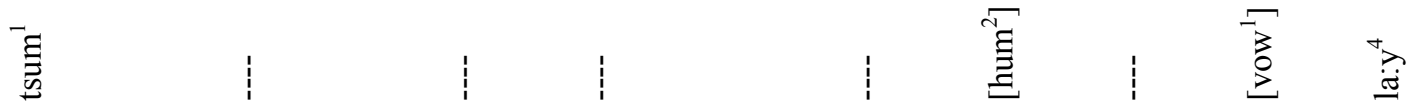

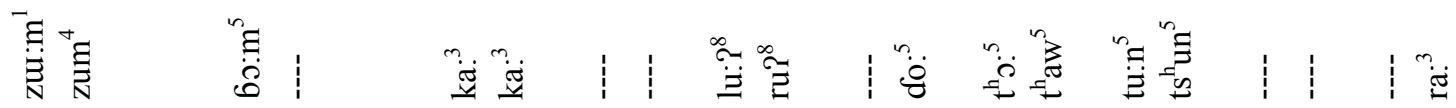

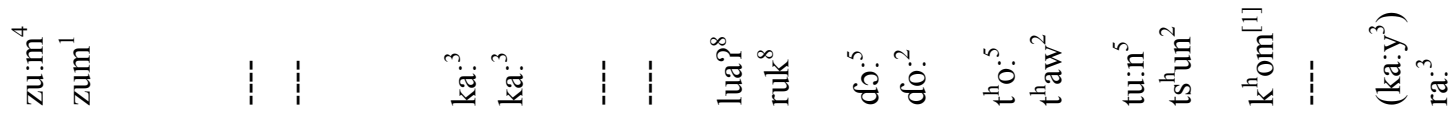

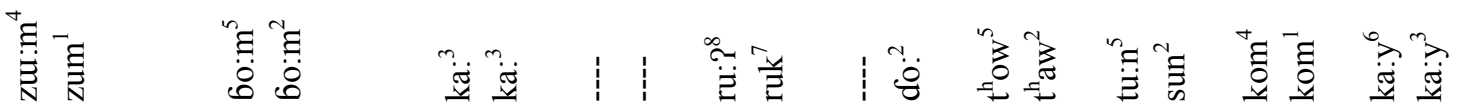

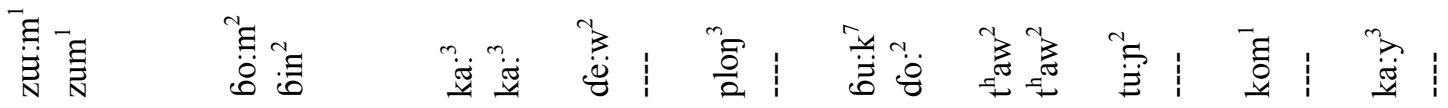

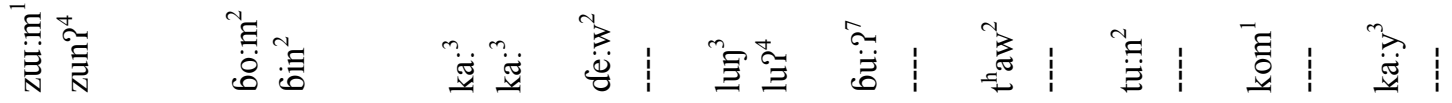

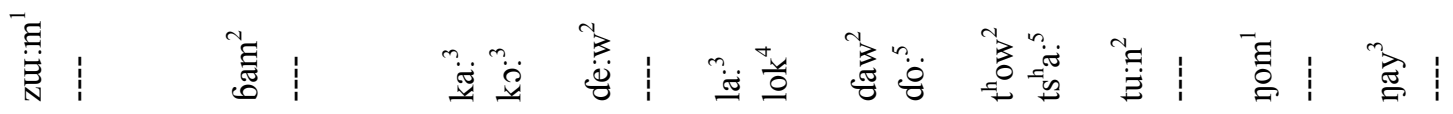

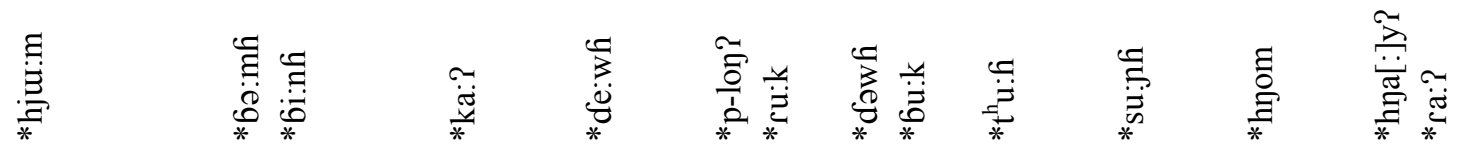

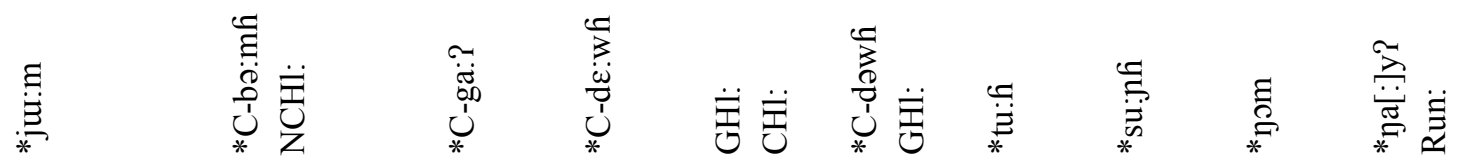

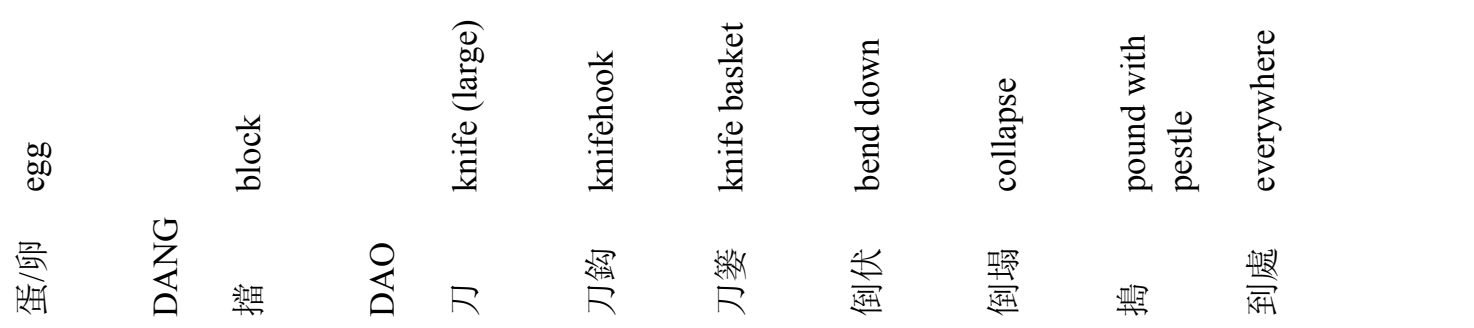




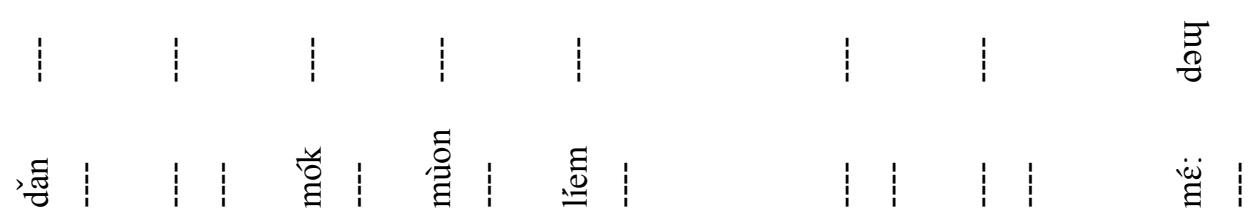

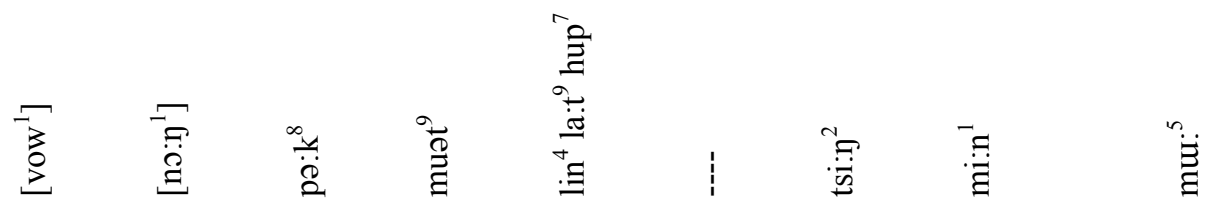

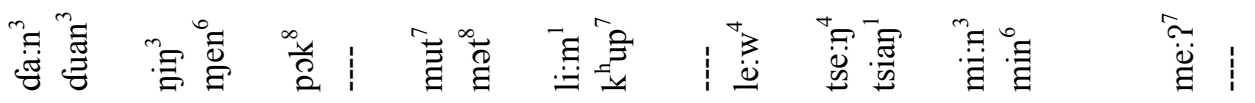

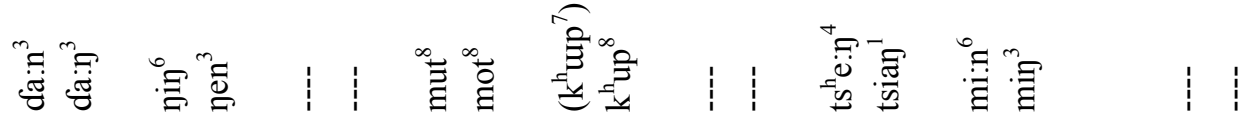

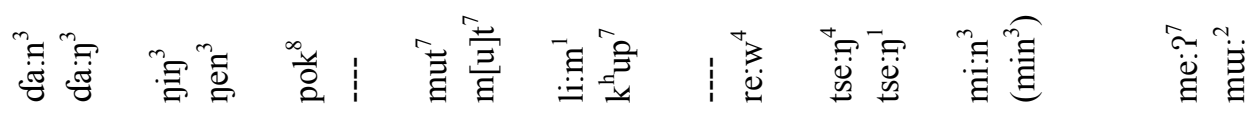

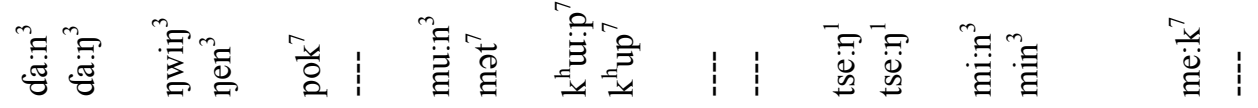

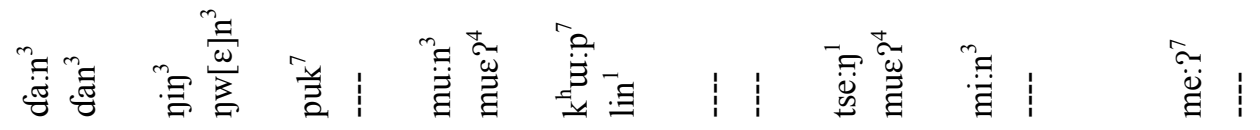

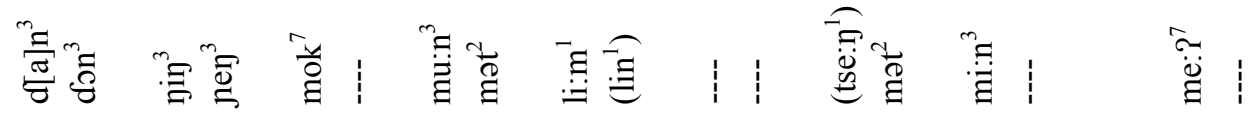

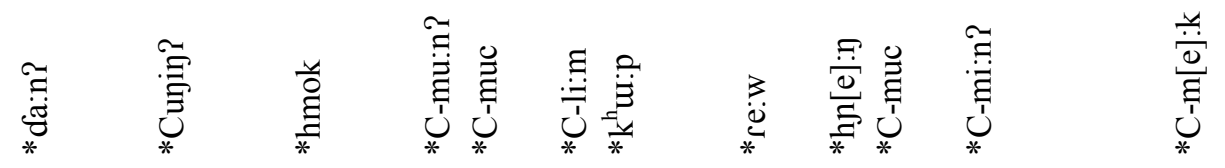

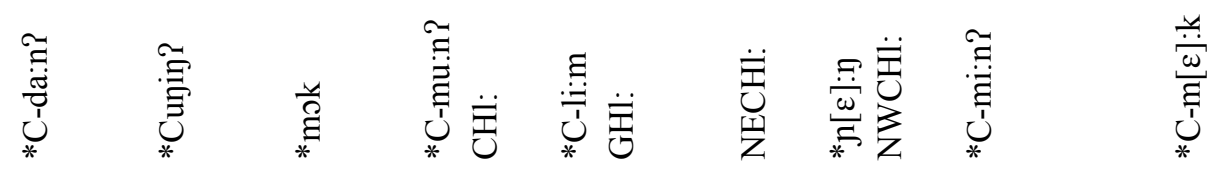

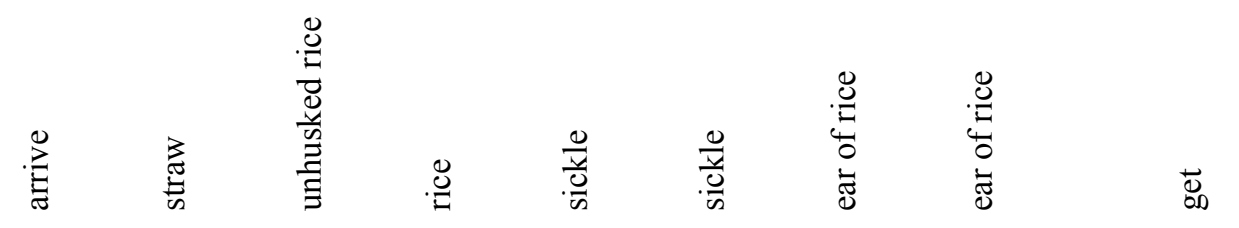

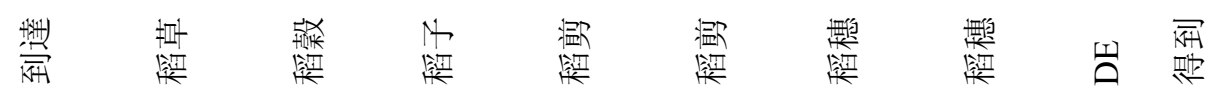




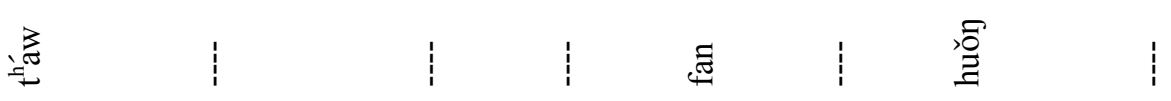

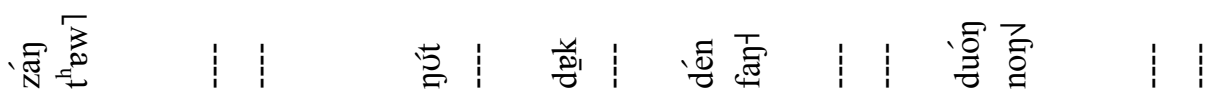

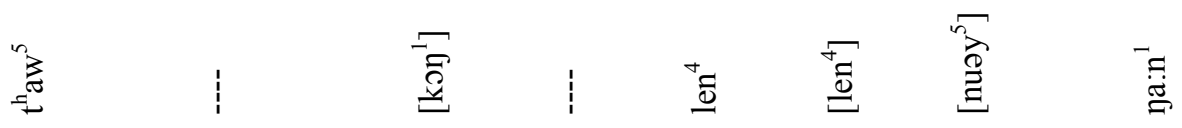

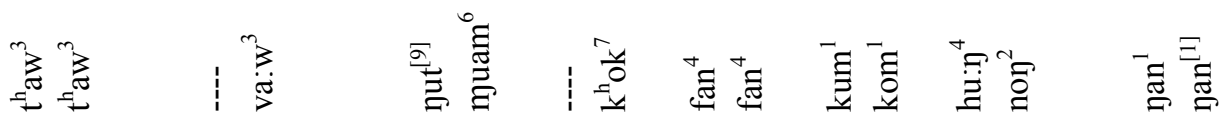

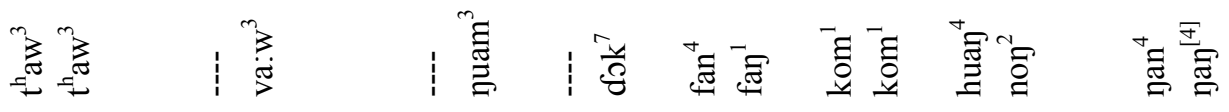

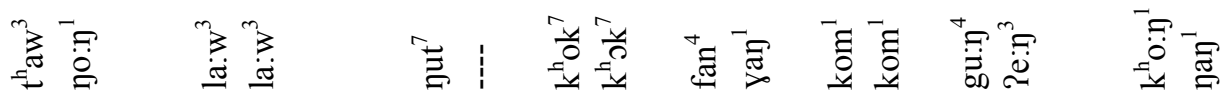

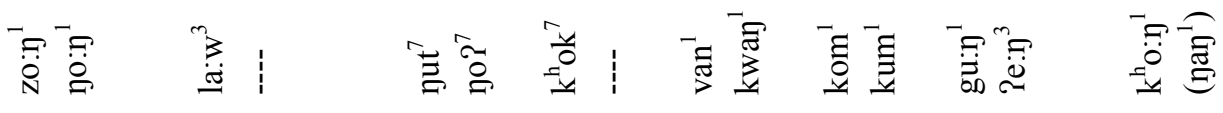

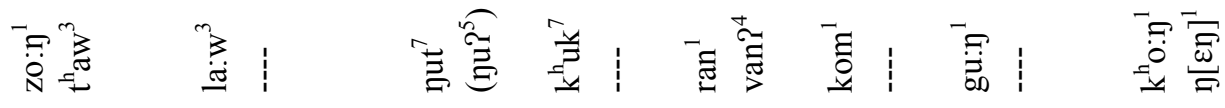

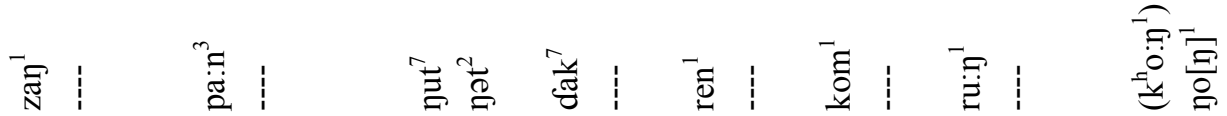

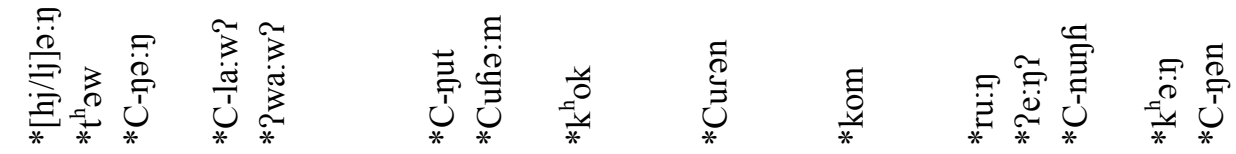

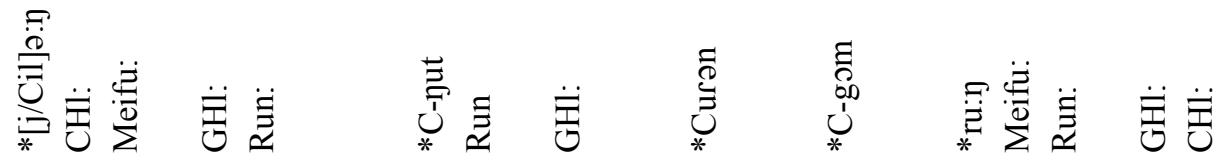

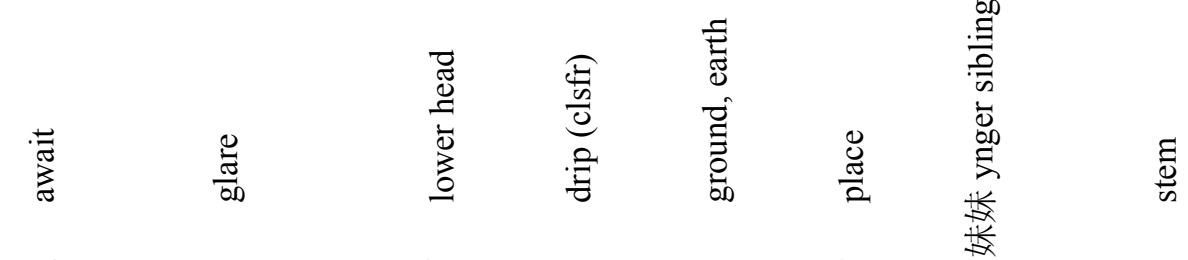

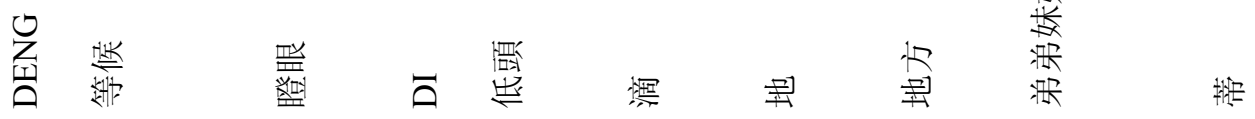




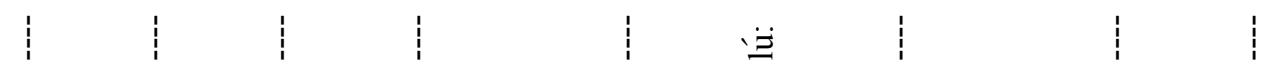

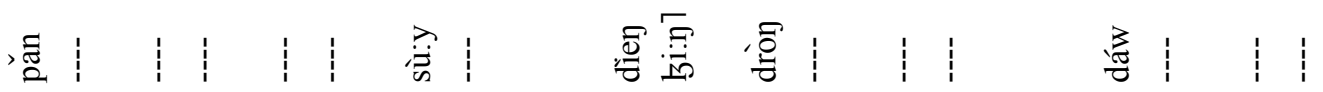

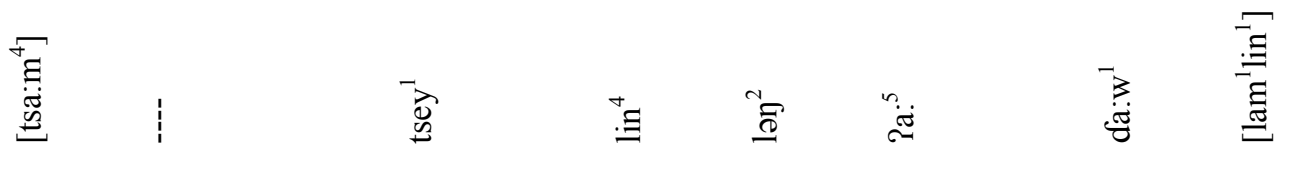

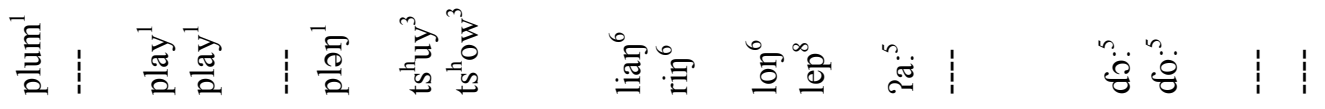

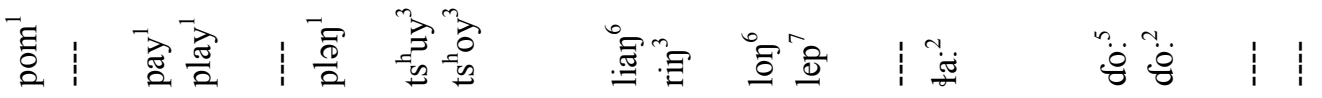

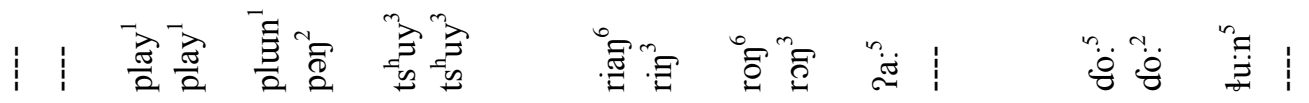

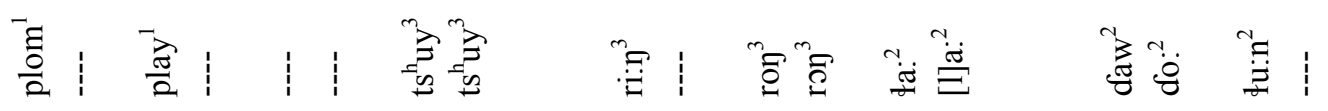

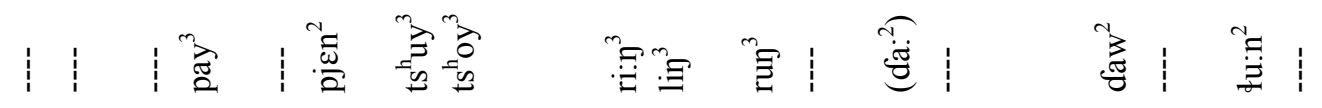

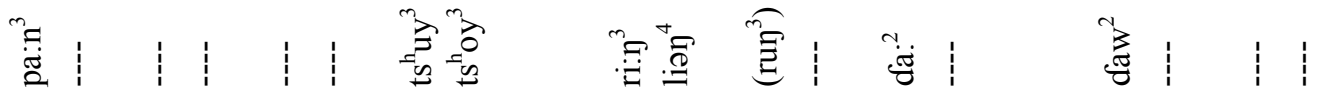

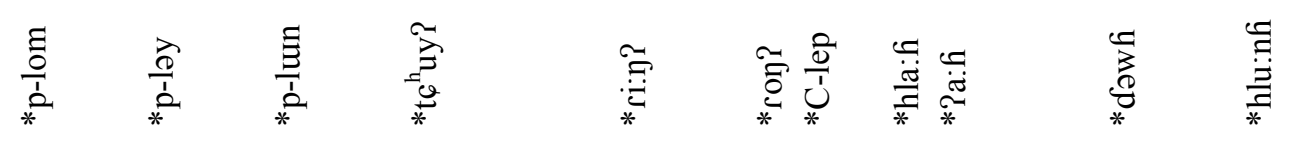

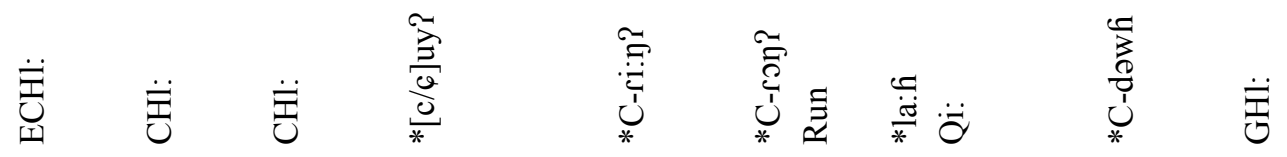

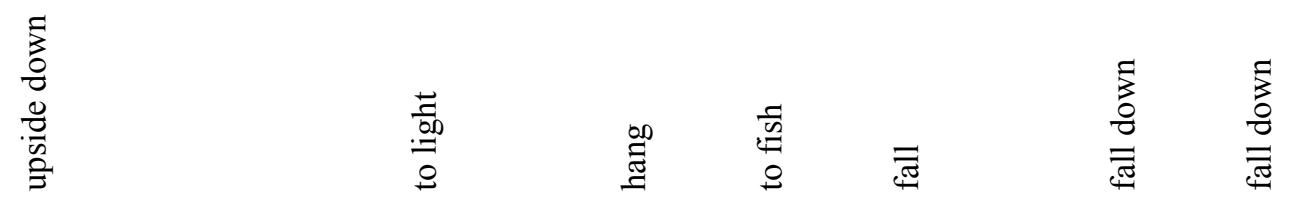

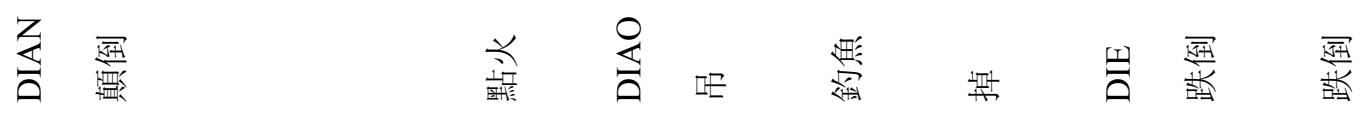




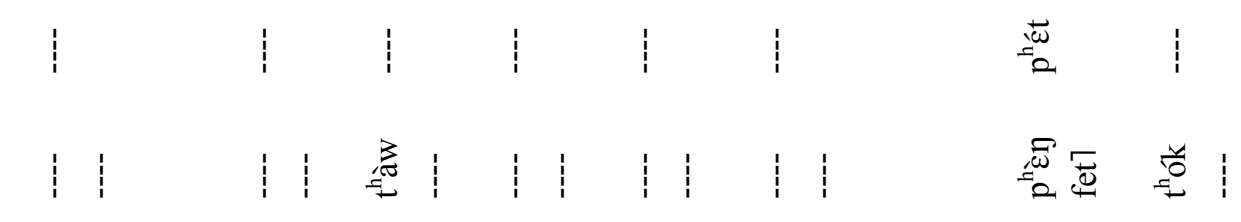

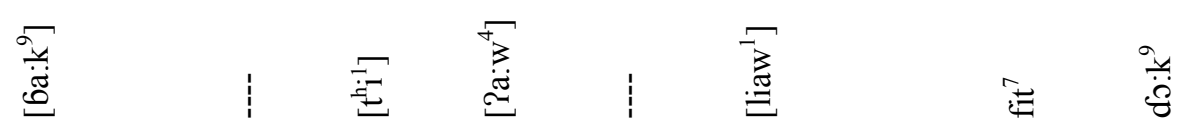

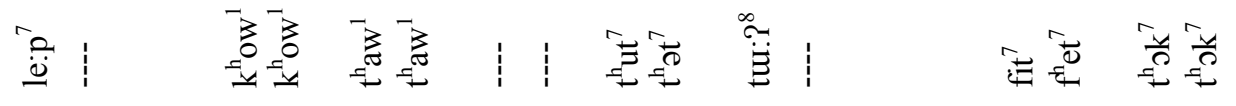

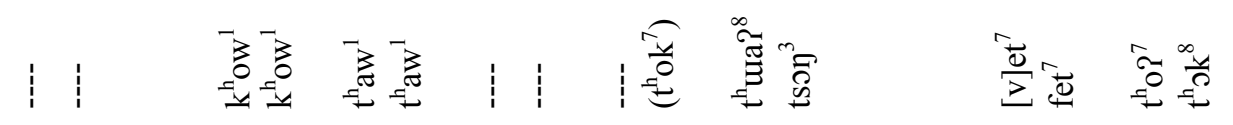

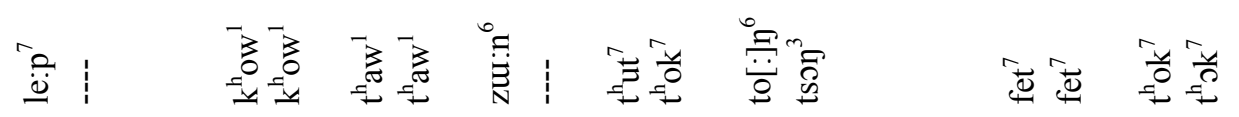

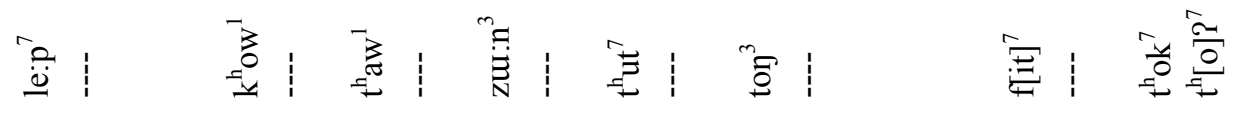

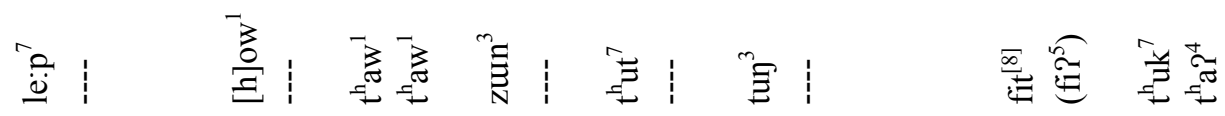

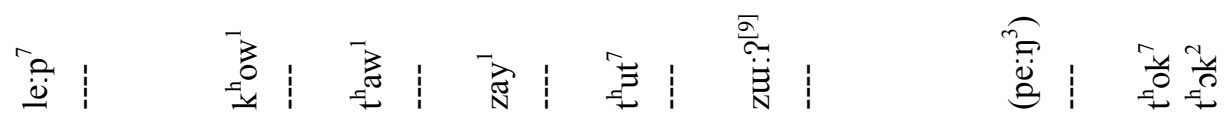

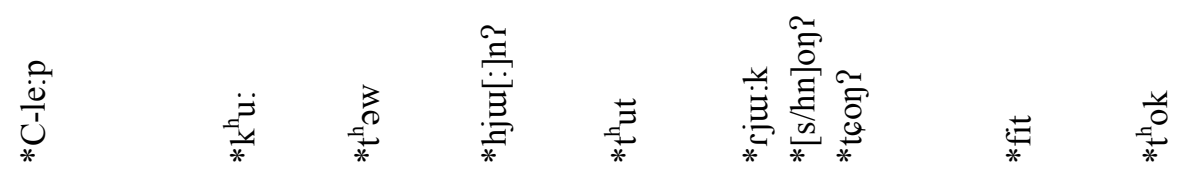

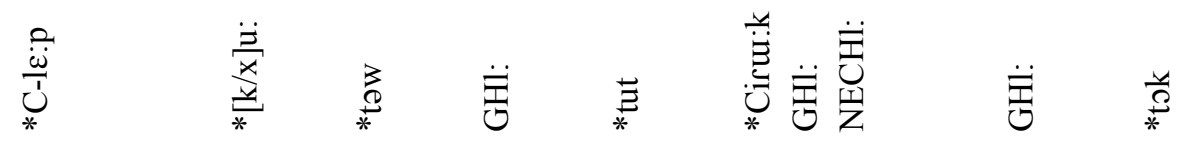

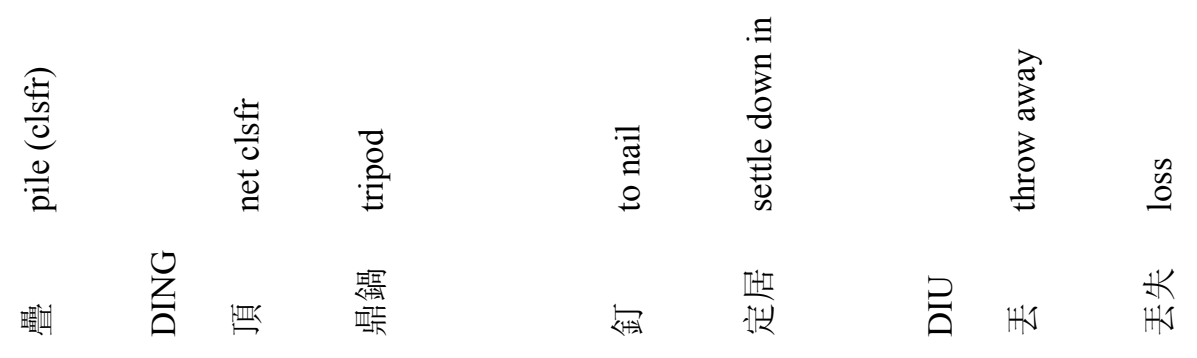




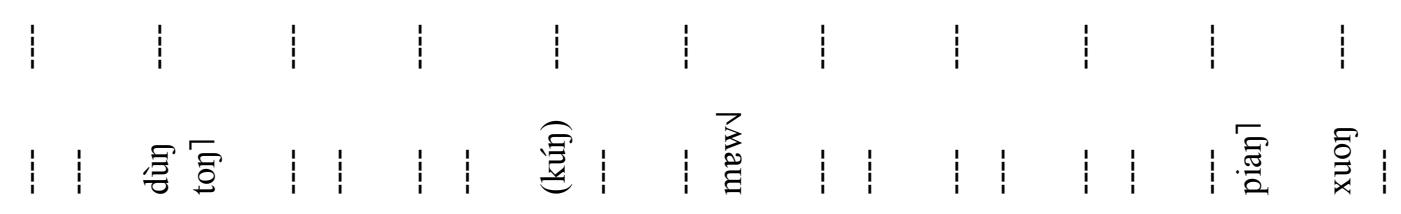

1

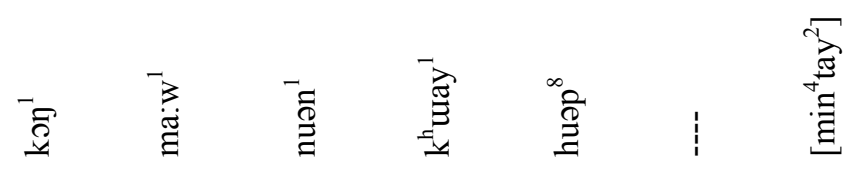

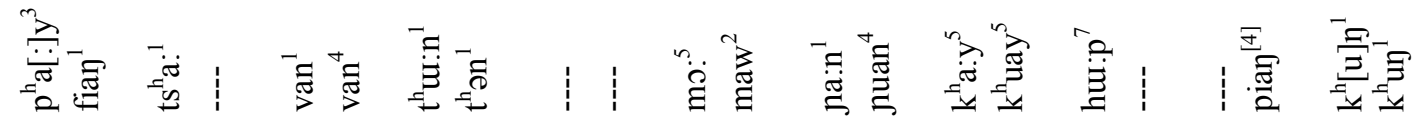

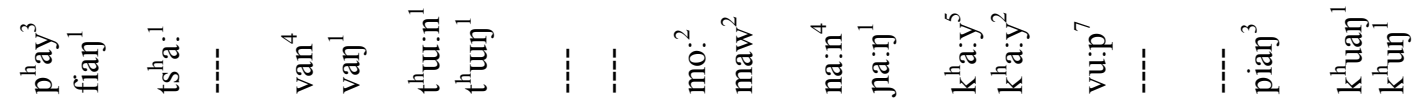

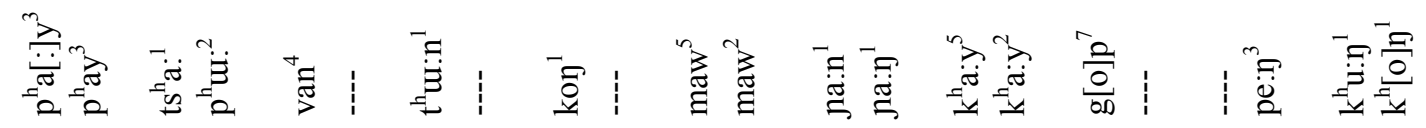

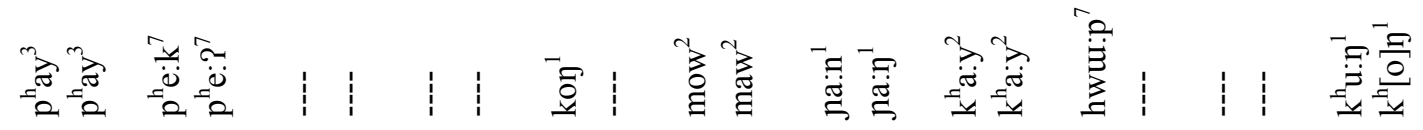

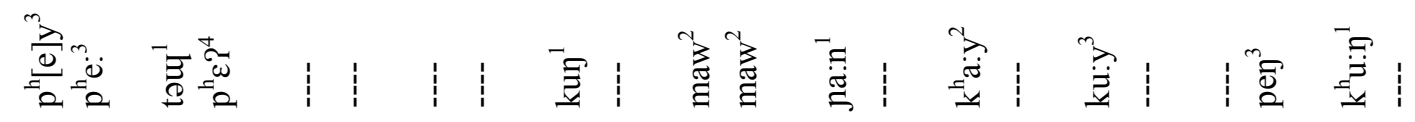

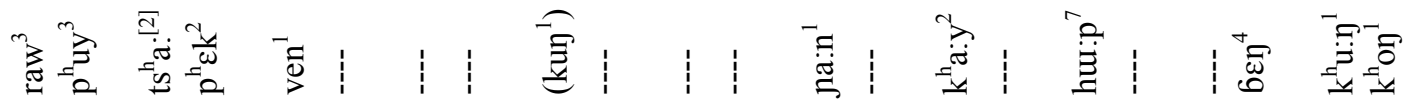

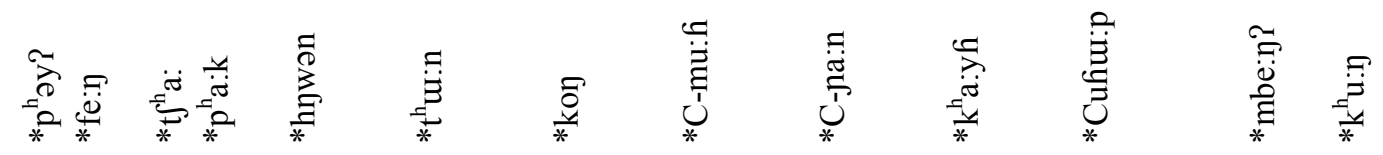

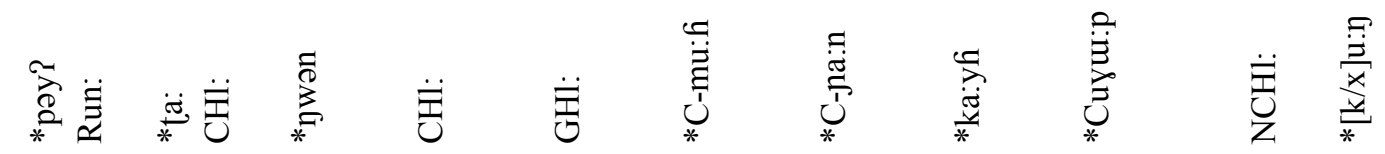

鯜 *

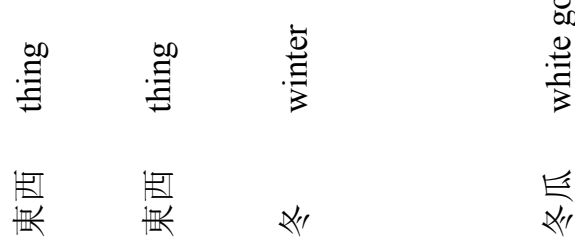

章

" 


\begin{tabular}{|c|c|c|c|c|c|c|}
\hline & 11 & 1 & ! & & 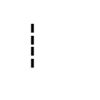 & \\
\hline 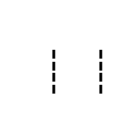 & 总 & 11 & 辛 & & 11 & 1 \\
\hline & 1 & 1 & 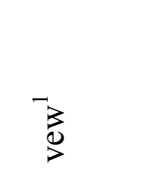 & & 䉕 & 言 \\
\hline 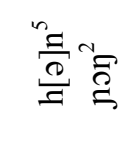 & 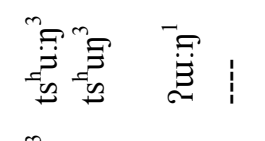 & 琶要 & 量 & 辛 & 善 & 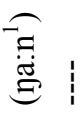 \\
\hline & 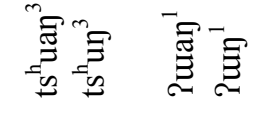 & 18 & 部 & F" & 意 & 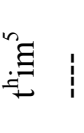 \\
\hline & 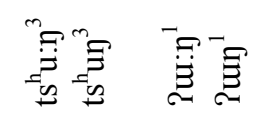 & is & 部 & 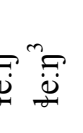 & 意 & 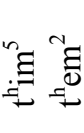 \\
\hline & 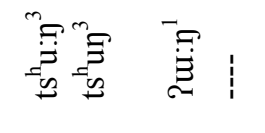 & 菅 & 童部 & 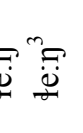 & 意 & 管: \\
\hline 13 & 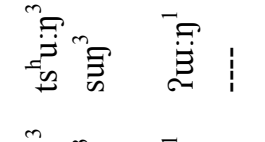 & is & 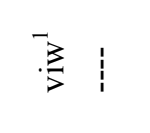 & "s & 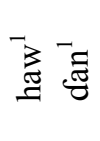 & 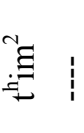 \\
\hline & 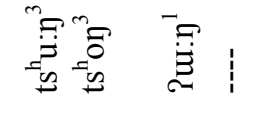 & 恶 & & : & 高 & E \\
\hline
\end{tabular}

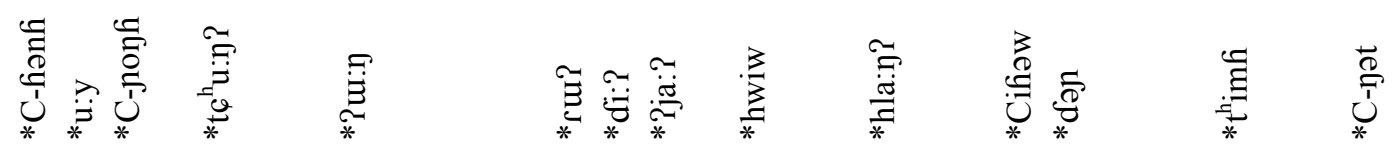

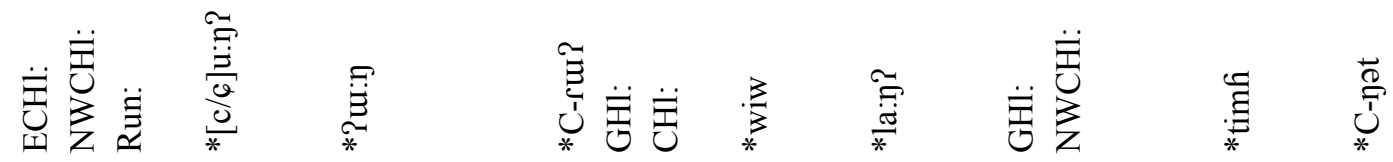

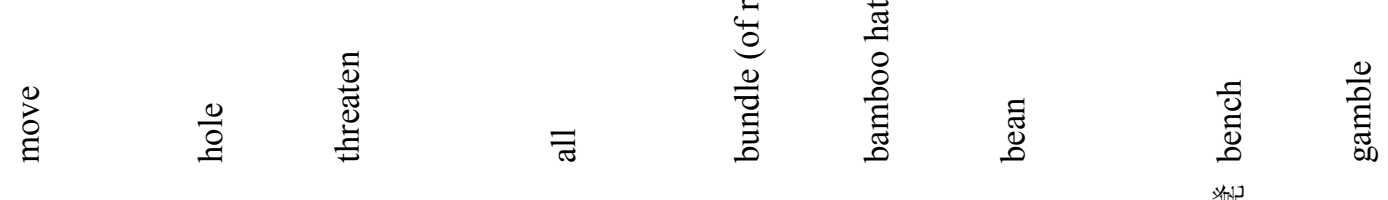

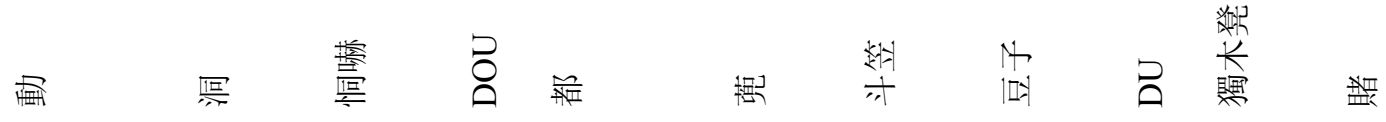




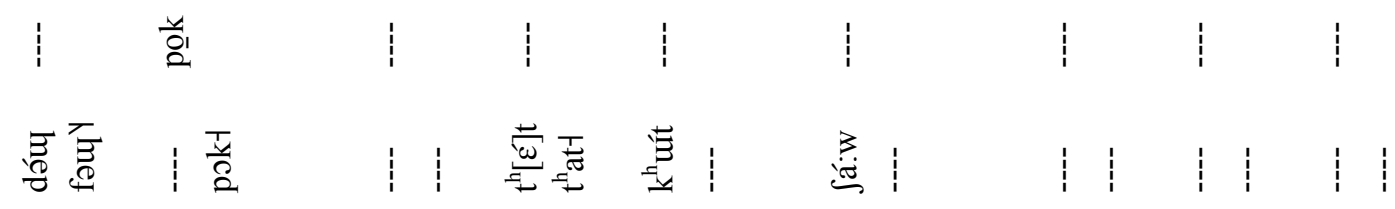

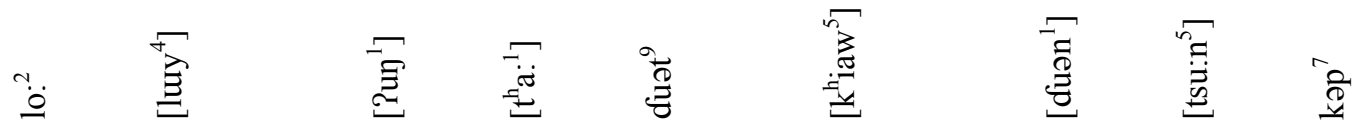

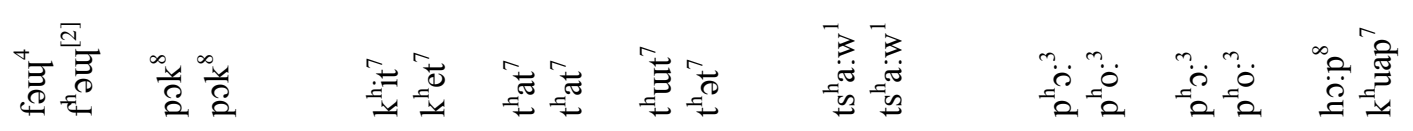

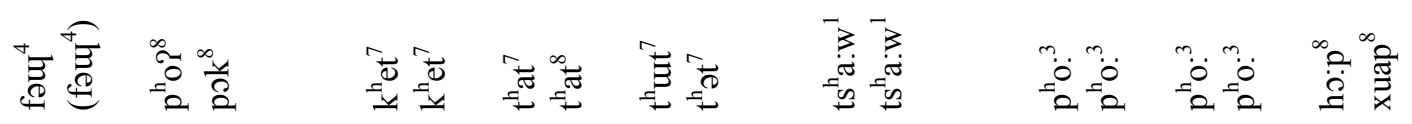

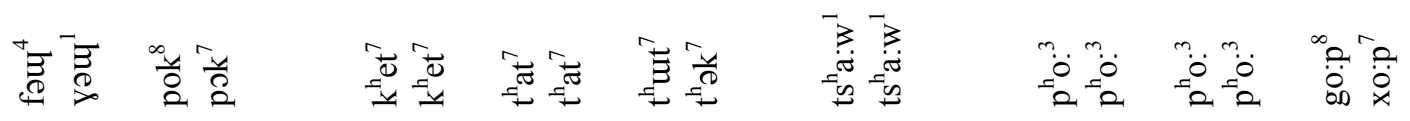

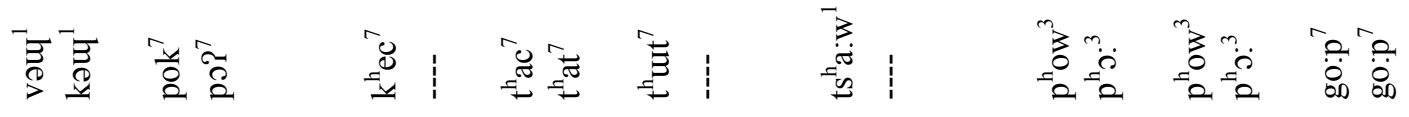

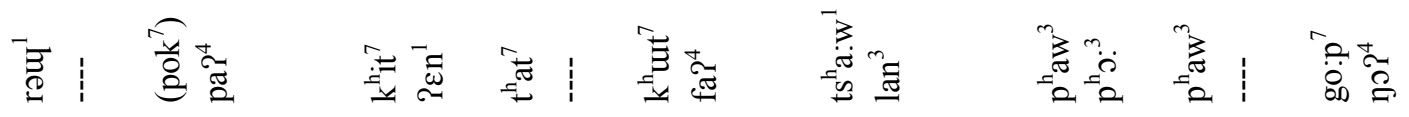

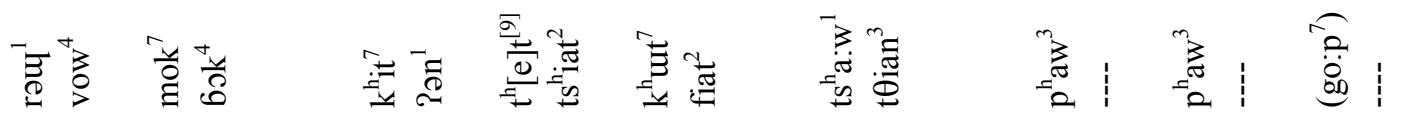

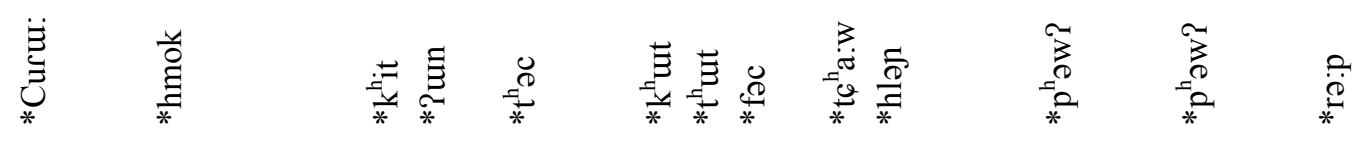

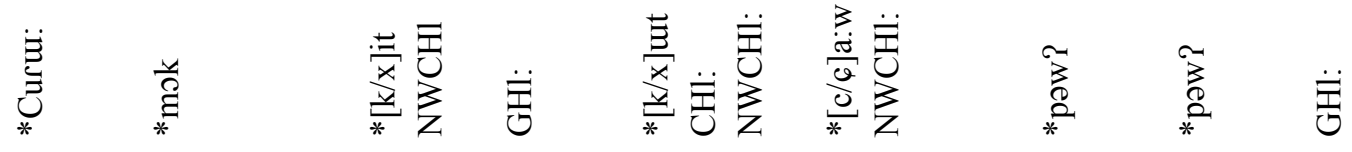

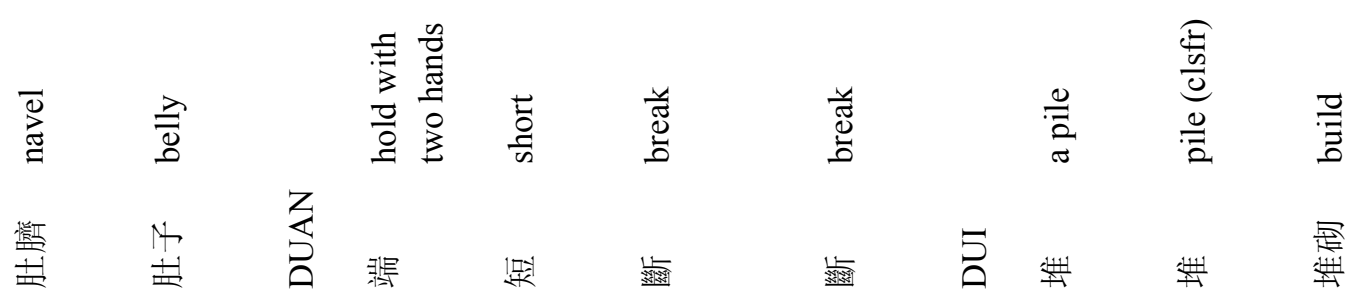




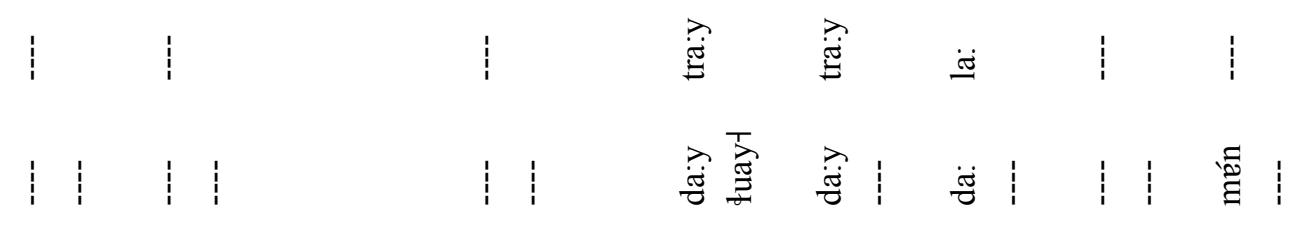

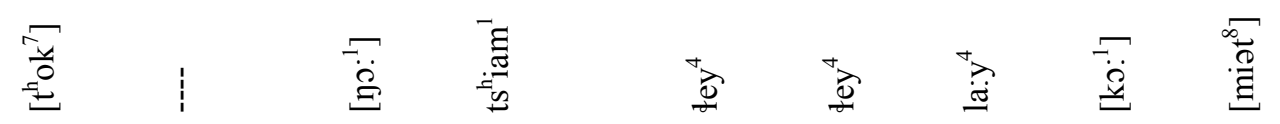

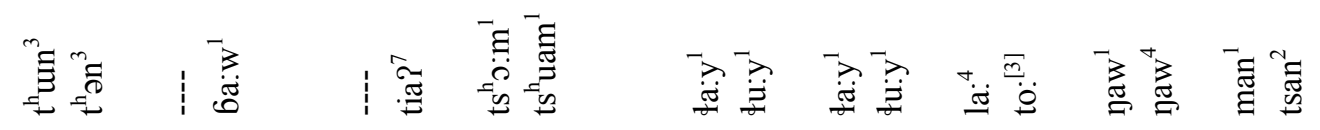

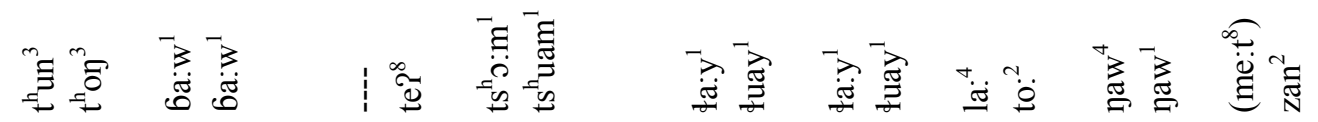

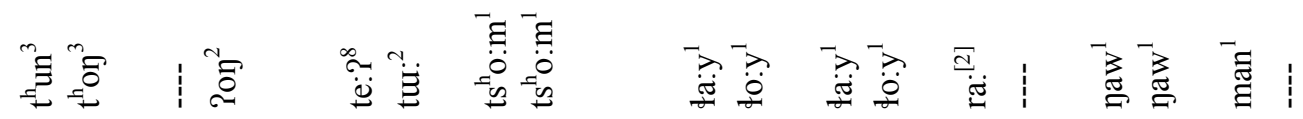

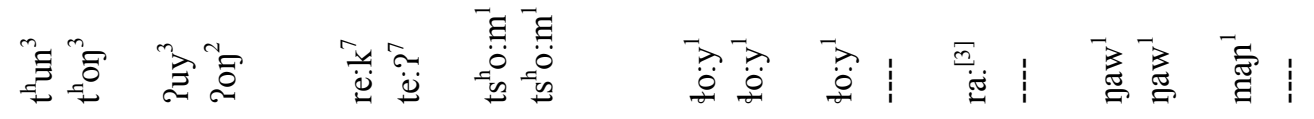

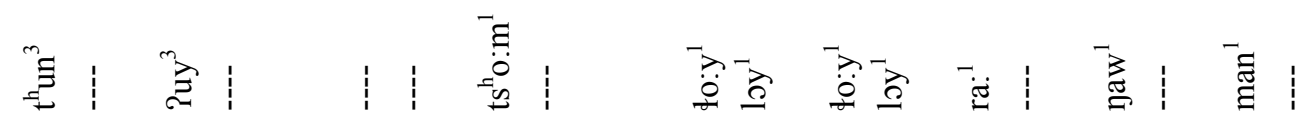

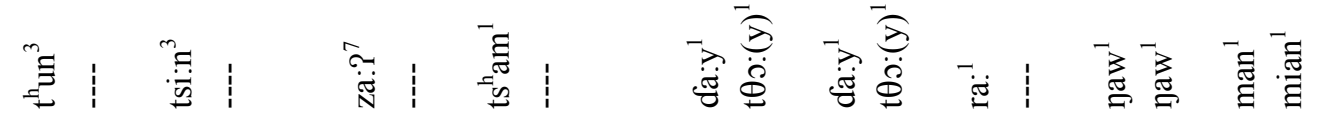

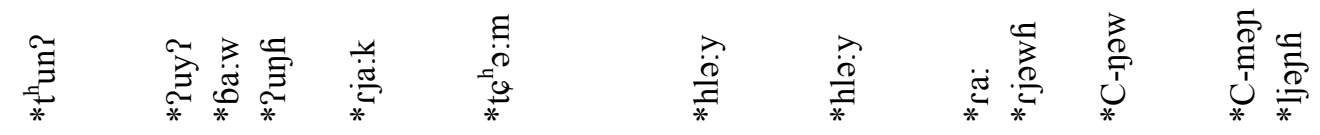

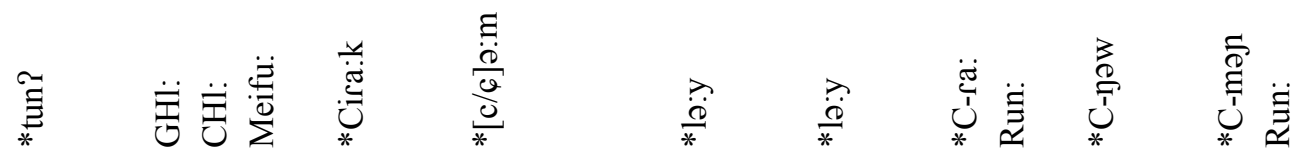

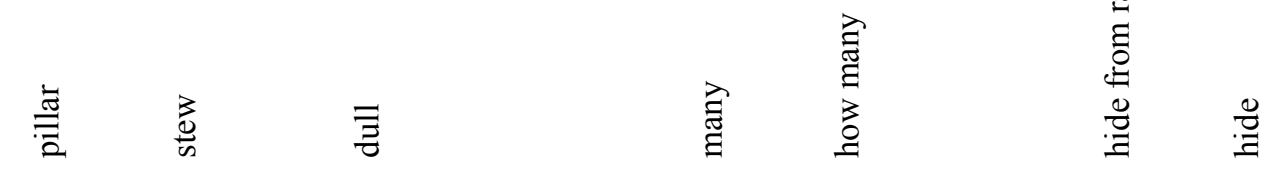

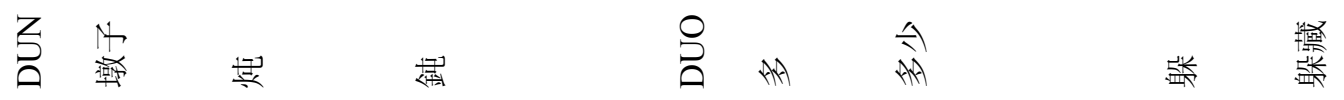




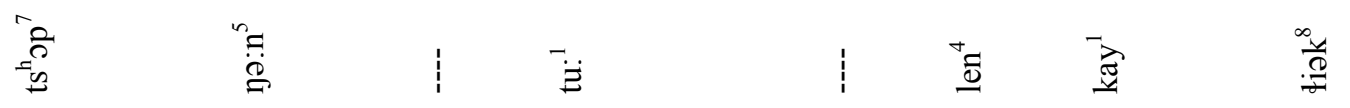

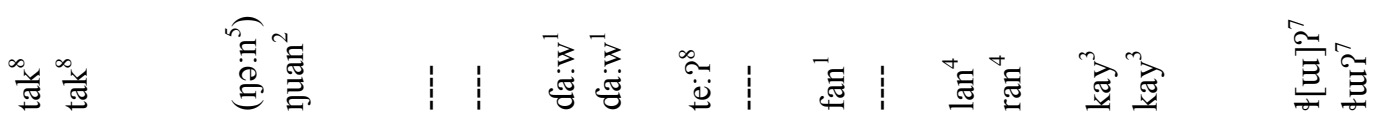

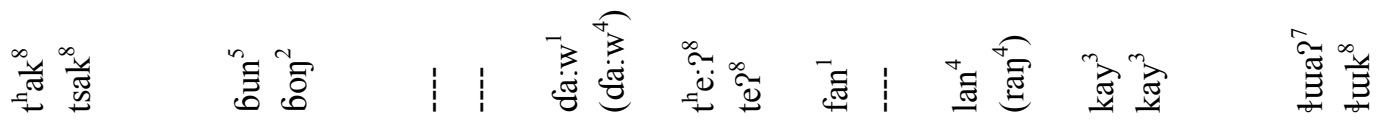

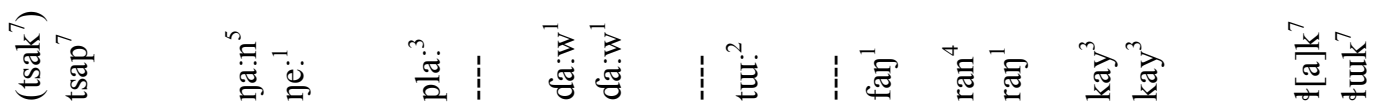

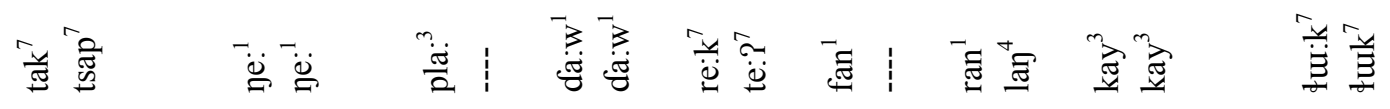

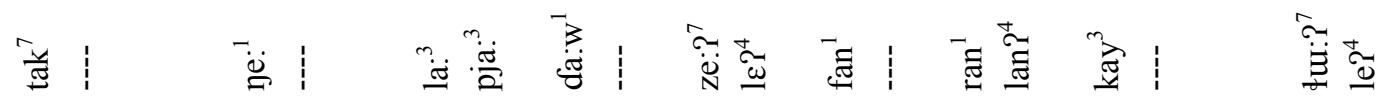

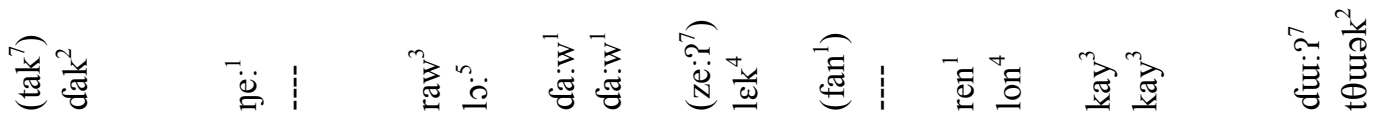

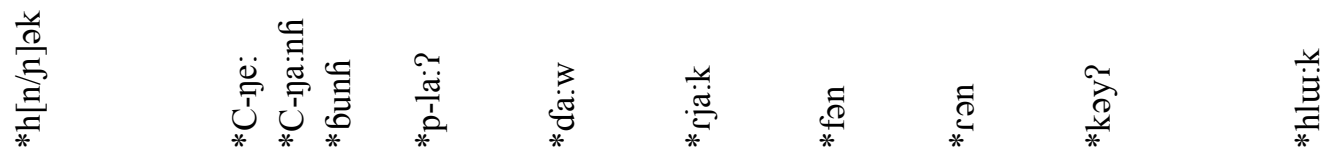

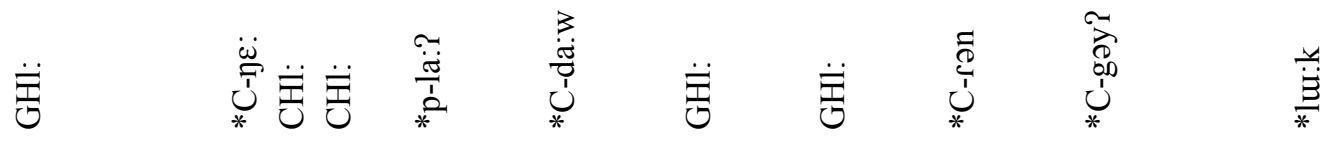
\%
荵
咅产
墨高

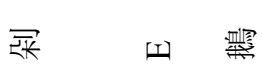

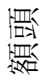
州葆

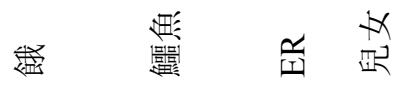




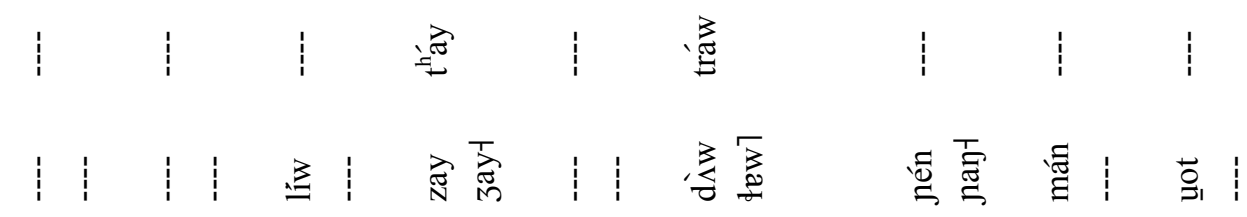

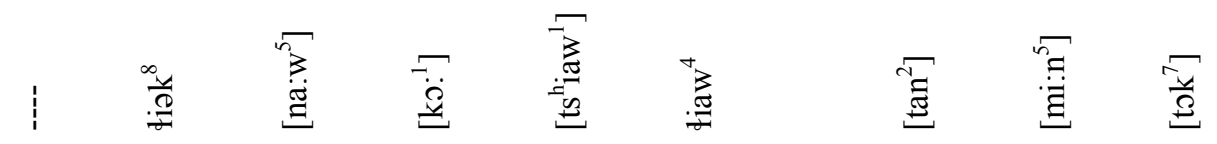

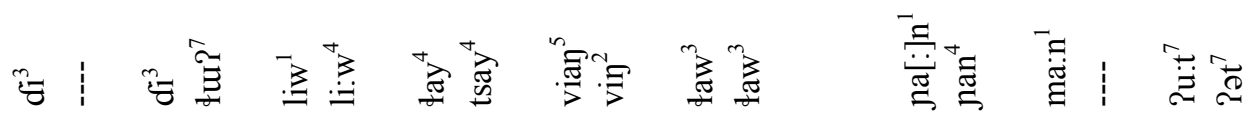

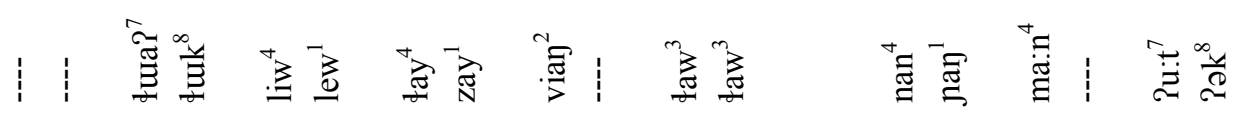

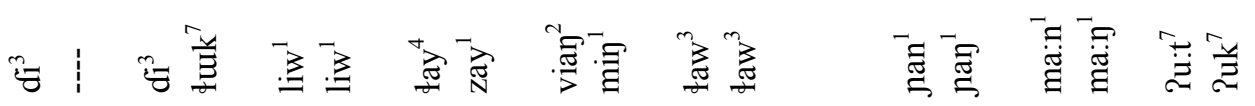

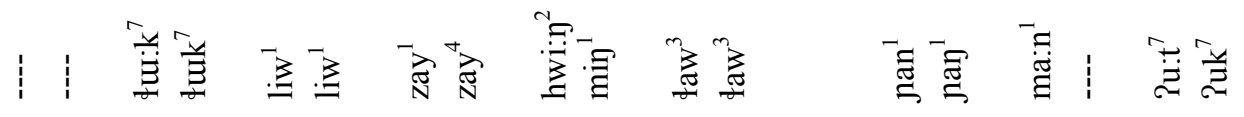

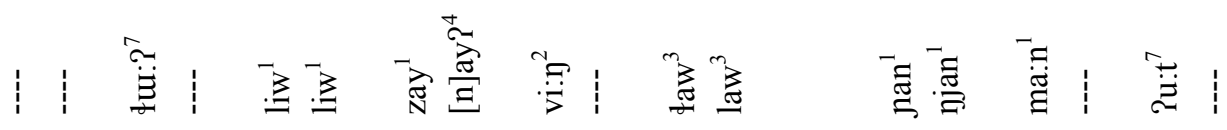

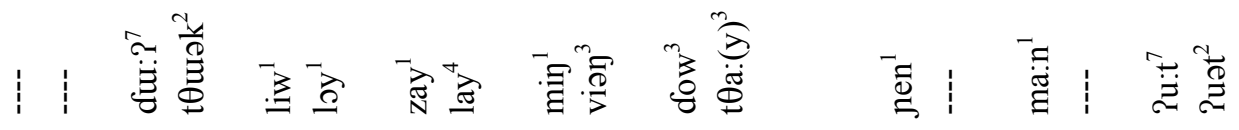

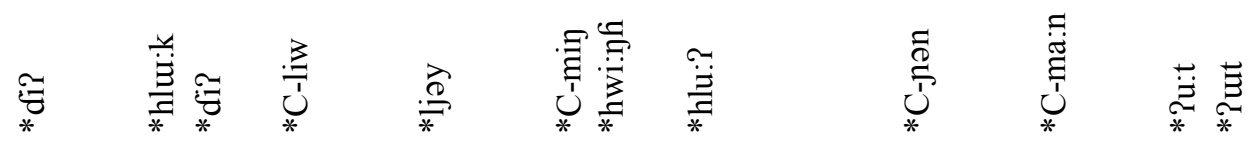

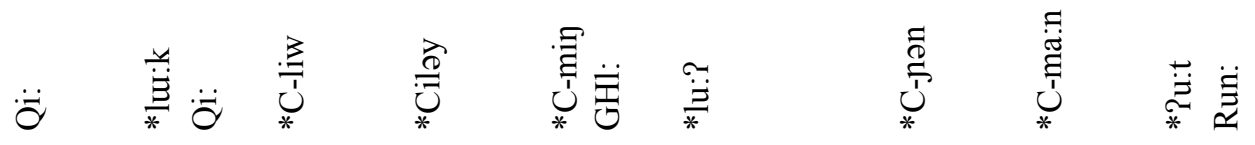

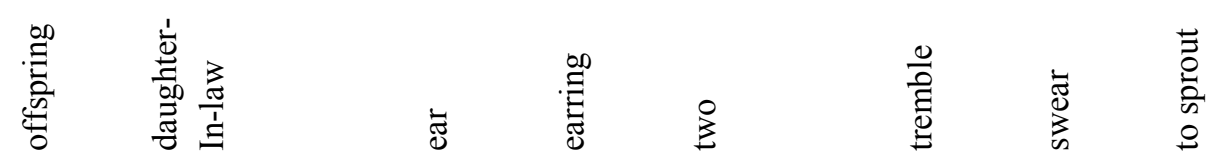

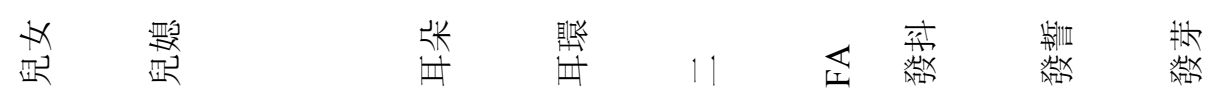




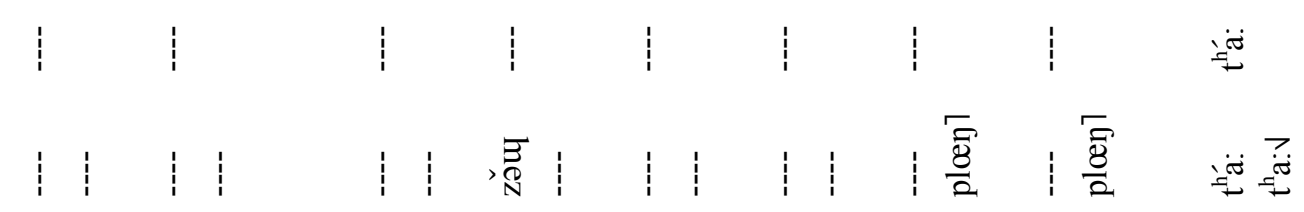

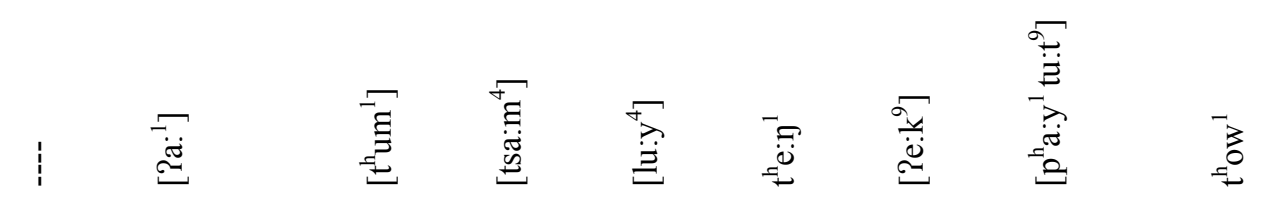

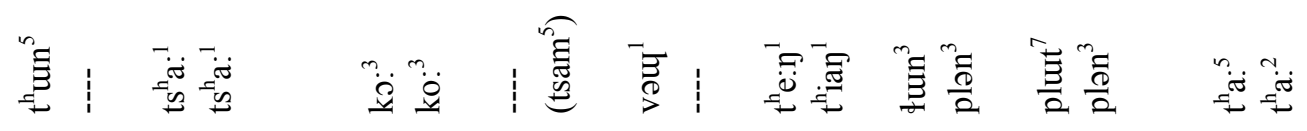

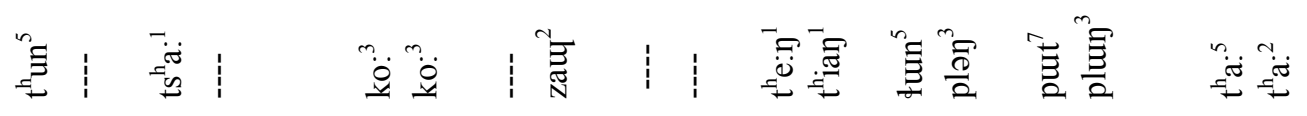

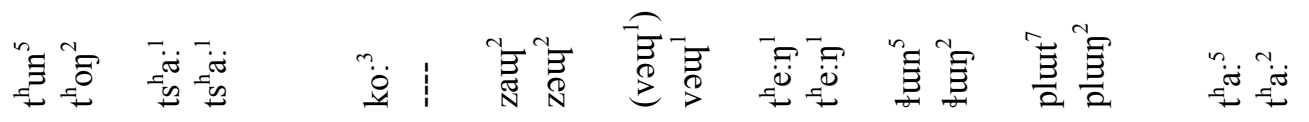

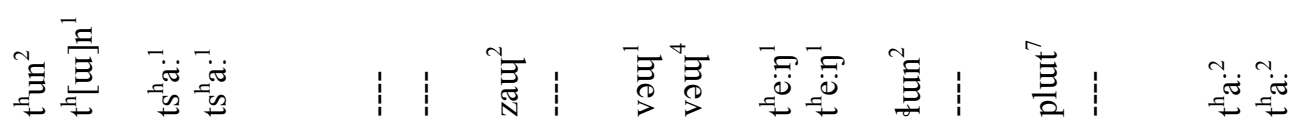

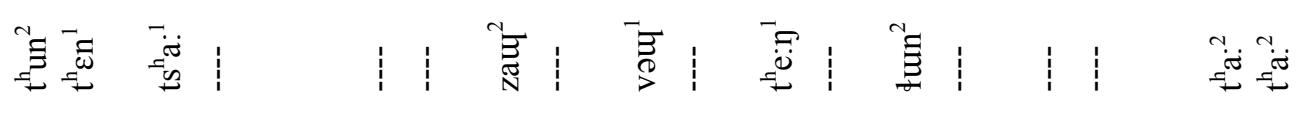

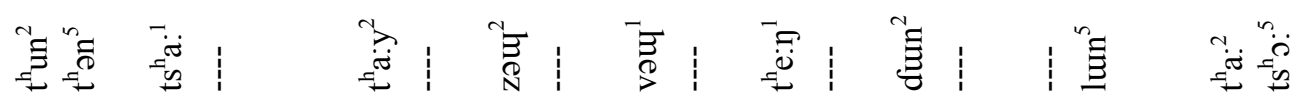

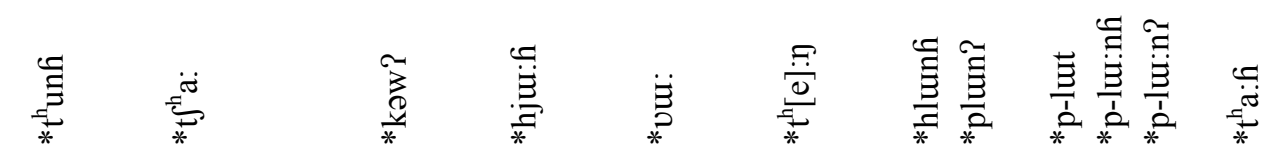

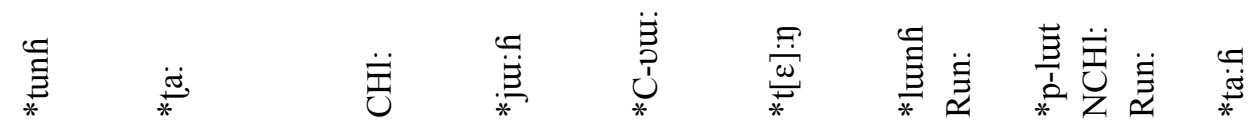

毵

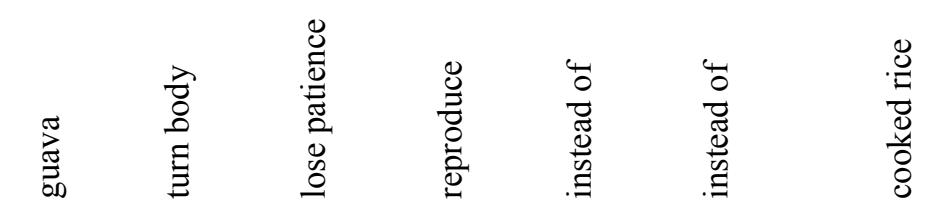

物

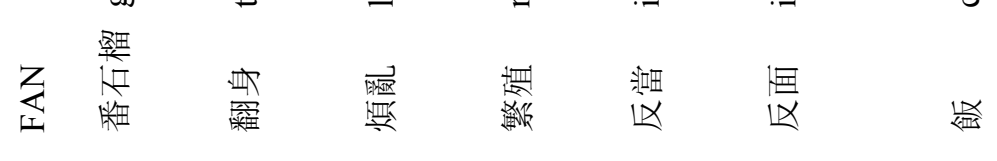




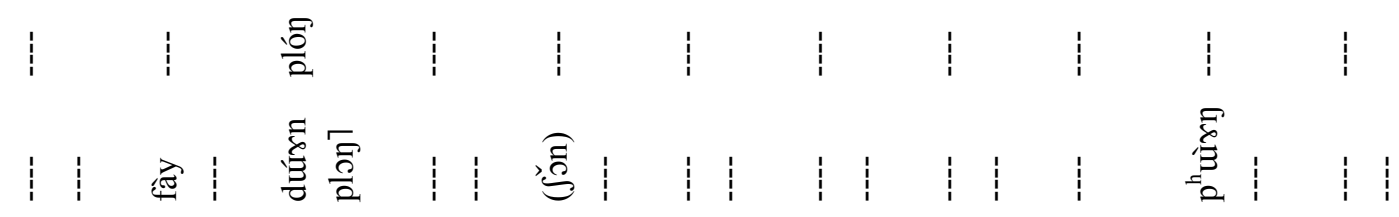

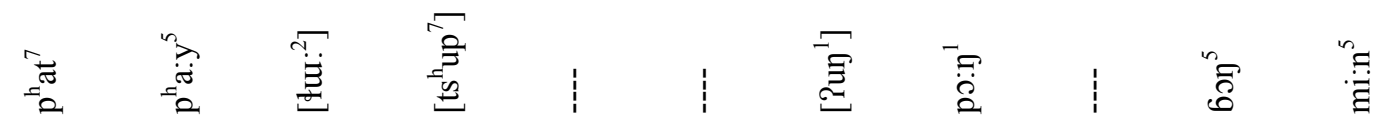

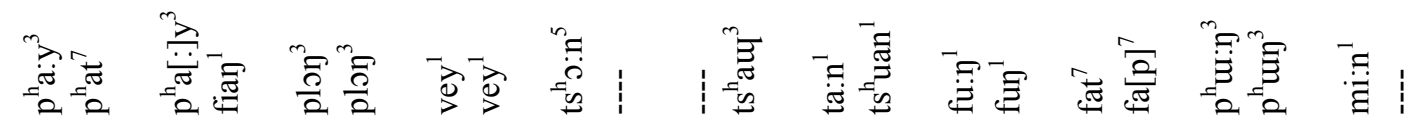

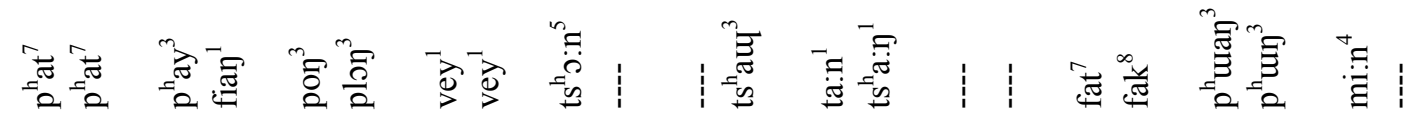

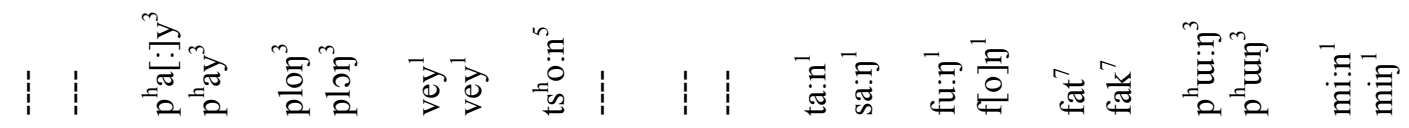

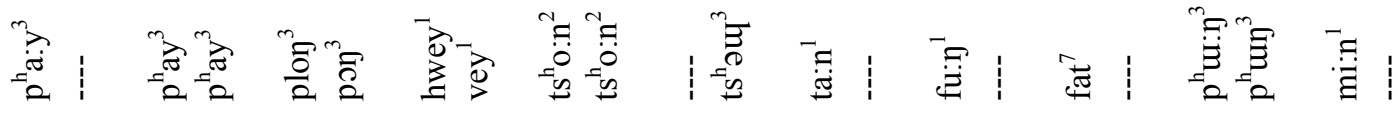

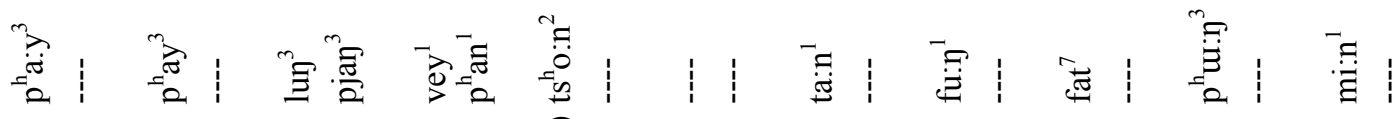

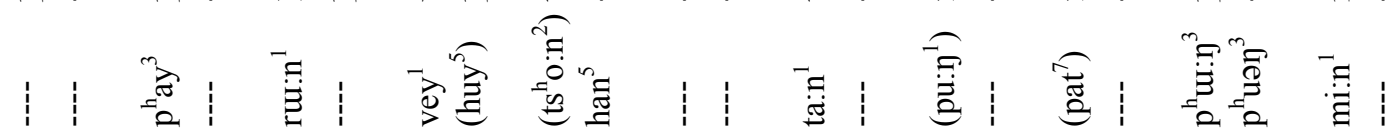

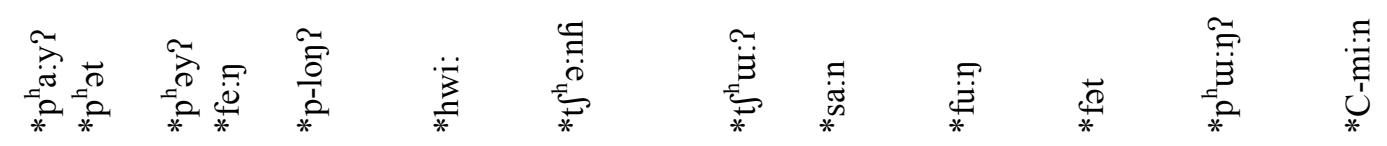

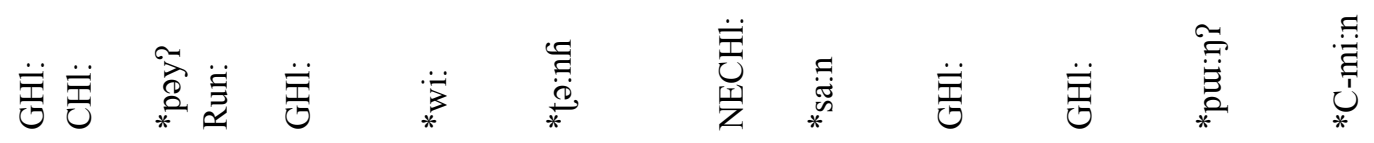

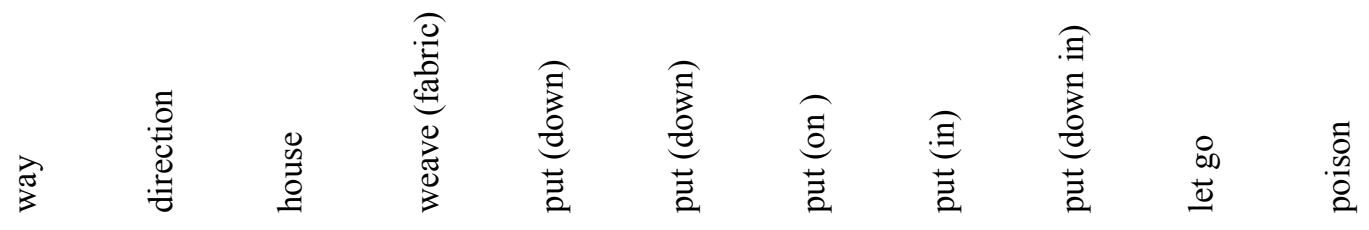

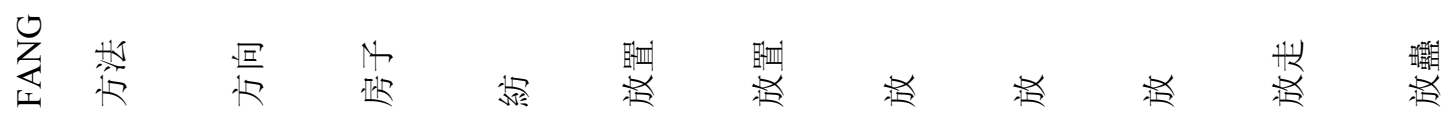




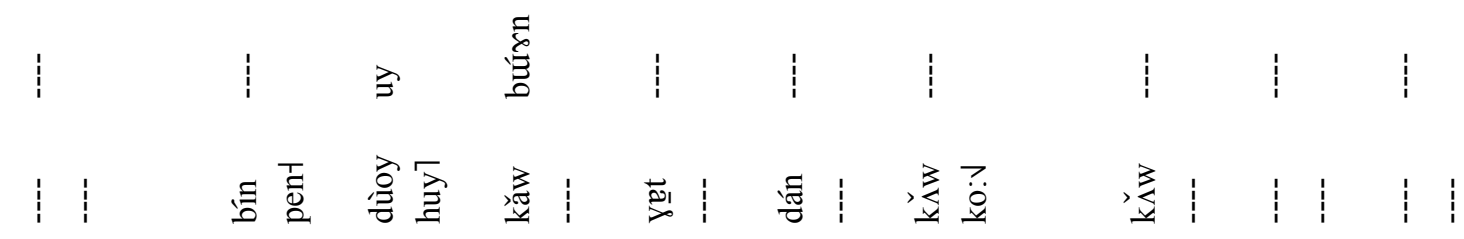

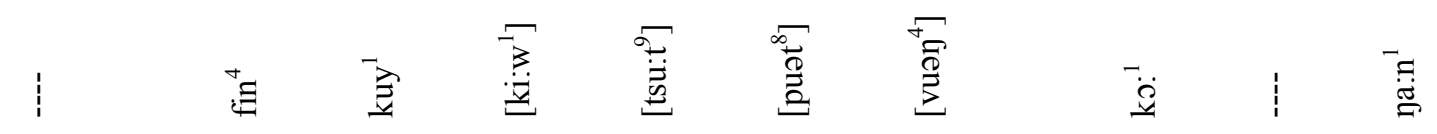

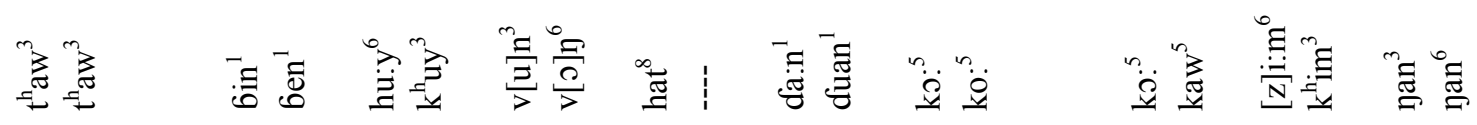

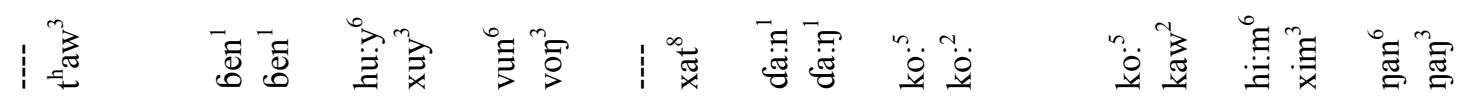

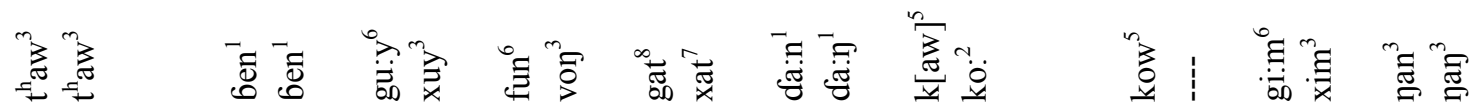

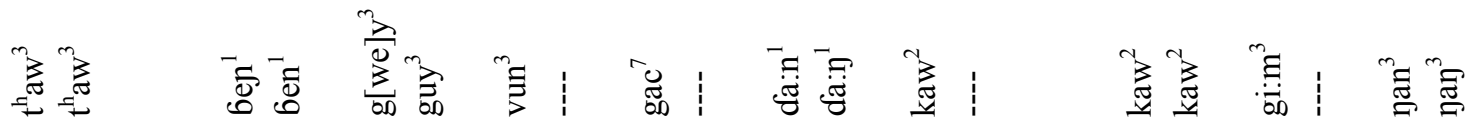

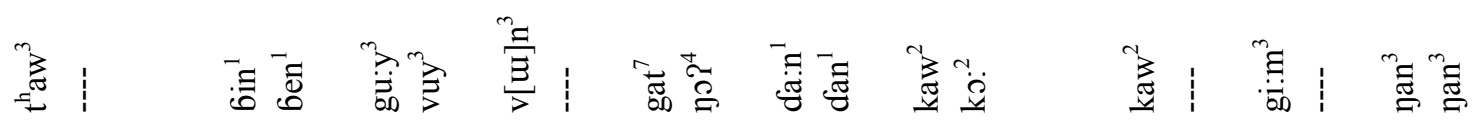

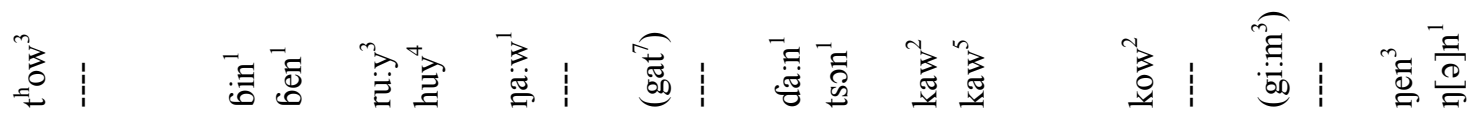

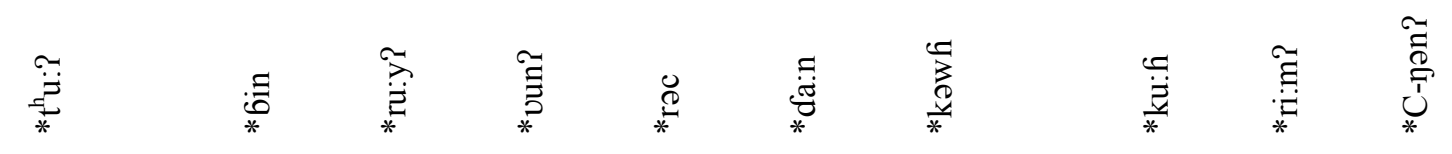

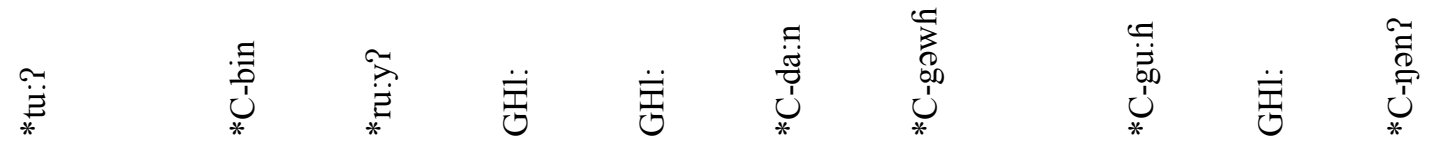

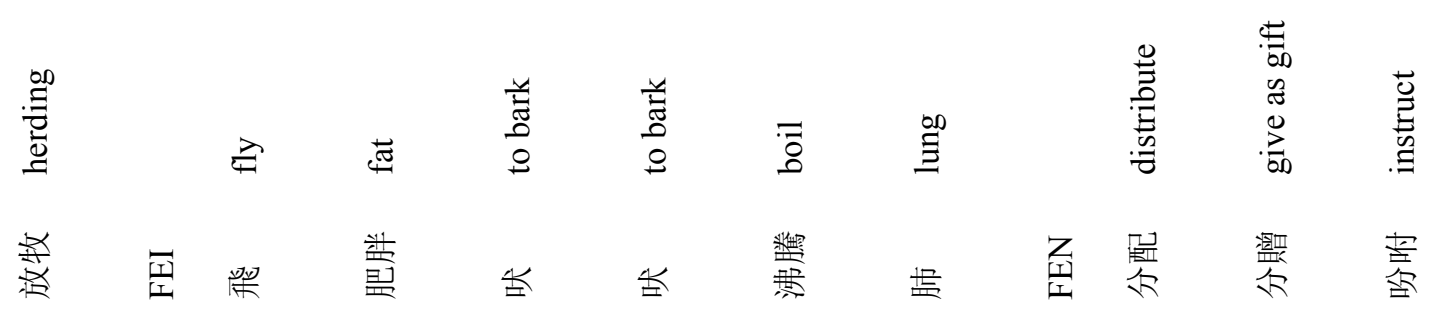


$1 \quad 1$

$=1 \quad 1 \quad \mid$

) 产

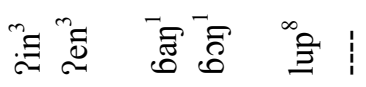

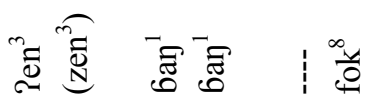

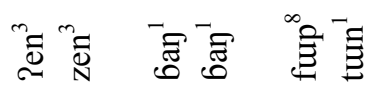

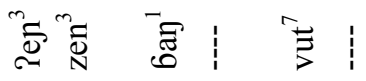

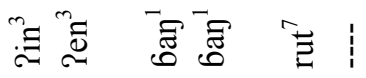

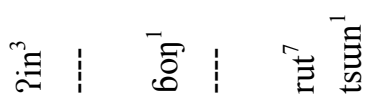

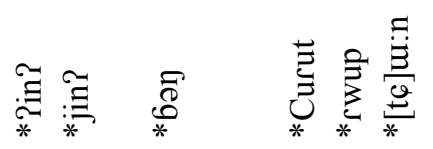

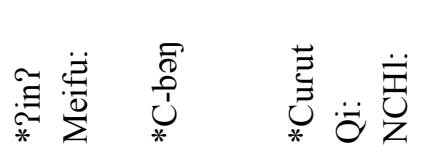

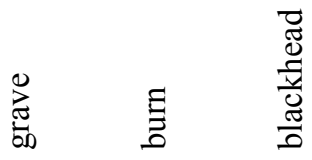

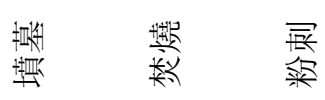

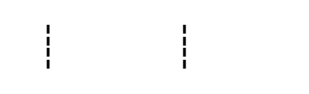

登

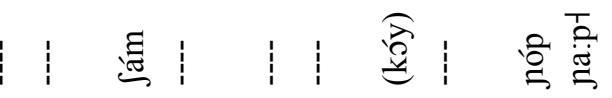

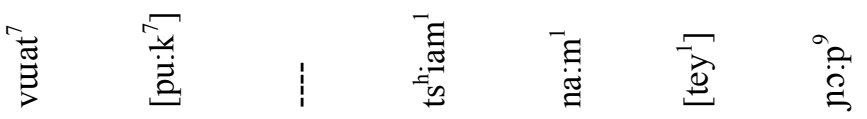

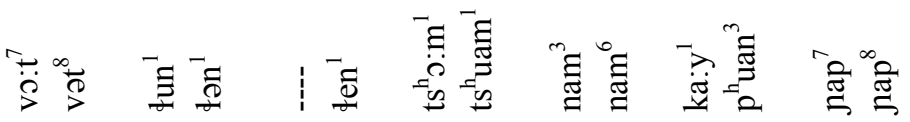

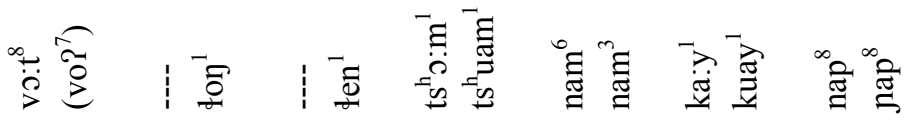

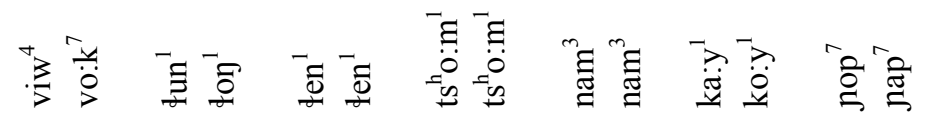

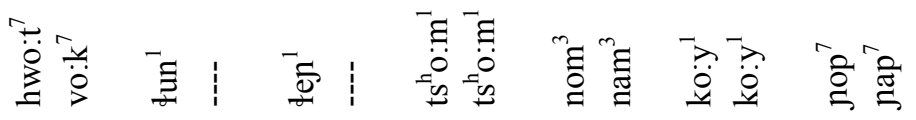

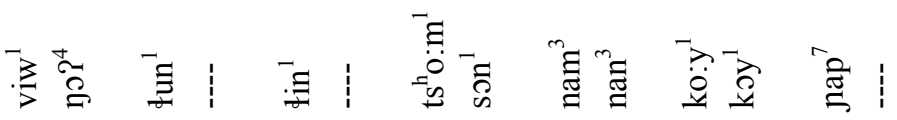

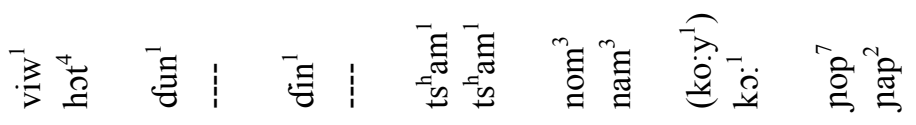

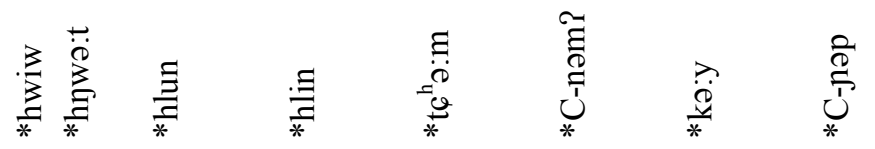

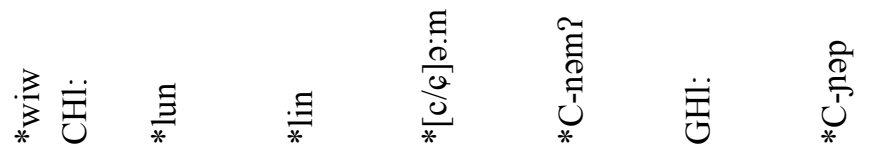

䓂高高

흥

勇

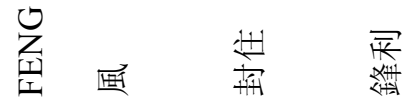

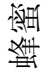

器 


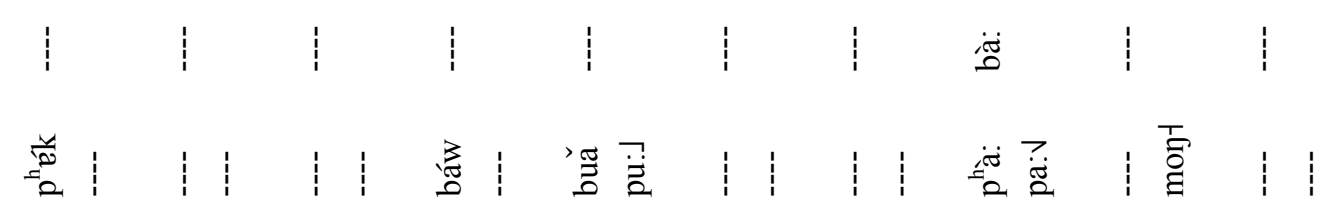

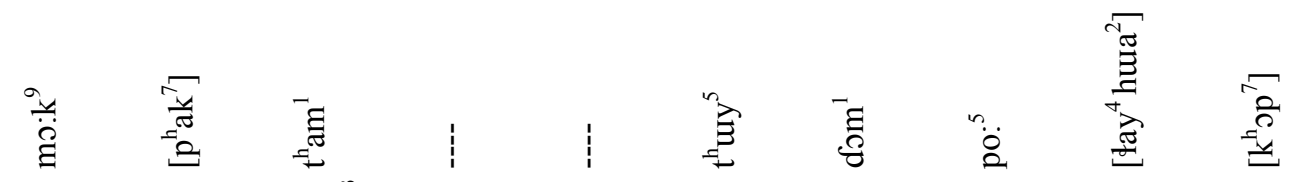

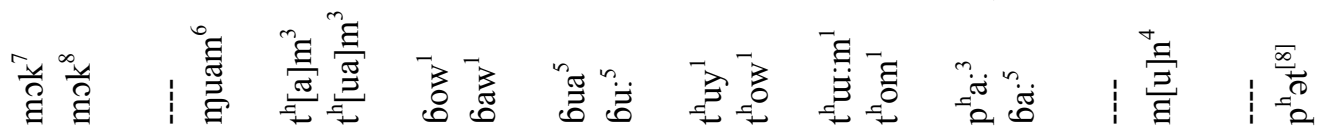

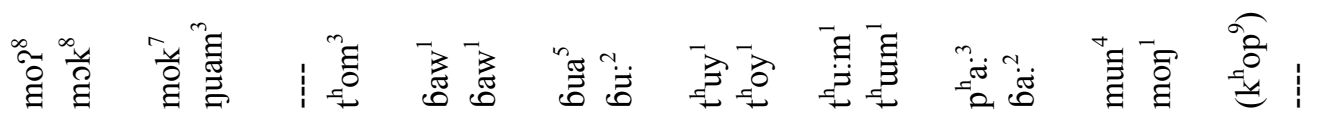

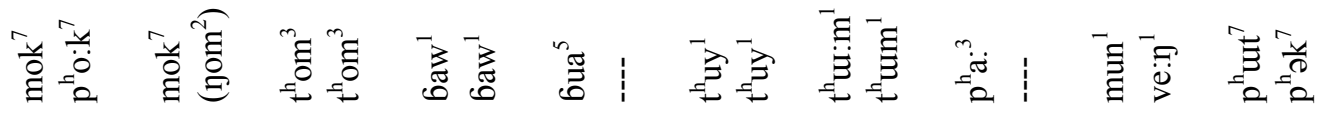

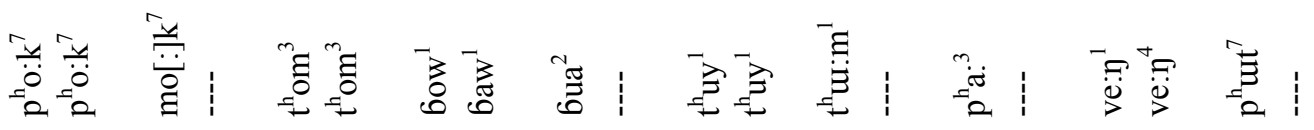

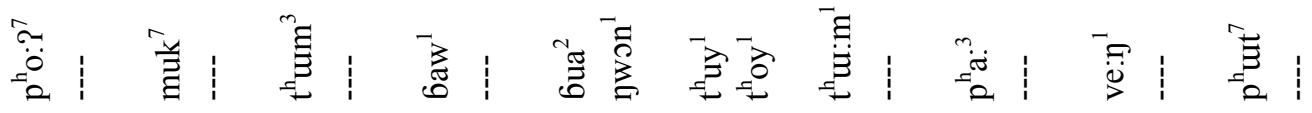

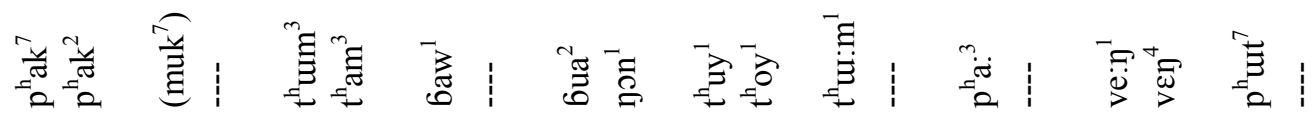

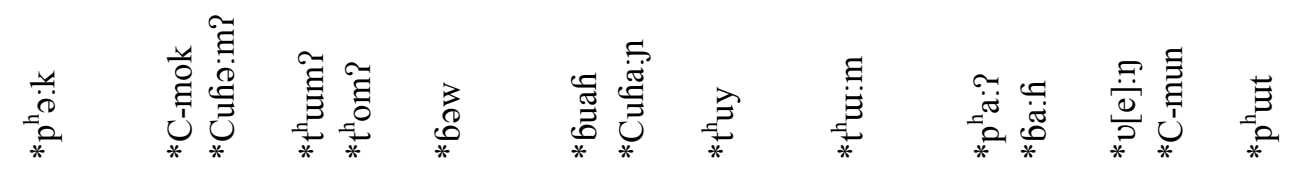

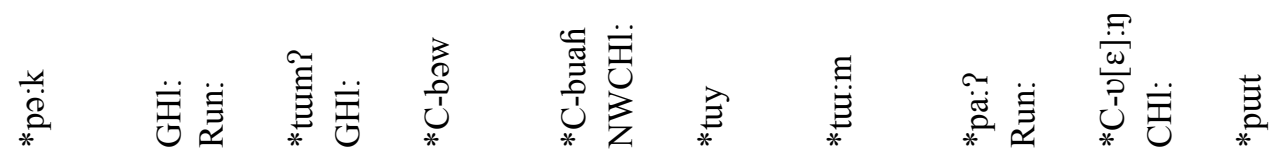

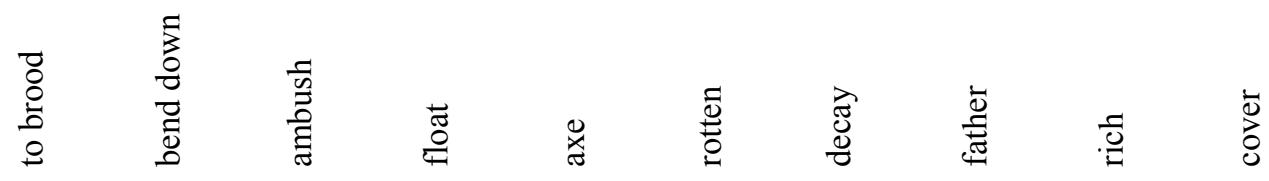

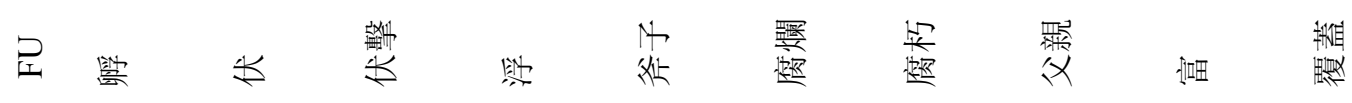




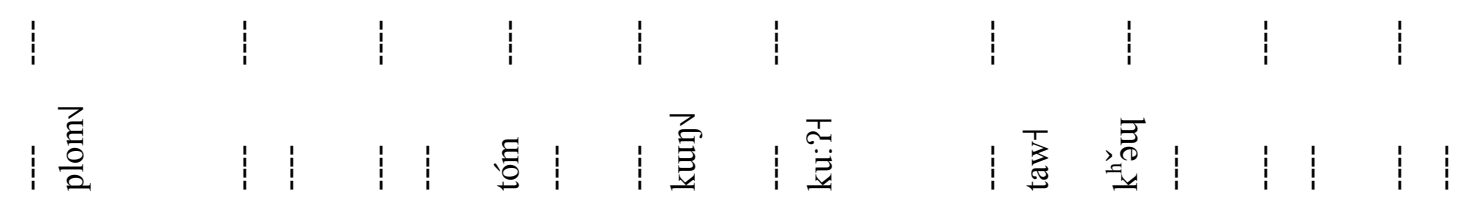

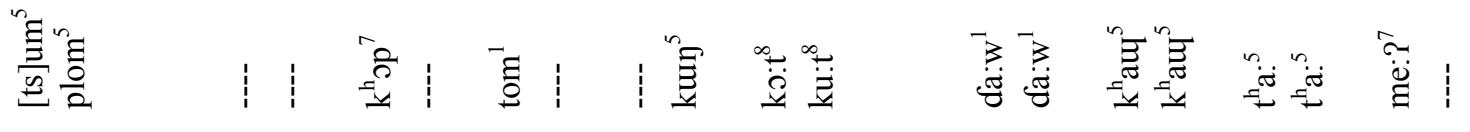

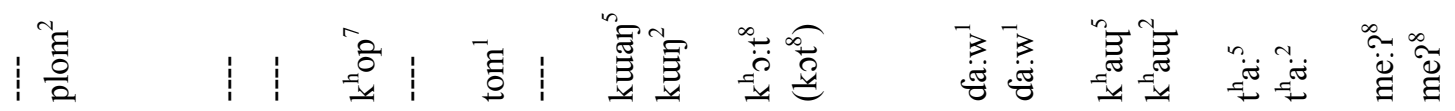

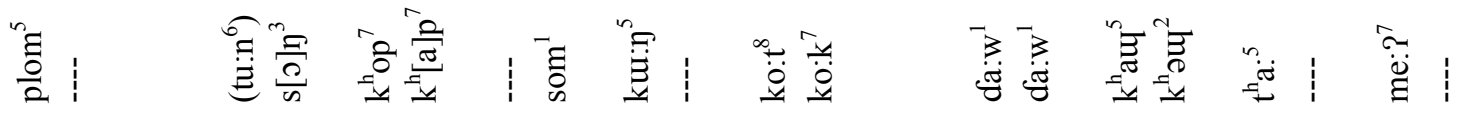

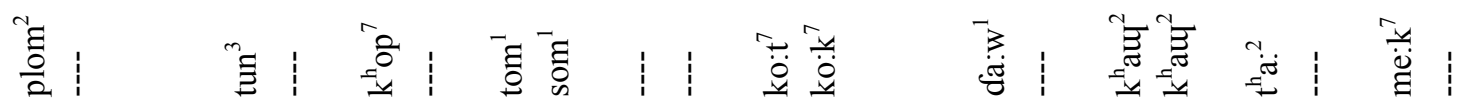

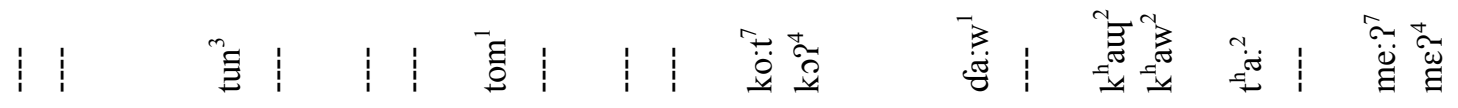

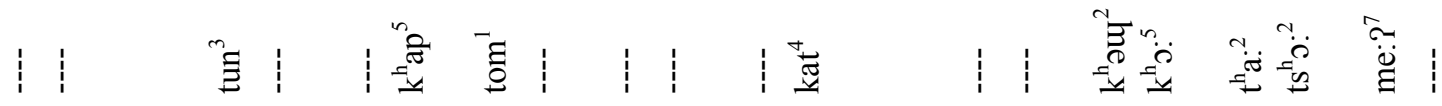

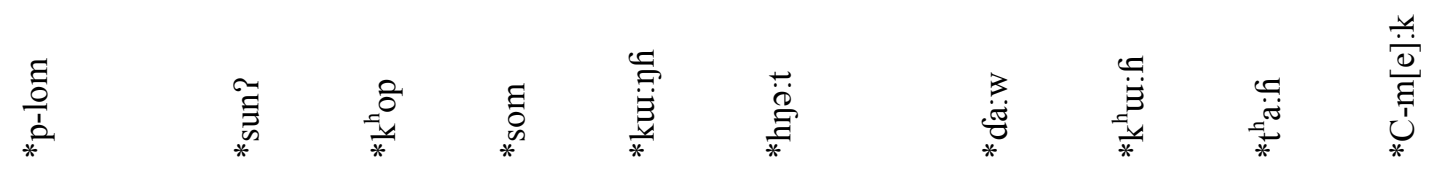

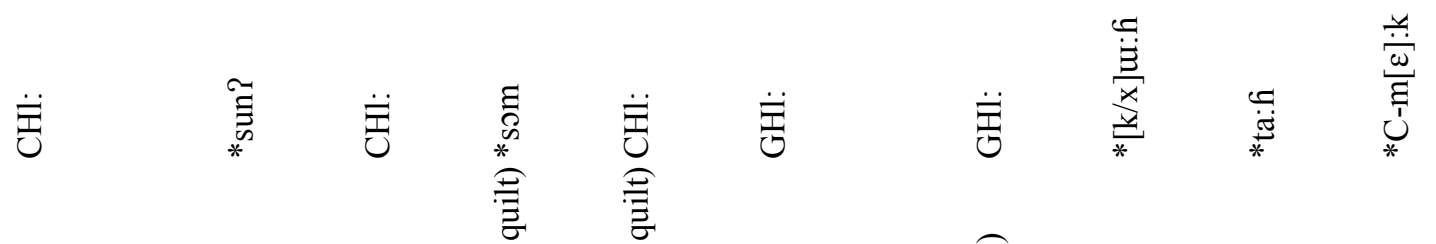

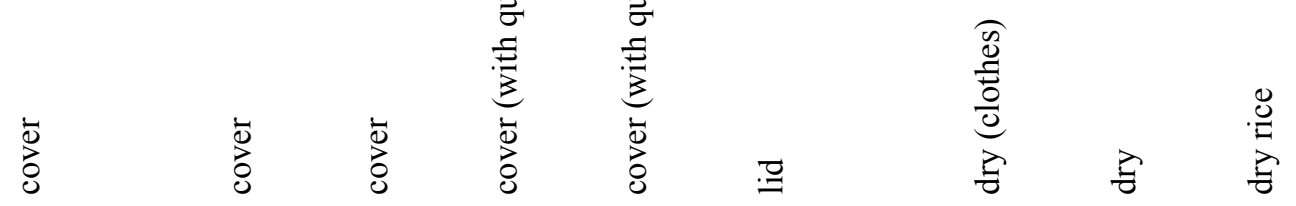

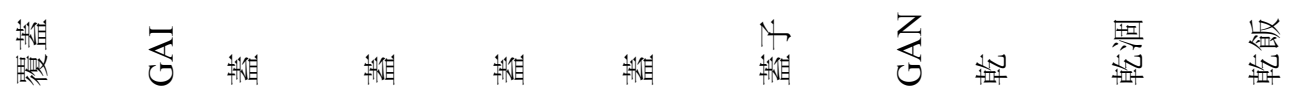




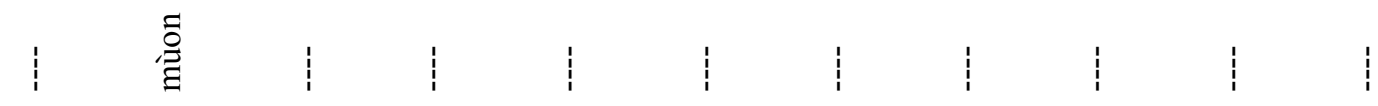

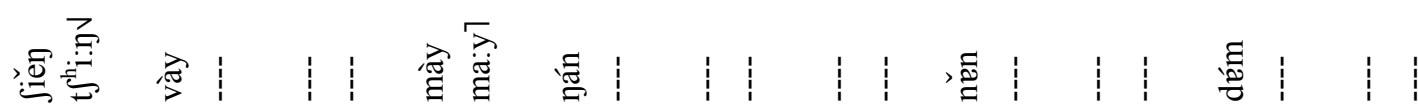

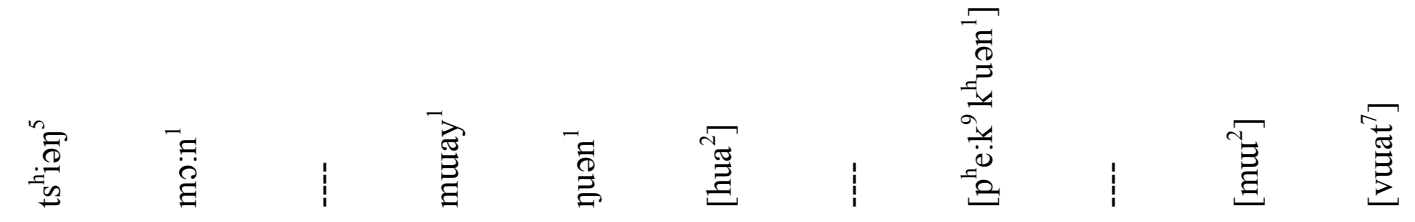
丞苛

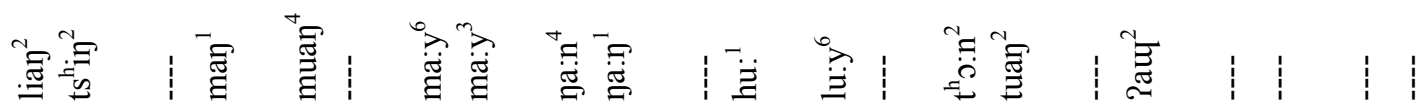

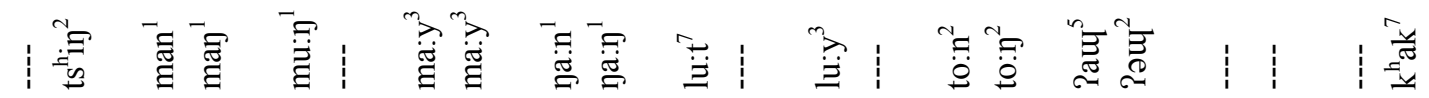

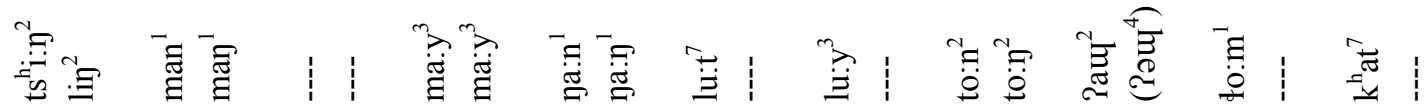

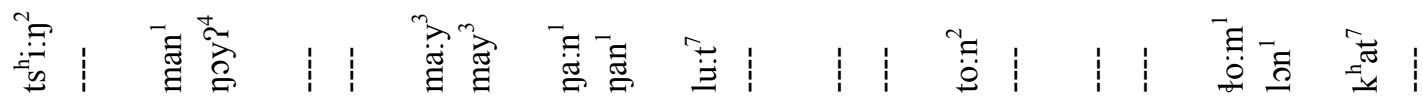

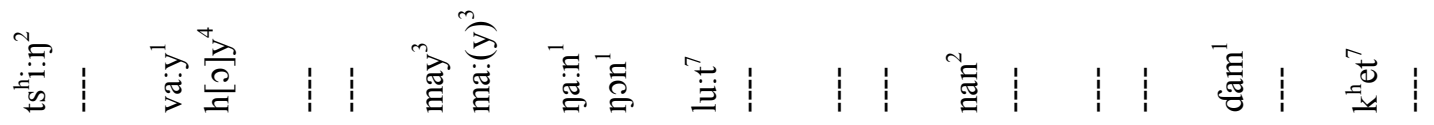

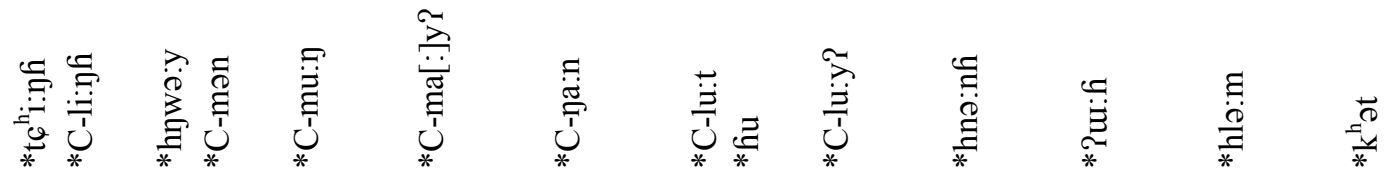

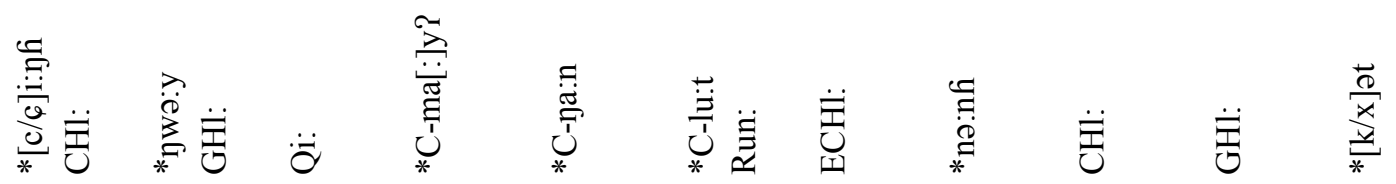

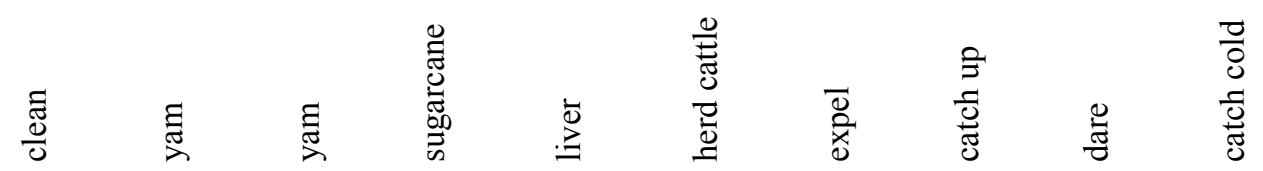

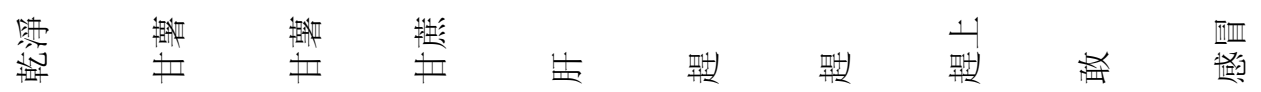




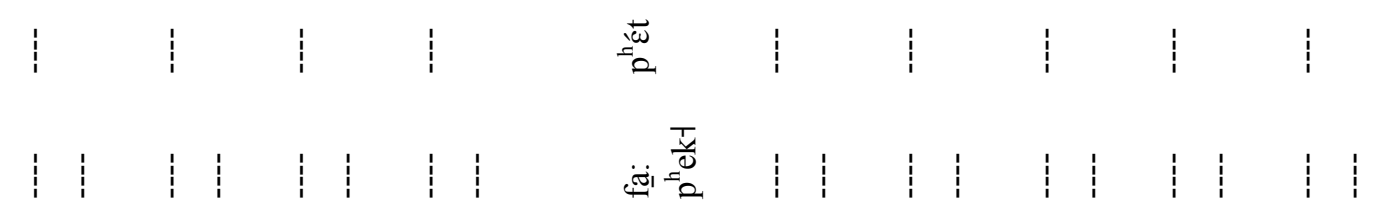

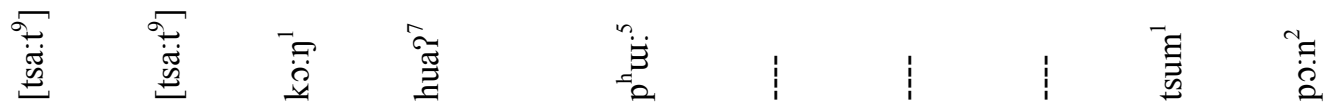

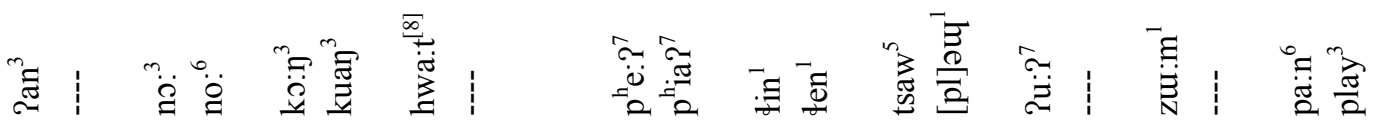

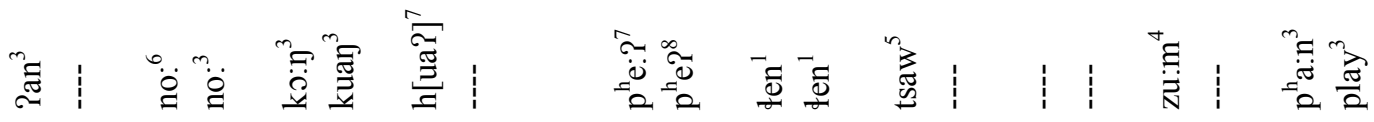

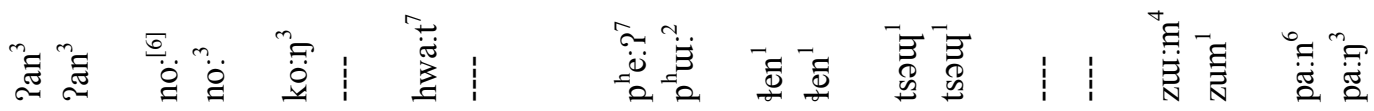

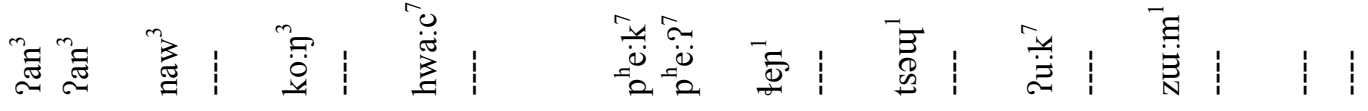

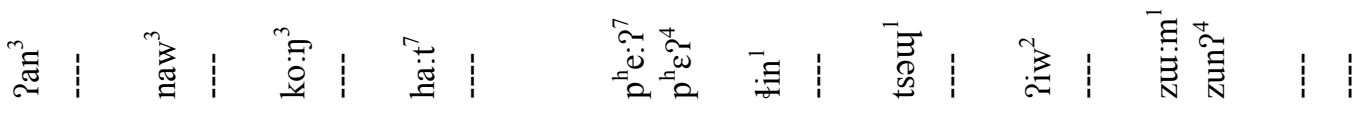

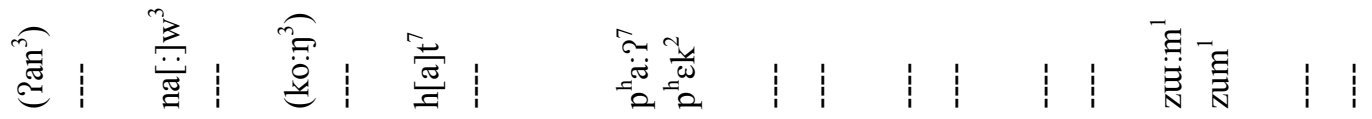

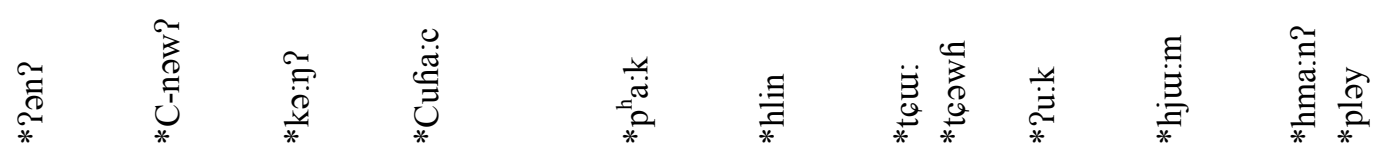

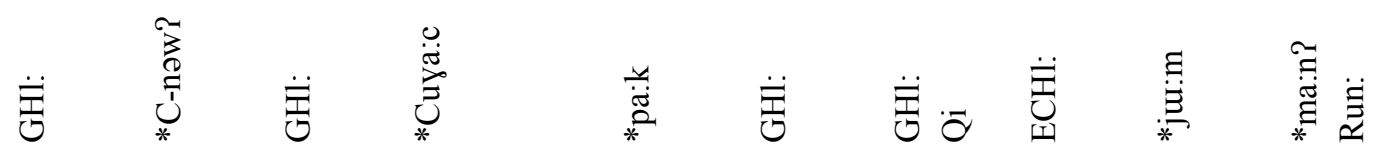

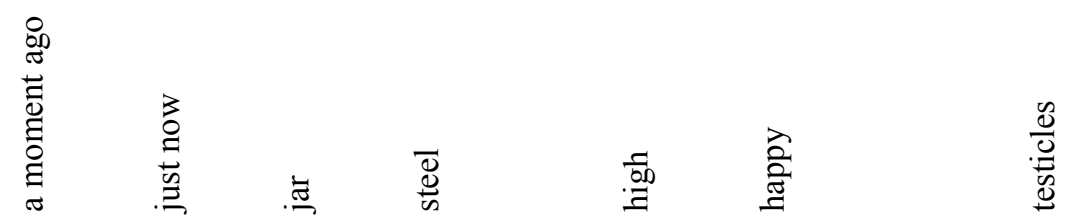

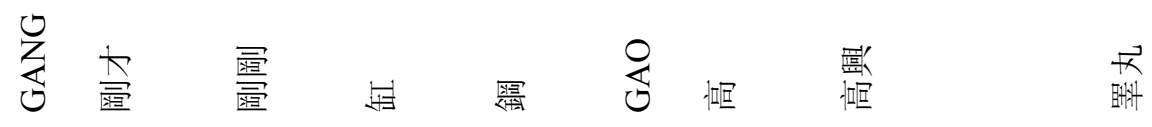




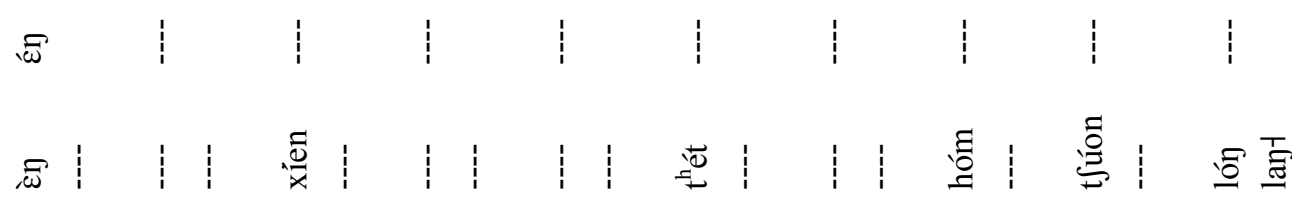

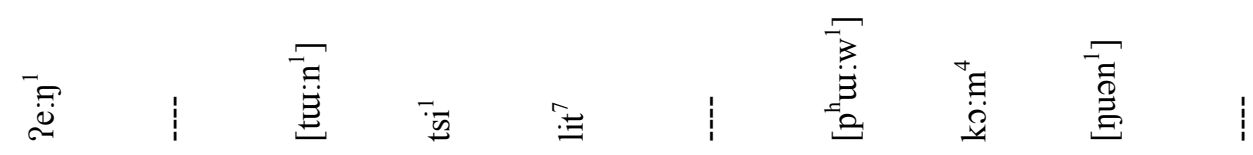

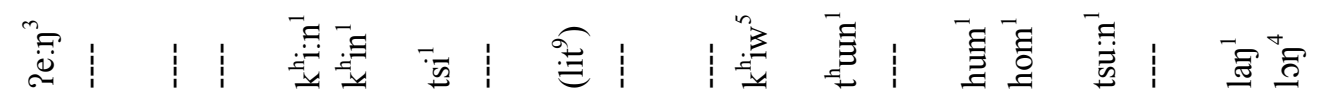

勇|| |

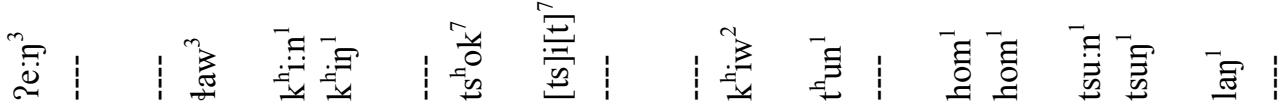

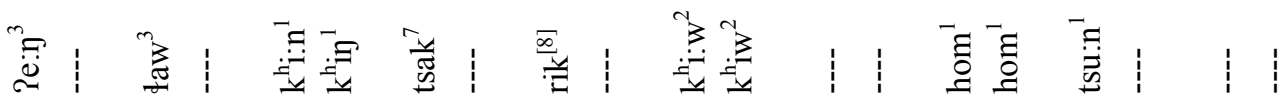

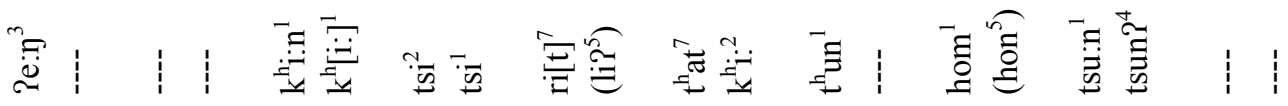

莒

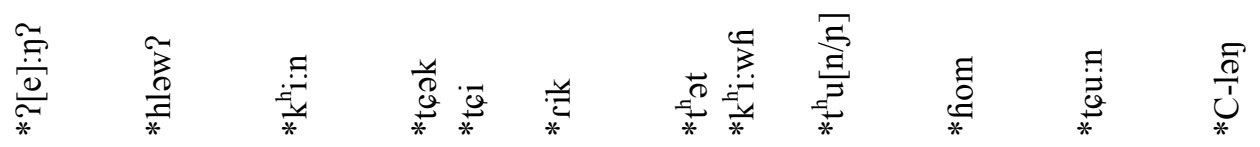

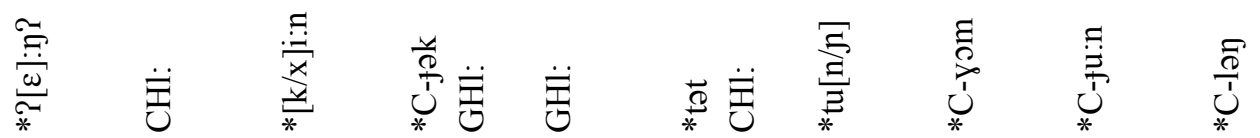

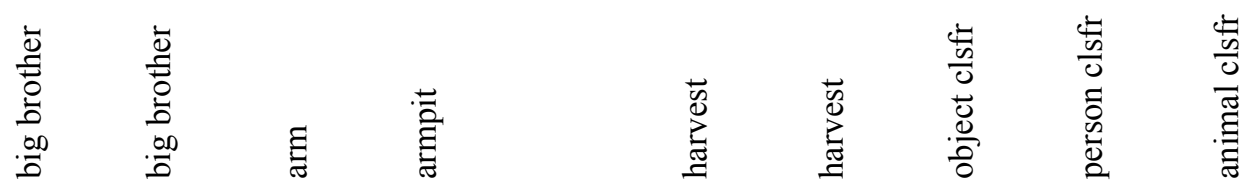

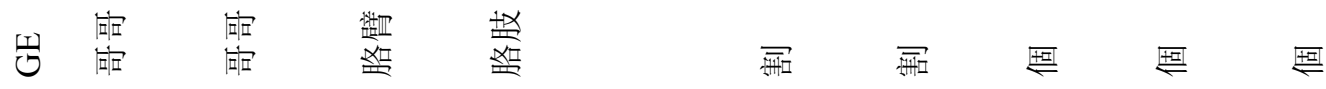




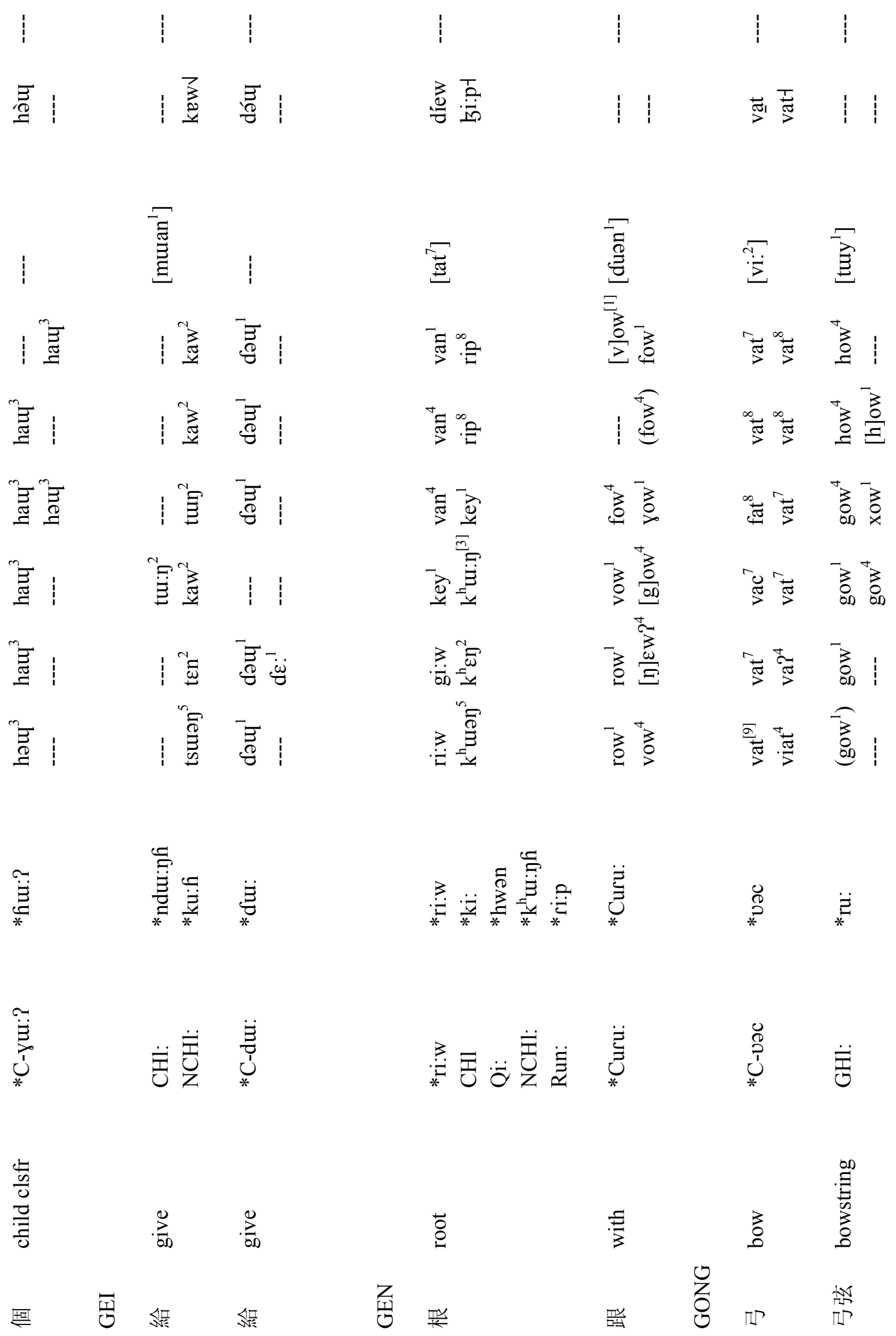


$11+111111$

造| 窝:

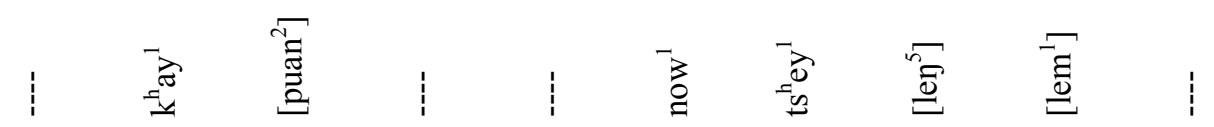

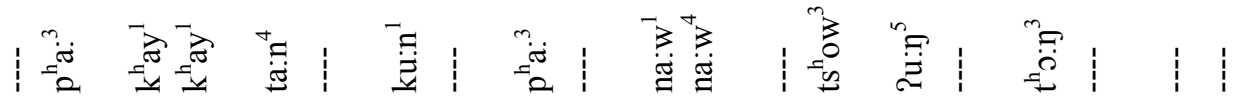

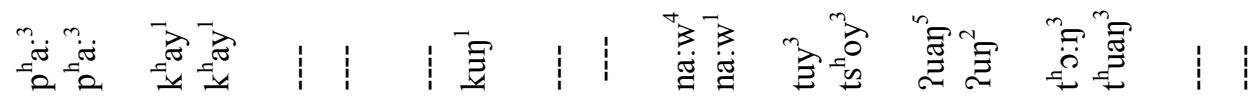

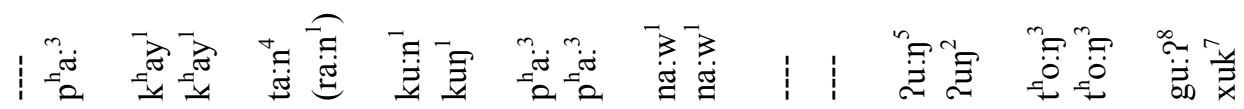

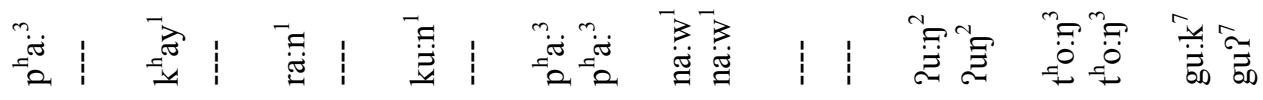

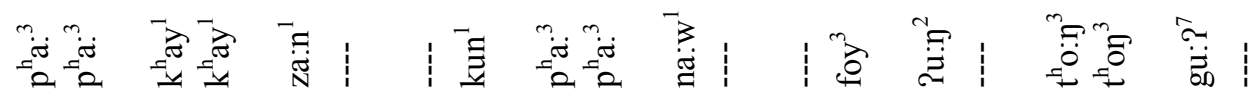

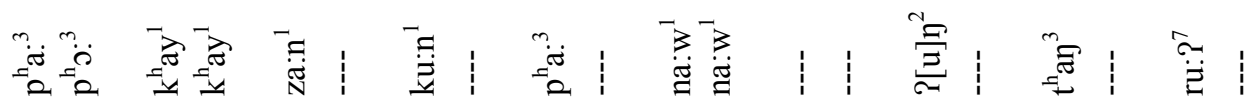

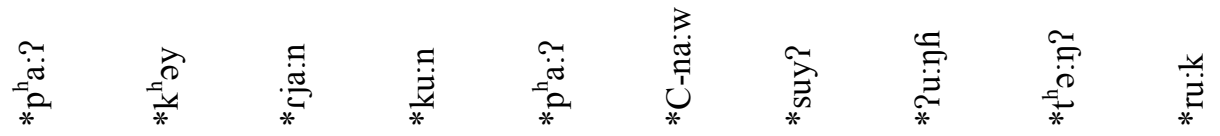

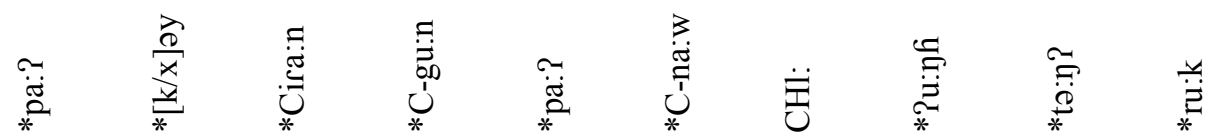

高

高

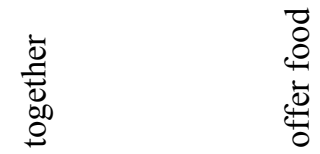

䈍

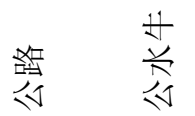

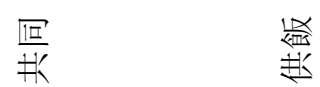




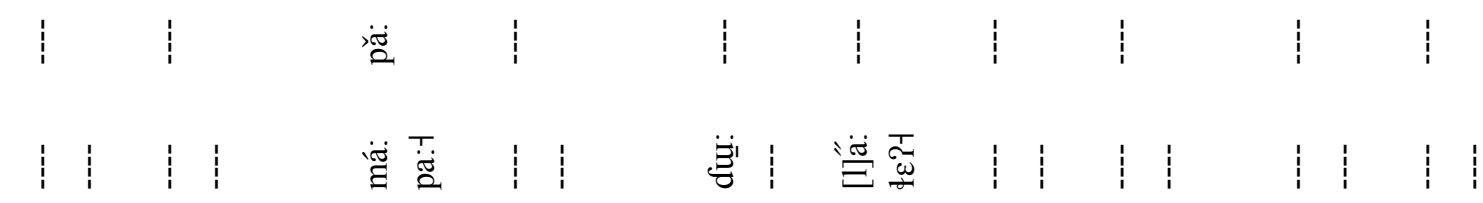

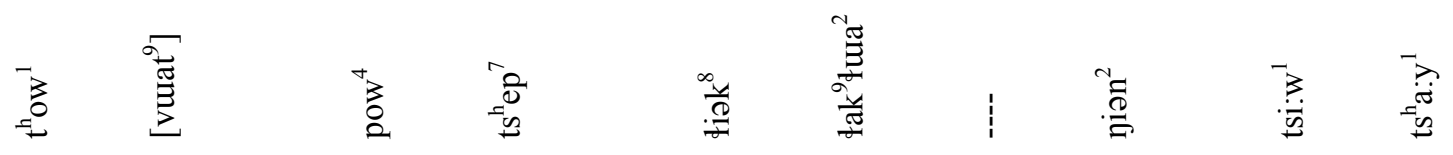

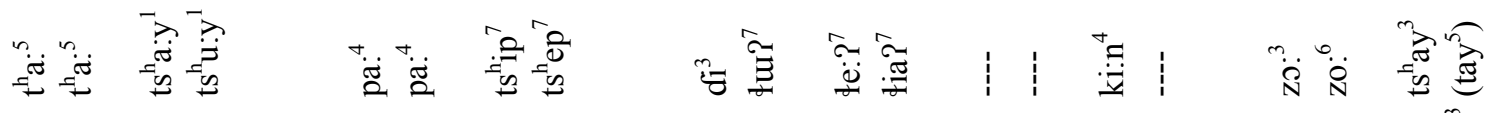

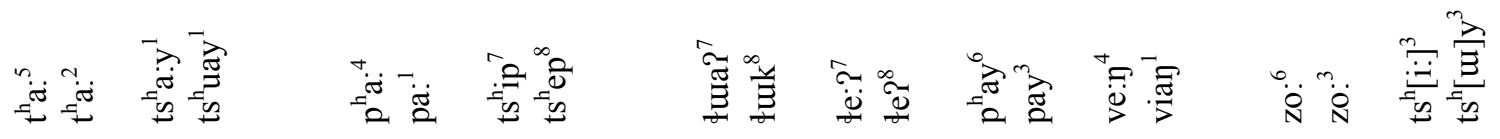

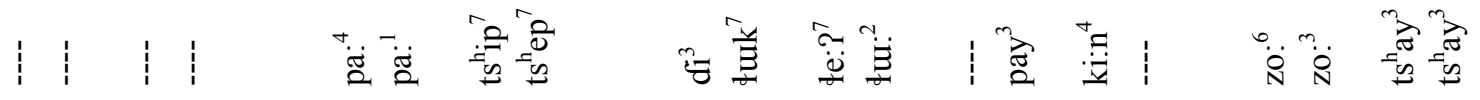

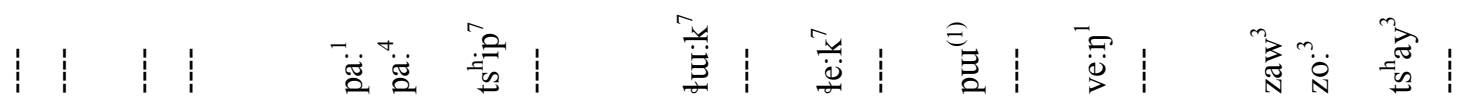

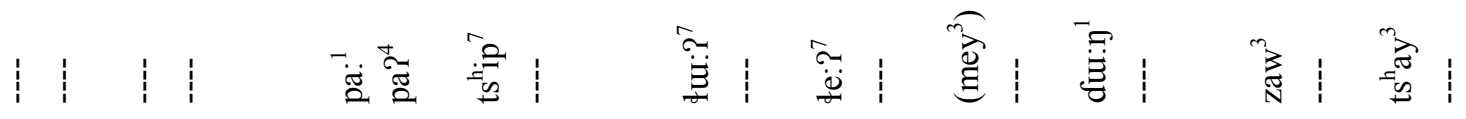

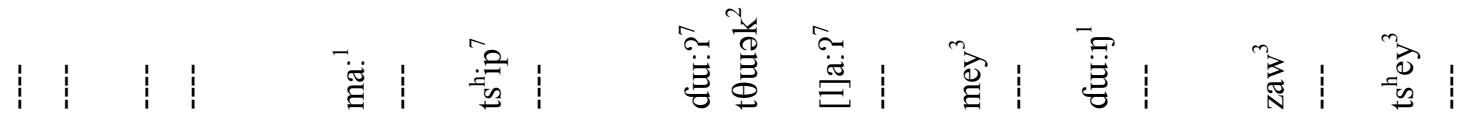

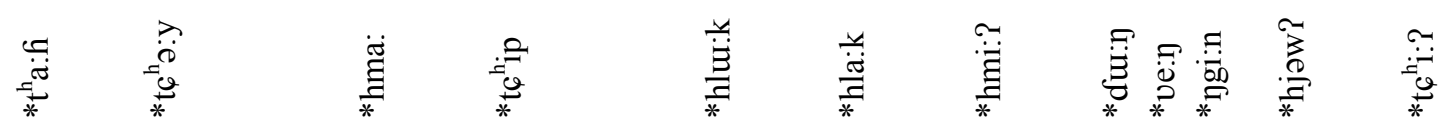

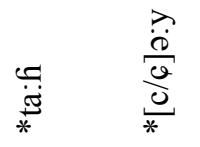

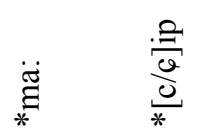
$\stackrel{4}{*}$

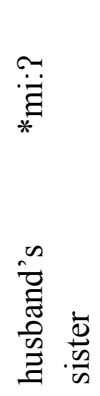

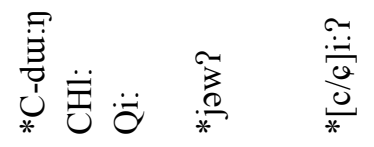
总
임

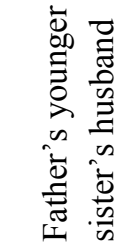

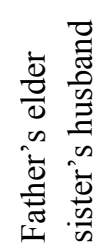

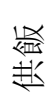

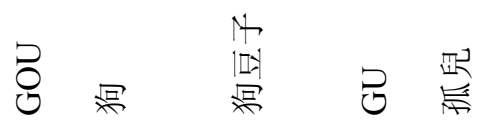
标

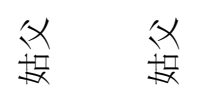




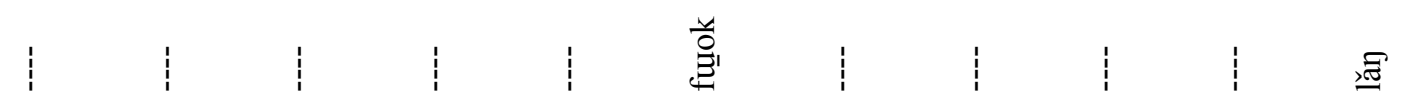

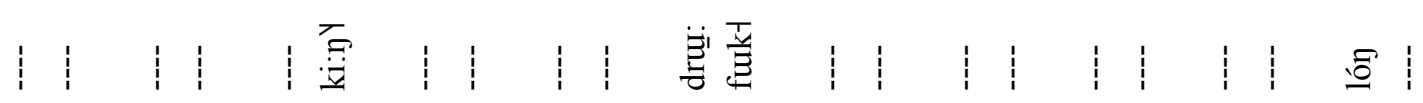

盈|

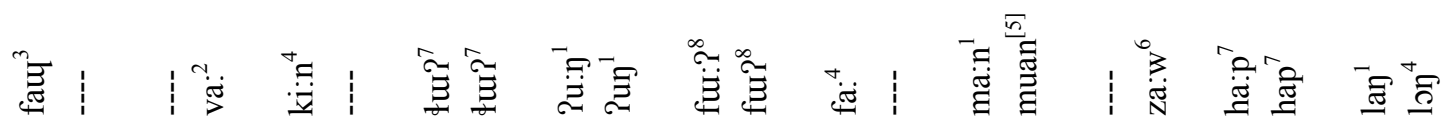

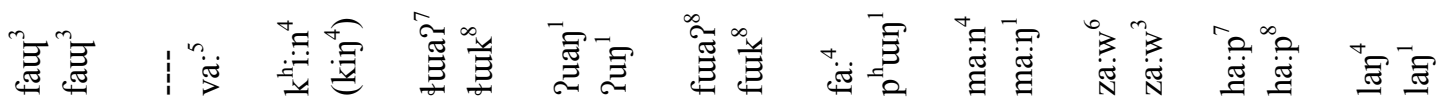

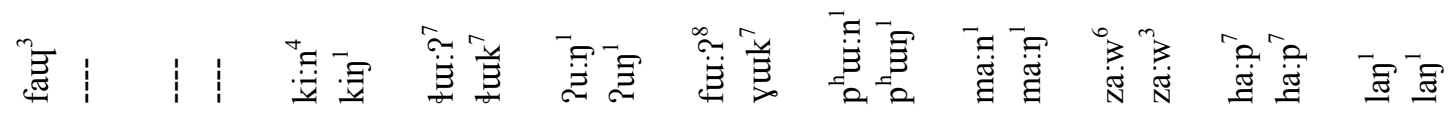

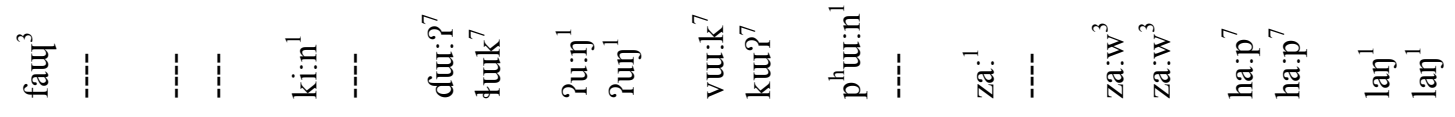

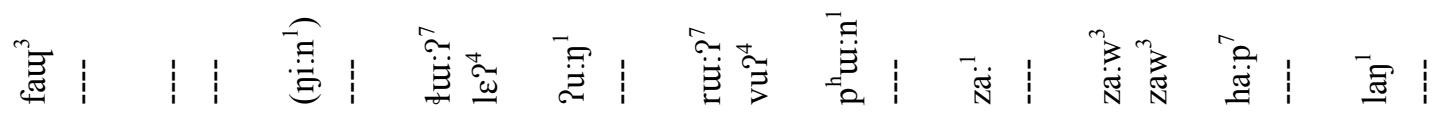

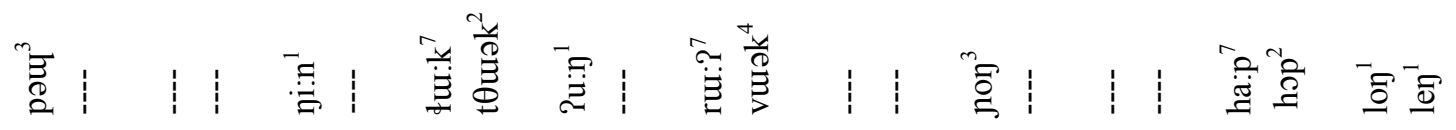

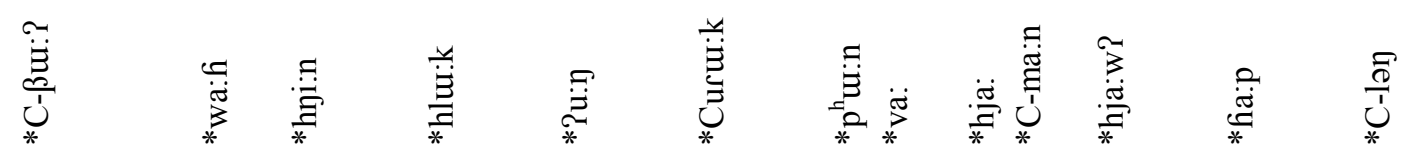

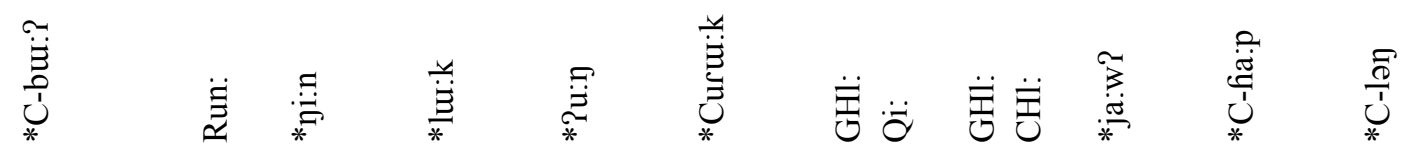
$\begin{array}{llllll} & & & & & \\ \end{array}$ 


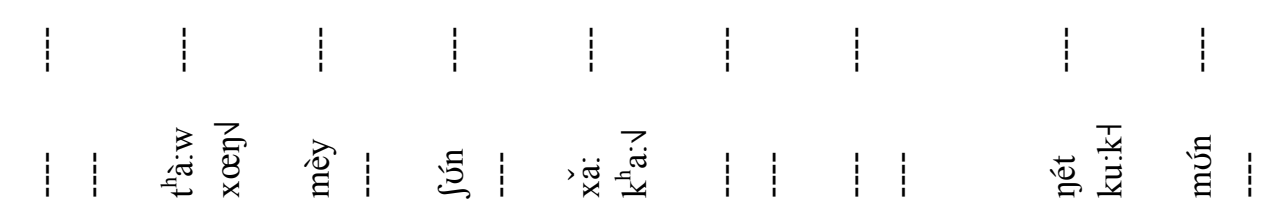

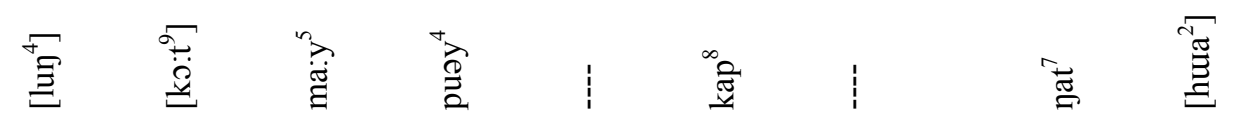

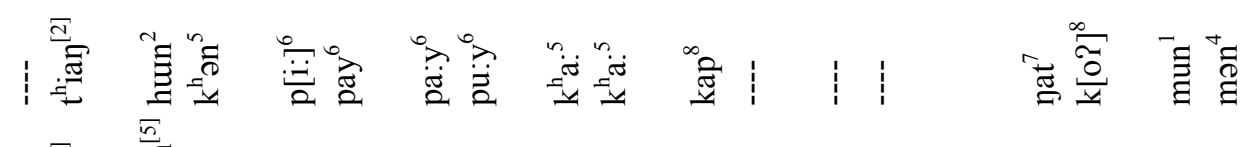

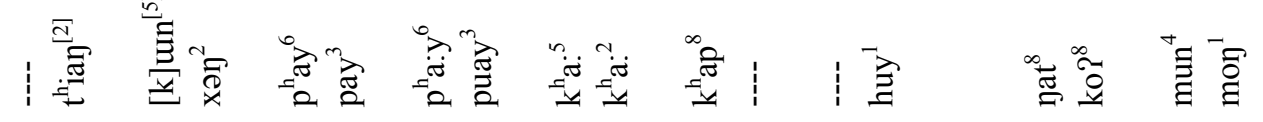

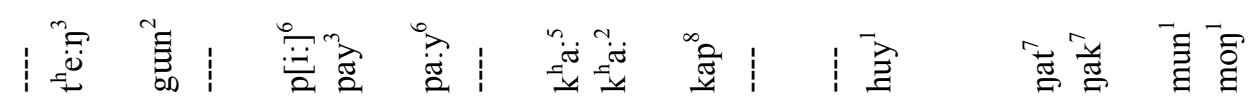

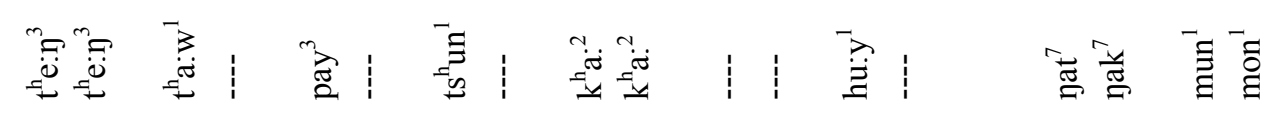

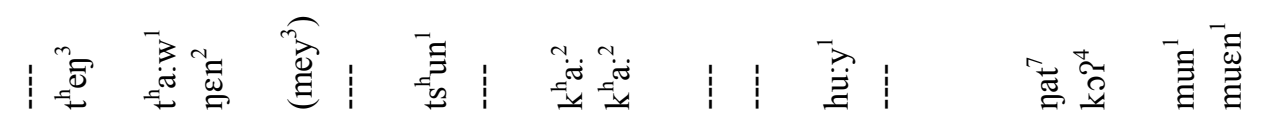

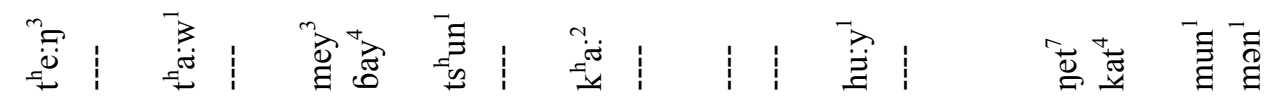

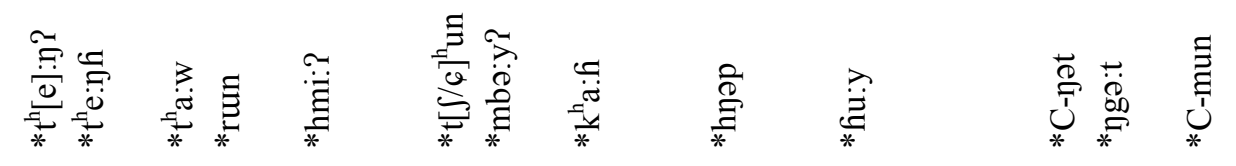

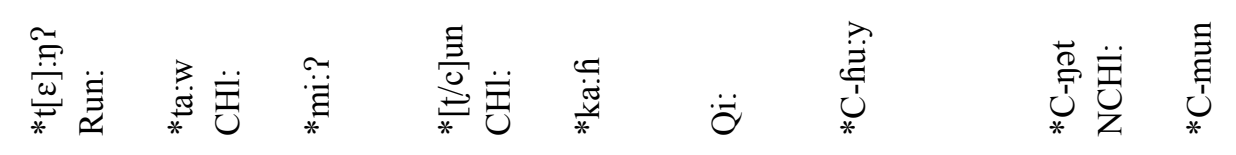

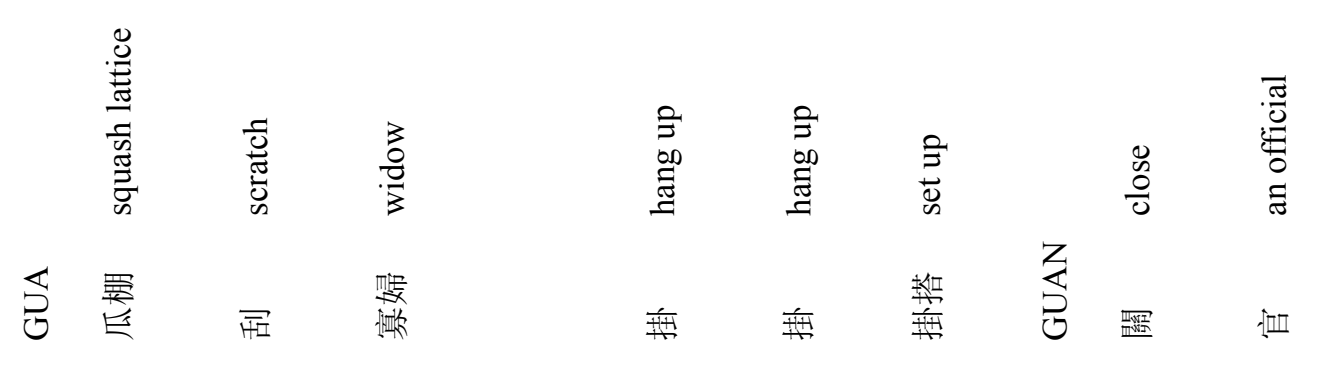




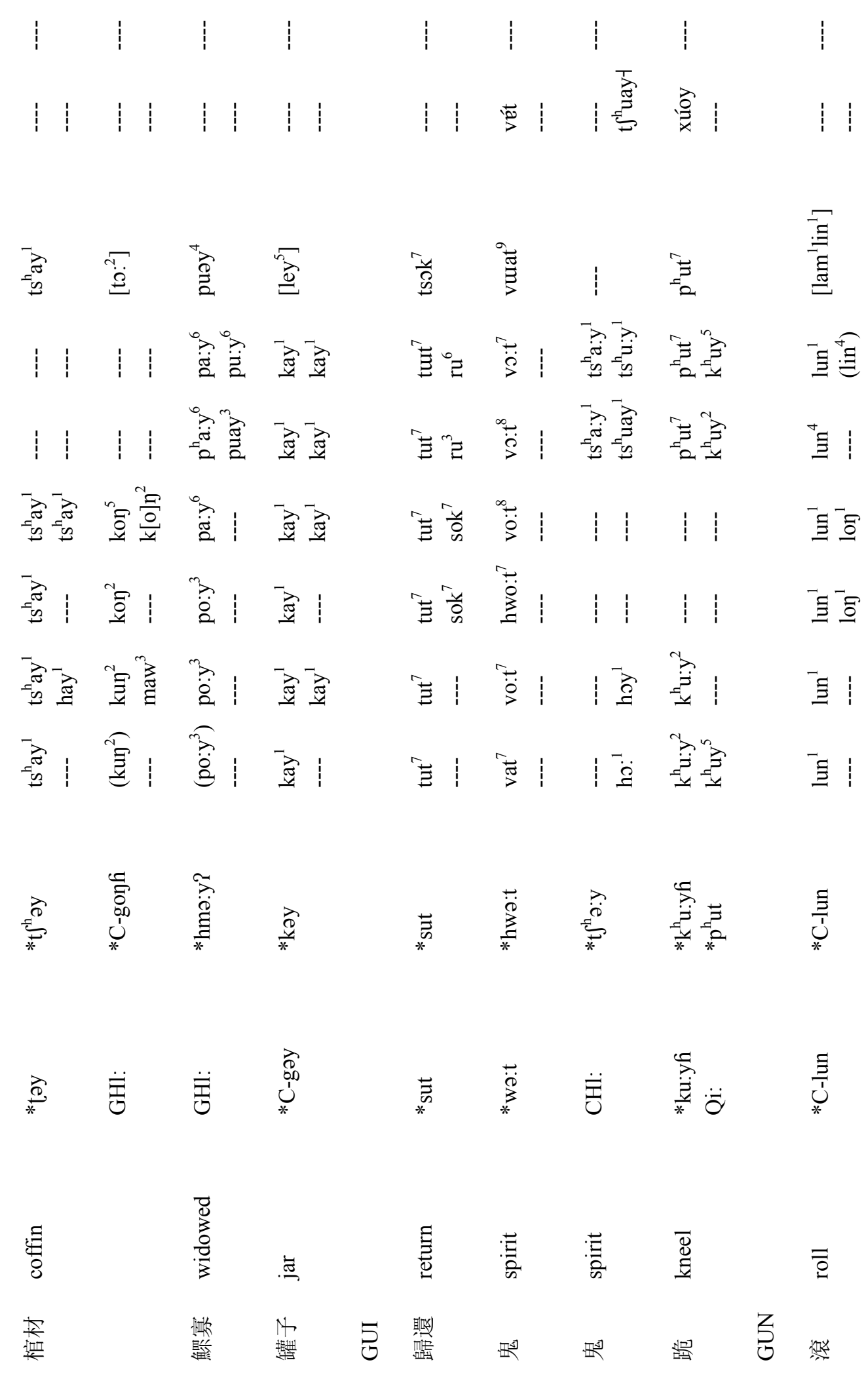




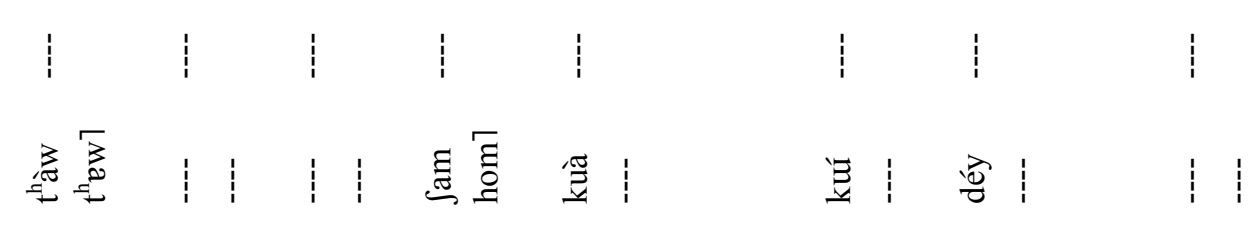

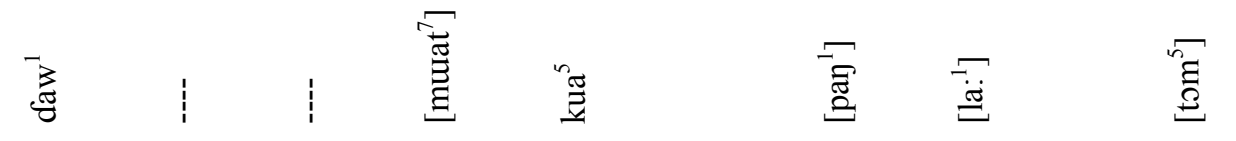

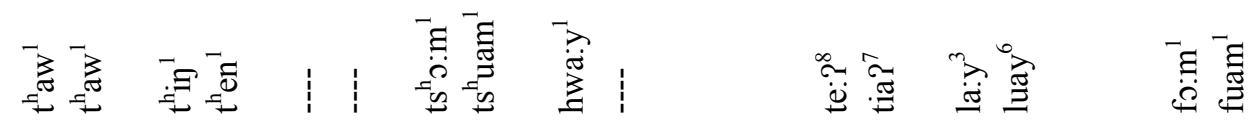

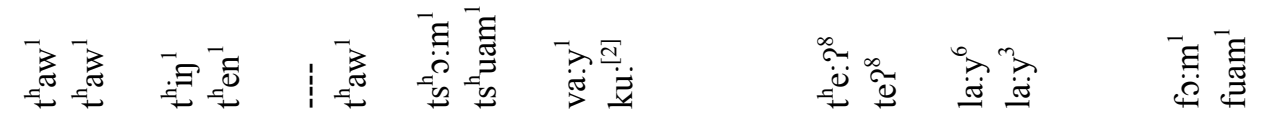

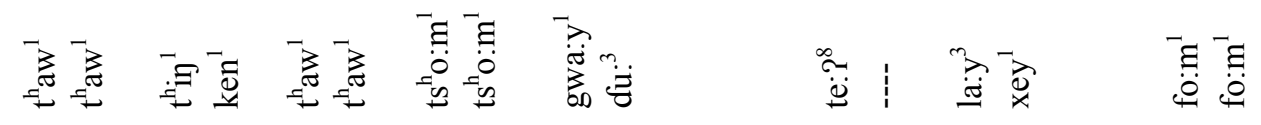

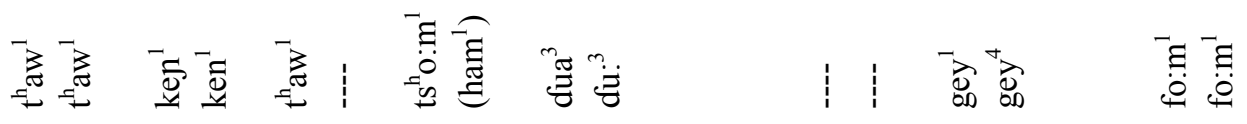

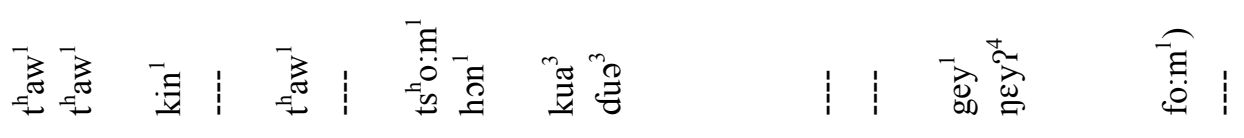

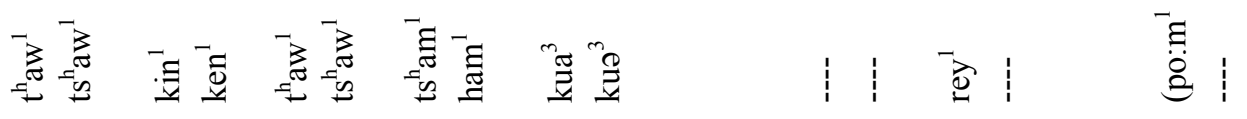

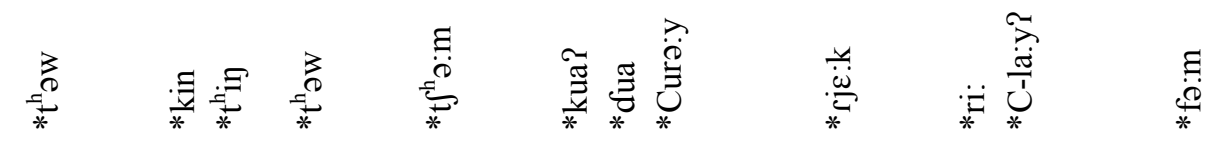

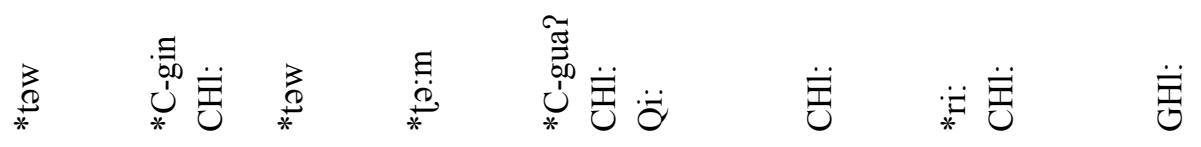

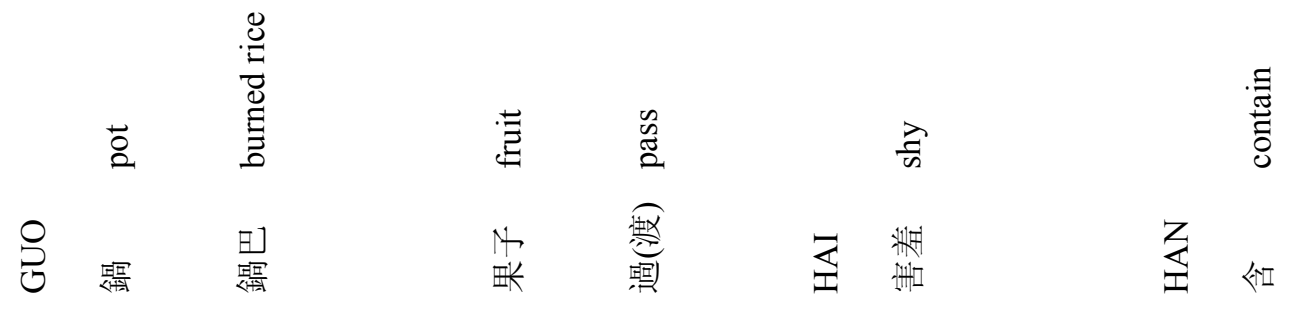




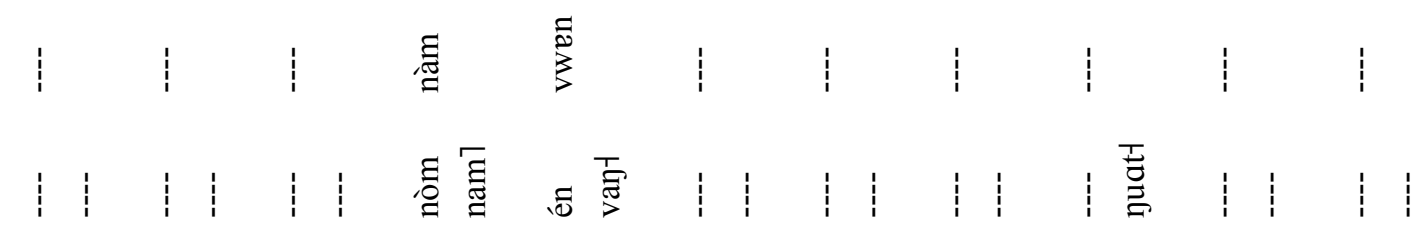

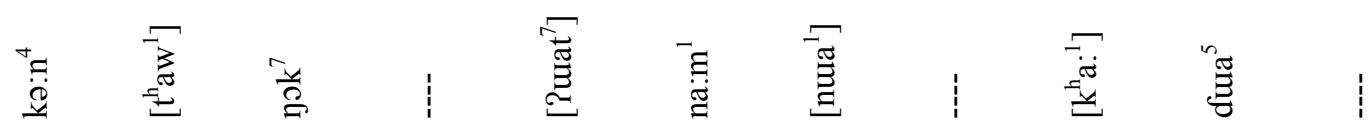

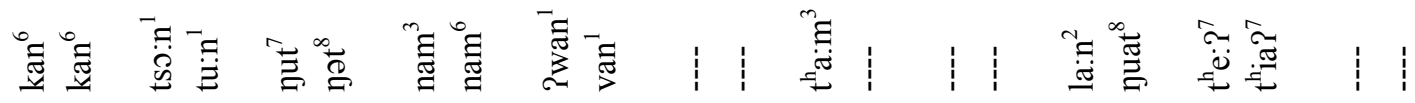

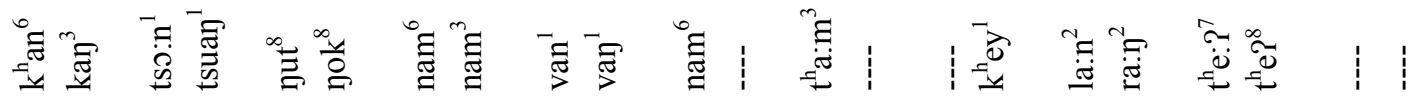

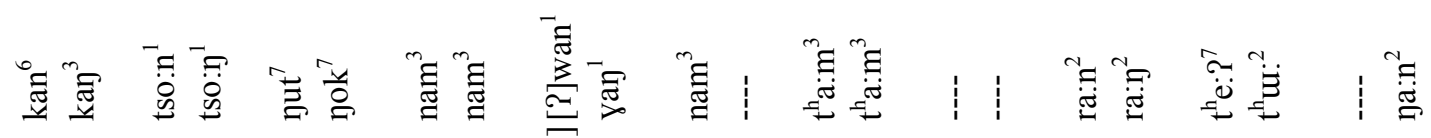

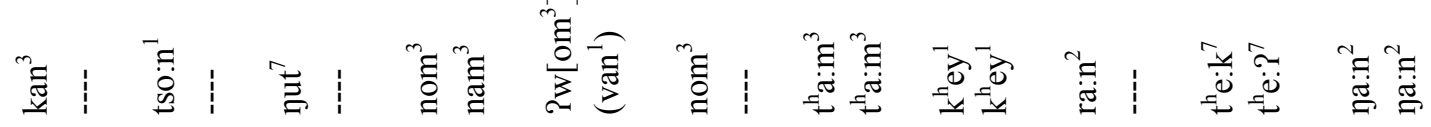

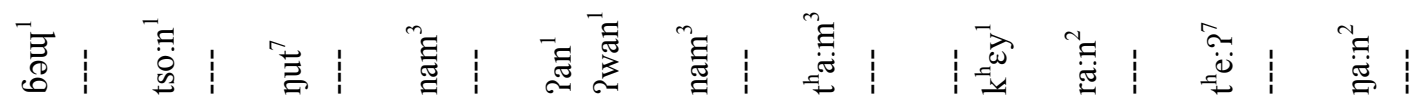

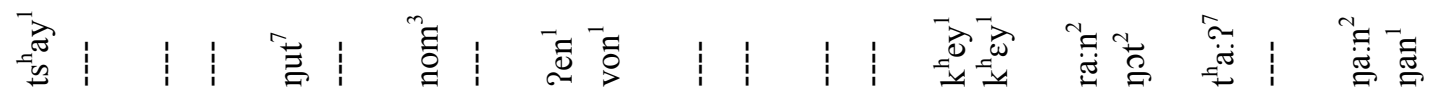

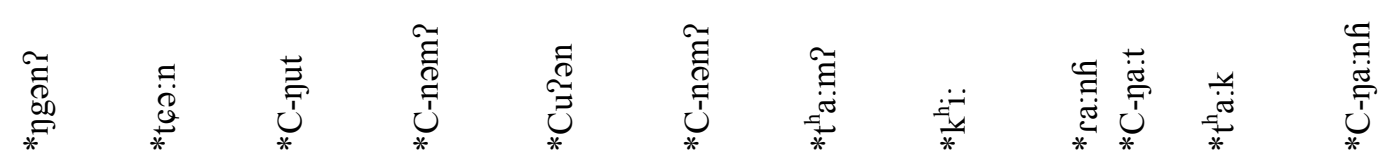

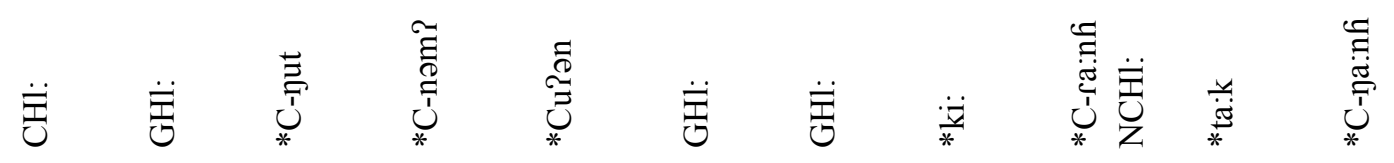

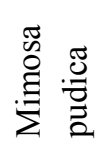

捑

将स

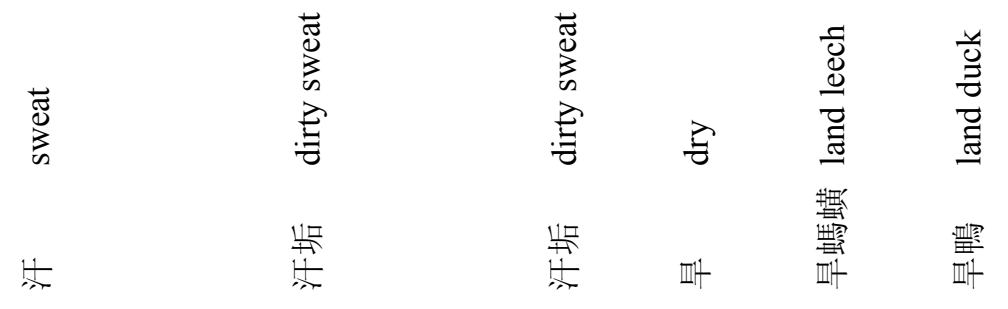




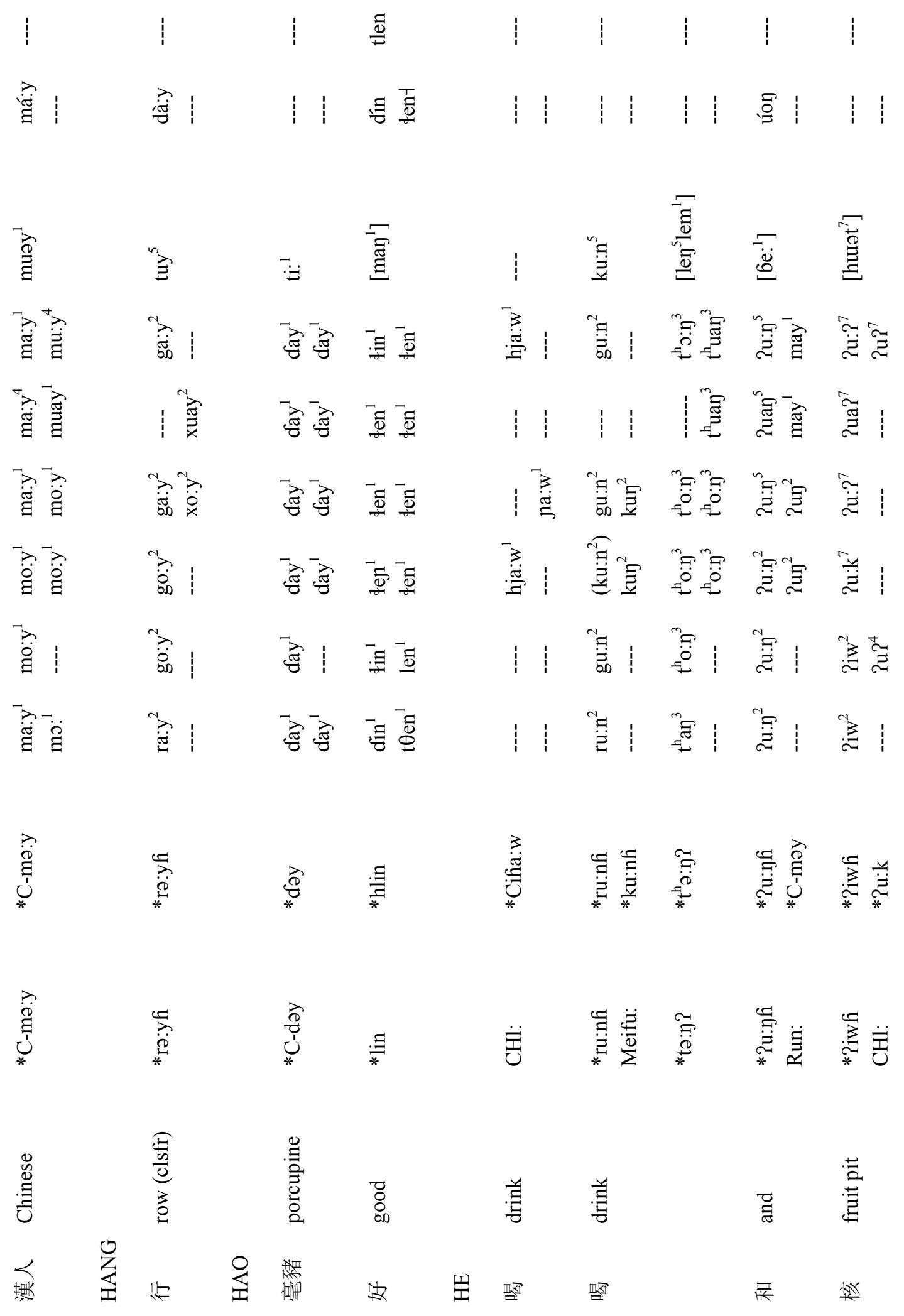




$$
\begin{aligned}
& 1 \quad 1 \quad 1 \text { 泾 } 111
\end{aligned}
$$

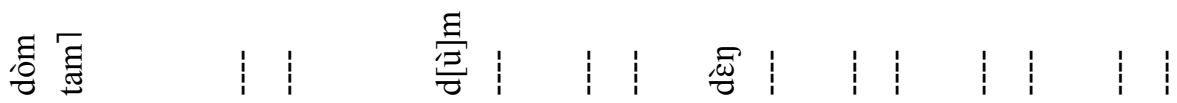

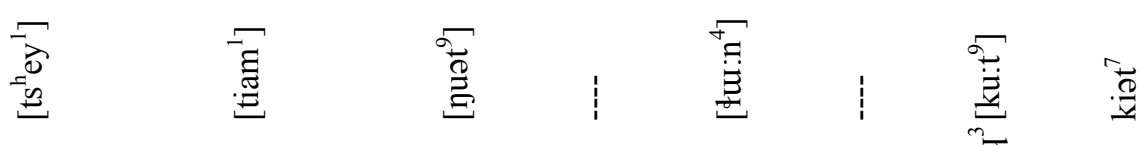

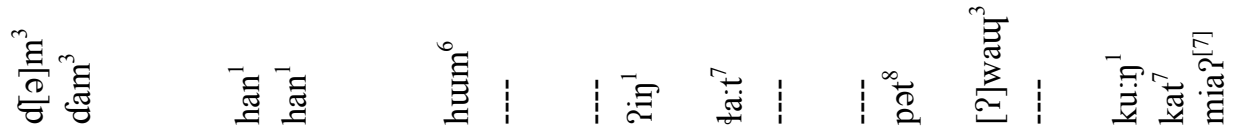

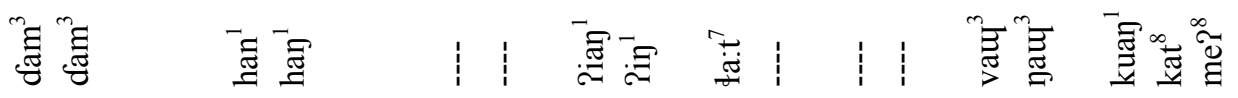

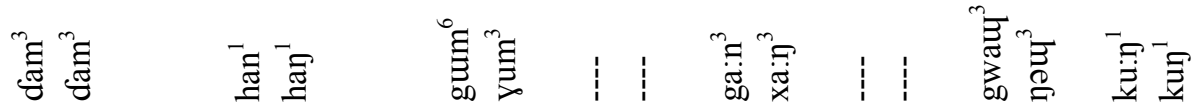

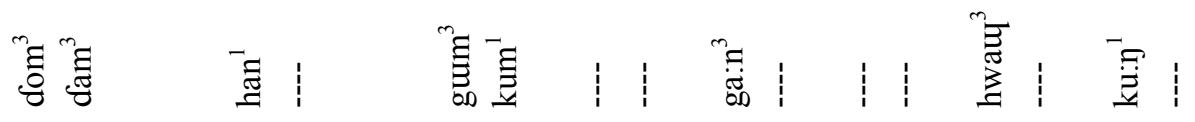

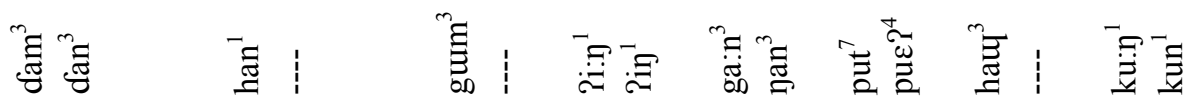

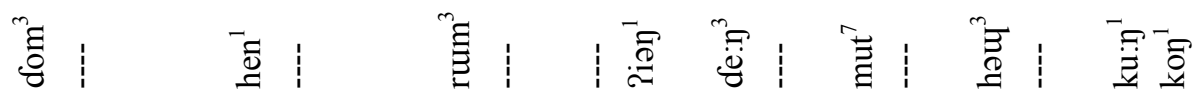

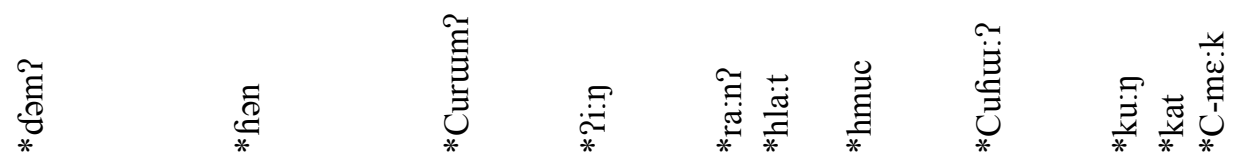

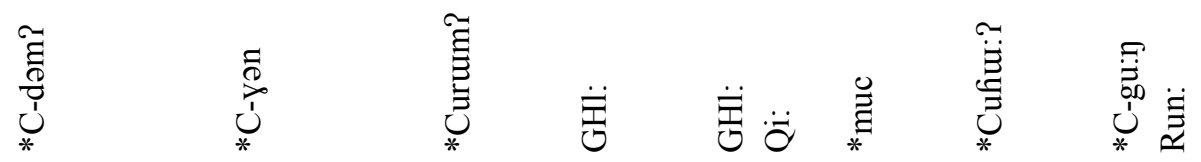

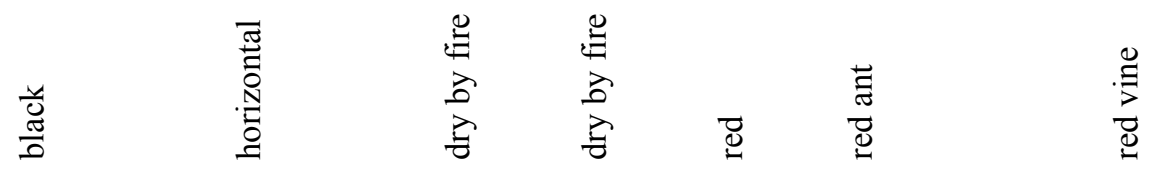

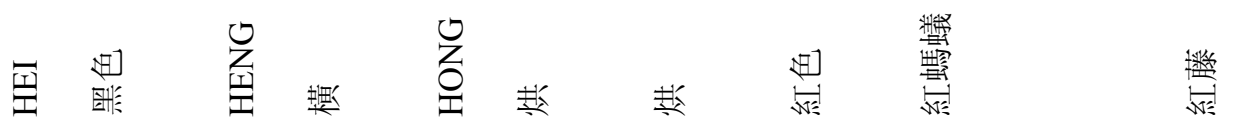




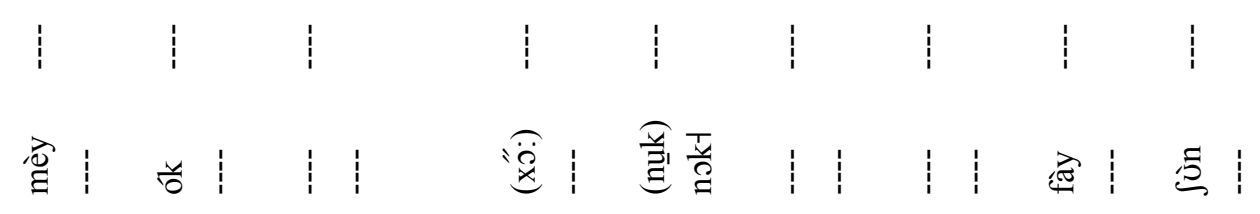

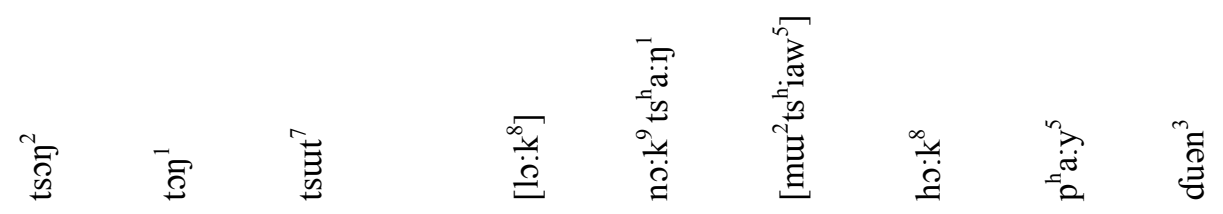

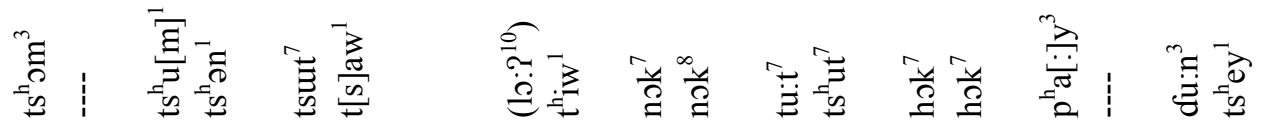

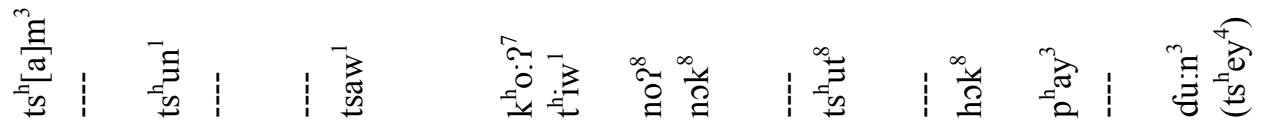

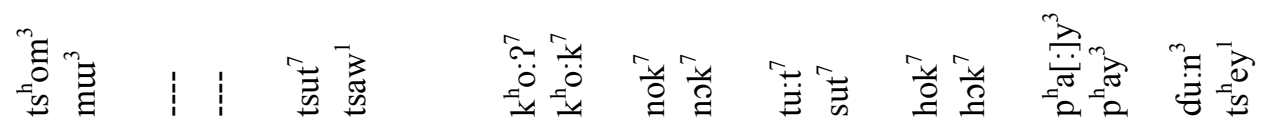

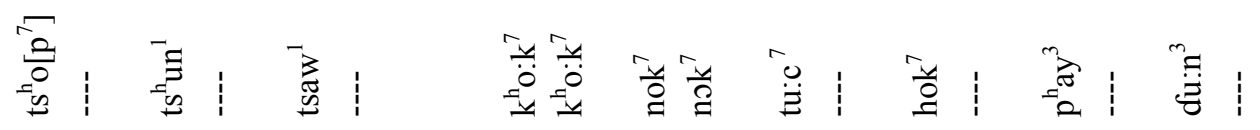

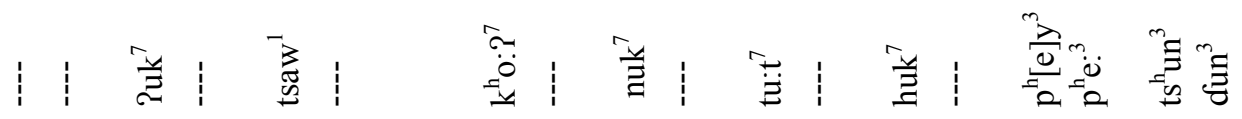

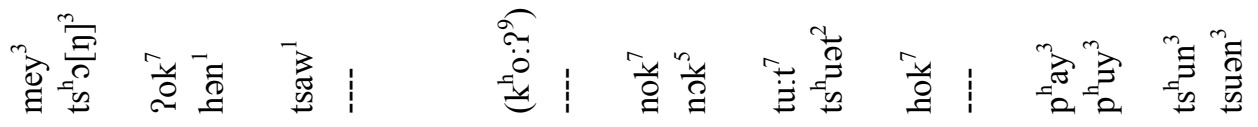

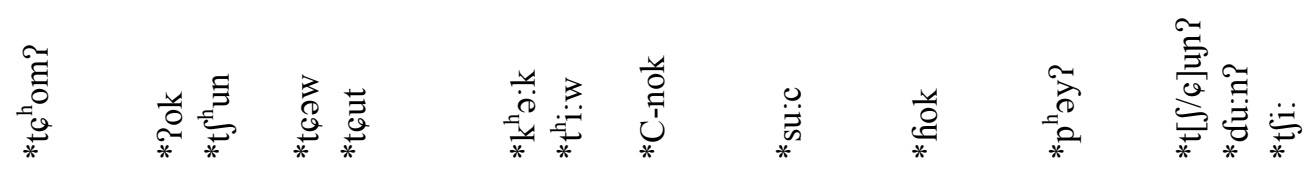

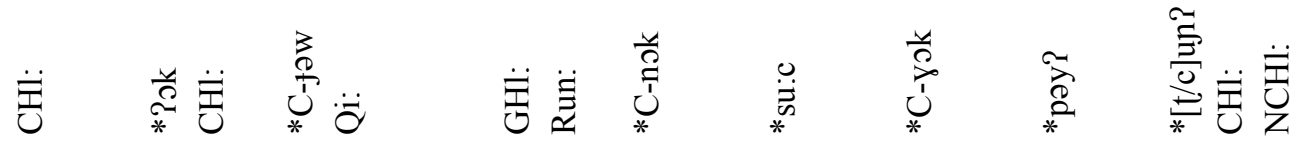

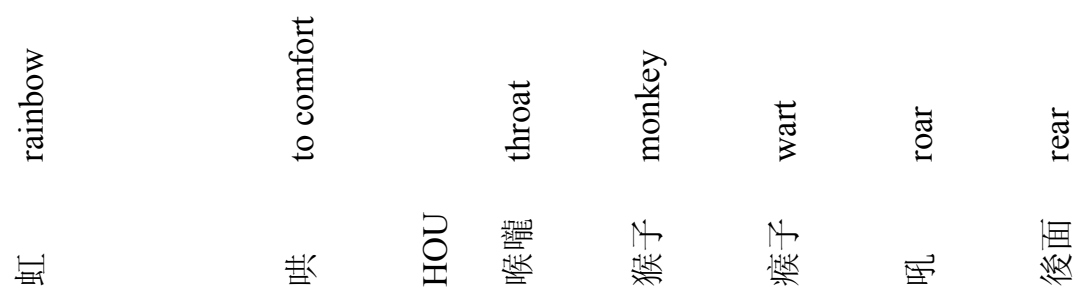




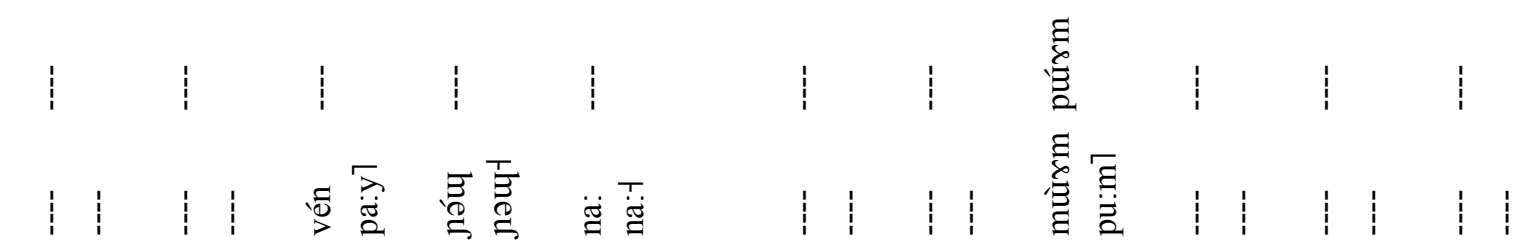

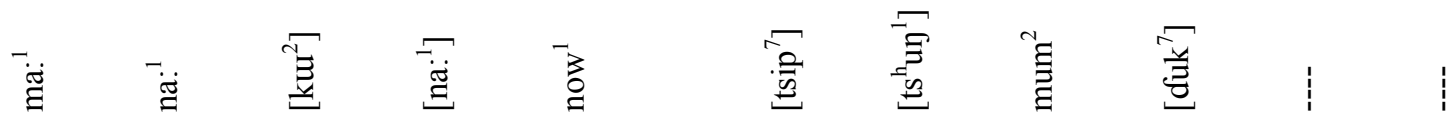

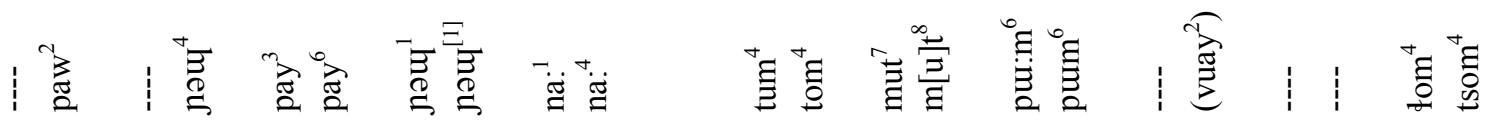

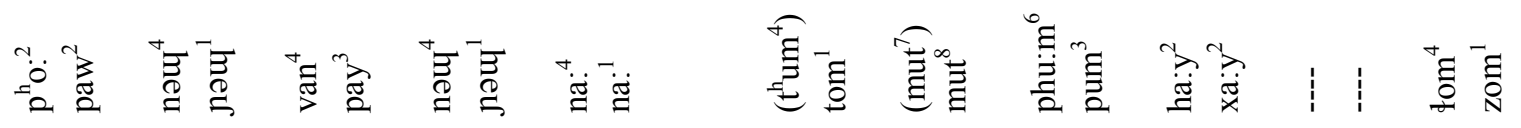

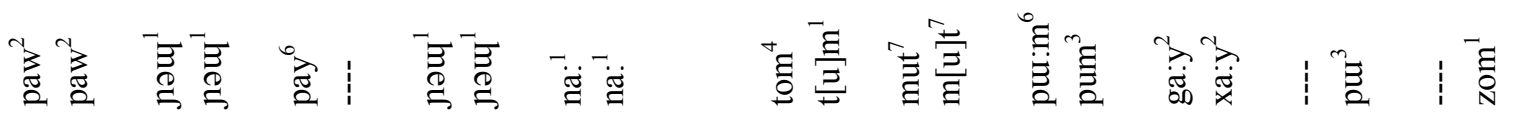

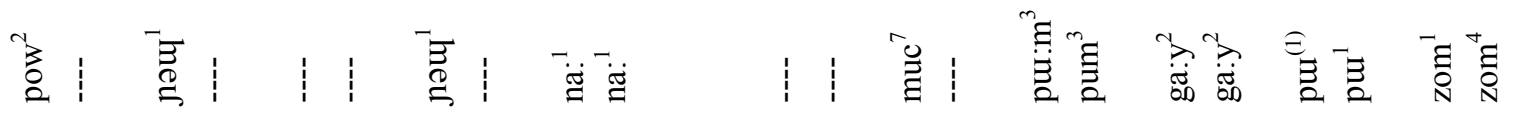

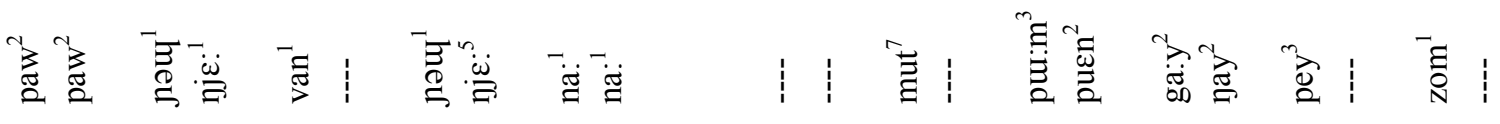

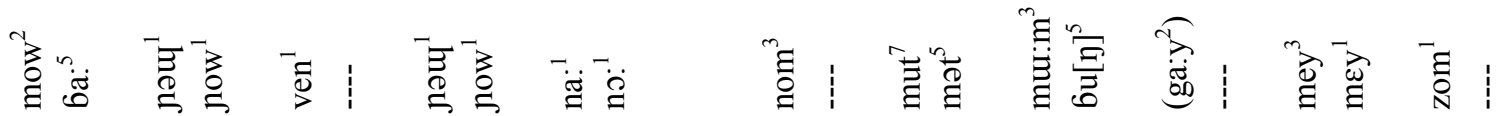

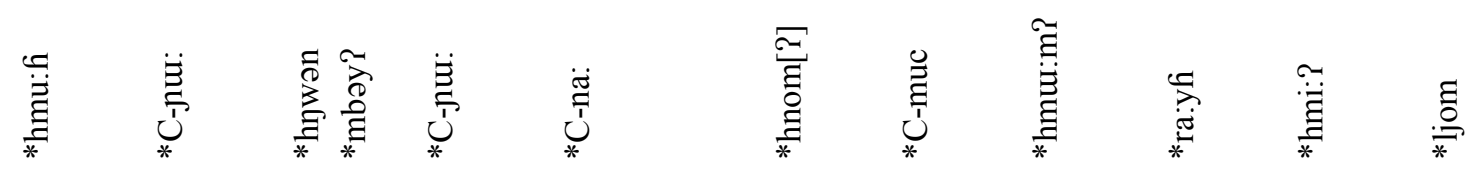

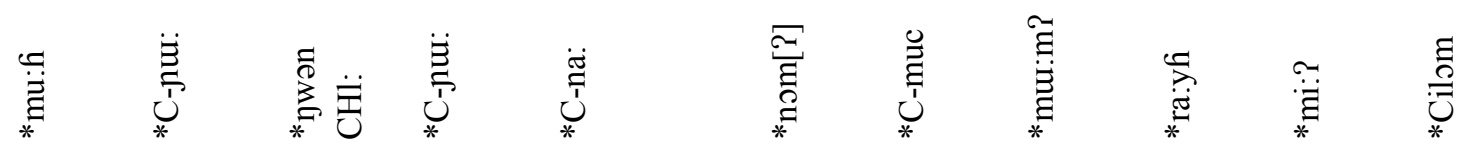

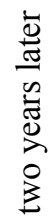

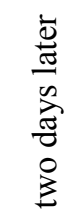

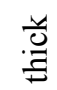
$\stackrel{\star}{0}$

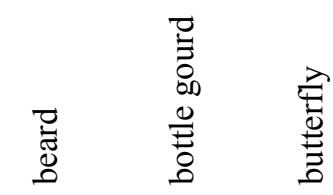
这
低
㽖召要

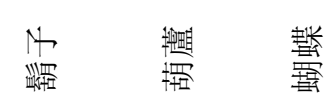




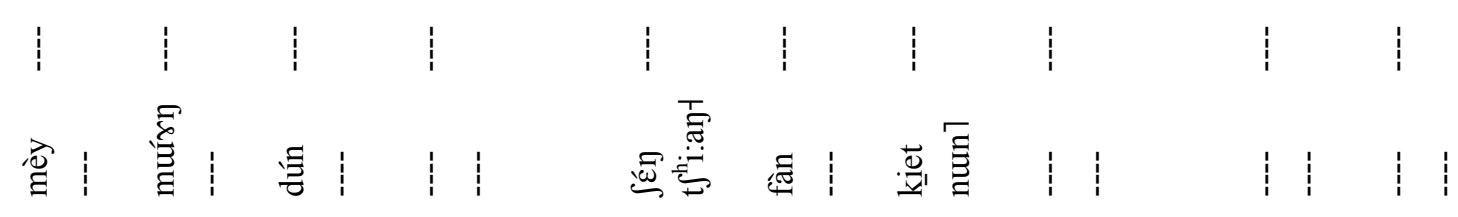

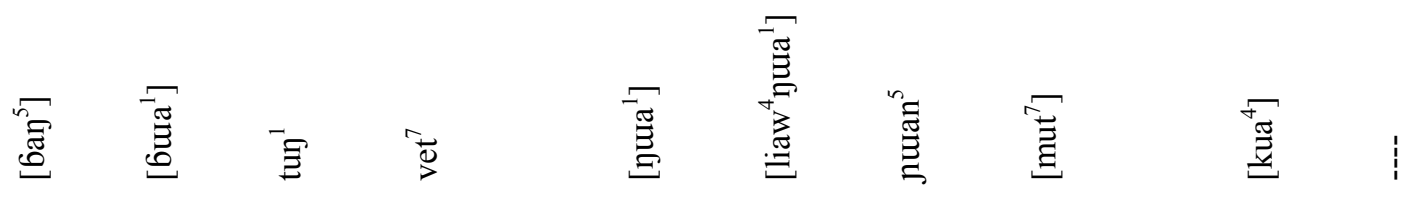

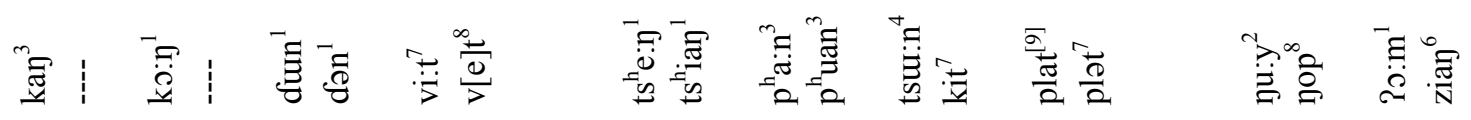

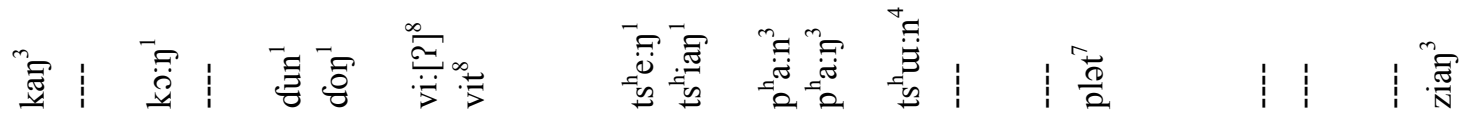

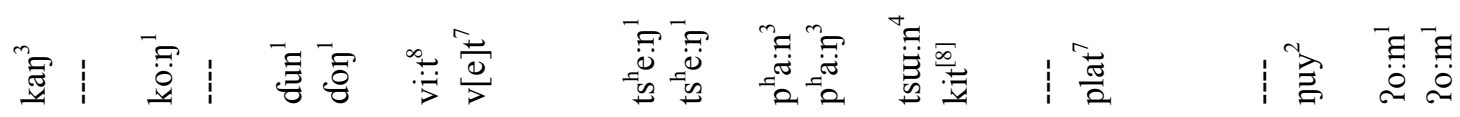

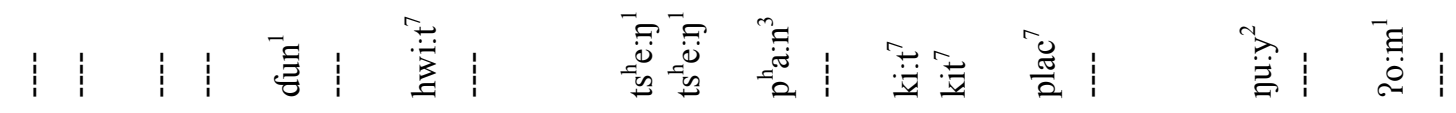

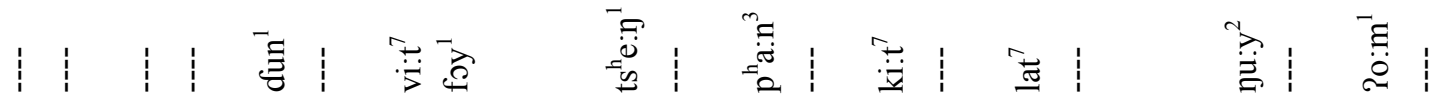

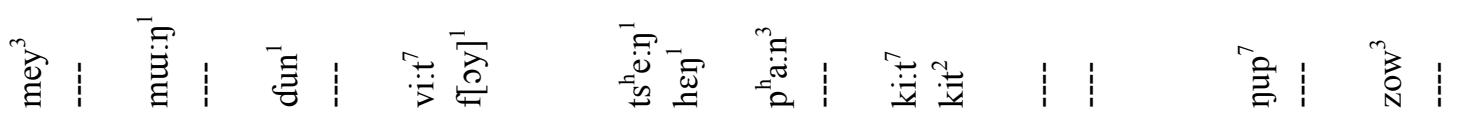

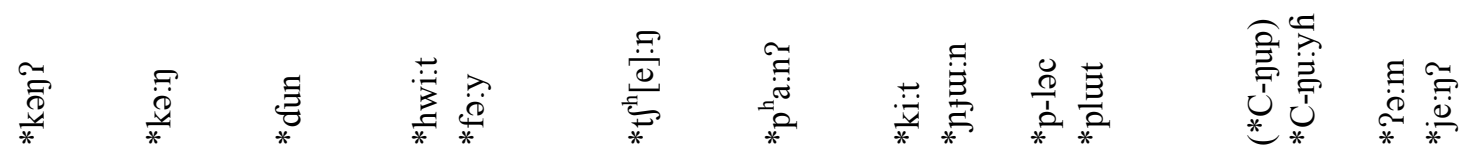

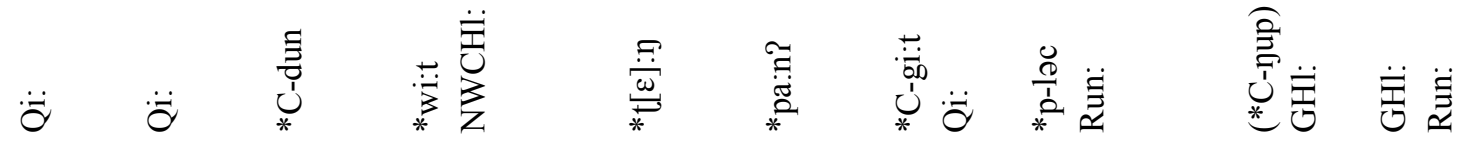

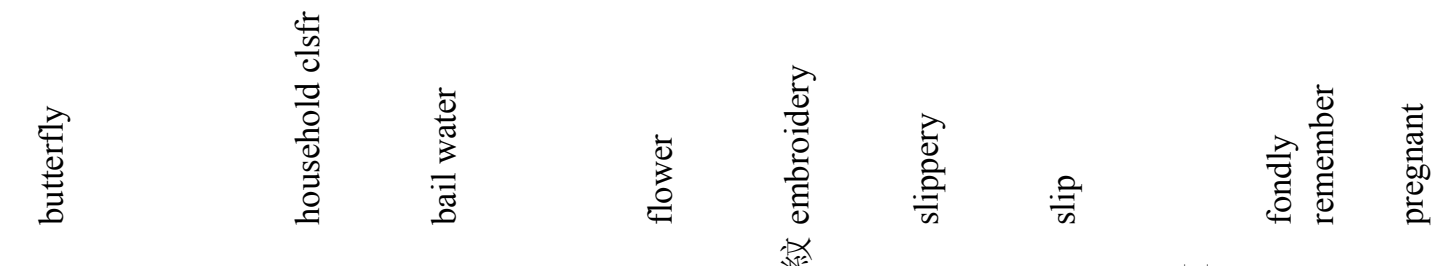

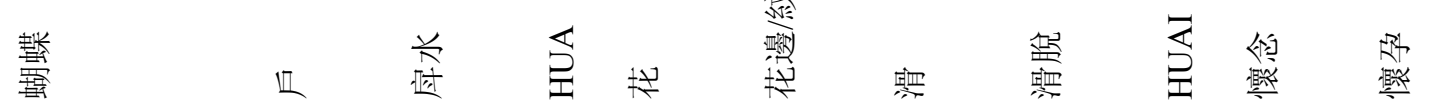




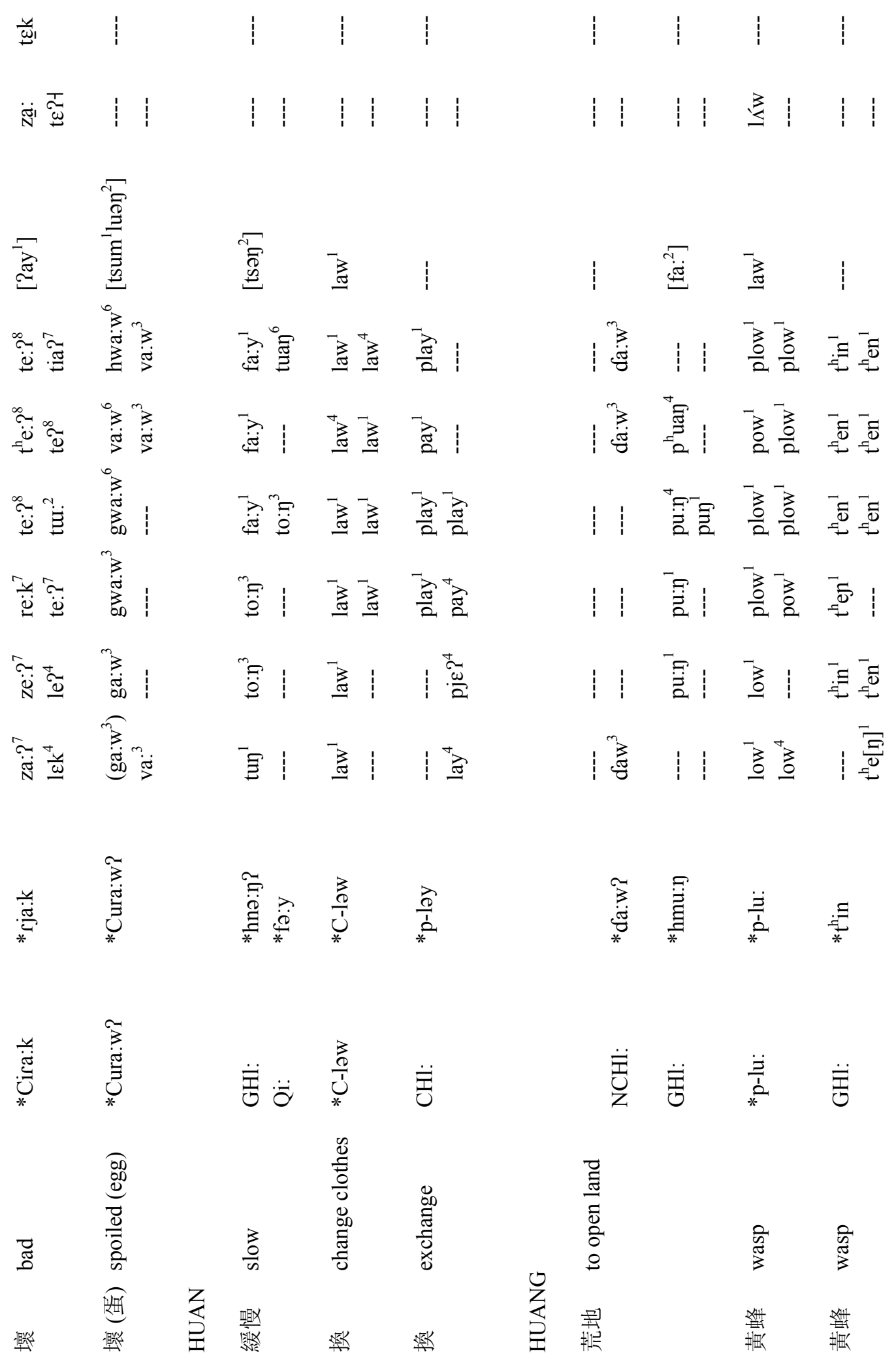




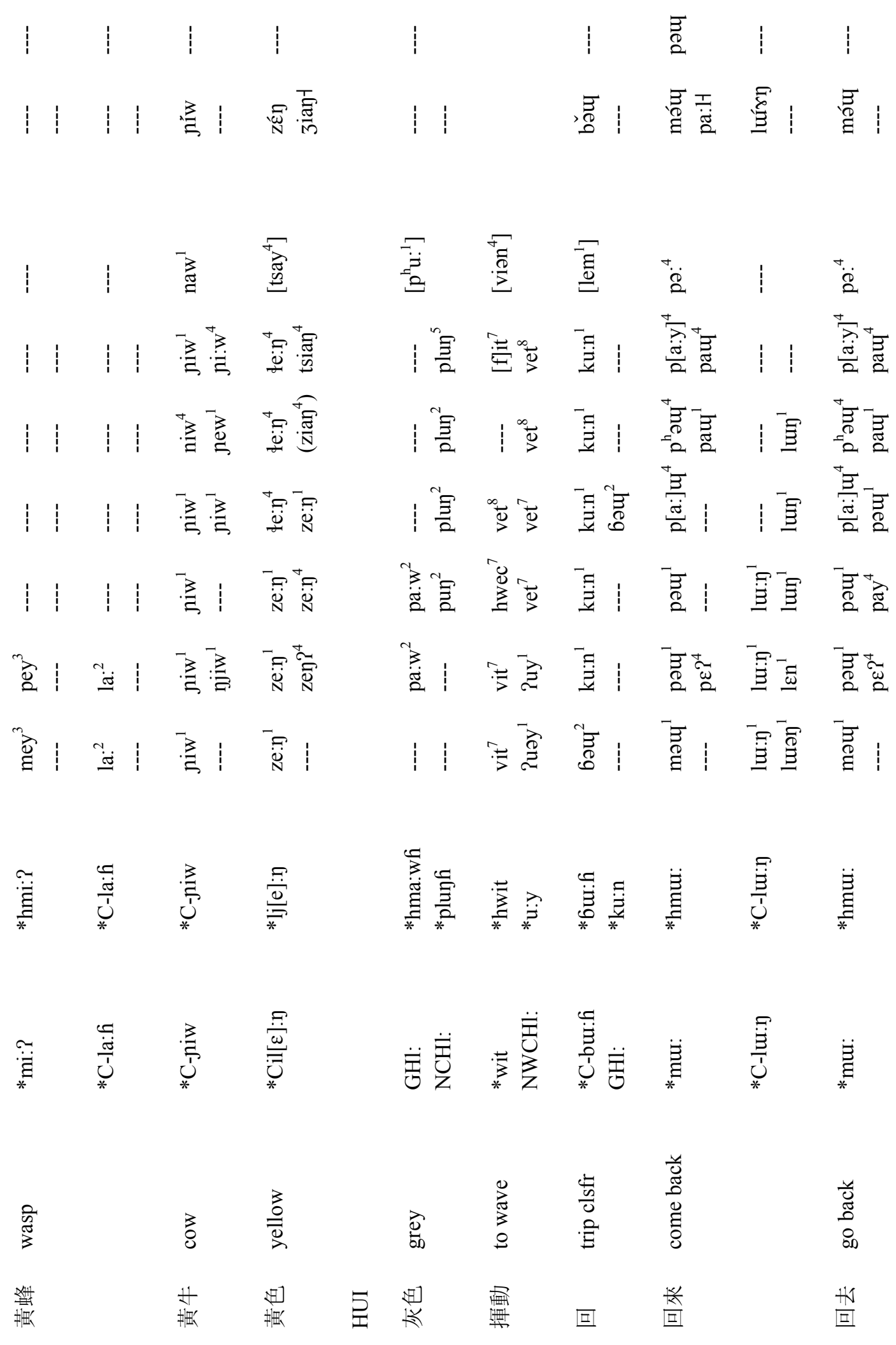




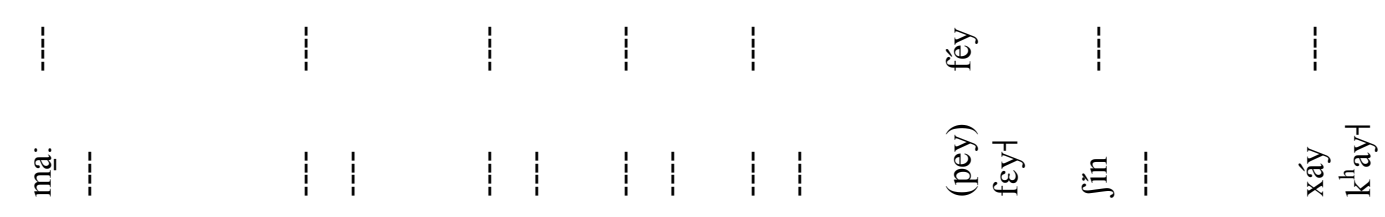

登

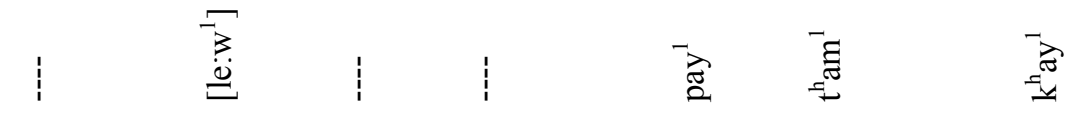

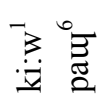

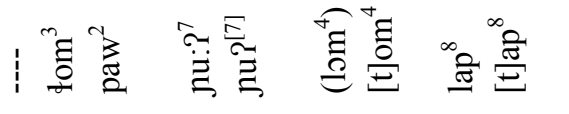

矛死

死无

$\stackrel{n}{z}^{z}$

苞莕

氙

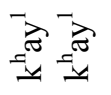

葛疍

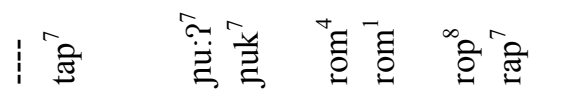

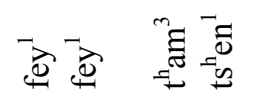

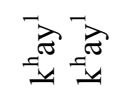

葛

กิำ

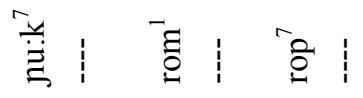

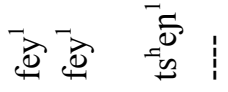

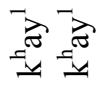

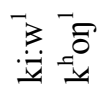

छิ।

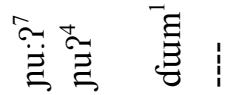

ָี

离离宓

$\underset{\sim}{\nexists \approx}=$

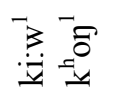

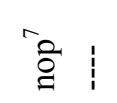

芯! I

㐫:

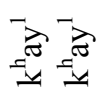

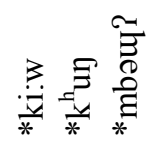

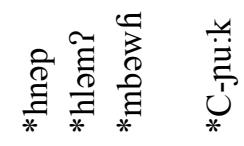

$\stackrel{0}{*} \stackrel{0}{*}$

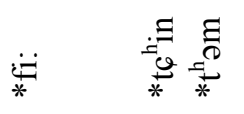

$=\frac{\overrightarrow{0}}{*}$

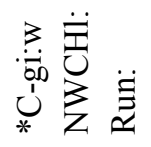

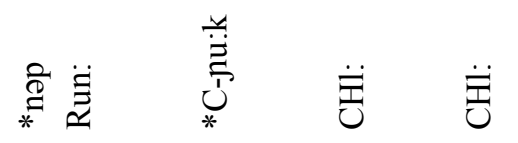

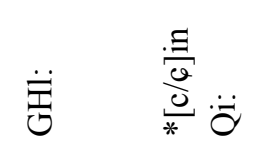

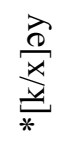

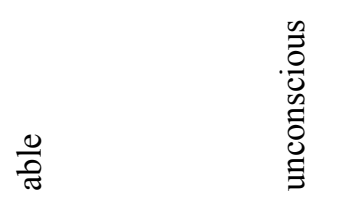

$\stackrel{\overrightarrow{0}}{\frac{0}{0}} \quad \stackrel{x}{\Xi}$

๑

$\frac{\frac{1}{0}}{\frac{1}{0}}$

睓

点糔

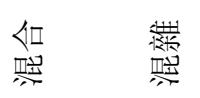

吕 
$\begin{array}{llllllllllll}1 & 1 & 1 & 1 & 1 & 1 & 1 & 1 & 1 & 1 & 1 \\ 11 & 1 & 1 & 1 & 1 & 1 & 1 & 1 & 1 & 1 & 1 & 1\end{array}$

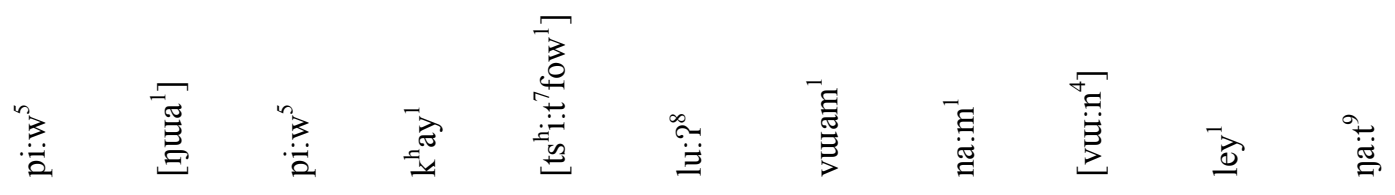

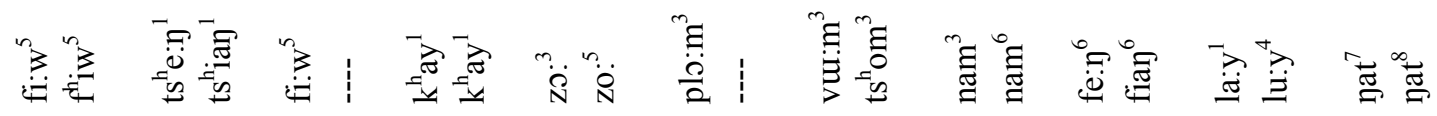

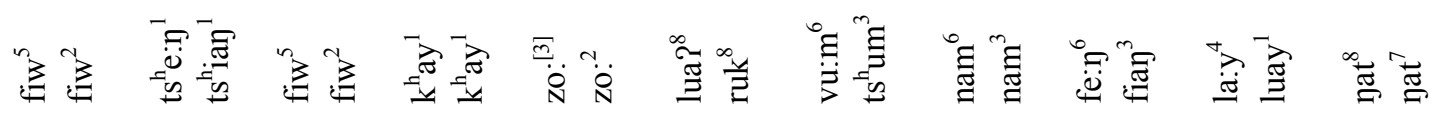

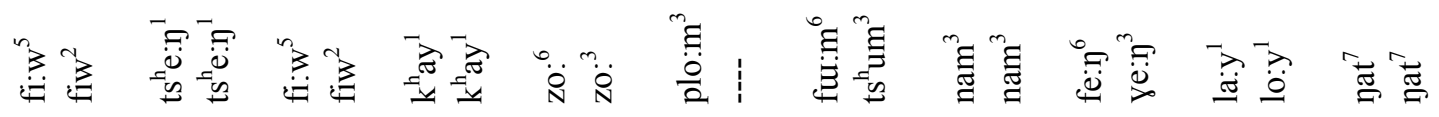

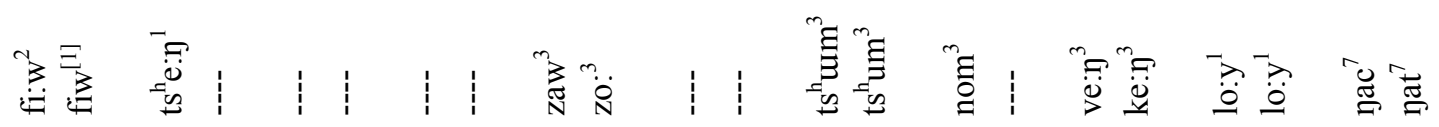

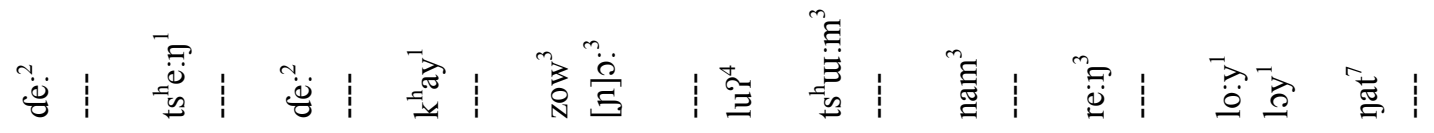

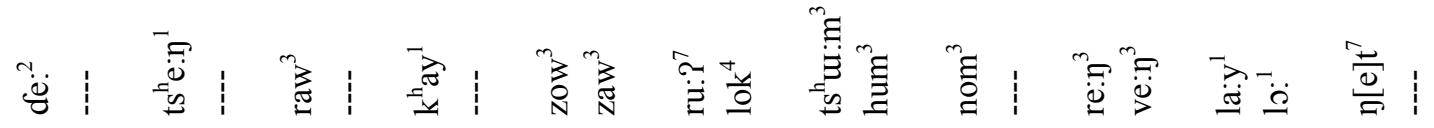

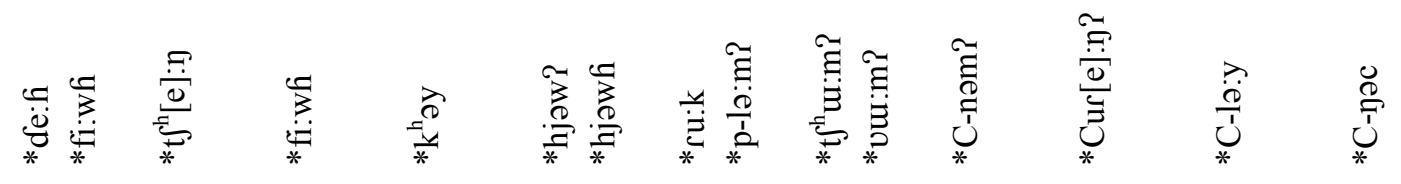

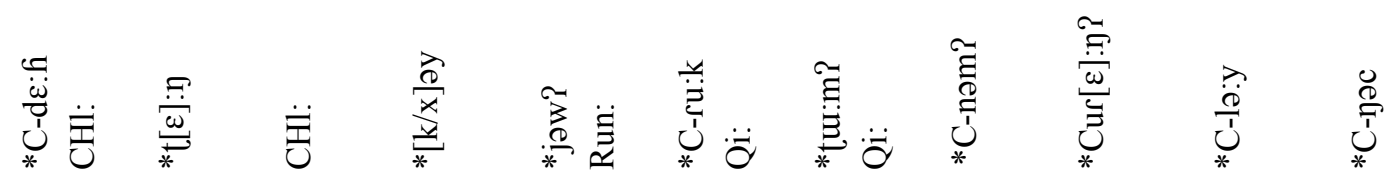

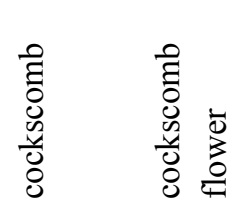

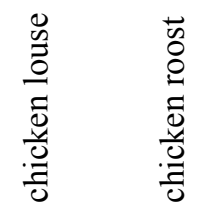

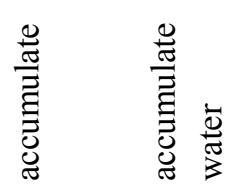
量
碞蓄

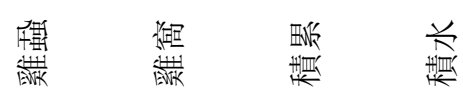

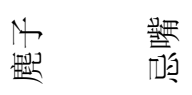




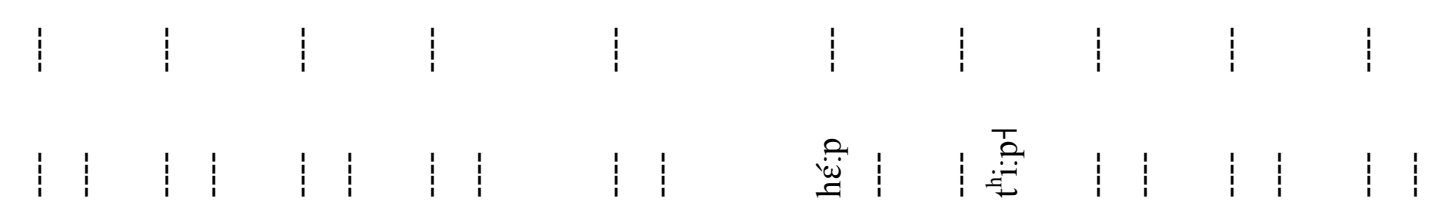

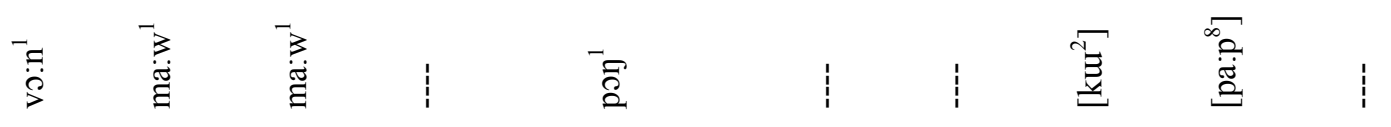

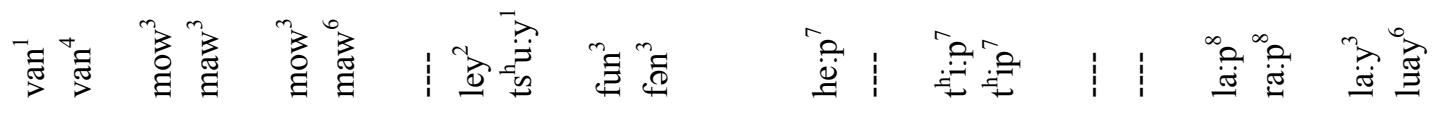

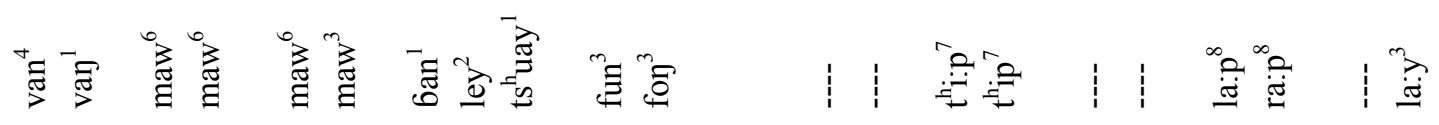

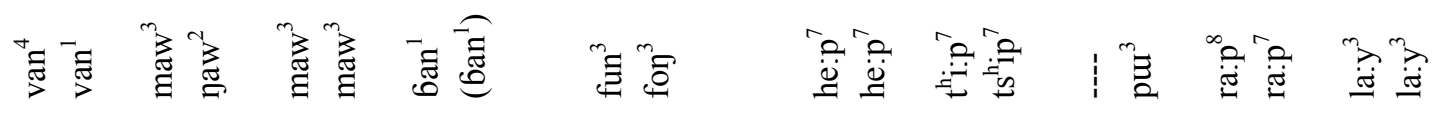

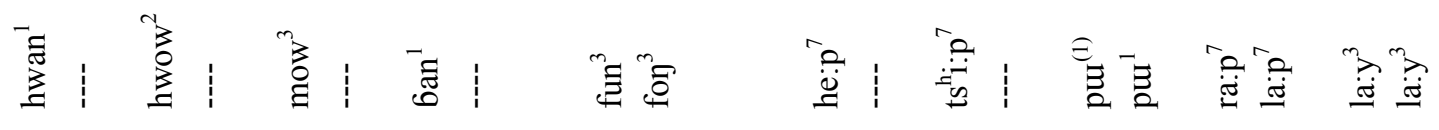

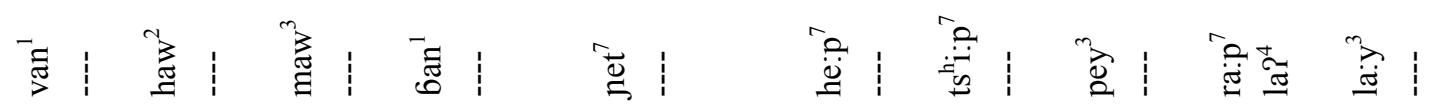

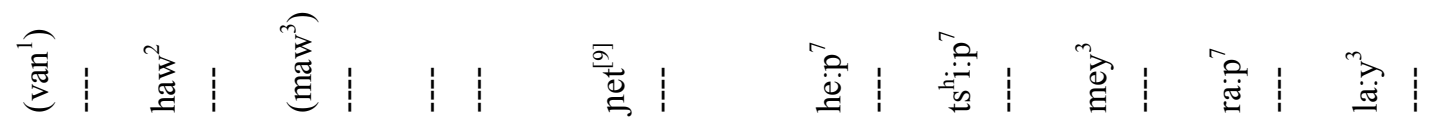

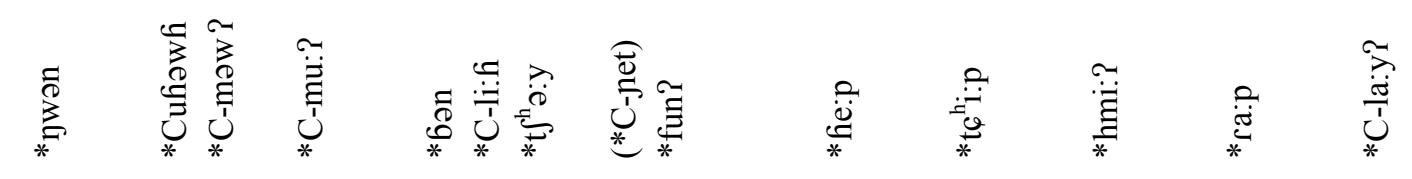

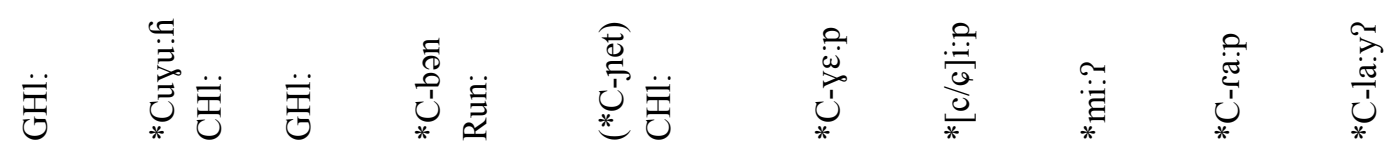

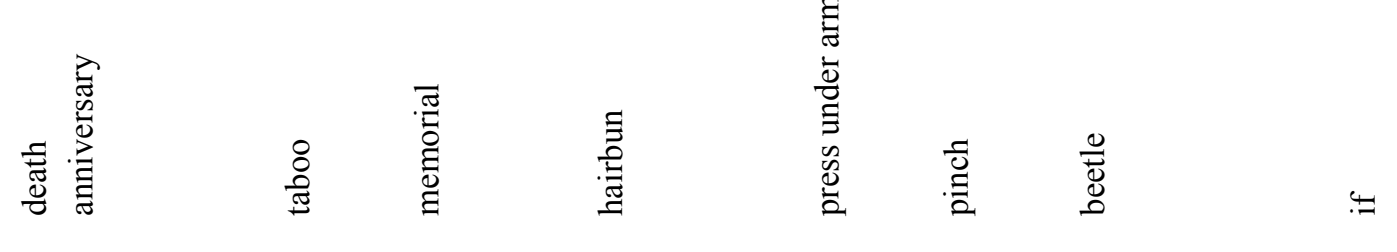

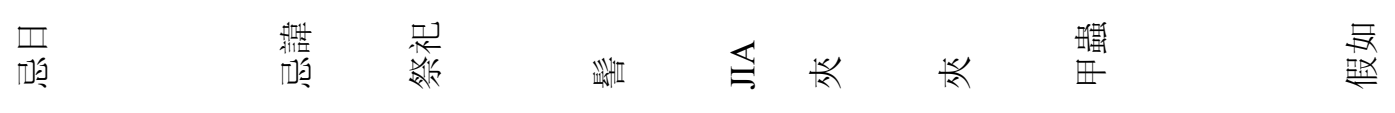




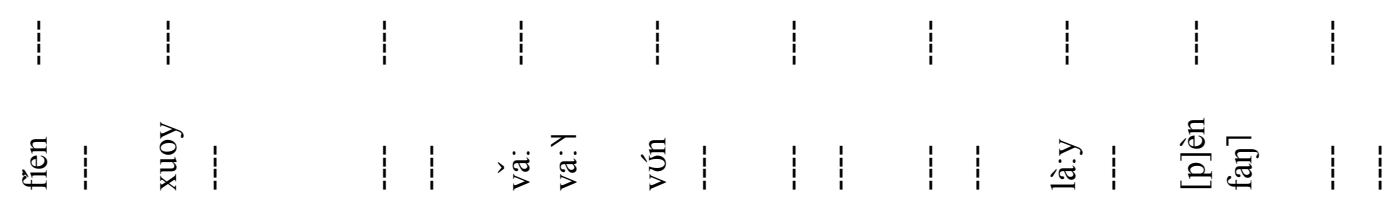

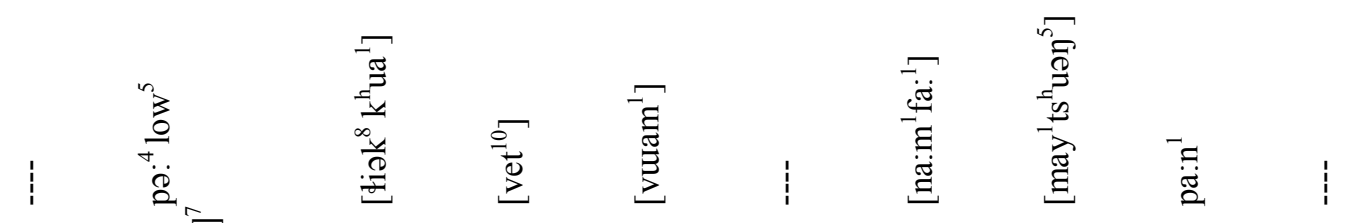

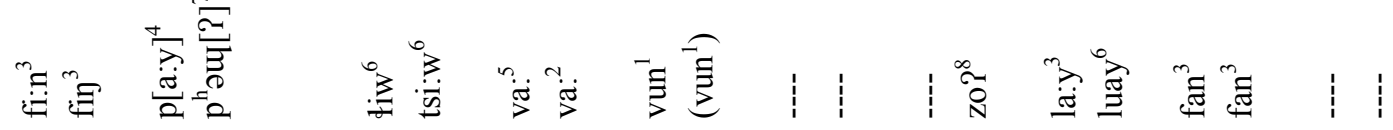

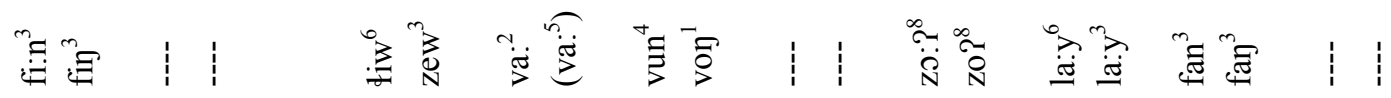

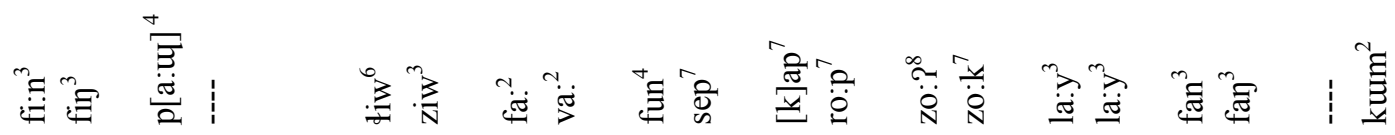

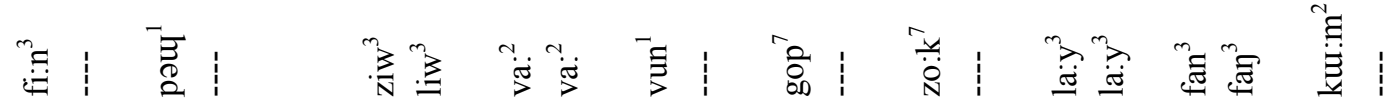

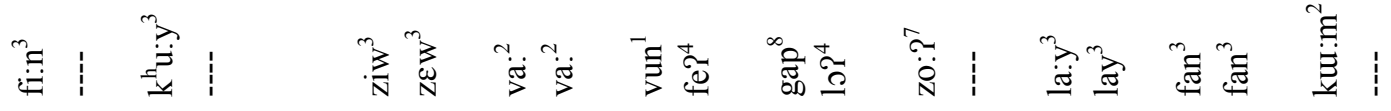

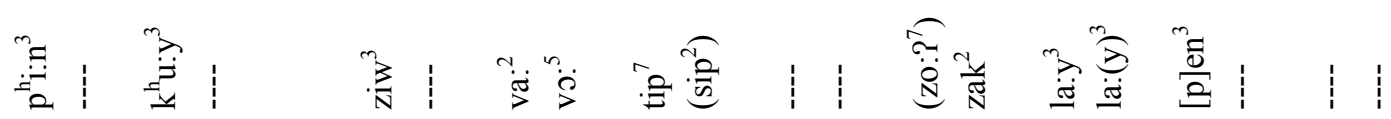

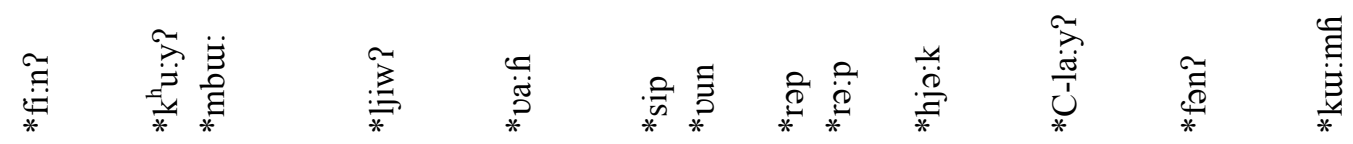

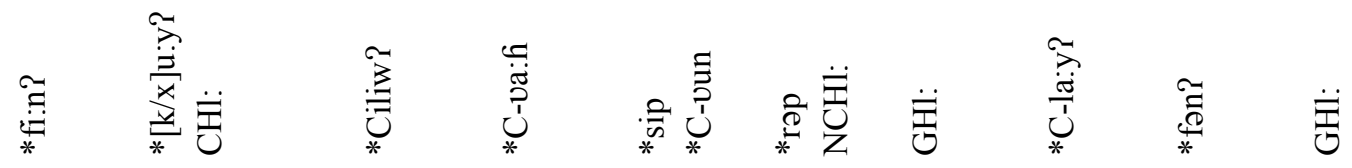

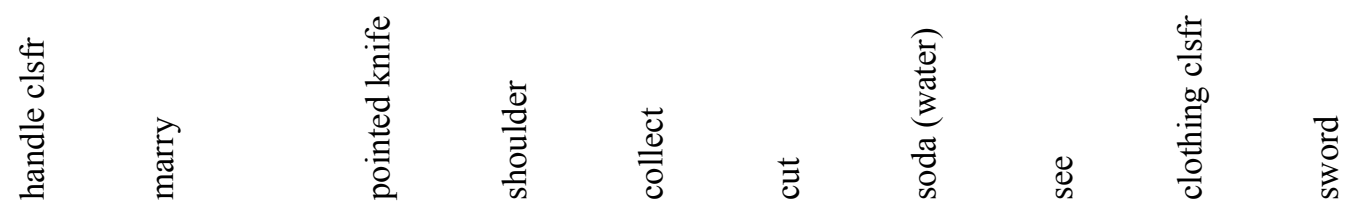

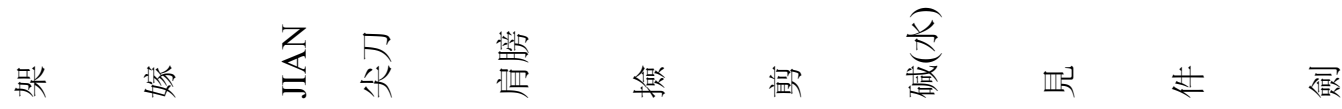




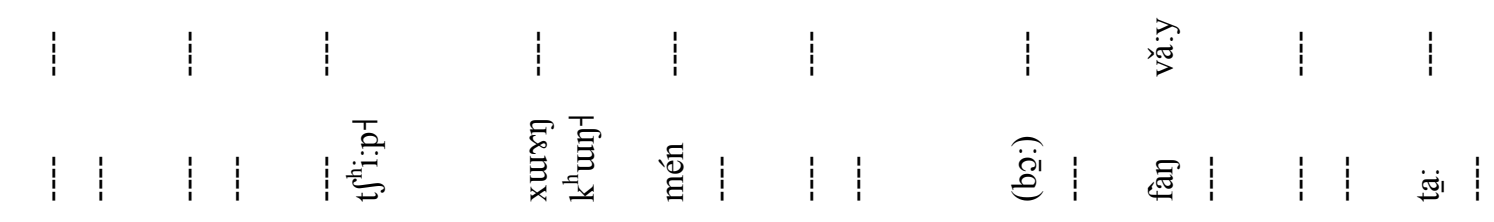

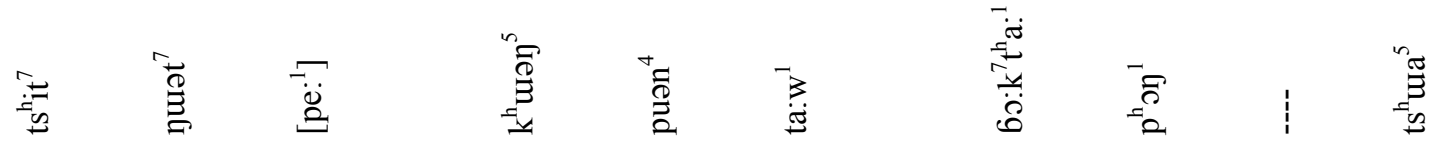

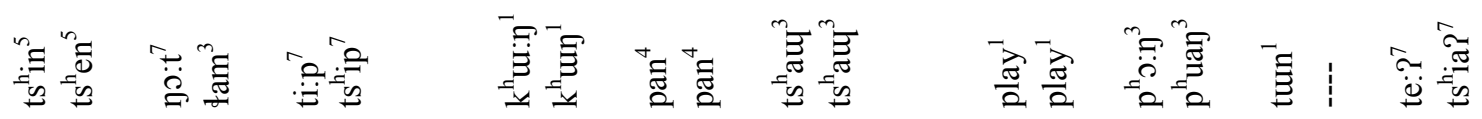

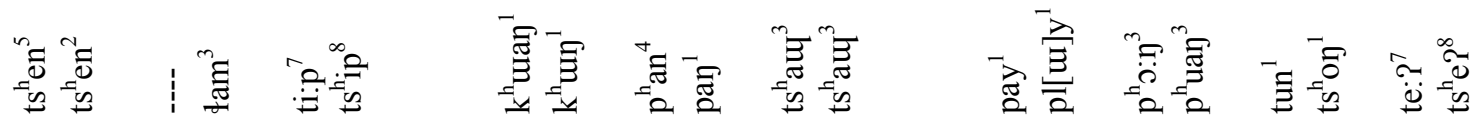

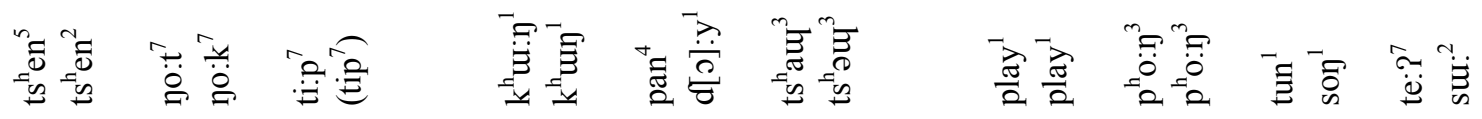

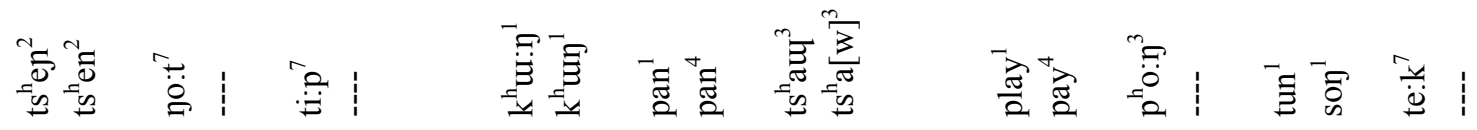

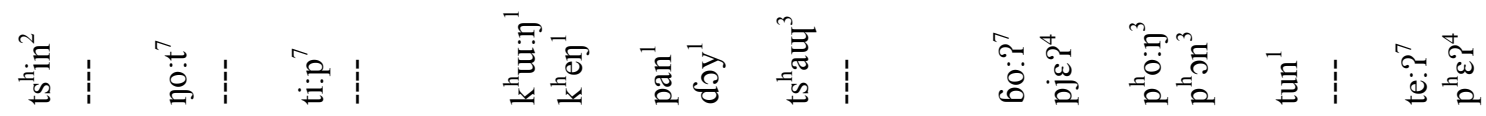

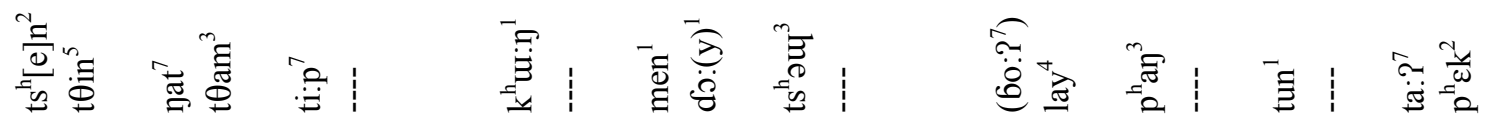

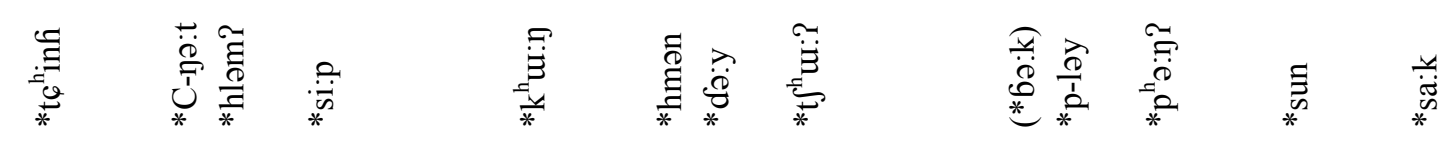

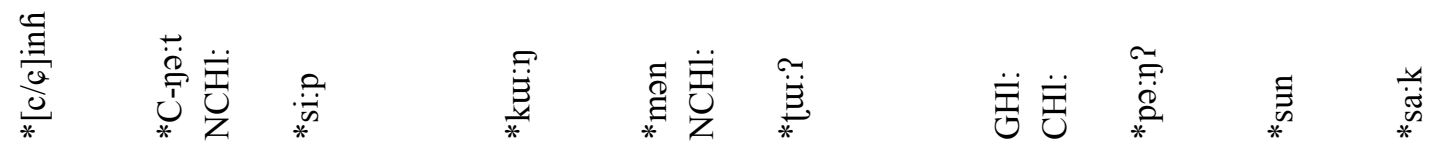

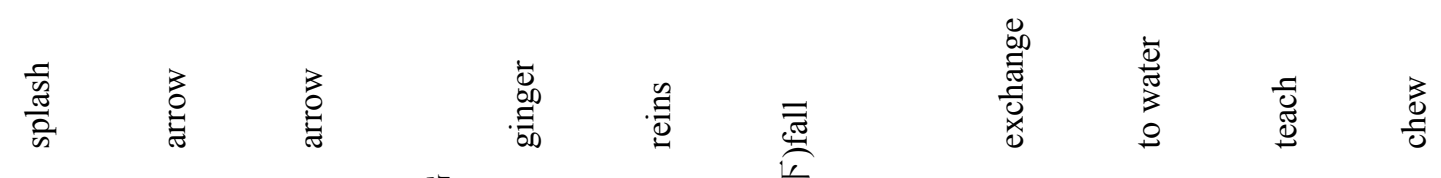

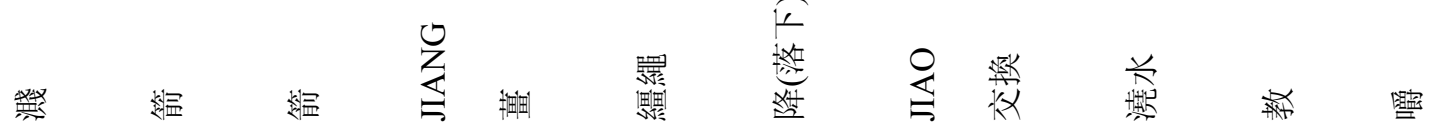


$1+1+101011$

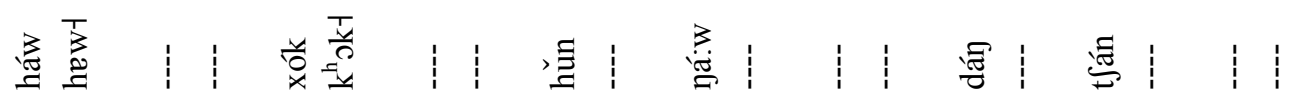

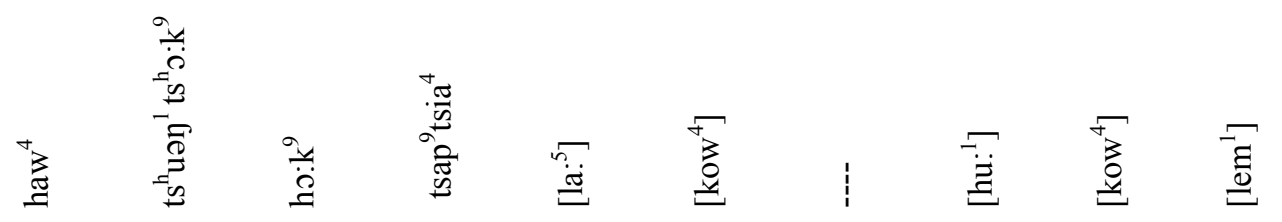

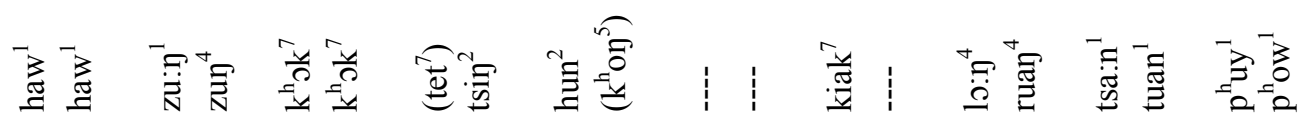

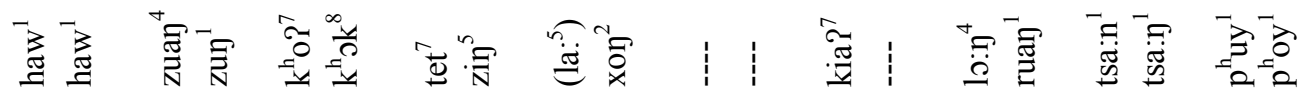

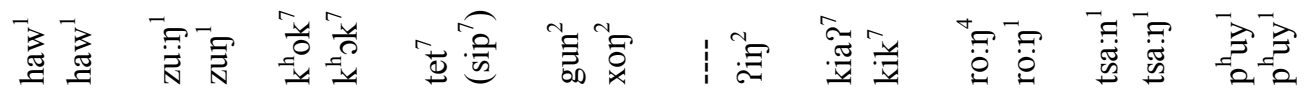

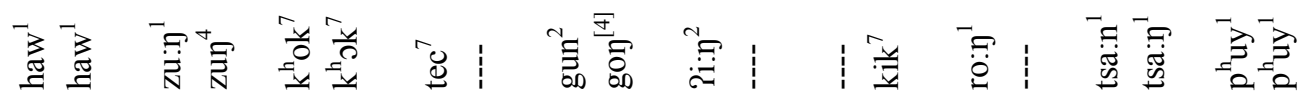

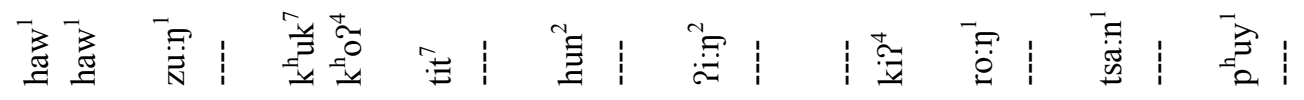

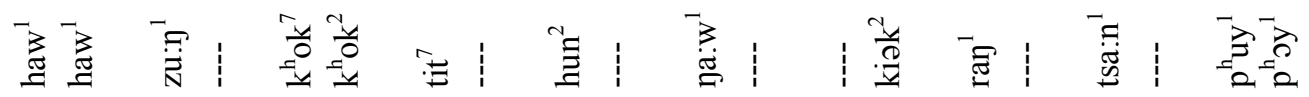

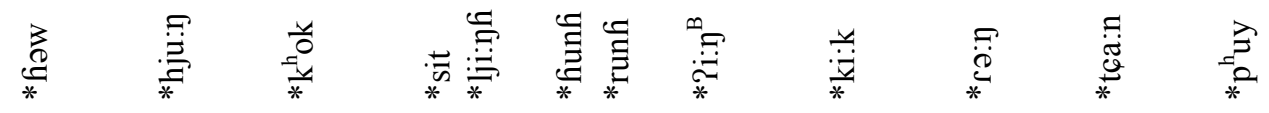

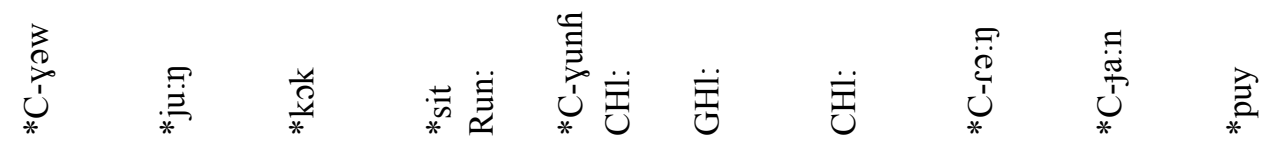

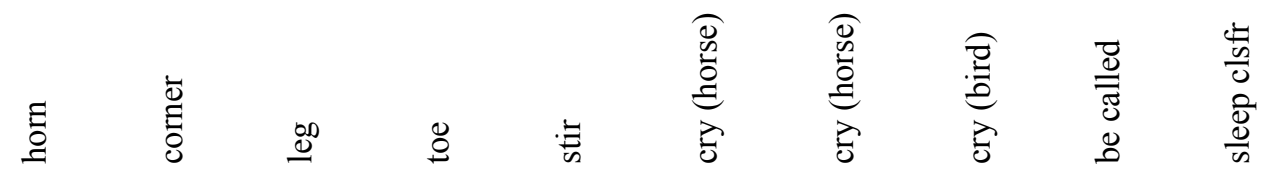

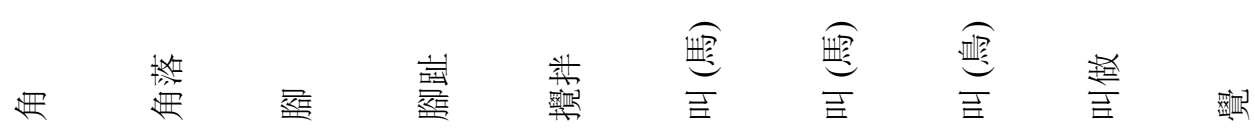




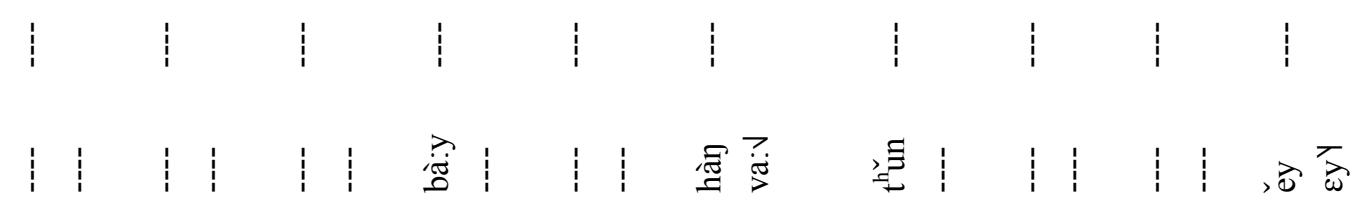

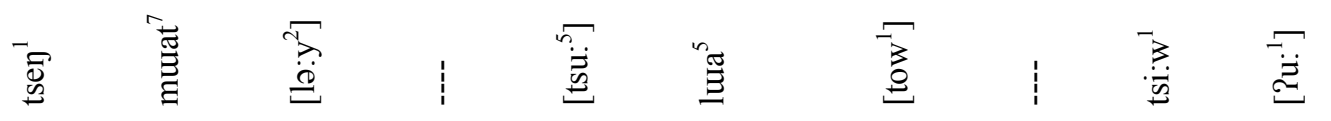

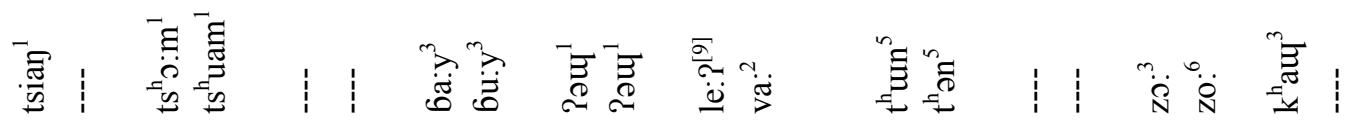

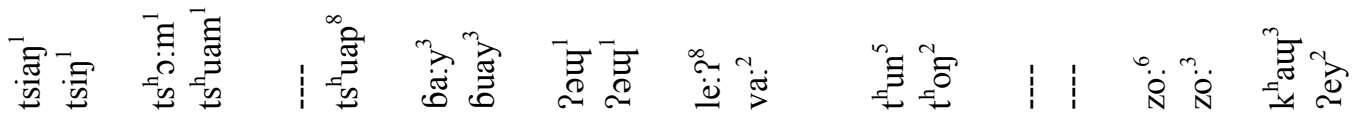

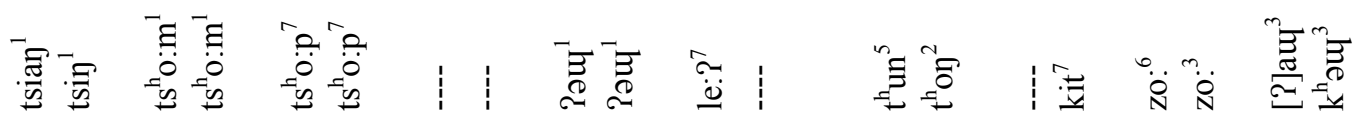

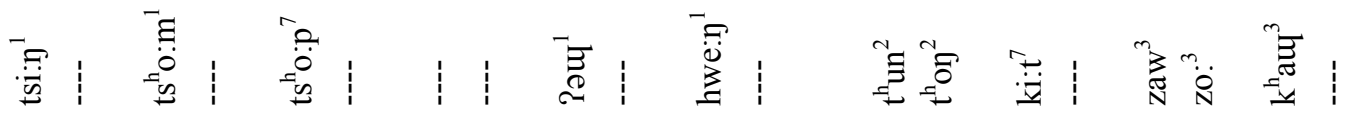

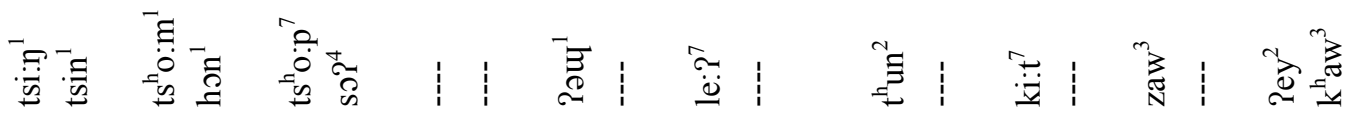

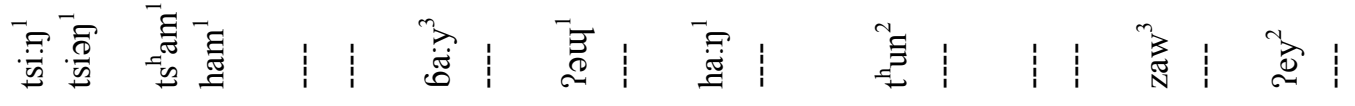

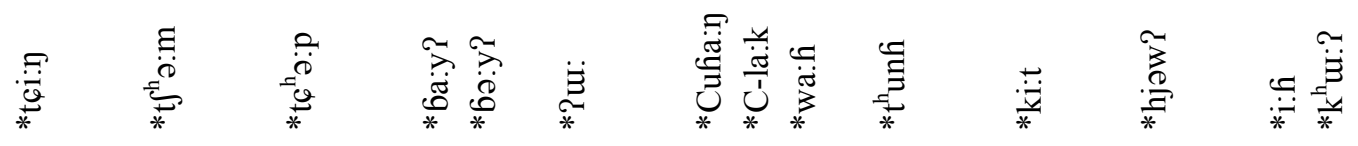

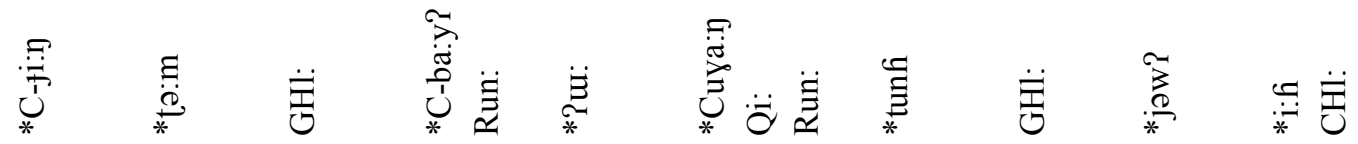

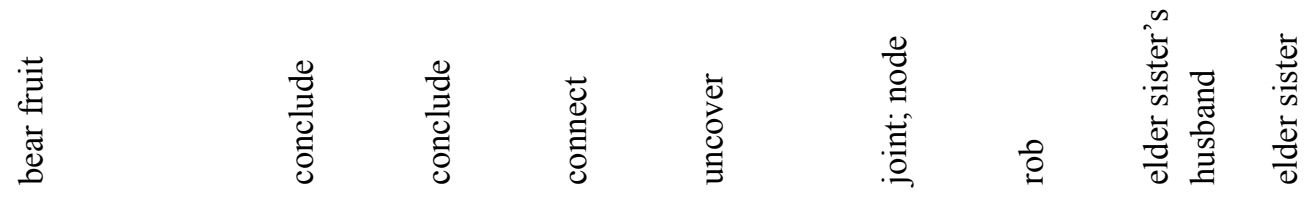

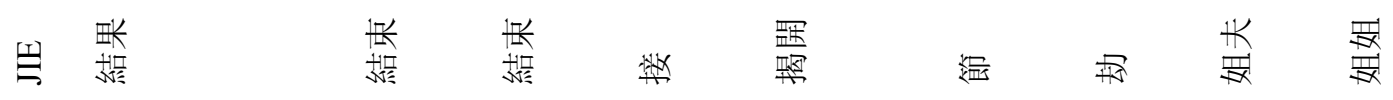




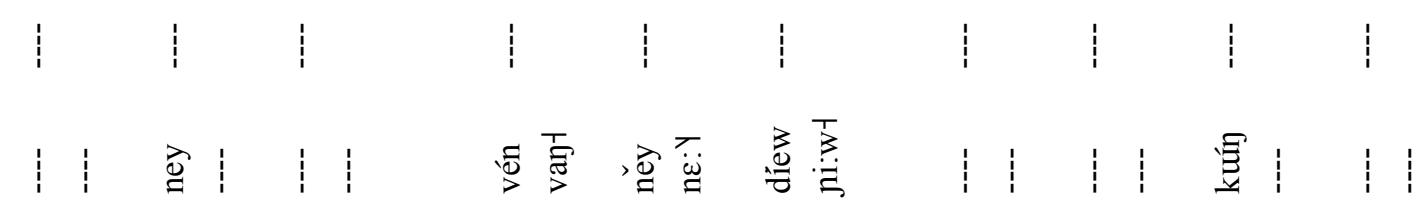

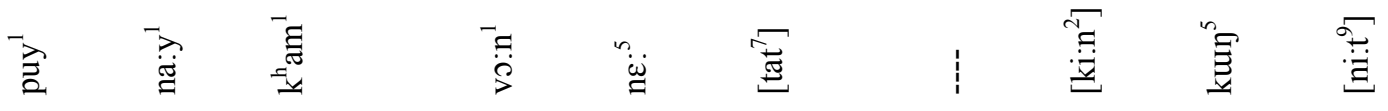

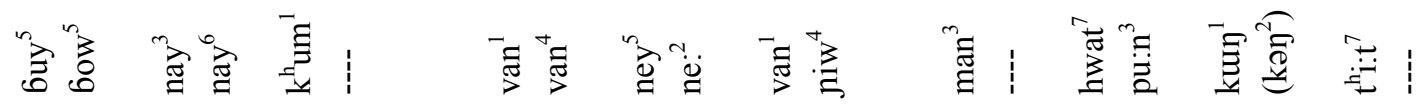

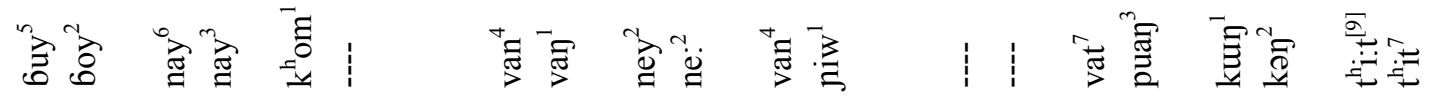

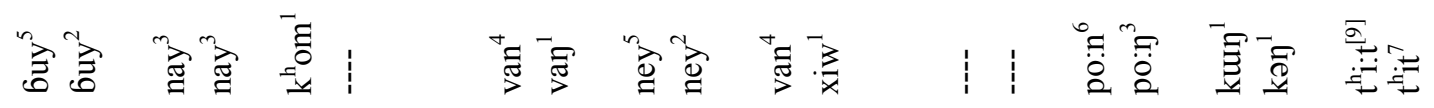

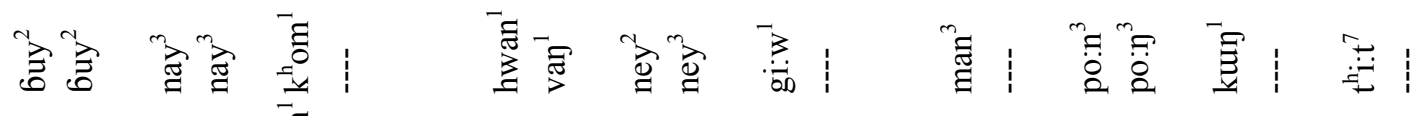

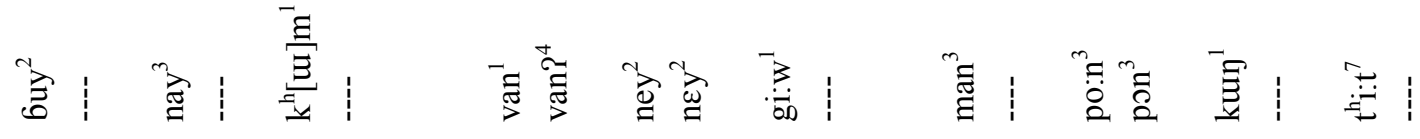

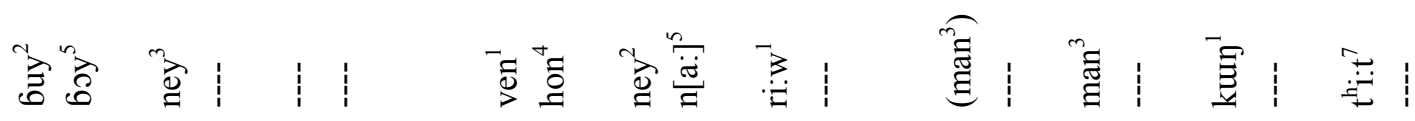

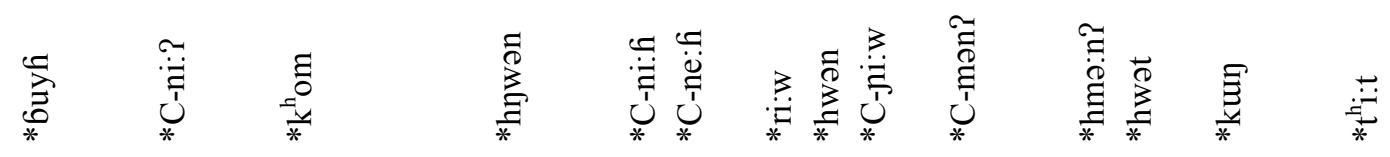

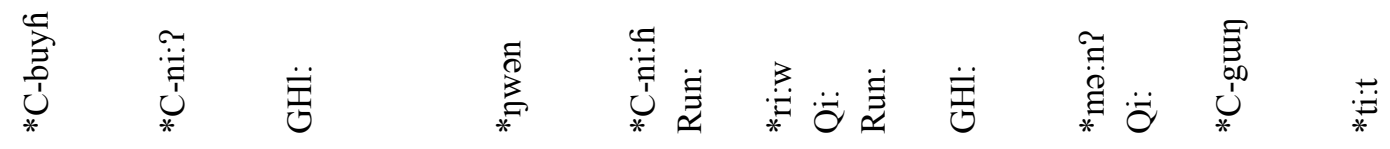

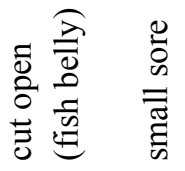
용
I

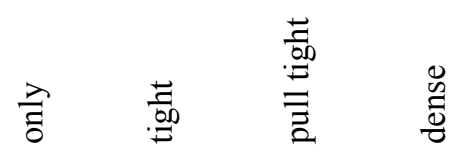
镸紫
学炎

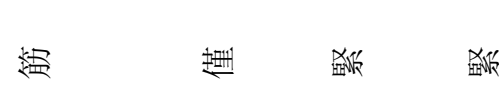




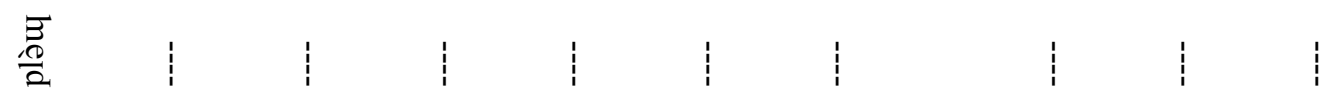

畫突

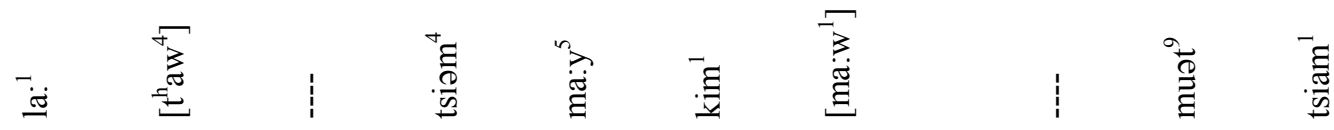

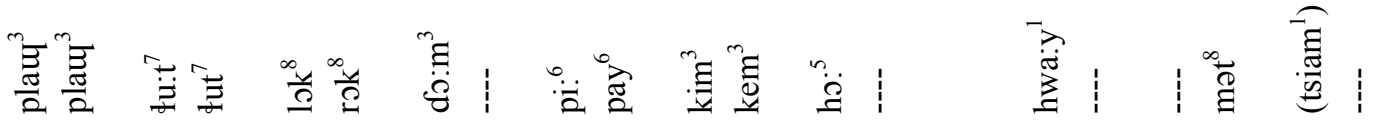

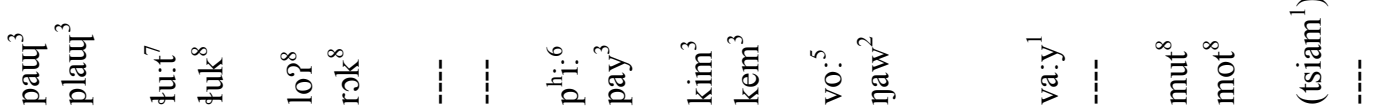

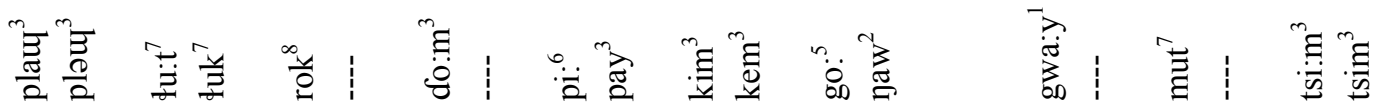

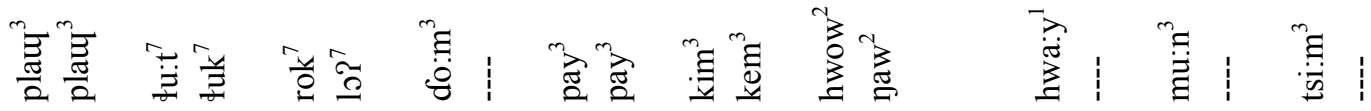

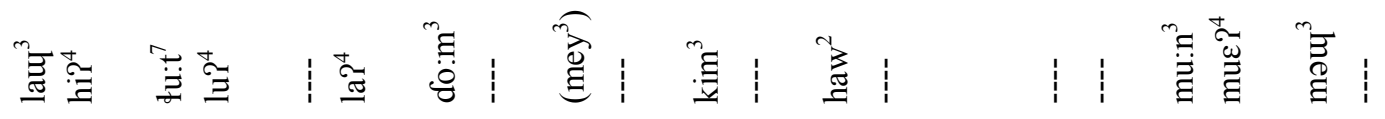

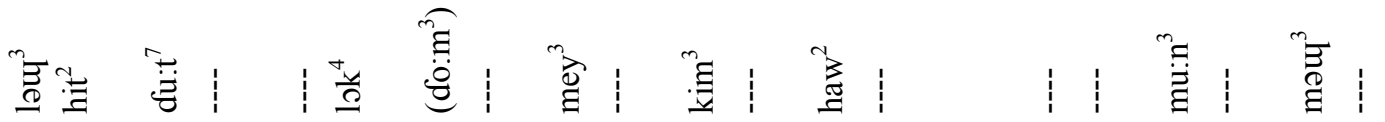

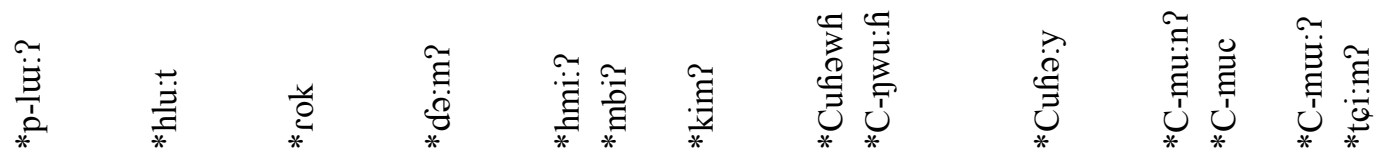

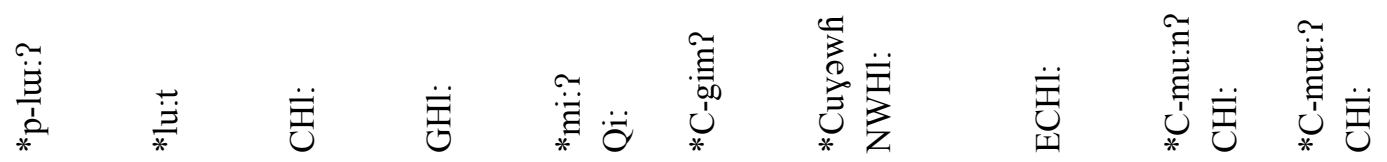

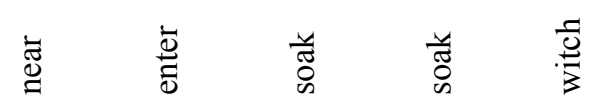

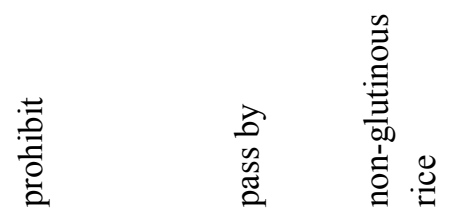

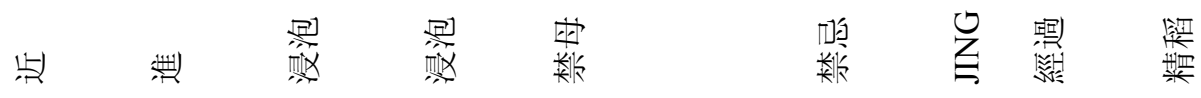




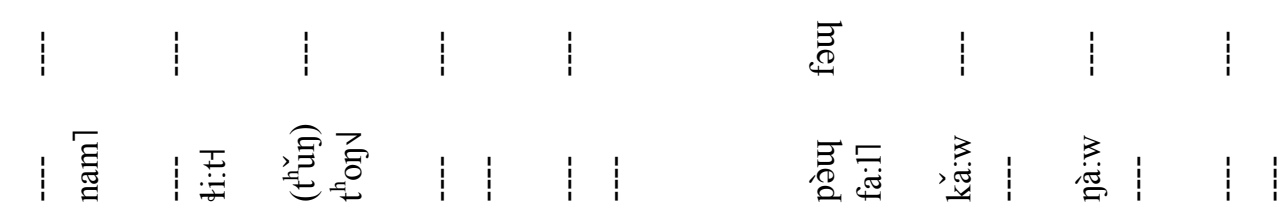

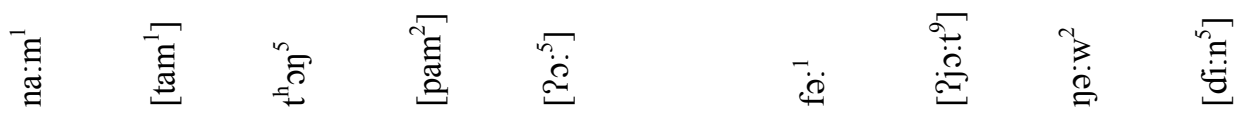

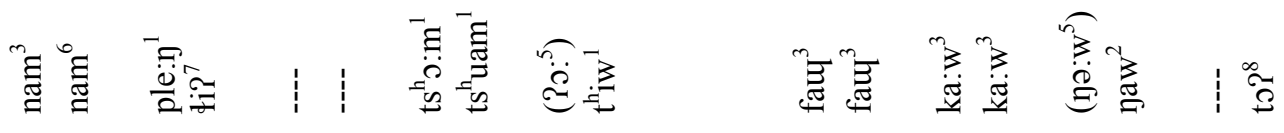

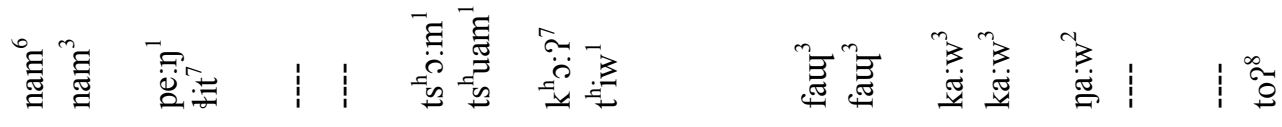

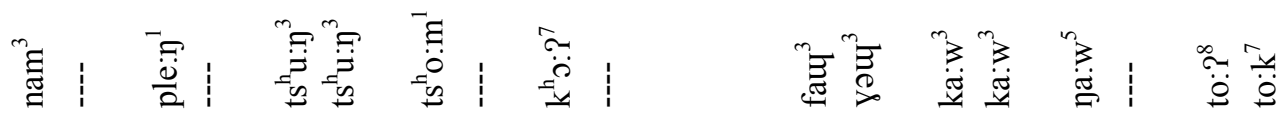

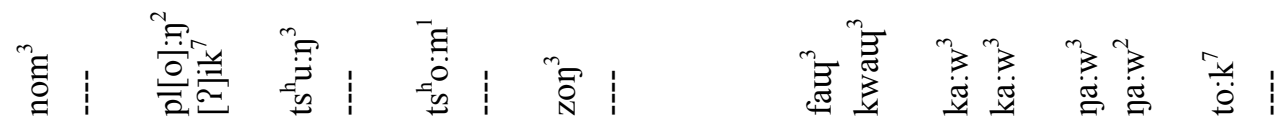

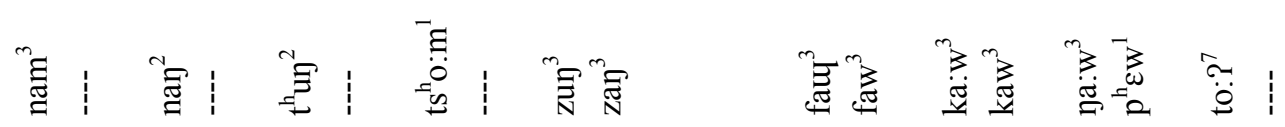

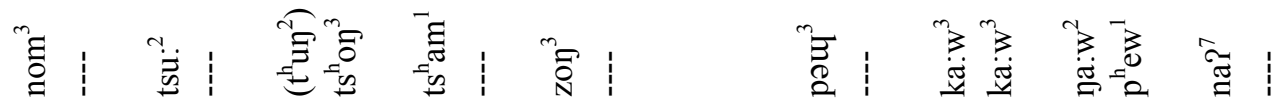

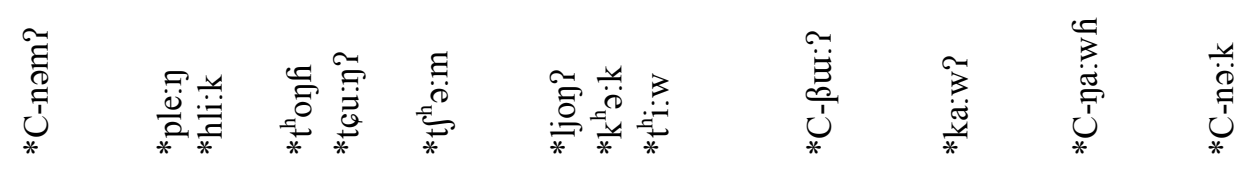

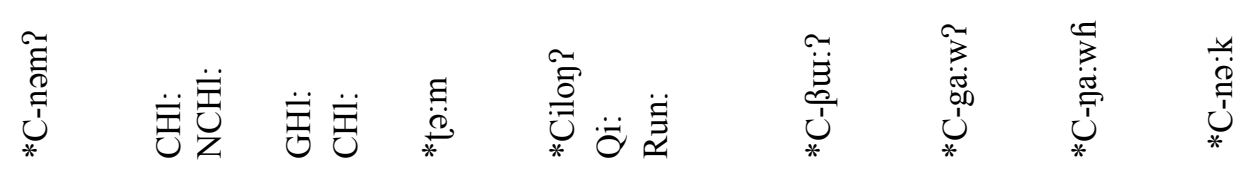

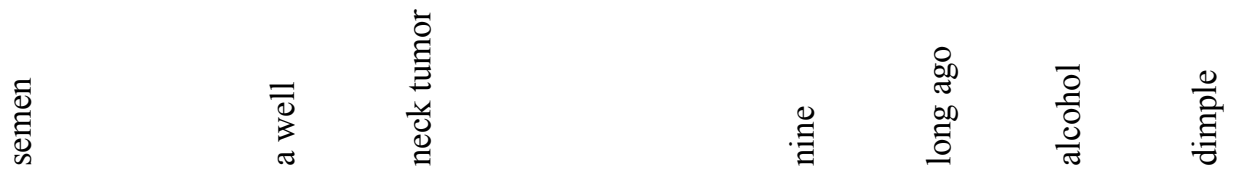

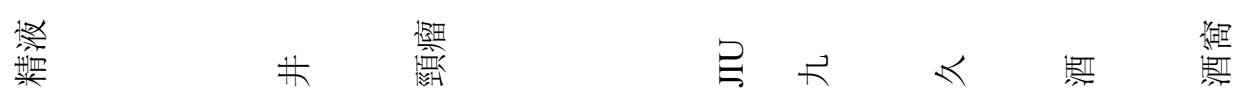




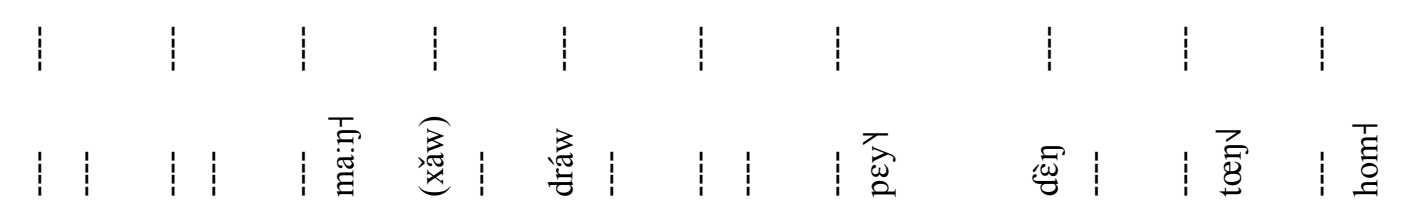

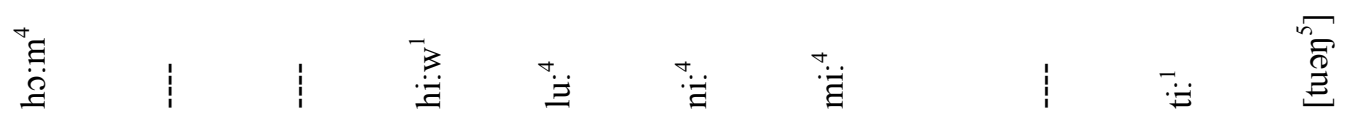

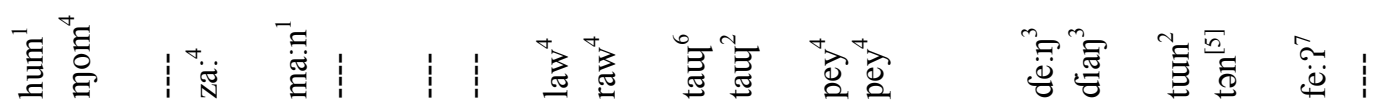

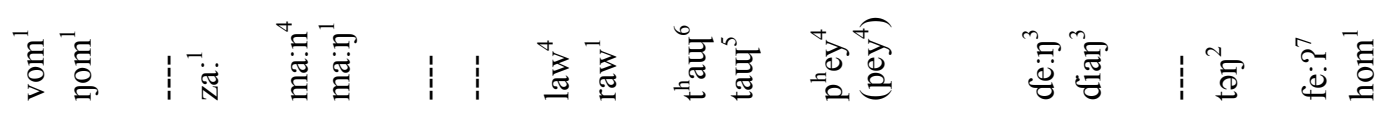

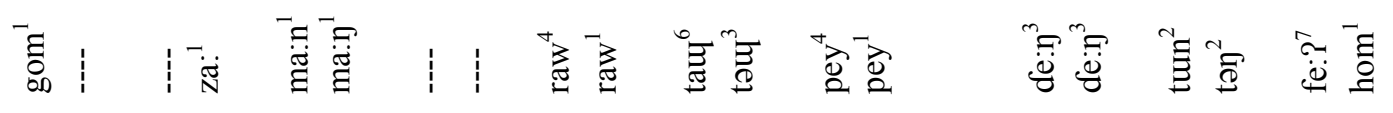

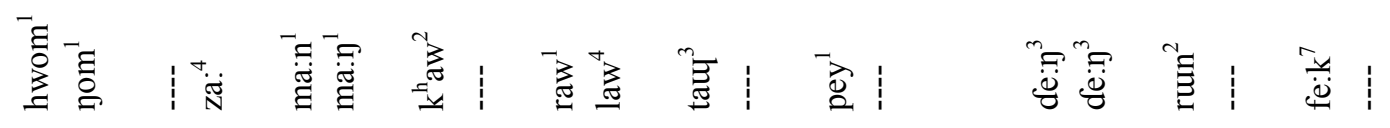

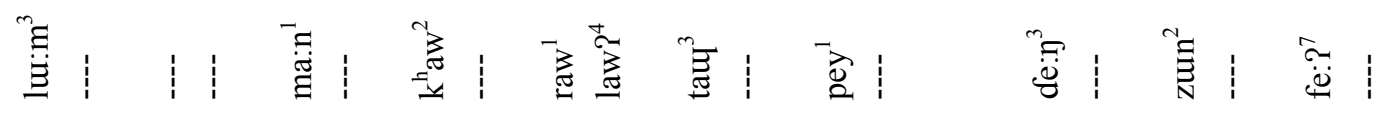

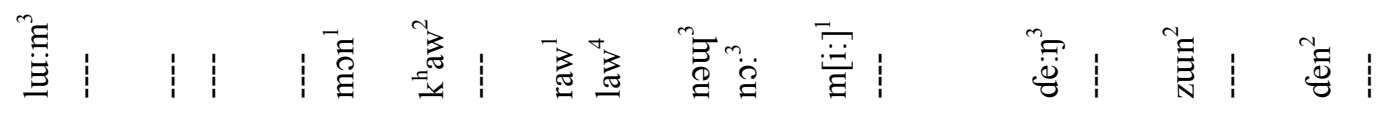

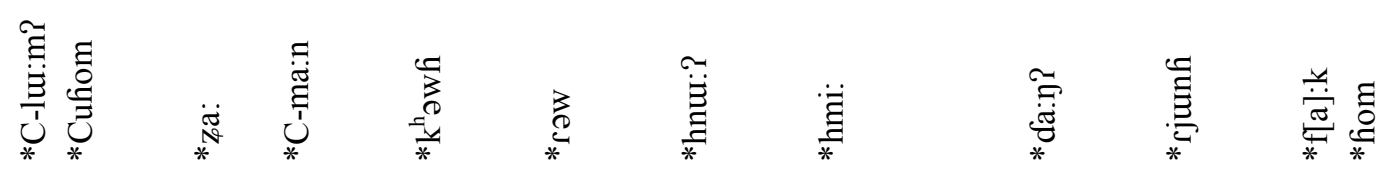

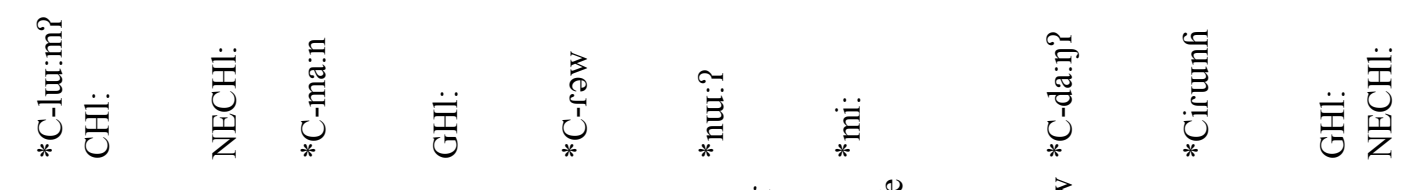

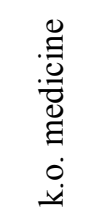

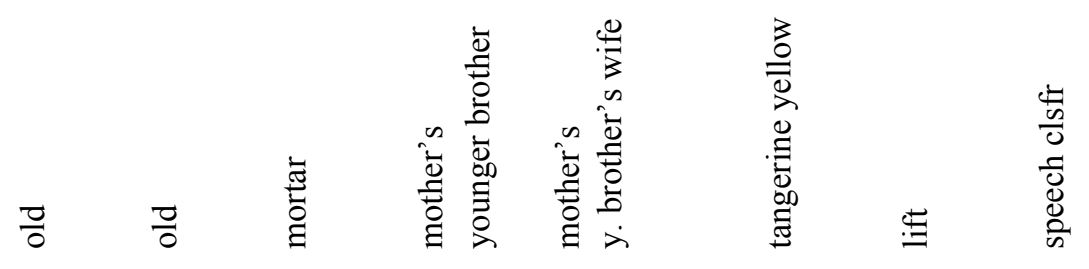

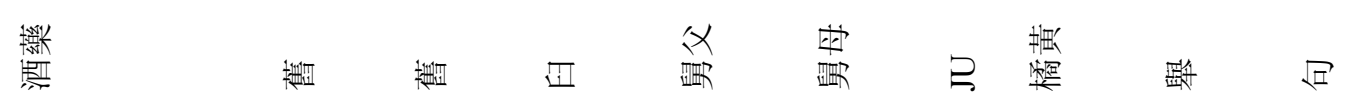




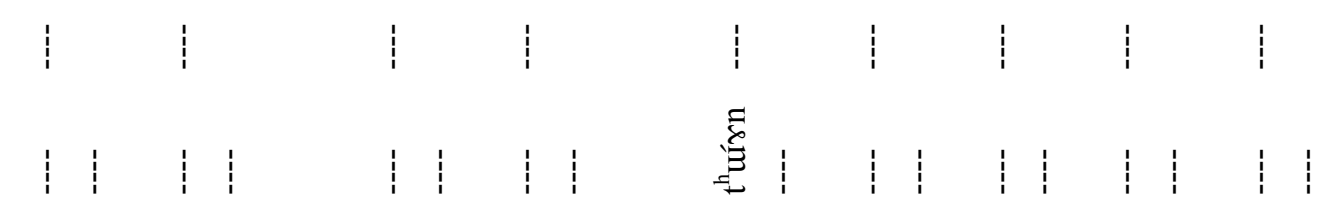

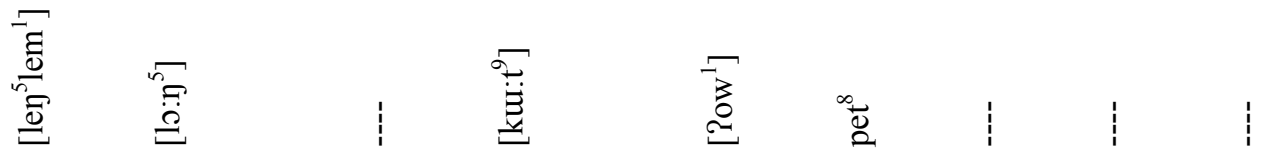

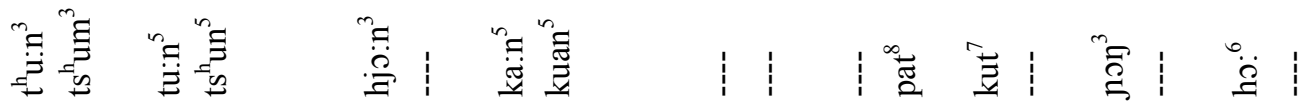

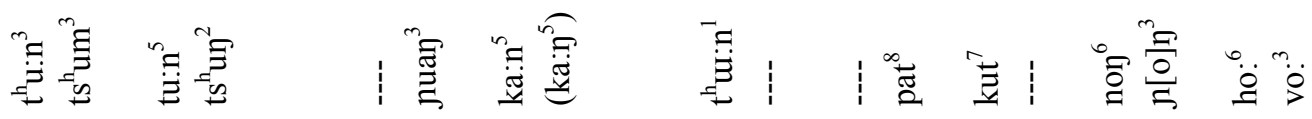

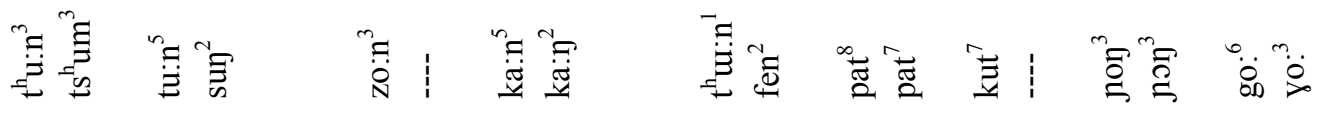

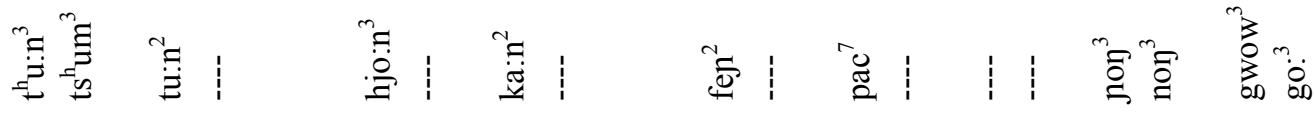

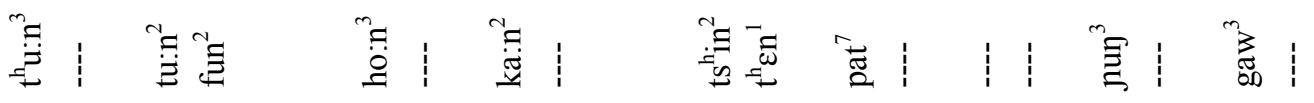

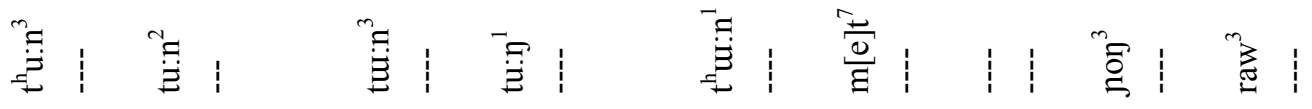

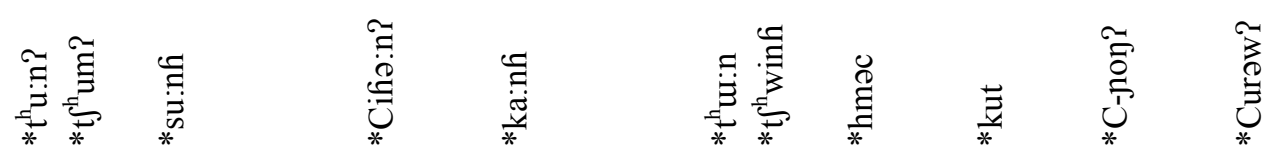

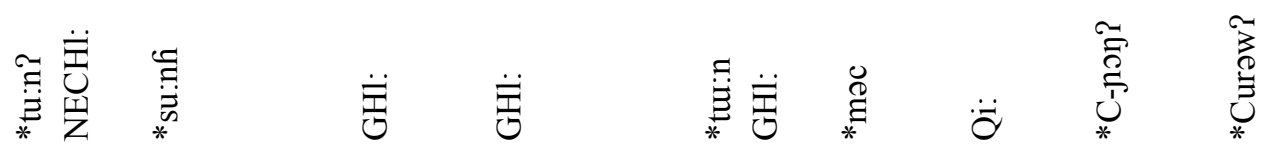

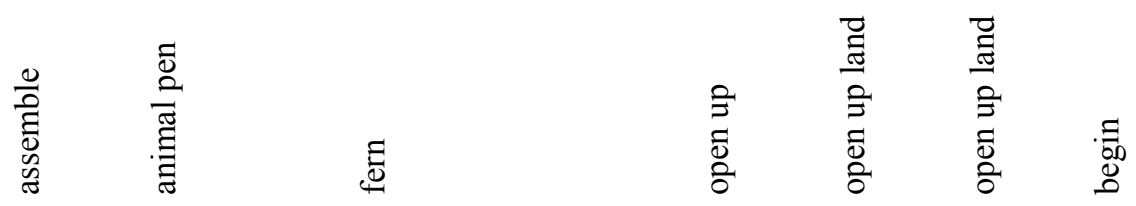

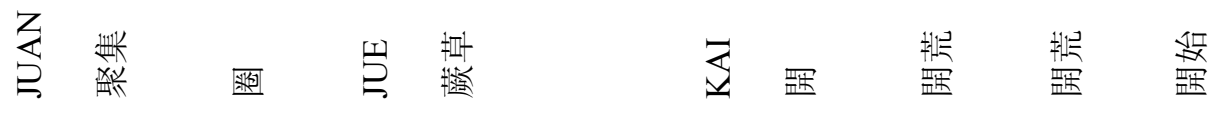




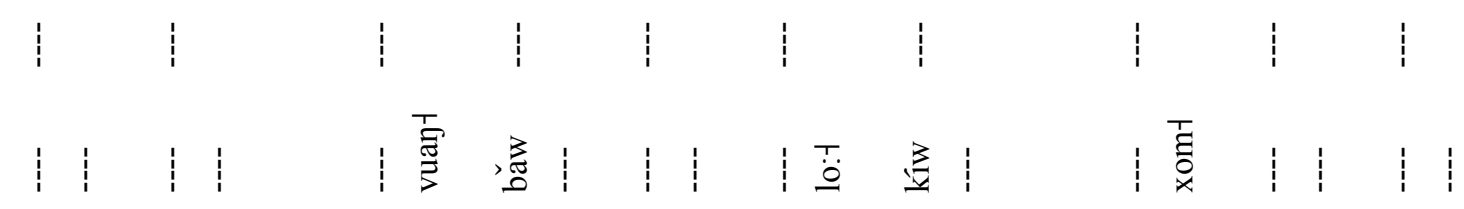

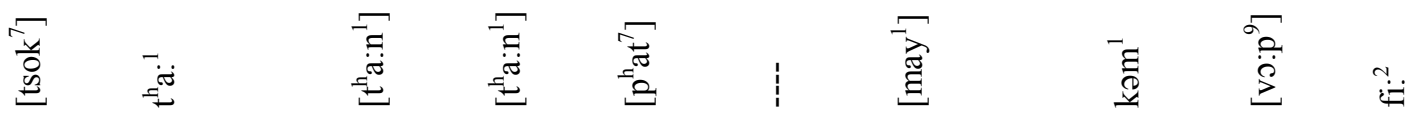

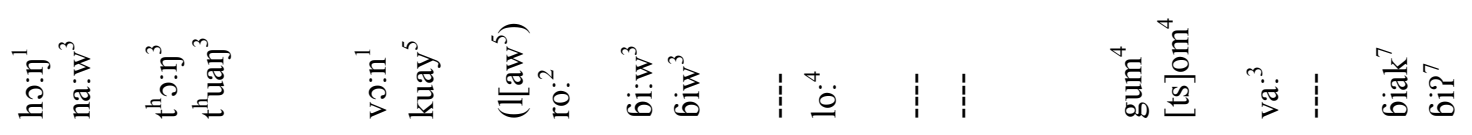

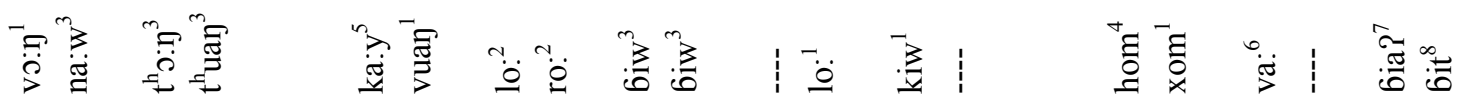

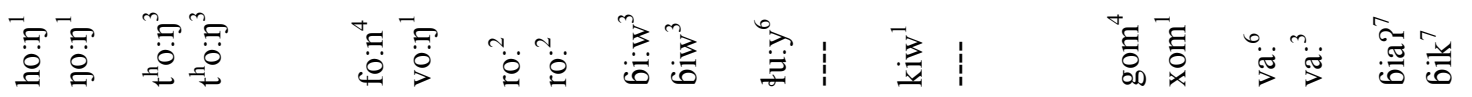

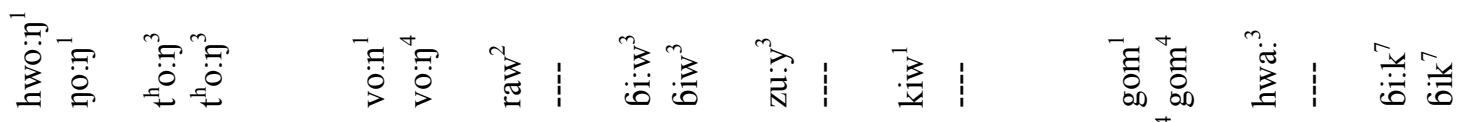

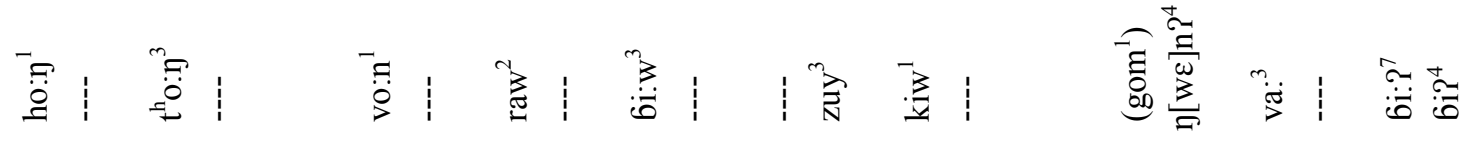

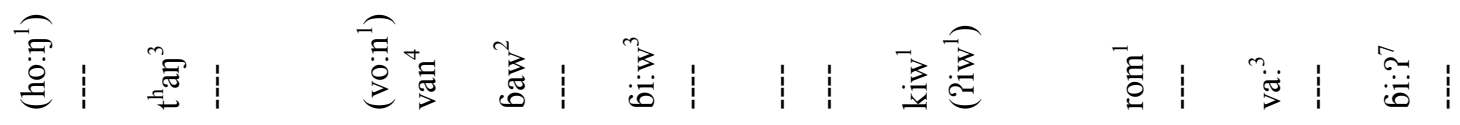

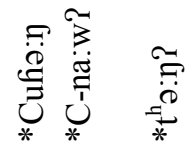

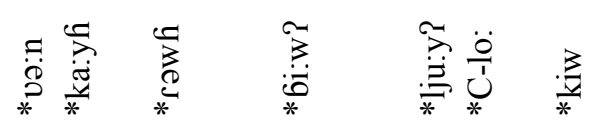

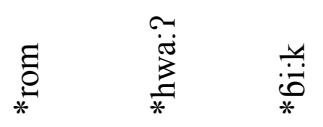

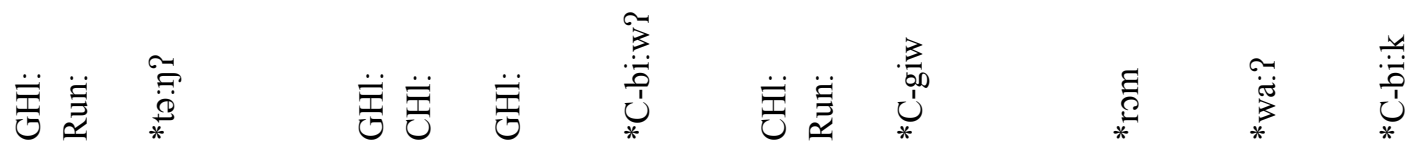

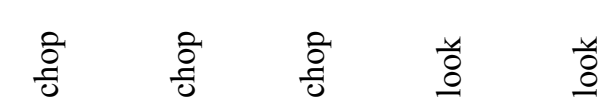

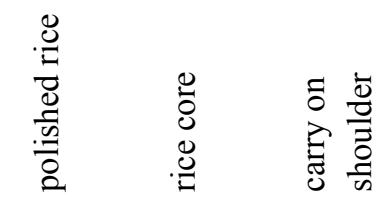

漬

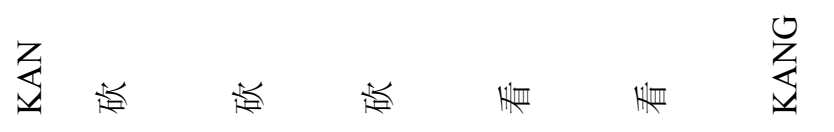
泉齿 


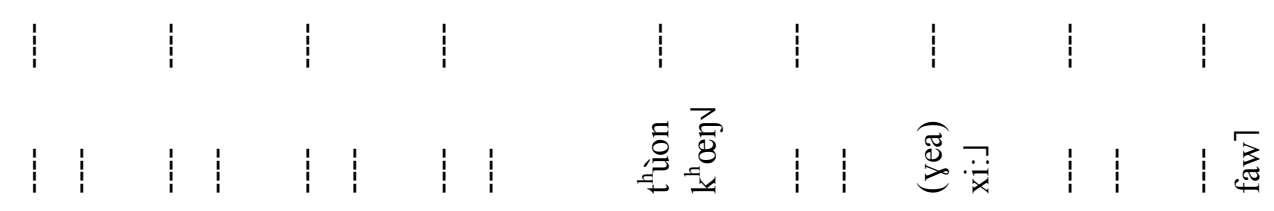

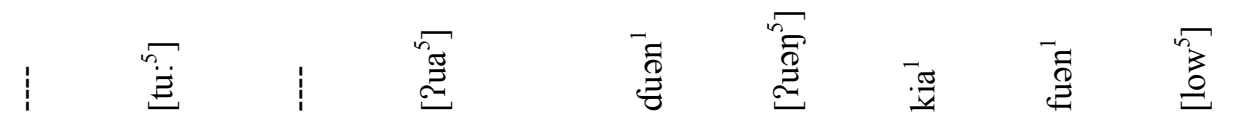

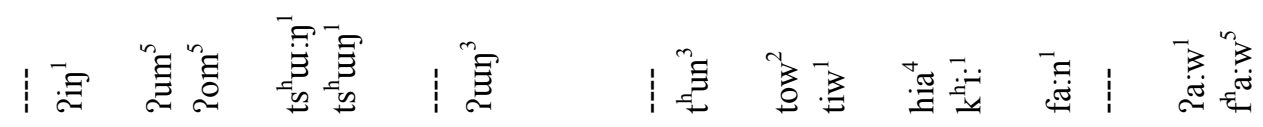

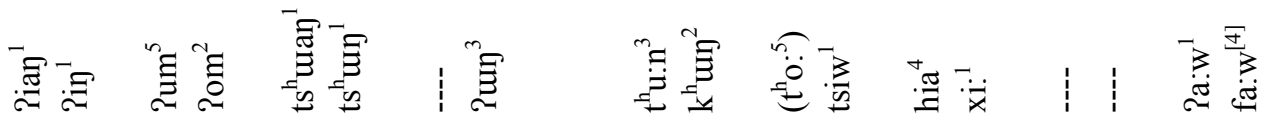

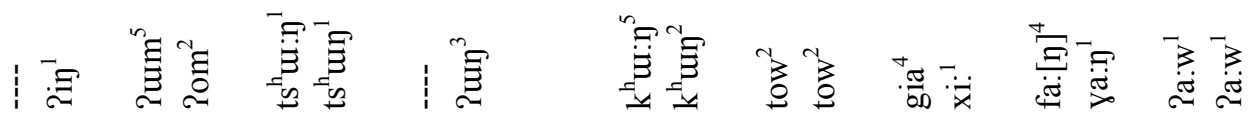

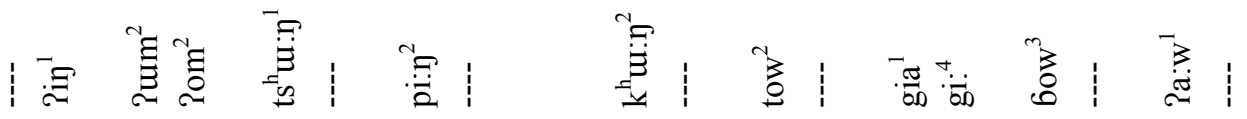

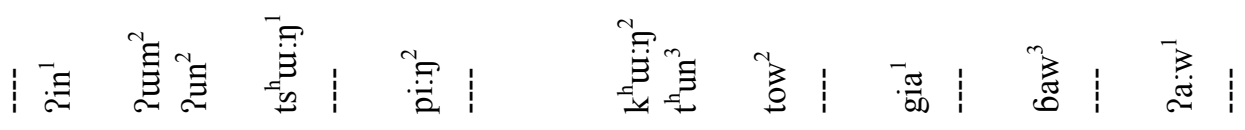

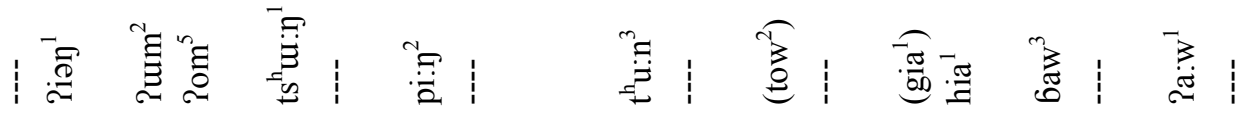

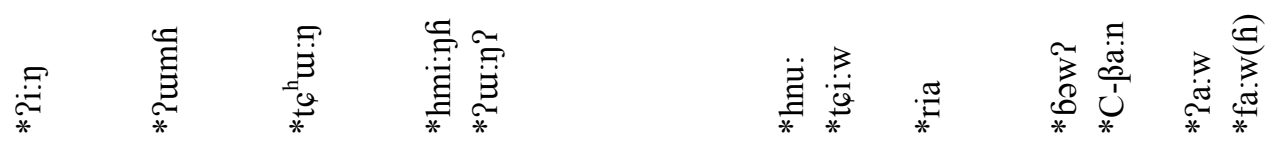

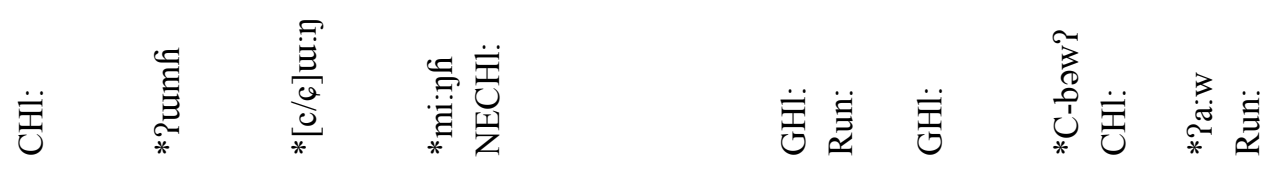

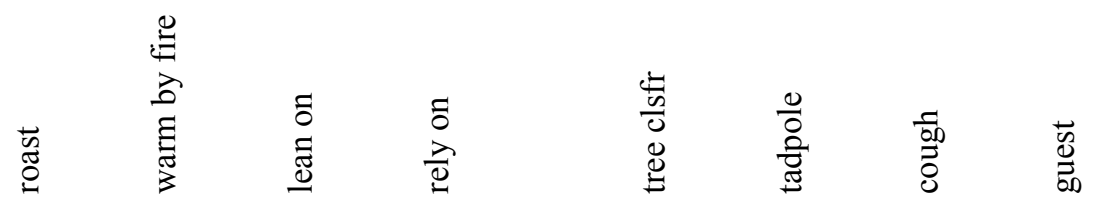

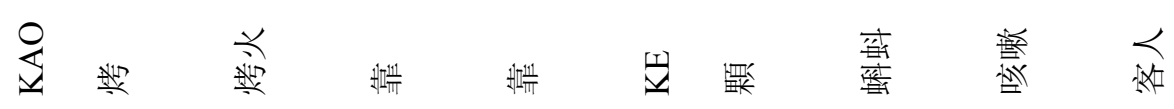




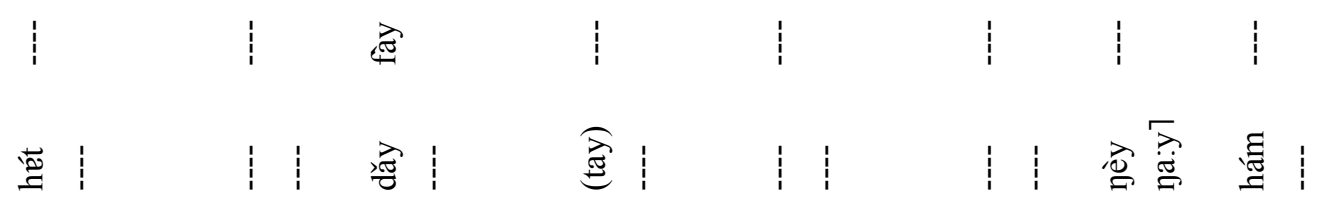

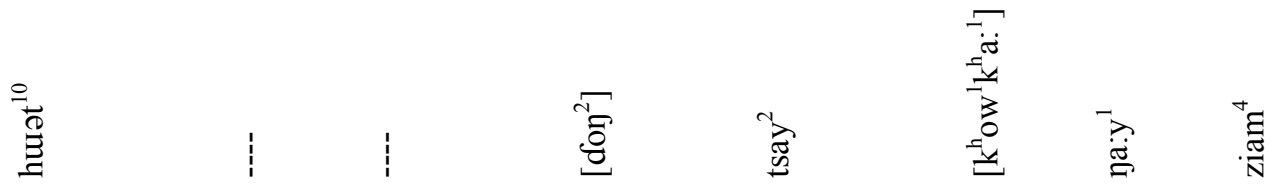

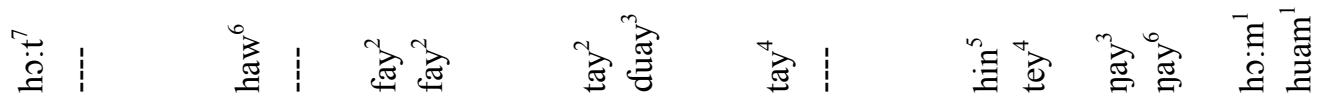

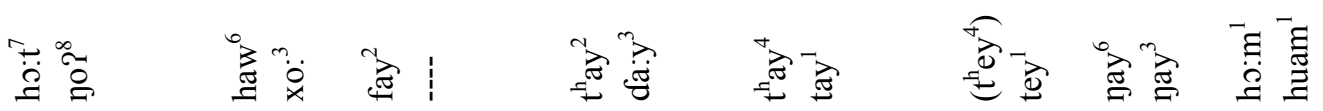

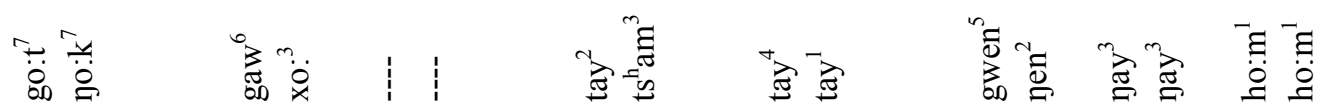

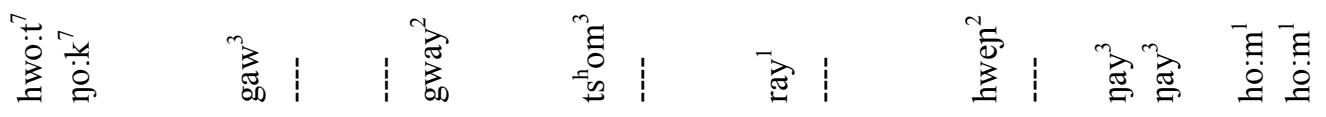

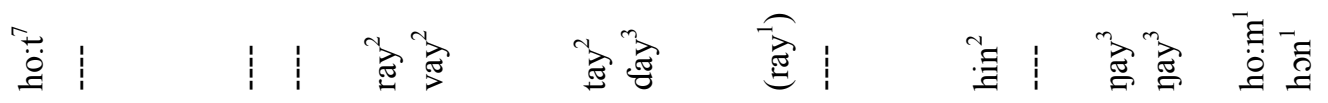

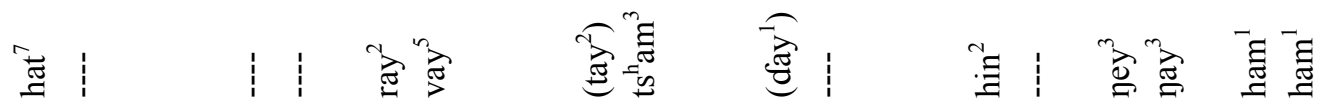

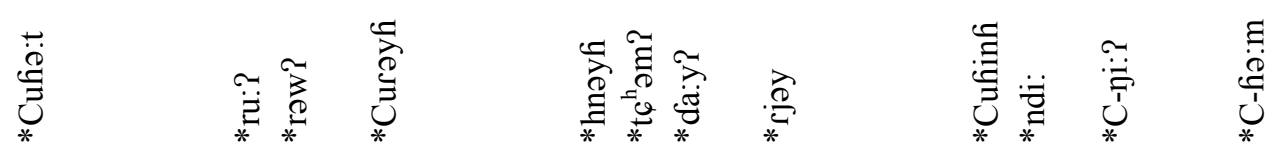

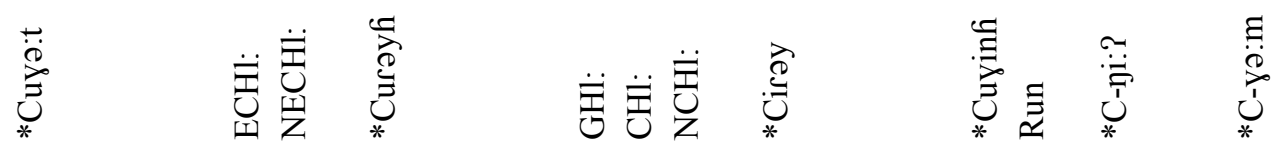

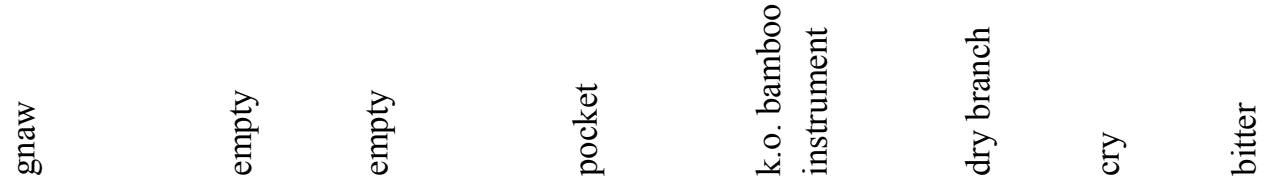

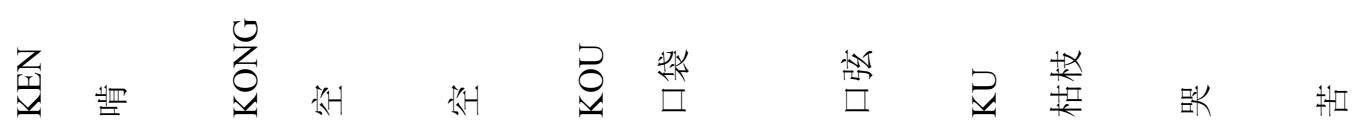




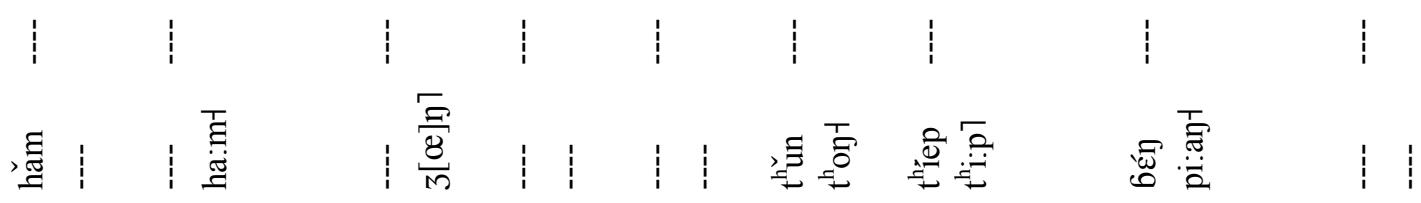

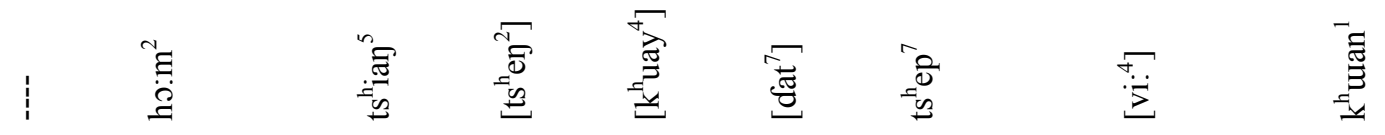

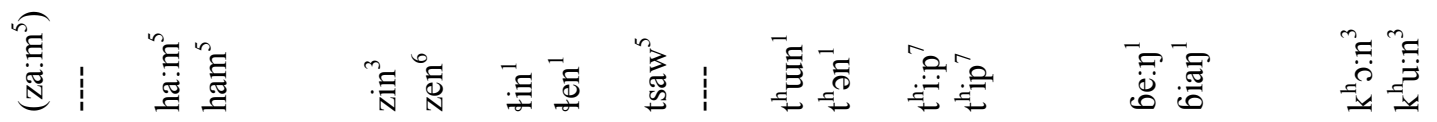

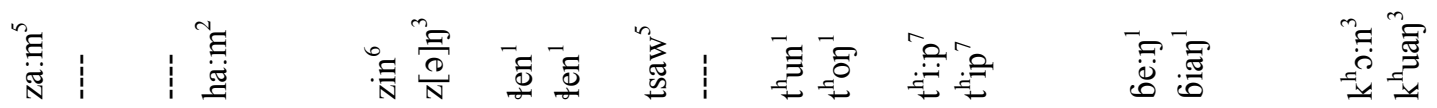

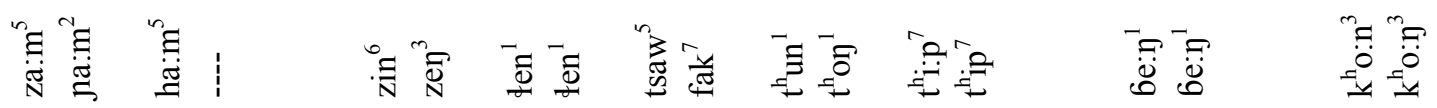

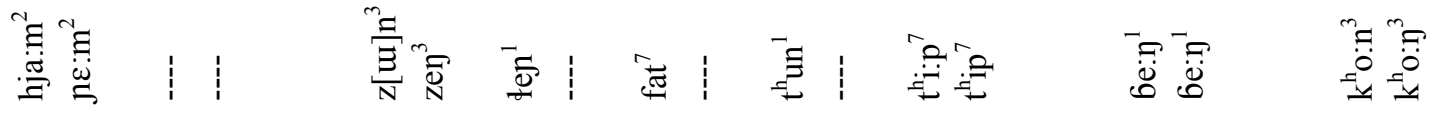

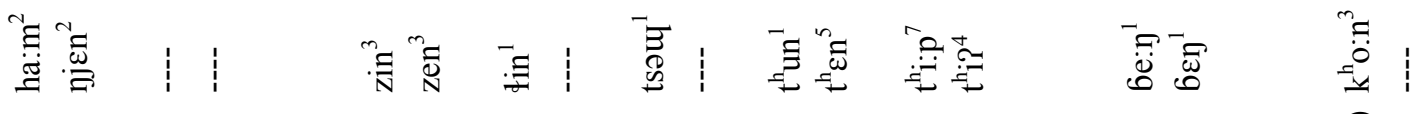

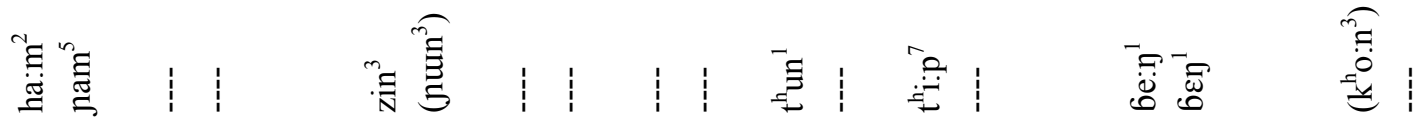

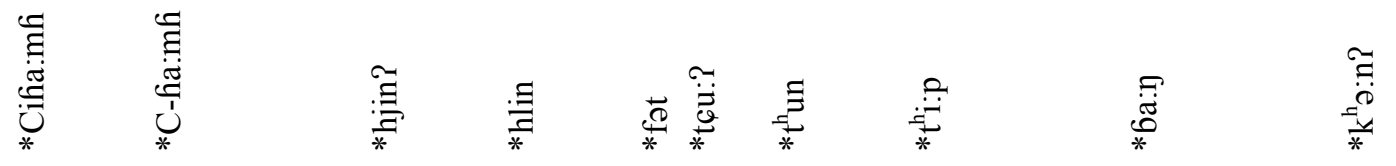

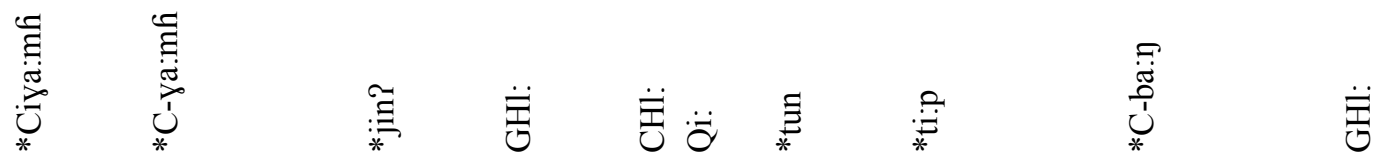

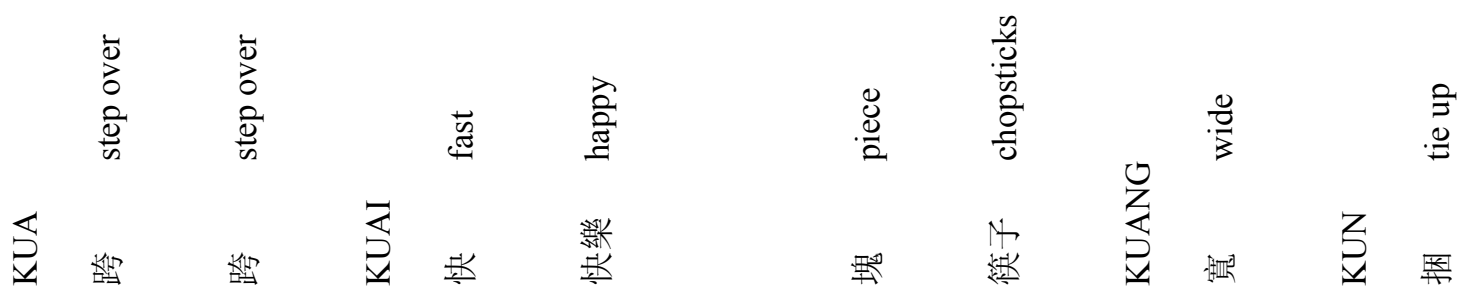




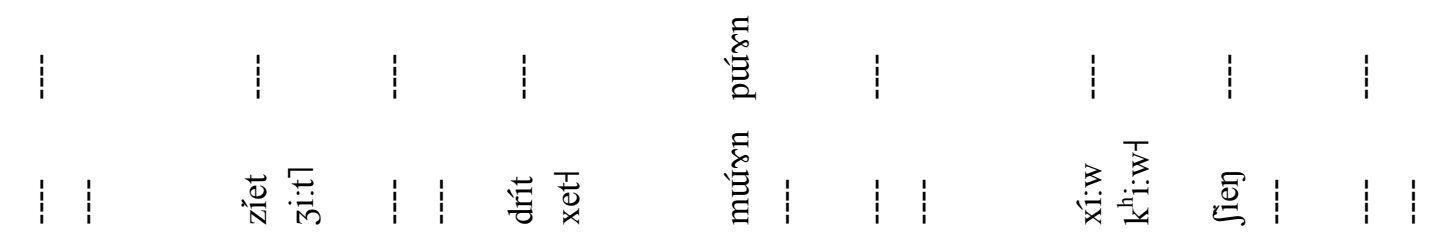

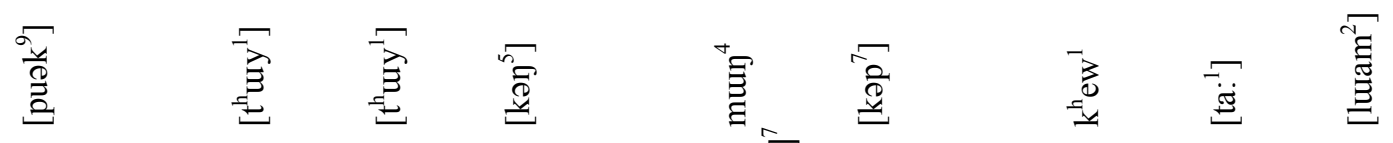
尊粱

瀶泀

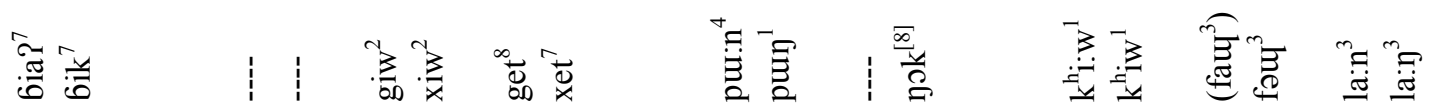

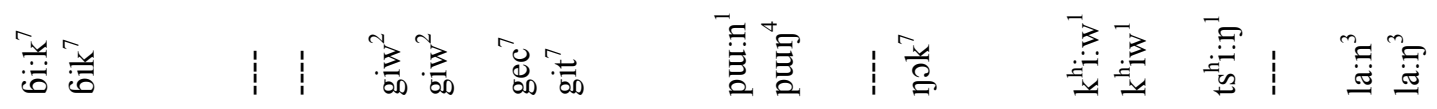

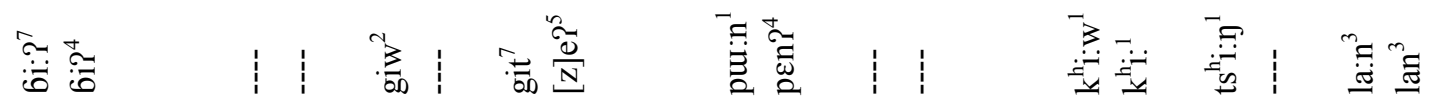

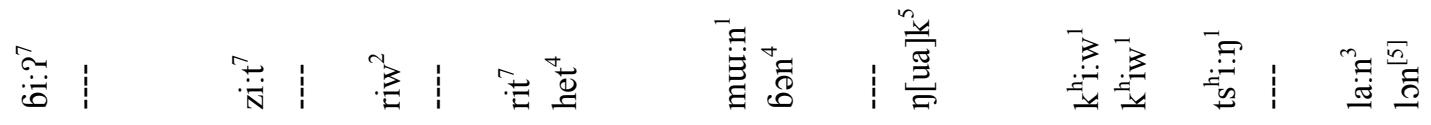

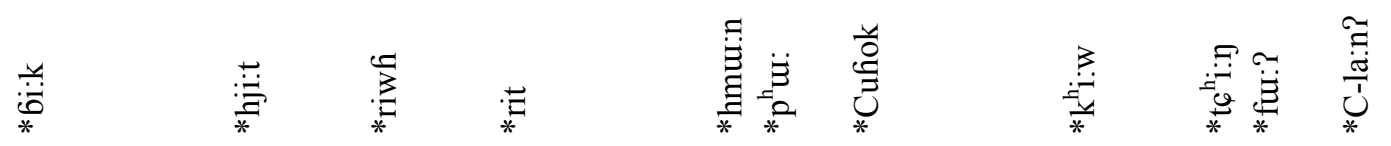

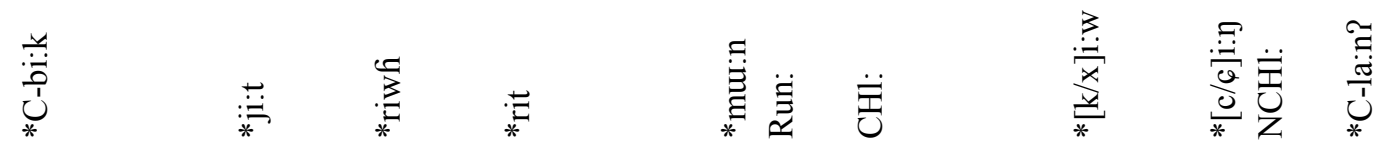

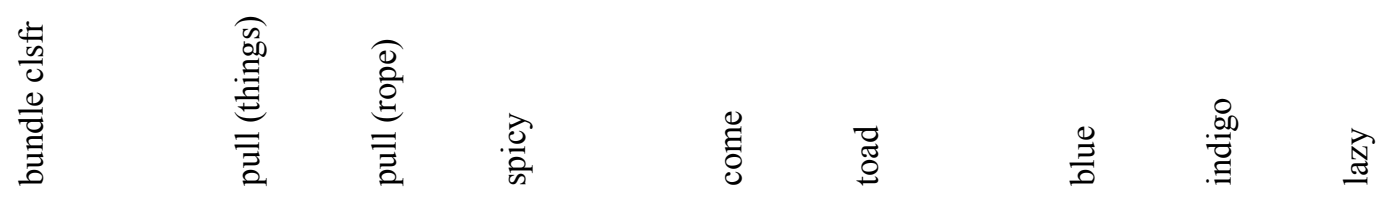

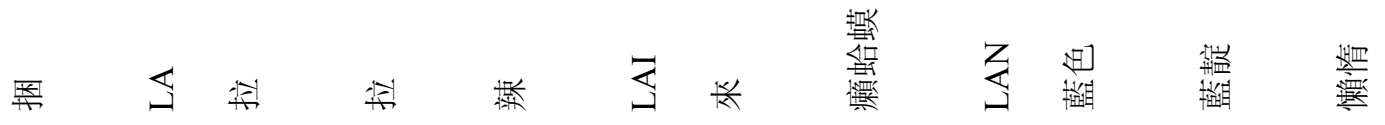




\begin{tabular}{|c|c|c|c|c|c|c|}
\hline 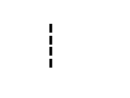 & 1 & 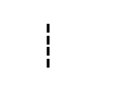 & 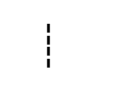 & 1 & 录 & 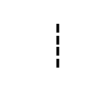 \\
\hline$:$ & \begin{tabular}{l|l|l} 
& 5 \\
\end{tabular} & 镸: & 恣 & 1 & 量量 & $:$ \\
\hline 离 & $\stackrel{\bar{\Xi}}{\ddot{E}}$ & 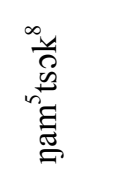 & $\ddot{\ddot{\theta}}$ & 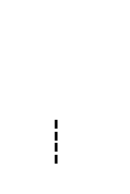 & 峷 & $\stackrel{\circ}{\underline{\text { I }}}$ \\
\hline : & 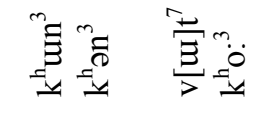 & 言: & 咲莒 & 寻": & 棒焉 & 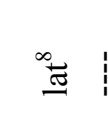 \\
\hline 1 & $1: \quad \begin{array}{l}0 \\
1\end{array}$ & 言: & 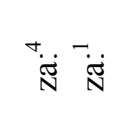 & 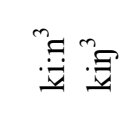 & 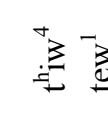 & 旅 \\
\hline 惫焉 & 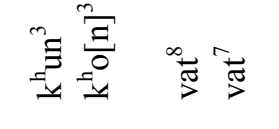 & 唇営 & 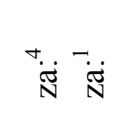 & 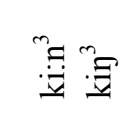 & 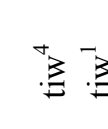 & $\underset{\bar{y}}{\infty}$ \\
\hline 胥 & 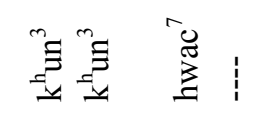 & 言: & 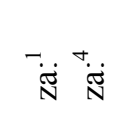 & 解: & 寻 & 巳̈: \\
\hline 部 & 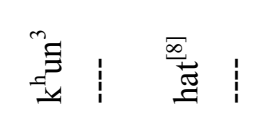 & 謤: & 离营 & : & 青毫 & $\frac{\Phi}{\square}$ \\
\hline & 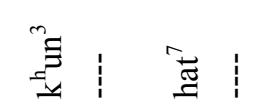 & 言: & ن & : & 寻䓪 & च् \\
\hline
\end{tabular}

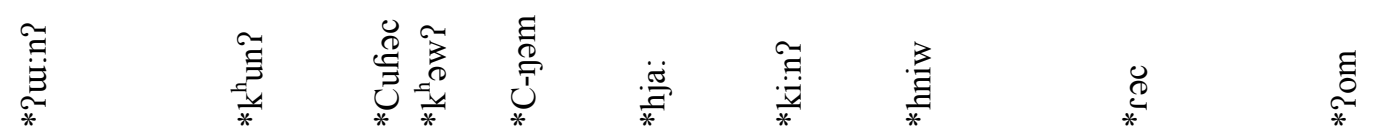

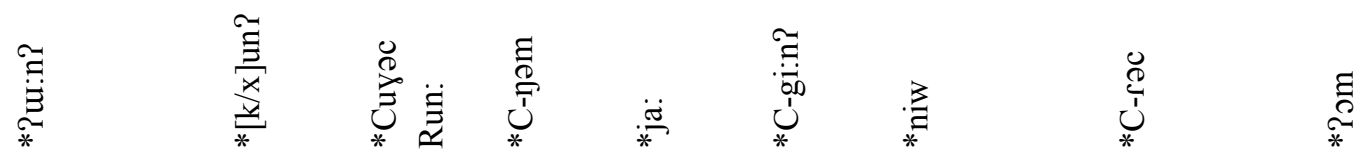

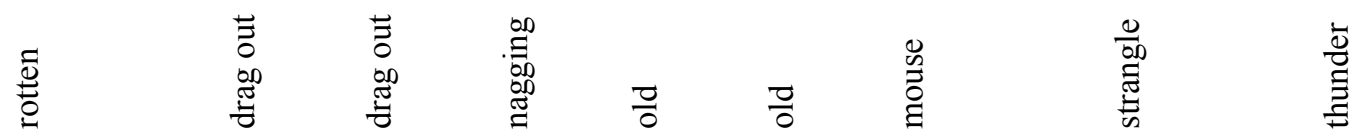

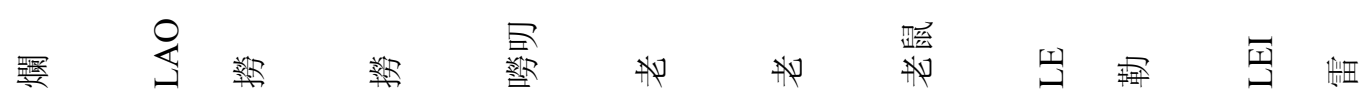




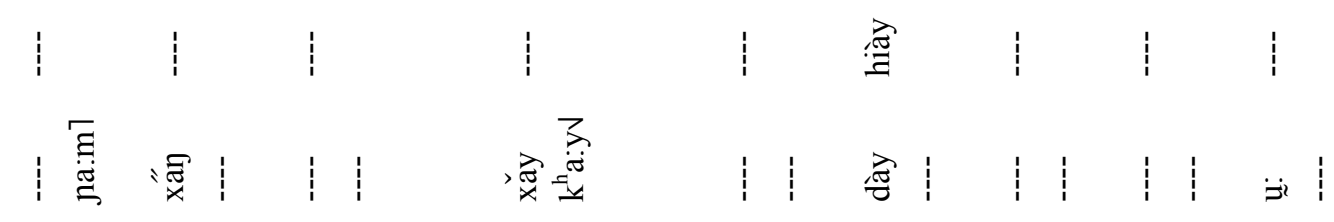

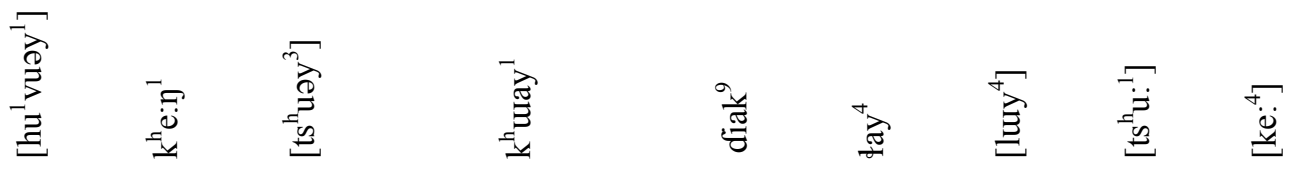

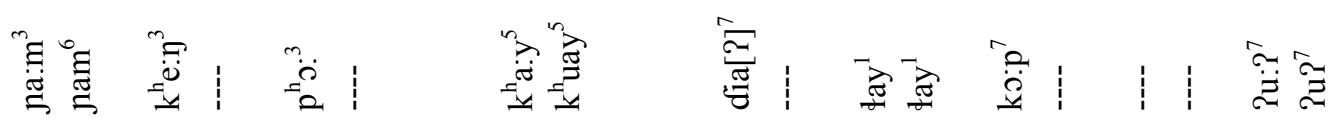

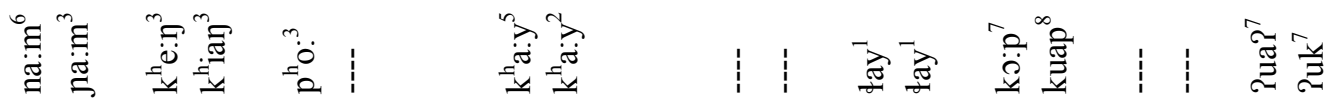

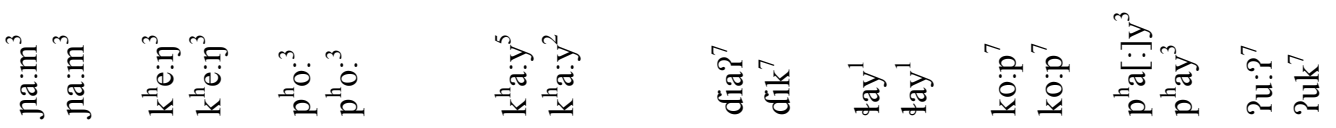

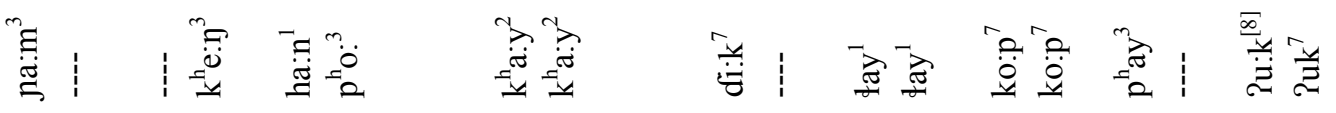

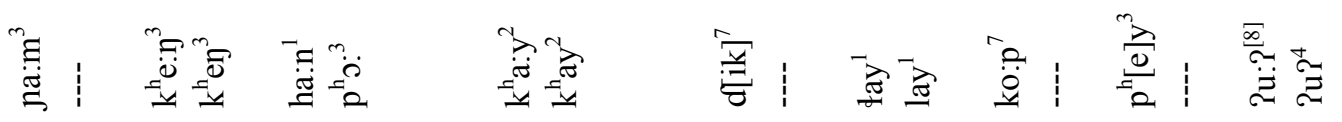

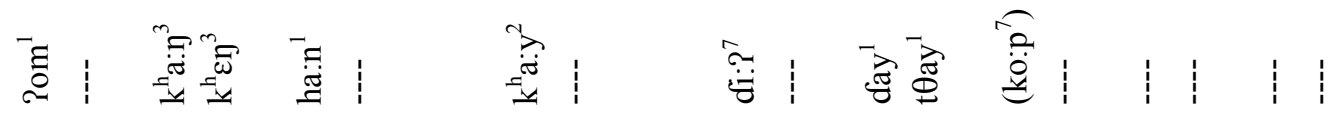

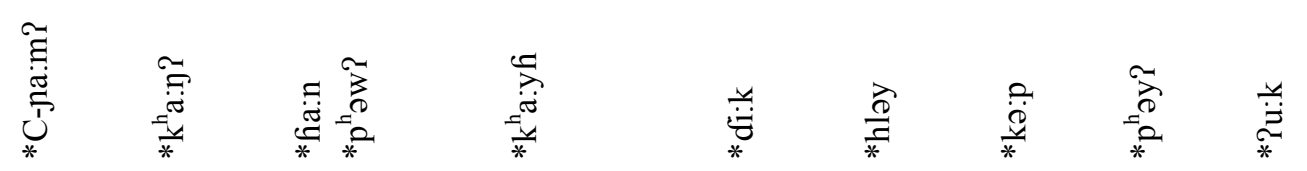

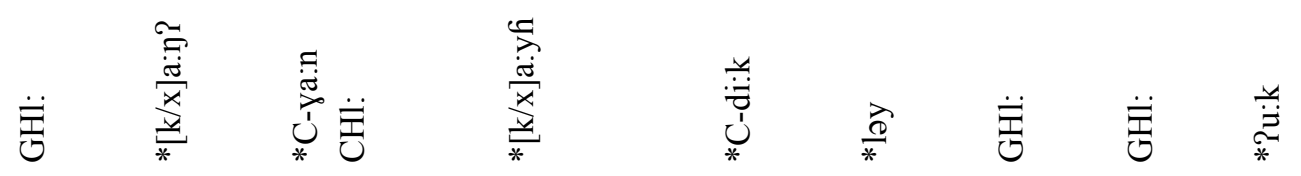

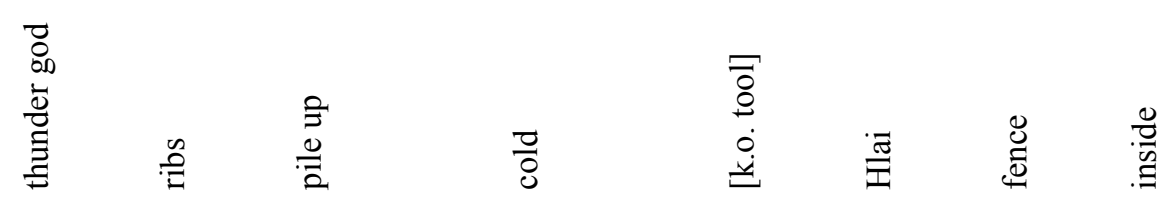

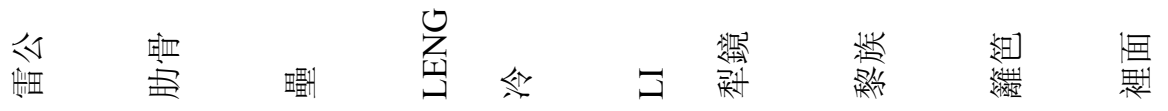




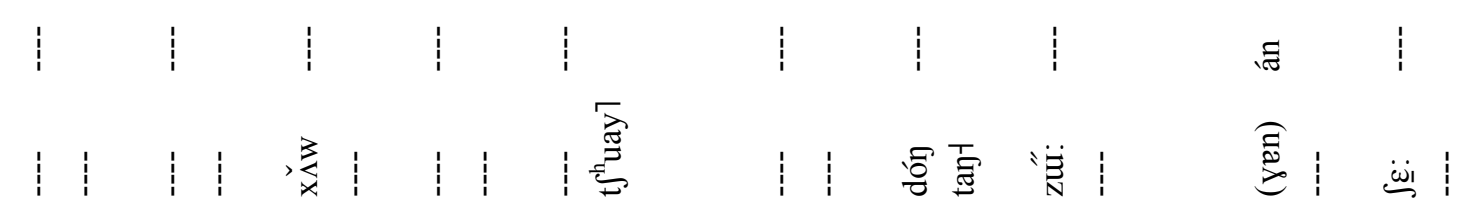

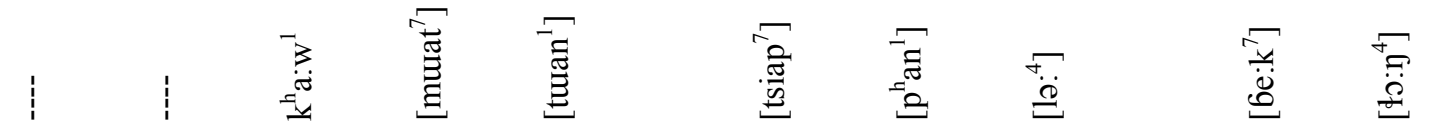

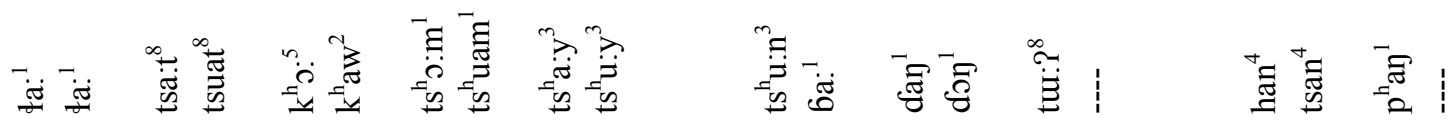

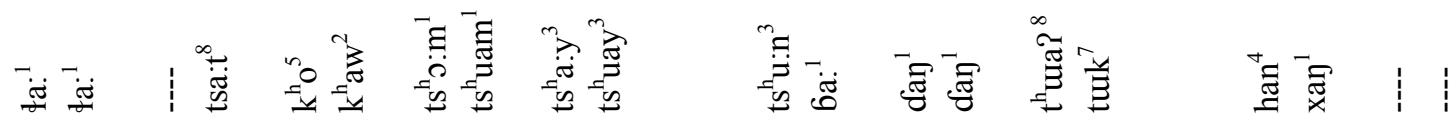

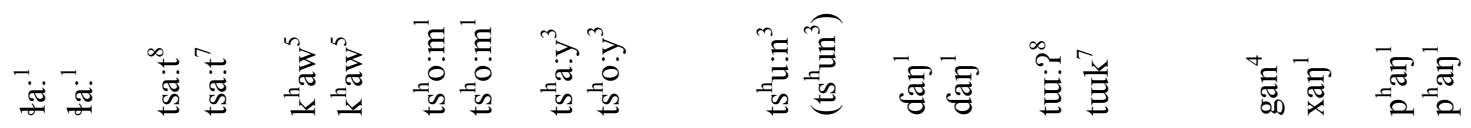

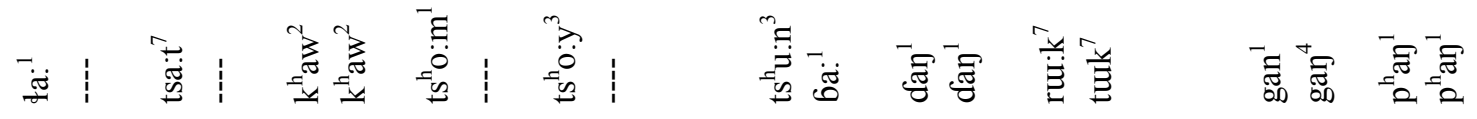

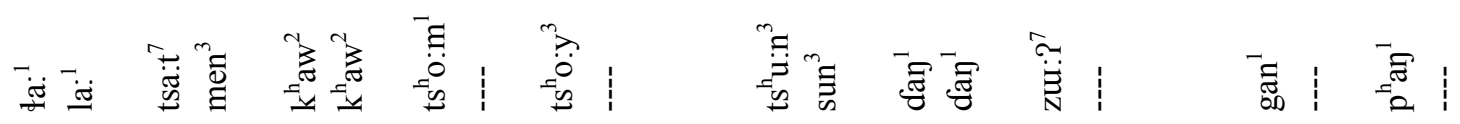

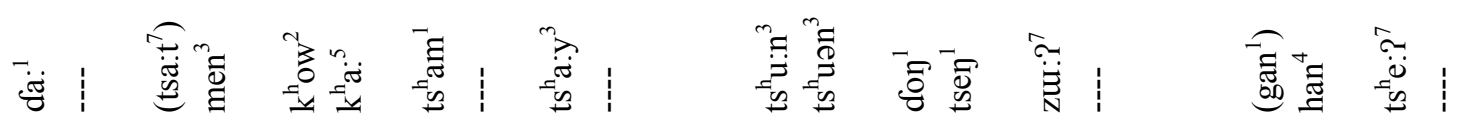

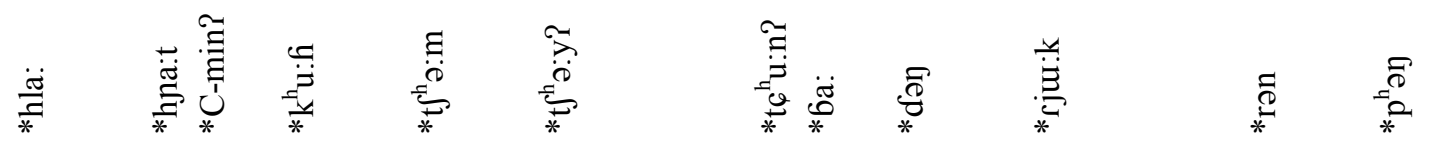

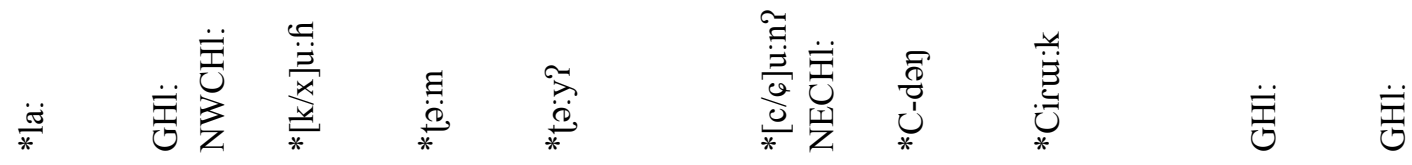

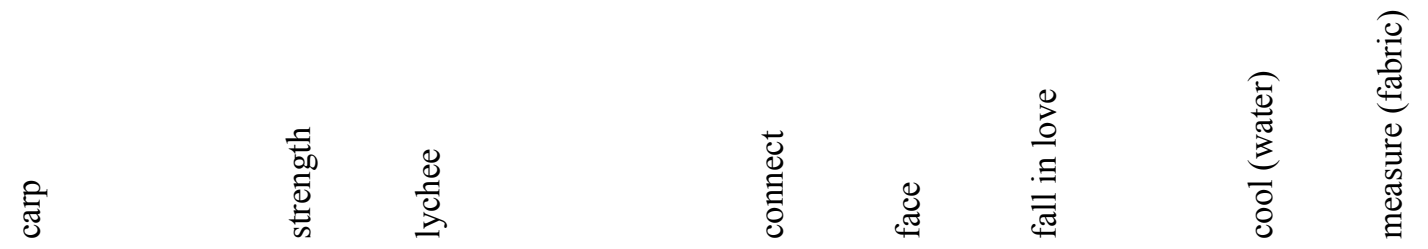

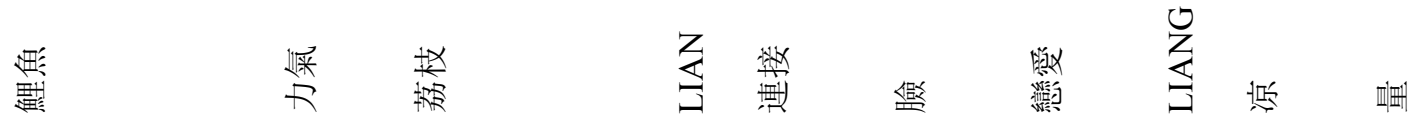




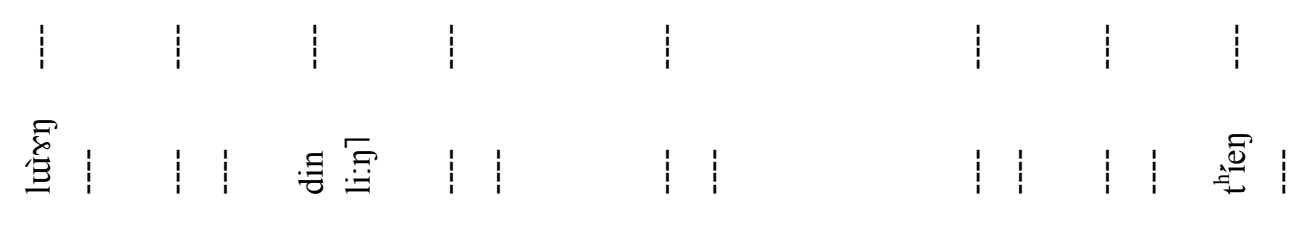

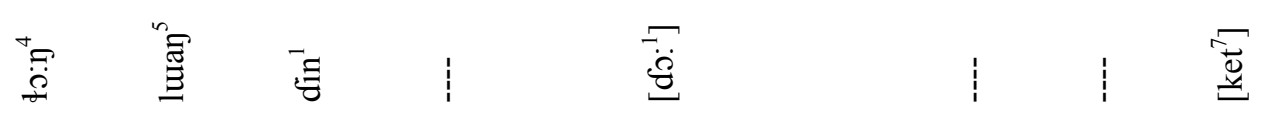

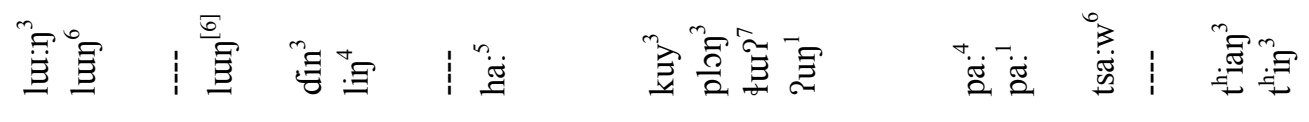

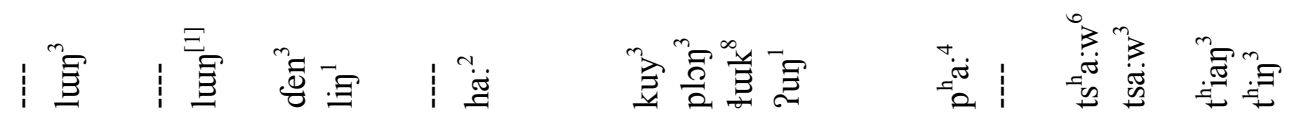

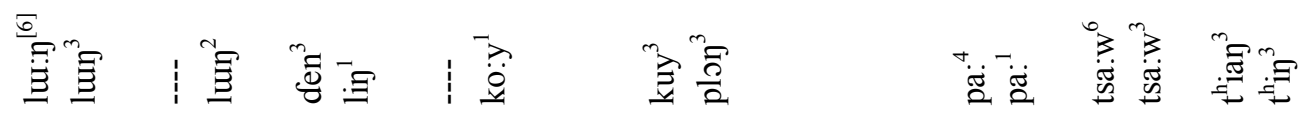

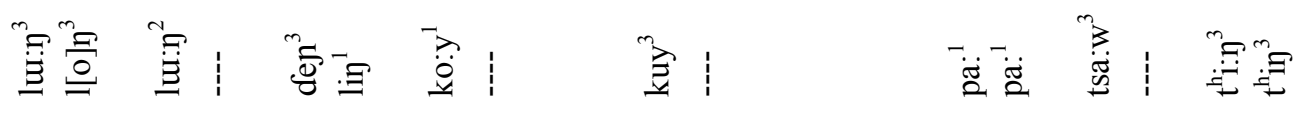

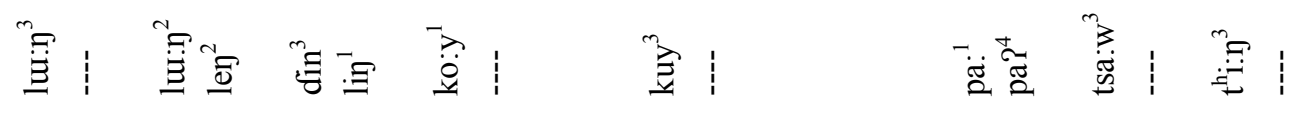

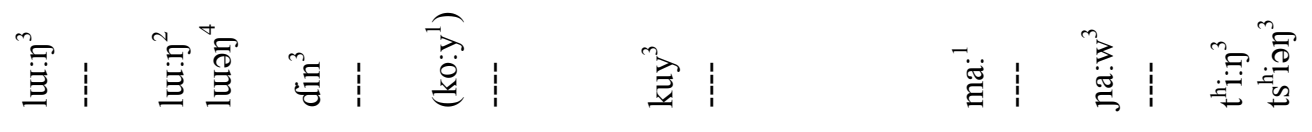

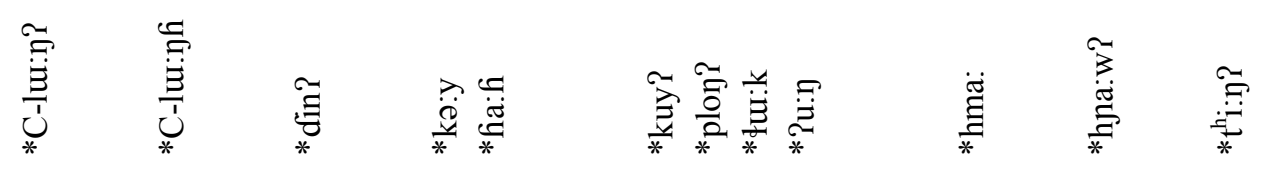

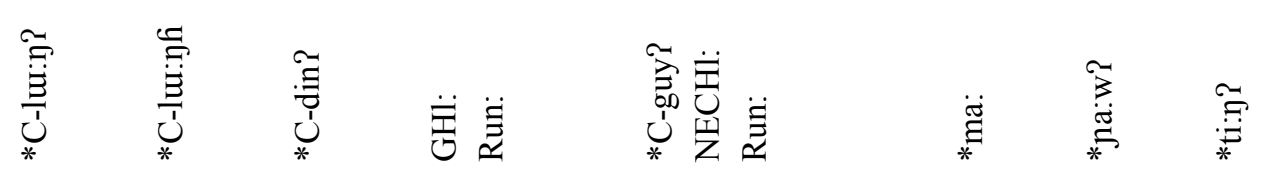

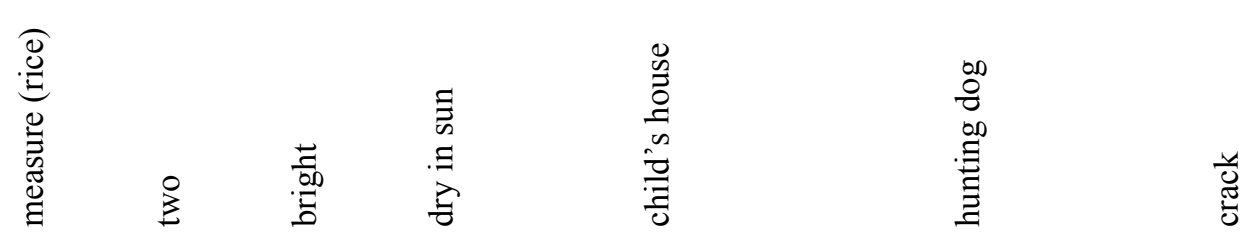

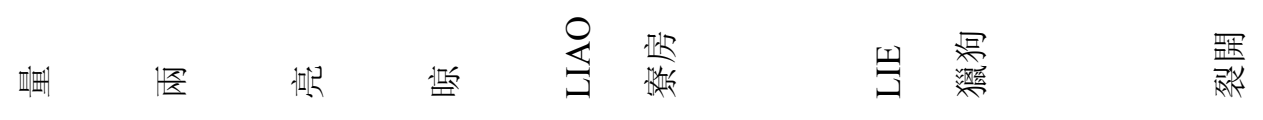




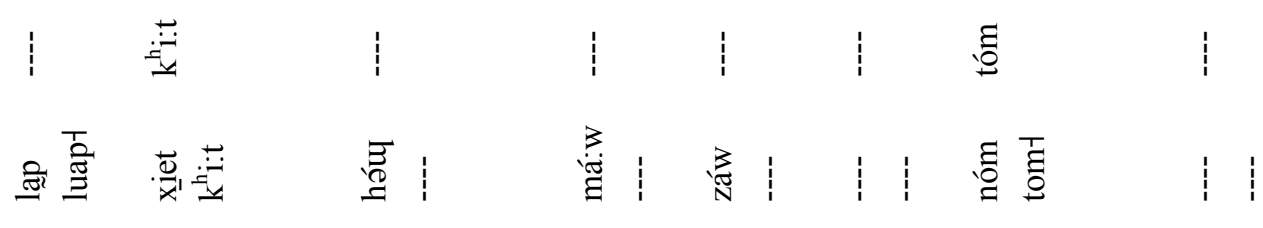

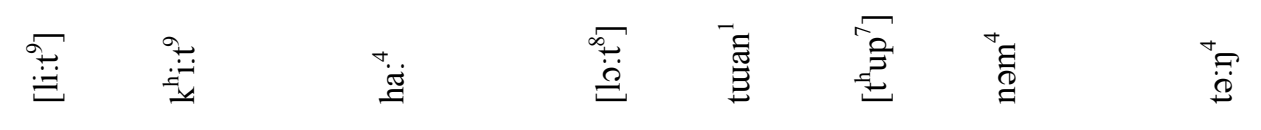

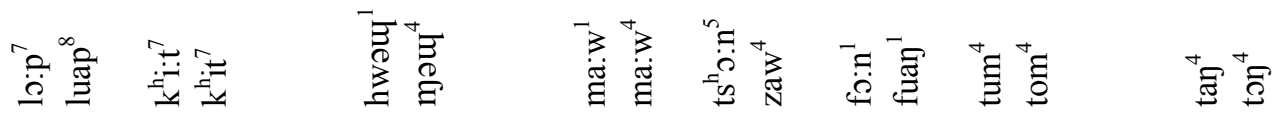

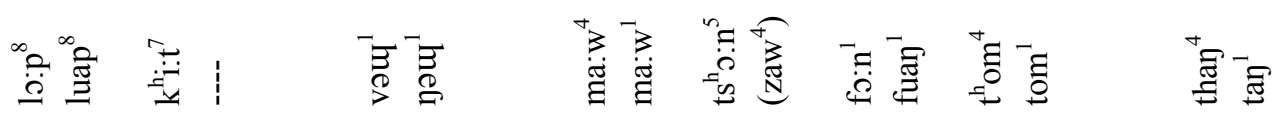

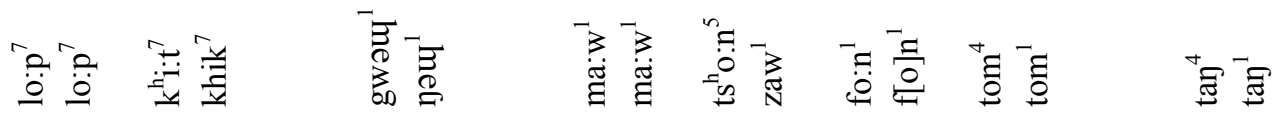

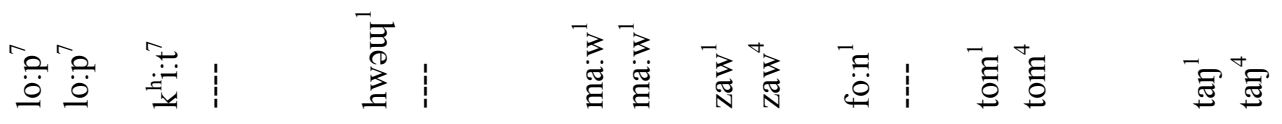

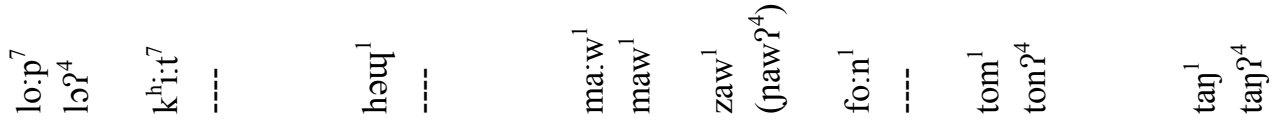

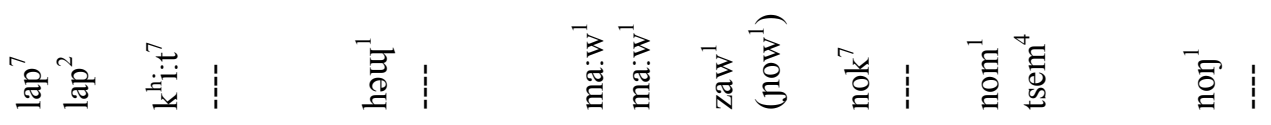

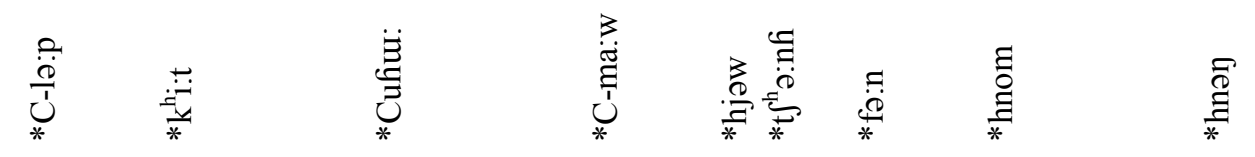

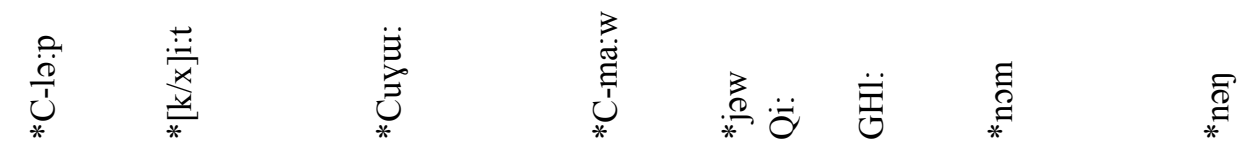

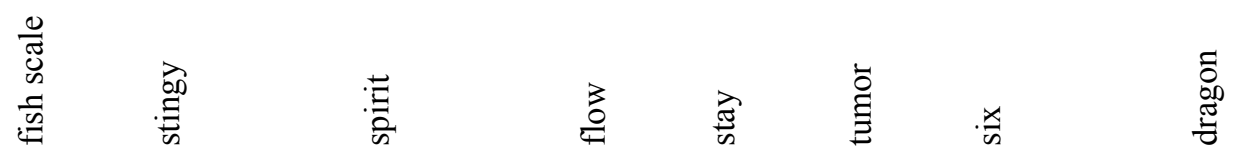

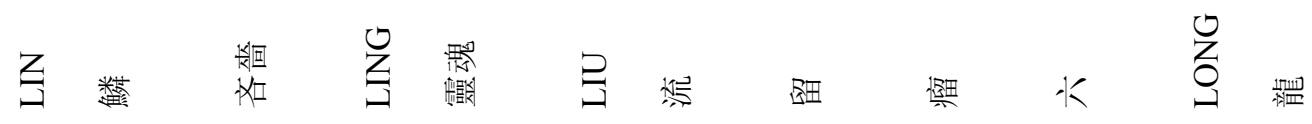




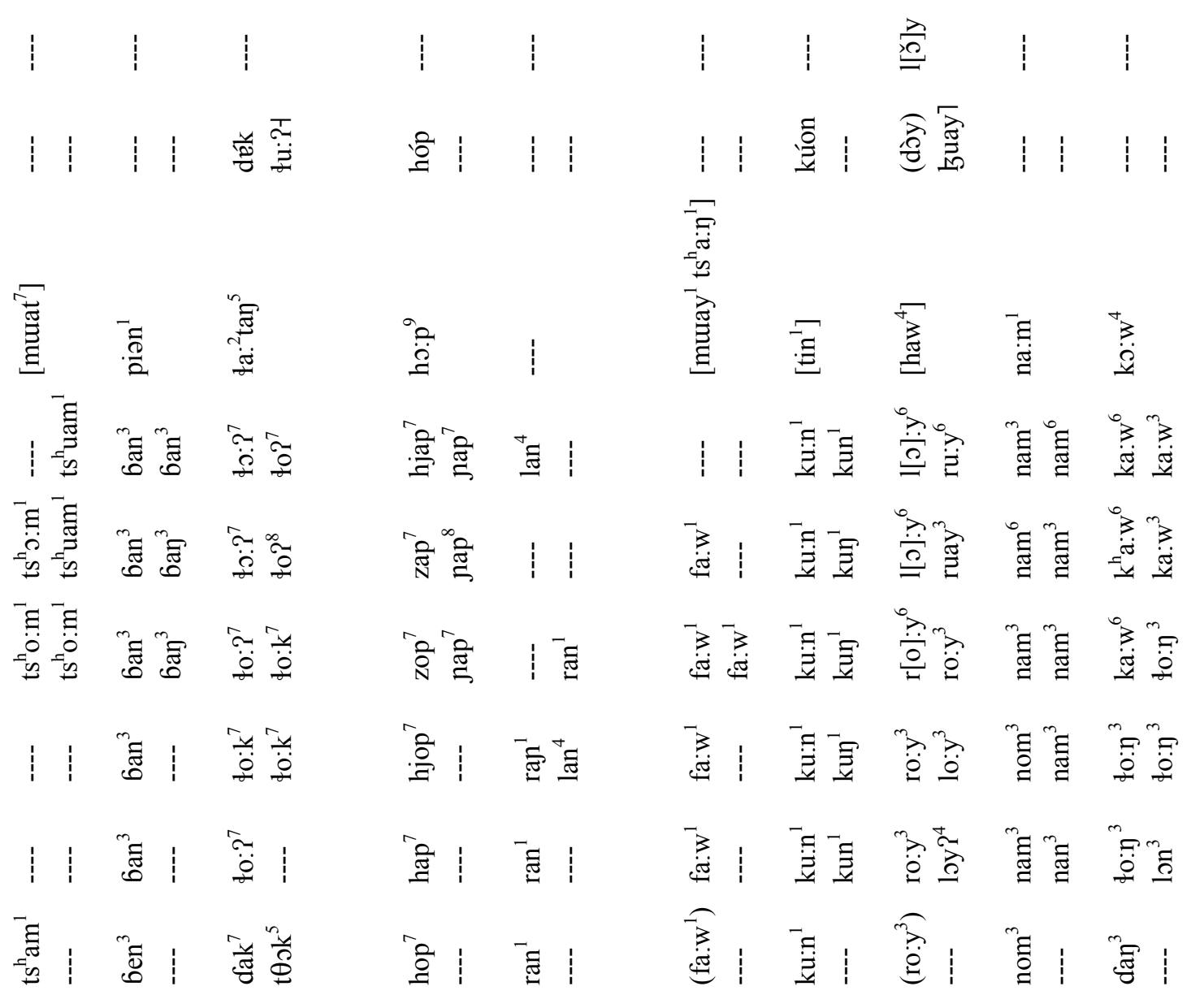

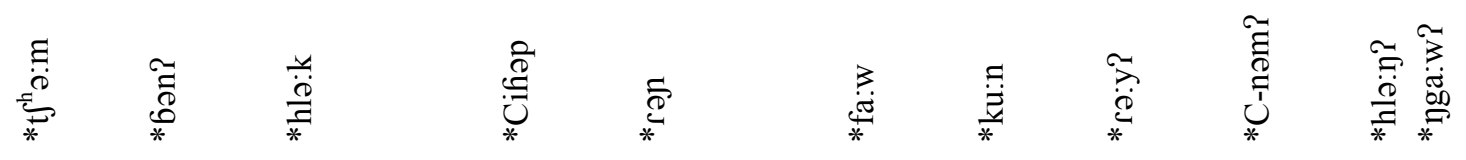

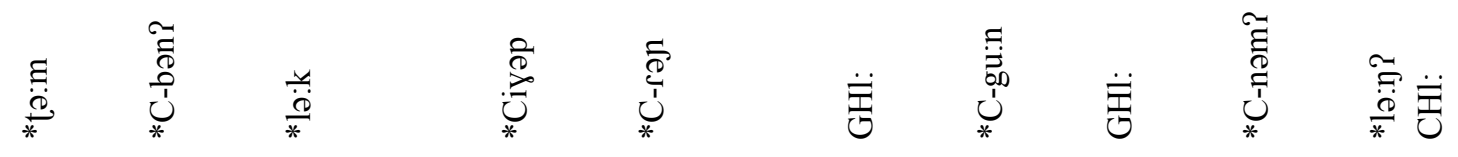

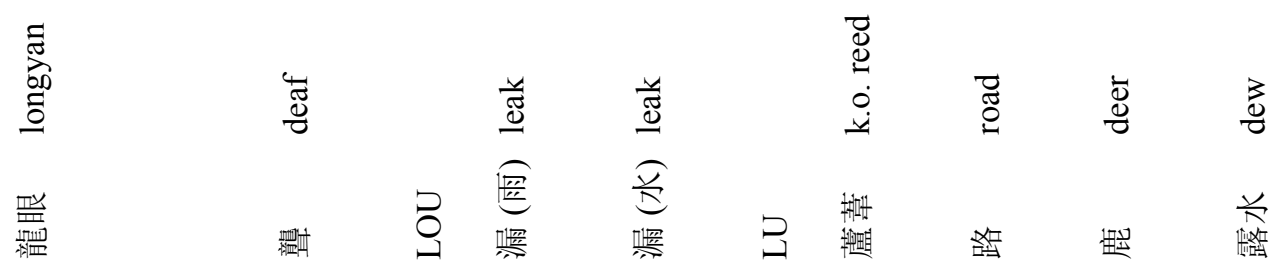




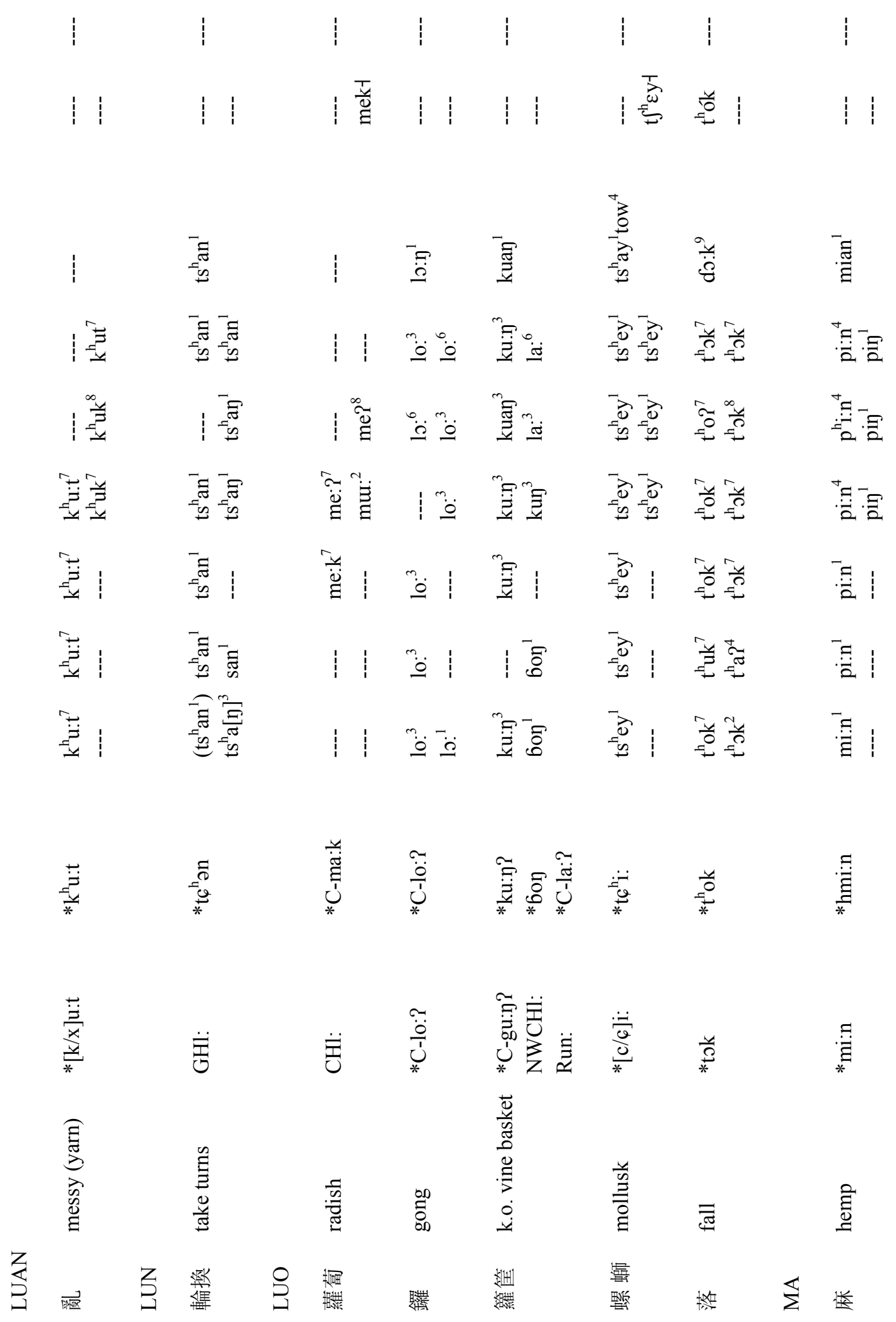




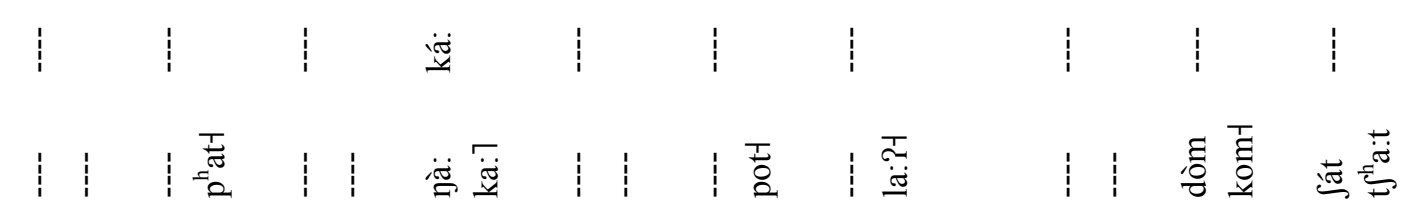

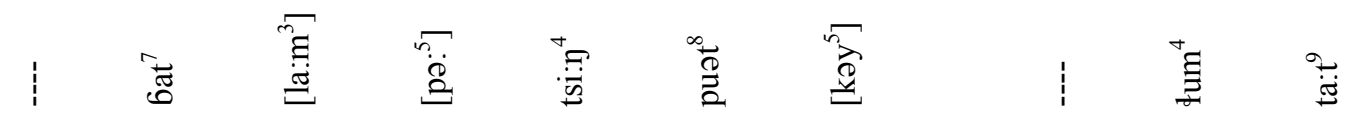

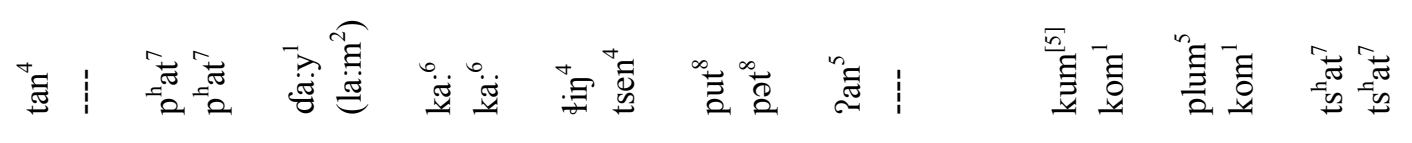

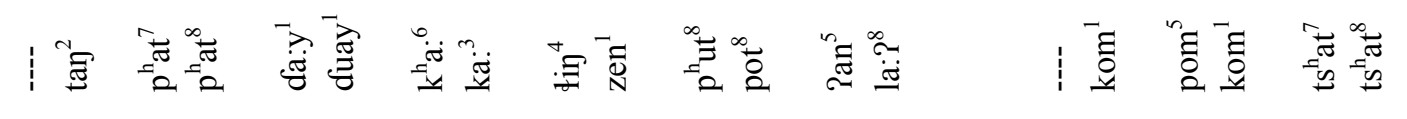

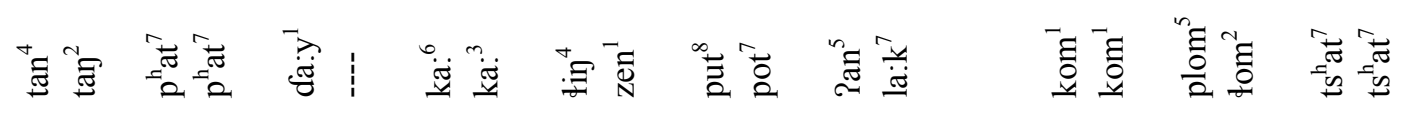

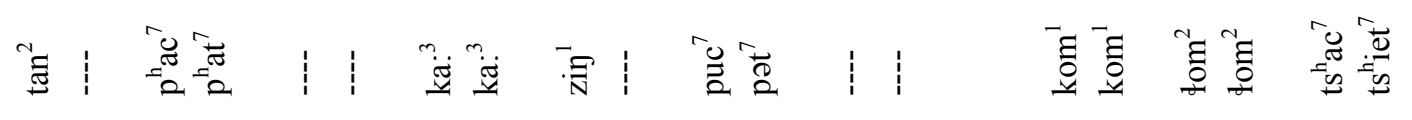

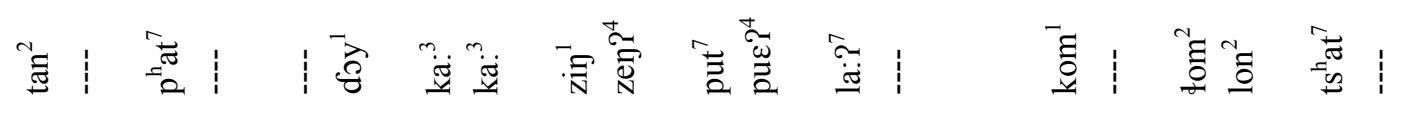

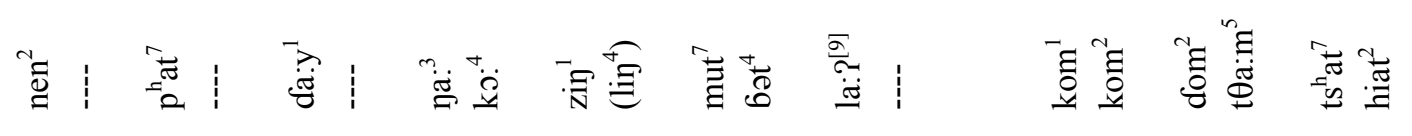

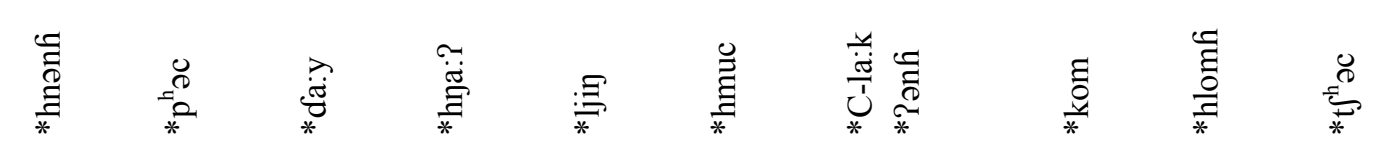

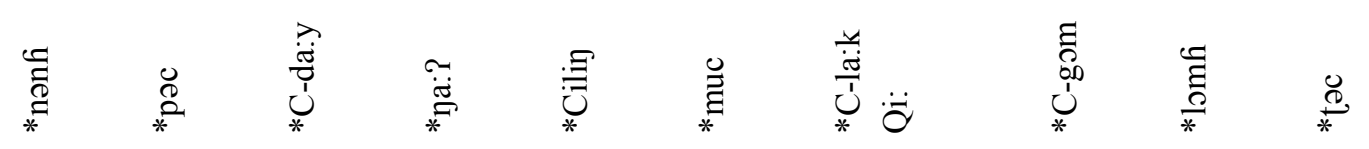

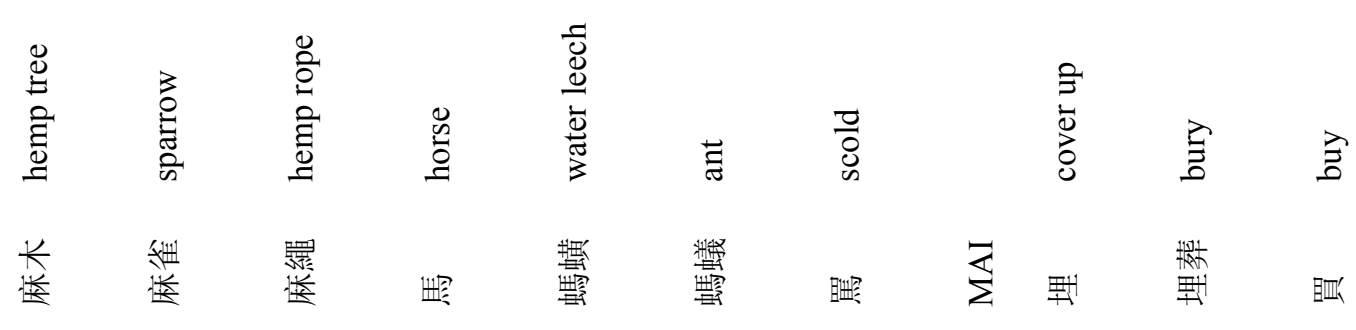




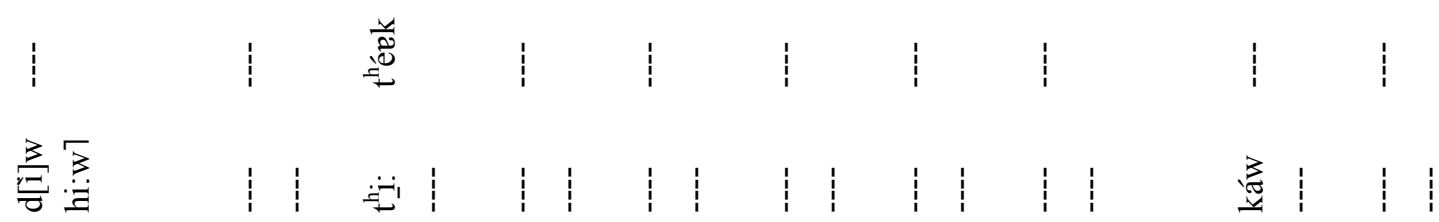

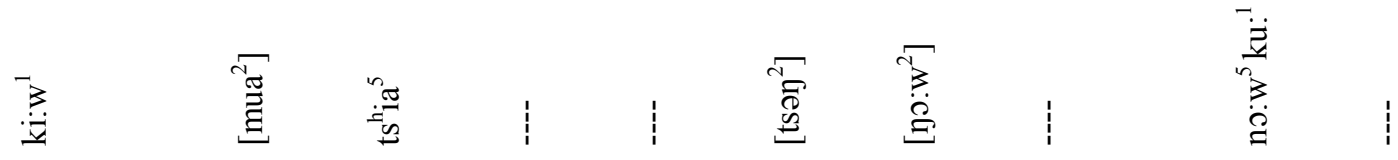

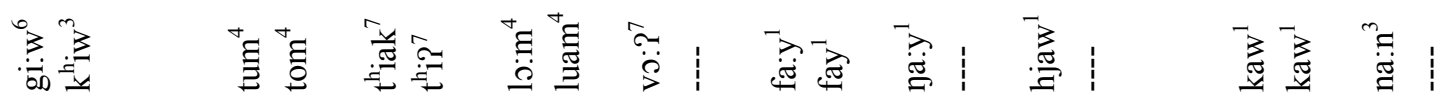

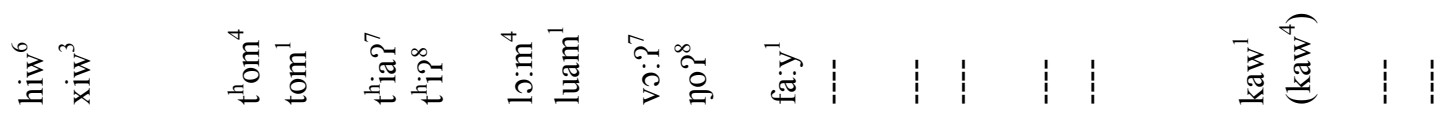

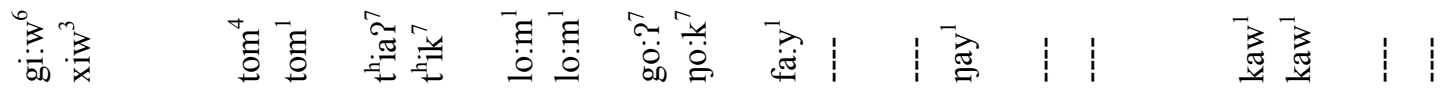

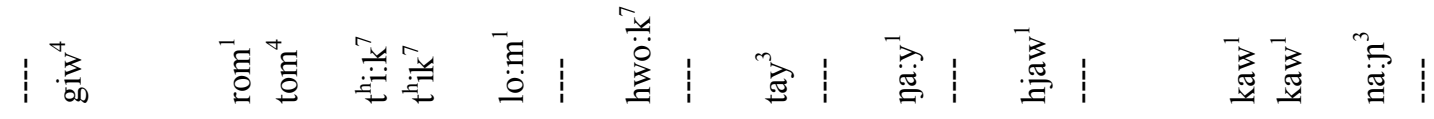

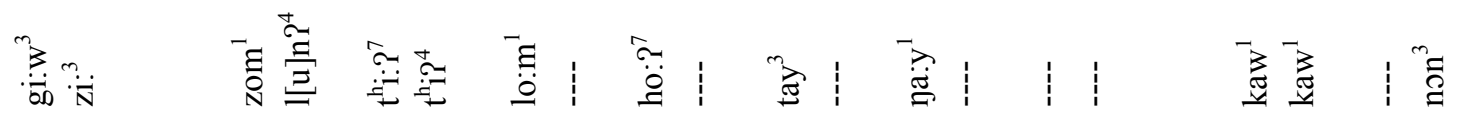

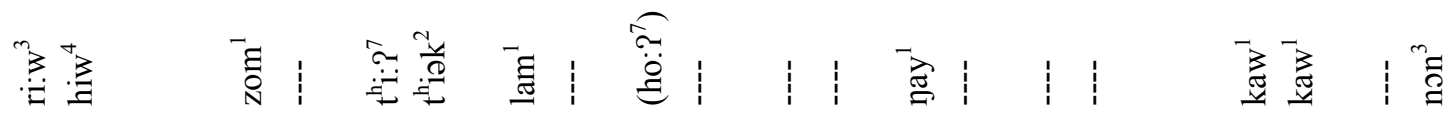

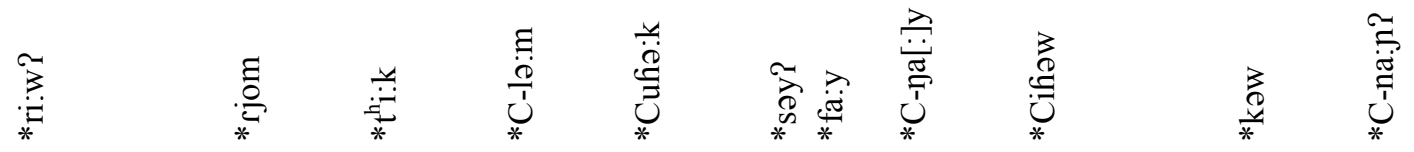

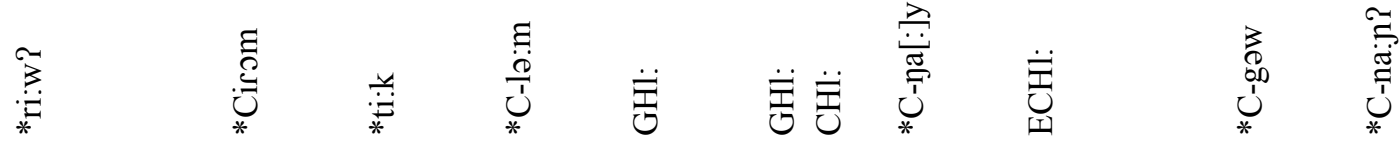

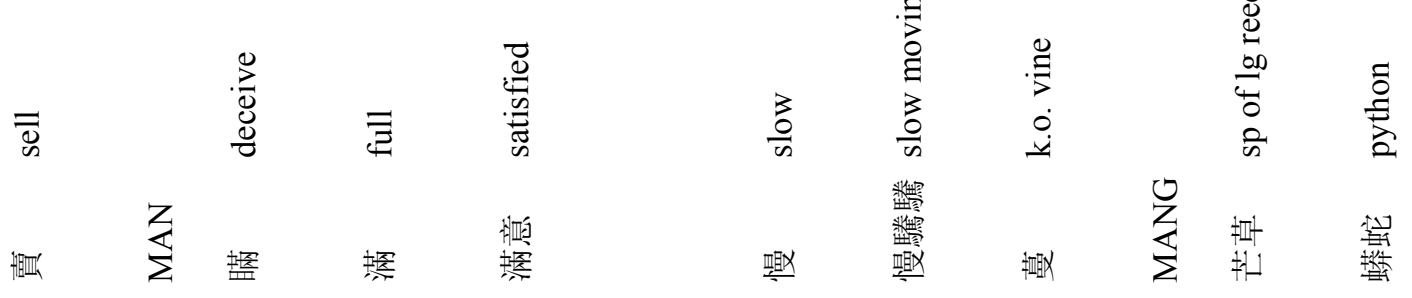




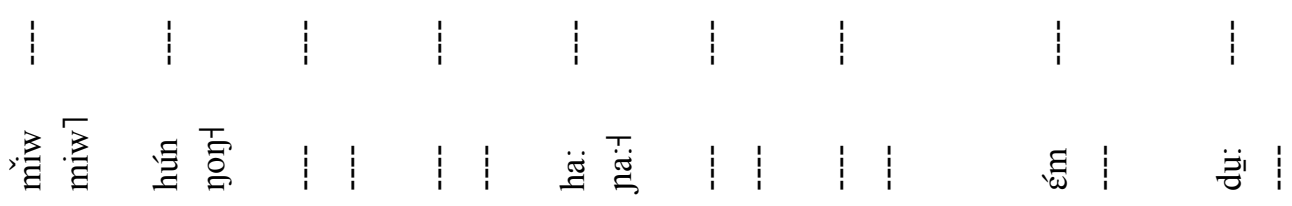

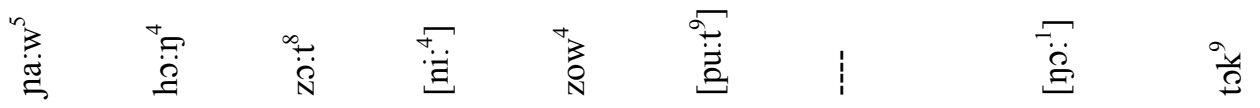

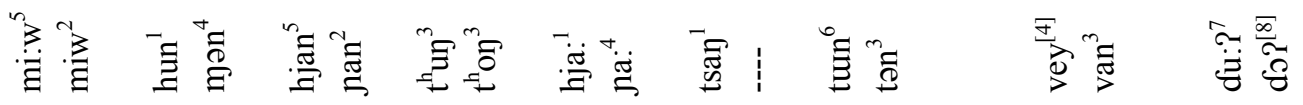

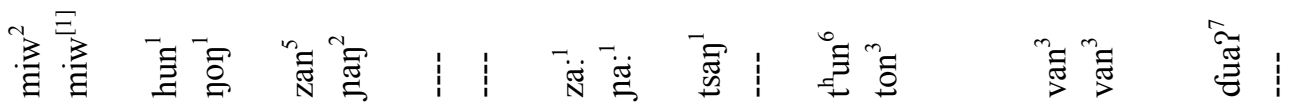

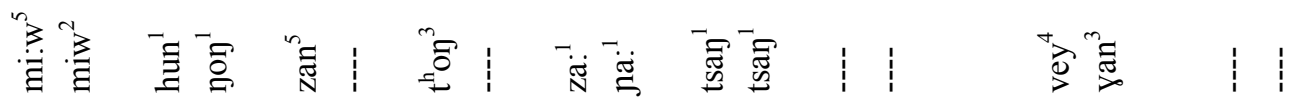

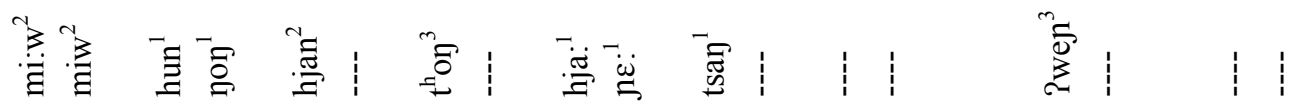

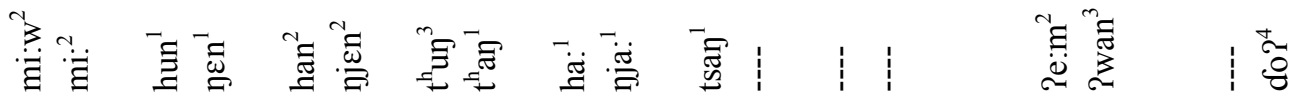

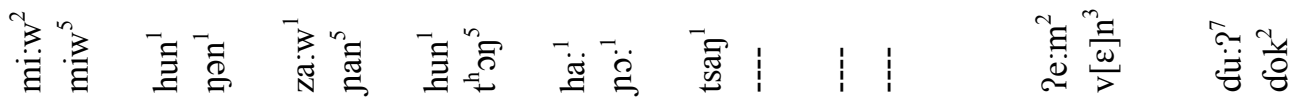

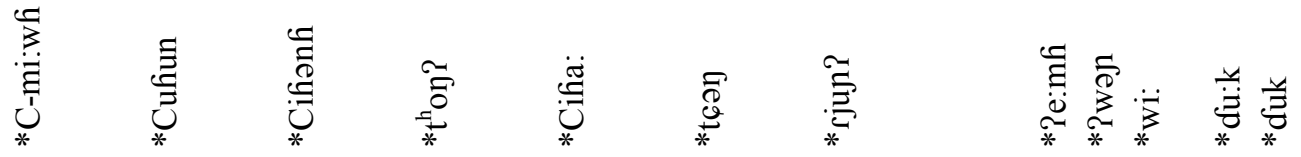

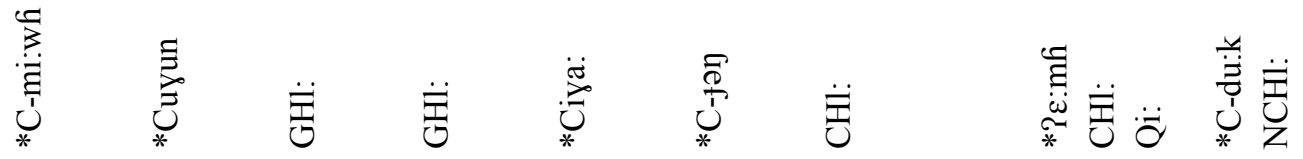

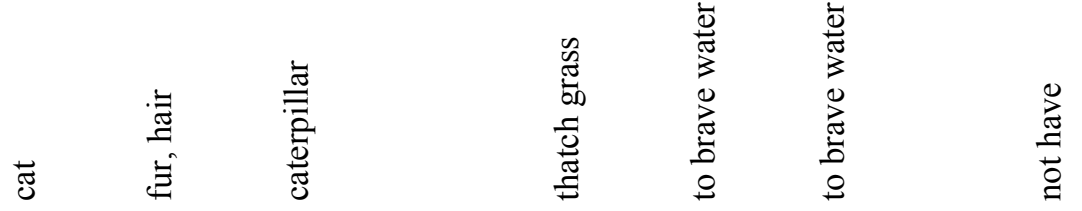

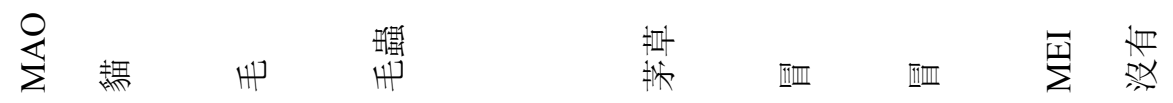




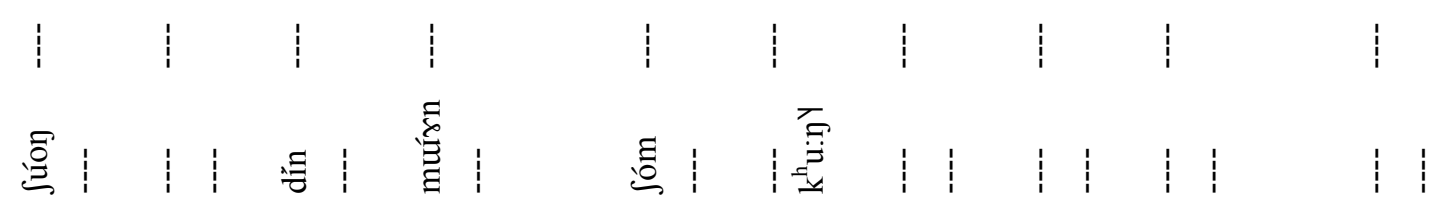

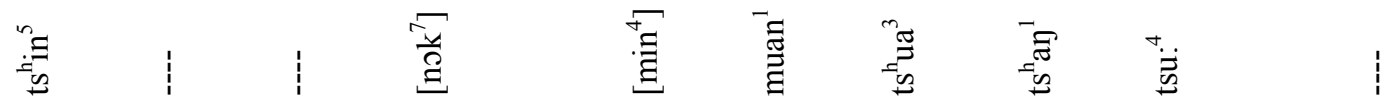

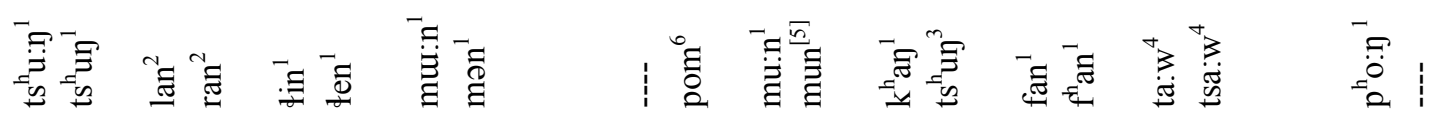

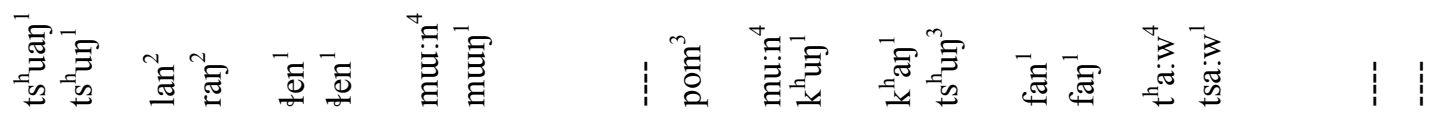

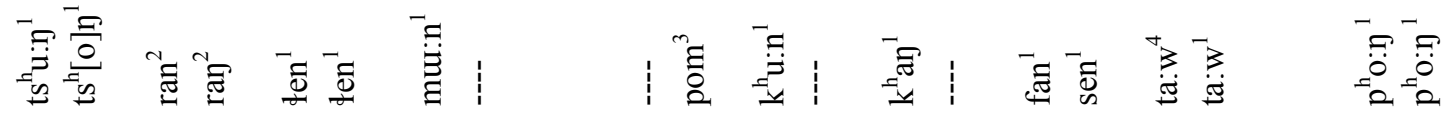

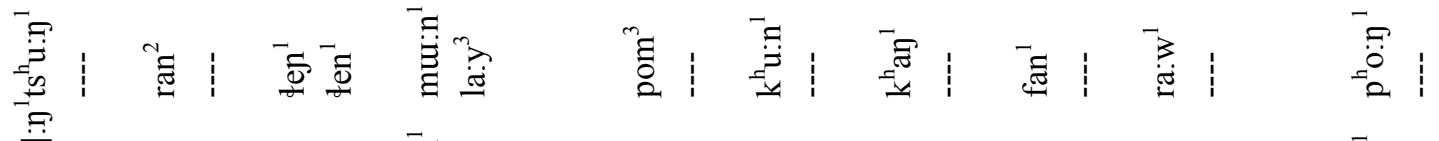

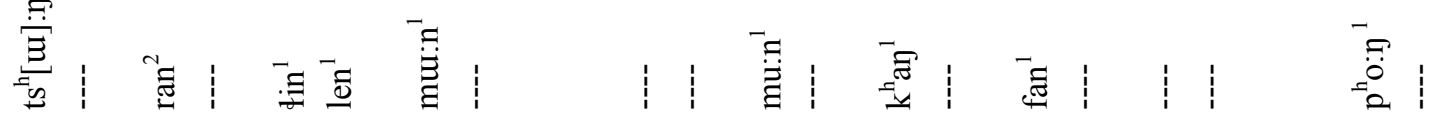

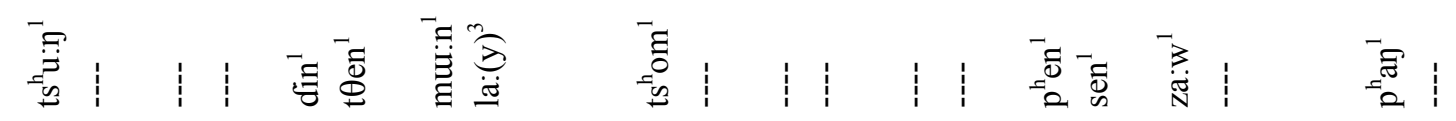

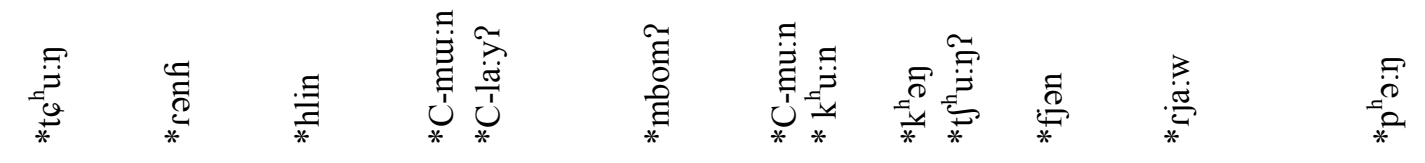

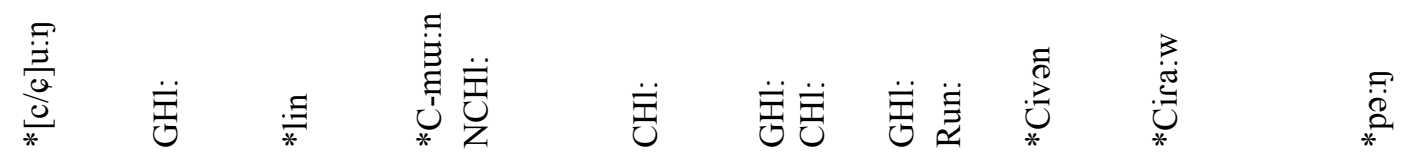
$\frac{\sharp}{20}$
总
高

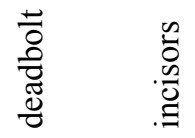
逃嘕

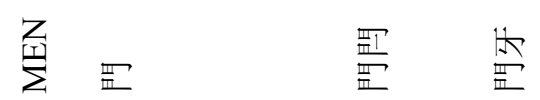
茎
$\frac{\hbar}{0}$
梳 


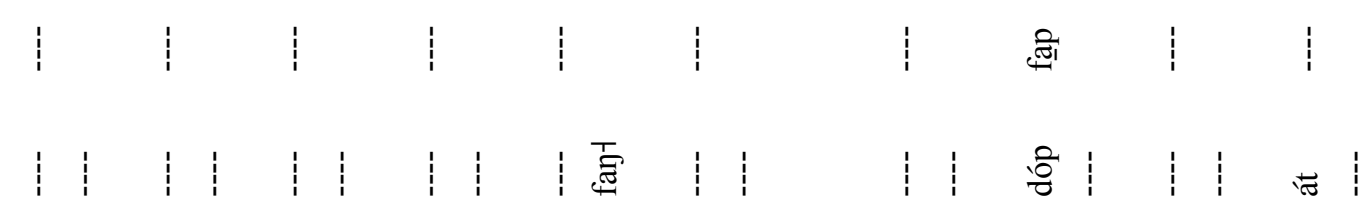

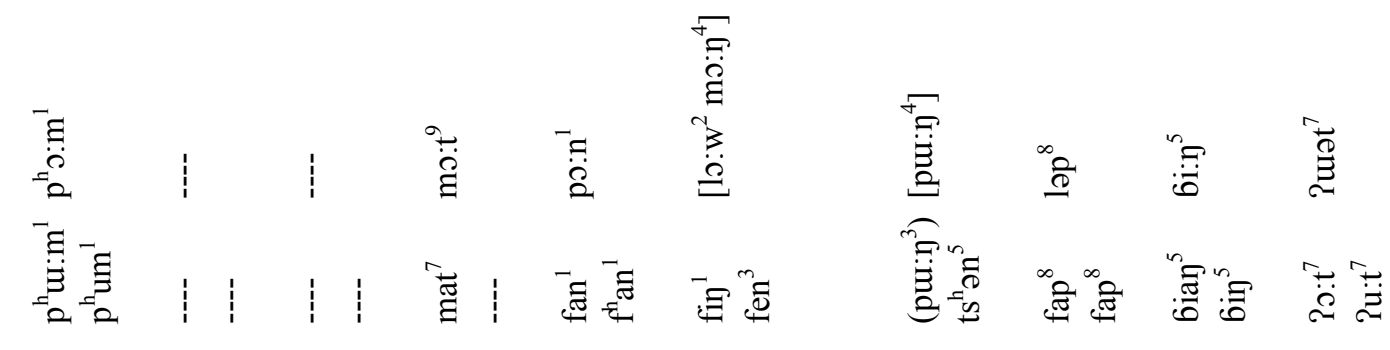

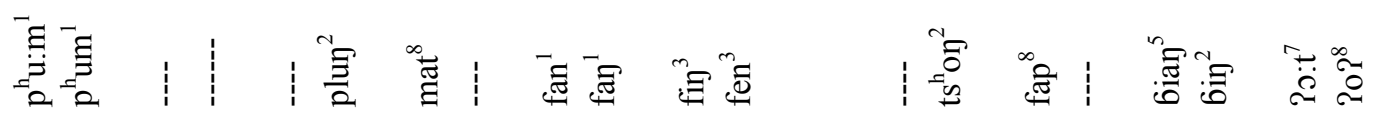

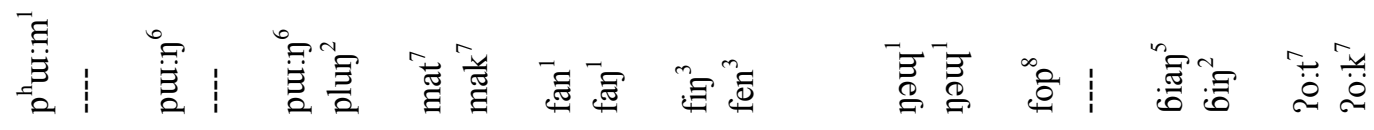

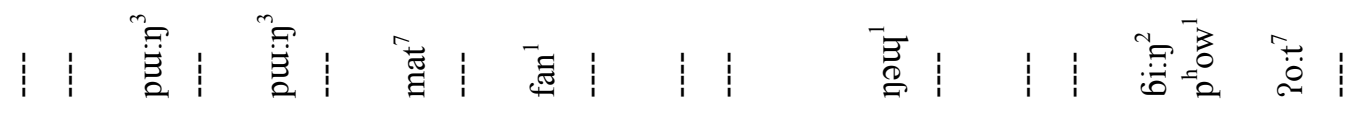

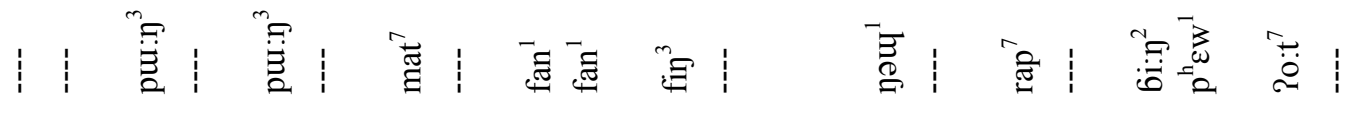

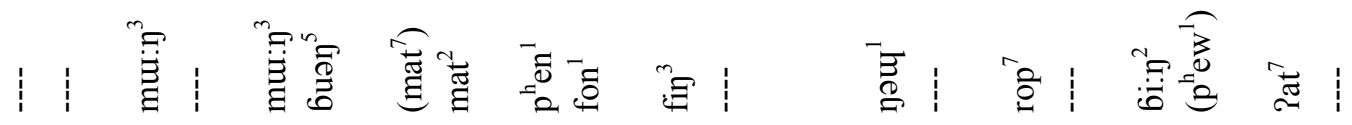

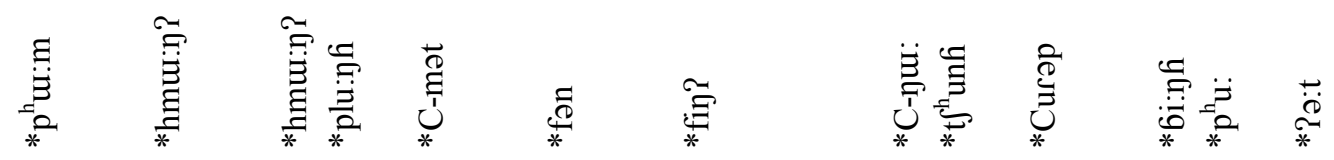

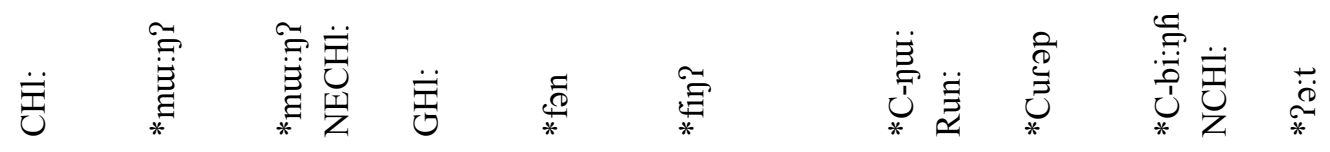

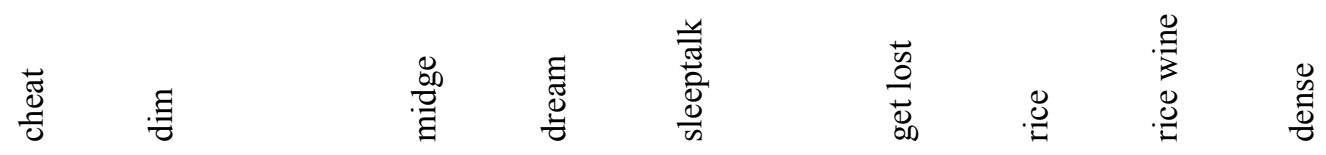

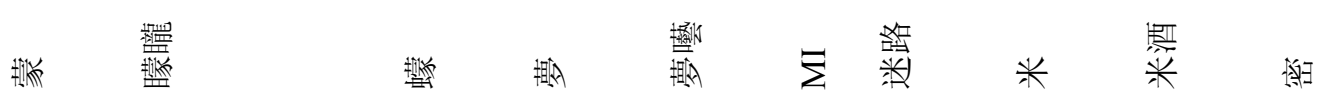




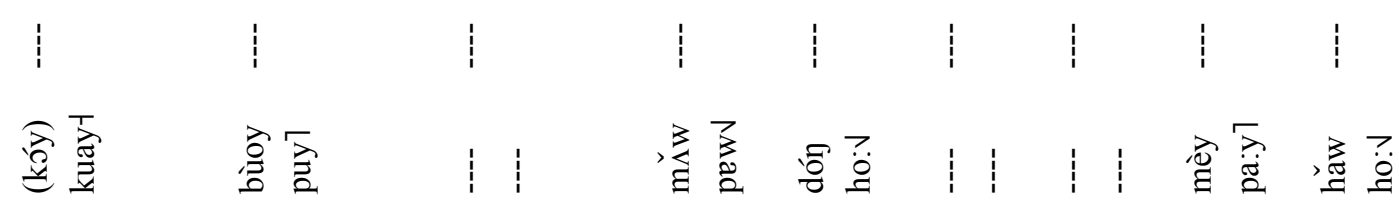

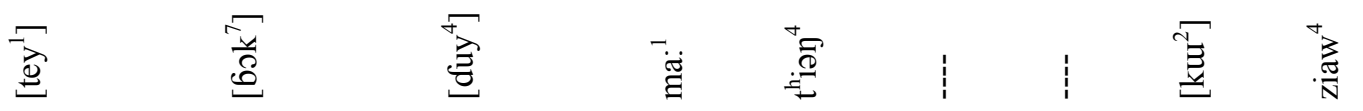

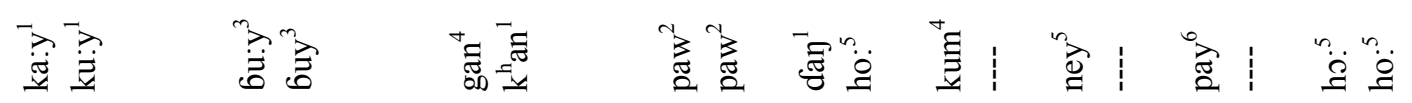

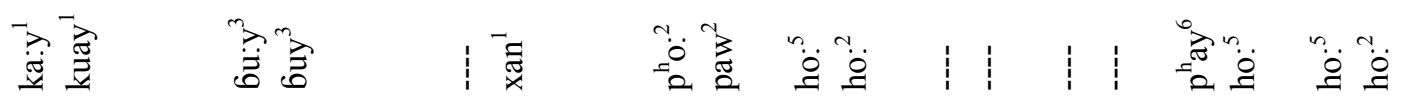

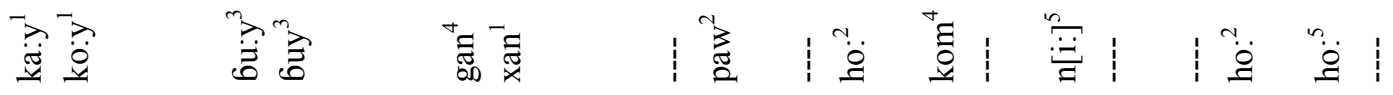

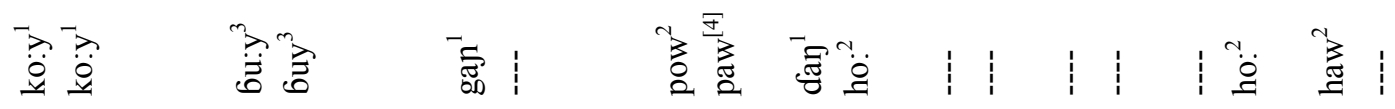

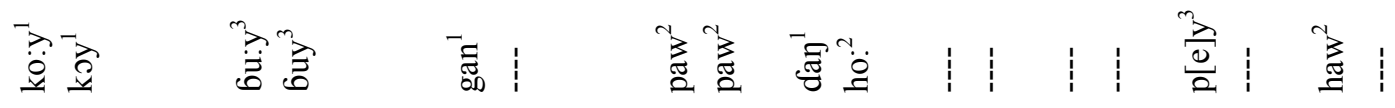

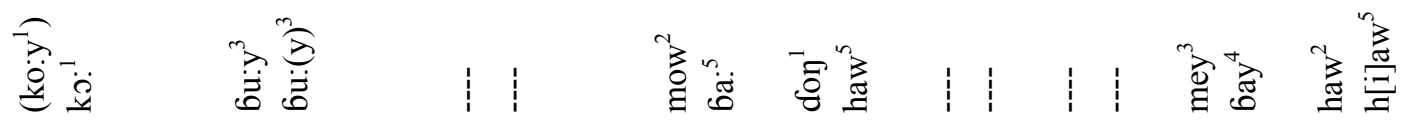

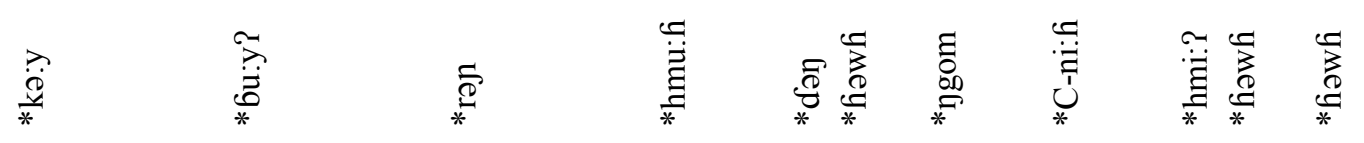

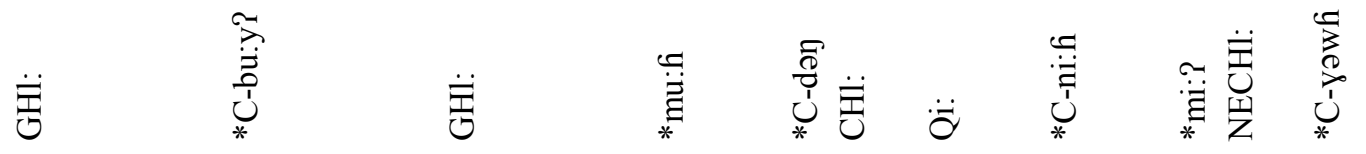

๕

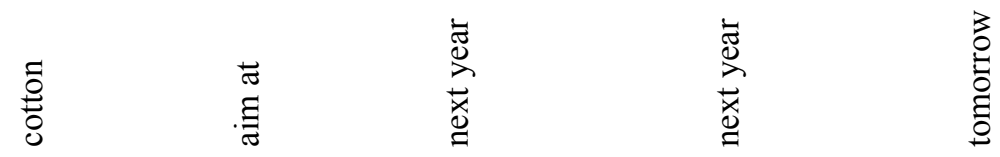

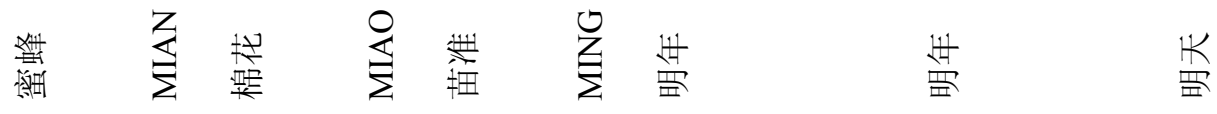




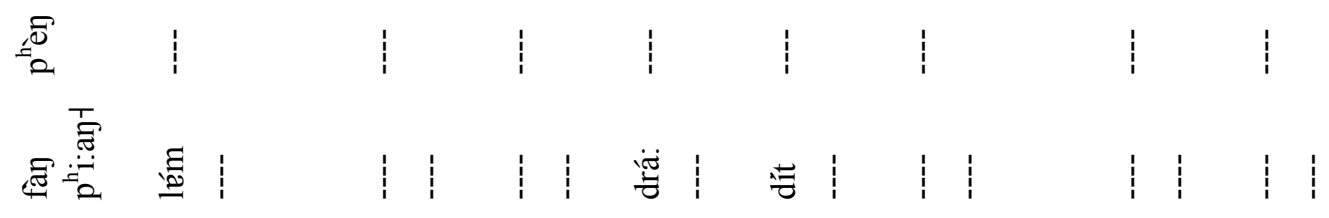

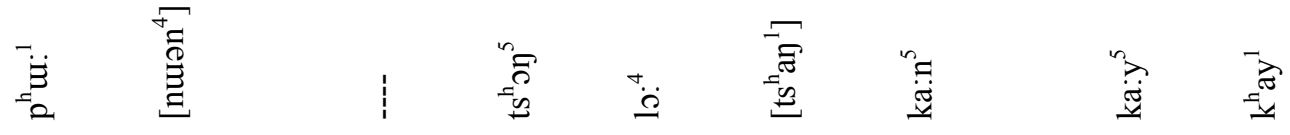

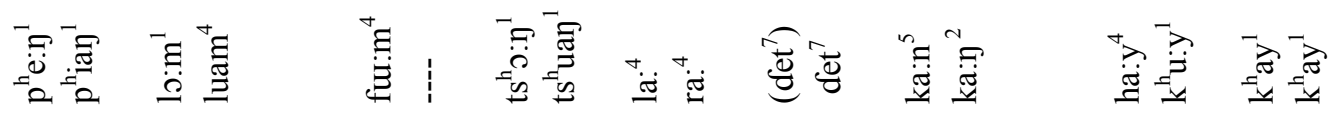

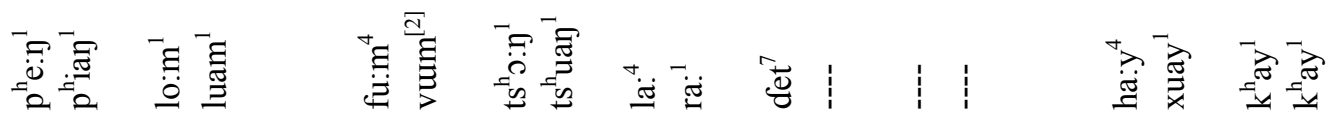

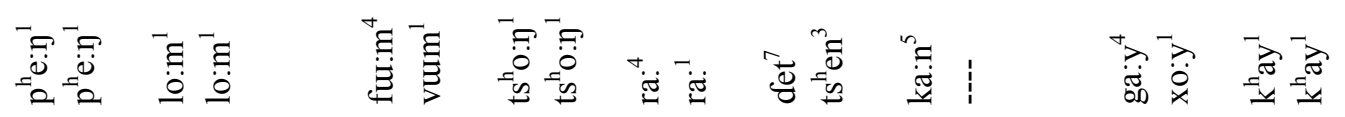

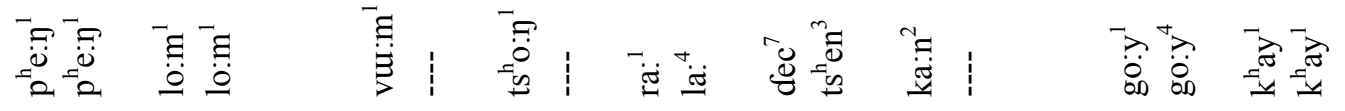

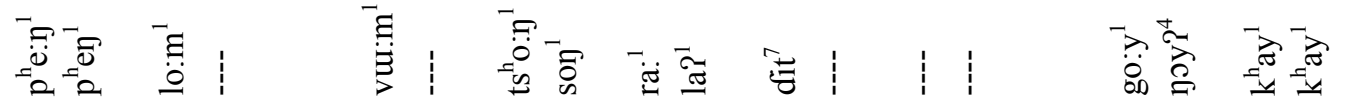

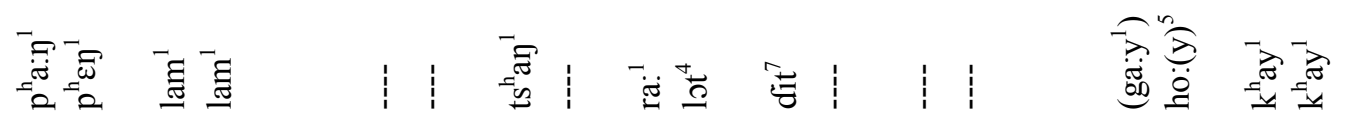

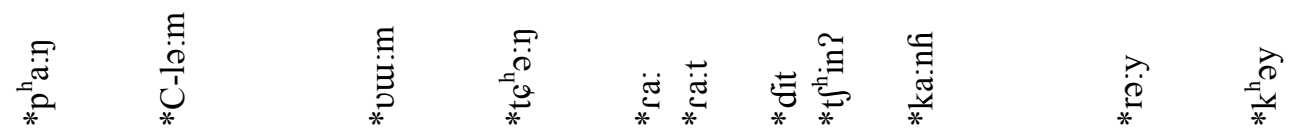

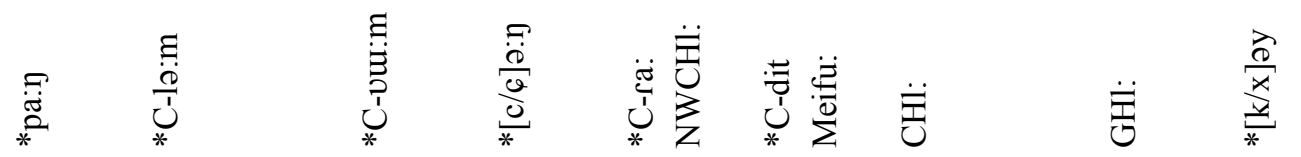

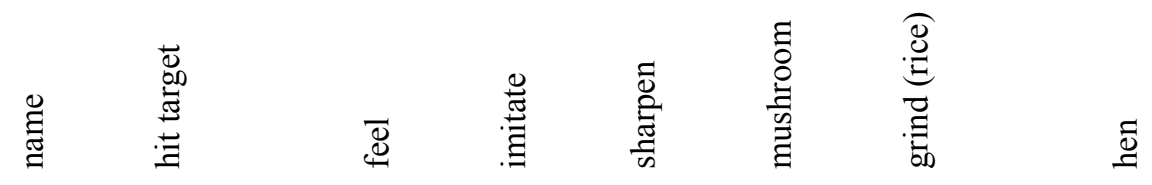

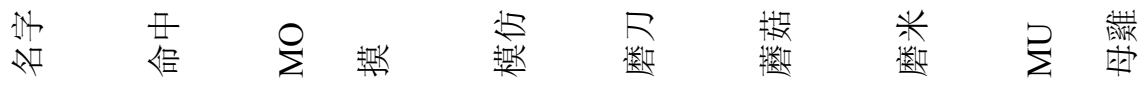




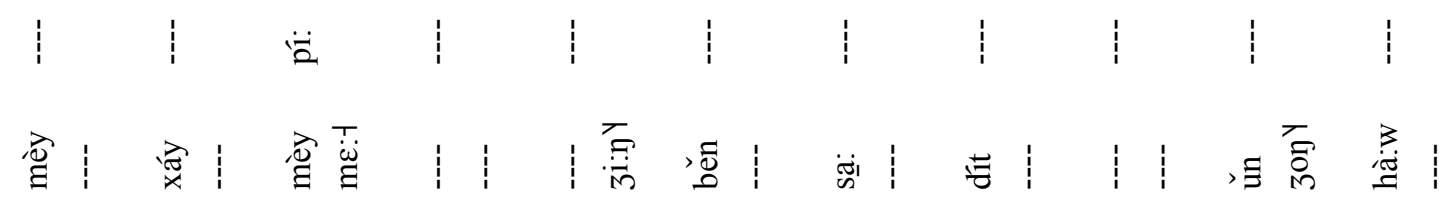

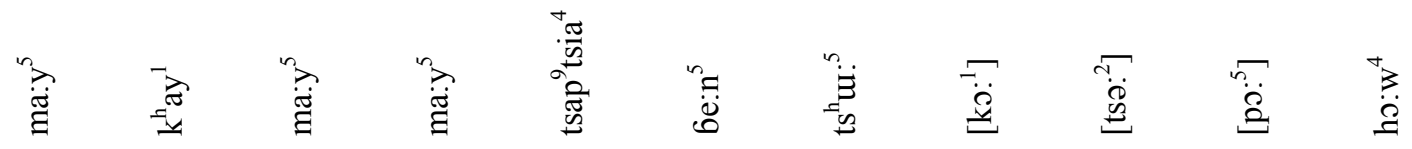

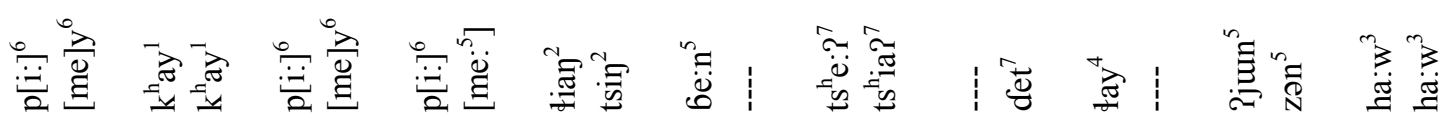

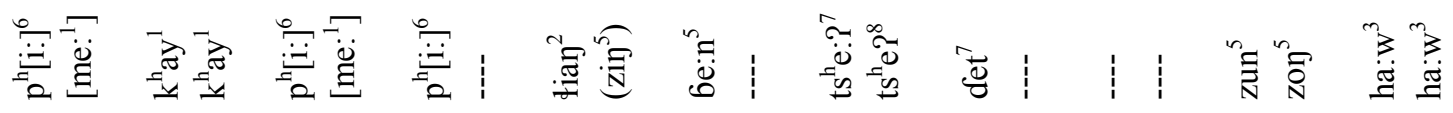

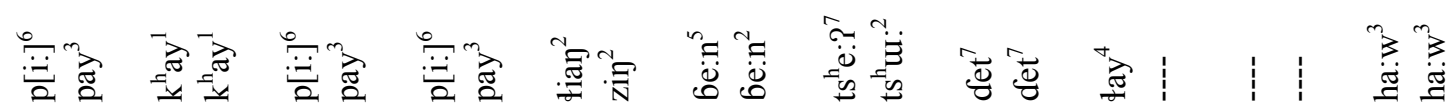

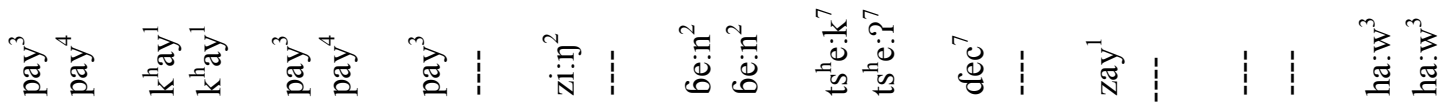

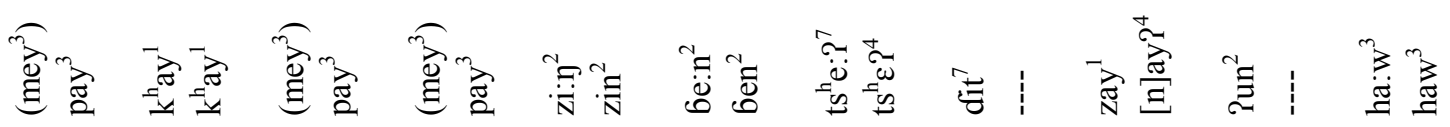

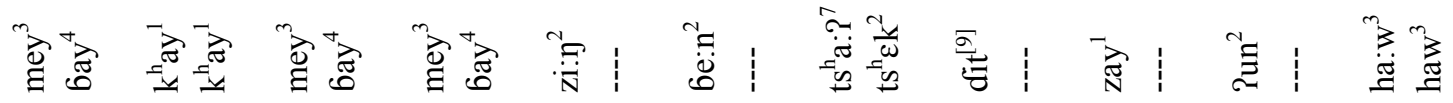

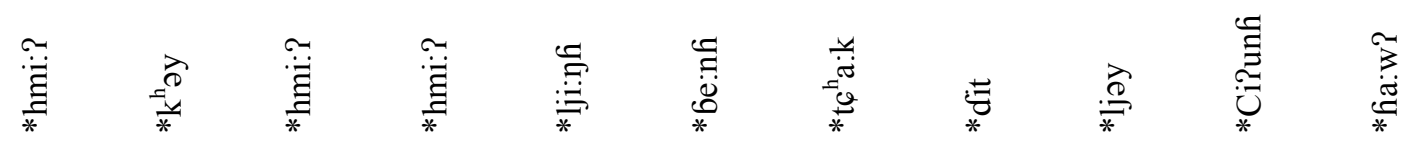

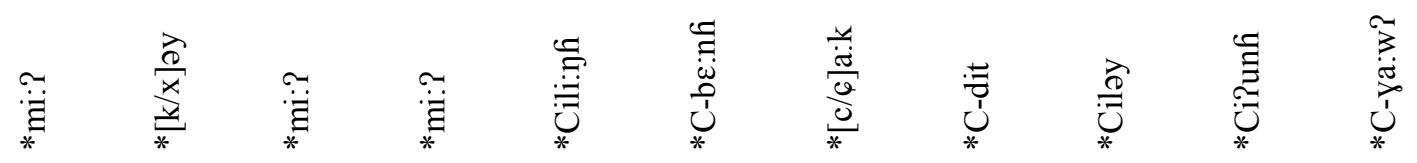

高

毫旁

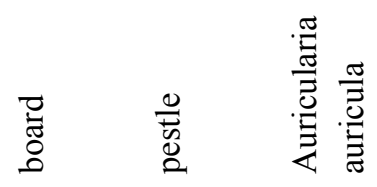

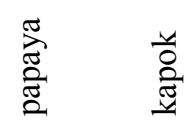

轓

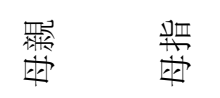

罢萃菜

昱罯 


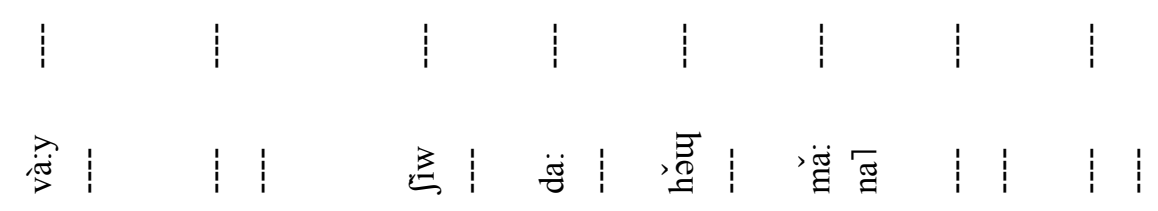

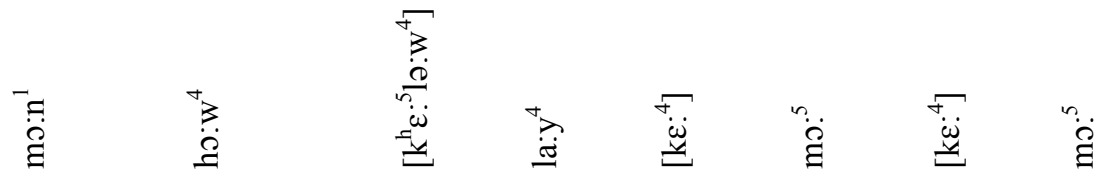

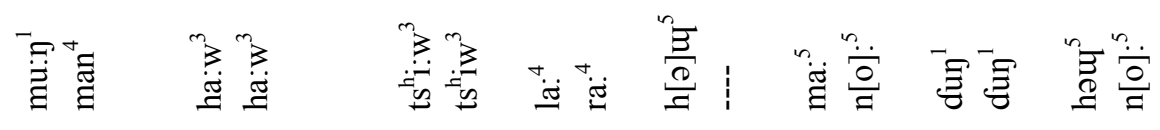

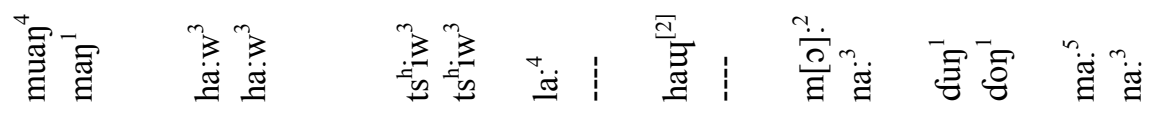

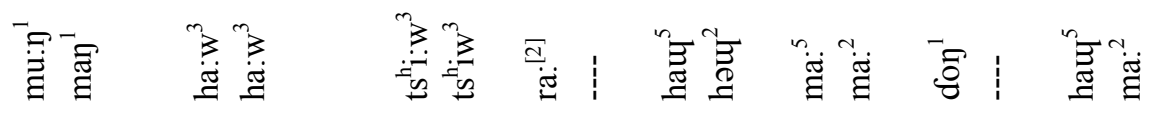

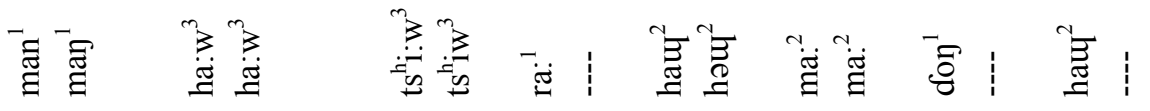

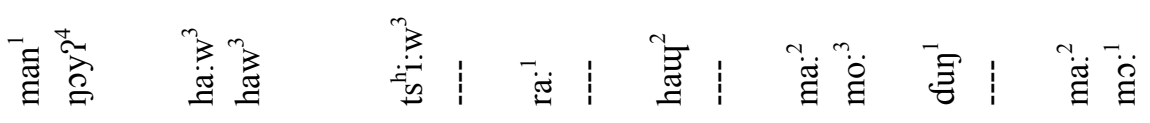

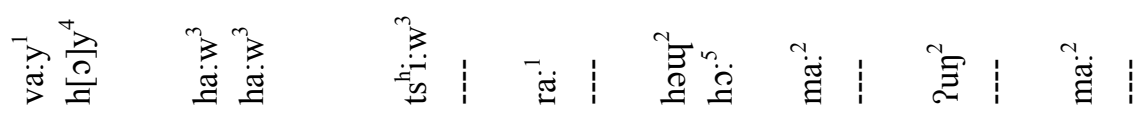

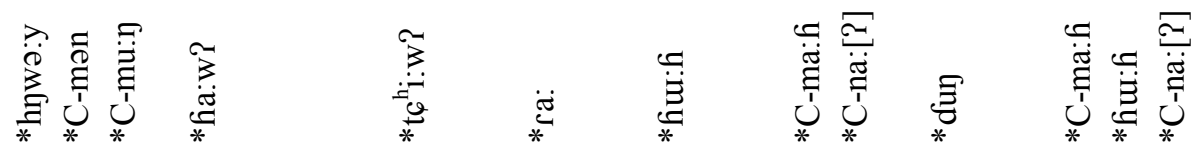

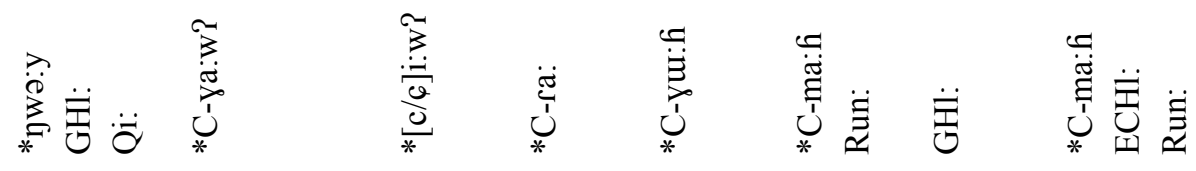

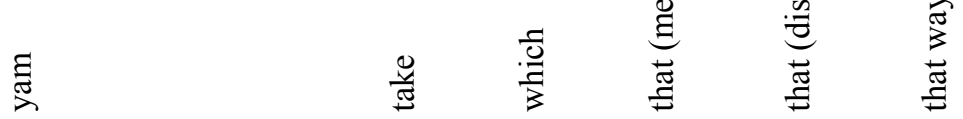

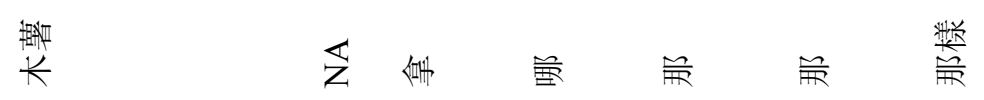




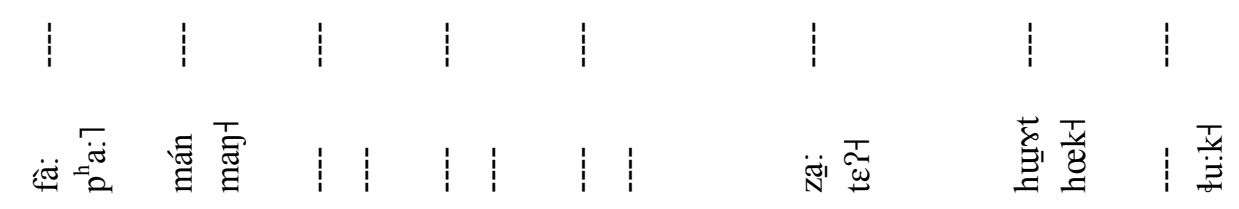

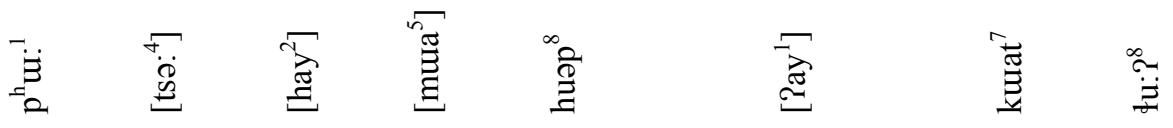

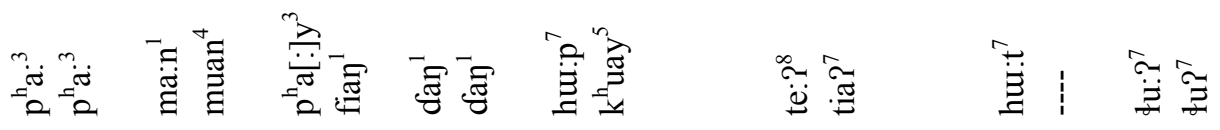

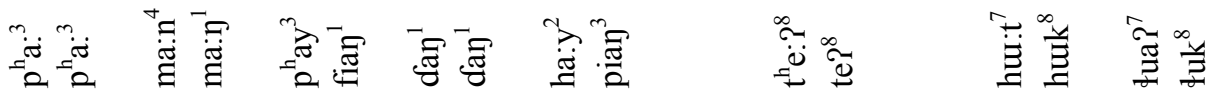

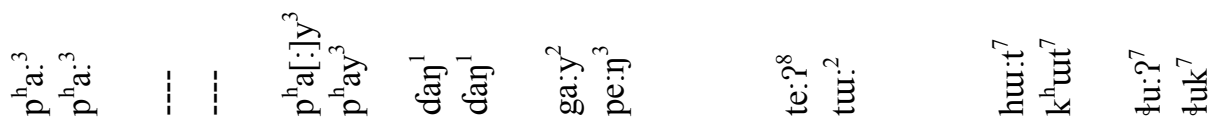

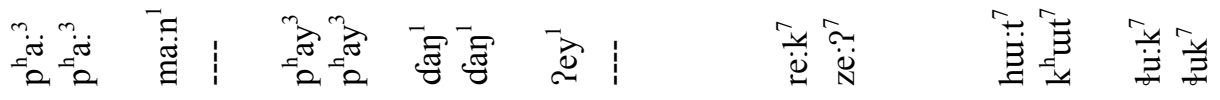

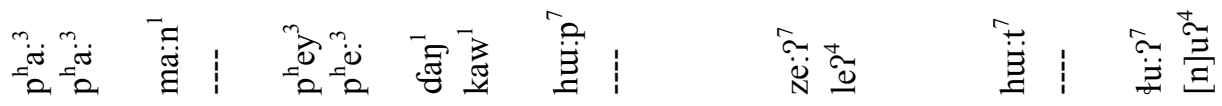

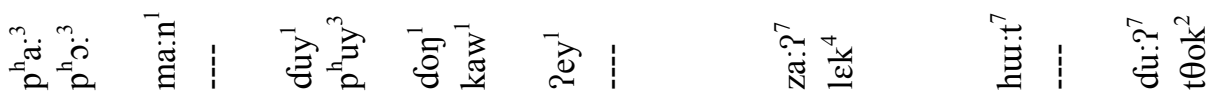

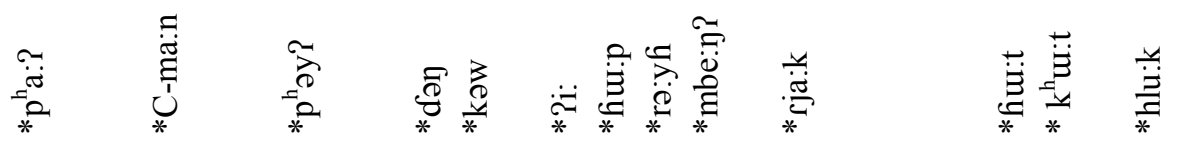

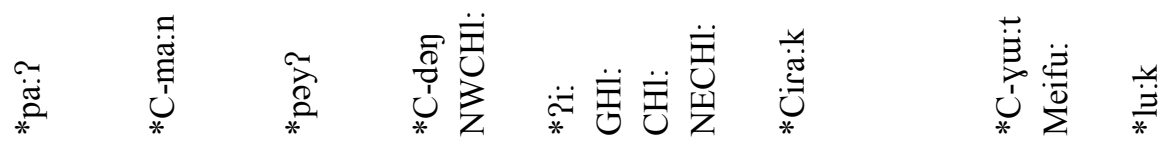

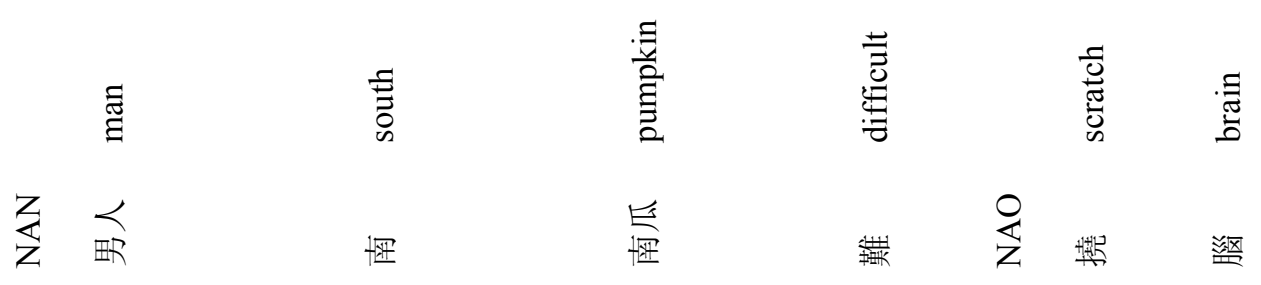




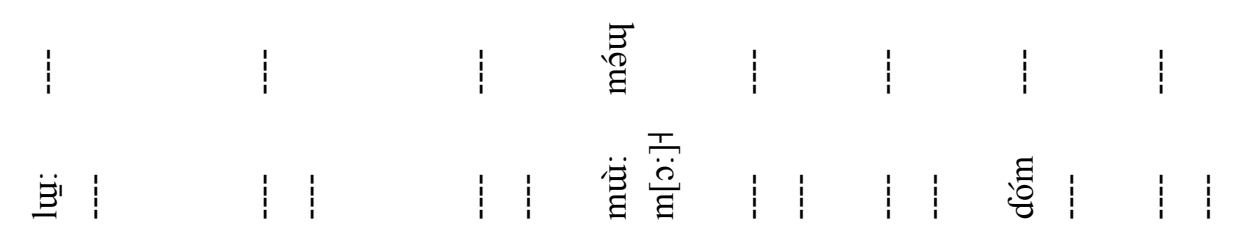

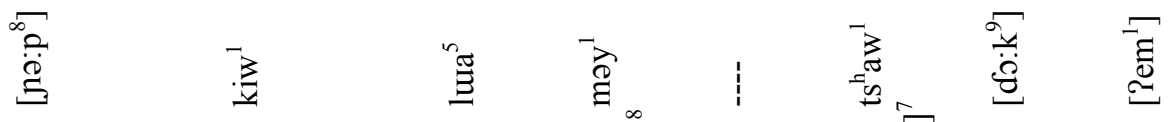

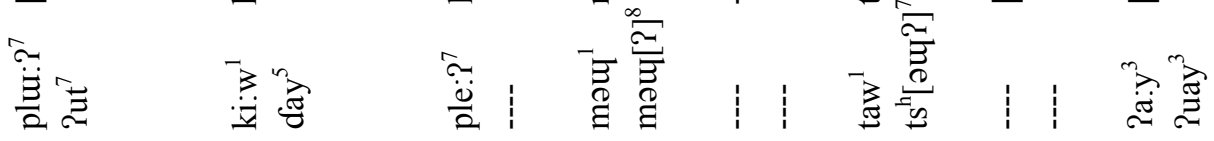

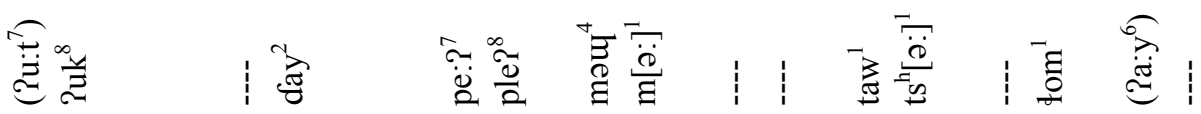

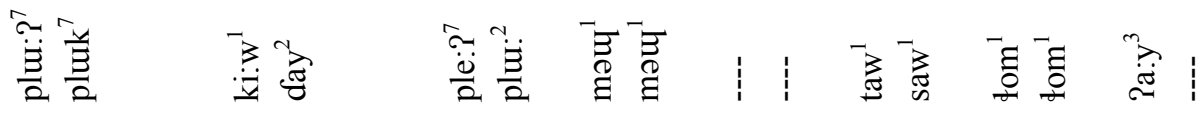

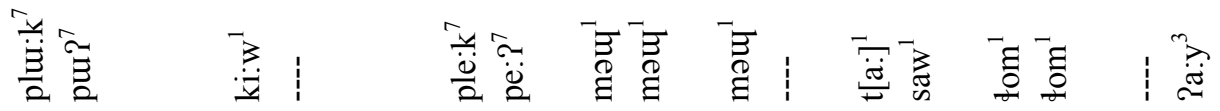

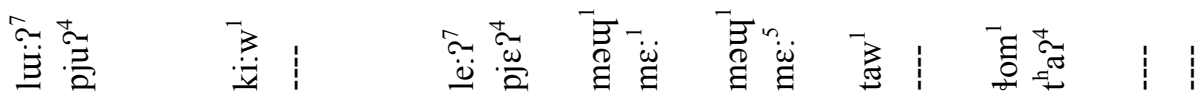

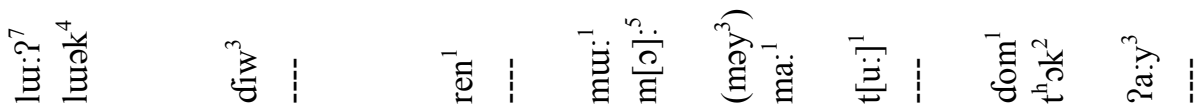

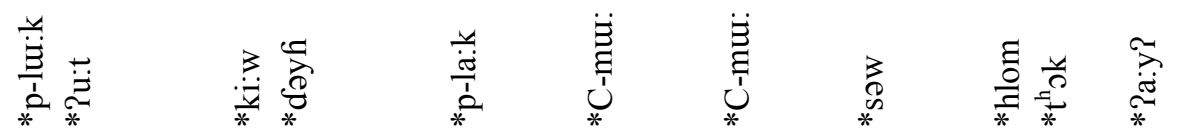

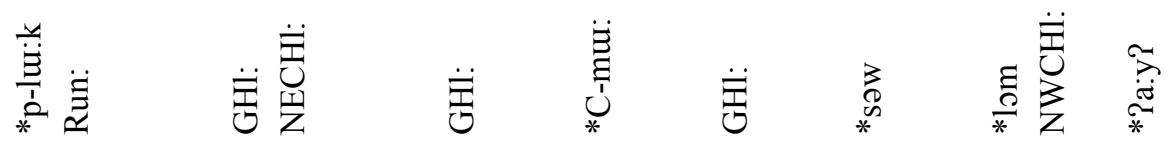

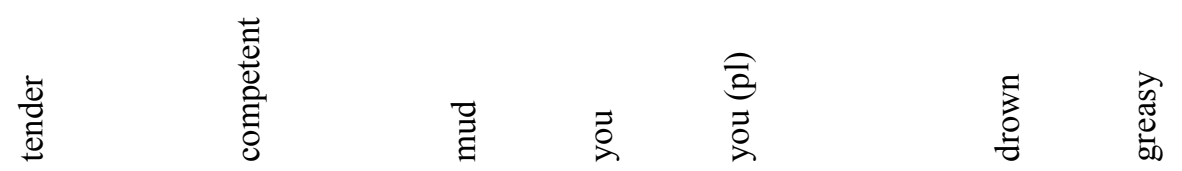

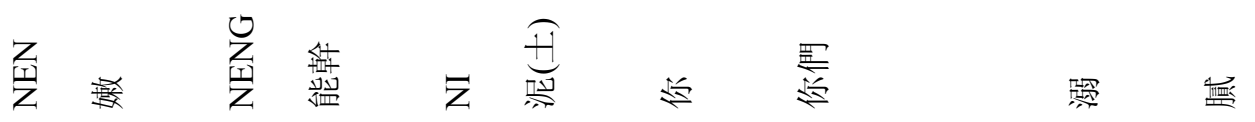




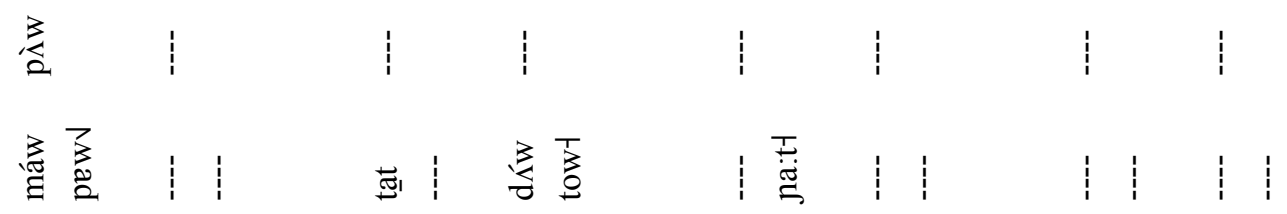

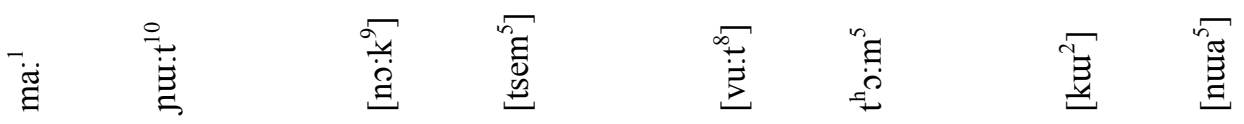

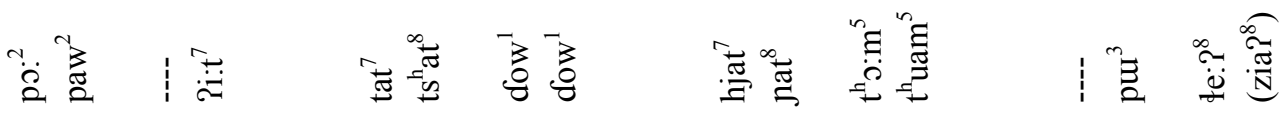

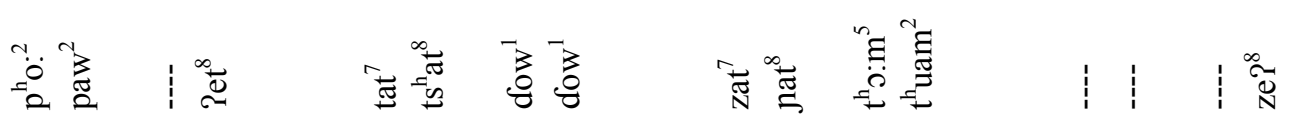

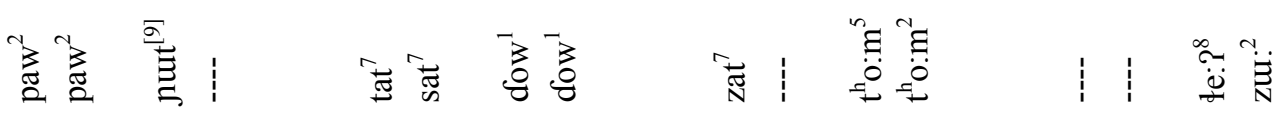

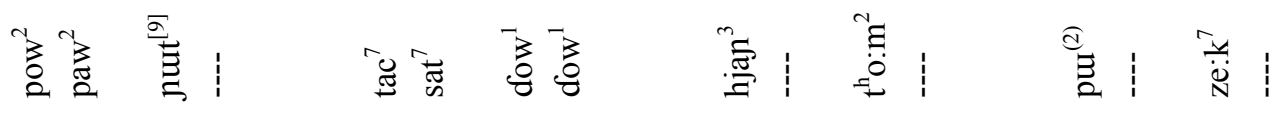

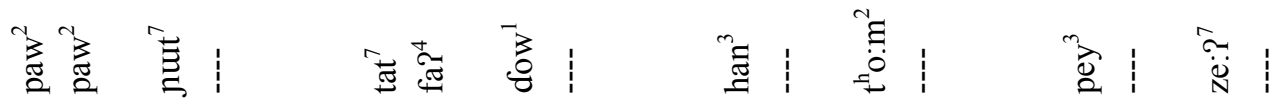

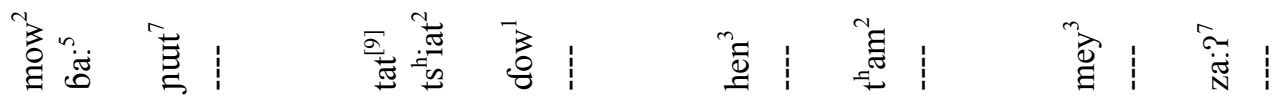

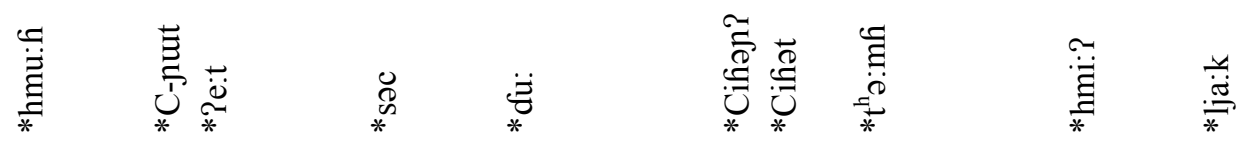

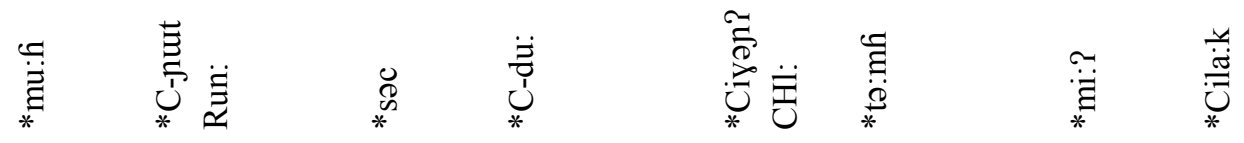

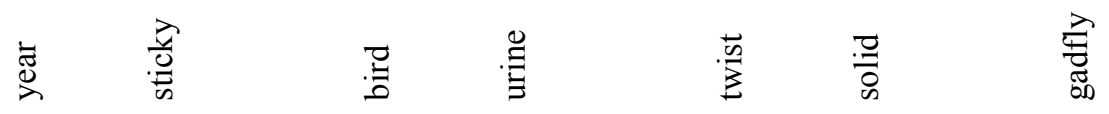

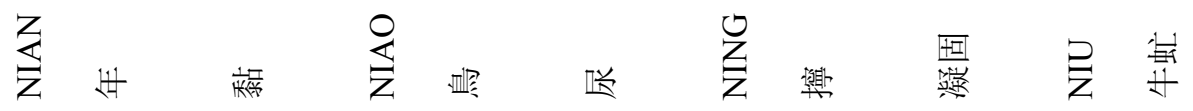




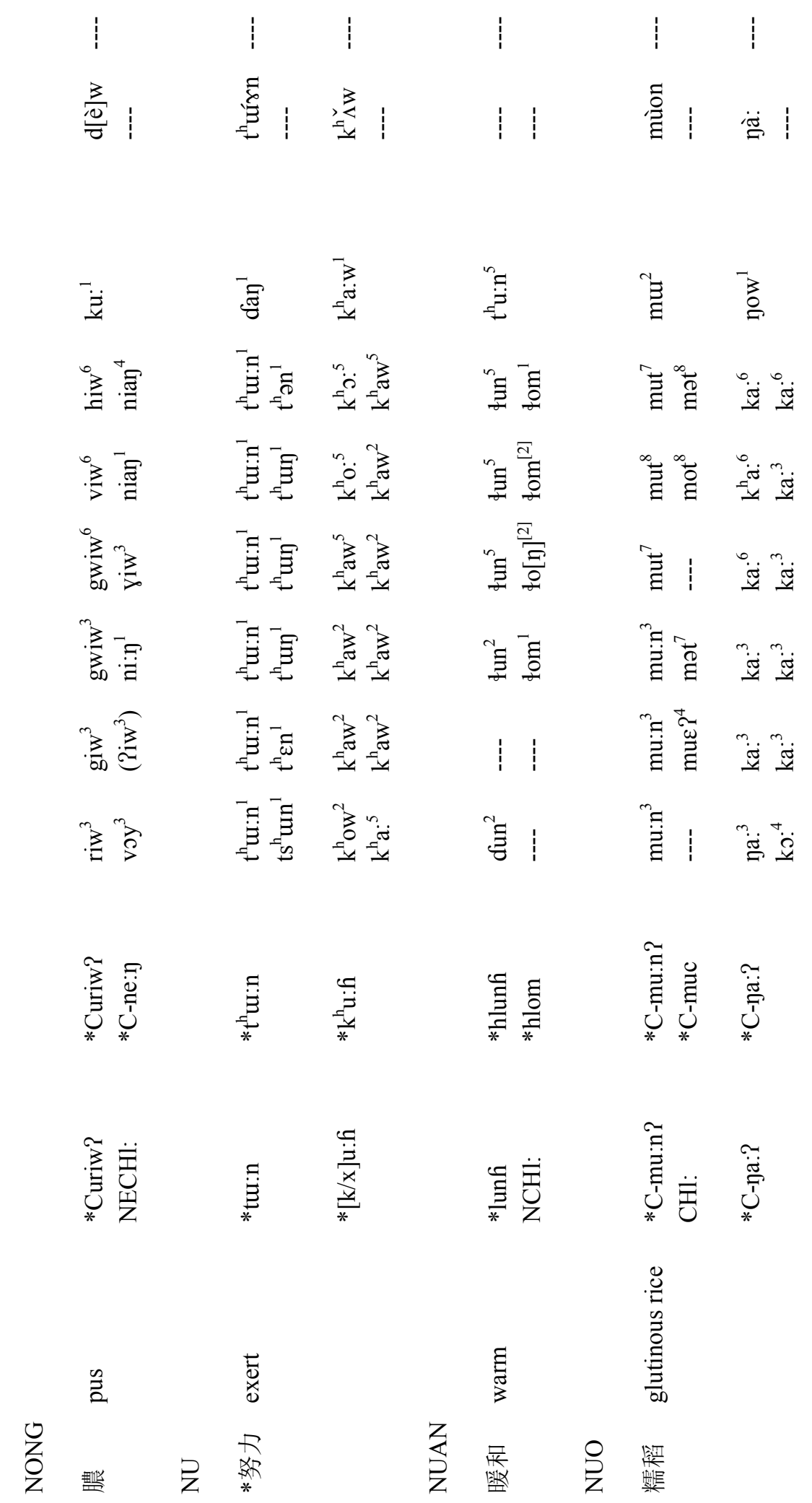




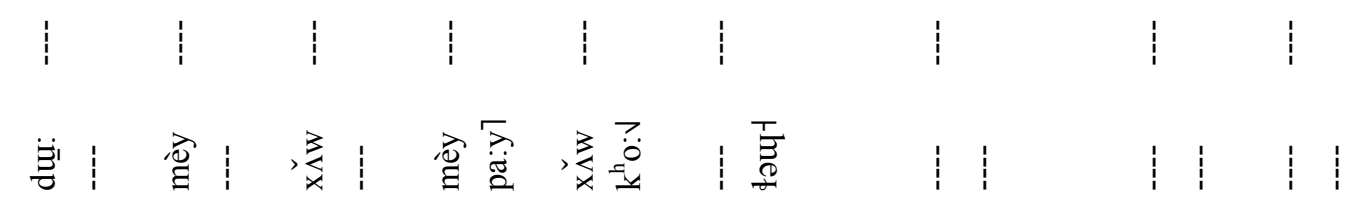

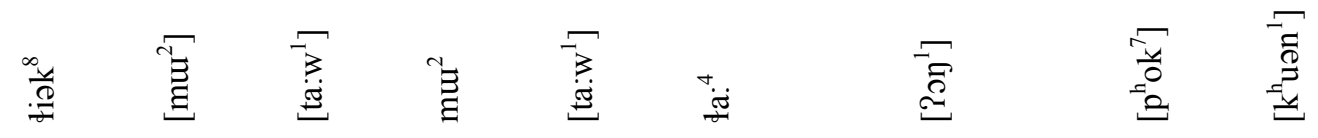

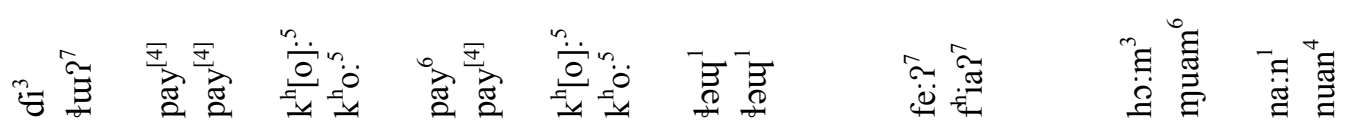

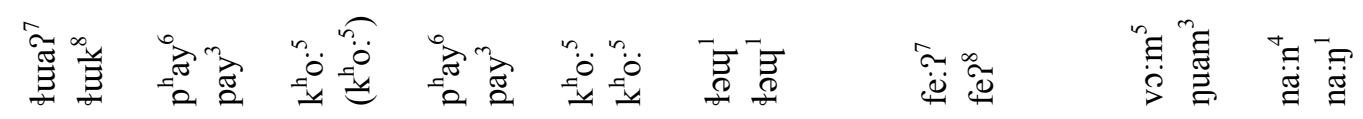

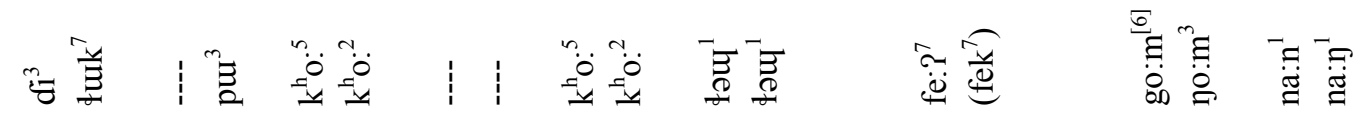

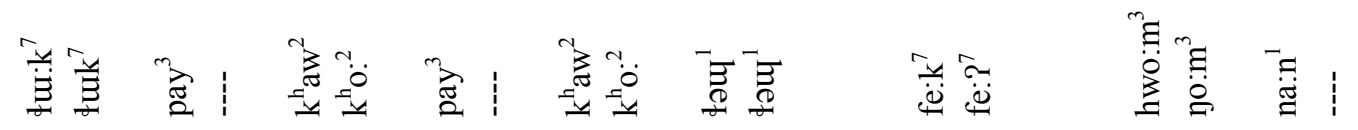

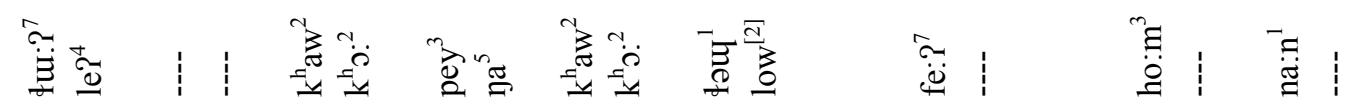

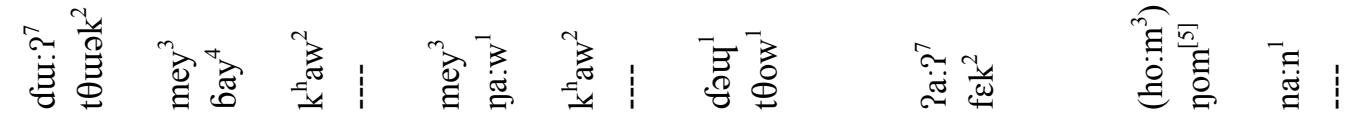

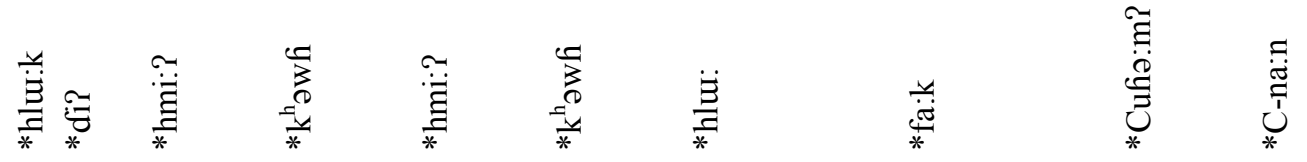

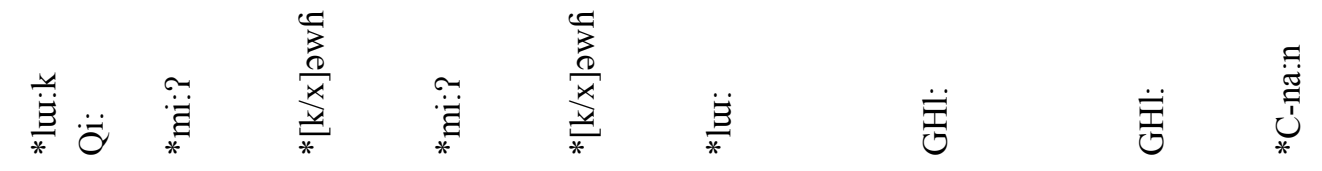

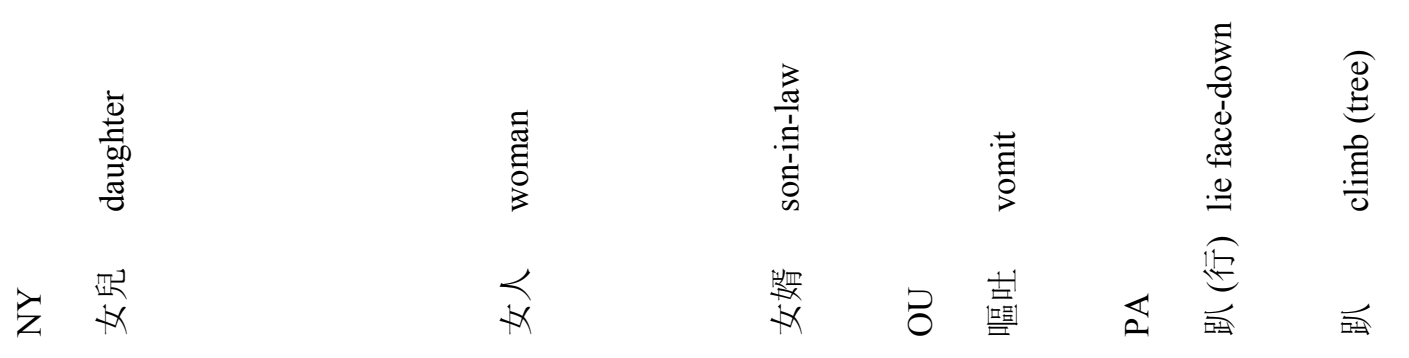




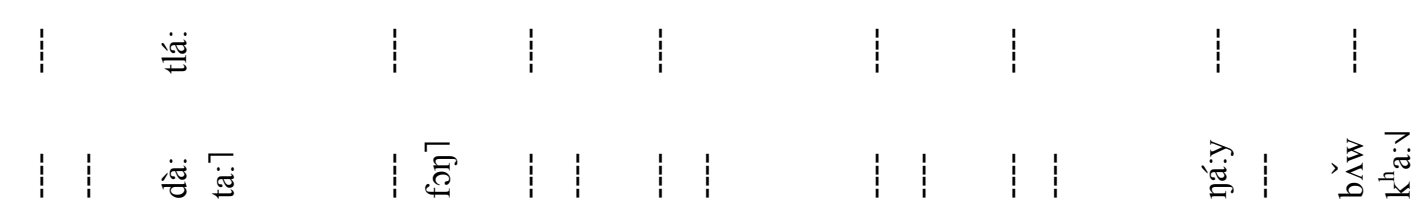

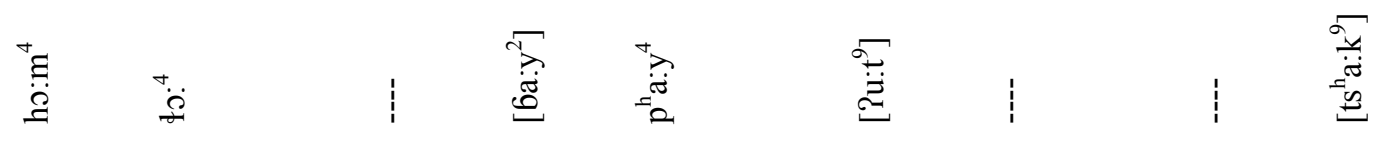

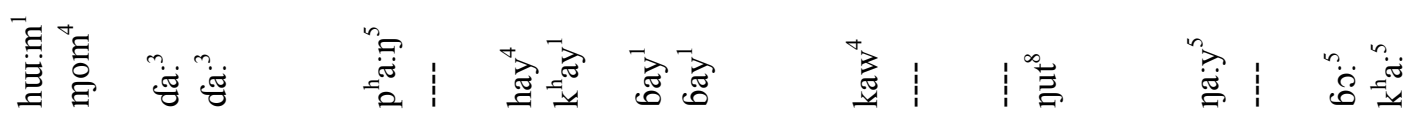

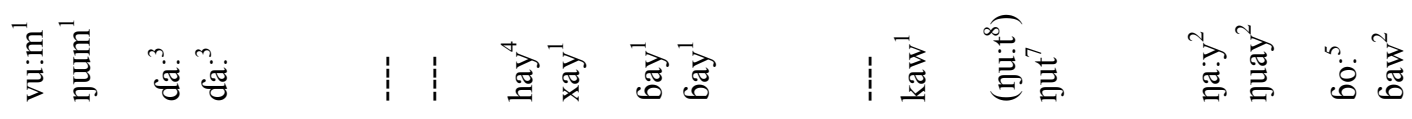

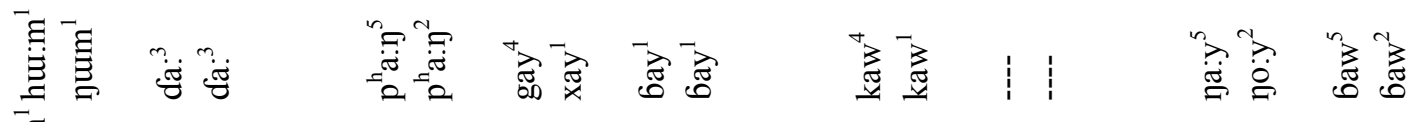

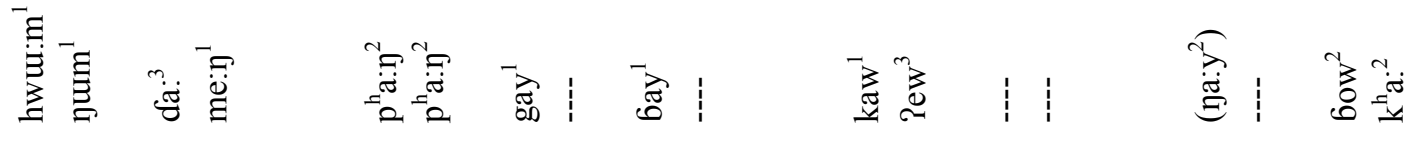

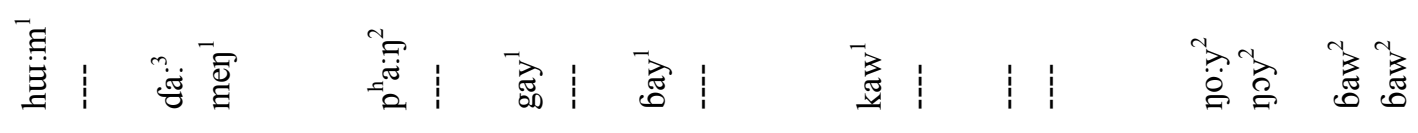

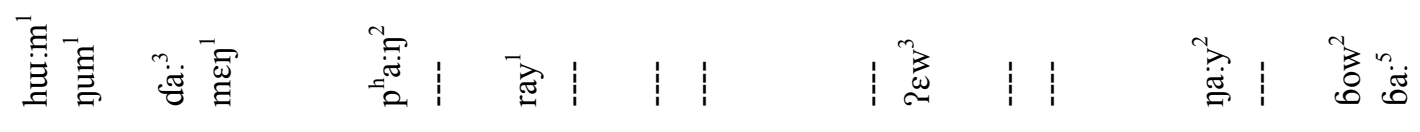

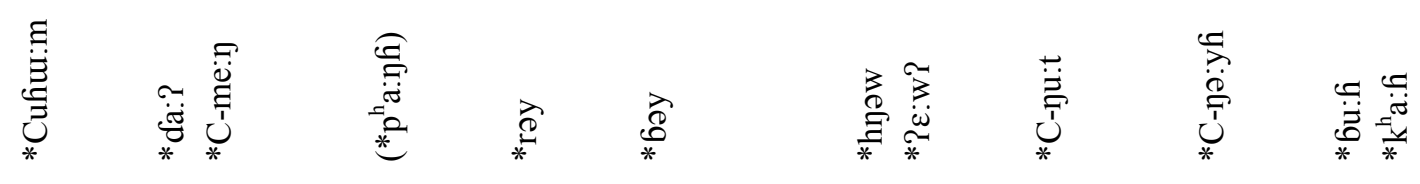

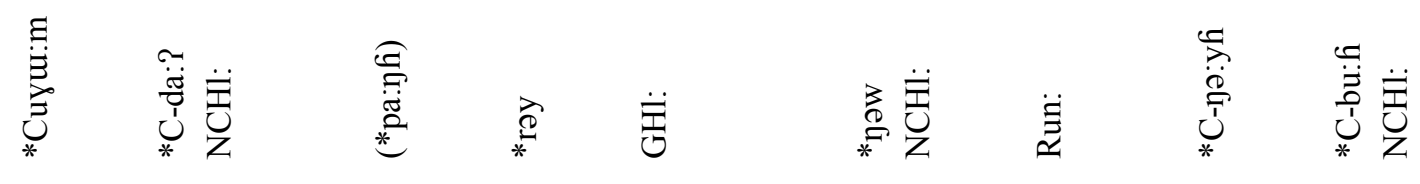

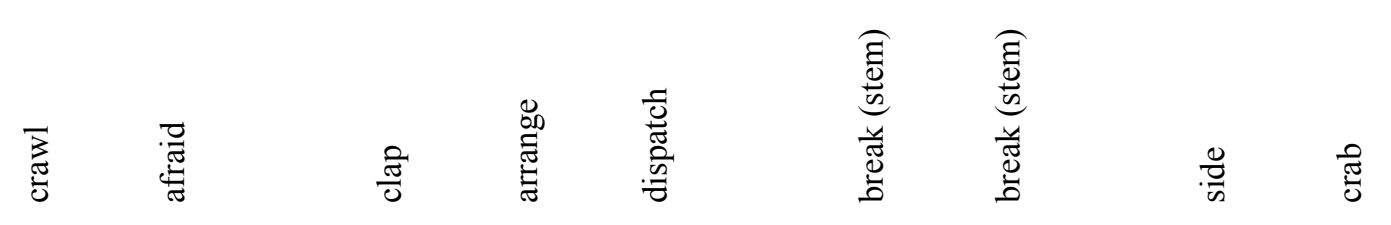

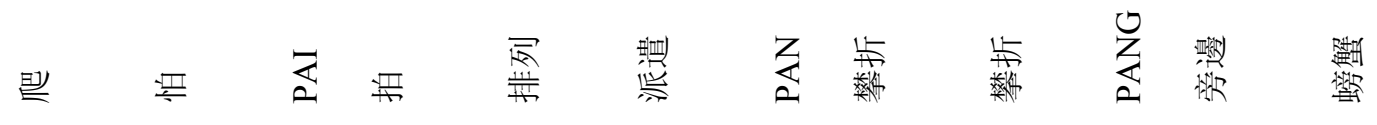




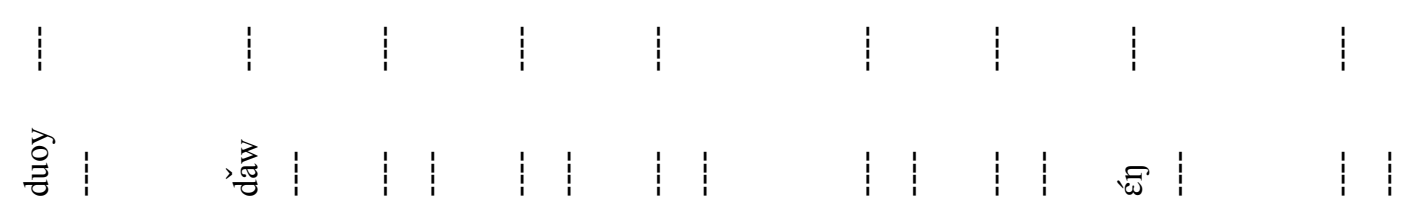

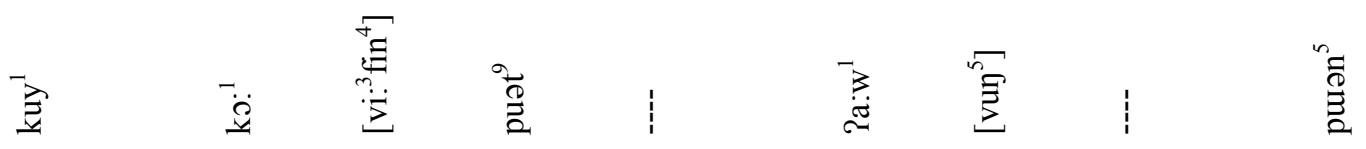

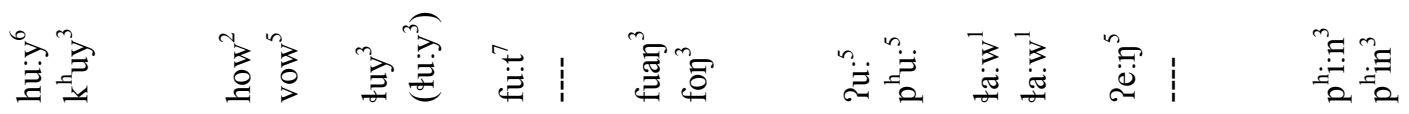

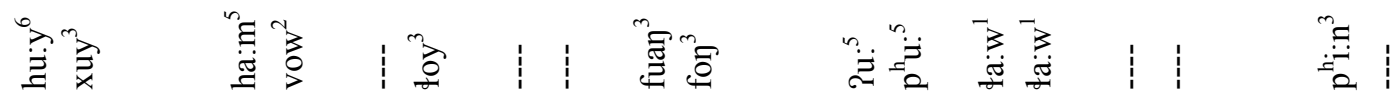

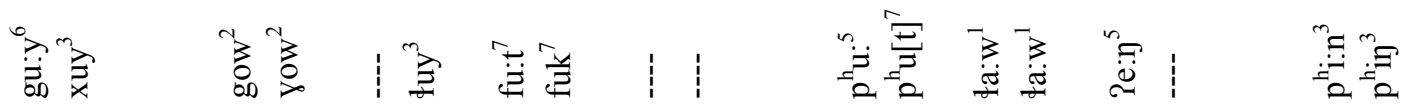

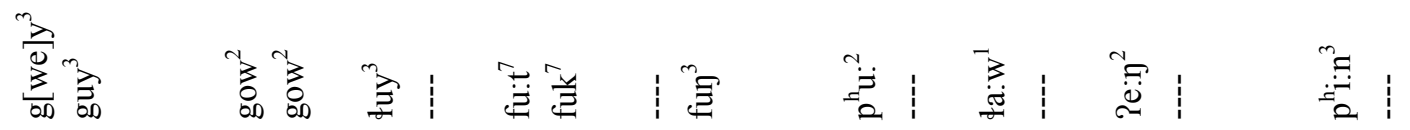

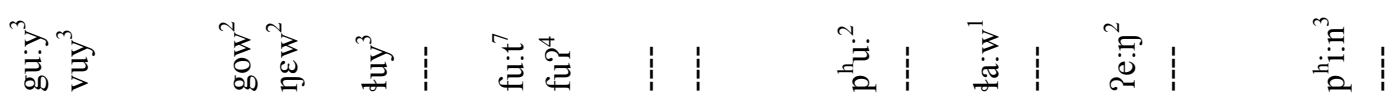

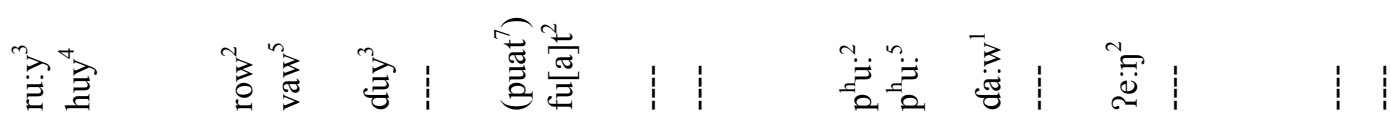

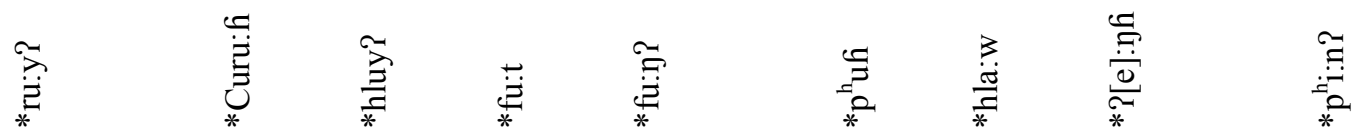

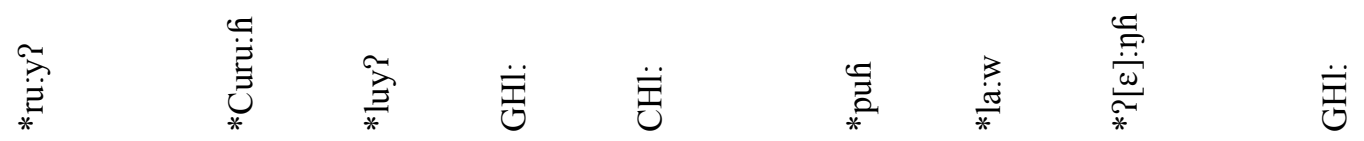

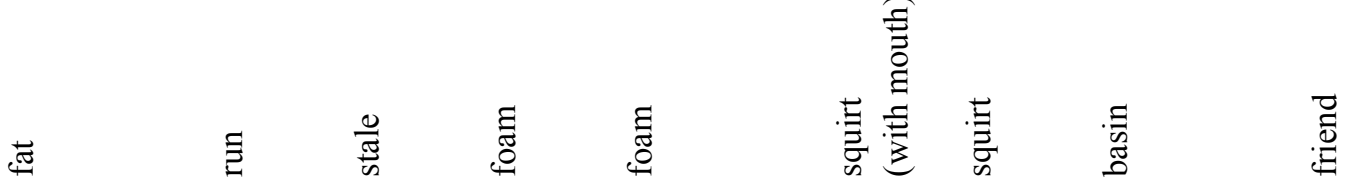

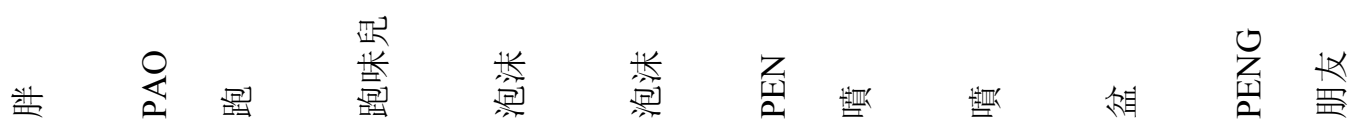




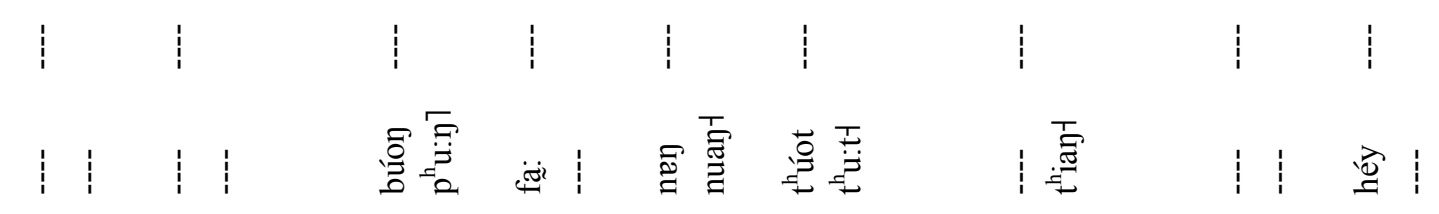

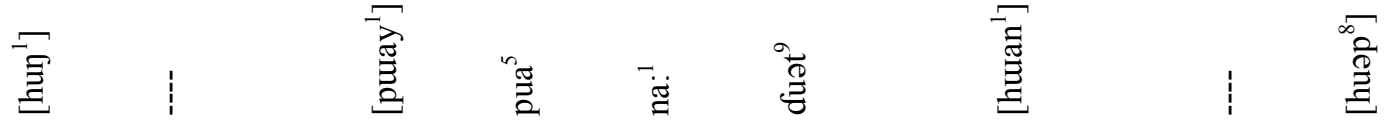

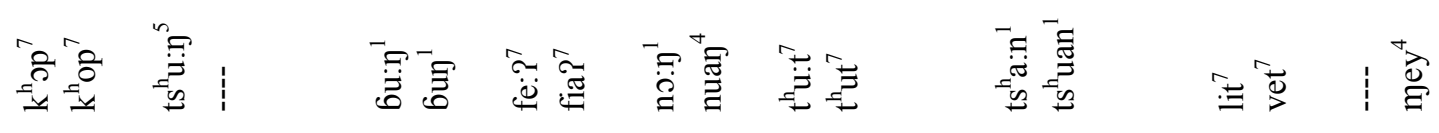

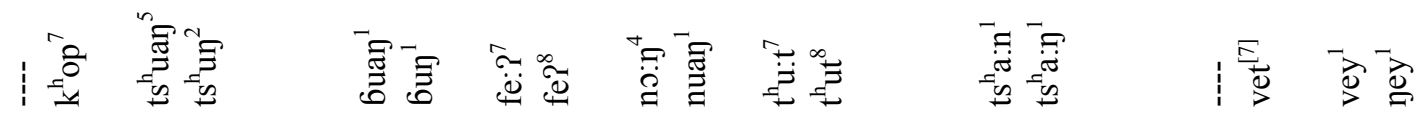

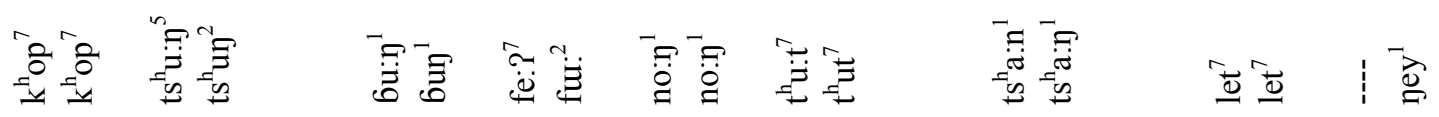

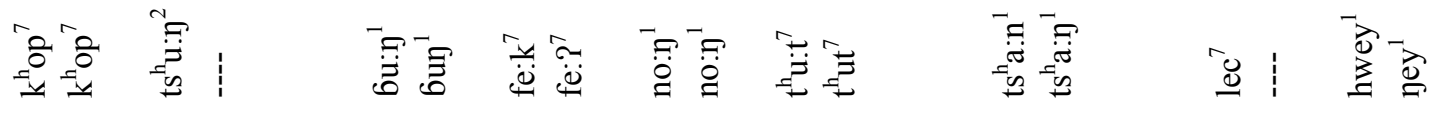

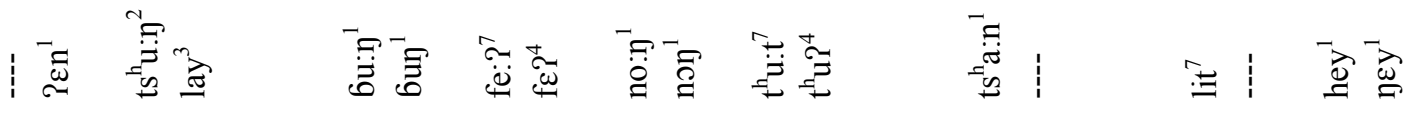

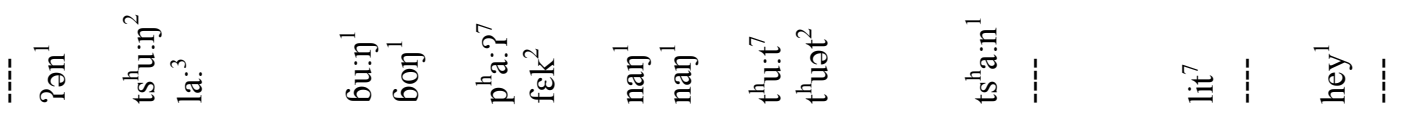

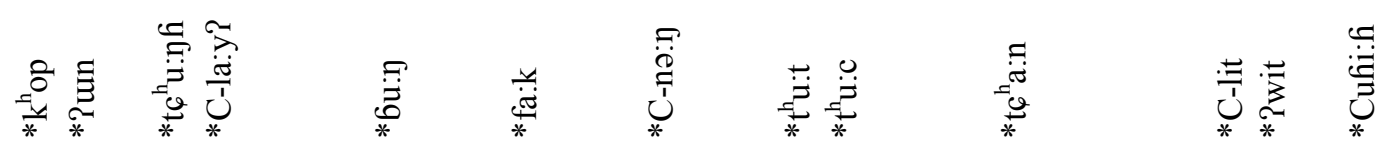

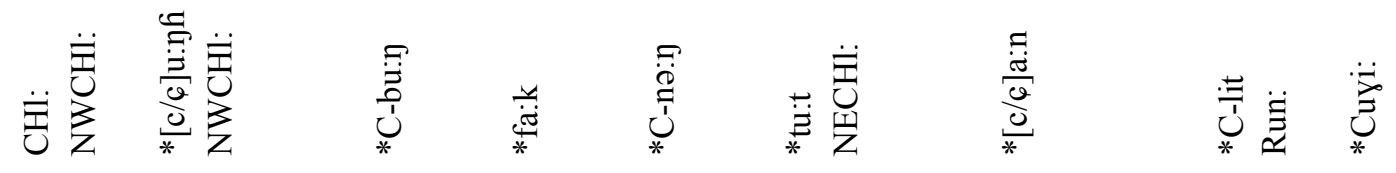

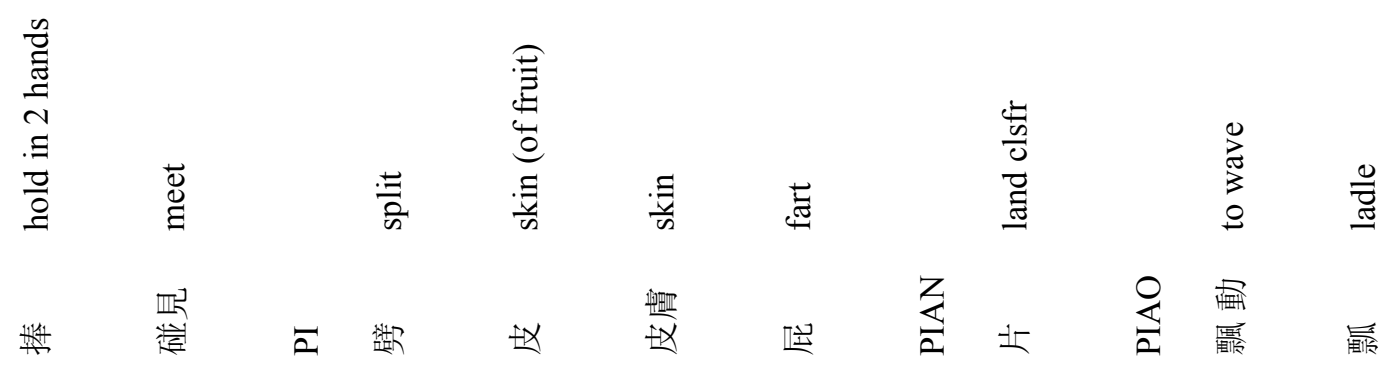




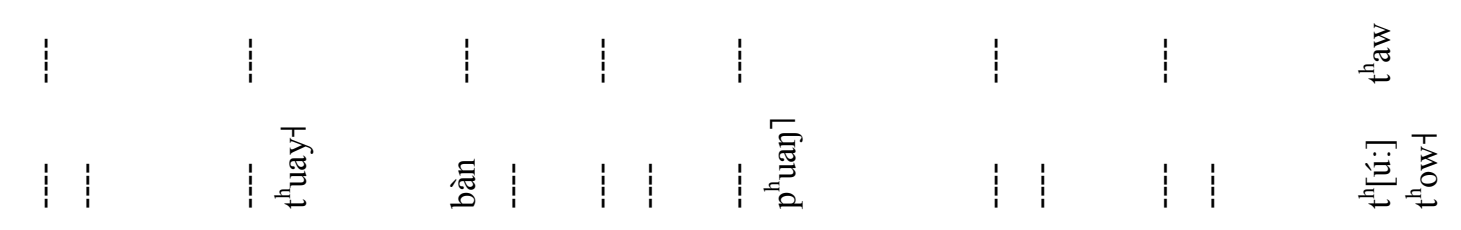

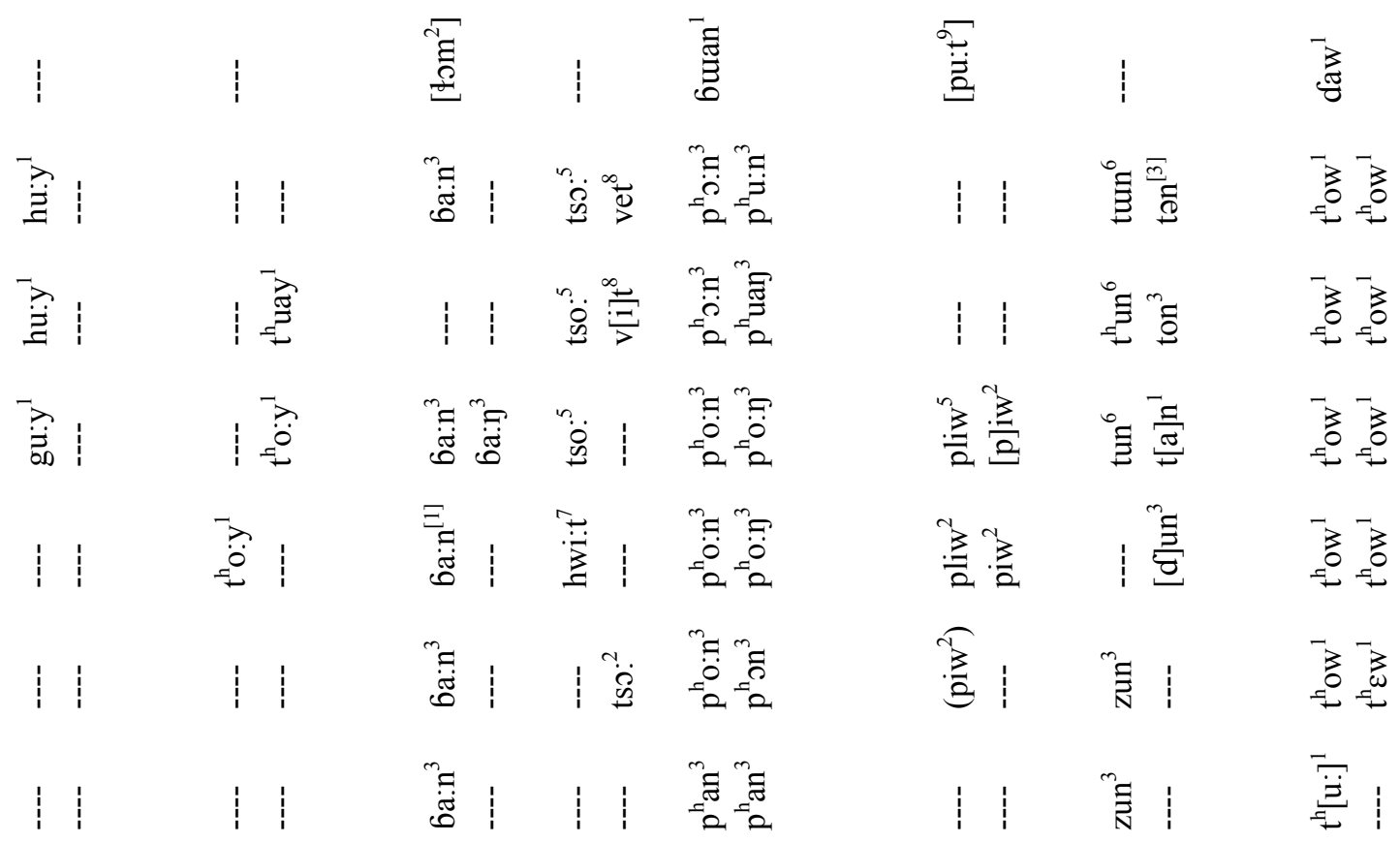

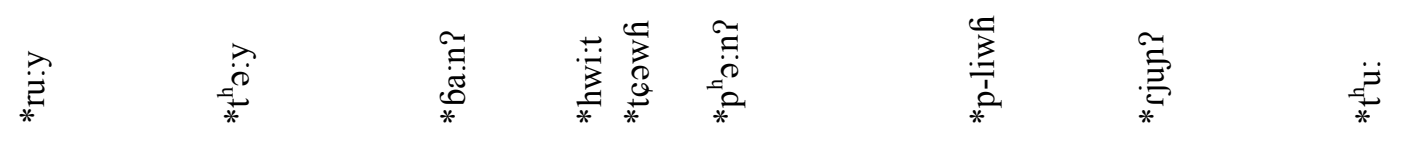

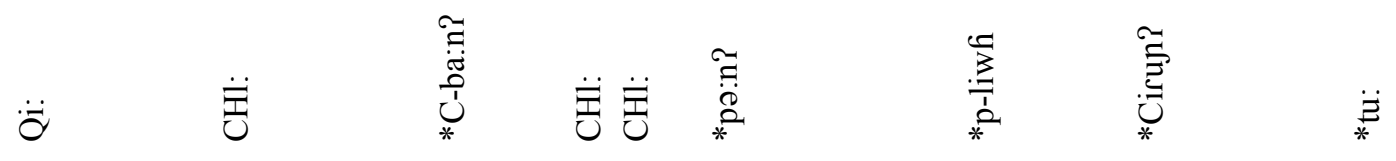

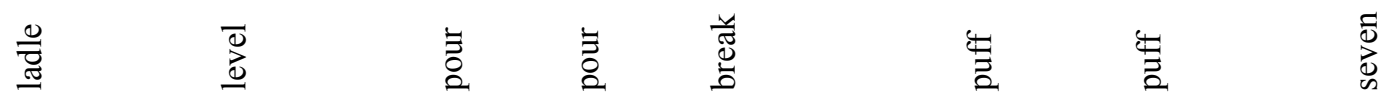

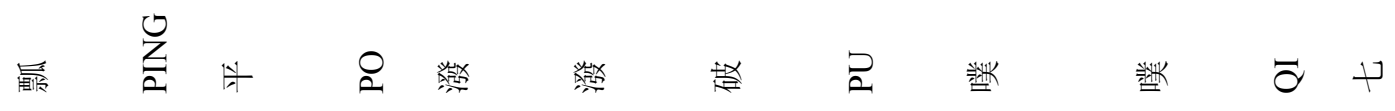




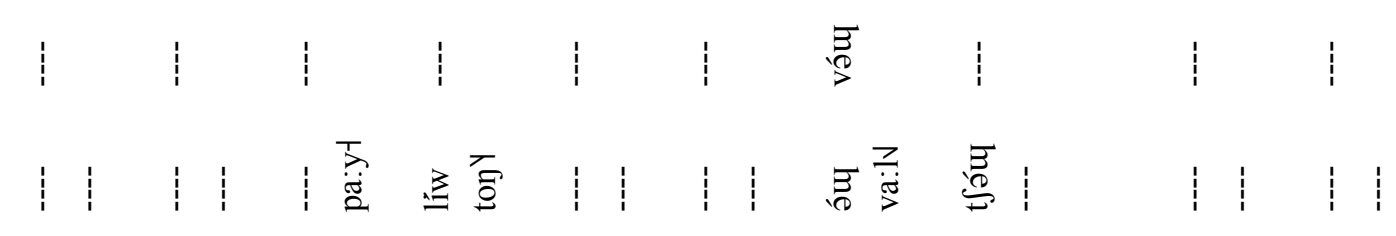

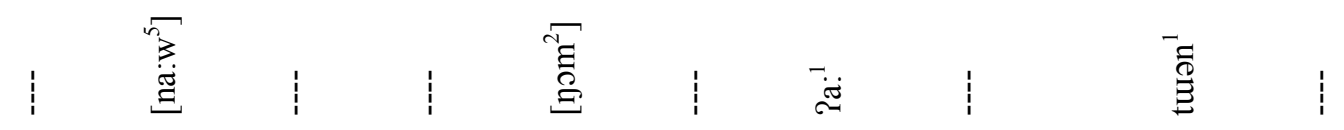

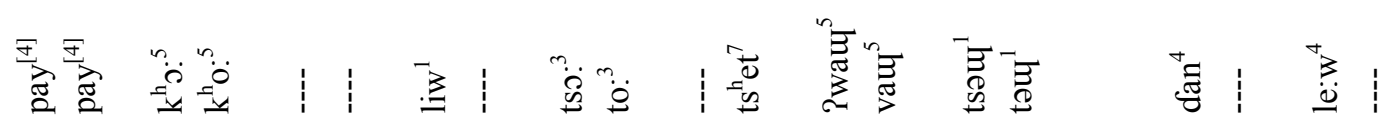

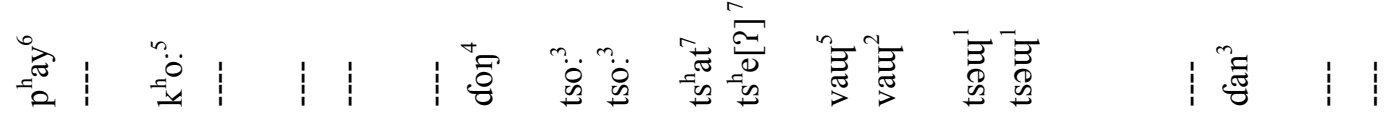

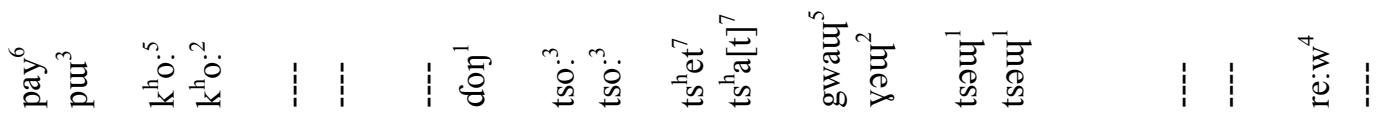

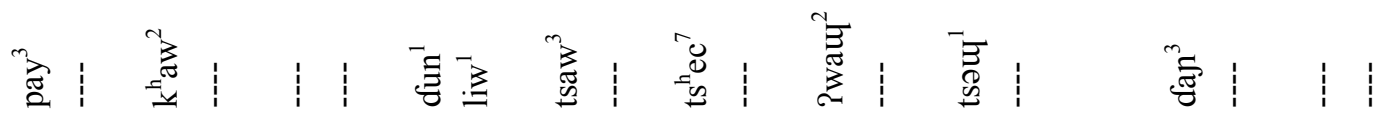

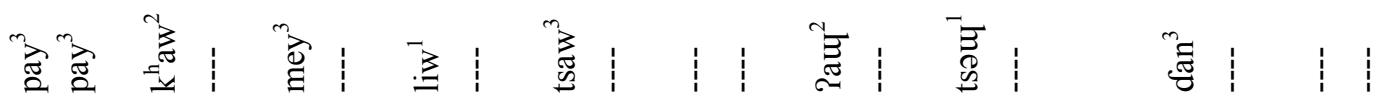

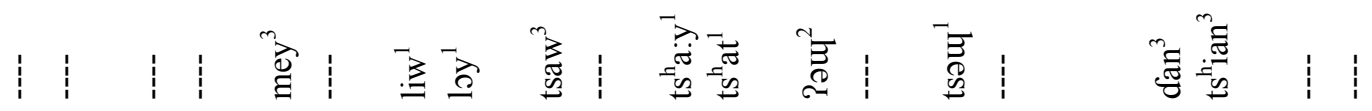

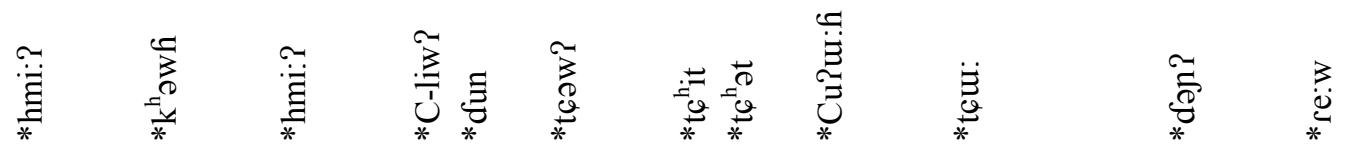

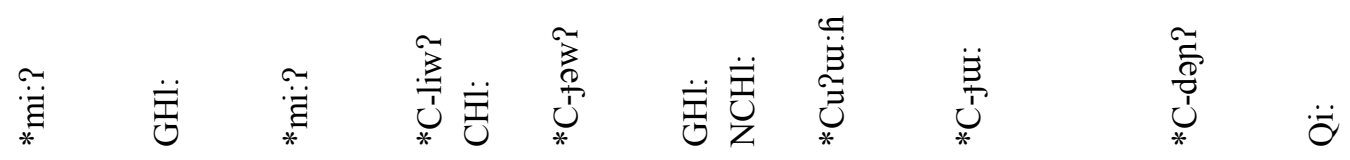

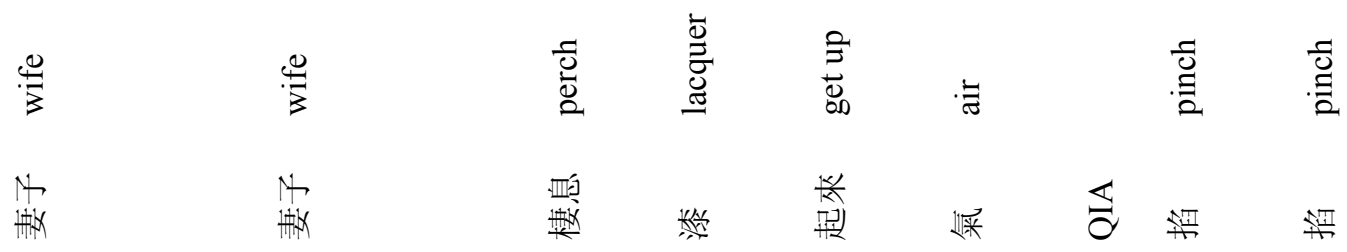




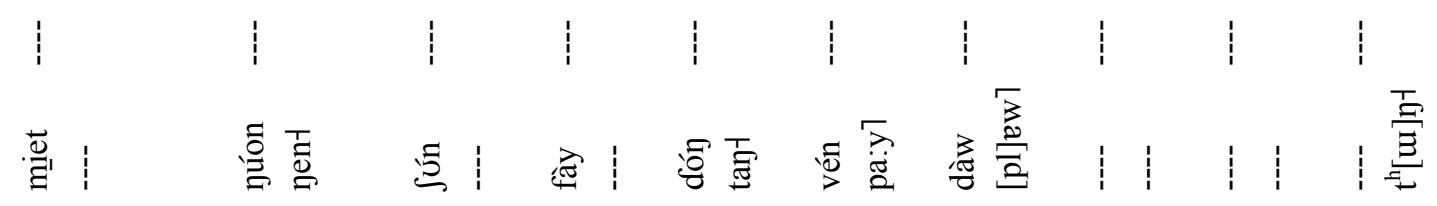

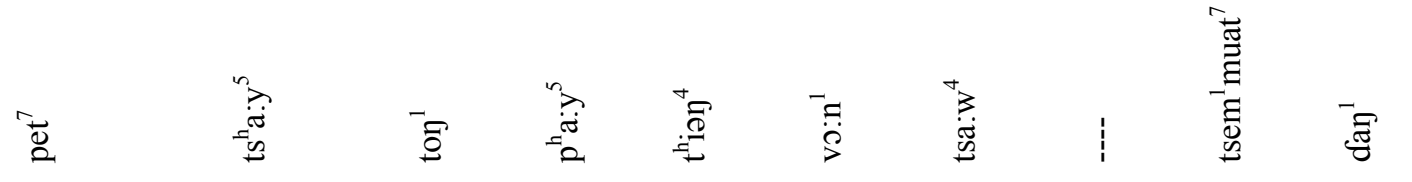

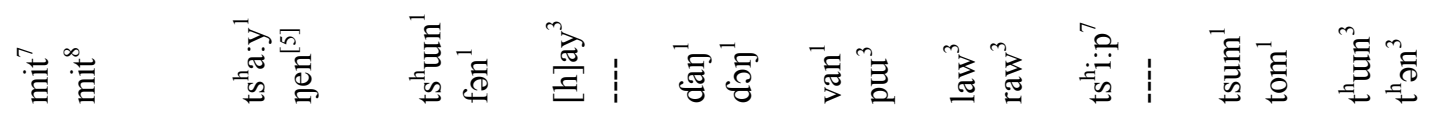

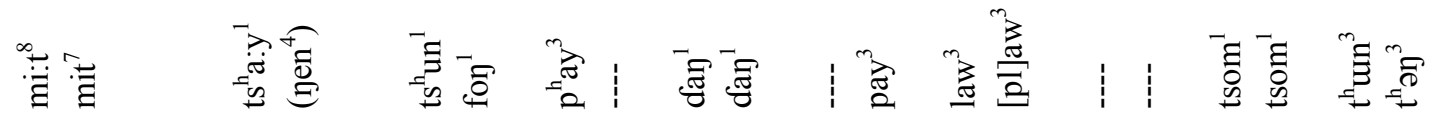

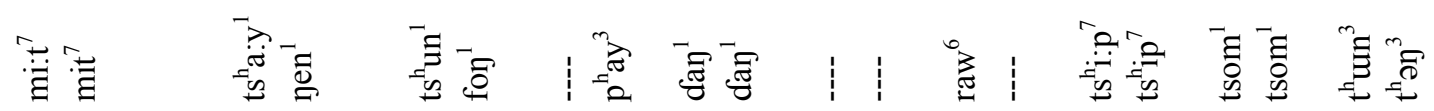

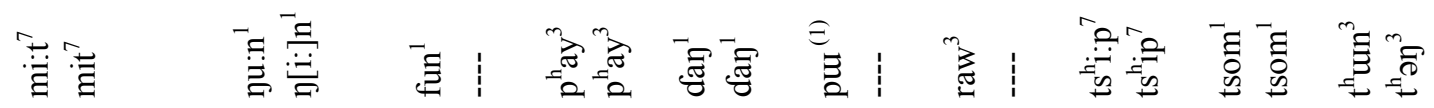

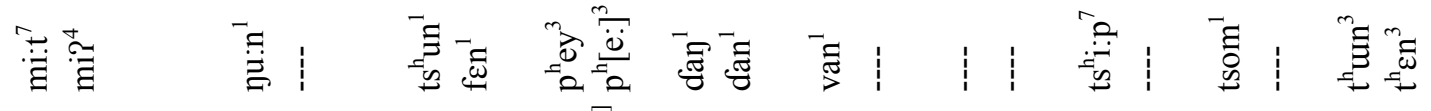

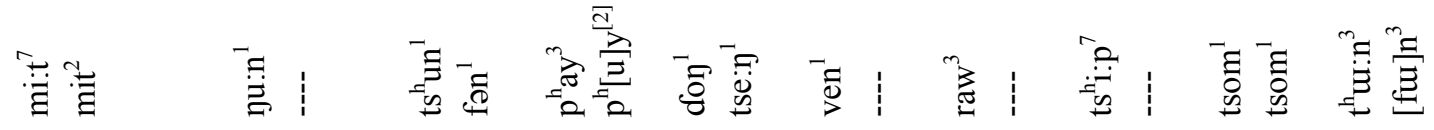

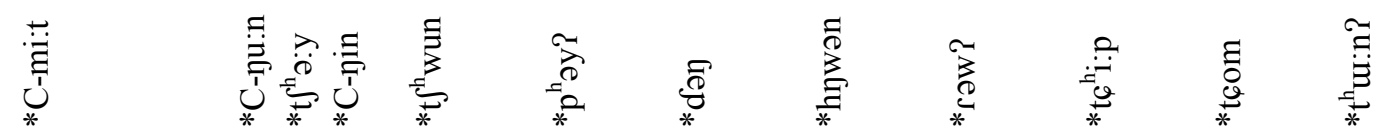

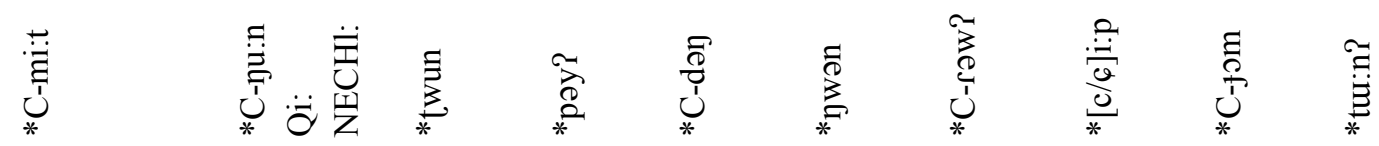

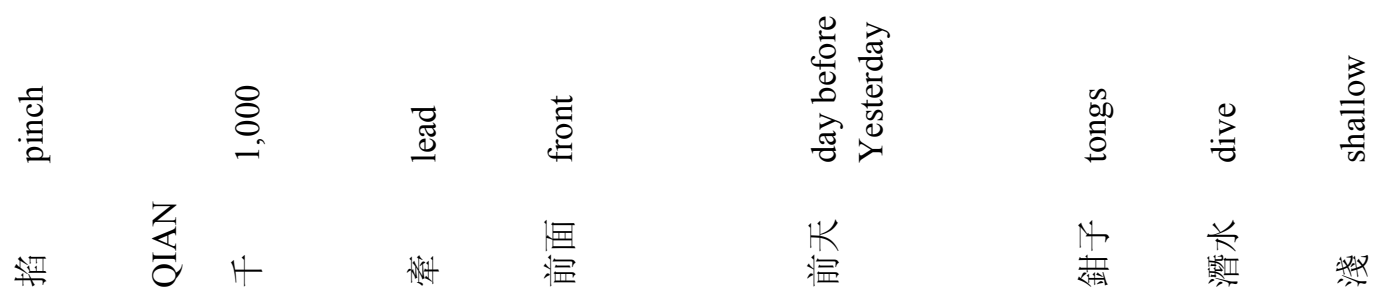




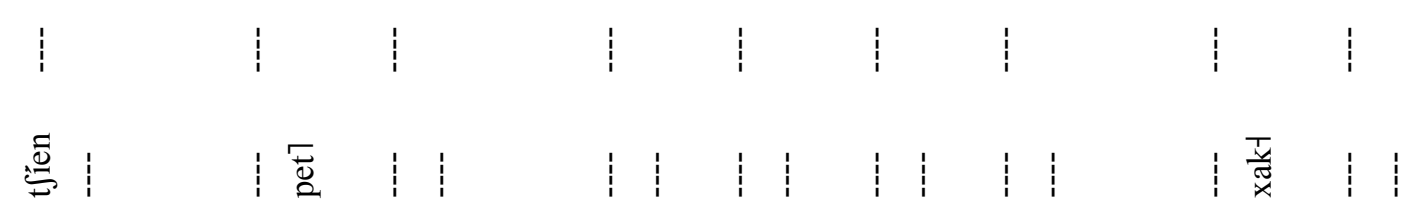

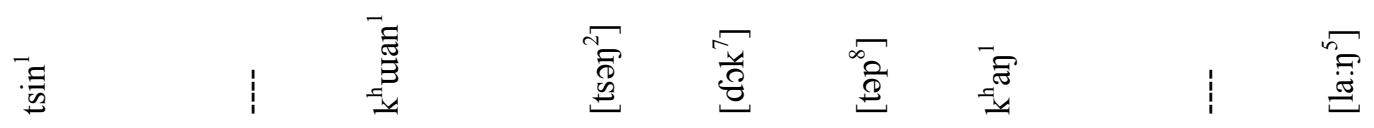

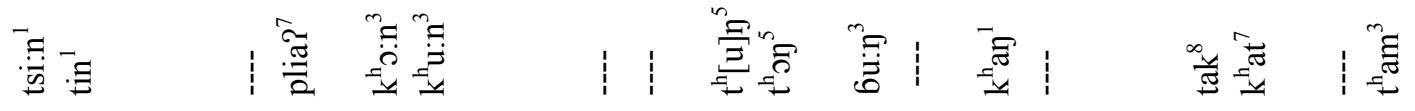

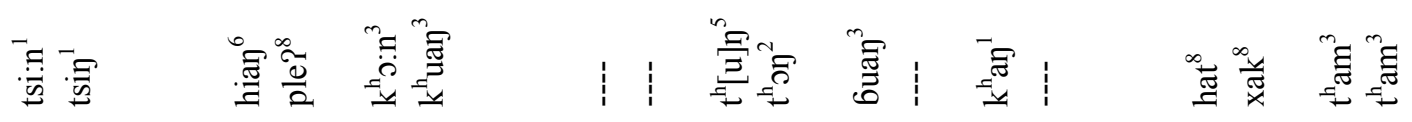

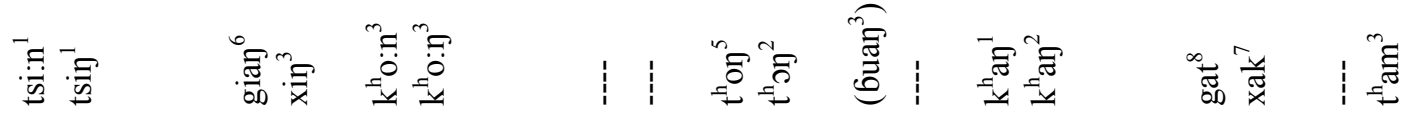

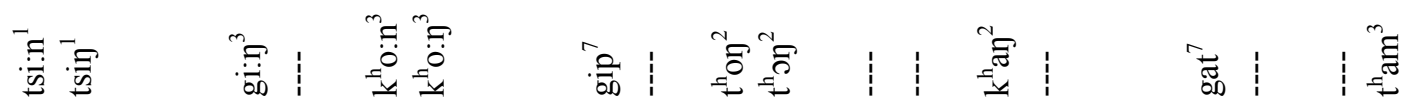

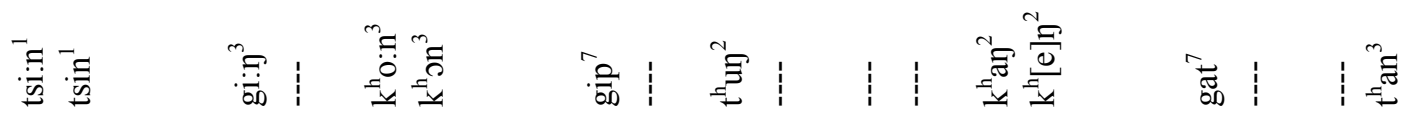

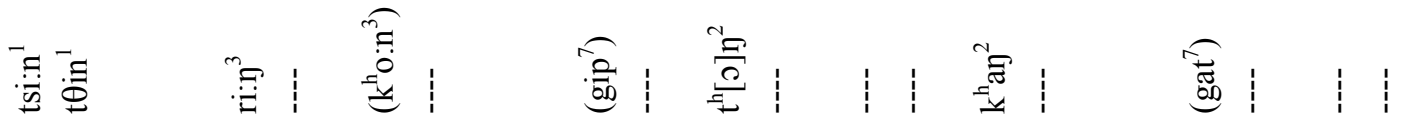

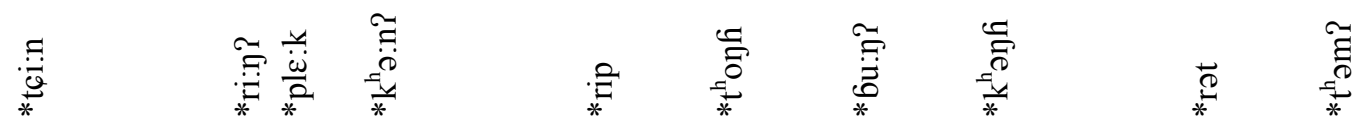

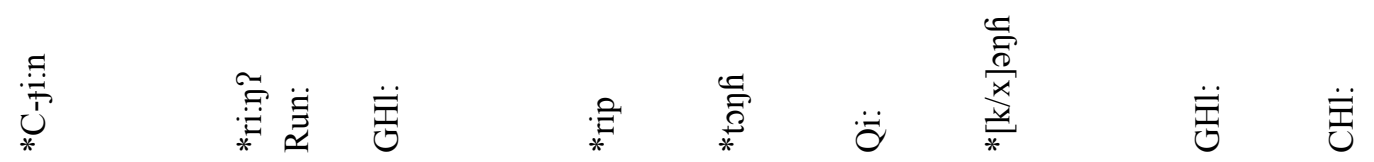

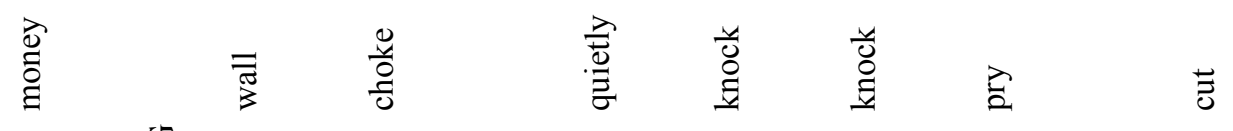

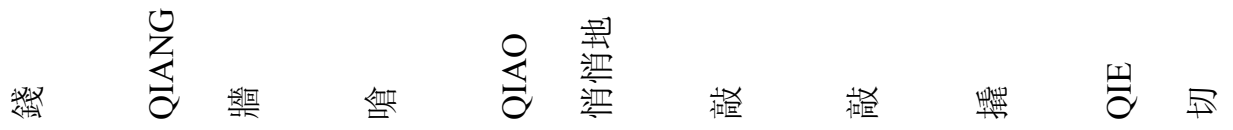




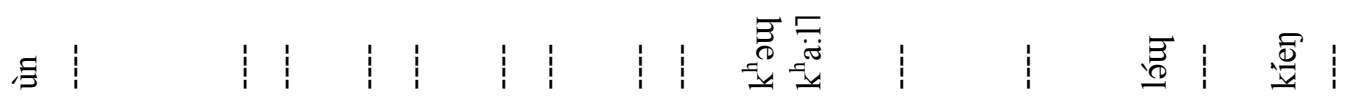

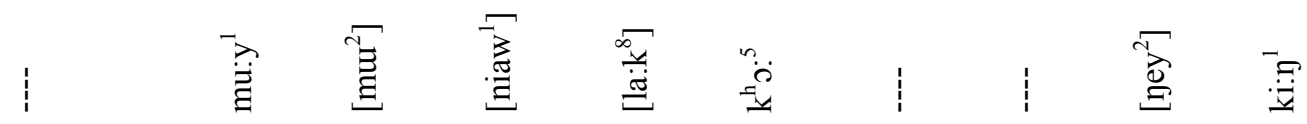

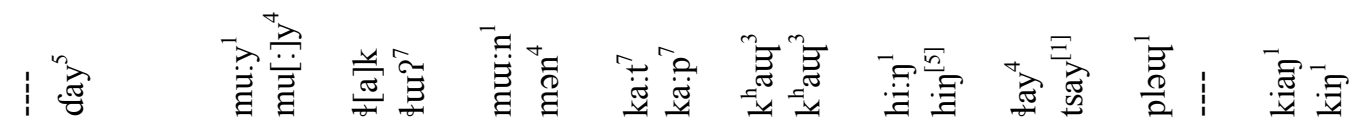

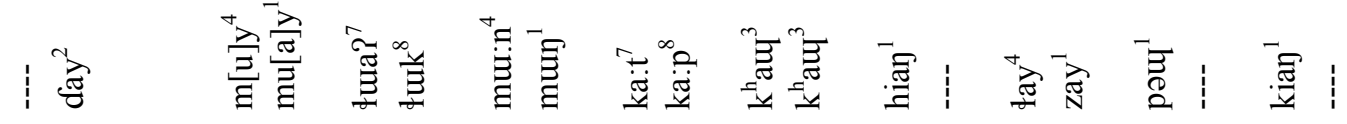

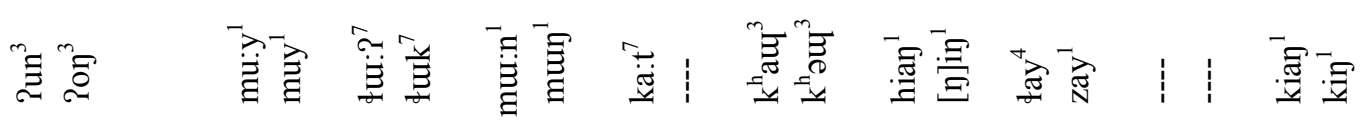

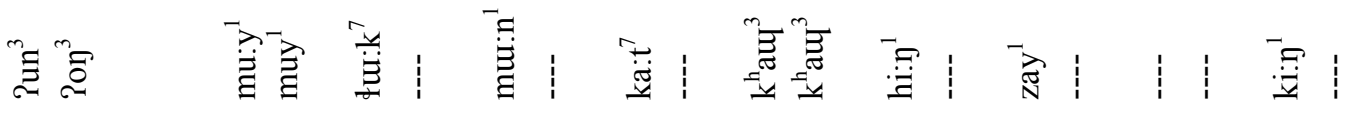

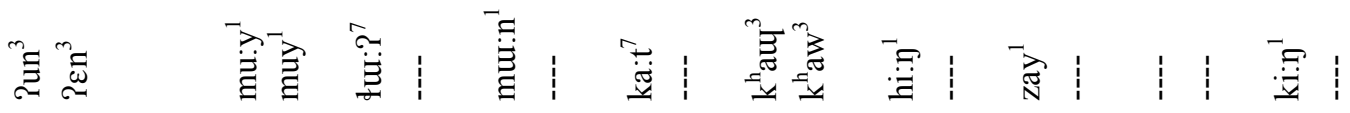

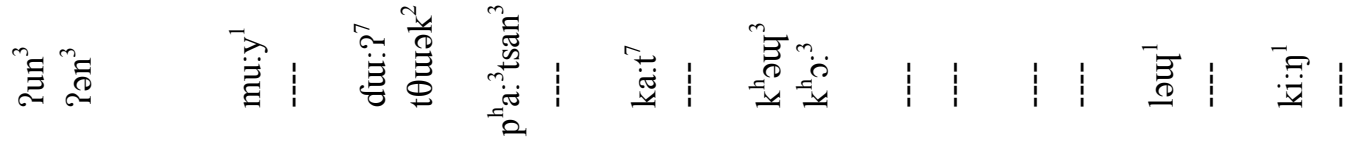

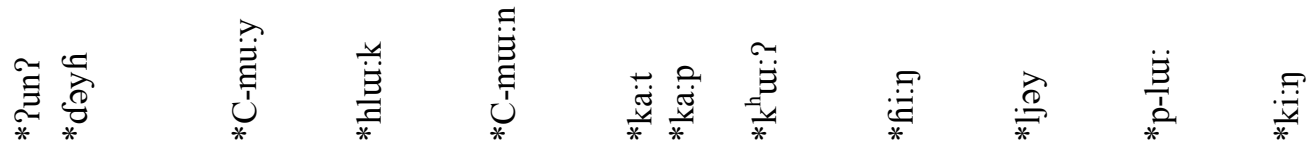

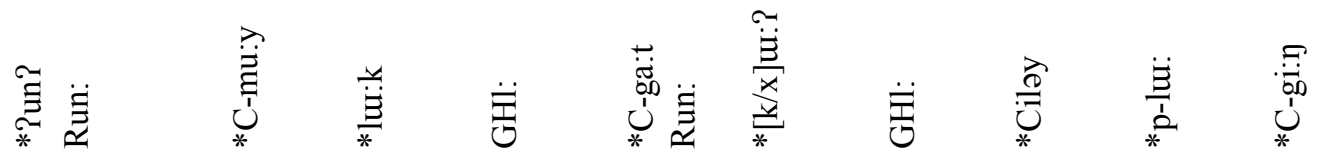

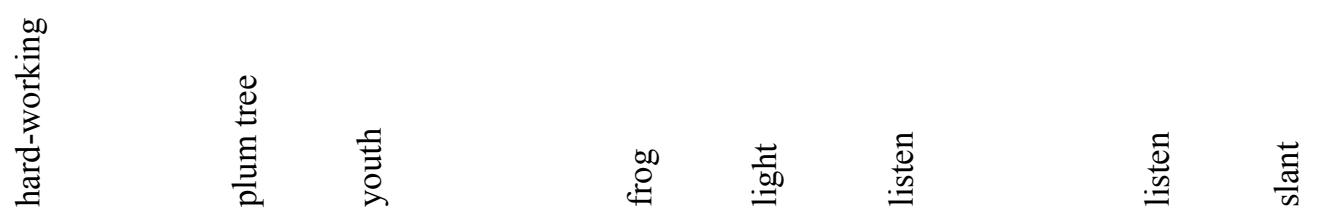

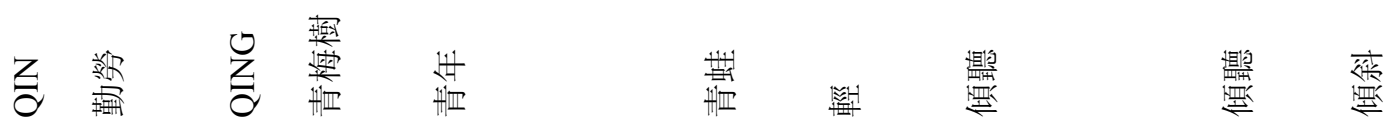




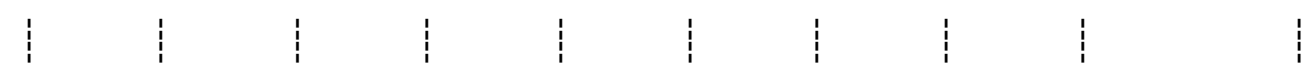

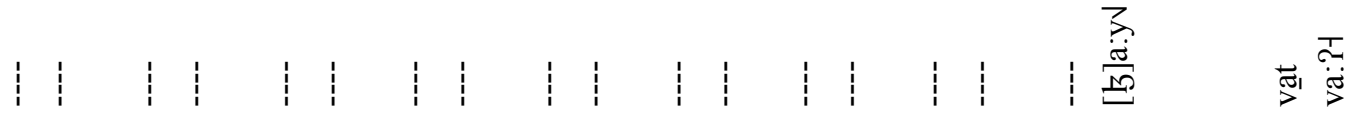

|

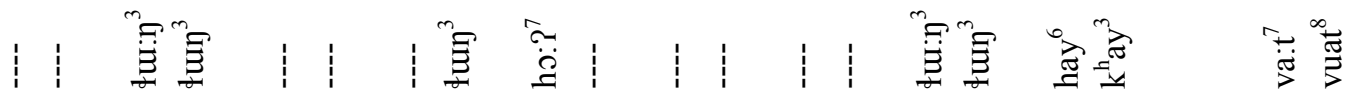

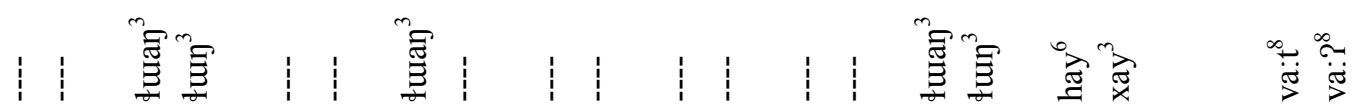

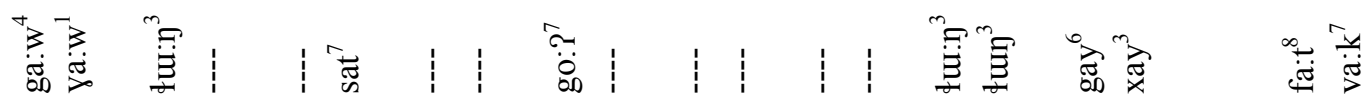

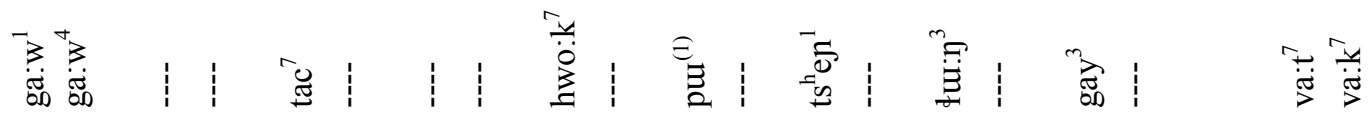

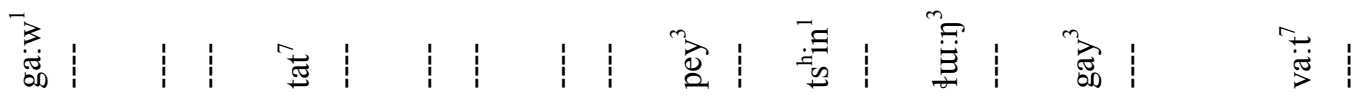

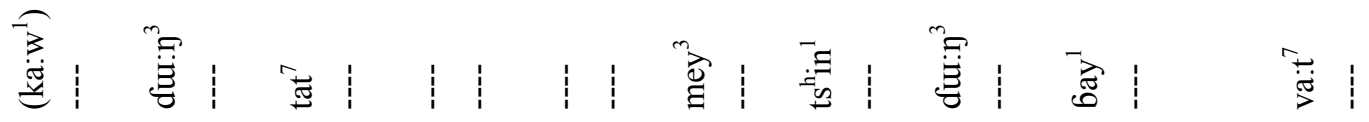

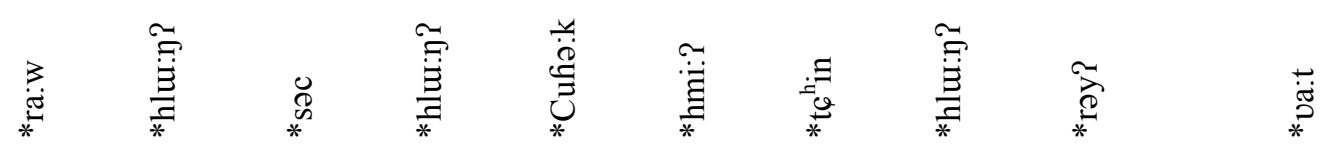

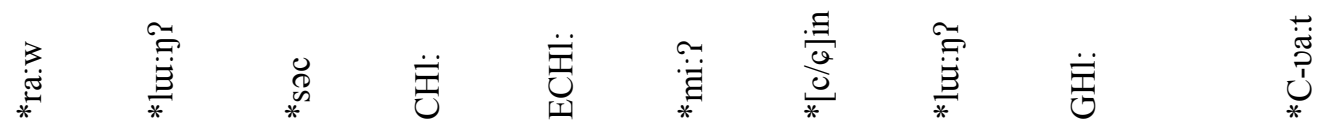

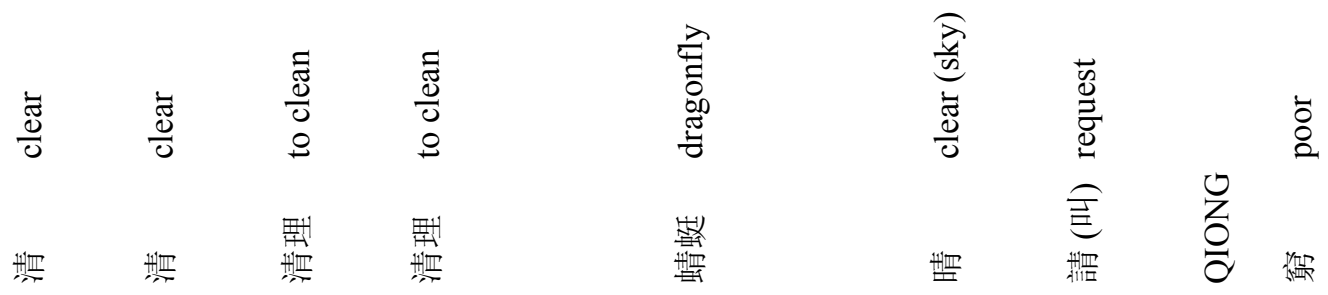




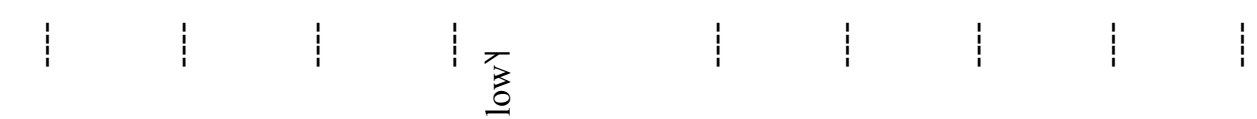

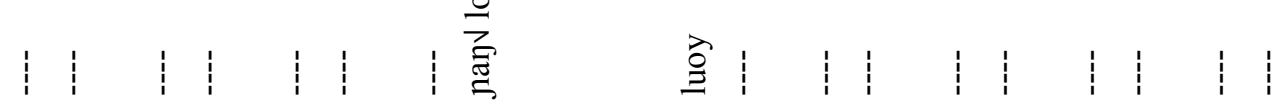

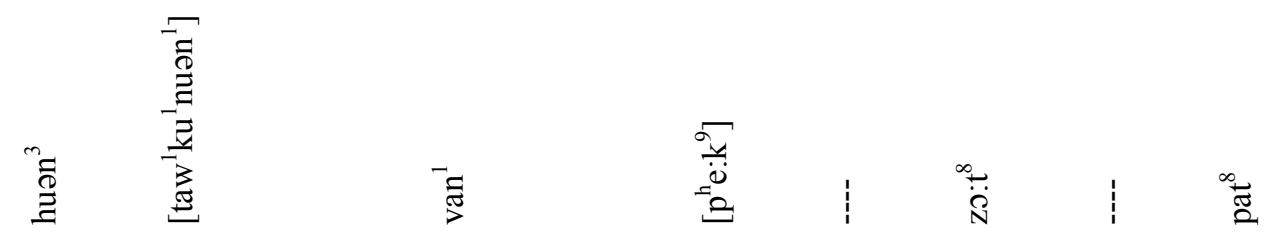

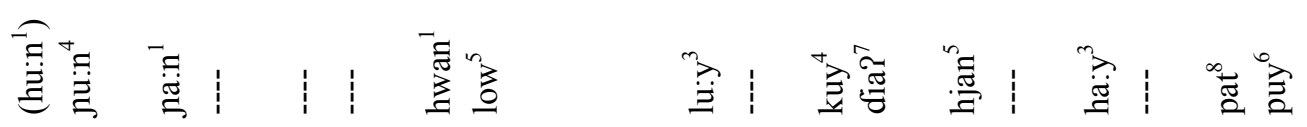

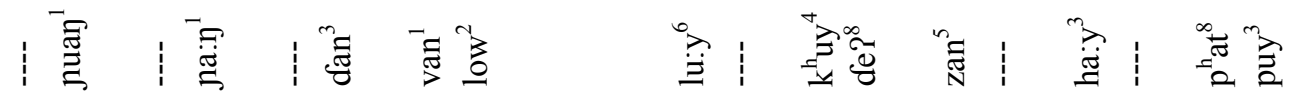

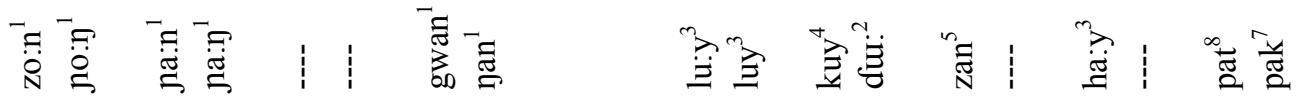

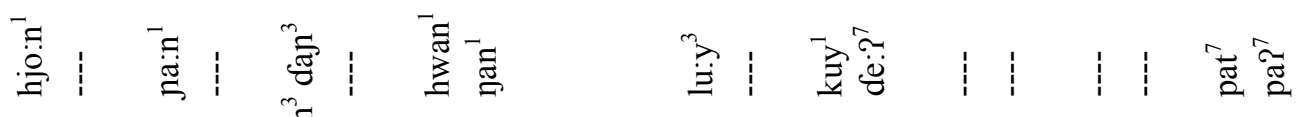

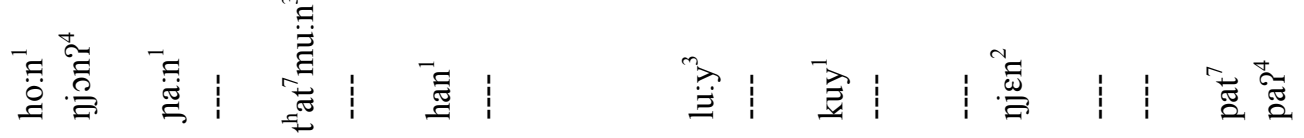

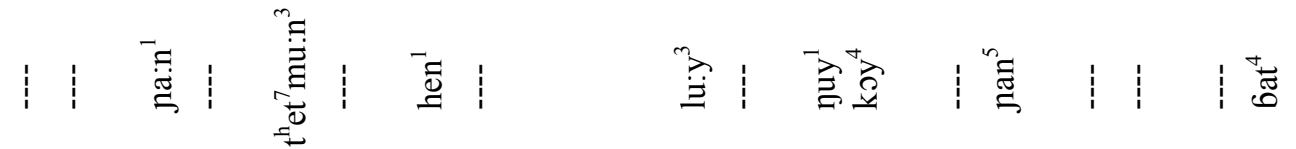

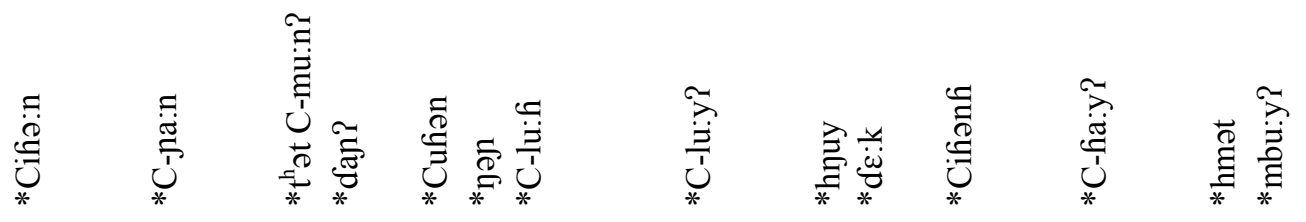

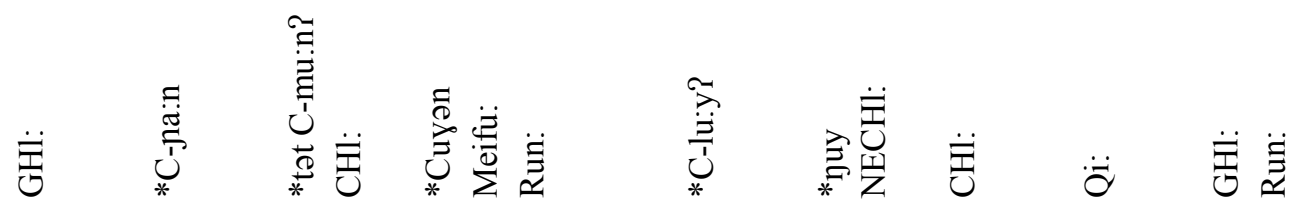

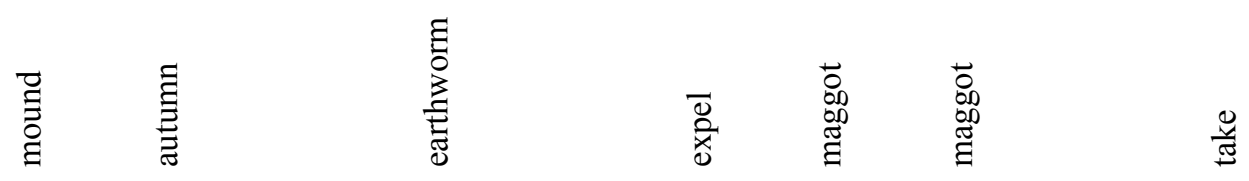

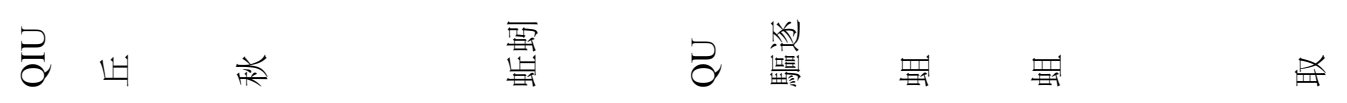




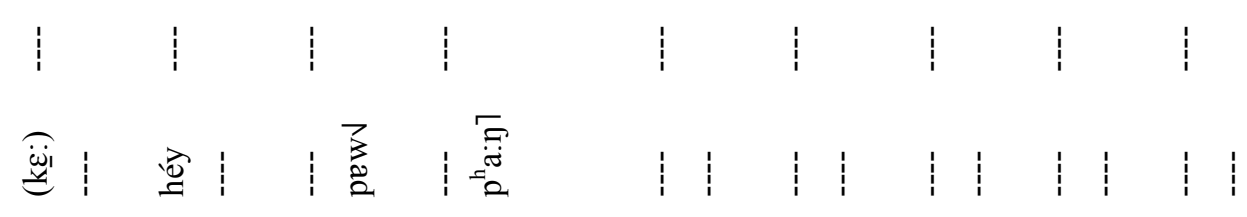

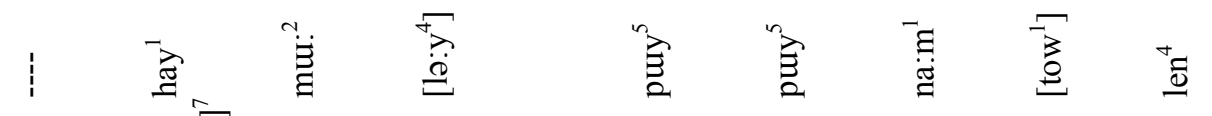

苛宽

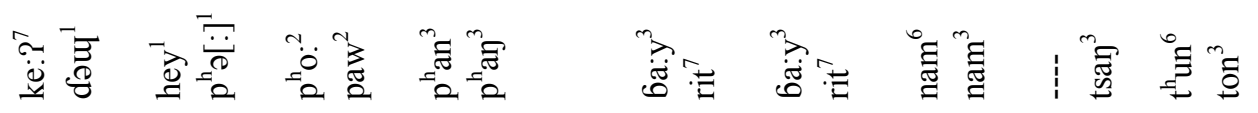

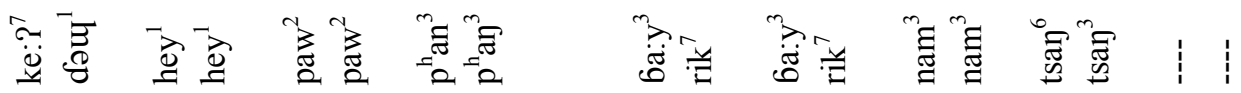

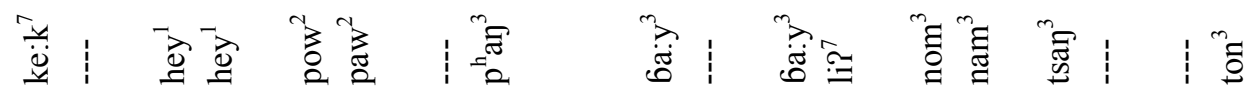

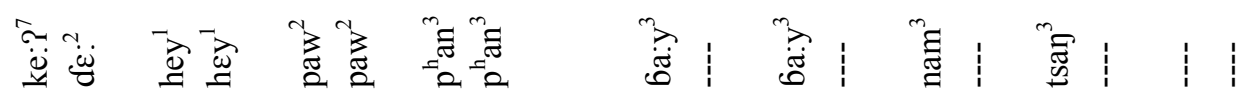

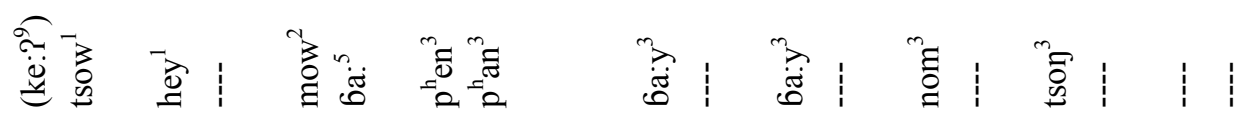

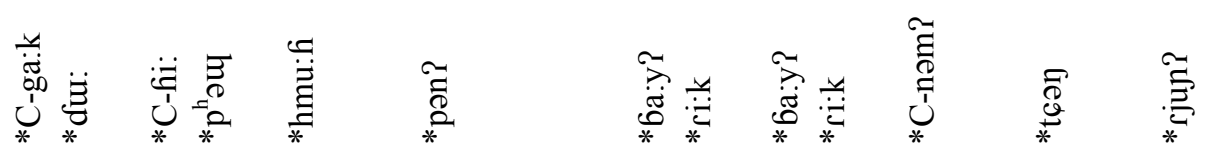

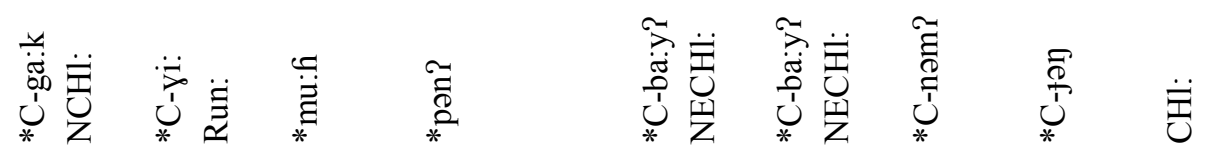

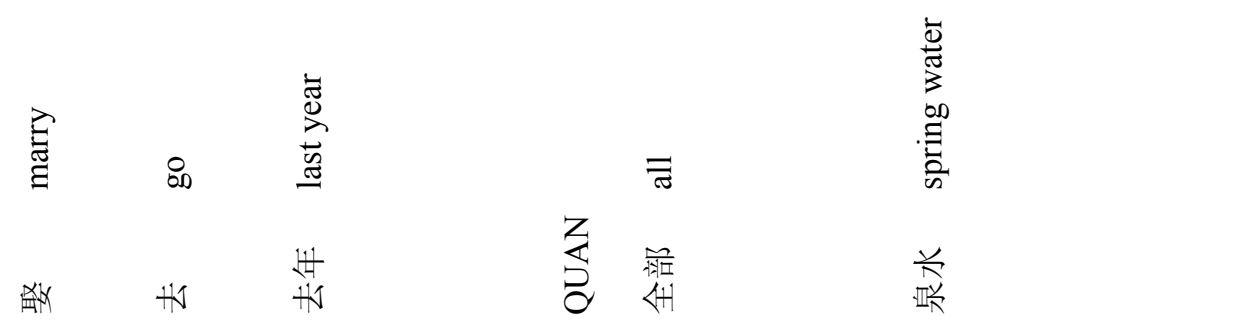




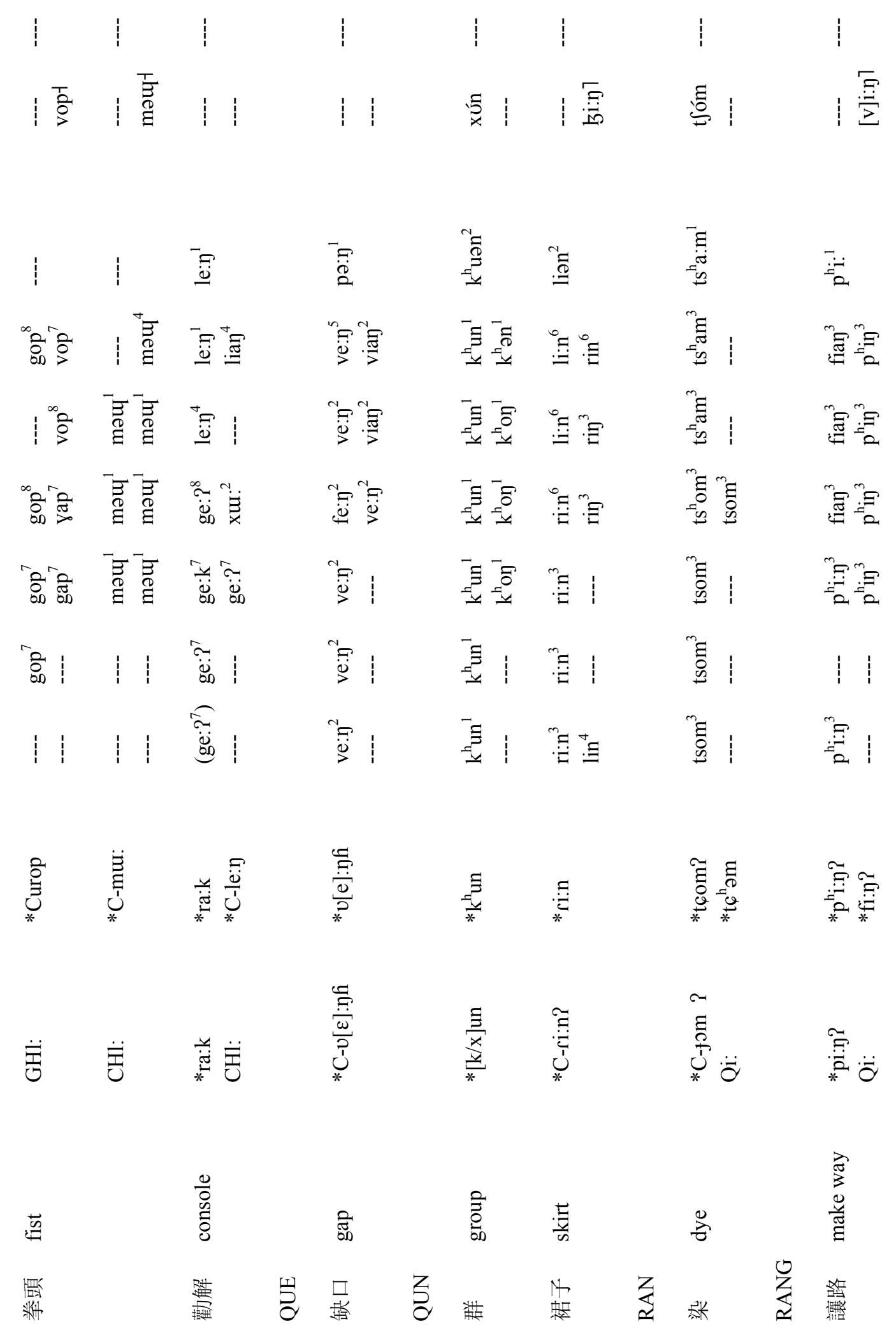




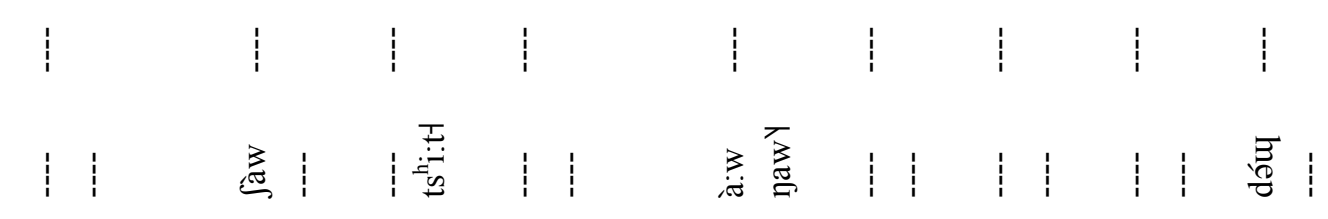

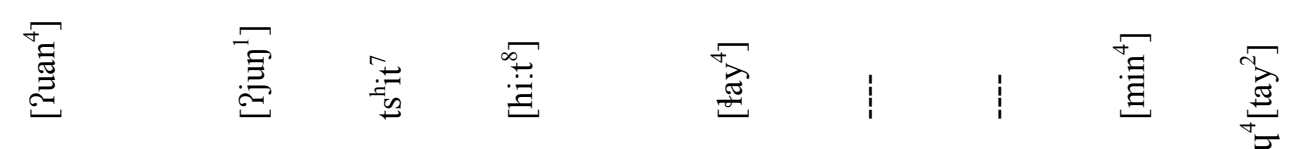

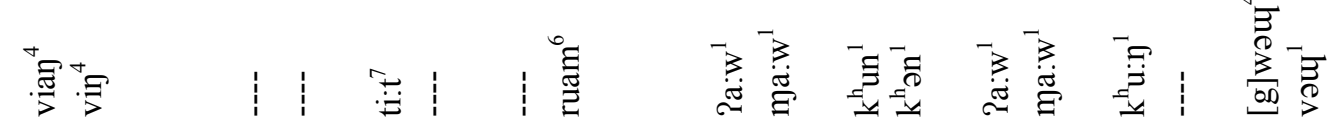

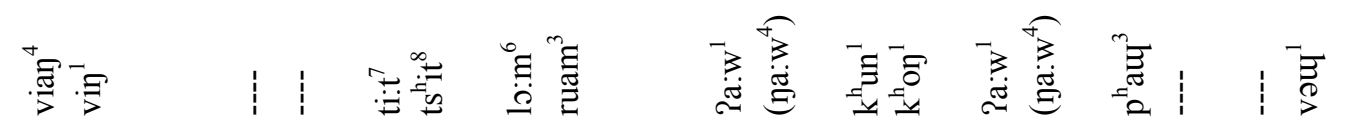

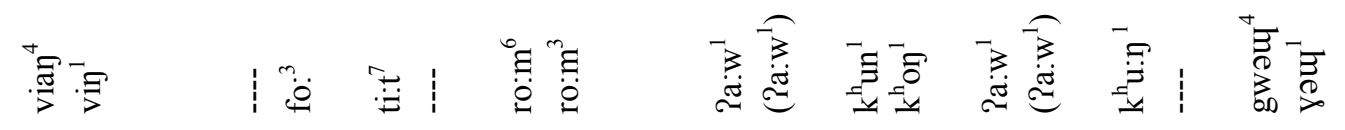

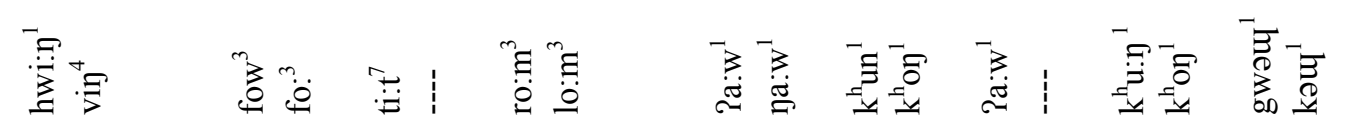

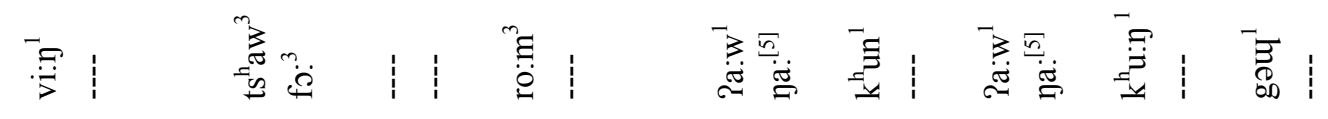

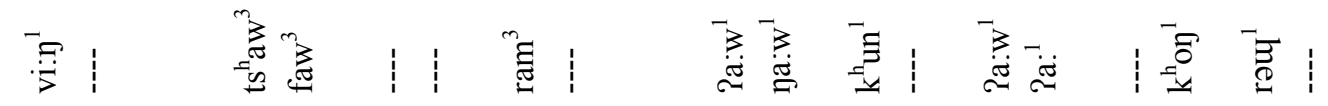

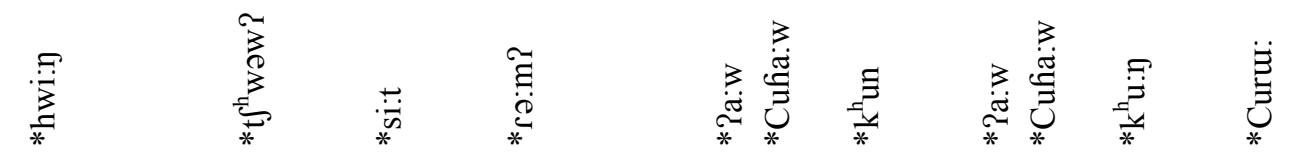

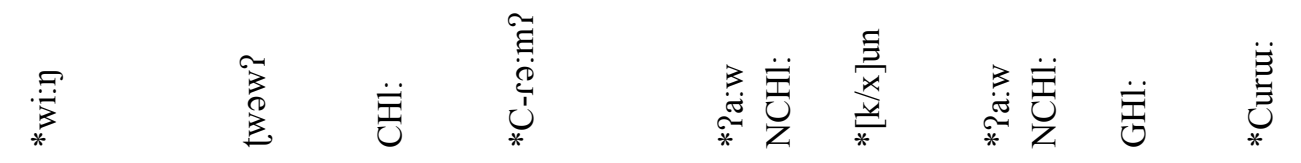

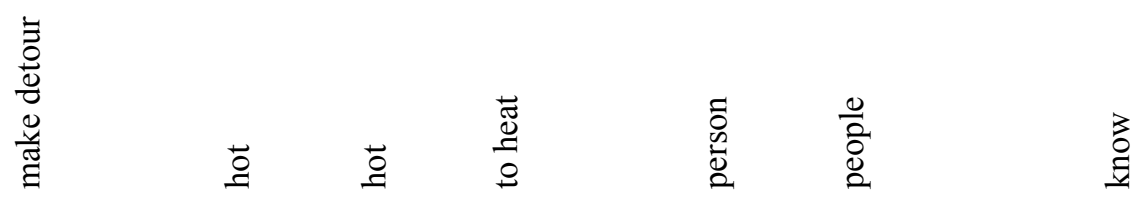

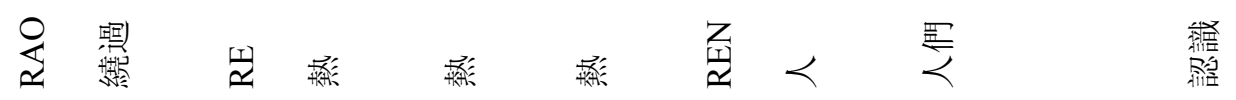




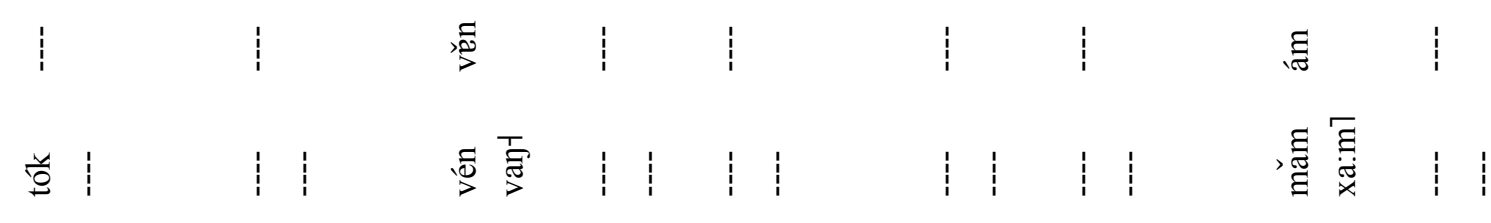

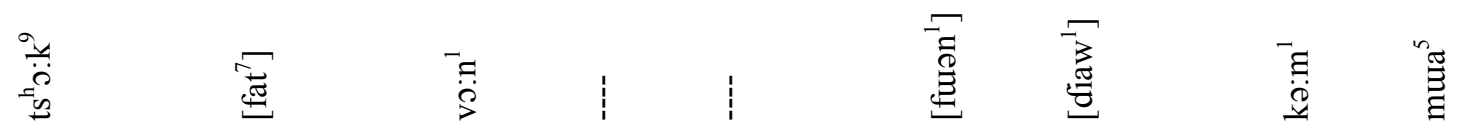

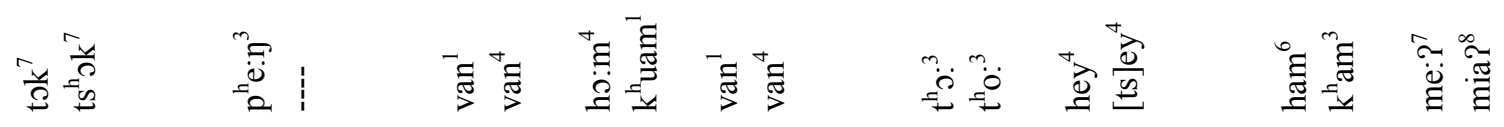

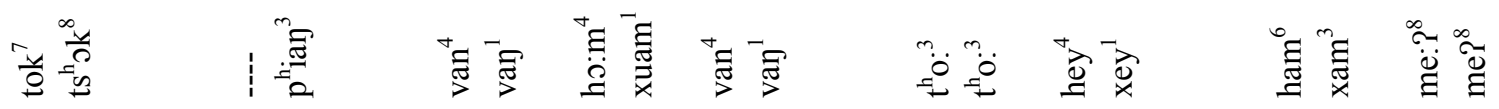

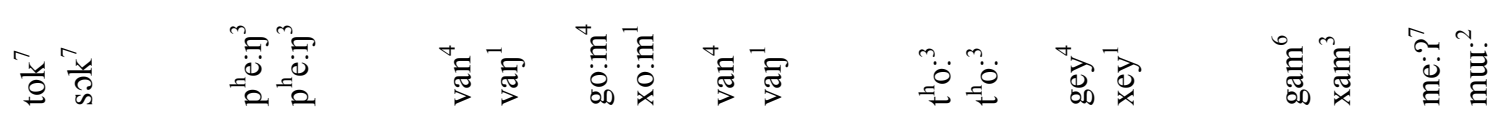

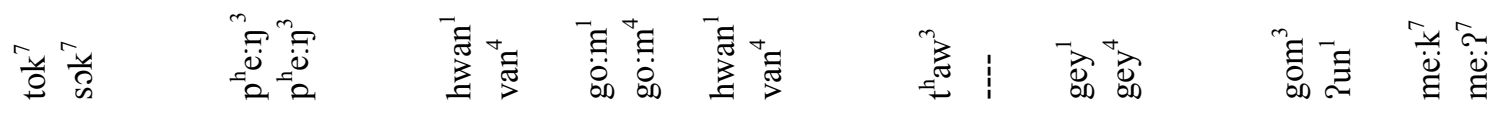

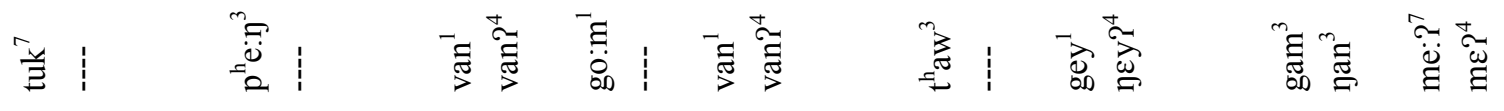

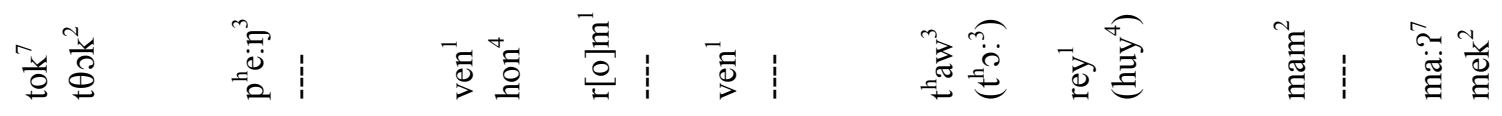

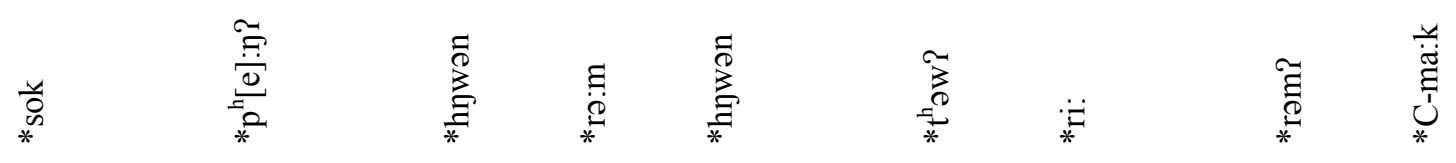

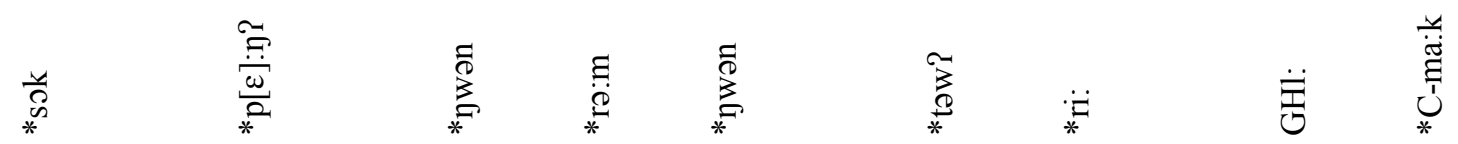

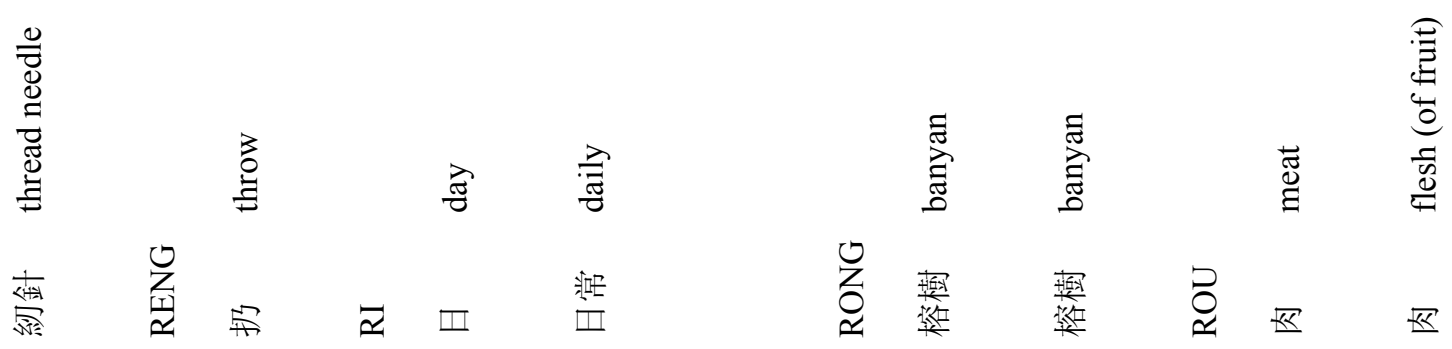




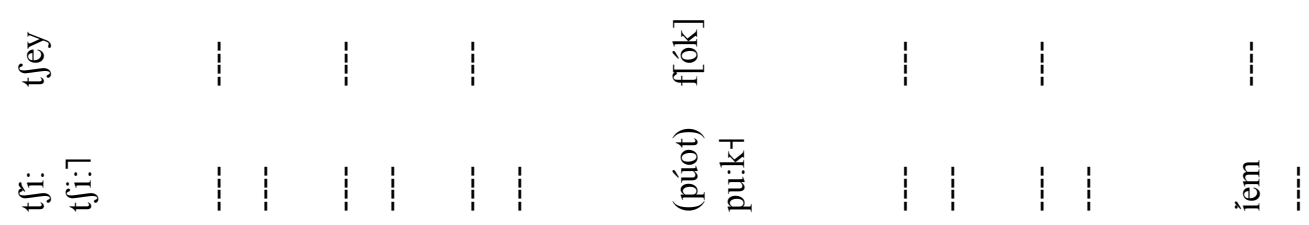

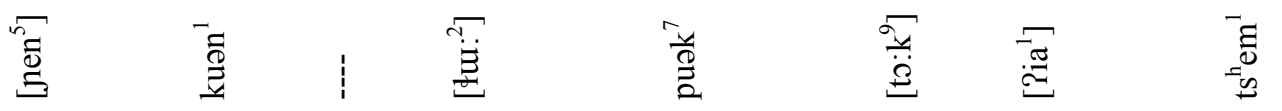

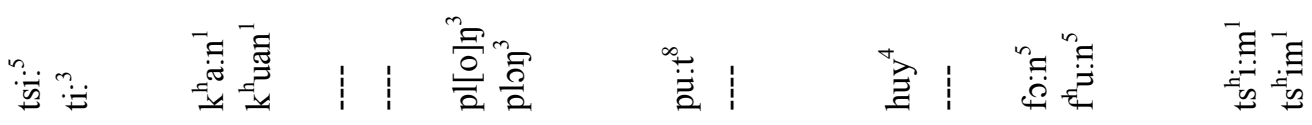

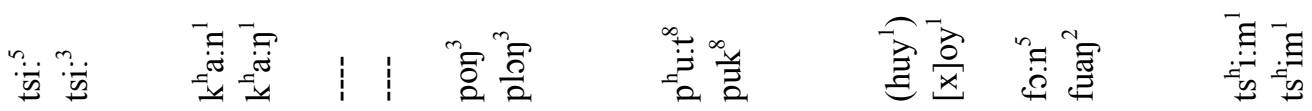

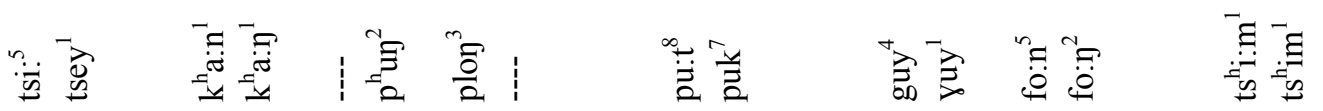

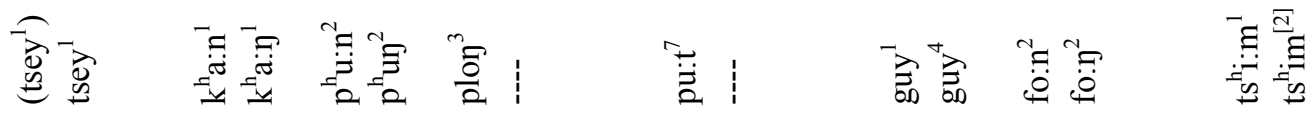

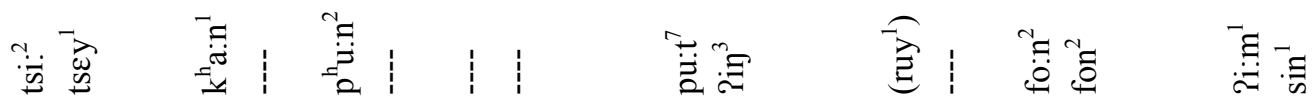

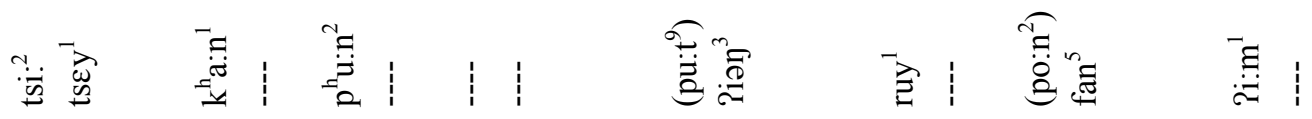

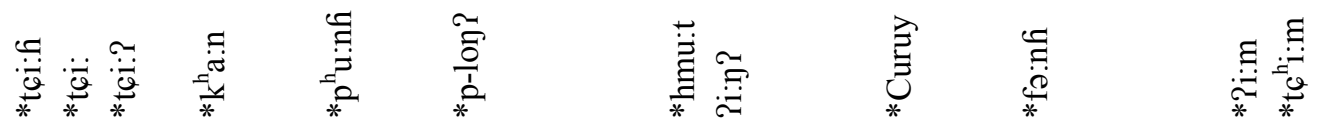

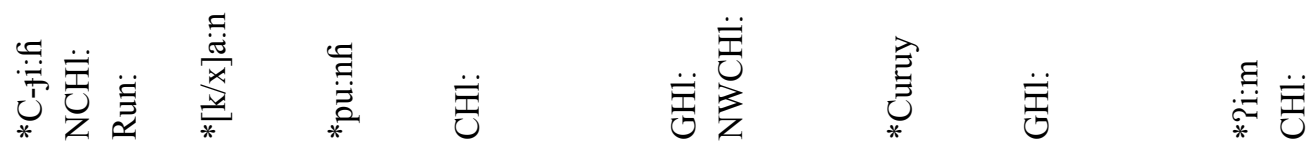

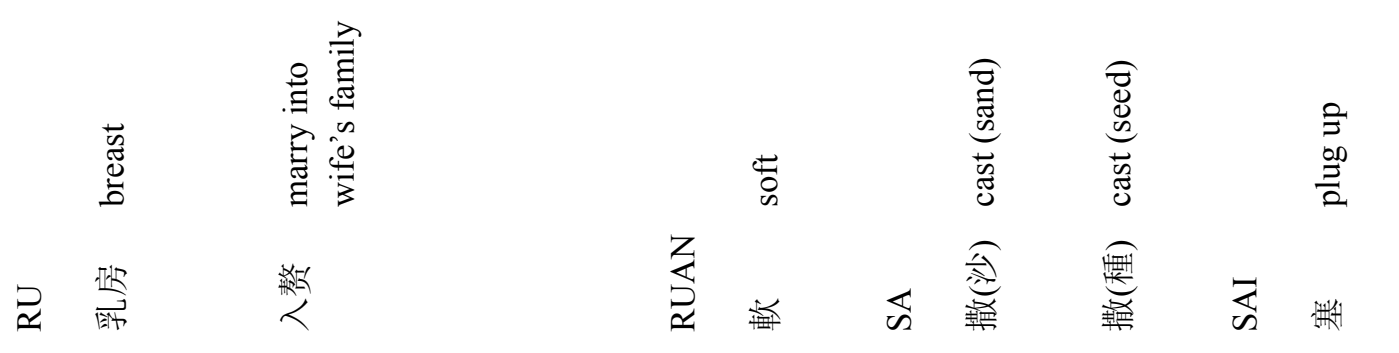




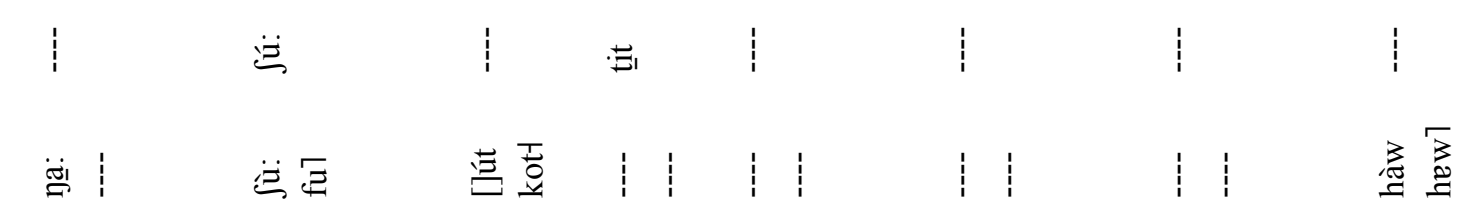

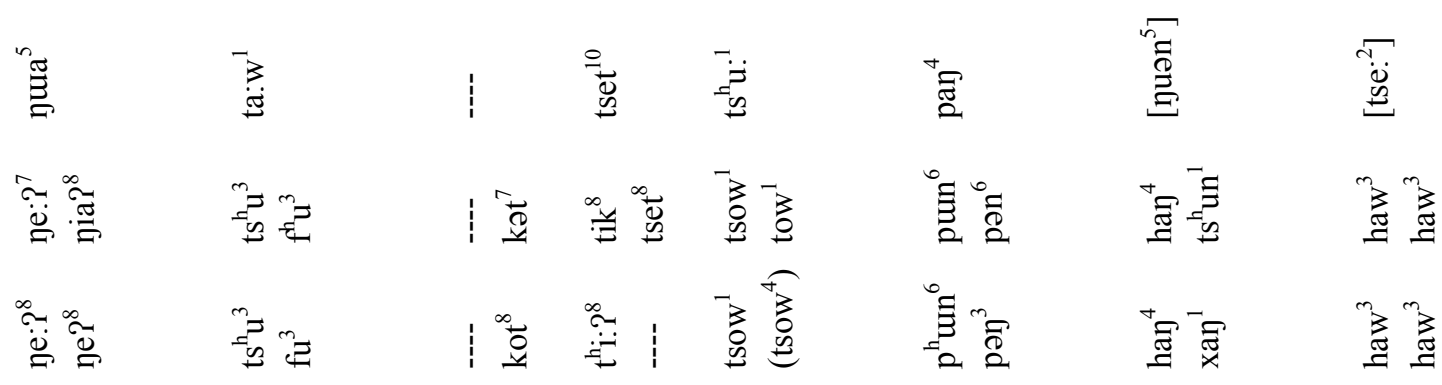

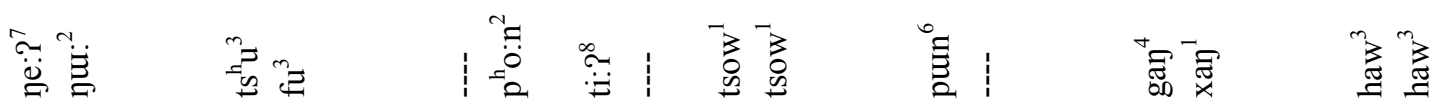

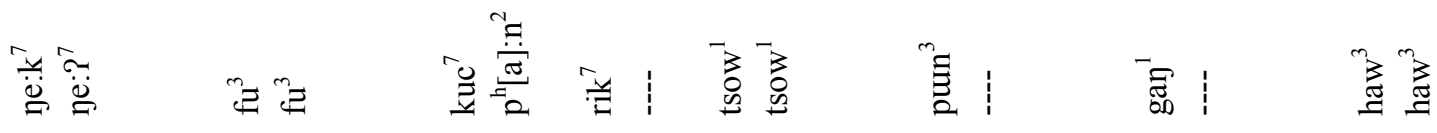

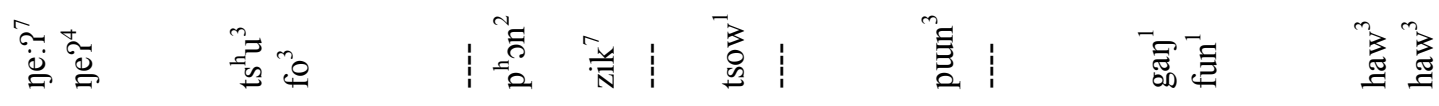

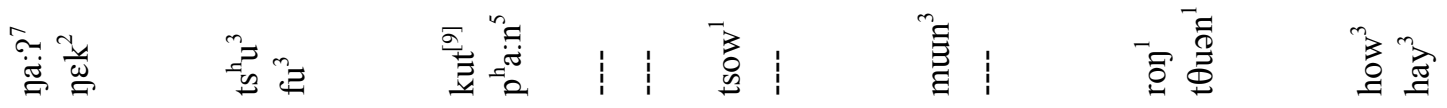

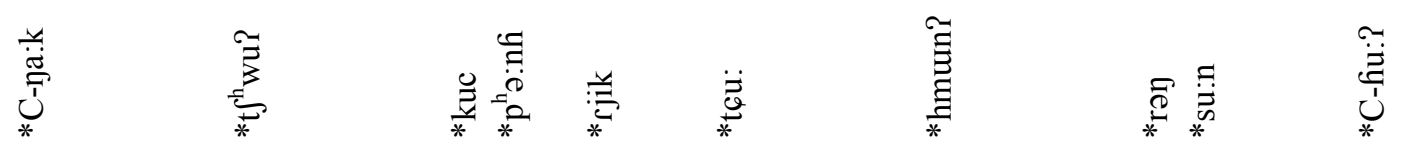

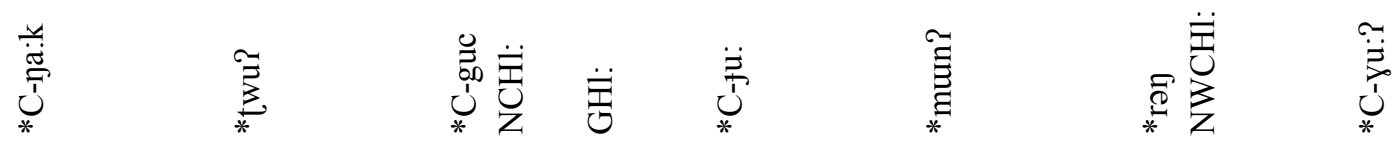

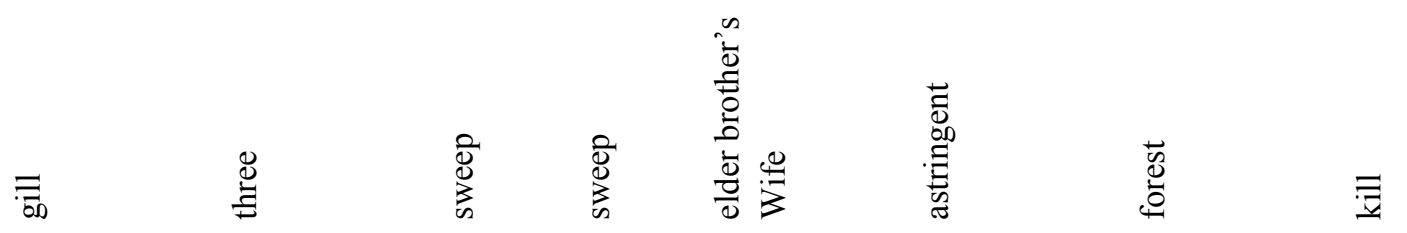

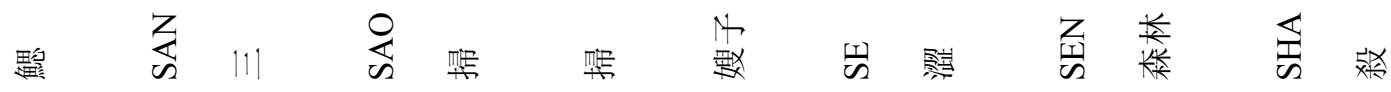




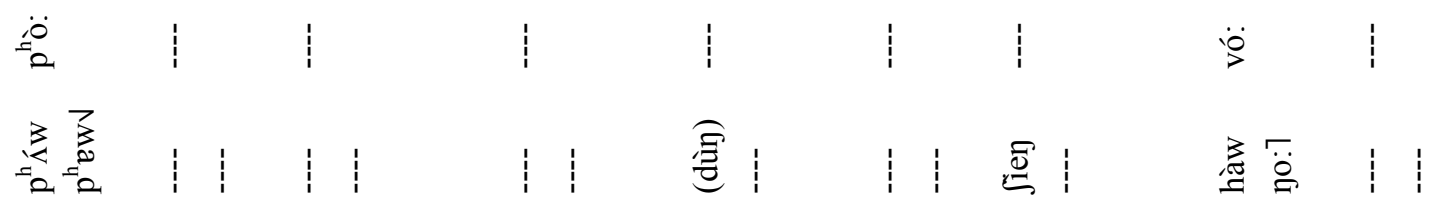

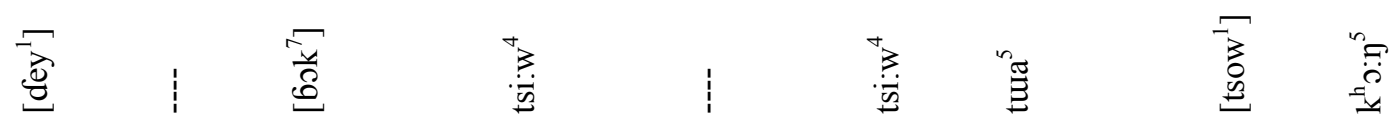

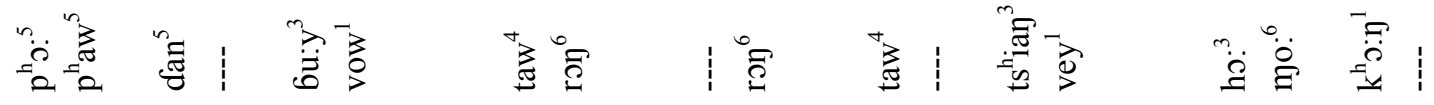

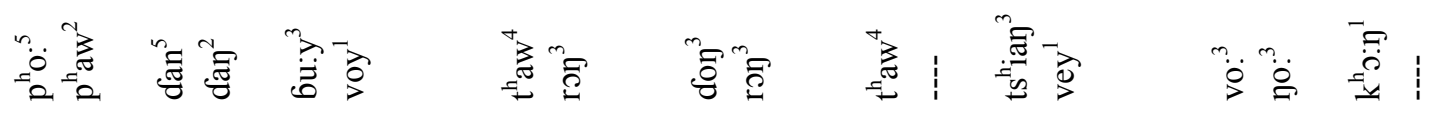

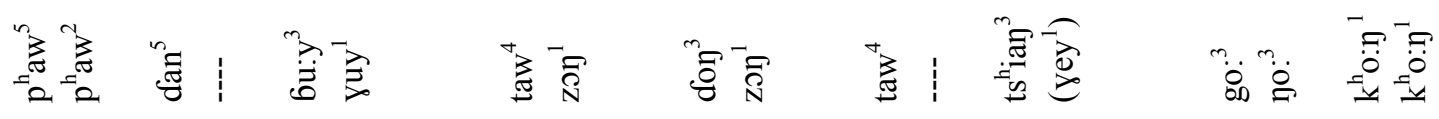

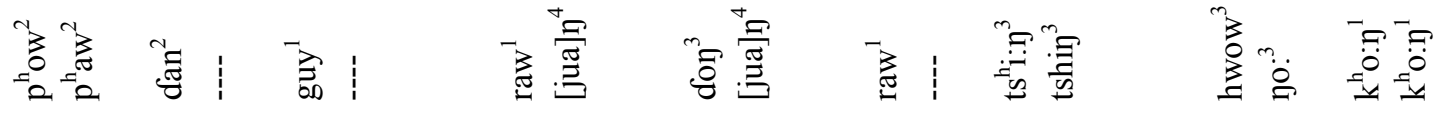

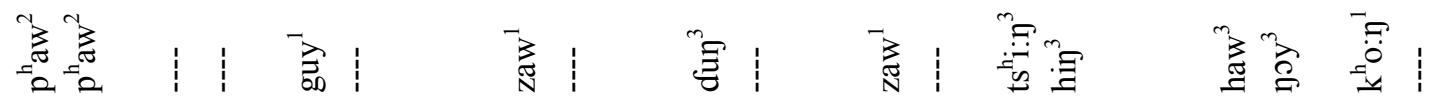

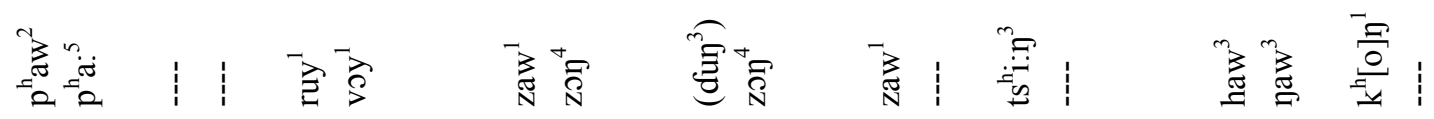

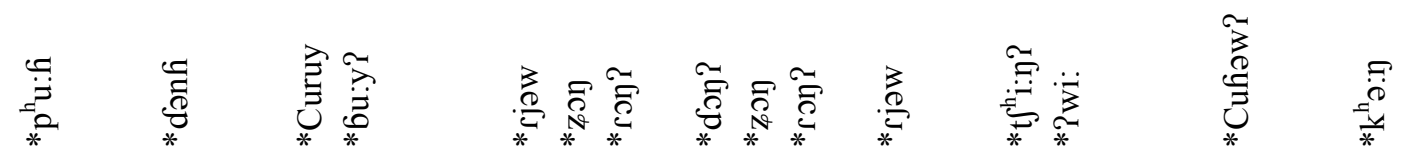

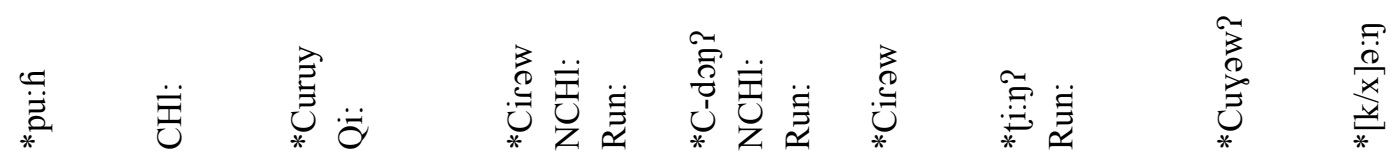

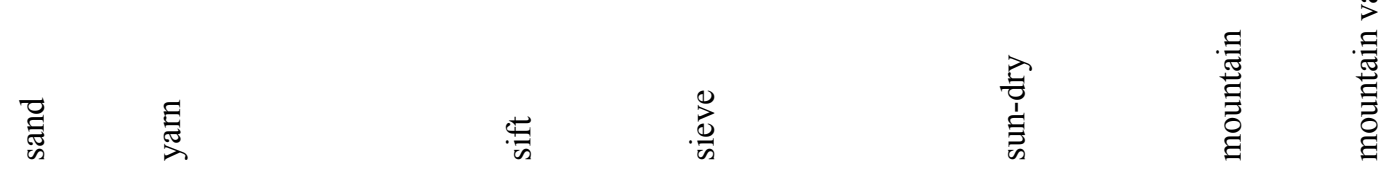

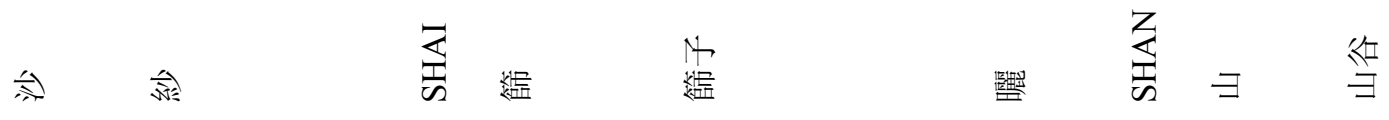


\begin{tabular}{l|l|l|l|l|l|}
1 & 1 & 1 & 1
\end{tabular}

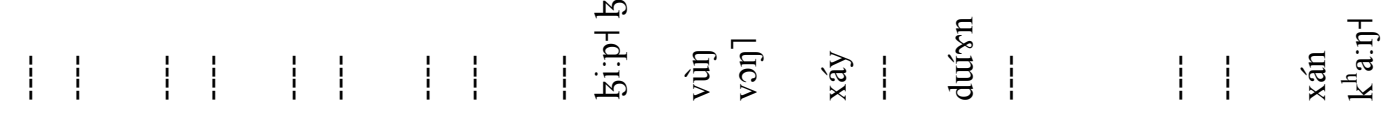

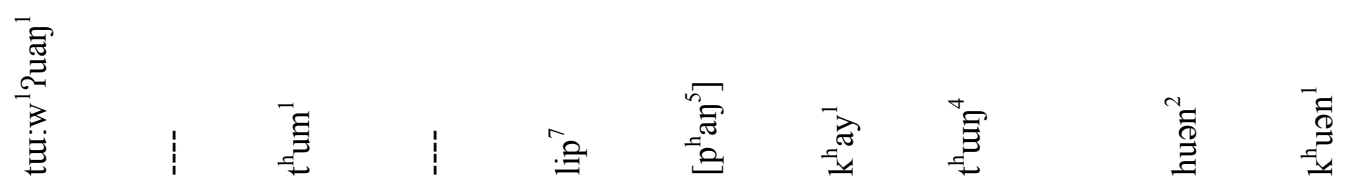

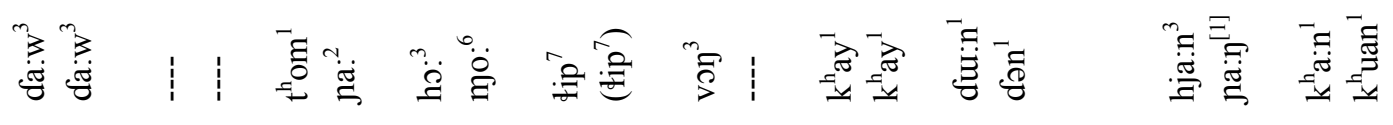

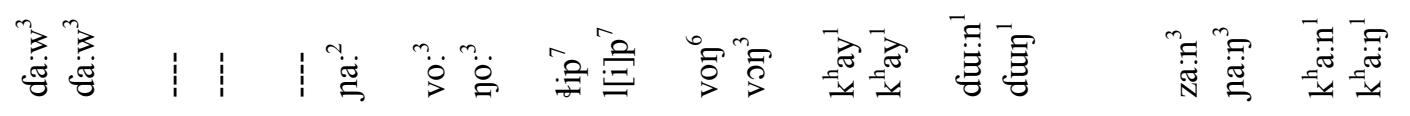

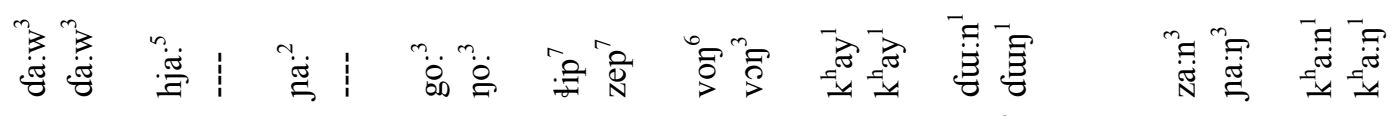

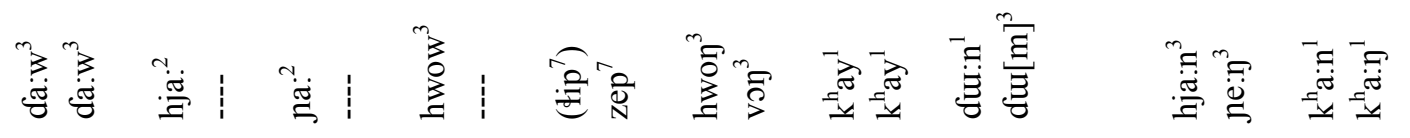

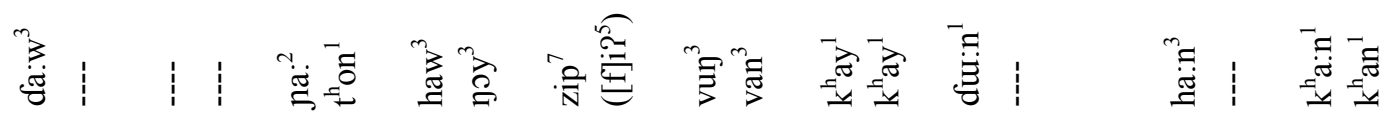

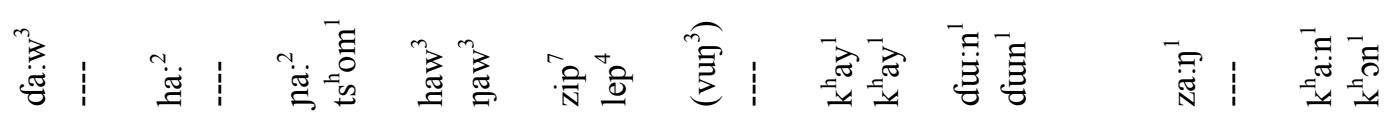

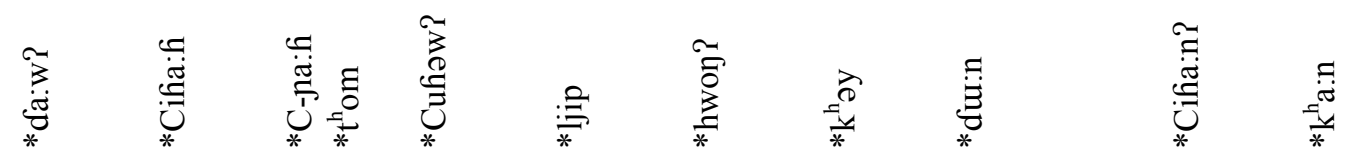

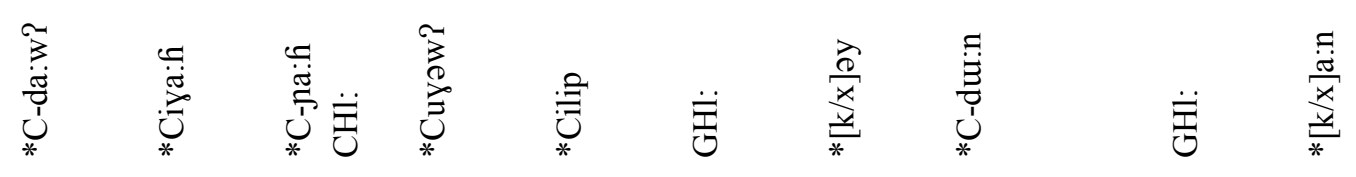

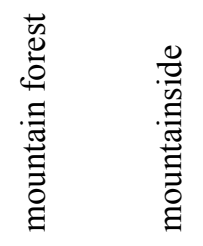

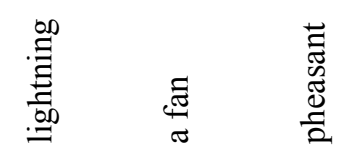

差䇺

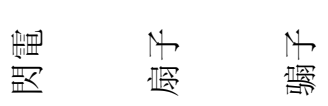

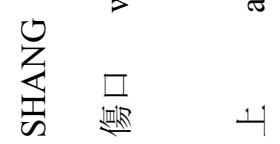


$1+1 \quad 1 \quad 1 \quad 1010$

|

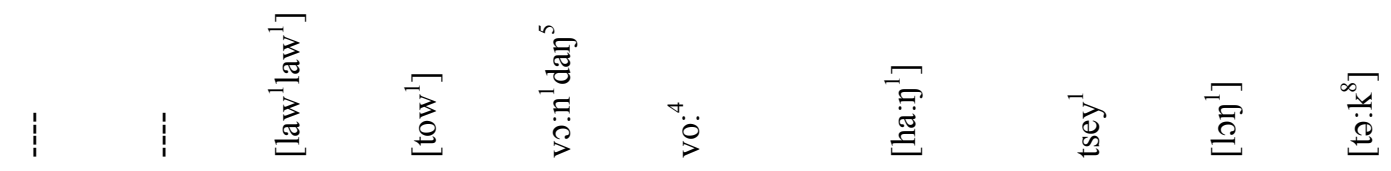

|

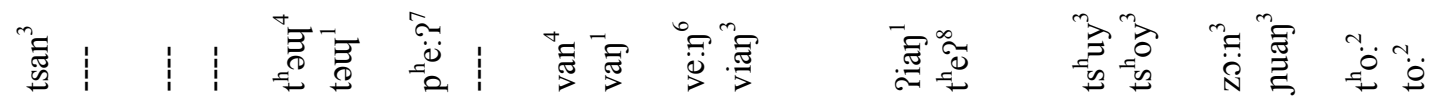

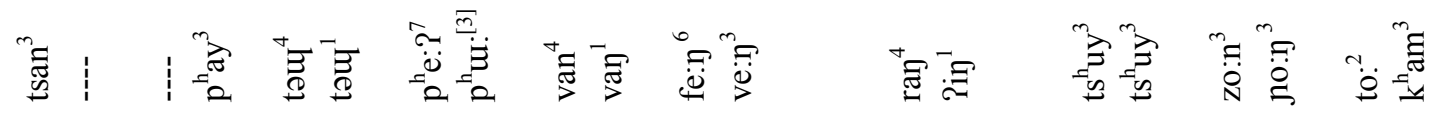

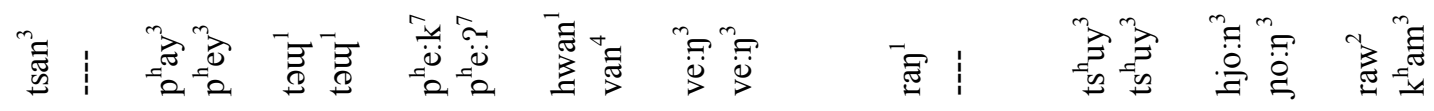

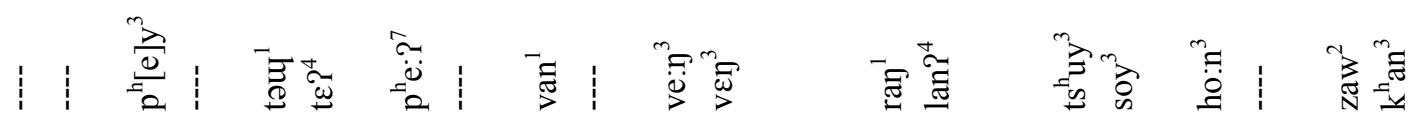

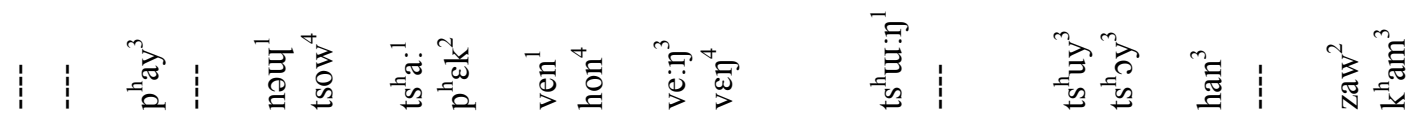

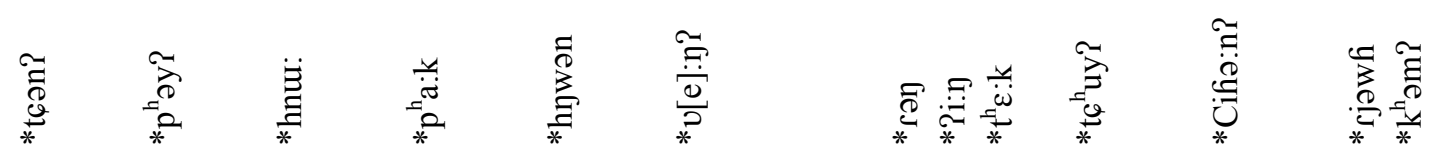

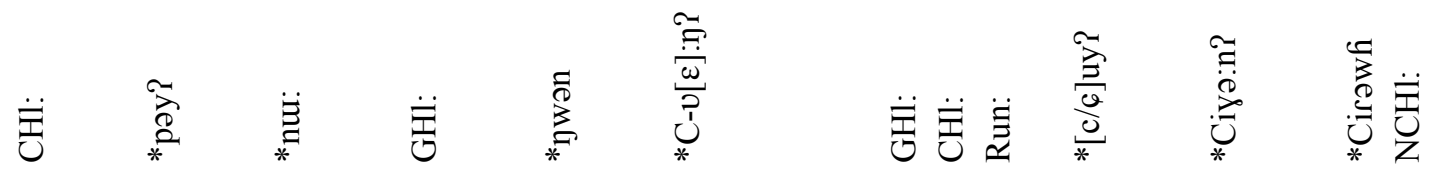

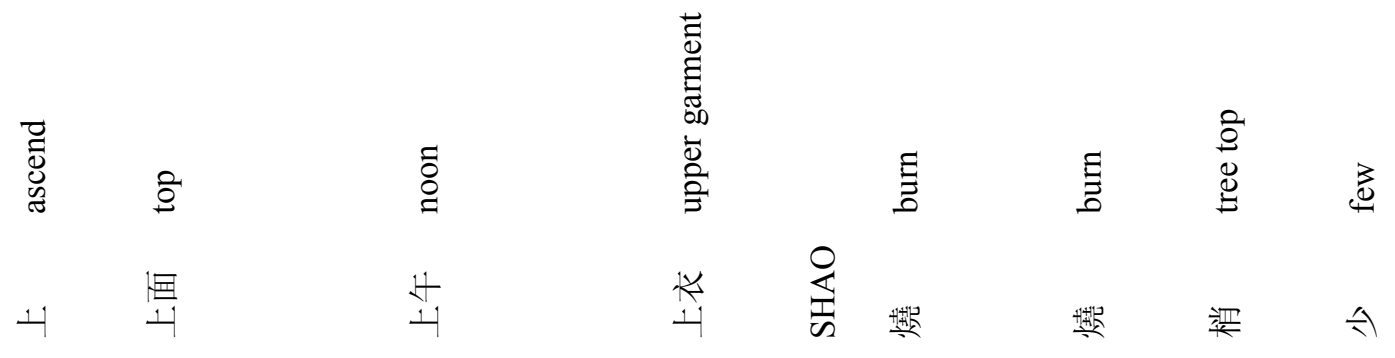




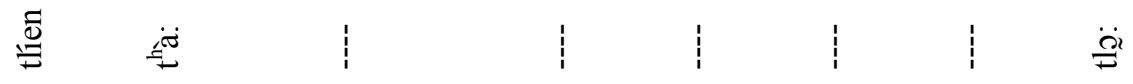

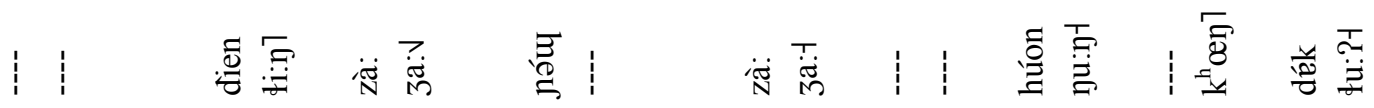

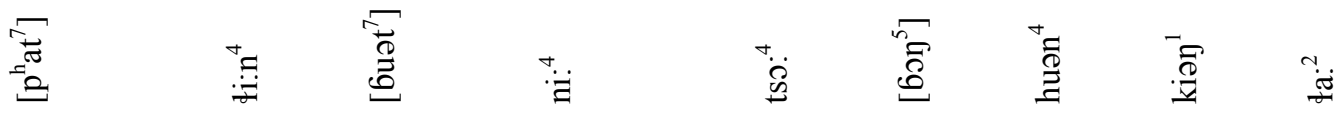

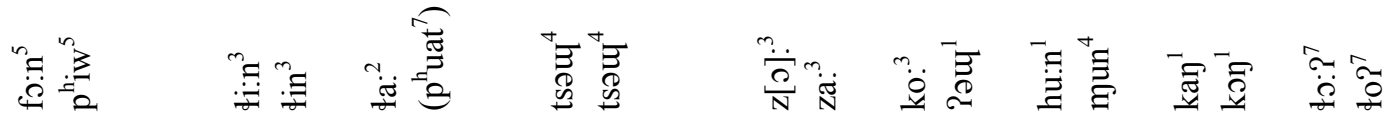

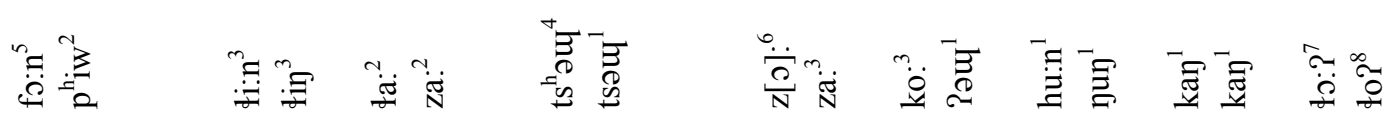

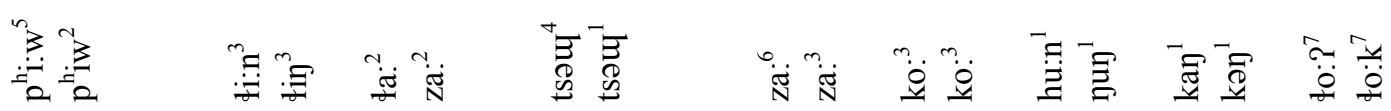

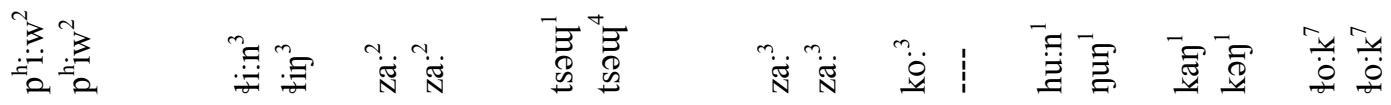

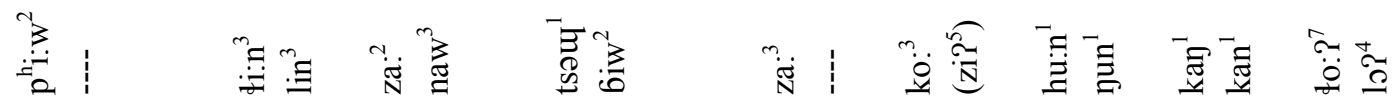

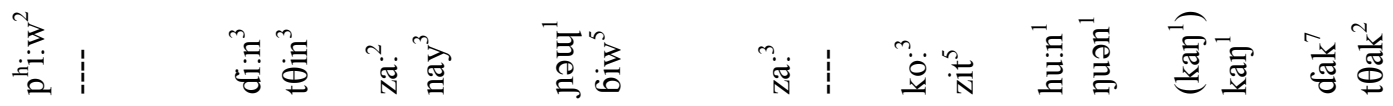

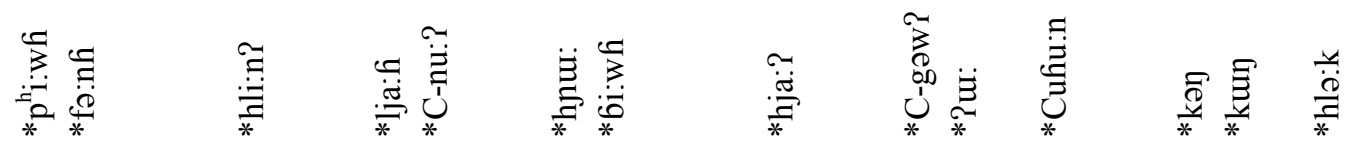

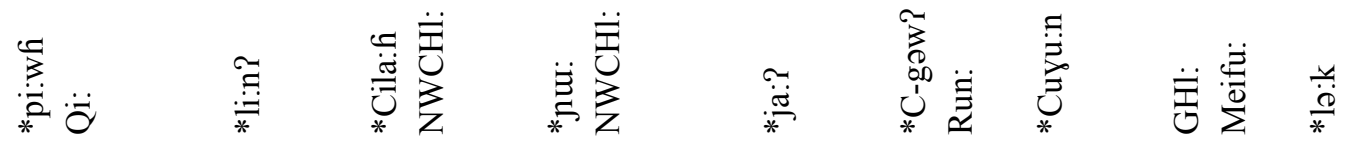

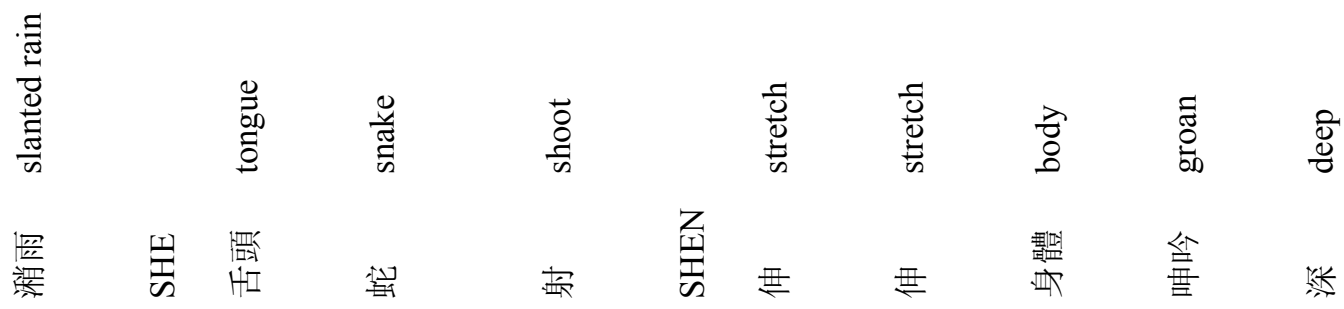




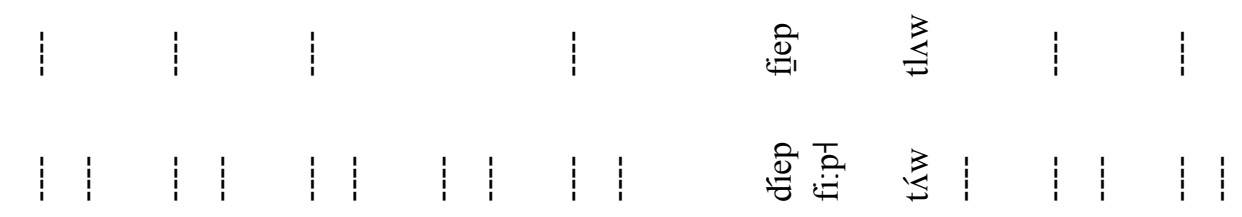

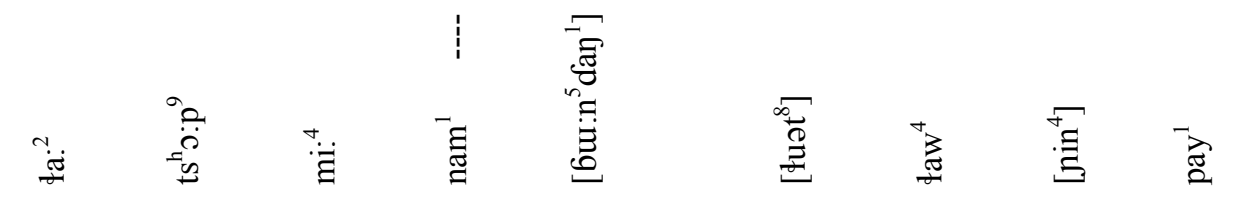

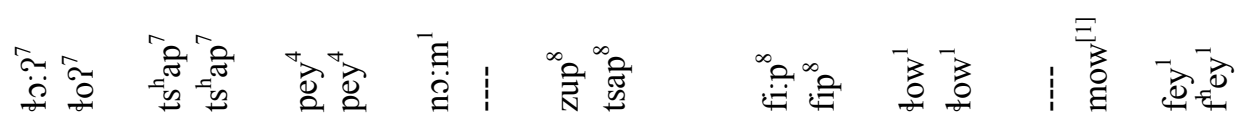

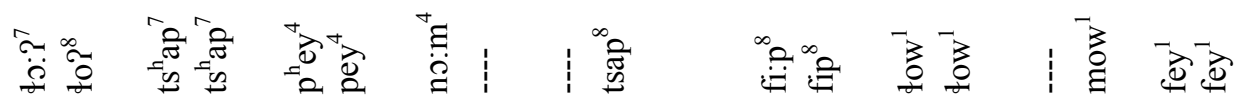

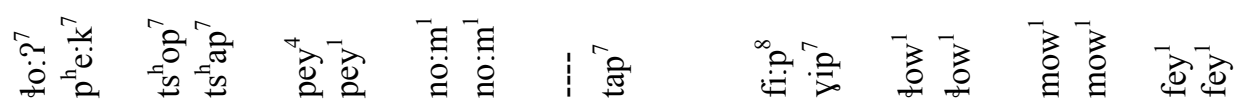

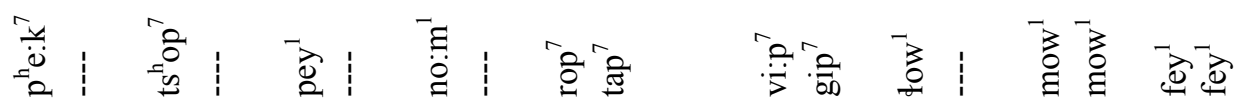

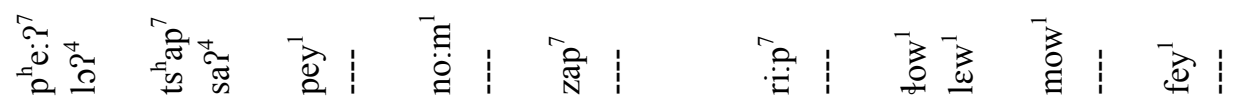

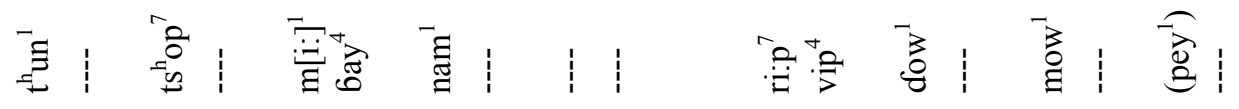

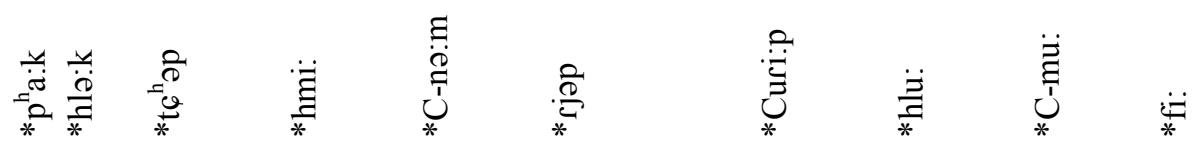

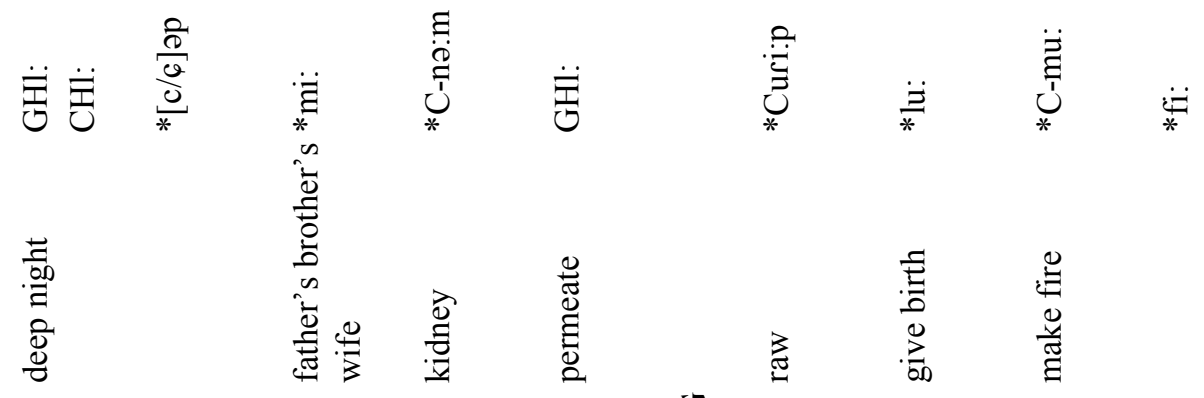

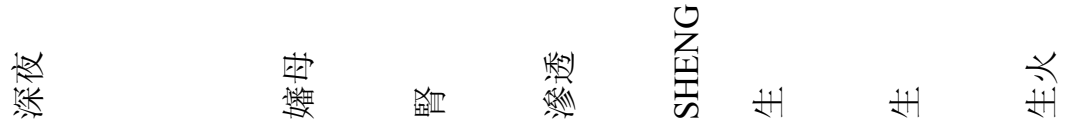


$1+1+1 \quad 1 \quad 1 \quad 1 \quad 1$

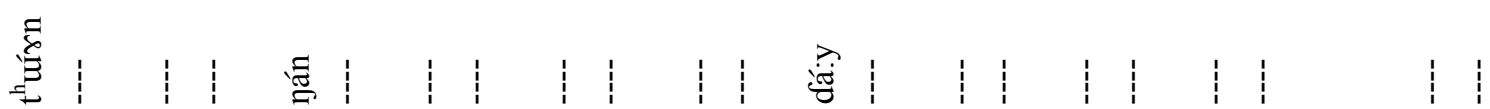

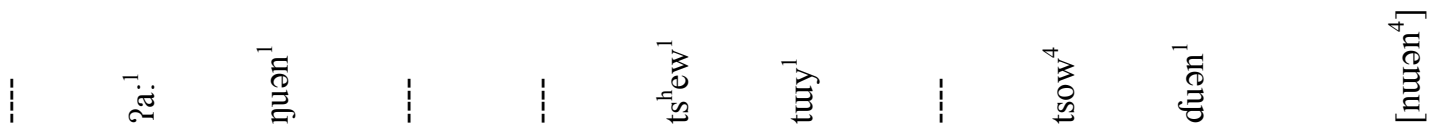

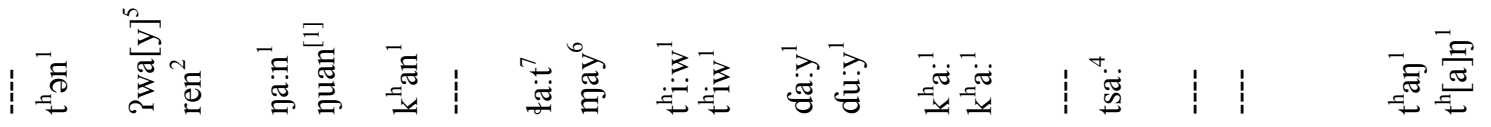

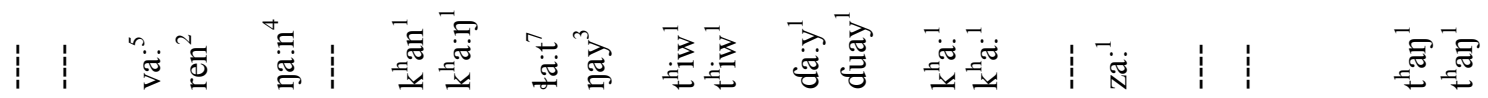

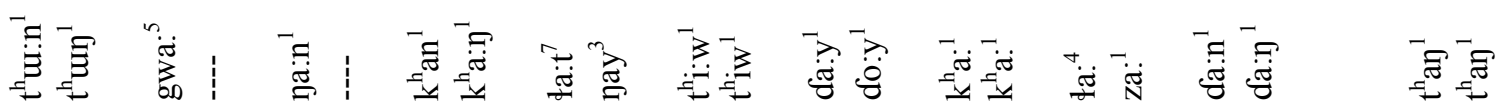

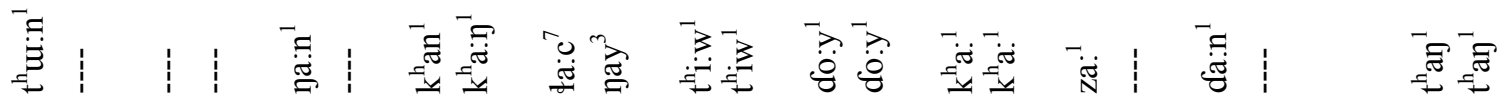

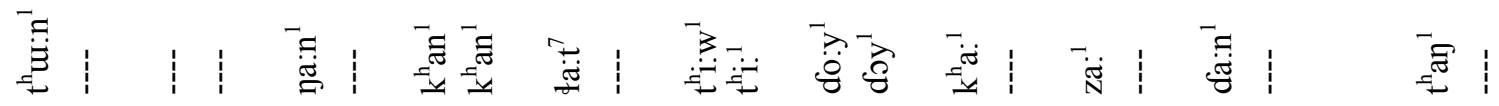

苛 $\quad$ |

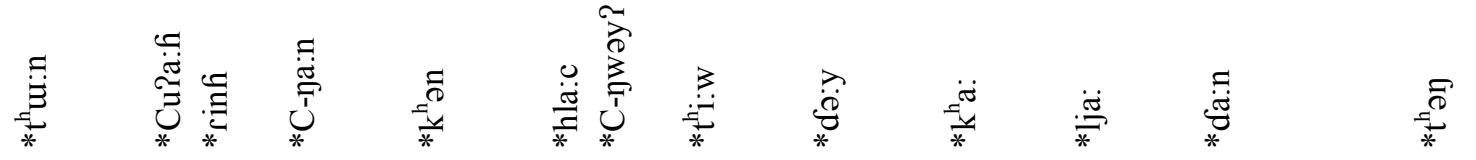

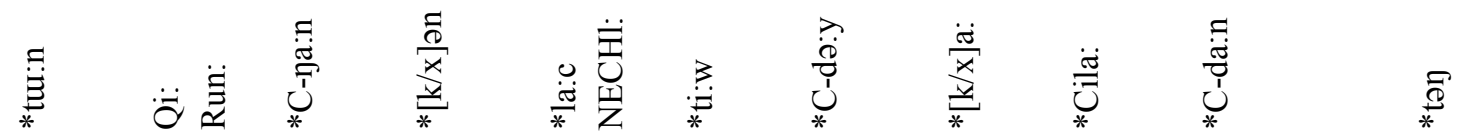

突

莱
$\stackrel{\square}{\Xi}$

焰

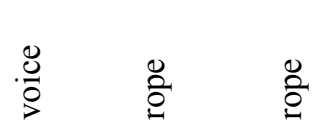

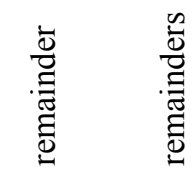

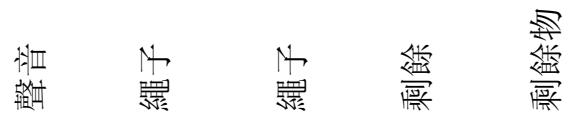

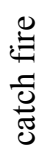

茫 获 


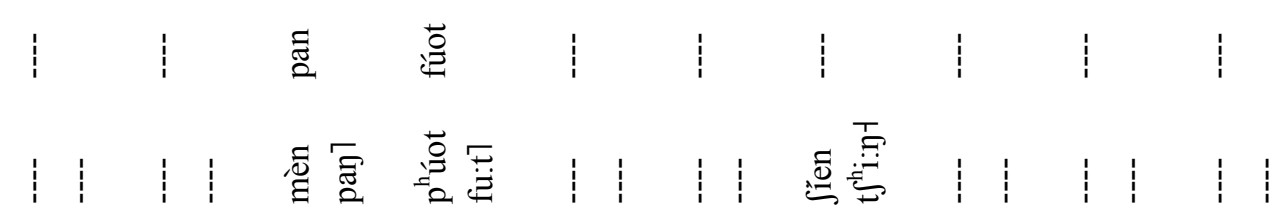

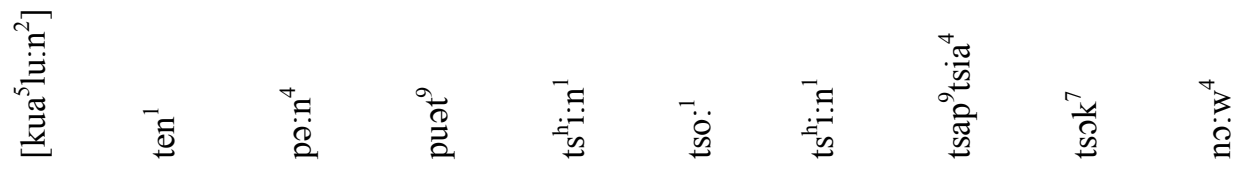

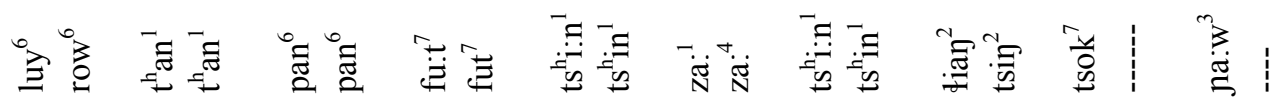

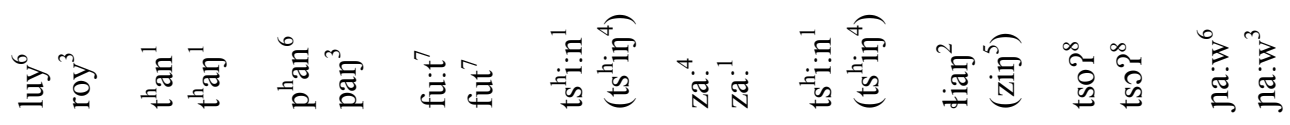

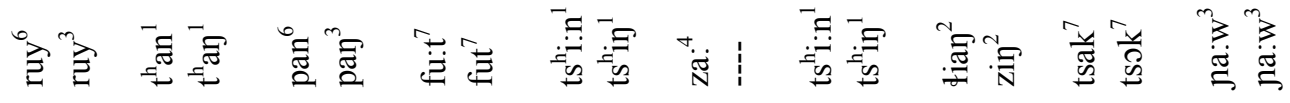

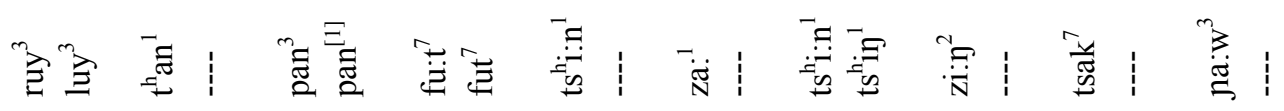

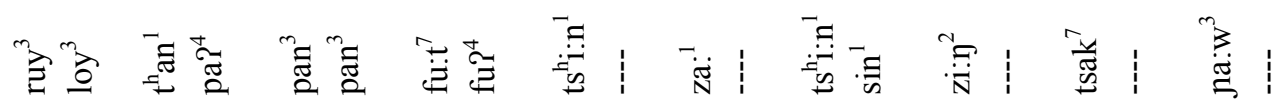

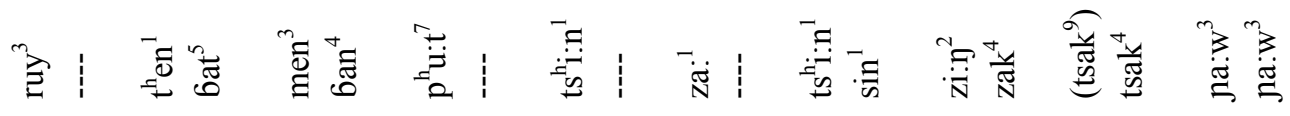

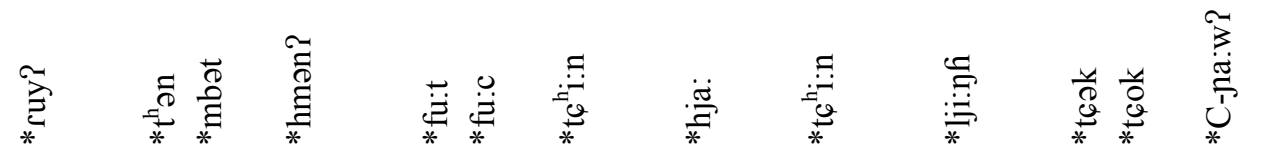

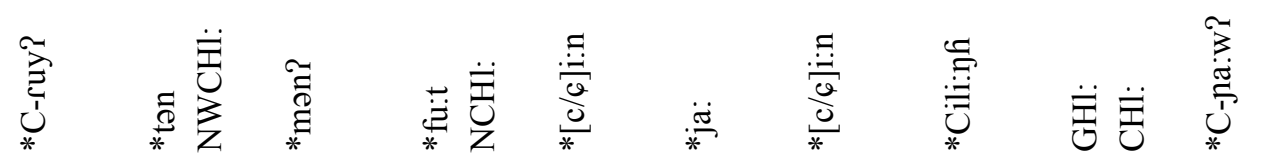
眷
高高高

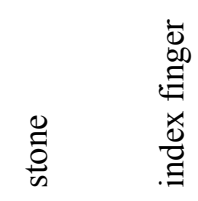

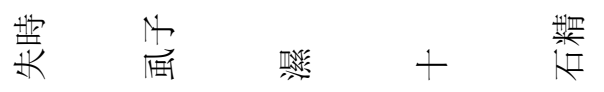
塄照 


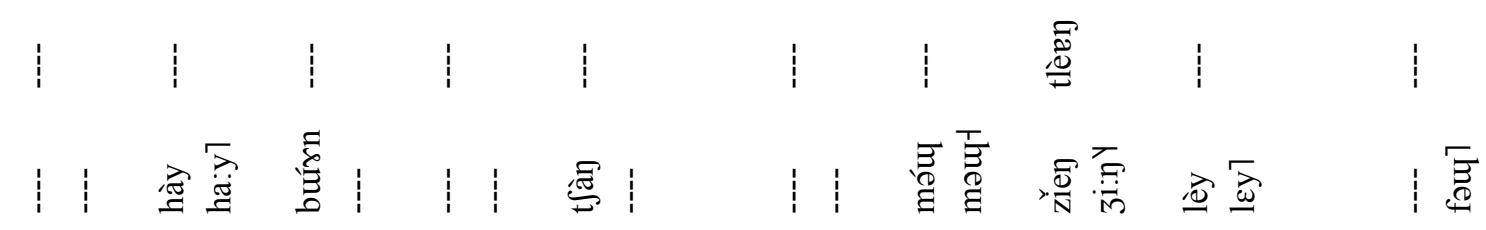

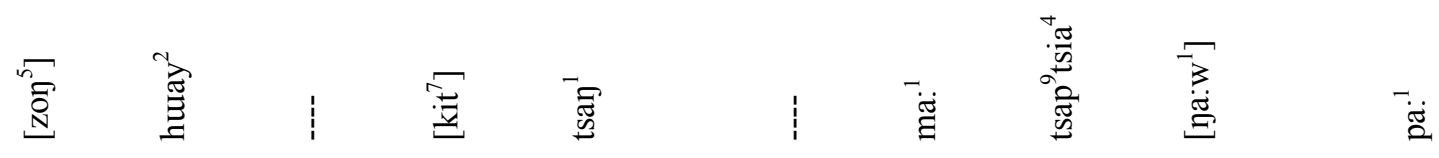

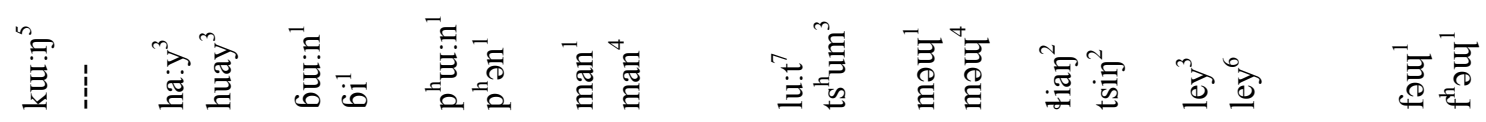

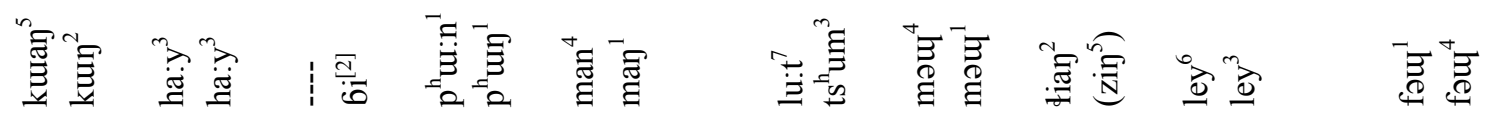

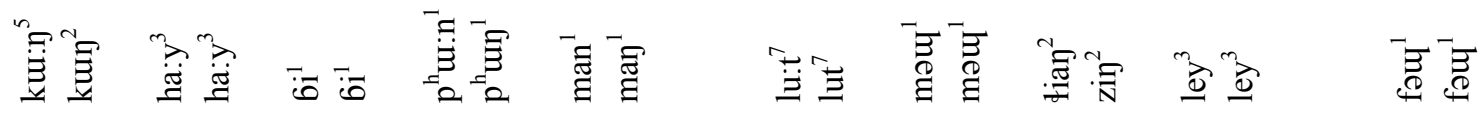

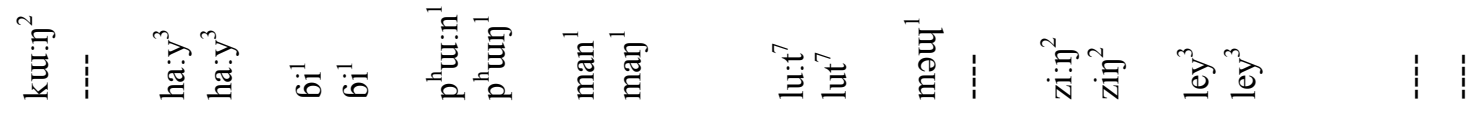

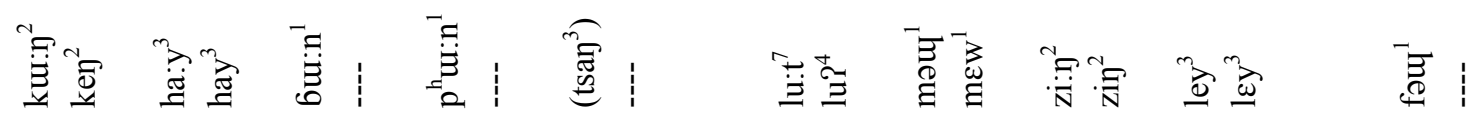

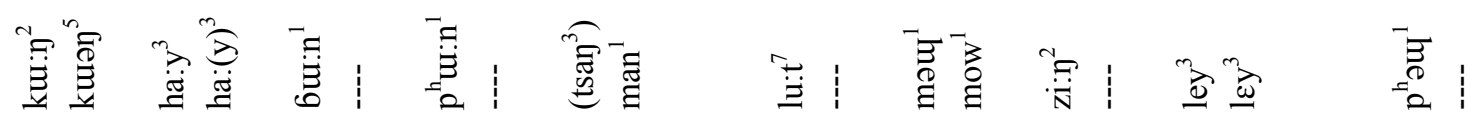

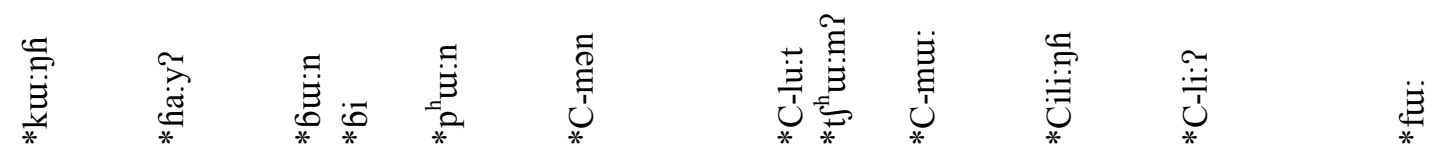

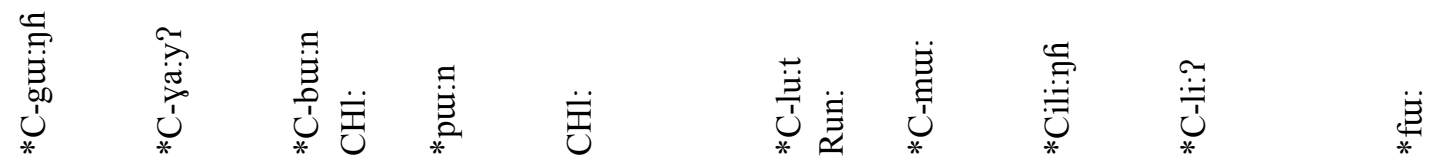

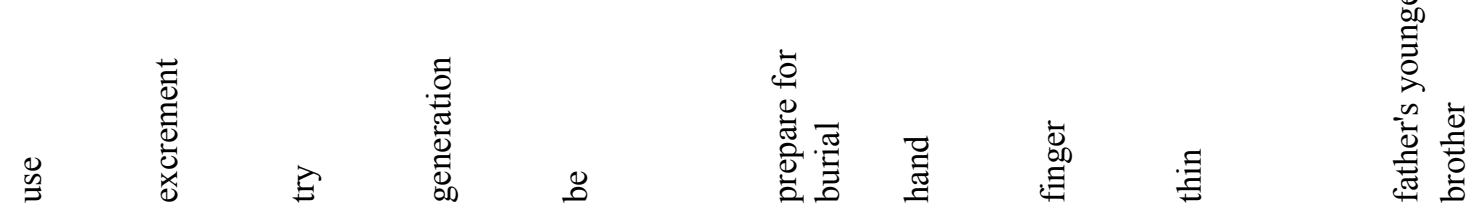

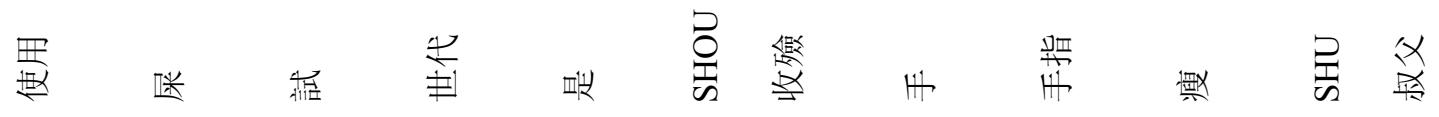




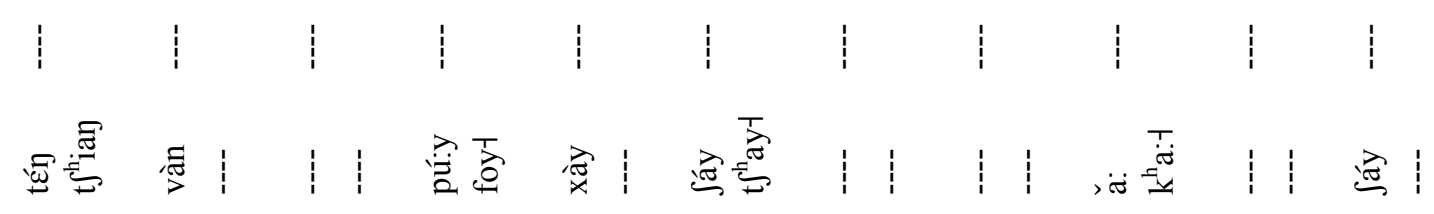

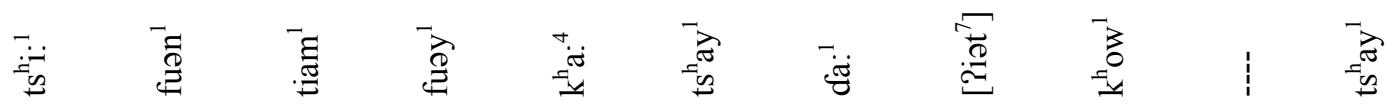

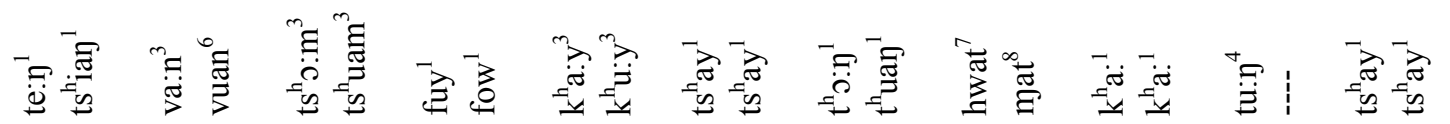

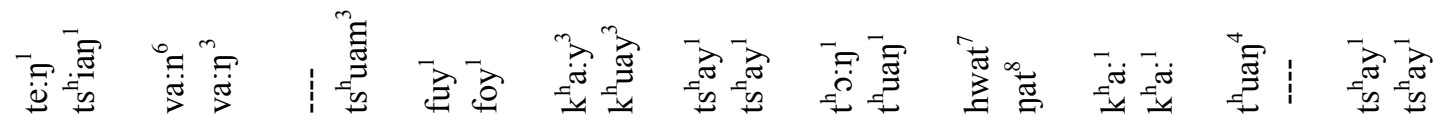

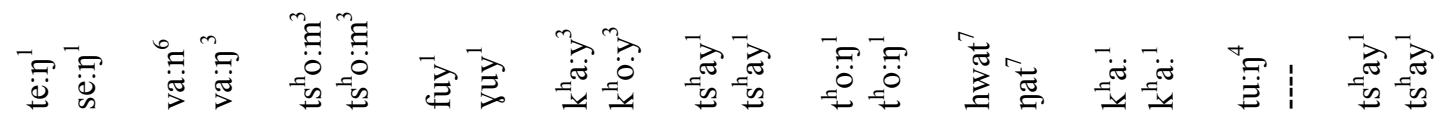

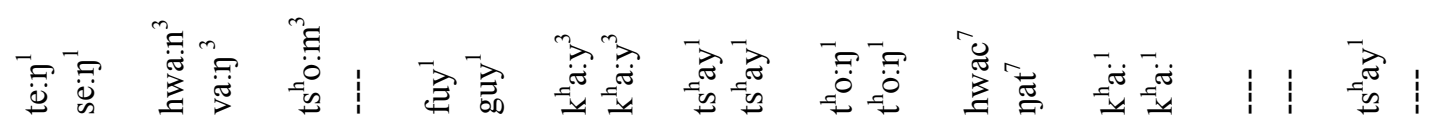

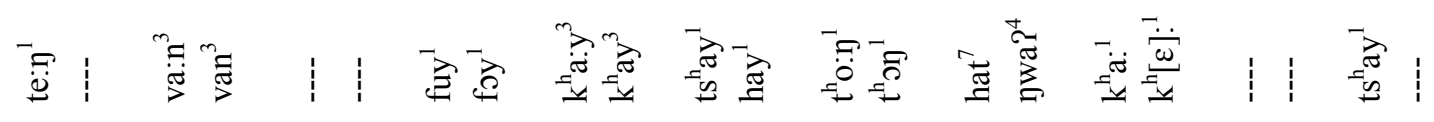

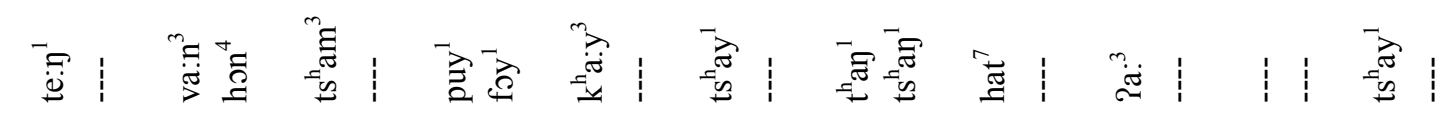

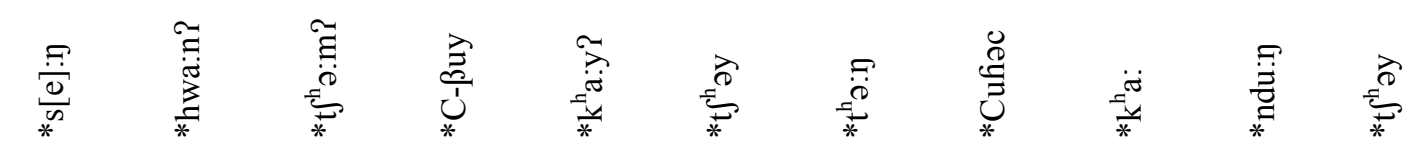

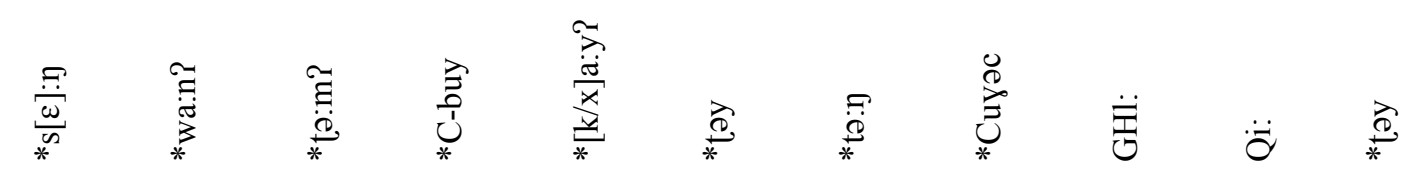

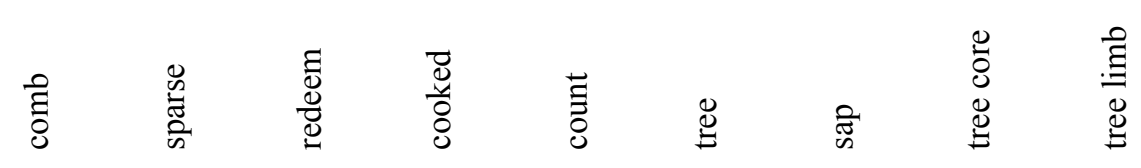

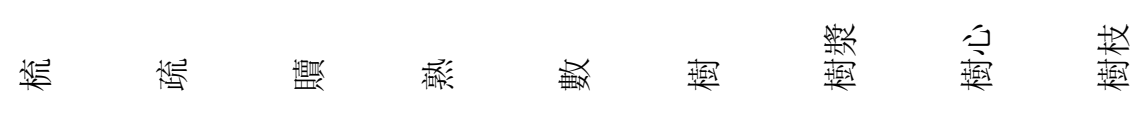




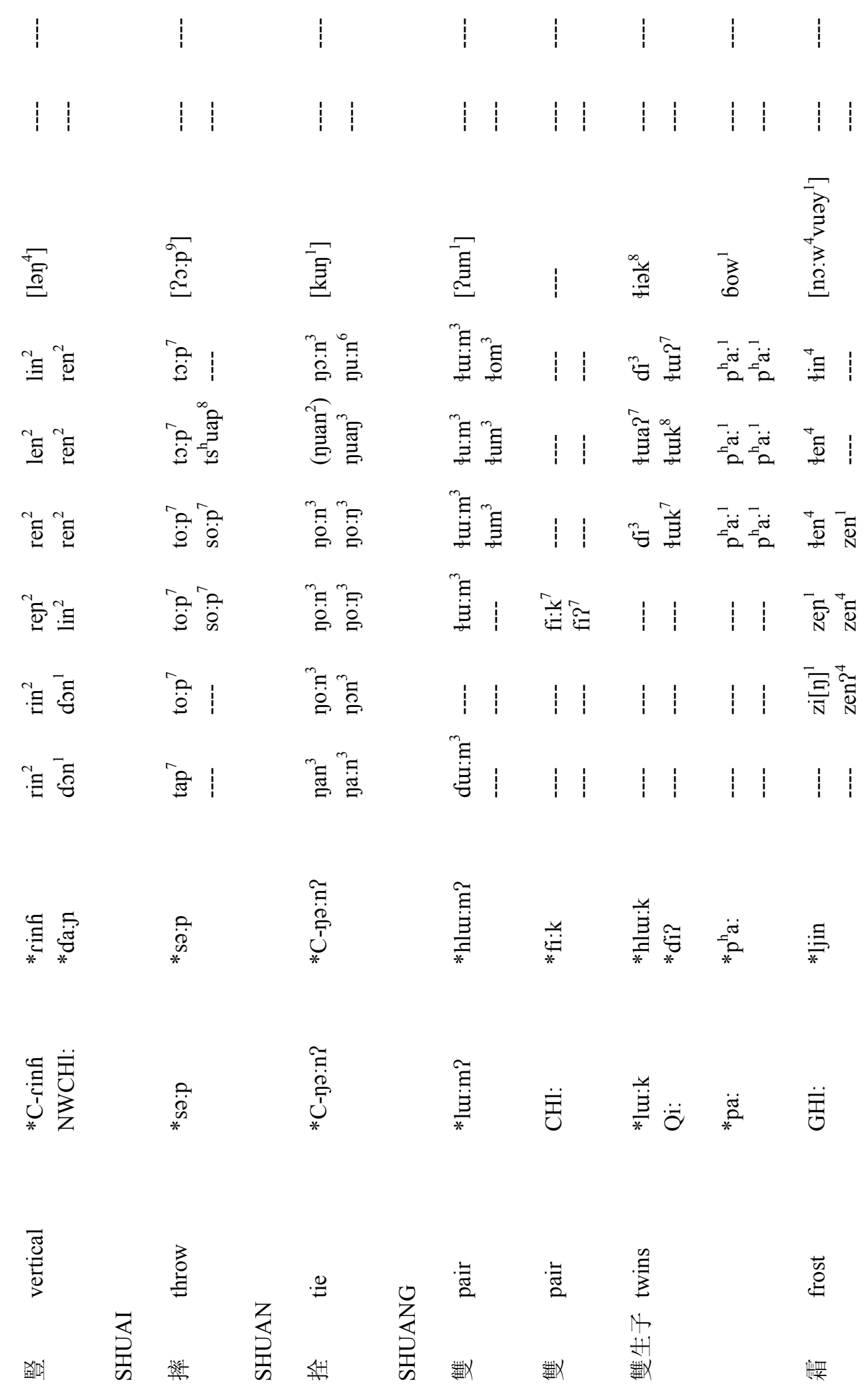


$1\left|\frac{1}{9}\right| 1|1| 1 \mid$

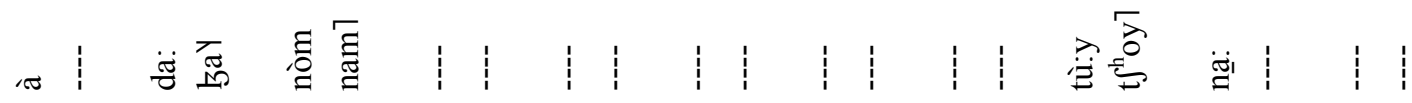

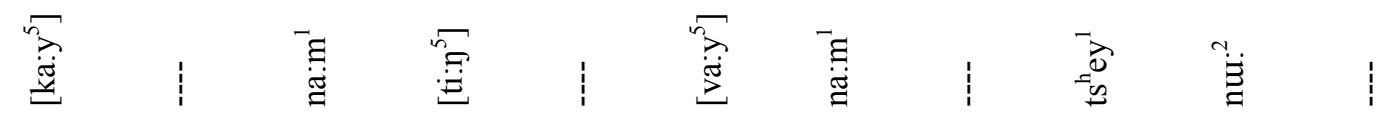

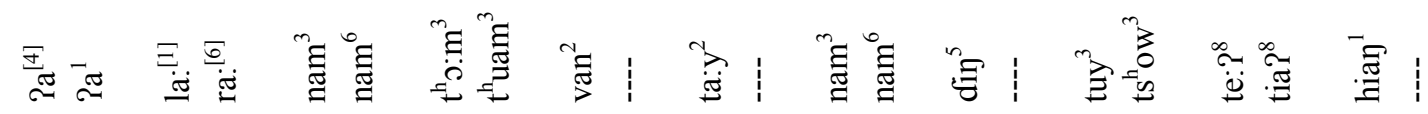

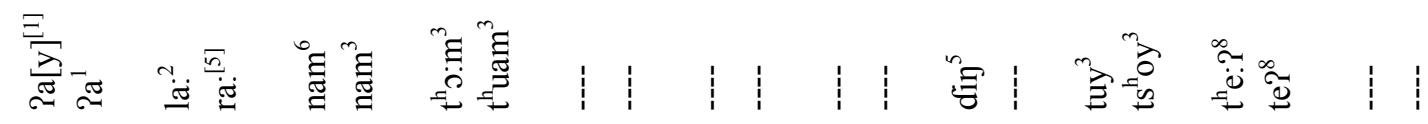

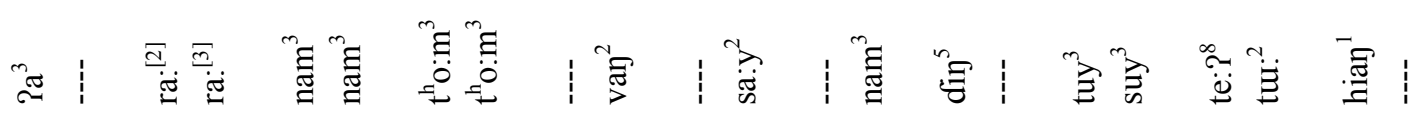

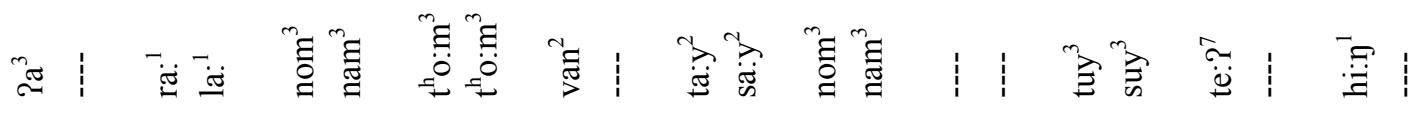

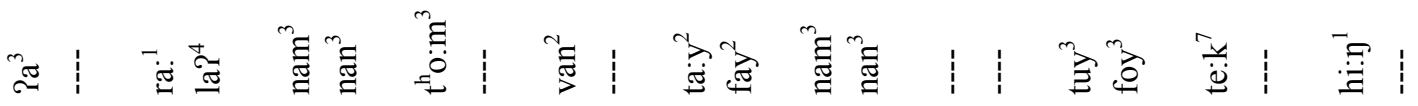

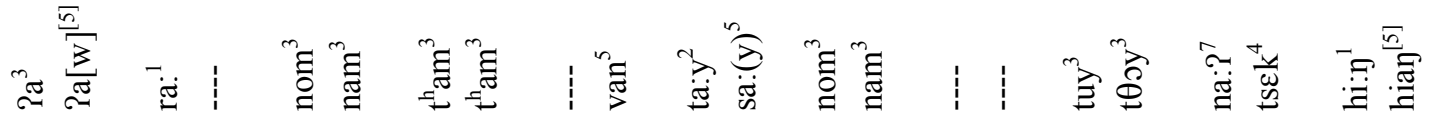

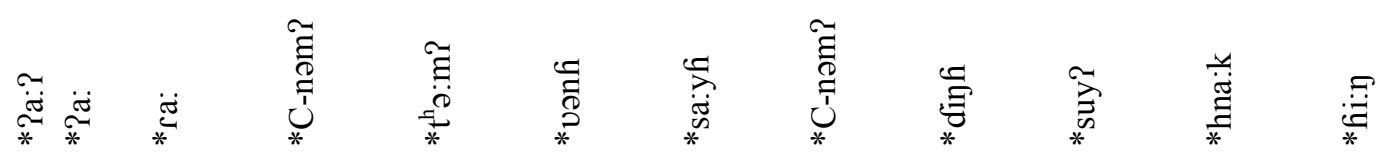

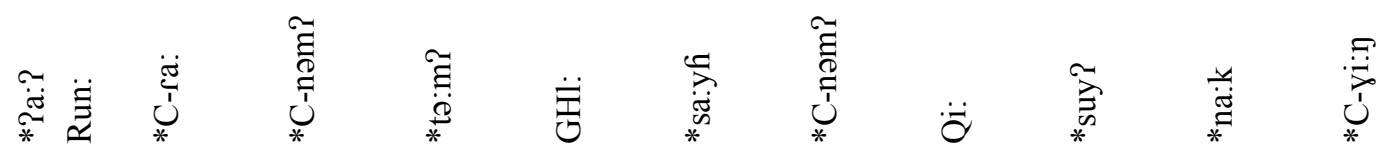
$\stackrel{\circ}{\frac{2}{3}}$

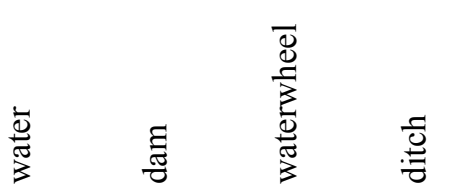

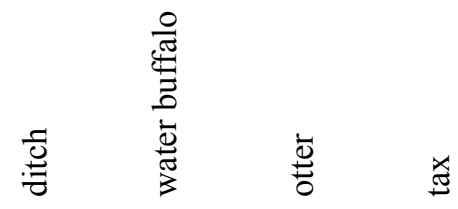

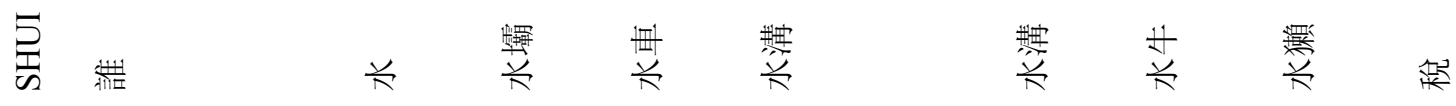




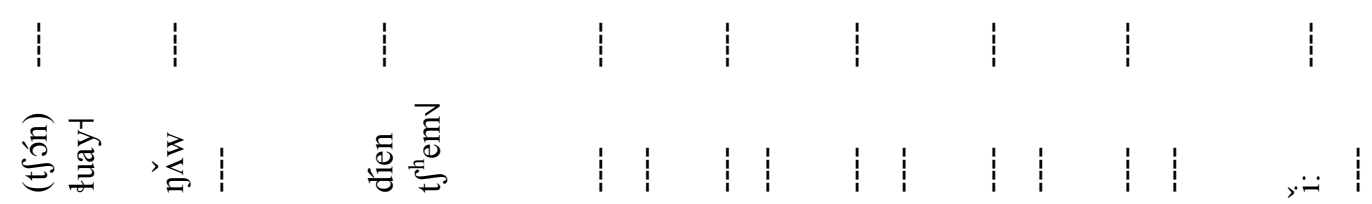

\begin{tabular}{|c|c|c|c|c|c|}
\hline 弟 & 羪 & 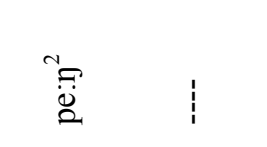 & 豆 & $\frac{\infty}{\underline{p}}$ & \\
\hline 的童 & 㕝 & 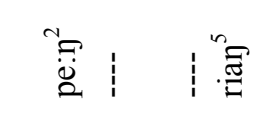 & 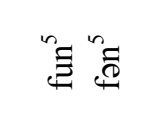 & ชิ & \\
\hline 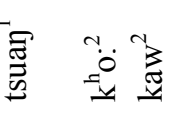 & 讋 & 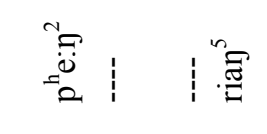 & : & 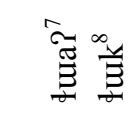 & \\
\hline 量基 & 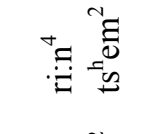 & 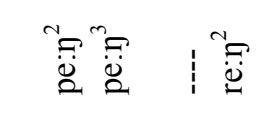 & 管要 & \&章 & \\
\hline 拿拿 & 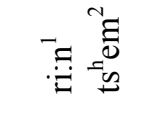 & 要: & & 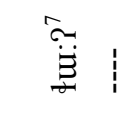 & \\
\hline 䇺拿 & 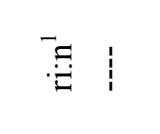 & 覀: 11 & 罢 & 音 & \\
\hline 管 & 到 & 萻: & & 蓆 & \\
\hline
\end{tabular}

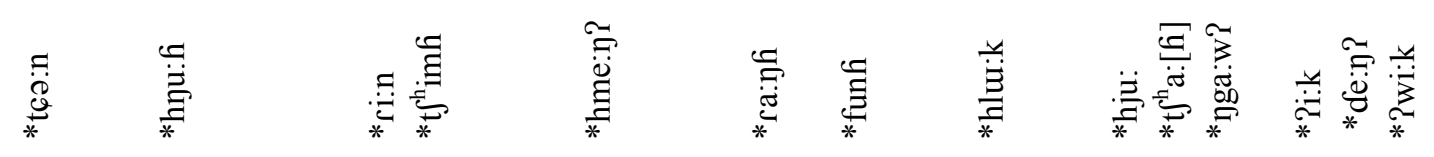

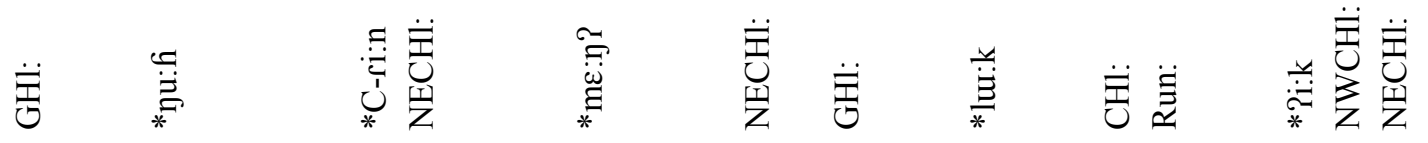

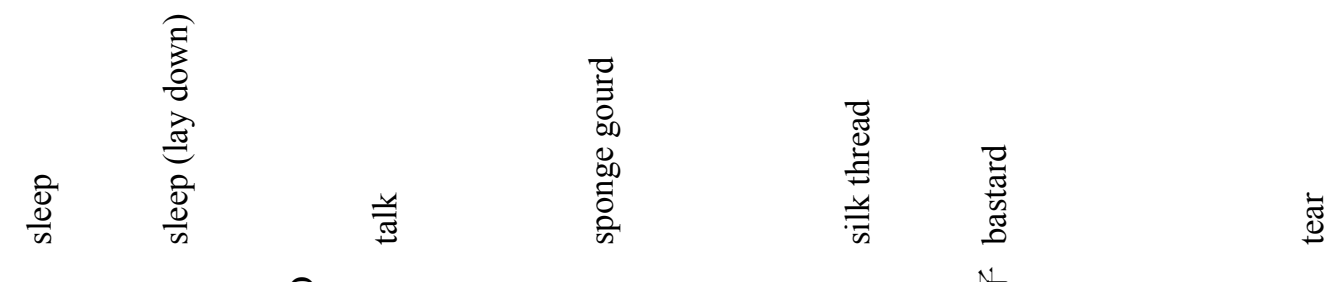

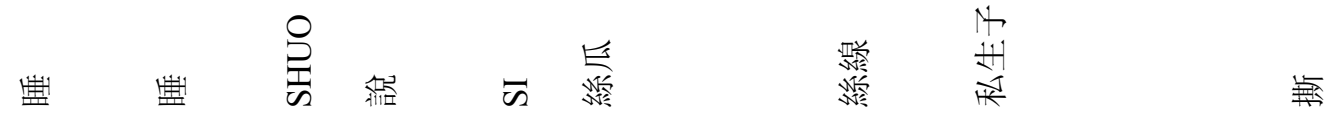




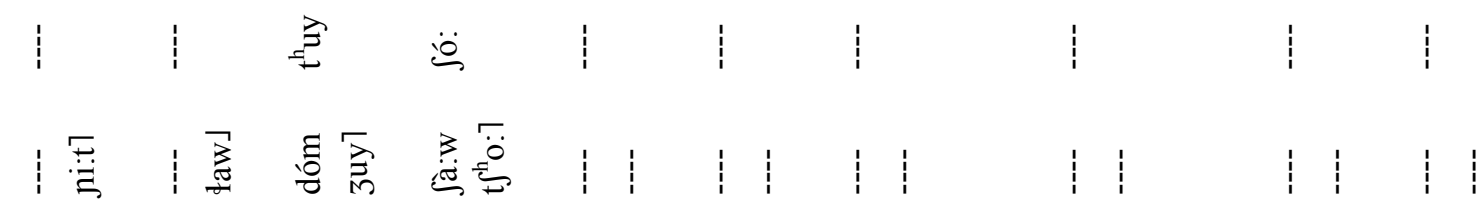

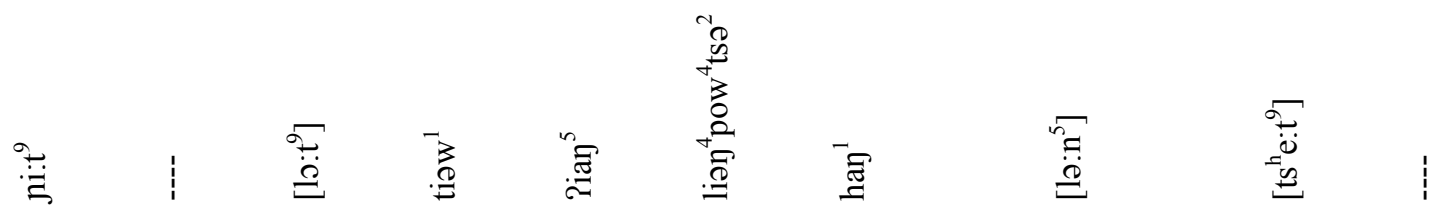

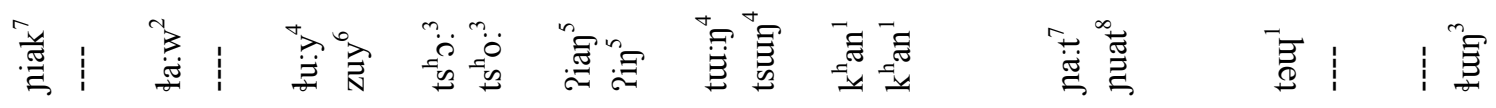

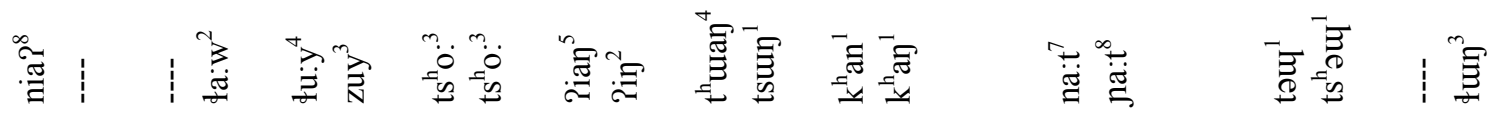

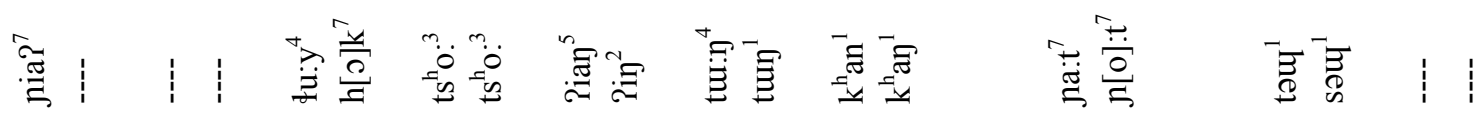

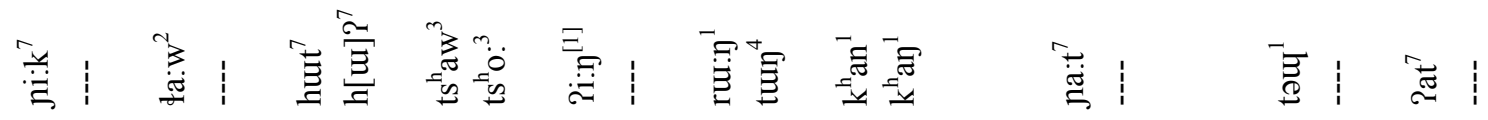

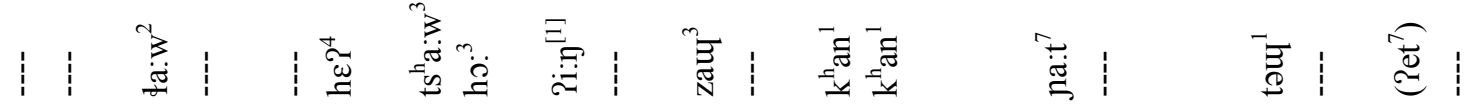

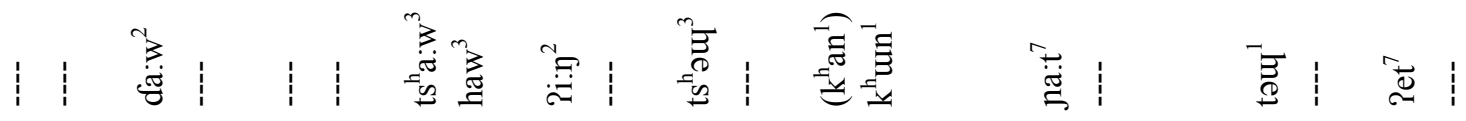

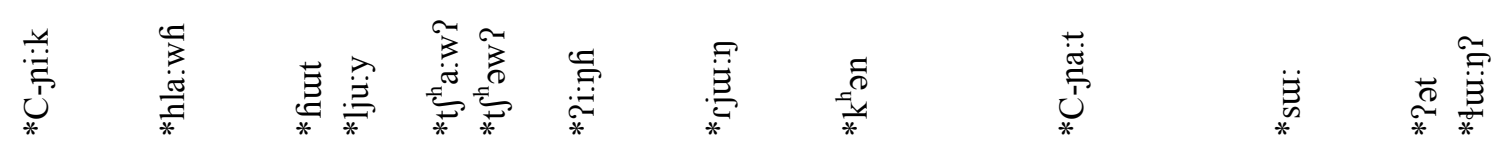

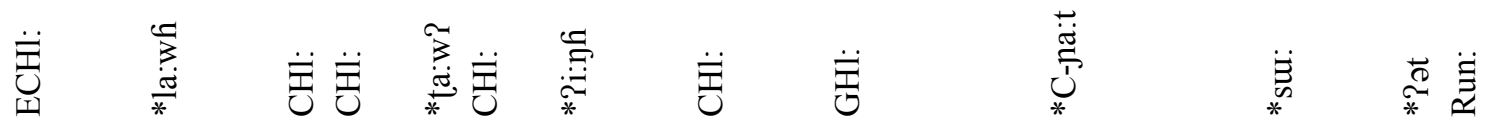

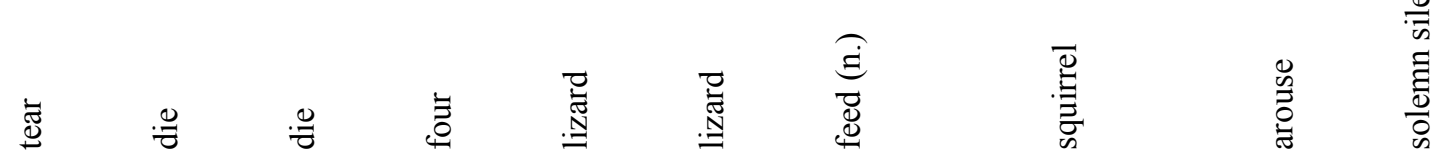

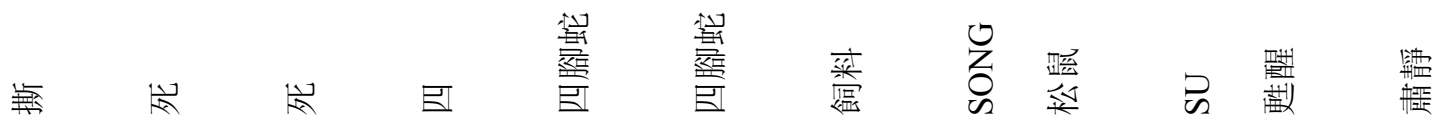




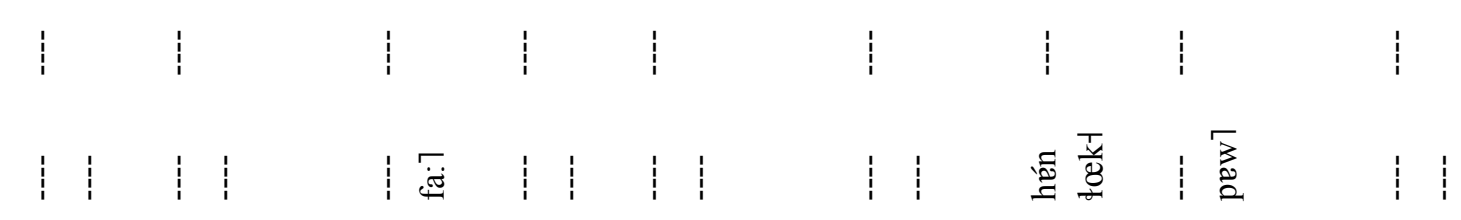

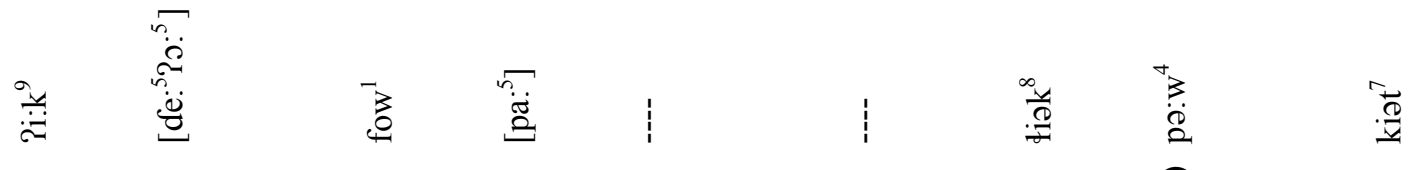

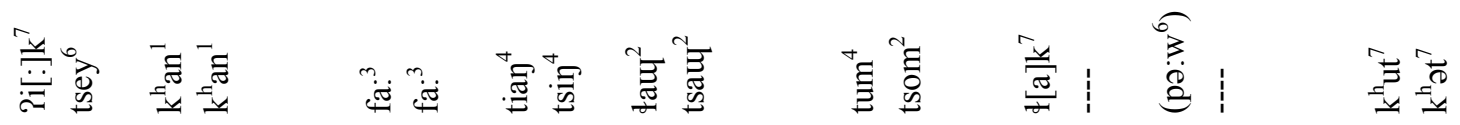

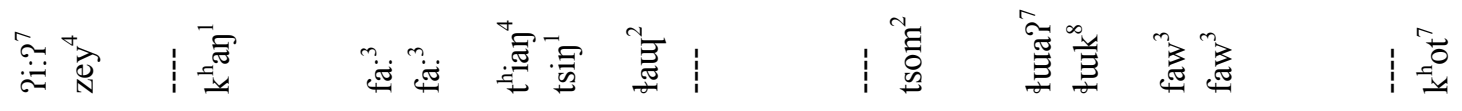

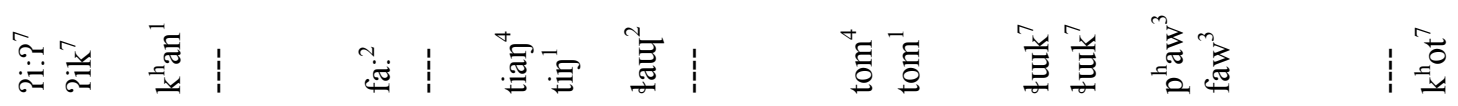

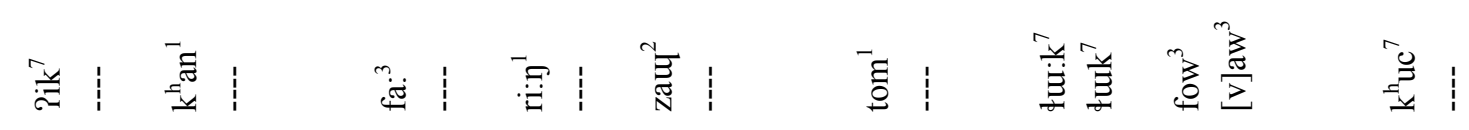

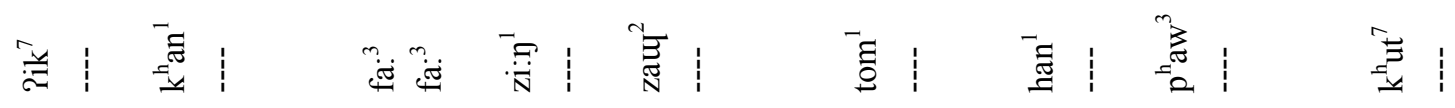

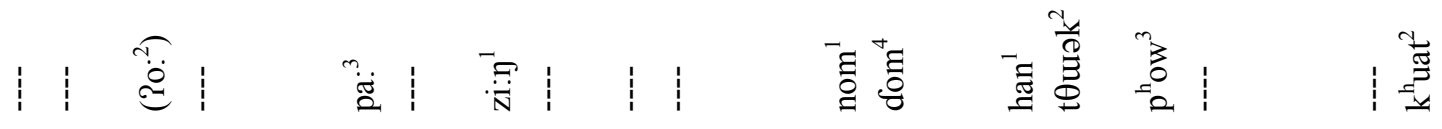

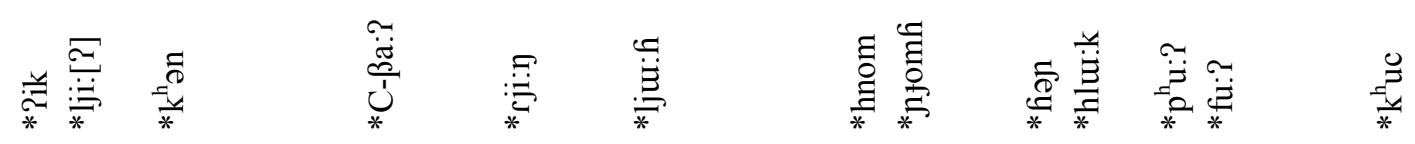

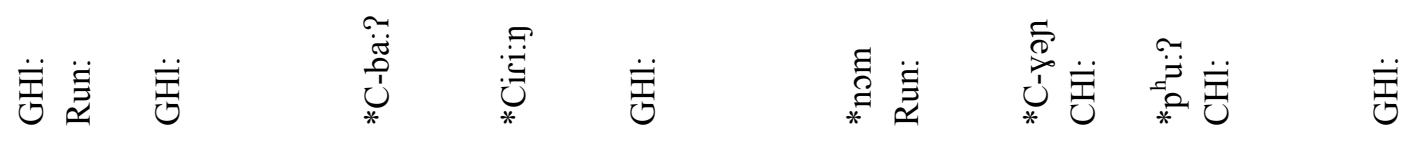

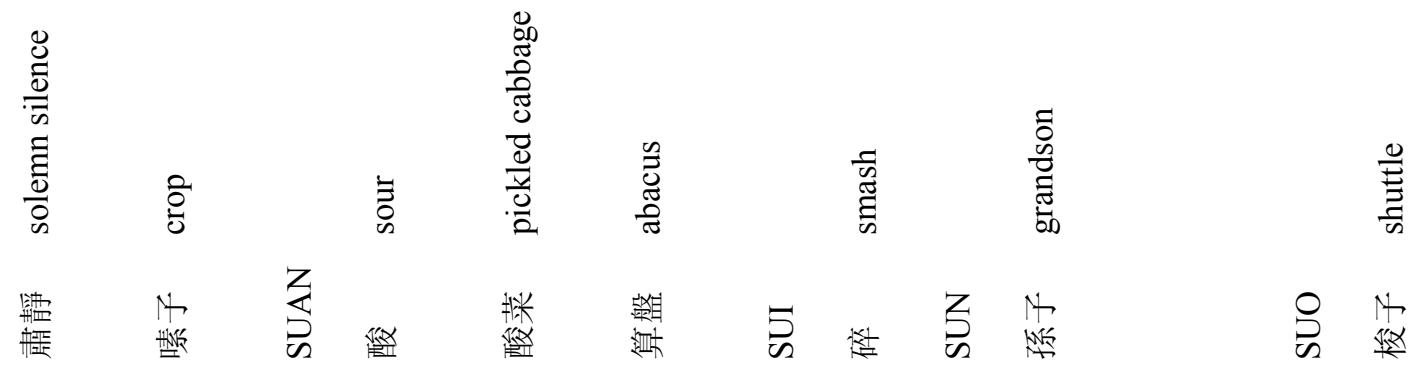




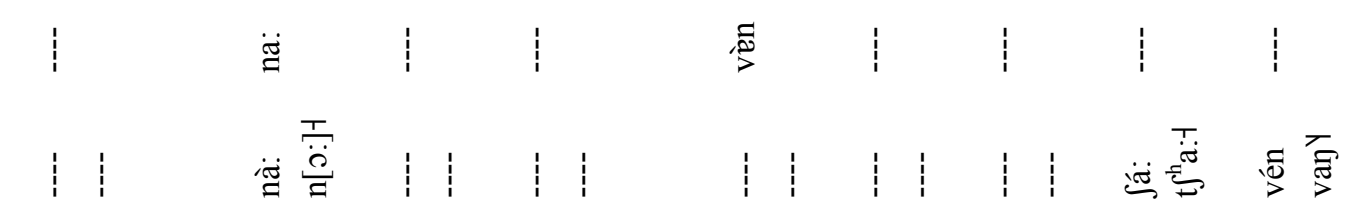

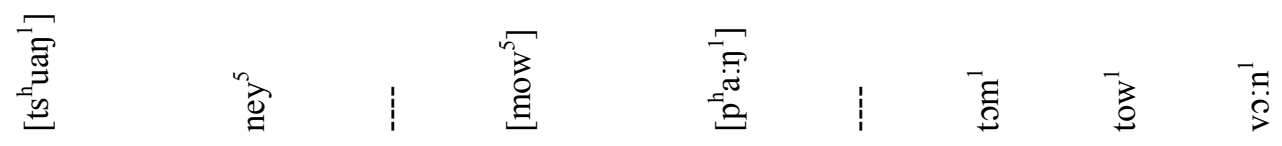

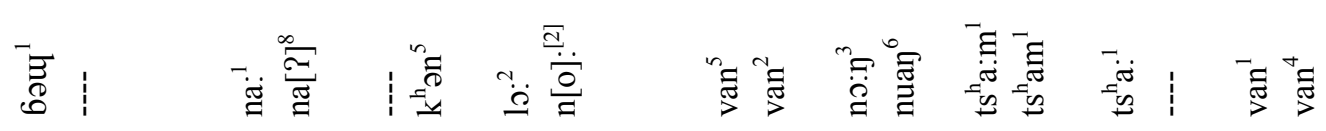

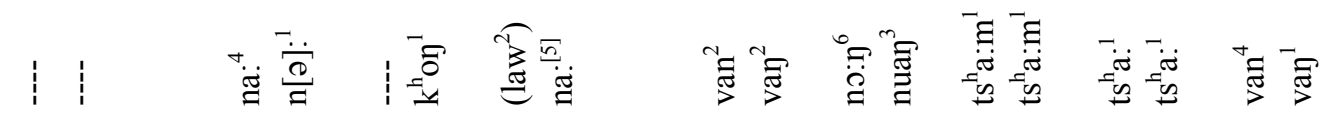

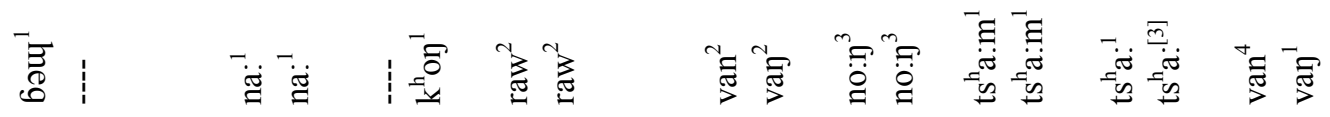

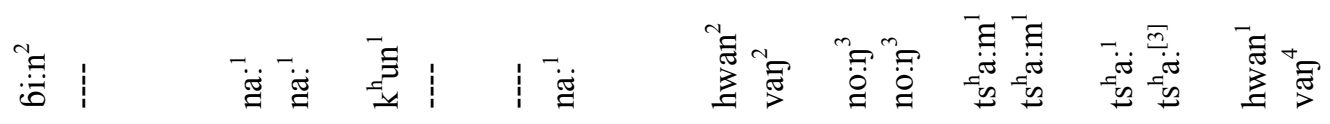

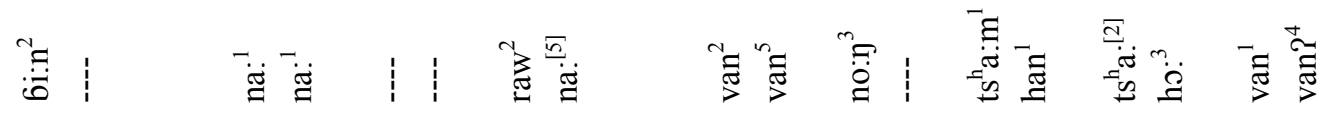

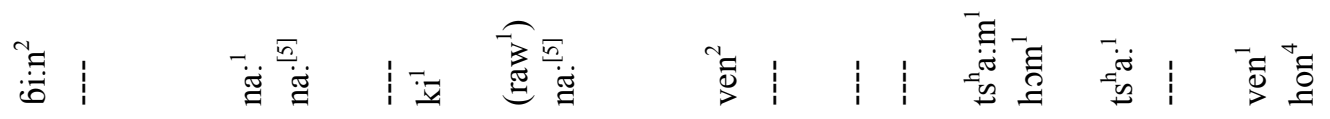

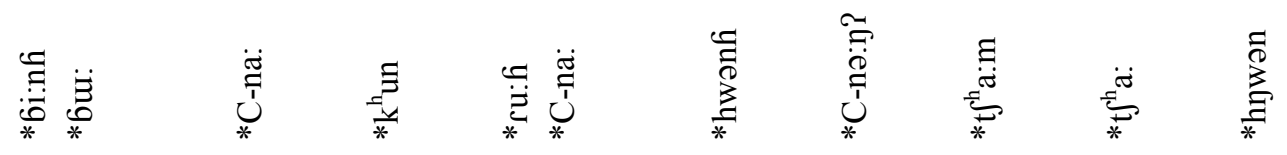

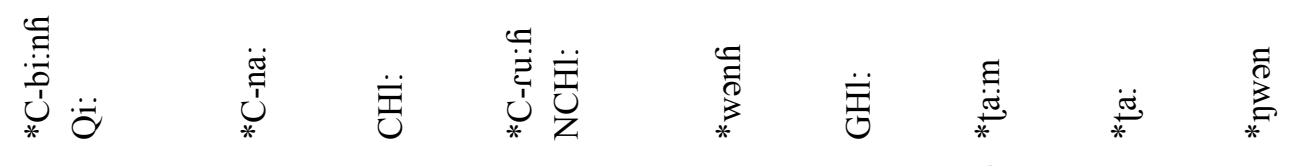

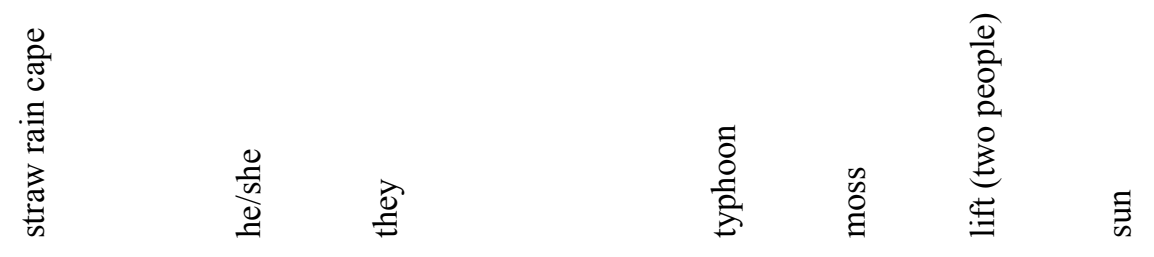

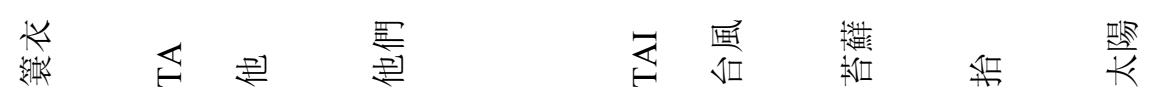


$1+1+11111$

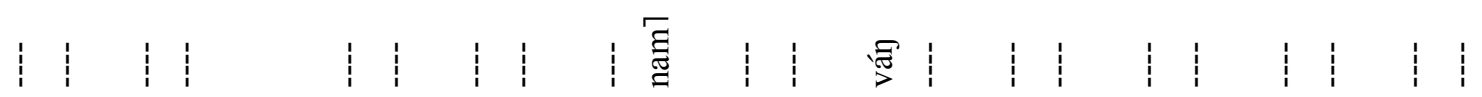

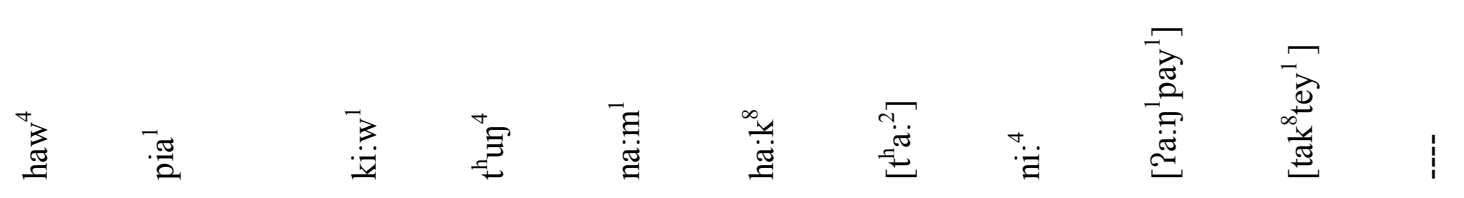

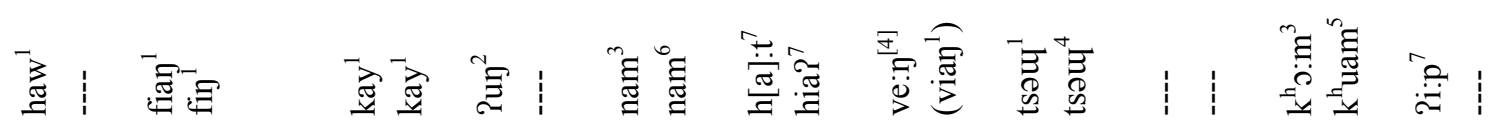

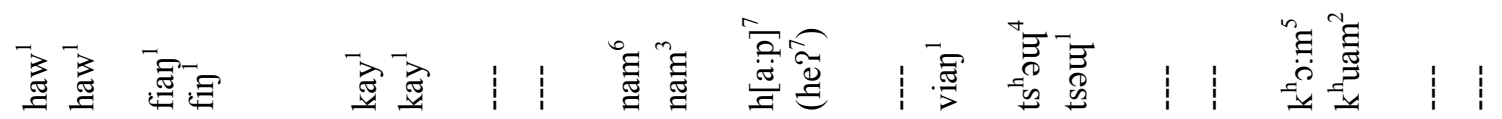

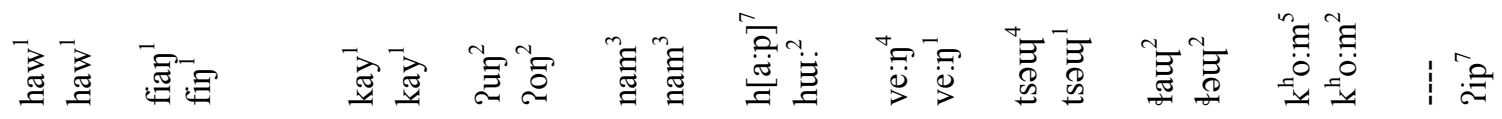

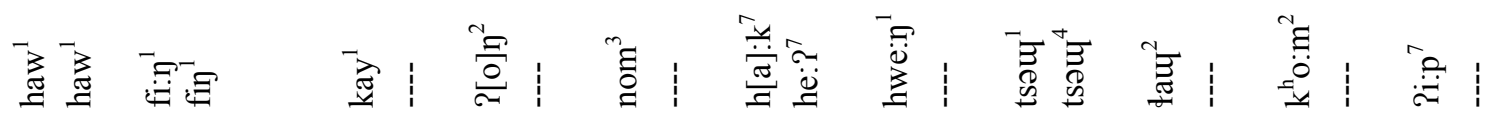

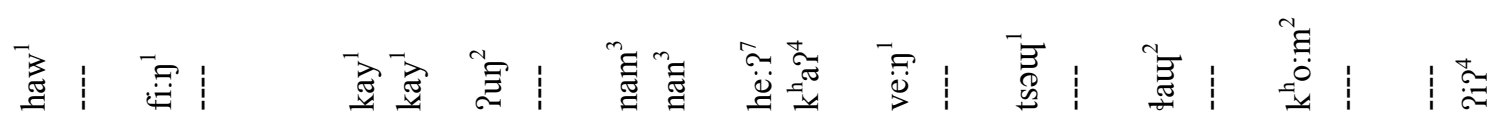

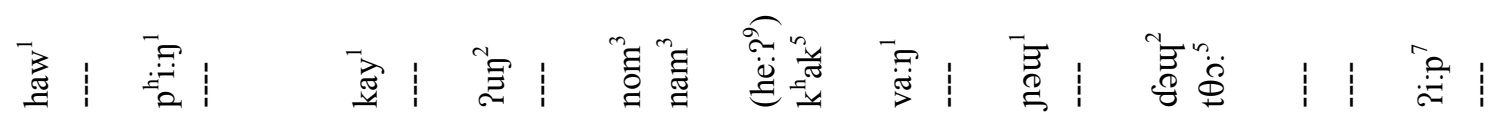

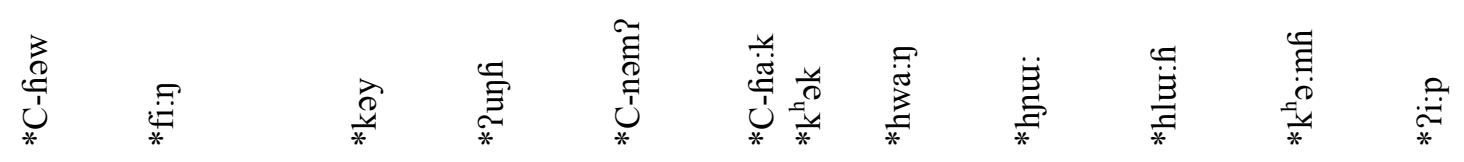

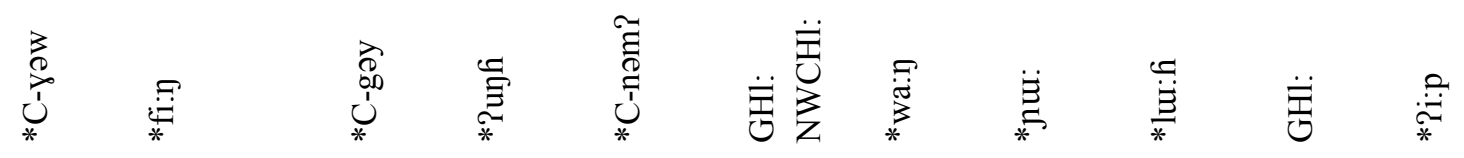

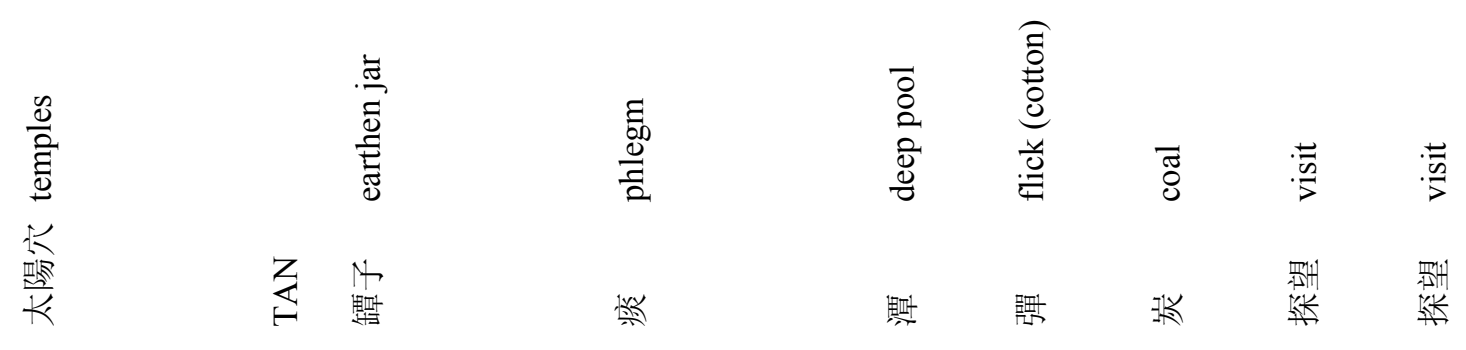




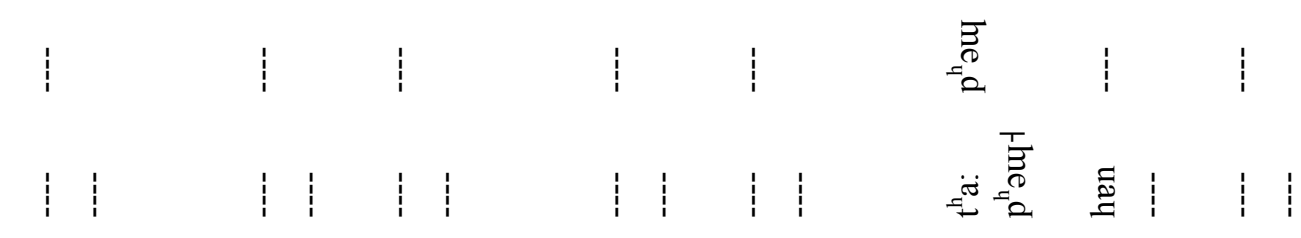

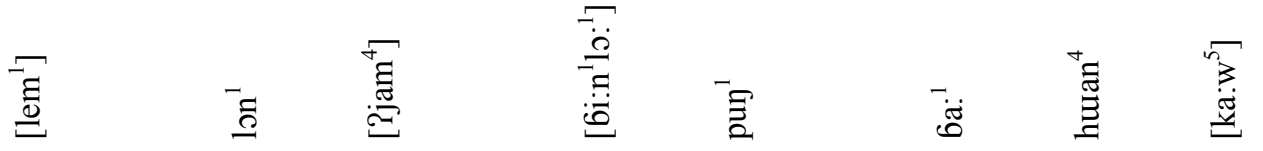

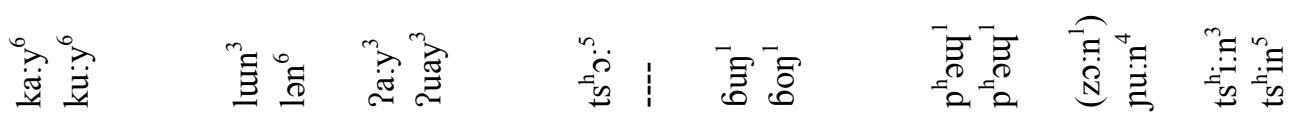

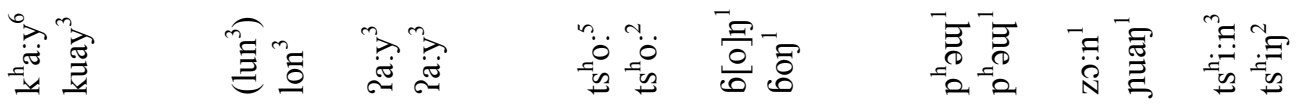

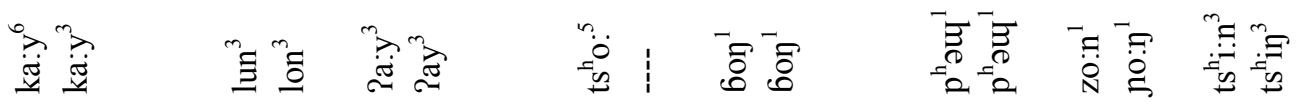

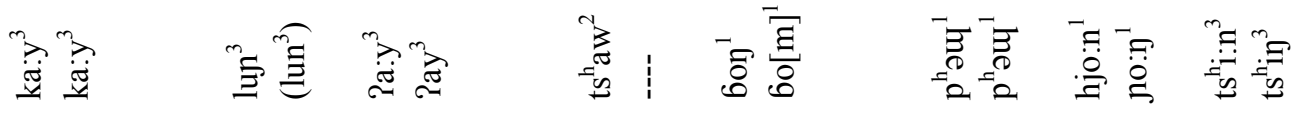

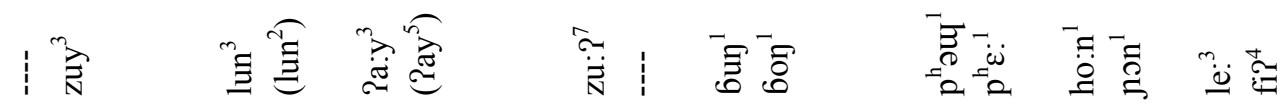

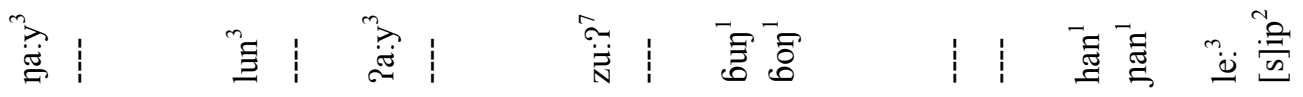

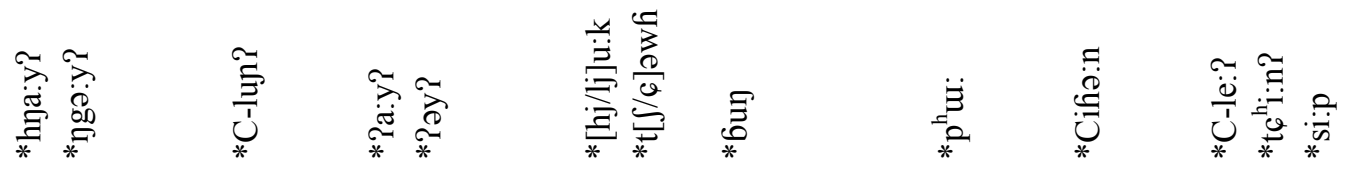

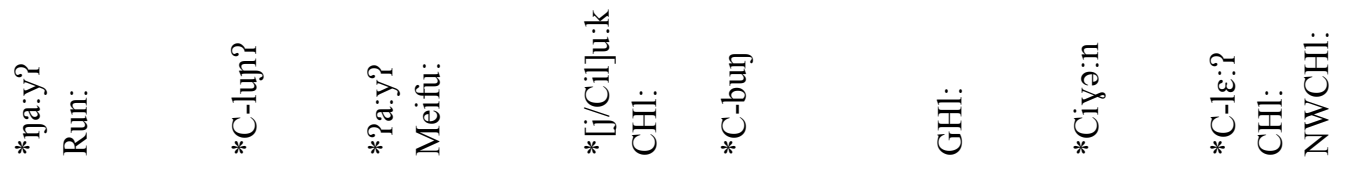

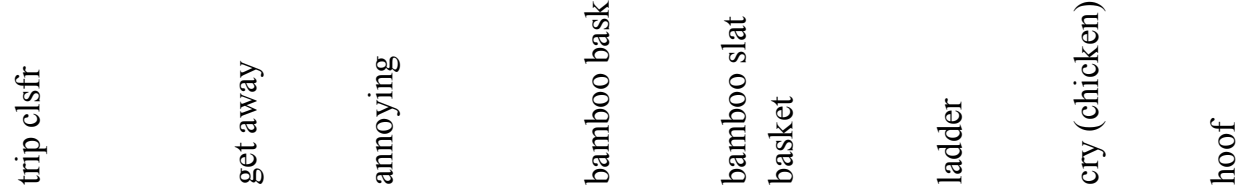

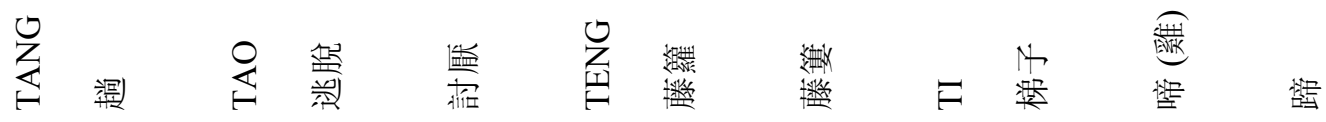




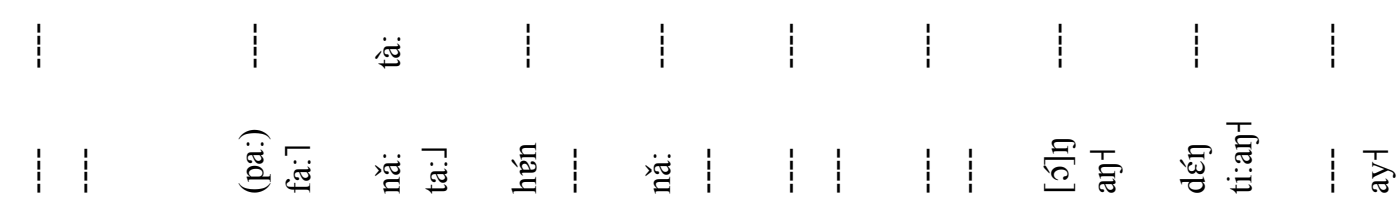

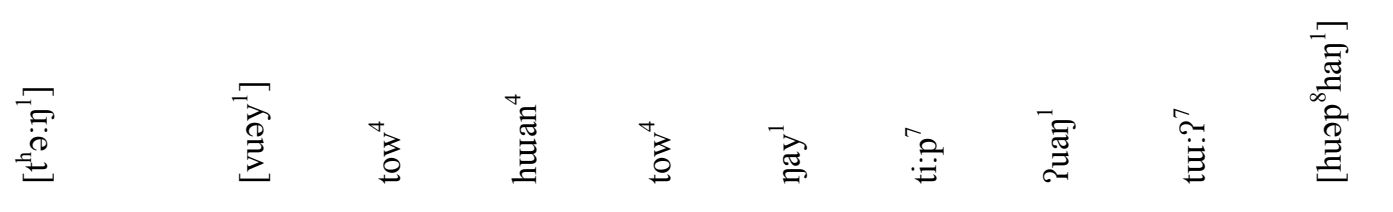

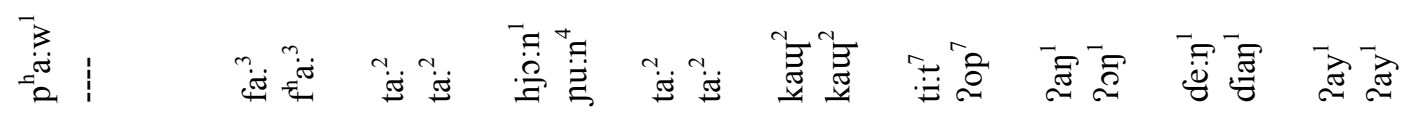

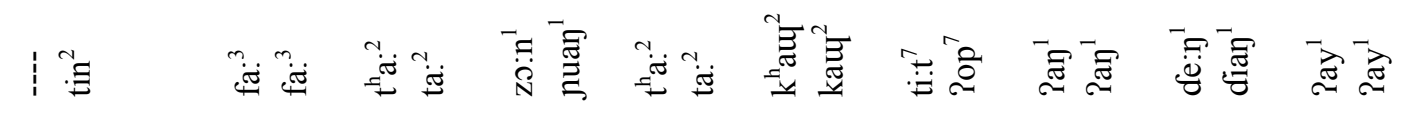

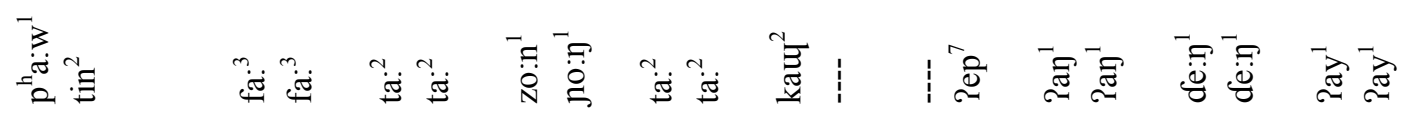

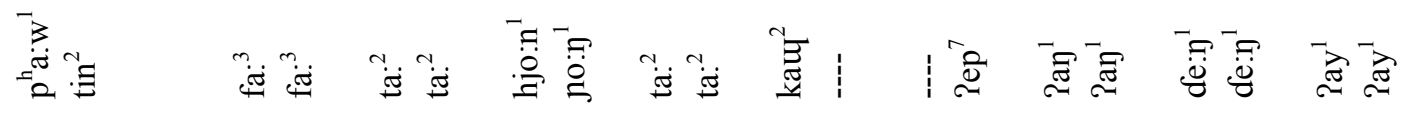

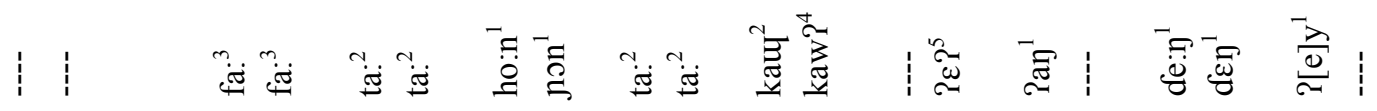

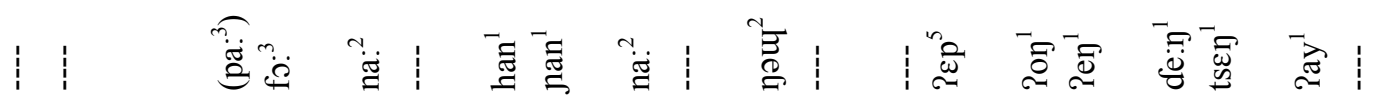

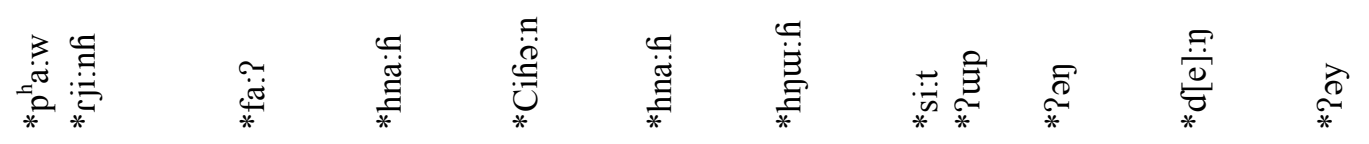

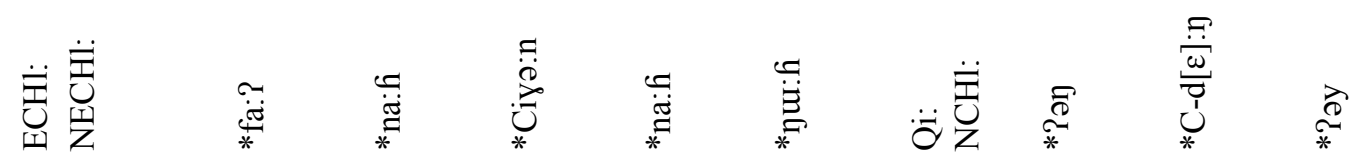

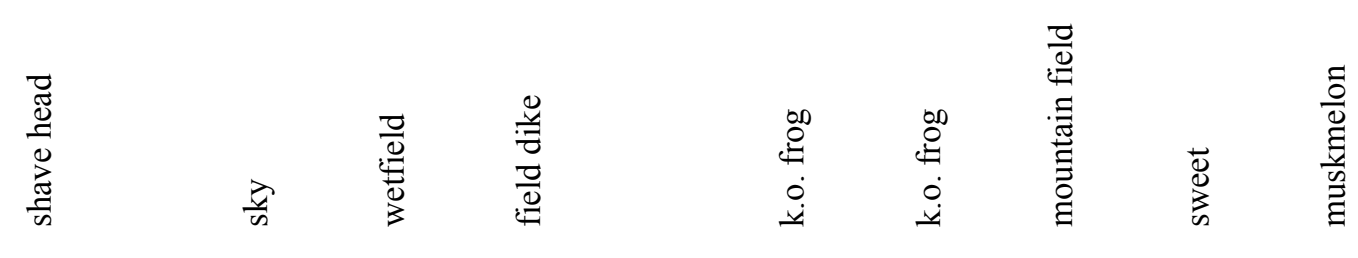

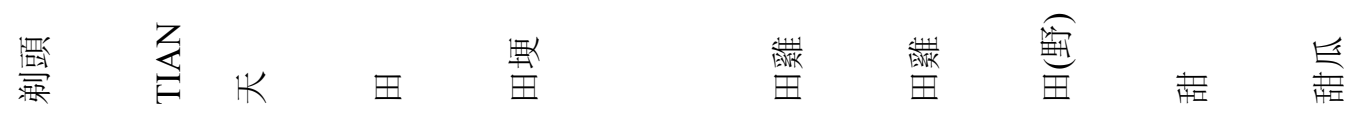




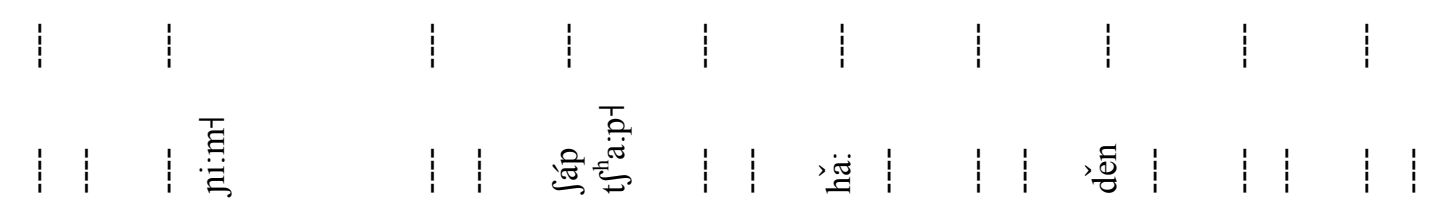

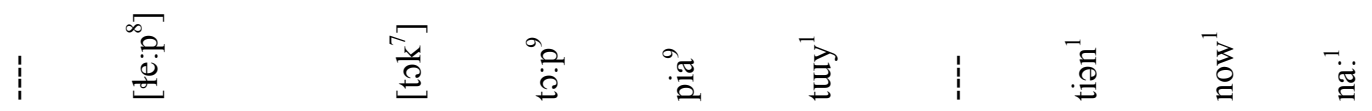

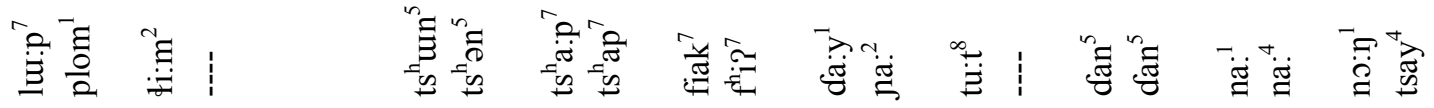

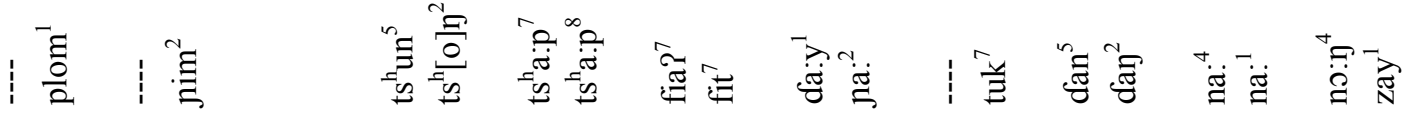

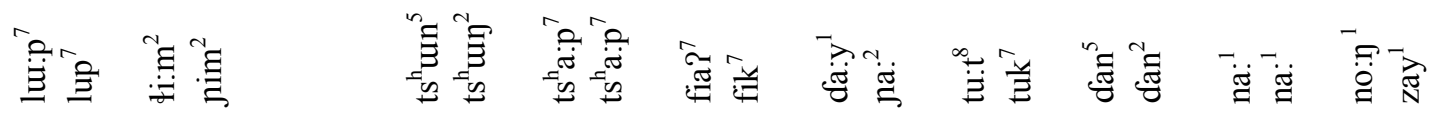

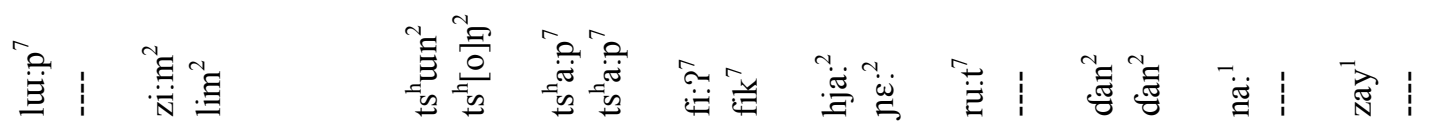

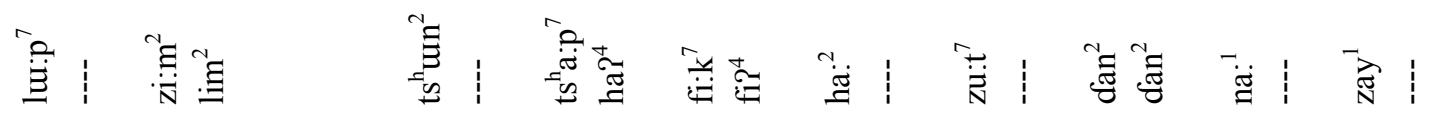

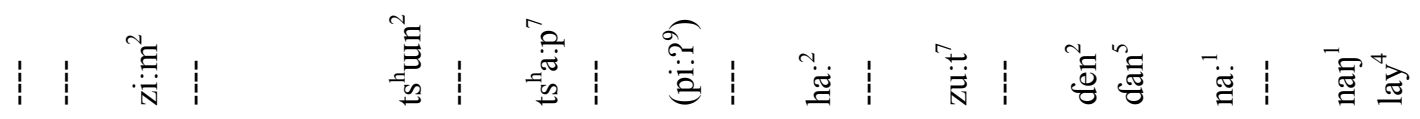

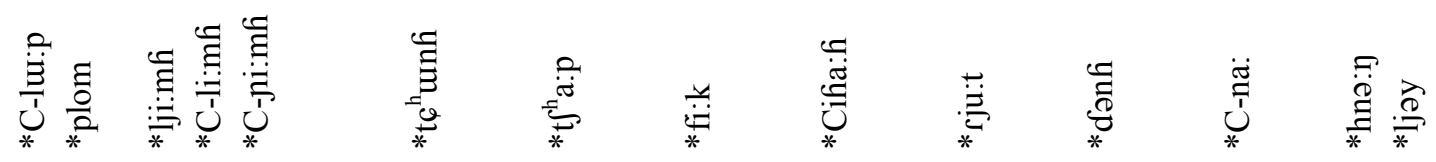

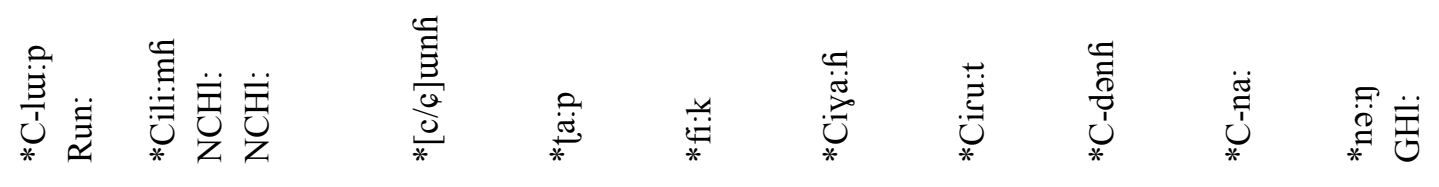

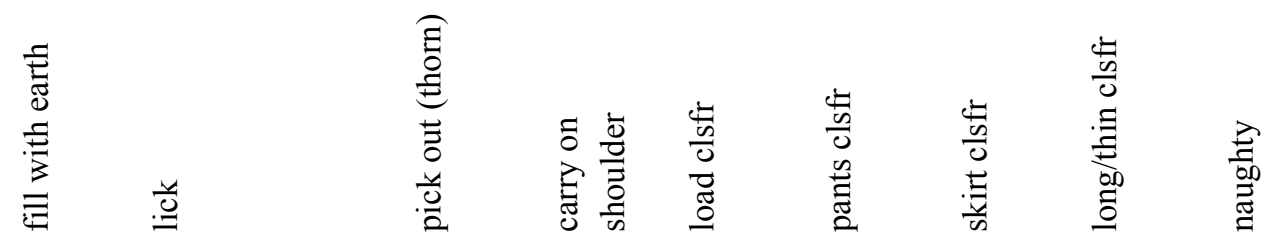

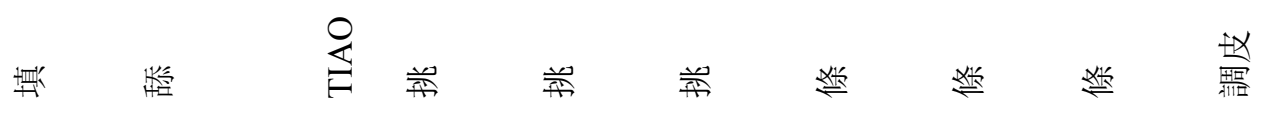




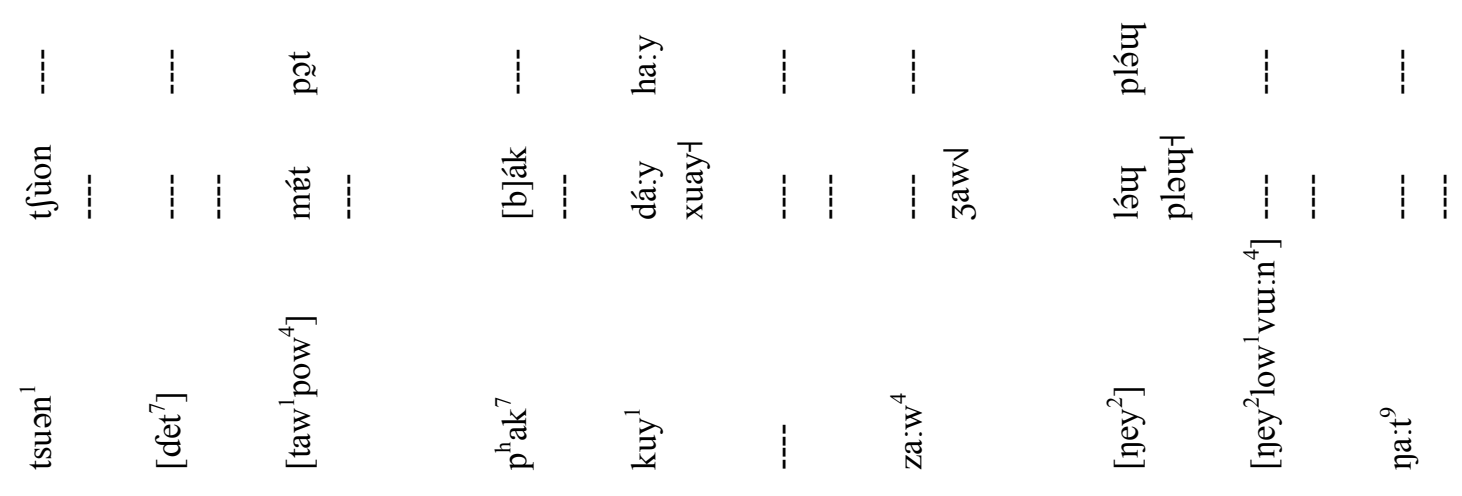

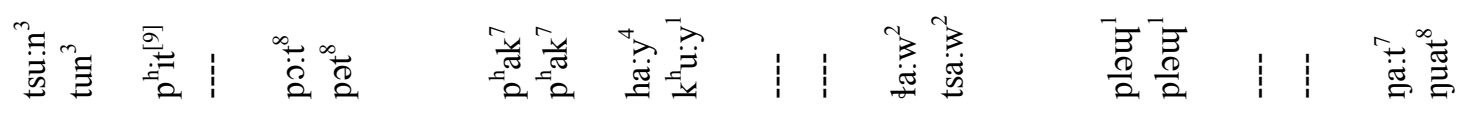

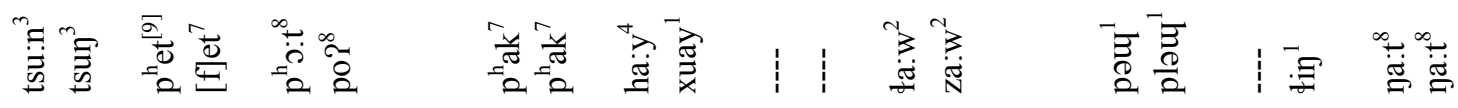

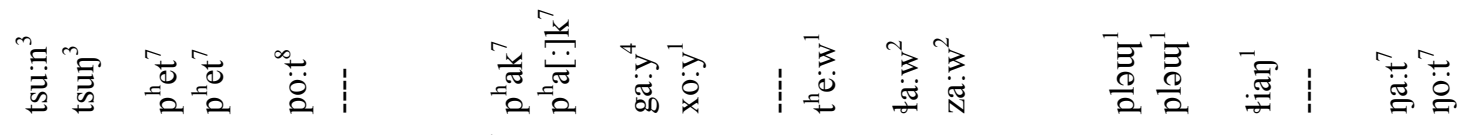

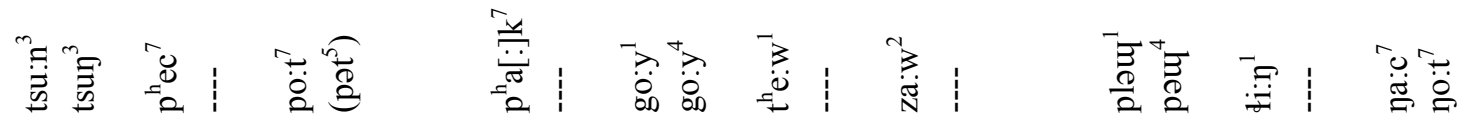

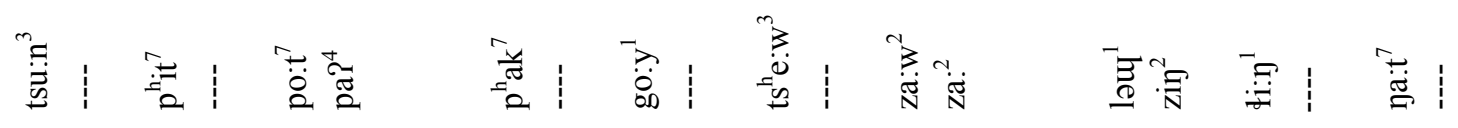

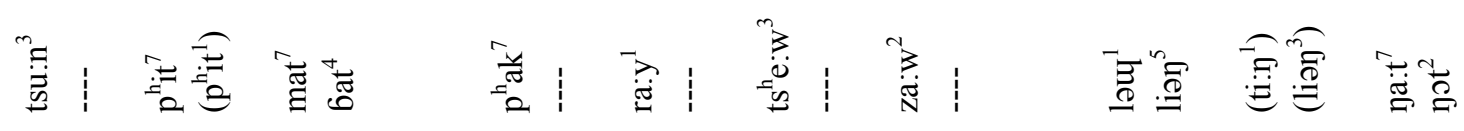

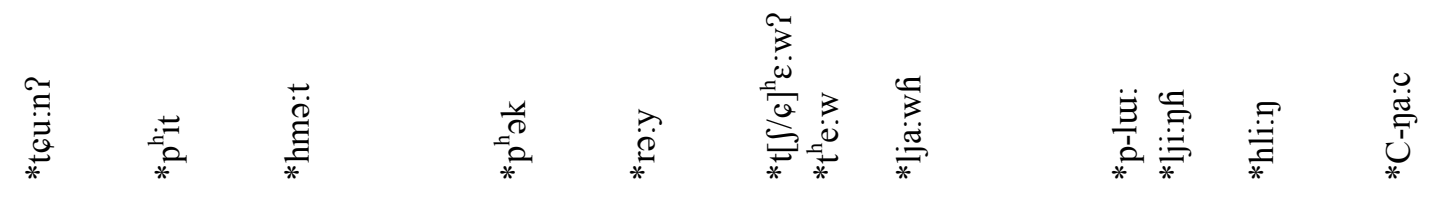

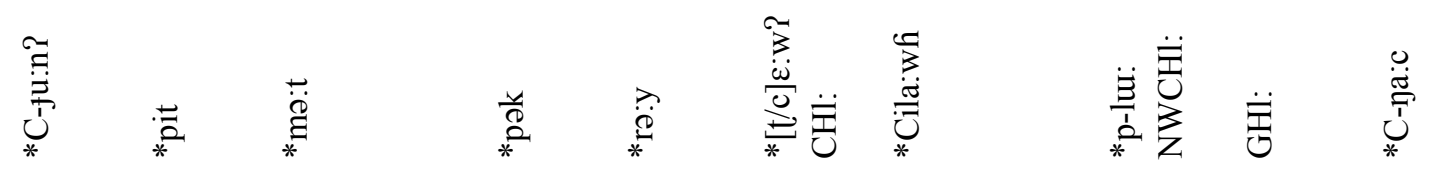

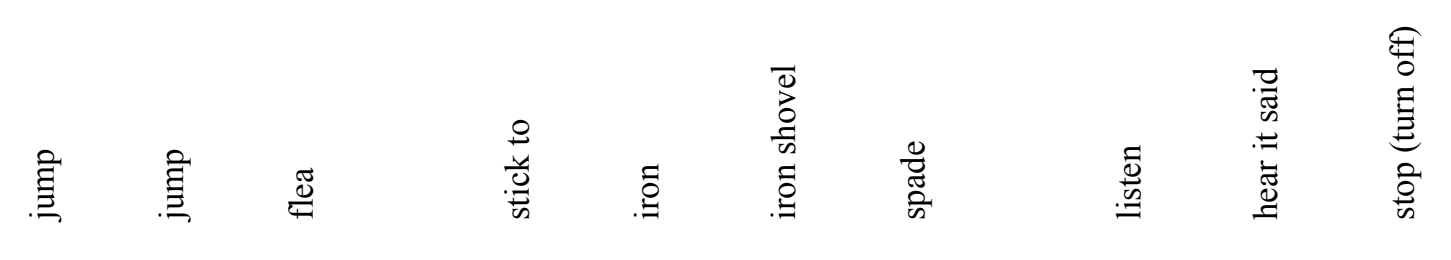

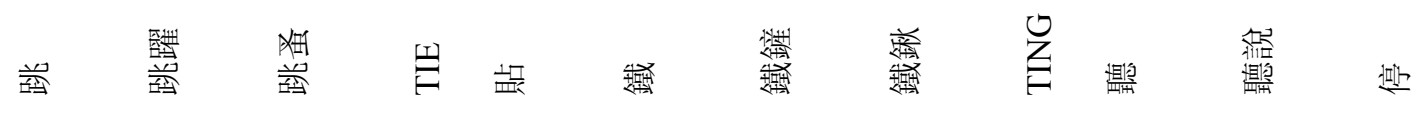




$$
\begin{aligned}
& 1 \quad 1 \quad 1 \quad 1 \quad 10
\end{aligned}
$$

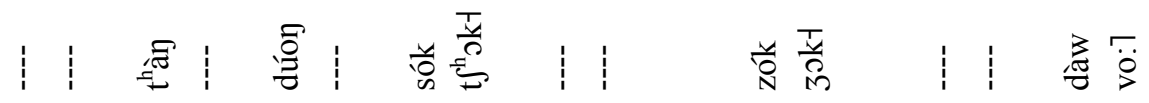

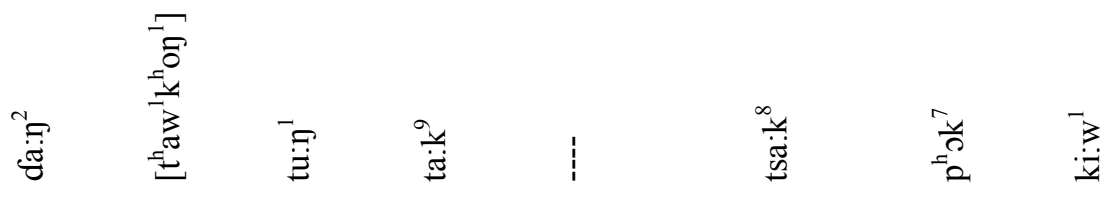

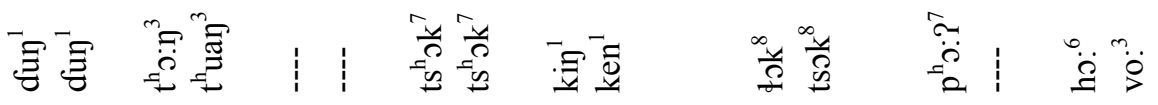

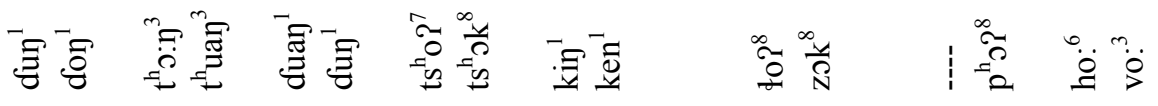

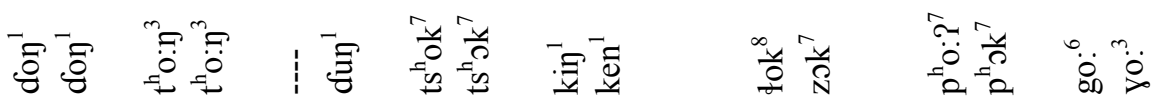

$$
\begin{aligned}
& \text { 要| }
\end{aligned}
$$

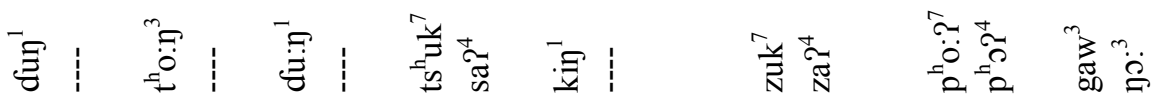

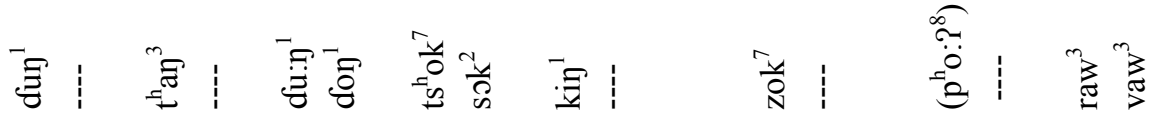

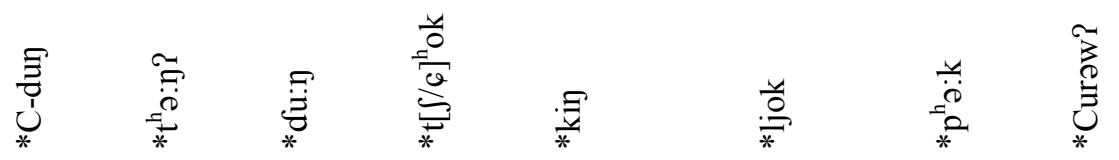

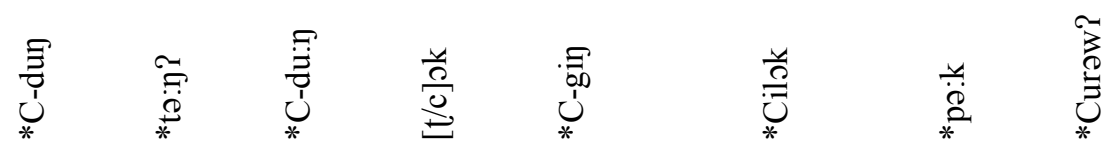

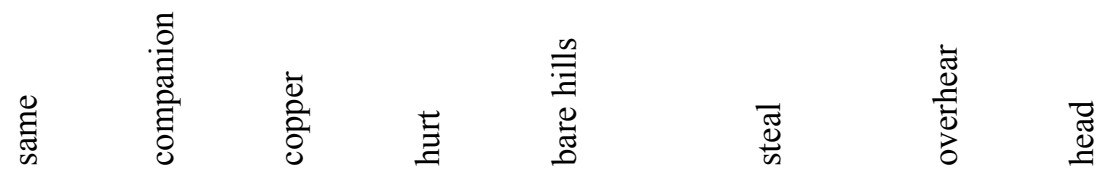

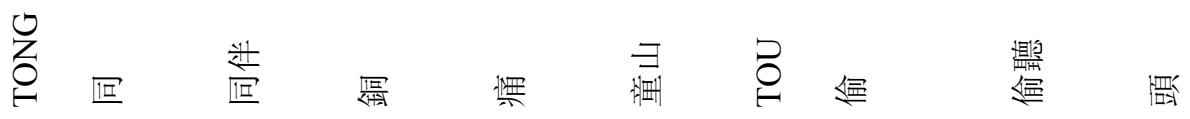




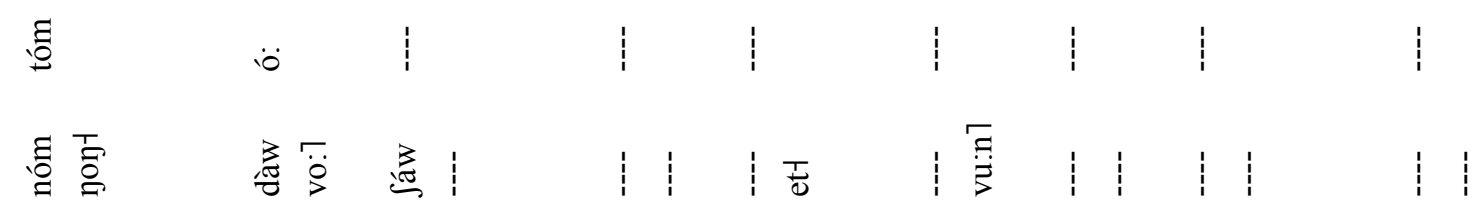

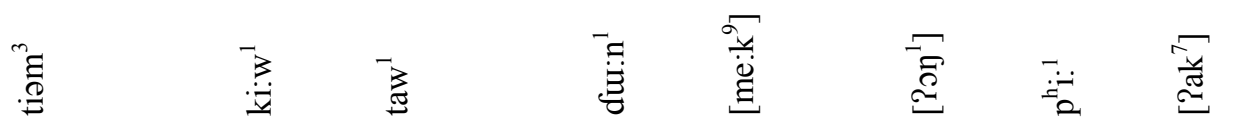

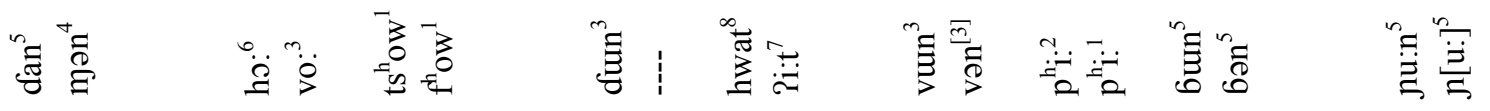

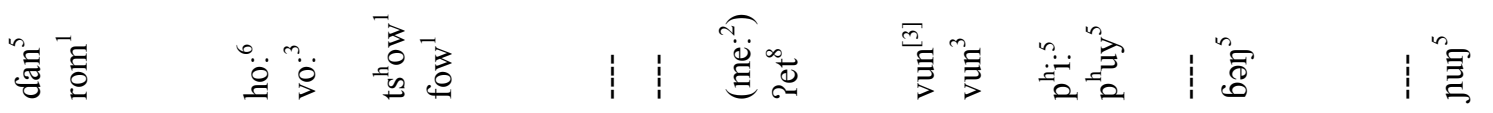

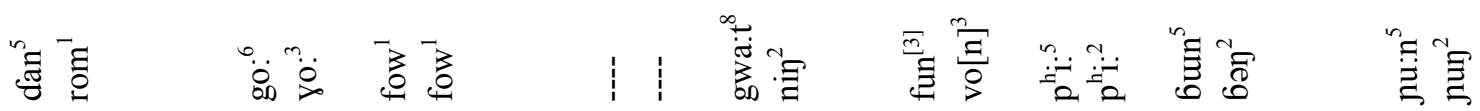

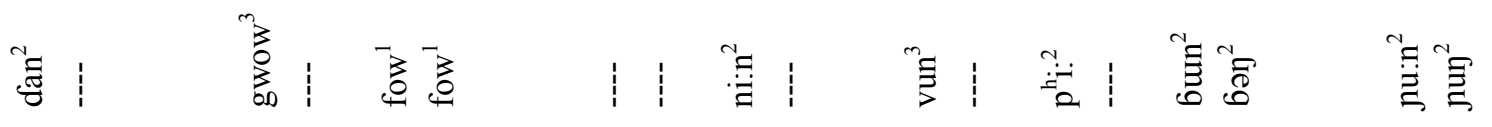

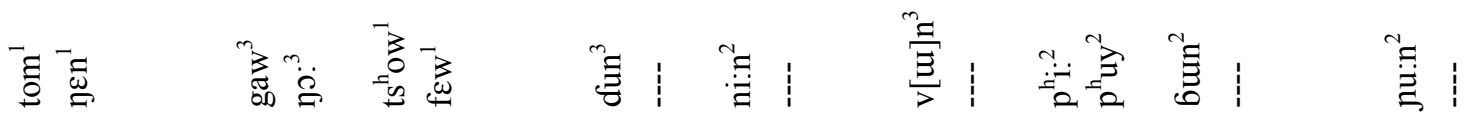

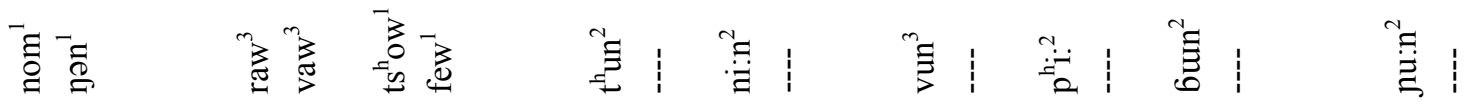

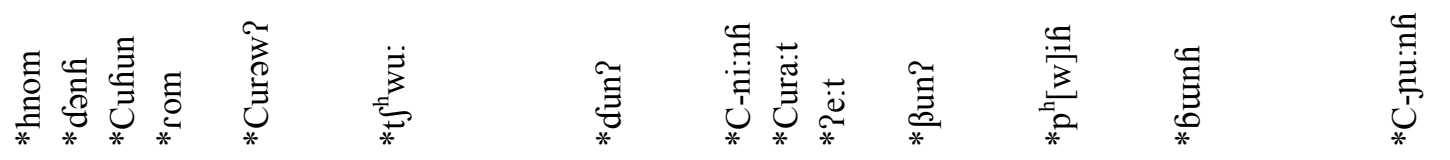

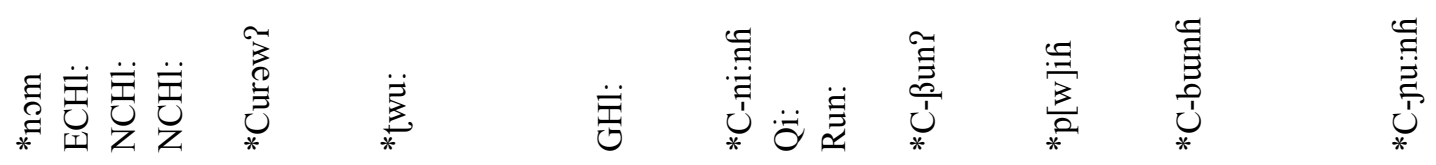

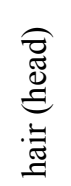

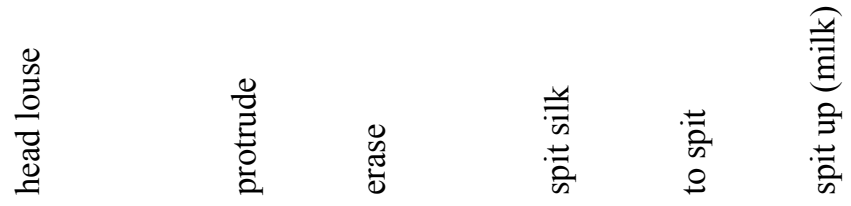

䇏

监住:

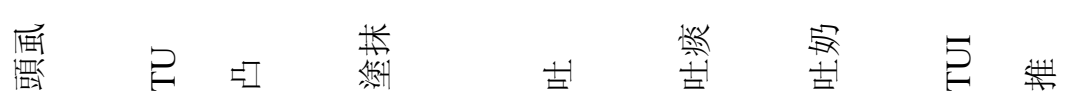




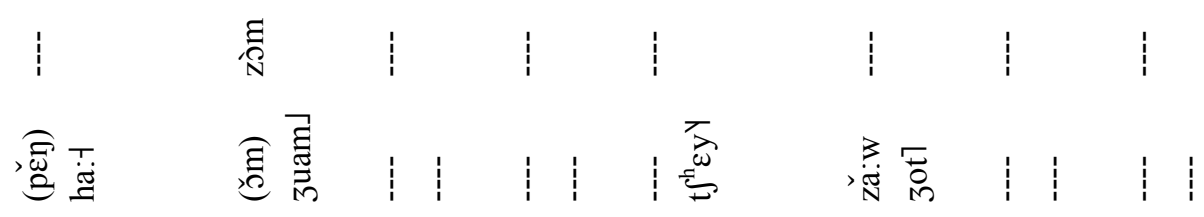

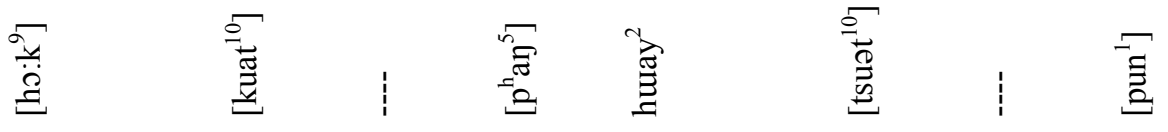

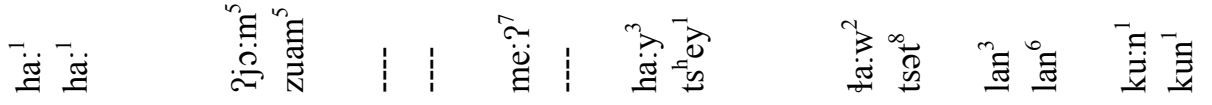

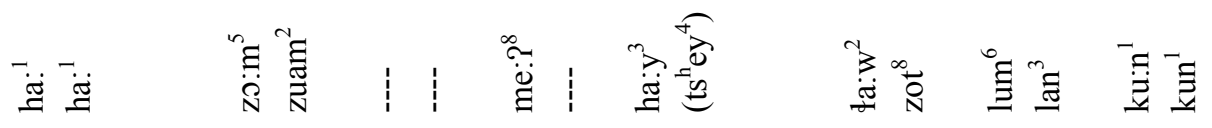

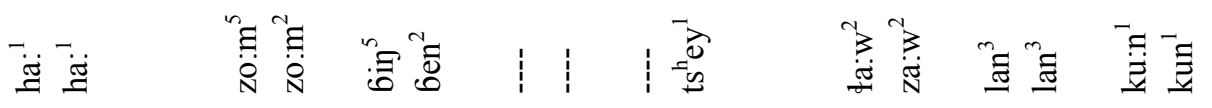

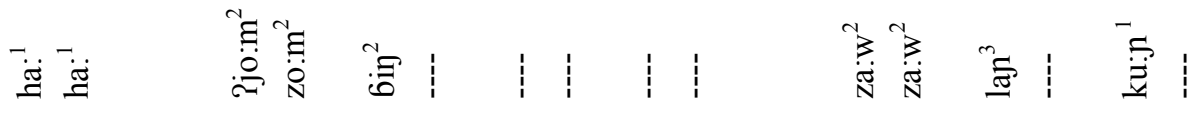

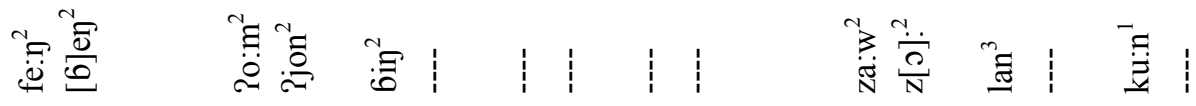

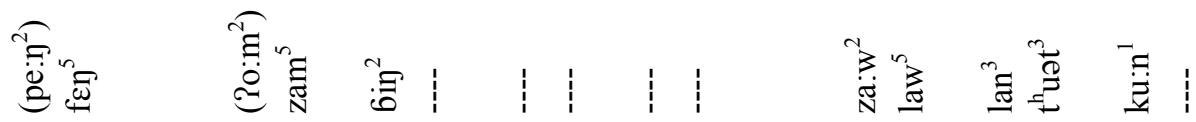

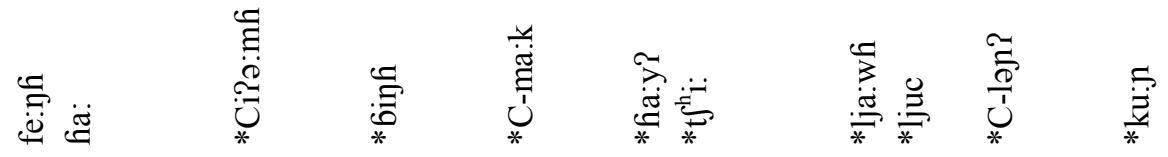

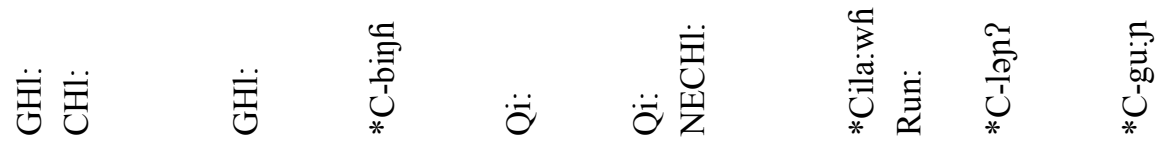

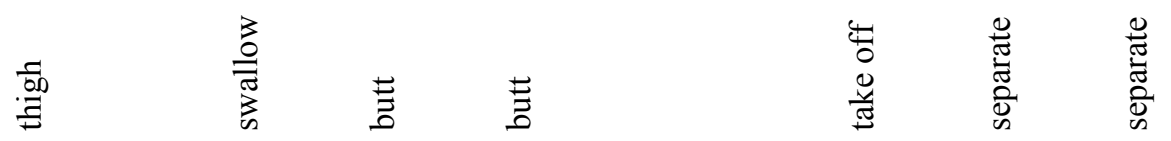

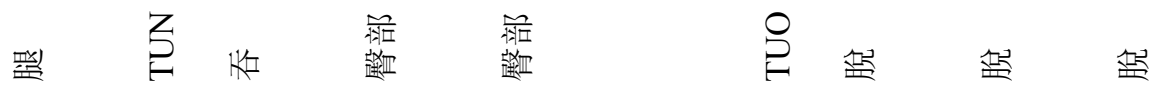




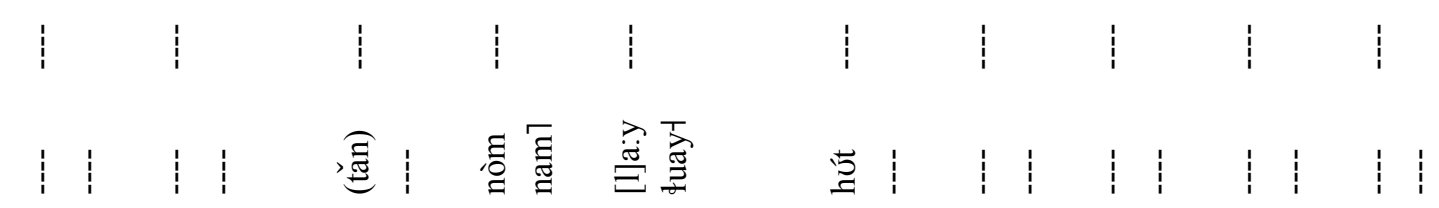

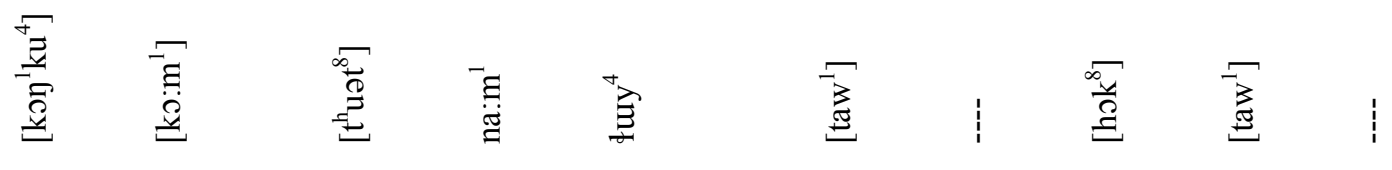

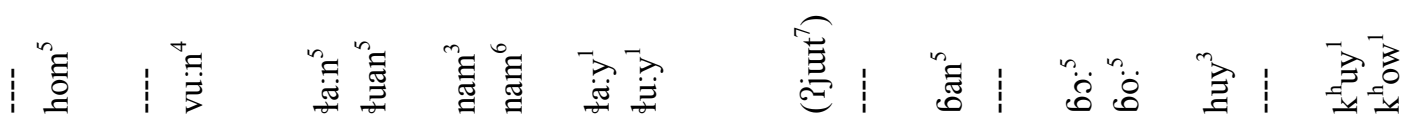

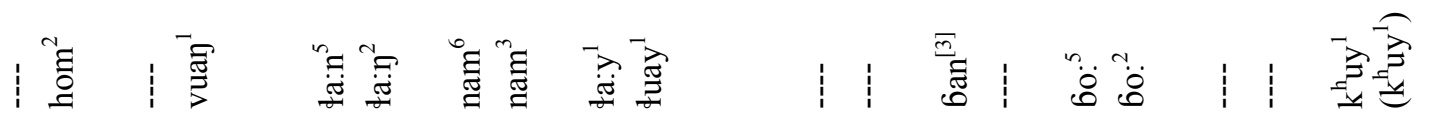

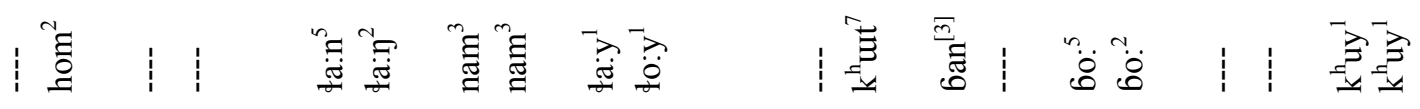

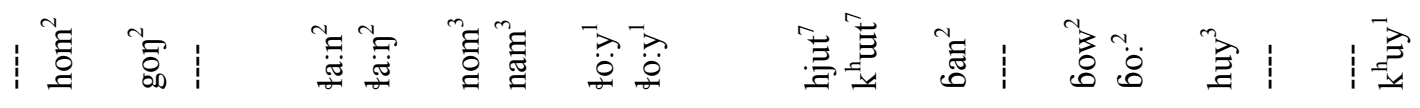

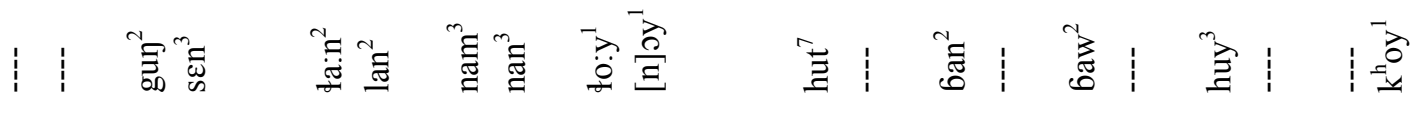

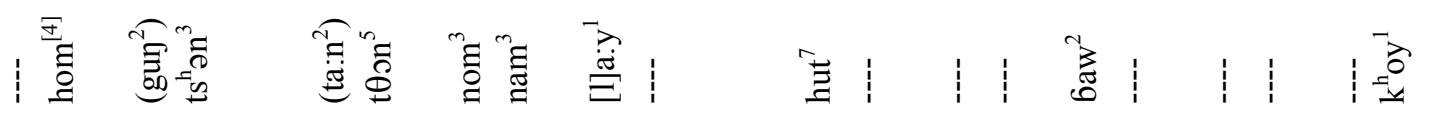

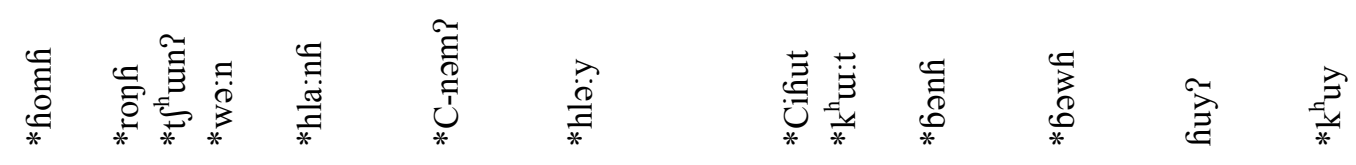

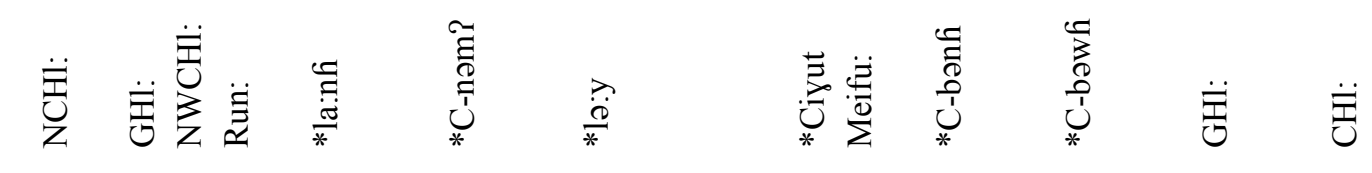

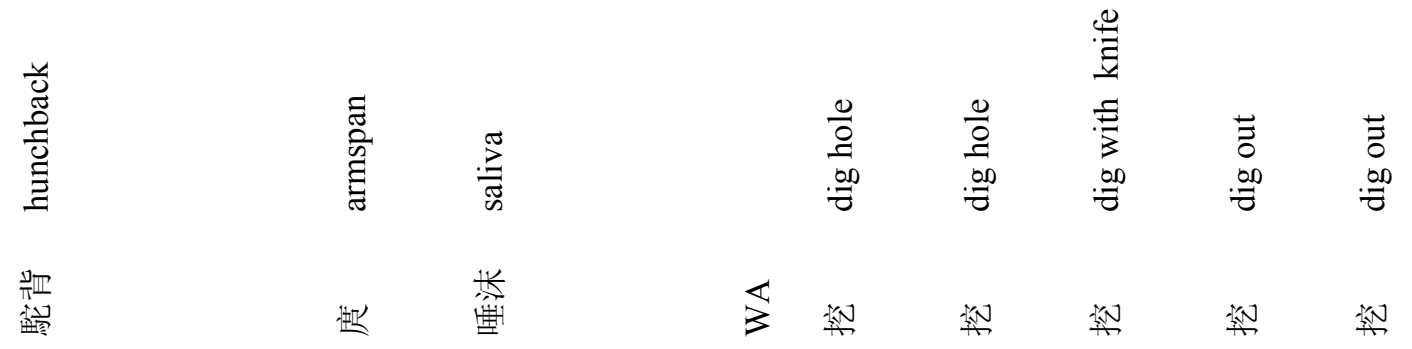



1
1111
$1 \quad 1$

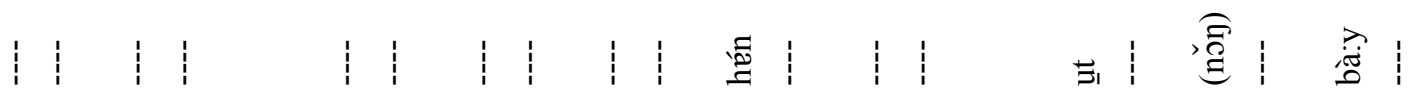
1

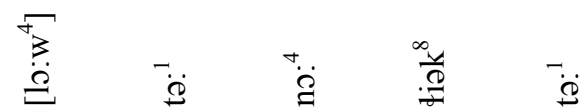

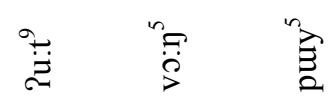
蛋

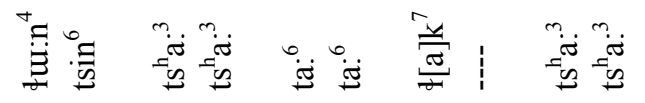

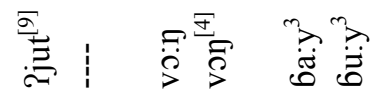

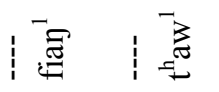

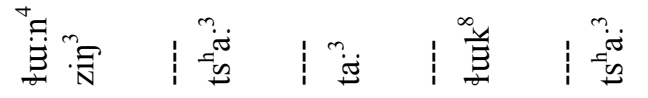

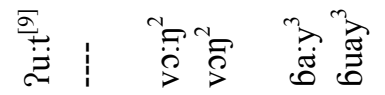
资|

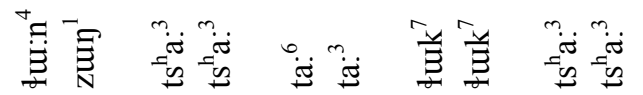

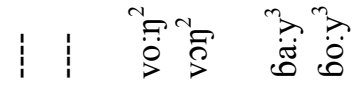
$1: 1$

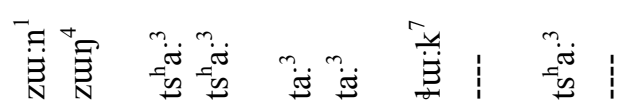

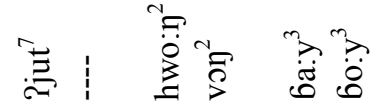
证 11

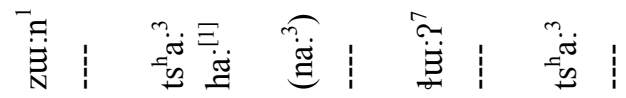

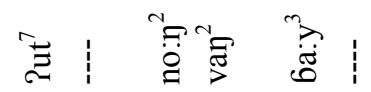
111

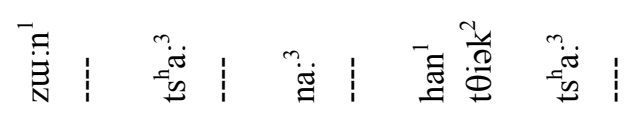

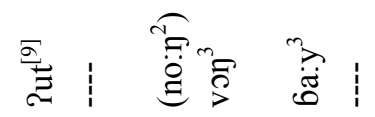

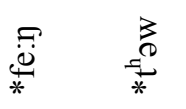

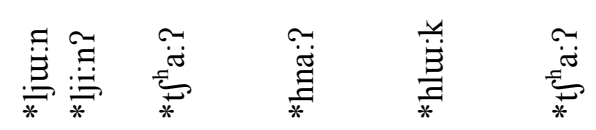

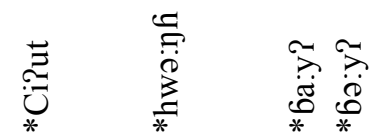
㡺 弟

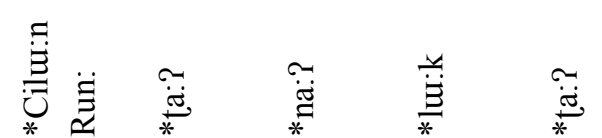

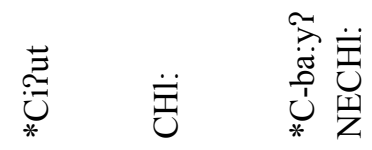
$\cong$

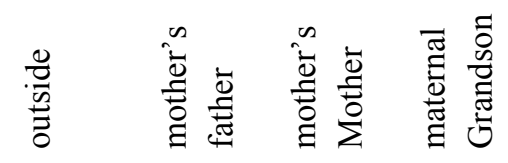

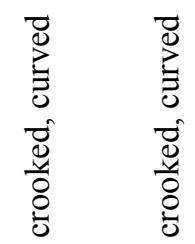
运

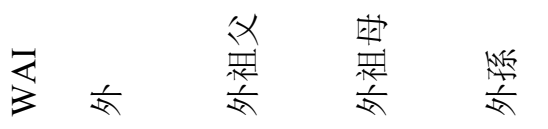
登跑 


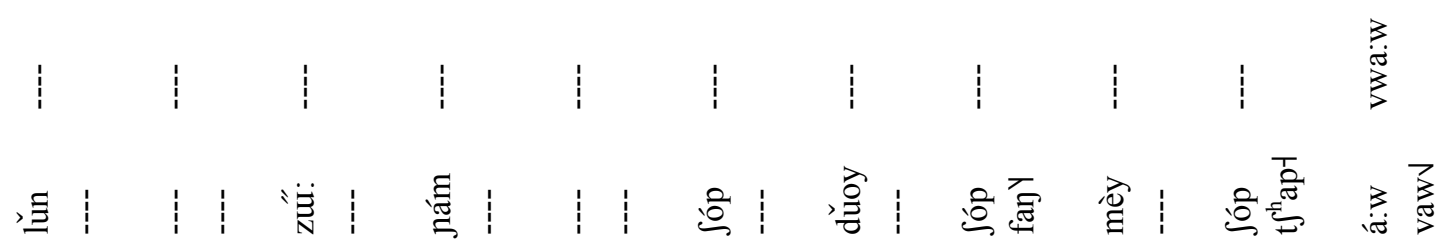

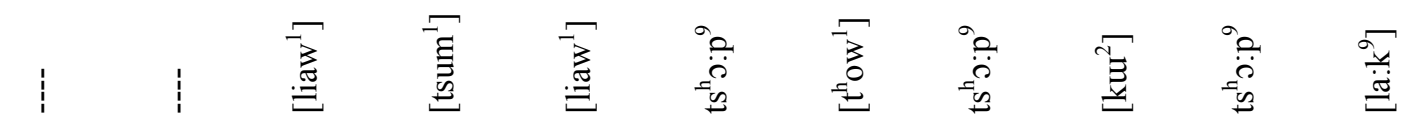

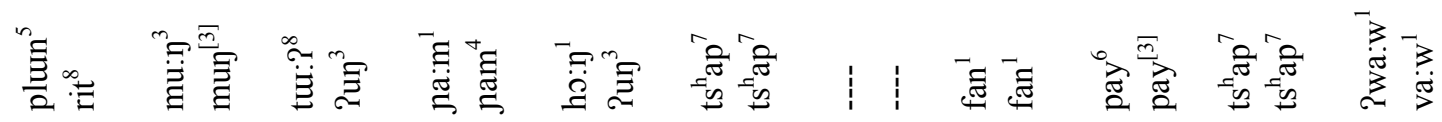

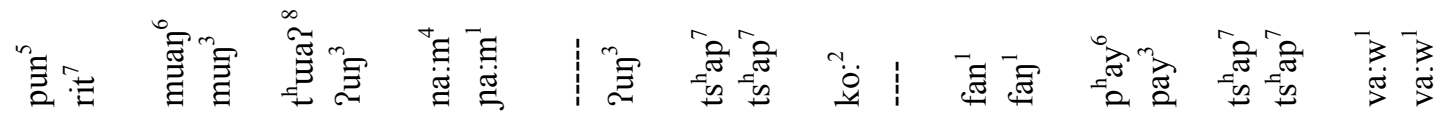

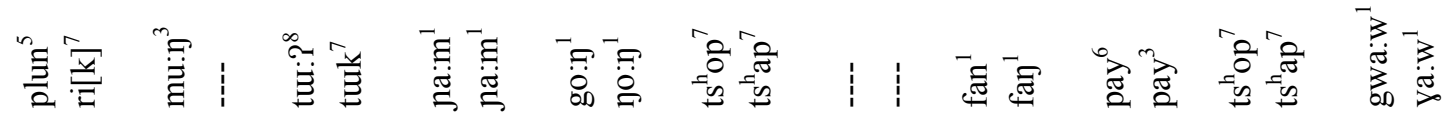

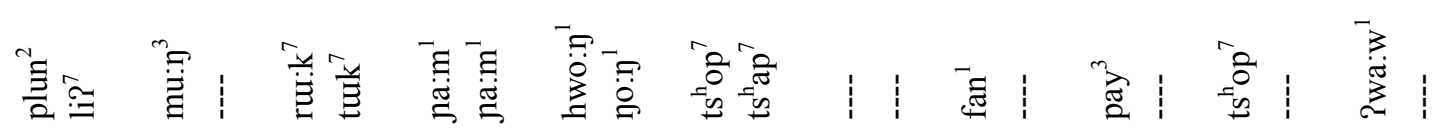

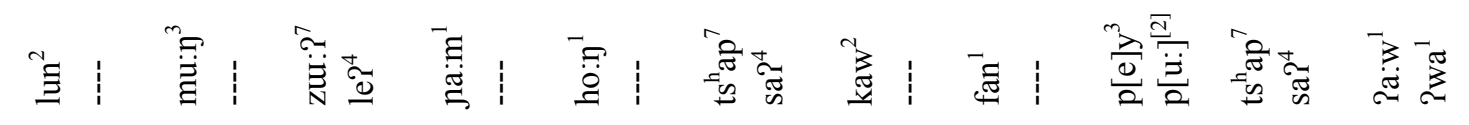

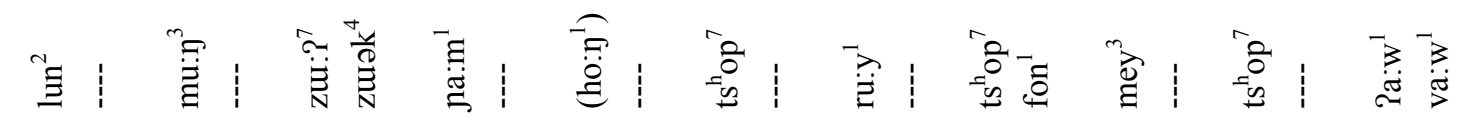

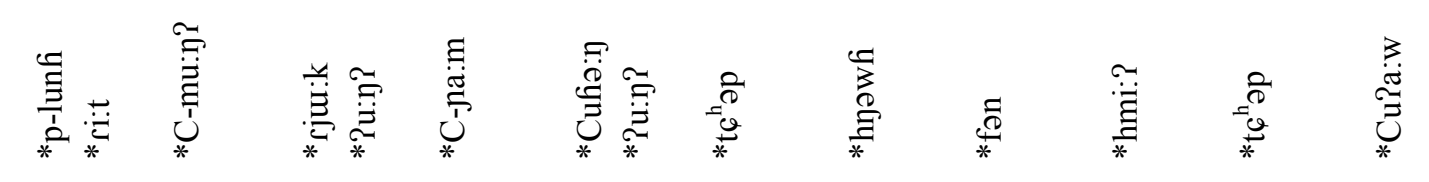

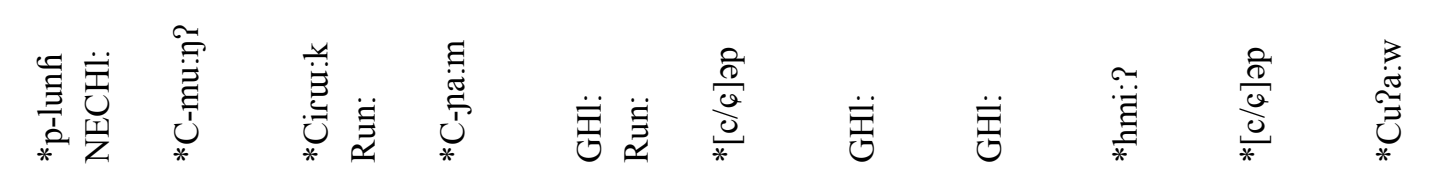

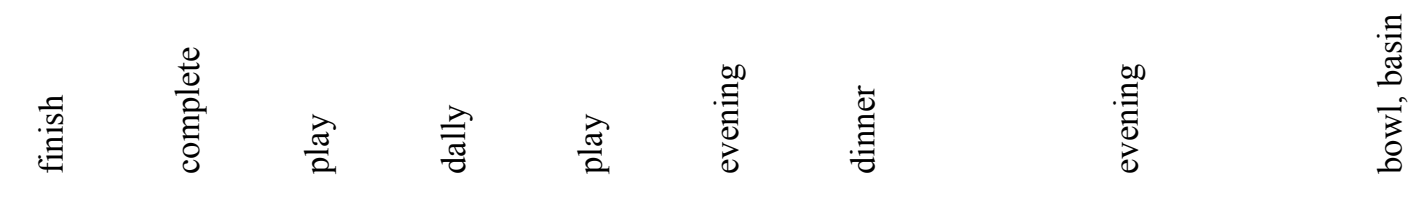

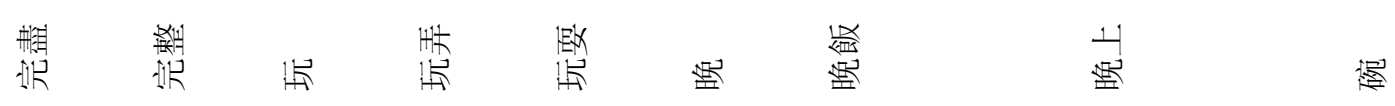




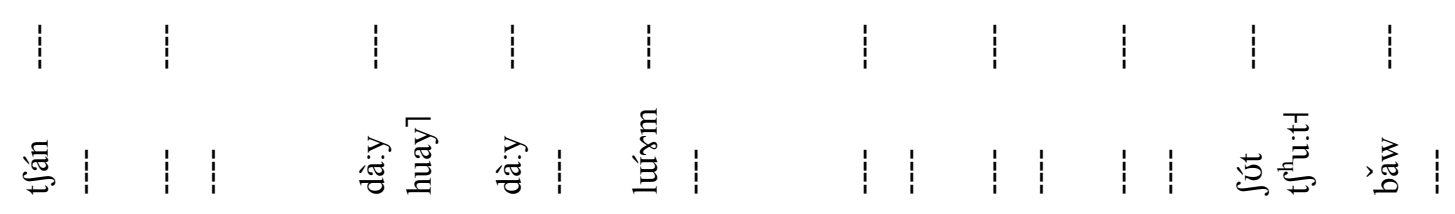

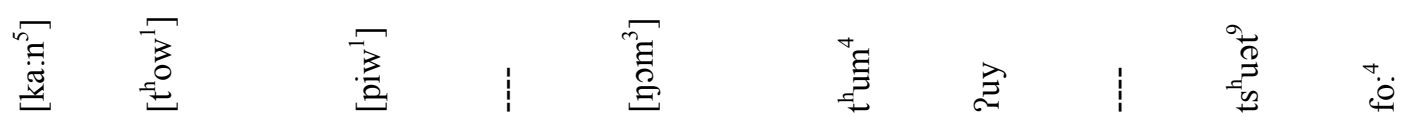

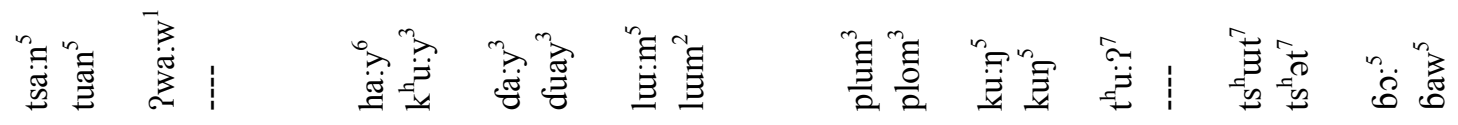

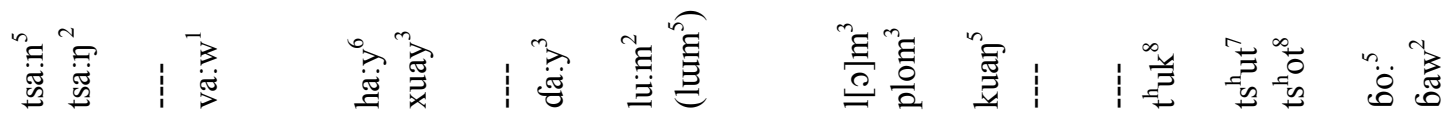

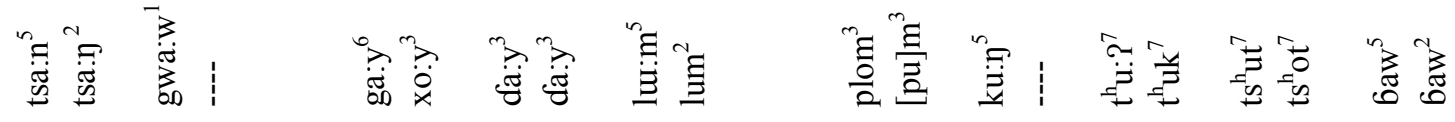

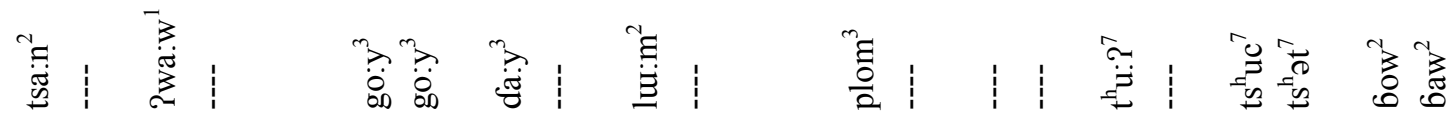

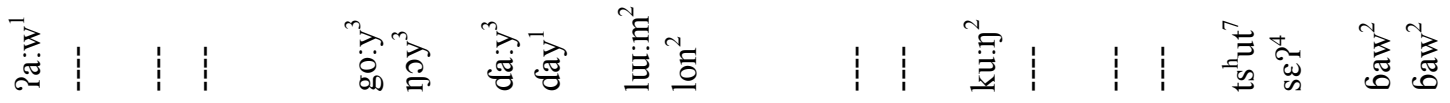

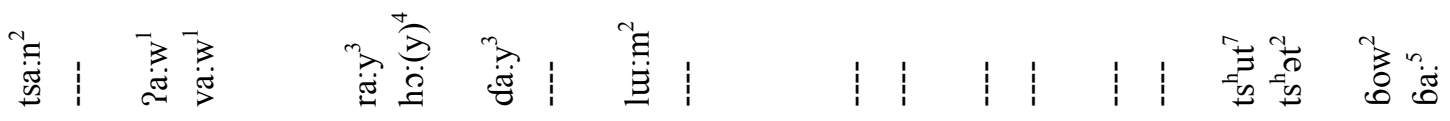

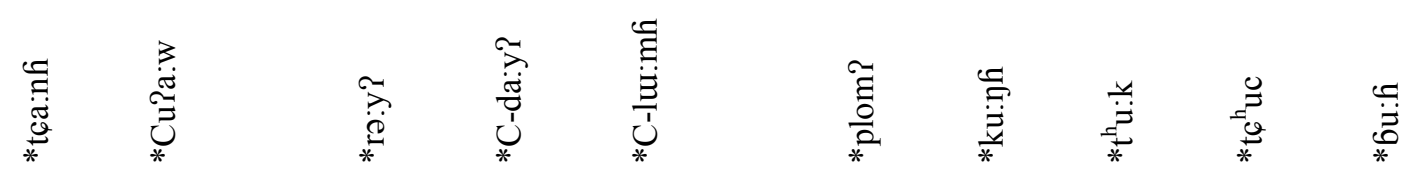

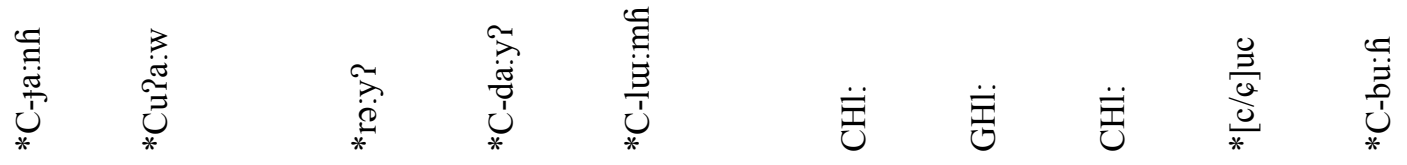

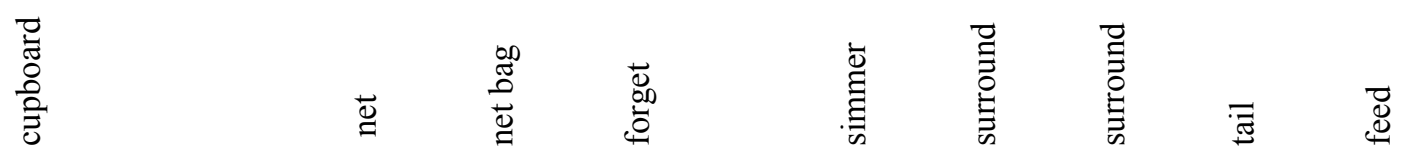

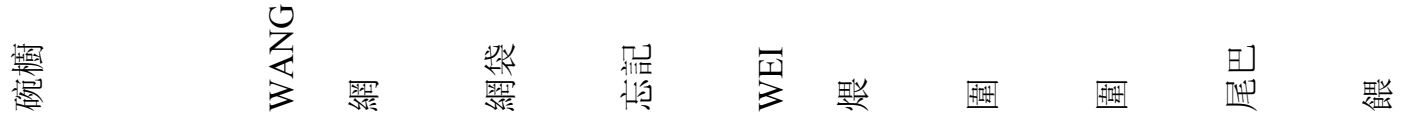




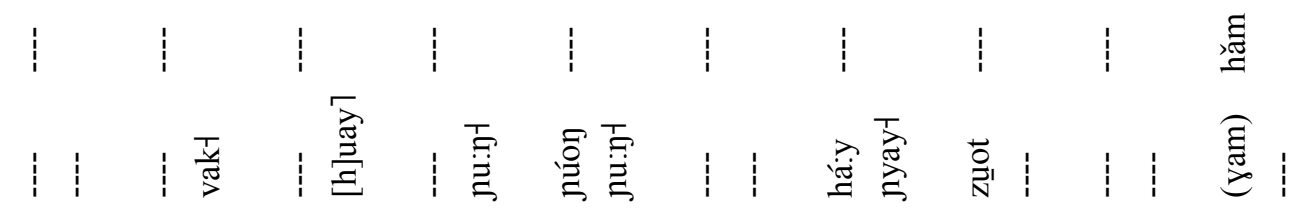

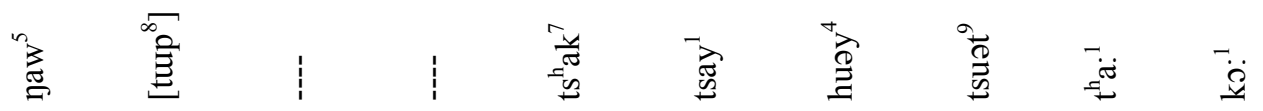

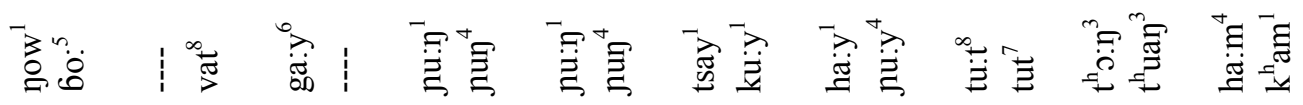

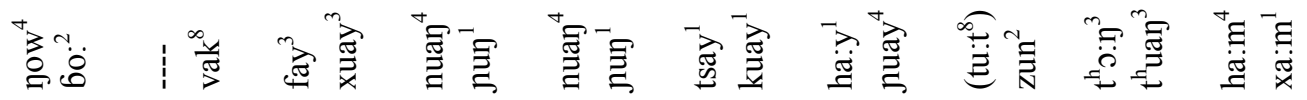

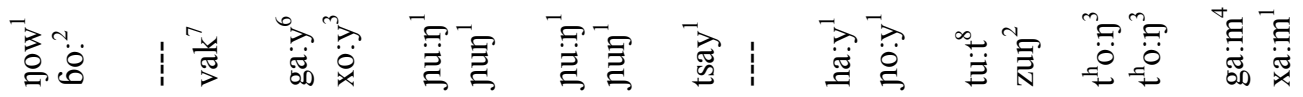

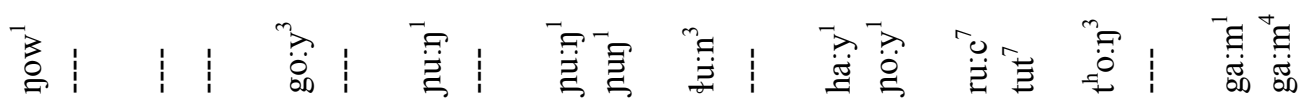

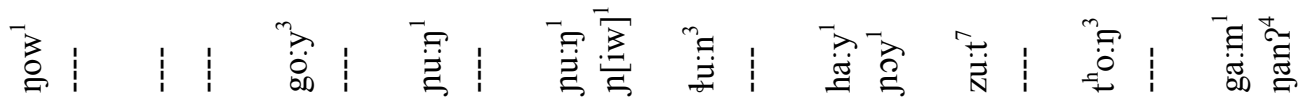

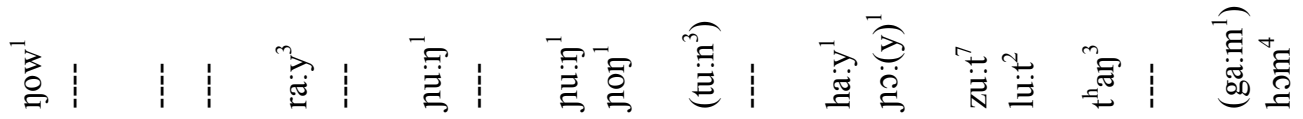

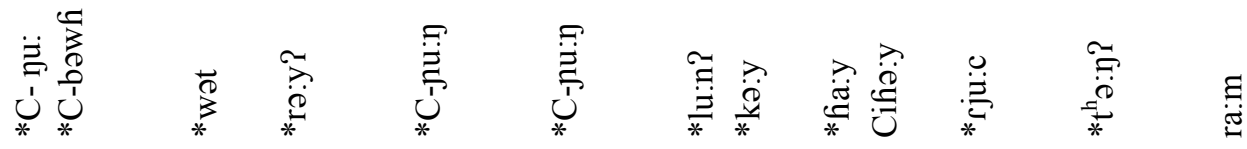

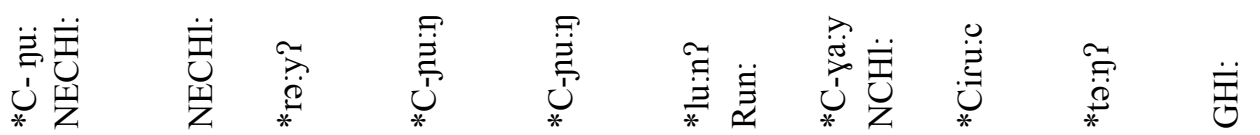

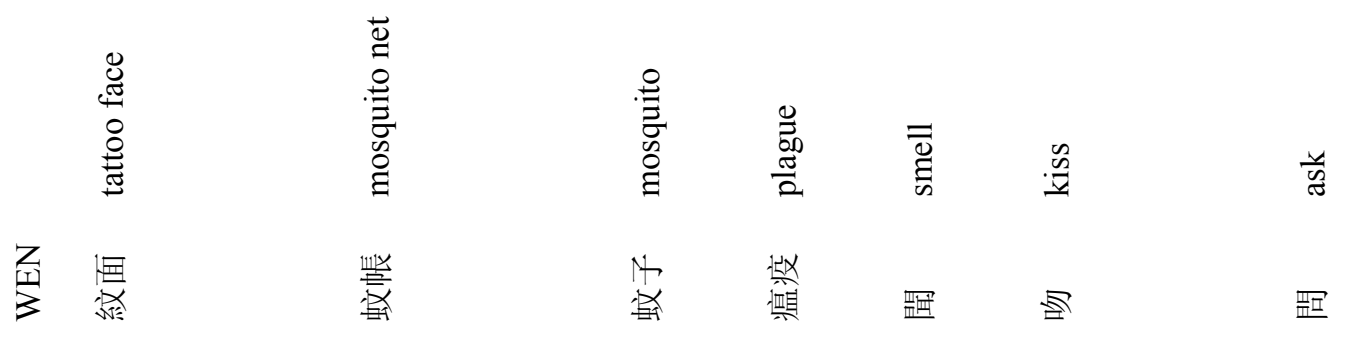




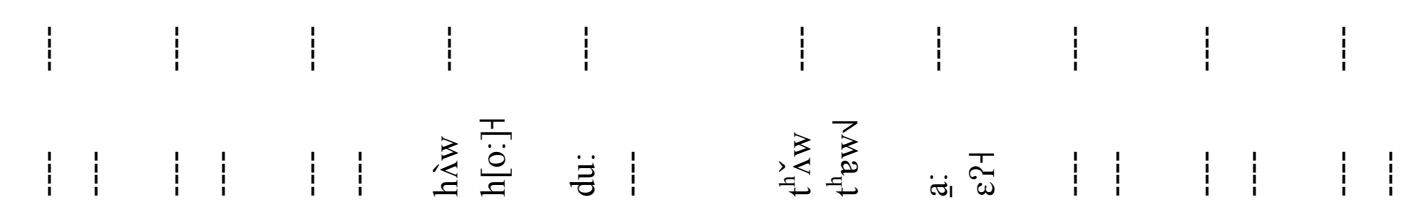

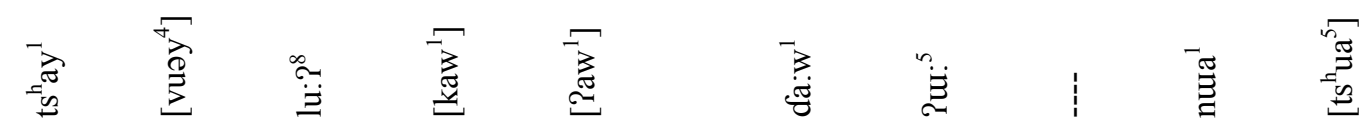

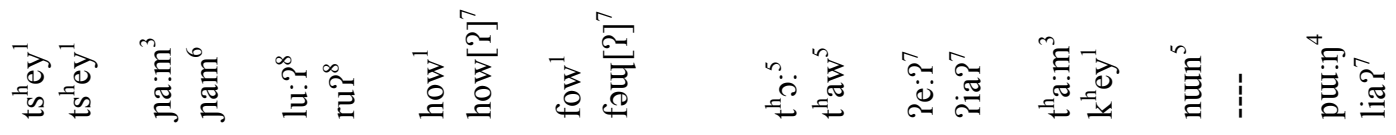

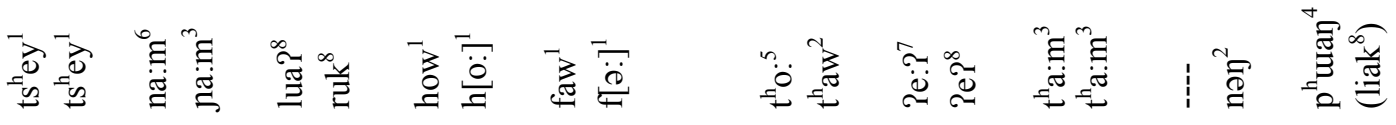

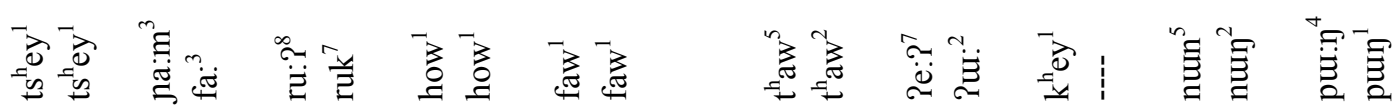

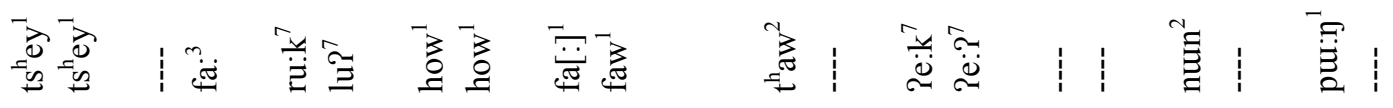

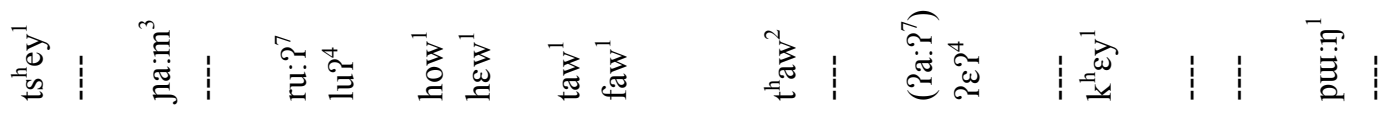

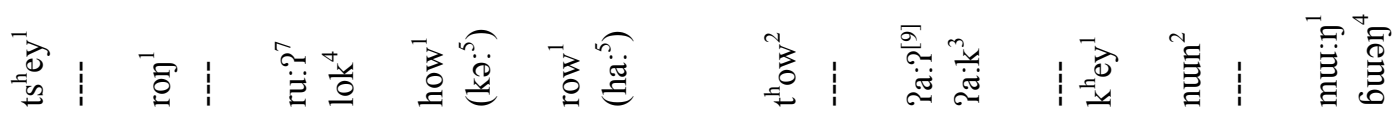

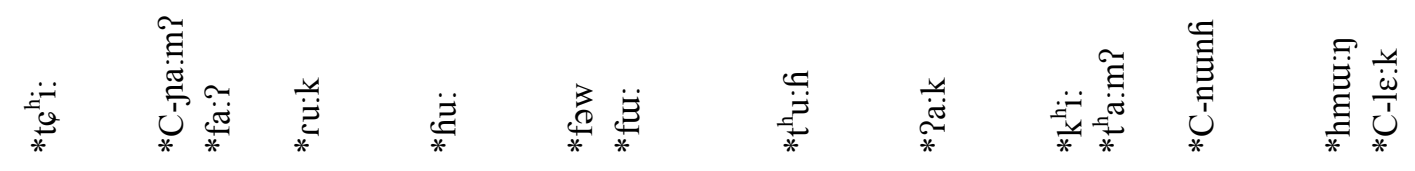

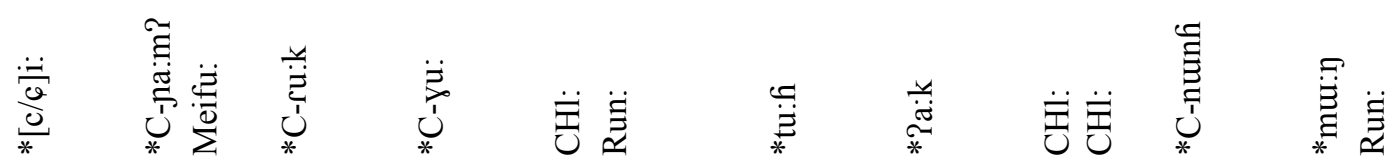

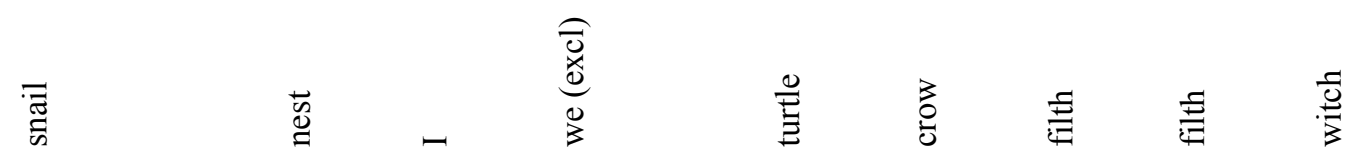

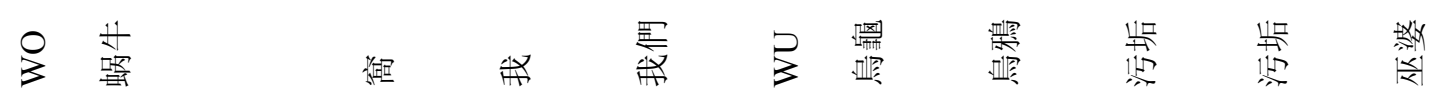




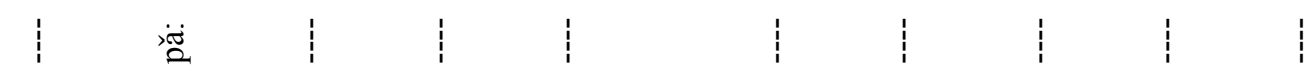

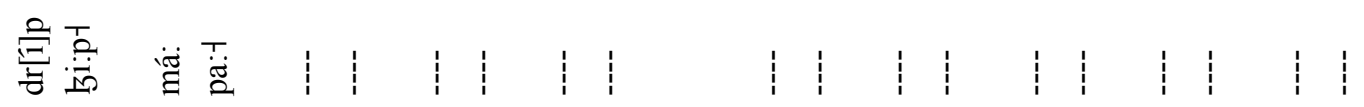

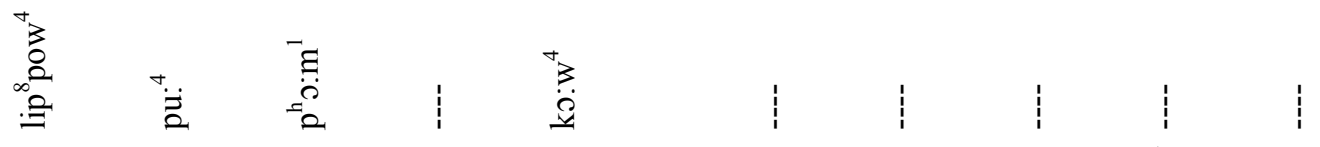

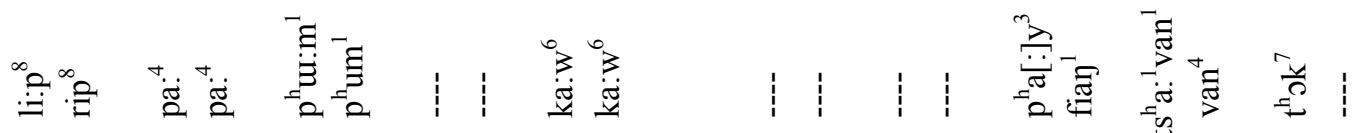

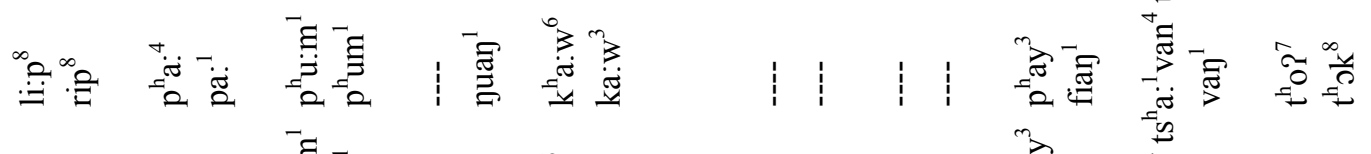

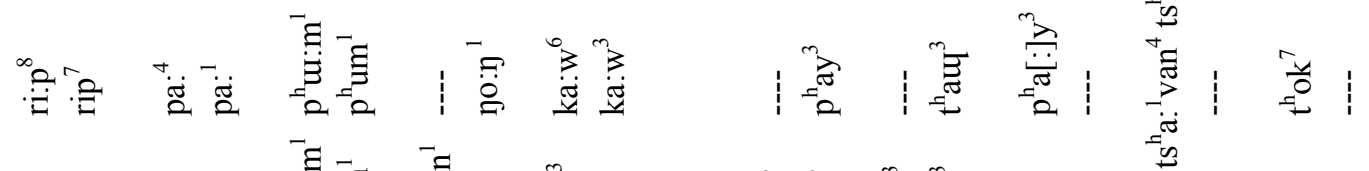

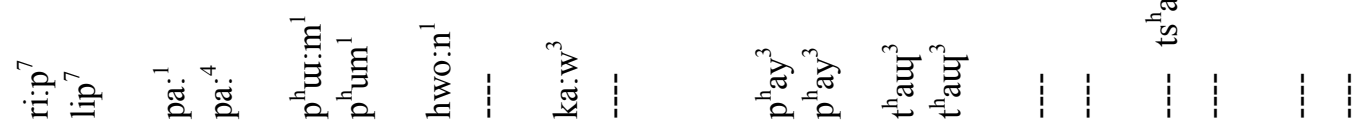

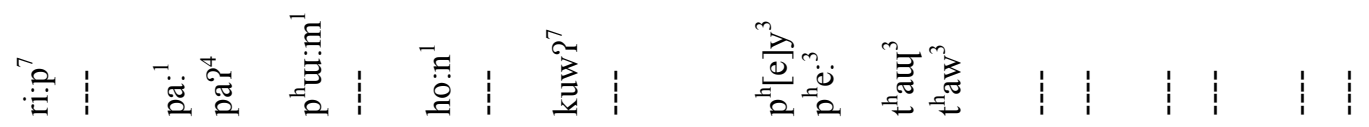

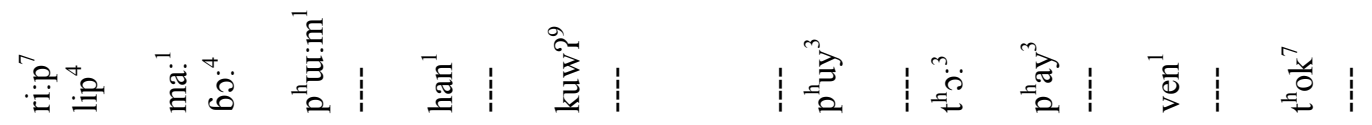

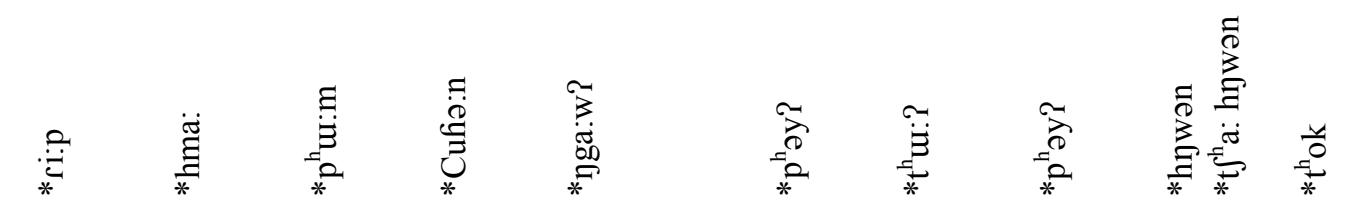

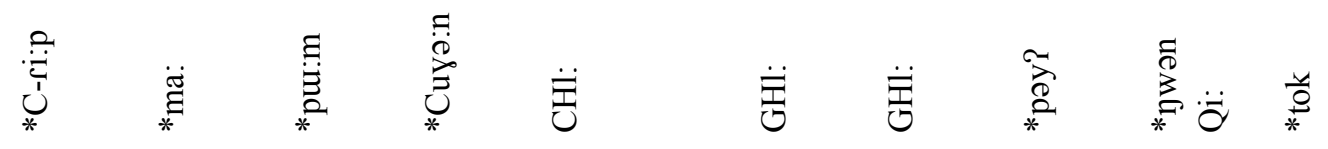

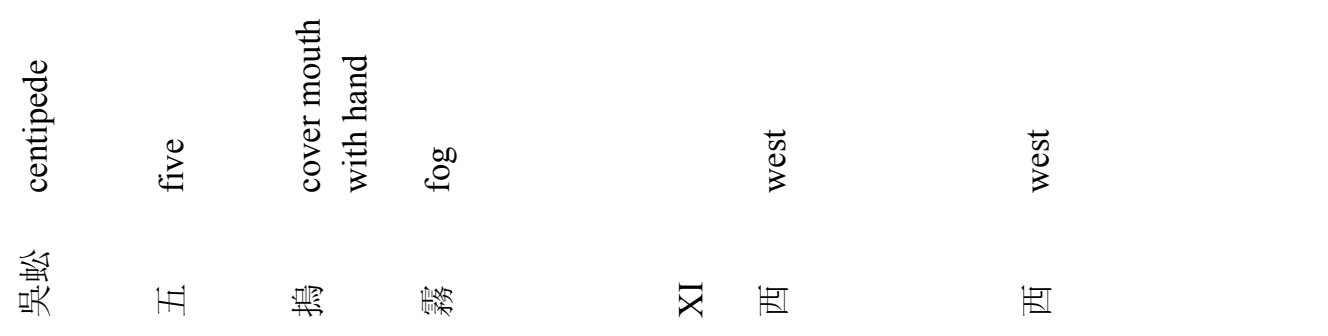




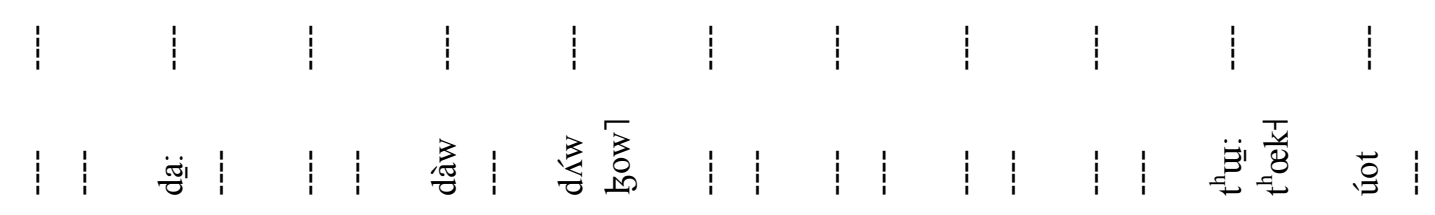

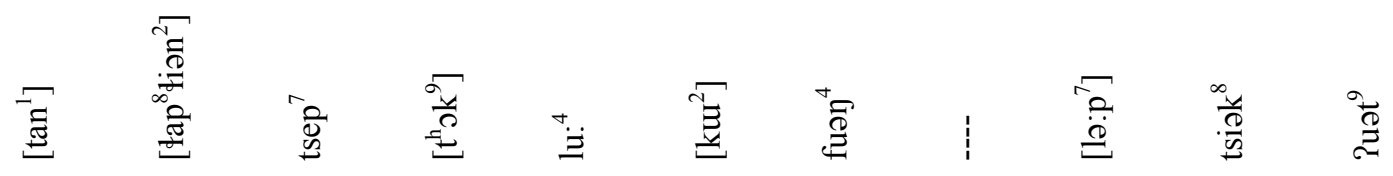

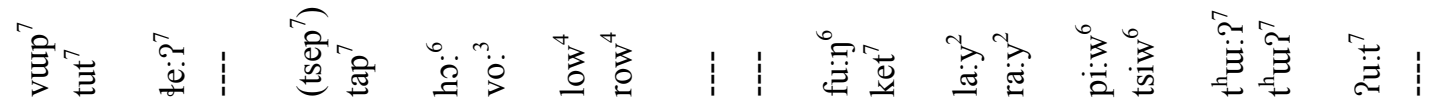

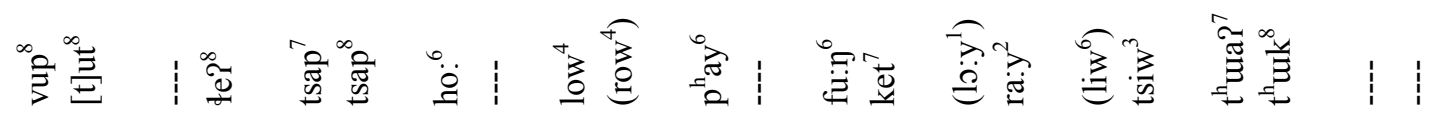

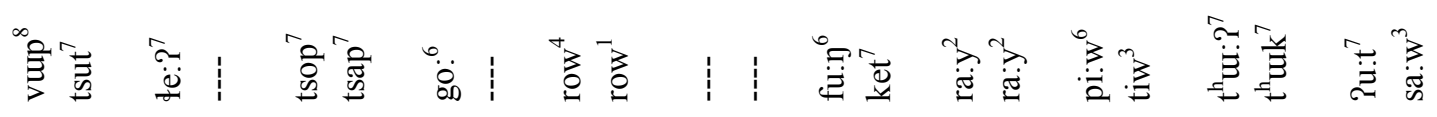

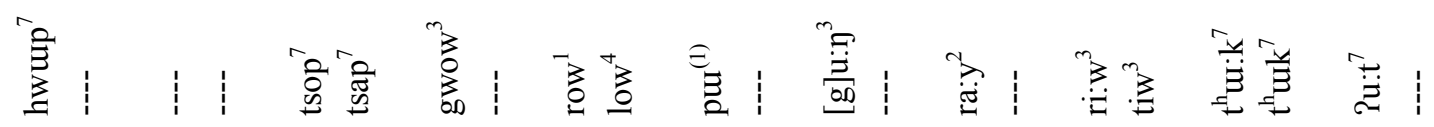

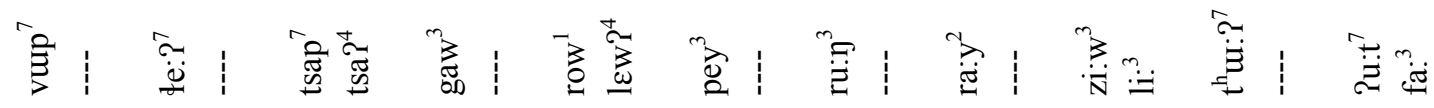

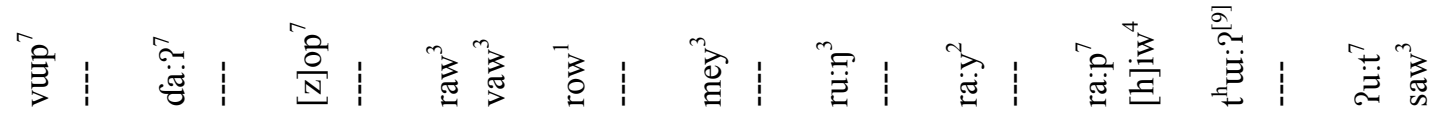

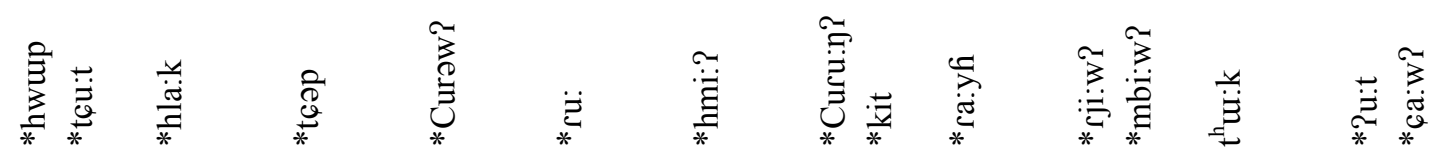

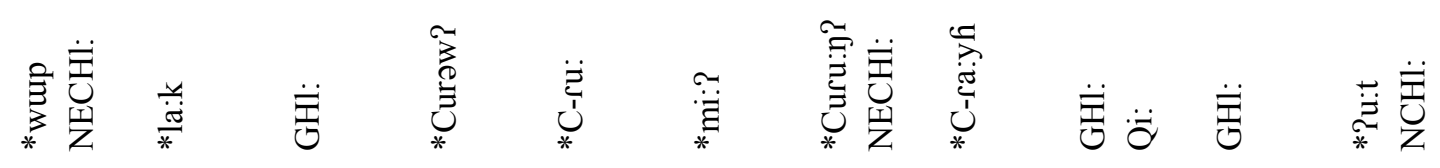

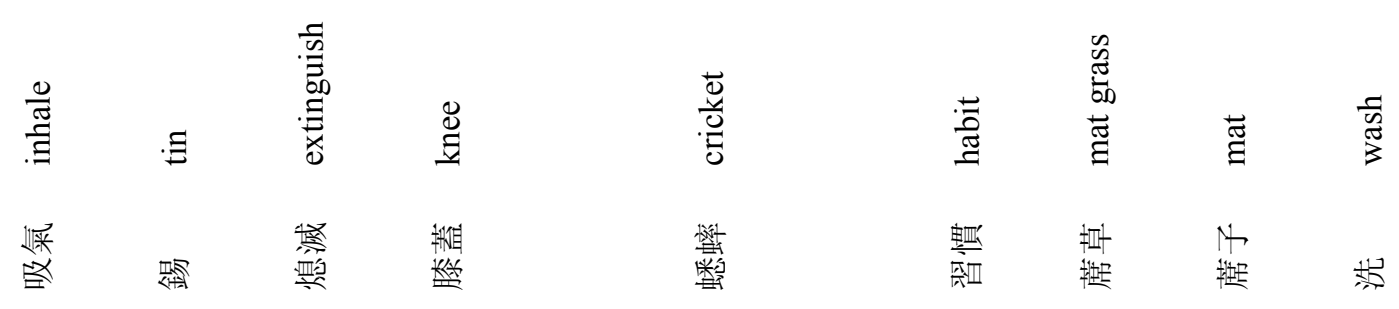




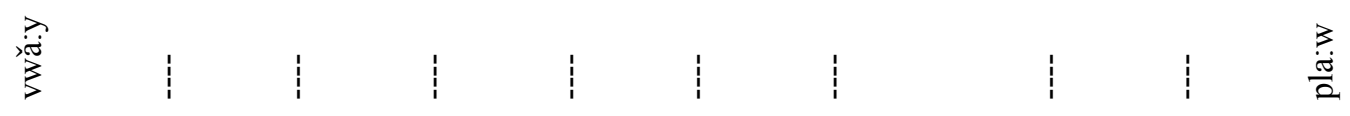

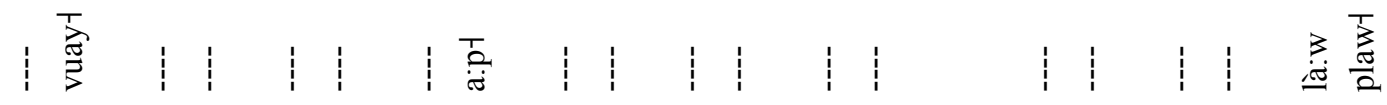

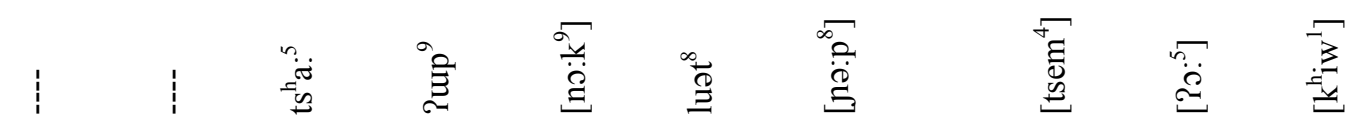

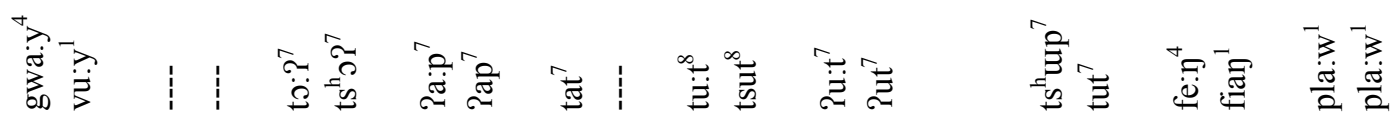

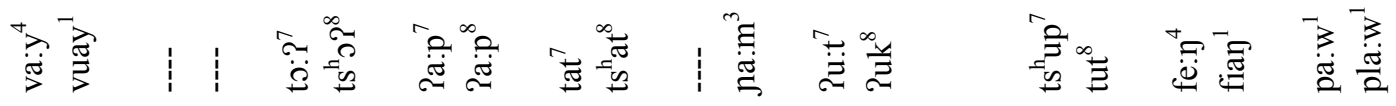

嚄|

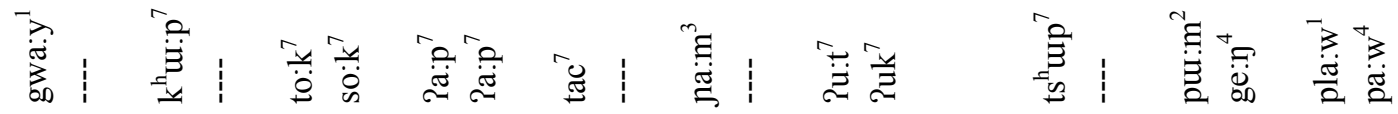

|1 1 ||

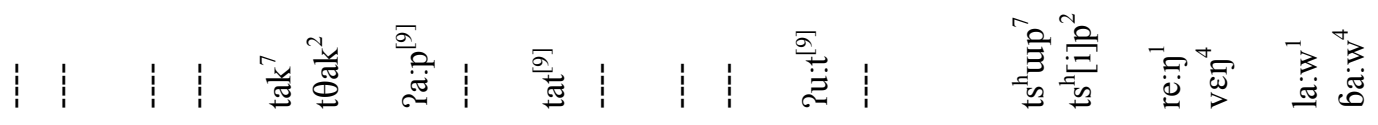

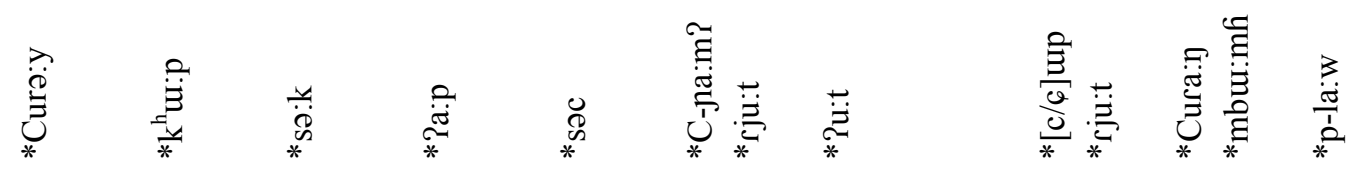

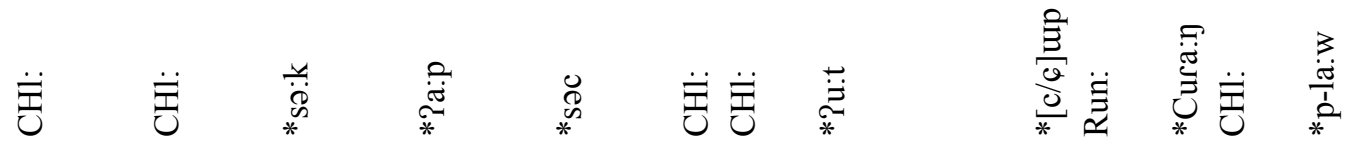

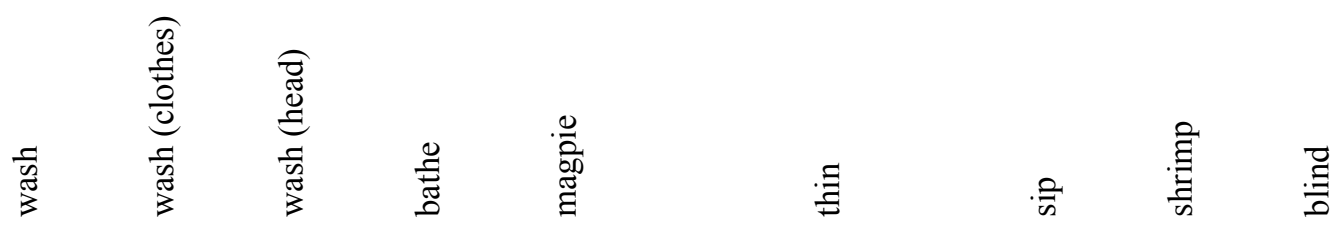

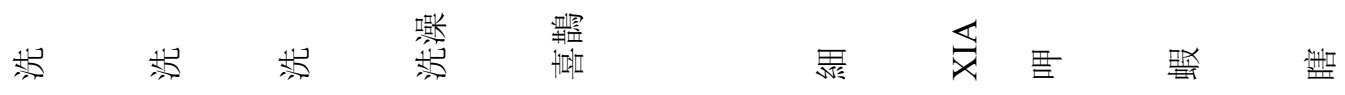




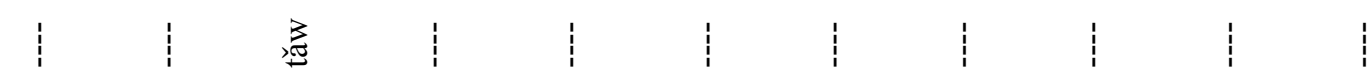

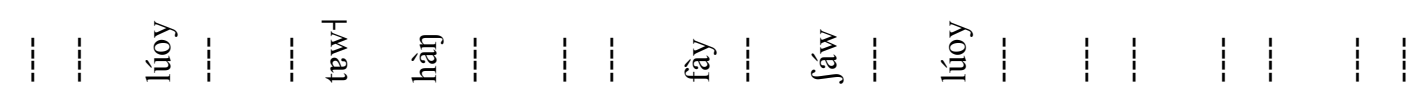

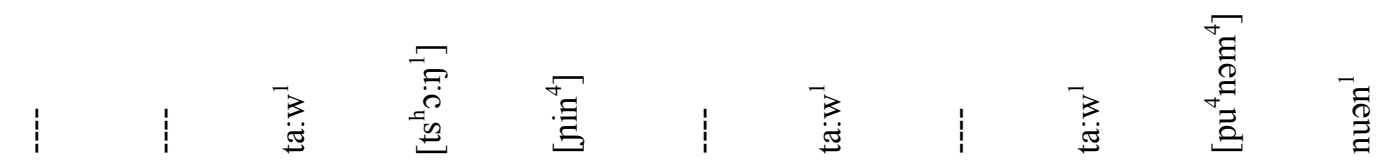

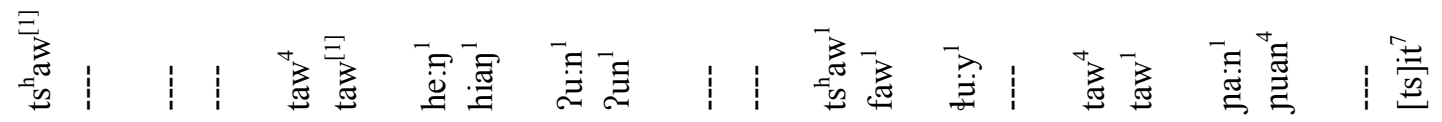

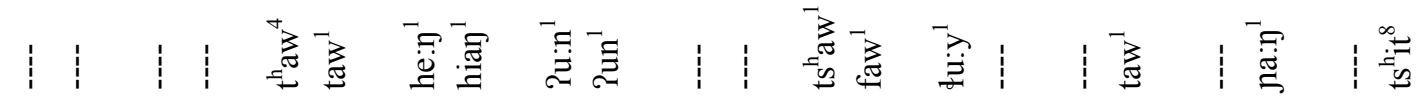

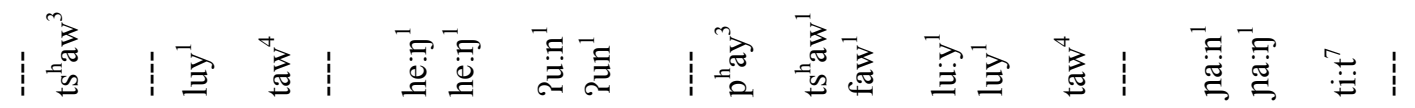

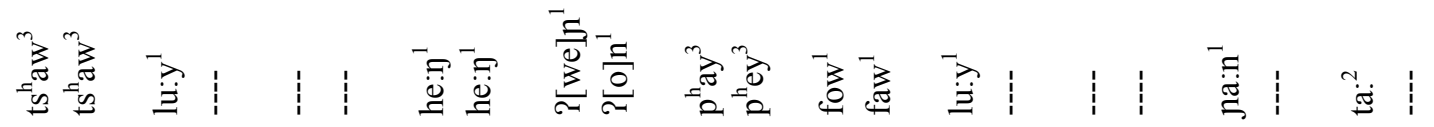

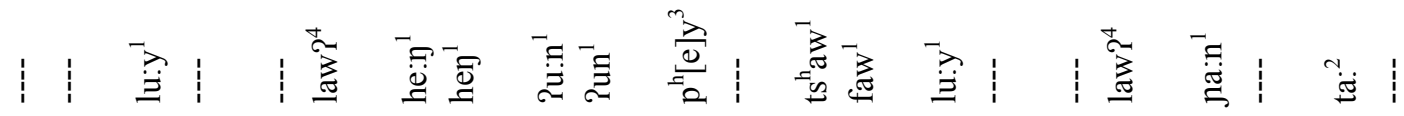

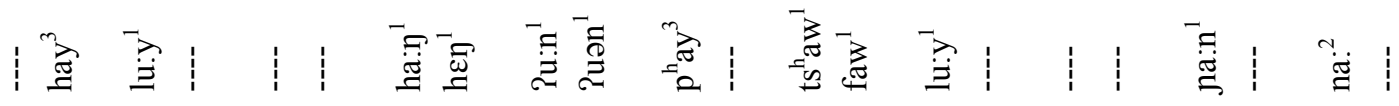

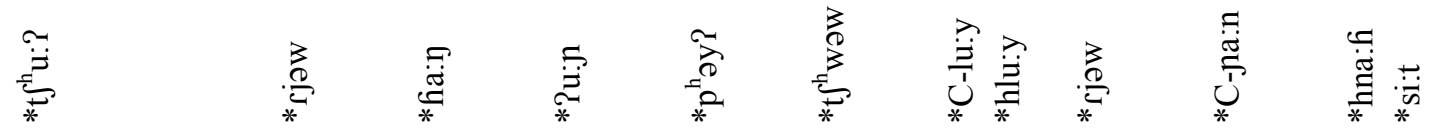

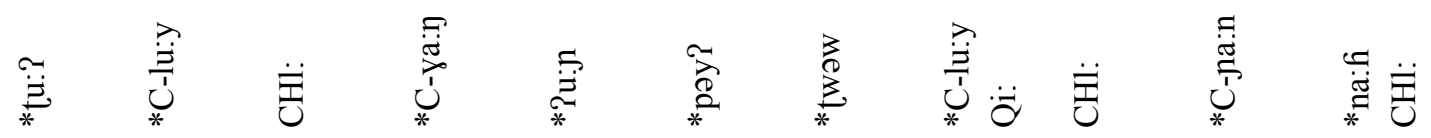

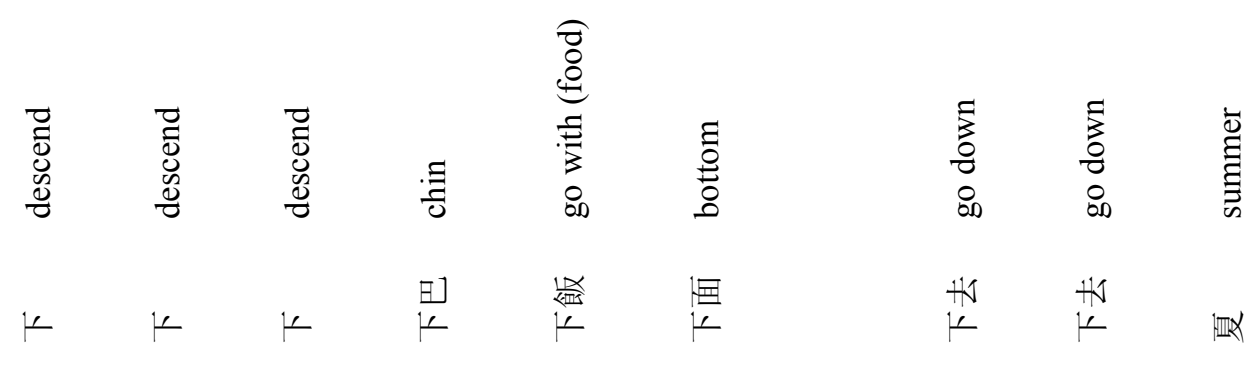




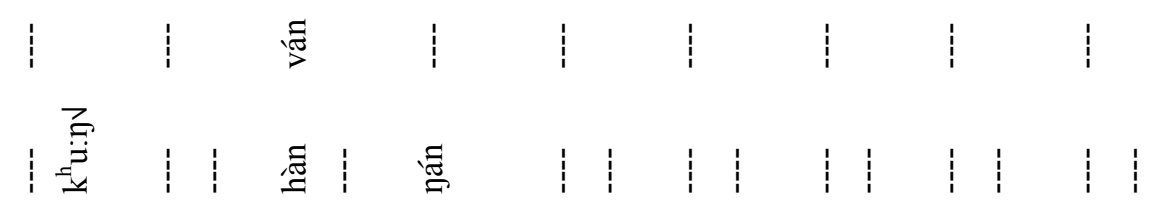

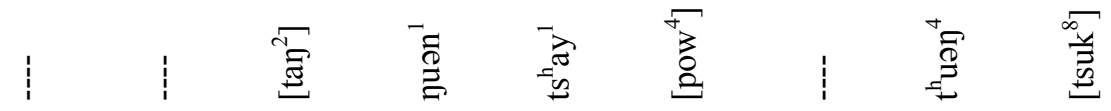

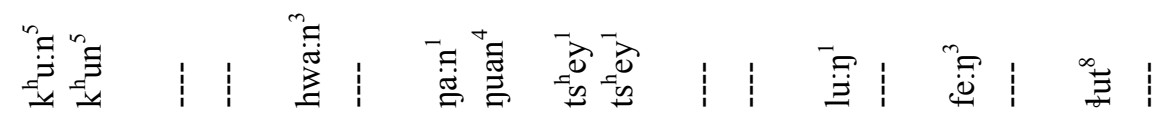

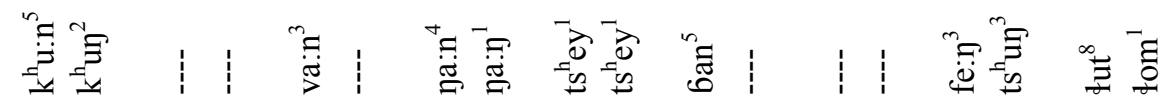

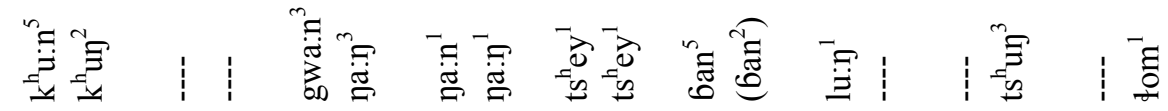

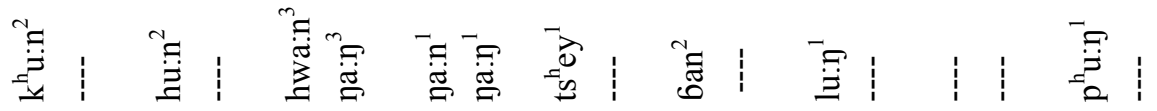

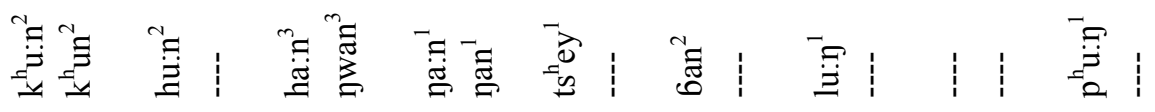

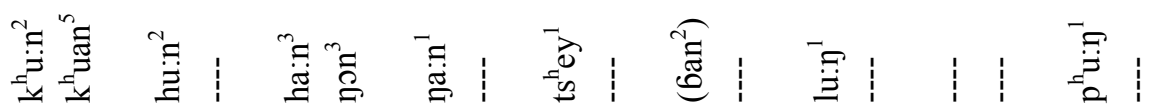

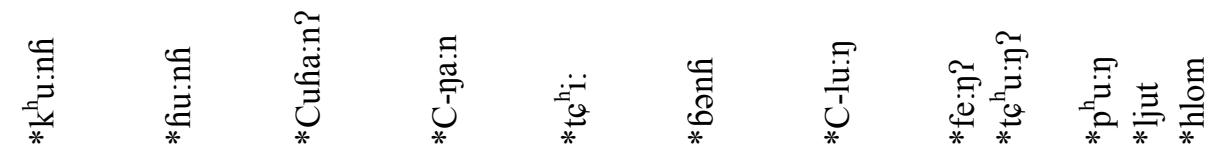

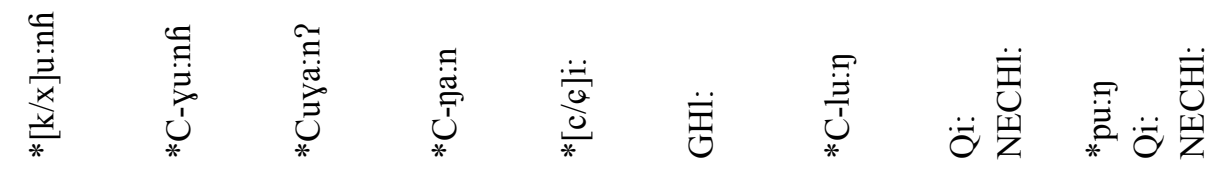

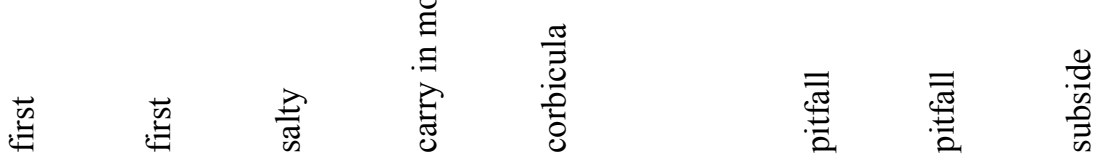

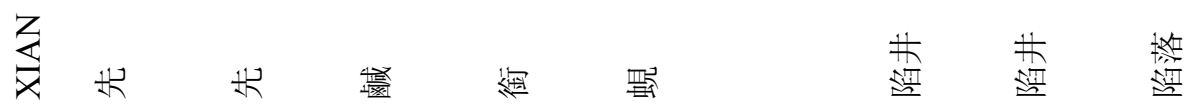




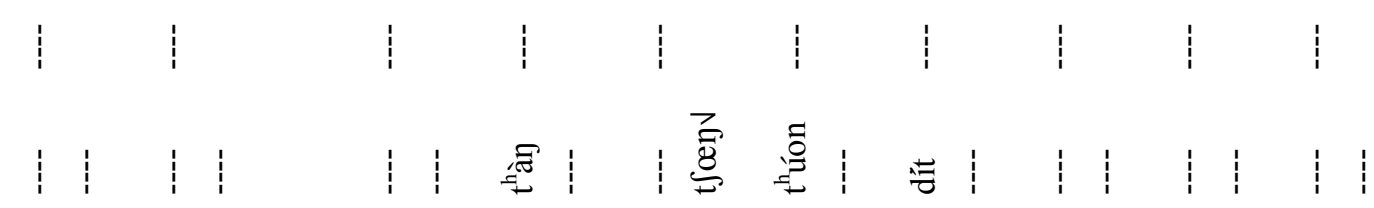

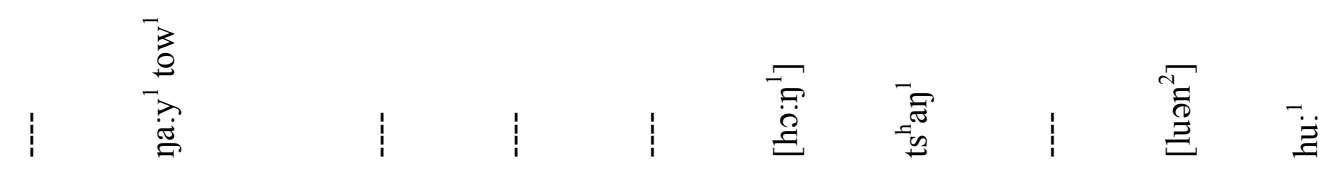

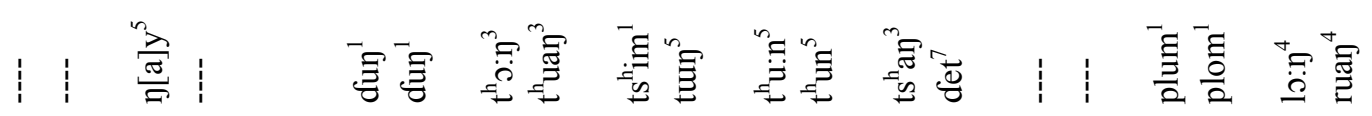

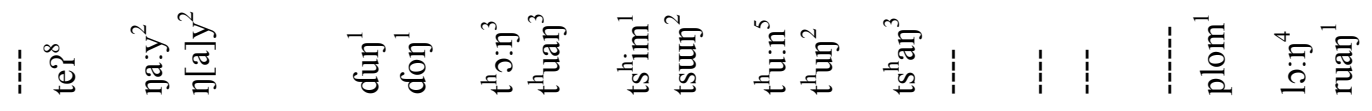

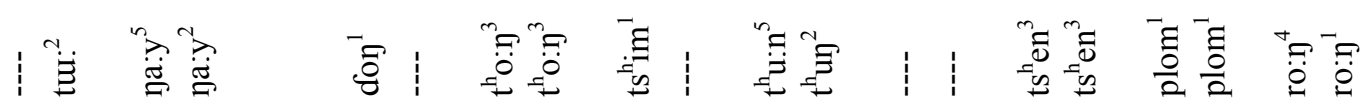

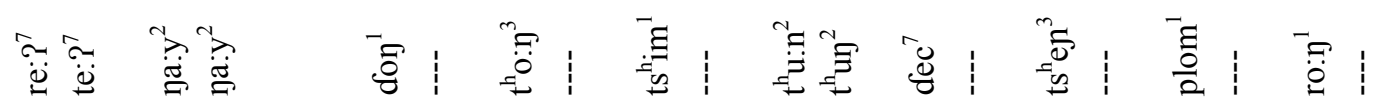

|

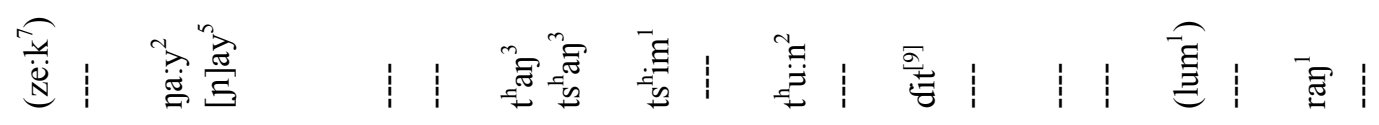

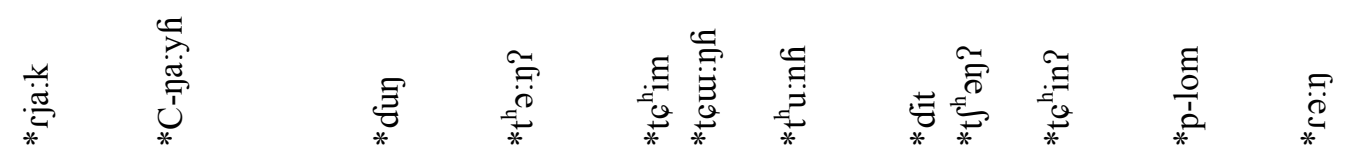

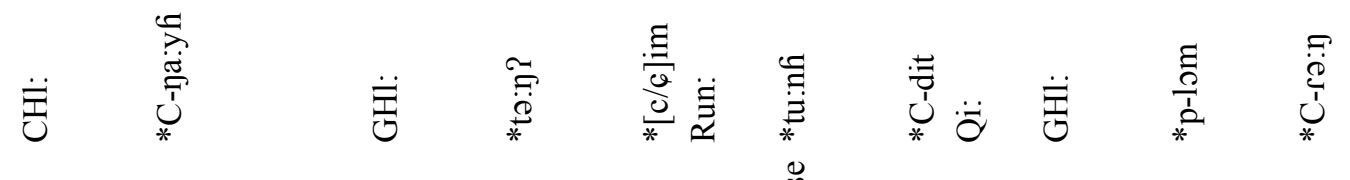

言

鄫

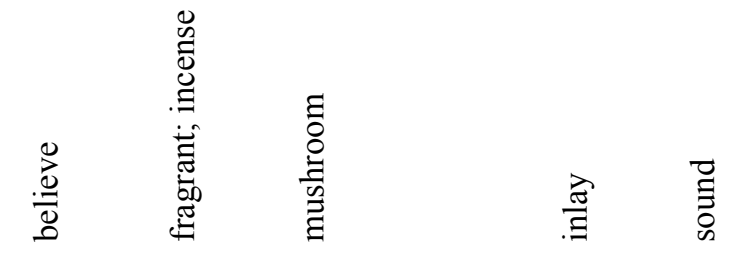

熼

美蛋

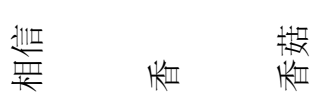

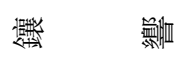




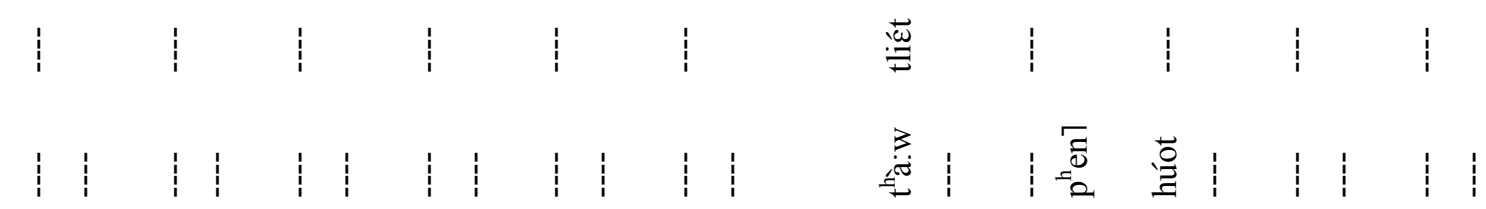

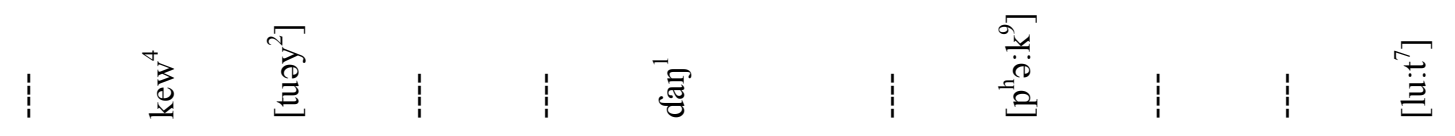

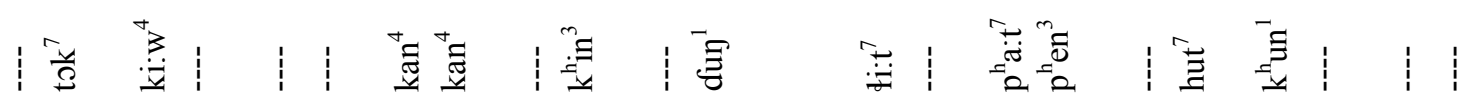

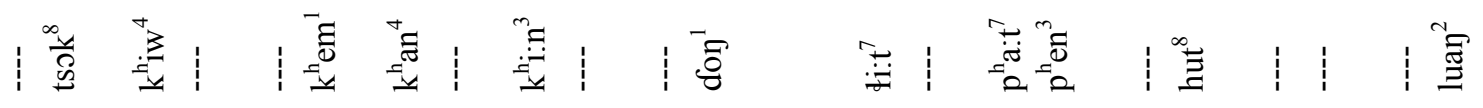

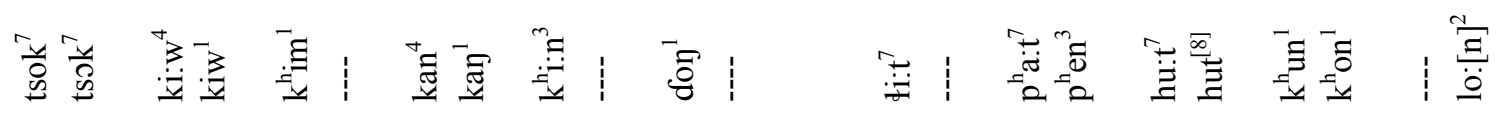

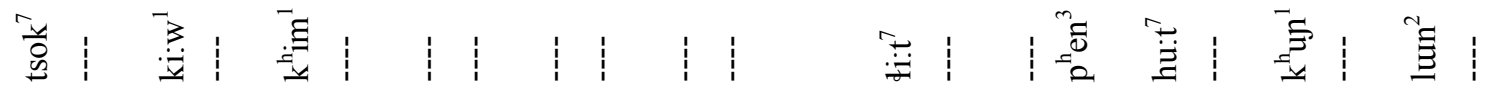

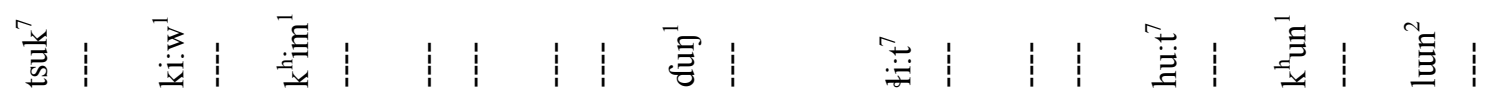

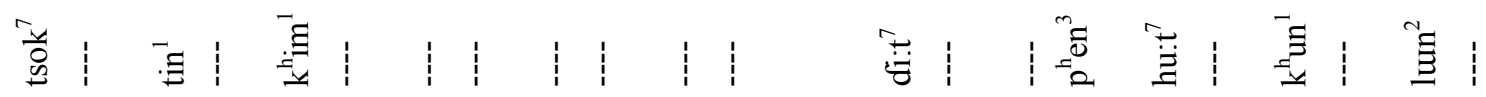

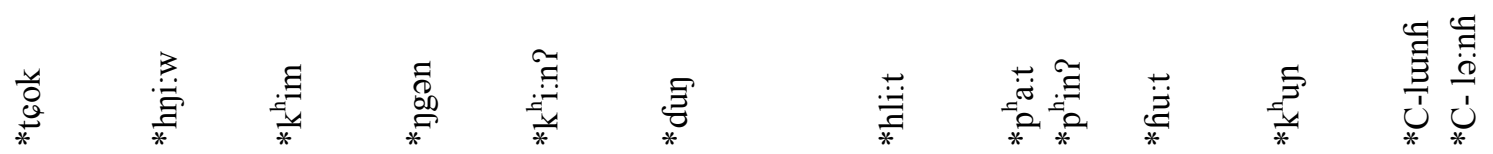

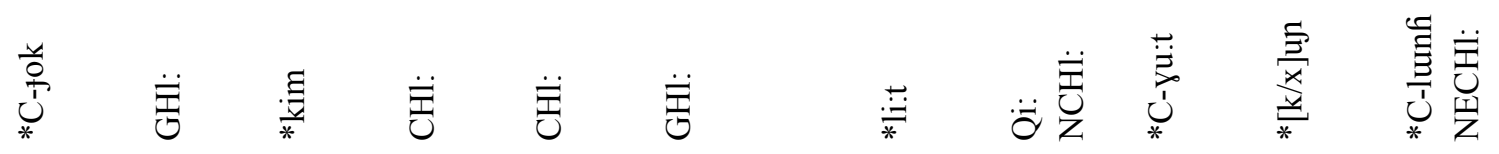

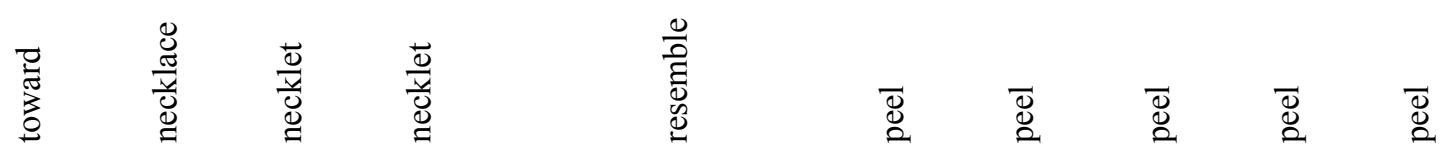

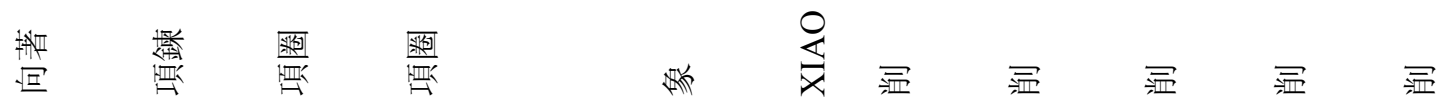




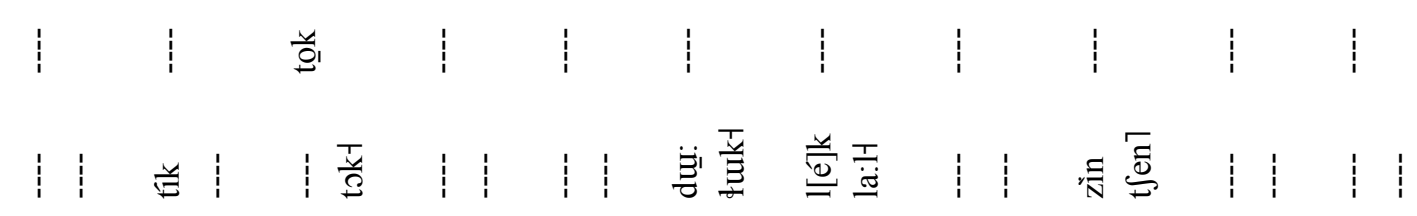

|

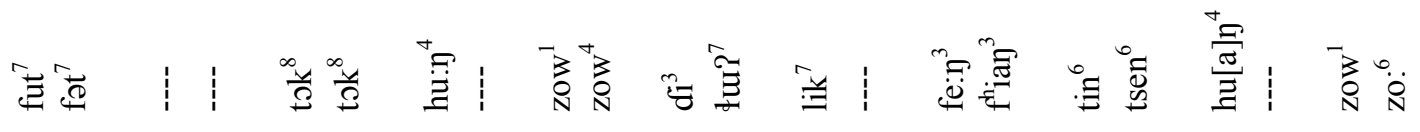

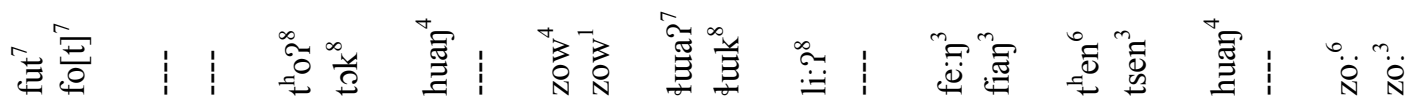

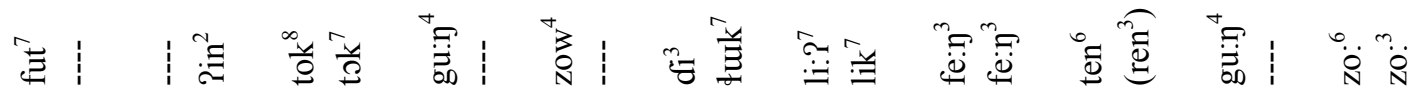

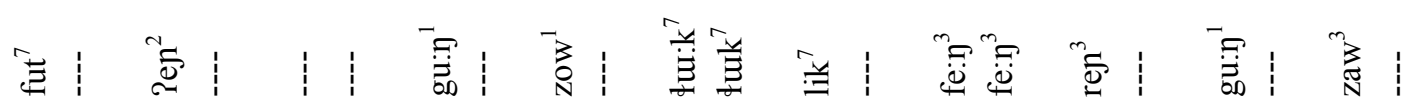

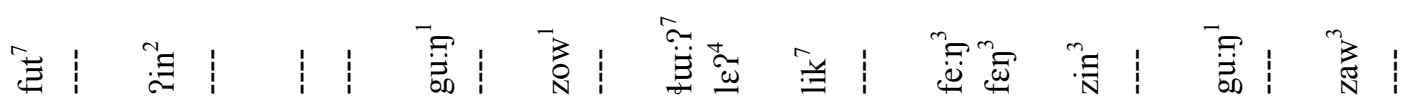

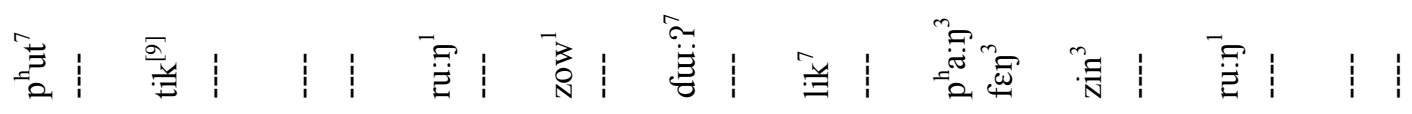

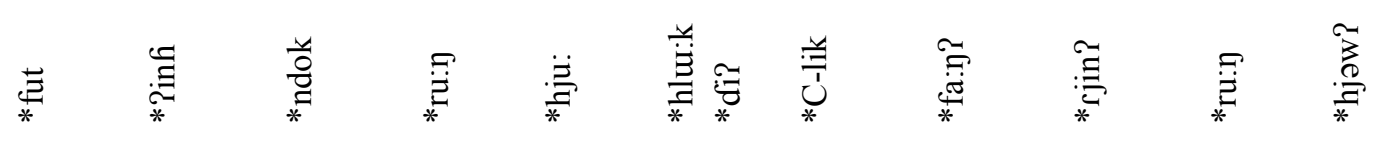

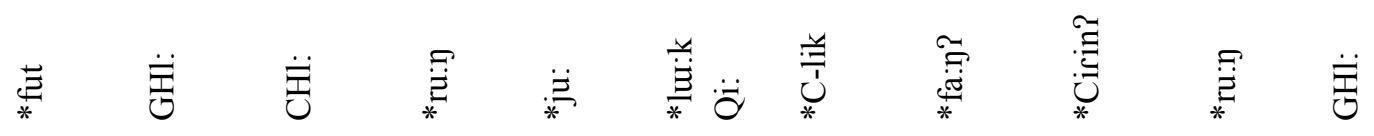

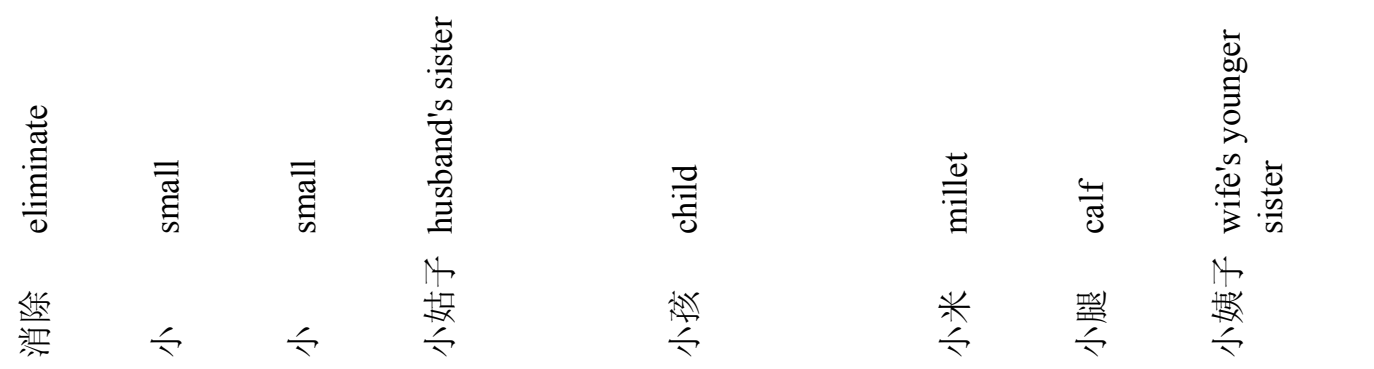




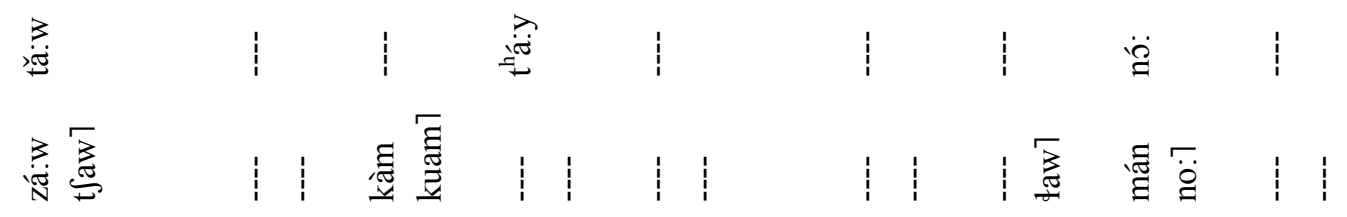

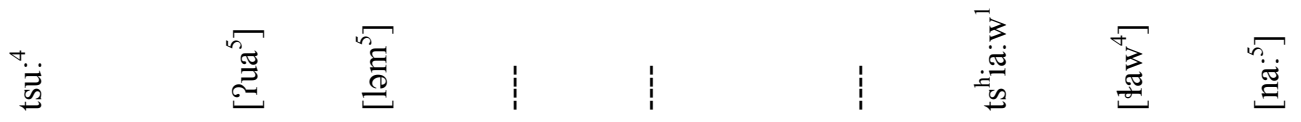

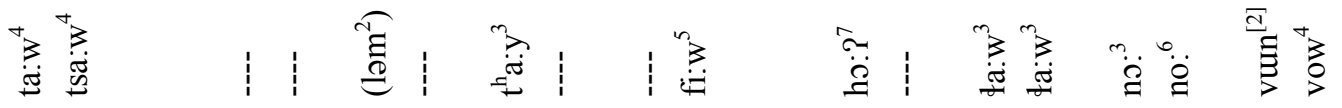

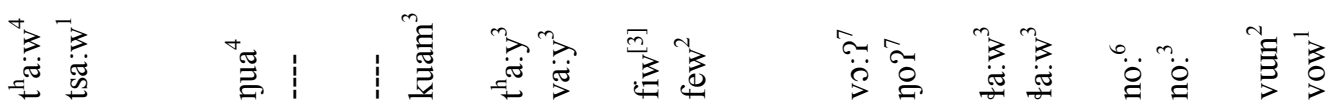

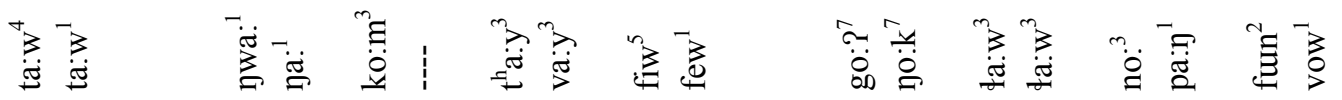

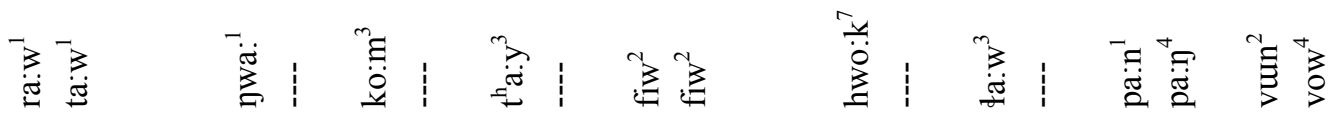

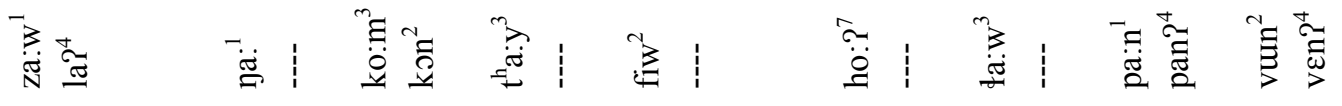

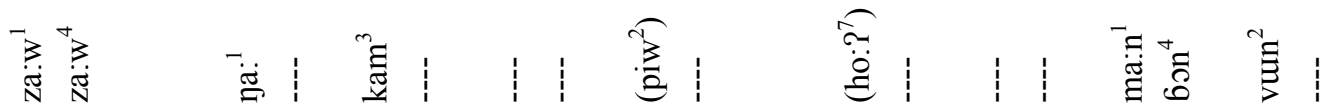

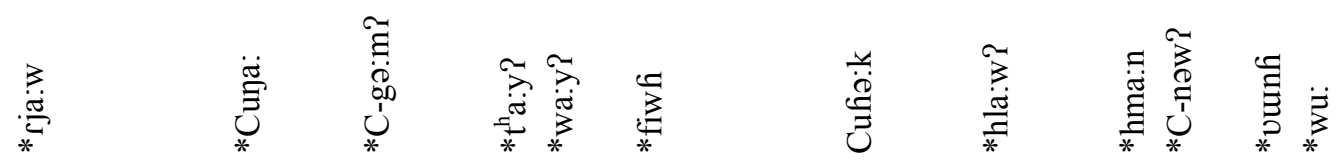

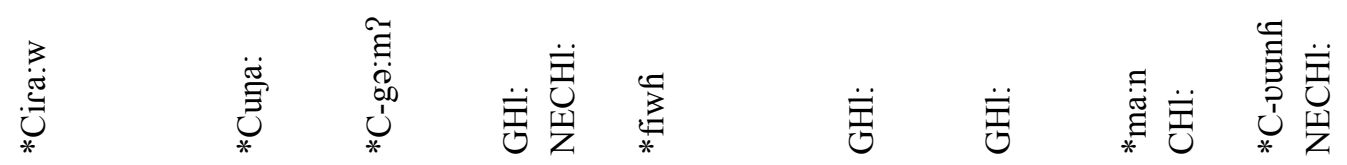

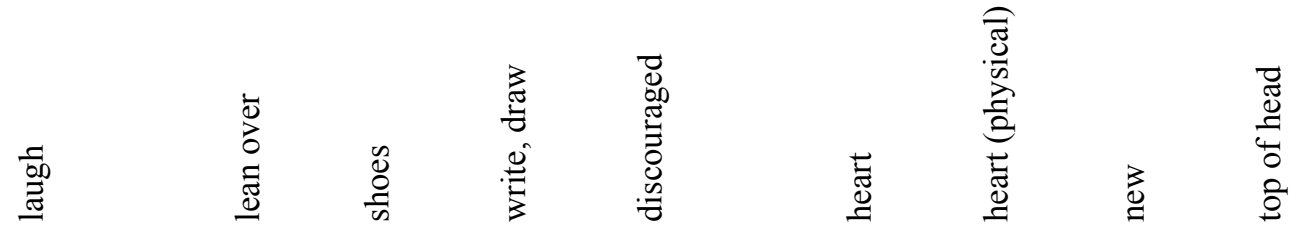

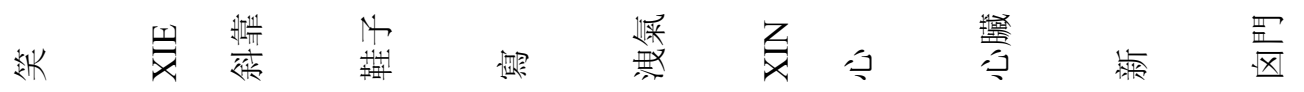




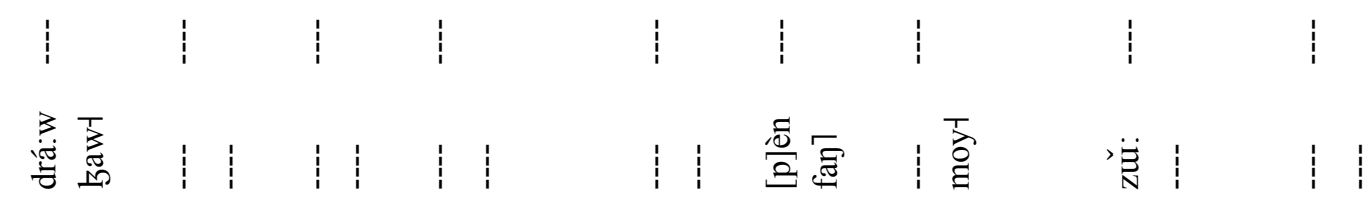

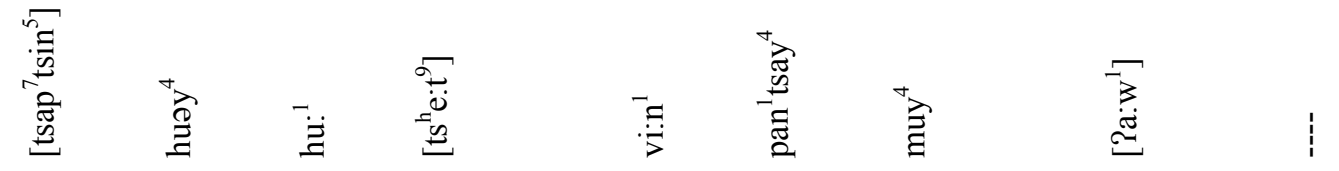

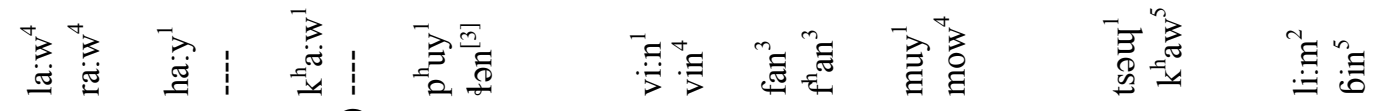

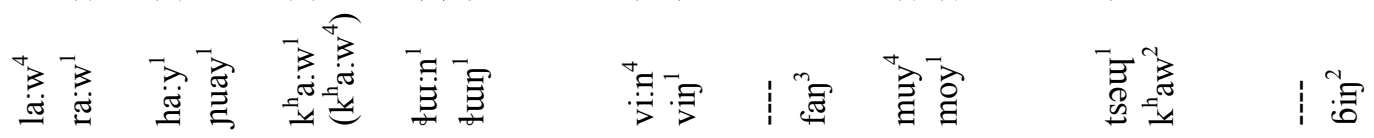

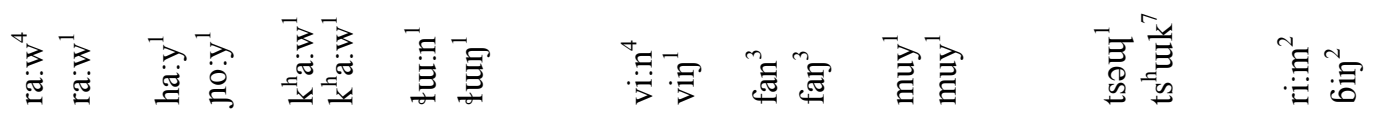

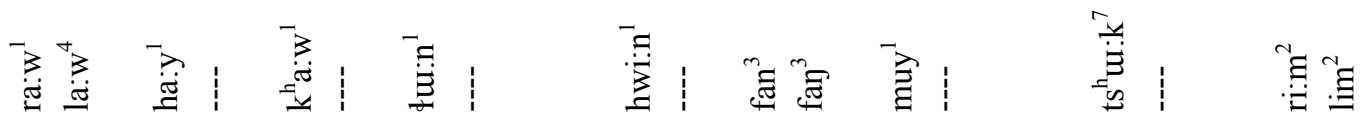

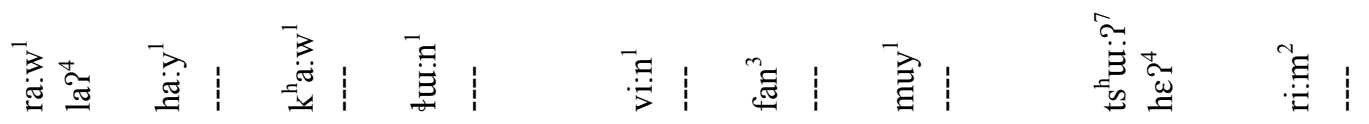

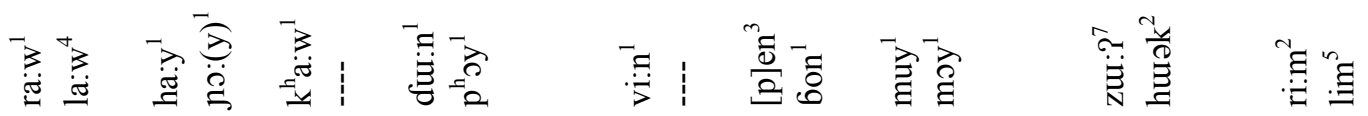

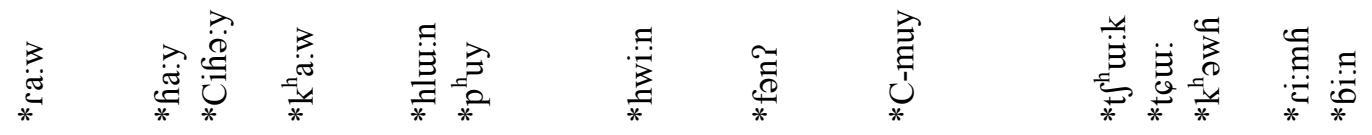

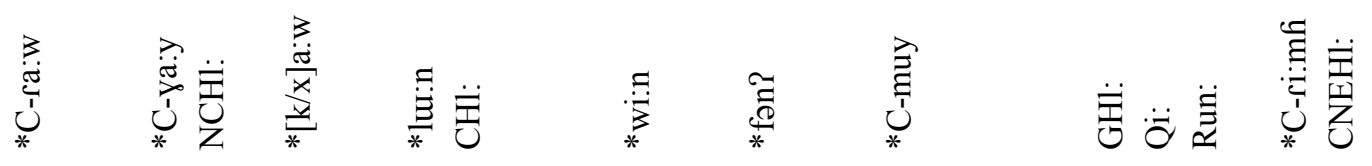

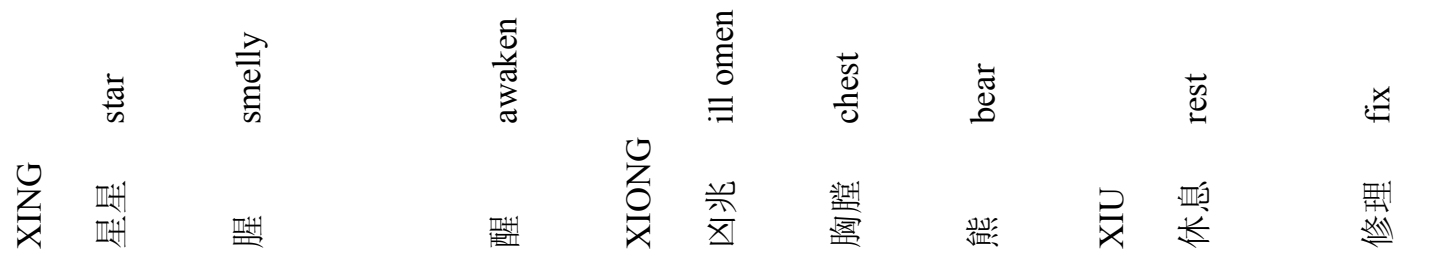




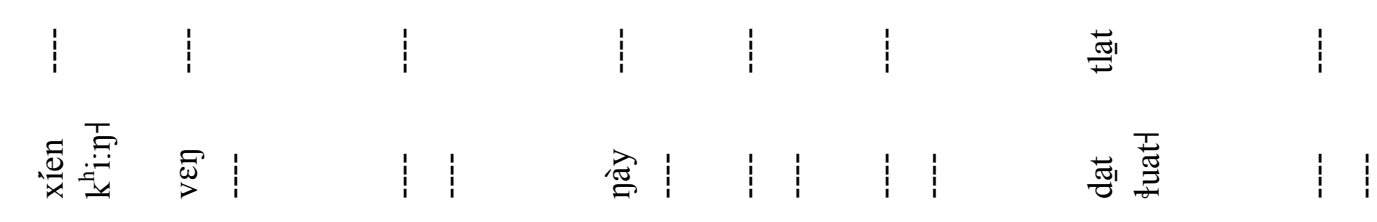

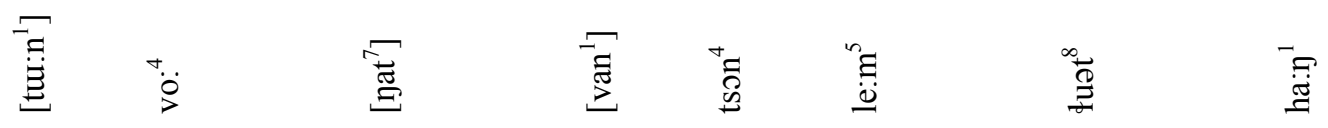

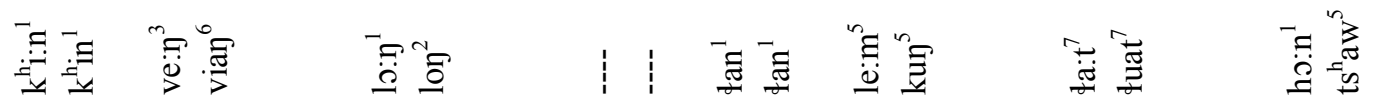

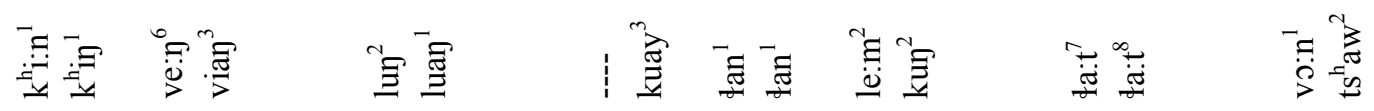

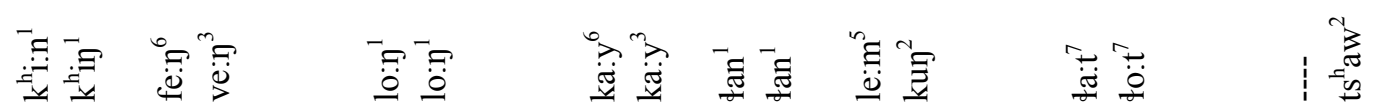

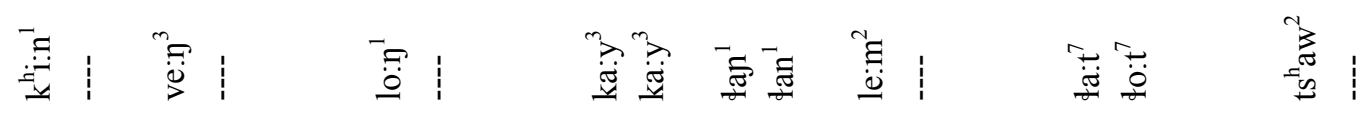

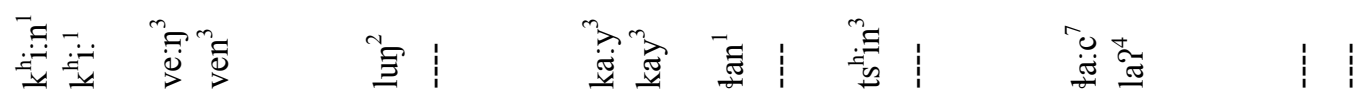

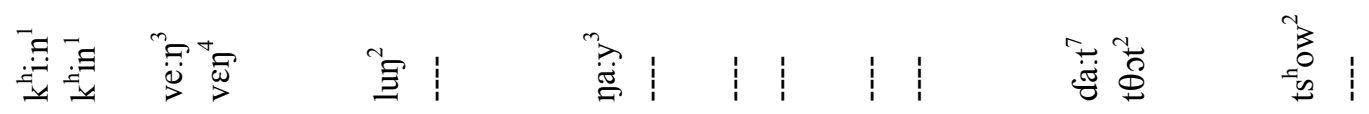

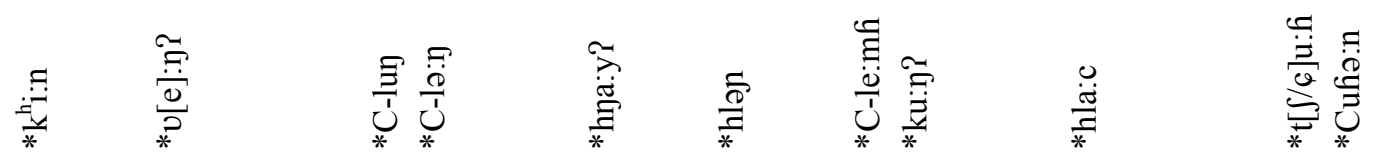

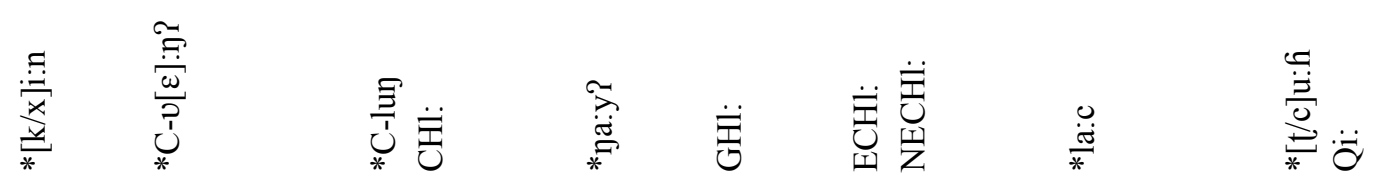

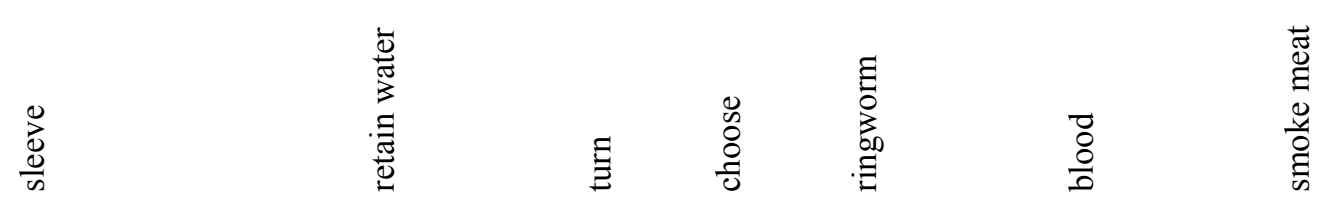

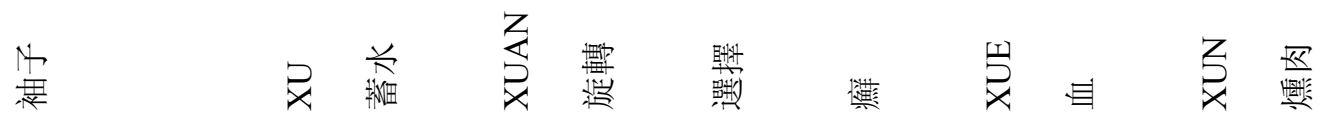


\begin{tabular}{l|l|l|l|l|l|l}
1 & 1 & 1 & 1 & & 1 & 1 \\
1 & $\mid 1$
\end{tabular}

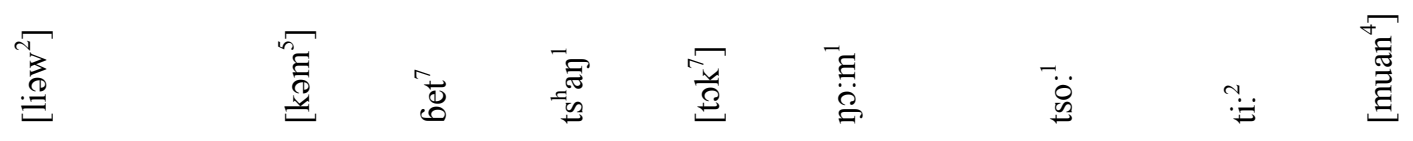

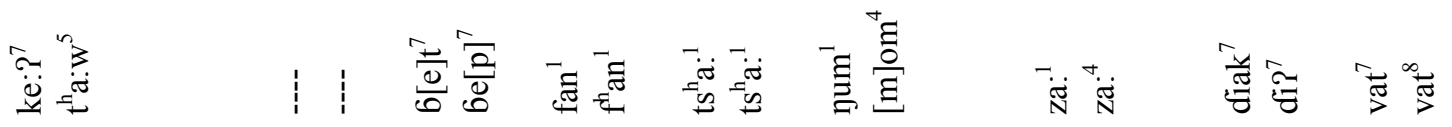

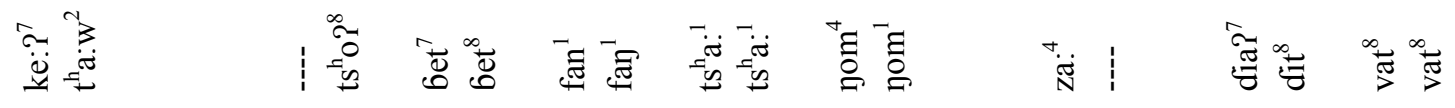

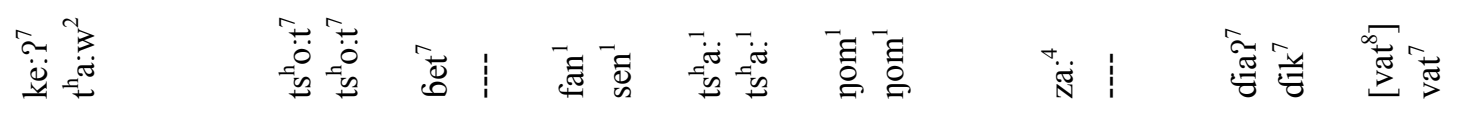

㟥|

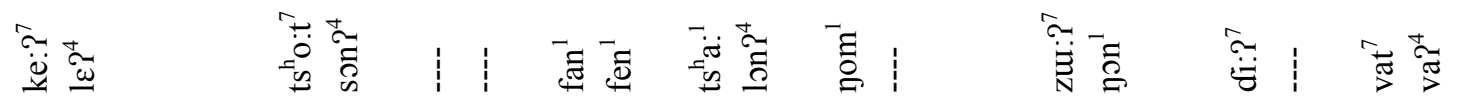

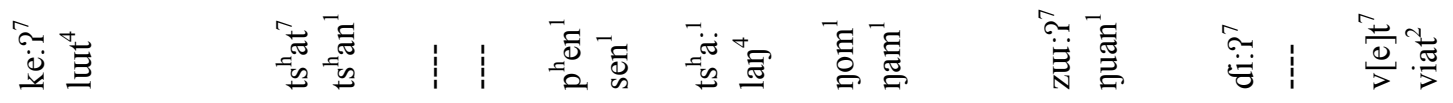

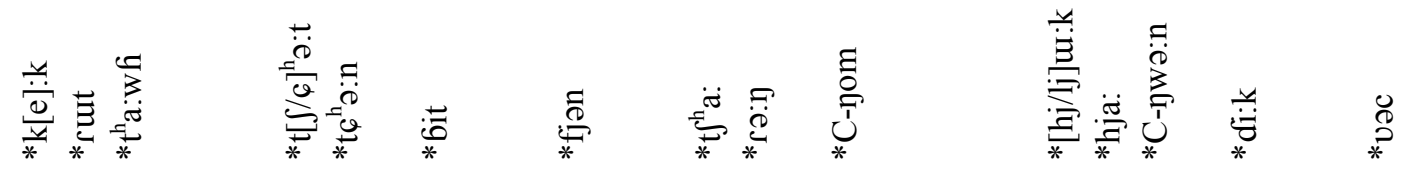

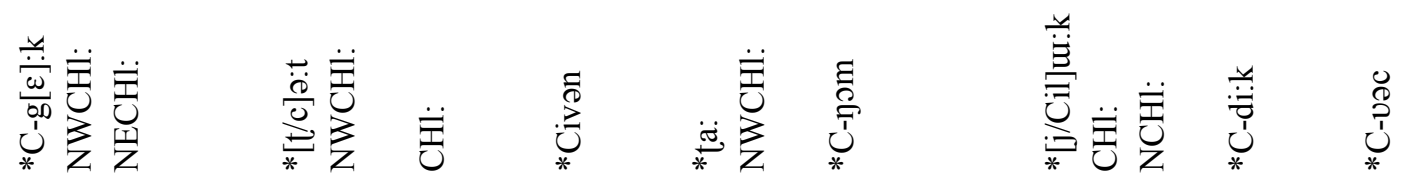

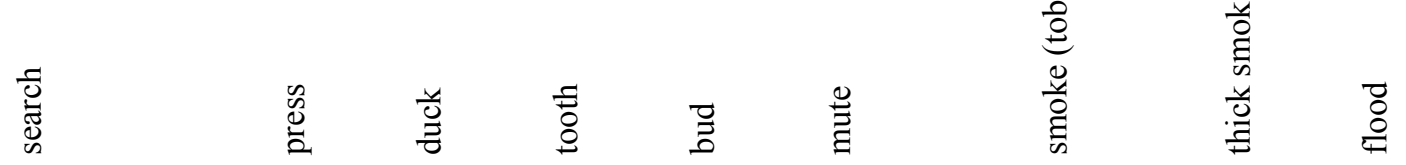

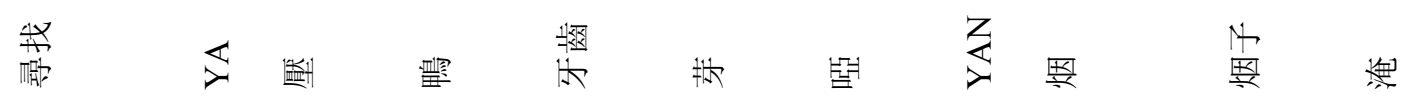


$11+1|1| 111$

童

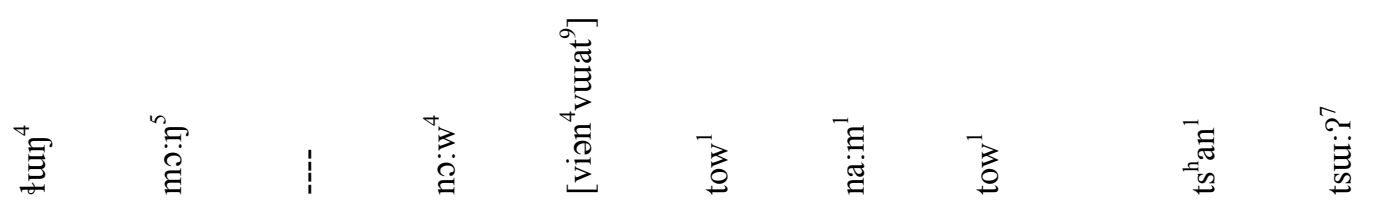

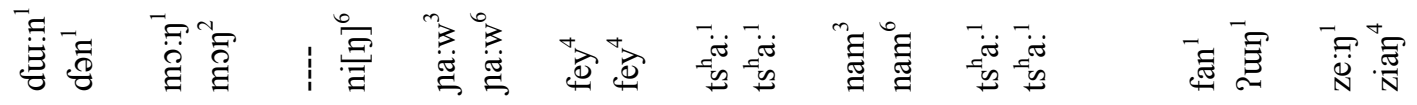

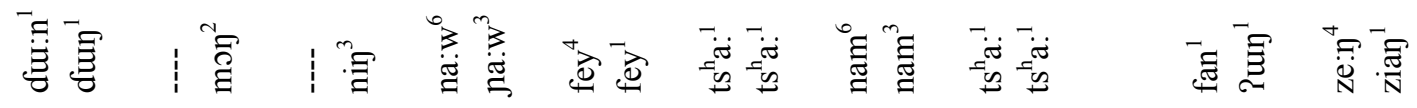

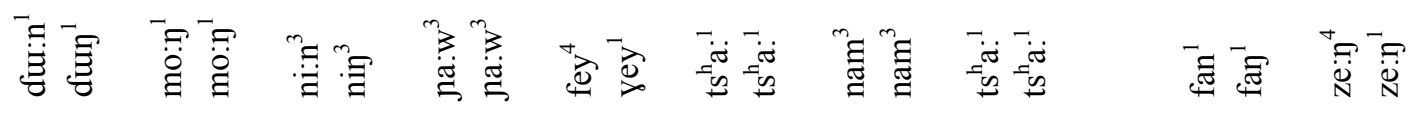

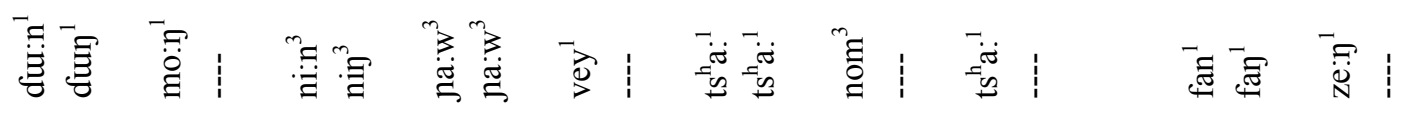

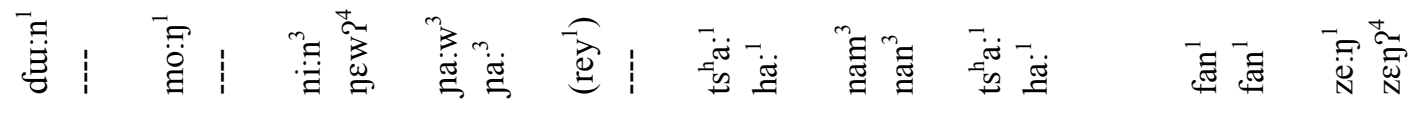

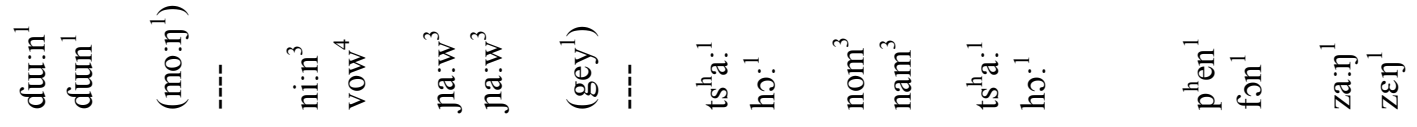

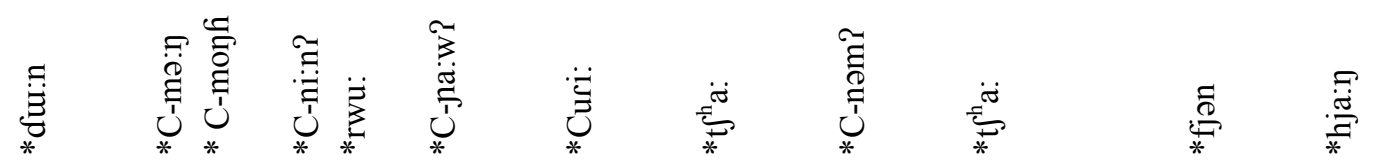

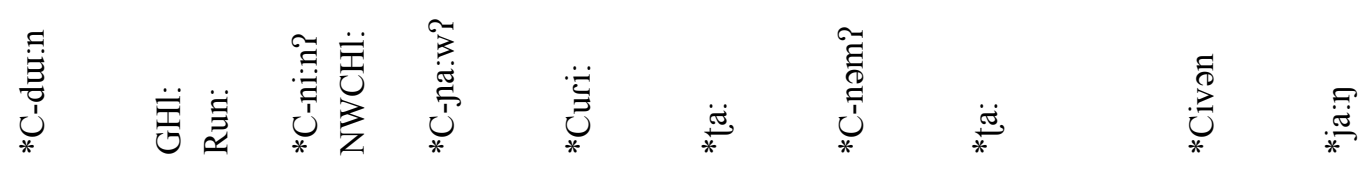

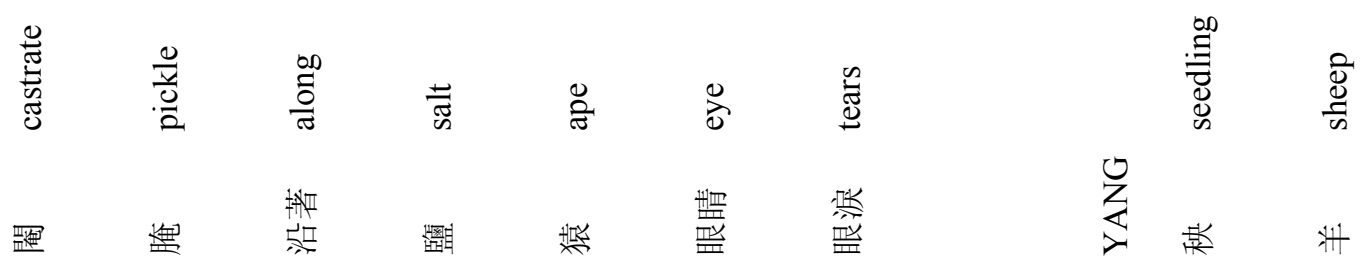




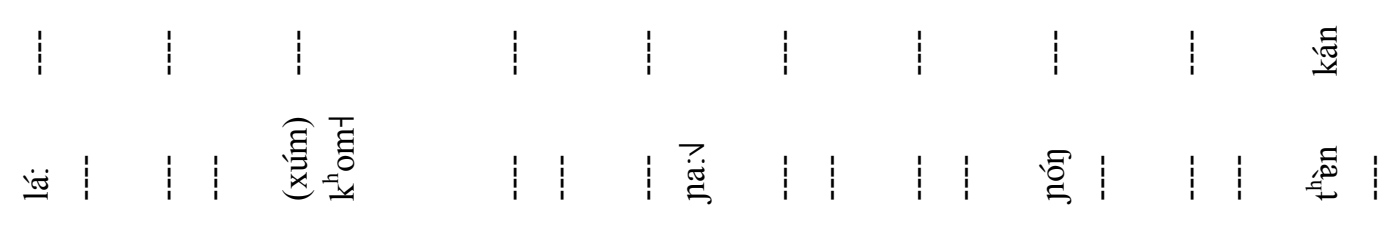

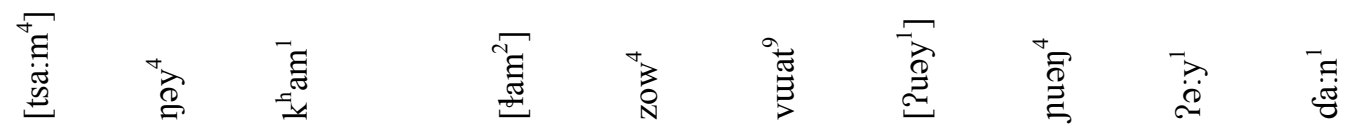

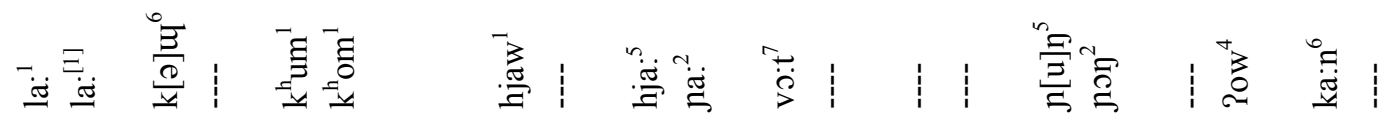

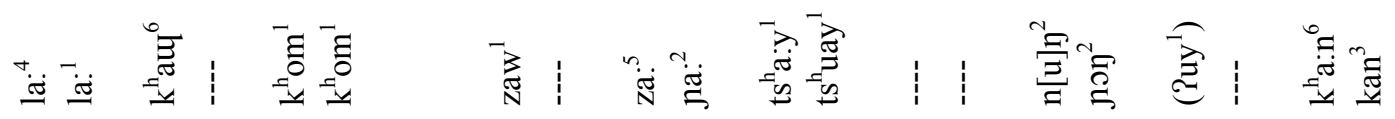

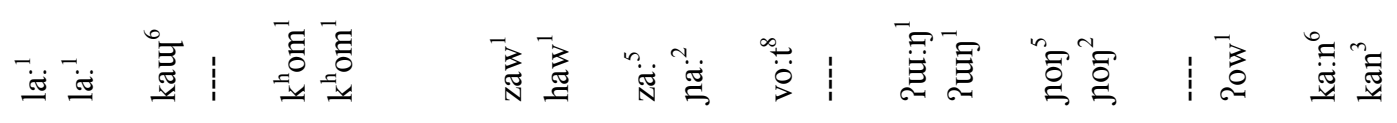

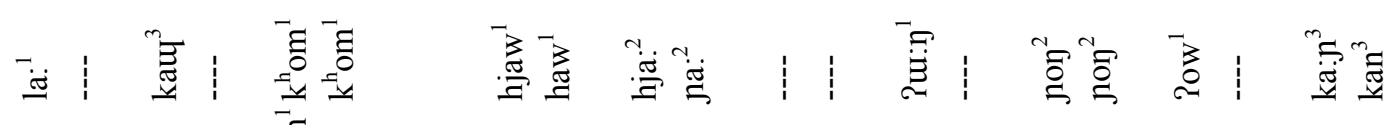

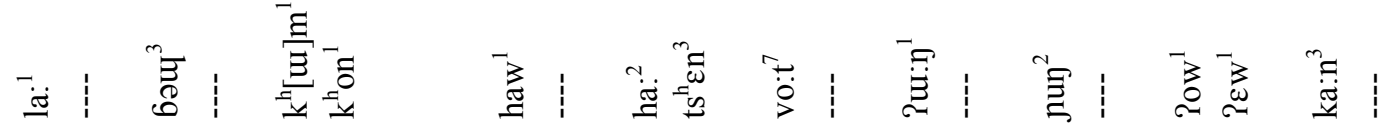

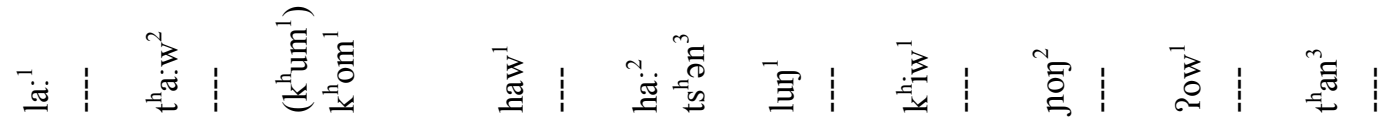

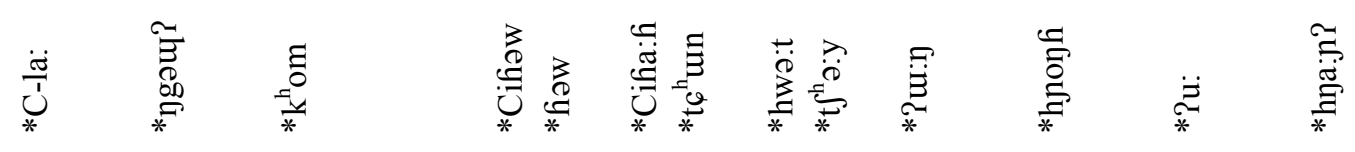

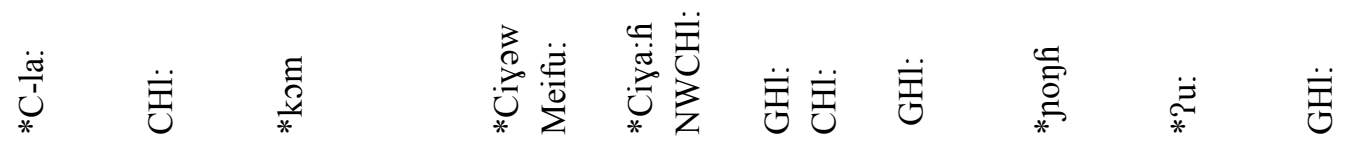

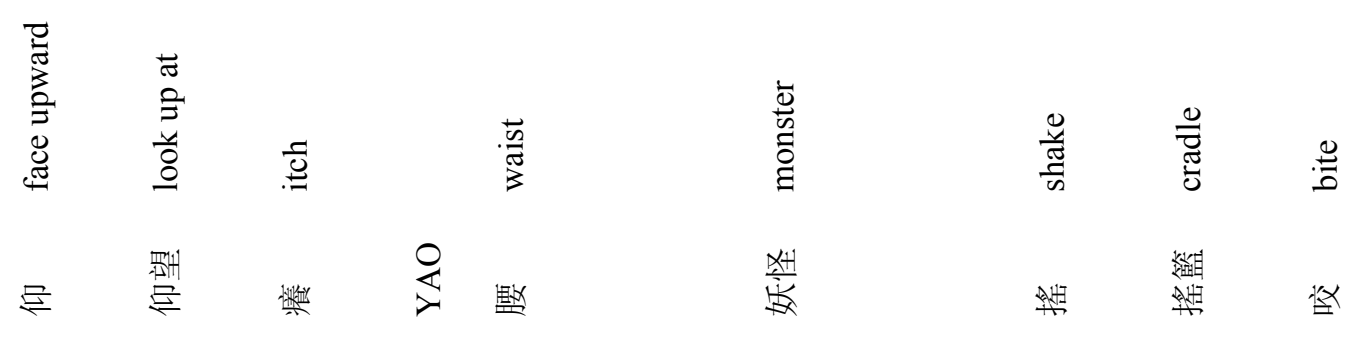




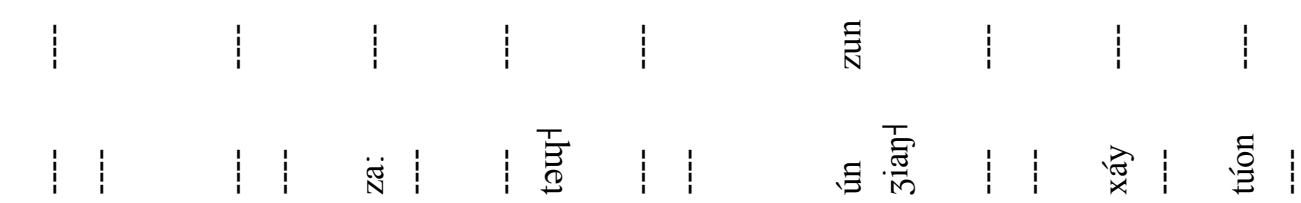

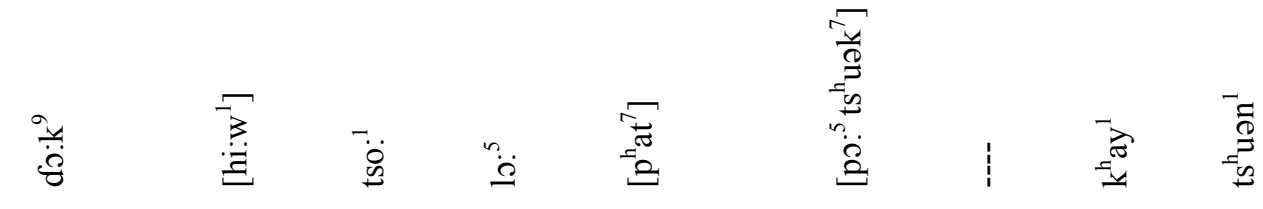

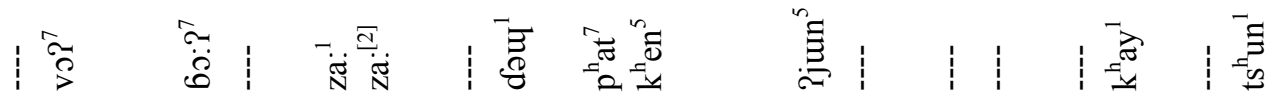

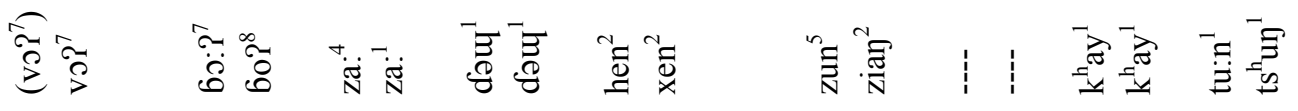

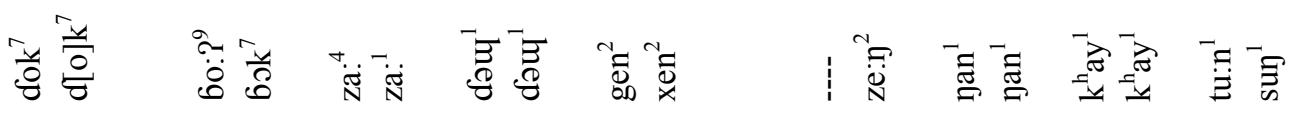

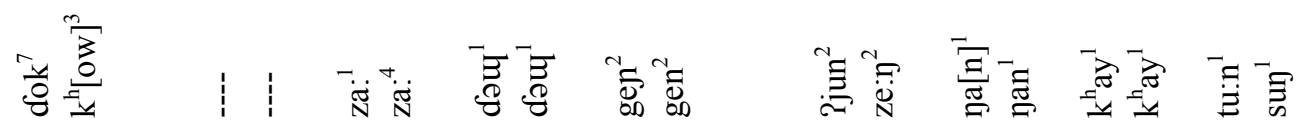

鸢鸢

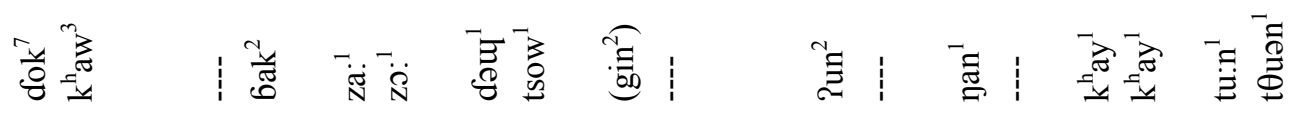

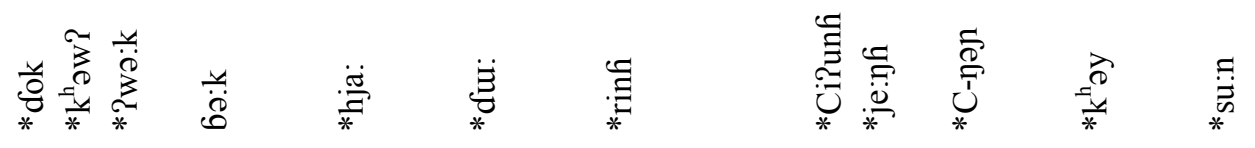

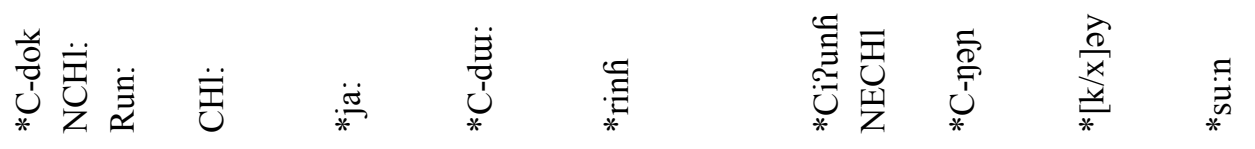

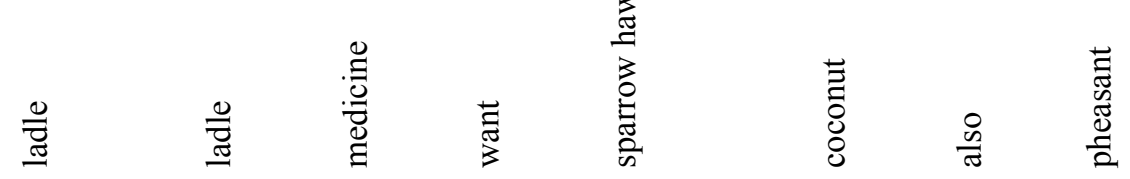

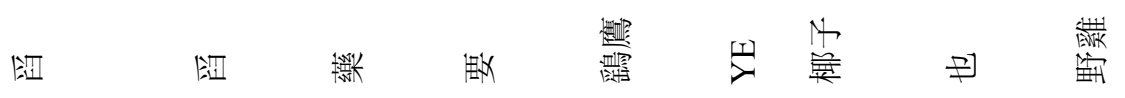




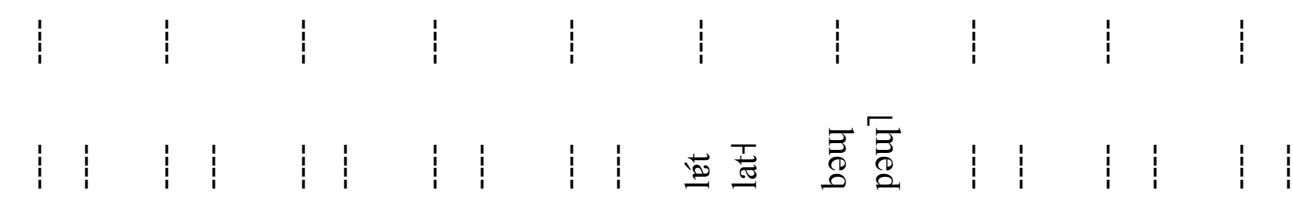

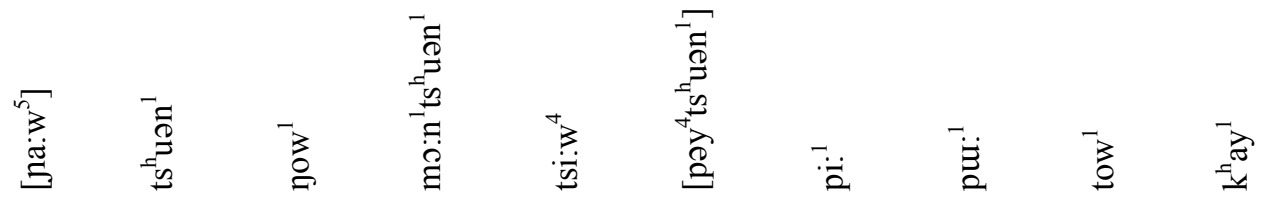

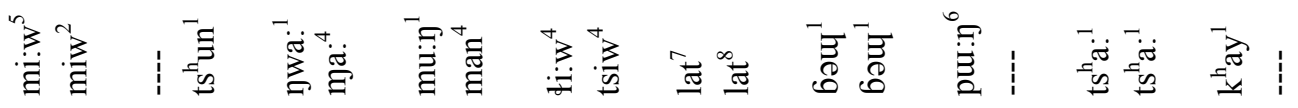

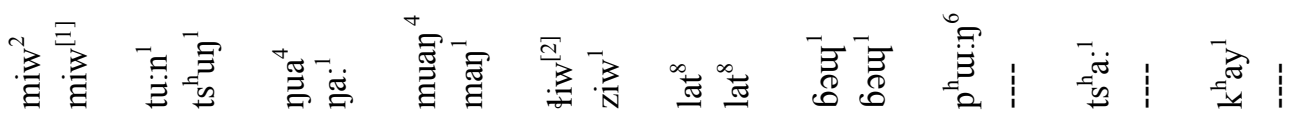

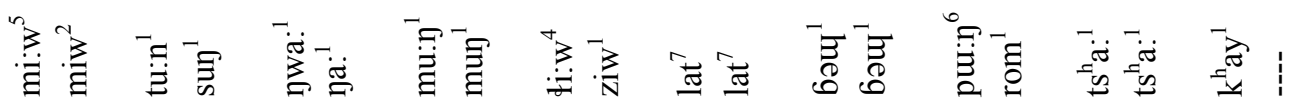

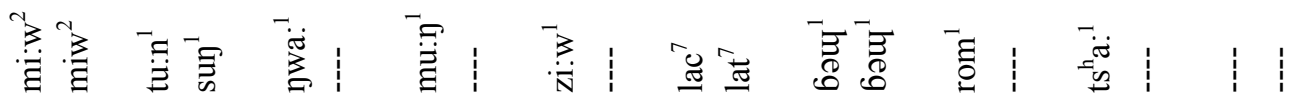

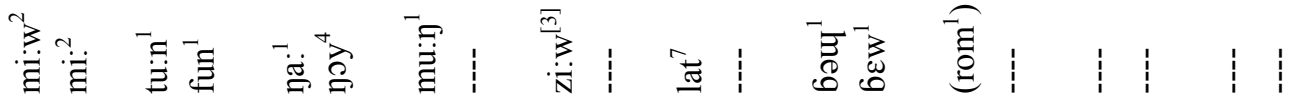

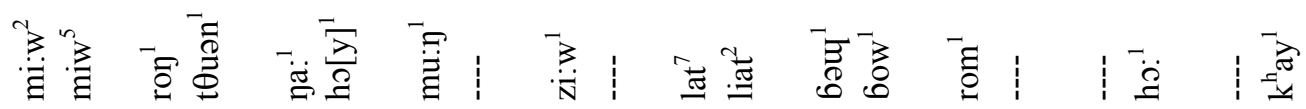

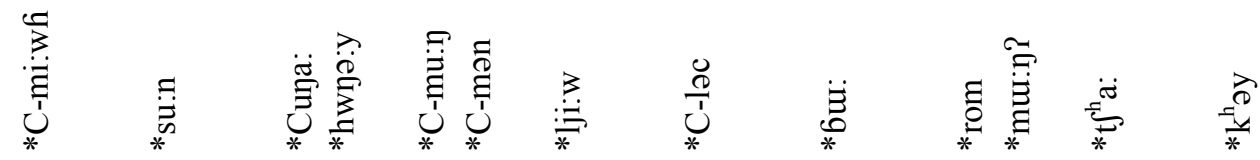

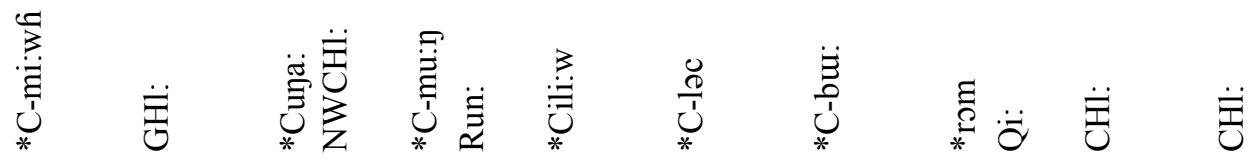

$\frac{\pi}{\frac{\pi}{3}}$

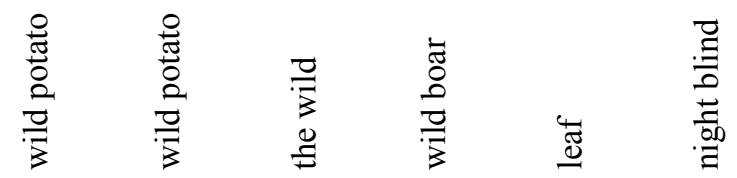

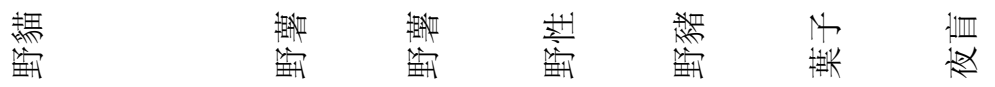




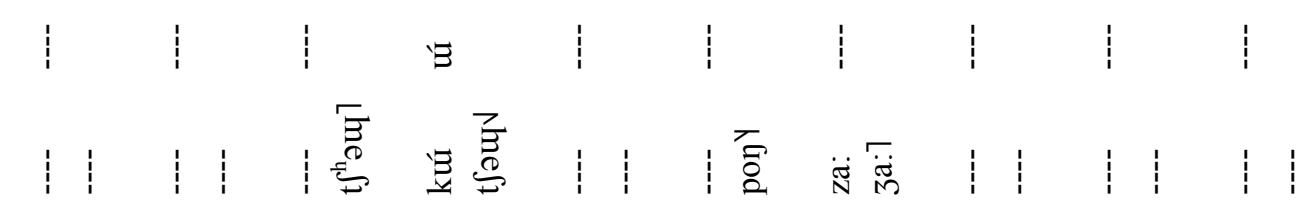

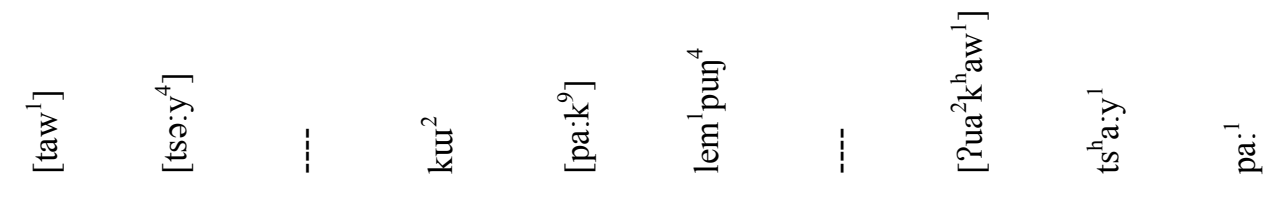

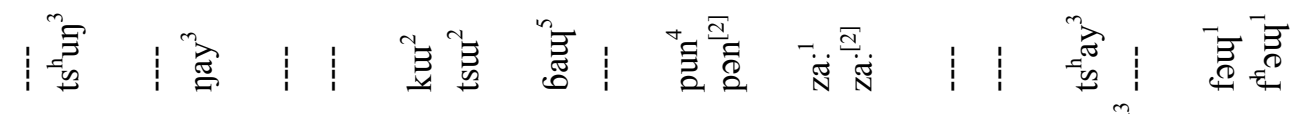

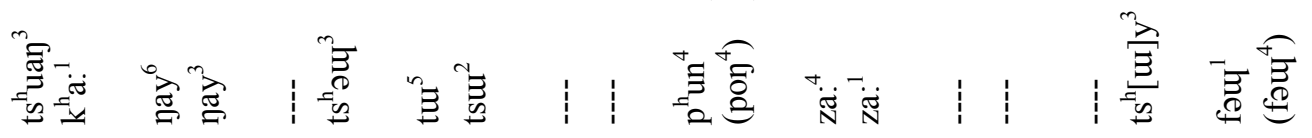

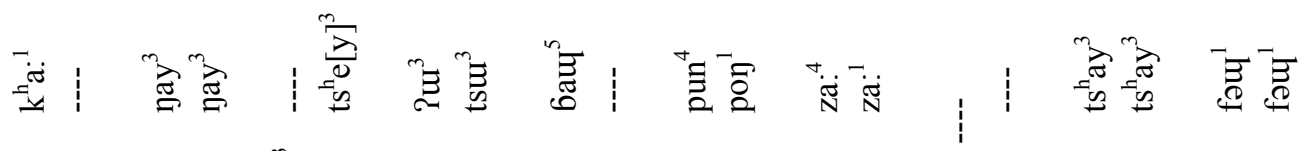

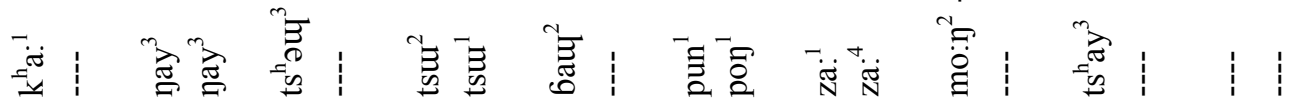

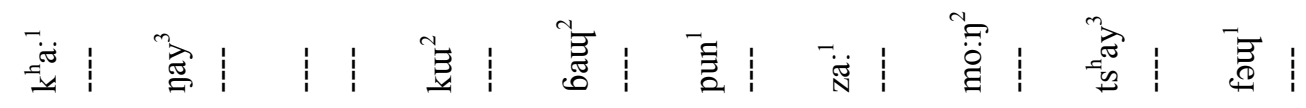

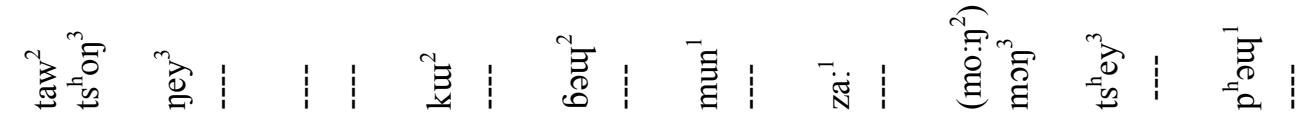

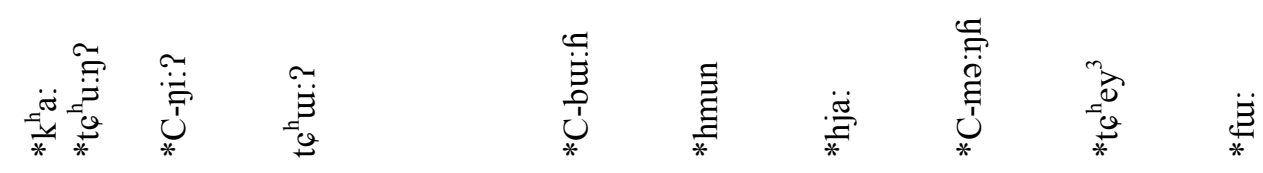

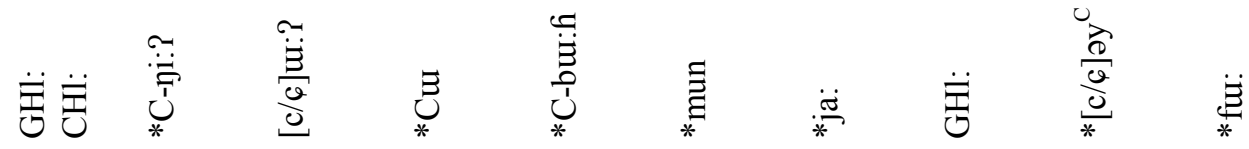

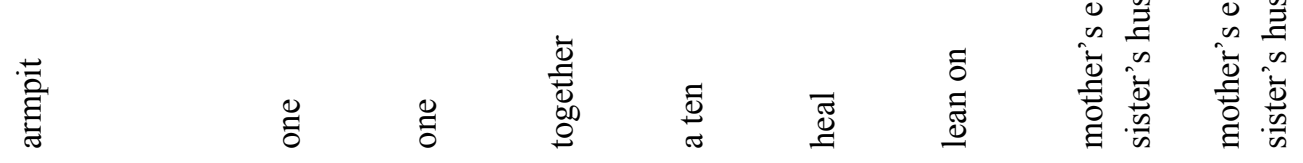

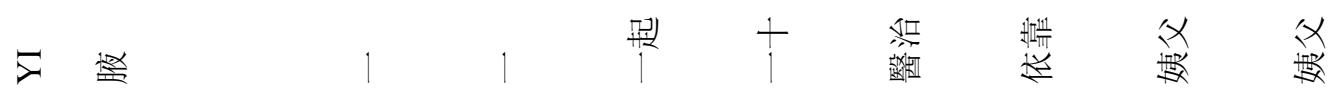




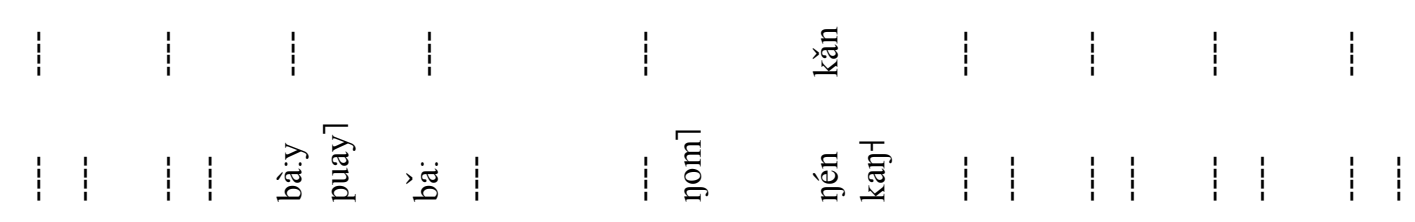

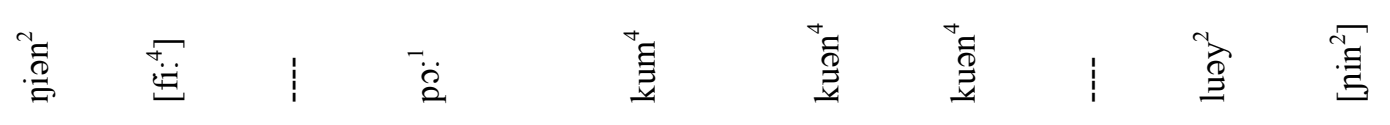

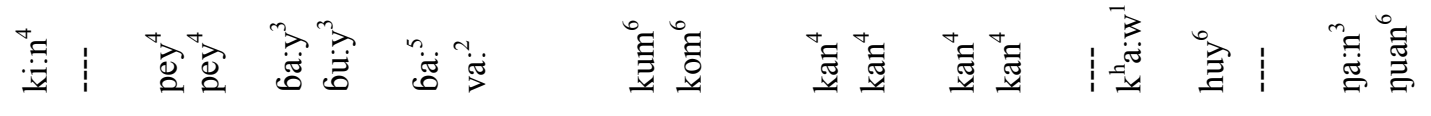

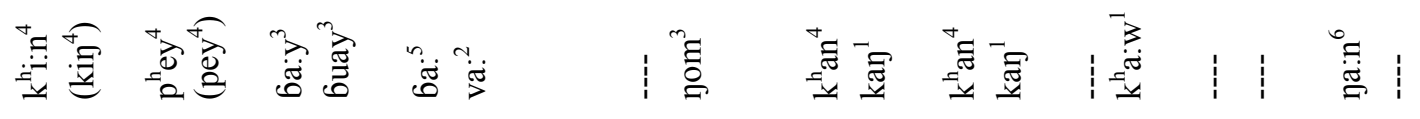

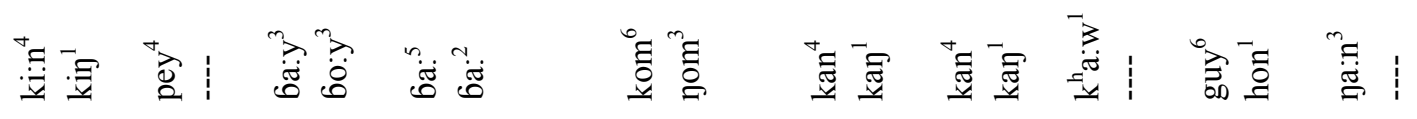

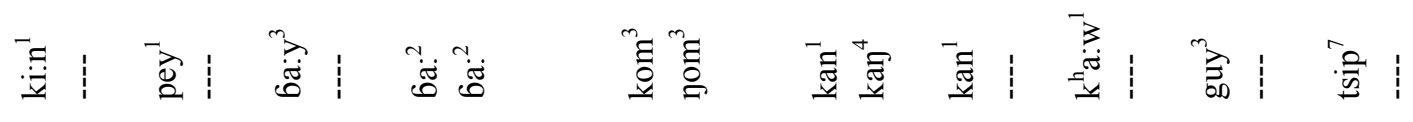

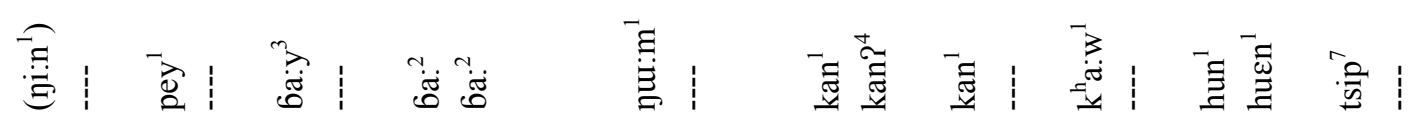

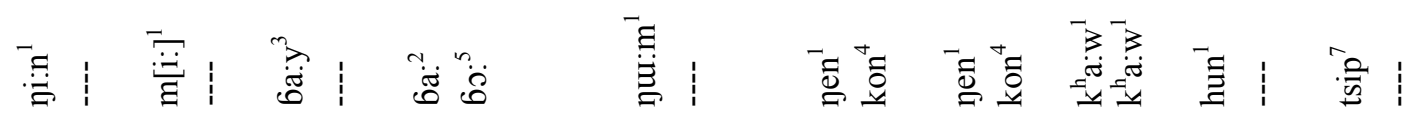

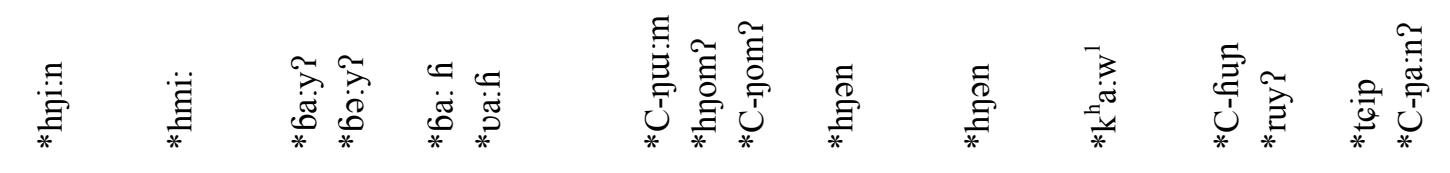

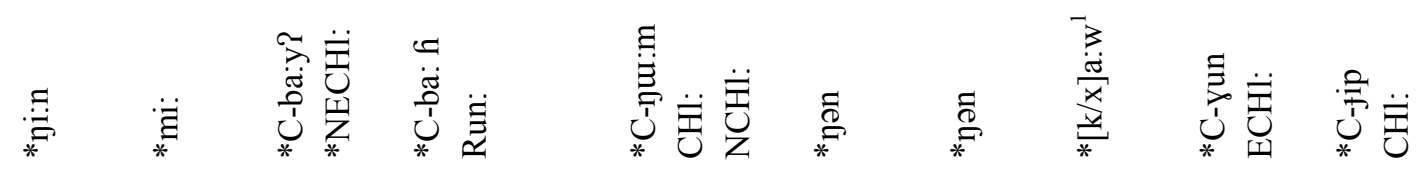

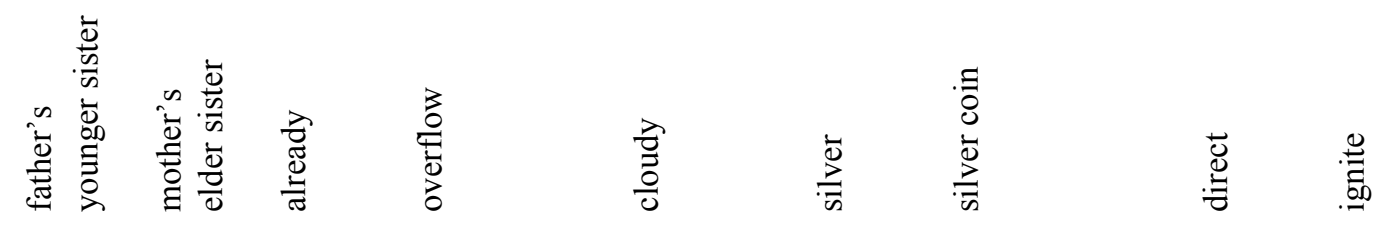

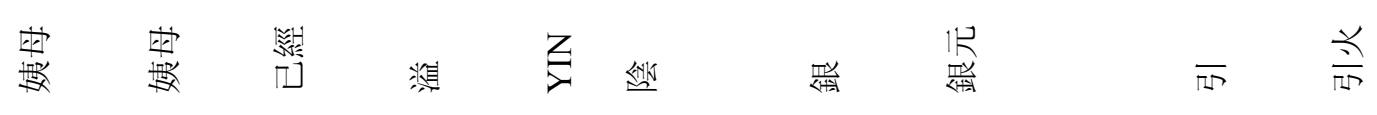




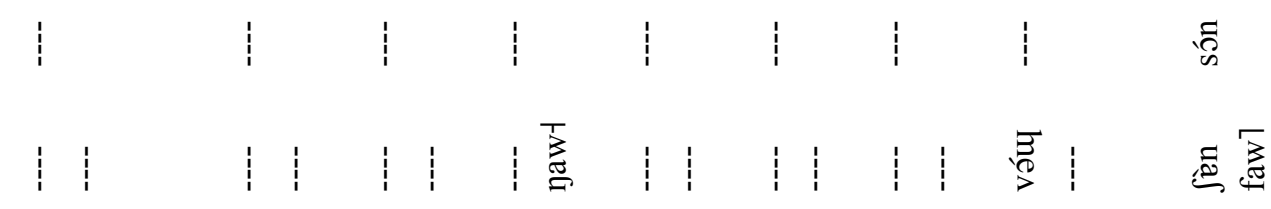

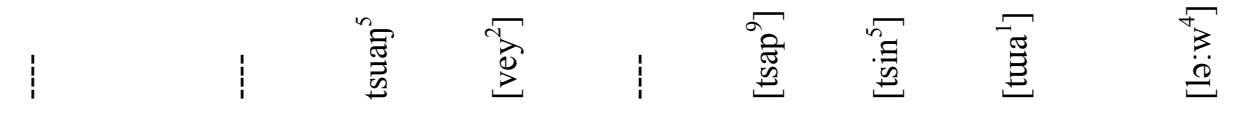

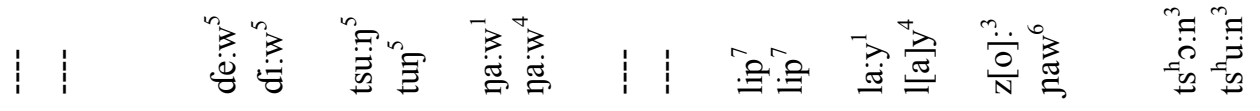

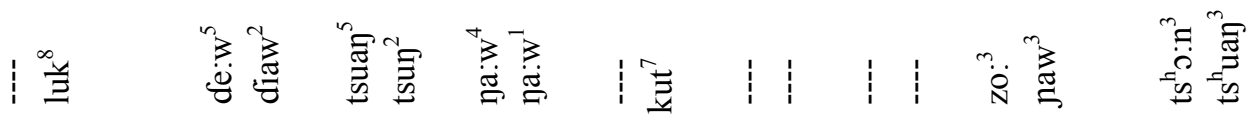

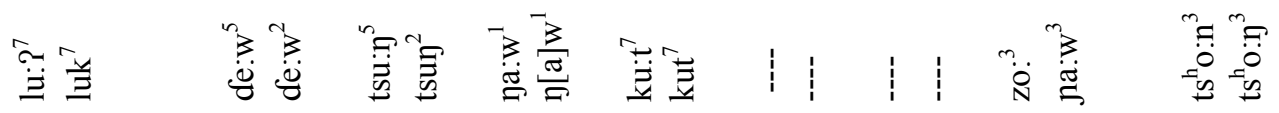

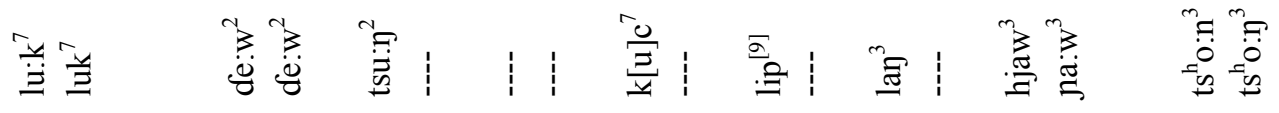

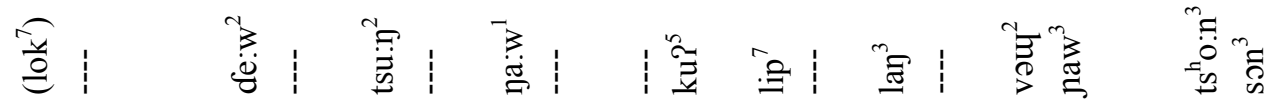

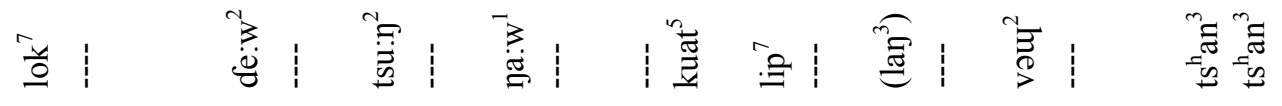

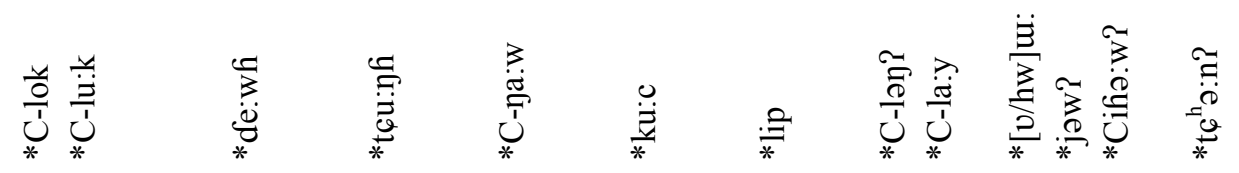

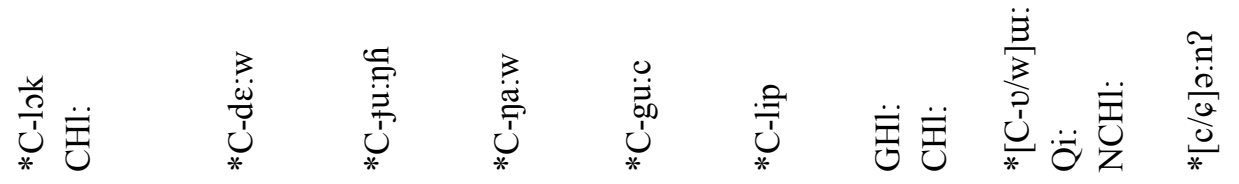

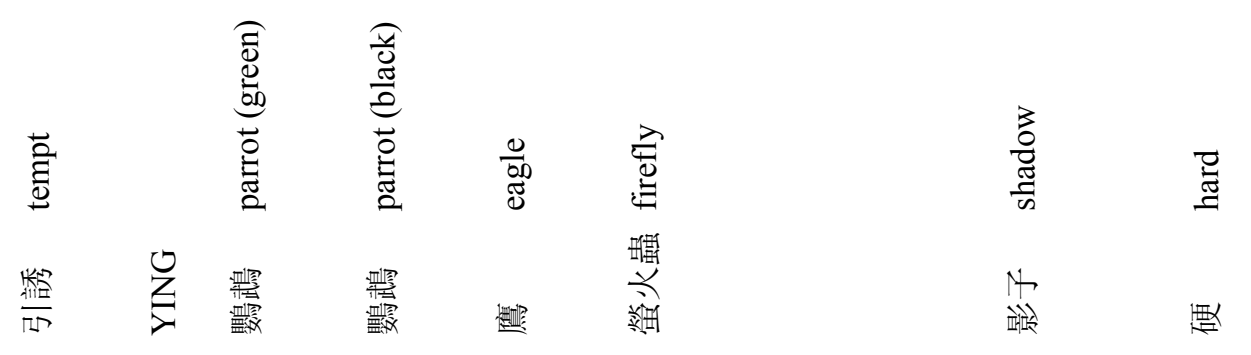




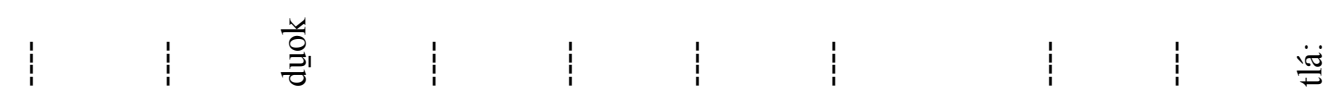

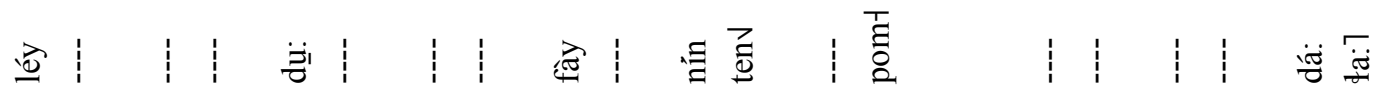

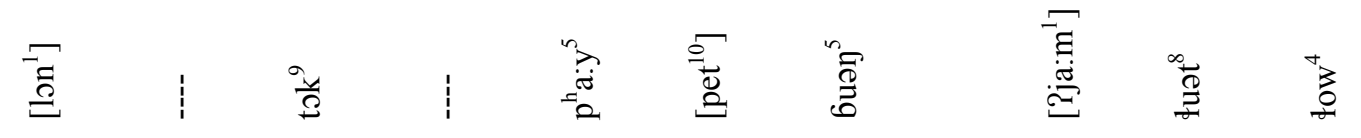

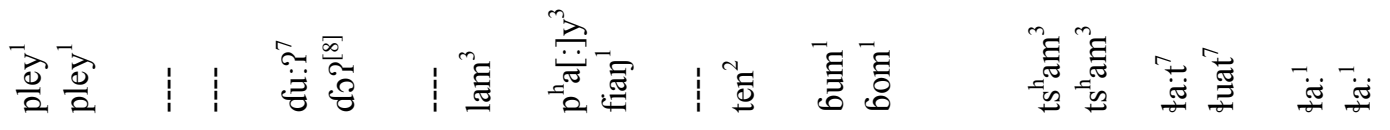

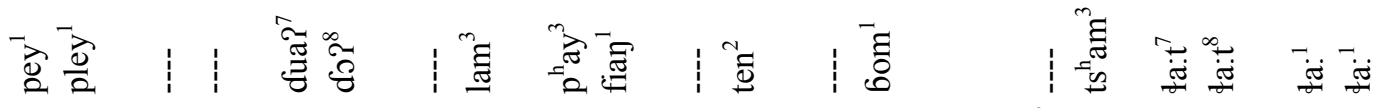

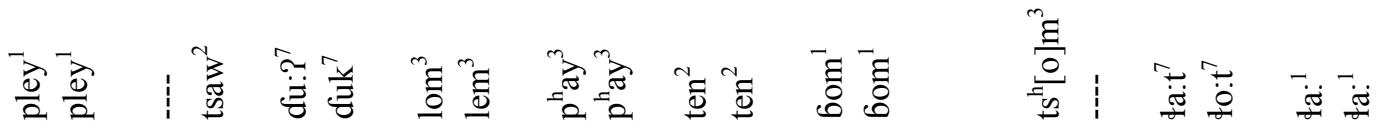

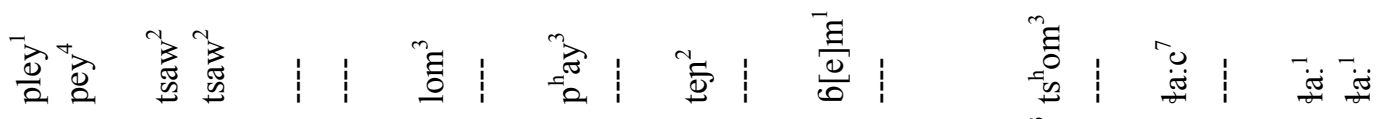

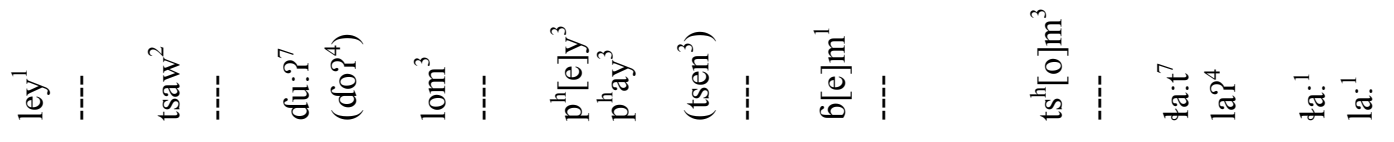

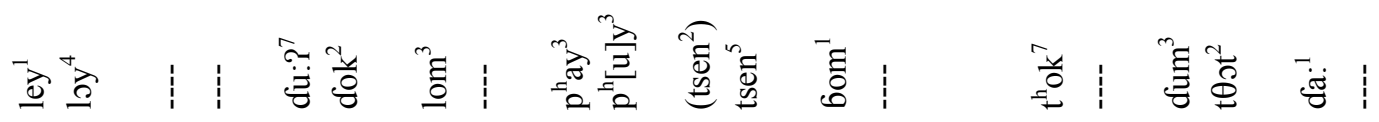

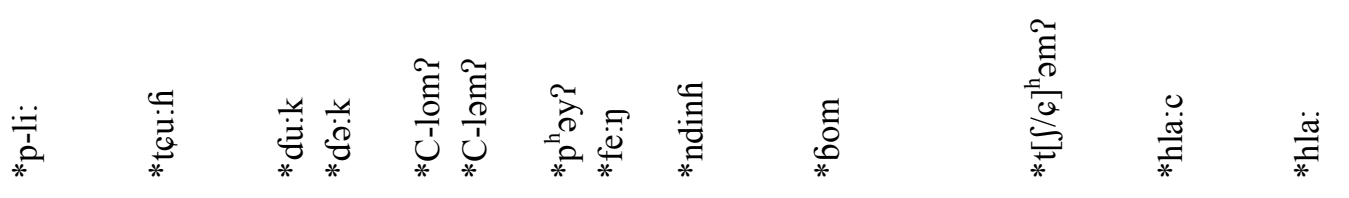

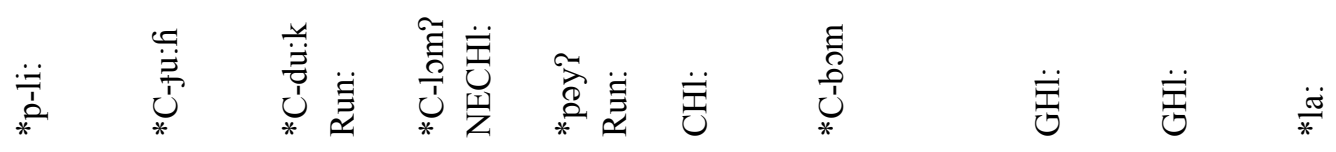

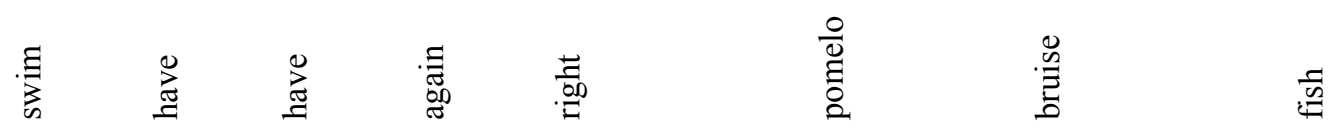

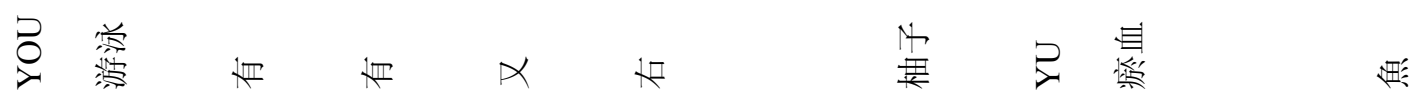




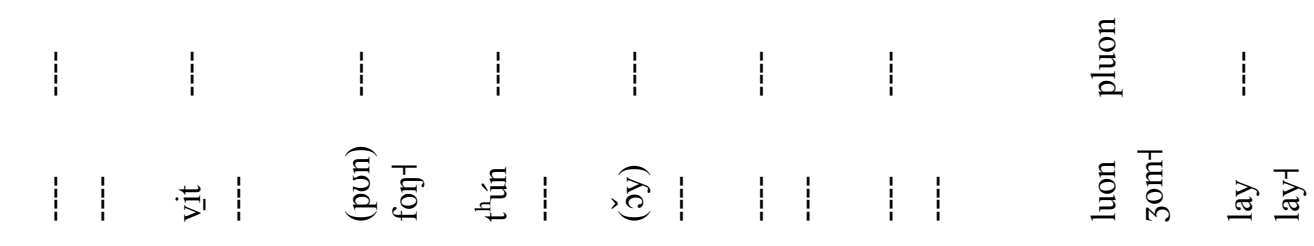

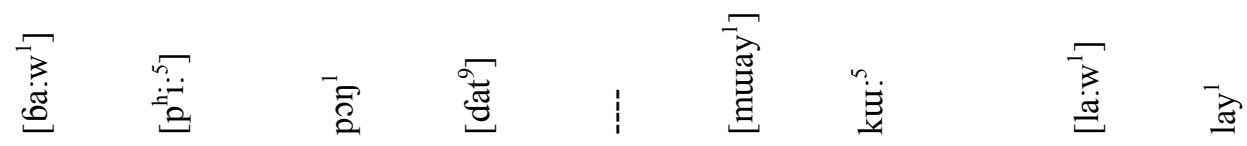

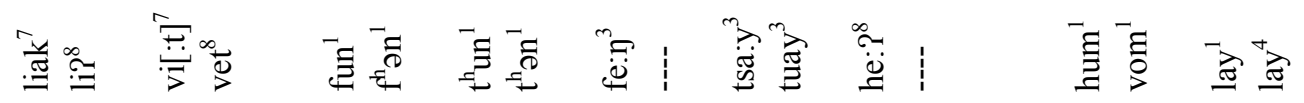

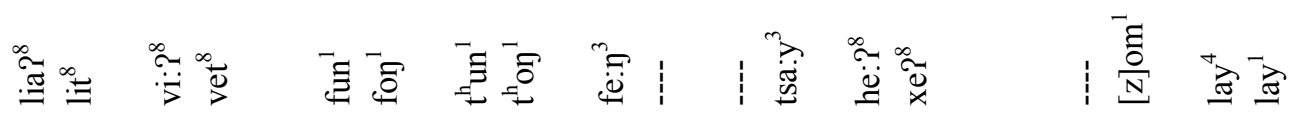

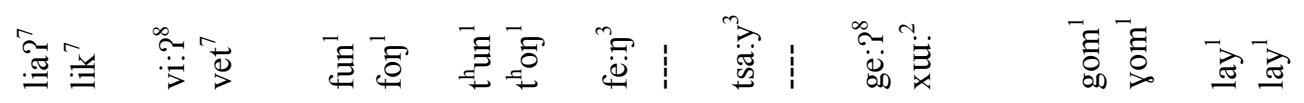

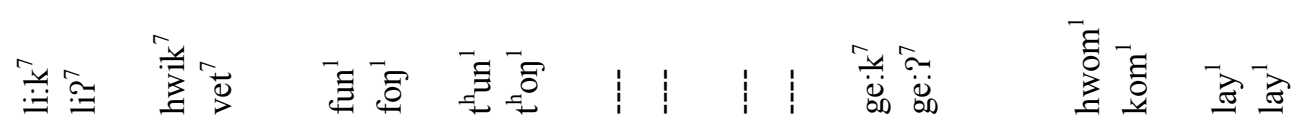

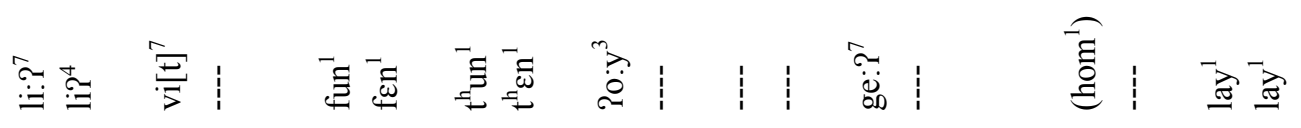

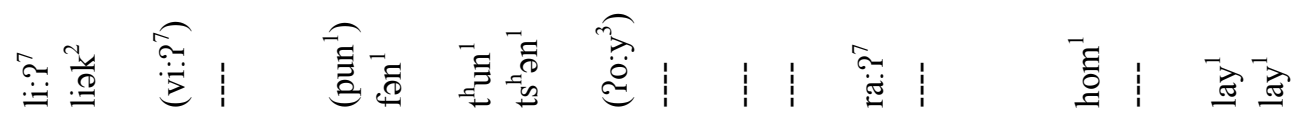

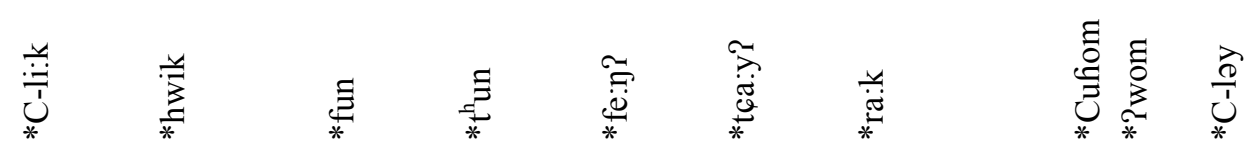

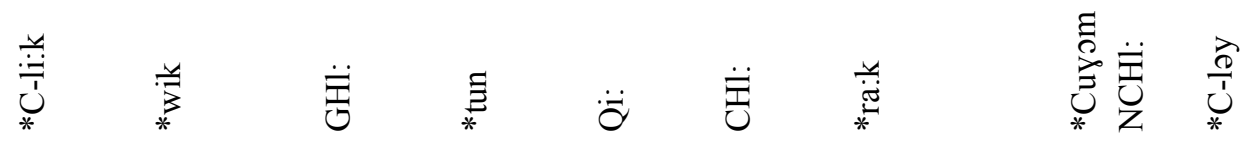

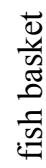

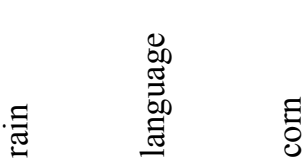

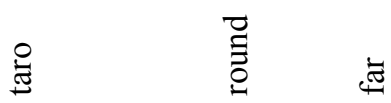

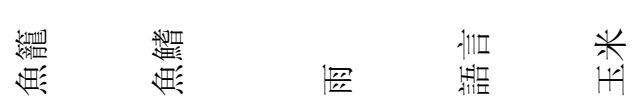

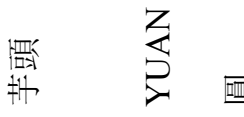
陉装 


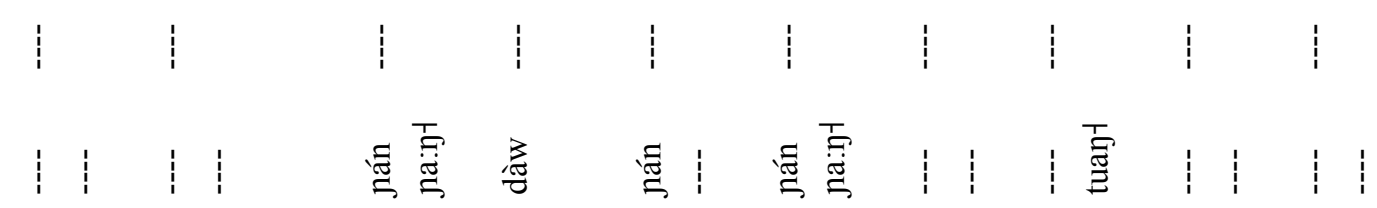

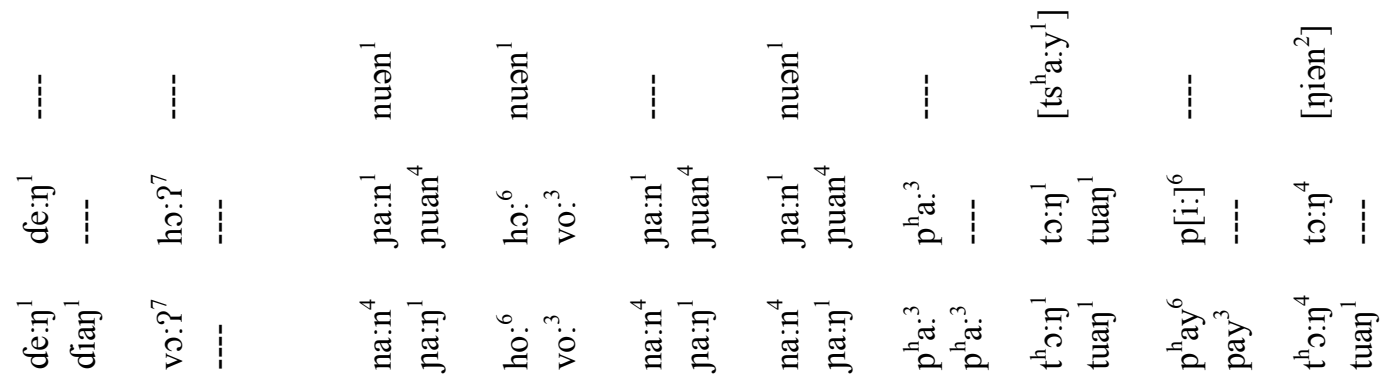

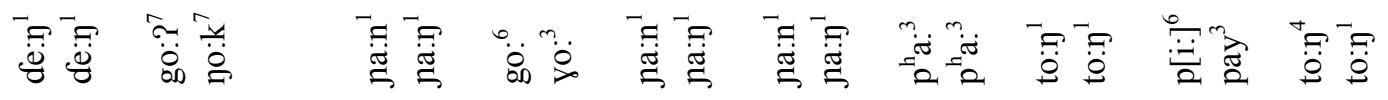

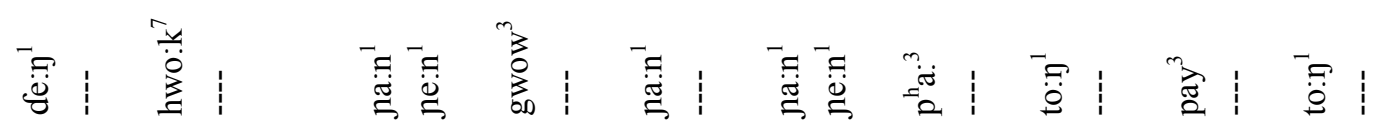

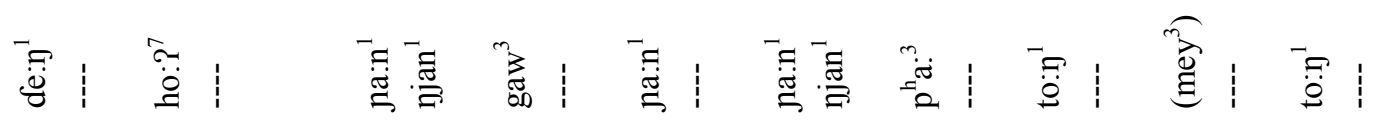

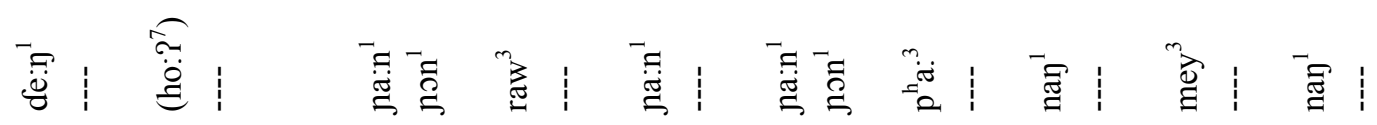

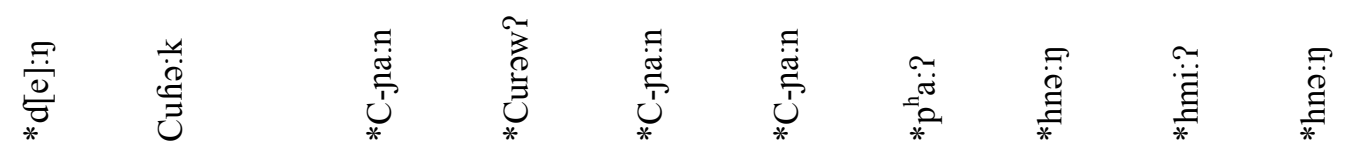

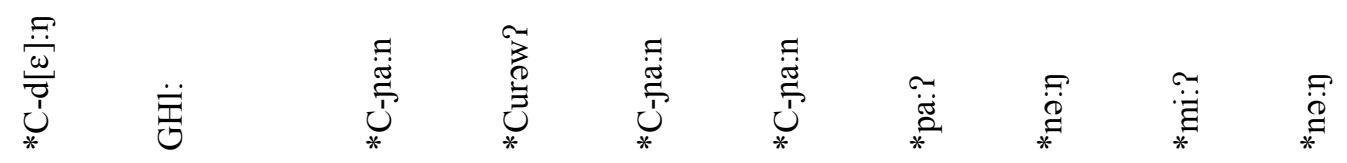

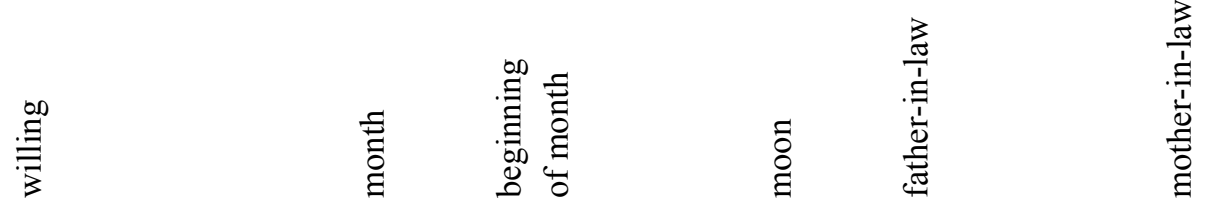

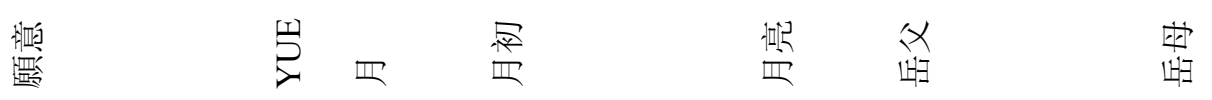


兽道

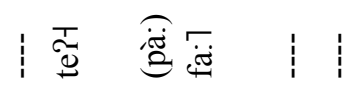

|

1

急旁

勇

1 言

롱

霆

㿣要

$\underset{3}{3}$

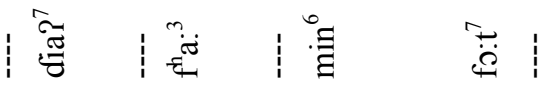

重童

整高

" 焉"

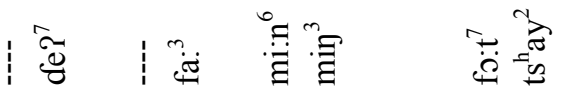

咅咅

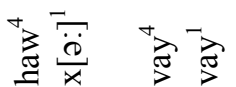

要

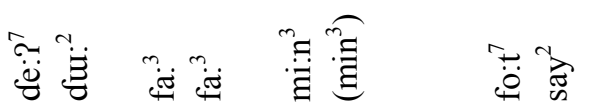

童童

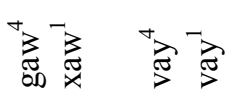

惑第

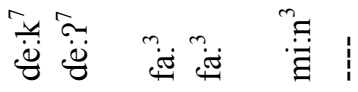

全拿

咅?

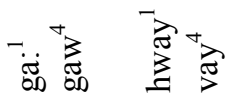

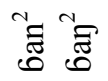

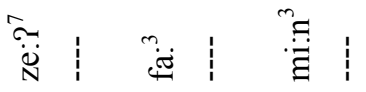

言

蛋咅

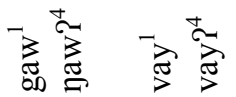

覧言

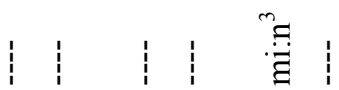

츨

管

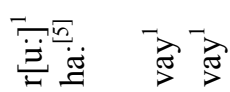

章焉

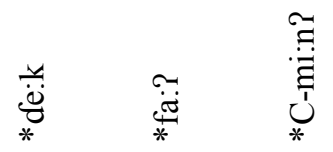

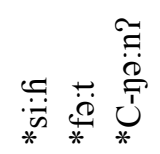

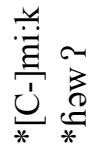

$\ddot{*} \quad \frac{\overrightarrow{0}}{*}$

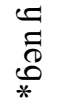

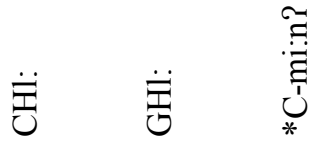

乐泀

晜泀

$\underset{*}{*} \stackrel{\overrightarrow{3}}{*}$

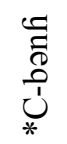

$\frac{\overline{0}}{0}$

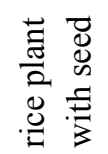

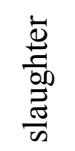

$\widehat{0}$
$\stackrel{0}{0}$
0

总

高

文 粗

鼠出

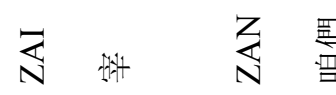

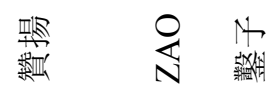




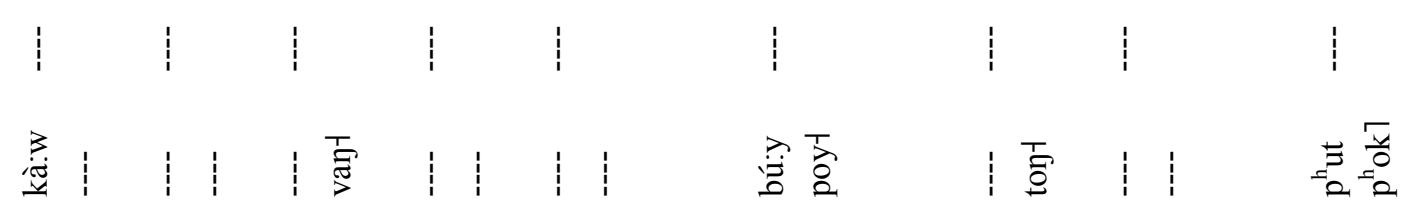

常

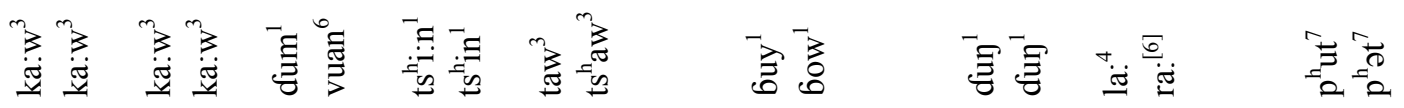

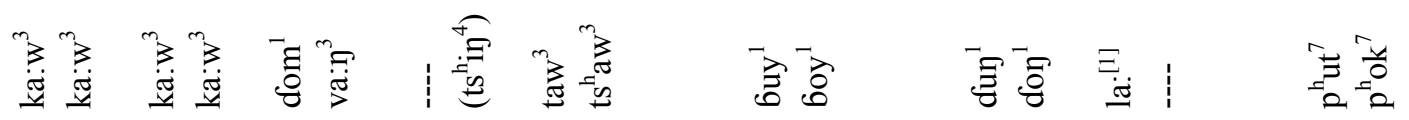

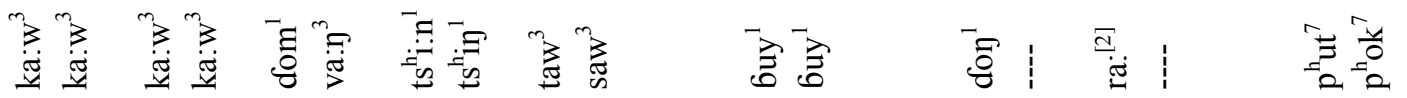

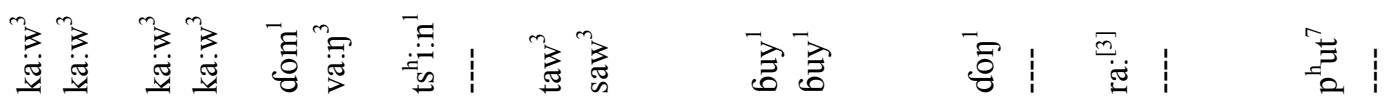

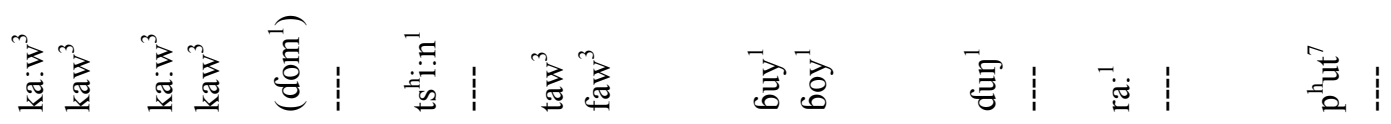

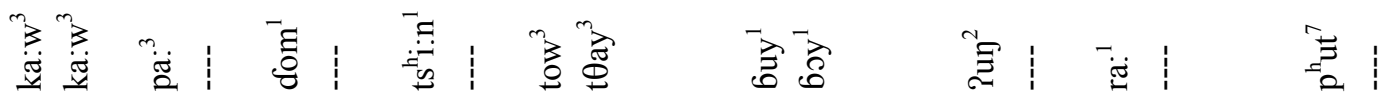

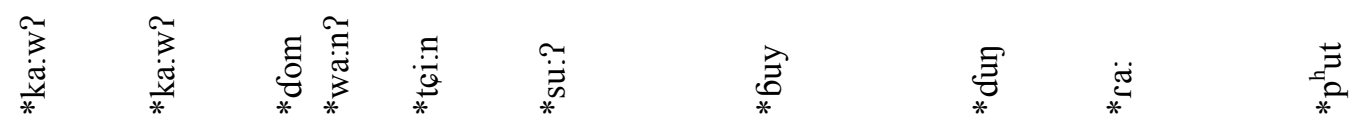

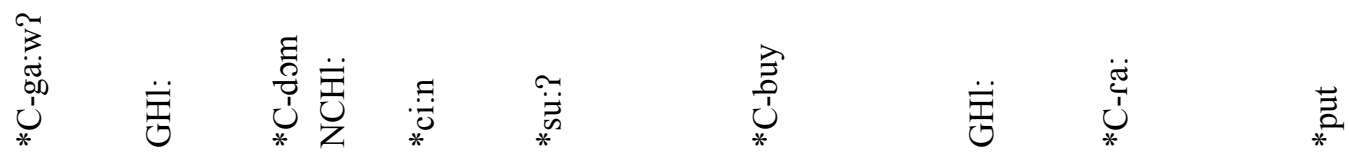

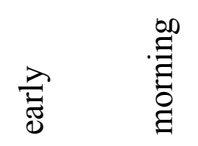
总

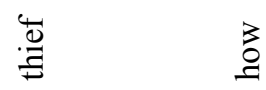
파 $\quad$ 䟵

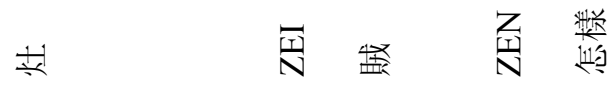
蛋高

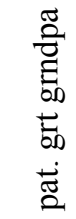




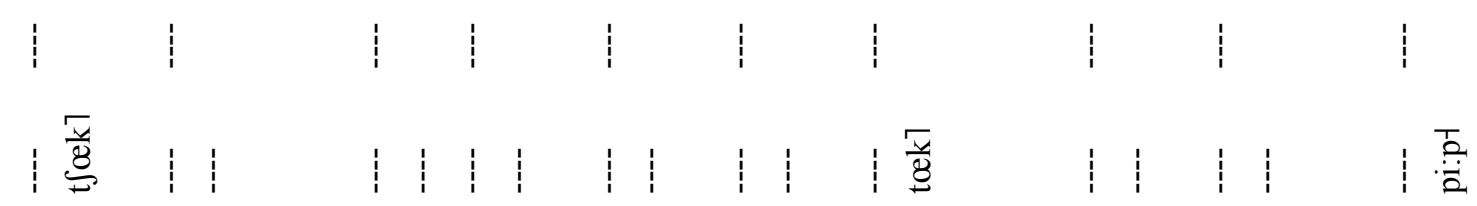

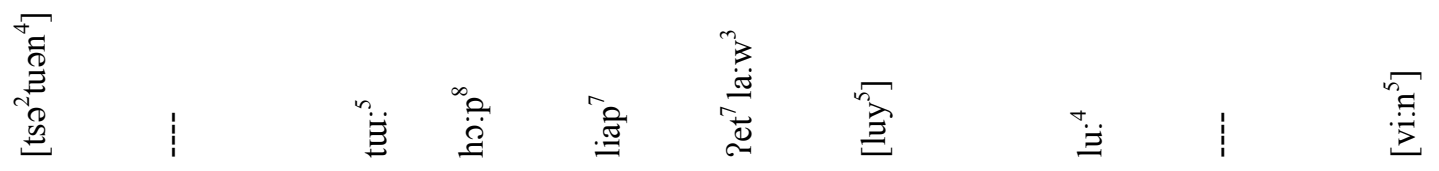

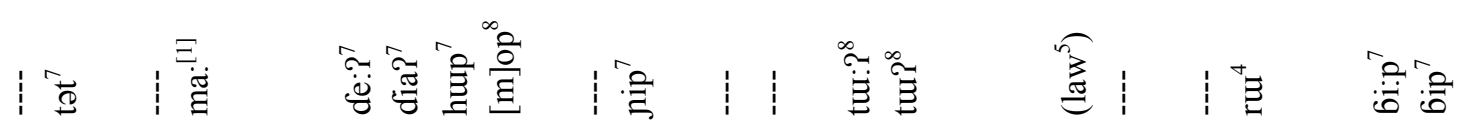

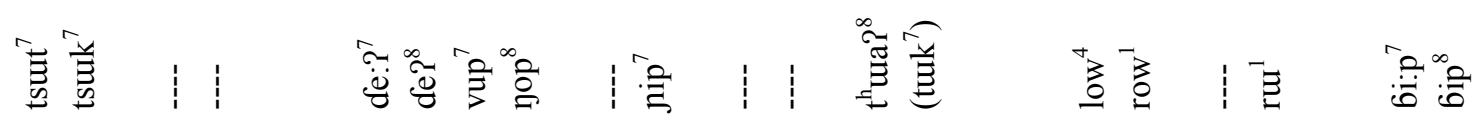

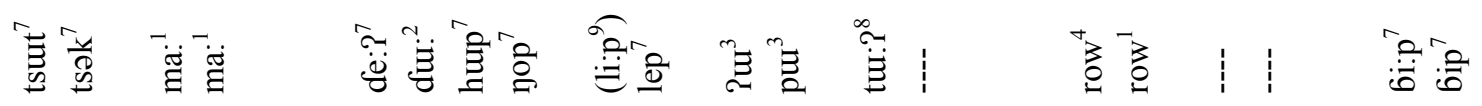

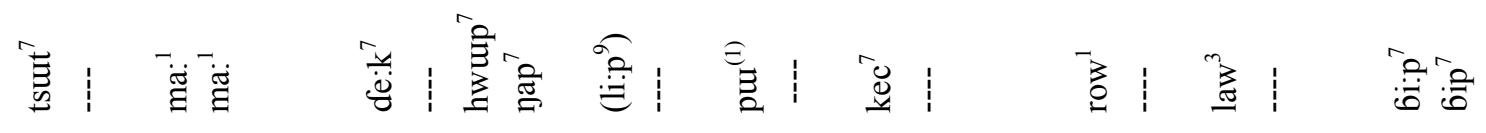

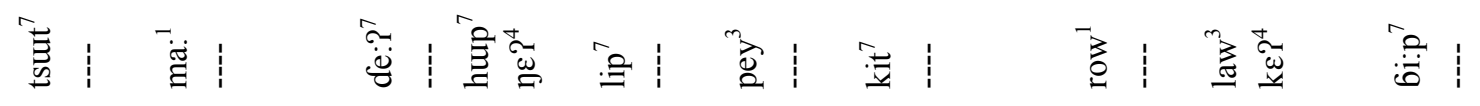

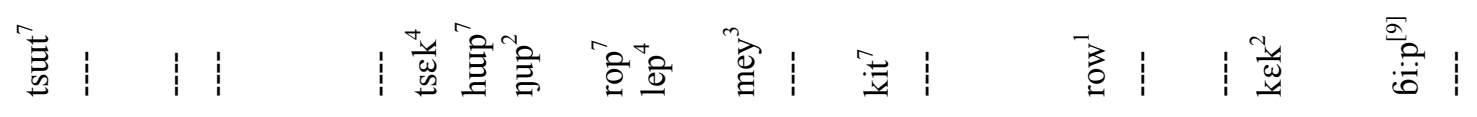

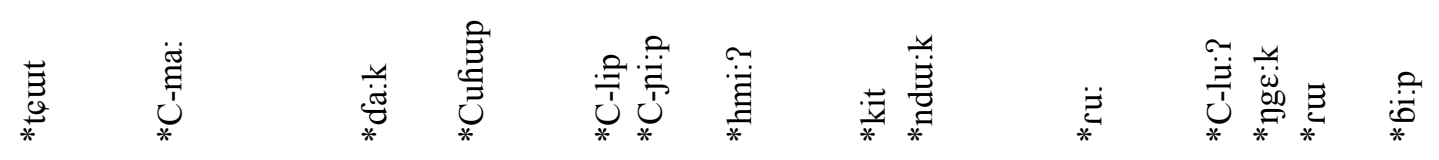

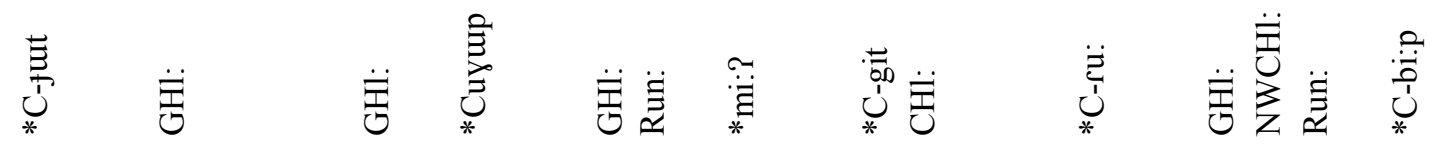

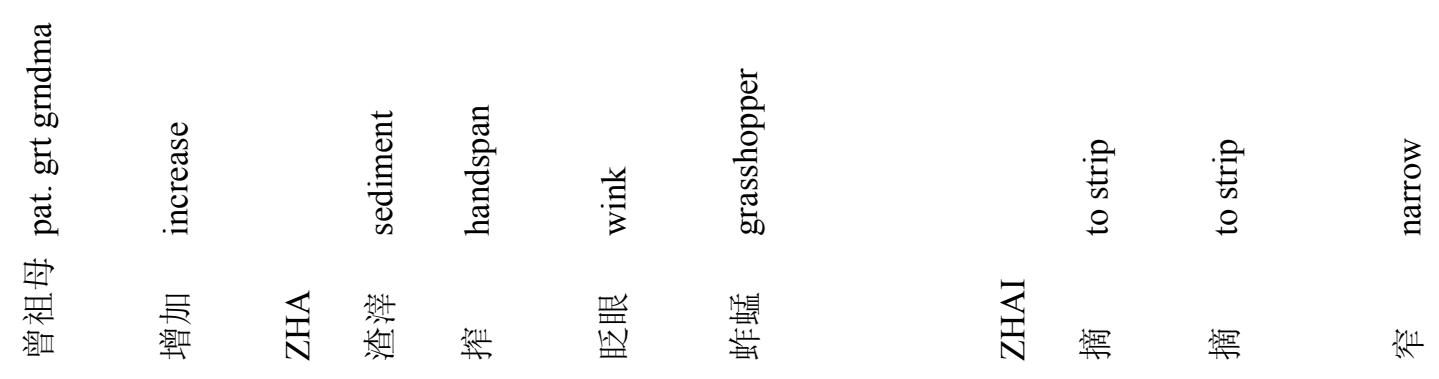




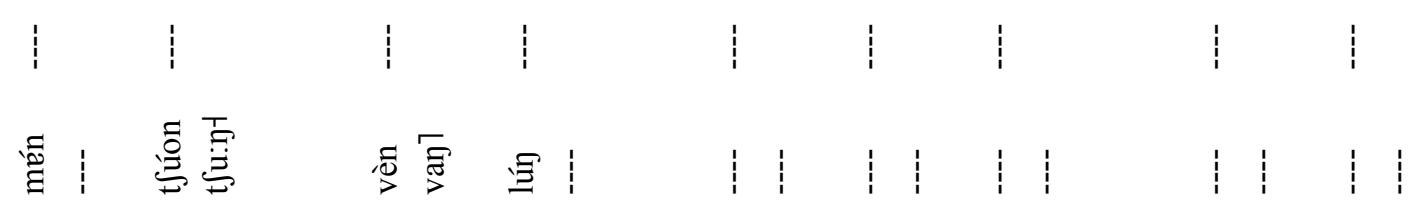

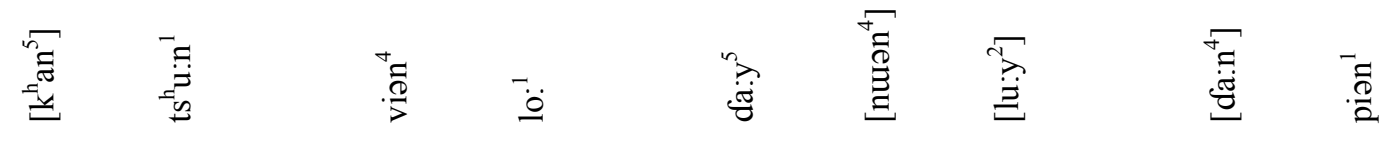

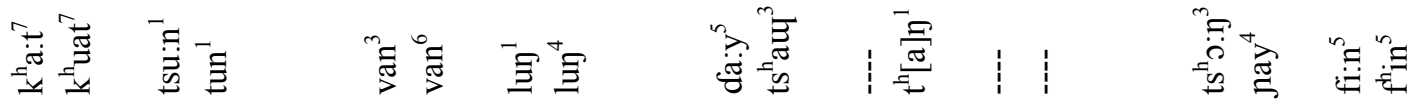

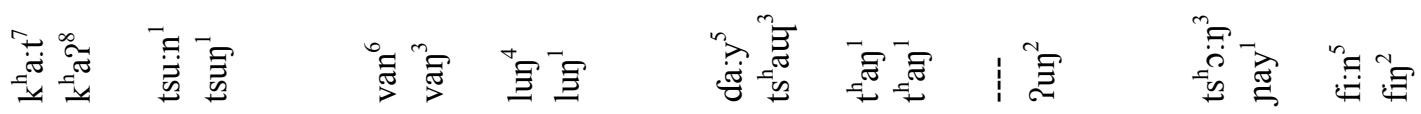

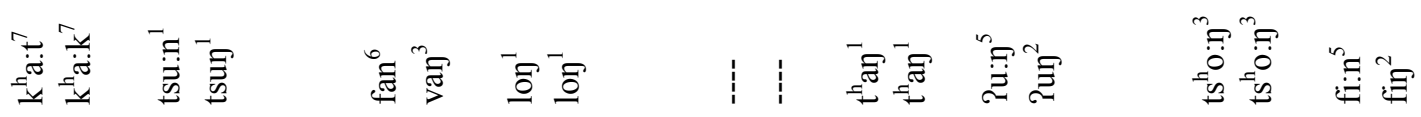

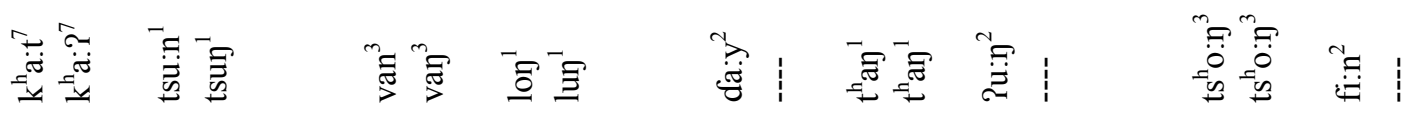

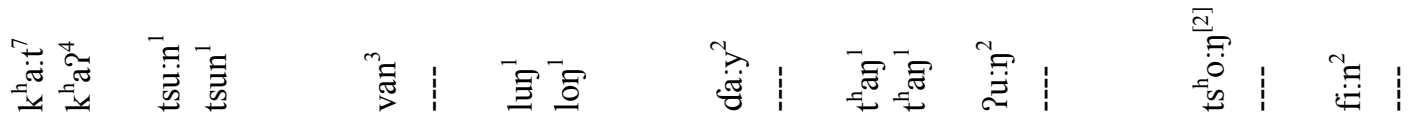

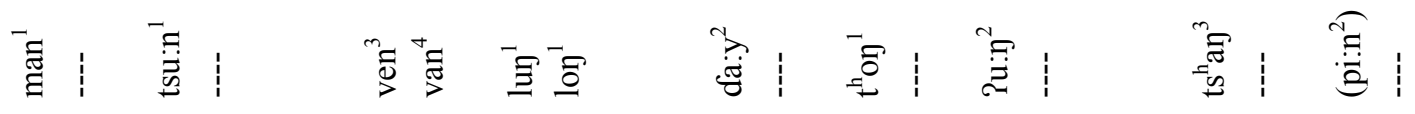

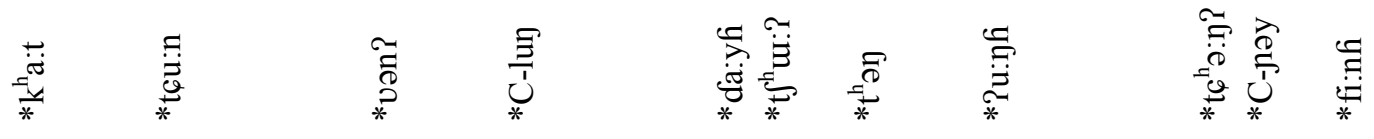

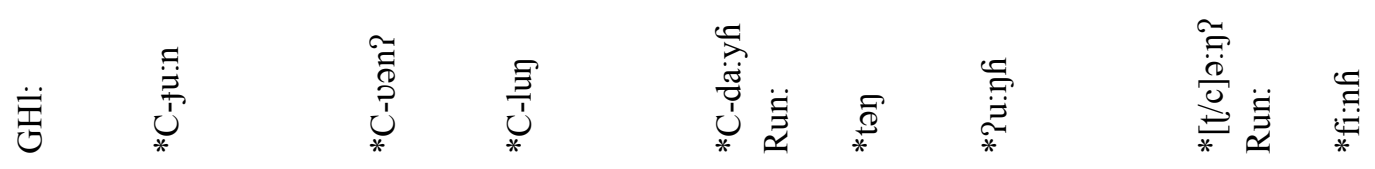

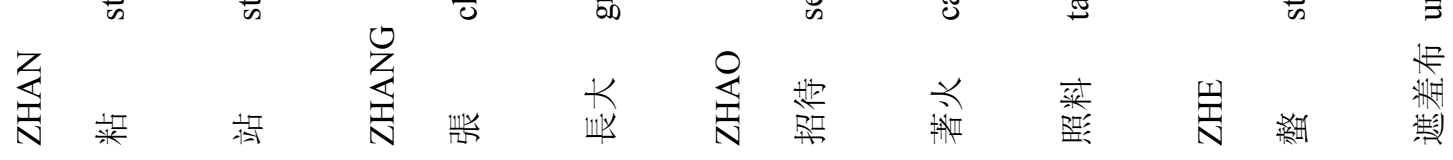




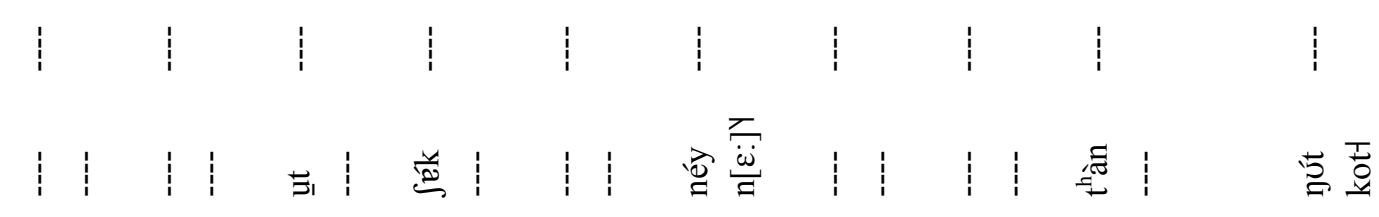

䨪|

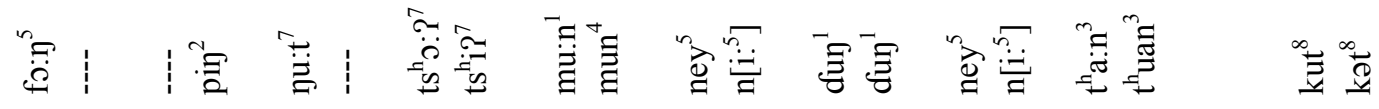

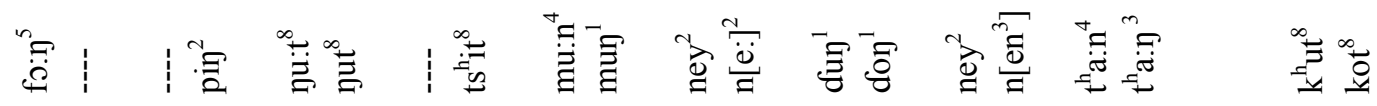

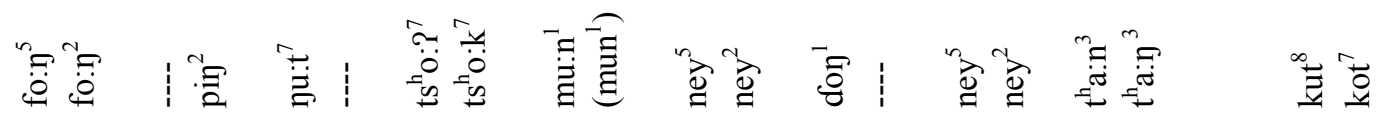

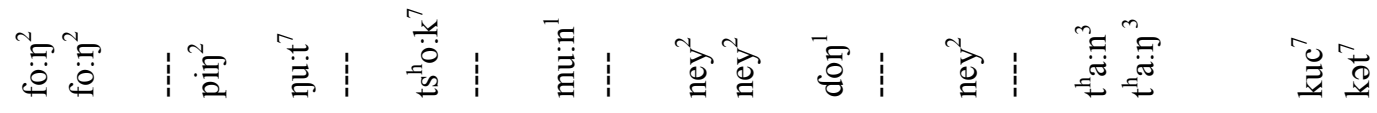

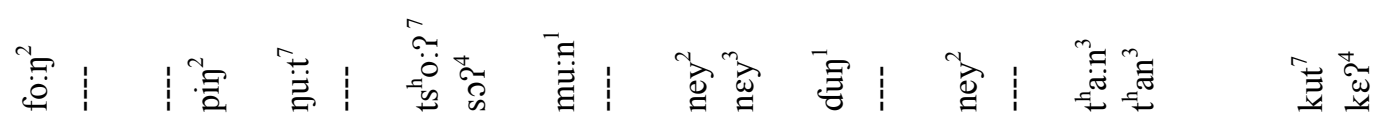

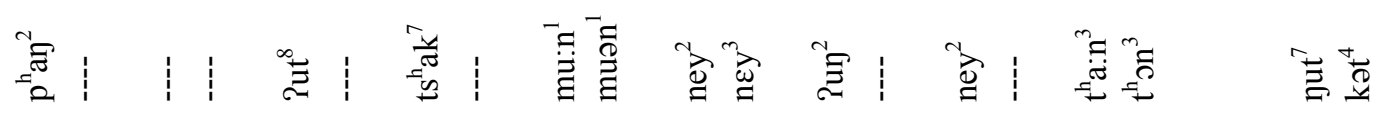

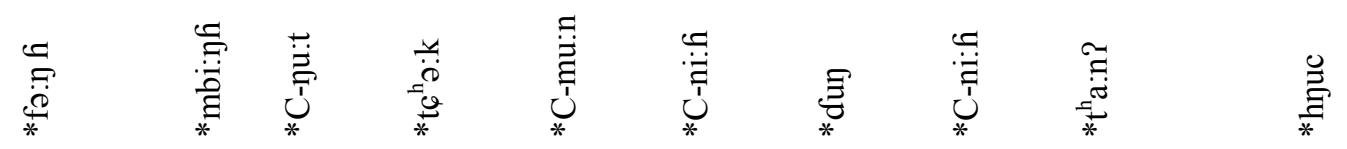

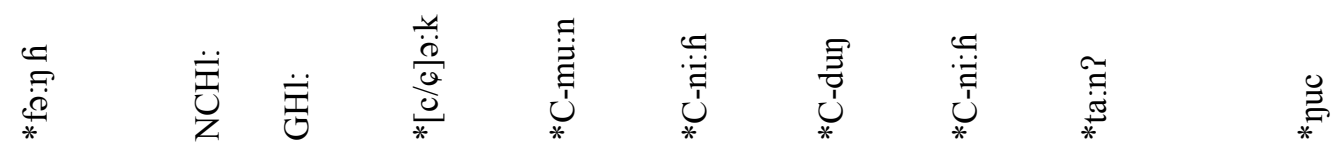

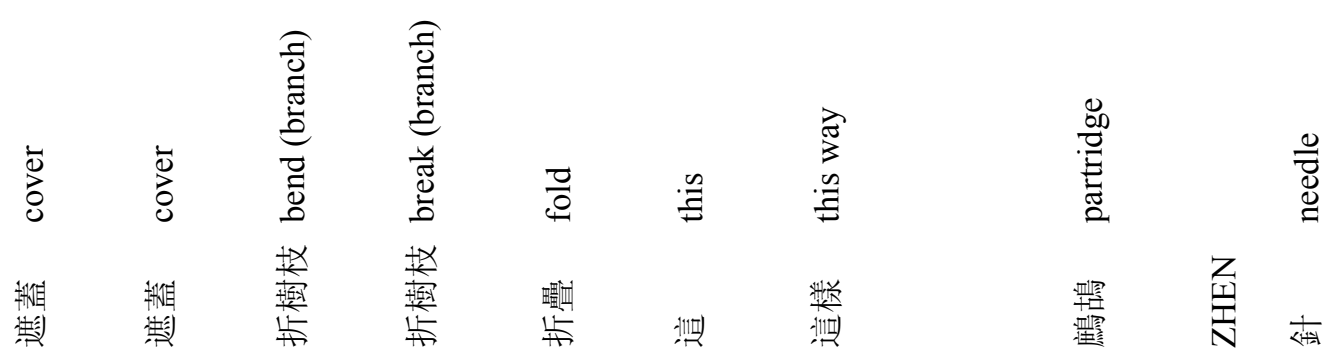




\begin{tabular}{l|l|l|l|l|l} 
& $\mid$ & $\mid$ & 1 & 1 & 1
\end{tabular}

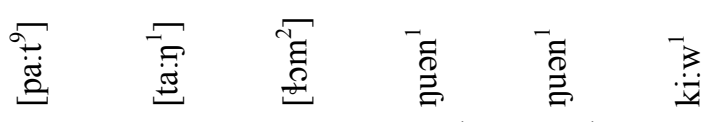

尊

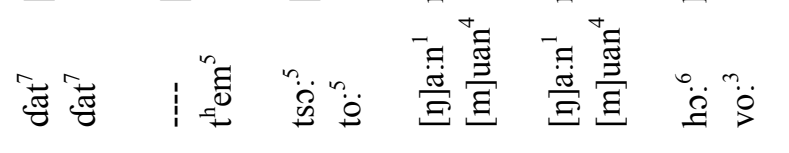

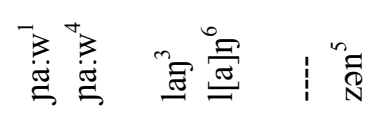

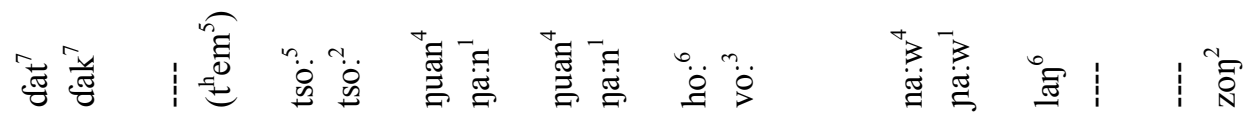

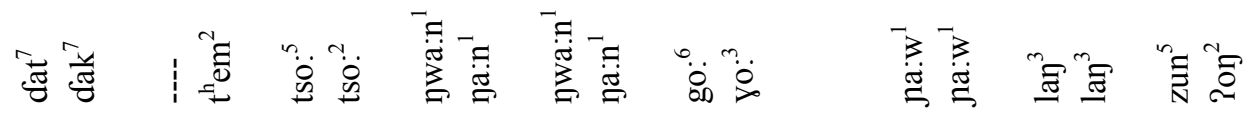

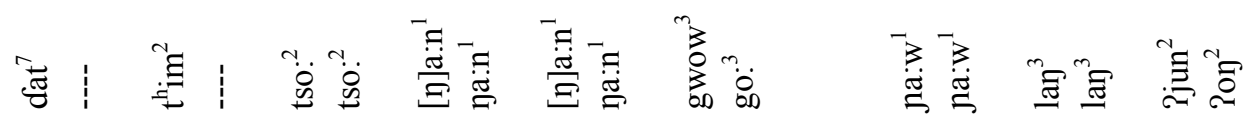

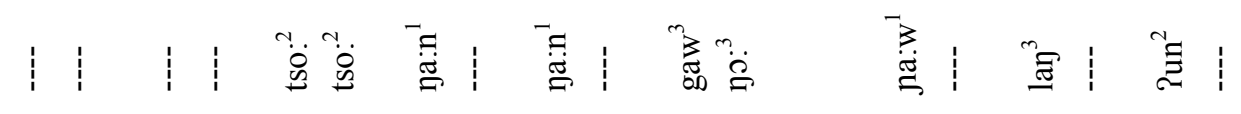

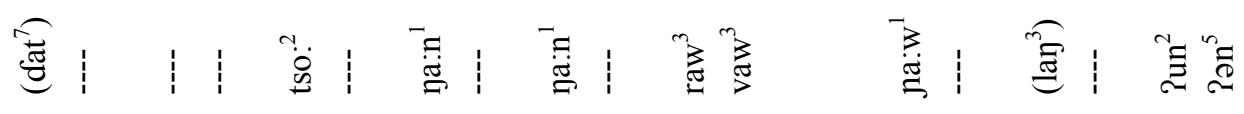

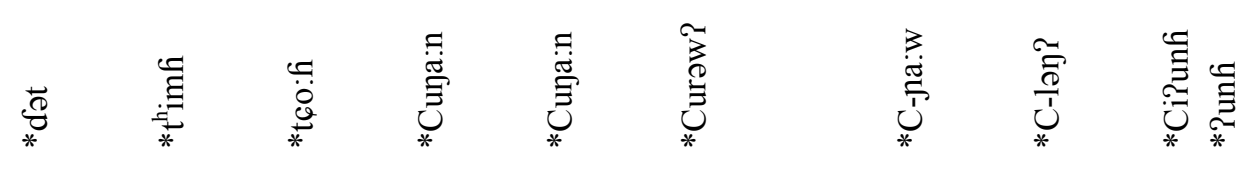

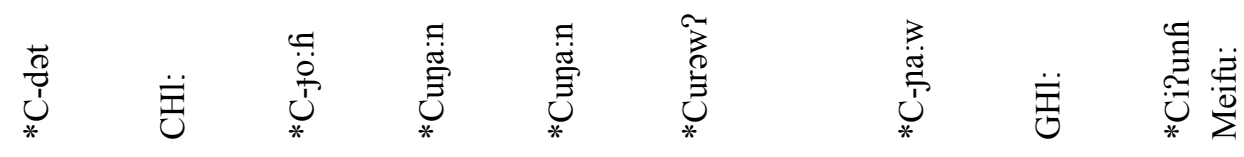

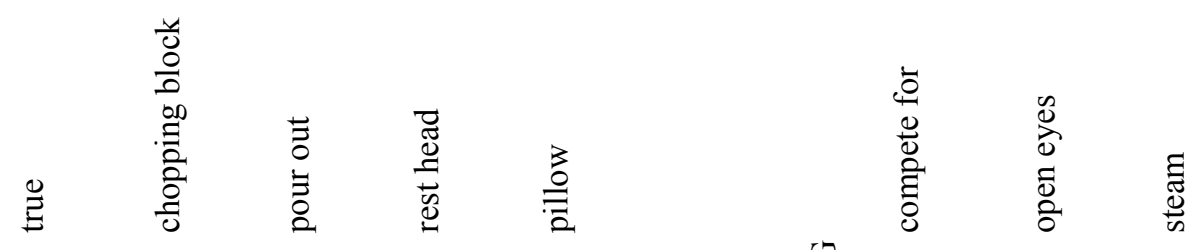

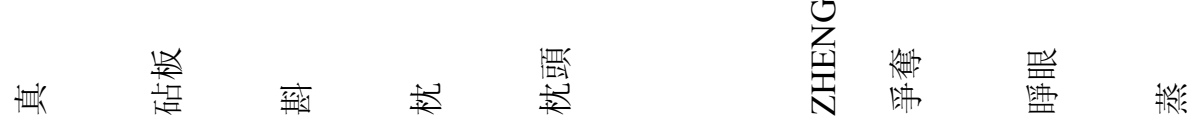




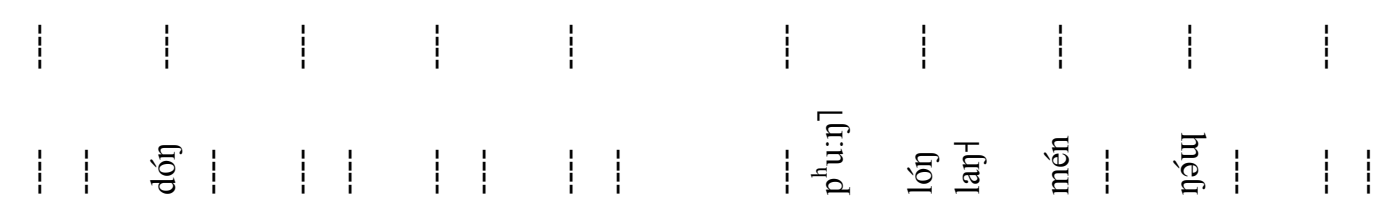

震要|

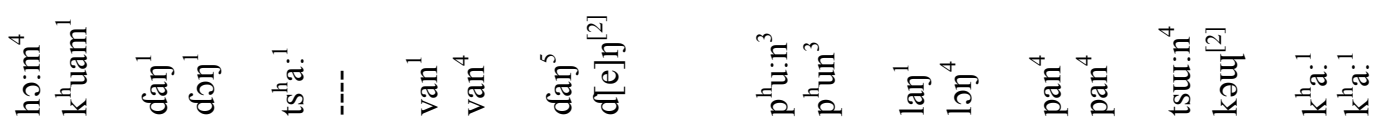

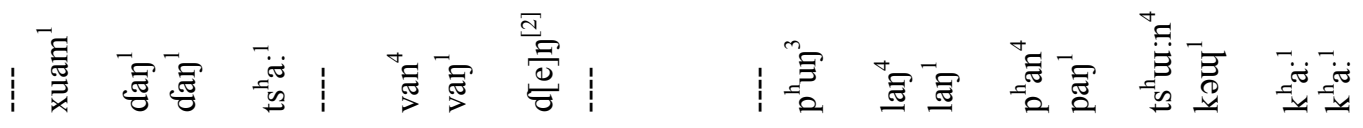

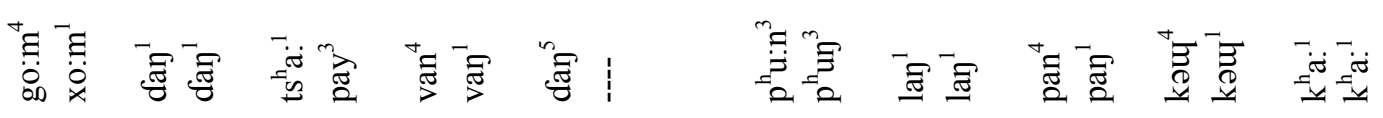

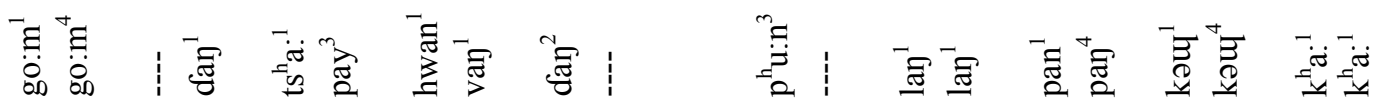

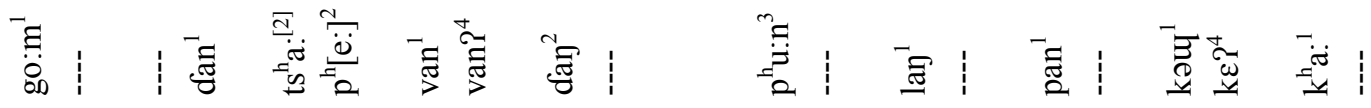

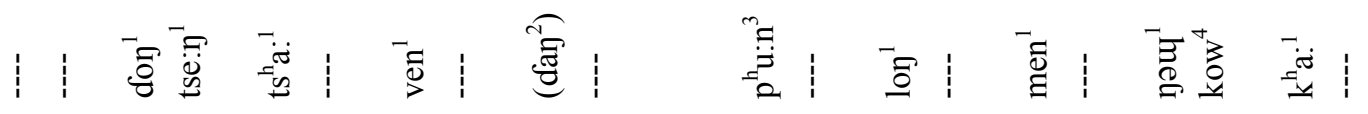

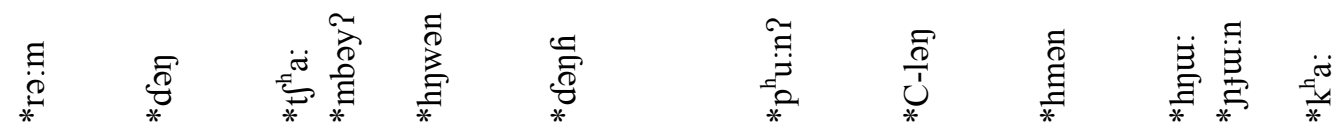

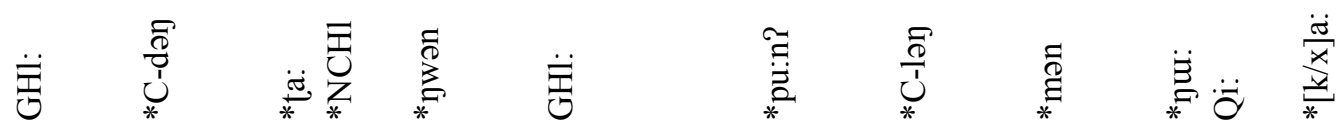

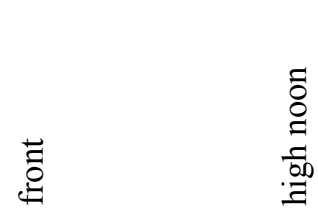

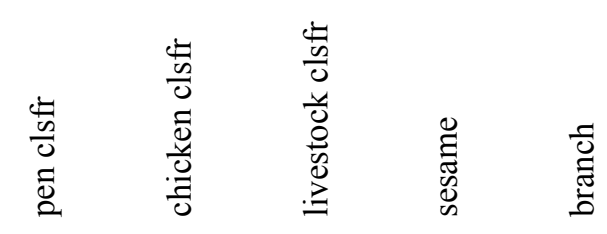
鯧获

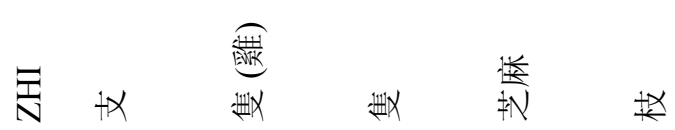




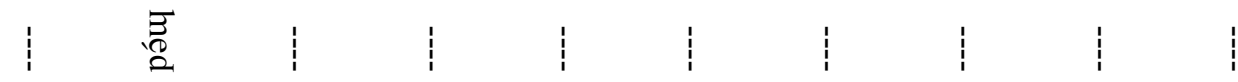

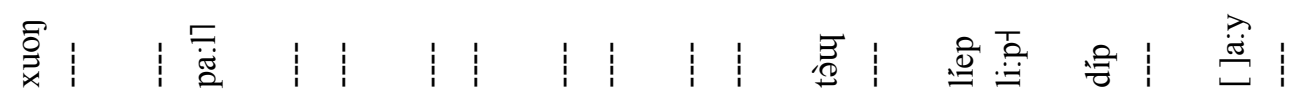

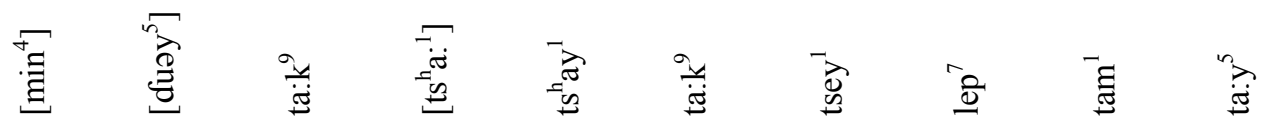

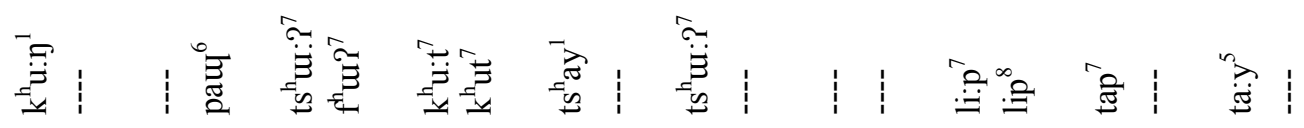

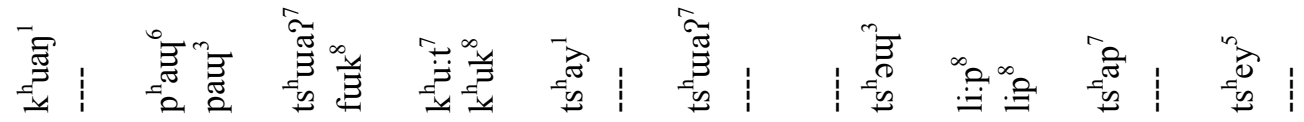

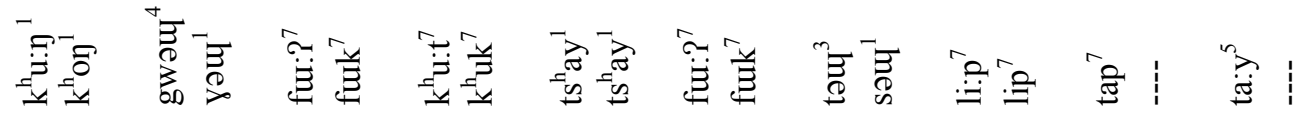

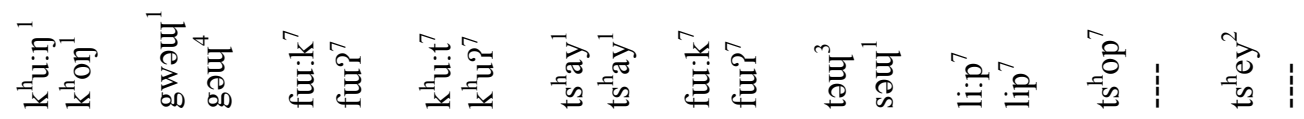

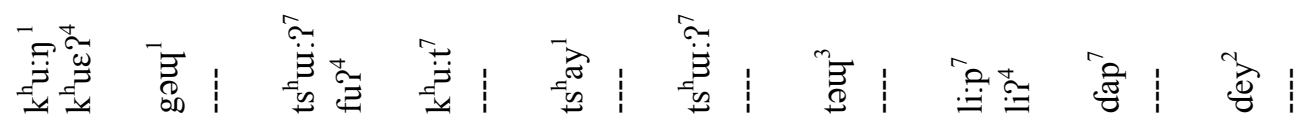

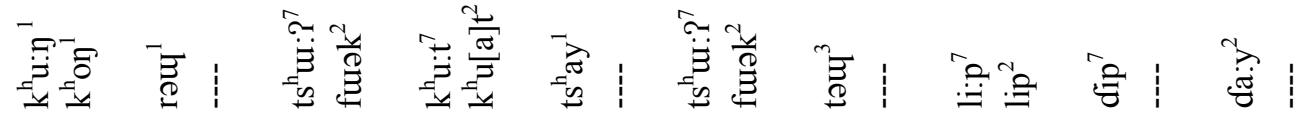

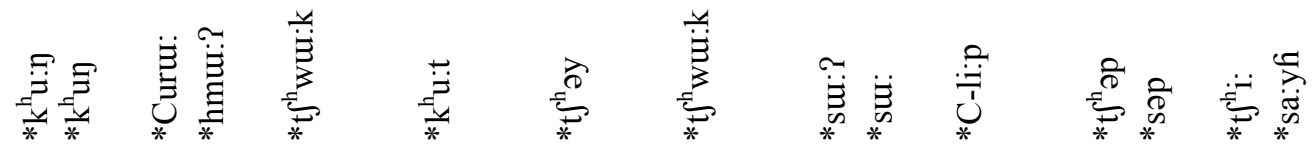

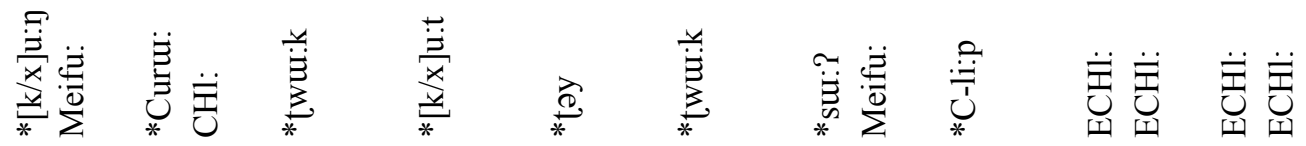

言

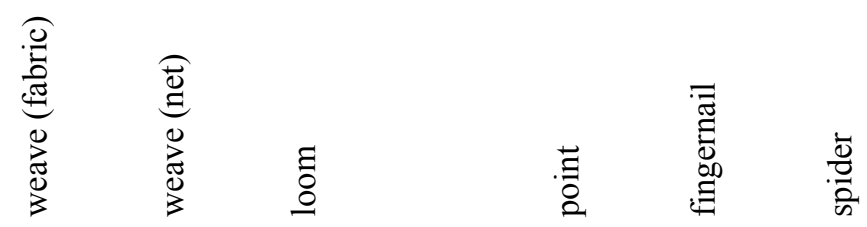

傮

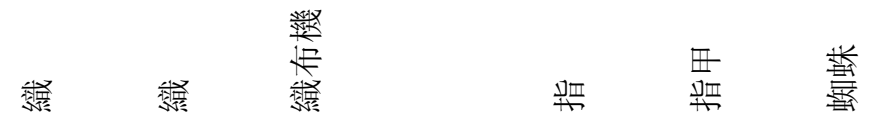




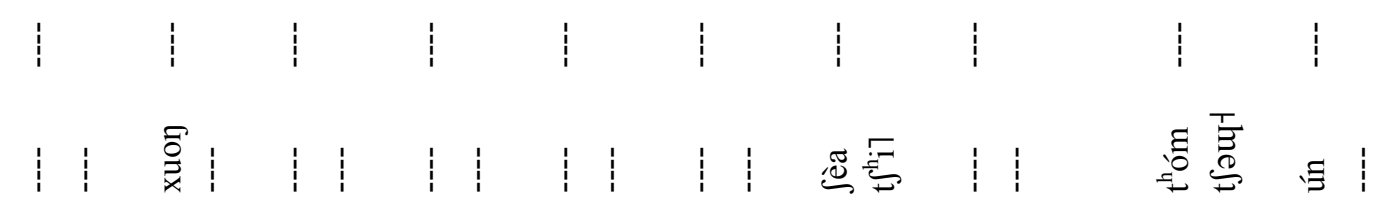

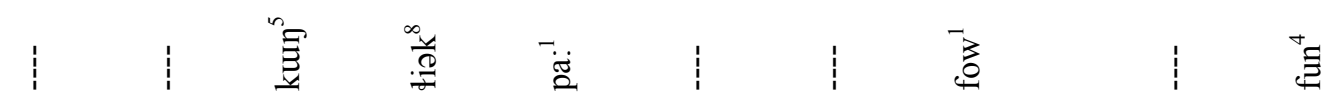

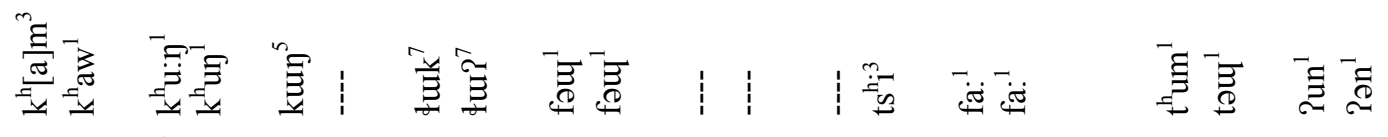

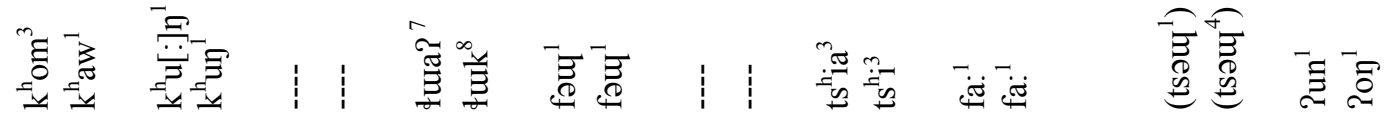

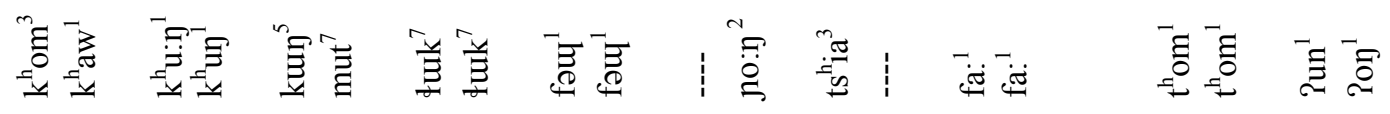

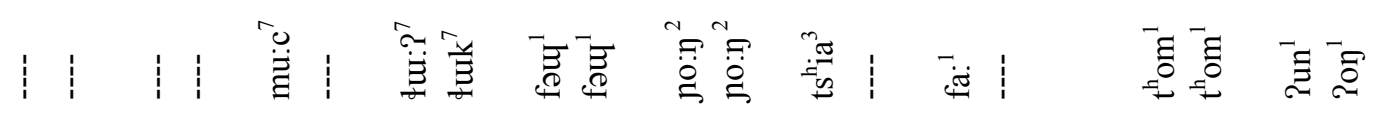

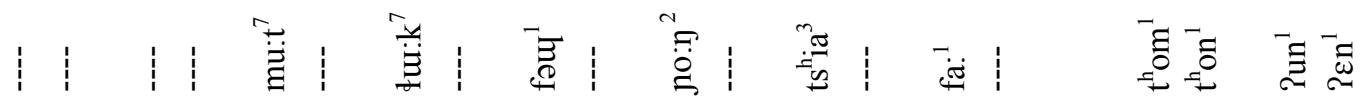

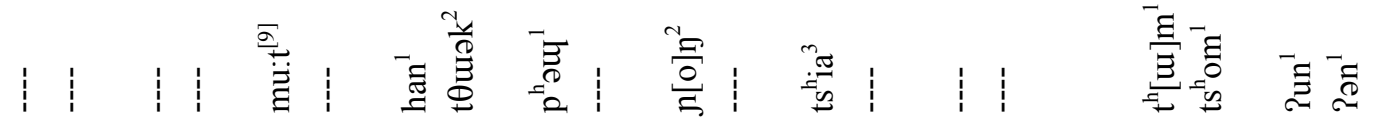

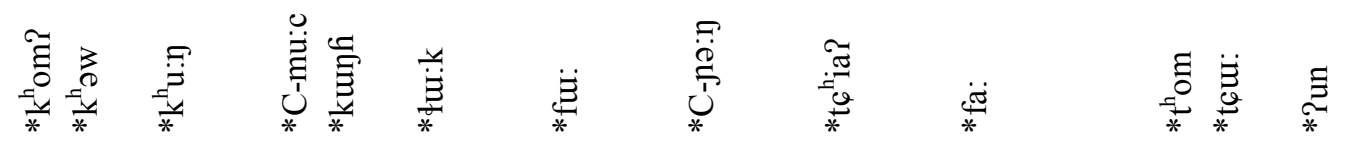

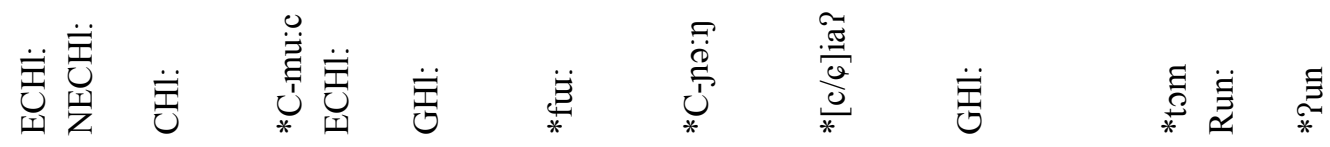

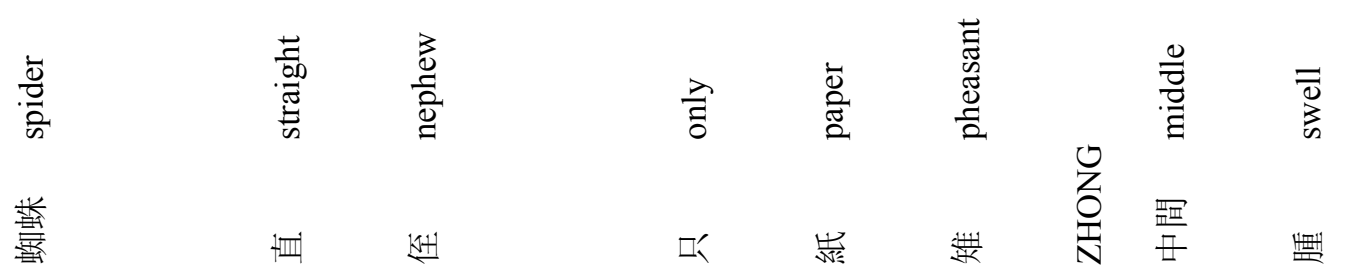




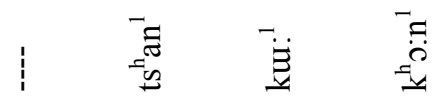

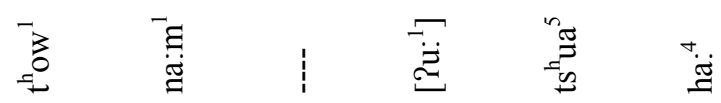

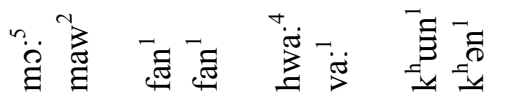

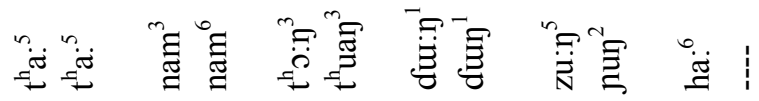

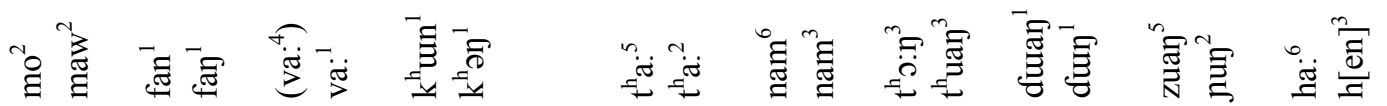

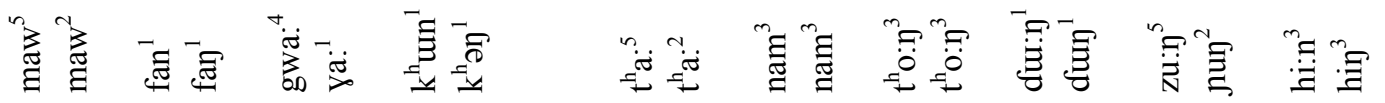

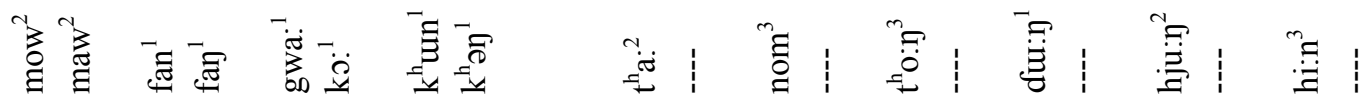

芯: 寻志

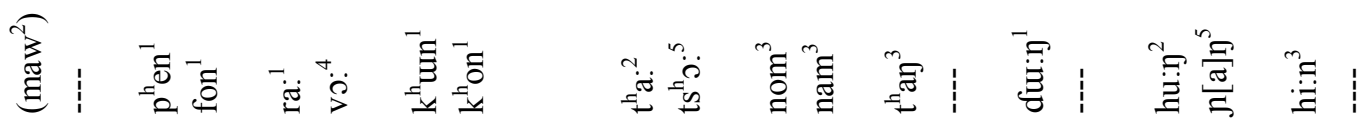

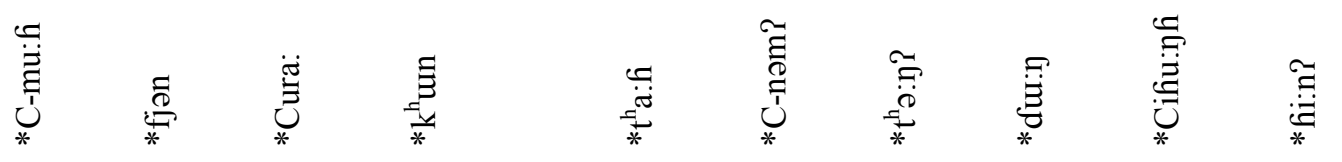

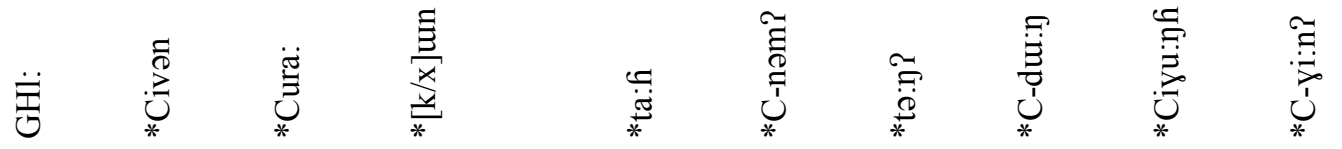

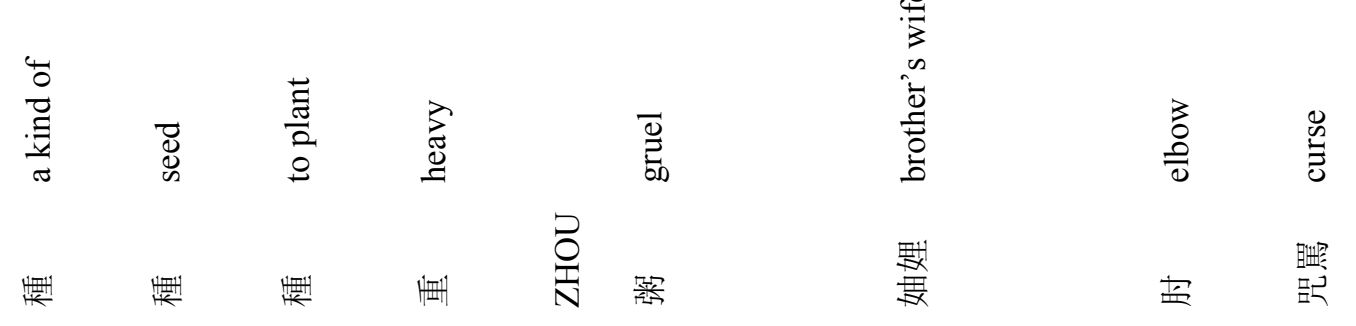




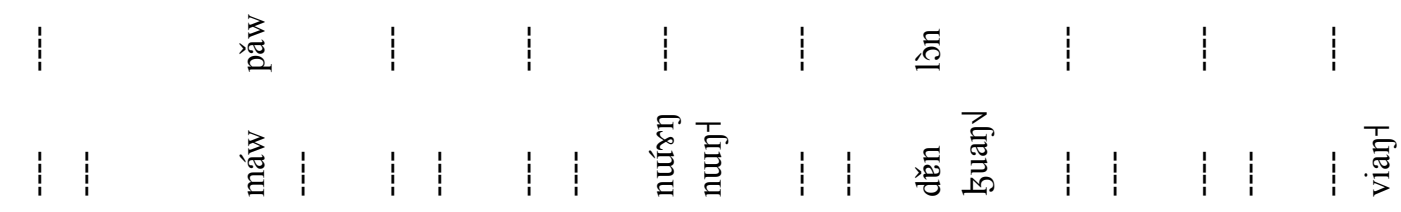

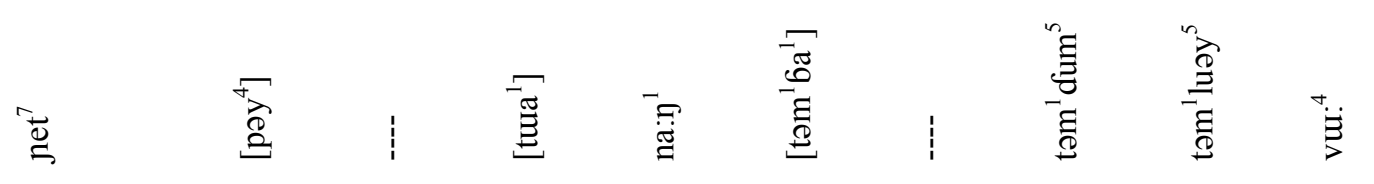

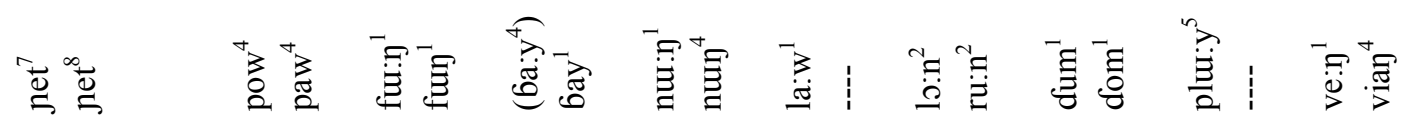

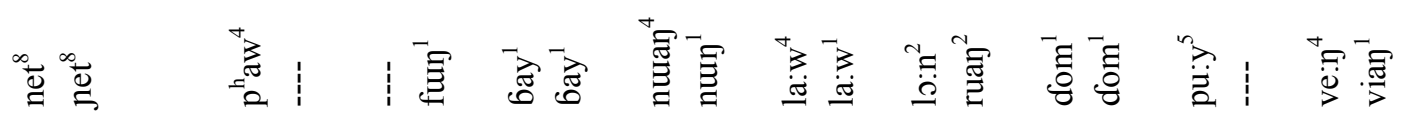

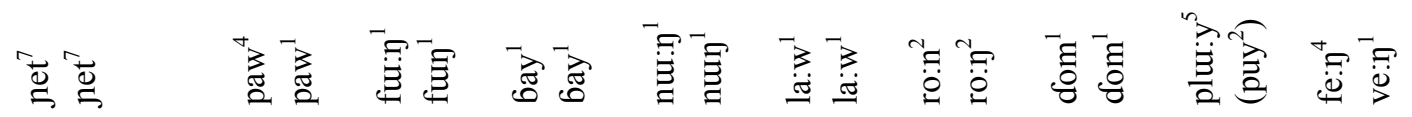

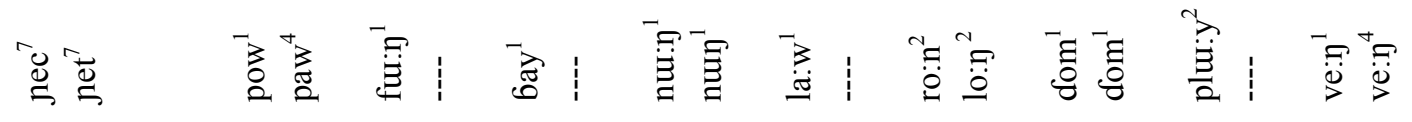

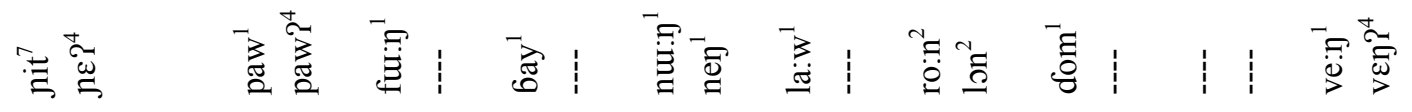

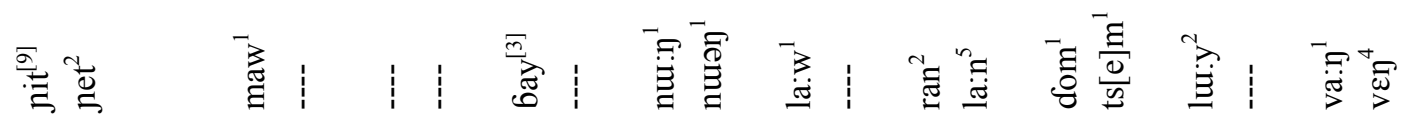

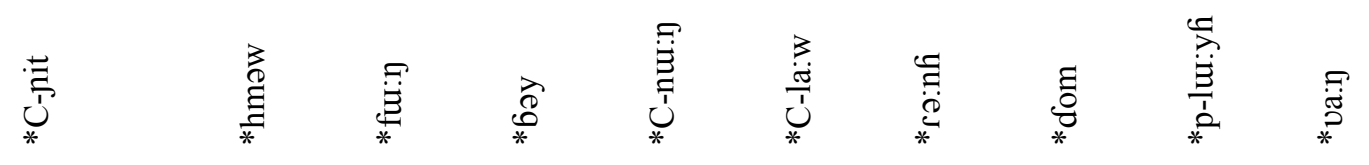

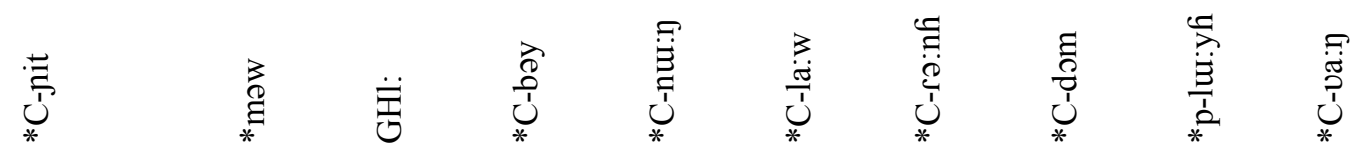

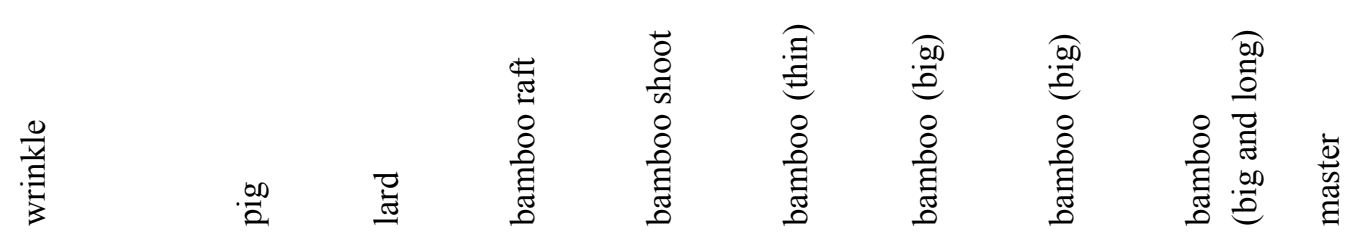

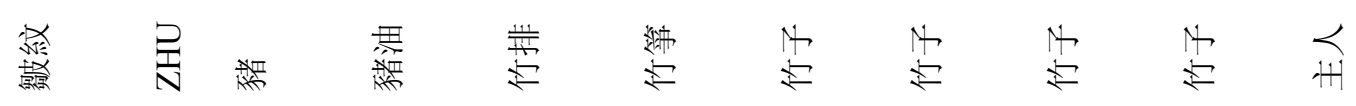




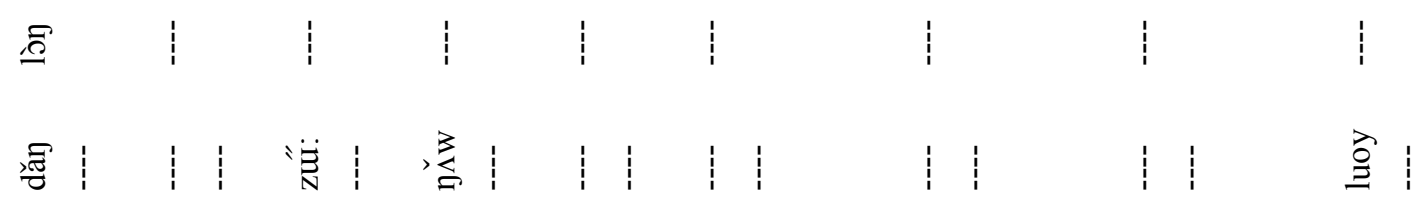

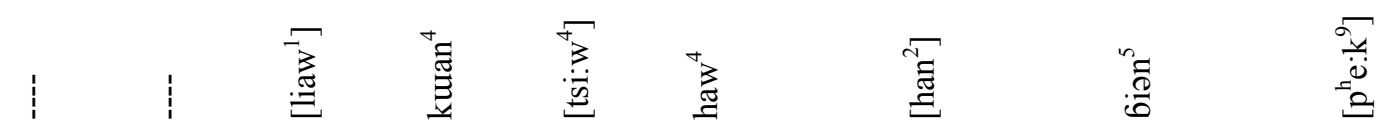

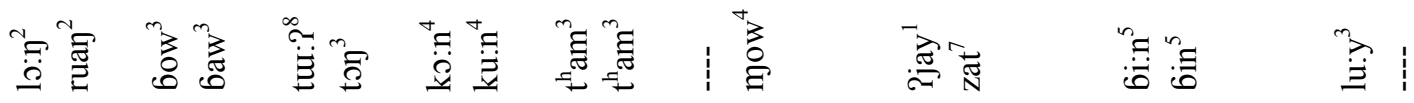

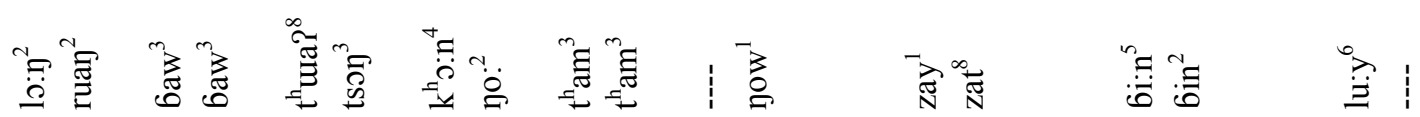

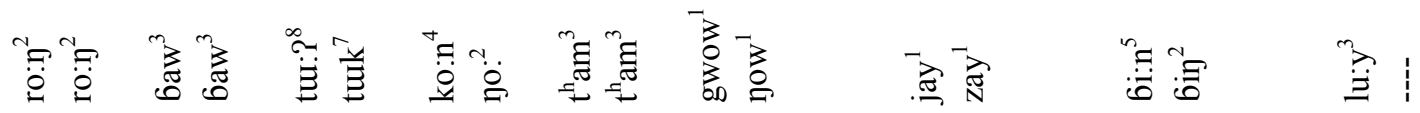

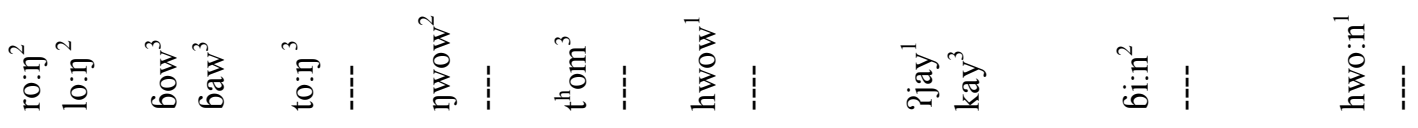

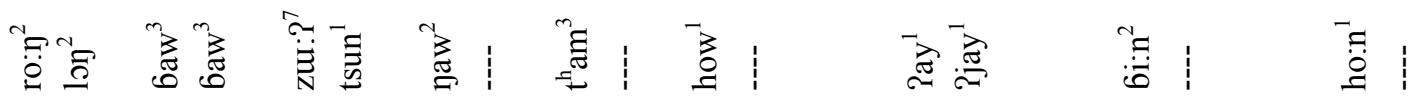

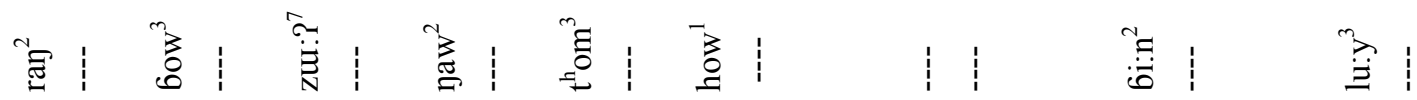

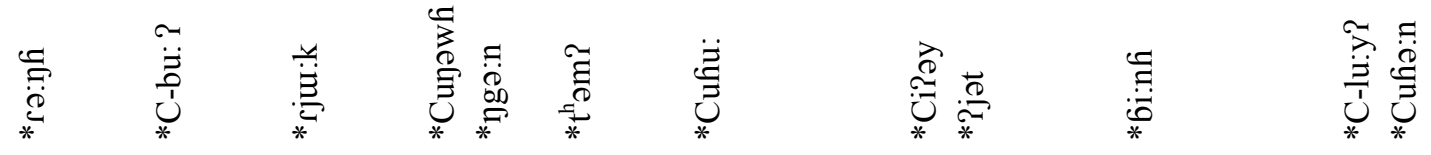

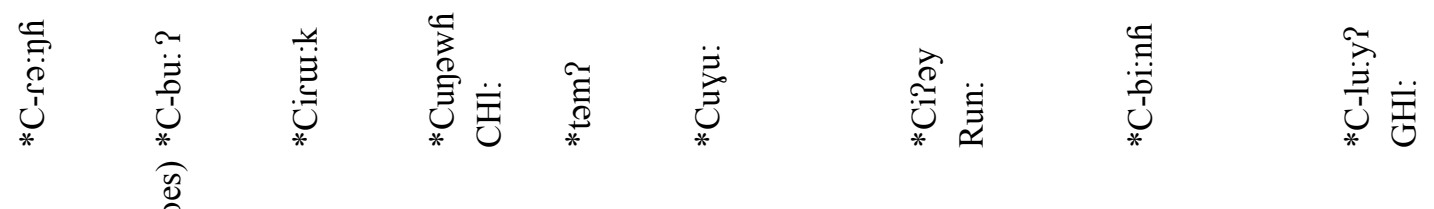

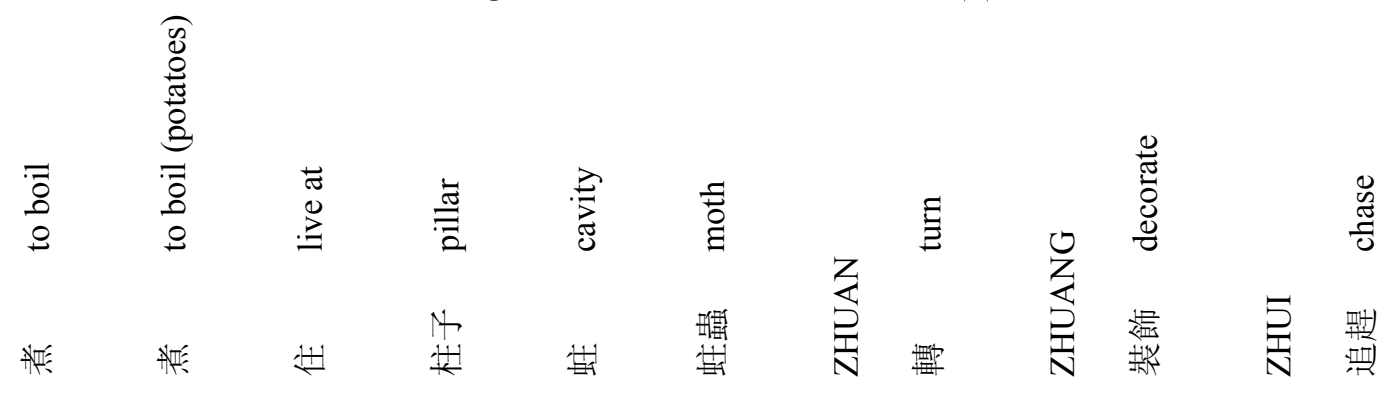




$$
\begin{aligned}
& 111110111
\end{aligned}
$$

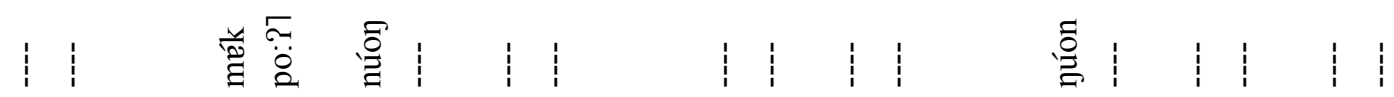

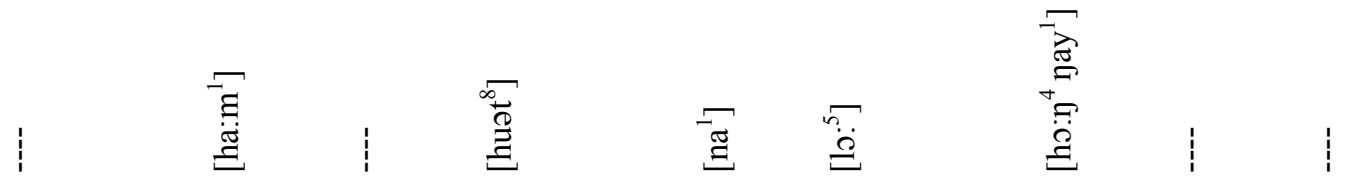

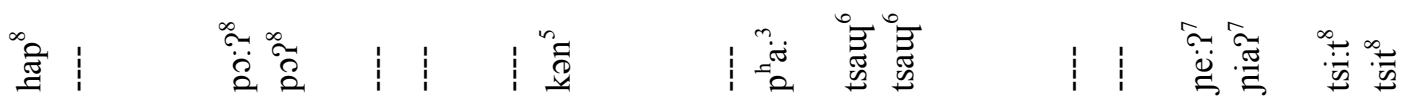

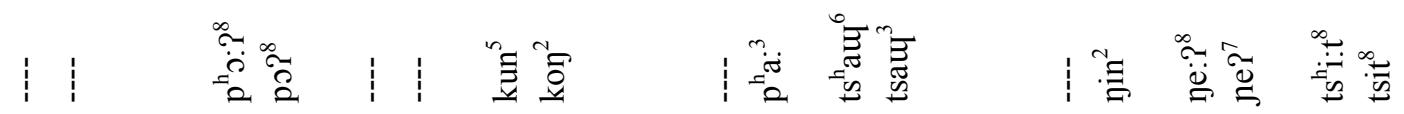

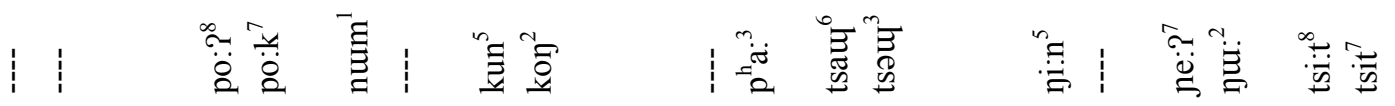

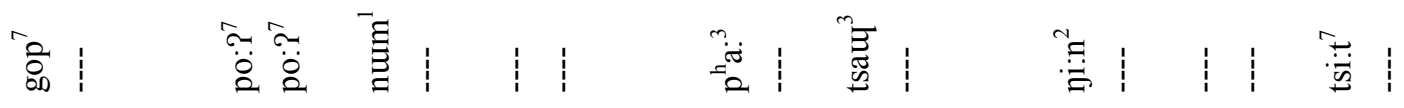

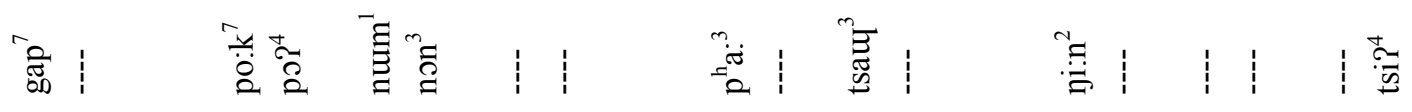

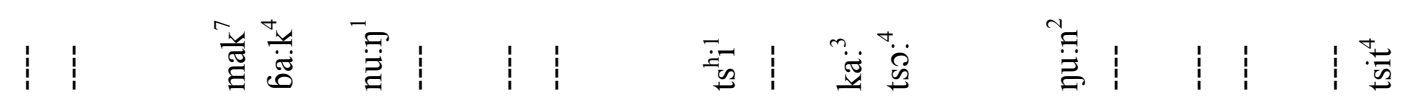

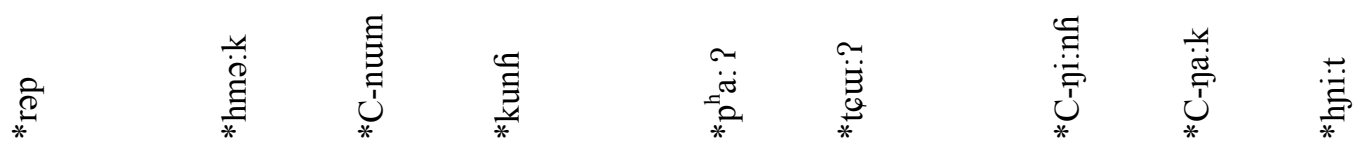

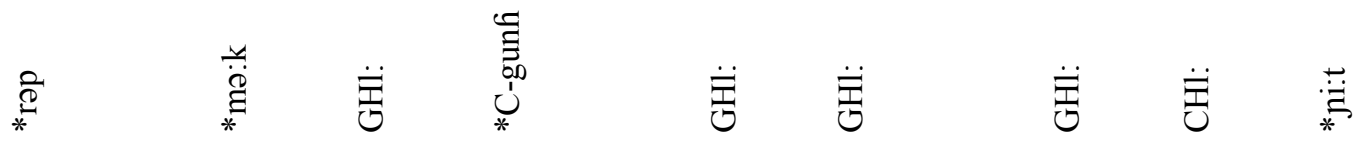

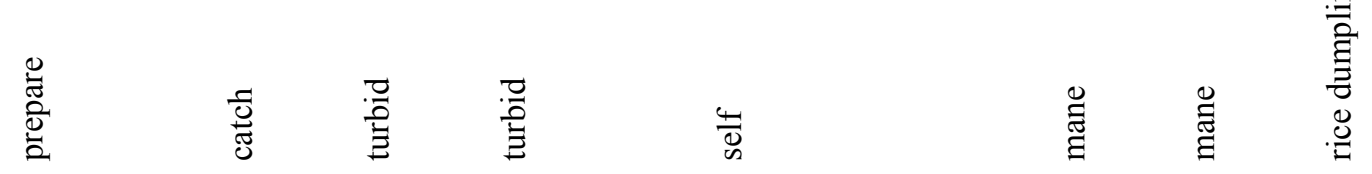

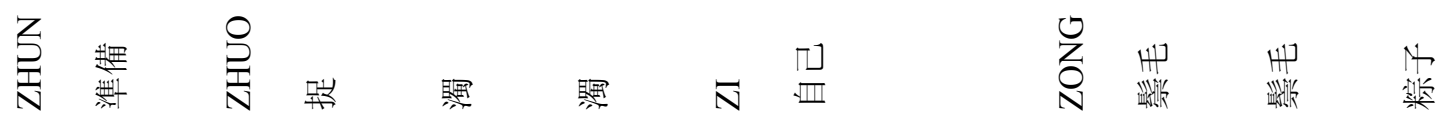




$$
\begin{aligned}
& 1+1+1 \quad 1 \quad 1
\end{aligned}
$$

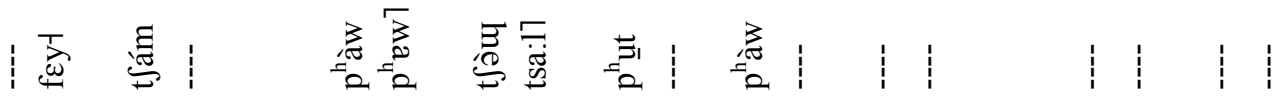

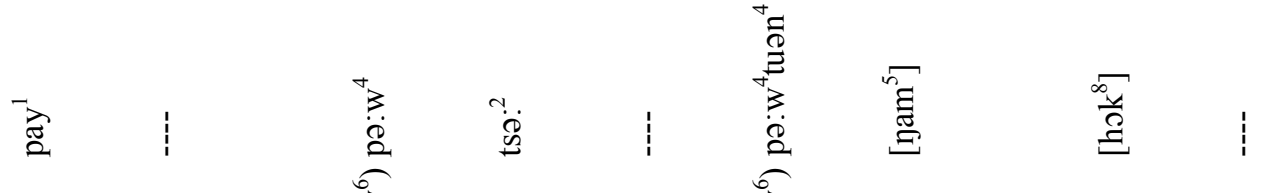

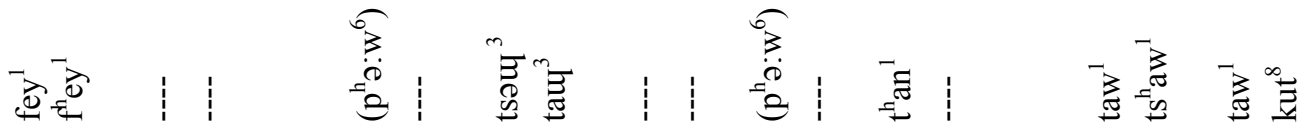

$$
\begin{aligned}
& \text { 氙离 } \\
& \text { 氙离 } 1 \text { | } \\
& \text { 氞怘 }
\end{aligned}
$$

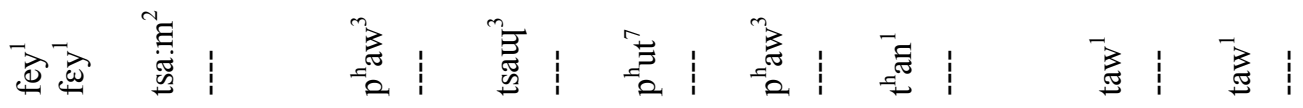

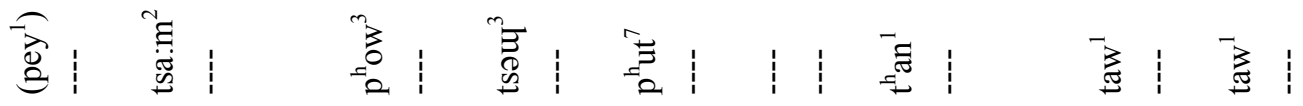

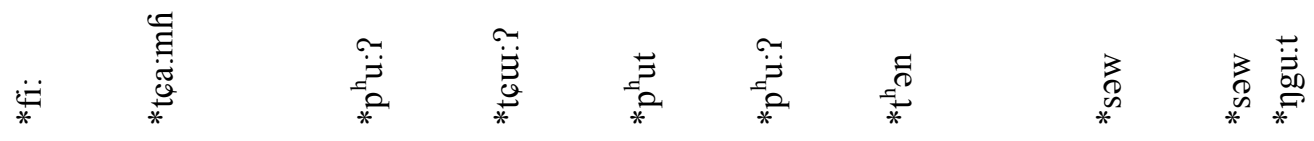

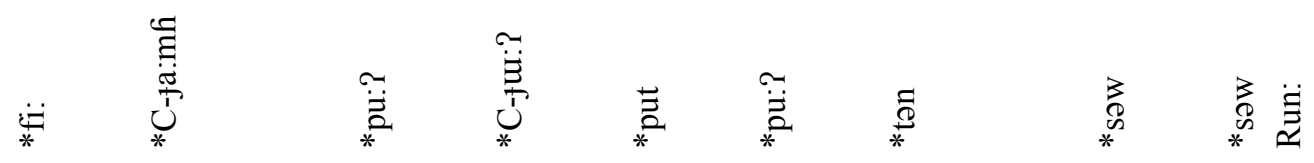

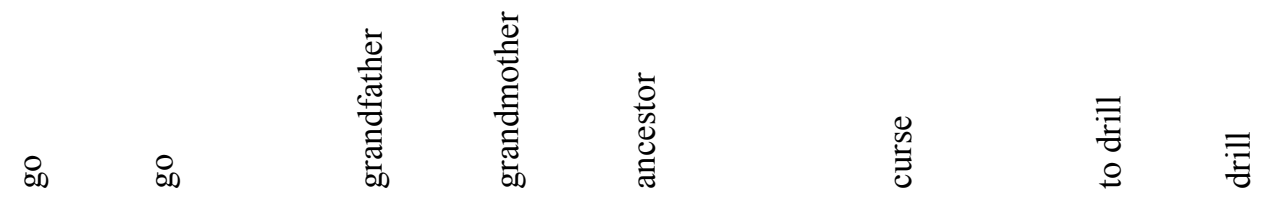

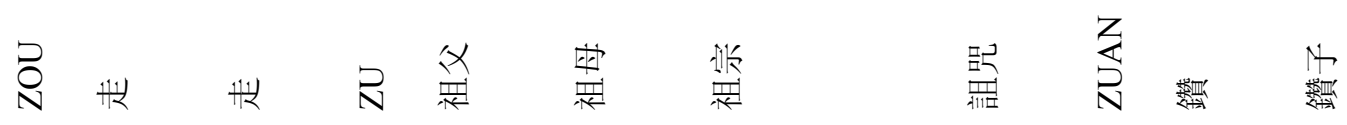




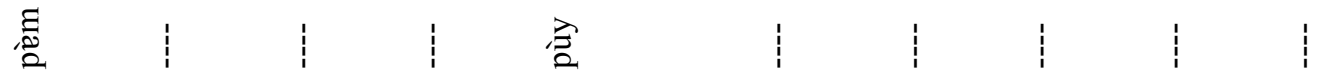

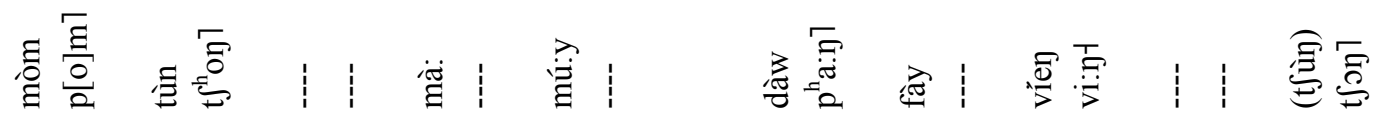

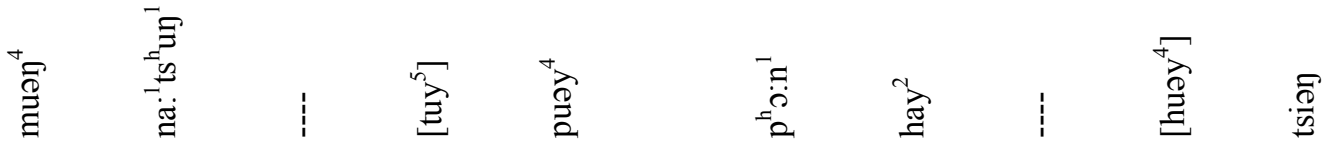

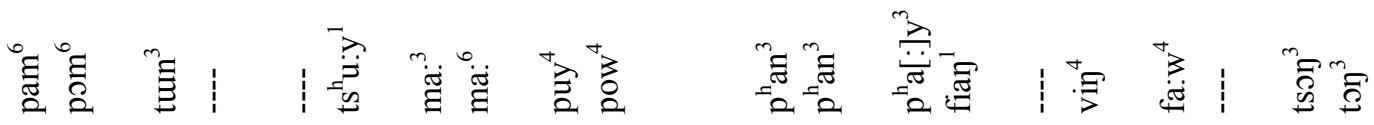

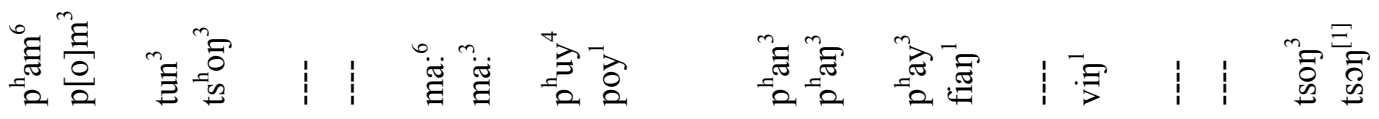

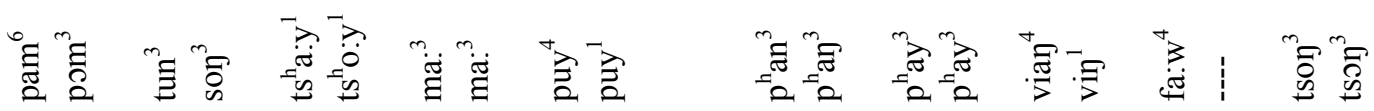

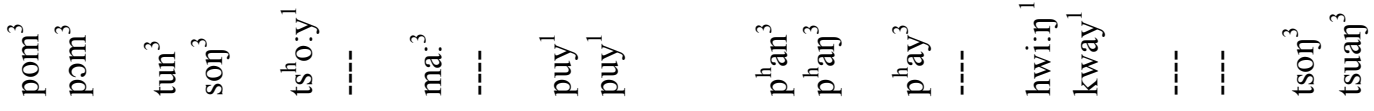

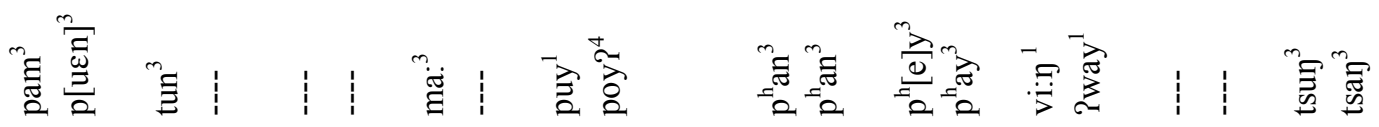

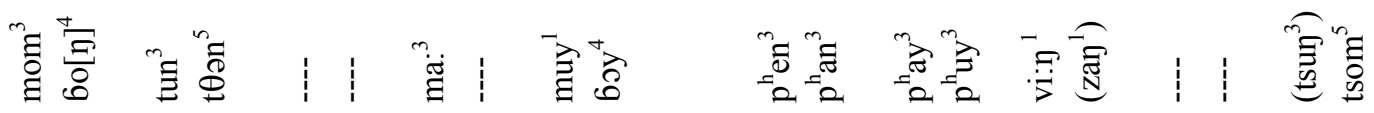

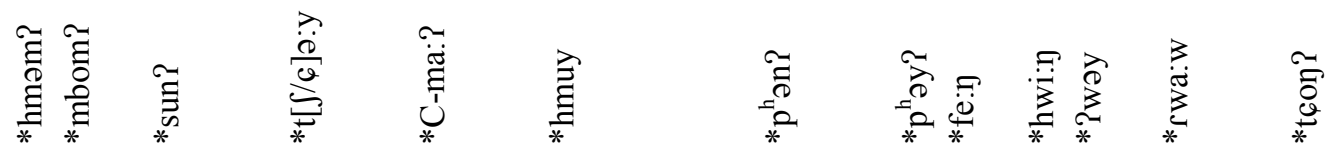

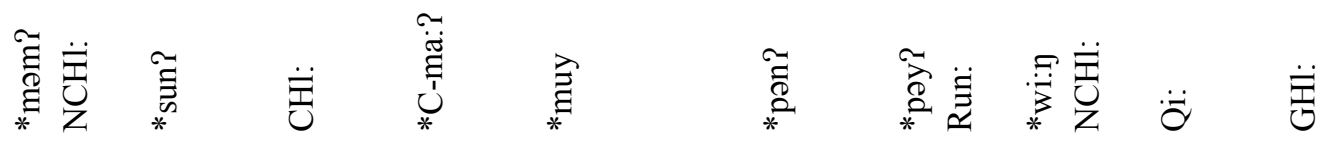

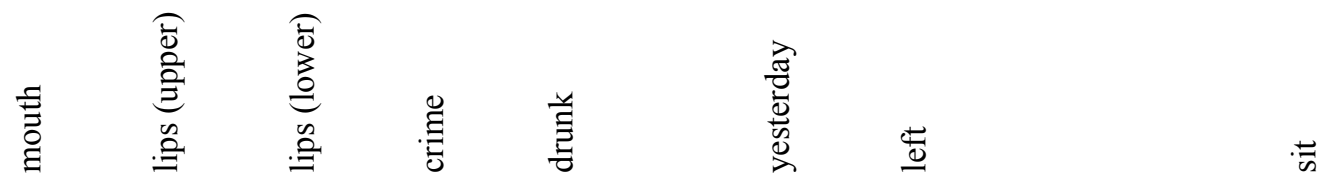

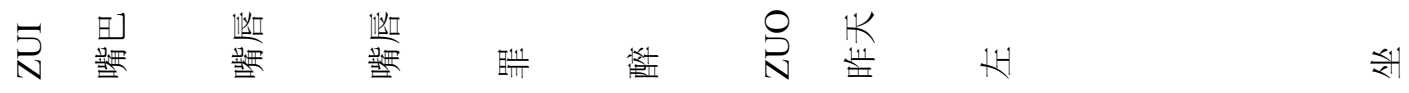


익

$\ddot{\vec{s}}$

ब

๙ิ อิ

$\stackrel{\infty}{0}$ 希

$\frac{n}{3} \frac{y}{3}$

拣

芦

苛

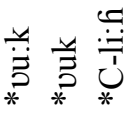

*

용

趧 


\section{References}

Austronesian basic vocabulary database. University of Auckland. http://language.psy.auckland.ac.nz/austronesian/

Benedict, Paul K. 1942. Thai, Kadai, and Indonesian: A new alignment in Southeastern Asia. American Anthropologist, n.s. 44: 576-601.

----. 1975. Austro-Thai: Language and culture with a glossary of roots. New Haven: HRAF Press.

Bhaskararao, P. \& Peter Ladefoged. 1991. Two types of voiceless nasals. Journal of the International Phonetic Association 21.2: 80-88.

Blevins, Juliette. 2004. Evolutionary phonology : the emergence of sound patterns. Cambridge; New York: Cambridge University Press.

Blust, Robert. 1999. Subgrouping, circularity, and extinction: Some issues in Austronesian comparative linguistics. In Selected Papers from the Eighth International Conference on Austronesian Linguistics. Edited by Elizabeth Zeitoun and Paul Jen-Kuei Li. Symposium series of the Institute of Linguistics no. 1. Taipei: Academica Sinica: 31-94.

Bradley, David. 1978. Proto-Loloish. London: Curzon Press.

Bradley, Travis G. and Ann Marie Delforge. 2006. Historical Romance Linguistics: Retrospectives and Perspectives. Edited by Deborah Arteaga and Randall Gess. Amsterdam: John Benjamins.

Bybee, Joan. 2001. Phonology and Language Use. Cambridge; New York : Cambridge University Press.

Campbell, Lyle. 2004. Historical Linguistics: An Introduction. Cambridge: The MIT Press.

Cho, Young-mee \& Tracy Holloway King. 1996. Semi-syllables and Georgian clusters. TREND VI, Stanford University.

----. 2003. Semi-syllables and universal syllabification. In The Syllable in Optimality Theory. Edited by Caroline Féry \& Ruben van de Vijver. Cambridge; New York : Cambridge University Press: 183-212.

Crothers, John. 1978. Typology and universals of vowel systems. In Greenberg, Joseph H., ed. 1978. Universals of human language 2: Phonology. Stanford: Stanford University Press. Pp. 93-152.

Edmondson, Jerold A. To appear 2007. The power of language over the past: Tai settlement and Tai linguistics in southern China and northern Vietnam. In Studies in Southeast Asian Linguistics. Edtied by J. G. Harris, S. Burusphat, and James E. Harris. Bangkok.

Edmondson, Jerold A., John H. Esling, Jimmy G. Harris, and James Wei. 2004. Sui phonetics. MonKhmer Studies XXXIV: 47-66.

Edmondson, Jerold A. \& David B Solnit, eds. 1988. Comparative Kadai: Linguistic Studies Beyond Tai. Arlington: Summer Institute of Linguistics, University of Texas.

----. 1997. Comparative Kadai: The Tai Branch. Arlington: Summer Institute of Linguistics, University of Texas. 
Enfield, N.J. 2005. Areal Linguistics and Mainland Southeast Asia. In Annual Review of Anthropology 34: 181-206.

Ferlus, Michel. 1992a. Histoire abregee de l'evolution des consonnes initiales du vietnamien. Mon-Khmer Studies XX: 111-126.

----. 1992b. Essai de phonetique historique du Khmer. Mon-Khmer Studies XXI: 57-90.

Fu Guangzhong. 1997. Hainan Cunhua. Guangzhou: Huanan Ligong Daxue Chubanshe.

Fu Zhennan. 1990. A dialect island of Li --- Natou hua. Minzu Yuwen 4: 14-18.

Gedney, William J. 1989. Selected Papers on Comparative Tai Studies. Ann Arbor: Central South and Southeastern Asian Studies, University of Michigan.

Handel, Zev. 1998. The Medial Systems of Old Chinese and Proto-Sino-Tibetan. Doctoral Dissertation, University of California, Berkeley.

----. 2003. Northern Min tone values and the reconstruction of "softened initials." Language and Linguistics 4.1: 47-84.

Hansell, Mark. 1988. The Relation of Be to Tai: Evidence from Tones and Initials. In Comparative Kadai: Linguistic studies beyond Tai. Edited by Jerold A. Edmondson and David B. Solnit. Summer Institute of Linguistics and The University of Texas at Arlington Publications in Linguistics No. 86: $239-288$.

Harris-Northall, Raymond. 1990. Weakening Processes in the History of Spanish Consonants. New York: Routledge.

Haudricourt, Andre G. 1984. La tonologie du Li de Hainan. Minzu Yuwen 4: 17-25.

----. 1989. La tonologie du Li de Hainan. In South-East Asian Linguistics: Essays in honour of Eugenie J. A. Henderson. Edited by Jeremy H. C. S. Davidson. University of London: School of Oriental and African Studies.

Hayes, Bruce. 1995. Metrical Stress Theory: Principles and Case Studies. University of Chicago Press.

Hayes, La Vaughn. 1983. The mutation of *R in Thavung. Mon-Khmer Studies XI: 83-100.

Honda, Koichi. 2005. Tone correspondences and tonogenesis in Vietic. In Papers from the 15th annual meeting of the Southeast Asian Linguistics Society (SEALS XV). Edited by Paul Sidwell. Canberra: Pacific Linguistics: 177-192.

Jonsson, Nanna L. 1991. Proto Southwestern Tai. Doctoral dissertation, State University of New York at Albany.

Kingston, John. 2005. The Phonetics of Athabaskan Tonogenesis. In Athabaskan Prosody. Edited by Sharon L. Hargus and Keren D. Rice. John Benjamins: 137-184.

Kiparsky, Paul. Syllables and Moras in Arabic. In The Syllable in Optimality Theory. Edited by Caroline Féry \& Ruben van de Vijver. Cambridge; New York : Cambridge University Press: 183-212. 
Kosaka, Ryuichi. 1996. On the genetic location of the Hlai language. Language, Area and Culture Studies No. 2. Graduate School, Tokyo University of Foreign Studies: 15-58.

Kwok, Bit-chee. 2006. The Role of Language Strata in Language Evolution: Three Hainan Min Dialects. Journal of Chinese Linguistics 34.2: 147-182.

Ladefoged, Peter \& Ian Maddieson. 1996. The Sounds of the World's Languages. Oxford: Blackwell Publishers, Ltd.

Lavoie, Lisa Marie. 1999. Phonological Patterns and Phonetic Manifestations of Consonant Weakening. PhD dissertation, Cornell University.

Li Fangkuei. 1977. A handbook of comparative Tai. Honolulu: The University Press of Hawaii.

Li Rong. 1998. Haikou fangyan cidian. Nanjing: Jiangsu Jiaoyu Chubanshe.

Liang Min \& Zhang Junru. 1996. Dongtaiyuzu Gailun. Beijing: Zhongguo Shehui Kexue Chubanshe.

Maddieson, Ian. 1984. Patterns of Sounds. Cambridge University Press.

Mathangwane, Joyce T. 1999. Ikalanga Phonetics and Phonology: A Synchronic and Diachronic Study. Stanford: CSLI Publications.

Matisoff, James A. 1973. 'Tonogenesis in Southeast Asia'. In Larry M. Hyman (ed.), Consonant types and tone (Southern California Occasional Papers in Linguistics 1), 71-95. Los Angeles: University of Southern California.

----. 1975. Rhinoglottophilia: the mysterious connection between nasality and glottality. In Nasalfest: Papers from a Symposium on Nasals and Nasalization. Edited by Charles Ferguson, Larry M. Hyman, and John Ohala. Stanford University Language Universals Project. Stanford, California: 265-87.

----. 1988. Proto-Hlai initials and tones: a first approximation. In Comparative Kadai: Linguistic studies beyond Tai. Edited by Jerold A. Edmondson and David B. Solnit. Summer Institute of Linguistics and The University of Texas at Arlington Publications in Linguistics No. 86: 289-321.

Mortensen, David. 2006. Tonally Conditioned Vowel Raising in Shuijingping Hmong. Talk delivered at the $80^{\text {th }}$ annual meeting of the LSA, Albequerque, New Mexico, on January $6^{\text {th }}$.

Myers, James, and Jane Tsay. 2003. A formal functional model of tone. Language and Linguistics 4: 105138.

Norman, Jerry. 1969. The Kienyang Dialect of Fukien. Ph.D. Dissertation: University of California, Berkeley.

----. 1986. The origins of the Proto-Min softened stops. Contributions to Sino-Tibetan studies. Edited by John McCoy and Timothy Light. Cornell Linguistic Contributions 5. Leiden: E. J. Brill: 375-384.

Ostapirat, Weera. 1993a. Proto-Hlai Vowel System. Masters thesis, Mahidol University.

----. 1993b. Evidences of Hlai dental and velar clusters from Savina’s Day variety. Mon-Khmer Studies XXIII: $133-137$. 
----. 1996. Kadai dummy *-u. Mon-Khmer Studies XXVI: 79-89.

----. 1999. Proto-Kra. Doctoral dissertation, University of Michigan.

----. 2004. Proto-Hlai Sound System and Lexicons. In Studies on Sino-Tibetan Languages: Papers in Honor of Professor Hwang-cherng Gong on His Seventieth Birthday. Edited by Ying-chin Lin, Fang-min Hsu, Chun-chih Lee, Jackson T.-S. Sun, Hsiu-fang Yang, and Dah-an Ho. Institute of Linguistics. Academia Sinica, Taipei, Taiwan: 121-175.

----. 2005. Kra-Dai and Austronesian: notes on phonological correspondences and vocabulary distribution. In The Peopling of East Asia: Putting together archaeology, linguistics and genetics. Edited by Laurent Sagart, Roger Blench, and Alicia Sanchez-Mazas. New York: RoutledgeCurzon: 107-131.

Ouyang Jueya. 1992. Li-Han Cidian. Chengdu: Sichuan Minzu Chubanshe.

----. 1998. Cunhua. Zhongguo xinfaxian yuyan yanjiu congshu. Shanghai: Shanghai Yuandong Chubanshe.

Ouyang Jueya \& Fu Zhennan. 1988. On the Issues of the Genetic Classification of Cunhua on Hainan Island. Minzu Yuwen 1: 8-17.

Ouyang Jueya \& Zheng Yiqing. 1980. A brief description of Li (Hainan). Chinese Minority People’s Language, basic description series. Beijing.

----. 1983. Liyu diaocha yanjiu. Beijing: Zhongguo Shehui Kexue Chubanshe: Xinhua shudian Beijing faxingsuo faxing.

Peiros, Ilia. 1998. Comparative Linguistics in Southeast Asia. Canberra: Pacific Linguistics series C-142.

Pittayaporn, Pittayawat. 2007. Directionality of Tone Change. In Proceedings of the ICPhS XVI, August 6-10, Saarbrucken, Germany.

----. Reconstruction of Southwest Tai. Ms.

Prince, Alan. 1990. Quantitative consequences of rhythmic organization. Chicago Linguistic Society, 26.2: 355-98.

Savina. Francois M. 1931. Lexique Dai-Francais accompagne d'un petit lexique Francais-Day et d'un tableau des differences dialects. Bulletin de l'ecole Francais d'Extreme-Orient 31: 103-199. Schuessler, Axel. 2007. ABC Etymological Dictionary of Old Chinese. Honolulu: University of Hawai'i Press.

Shafer, Robert. 1957. Quelque equations phonetiques pour les langues li d'Hainan. Rocznik Orientalistyczny 21: 385-407.

Shaw, Patricia. 1993. The prosodic constituency of minor syllables. WCCFL 12: 117-132.

Shearer, Walter \& Sun Hongkai. 2002. Speakers of the non-Han dialects of China. Chinese studies vol. 20. Lewiston: The Edwin Mellen Press.

Shintani, Tadahiko L. A. 1991. Preglottalized Consonants in the Languages of Hainan Island, China. Journal of Asian and African Studies 41: 1-10. 
Sidwell, Paul. 2000. Proto South Bahnaric: a reconstruction of a Mon-Khmer language of Indo-China. Canberra: Pacific Linguistics 501.

Sidwell, Paul \& Pascale Jacq. A Handbook of Comparative Bahnaric: volume 1 - West Bahnaric. Canberra: Pacific Linguistics 551.

Smith, Norval. 1997. Shrinking and Hopping Vowels in Northern Cape York: Minimally Different Systems. In Variation, Change, and Phonological Theory. Edited by Hinskens, Frans, Roeland van Hout and W. Leo Wetzels. Amsterdam: John Benjamins: 267-302.

Solnit, David B. 1982. Linguistic Contact in ancient Southern China: the case of Hainan Chinese, Be and Vietnamese. Proceedings of the eighth annual meeting of the Berkeley Linguistics Society: 13-15 February, 1982. Edited by Macaulay, Monica, Orin Gensler, Claudia Brugman, Inese Civkulis, Amy Dahlstrom, Katherine Krile, and Rob Sturm.

----. 1988. The Position of Lakkia within Kadai. In Comparative Kadai: Linguistic studies beyond Tai. Edited by Jerold A. Edmondson and David B. Solnit. Summer Institute of Linguistics and The University of Texas at Arlington Publications in Linguistics No. 86: 219-238.

Svantesson, Jan-Olof. 1983. Kammu phonology and morphology. Lund: Liber Förlag.

Theraphan, L.-Thongkum. 1992. A preliminary reconstruction of Proto-Lakkja (Cha Shan Yao). MonKhmer Studies, 20: 57-90.

Thompson, Laurence C. 1976. Proto-Viet-Muong Phonology. In Austroasiatic Studies: Part II. Edited by Philip N. Jenner, Laurence C. Thompson, \& Stanley Starosta. Honolulu: The University of Hawai'i Press.

Thurgood, Graham. 1988a. Notes on the reconstruction of Kam-Sui. In Comparative Kadai: Linguistic studies beyond Tai. Edited by Jerold A. Edmondson and David B. Solnit. Summer Institute of Linguistics and The University of Texas at Arlington Publications in Linguistics No. 86: 179-218.

----. 1988b. K-prefixes in Kam-Sui and Kadai: some notes. In Languages and History in East Asia: Festschrift for Tatsuo Nishida on the Occasion of his 60th Birthday. Edited by Paul K. Eguchi, Yukio Fujimoto, Nobuyoshi Fukuhara, Masura Hashimoto, Koichi Miyamoto, Atsuchi Iwamoto, Tatsuo Kondo, Masaoki Miyamoto, Osamu Sakiyama, Akihiro Sato, David Sell, Norio Shibata, Ken-ichiro Shirai, Mashiro Shogaito, Shiro Yabu and Kazuhiko Yoshida. Kyoto: Shokado: 229235.

----. 1991. Proto-Hlai (Li): a look at the initials, tones, and finals. In Kadai: Discussions in Kadai and SE Asian Linguistics III: 1-49.

----. 1992. The aberrancy of the Jiamao dialect of Hlai: speculation on its origins and history. In Papers from the first annual meeting of the Southeast Asian Linguistics Society. Edited by Martha Ratliff and Eric Schiller. Arizona State University Program for Southeast Asian Studies: 417-433.

----. 1994. Tai-Kadai and Austronesian: The nature of the Historical Relationship. Oceanic Linguistics 33.2: $346-368$.

---- 1999. From ancient Cham to modern dialects: two thousand years of language contact and change. Honolulu: The University of Hawaii Press. 
----. 2002. Vietnamese and tonogenesis: revising the model and the analysis. Diachronica 19.2: 333-363.

Wang Fushi \& Mao Zhongwu. 1995. Miao-Yao-Yu Guin Goutz. Zhongguo Shehui Kexue Chubanshe. Beijing.

Wang Li \& Qian Sun. 1951. First steps in the White Sand Li language of Hainan . Lingnan Science Journal 2.11: 253-300.

Weidert, Alfons. 1987. Tibeto-Burman Tonology. Amsterdam: John Benjamins.

Yip, Moira. 2002. Tone. Cambridge: Cambridge University Press.

Zheng, Yiqing \& Ouyang Jueya. 1993. Li Han cidian. Chengdu: Sichuan Minzu Chubanshe.

Zorc, R. David. 1995. A glossary of Austronesian reconstructions. In Comparative Austronesian dictionary: an introduction to Austronesian studies. Edited by Darrell T. Tryon. New York: Mouton de Gruyter. Part 1: Fascicle 2: 1106-1197. 